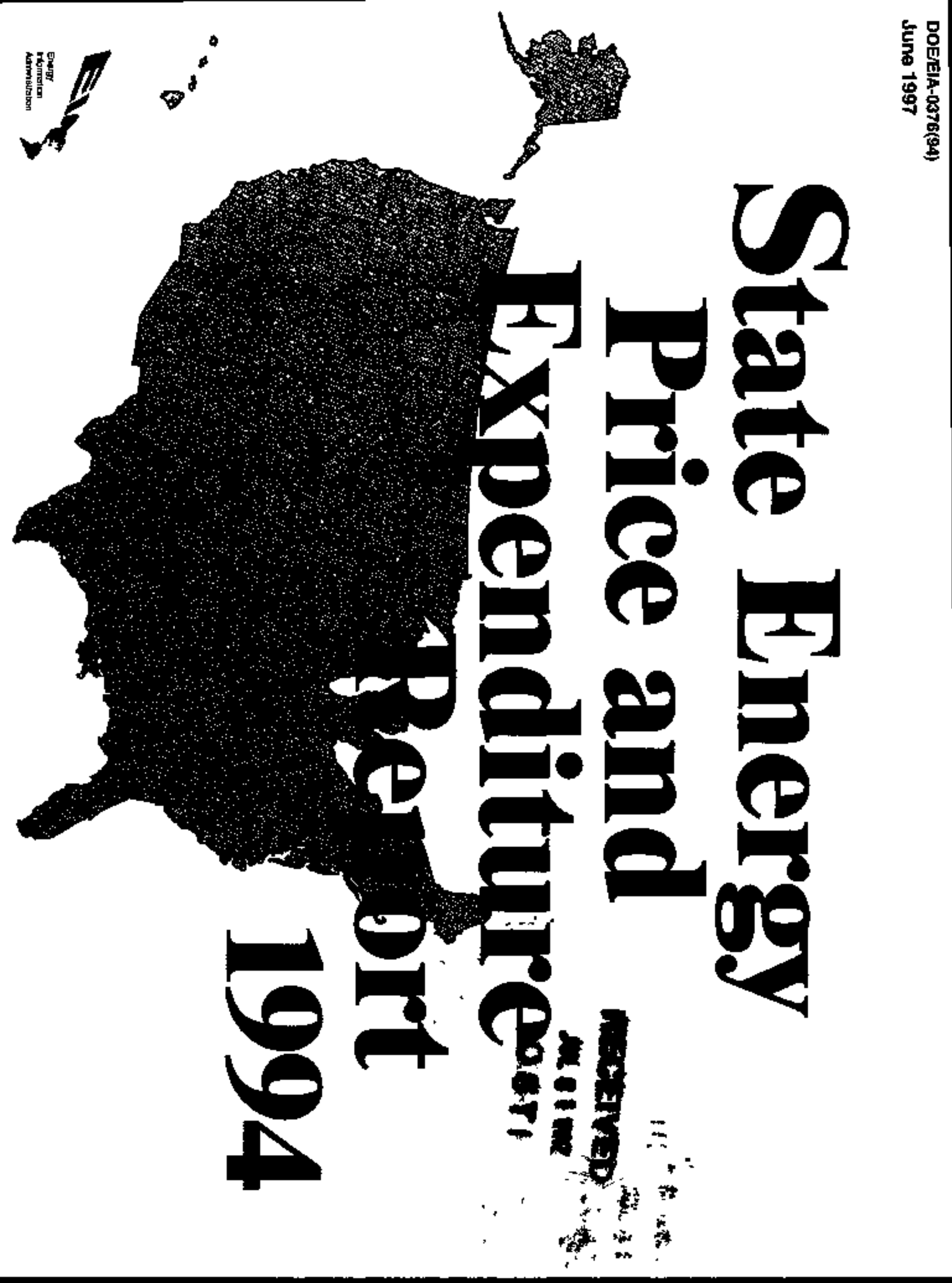




\section{State Energy Price and Expenditure Aeport 1994}

The State Energy Price and Expenditure Report 1994 presents energy price and expenditure estimates for the 50 States, the District of Columbia, and the United States for 1970 through 1994. The estimateg are presented by energy source ( $t . g_{r}$ petroleum, natural gas, coal, and electricity) and by major consuming sector. This publication is an enhanced update of the State Energy Price and Expenditure Report 1993 (published December 1995).

Publication of this report is in keeping with responsibilities given the Energy Information Administration (ElA) in Public Law 95-91 (Department of Energy Organization Act), which states, in part, in Section 205(a)(2) that:

The Admintistrator shall be respongible for carrying out a central, comprehensive, atd ustified erergy data and information program which will collect, evaluate, assemble, analyze and disserrinate data and linformatton....

The State Energy Price and Expenditure Report is a part of EIA's historical in tegrated energy data program and is intended to provide historical energy information to Members of Cougress. Federal and State agencies, and the general public.

\section{Contacts}

The Stute Energy Price and Expenditure Repon is prepared by the Energy Information Administration under the direction of W. Calvin Kilgore, Dírector of the Office of Energy Markets and End Use, 202-586-1617, and Katherine E. Seiferlein, Chief of the Integrated Statistics Branch, 202-586-5692.

Questions concerning the contents of the State Energy Price and Expenditure Repart may be referted to:

$\begin{array}{llc}\text { Julia Hutchins } & 202-586-5138 & \text { jbutchin@.eia.doe.gov } \\ \text { Thomas Leckey } & 202-586-9413 & \text { teckey@eia.doe.gov } \\ \text { Roy Stanley } & 202-586-5839 & \text { rstanley eia.doe.gov } \\ \text { The Division fax number is 202-586-0018. }\end{array}$

Released for printing: June 17,1997 GPO Stock No: 061-003-00995-6

Pronted with soy ank on recroded papes

\section{Ordering Information}

This and other EIA publications may be purchased from the Superintendent of Documents, U.S. Government Printing Office. An order form for this publication is provided in the back of this report. Orders may be directed to:

Superintendent of Documetsts

U.S. Government Pinting Office

P.O. Box 371954

Pittsburgh, PA 15250-7954

$202-512-1800$

Fax: 202-512-2250

8 a.m. to $4: 30$ p.m., ezstem time, M-F

Complimentary subscriptions and single issues are evailable to certain groups of subscribers, such as public and acaitemic libraries; Federal, State, local, and foreign governments; ElA survey respondents; and the media. For further information and for answers to questions on energy statistics, please contact EIA's National Energy lnformation Center at:

National Energy Information Center, El-231

Energy Information Administration

Forrestal Building, Room if-048

Washingtor, DC 20585

202-586-8800

Internet E-Mail: infocte@eia,doe,gov

Fax: 202-586-0727

TTY: For people who are deaf or hard of hearing: 202-586-1181

9 a.m. to 5 p.m., eastern time, M-F

\section{Electronic Access}

The Stase Energy Price and Expenditure Report and data are available electronically on PC diskettes and at the Internet sites listed below. (See additional information on the inside back cover of this report.)

World Wide Web: http:/www.eia.doe.gov

Gopher Site: $\quad$ gopher.//gopher.tia,doe.gov

FTP Sile: $\quad$ frp://tip.ejadoe.gov

The teport and data are also available on EIA's CD-ROM, the Energy InfoDisc. See the ad and order form on the last page of this report. 


\section{DHSCLAAMER}

Portions of this document may be lllegible in electronic image produets. Images are produced from the best aroliable origtal document. 


\title{
State Energy Price and Expenditure Report
}

\section{4}

\author{
June 1997
}

Energy Information Admintiatration

Office of Energy Markets and End Use

U.S. Department of Energy

Washington, DC 20585

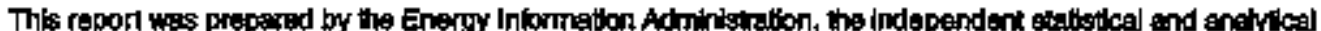
agancy wtthin the Department of Enorgy. The information contsined herein should not be construed as edroceting or rellecting any pollcy posstion of the Department of Energy or any other organtistion.

\section{MASTER}




\section{Contents}

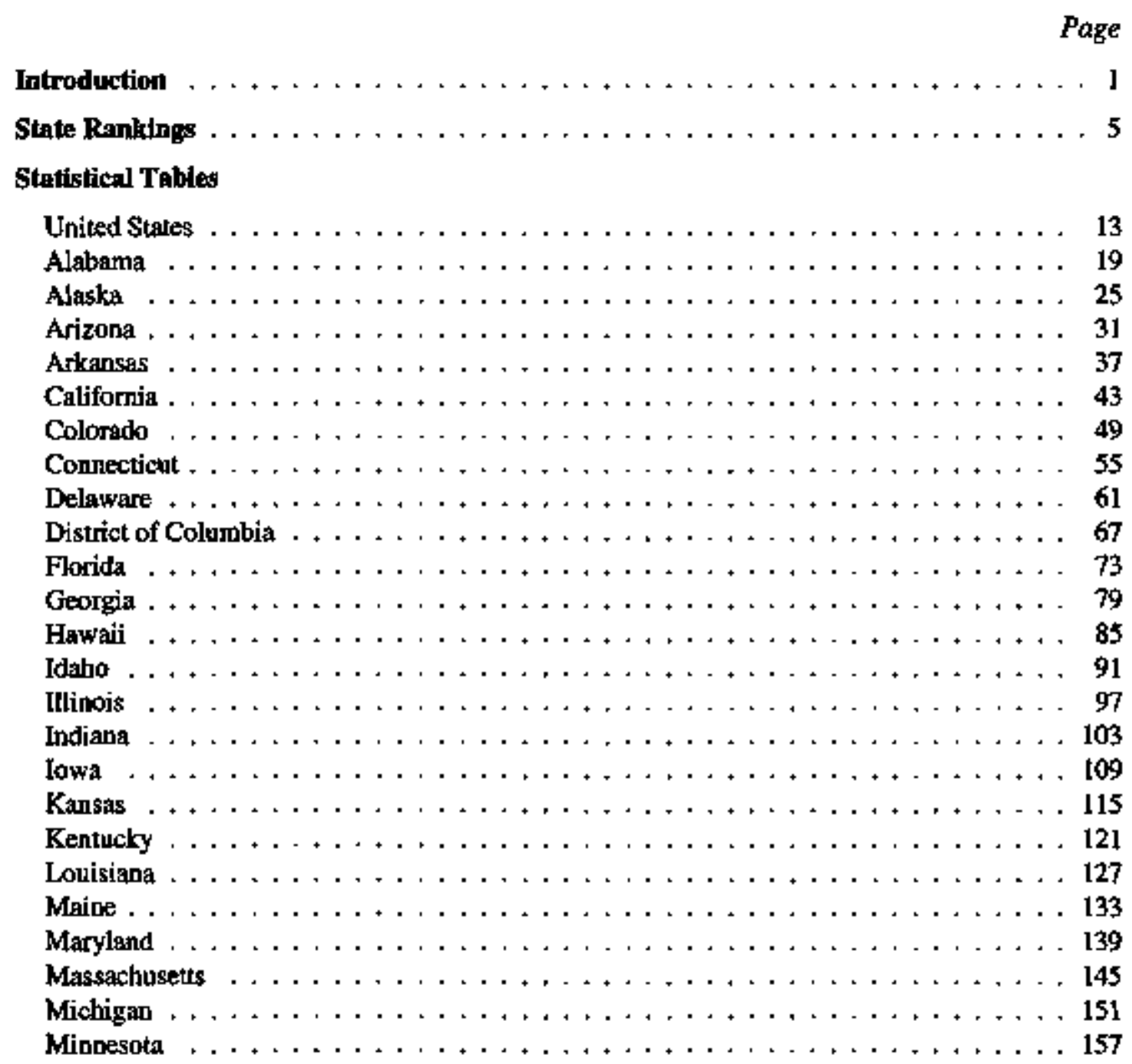

Energy Information Adminlatretion 
Statistical Tahles (continued)

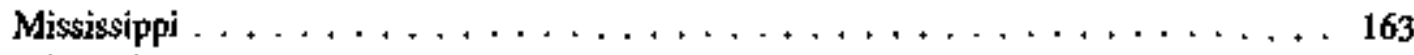

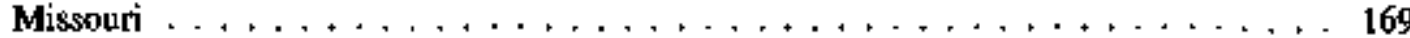

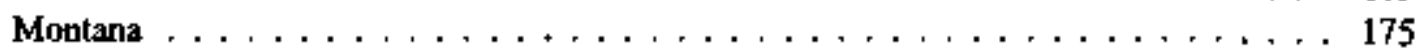

Nebraska . . . . . . . . . . . . . . . . . . . . . . . . . . I81

Nevada . . . . . . . . . . . . . . . . . . . . . .

New Hampshire $\ldots \ldots \ldots \ldots \ldots \ldots$

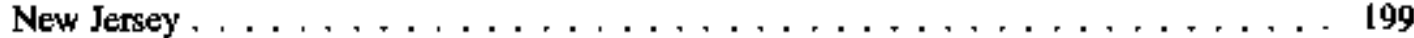

New Mexico . . . . . . . . . . . . . . . . . . . . . . . . . . . 205

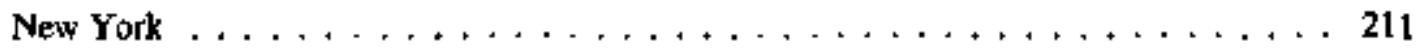

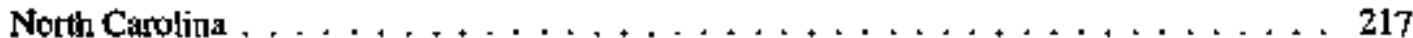

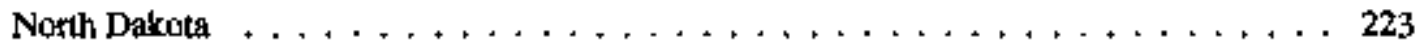

Ohio . . . . . . . . . . . . . . . . . . . . . . . . . . 229

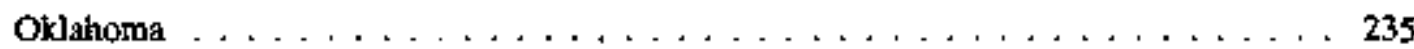

Oregon . . . . . . . . . . . . . . . . . . . . 241

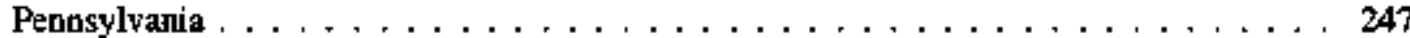

Rhode Island . . . . . . . . . . . . . . . . . . . . . . . 253

South Carolina . . . . . . . . . . . . . . . . . . . . . 259

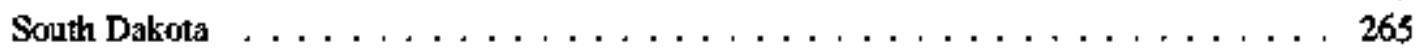

Tennessee . . . . . . . . . . . . . . . . . . . 271

Texas . . . . . . . . . . . . . . . . 277

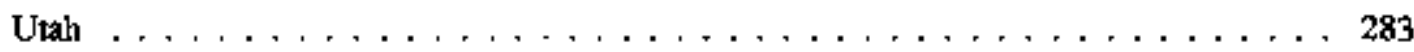

Vermont . . . . . . . . . . . . . . . . . . . . 289

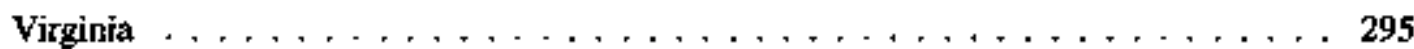

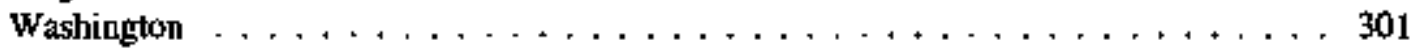

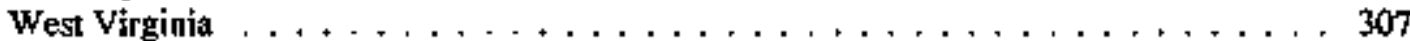

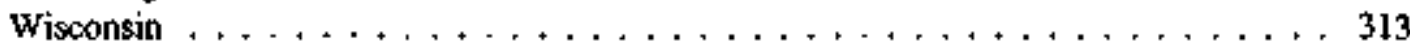

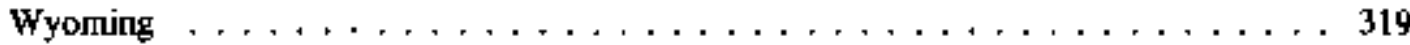

\section{Appendices}

A. Documentation . . . . . . . . . . . . . . . . . . . . 327

Section 1. Overview . . . . . . . . . . . . . . . 327

Section 2. Coal . . . . . . . . . . . . . . . . . . . . . 333

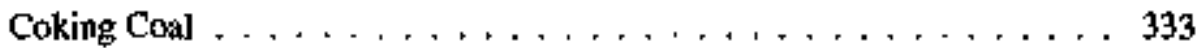

Steam Coal . . . . . . . . . . . . . . . . . . . . . 334

Coal Coke, Imports and Exports $\ldots \ldots \ldots \ldots \ldots \ldots \ldots \ldots$

Section 3. Natural Gas $\ldots \ldots \ldots \ldots \ldots \ldots$ 
Appendices (Continued)

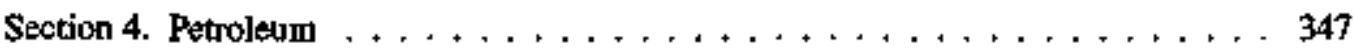

Asphalt and Road $\mathrm{Oil} \ldots \ldots \ldots \ldots \ldots \ldots \ldots \ldots \ldots$

Aviation Gasoline . . . . . . . . . . . . . . . . 348

Distillate Fuet $\ldots \ldots \ldots \ldots \ldots \ldots \ldots \ldots$

Heavy Oil (Utilities) . . . . . . . . . . . . . . . . . 363

Jet Fuel . . . . . . . . . . . . . . . . . . . 363

Kerosene . . . . . . . . . . . . . . . . 366

Light Oil (Utilities) . . . . . . . . . . . . . . . . . . . . . 369

Liquefied Petrolentm Gases . . . . . . . . . . . . . . . . 369

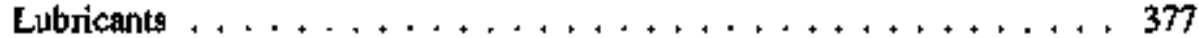

Motcr Gasoline . . . . . . . . . . . . . . . 378

Petroleum Coke (Utilities) $\ldots \ldots \ldots \ldots \ldots \ldots \ldots \ldots$

Residual Fuel $\ldots \ldots \ldots . . . \ldots \ldots$

Other Petroleam . . . . . . . . . . . . . . . 397

Section 5. Renewable Energy . . . . . . . . . . . . . . . . . 405

Section 6. Electricity . . . . . . . . . . . . . . . . . . . . . . 409

Electricity Consumed by End-Use Sectors . . . . . . . . . . 409

Nuckear Fuel for Generation of Electricity . . . . . . . . . . . 411

Section 7. Consumption Adjustments for Calculating Expenditures . . . . . . . . 413

B. Metric and Other Physical Conversion Factors . . . . . . . . . . . . . . 421

C. Summary of Changes Since the Stase Energy Price and Expenditure Report 1993 . . . 427

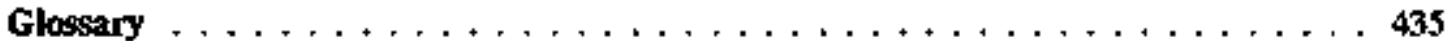




\section{Introduction}

The Stale Exergy Price and Expenditure Report (SEPER) presents energy price and expenditure estimates individually for the 50 States and the District of Columbia and in aggregate for the United States. The price and expenditure estimates developed in the State Energy Price and Expenditure Data System (SEPEDS) are provided by energy source and economic sector and are published for the years 1970 through 1994. Data for all years are available on personal computer diskettes, CDROM, and vis Internet. (See inside front and back covers.)

Consumption estimates used to calculate expenditures and the documentation for those estimates are taken from the State Energy Data Repors 1994, Consumption Estimaves (SEDR), published in October 1996. Expenditures are calculated by multiplying the price estimates by the constumption estimates, which are adjusted to remove process fuel; intermediate petroleum products; and other consumption that bas no direct fuel costs, i.e., bydroelectric, geothermal, wind, solar, and photovoltaic energy sources. See Section 7. "Consumption Adjustments for Calculating Expenditures," on page 413.

All expenditures are consumer expenditures; that is, thay represent estimates of money spent directly by consumers to purchase energy, generally including taxes. (See box on page 2.)

The documentation in Appendix A describes bow the price estimates are developed, including sources of data, methods of estimation, and conversion factors applied. Appendix B provides metric and other physical conversion factors for measures used in energy analyses.

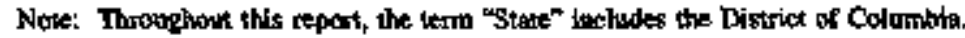

This report contains significant revisions since the last edition, the Stote Energy Price and Expenditure Report 1993, published in December 1995. The format of SEPER is completely redesigned to closely resernble $\$ E D R^{\prime} s$ format. Rotating the tables and doubling their number for each State makes it possible to present prices and expenditures for all years. Additional prices and expenditures for biofuels used in the residential (including coramercial) and the industrial sectors are added for 1990 through 1994. Distiltate fuel prices for the commercial and industrial sectors are improved for 1983 through 1994 by replacing the No. 2 distillate fuel oil price series, which was used in previous versions of SEPEDS, with a series that is an average price of all forms of distillate fuel. Data changes from the last report are summarized and explajned in detail in Appendix $\mathrm{C}$.

Although all prices and expenditures in this sepost are expressed in nominal doliars, a supplement to the 1993 data report entitled "Addjtional Measures of Energy Consumption, Expenditures, and Prices," provides energy expenditure estimates in constant 1992 dollars and fixed-wejght energy price indices in both nominal and real terms, along with an explanation of the methodology used to develop them. This supplement is available via Internet and in hard-copy from the National Energy Information Center. (See inside front cover.)

Reliable data for State-level prices rarely exist, especially as series that are consistent over a long period. Estimates and assumptions are applied to fill data gaps and to maintain consistent definitions in the data series over time. SEPEDS incorporates the most consistent series and procodures possible. Users of this report (and the electronic data files) should recognize the limitations imposed on the system due to changing and inadequate data sources. Estimates often are based on a variety of surrogate measures that are selected on the basis of availability, 
applicability as indicators, continuity over time, and consistency among the various energy commodities. Original source documents for data used in SEPEDS (cited in the SEPER documentation) include descriptions of collection methodologies, universes, imputation or adjustment techniques (if any), and errors associated with the individual processes. Due to the numerous collection forms and procedures associated with these reports, it is not possible to develop a mesningful numerical estimate of the overall errors of the enaterial published in SEPER.

\section{Taxes in SEPER}

While the objective is to provide price estimates that include all taxes. tho data sources used in SEPER do aot treat taxes iniformly. In general, where taxes are included in the source data, they are also included in SEPER. Where taxes are not included but can be separately estimated, they are generally added. In many cases, States and even some localities provide tax exemptions for various kinds of activities or classes of end users, and readers of this report shoutd be careful to consider these exceptions when making detailed comparisons. The Energy Information Administration (ELA) is continuing to analyze these cases to see if a better tepresentation can be made. A comprehensive and detailed study of taxes in EIA data is available in the report End-Use Tates: Current ElA Practices, DOEJEJA-0583 (Washizgton, DC, August 1994). The status of tax data in this edition of SEPER is summarized below and described more fully in the Appendix A documentation for each energy source and sector.

\section{End-Use Septors}

Coal. All steam coal ano coking coal prices include taxes in all years. Appropriately, coal imports and exports in the industrial sector do not include end-user taxes.

Natural Gas. Natural gas prices are intended to include all Federal, State, and local taxes, surcharges, and adjustments billed to consumers. However, sales and other taxes itemized directiy on customers' bills are frequently not reported as revenues and, therefore, are not included in calculating the prices.

Petroleum. Motor gasoline and diesel fuel prices include excise and other per-gallon taxes but do not include general sales taxes due to wide variation at the local level. Residual fuel oil, distillate fivel oil, and kerosene prices include sales taxes in all years. Residential liquefied petroleum gas prices include taxes in all years, but commercial, industrial, and transportation sector prices do not include taxes from 1985 forward. Jet fuel, aviation gasoline, asphalt and road oil, 1tbricants and other petroleum products do not include taxes. Other petroleum products are miscellaneous products, petrochemical feedstocks (naphtha, other oils, and still gas), industrial petrolenm coke, special naphthas, and waxes.

Biofuels. Biofuels prices for the residential (including commercial) and the industrial sectors include taxes.

Electrleity. Tares paid directly by electric utilities (rather than end nsers) are considered operating costs and are passed on to the end user's as part of the price. Depending on jurisdiction, taxes collected from end users and tumed over directly to govemment authority are not generally included in the reported rovenues and, therefore, are not included in calculating the prices.

\section{Electric Utitty Sector}

Coal, natural gas, petroleum coke, nuckear, and biomass fuels prices include atl taxes, transportation, and handling costs. There aro no direct fuel costs (or taxes) for bydroelectric, geothermal, centralized solar, or wind energy, Capital, operation, and maintenance costs and related taxes associated with these energy sources are incinded indirectly because electricity prices reflect their presence in the rate base. 
It is also important to note that, even within a State, a singte avesage price may bave limited meaning in that it represents a consumption-weighted average over a whole \$tate. For example, urtan and rural electricity prices can vary significantly from a State's weighted average, and prices in one region of a State may differ from those in another because of access to less expensive hydroelectricity. Diffetences within a State may be greater than differences among adjacent States. Thus, the principal value of the estimates in this teport lies in general comparisons arnong the States, interstate comparisons for a given year, and the analysis of trends over several years.

The five economic sectors used in SEPER correspond to those used in SEDR as follows:

- Residential Sector-The regidential sector is considered to consist of all private residences, whether occupied or vacant, owned or rented, including single-family homes, muttifamily housing units, and mobile homes. Secondary hornes, such as sumuner homes, are also included. Institutional housing, such as school dormitories, hospitals, and military barracks, generally are not included in the residential sector; they are included in the commercial sector.

- Commerial Sector-The commercial sector, as defined econonically $y_{4}$ consists of business establishments that are not engaged in transportation or in manufacturing or other types of industrial activity (agriculture, mining, or construction). Commercial establishments include hokels, motels, restaurants, wholesale businesses, retail stores, laundries, and other service enterprises; religious and nonprofit onganizations; health, social, and edtreational institutions; and Federal, State, and local governnents. Street lights, pumps, bridges, and public services are also included if the establishment operating them is considered commercial.

- Industrial Sector-The industria! sector comprises manufachuring industries, which make up the largest part of the sector, along with mining, construction, agriculture, fisheries, and forestry. Establishments in the sector range from steel mills, to small farms, to companies assembling electronic components.

- Transportation Sector-The transportation sector consists of privafe and public vehicles that move people and commodities. Included are automobiles, trucks, buses, motorcycles, railroads and railways (including streetcars), aireraft, ships, barges, and natural gas pipelines.

- Electric Utility Sector-The electric utility sector consists of privately and publicly owned establishments that generate, transmit, distribute, or sell electricity primarily for use by the public and meet the definition of an electric utility. Nonutility power producers are not included in the electric utility sector.

Although end-use aljocations of energy consumation and expenditures follow those guidelines as closely as possible, some data are collected by using different classifications. For example, electric utilities often ciassify commercial and industrial users by the quantity of electricity purchases rather than by the business activity of the purchaser. Agricultural use of natural gas is collected and reported in the commercial sector, rather than in the industrial sector. Since agricultural use of natural gas cannot be identified separately, it remains in the commercial sector in this report. Another example is master-metered condominiums, apartments, and buildings with a combination of residential and conunercial units. In many cases, billing and metering practices cause residential energy usage of electricity, natural gas, or fuel oil to be included in the commercial sector. In those cases, there is no basis for separating residential from commexcial use. Readers are advised to consult the $S E D R$ documentation for specific assumptions regarding the consumption estimates.

Where prices for an energy source and sector are not available, comparable prices art substituted. For example, the transportation sector motor gasoline prices are also applied to the commercial and industrial sectors. In some cases, the average of adjacent States' prices is assigned to a missing State price. The docamentation elaborates on these price assumptions.

Except where specified, it is not generally possible to describe the prices in this report as "wholesale" or "retail." The prices paid in each consuming sector are actually a combination of both sets of prices, depending on a number of closely interrelated factors, and the data reflect the combination of prices actually paid by each sector. Almost all residential sector prices are close to retail prices, reflecting the relatively small quantities of individual purchases and the increased costs of 
extensive, diffuse, and multilayered distribution systems. Similarly, in the transportation sector almost everyone pays the same rekal-like price for motor gasoline, regardless of volume purchased of location of purchase. Conversely, residual fuel oil prices in the transportation sector are certainly more wholesale-like as a result of large deliveries to bulk facilities in major ports. In the same manner, most large industrial and many large commercial expenditures can be thought of as near wholesale, frequently involving direct access to a producer or bulk distribution facility for very large quantities. Many smaller industrial and commercial facilities pay something much closer to retail prices as a result of the small quantities involved and their institutional distance from primary suppliers. Notanble exceptions to these relationships include natural gas and electric utilities, which typically establish fixed rates for each of several classes of service, depending on representative quantities, service factors, and distribution expenses. 
State Rankings 

Table 1. Energy Pricos and Expondliura Ranked by Stete, 1994

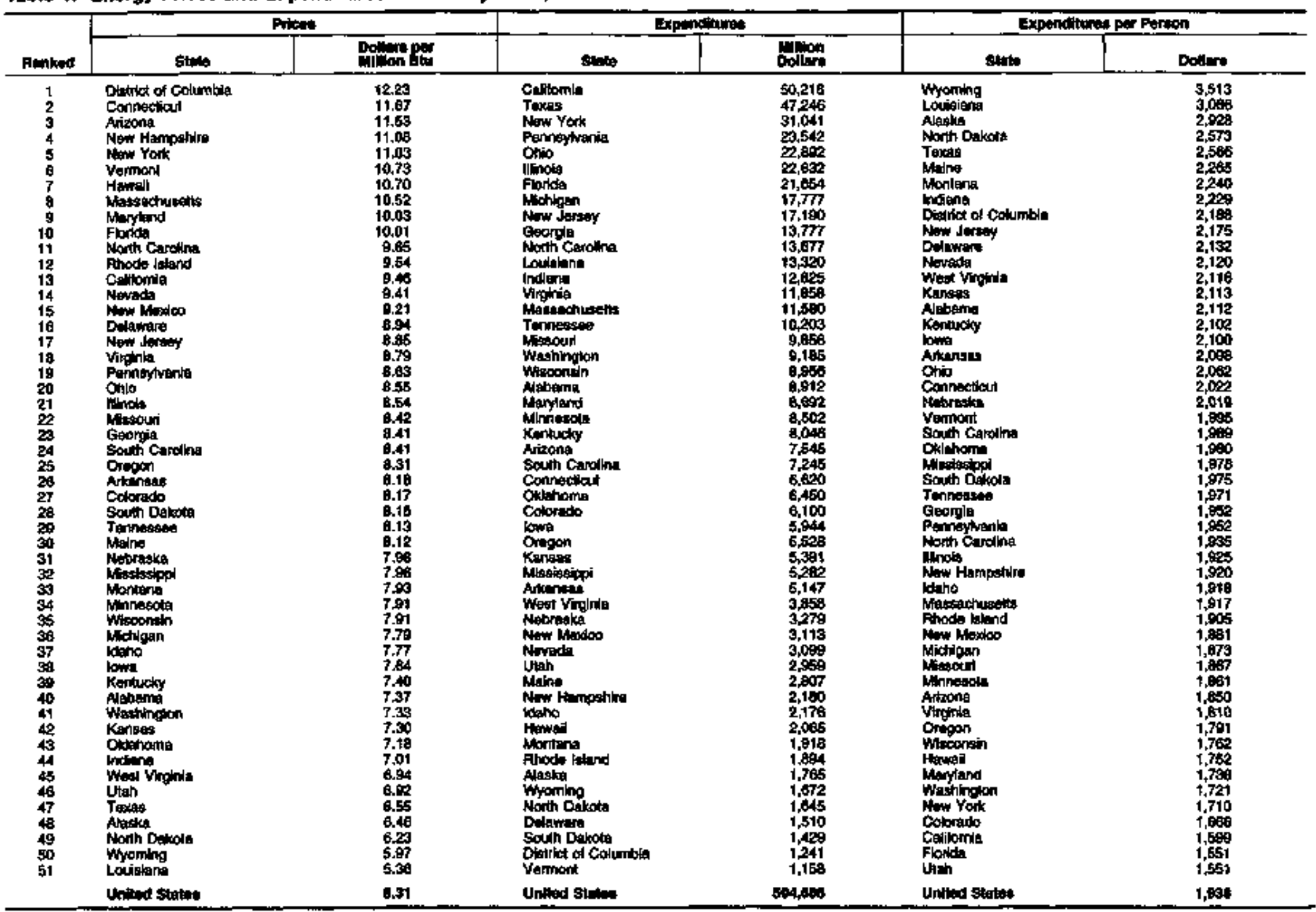

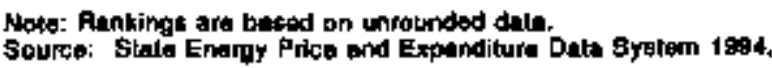


Table 2. Motor Coboline Prices and Expendifures Fanibed by Strite, T994

\begin{tabular}{|c|c|c|c|c|c|c|}
\hline \multirow[b]{2}{*}{ Praimed } & \multicolumn{2}{|c|}{ Puteen } & \multicolumn{2}{|c|}{ 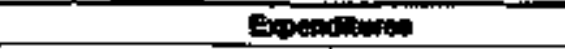 } & \multicolumn{2}{|c|}{ Expendiches pos Person } \\
\hline & 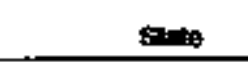 & 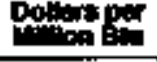 & sint & Dopister & sinte & 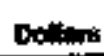 \\
\hline 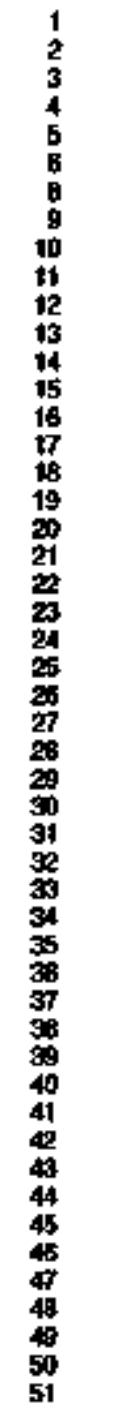 & 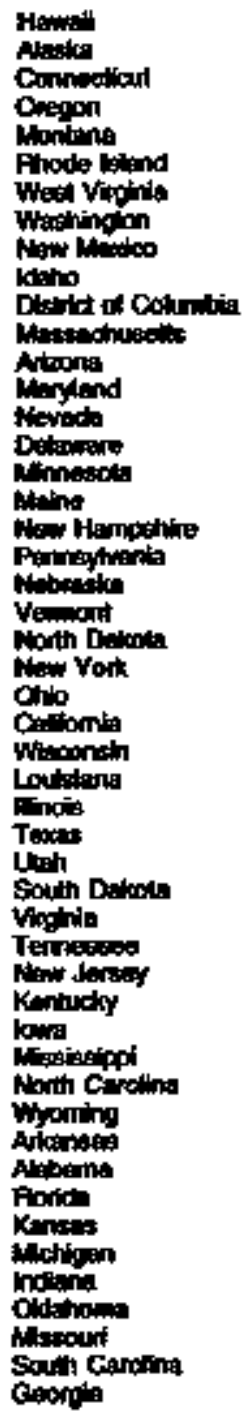 & 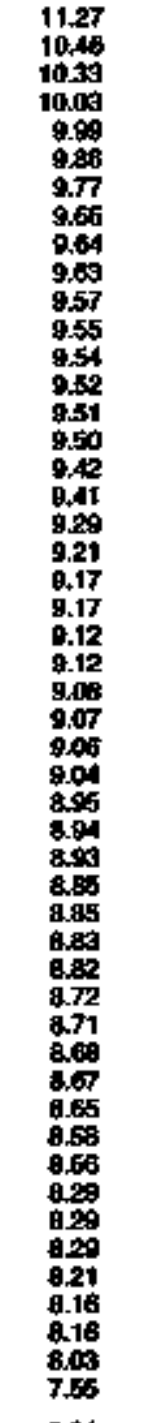 & 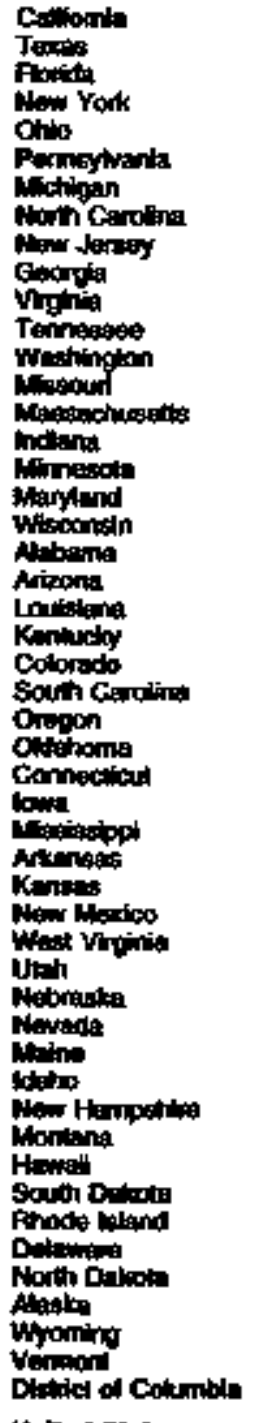 & 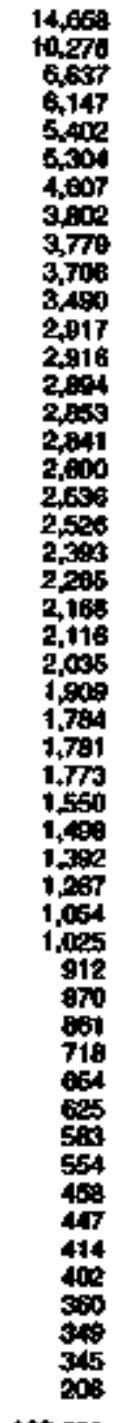 & 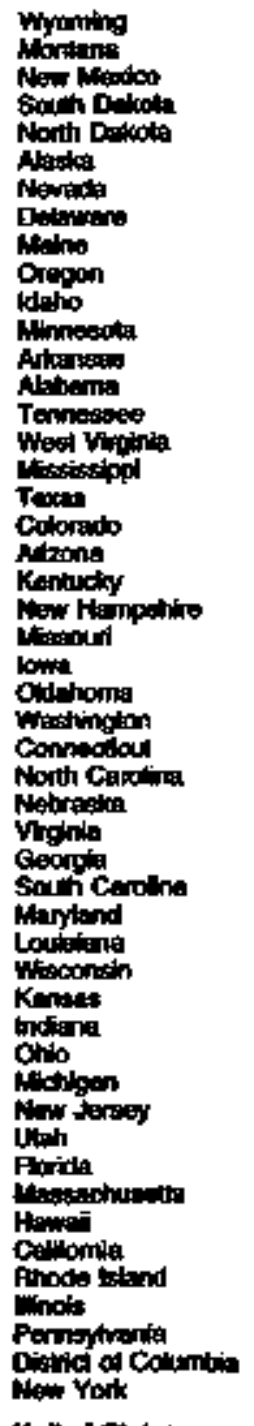 & 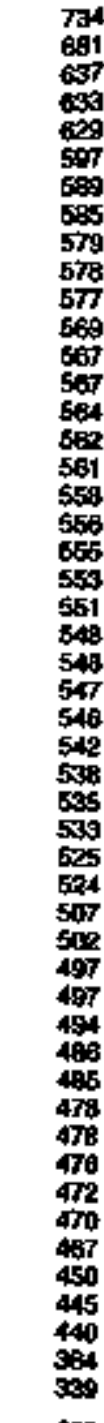 \\
\hline & تصفيt & ats & (t) & 124,900 & In'md 8t: & 490 \\
\hline
\end{tabular}

Nola: Fentings era based on mrounded data.

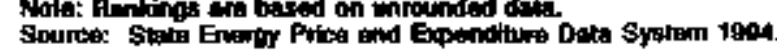


Table 3. Petroleum and Natural Gas Prices and Expondihures Fenked by Stete, 1 then

\begin{tabular}{|c|c|c|c|c|c|c|c|c|}
\hline \multirow[b]{3}{*}{ Fandoud } & \multicolumn{4}{|c|}{ 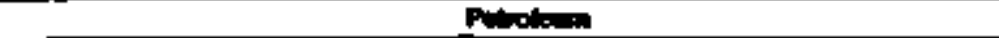 } & \multicolumn{4}{|c|}{ Notures otos } \\
\hline & \multicolumn{2}{|c|}{ Allows } & \multicolumn{2}{|c|}{ 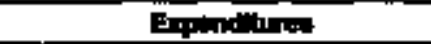 } & \multicolumn{2}{|c|}{ filot: } & \multicolumn{2}{|c|}{ 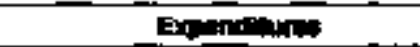 } \\
\hline & Stot: & Don: & st. & Dollon & מא:Bל & 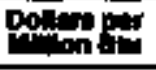 & ste & 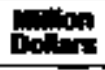 \\
\hline $\begin{array}{c}1 \\
2 \\
3 \\
4 \\
5 \\
6 \\
7 \\
8 \\
9 \\
10 \\
11 \\
19 \\
13 \\
14 \\
15 \\
15 \\
17 \\
19 \\
19 \\
20 \\
21 \\
22 \\
23 \\
23 \\
24 \\
25 \\
25 \\
27 \\
20 \\
29 \\
30 \\
31 \\
39 \\
33 \\
34 \\
35 \\
35 \\
37 \\
38 \\
39 \\
40 \\
41 \\
42 \\
43 \\
44 \\
45 \\
46 \\
47 \\
49 \\
49 \\
50 \\
51\end{array}$ & 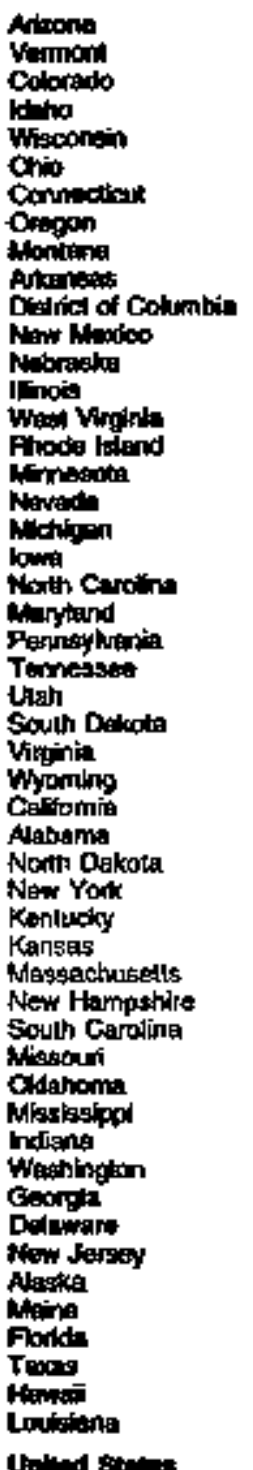 & 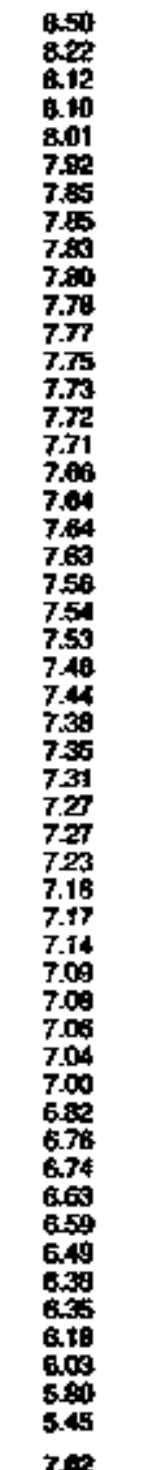 & 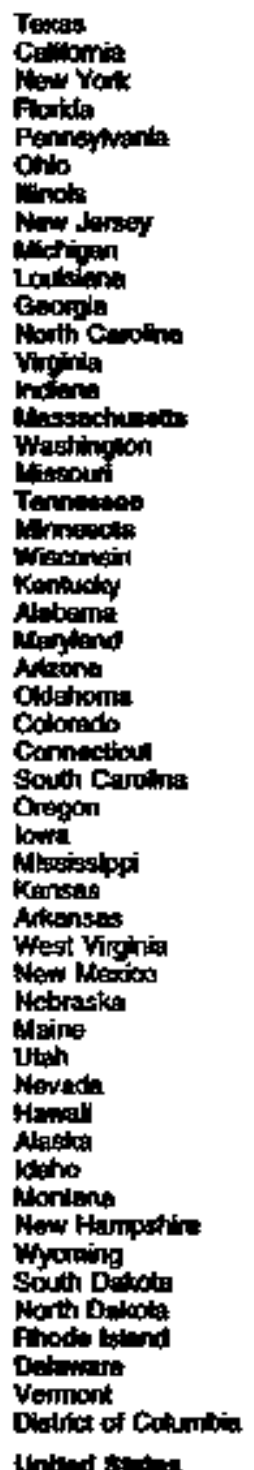 & 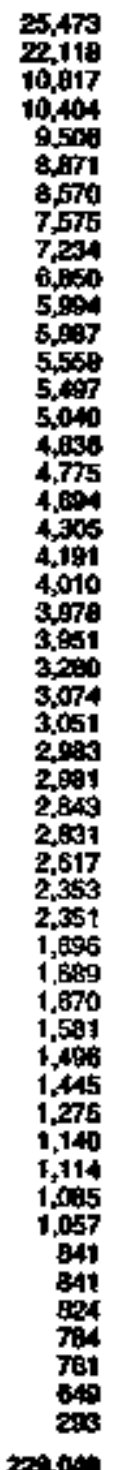 & 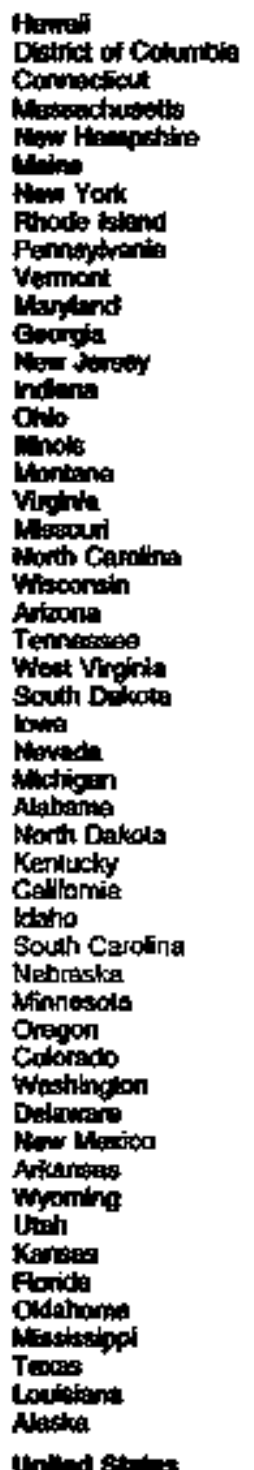 & 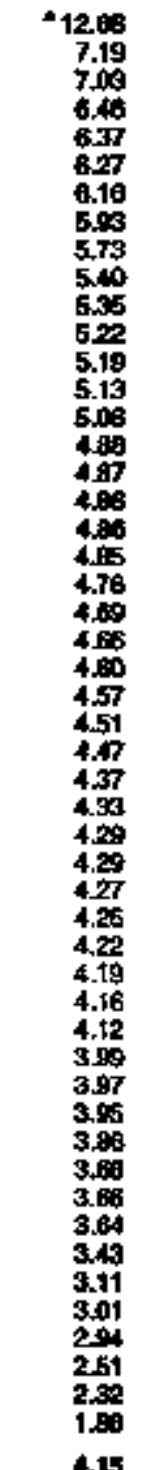 & 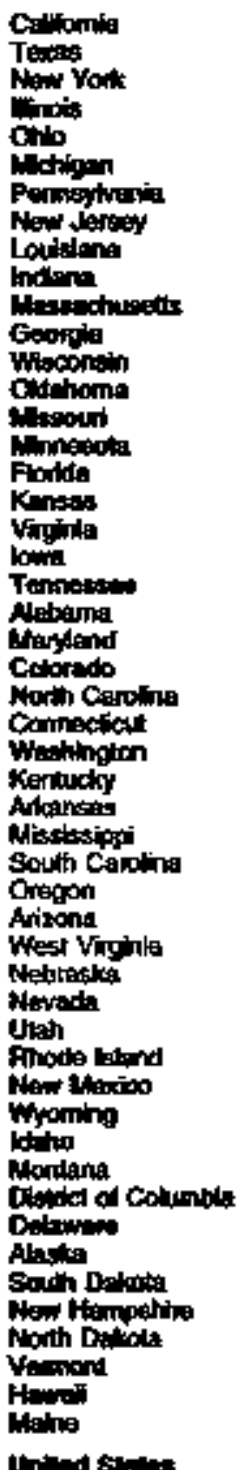 & 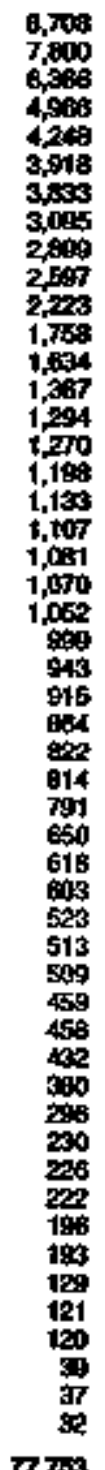 \\
\hline
\end{tabular}

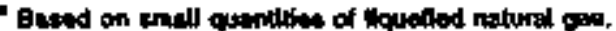

Nota: Ravtings arts basted on uninonded daln

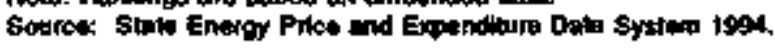


Table 4. Coal and Electricity Prices end Expenditures Ranked by State, 1004

\begin{tabular}{|c|c|c|c|c|c|c|c|c|}
\hline \multirow[b]{3}{*}{ Panlmod } & \multicolumn{4}{|c|}{ cons } & \multicolumn{4}{|c|}{ Enctrkiny } \\
\hline & \multicolumn{2}{|c|}{ Prolon: } & \multicolumn{2}{|c|}{ Expenditure: } & \multicolumn{2}{|c|}{ Prdoes } & \multicolumn{2}{|c|}{ Expetin: lures } \\
\hline & State & Dolleser per & stinte & Dollan & state & 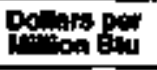 & statio & 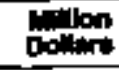 \\
\hline $\begin{array}{l}1 \\
2 \\
3 \\
4 \\
5 \\
6 \\
7 \\
9 \\
9 \\
10 \\
11 \\
12 \\
13 \\
14 \\
15 \\
16 \\
17 \\
18 \\
19 \\
20 \\
21 \\
22 \\
23 \\
24 \\
25 \\
26 \\
27 \\
28 \\
29 \\
30 \\
31 \\
32 \\
33 \\
34 \\
35 \\
36 \\
37 \\
39 \\
39 \\
40 \\
41 \\
42 \\
43 \\
44 \\
45 \\
43 \\
97 \\
49 \\
49 \\
50 \\
51\end{array}$ & 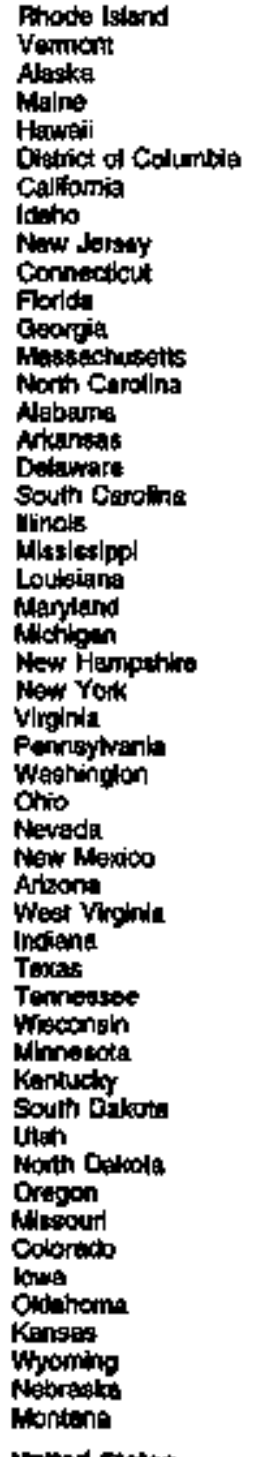 & 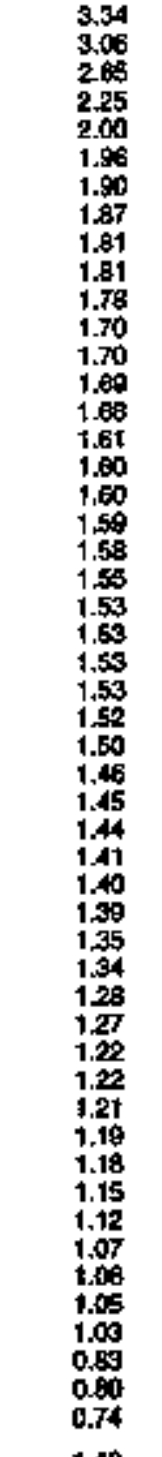 & 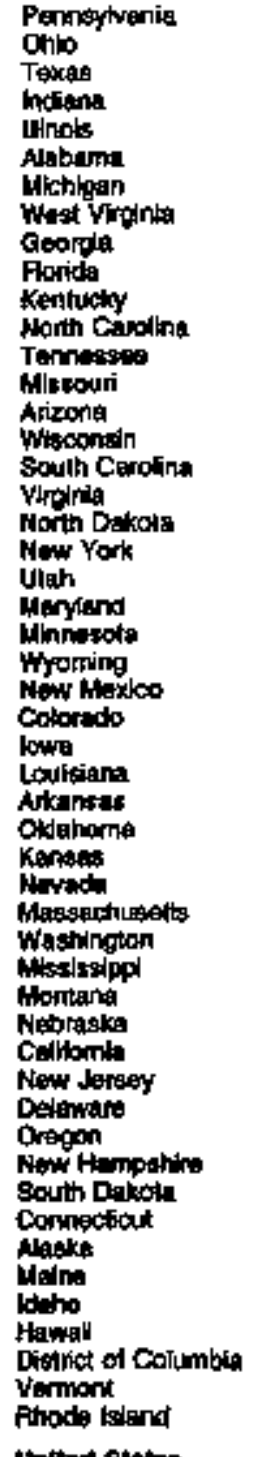 & 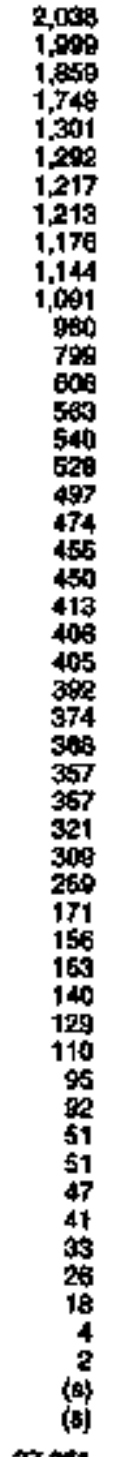 & 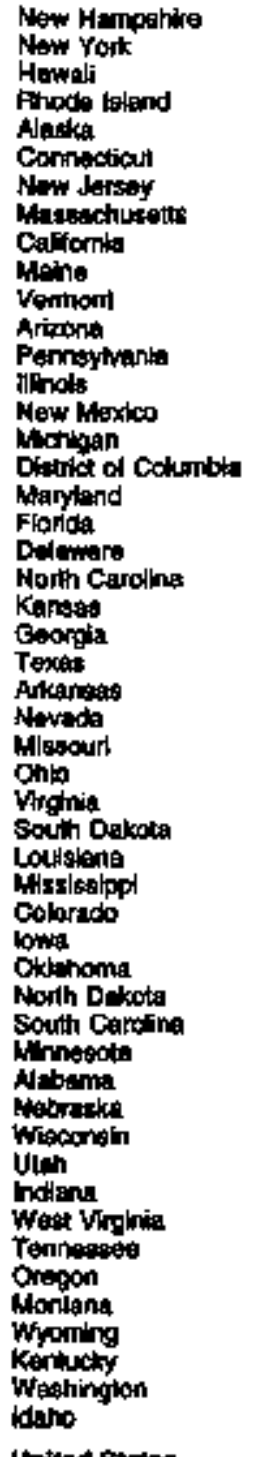 & 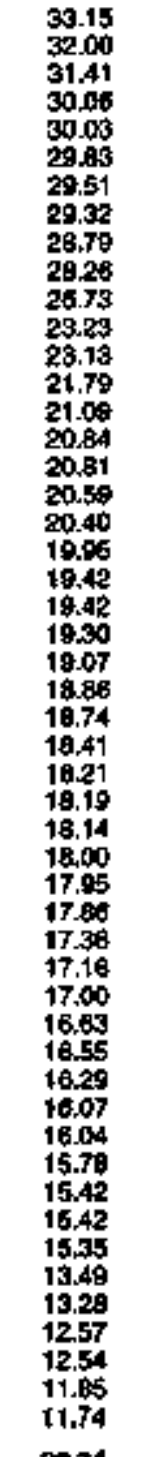 & 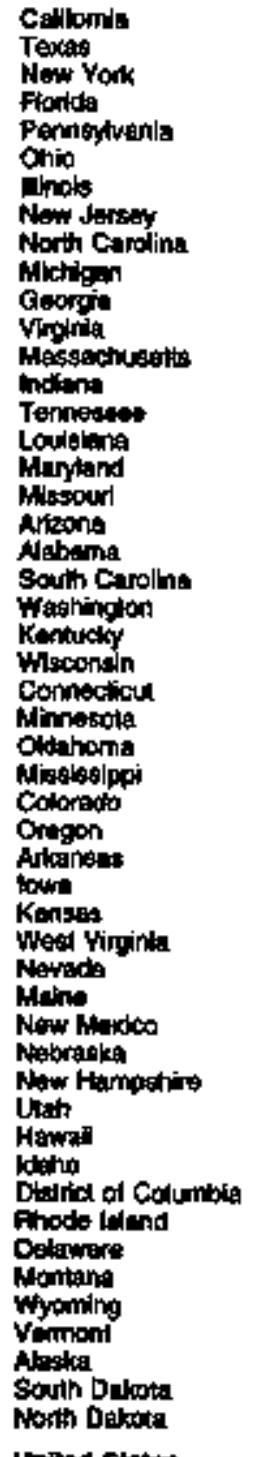 & 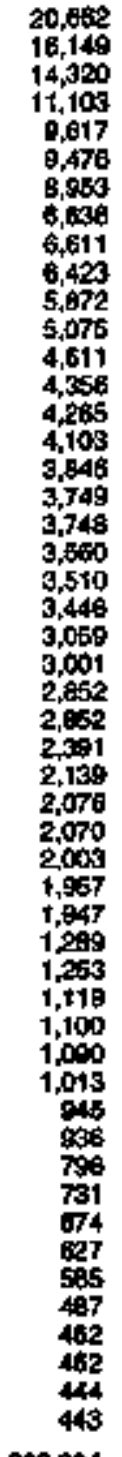 \\
\hline & Unitod stent: & 1.40 & 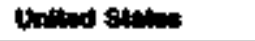 & 27 & Untind Strinis & 20.34 & Unined Stat & 200,004 \\
\hline
\end{tabular}

(o)=Vaius less then 0.5 militon dolfirs.

Nole; Fankings are based on unrounded dnto.

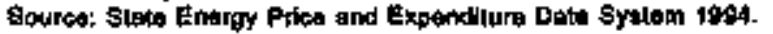


Statistical Tables 



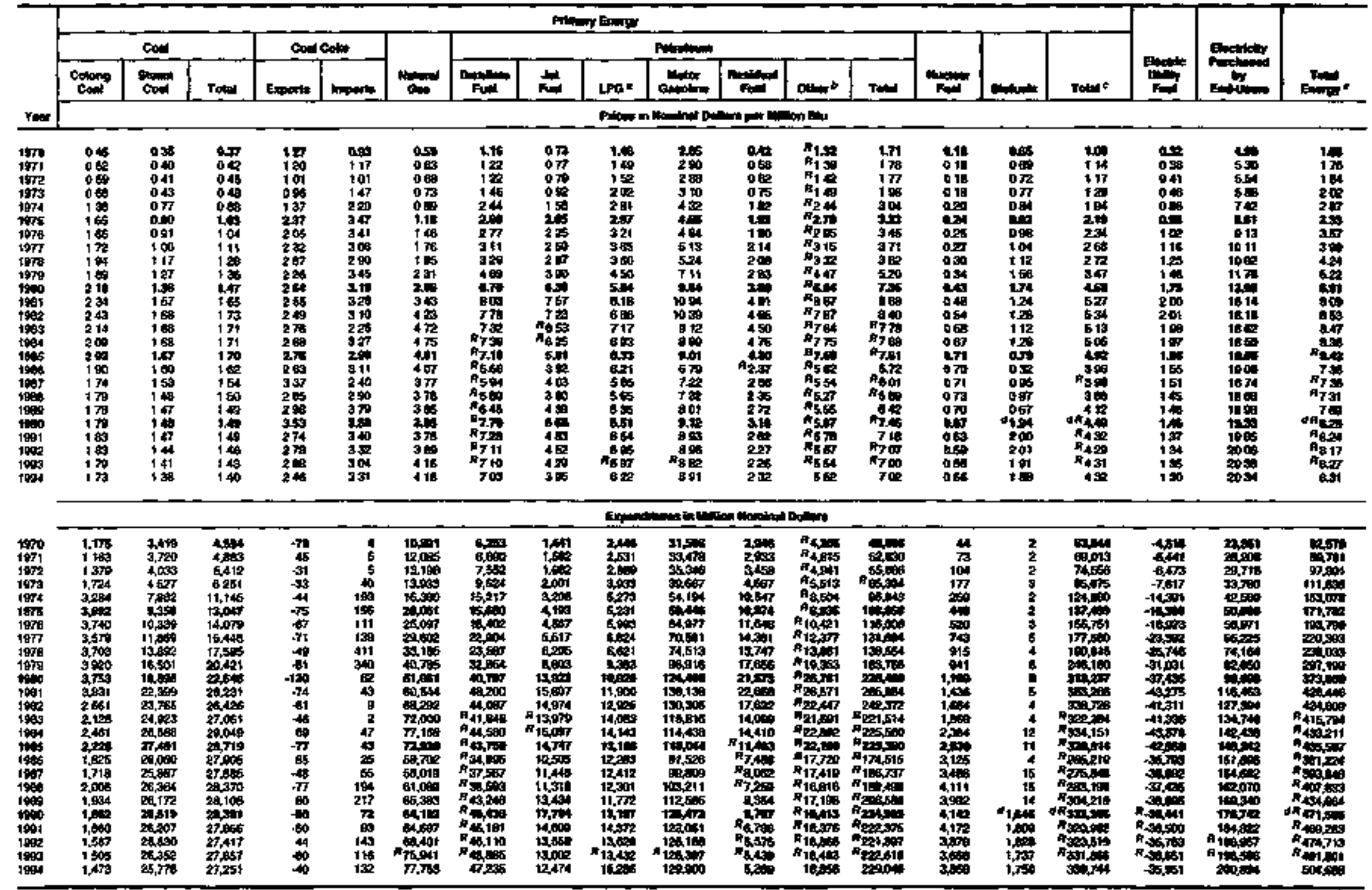

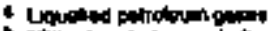

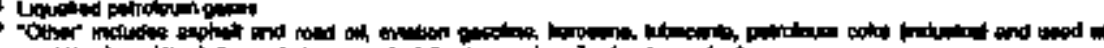

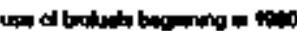

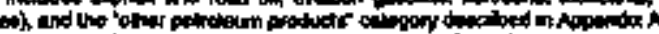

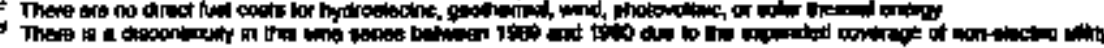




\begin{tabular}{|c|c|c|c|c|c|c|c|c|c|c|}
\hline \multirow[b]{4}{*}{ Year } & \multicolumn{8}{|c|}{ 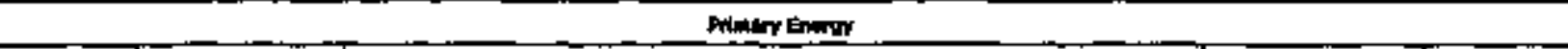 } & \multirow[b]{3}{*}{ anctioly } & \multirow[b]{3}{*}{ 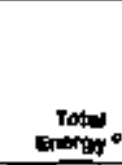 } \\
\hline & \multirow[b]{2}{*}{ 9. } & \multirow[b]{2}{*}{ 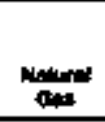 } & \multicolumn{4}{|c|}{ Patroleng } & \multirow[b]{2}{*}{ Bathowp } & \multirow[b]{2}{*}{ Tow } & & \\
\hline & & & 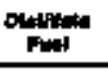 & Kenosunes & was & Tole & & & & \\
\hline & \multicolumn{10}{|c|}{ 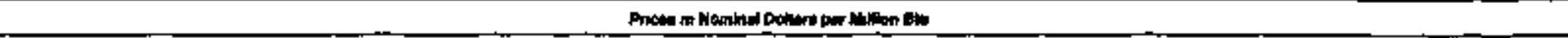 } \\
\hline \multirow[t]{2}{*}{ 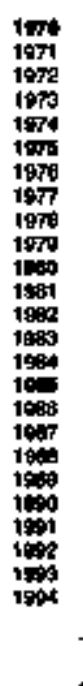 } & 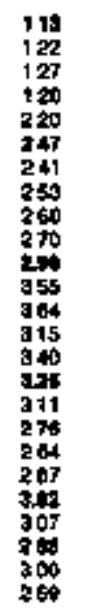 & 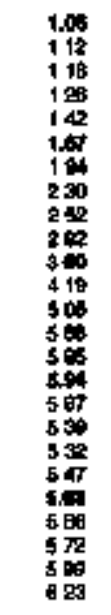 & 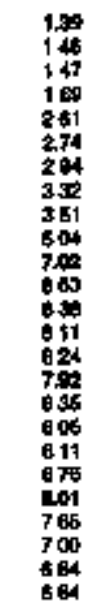 & 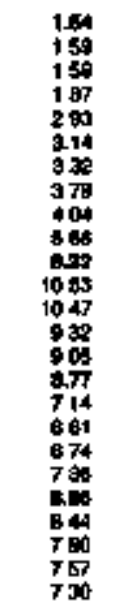 & 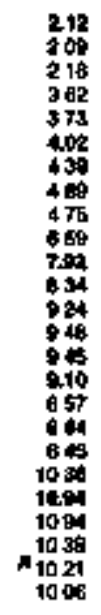 & 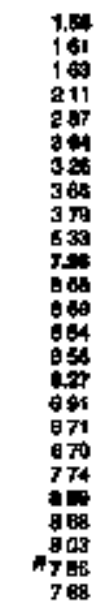 & 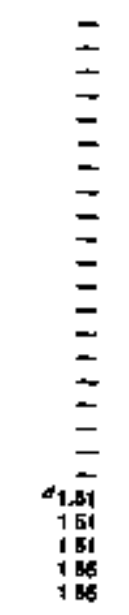 & 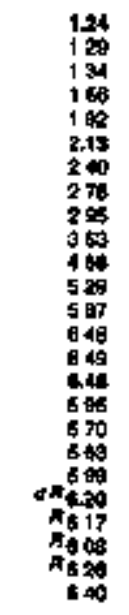 & 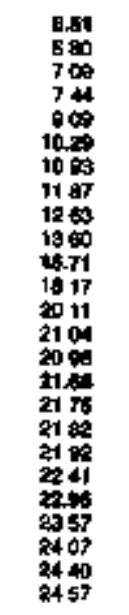 & 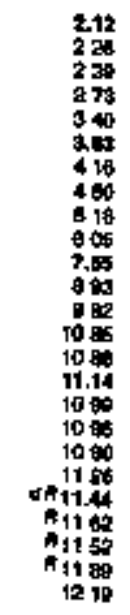 \\
\hline & \multicolumn{10}{|c|}{ 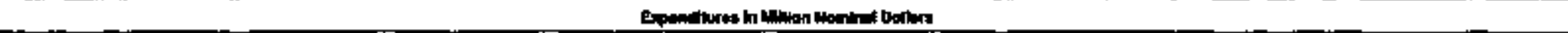 } \\
\hline 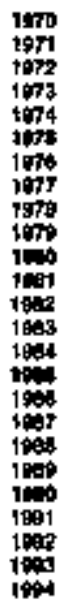 & 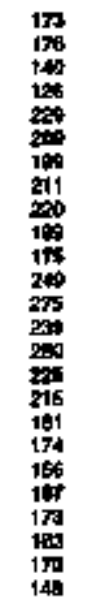 & 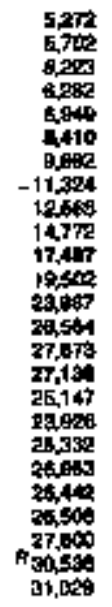 & 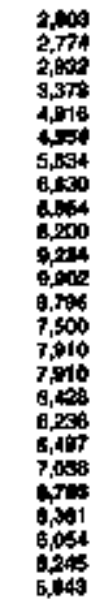 & 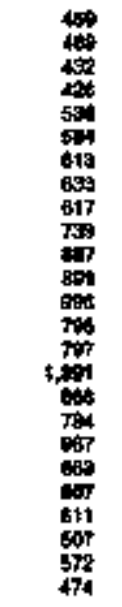 & 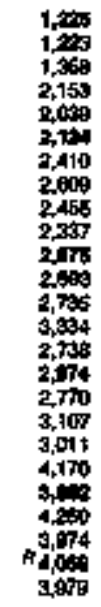 & 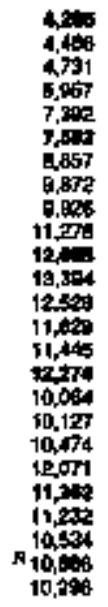 & 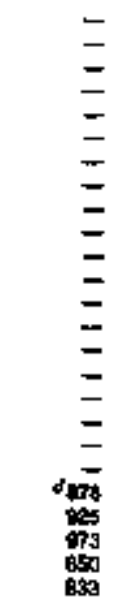 & 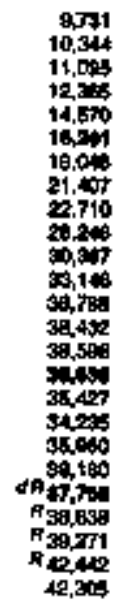 & 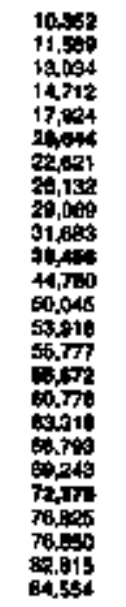 & 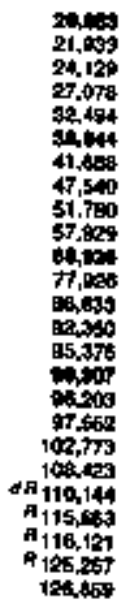 \\
\hline
\end{tabular}




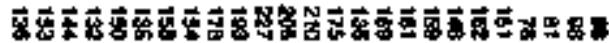

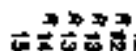

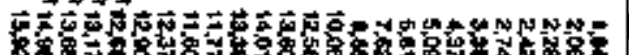

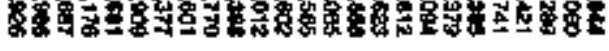

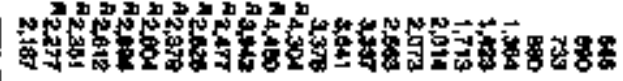

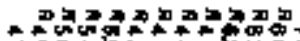

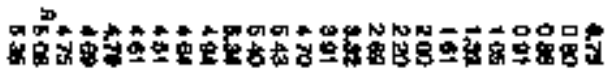

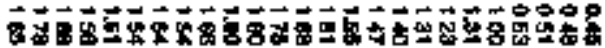

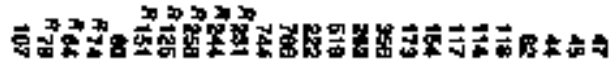

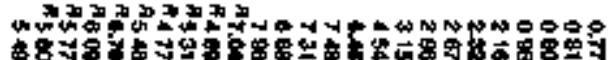

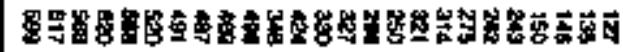

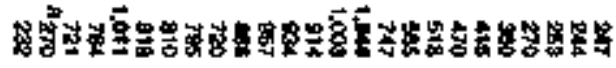

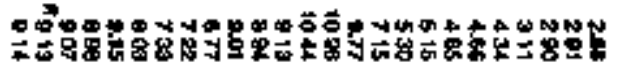

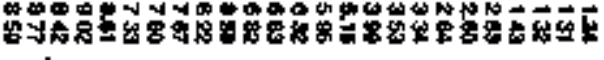

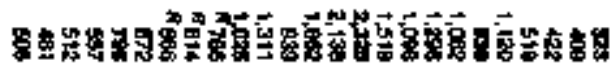

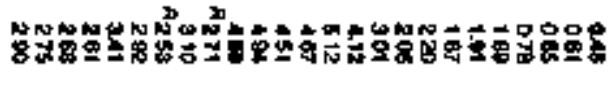

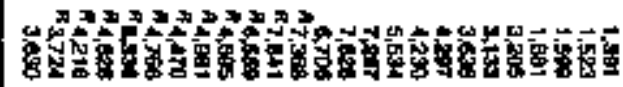

ind

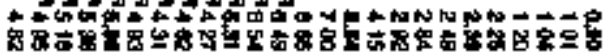

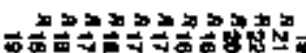

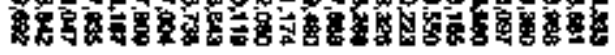

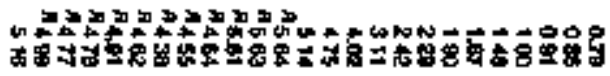

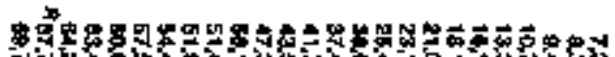

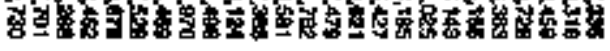

के

\$

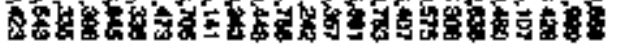

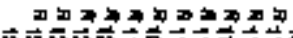

N

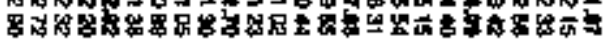

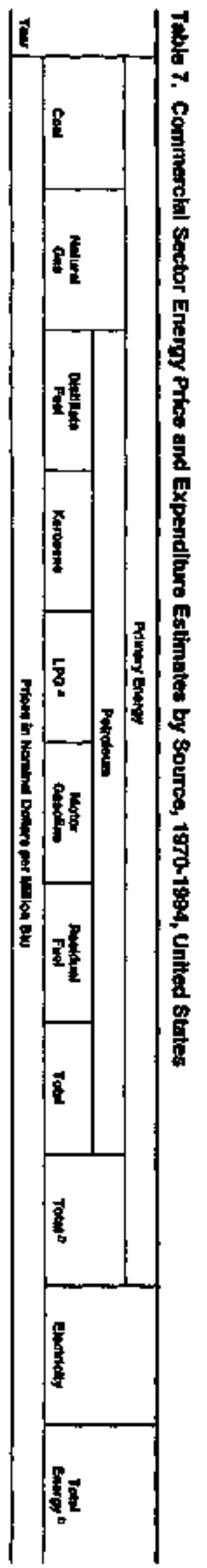

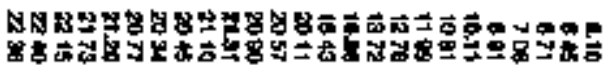

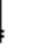




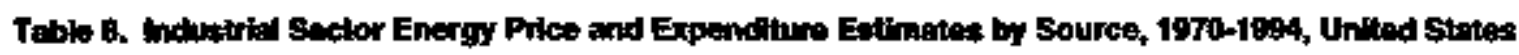

\begin{tabular}{|c|c|c|c|c|c|c|c|c|c|c|c|c|c|c|c|c|c|c|c|}
\hline \multirow[b]{4}{*}{$\mathrm{x}=$} & \multicolumn{17}{|c|}{ Phm } & \multirow[b]{3}{*}{ Bowerticin. } & \multirow[b]{3}{*}{$E_{n \rightarrow \infty}^{T}$} \\
\hline & \multicolumn{3}{|c|}{ 19 } & \multicolumn{2}{|c|}{$\cos 1 \cos \theta$} & \multirow[b]{2}{*}{ 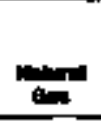 } & \multicolumn{9}{|c|}{ Pand } & \multirow[b]{2}{*}{ Datolumply } & \multirow[b]{2}{*}{ Tones 4} & & \\
\hline & sets & - & 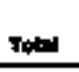 & Emank & 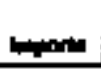 & & 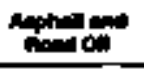 & and & xaranem & Let: & 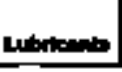 & 年 & minget & atser & Tate & & & & \\
\hline & \multicolumn{19}{|c|}{ 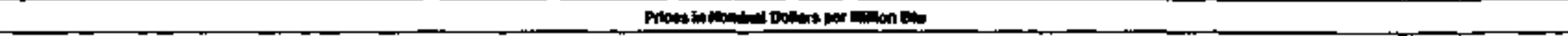 } \\
\hline \multirow[t]{2}{*}{ 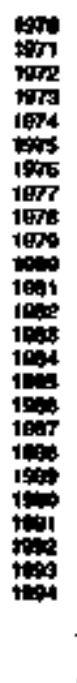 } & 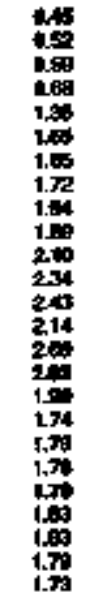 & 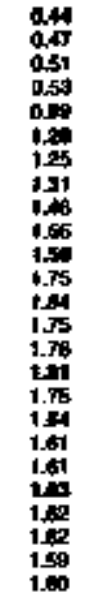 & 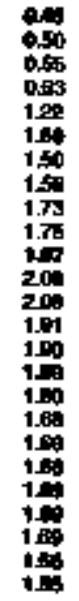 & 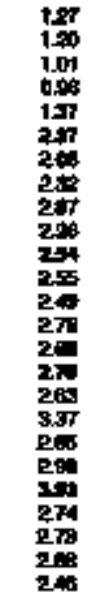 & 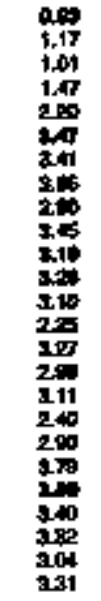 & 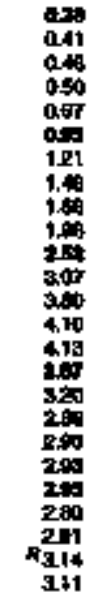 & 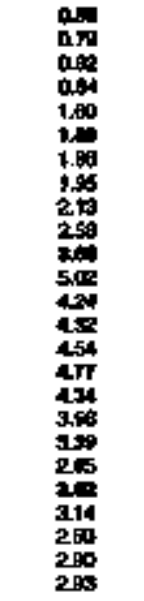 & 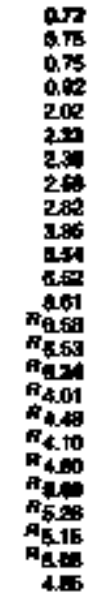 & 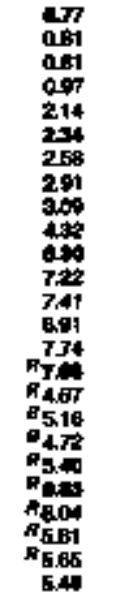 & 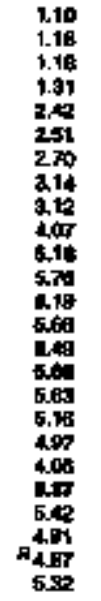 & 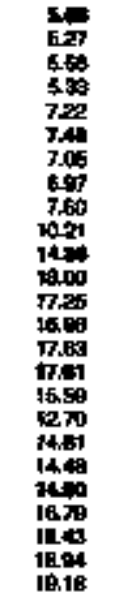 & 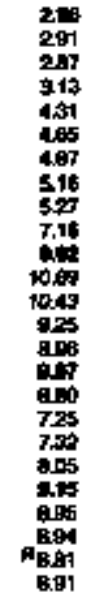 & 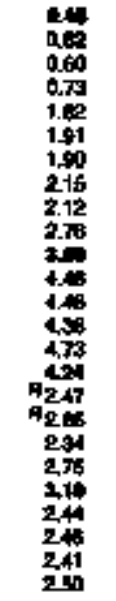 & 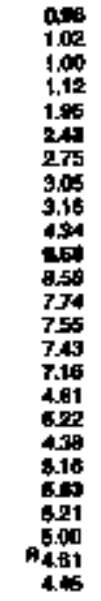 & 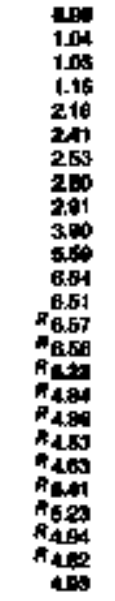 & $\begin{array}{l}= \\
= \\
= \\
= \\
= \\
= \\
= \\
= \\
= \\
= \\
= \\
\bar{z} \\
1.40 \\
1.35 \\
1.34\end{array}$ & 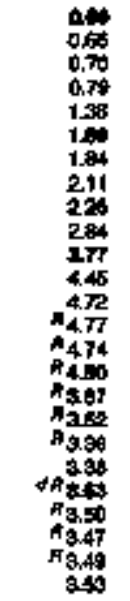 & 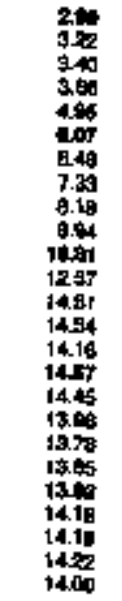 & 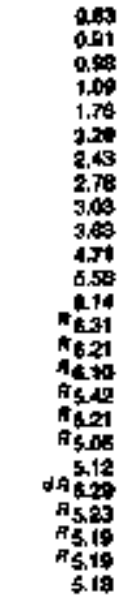 \\
\hline & \multicolumn{19}{|c|}{ 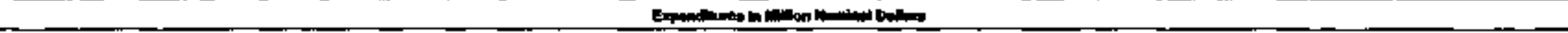 } \\
\hline 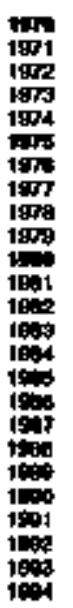 & 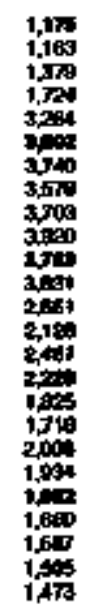 & 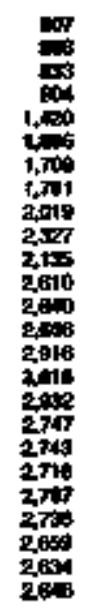 & 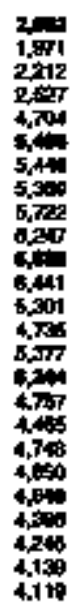 & 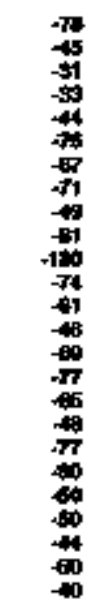 & 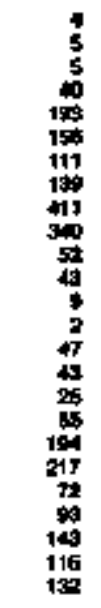 & 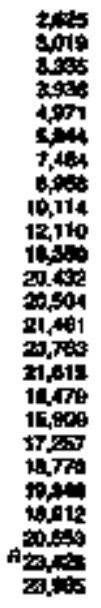 & 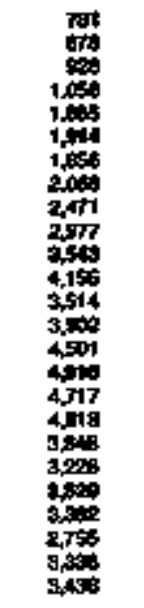 & 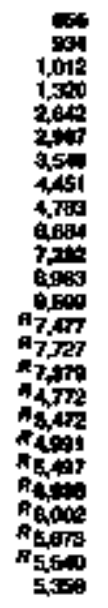 & 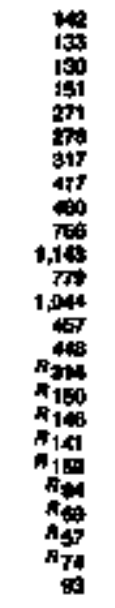 & 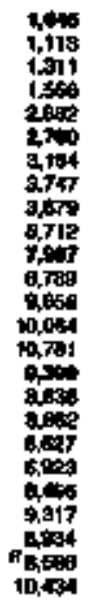 & 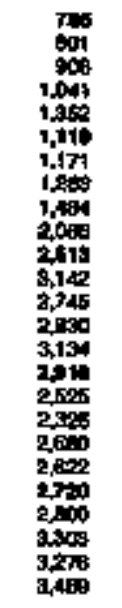 & 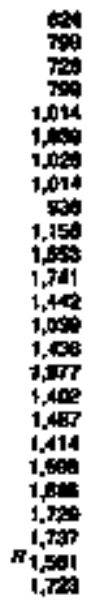 & 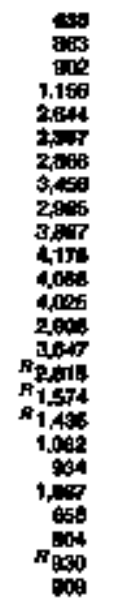 & 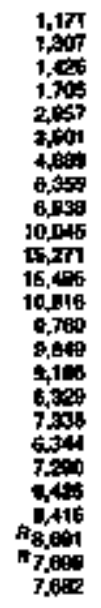 & 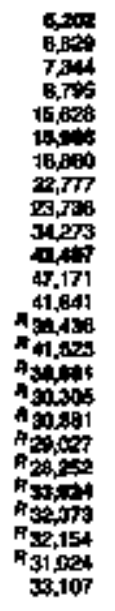 & 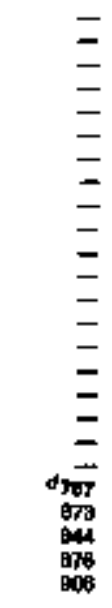 & 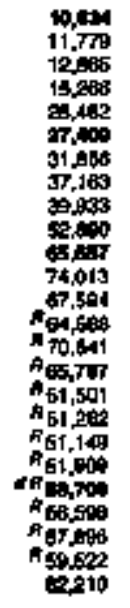 & 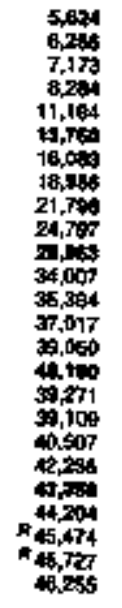 & 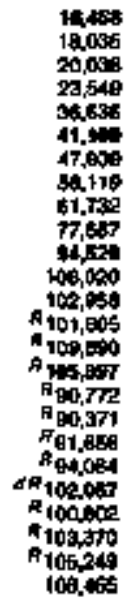 \\
\hline
\end{tabular}

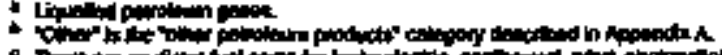

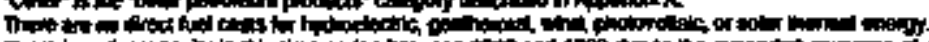

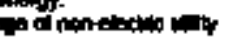

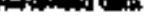

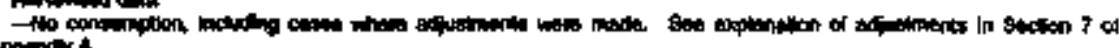
100

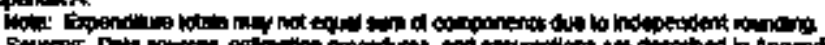

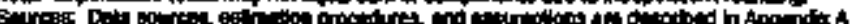




\begin{tabular}{|c|c|c|c|c|c|c|c|c|c|c|c|c|c|}
\hline \multirow[b]{4}{*}{$\underline{Y}=$} & \multicolumn{11}{|c|}{ Proming e-m } & \multirow[b]{3}{*}{ 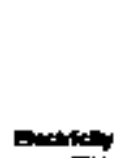 } & \multirow[b]{3}{*}{ Then } \\
\hline & \multirow[b]{2}{*}{$\cos$} & \multirow[b]{2}{*}{ Mand } & \multicolumn{8}{|c|}{ Ponolinen } & \multirow[b]{2}{*}{ Ton: } & & \\
\hline & & & 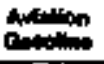 & onter & Fu & Letc: & 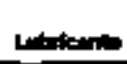 & 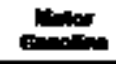 & |- & Toll & & & \\
\hline & \multicolumn{13}{|c|}{ 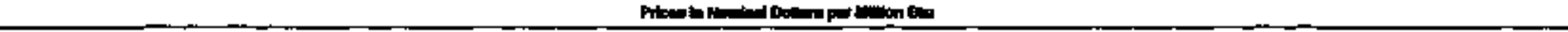 } \\
\hline \multirow[t]{2}{*}{ 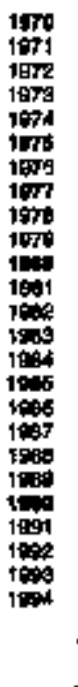 } & $\begin{array}{l}0.11 \\
0.40 \\
0.40 \\
0.60 \\
0.00 \\
1.20 \\
1.21 \\
1.27 \\
= \\
= \\
= \\
= \\
= \\
= \\
= \\
= \\
= \\
= \\
=\end{array}$ & $\begin{array}{l}= \\
= \\
= \\
= \\
= \\
= \\
= \\
= \\
= \\
= \\
= \\
= \\
\bar{z} \\
3.94 \\
304 \\
4.17\end{array}$ & 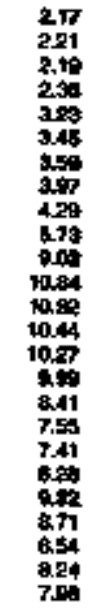 & 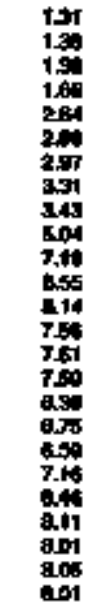 & 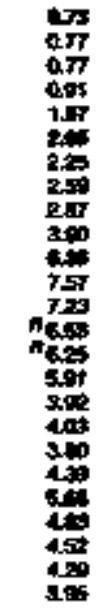 & 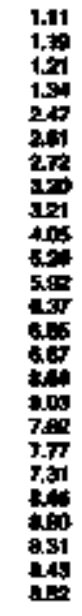 & 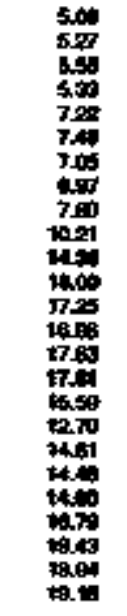 & 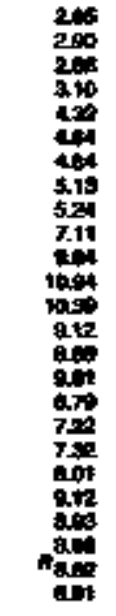 & 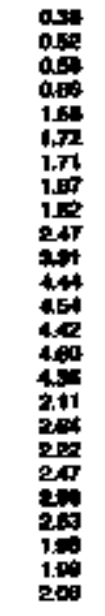 & 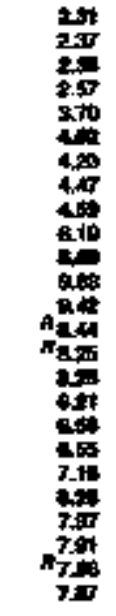 & 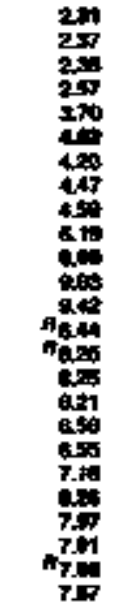 & 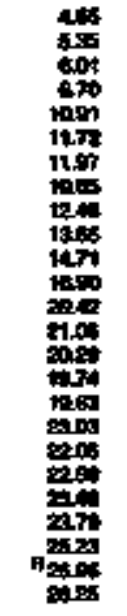 & 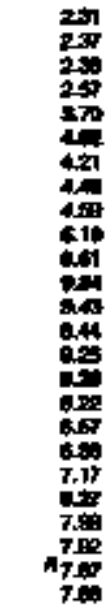 \\
\hline & \multicolumn{13}{|c|}{ 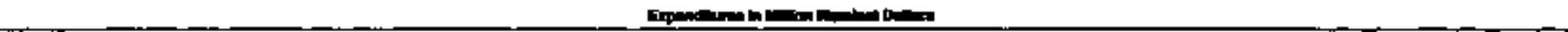 } \\
\hline 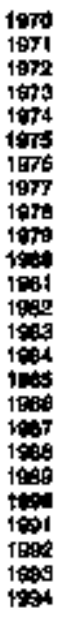 & $\begin{array}{l}3 \\
2 \\
2 \\
1 \\
2 \\
1 \\
0 \\
0 \\
= \\
= \\
= \\
= \\
= \\
= \\
= \\
= \\
= \\
= \\
= \\
= \\
=\end{array}$ & $\begin{array}{l}= \\
= \\
= \\
= \\
= \\
= \\
= \\
= \\
= \\
= \\
= \\
= \\
1 \\
z \\
4\end{array}$ & 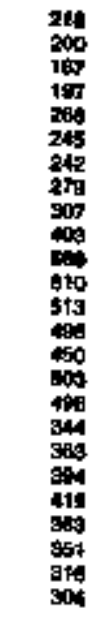 & 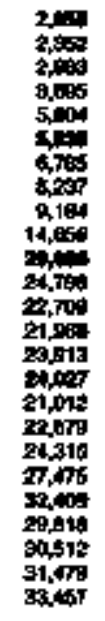 & 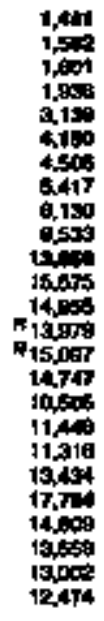 & 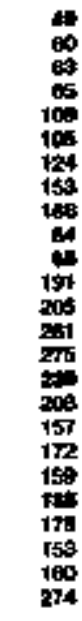 & 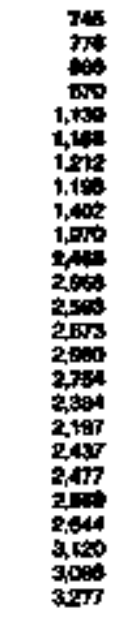 & 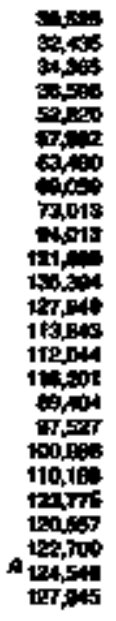 & 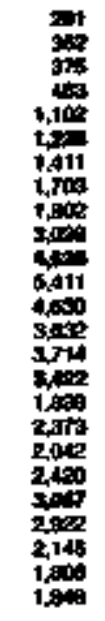 & 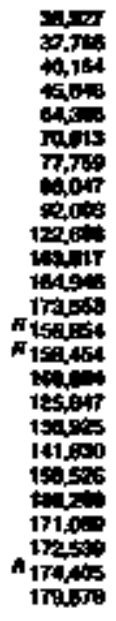 & 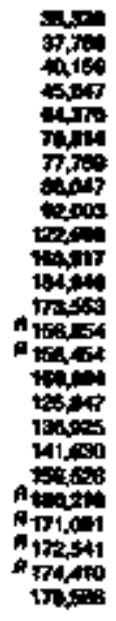 & 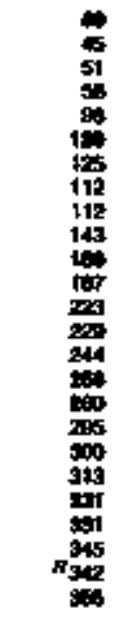 & 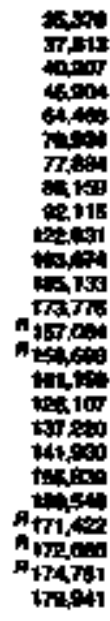 \\
\hline
\end{tabular}




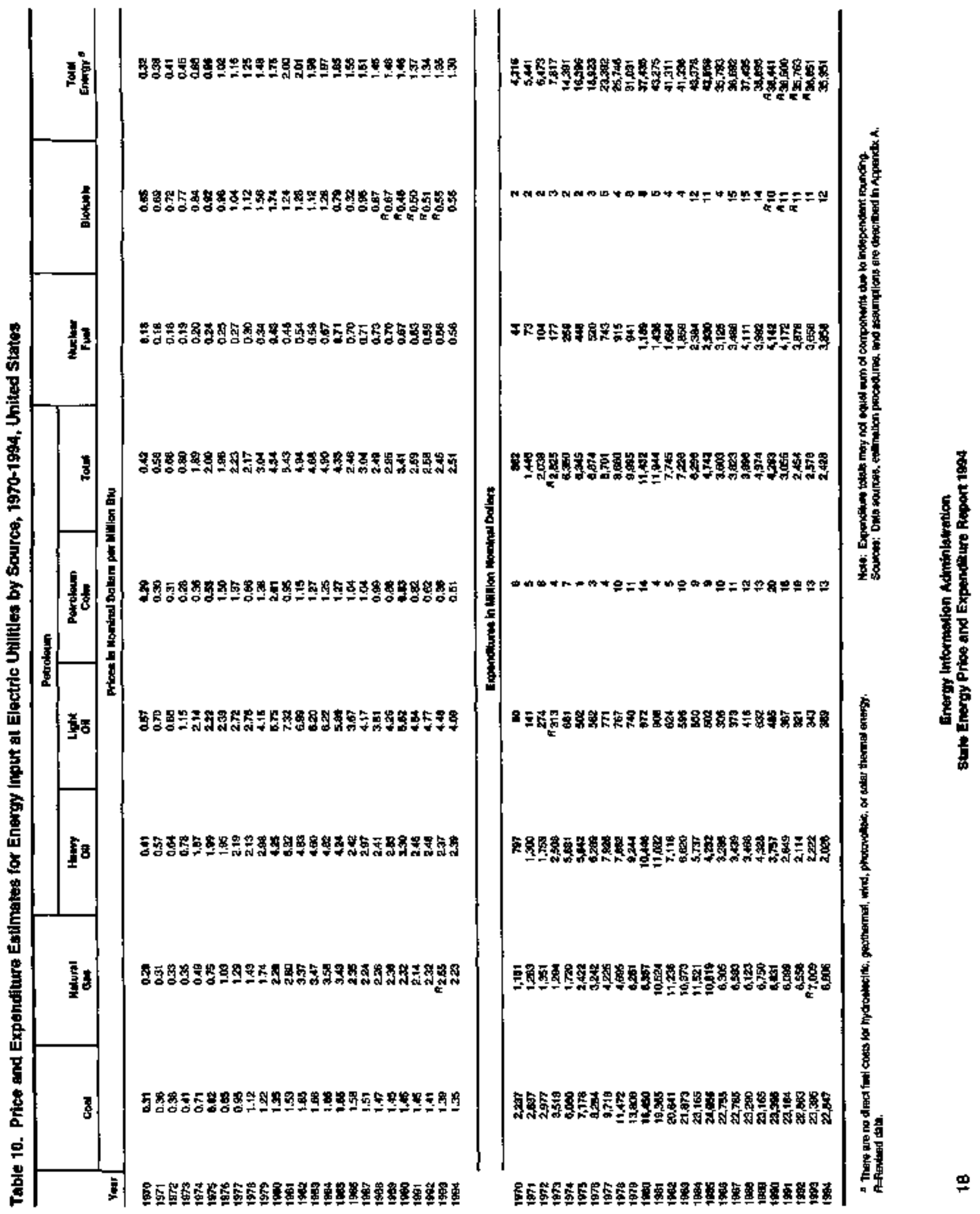

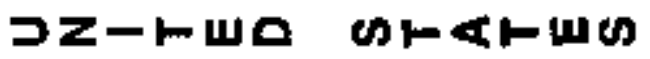


Tablo 11. Enorgy Prke ard Expendure Estimates by Souch, 1970-1994, Alabuma

\begin{tabular}{|c|c|c|c|c|c|c|c|c|c|c|c|c|c|c|c|c|c|}
\hline \multirow{2}{*}{ rar } & \multicolumn{14}{|c|}{ Promery Enopy } & \multirow{2}{*}{ 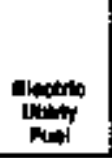 } & \multirow{2}{*}{ 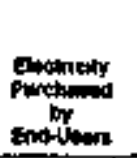 } & \multirow{2}{*}{ : } \\
\hline & \multicolumn{3}{|c|}{ Cast } & nowis & \multicolumn{7}{|c|}{ Prom } & mesen & 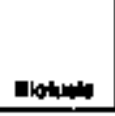 & $70+1)^{E}$ & & & \\
\hline 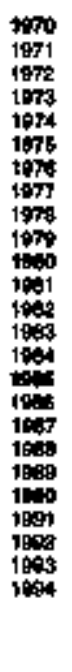 & 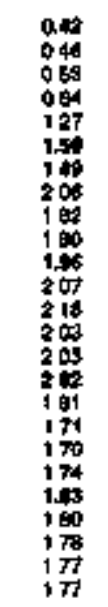 & 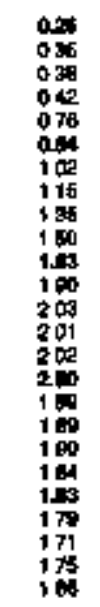 & 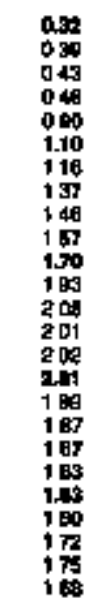 & 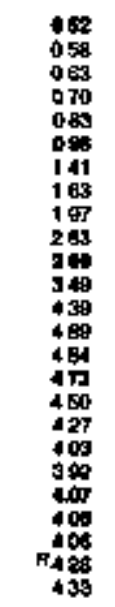 & 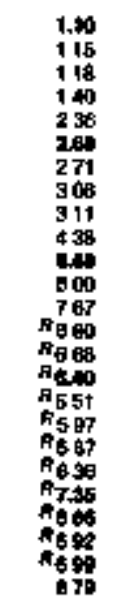 & 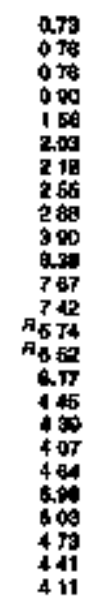 & 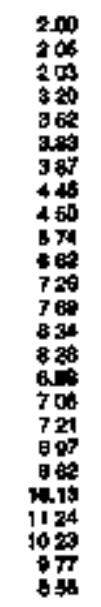 & 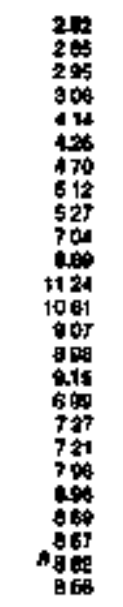 & 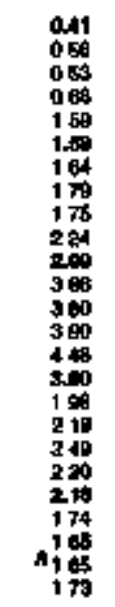 & 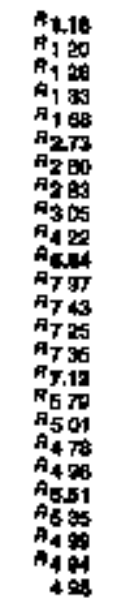 & 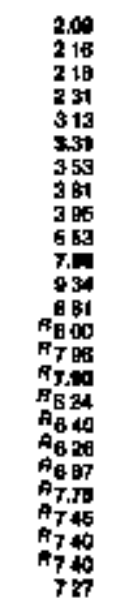 & 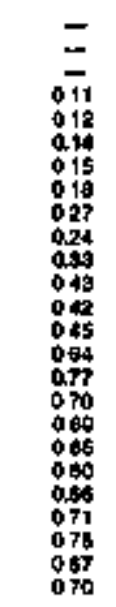 & 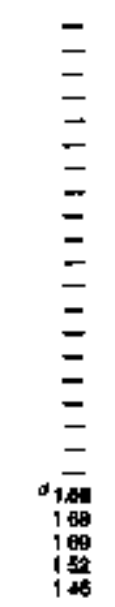 & 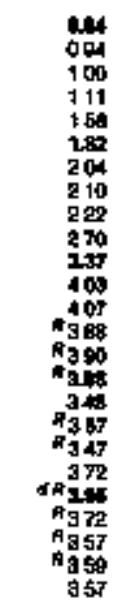 & 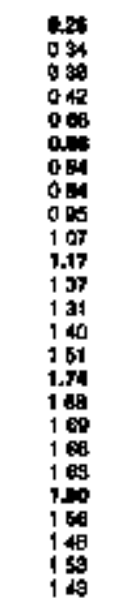 & 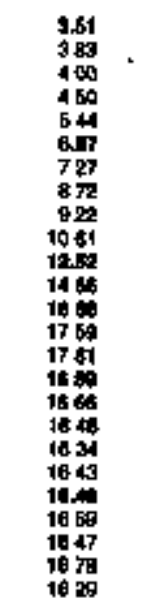 & 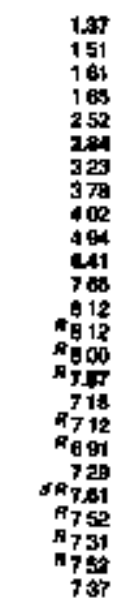 \\
\hline 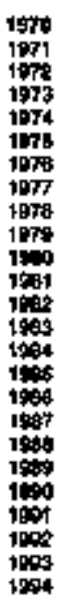 & 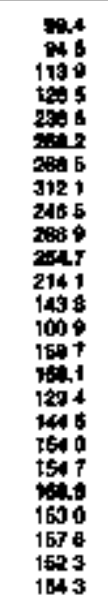 & 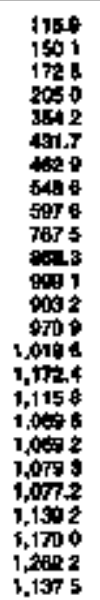 & 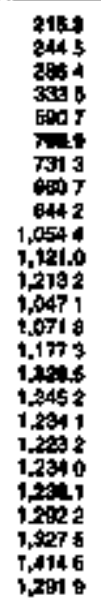 & 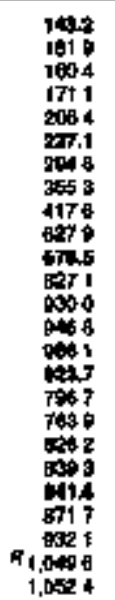 & 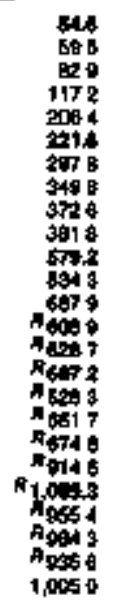 & 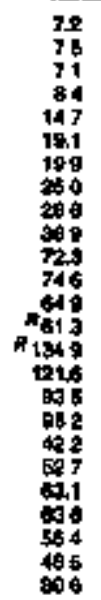 & 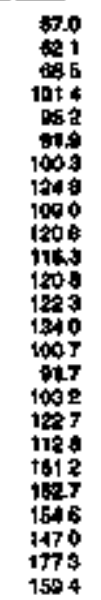 & 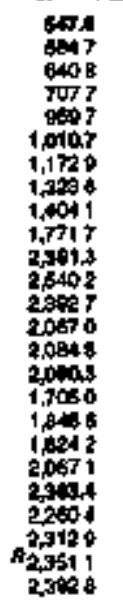 & 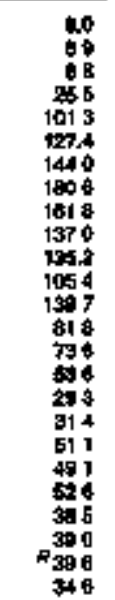 & 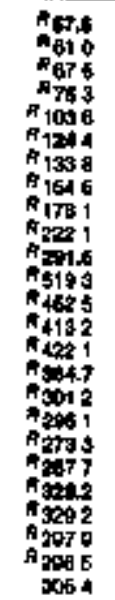 & 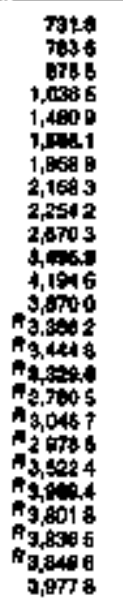 & 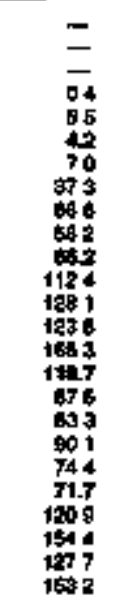 & 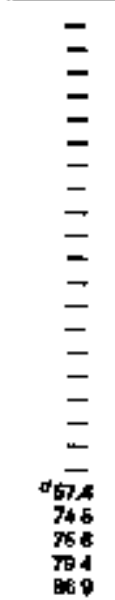 & 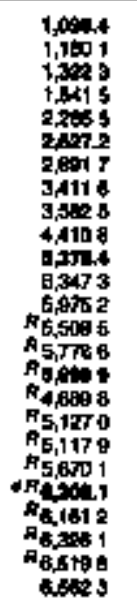 & 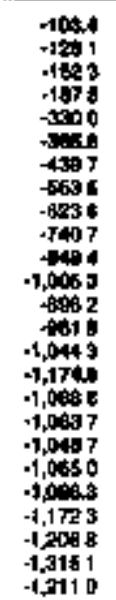 & 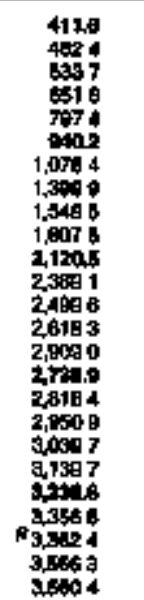 & 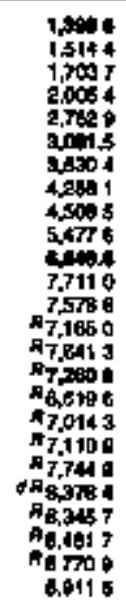 \\
\hline
\end{tabular}

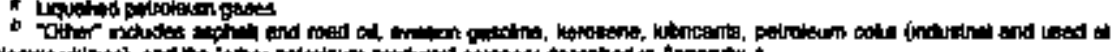

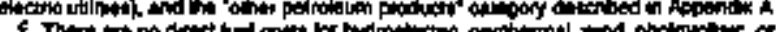

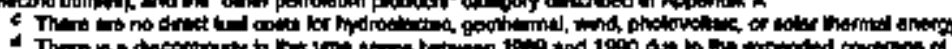

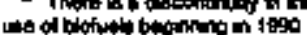

Arformed dete apondix $A$

管

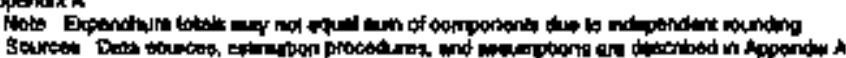

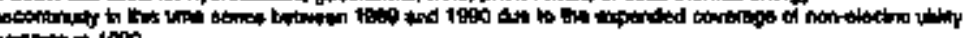




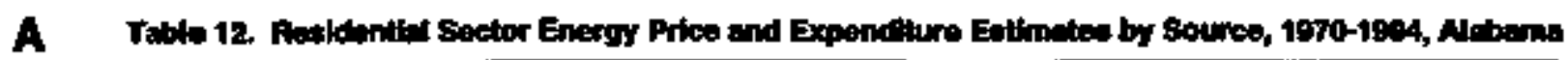

\begin{tabular}{|c|c|c|c|c|c|c|c|c|c|c|}
\hline & \multicolumn{8}{|c|}{ 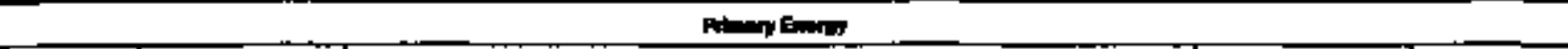 } & \multirow[b]{3}{*}{ Dy } & \multirow[b]{3}{*}{ ה } \\
\hline & \multirow[b]{2}{*}{ الוs } & \multirow[b]{2}{*}{$m$} & \multicolumn{4}{|c|}{ Panmang } & \multirow[b]{2}{*}{ mank } & \multirow[b]{2}{*}{ 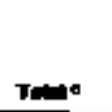 } & & \\
\hline & & & $\mathrm{F}=$ & rom & $\mathbf{m e}$ & החומד & & & & \\
\hline 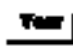 & \multicolumn{10}{|c|}{ 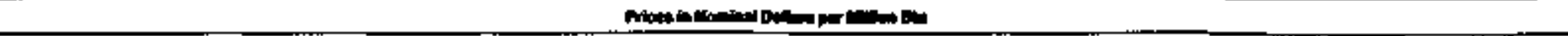 } \\
\hline \multirow[t]{2}{*}{ 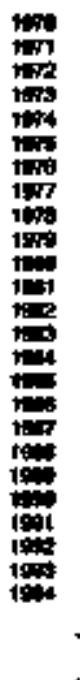 } & 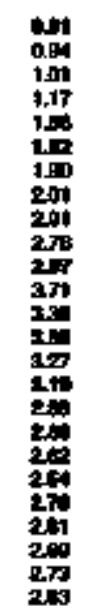 & 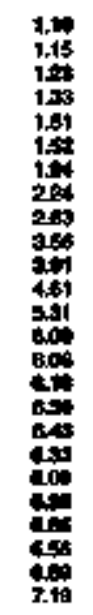 & 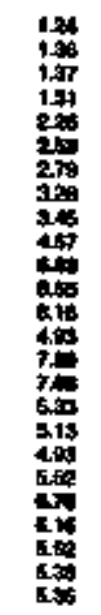 & 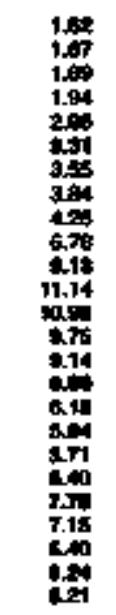 & 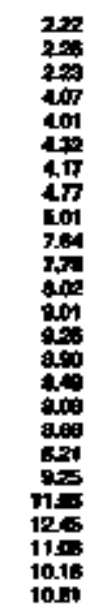 & 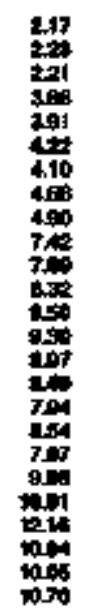 & 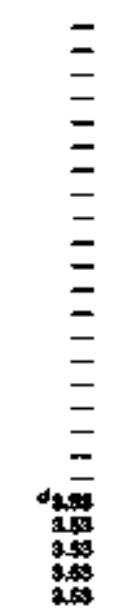 & 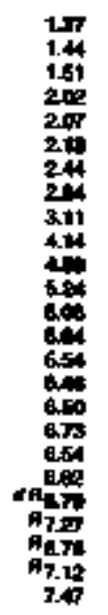 & 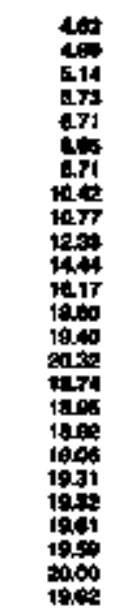 & 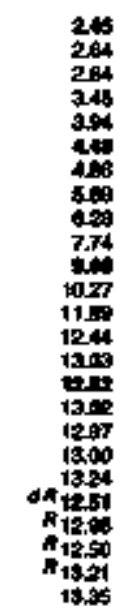 \\
\hline & \multicolumn{10}{|c|}{ 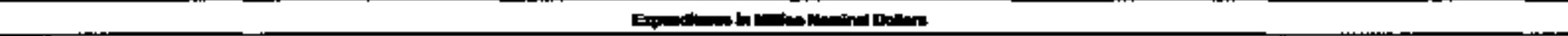 } \\
\hline 禹 & 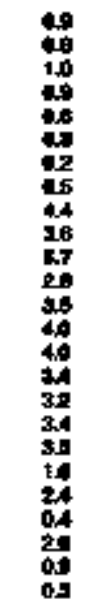 & 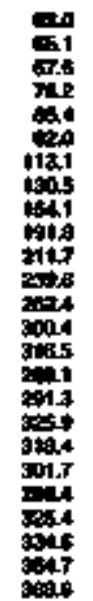 & 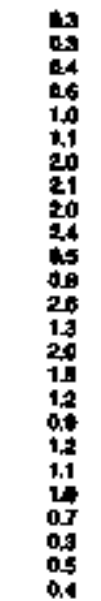 & 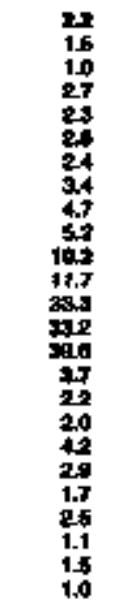 & 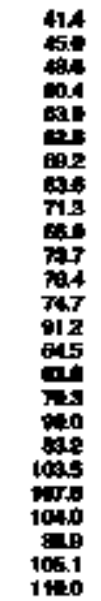 & 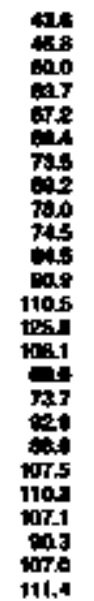 & $\begin{array}{l}= \\
= \\
= \\
= \\
= \\
= \\
= \\
= \\
= \\
= \\
= \\
= \\
9=5 \\
220 \\
301 \\
17 \pm\end{array}$ & 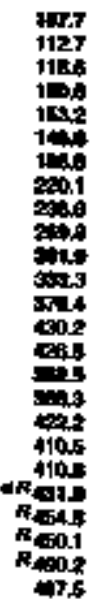 & 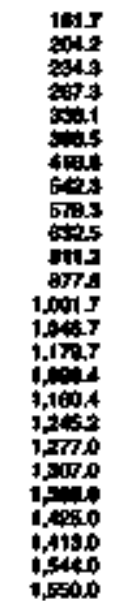 & 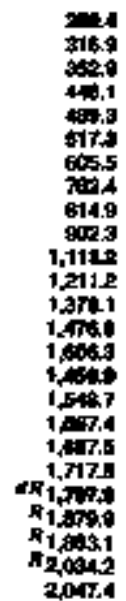 \\
\hline
\end{tabular}

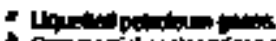

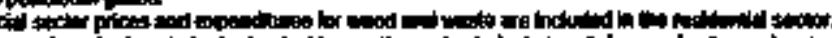

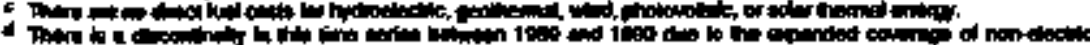

Printiod ons

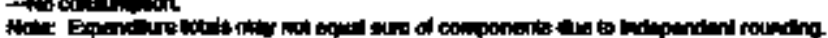

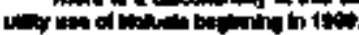

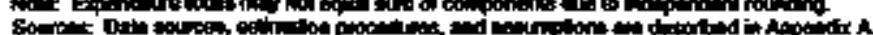




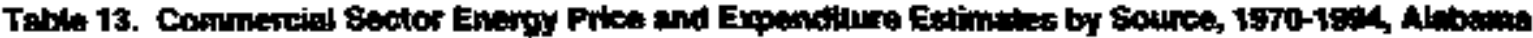

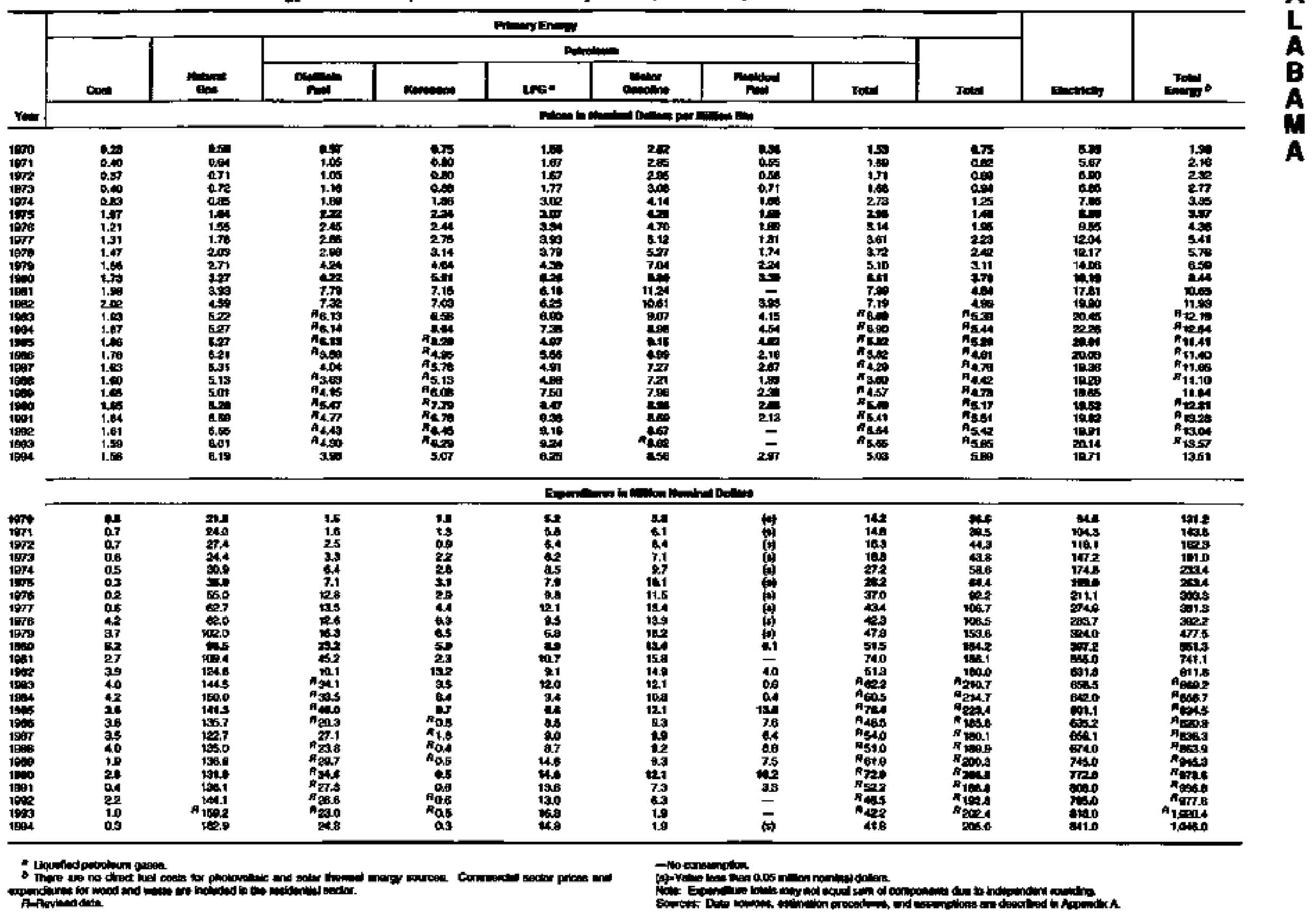


A Table 14. inclustrital Swetor Energy Price and Expenditure Eetimater by Source, 1970-1994, Alabsma

\begin{tabular}{|c|c|c|c|c|c|c|c|c|c|c|c|c|c|c|c|c|c|}
\hline \multirow[b]{4}{*}{$r=r$} & \multicolumn{15}{|c|}{ Minary Endif } & \multirow[b]{3}{*}{ Bnotiolvy } & \multirow[b]{3}{*}{ Gow } \\
\hline & \multicolumn{3}{|c|}{ con } & \multirow[b]{2}{*}{ avid } & \multicolumn{9}{|c|}{ Prtrolsm } & \multirow[b]{2}{*}{ Bathinc } & \multirow[b]{2}{*}{ Tot:=10 } & & \\
\hline & contant & cons & Tـ & & Arpher ond & مإ- & Feromene & LPQ: & Lubromita: & 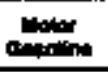 & minim & atase & Towat & & & & \\
\hline & \multicolumn{17}{|c|}{ 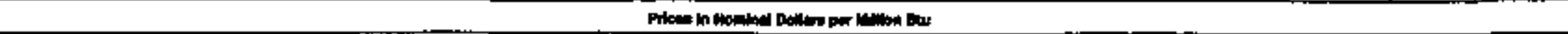 } \\
\hline \multirow[t]{2}{*}{ 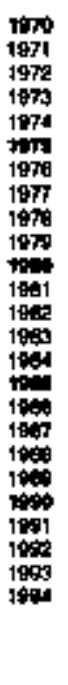 } & 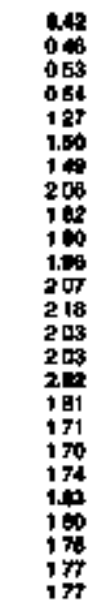 & 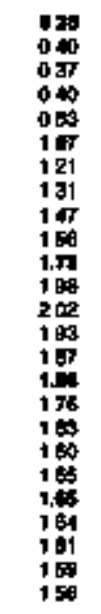 & 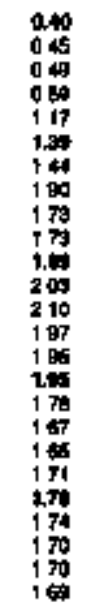 & 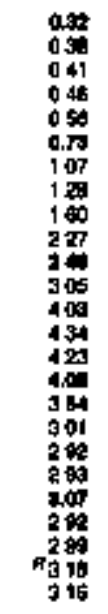 & 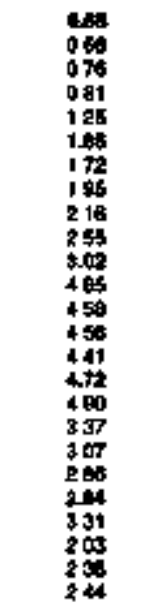 & 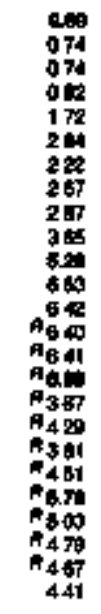 & 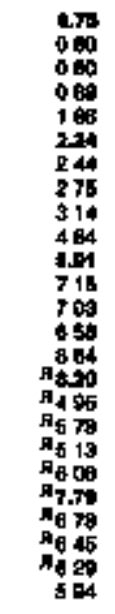 & 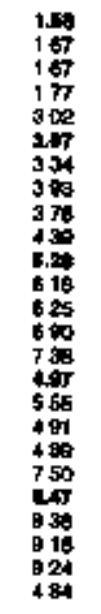 & 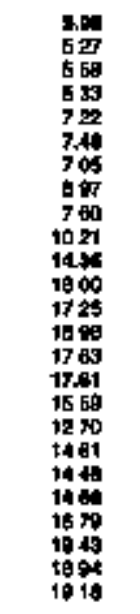 & 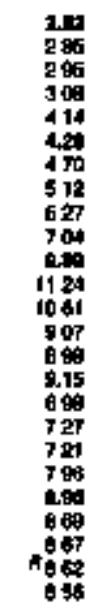 & 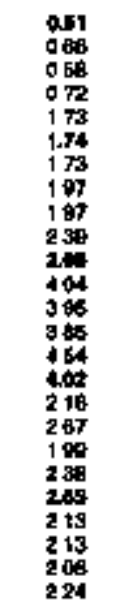 & 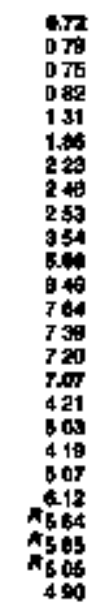 & 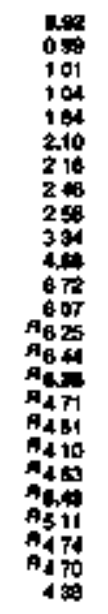 & 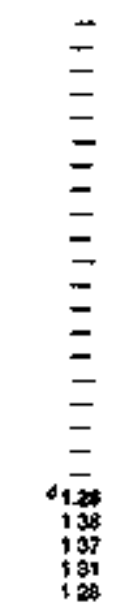 & 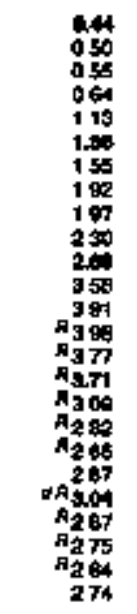 & 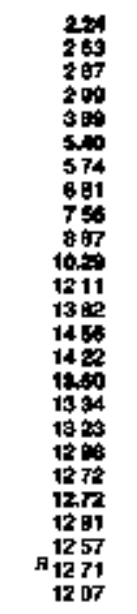 & 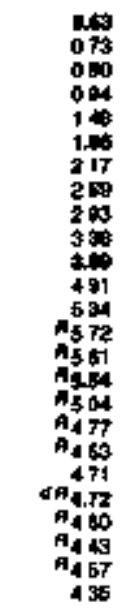 \\
\hline & \multicolumn{17}{|c|}{ 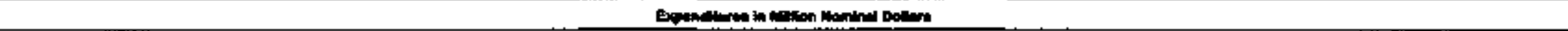 } \\
\hline 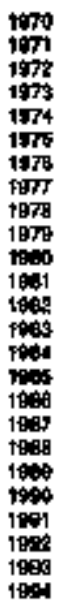 & 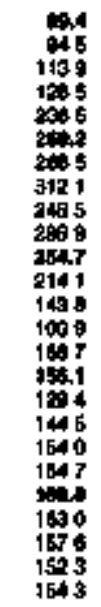 & 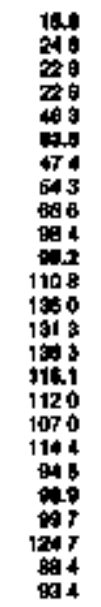 & 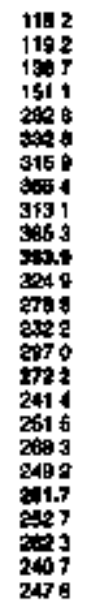 & 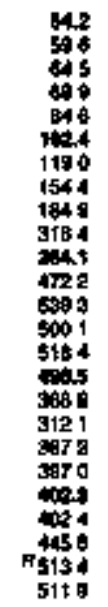 & 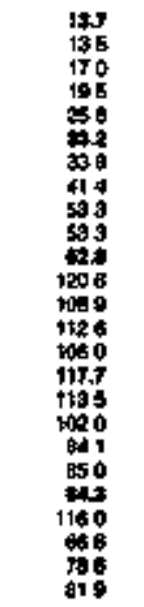 & 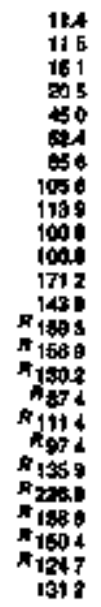 & 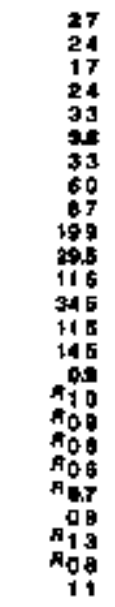 & 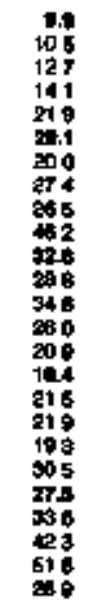 & 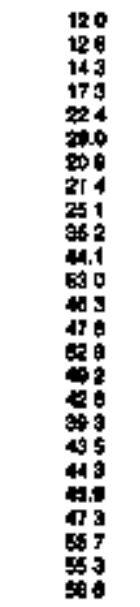 & 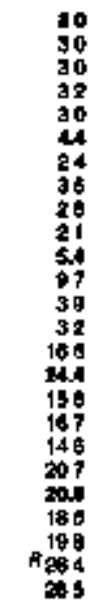 & 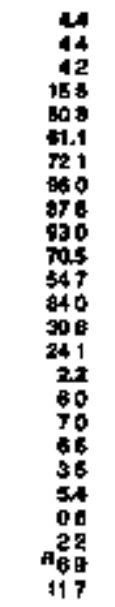 & 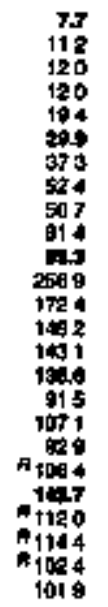 & 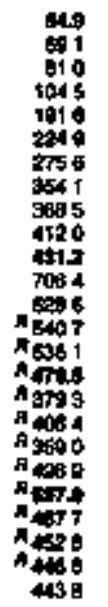 & 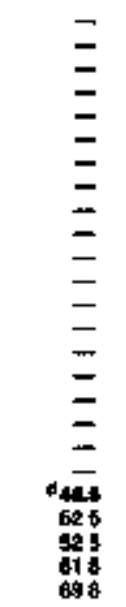 & 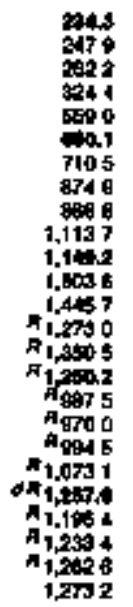 & 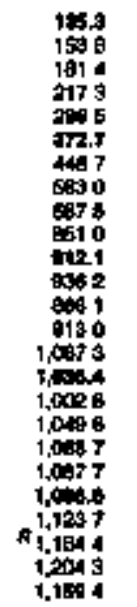 & 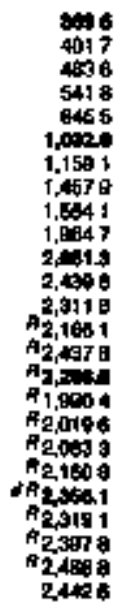 \\
\hline
\end{tabular}

: Lqueded potolinen pases

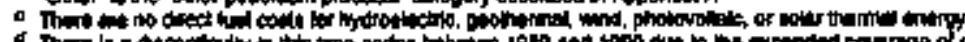

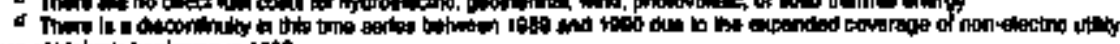

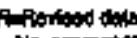

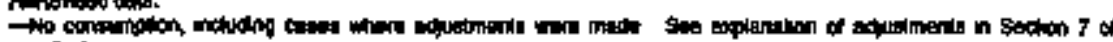
Apencia

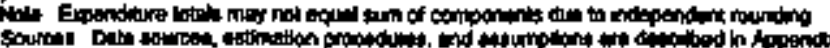

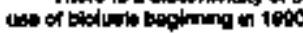


Table 16. Tramaportation Sector Energy Prios and Empendture Estlmates by Source, 1970-1984, Alabema

\begin{tabular}{|c|c|c|c|c|c|c|c|c|c|c|c|c|c|}
\hline \multirow[b]{4}{*}{ Kar } & \multicolumn{11}{|c|}{ Pntmey tinary } & \multirow[b]{3}{*}{ Evotididy } & \multirow[b]{3}{*}{ Eniming } \\
\hline & & \multirow[b]{2}{*}{ Nimured } & \multicolumn{8}{|c|}{ Phimoing } & \multirow[b]{2}{*}{ Totat } & & \\
\hline & cont & & Avinen & Potion & Pow & $\mathbf{L a *}$ & 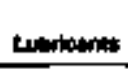 & 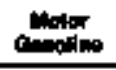 & Pinglowit & Toll & & & \\
\hline & \multicolumn{13}{|c|}{ 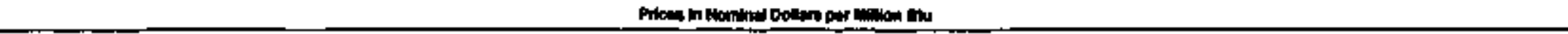 } \\
\hline \multirow[t]{2}{*}{ 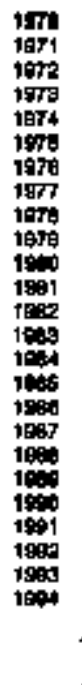 } & 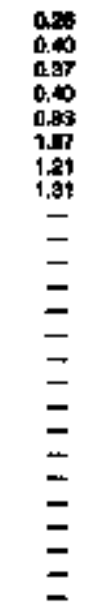 & $\begin{array}{l}= \\
= \\
= \\
= \\
= \\
= \\
= \\
= \\
= \\
= \\
= \\
\bar{z} \\
0.72 \\
\overline{0.70} \\
0.7\end{array}$ & 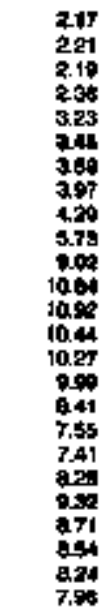 & 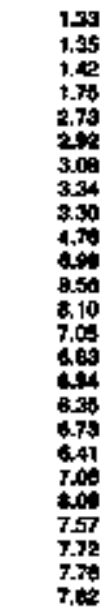 & 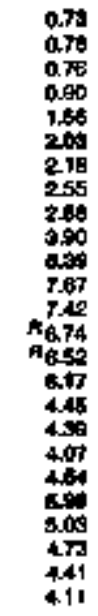 & 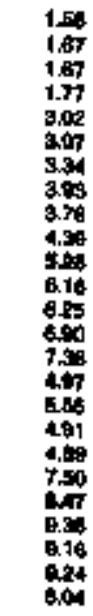 & 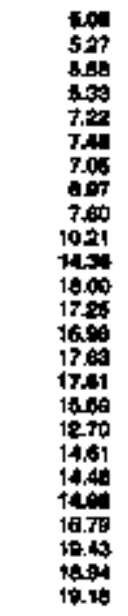 & 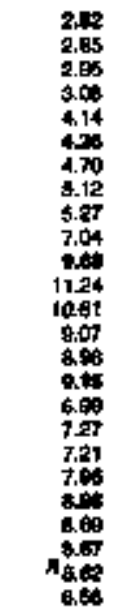 & 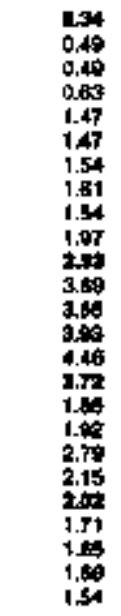 & 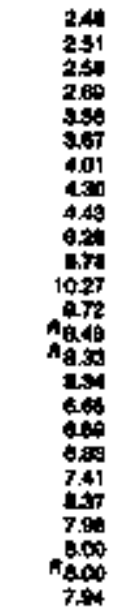 & 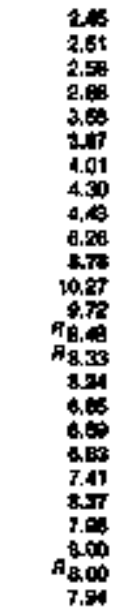 & $\begin{array}{l}= \\
= \\
= \\
= \\
= \\
= \\
= \\
= \\
= \\
= \\
= \\
= \\
=\end{array}$ & 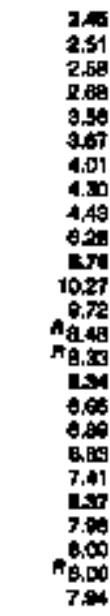 \\
\hline & \multicolumn{13}{|c|}{ 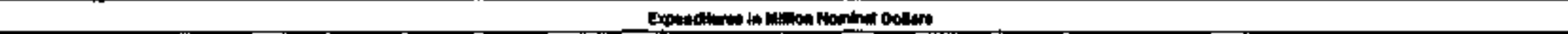 } \\
\hline 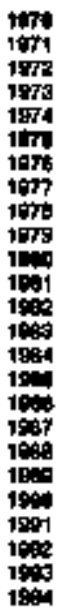 & $\begin{array}{l}0.1 \\
0.1 \\
0.2 \\
0.1 \\
0.1 \\
00 \\
0=0 \\
0=0 \\
= \\
= \\
= \\
= \\
= \\
= \\
= \\
= \\
= \\
= \\
=\end{array}$ & $\begin{array}{l}= \\
= \\
= \\
= \\
= \\
= \\
= \\
= \\
= \\
= \\
= \\
0 \\
0 \\
(65) \\
00 \\
0=0\end{array}$ & 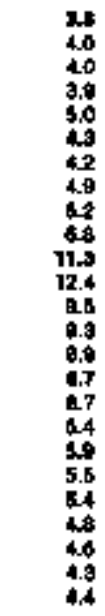 & 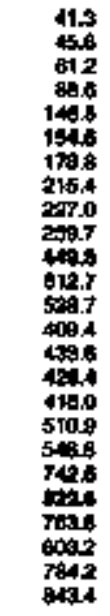 & 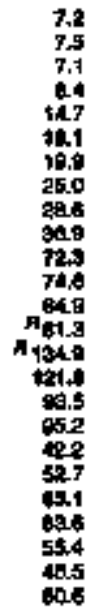 & 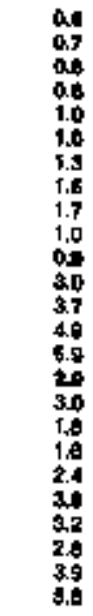 & 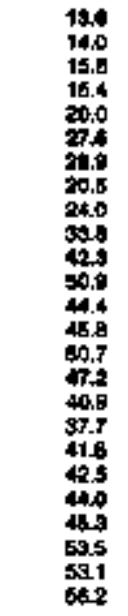 & 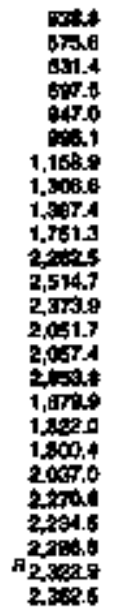 & 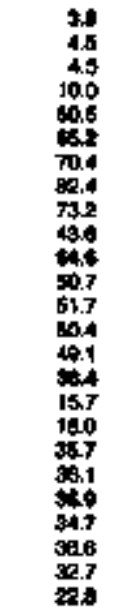 & 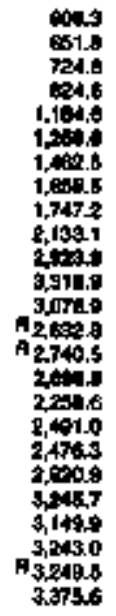 & 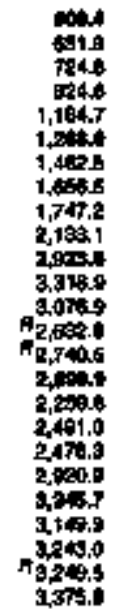 & $\begin{array}{l}= \\
= \\
= \\
= \\
= \\
= \\
= \\
= \\
= \\
= \\
= \\
= \\
=\end{array}$ & 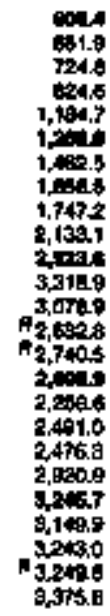 \\
\hline
\end{tabular}




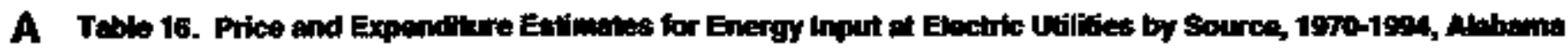

\begin{tabular}{|c|c|c|c|c|c|c|c|c|c|}
\hline \multirow[b]{3}{*}{ Yw } & \multirow[b]{2}{*}{ com } & \multirow[b]{2}{*}{ لגant } & \multicolumn{4}{|c|}{ manen. } & \multirow[b]{2}{*}{ ment } & \multirow[b]{2}{*}{ Niming } & \multirow[b]{2}{*}{ " } \\
\hline & & & "여 & 너 & תומה & notet & & & \\
\hline & \multicolumn{9}{|c|}{ 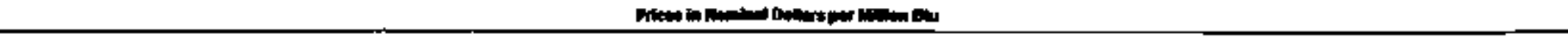 } \\
\hline \multirow[t]{2}{*}{ 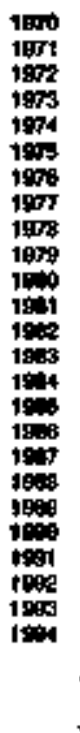 } & 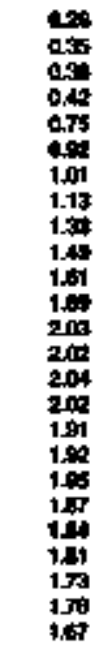 & 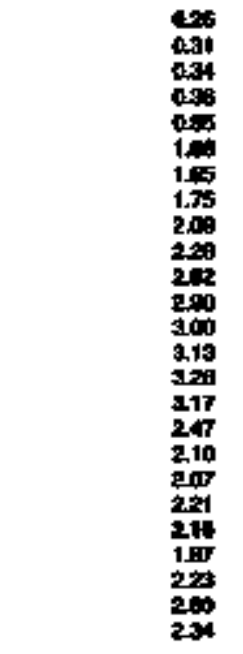 & $\begin{array}{l}= \\
= \\
= \\
20 \\
1.78 \\
1.78 \\
= \\
= \\
= \\
= \\
= \\
= \\
= \\
= \\
=\end{array}$ & 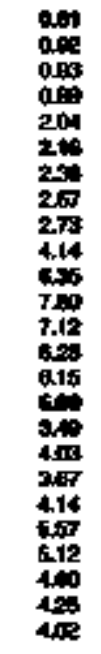 & 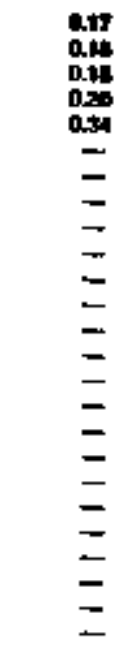 & 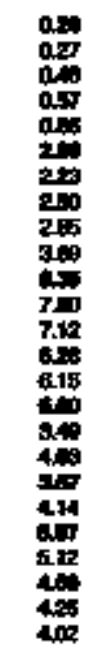 & 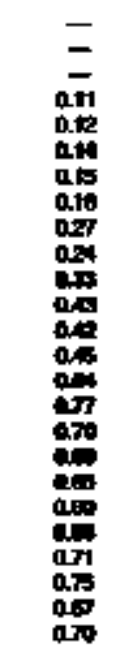 & $\begin{array}{l}= \\
= \\
= \\
= \\
= \\
= \\
= \\
= \\
= \\
= \\
= \\
= \\
= \\
=\end{array}$ & 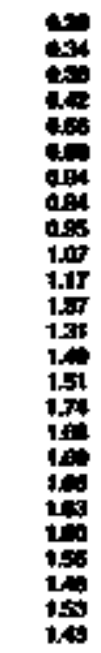 \\
\hline & \multicolumn{9}{|c|}{ 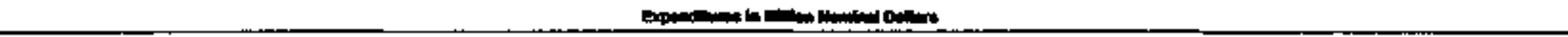 } \\
\hline 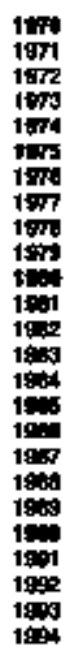 & 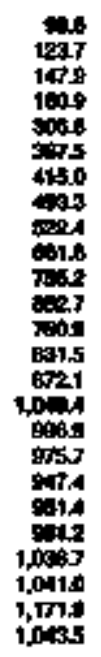 & 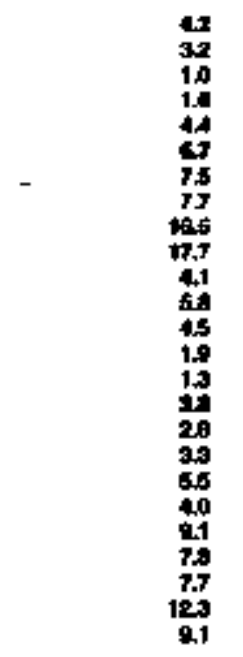 & $\begin{array}{l}z \\
z \\
= \\
9.5 \\
1.5 \\
2.1 \\
64 \\
= \\
= \\
= \\
= \\
= \\
= \\
= \\
= \\
=\end{array}$ & 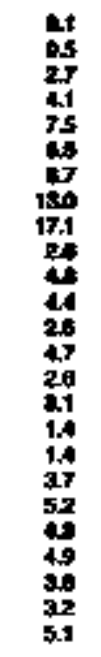 & $\begin{array}{l}0 \\
0 \\
0=0 \\
0= \\
= \\
= \\
= \\
= \\
= \\
= \\
= \\
= \\
= \\
= \\
= \\
= \\
=\end{array}$ & 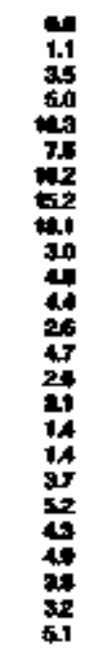 & 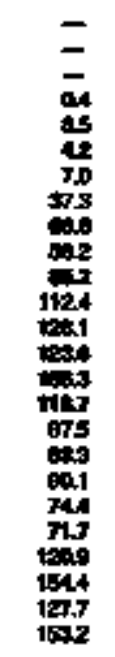 & $\begin{array}{l}= \\
= \\
= \\
= \\
= \\
= \\
= \\
= \\
= \\
= \\
= \\
= \\
= \\
=\end{array}$ & 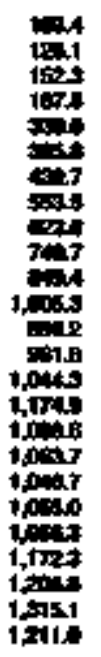 \\
\hline
\end{tabular}

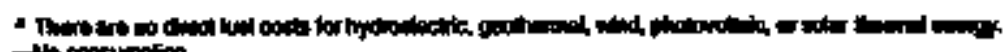
tis consumpion.

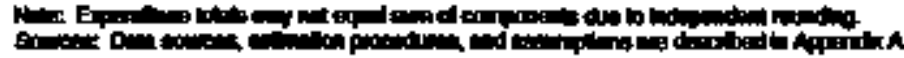




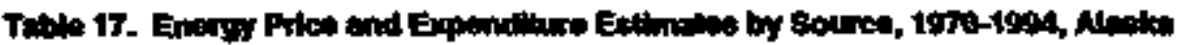

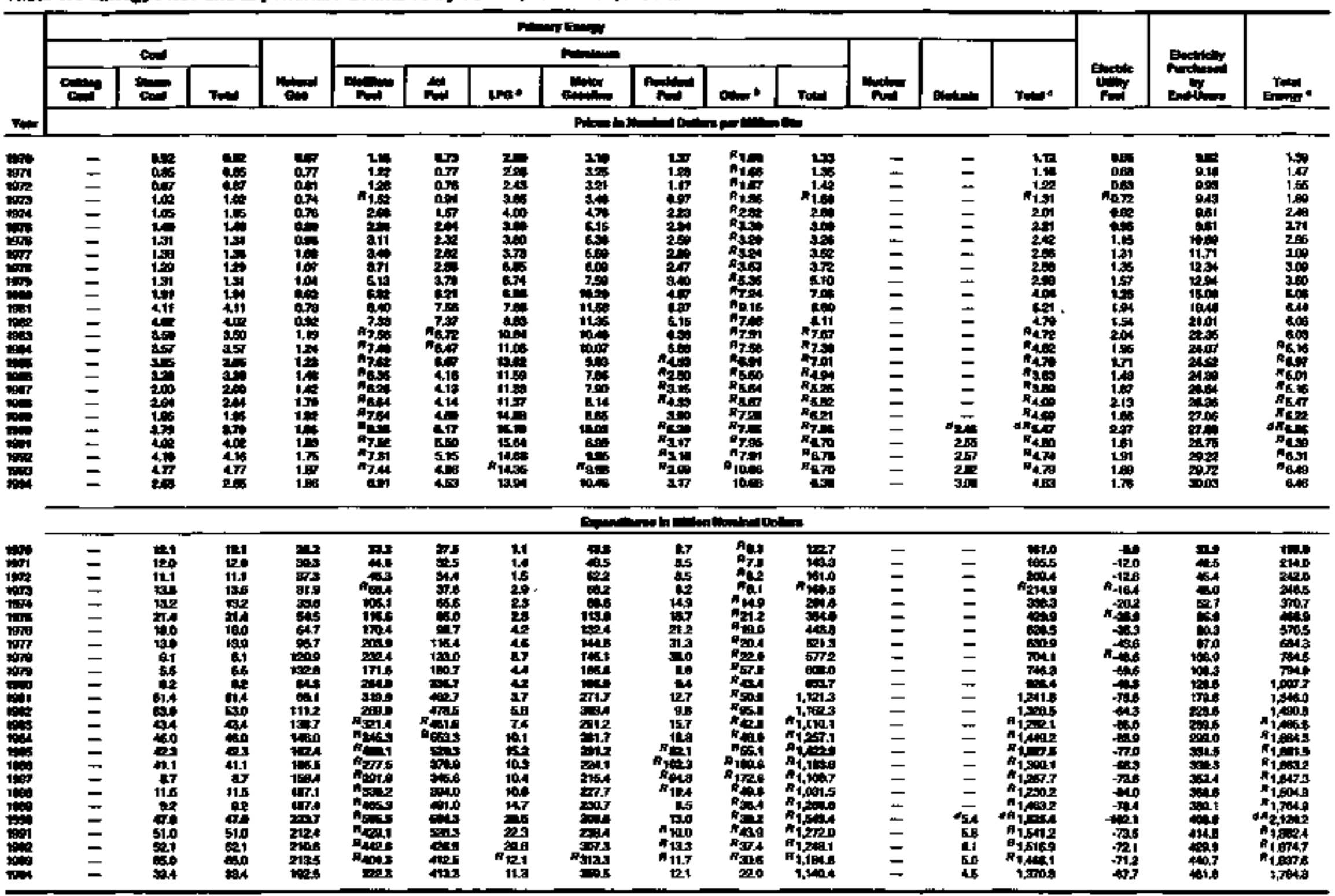

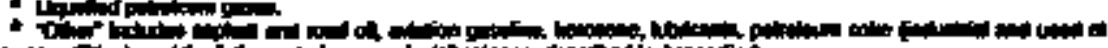

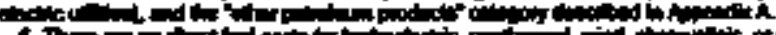

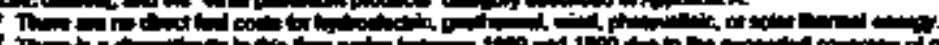

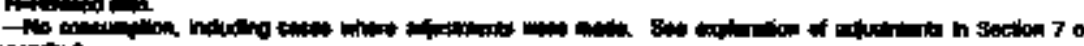

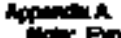

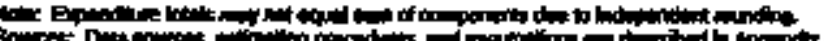

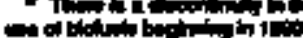


A Table 18. Aasidentle Sector Enmgy Price and Expenditurs Estimetes by Source, 1970-1994, Alagika

\begin{tabular}{|c|c|c|c|c|c|c|c|c|c|c|}
\hline \multirow[b]{4}{*}{$y=$} & \multicolumn{8}{|c|}{ Alomeng Enowity } & \multirow[b]{3}{*}{ Besturity } & \multirow[b]{3}{*}{ 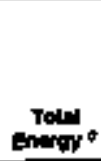 } \\
\hline & \multirow[b]{2}{*}{ can } & \multirow[b]{2}{*}{ wis } & \multicolumn{4}{|c|}{ Polromenn } & \multirow[b]{2}{*}{ ofohminto } & \multirow[b]{2}{*}{ Tokter } & & \\
\hline & & & Pats & Konant & LPa* & 7000 & & & & \\
\hline & \multicolumn{10}{|c|}{ 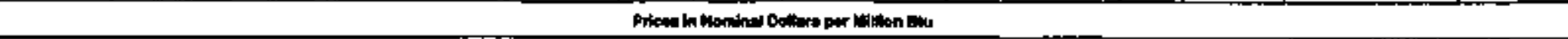 } \\
\hline \multirow[t]{2}{*}{ 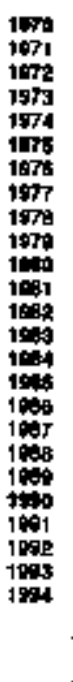 } & 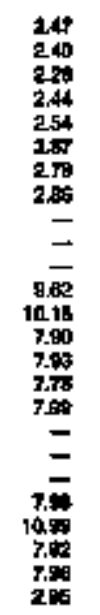 & 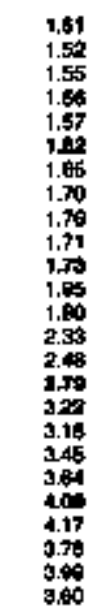 & 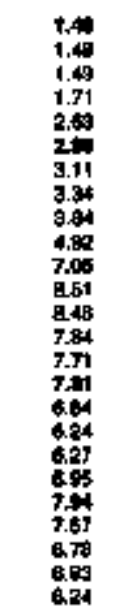 & 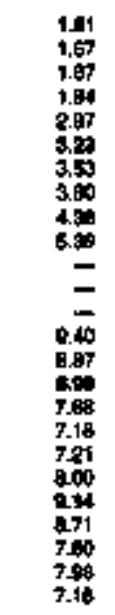 & 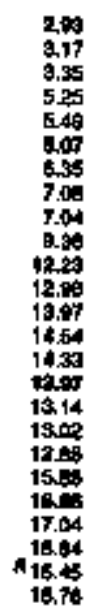 & 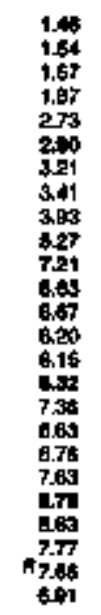 & $\begin{array}{l}= \\
= \\
= \\
= \\
= \\
= \\
= \\
= \\
= \\
= \\
\bar{z} \\
0.75 \\
4.75 \\
4.75 \\
4.75\end{array}$ & 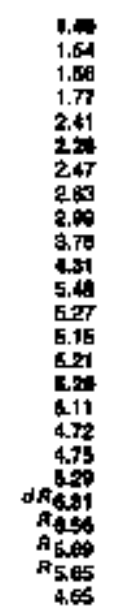 & 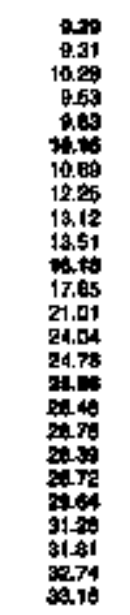 & 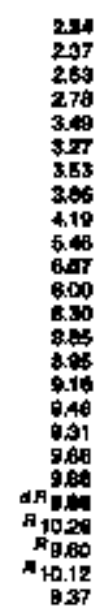 \\
\hline & \multicolumn{10}{|c|}{ 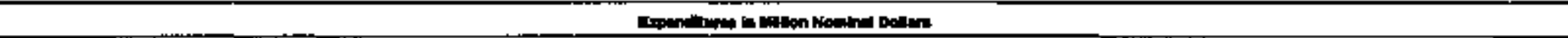 } \\
\hline 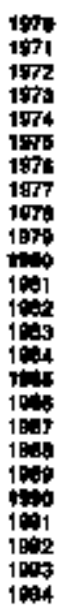 & 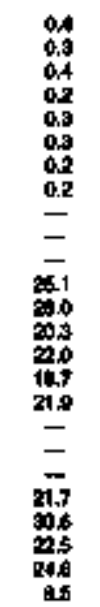 & 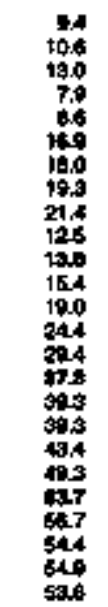 & 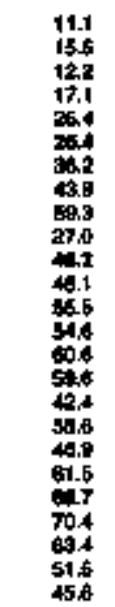 & 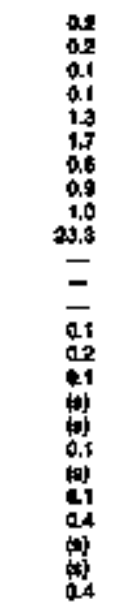 & 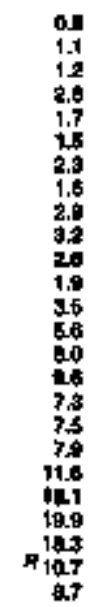 & 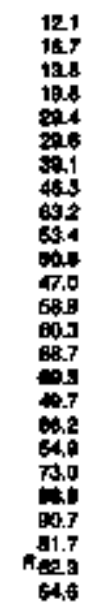 & 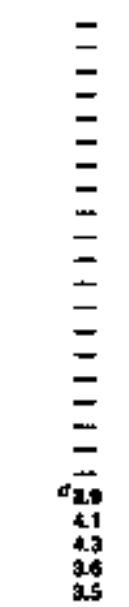 & 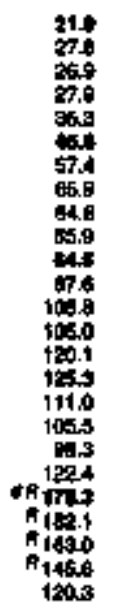 & 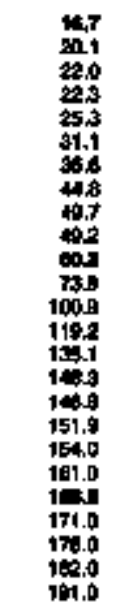 & 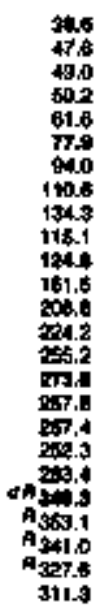 \\
\hline
\end{tabular}

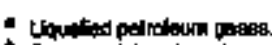

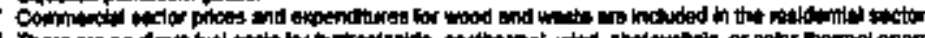

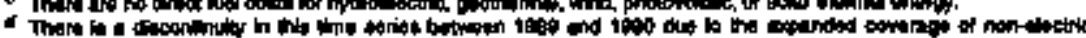

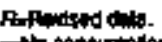

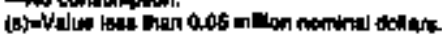

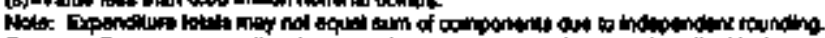

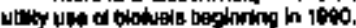


Table 19. Commercial Sector Energy Price and Expenditure Enilmatea by Soure, 1970-1934, Alegke

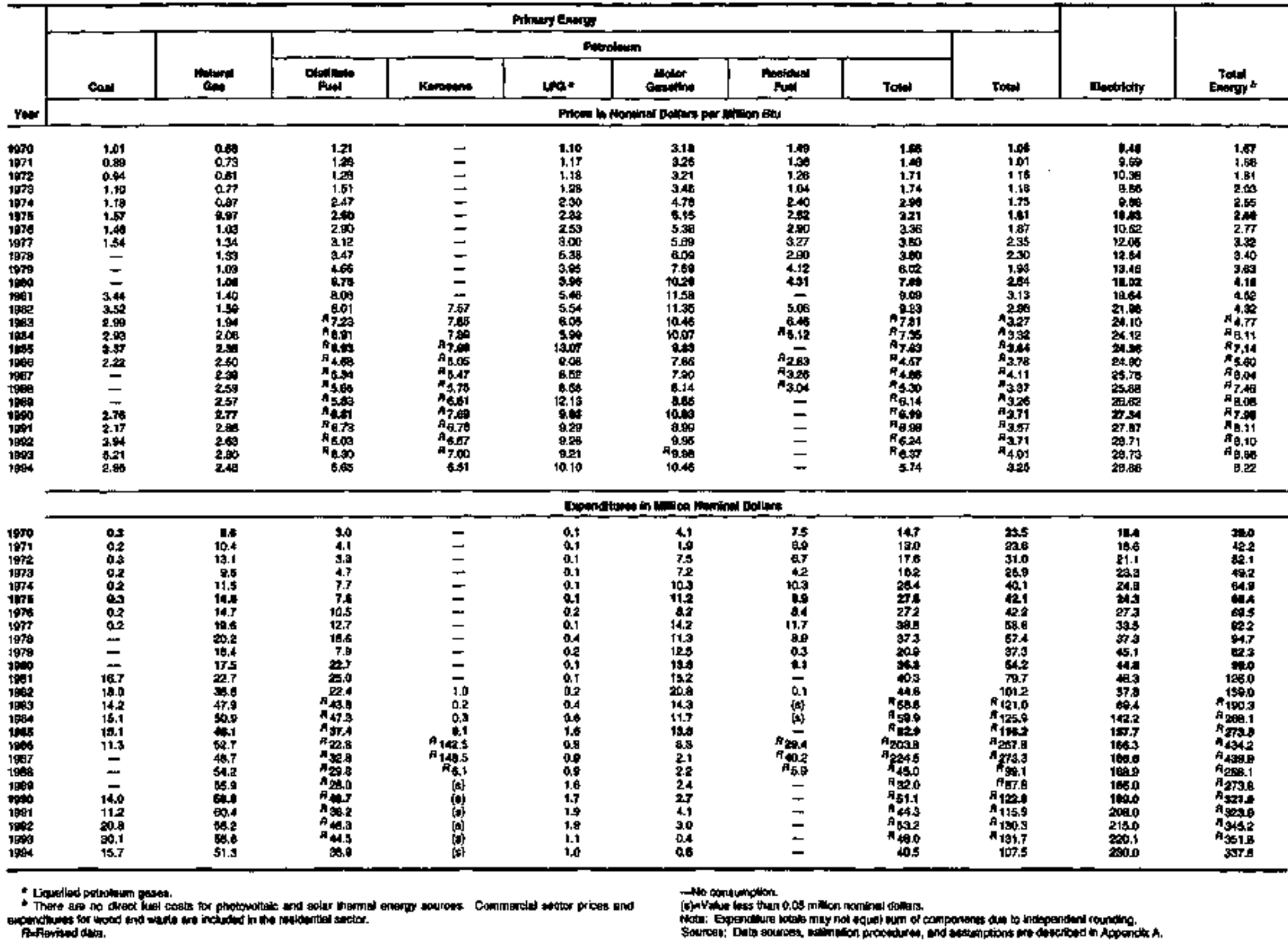




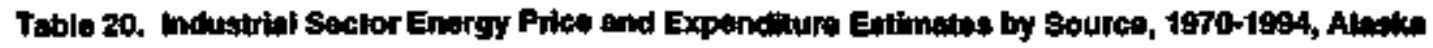

A

$\mathbf{S}$

$\mathbf{A}$

\begin{tabular}{|c|c|c|c|c|c|c|c|c|c|c|c|c|c|c|c|c|c|}
\hline & \multicolumn{15}{|c|}{ Ping } & \multirow[b]{3}{*}{ Entan } & \multirow[b]{3}{*}{ : } \\
\hline & \multicolumn{3}{|c|}{ con } & \multirow[b]{2}{*}{ the } & \multicolumn{9}{|c|}{ Putrolen:en } & \multirow[b]{2}{*}{ 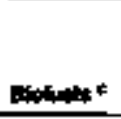 } & \multirow[b]{2}{*}{ Totey I } & & \\
\hline & Complo & (1) & Thell & & 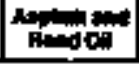 & 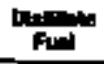 & 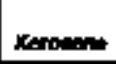 & ING: & 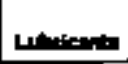 & " & 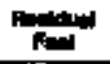 & Det: & 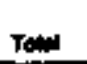 & & & & \\
\hline har & \multicolumn{17}{|c|}{ 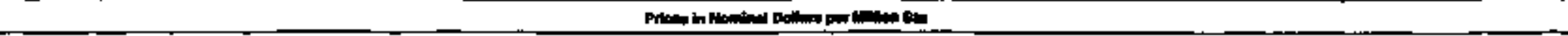 } \\
\hline 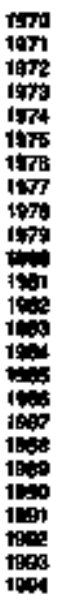 & $\begin{array}{l}= \\
= \\
= \\
= \\
= \\
= \\
= \\
= \\
= \\
= \\
= \\
= \\
=\end{array}$ & $\begin{array}{l}100 \\
0.80 \\
0.04 \\
1.10 \\
1.10 \\
1.5 \\
1.40 \\
= \\
= \\
= \\
= \\
= \\
= \\
= \\
= \\
= \\
= \\
5.21 \\
2.10\end{array}$ & 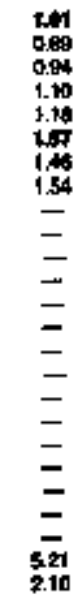 & 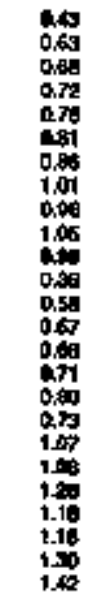 & 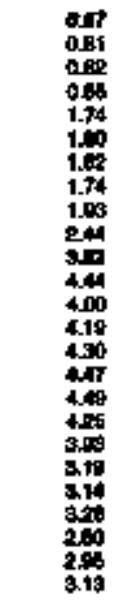 & 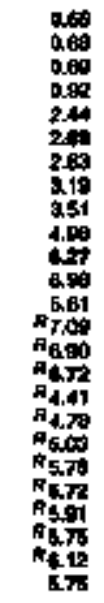 & 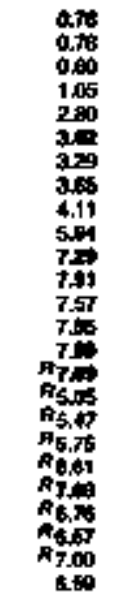 & 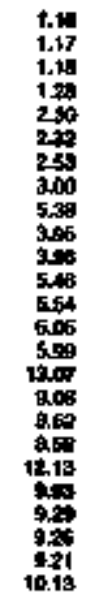 & 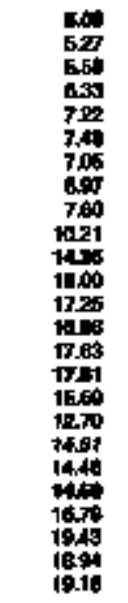 & 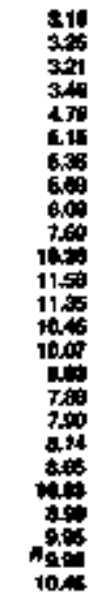 & 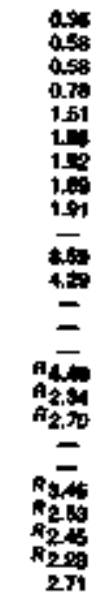 & 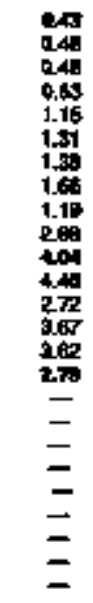 & 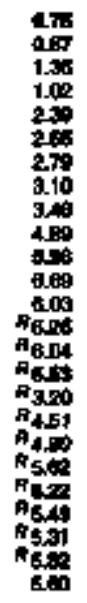 & $\begin{array}{l}= \\
= \\
= \\
= \\
= \\
= \\
= \\
= \\
= \\
\bar{z} \\
0 \\
1.24 \\
1.24 \\
1.94\end{array}$ & 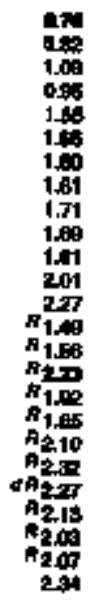 & 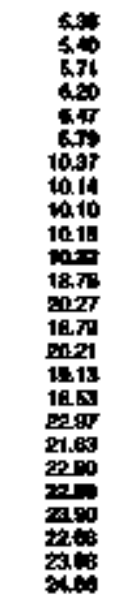 & 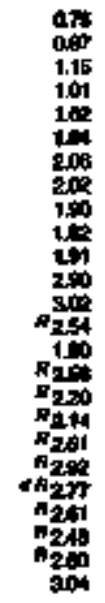 \\
\hline
\end{tabular}

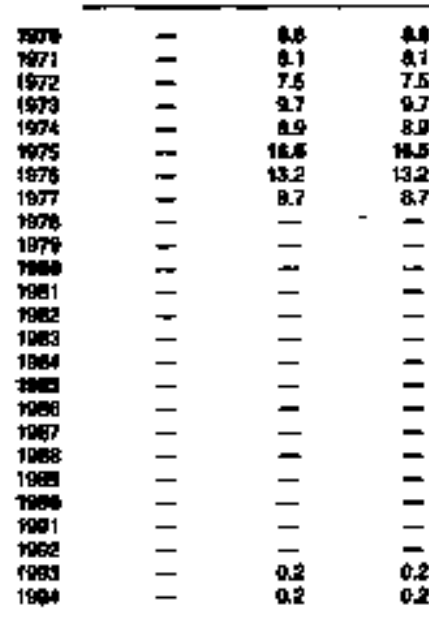

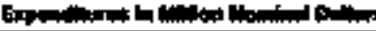

1.1
69
6.5
7.4

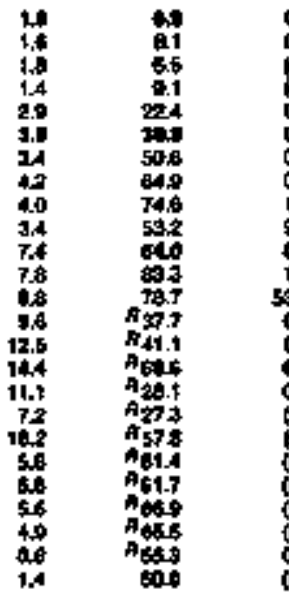

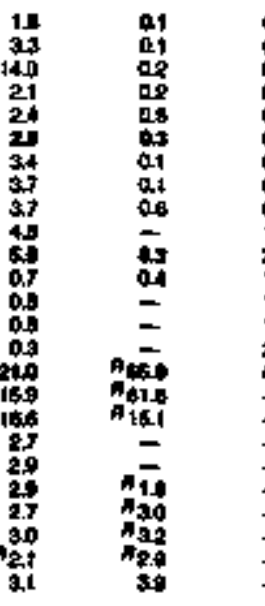

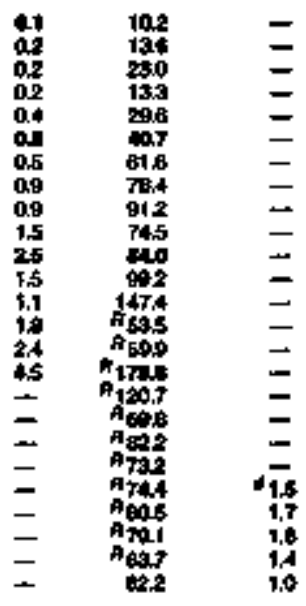

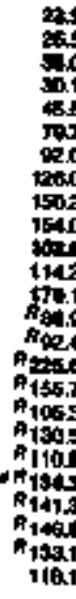

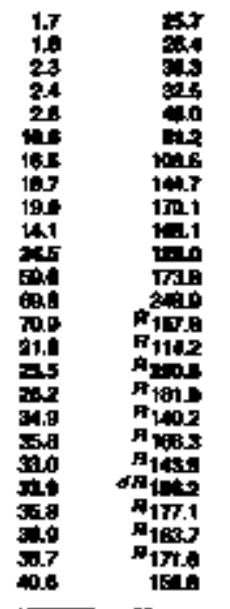




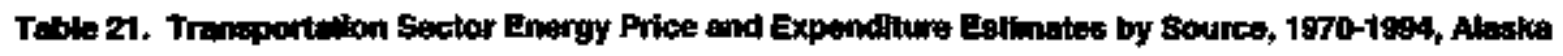

\begin{tabular}{|c|c|c|c|c|c|c|c|c|c|c|c|c|c|}
\hline \multirow[b]{4}{*}{$\mathbf{Y}_{\boldsymbol{n}}$} & \multicolumn{11}{|c|}{ Fitany Em } & \multirow[b]{3}{*}{ Inechituty } & \multirow[b]{3}{*}{ Tost } \\
\hline & \multirow[b]{2}{*}{ البس } & \multirow[b]{2}{*}{ tim } & \multicolumn{8}{|c|}{ 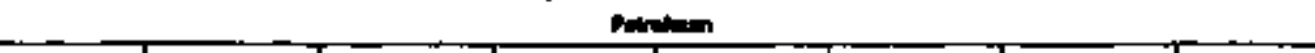 } & \multirow[b]{2}{*}{ Toter } & & \\
\hline & & & 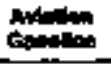 & 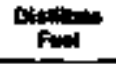 & لئ & tre. & 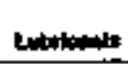 & rinter & 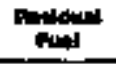 & Tan & & & \\
\hline & \multicolumn{13}{|c|}{ 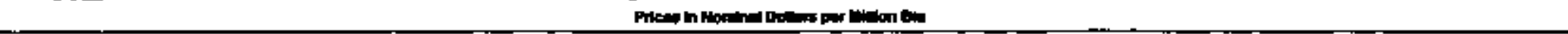 } \\
\hline \multirow[t]{2}{*}{ 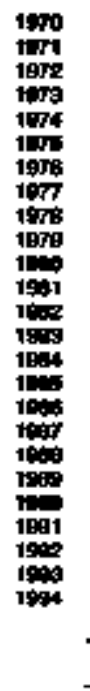 } & $\begin{array}{l}1.90 \\
0.60 \\
0.04 \\
1.10 \\
1.50 \\
1.59 \\
1.90 \\
= \\
= \\
= \\
= \\
= \\
= \\
= \\
= \\
= \\
= \\
=\end{array}$ & $\begin{array}{l}= \\
= \\
= \\
= \\
= \\
= \\
= \\
= \\
= \\
= \\
= \\
= \\
= \\
=\end{array}$ & 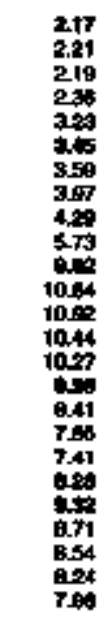 & 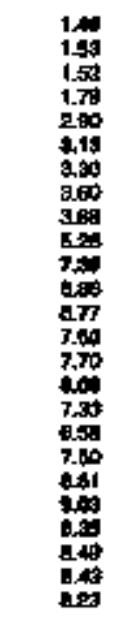 & 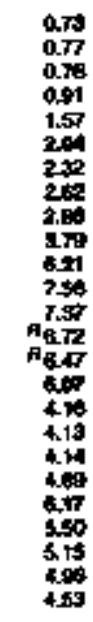 & 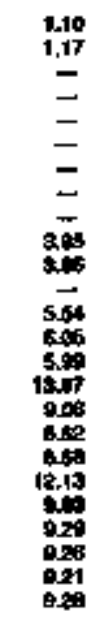 & 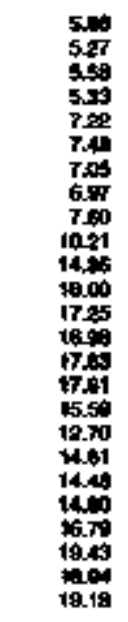 & 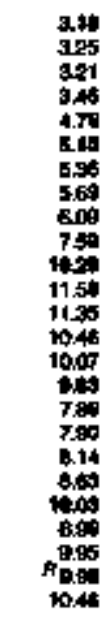 & 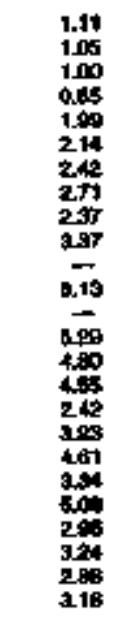 & 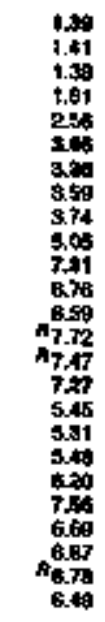 & 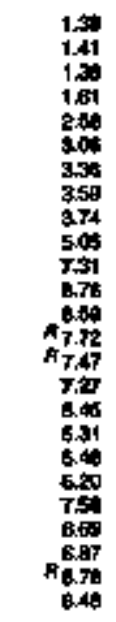 & $\begin{array}{l}= \\
= \\
= \\
= \\
= \\
= \\
= \\
= \\
= \\
= \\
= \\
= \\
= \\
= \\
=\end{array}$ & 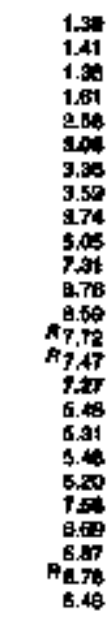 \\
\hline & \multicolumn{13}{|c|}{ 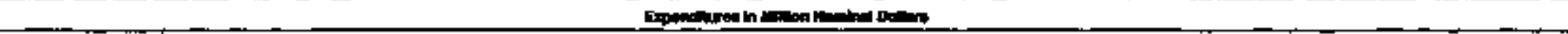 } \\
\hline 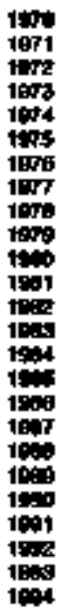 & $\begin{array}{l}0 \\
0 \\
0 \\
0 \\
0 \\
= \\
= \\
= \\
= \\
= \\
= \\
= \\
= \\
= \\
=\end{array}$ & $\begin{array}{l}= \\
= \\
= \\
= \\
= \\
= \\
= \\
= \\
= \\
= \\
= \\
= \\
= \\
= \\
=\end{array}$ & 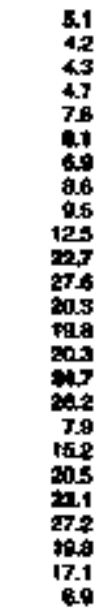 & 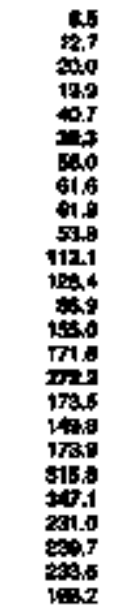 & 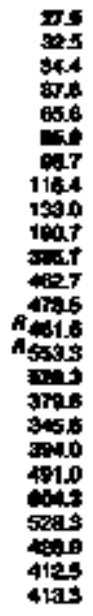 & 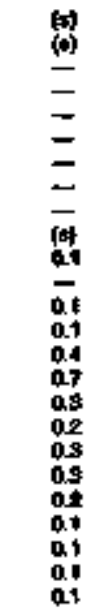 & 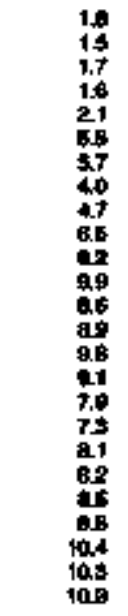 & 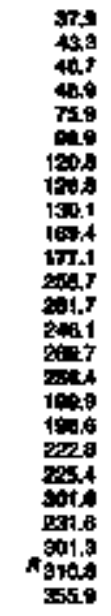 & 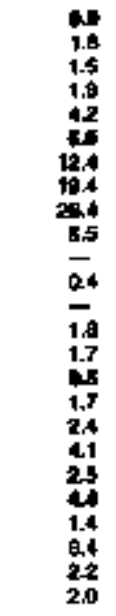 & 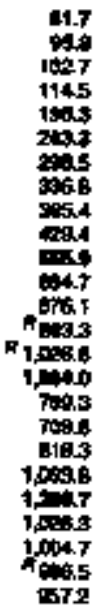 & 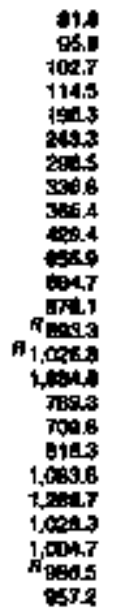 & $\begin{array}{l}= \\
= \\
= \\
= \\
= \\
= \\
= \\
= \\
= \\
= \\
= \\
= \\
= \\
= \\
=\end{array}$ & 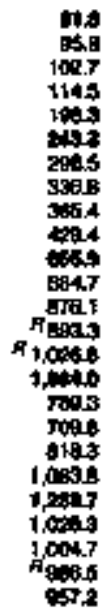 \\
\hline
\end{tabular}

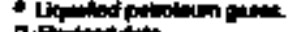

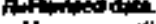
apoinotion

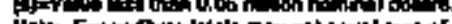

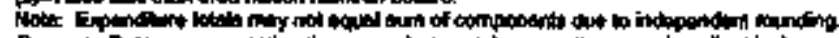

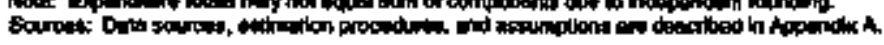


A Table 22. Prlee and Expenditure Estimates for Energy Input at Electric Utillites by Source, 1970-1994, Alaska

\begin{tabular}{|c|c|c|c|c|c|c|c|c|c|}
\hline \multirow[b]{3}{*}{ reap } & & \multirow[b]{2}{*}{ Mround } & \multicolumn{4}{|c|}{ Puroming } & \multirow[b]{2}{*}{ Pusul } & \multirow[b]{2}{*}{ Botheme } & \multirow[b]{2}{*}{ trolsy, } \\
\hline & cos & & thens & 내 & Dovolum & Total & & & \\
\hline & \multicolumn{9}{|c|}{ 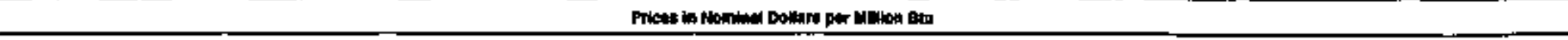 } \\
\hline \multirow[t]{2}{*}{ 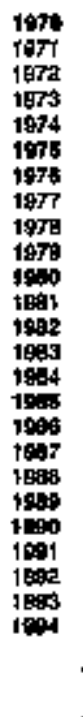 } & 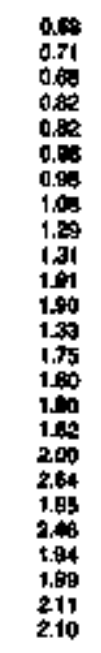 & 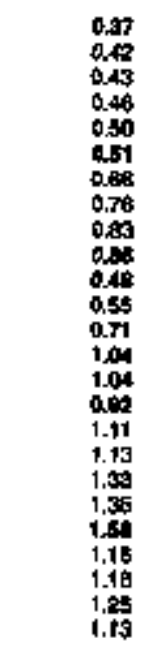 & 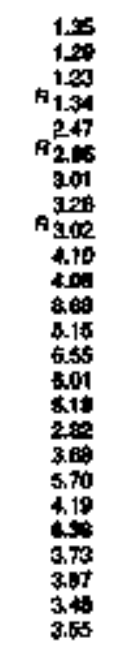 & 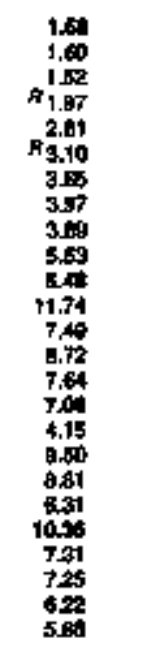 & $\begin{array}{l}= \\
z \\
z \\
z \\
= \\
z \\
= \\
= \\
= \\
= \\
= \\
= \\
=\end{array}$ & 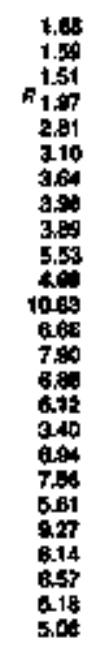 & $\begin{array}{l}= \\
= \\
= \\
= \\
= \\
= \\
= \\
= \\
= \\
= \\
= \\
= \\
=\end{array}$ & $\begin{array}{l}= \\
= \\
= \\
= \\
= \\
= \\
= \\
= \\
= \\
= \\
= \\
=\end{array}$ & 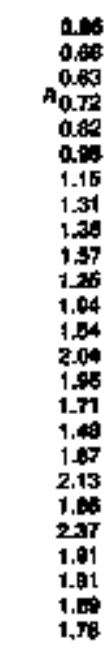 \\
\hline & \multicolumn{9}{|c|}{ 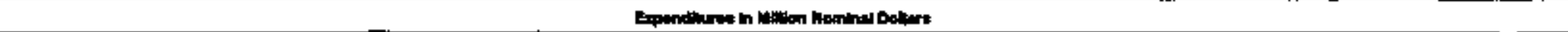 } \\
\hline 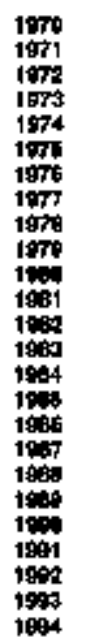 & 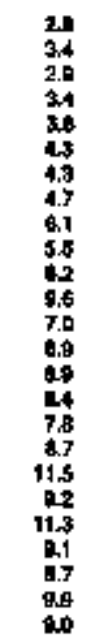 & 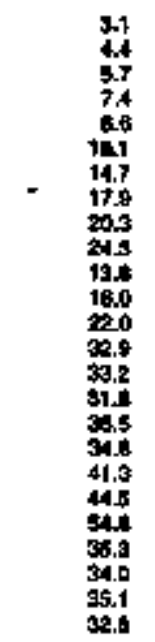 & 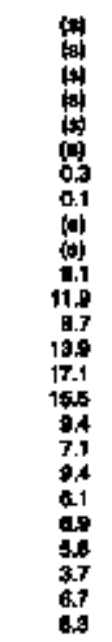 & 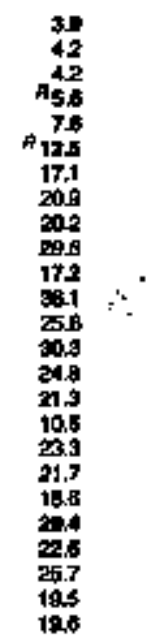 & $\begin{array}{l}z \\
z \\
z \\
z \\
z \\
= \\
= \\
= \\
= \\
= \\
= \\
= \\
=\end{array}$ & 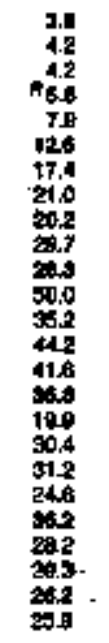 & $\begin{array}{l}z \\
= \\
= \\
= \\
= \\
= \\
= \\
= \\
= \\
= \\
= \\
= \\
=\end{array}$ & $\begin{array}{l}= \\
= \\
= \\
= \\
= \\
= \\
= \\
= \\
= \\
= \\
= \\
= \\
=\end{array}$ & 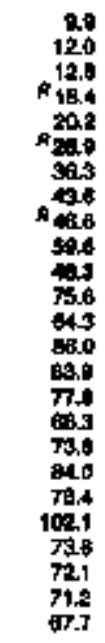 \\
\hline
\end{tabular}

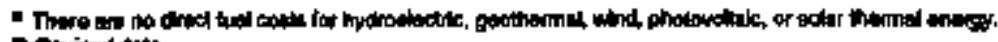
$\rightarrow 0$

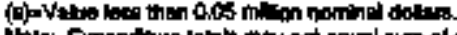

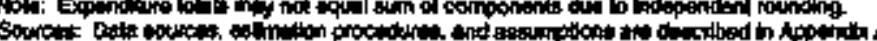


Tab6 23. Energy Price and Expendture Entim:etes by Source, 1970-1994, Artzon:

\begin{tabular}{|c|c|c|c|c|c|c|c|c|c|c|c|c|c|c|c|c|c|}
\hline \multirow[b]{4}{*}{ Yow } & \multicolumn{14}{|c|}{ Mnery then } & \multirow{3}{*}{ trictic } & \multirow{3}{*}{ 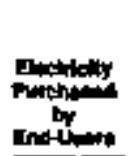 } & \multirow[b]{3}{*}{ Totist: } \\
\hline & \multicolumn{3}{|c|}{ Cost } & \multirow[b]{2}{*}{ 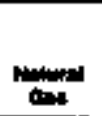 } & \multicolumn{7}{|c|}{ 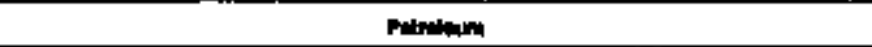 } & \multirow[b]{2}{*}{ Full } & \multirow[b]{2}{*}{ Motume } & \multirow[b]{2}{*}{ retoda } & & & \\
\hline & 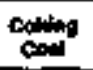 & con & Totw & & מה & fw & LPo: & novor & 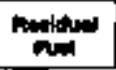 & tomen" & Totel & & & & & & \\
\hline & \multicolumn{17}{|c|}{ 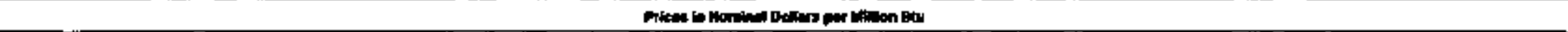 } \\
\hline \multirow[t]{2}{*}{ 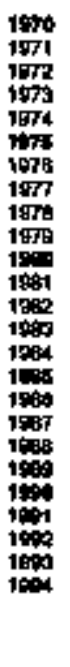 } & $\begin{array}{l}= \\
= \\
= \\
= \\
= \\
= \\
= \\
= \\
= \\
= \\
= \\
= \\
= \\
= \\
=\end{array}$ & 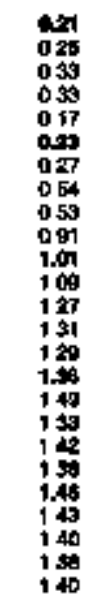 & 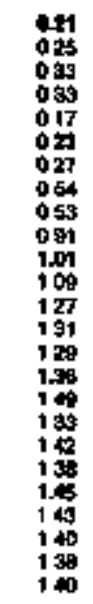 & 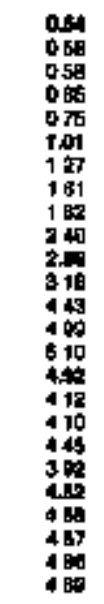 & 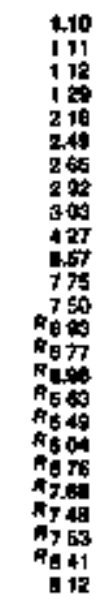 & 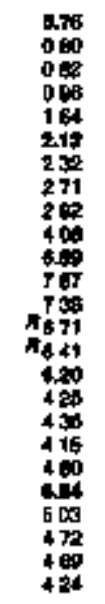 & 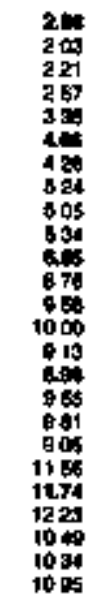 & 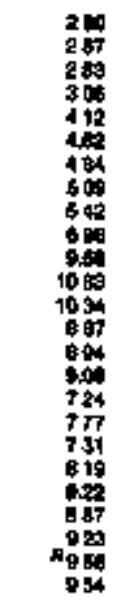 & 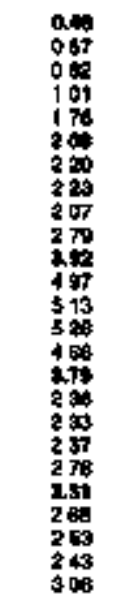 & 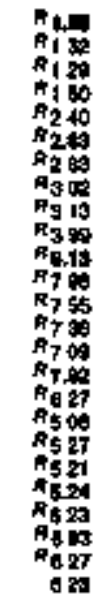 & 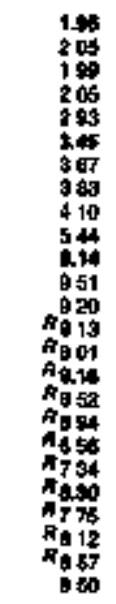 & 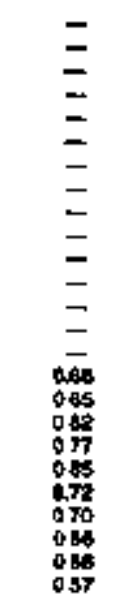 & $\begin{array}{l}= \\
= \\
= \\
= \\
= \\
= \\
= \\
= \\
= \\
= \\
= \\
= \\
= \\
922 \\
321 \\
324 \\
304\end{array}$ & 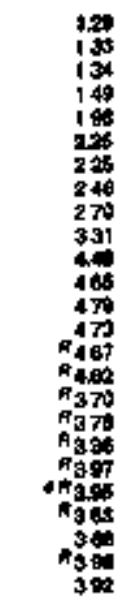 & 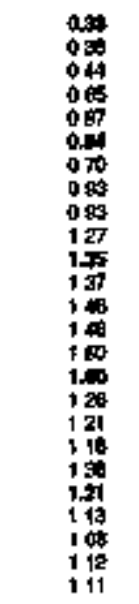 & 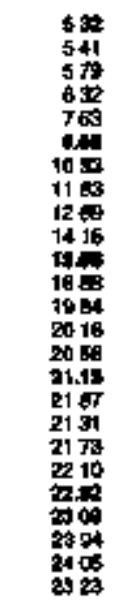 & 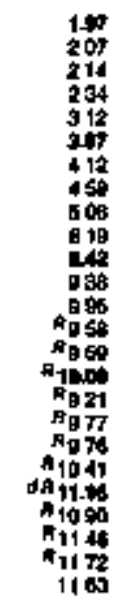 \\
\hline & \multicolumn{17}{|c|}{ 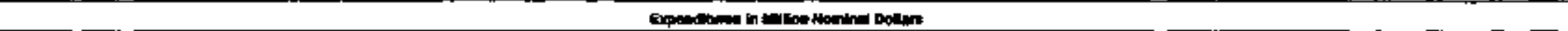 } \\
\hline 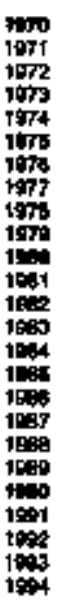 & $\begin{array}{l}= \\
= \\
= \\
= \\
= \\
= \\
= \\
= \\
= \\
= \\
= \\
= \\
=\end{array}$ & 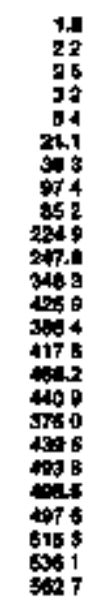 & 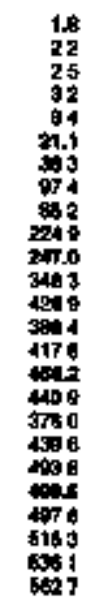 & 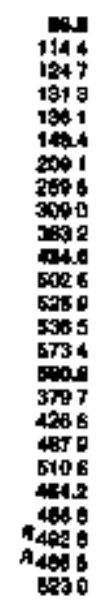 & 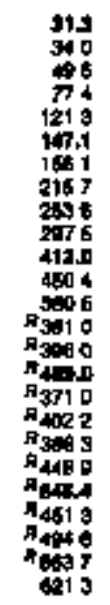 & 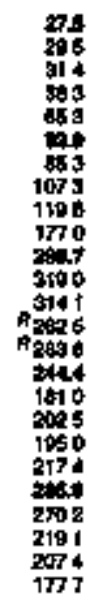 & 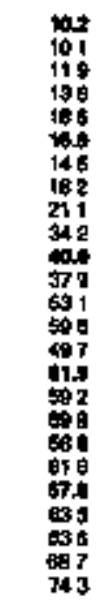 & 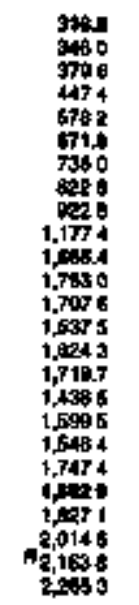 & 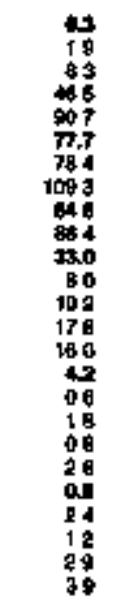 & 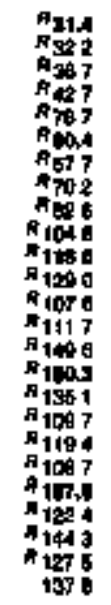 & 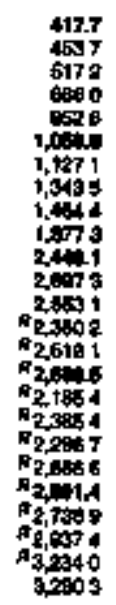 & 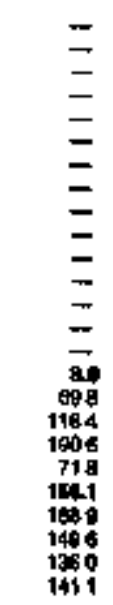 & $\begin{array}{l}= \\
= \\
= \\
= \\
= \\
= \\
= \\
= \\
= \\
= \\
= \\
=20 \\
221 \\
227 \\
215 \\
215\end{array}$ & 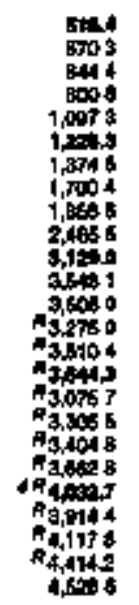 & 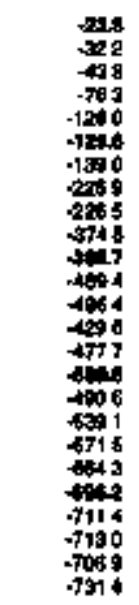 & 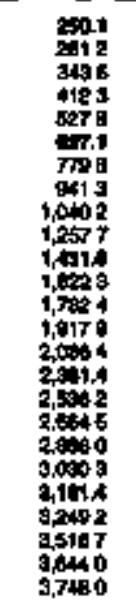 & 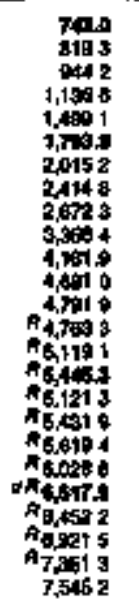 \\
\hline & & & & & & & & & & Exa & & & & 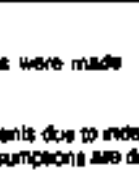 & 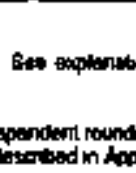 & of atyonest & 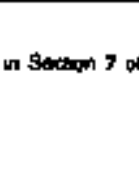 \\
\hline
\end{tabular}


Table 24. Aagidarilied Soctor Energy Price and Expenditure Esllmatos by Source, 1970-1994, Arizong

\begin{tabular}{|c|c|c|c|c|c|c|c|c|c|c|}
\hline \multirow[b]{4}{*}{$r=$} & \multicolumn{8}{|c|}{ Miner trenmpr } & \multirow[b]{3}{*}{ Enetrians } & \multirow[b]{3}{*}{ Exim } \\
\hline & \multirow[b]{2}{*}{ can } & \multirow[b]{2}{*}{ 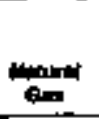 } & \multicolumn{4}{|c|}{ Putrainen } & \multirow[b]{2}{*}{ 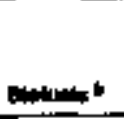 } & \multirow[b]{2}{*}{ Totad } & & \\
\hline & & & 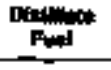 & Lي & $\mathbf{L G}$ & $T=1$ & & & & \\
\hline & \multicolumn{10}{|c|}{ 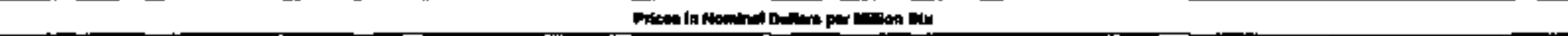 } \\
\hline \multirow[t]{2}{*}{ 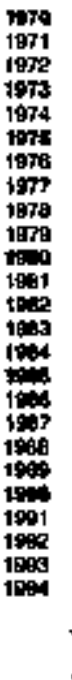 } & 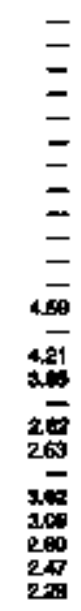 & 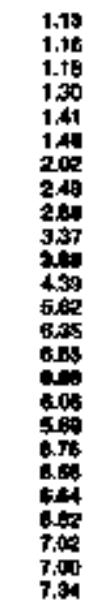 & 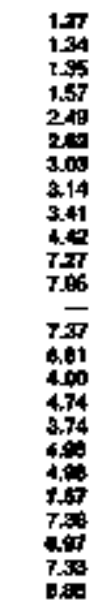 & 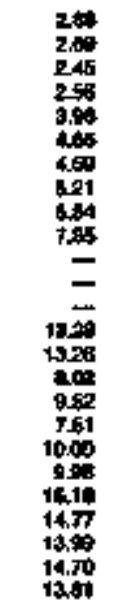 & 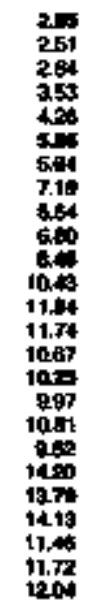 & 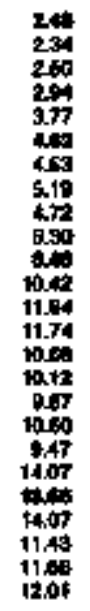 & $\begin{array}{r}= \\
= \\
= \\
= \\
= \\
= \\
= \\
= \\
= \\
= \\
= \\
4 \overline{5} \\
4,55 \\
4,75\end{array}$ & 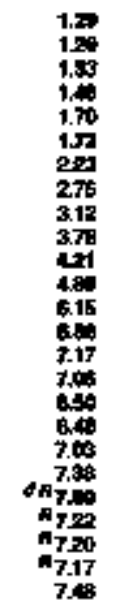 & 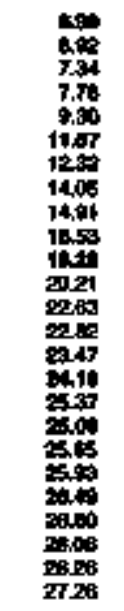 & 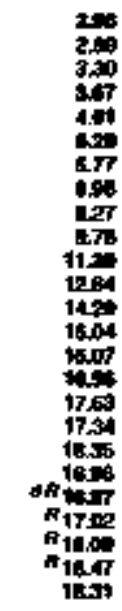 \\
\hline & \multicolumn{10}{|c|}{ 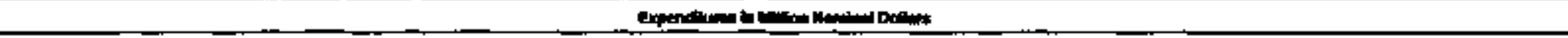 } \\
\hline 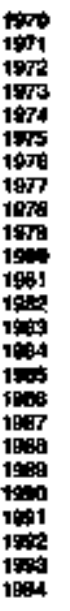 & 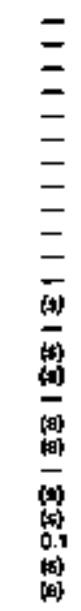 & 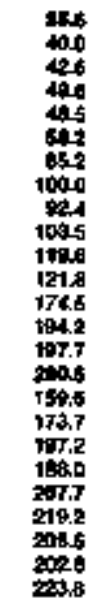 & 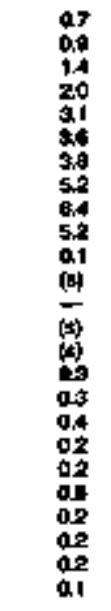 & $\begin{array}{l}1.1 \\
0.8 \\
0.6 \\
0.8 \\
20 \\
20 \\
42 \\
50 \\
54 \\
2.7 \\
= \\
= \\
0.9 \\
02 \\
01 \\
0.2 \\
0.1 \\
0.2 \\
091 \\
0.1 \\
0.1 \\
02 \\
0.1 \\
0.1\end{array}$ & 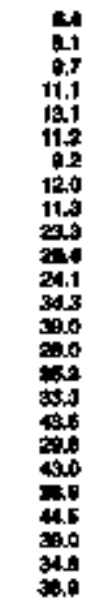 & 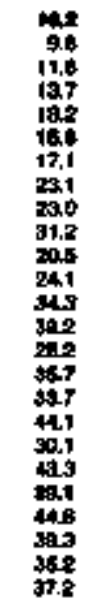 & $\begin{array}{l}= \\
= \\
= \\
= \\
= \\
= \\
= \\
= \\
= \\
= \\
= \\
= \\
0104 \\
173 \\
172 \\
170\end{array}$ & 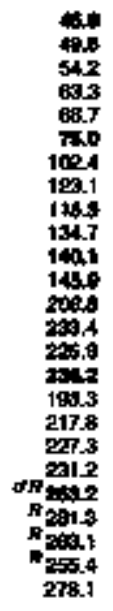 & 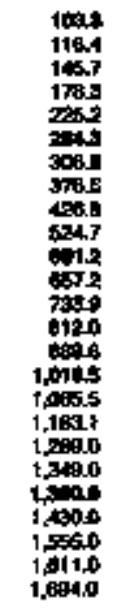 & 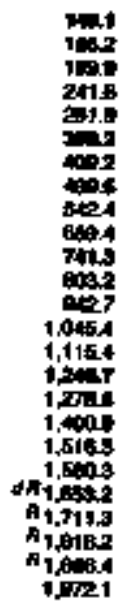 \\
\hline
\end{tabular}


Table 25. Commercial Sector Entroy Prico and Exponditure Estimatien by Sotre, 1970-1994, Arbona

\begin{tabular}{|c|c|c|c|c|c|c|c|c|c|c|c|}
\hline & \multicolumn{9}{|c|}{ Primery energy } & \multirow[b]{3}{*}{ enctinaty } & \multirow[b]{3}{*}{ Thets } \\
\hline & \multirow[b]{2}{*}{ con } & \multirow[b]{2}{*}{ דות } & \multicolumn{6}{|c|}{ 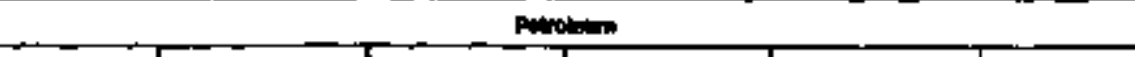 } & \multirow[b]{2}{*}{ Totit } & & \\
\hline \multirow[b]{2}{*}{ ner } & & & 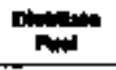 & 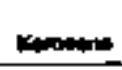 & 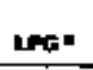 & ה: & Pnimat & 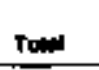 & & & \\
\hline & \multicolumn{11}{|c|}{ 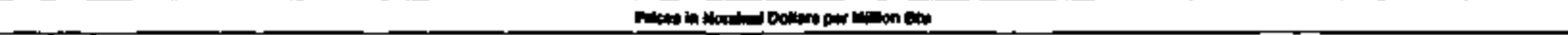 } \\
\hline \multirow[t]{2}{*}{ 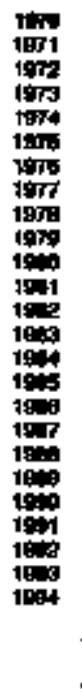 } & 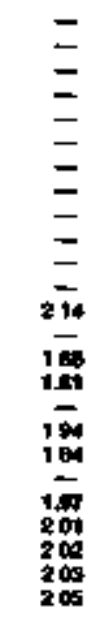 & 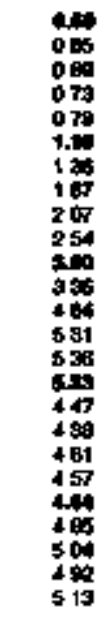 & 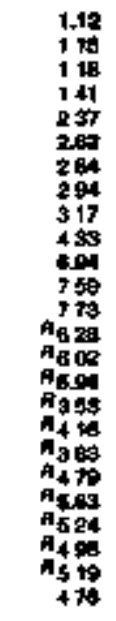 & 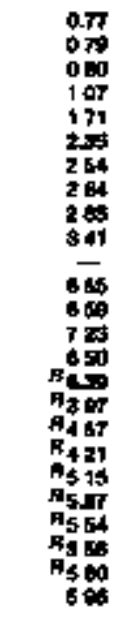 & 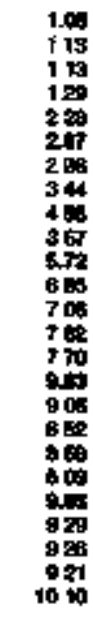 & 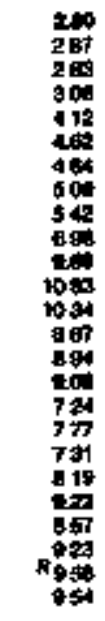 & 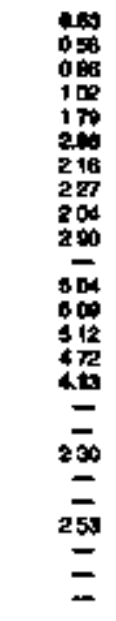 & 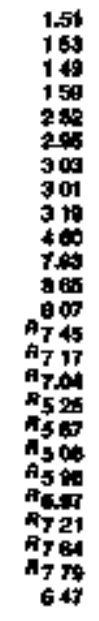 & 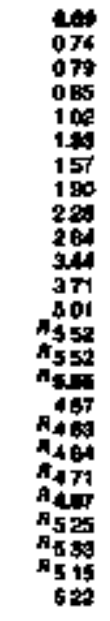 & 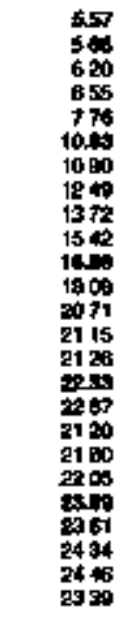 & 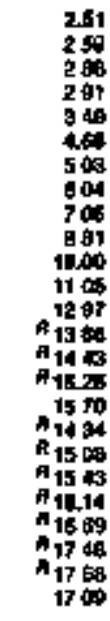 \\
\hline & \multicolumn{11}{|c|}{ 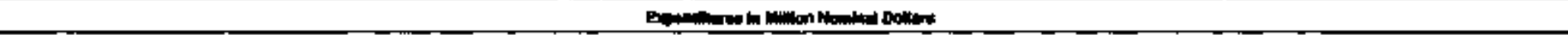 } \\
\hline 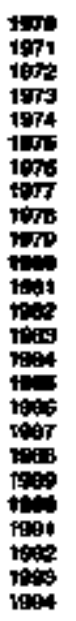 & 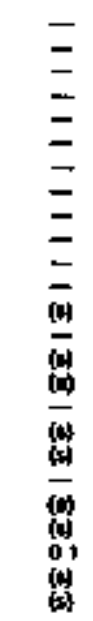 & 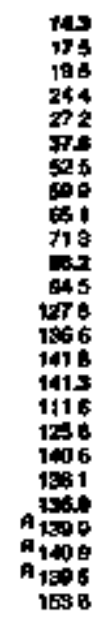 & 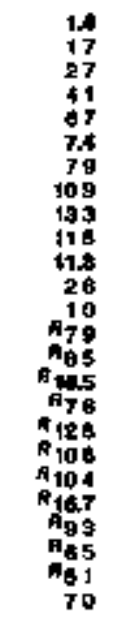 & 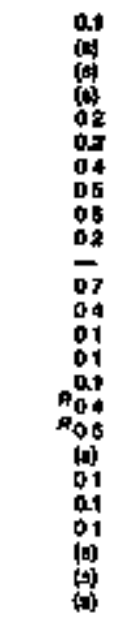 & $\begin{array}{l}06 \\
06 \\
07 \\
07 \\
18 \\
08 \\
09 \\
10 \\
18 \\
22 \\
24 \\
26 \\
36 \\
48 \\
36 \\
58 \\
53 \\
61 \\
47 \\
43 \\
45 \\
52 \\
56 \\
46 \\
55\end{array}$ & 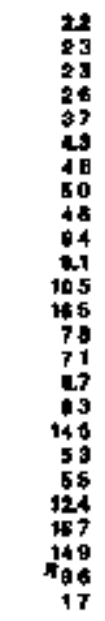 & 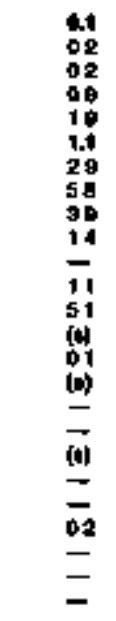 & 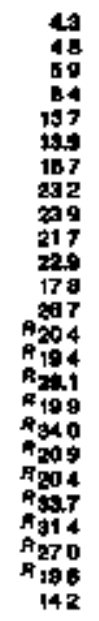 & 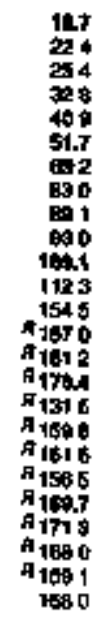 & 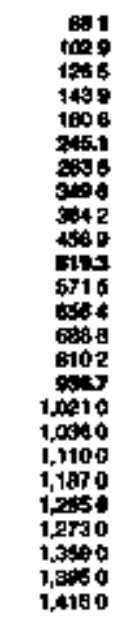 & 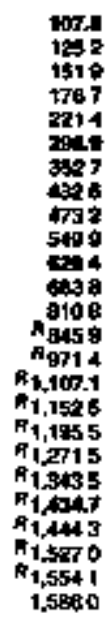 \\
\hline
\end{tabular}


Tabto 26. Industriel Sector Energy Pelce and Expenditure Esalmates by Souren, 1670-1994, Arizone

\begin{tabular}{|c|c|c|c|c|c|c|c|c|c|c|c|c|c|c|c|c|c|}
\hline \multirow[b]{4}{*}{ Vorr } & \multicolumn{15}{|c|}{ Pustry langy } & \multirow[b]{3}{*}{ Anotion } & \multirow[b]{3}{*}{ Toris: } \\
\hline & \multicolumn{3}{|c|}{ Con } & \multirow[b]{2}{*}{ 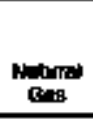 } & \multicolumn{9}{|c|}{ Pubolinen } & \multirow[b]{2}{*}{ 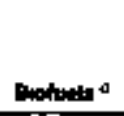 } & \multirow[b]{2}{*}{ Tolate } & & \\
\hline & cons & 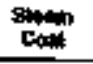 & To네 & & $\begin{array}{c}\text { Alphert and } \\
\text { heos on }\end{array}$ & $\begin{array}{c}\text { Dawam } \\
\text { Fust }\end{array}$ & Karenter & LPQ* & turdonsts & canoling & Mondum & ans" & Iotal & & & & \\
\hline & \multicolumn{17}{|c|}{ 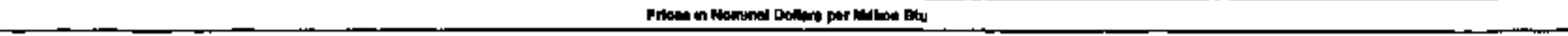 } \\
\hline \multirow[t]{2}{*}{ 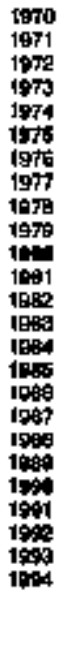 } & $\begin{array}{l}= \\
= \\
= \\
= \\
= \\
= \\
= \\
= \\
= \\
= \\
= \\
= \\
= \\
=\end{array}$ & 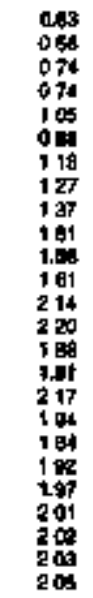 & 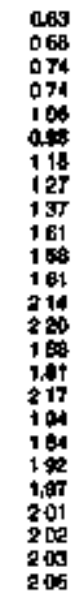 & 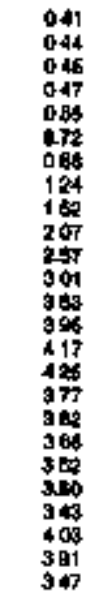 & 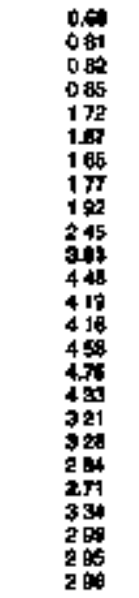 & 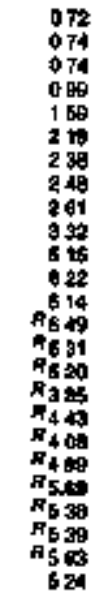 & 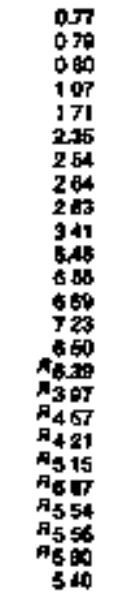 & 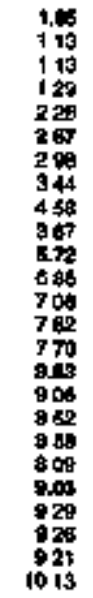 & 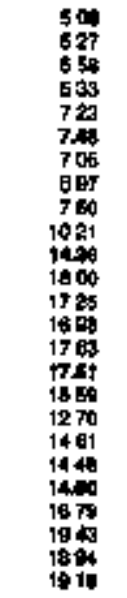 & 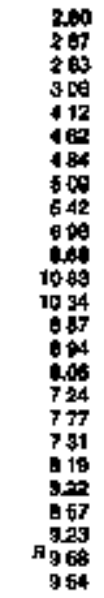 & 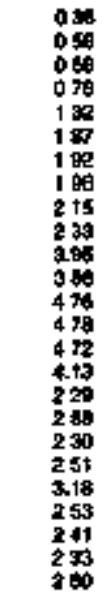 & 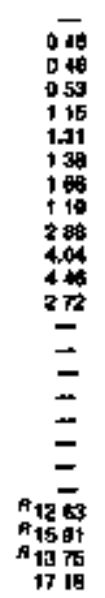 & 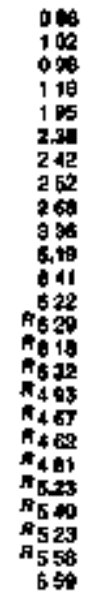 & $\begin{array}{l}= \\
= \\
= \\
= \\
= \\
= \\
= \\
= \\
= \\
= \\
= \\
= \\
\bar{z} \\
140 \\
146 \\
149\end{array}$ & 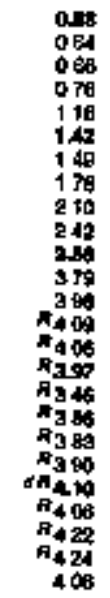 & 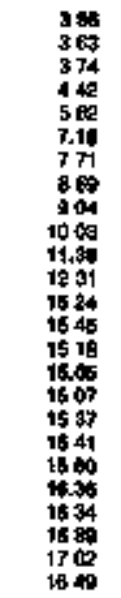 & 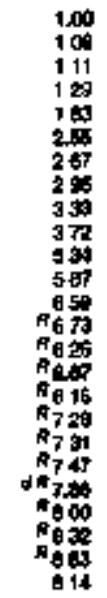 \\
\hline & \multicolumn{17}{|c|}{ 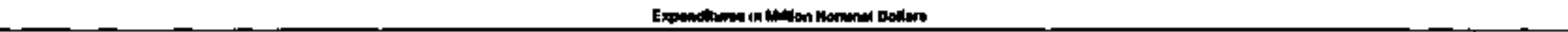 } \\
\hline 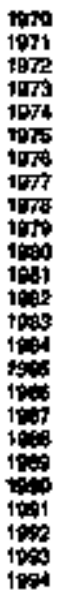 & $\begin{array}{l}= \\
z \\
= \\
z \\
z \\
z \\
= \\
= \\
= \\
= \\
= \\
= \\
= \\
= \\
=\end{array}$ & 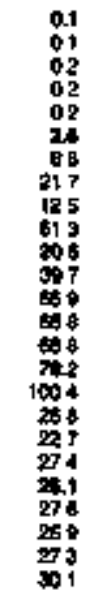 & 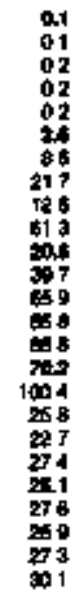 & 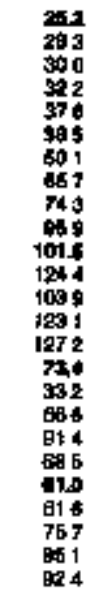 & 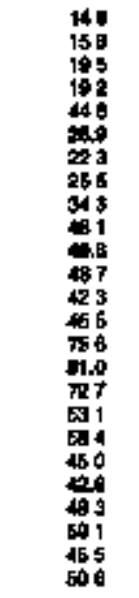 & 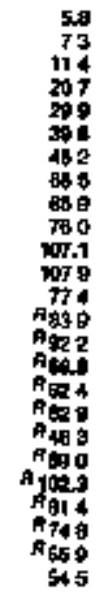 & 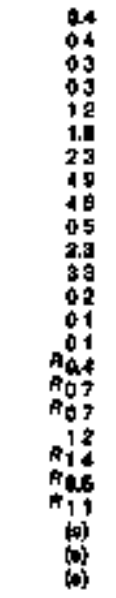 & $\begin{array}{l}1.0 \\
10 \\
12 \\
15 \\
98 \\
19 \\
36 \\
46 \\
64 \\
86 \\
165 \\
74 \\
193 \\
124 \\
145 \\
175 \\
170 \\
193 \\
209 \\
124 \\
120 \\
110 \\
172 \\
270 \\
291\end{array}$ & 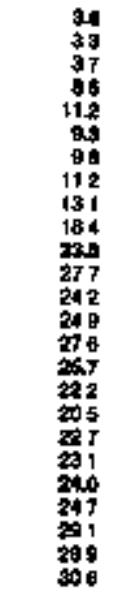 & $\begin{array}{r}17 \\
99 \\
70 \\
73 \\
103 \\
107 \\
06 \\
160 \\
104 \\
126 \\
117 \\
154 \\
116 \\
896 \\
117 \\
192 \\
158 \\
165 \\
158 \\
180 \\
242 \\
168 \\
169 \\
1170 \\
164\end{array}$ & $\begin{array}{l}.1 \\
03 \\
06 \\
12 \\
21 \\
12 \\
32 \\
46 \\
50 \\
17 \\
34 \\
04 \\
04 \\
02 \\
04 \\
04 \\
05 \\
03 \\
05 \\
01 \\
09 \\
19 \\
10 \\
86 \\
07\end{array}$ & 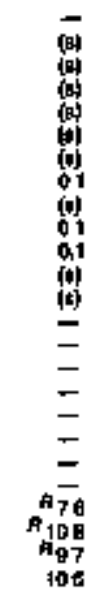 & 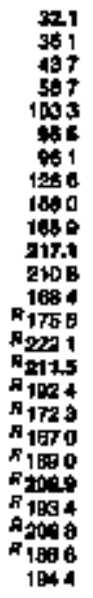 & $\begin{array}{l}\bar{z} \\
\bar{z} \\
\bar{z} \\
\bar{z} \\
= \\
\bar{z} \\
= \\
= \\
= \\
= \\
441 \\
49 \\
43 \\
45\end{array}$ & 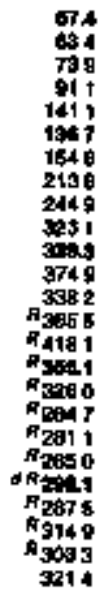 & 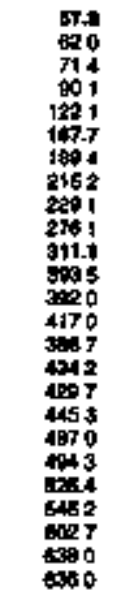 & 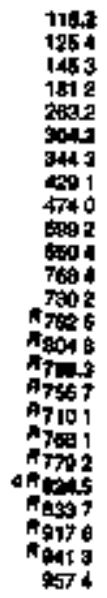 \\
\hline
\end{tabular}

Lapiod pemom oktas

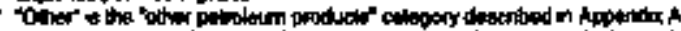

nermel enoroy

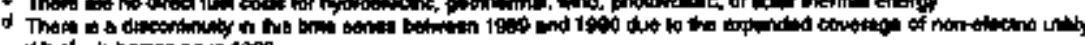
Antomed on

Appongin

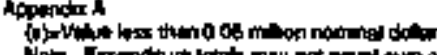

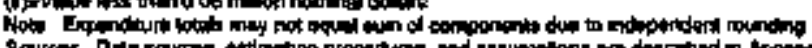

Enargy hiomentlon Adminletrallon 
Tablo 27. Tremeportetion Soctor Energy Price and Expenditure Estimated by sourte, 1970-1994, Artona

\begin{tabular}{|c|c|c|c|c|c|c|c|c|c|c|c|c|c|}
\hline \multirow[b]{4}{*}{ זעו } & \multicolumn{11}{|c|}{ Pinuny teraror. } & \multirow[b]{3}{*}{ emontety } & \multirow[b]{3}{*}{$\begin{array}{l}\text { Tots: } \\
\text { Energy }\end{array}$} \\
\hline & \multirow[b]{2}{*}{ cont } & \multirow[b]{2}{*}{ Non } & \multicolumn{8}{|c|}{ Ratrangen } & \multirow[b]{2}{*}{ Totod } & & \\
\hline & & & Animiton & $\begin{array}{l}\text { Dintines } \\
\text { Fut }\end{array}$ & 呚 & Let: & Lutipantin & 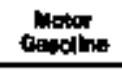 & 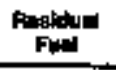 & Totod & & & \\
\hline & \multicolumn{13}{|c|}{ 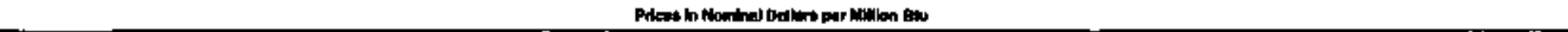 } \\
\hline \multirow[t]{2}{*}{ 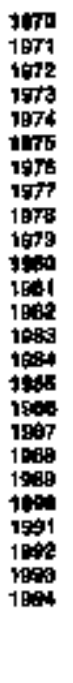 } & $\begin{array}{l}0,15 \\
056 \\
074 \\
074 \\
105 \\
0.19 \\
190 \\
19 \\
= \\
= \\
= \\
= \\
= \\
= \\
= \\
= \\
= \\
=\end{array}$ & $\begin{array}{l}= \\
= \\
= \\
= \\
= \\
= \\
= \\
= \\
= \\
= \\
= \\
= \\
= \\
373 \\
300 \\
340 \\
300\end{array}$ & 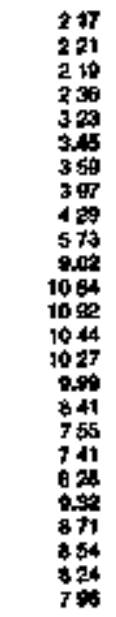 & 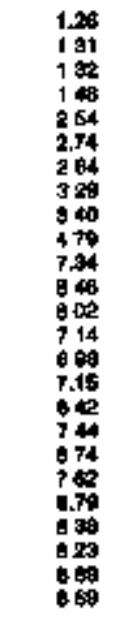 & 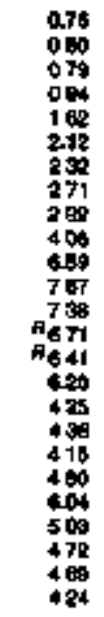 & 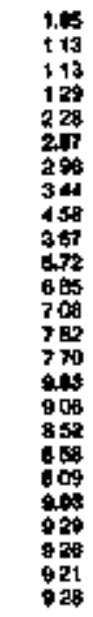 & 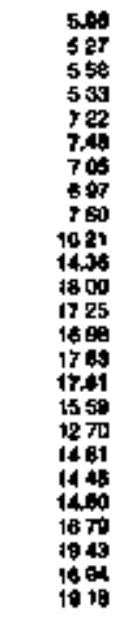 & 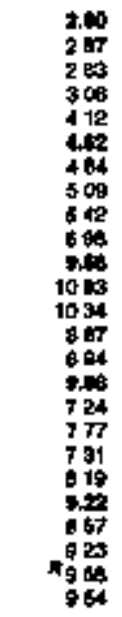 & $\begin{array}{l}= \\
= \\
= \\
= \\
= \\
= \\
= \\
= \\
= \\
= \\
= \\
= \\
= \\
=\end{array}$ & 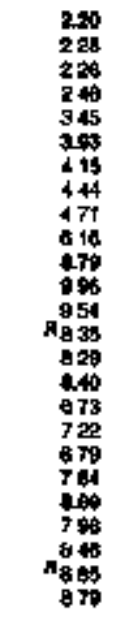 & 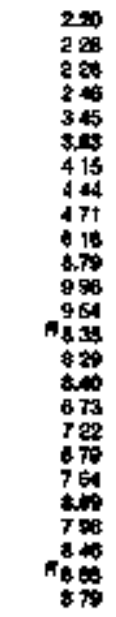 & $\begin{array}{l}= \\
= \\
= \\
= \\
= \\
= \\
= \\
= \\
= \\
= \\
= \\
= \\
= \\
=\end{array}$ & 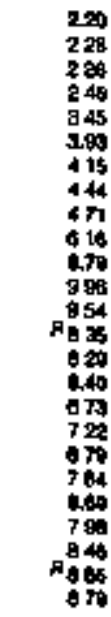 \\
\hline & \multicolumn{13}{|c|}{ 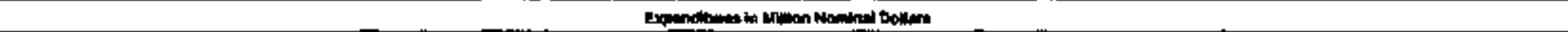 } \\
\hline 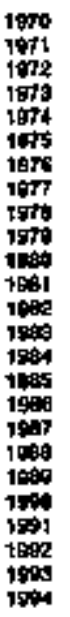 & 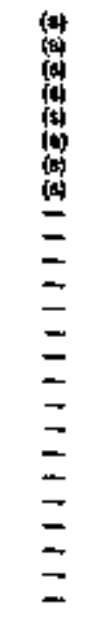 & $\begin{array}{l}= \\
= \\
= \\
= \\
= \\
= \\
= \\
= \\
= \\
= \\
= \\
= \\
= \\
= \\
01 \\
02 \\
02 \\
02\end{array}$ & $\begin{array}{c}47 \\
47 \\
45 \\
43 \\
68 \\
64 \\
81 \\
75 \\
74 \\
107 \\
128 \\
123 \\
86 \\
68 \\
68 \\
94 \\
86 \\
79 \\
76 \\
98 \\
04 \\
93 \\
93 \\
68 \\
53 \\
57\end{array}$ & 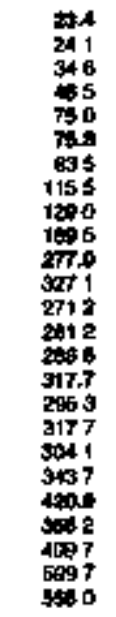 & 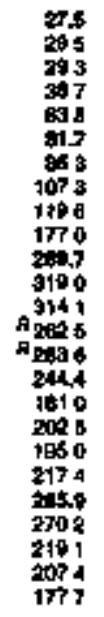 & $\begin{array}{l}02 \\
03 \\
03 \\
03 \\
05 \\
08 \\
06 \\
07 \\
18 \\
01 \\
14 \\
36 \\
28 \\
38 \\
38 \\
48 \\
28 \\
18 \\
90 \\
19 \\
18 \\
19 \\
19 \\
18 \\
28\end{array}$ & 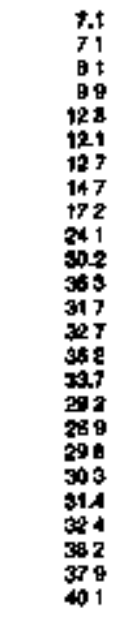 & 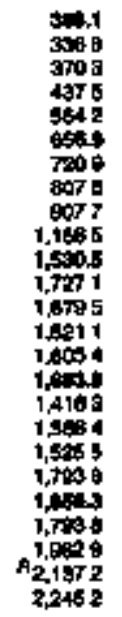 & $\begin{array}{l}= \\
= \\
= \\
= \\
= \\
= \\
= \\
= \\
= \\
= \\
= \\
= \\
= \\
=\end{array}$ & 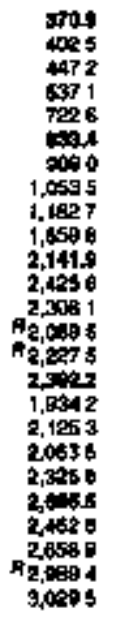 & 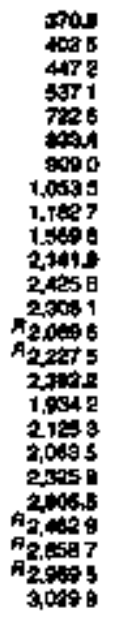 & $\begin{array}{l}= \\
= \\
= \\
= \\
= \\
= \\
= \\
= \\
= \\
= \\
= \\
= \\
= \\
=\end{array}$ & 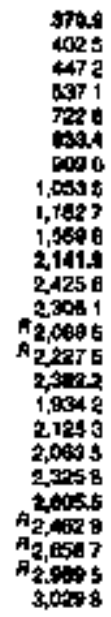 \\
\hline
\end{tabular}

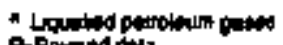

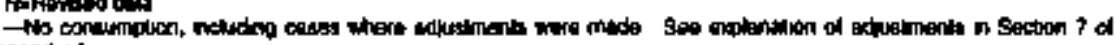

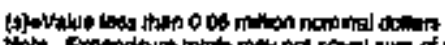

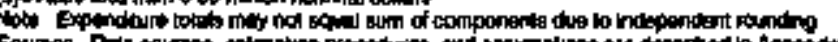
axpinter 


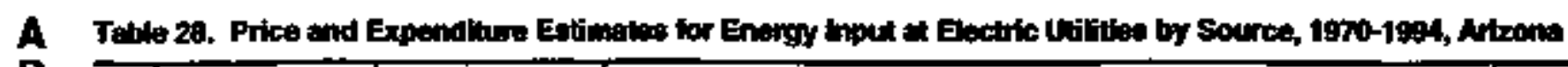

\begin{tabular}{|c|c|c|c|c|c|c|c|c|c|}
\hline \multirow[b]{3}{*}{ now } & \multirow[b]{2}{*}{ coet } & \multirow[b]{2}{*}{ 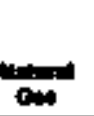 } & \multicolumn{4}{|c|}{ m } & \multirow[b]{2}{*}{ nent } & \multirow[b]{2}{*}{ and } & \multirow[b]{2}{*}{ 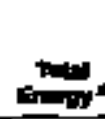 } \\
\hline & & & 여 & Lit & 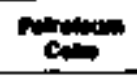 & שה & & & \\
\hline & \multicolumn{9}{|c|}{ 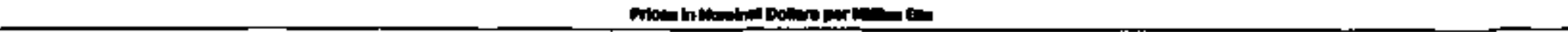 } \\
\hline \multirow[t]{2}{*}{ 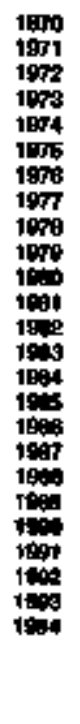 } & 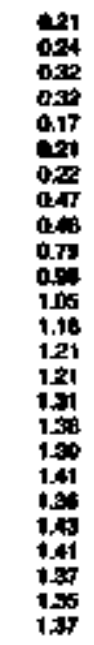 & 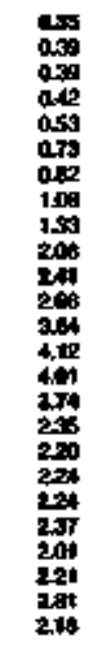 & 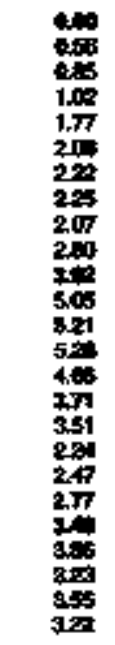 & 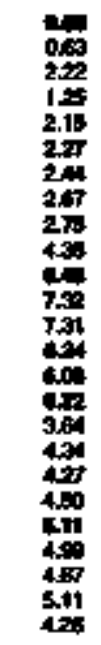 & $\begin{array}{l}z \\
z \\
z= \\
= \\
= \\
= \\
= \\
= \\
= \\
= \\
= \\
=\end{array}$ & 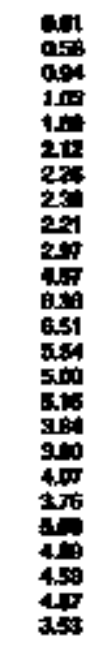 & 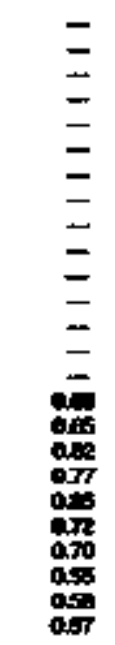 & $\begin{array}{l}z \\
z \\
z \\
z \\
z \\
z \\
= \\
= \\
= \\
= \\
= \\
= \\
=\end{array}$ & 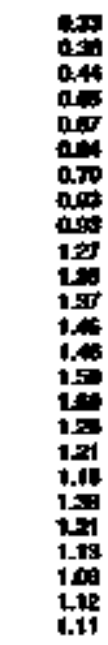 \\
\hline & \multicolumn{9}{|c|}{ 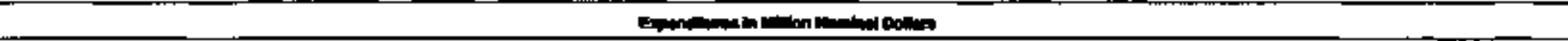 } \\
\hline 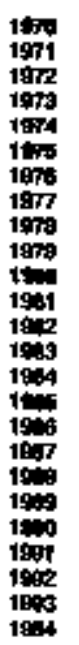 & 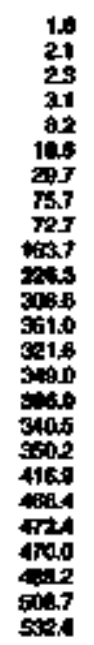 & 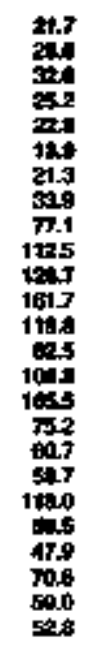 & 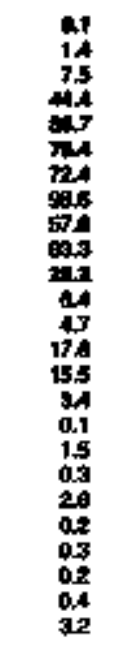 & 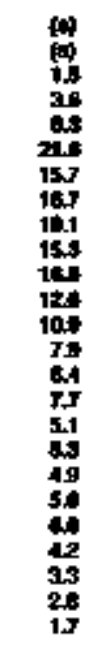 & $\begin{array}{l}z \\
z \\
= \\
z \\
= \\
= \\
= \\
= \\
= \\
= \\
= \\
z\end{array}$ & 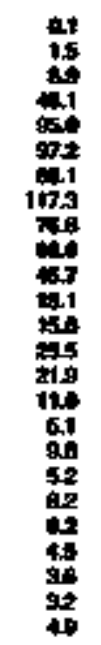 & $\begin{array}{l}= \\
= \\
= \\
= \\
= \\
= \\
= \\
= \\
\bar{z} \\
\mathbf{4 0}\end{array}$ & $\begin{array}{l}z= \\
z \\
= \\
= \\
= \\
= \\
= \\
= \\
= \\
z \\
z \\
z \\
z\end{array}$ & 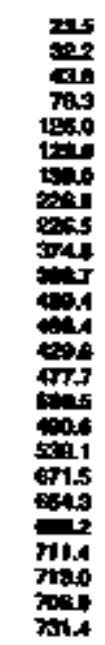 \\
\hline
\end{tabular}

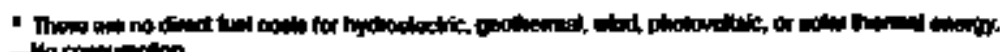

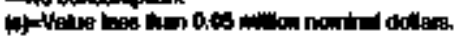




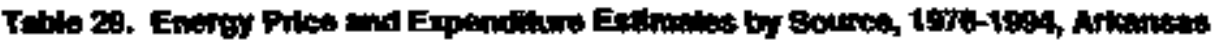

\begin{tabular}{|c|c|c|c|c|c|c|c|c|c|c|c|c|c|c|c|c|c|}
\hline & \multicolumn{14}{|c|}{ Promengen } & \multirow{3}{*}{ Entix } & \multirow{3}{*}{ dition } & \multirow[b]{3}{*}{ Totis } \\
\hline & \multicolumn{3}{|c|}{ and } & & \multicolumn{7}{|c|}{ mantion } & \multirow[b]{2}{*}{ mucter } & \multirow[b]{2}{*}{ Butum } & \multirow[b]{2}{*}{$T=16$} & & & \\
\hline & ath & - & $T=$ & 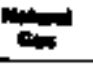 & Find & f & wa & שה & 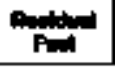 & De & Totid & & & & & & \\
\hline$x$ & \multicolumn{17}{|c|}{ 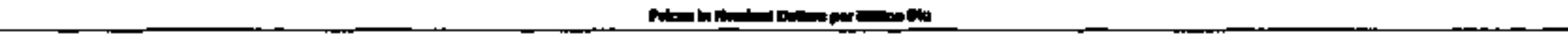 } \\
\hline \multirow[t]{2}{*}{ (1) } & $\begin{array}{l}= \\
z \\
z \\
z \\
z \\
z \\
= \\
= \\
= \\
= \\
= \\
= \\
= \\
=\end{array}$ & 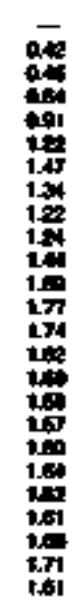 & 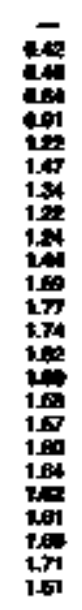 & 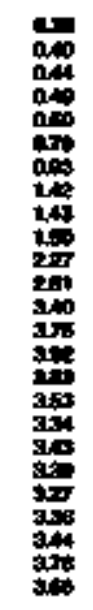 & 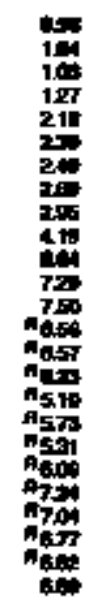 & 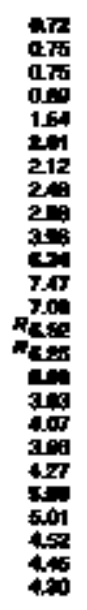 & 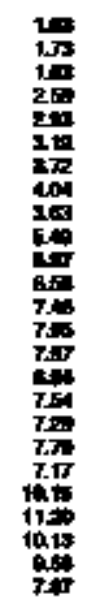 & 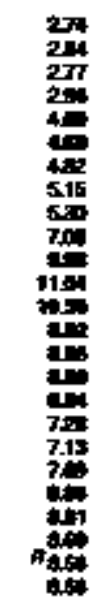 & 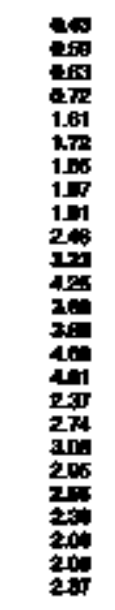 & 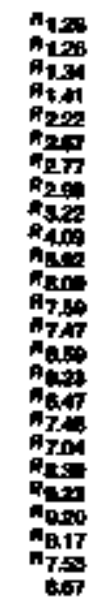 & 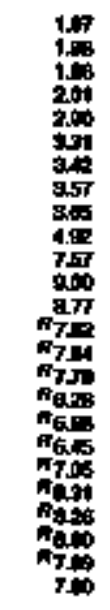 & 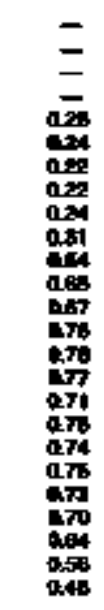 & $\begin{array}{l}= \\
= \\
= \\
= \\
= \\
= \\
= \\
= \\
= \\
= \\
= \\
=15 \\
1.5 \\
1.50\end{array}$ & 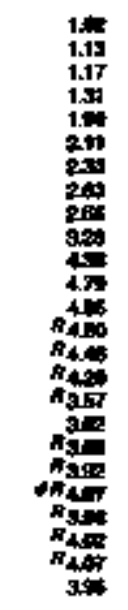 & 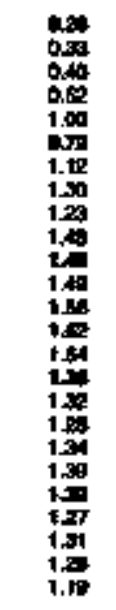 & 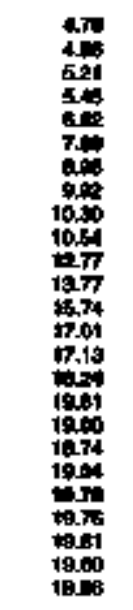 & 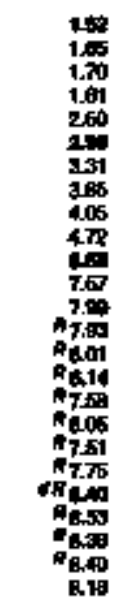 \\
\hline & \multicolumn{17}{|c|}{ 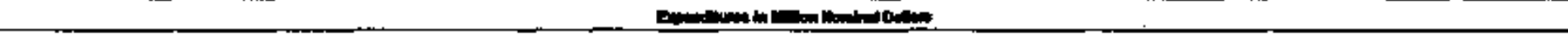 } \\
\hline 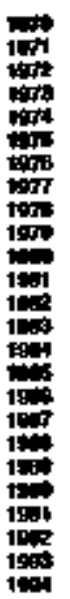 & $\begin{array}{l}= \\
= \\
= \\
= \\
= \\
= \\
= \\
= \\
= \\
= \\
= \\
= \\
=\end{array}$ & 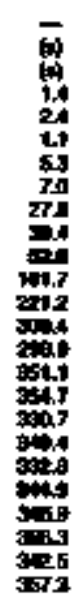 & 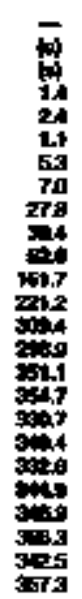 & 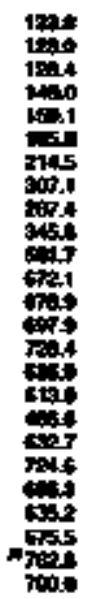 & 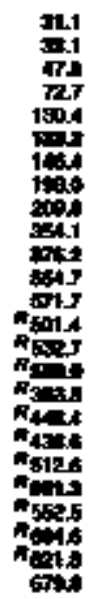 & 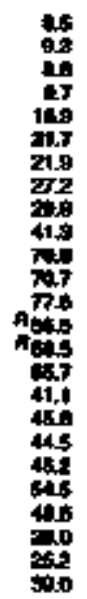 & 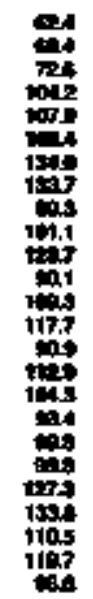 & 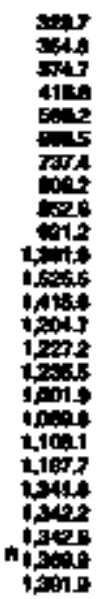 & 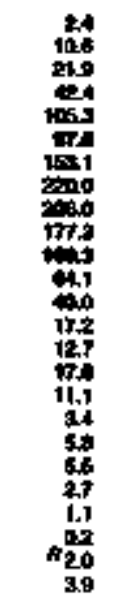 & 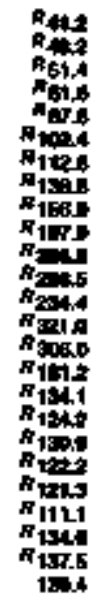 & 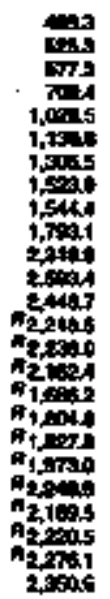 & 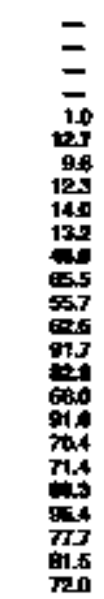 & $\begin{array}{l}= \\
= \\
= \\
= \\
= \\
= \\
= \\
= \\
= \\
= \\
= \\
=5 \\
5=1 \\
3.7 \\
3=3\end{array}$ & 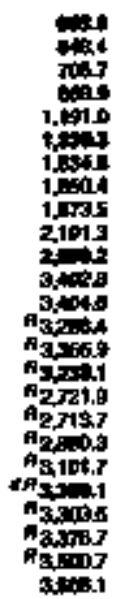 & 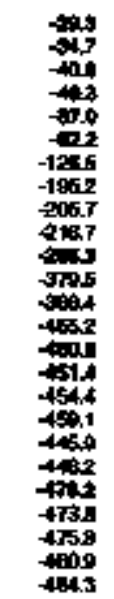 & 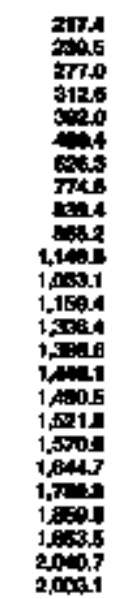 & 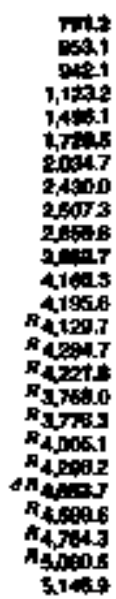 \\
\hline
\end{tabular}

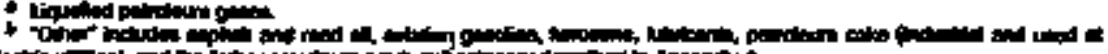

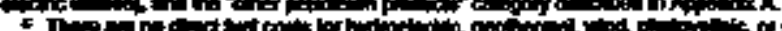

Tom

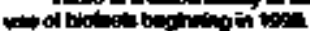

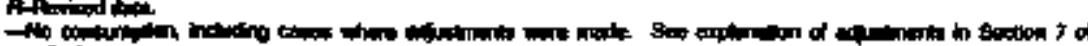

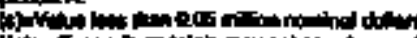

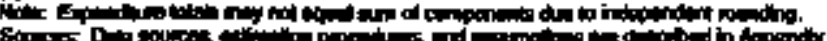




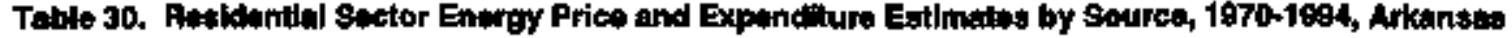

\begin{tabular}{|c|c|c|c|c|c|c|c|c|c|c|}
\hline \multirow[b]{4}{*}{ אמר } & \multicolumn{8}{|c|}{ 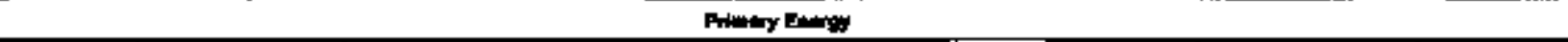 } & \multirow[b]{3}{*}{ Puctoleth } & \multirow[b]{3}{*}{ Tod } \\
\hline & \multirow[b]{2}{*}{ con } & \multirow[b]{2}{*}{ mand } & \multicolumn{4}{|c|}{ Prim } & \multirow[b]{2}{*}{ Dotuly" } & \multirow[b]{2}{*}{$T \rightarrow \mathbf{N}^{\sigma}$} & & \\
\hline & & & Otaming & 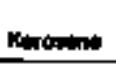 & LOE= & Tall & & & & \\
\hline & \multicolumn{10}{|c|}{ 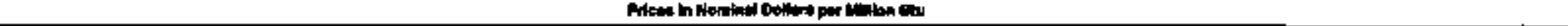 } \\
\hline \multirow[t]{2}{*}{ 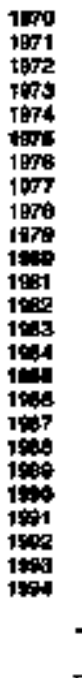 } & 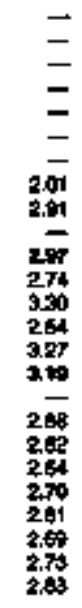 & 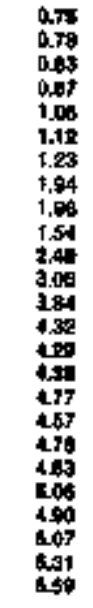 & 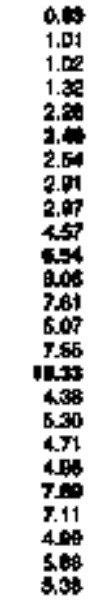 & 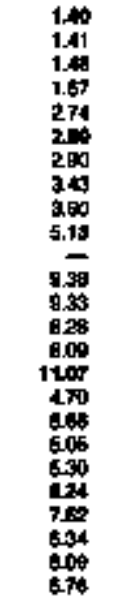 & 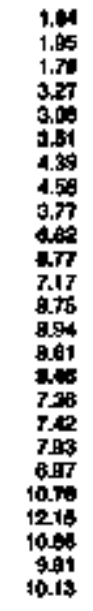 & 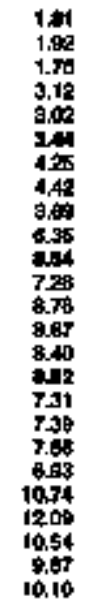 & $\begin{array}{l}= \\
= \\
= \\
= \\
= \\
= \\
= \\
= \\
= \\
= \\
= \\
= \\
= \\
0.5 \\
3.5 \\
3.50 \\
3.50\end{array}$ & 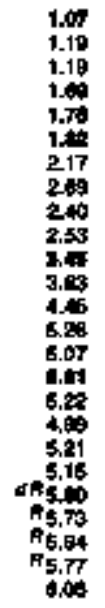 & 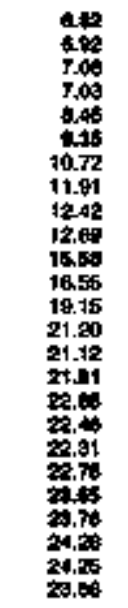 & 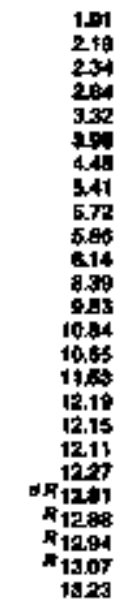 \\
\hline & \multicolumn{10}{|c|}{ 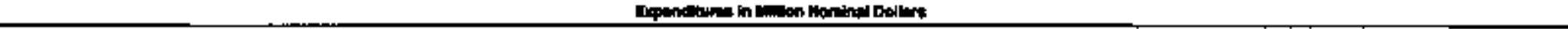 } \\
\hline 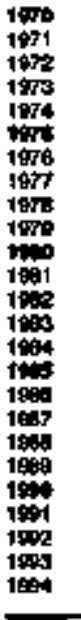 & 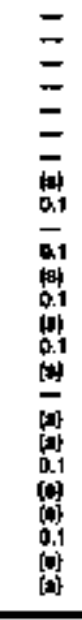 & 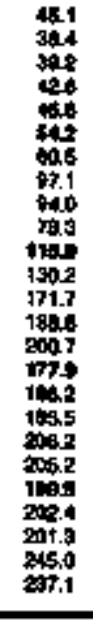 & 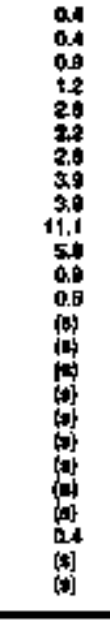 & 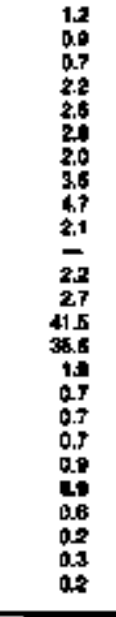 & 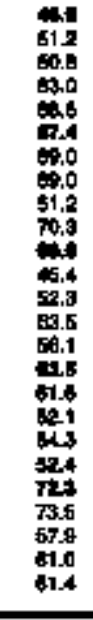 & 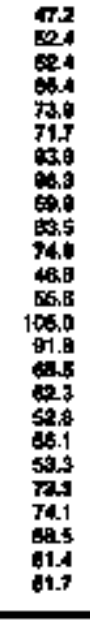 & $\begin{array}{l}= \\
= \\
= \\
= \\
= \\
= \\
= \\
= \\
= \\
= \\
=0.1 \\
7.5 \\
8.7\end{array}$ & 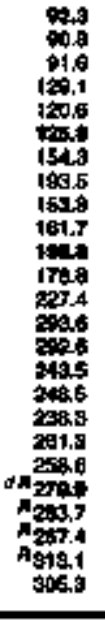 & 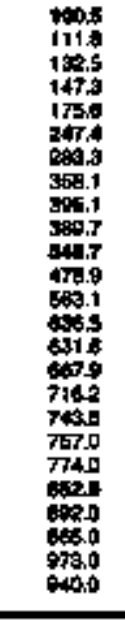 & 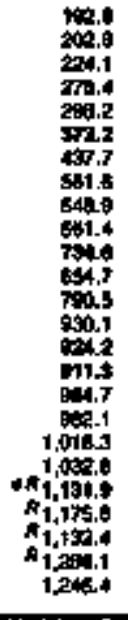 \\
\hline
\end{tabular}

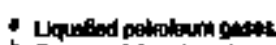

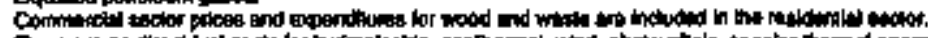

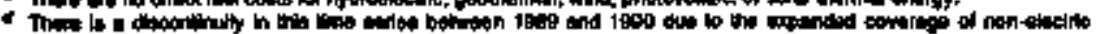

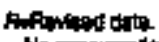

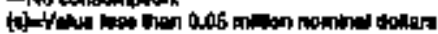

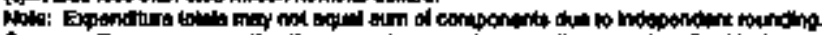

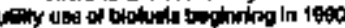




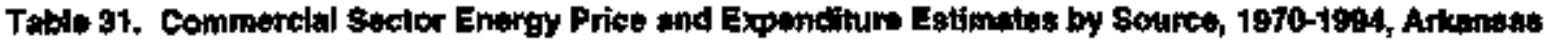

\begin{tabular}{|c|c|c|c|c|c|c|c|c|c|c|c|}
\hline \multirow[b]{4}{*}{$\mathrm{Yar}$} & \multicolumn{9}{|c|}{ Pumary enery } & \multirow[b]{3}{*}{ Butrikiks } & \multirow[b]{3}{*}{ Tow } \\
\hline & \multirow[b]{2}{*}{ coed } & \multirow[b]{2}{*}{ Mation } & \multicolumn{6}{|c|}{ Andanm } & \multirow[b]{2}{*}{ Tot:! } & & \\
\hline & & & 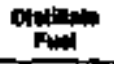 & Monotain & LPG" & 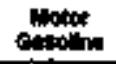 & Ponatud & Totel & & & \\
\hline & \multicolumn{11}{|c|}{ 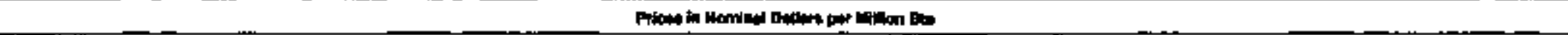 } \\
\hline \multirow[t]{2}{*}{ 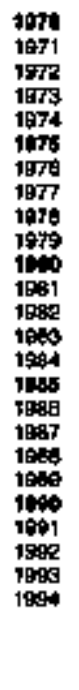 } & 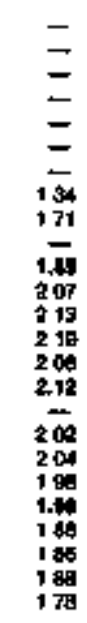 & 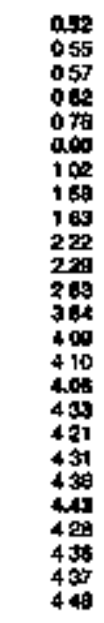 & 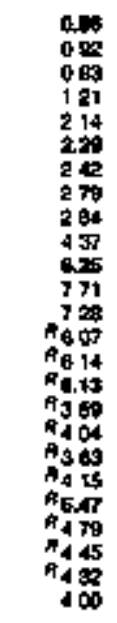 & 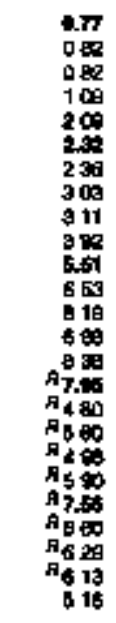 & 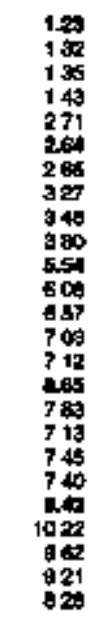 & 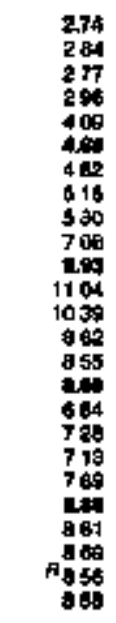 & 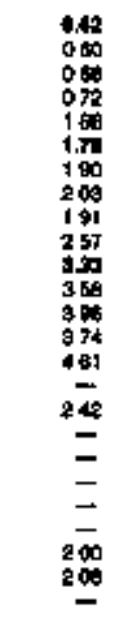 & 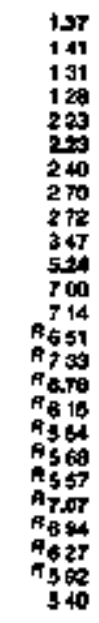 & 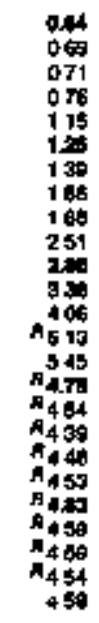 & 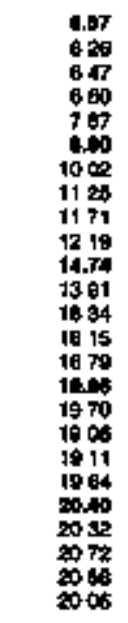 & 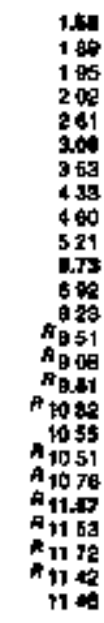 \\
\hline & \multicolumn{11}{|c|}{ 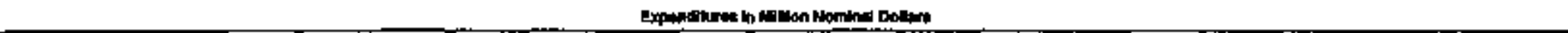 } \\
\hline 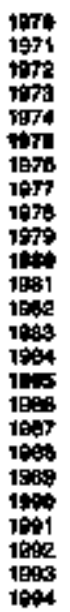 & 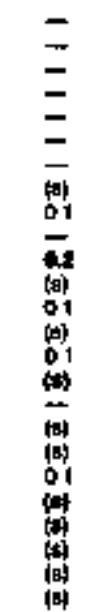 & 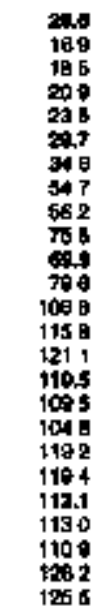 & 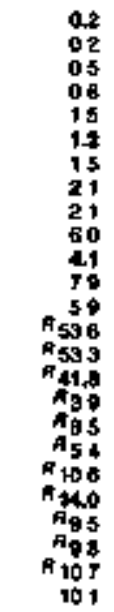 & 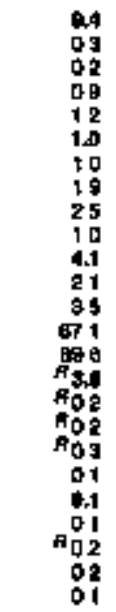 & 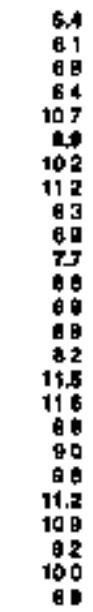 & $\begin{array}{l}20 \\
20 \\
19 \\
20 \\
27 \\
96 \\
37 \\
39 \\
50 \\
67 \\
106 \\
100 \\
81 \\
81 \\
41 \\
85 \\
42 \\
50 \\
48 \\
44 \\
16 \\
37 \\
33 \\
13 \\
13\end{array}$ & 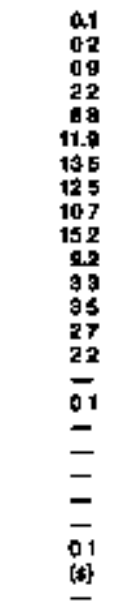 & 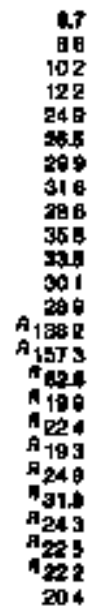 & 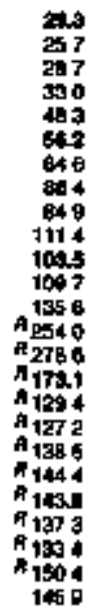 & 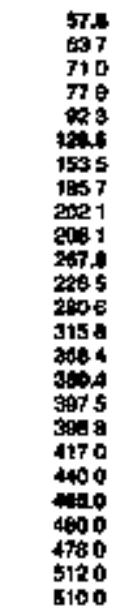 & 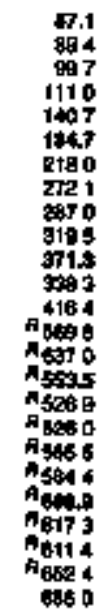 \\
\hline
\end{tabular}




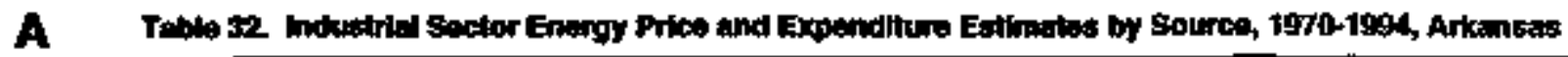

\begin{tabular}{|c|c|c|c|c|c|c|c|c|c|c|c|c|c|c|c|c|c|}
\hline & \multicolumn{15}{|c|}{ 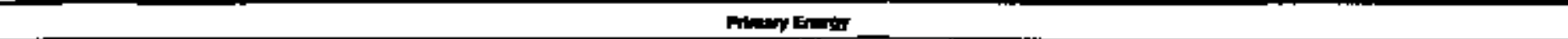 } & \multirow[b]{3}{*}{ Ancticeny } & \multirow[b]{3}{*}{ Thens } \\
\hline & \multicolumn{3}{|c|}{ נons } & \multirow[b]{2}{*}{$\operatorname{man}$} & \multicolumn{9}{|c|}{ 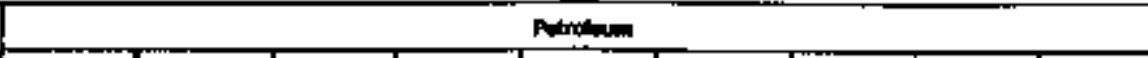 } & \multirow[b]{2}{*}{ Donmente } & \multirow[b]{2}{*}{$T=0$} & & \\
\hline & cons & con & Tent & & 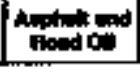 & Ant & Kanome & $\mathbf{c o s}$ & Lutoent & (1) & Randuen & cons & tol & & & & \\
\hline$y=$ & \multicolumn{17}{|c|}{ 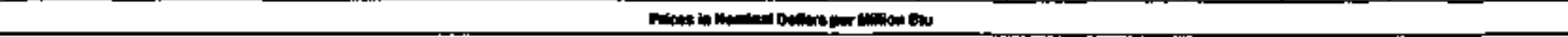 } \\
\hline 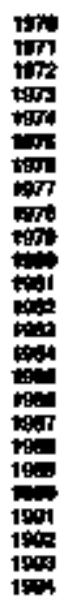 & $\begin{array}{l}= \\
= \\
= \\
= \\
= \\
= \\
= \\
= \\
= \\
= \\
= \\
= \\
=\end{array}$ & 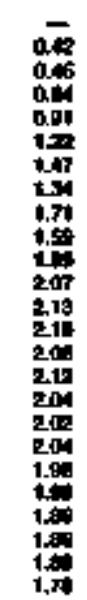 & 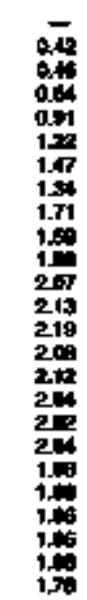 & 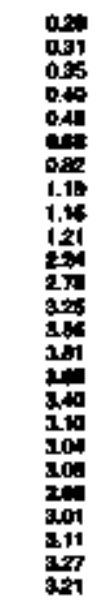 & 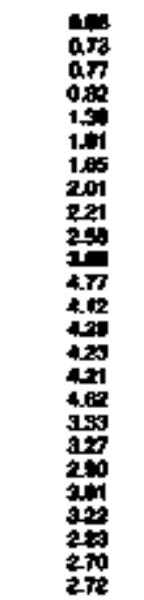 & 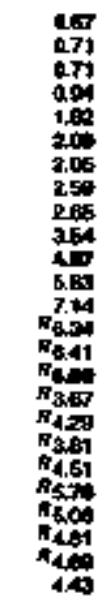 & 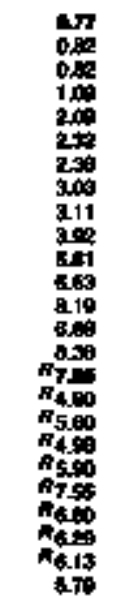 & 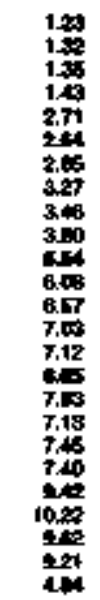 & 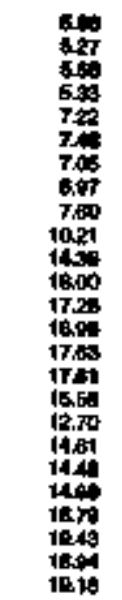 & 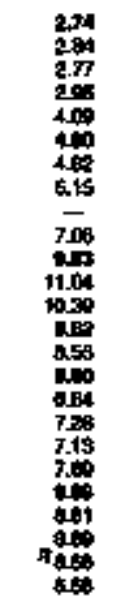 & 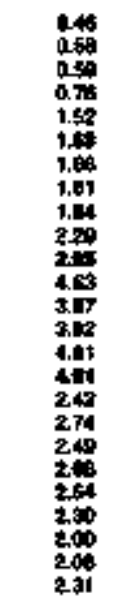 & 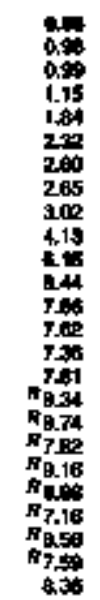 & 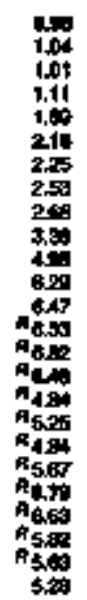 & 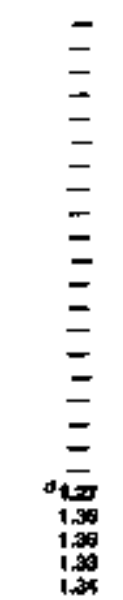 & 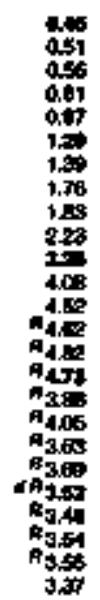 & 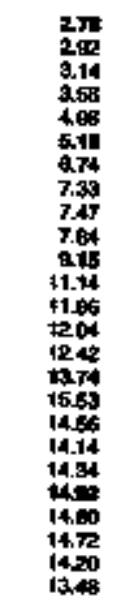 & 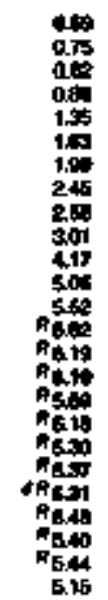 \\
\hline
\end{tabular}

\begin{tabular}{|c|c|c|c|c|c|c|c|c|c|c|c|c|c|c|c|c|c|}
\hline & & & & & & & & ي & n n n & $m-100 m$ & & & & & & & \\
\hline 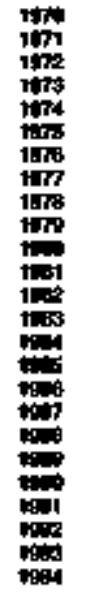 & $\begin{array}{l}= \\
= \\
= \\
= \\
= \\
= \\
= \\
= \\
= \\
= \\
= \\
= \\
=\end{array}$ & 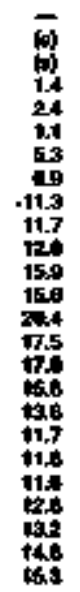 & 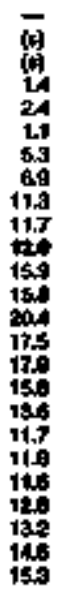 & 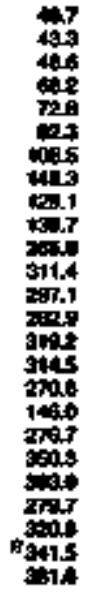 & 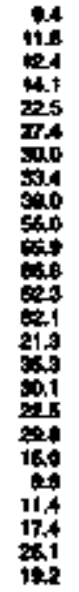 & 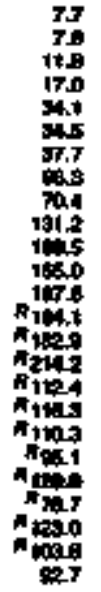 & 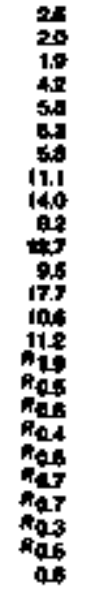 & 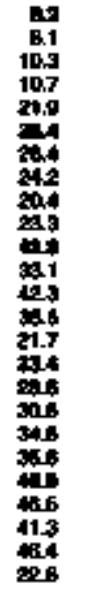 & 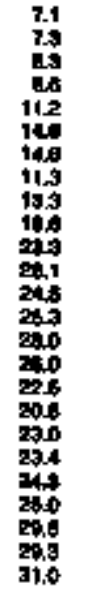 & 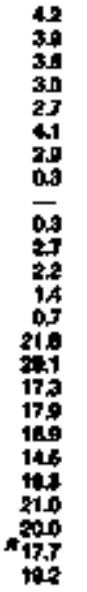 & 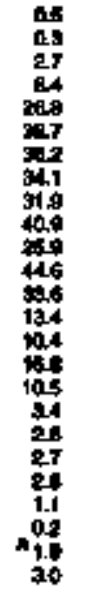 & 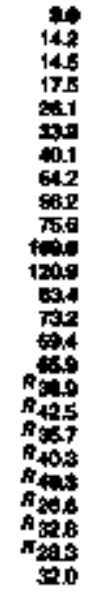 & 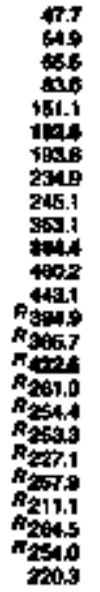 & 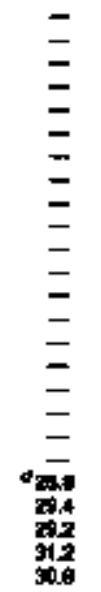 & 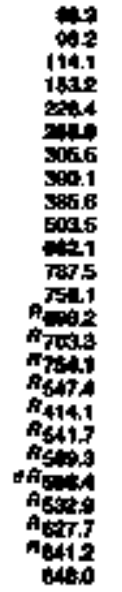 & 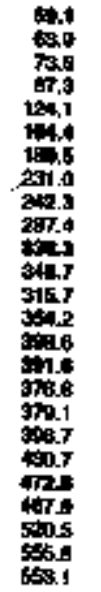 & 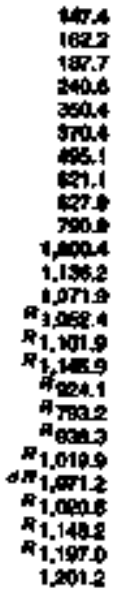 \\
\hline
\end{tabular}




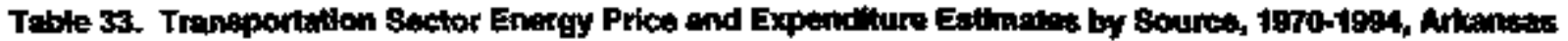

\begin{tabular}{|c|c|c|c|c|c|c|c|c|c|c|c|c|c|}
\hline \multirow[b]{4}{*}{$x=$} & \multicolumn{11}{|c|}{ Phtap En } & \multirow[b]{3}{*}{ Drater } & \multirow[b]{3}{*}{${ }_{E=1}^{T+1}$} \\
\hline & \multirow[b]{2}{*}{ on } & \multirow[b]{2}{*}{ Dand } & \multicolumn{8}{|c|}{ Antan } & \multirow[b]{2}{*}{ Tot } & & \\
\hline & & & 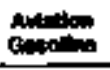 & Fin: & 呈 & uns: & 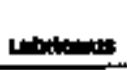 & 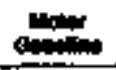 & Pit & rotid & & & \\
\hline & \multicolumn{13}{|c|}{ 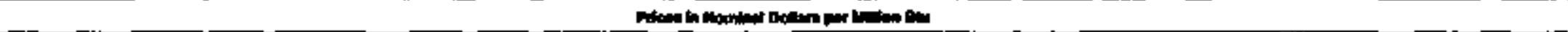 } \\
\hline \multirow[t]{2}{*}{ 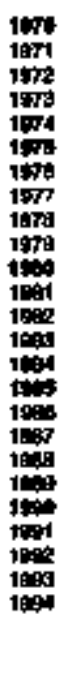 } & $\begin{array}{l}0= \\
0.09 \\
0.04 \\
0.91 \\
102 \\
1.30 \\
= \\
= \\
= \\
= \\
= \\
= \\
= \\
= \\
= \\
= \\
=\end{array}$ & $\begin{array}{l}= \\
= \\
z \\
z \\
z \\
z \\
= \\
= \\
= \\
= \\
= \\
= \\
= \\
= \\
=\end{array}$ & 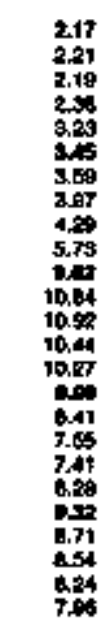 & 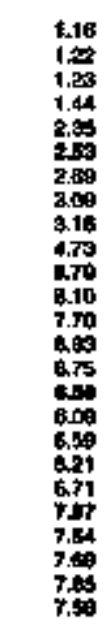 & 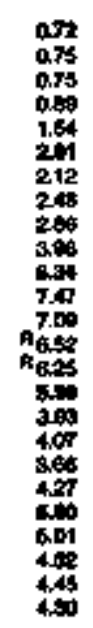 & 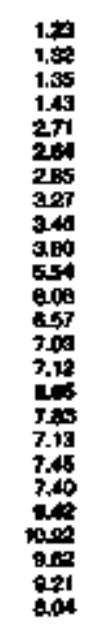 & 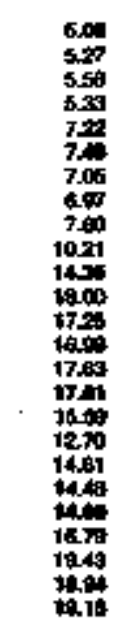 & 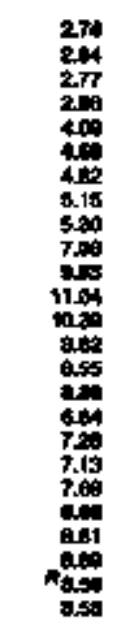 & $\begin{array}{l}= \\
= \\
0 \\
10 \\
10 \\
= \\
17 \\
= \\
= \\
= \\
= \\
= \\
= \\
= \\
= \\
=\end{array}$ & 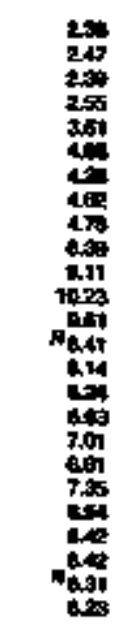 & 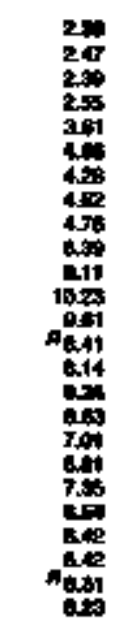 & $\begin{array}{l}= \\
= \\
= \\
= \\
= \\
= \\
= \\
= \\
= \\
= \\
= \\
= \\
= \\
=\end{array}$ & 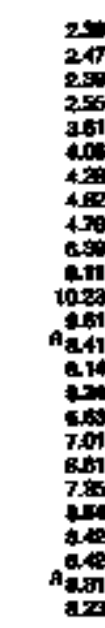 \\
\hline & \multicolumn{13}{|c|}{ 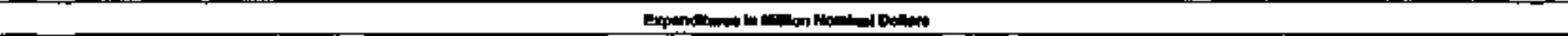 } \\
\hline 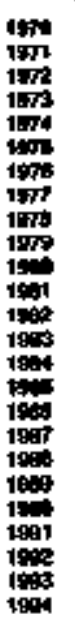 & $\begin{array}{l}= \\
0 \\
0 \\
0 \\
= \\
= \\
= \\
= \\
= \\
= \\
= \\
= \\
= \\
= \\
=\end{array}$ & $\begin{array}{l}= \\
= \\
= \\
= \\
= \\
= \\
= \\
= \\
= \\
= \\
= \\
= \\
=\end{array}$ & 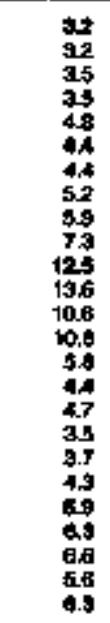 & 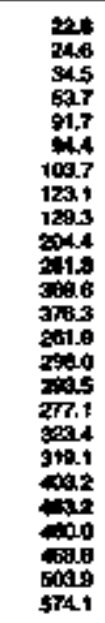 & 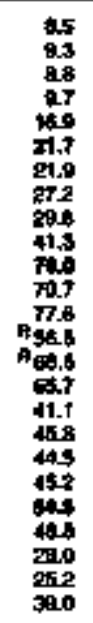 & 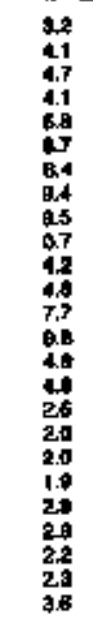 & 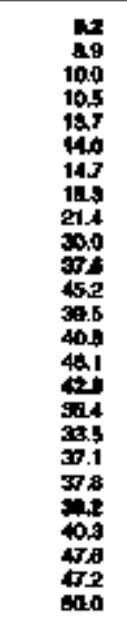 & 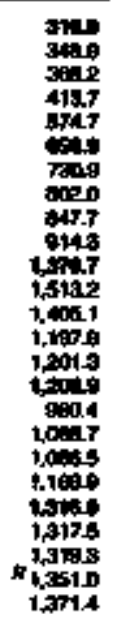 & 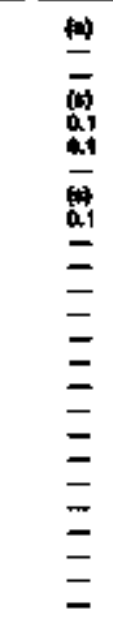 & 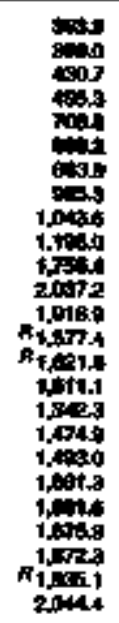 & 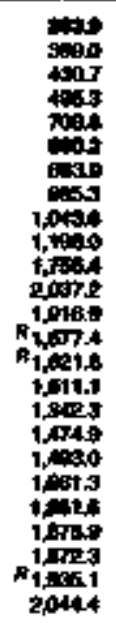 & $\begin{array}{l}= \\
= \\
= \\
= \\
= \\
= \\
= \\
= \\
= \\
= \\
= \\
= \\
= \\
=\end{array}$ & 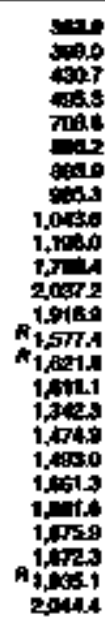 \\
\hline
\end{tabular}


A Table 34. Price and Expenditure Estimates for Energy input at Electric Utulties by Source, 1970-1994, Arkansas

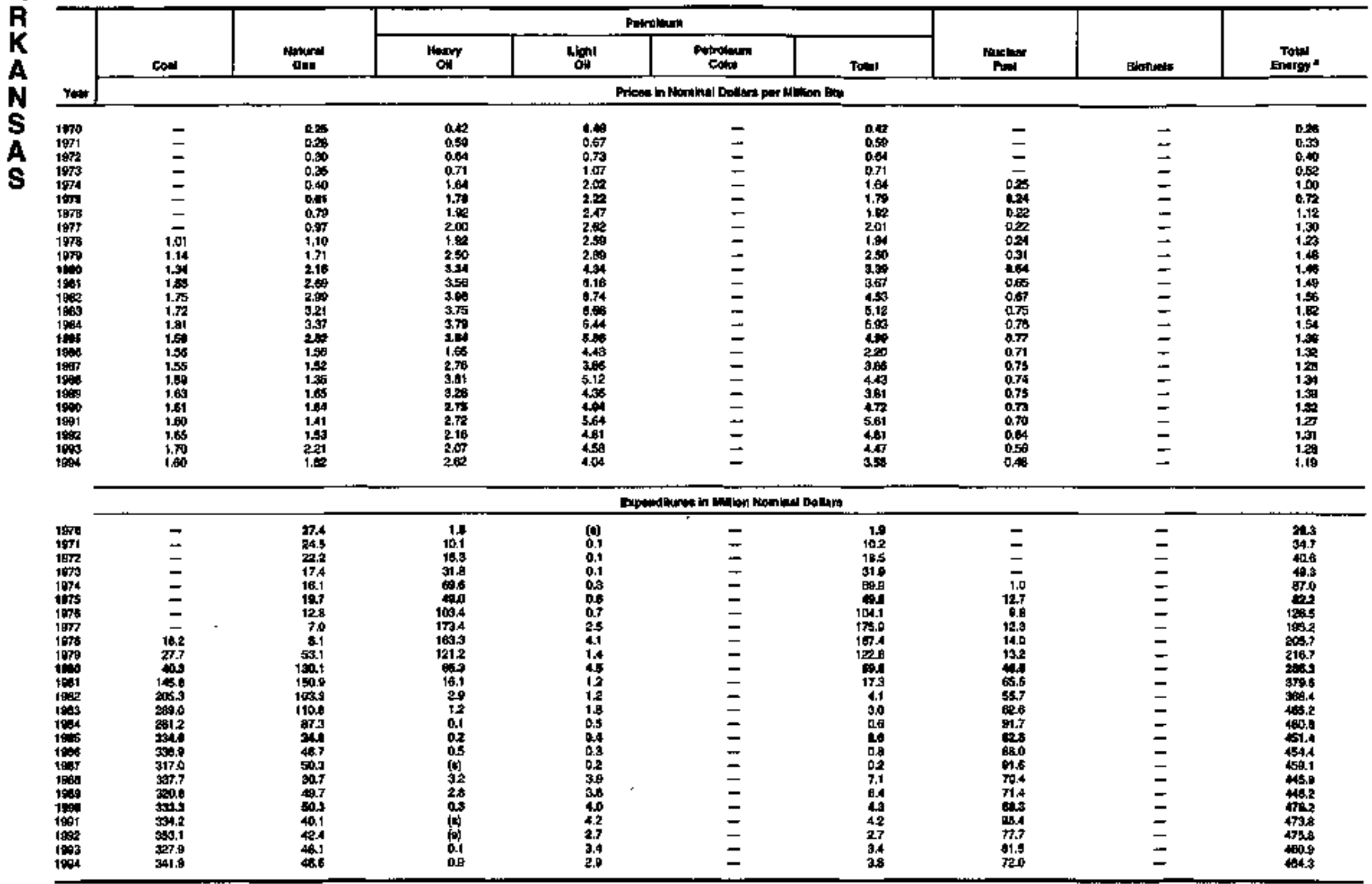

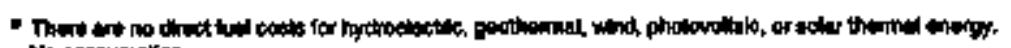

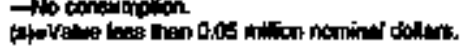

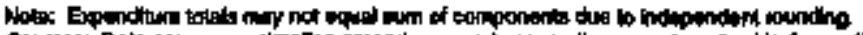

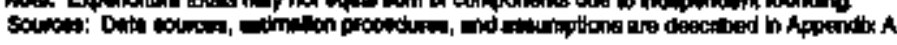




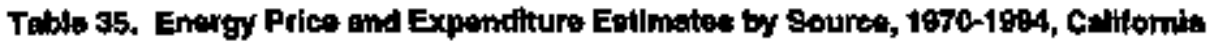

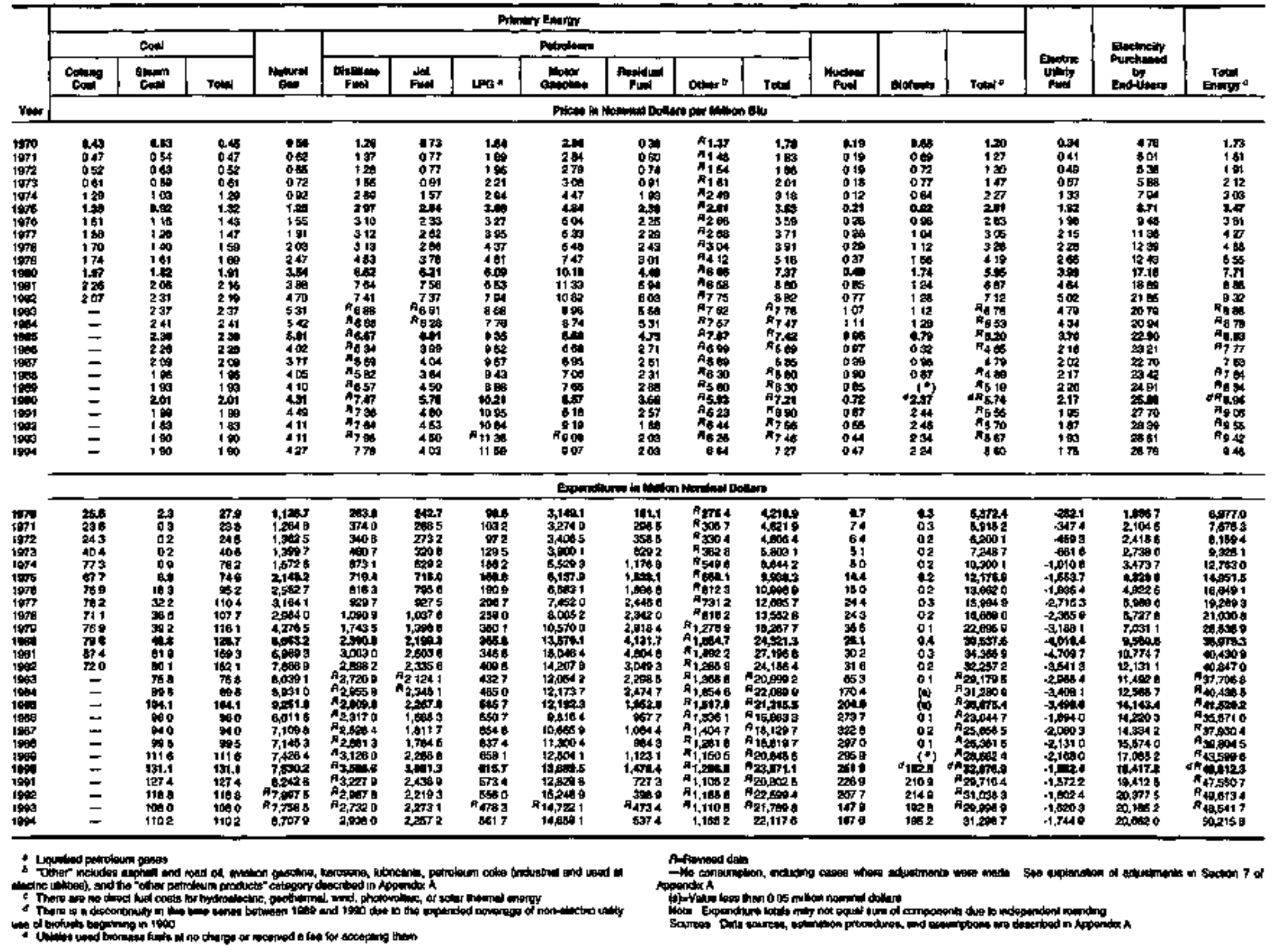




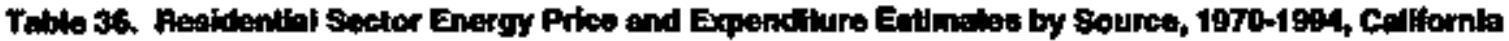

\begin{tabular}{|c|c|c|c|c|c|c|c|c|c|c|}
\hline \multirow[b]{4}{*}{ Yex } & \multicolumn{8}{|c|}{ M $x$ t } & \multirow[b]{3}{*}{ 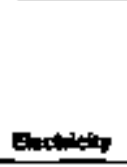 } & \multirow{3}{*}{ Fin: } \\
\hline & & & \multicolumn{4}{|c|}{ Fitroline } & \multirow[b]{2}{*}{ Dobines } & \multirow[b]{2}{*}{$\operatorname{Tim}_{\mathbf{n}} \mathbf{x}$} & & \\
\hline & Con & - & Fin & 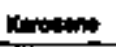 & tros & $=$ & & & & \\
\hline & \multicolumn{10}{|c|}{ 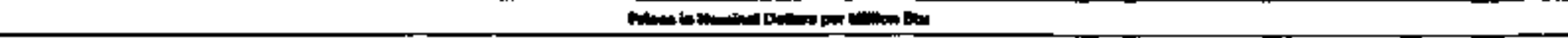 } \\
\hline \multirow[t]{2}{*}{ 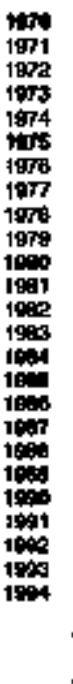 } & 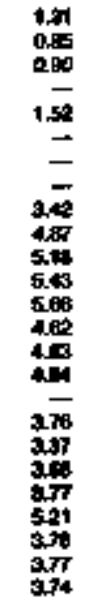 & 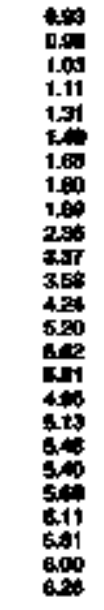 & 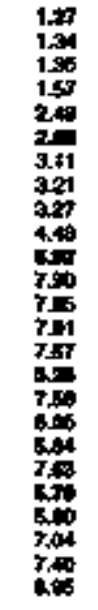 & 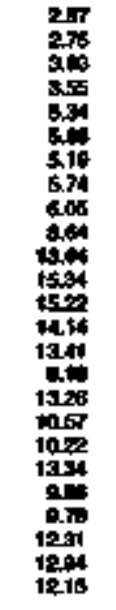 & 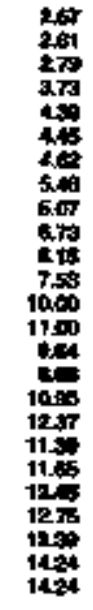 & 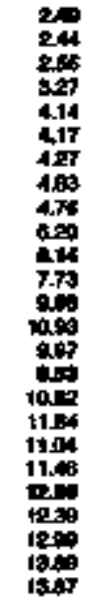 & $\begin{array}{l}= \\
= \\
= \\
= \\
= \\
= \\
= \\
= \\
= \\
= \\
= \\
= \\
47 \\
4 \pi \\
4.75\end{array}$ & 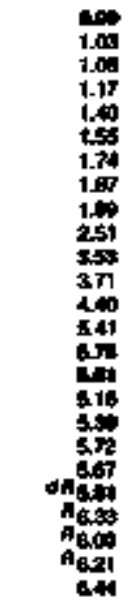 & 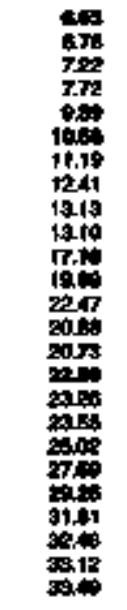 & 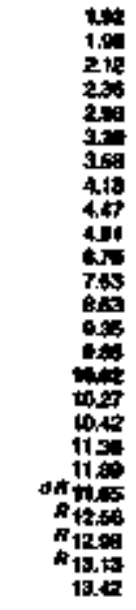 \\
\hline & \multicolumn{10}{|c|}{ 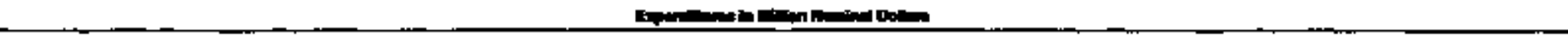 } \\
\hline 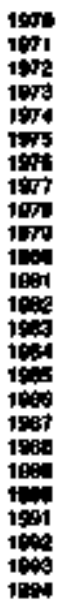 & 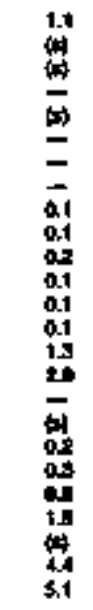 & 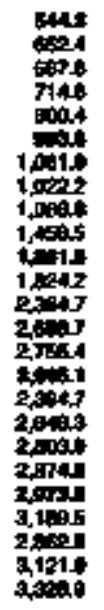 & 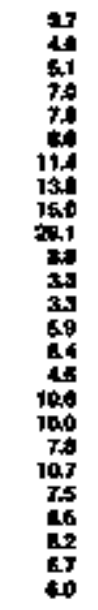 & 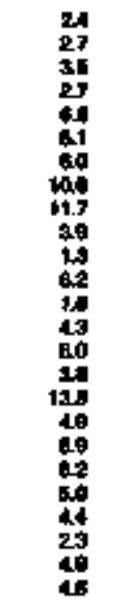 & 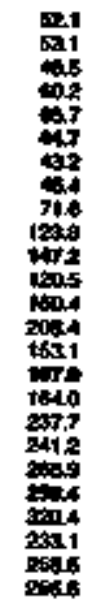 & 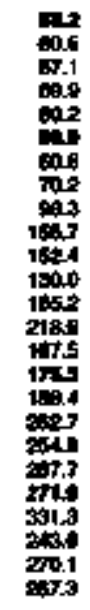 & 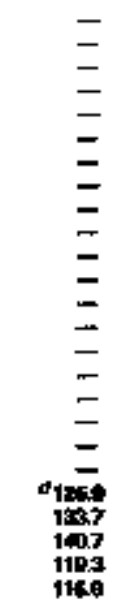 & 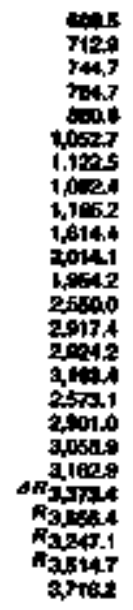 & 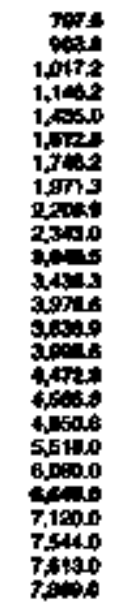 & 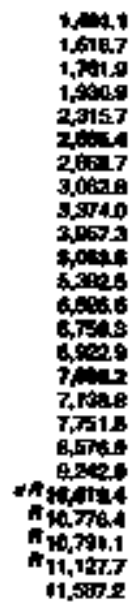 \\
\hline
\end{tabular}




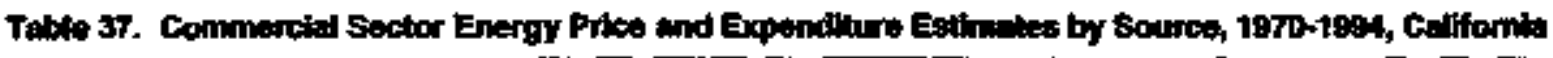

\begin{tabular}{|c|c|c|c|c|c|c|c|c|c|c|c|}
\hline \multirow[b]{4}{*}{$\mathrm{K}_{\mathrm{r}}$} & \multicolumn{9}{|c|}{ 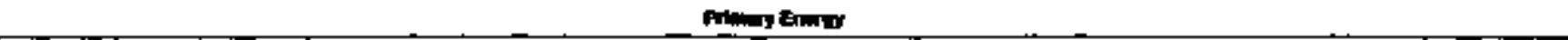 } & \multirow[b]{3}{*}{ enterlety } & \multirow[b]{3}{*}{ 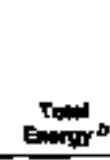 } \\
\hline & & \multirow[b]{2}{*}{ mantin } & \multicolumn{6}{|c|}{ 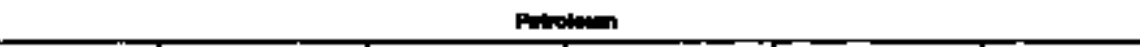 } & \multirow[b]{2}{*}{$T=1$} & & \\
\hline & $\infty$ & & 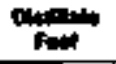 & 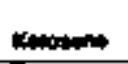 & tno: & 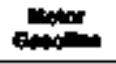 & 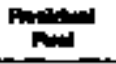 & 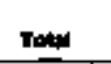 & & & \\
\hline & \multicolumn{11}{|c|}{ 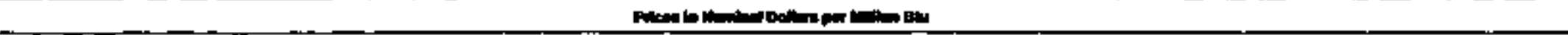 } \\
\hline \multirow[t]{2}{*}{ 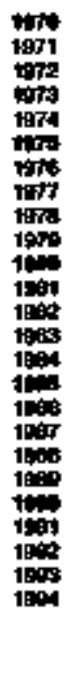 } & 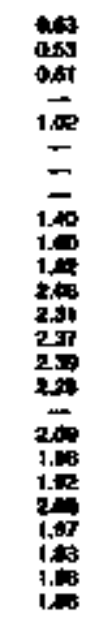 & 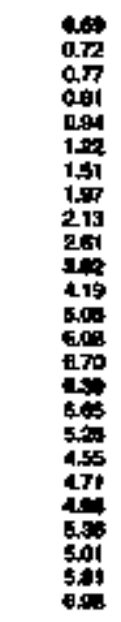 & 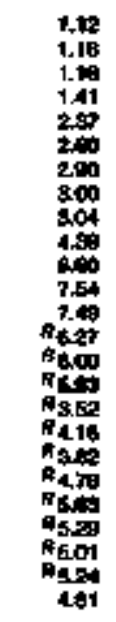 & 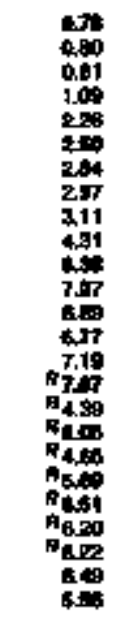 & 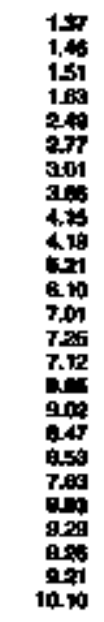 & 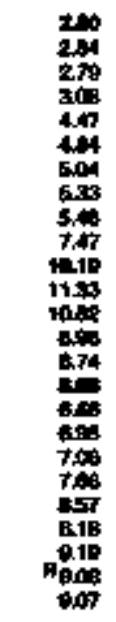 & 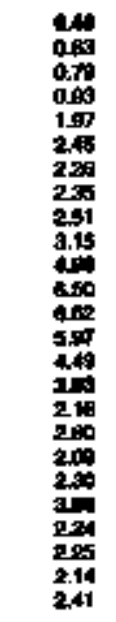 & 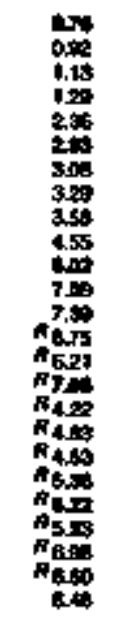 & 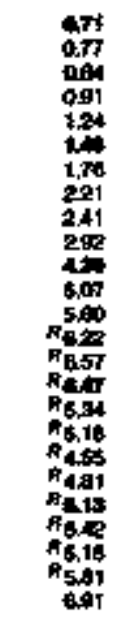 & 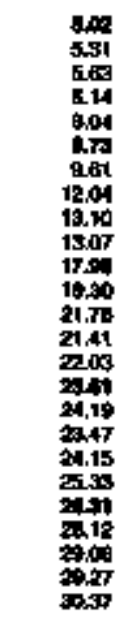 & 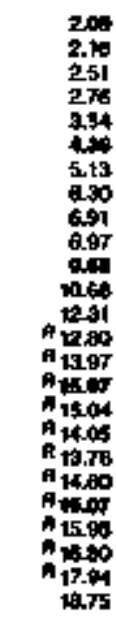 \\
\hline & \multicolumn{11}{|c|}{ 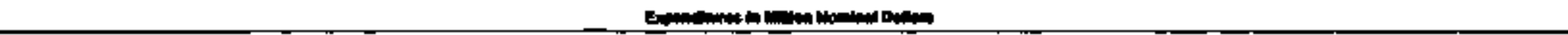 } \\
\hline 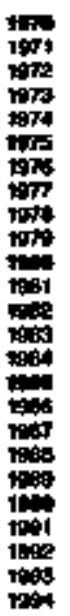 & 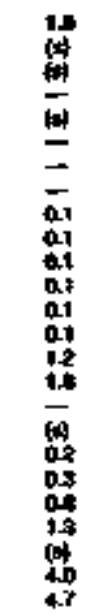 & 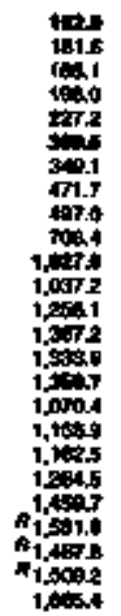 & 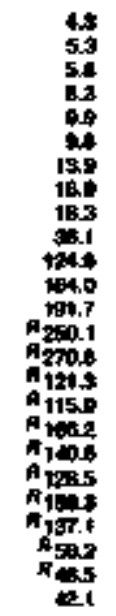 & 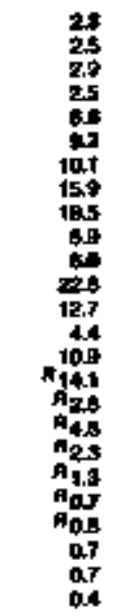 & 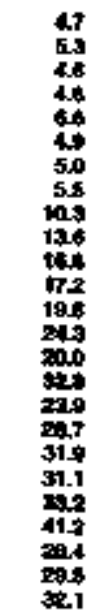 & 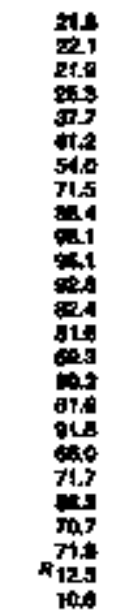 & 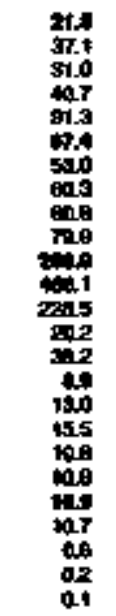 & 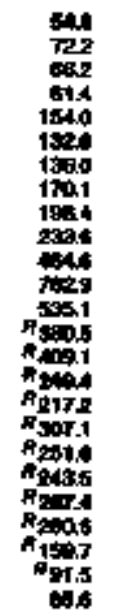 & 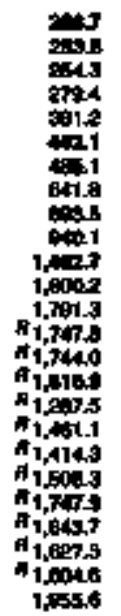 & 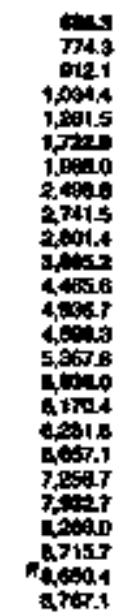 & 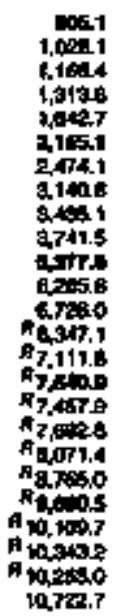 \\
\hline
\end{tabular}


Table 38. Industrlal Sactor Enargy Price and Expondlume Estimalo by Source, 1870-19a4, Califormid

\begin{tabular}{|c|c|c|c|c|c|c|c|c|c|c|c|c|c|c|c|c|c|}
\hline & \multicolumn{15}{|c|}{ Mtrey Enewory } & \multirow[b]{3}{*}{ Eugtomitr } & \multirow[b]{3}{*}{ Totor } \\
\hline & \multicolumn{3}{|c|}{$\cos$} & \multirow[b]{2}{*}{ 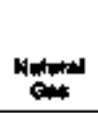 } & \multicolumn{9}{|c|}{ Potrolowa } & \multirow[b]{2}{*}{ 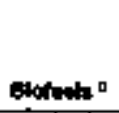 } & \multirow[b]{2}{*}{ Tolns: } & & \\
\hline & Colvang & Som & Totw & & rethen ind & Put" & tonominats & LPOS & Lenewenta & artor & Finglud & Otwers & Tont & & & & \\
\hline$Y$ in & \multicolumn{17}{|c|}{ 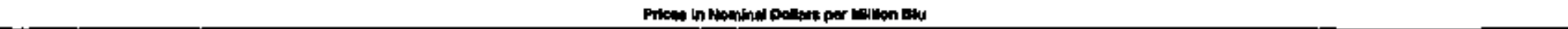 } \\
\hline \multirow[t]{2}{*}{ 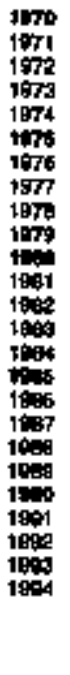 } & $\begin{array}{l}040 \\
047 \\
052 \\
061 \\
129 \\
190 \\
141 \\
158 \\
170 \\
174 \\
199 \\
2207 \\
207 \\
= \\
= \\
= \\
= \\
= \\
= \\
= \\
=\end{array}$ & 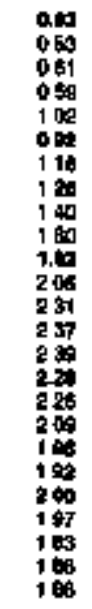 & 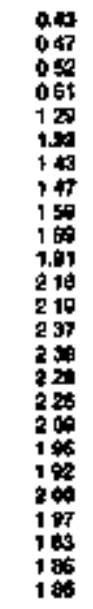 & 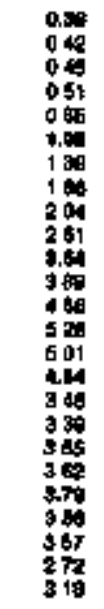 & 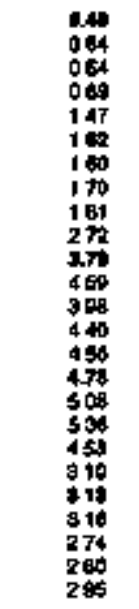 & 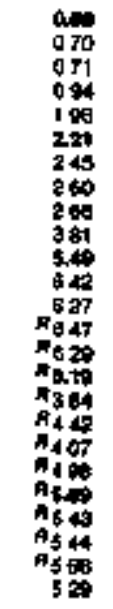 & 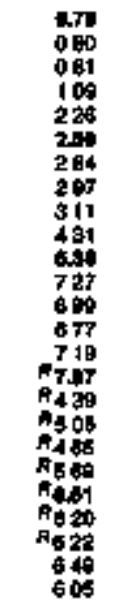 & 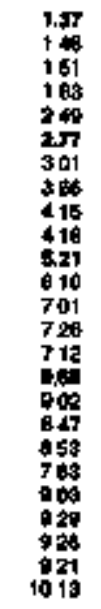 & 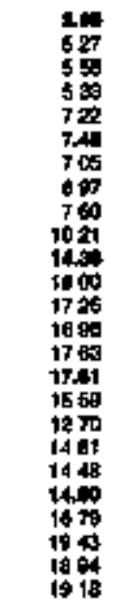 & 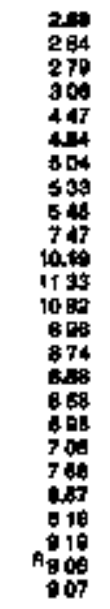 & 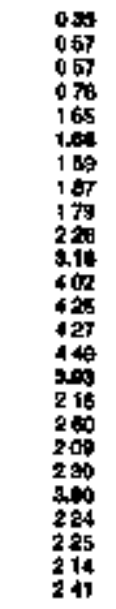 & 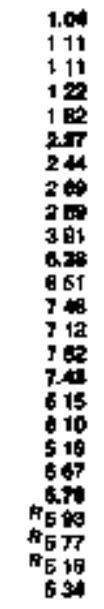 & 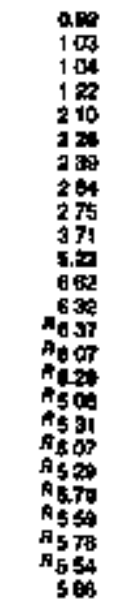 & $\begin{array}{l}= \\
= \\
= \\
= \\
= \\
= \\
= \\
= \\
= \\
= \\
= \\
= \\
1=0 \\
132 \\
128 \\
128 \\
130\end{array}$ & 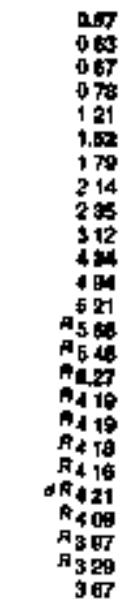 & 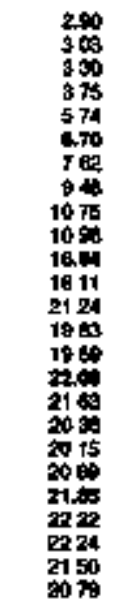 & 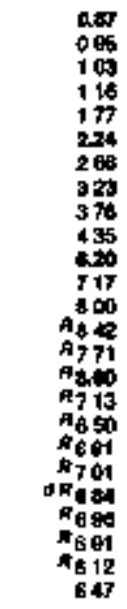 \\
\hline & \multicolumn{17}{|c|}{ 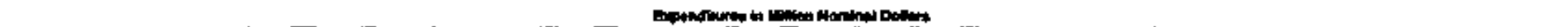 } \\
\hline 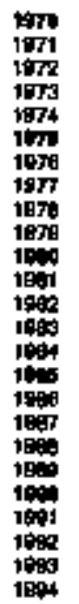 & $\begin{array}{l}256 \\
206 \\
243 \\
404 \\
775 \\
979 \\
760 \\
762 \\
711 \\
769 \\
701 \\
974 \\
720 \\
= \\
= \\
= \\
= \\
= \\
= \\
= \\
=\end{array}$ & 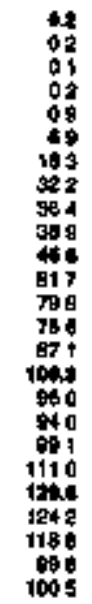 & 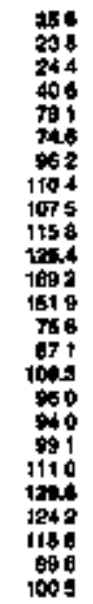 & 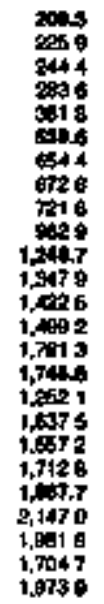 & 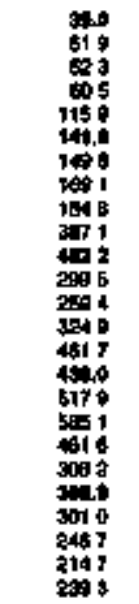 & 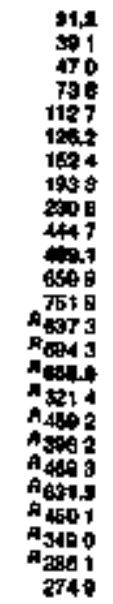 & 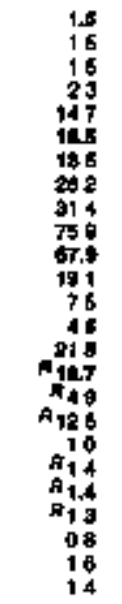 & 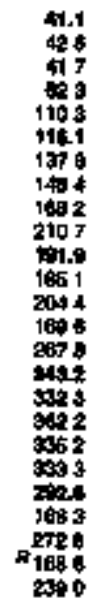 & 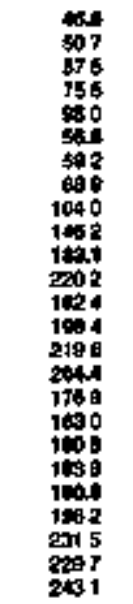 & 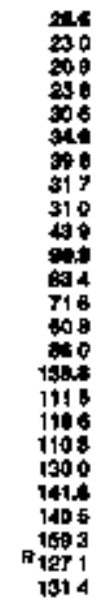 & 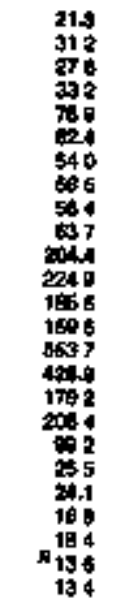 & 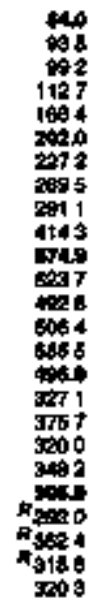 & 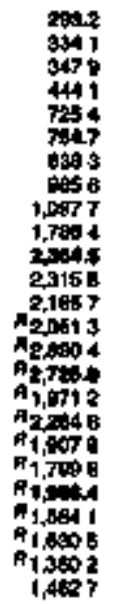 & $\begin{array}{l}= \\
= \\
= \\
= \\
= \\
= \\
= \\
= \\
= \\
= \\
= \\
0 \\
\pi 19 \\
742 \\
703\end{array}$ & 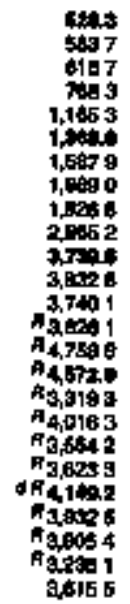 & 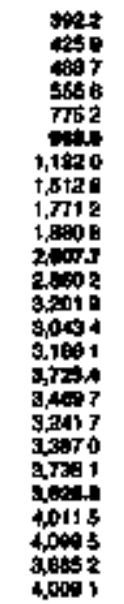 & 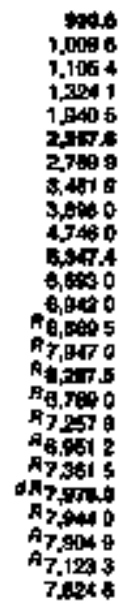 \\
\hline
\end{tabular}

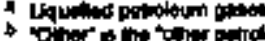

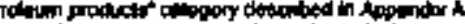

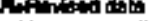

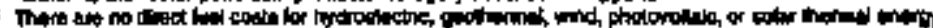

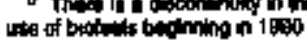


Table 39. Trensportatlon Sector Encray Price end Expenditure Estimates by Soumbe, 19ro-1994, Callomia

\begin{tabular}{|c|c|c|c|c|c|c|c|c|c|c|c|c|c|}
\hline \multirow[b]{4}{*}{ var } & \multicolumn{11}{|c|}{ 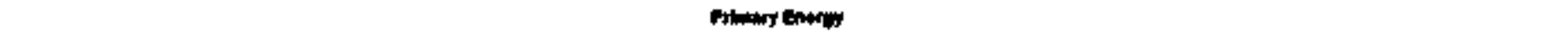 } & \multirow[b]{3}{*}{ Inotiothr } & \multirow[b]{3}{*}{ Ty } \\
\hline & \multirow[b]{2}{*}{ cont } & \multirow[b]{2}{*}{ Nam } & \multicolumn{8}{|c|}{ Rintingh } & \multirow[b]{2}{*}{ Total } & & \\
\hline & & & Avadion & 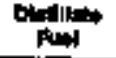 & fint & LPQ= & 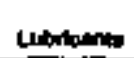 & 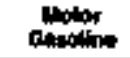 & 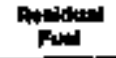 & Tall & & & \\
\hline & \multicolumn{13}{|c|}{ 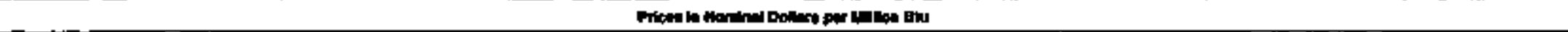 } \\
\hline \multirow[t]{2}{*}{ 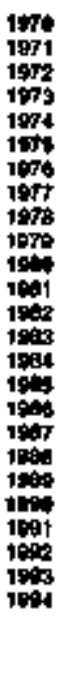 } & $\begin{array}{l}0.00 \\
0.50 \\
0.61 \\
0.50 \\
1.08 \\
0.00 \\
1.10 \\
1.20 \\
= \\
= \\
= \\
= \\
= \\
= \\
= \\
= \\
= \\
= \\
=\end{array}$ & $\begin{array}{l}= \\
= \\
= \\
= \\
= \\
= \\
= \\
= \\
= \\
= \\
= \\
= \\
450 \\
508 \\
408\end{array}$ & 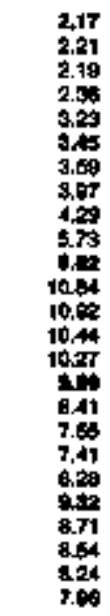 & 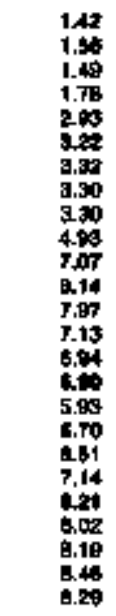 & 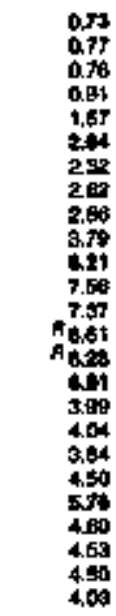 & 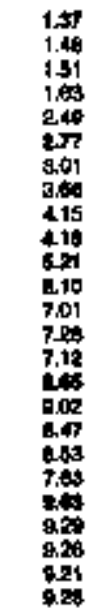 & 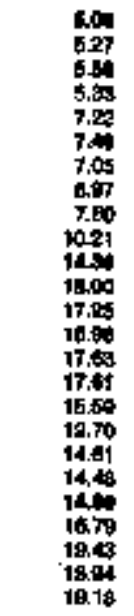 & 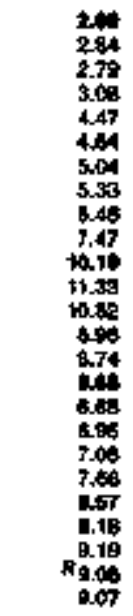 & 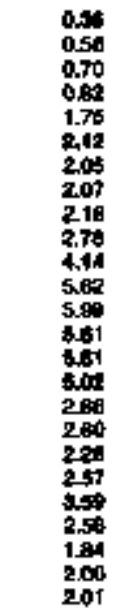 & 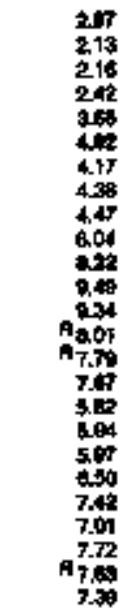 & 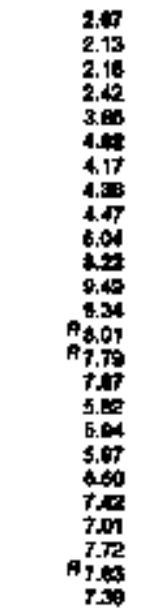 & 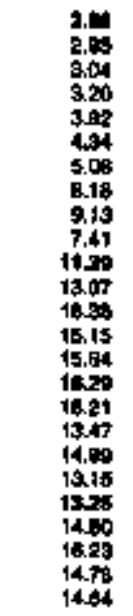 & 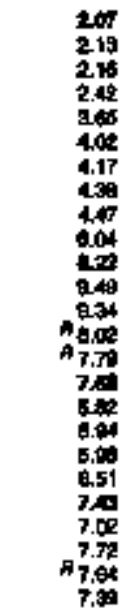 \\
\hline & \multicolumn{13}{|c|}{ 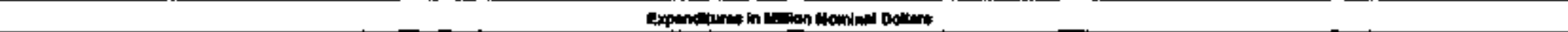 } \\
\hline 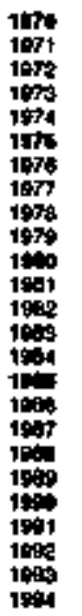 & 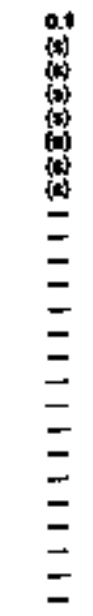 & $\begin{array}{l}= \\
= \\
= \\
= \\
= \\
= \\
= \\
= \\
= \\
= \\
= \\
= \\
= \\
01 \\
01 \\
02 \\
2.8\end{array}$ & 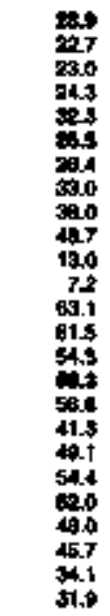 & 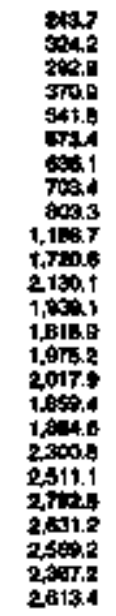 & 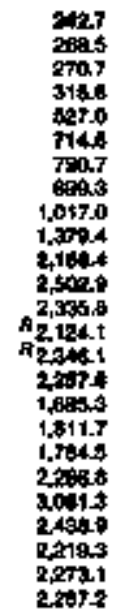 & 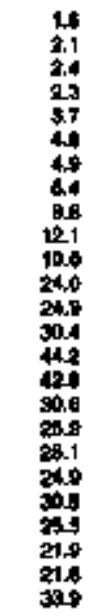 & 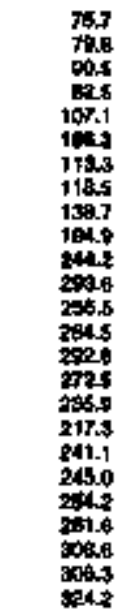 & 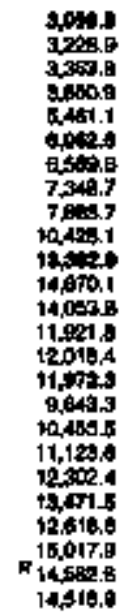 & 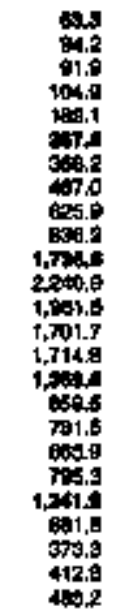 & 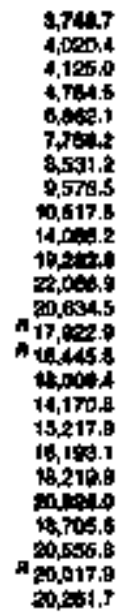 & 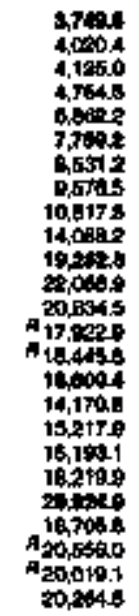 & 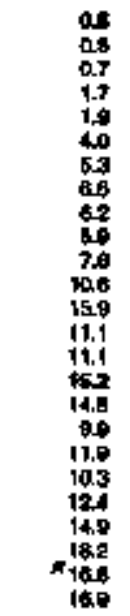 & 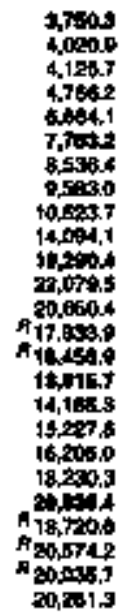 \\
\hline
\end{tabular}

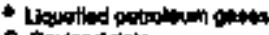

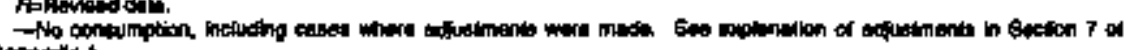

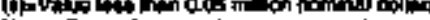

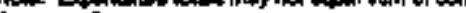
(nt) 
C Table 40. Price and Expendlume Estimetes for Enorgy mput at Eloctite Utilitis by Sosree, 1970-1994, Califomia

\begin{tabular}{|c|c|c|c|c|c|c|c|c|c|}
\hline \multirow[b]{3}{*}{ ve } & \multirow[b]{2}{*}{ cond } & \multirow[b]{2}{*}{ matid } & \multicolumn{4}{|c|}{ 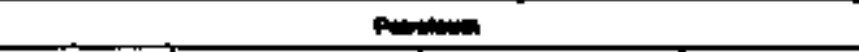 } & \multirow[b]{2}{*}{ "וה" } & \multirow[b]{2}{*}{ (1) } & \multirow[b]{2}{*}{ 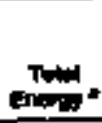 } \\
\hline & & & an & 낭 & مئم & Totor & & & \\
\hline & \multicolumn{9}{|c|}{ 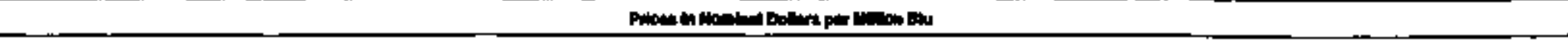 } \\
\hline \multirow[t]{2}{*}{ 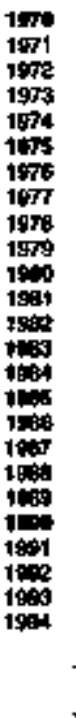 } & 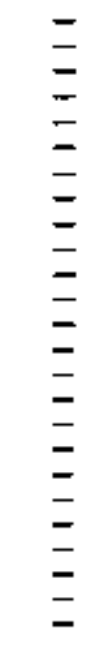 & 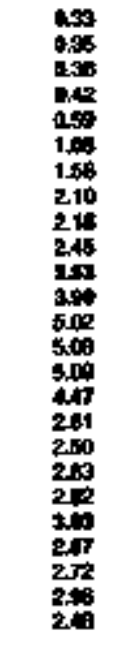 & 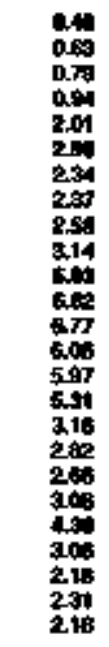 & 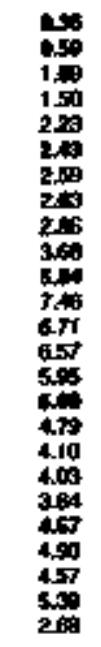 & $\begin{array}{l}= \\
= \\
= \\
= \\
= \\
= \\
= \\
= \\
= \\
= \\
= \\
= \\
= \\
=\end{array}$ & 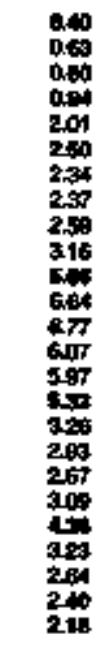 & 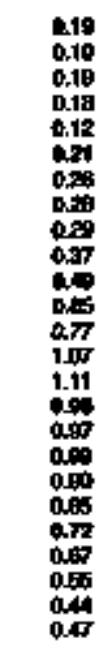 & 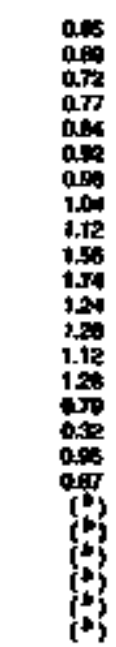 & 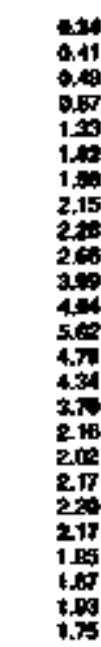 \\
\hline & \multicolumn{9}{|c|}{ 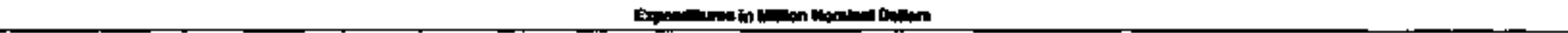 } \\
\hline 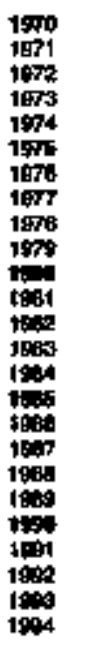 & $\begin{array}{l}= \\
= \\
z \\
= \\
= \\
= \\
= \\
= \\
= \\
= \\
= \\
= \\
= \\
=\end{array}$ & 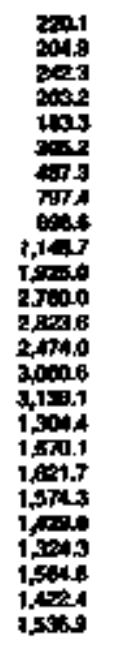 & 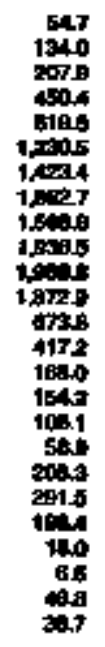 & 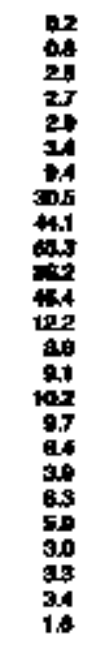 & $\begin{array}{l}= \\
= \\
= \\
= \\
= \\
= \\
= \\
= \\
= \\
= \\
= \\
= \\
= \\
=\end{array}$ & 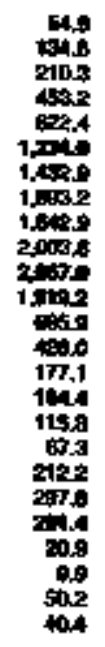 & 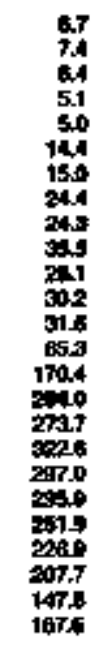 & 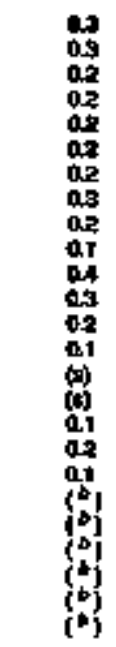 & 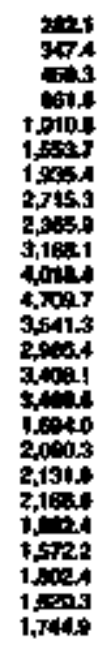 \\
\hline
\end{tabular}

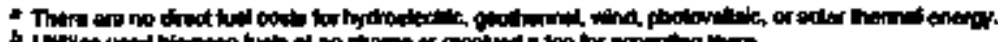

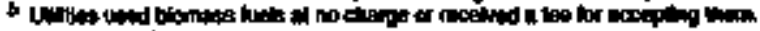

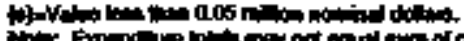

No can:aripion.

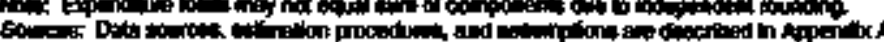




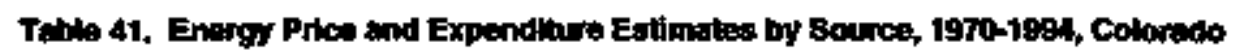

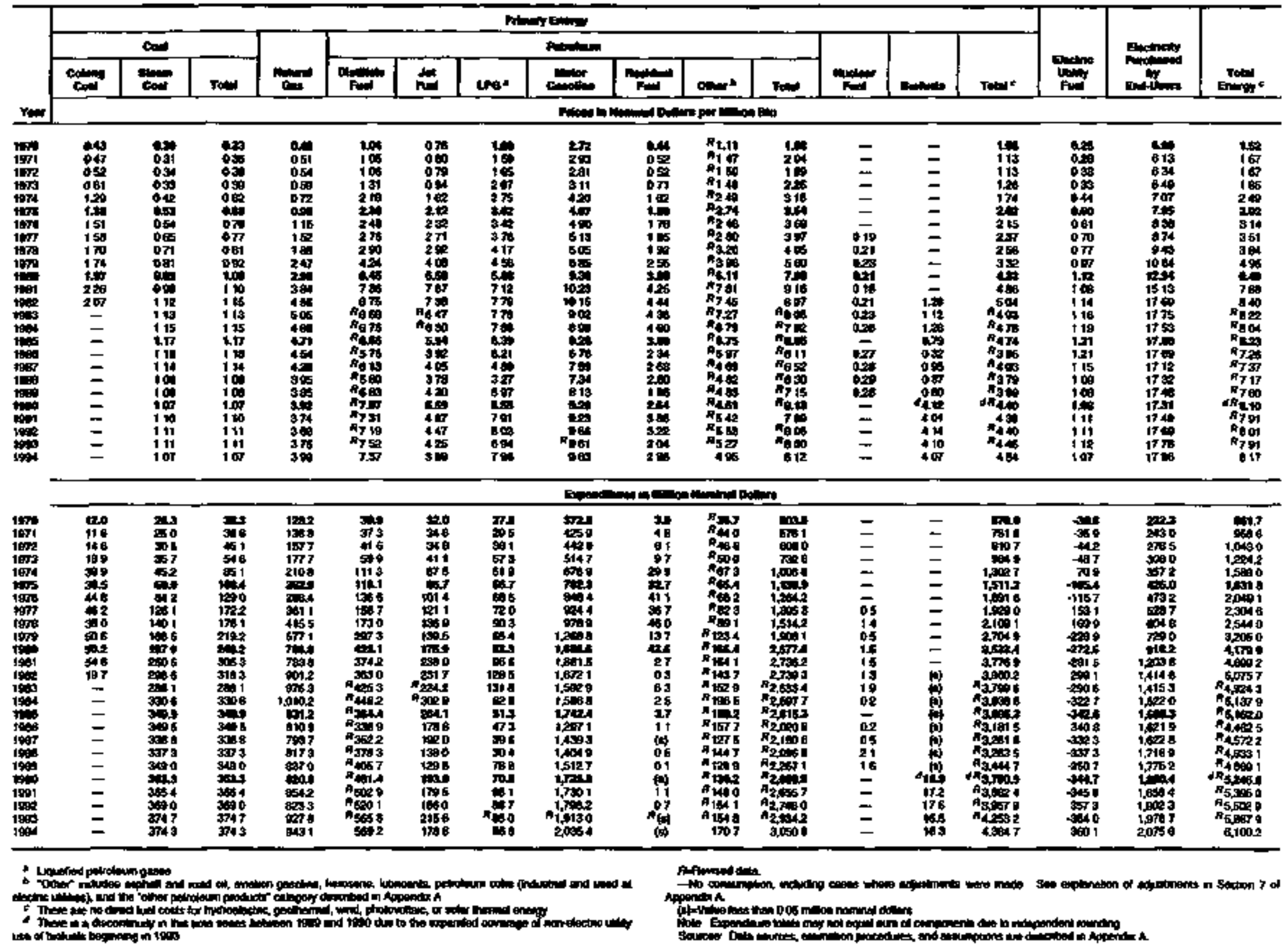


Tablo 42. Reeldential Sector Enargy Price and Expondthure Eatimates by Source, 1870-1994, Colorado

\begin{tabular}{|c|c|c|c|c|c|c|c|c|c|c|}
\hline \multirow[b]{4}{*}{$Y=$} & \multicolumn{8}{|c|}{ Phinny Ennory. } & \multirow[b]{3}{*}{ Enctivaty } & \multirow[b]{3}{*}{ Enerots } \\
\hline & & \multirow[b]{2}{*}{ 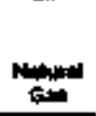 } & \multicolumn{4}{|c|}{ Patrolowin } & \multirow[b]{2}{*}{ Dlotum } & \multirow[b]{2}{*}{ tolv' } & & \\
\hline & $\cos$ & & 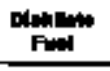 & Knrowase & LPQ* & Tolp & & & & \\
\hline & \multicolumn{10}{|c|}{ 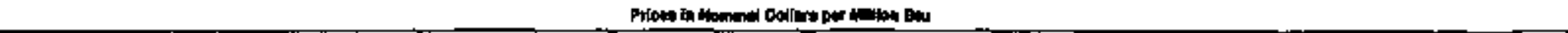 } \\
\hline \multirow[t]{2}{*}{ 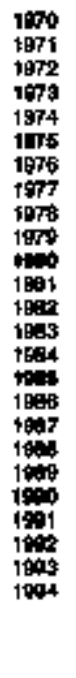 } & 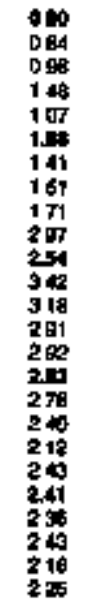 & 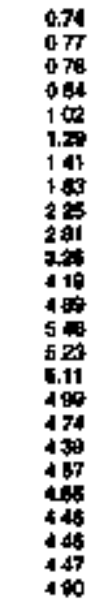 & 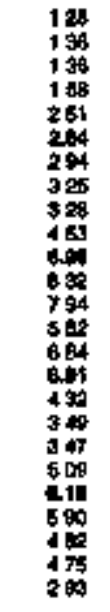 & 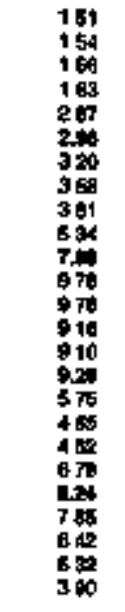 & 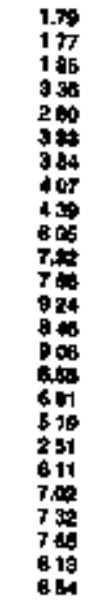 & 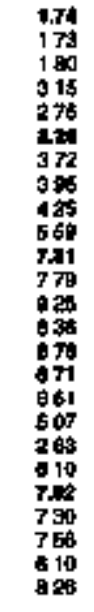 & $\begin{array}{l}= \\
= \\
= \\
= \\
= \\
= \\
= \\
= \\
= \\
= \\
= \\
49 \\
476 \\
475 \\
476\end{array}$ & 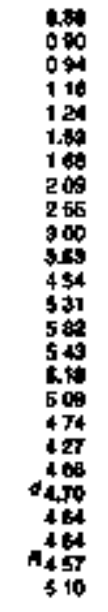 & 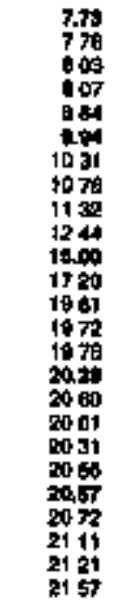 & 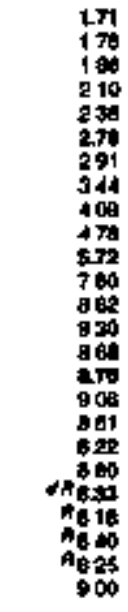 \\
\hline & \multicolumn{10}{|c|}{ 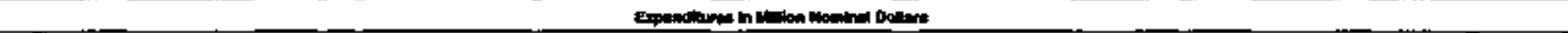 } \\
\hline 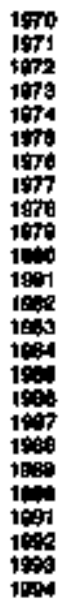 & $\begin{array}{l}14 \\
14 \\
17 \\
20 \\
09 \\
02 \\
00 \\
10 \\
25 \\
40 \\
19 \\
85 \\
90 \\
10 \\
20 \\
19 \\
22 \\
16 \\
15 \\
12 \\
19 \\
12 \\
10 \\
08 \\
04\end{array}$ & 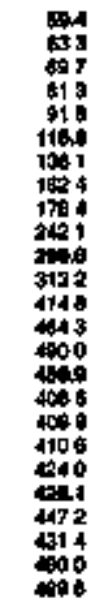 & $\begin{array}{l}11 \\
16 \\
17 \\
23 \\
43 \\
45 \\
40 \\
55 \\
55 \\
90 \\
31 \\
26 \\
27 \\
46 \\
66 \\
49 \\
18 \\
12 \\
11 \\
12 \\
10 \\
08 \\
06 \\
00 \\
04\end{array}$ & 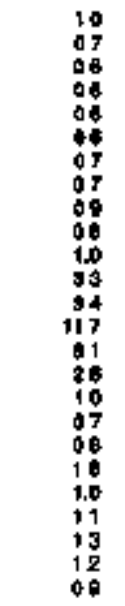 & 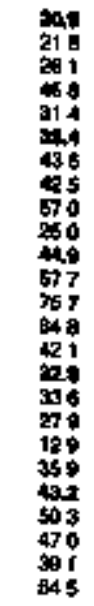 & 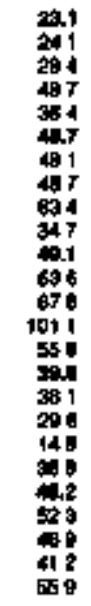 & 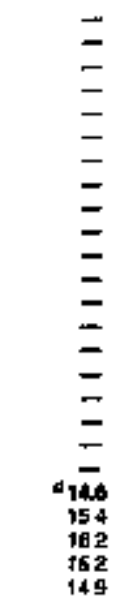 & 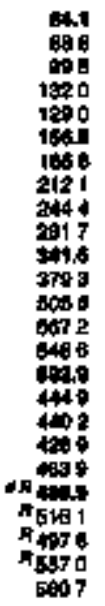 & 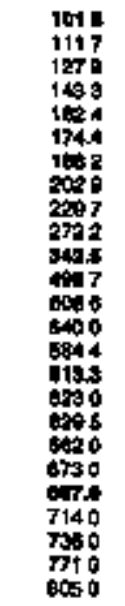 & 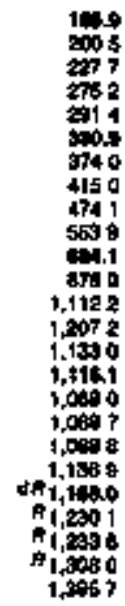 \\
\hline
\end{tabular}

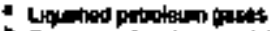

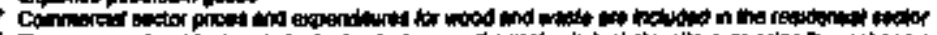

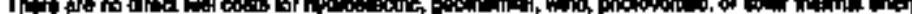

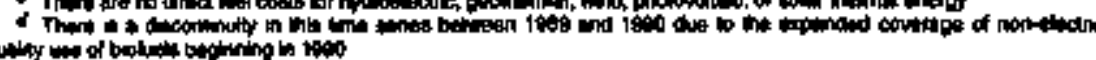

Anflowaed dot

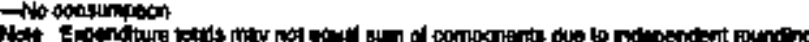

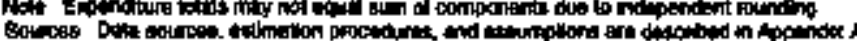

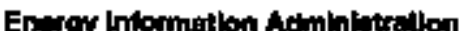

Stato Enorgy Prlew and Expenditure Repont 1 sed 
TE

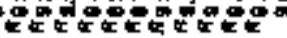

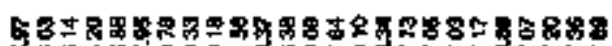

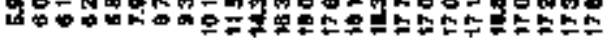

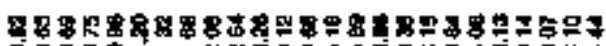

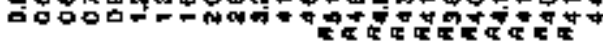

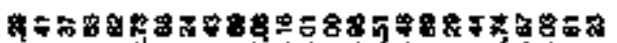

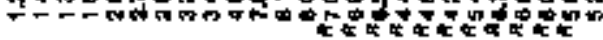

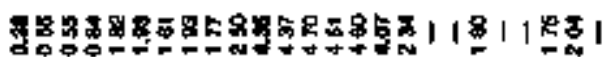

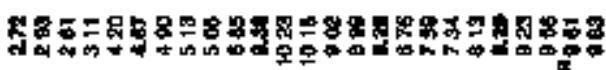

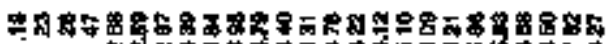

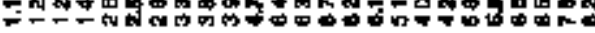

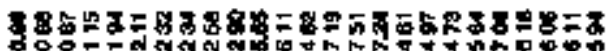

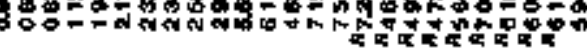

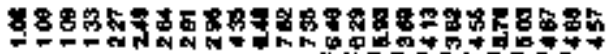

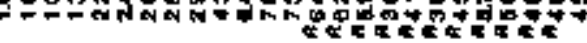

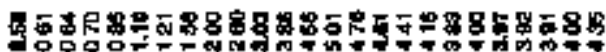

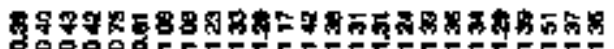

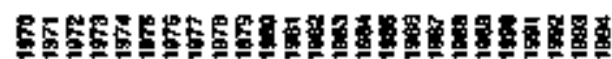

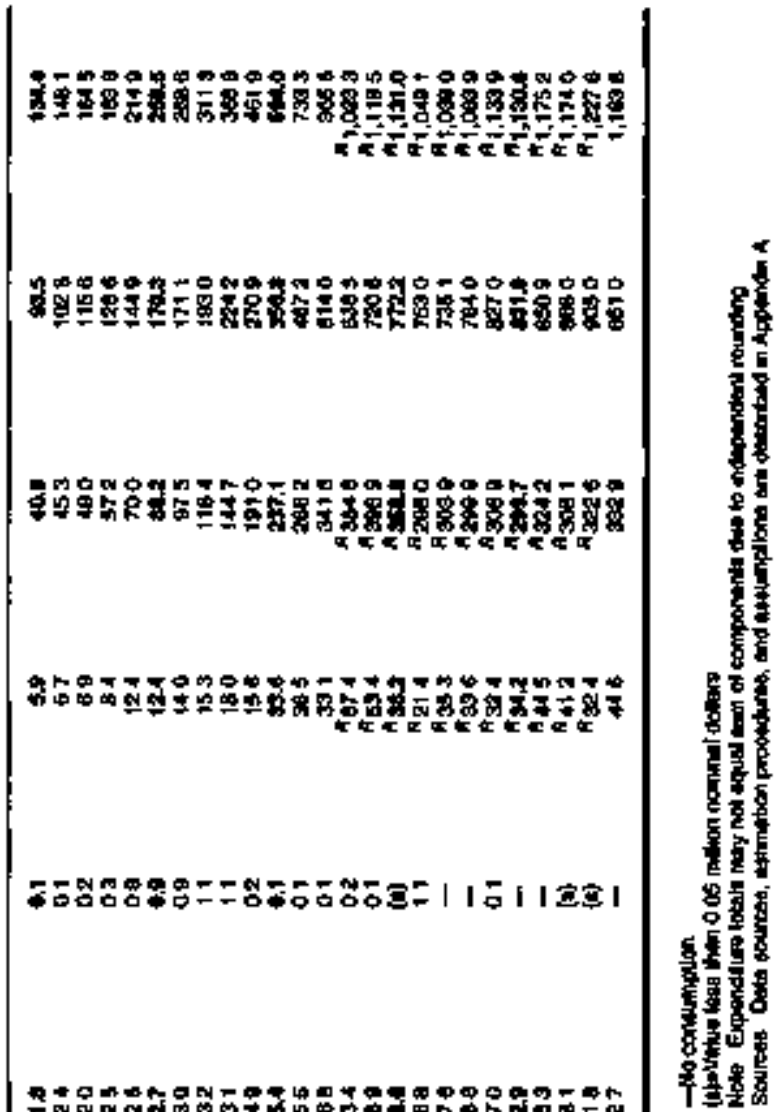

5

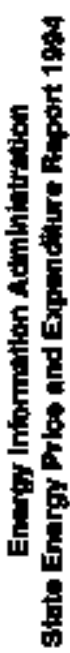

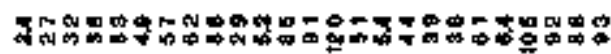

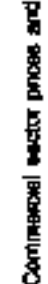

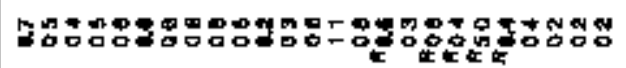

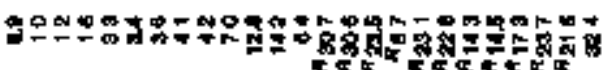

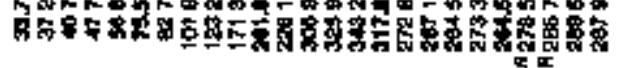

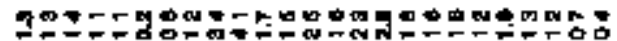

5 


\begin{tabular}{|c|c|c|c|c|c|c|c|c|c|c|c|c|c|c|c|c|c|}
\hline & \multicolumn{15}{|c|}{ 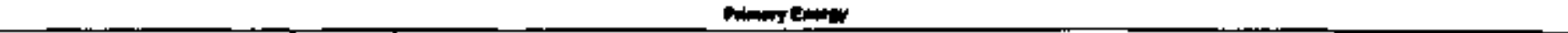 } & \multirow[b]{3}{*}{ Eximely } & \multirow[b]{3}{*}{ 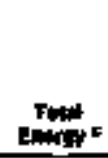 } \\
\hline & \multicolumn{3}{|c|}{ 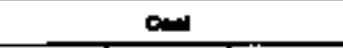 } & \multirow[b]{2}{*}{ 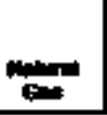 } & \multicolumn{9}{|c|}{ Parotan } & \multirow[b]{2}{*}{ 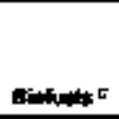 } & \multirow[b]{2}{*}{ 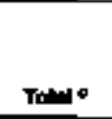 } & & \\
\hline & החיר & cont & Int & & 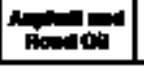 & Fid & | & Les: & Lenter: & 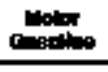 & 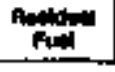 & Oumb & rolken & & & & \\
\hline$x_{n=1}$ & \multicolumn{17}{|c|}{ 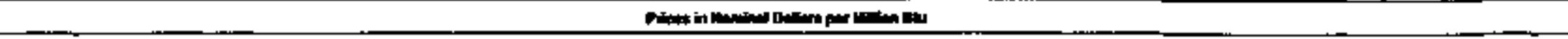 } \\
\hline \multirow[t]{2}{*}{ 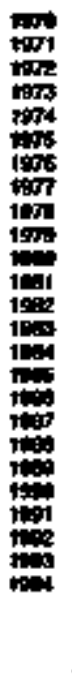 } & 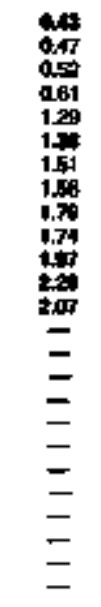 & 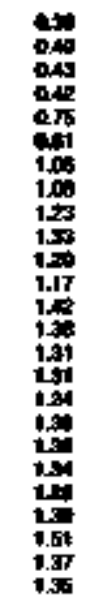 & 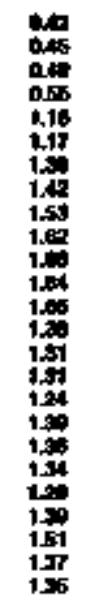 & 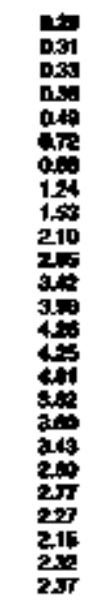 & 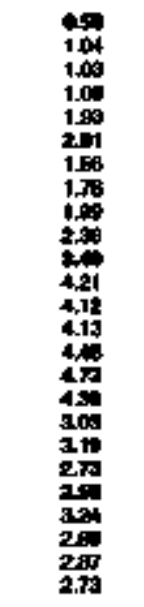 & 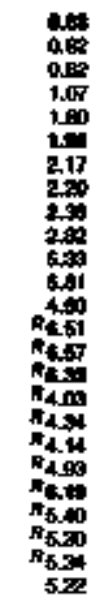 & 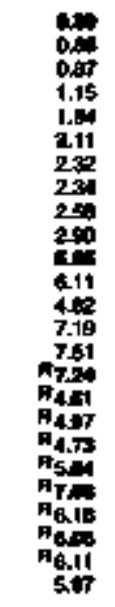 & 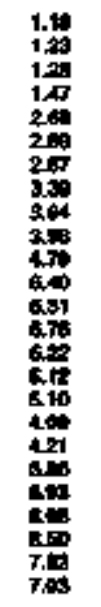 & 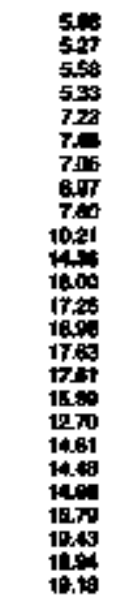 & 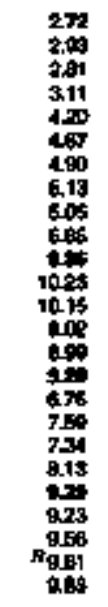 & 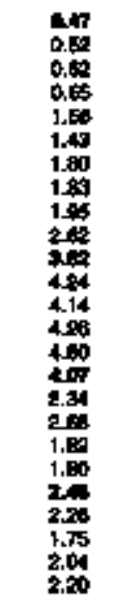 & 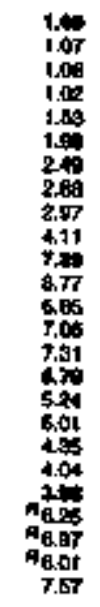 & 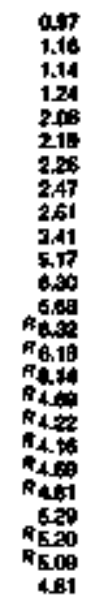 & 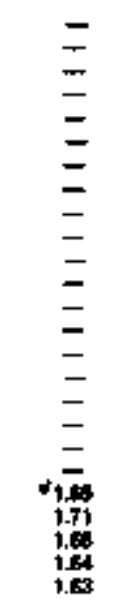 & 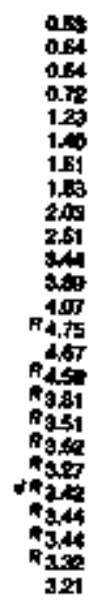 & 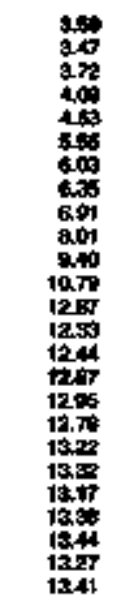 & 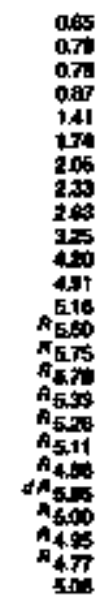 \\
\hline & \multicolumn{17}{|c|}{ 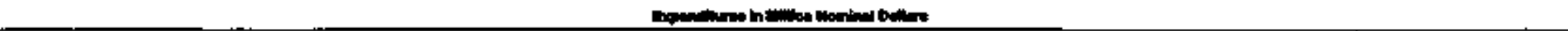 } \\
\hline 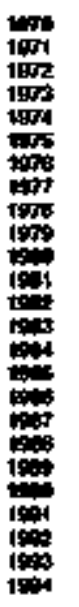 & 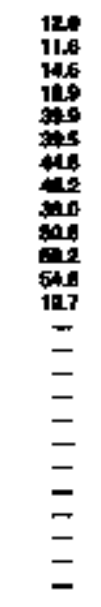 & 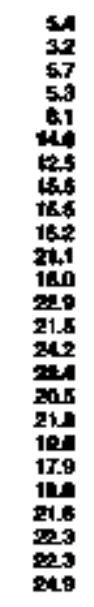 & 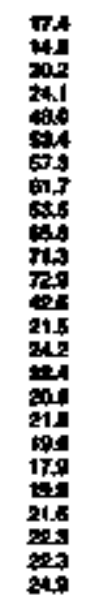 & 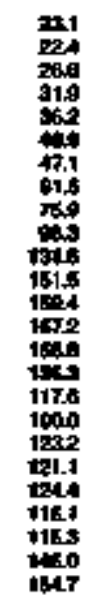 & 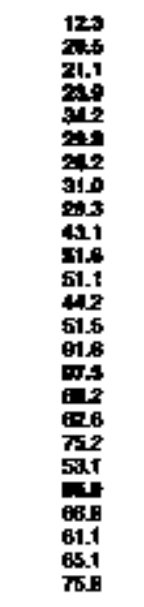 & 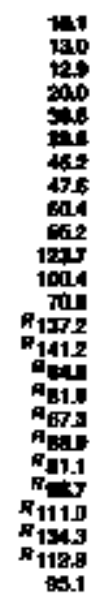 & 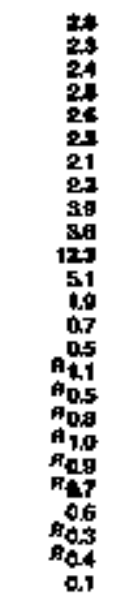 & 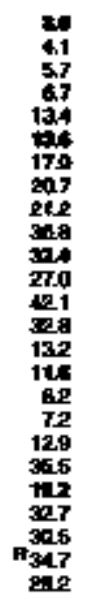 & 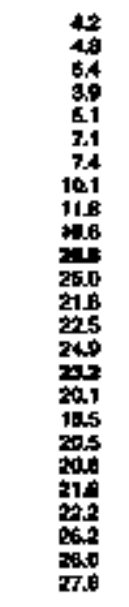 & 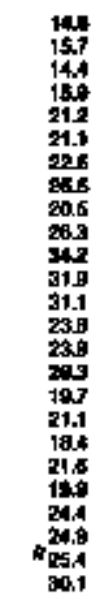 & 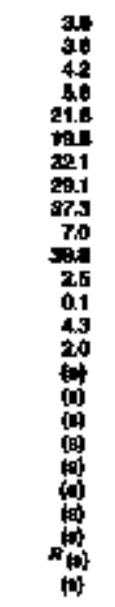 & 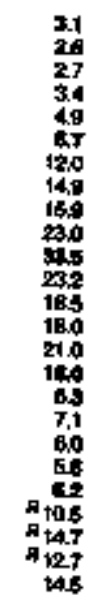 & 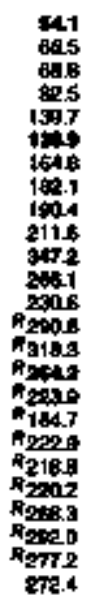 & $\begin{array}{l}z \\
z \\
z \\
z \\
z \\
z \\
z \\
z \\
z \\
z \\
z \\
z \\
4.3 \\
1.4 \\
1.4\end{array}$ & 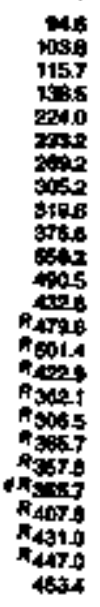 & 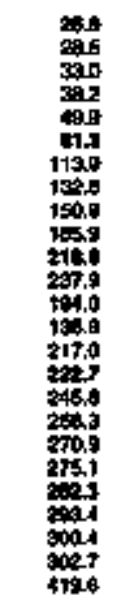 & 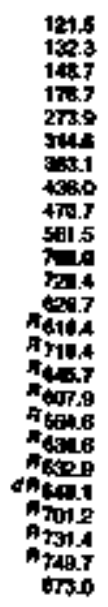 \\
\hline
\end{tabular}

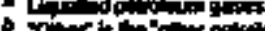

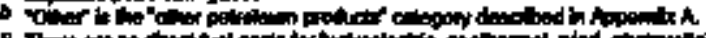

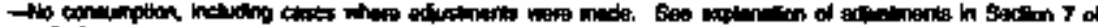

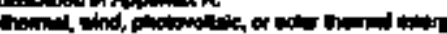

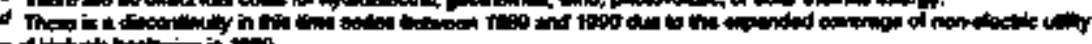

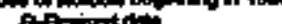

Apendix

(a)

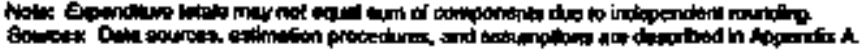


Table 45. Transpontotion Sector Energy Price and Expendhure Ettinnutoe by Sounse, 1970-1994, Coloredo

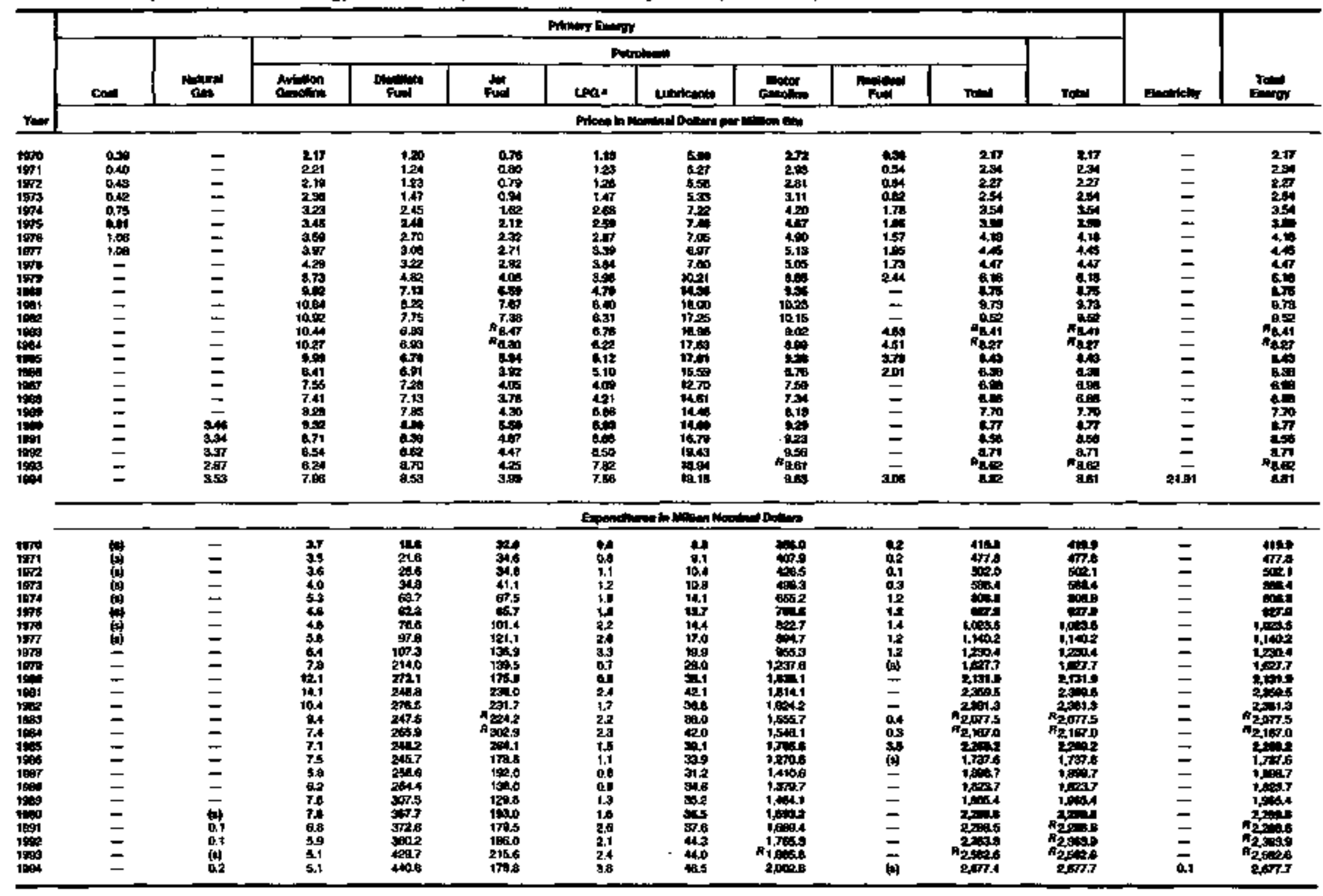


C Tablo 46. Price and Expenditure Estimates for Energy input at Electric Utelities by Source, 1970-1994, Colorado

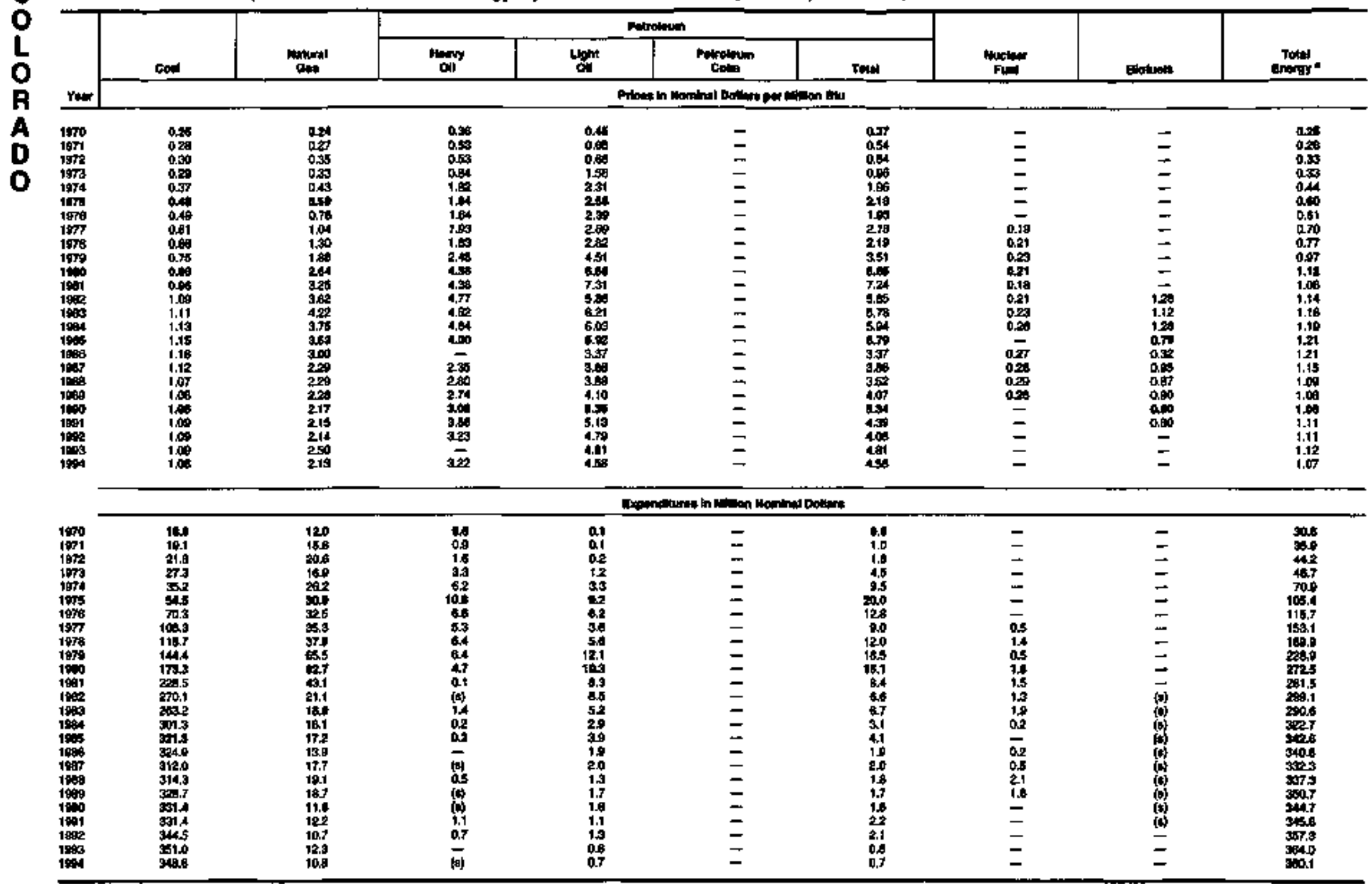

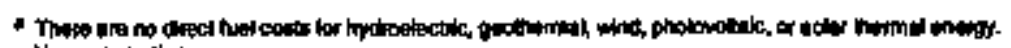
$\rightarrow$ Naconatimpiom

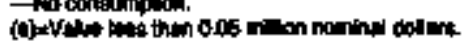

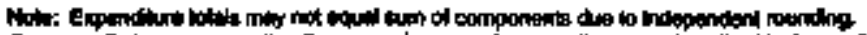

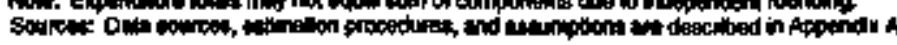


Table 47. Enargy Prico and Expenditure Estinutes by Boures, 1970-1994, Connoticul

\begin{tabular}{|c|c|c|c|c|c|c|c|c|c|c|c|c|c|c|c|c|c|}
\hline \multirow[b]{4}{*}{ rest } & \multicolumn{14}{|c|}{ Primery enery } & \multirow{3}{*}{ tome } & \multirow{3}{*}{ 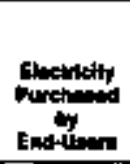 } & \multirow[b]{3}{*}{ Tom } \\
\hline & \multicolumn{3}{|c|}{ coll } & \multirow[b]{2}{*}{ tim } & \multicolumn{7}{|c|}{ Putrolenn } & \multirow[b]{2}{*}{ Finer } & \multirow[b]{2}{*}{ motints: } & \multirow[b]{2}{*}{ Toted } & & & \\
\hline & Conding & Singen & Totid & & Diver & 苾 & Lra. & (1) & 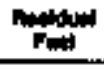 & Oothers & Ton: & & & & & & \\
\hline & \multicolumn{17}{|c|}{ 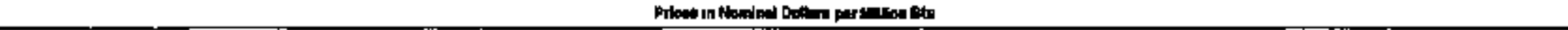 } \\
\hline \multirow[t]{2}{*}{ 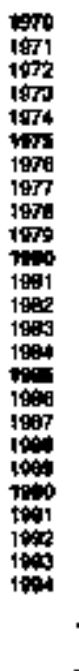 } & $\begin{array}{l}= \\
z \\
z \\
z \\
z \\
= \\
= \\
= \\
= \\
= \\
= \\
= \\
= \\
= \\
=\end{array}$ & 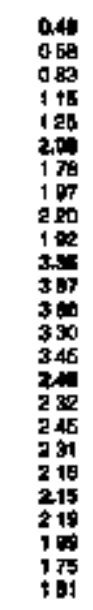 & 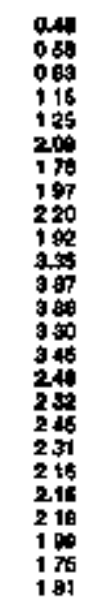 & 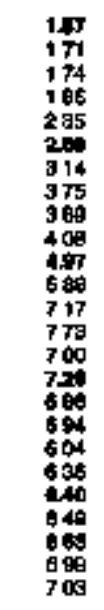 & 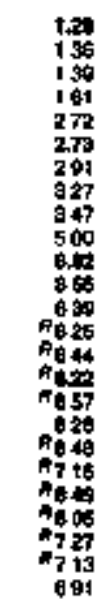 & 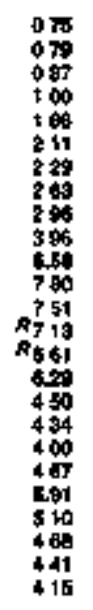 & 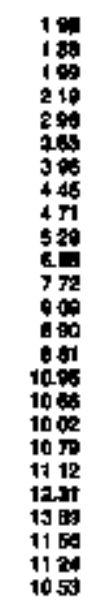 & 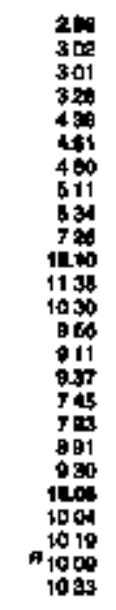 & 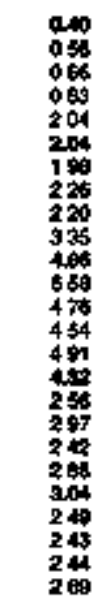 & 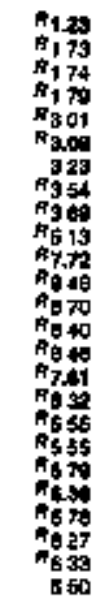 & 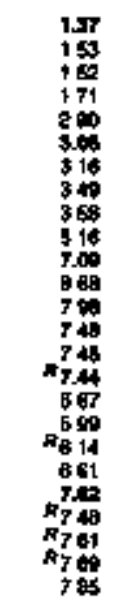 & 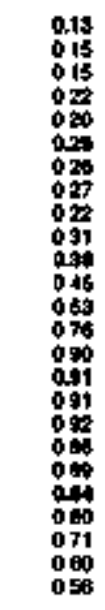 & 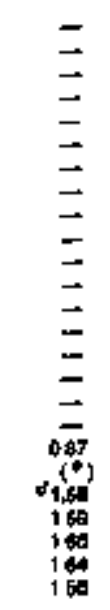 & 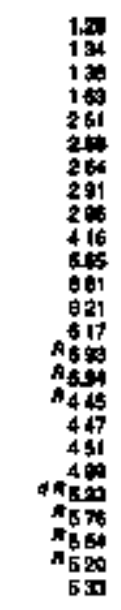 & 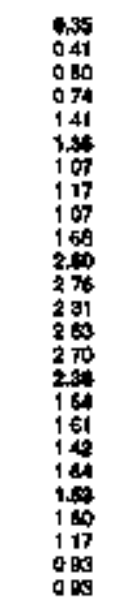 & 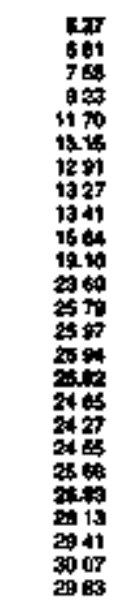 & 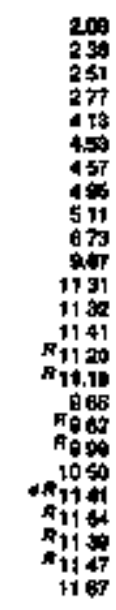 \\
\hline & \multicolumn{17}{|c|}{ 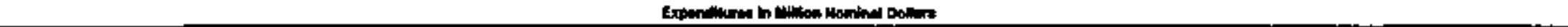 } \\
\hline 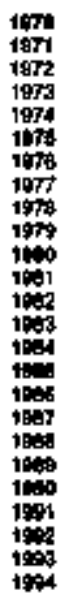 & $\begin{array}{l}= \\
z \\
= \\
= \\
= \\
= \\
= \\
= \\
= \\
= \\
= \\
= \\
= \\
z\end{array}$ & 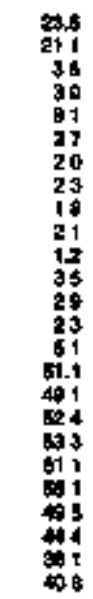 & $\begin{array}{r}24.5 \\
211 \\
35 \\
30 \\
51 \\
27 \\
20 \\
23 \\
18 \\
21 \\
11 \\
36 \\
28 \\
23 \\
51 \\
61.1 \\
491 \\
524 \\
593 \\
511 \\
5.1 \\
498 \\
444 \\
381 \\
408\end{array}$ & 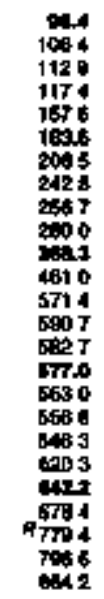 & 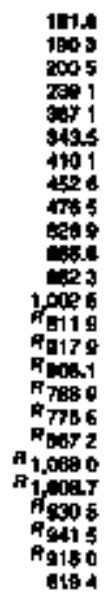 & 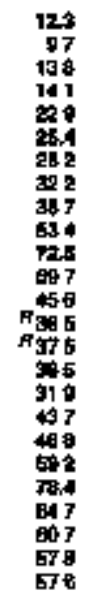 & 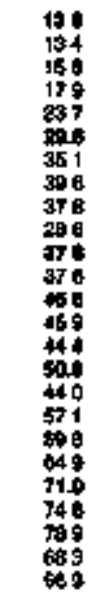 & 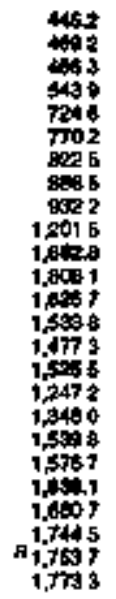 & 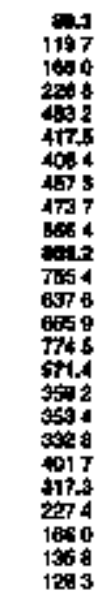 & 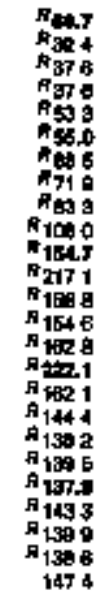 & 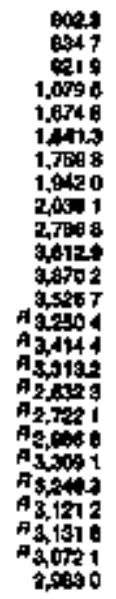 & 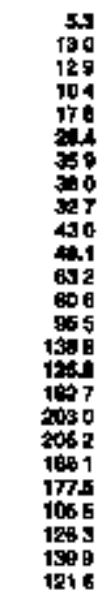 & $\begin{array}{l}= \\
= \\
= \\
= \\
= \\
= \\
= \\
= \\
= \\
= \\
= \\
12 \\
140 \\
245 \\
249 \\
244 \\
203\end{array}$ & 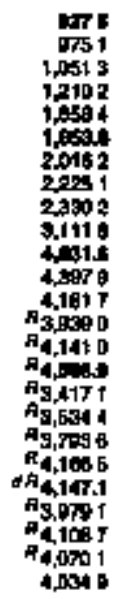 & 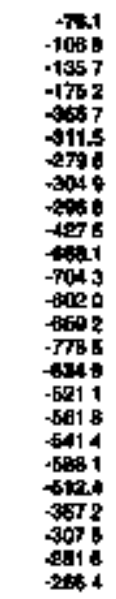 & 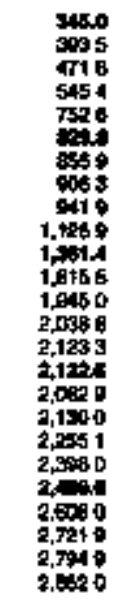 & 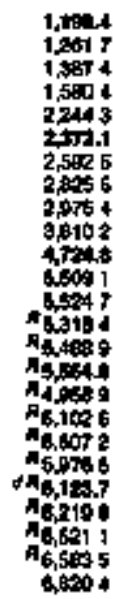 \\
\hline
\end{tabular}

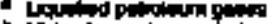

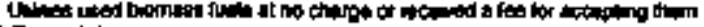

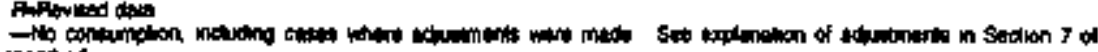

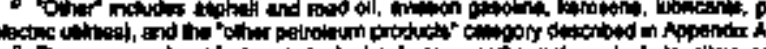

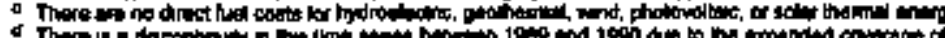

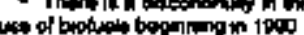

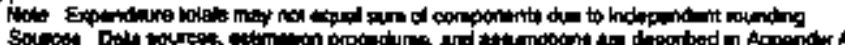




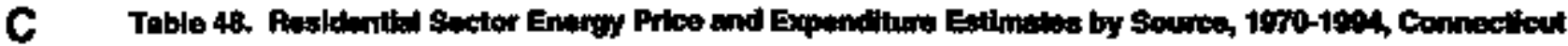

\begin{tabular}{|c|c|c|c|c|c|c|c|c|c|c|}
\hline \multirow[b]{4}{*}{$x=$} & \multicolumn{8}{|c|}{ PAm } & \multirow[b]{3}{*}{ Bnisy } & \multirow[b]{3}{*}{ C } \\
\hline & \multirow[b]{2}{*}{ conf } & \multirow[b]{2}{*}{ 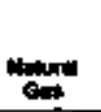 } & \multicolumn{4}{|c|}{ שרומוn } & \multirow[b]{2}{*}{ 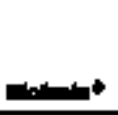 } & \multirow[b]{2}{*}{ For } & & \\
\hline & & & min & ranum & Leo: & Tow & & & & \\
\hline & \multicolumn{10}{|c|}{ 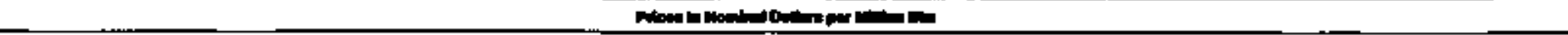 } \\
\hline \multirow[t]{2}{*}{ 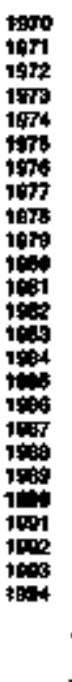 } & 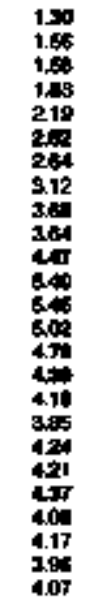 & 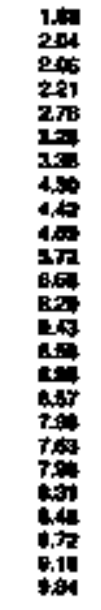 & 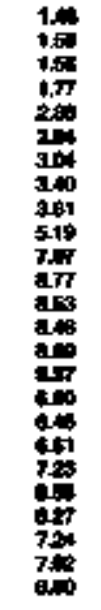 & 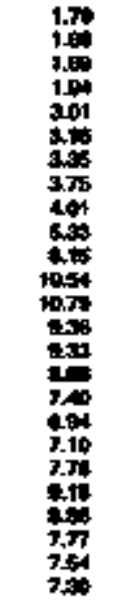 & 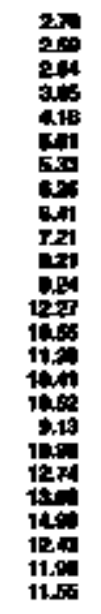 & 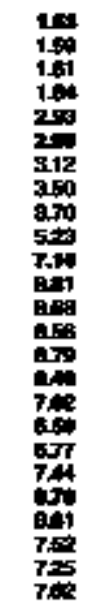 & 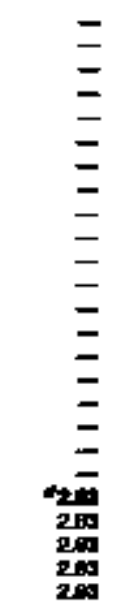 & 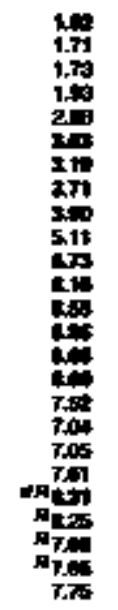 & 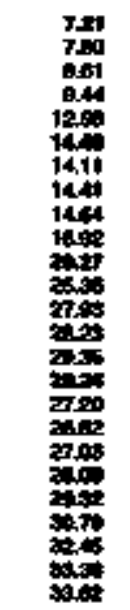 & 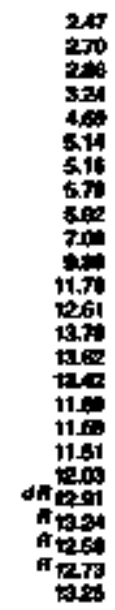 \\
\hline & \multicolumn{10}{|c|}{ 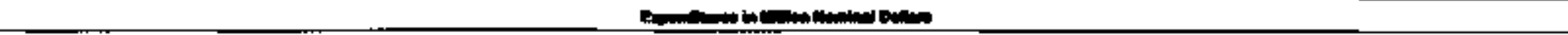 } \\
\hline 19: & 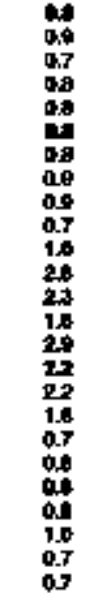 & 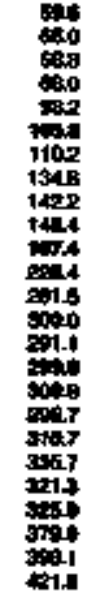 & 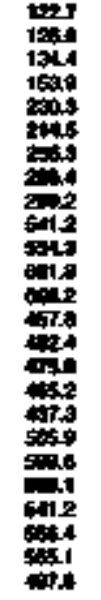 & 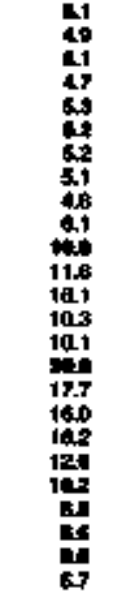 & 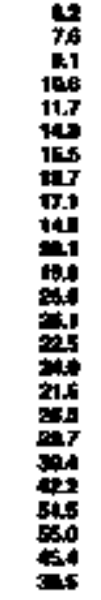 & 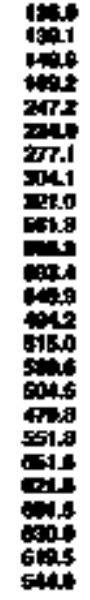 & 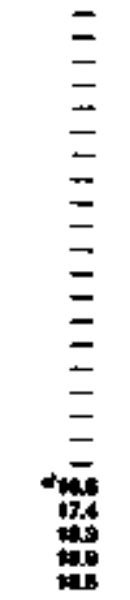 & 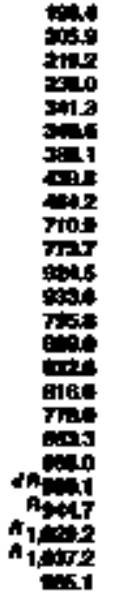 & 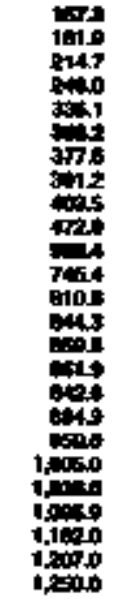 & 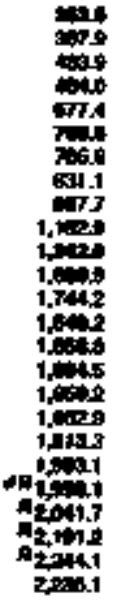 \\
\hline
\end{tabular}

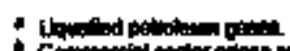

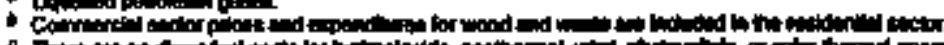

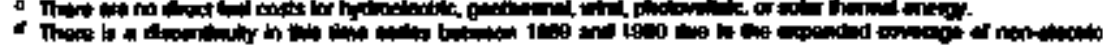

Antomenter

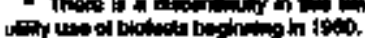

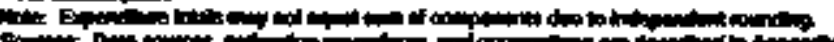




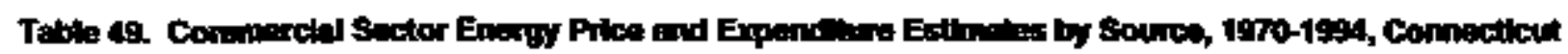

\begin{tabular}{|c|c|c|c|c|c|c|c|c|c|c|c|}
\hline \multirow[b]{4}{*}{ | } & \multicolumn{9}{|c|}{ M } & \multirow[b]{3}{*}{ Antring } & \multirow[b]{3}{*}{ 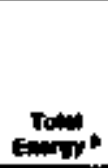 } \\
\hline & & \multirow[b]{2}{*}{ (1) } & \multicolumn{6}{|c|}{ 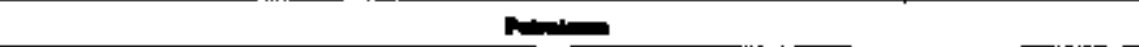 } & \multirow[b]{2}{*}{ Tols } & & \\
\hline & and & & hand & 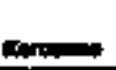 & ua* & 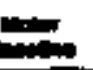 & 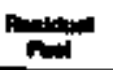 & Tet: & & & \\
\hline & \multicolumn{11}{|c|}{ 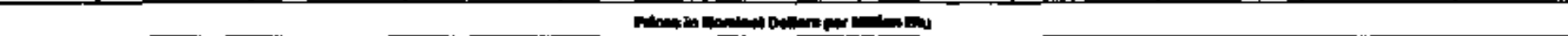 } \\
\hline \multirow[t]{2}{*}{ 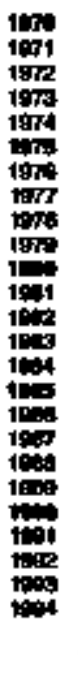 } & 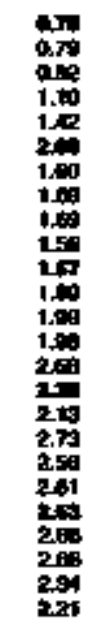 & 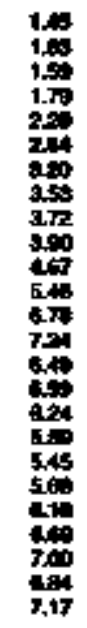 & 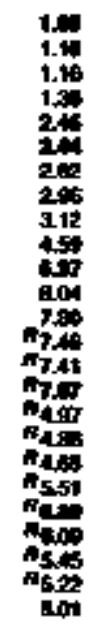 & 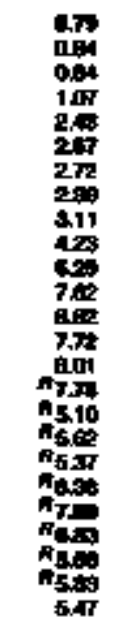 & 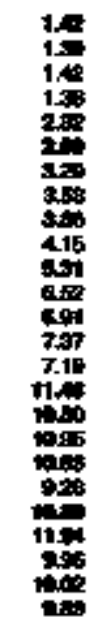 & 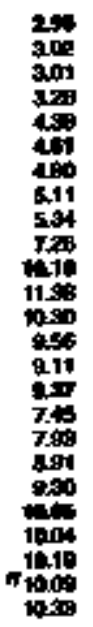 & 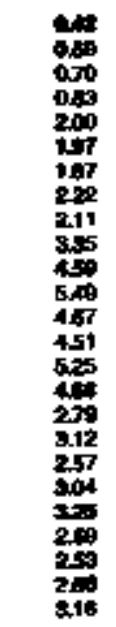 & 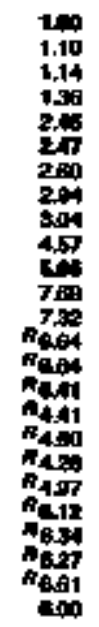 & 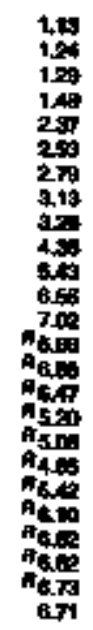 & 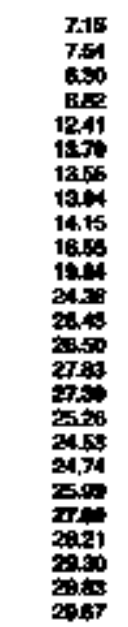 & 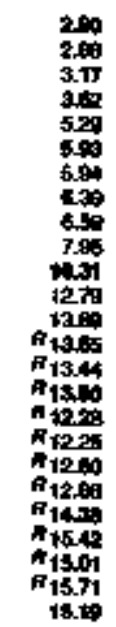 \\
\hline & \multicolumn{11}{|c|}{ ב } \\
\hline 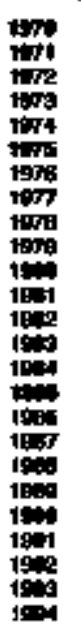 & 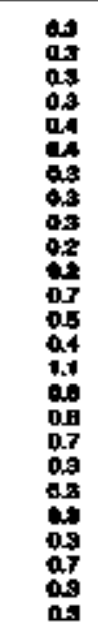 & 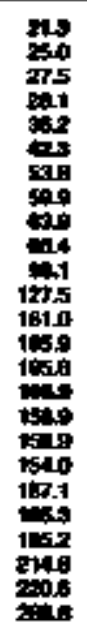 & 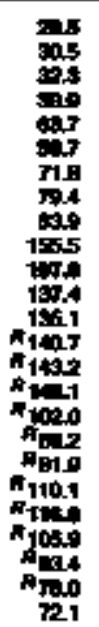 & 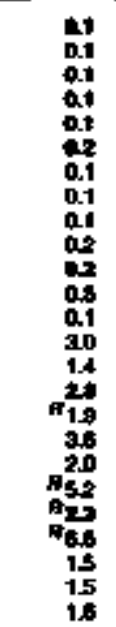 & 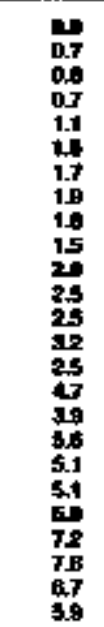 & 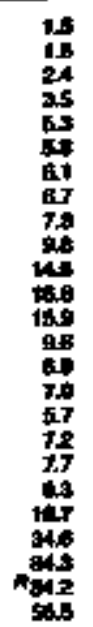 & 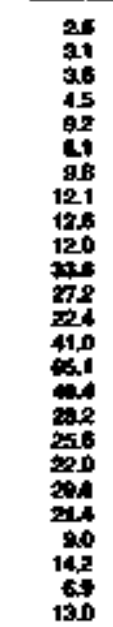 & 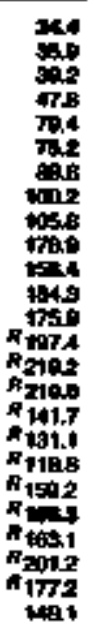 & 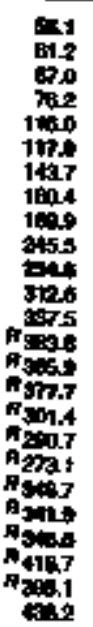 & 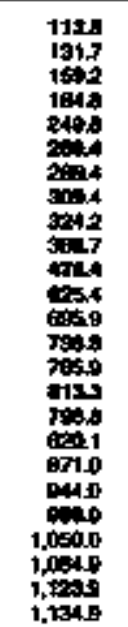 & 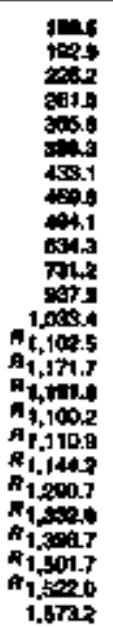 \\
\hline
\end{tabular}

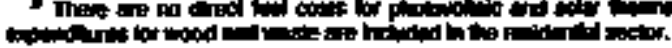

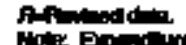

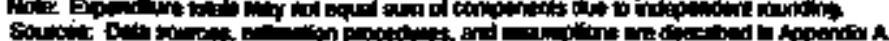




\begin{tabular}{|c|c|c|c|c|c|c|c|c|c|c|c|c|c|c|c|c|c|}
\hline \multirow[b]{4}{*}{ sum } & \multicolumn{15}{|c|}{ Pnawrengog } & \multirow[b]{3}{*}{ Bqotoroty } & \multirow[b]{3}{*}{ Tomit } \\
\hline & \multicolumn{3}{|c|}{ coll } & \multirow[b]{2}{*}{ mard } & \multicolumn{9}{|c|}{ molwom } & \multirow[b]{2}{*}{ Brotume: } & \multirow[b]{2}{*}{ Tot:- } & & \\
\hline & cotong & cont & Tow & & 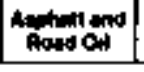 & Fim & Non:mers: & Lم:4 & Leorlewing & motsert & 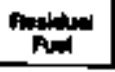 & ome & Tow & & & & \\
\hline & \multicolumn{17}{|c|}{ 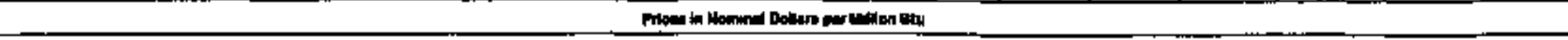 } \\
\hline \multirow[t]{2}{*}{ 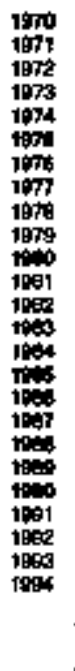 } & $\begin{array}{l}= \\
z \\
z \\
z \\
z \\
z \\
z \\
z \\
= \\
= \\
= \\
= \\
z\end{array}$ & 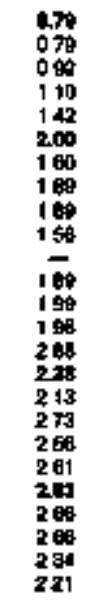 & 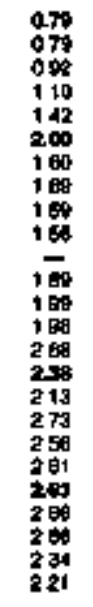 & 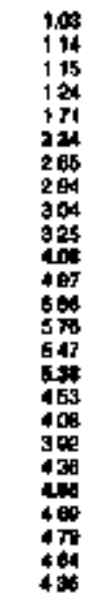 & 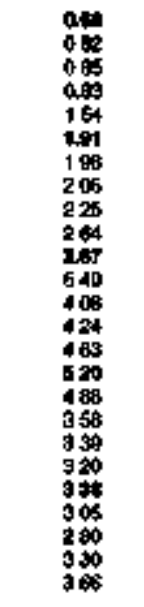 & 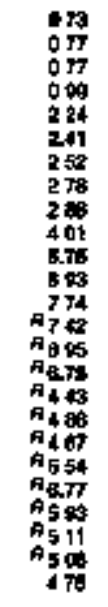 & 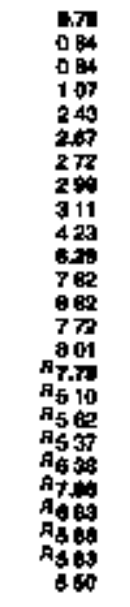 & 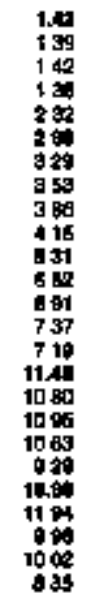 & 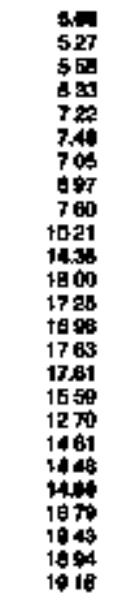 & 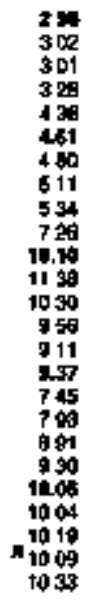 & 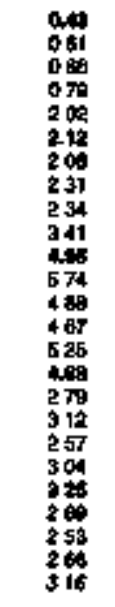 & 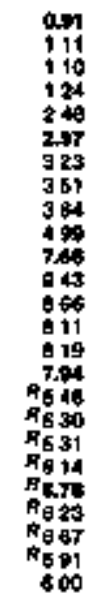 & 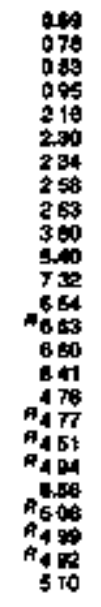 & 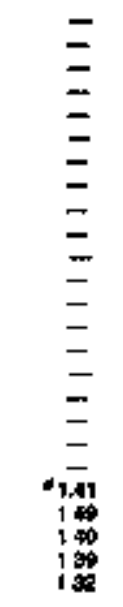 & 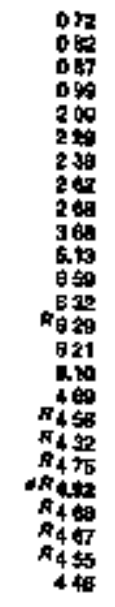 & 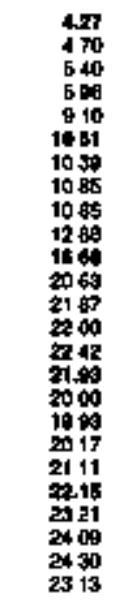 & 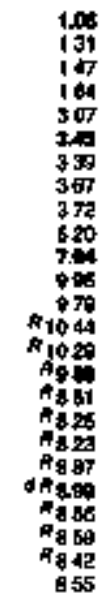 \\
\hline & \multicolumn{17}{|c|}{ 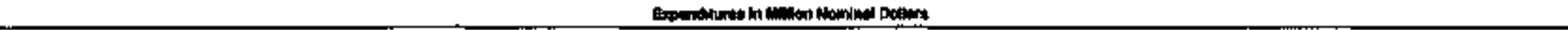 } \\
\hline 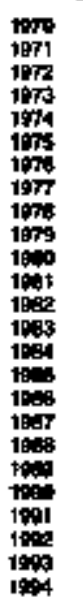 & $\begin{array}{l}= \\
z \\
z \\
= \\
z \\
= \\
= \\
= \\
= \\
= \\
= \\
z\end{array}$ & 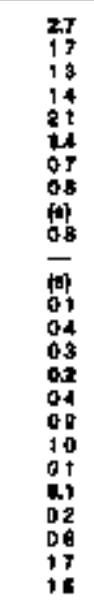 & 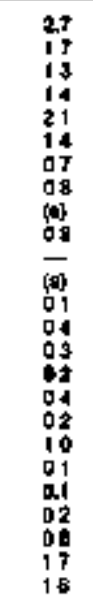 & 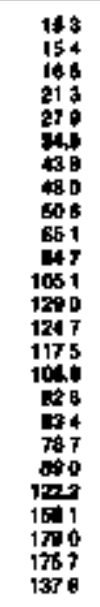 & 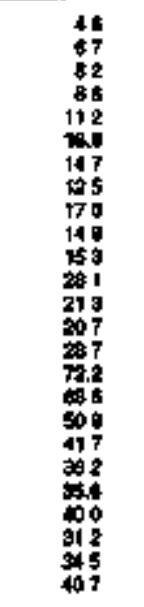 & 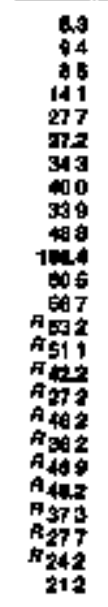 & 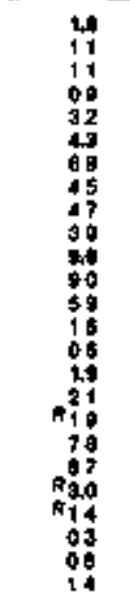 & 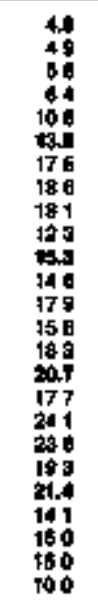 & 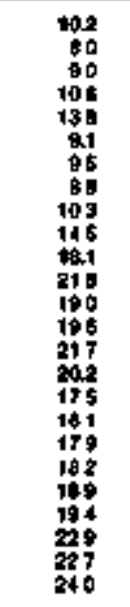 & 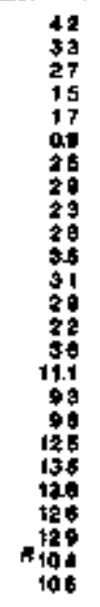 & 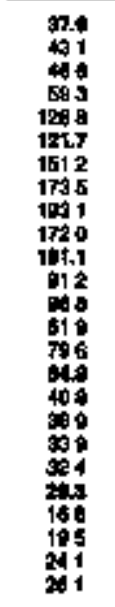 & 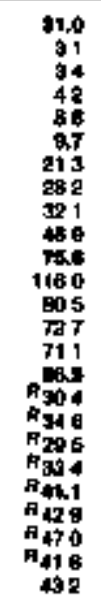 & 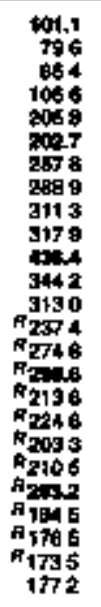 & 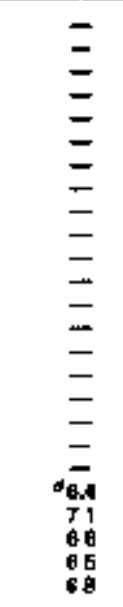 & 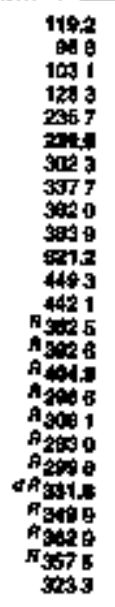 & 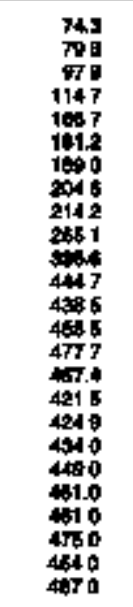 & 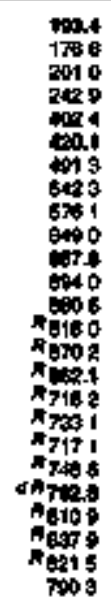 \\
\hline
\end{tabular}

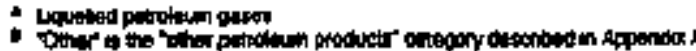

然

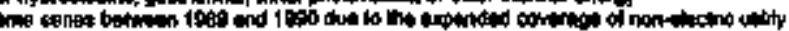

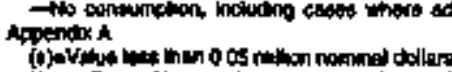

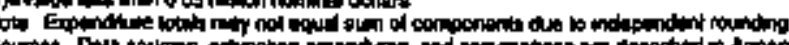

Arominged ont 
Table 51. Tranaportation Sector Eneroy Prke and Expenditure Esimatea by Sounte, 1970-1954, Connectiput

\begin{tabular}{|c|c|c|c|c|c|c|c|c|c|c|c|c|c|}
\hline & \multicolumn{11}{|c|}{ Priney tearof } & \multirow[b]{3}{*}{ Enectolity } & \multirow[b]{3}{*}{ Tration } \\
\hline & & \multirow[b]{2}{*}{ Nonternt } & \multicolumn{8}{|c|}{ Purotan } & \multirow[b]{2}{*}{ Totw } & & \\
\hline & con & & Alation & Pind & 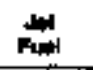 & LPG & Lunvicumits & (1) & Areldual & $T$ & & & \\
\hline$Y=r$ & \multicolumn{13}{|c|}{ 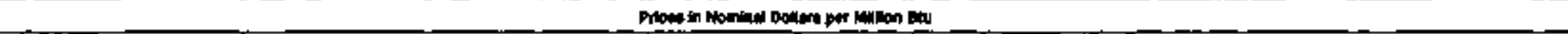 } \\
\hline \multirow[t]{2}{*}{ 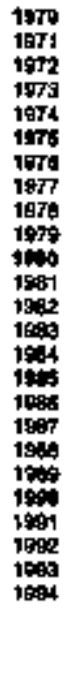 } & $\begin{array}{l}0.75 \\
078 \\
0.98 \\
110 \\
100 \\
200 \\
100 \\
100 \\
= \\
= \\
= \\
= \\
= \\
= \\
= \\
= \\
= \\
= \\
=\end{array}$ & $\begin{array}{l}= \\
= \\
= \\
= \\
= \\
= \\
= \\
= \\
= \\
= \\
= \\
= \\
1211 \\
873 \\
751\end{array}$ & 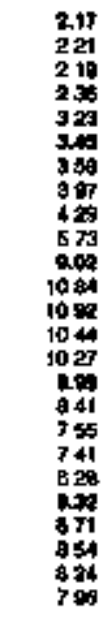 & 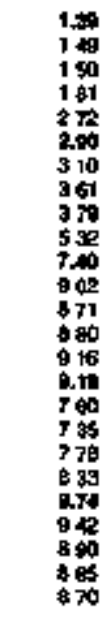 & 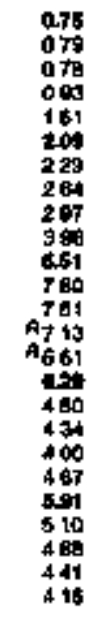 & 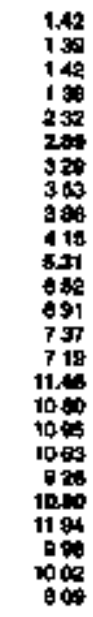 & 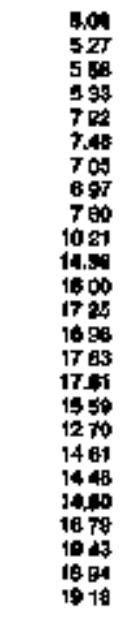 & 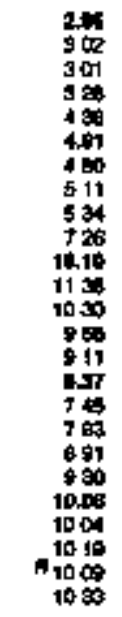 & 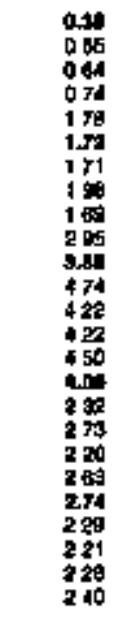 & 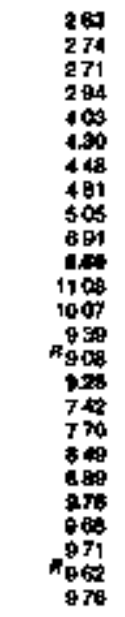 & 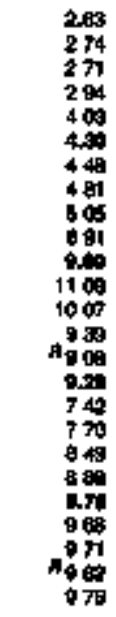 & $\begin{array}{l}= \\
= \\
= \\
= \\
= \\
= \\
= \\
= \\
= \\
= \\
= \\
=\end{array}$ & 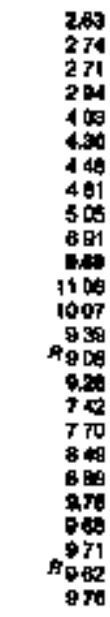 \\
\hline & \multicolumn{13}{|c|}{ 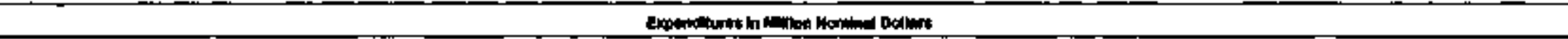 } \\
\hline 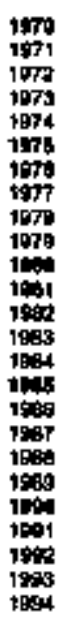 & 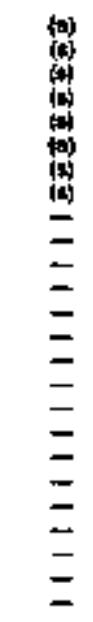 & $\begin{array}{l}= \\
= \\
= \\
= \\
= \\
= \\
= \\
= \\
= \\
= \\
= \\
\vec{y} \\
09\end{array}$ & 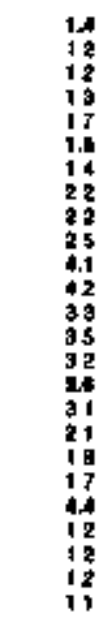 & 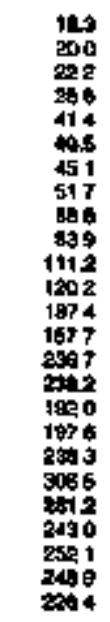 & 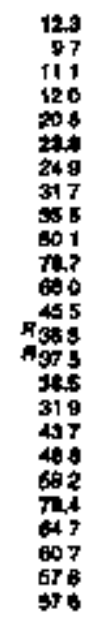 & $\begin{array}{l}0.1 \\
01 \\
01 \\
02 \\
03 \\
0.3 \\
04 \\
05 \\
00 \\
03 \\
0.3 \\
010 \\
06 \\
07 \\
11 \\
13 \\
09 \\
09 \\
12 \\
11 \\
11 \\
17 \\
12 \\
12 \\
15\end{array}$ & 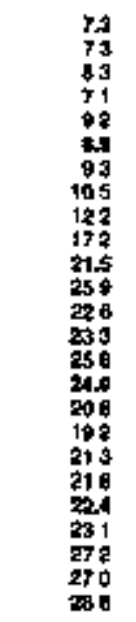 & 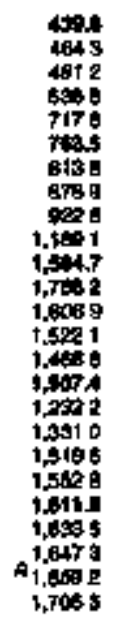 & 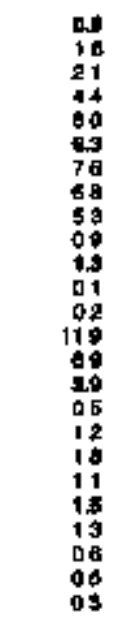 & 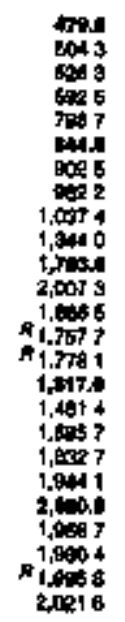 & 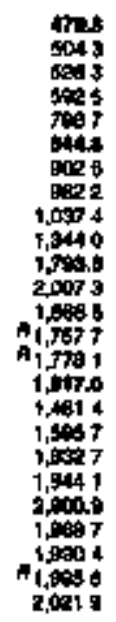 & $\begin{array}{l}= \\
= \\
= \\
= \\
= \\
= \\
= \\
= \\
= \\
= \\
= \\
= \\
=\end{array}$ & 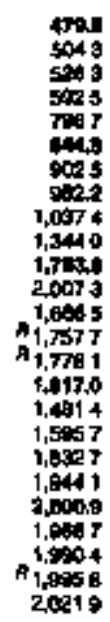 \\
\hline
\end{tabular}


C Toble 52 Price end Expendhure Eetimates for Ensrgy Inpul at Electric Utillies by \$ource, 1970-1984, Conneclicut

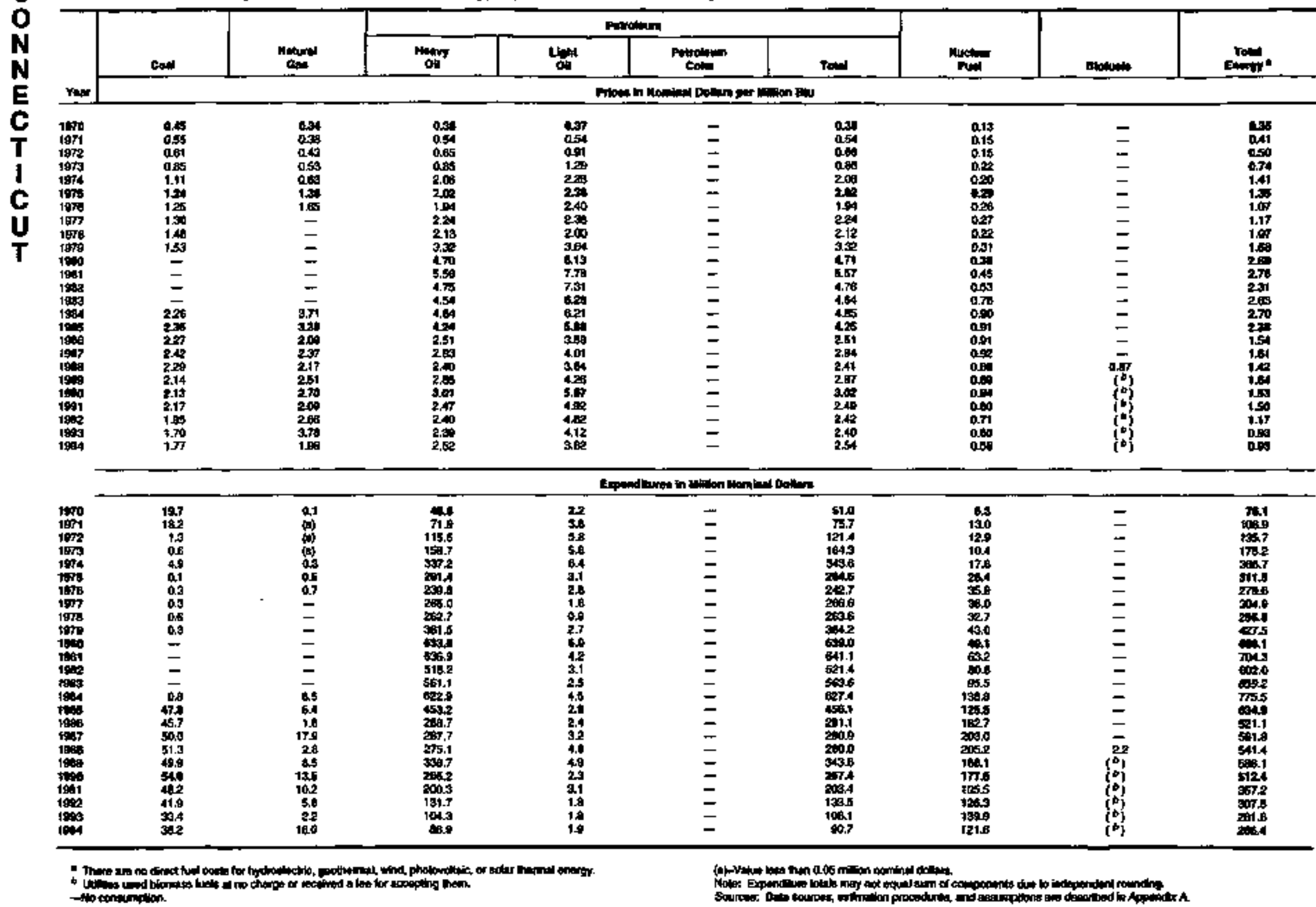




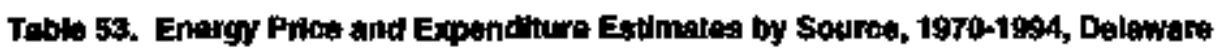

\begin{tabular}{|c|c|c|c|c|c|c|c|c|c|c|c|c|c|c|c|c|c|}
\hline & \multicolumn{14}{|c|}{ Prominy Enorgy } & \multirow[b]{3}{*}{$\begin{array}{l}\text { Evectent } \\
\text { Funtrit }\end{array}$} & \multirow{3}{*}{ Doctutly } & \multirow[b]{3}{*}{$\begin{array}{l}\text { Tot: } \\
\text { Enm- }\end{array}$} \\
\hline & \multicolumn{3}{|c|}{ שו } & \multirow[b]{2}{*}{ מוn } & \multicolumn{7}{|c|}{ Petroman } & \multirow[b]{2}{*}{ Muchl } & \multirow[b]{2}{*}{ Fifuere } & \multirow[b]{2}{*}{ Toesede } & & & \\
\hline & conem & son & Tolw & & 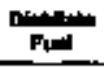 & 样 & LPR: & 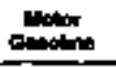 & Pade & Other" & Total & & & & & & \\
\hline $\mathbf{m}$ & \multicolumn{17}{|c|}{ 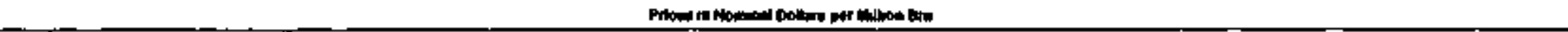 } \\
\hline \multirow[t]{2}{*}{ 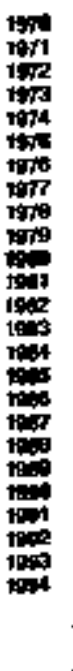 } & $\begin{array}{l}z \\
z \\
z \\
z \\
z \\
z \\
= \\
= \\
= \\
= \\
= \\
= \\
z \\
z\end{array}$ & 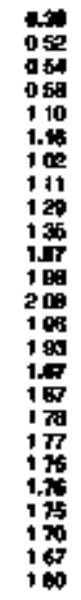 & 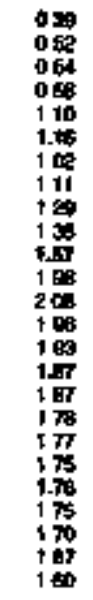 & 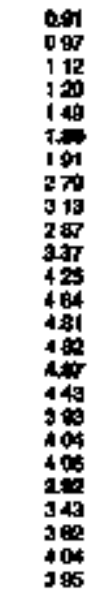 & 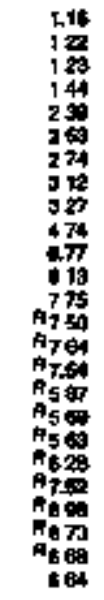 & 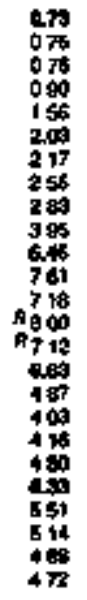 & 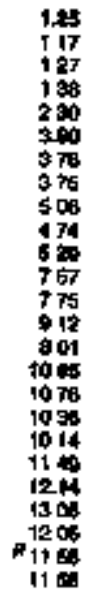 & 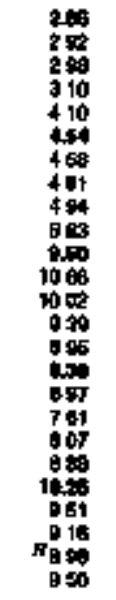 & 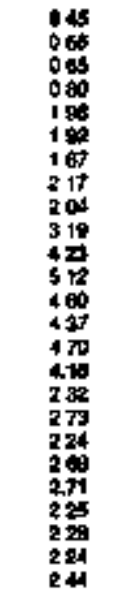 & 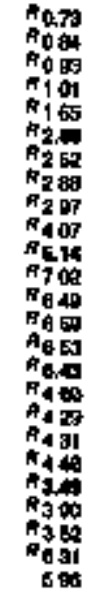 & 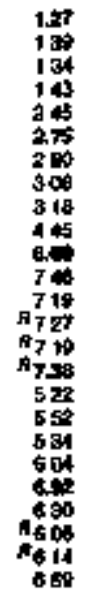 & $\begin{array}{l}= \\
= \\
= \\
= \\
= \\
= \\
= \\
= \\
= \\
= \\
= \\
= \\
= \\
=\end{array}$ & $\begin{array}{l}= \\
= \\
= \\
= \\
= \\
= \\
= \\
= \\
= \\
= \\
= \\
24 \\
240 \\
240 \\
244 \\
245\end{array}$ & 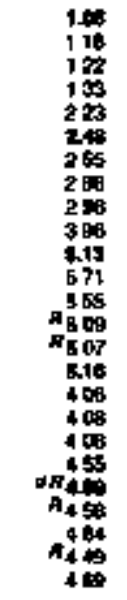 & 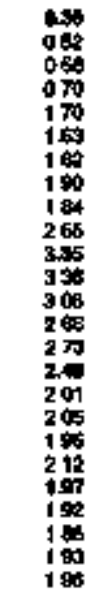 & 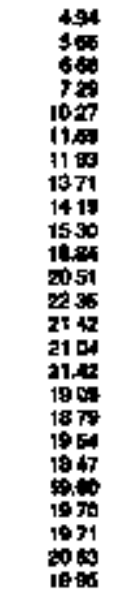 & 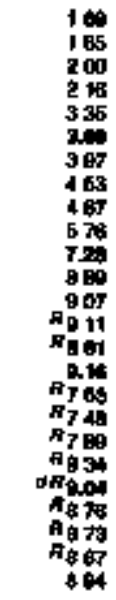 \\
\hline & \multicolumn{17}{|c|}{ 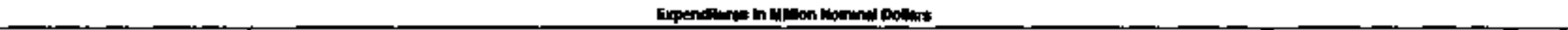 } \\
\hline 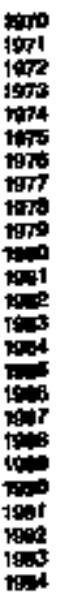 & $\begin{array}{l}z \\
z \\
z \\
z \\
z \\
z \\
z \\
z \\
= \\
z \\
z \\
z \\
z \\
z\end{array}$ & 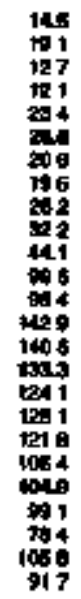 & 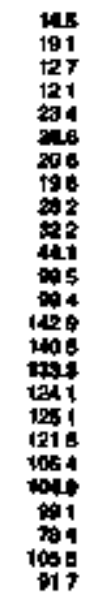 & 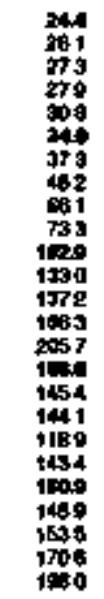 & 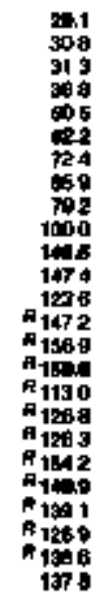 & 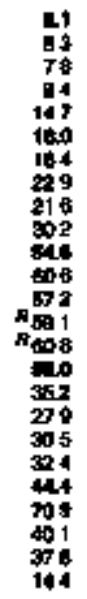 & 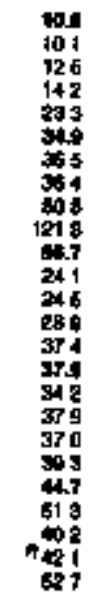 & 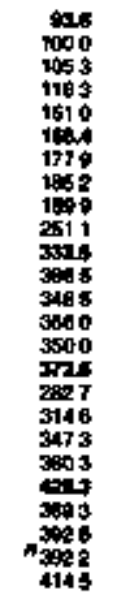 & 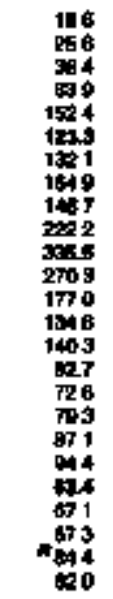 & 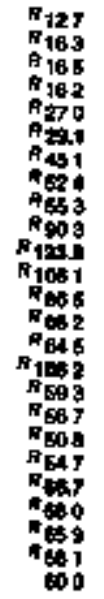 & 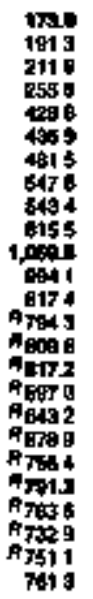 & $\begin{array}{l}= \\
= \\
= \\
= \\
= \\
= \\
= \\
= \\
= \\
= \\
= \\
= \\
= \\
=\end{array}$ & $\begin{array}{l}= \\
= \\
= \\
= \\
= \\
= \\
= \\
= \\
= \\
= \\
=14 \\
41 \\
42 \\
44\end{array}$ & 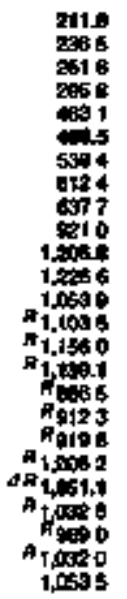 & 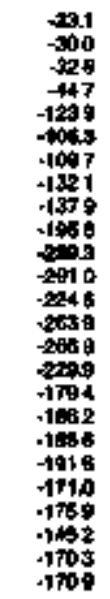 & 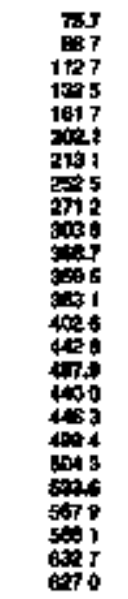 & 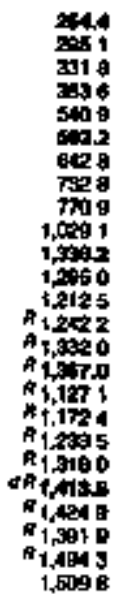 \\
\hline
\end{tabular}

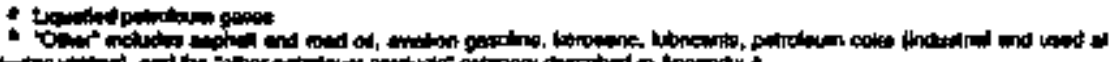

Anoviond to

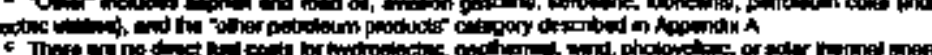

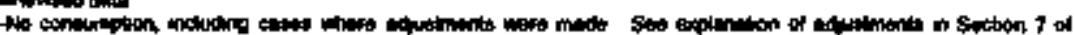

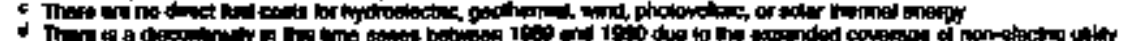
apendik $A$

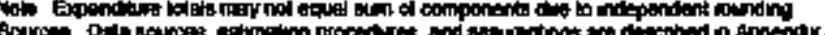

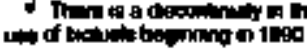




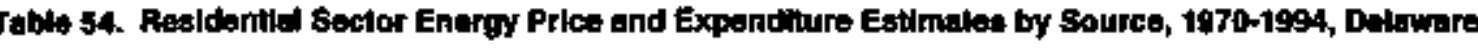

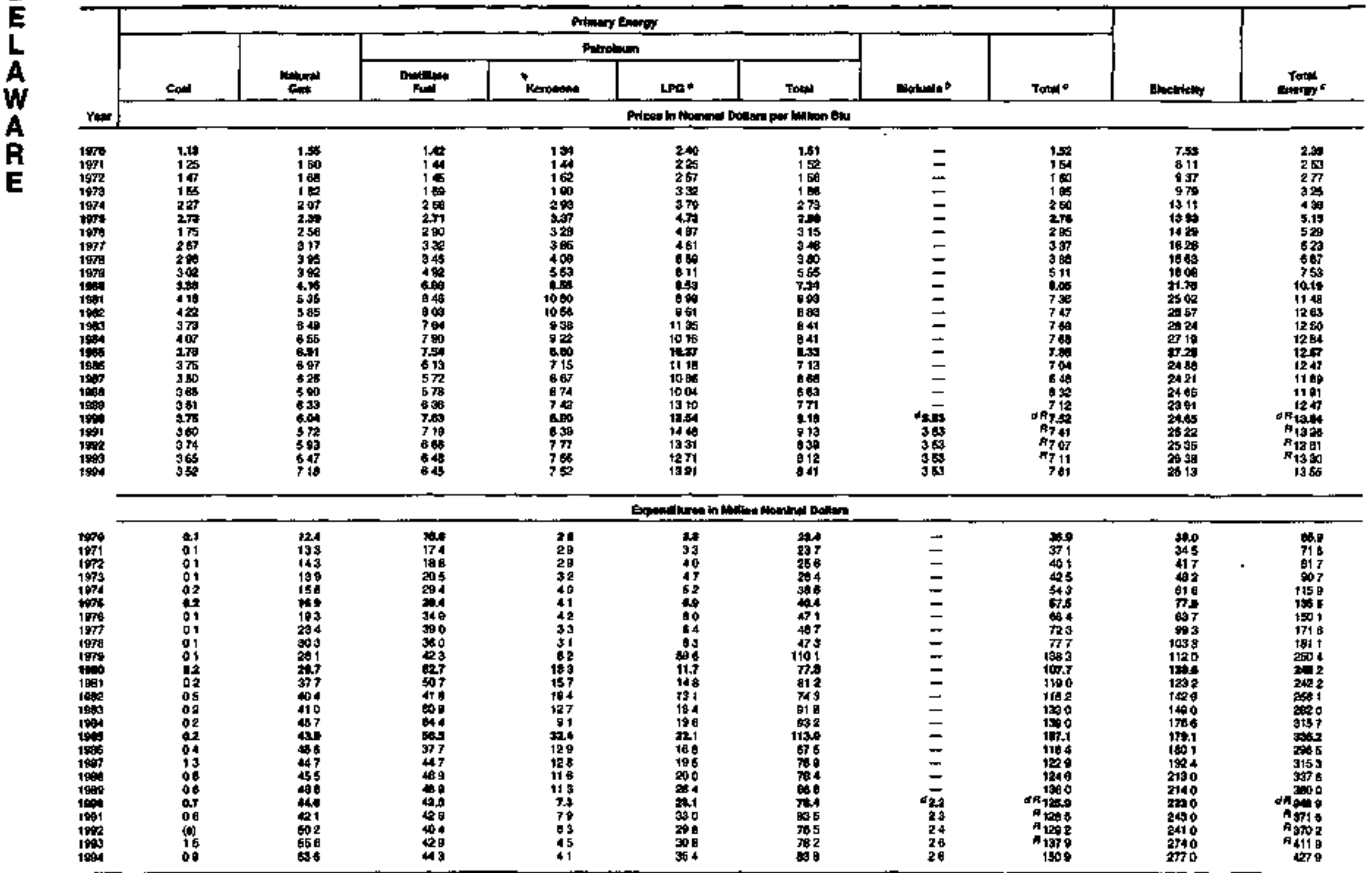

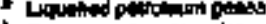

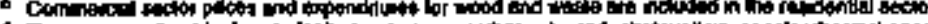

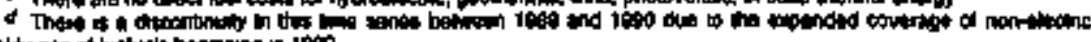

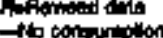

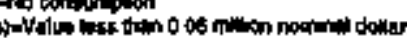

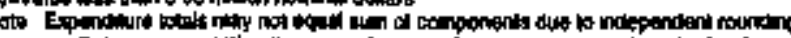

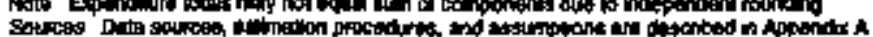

Encroy Intornation Adrilaistration 


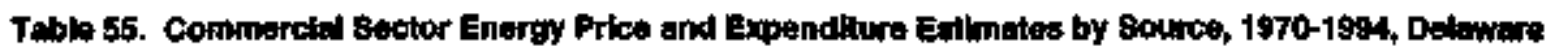

\begin{tabular}{|c|c|c|c|c|c|c|c|c|c|c|c|}
\hline \multirow[b]{4}{*}{ Yom } & \multicolumn{9}{|c|}{ Whery Energy } & \multirow[b]{3}{*}{ Deforlopy } & \multirow[b]{3}{*}{ rots } \\
\hline & \multirow[b]{2}{*}{ con } & \multirow[b]{2}{*}{ morst } & \multicolumn{6}{|c|}{ 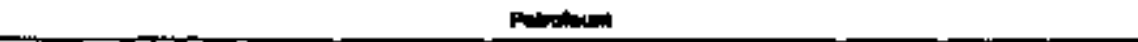 } & \multirow[b]{2}{*}{ Tot: } & & \\
\hline & & & 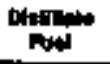 & 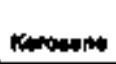 & LPAS & 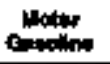 & 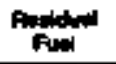 & Tow & & & \\
\hline & \multicolumn{11}{|c|}{ 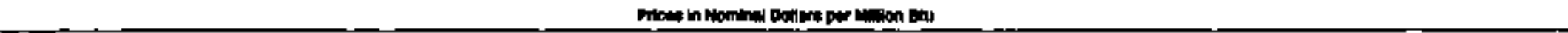 } \\
\hline \multirow[t]{2}{*}{ 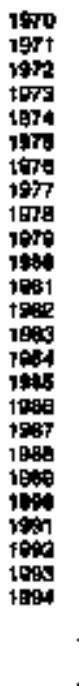 } & 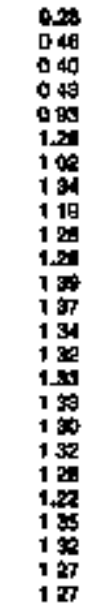 & 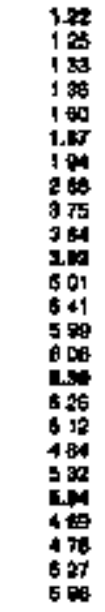 & 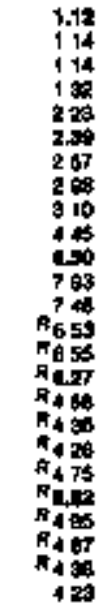 & 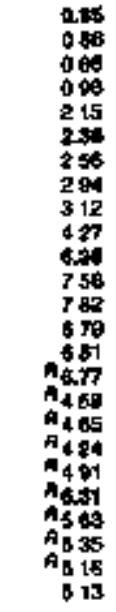 & 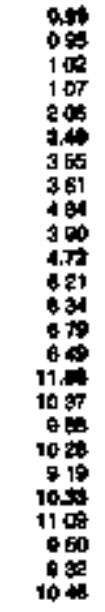 & 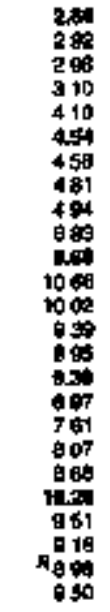 & 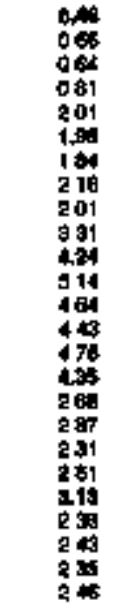 & 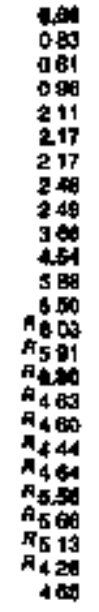 & 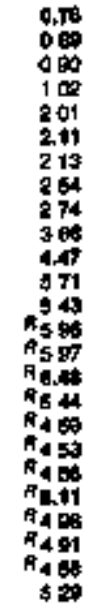 & 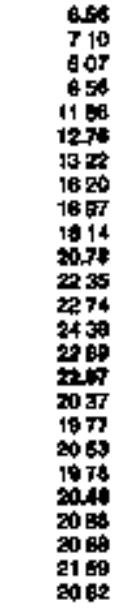 & 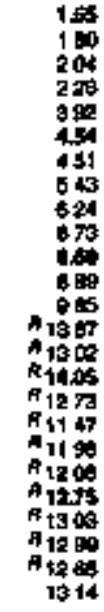 \\
\hline & \multicolumn{11}{|c|}{ 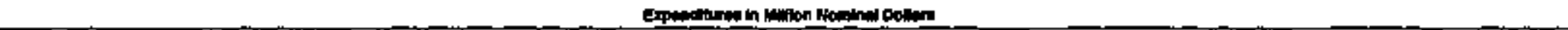 } \\
\hline 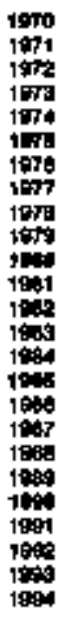 & 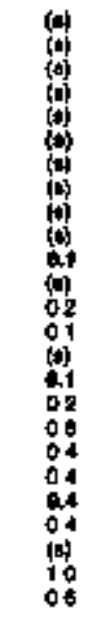 & 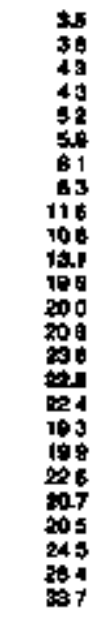 & 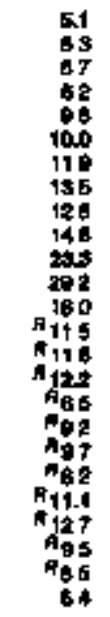 & $\begin{array}{l}02 \\
02 \\
02 \\
02 \\
04 \\
04 \\
04 \\
04 \\
03 \\
09 \\
03 \\
03 \\
03 \\
03 \\
02 \\
20 \\
04 \\
04 \\
04 \\
02 \\
04 \\
04 \\
09 \\
02 \\
02\end{array}$ & $\begin{array}{l}09 \\
02 \\
03 \\
03 \\
05 \\
09 \\
10 \\
09 \\
11 \\
67 \\
19 \\
10 \\
15 \\
19 \\
22 \\
42 \\
26 \\
31 \\
38 \\
33 \\
36 \\
46 \\
36 \\
40 \\
47\end{array}$ & $\begin{array}{l}04 \\
04 \\
03 \\
03 \\
08 \\
09 \\
09 \\
09 \\
09 \\
14 \\
29 \\
20 \\
51 \\
20 \\
13 \\
17 \\
14 \\
17 \\
17 \\
19 \\
19 \\
17 \\
17 \\
04 \\
04\end{array}$ & 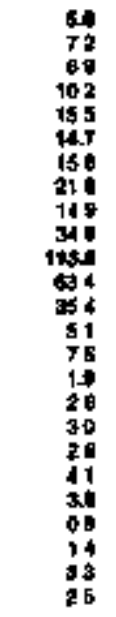 & 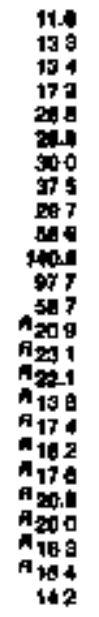 & 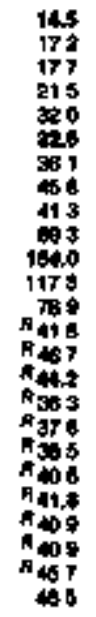 & 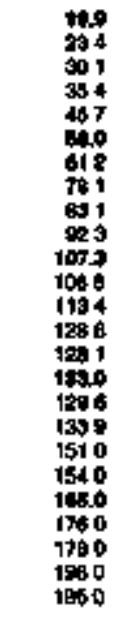 & 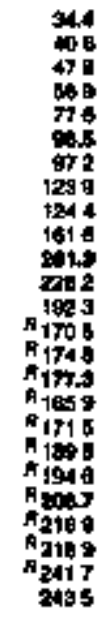 \\
\hline
\end{tabular}

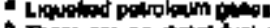

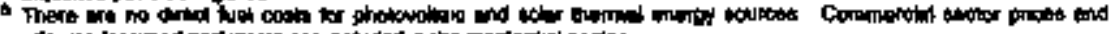

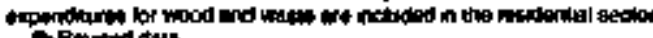

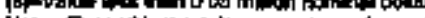

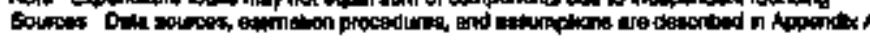


Table 56. Indubtrlal Sector Energy Price and Expendlume Estimates by source, 1970-1994, Delaware

\begin{tabular}{|c|c|c|c|c|c|c|c|c|c|c|c|c|c|c|c|c|c|}
\hline & \multicolumn{15}{|c|}{ Punary Entigy } & \multirow[b]{3}{*}{ dntritr } & \multirow[b]{3}{*}{ 1) } \\
\hline & \multicolumn{3}{|c|}{ Goed } & \multirow[b]{2}{*}{ 1) } & \multicolumn{9}{|c|}{ Patrowere } & \multirow[b]{2}{*}{ olingoke } & \multirow[b]{2}{*}{ Thise } & & \\
\hline & $\begin{array}{l}\text { Coland } \\
\text { Cood }\end{array}$ & הירוg & Tot & & $\begin{array}{c}\text { Aaphin and } \\
\text { Revit on }\end{array}$ & Fut & Korature & Lee* & 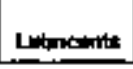 & andine & 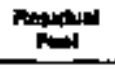 & anot & Total & & & & \\
\hline$\underline{Y=1}$ & \multicolumn{17}{|c|}{ 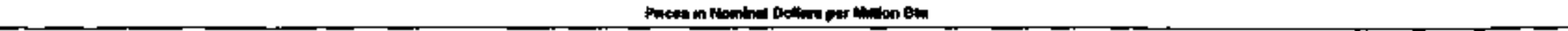 } \\
\hline \multirow[t]{2}{*}{ 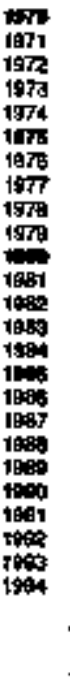 } & $\begin{array}{l}= \\
= \\
= \\
= \\
= \\
= \\
= \\
= \\
= \\
= \\
= \\
= \\
= \\
= \\
=\end{array}$ & 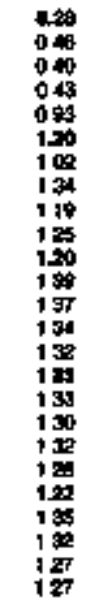 & 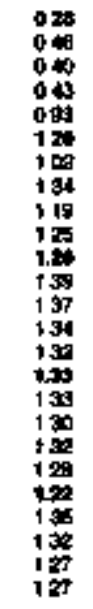 & 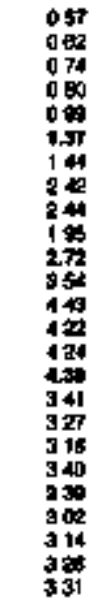 & 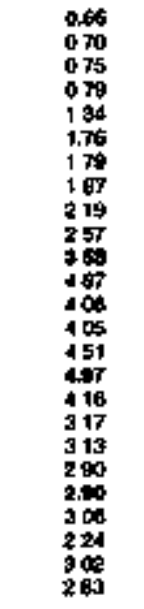 & 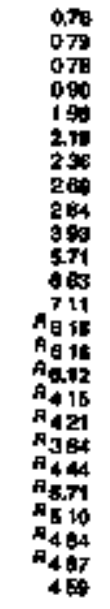 & 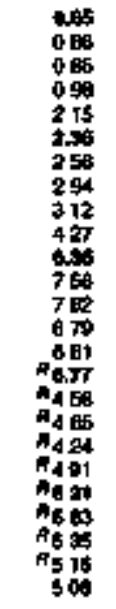 & 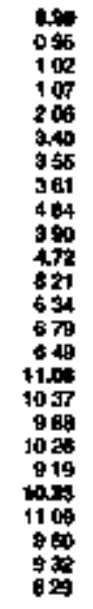 & 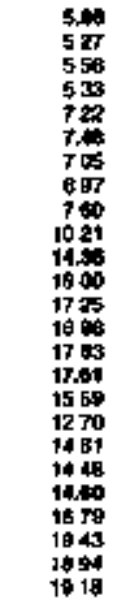 & 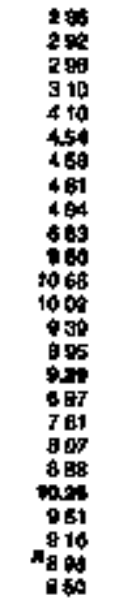 & 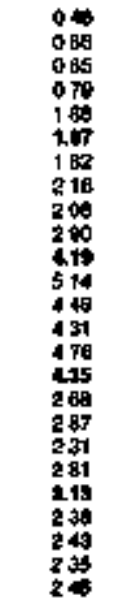 & 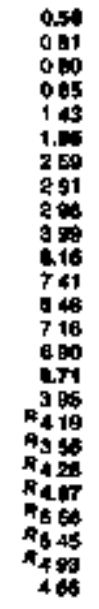 & 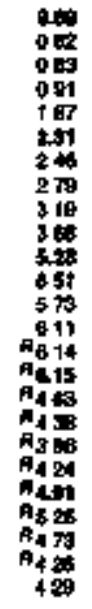 & $\begin{array}{l}= \\
= \\
= \\
= \\
= \\
= \\
= \\
= \\
= \\
= \\
= \\
\bar{z} \\
10 \\
100\end{array}$ & 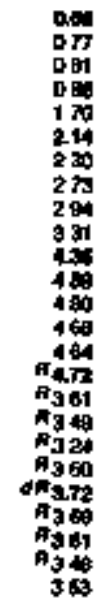 & 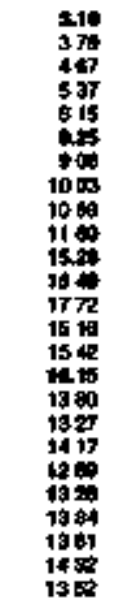 & 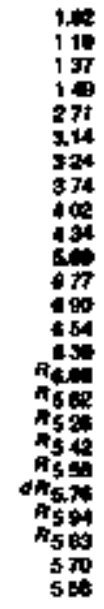 \\
\hline & \multicolumn{17}{|c|}{ 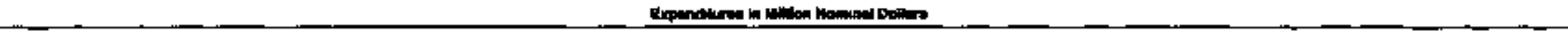 } \\
\hline 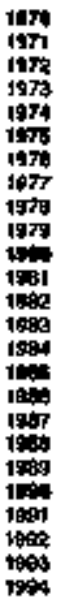 & $\begin{array}{l}= \\
= \\
= \\
= \\
= \\
= \\
= \\
= \\
= \\
= \\
= \\
= \\
=\end{array}$ & $\begin{array}{l}02 \\
03 \\
02 \\
03 \\
05 \\
08 \\
08 \\
06 \\
32 \\
04 \\
54 \\
60 \\
57 \\
60 \\
67 \\
70 \\
07 \\
70 \\
01 \\
85 \\
05 \\
70 \\
47 \\
85 \\
80\end{array}$ & $\begin{array}{l}0.3 \\
03 \\
09 \\
03 \\
05 \\
18 \\
06 \\
09 \\
92 \\
24 \\
54 \\
60 \\
67 \\
50 \\
81 \\
78 \\
67 \\
90 \\
81 \\
65 \\
05 \\
70 \\
47 \\
98 \\
40\end{array}$ & 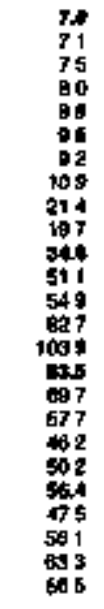 & 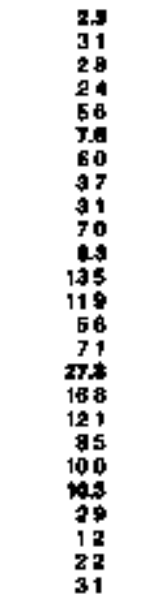 & 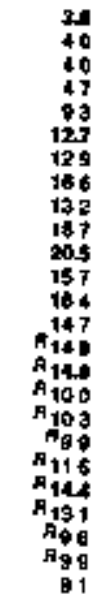 & 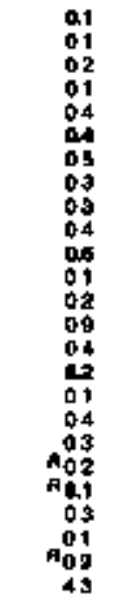 & $\begin{array}{r}65 \\
65 \\
62 \\
92 \\
174 \\
237 \\
281 \\
395 \\
494 \\
64 \\
46 \\
73 \\
97 \\
63 \\
154 \\
114 \\
120 \\
152 \\
191 \\
95 \\
126 \\
195 \\
65 \\
974 \\
124\end{array}$ & $\begin{array}{l}1.3 \\
13 \\
15 \\
15 \\
19 \\
14 \\
18 \\
32 \\
37 \\
82 \\
48 \\
78 \\
69 \\
79 \\
78 \\
79 \\
63 \\
58 \\
66 \\
68 \\
68 \\
70 \\
89 \\
82 \\
67\end{array}$ & $\begin{array}{r}14 \\
14 \\
15 \\
12 \\
81 \\
15 \\
14 \\
13 \\
12 \\
17 \\
17 \\
19 \\
15 \\
12 \\
27 \\
27 \\
22 \\
23 \\
24 \\
30 \\
26 \\
25 \\
25 \\
930 \\
32\end{array}$ & 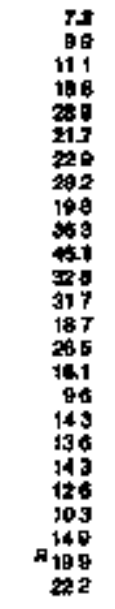 & 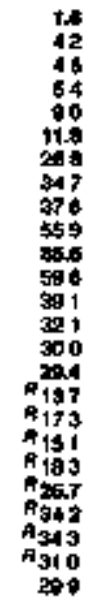 & 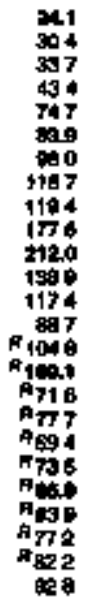 & $\begin{array}{l}= \\
= \\
= \\
= \\
= \\
= \\
= \\
= \\
= \\
= \\
= \\
= \\
01.7 \\
19 \\
18 \\
10\end{array}$ & 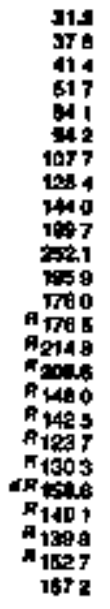 & 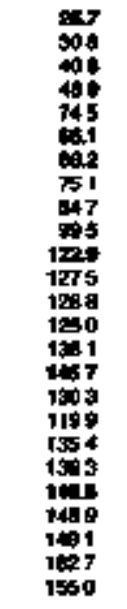 & 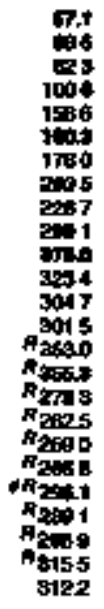 \\
\hline
\end{tabular}

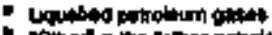

Amentood dita

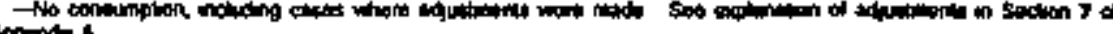

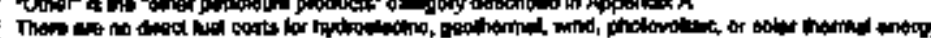

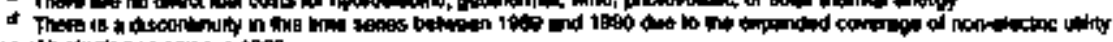
raperite 1

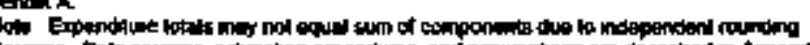

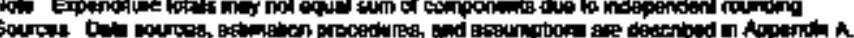

Encrgy Inlormation idmintitratton 


\begin{tabular}{|c|c|c|c|c|c|c|c|c|c|c|c|c|c|}
\hline \multirow[b]{4}{*}{$\mathbf{x}=\mathbf{m}$} & \multicolumn{11}{|c|}{ 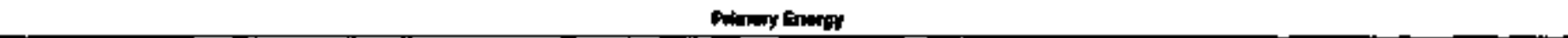 } & \multirow[b]{3}{*}{ Dectioly } & \multirow[b]{3}{*}{ Then } \\
\hline & \multirow[b]{2}{*}{ Cow } & \multirow[b]{2}{*}{ ming } & \multicolumn{8}{|c|}{ Nuthiming } & \multirow[b]{2}{*}{ Tont } & & \\
\hline & & & 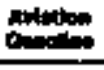 & onstion & Hines & 느뭉 & Lingleavin & chentions & Funt & Tow & & & \\
\hline & \multicolumn{13}{|c|}{ 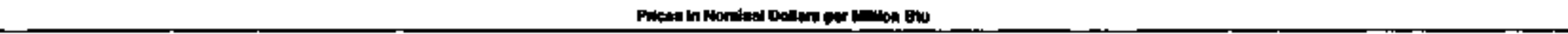 } \\
\hline \multirow[t]{2}{*}{ 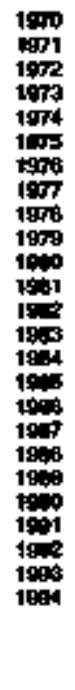 } & $\begin{array}{l}1210 \\
040 \\
040 \\
04 \\
08 \\
19 \\
130 \\
= \\
= \\
= \\
= \\
= \\
= \\
= \\
= \\
= \\
=\end{array}$ & $\begin{array}{l}= \\
= \\
= \\
= \\
= \\
= \\
= \\
= \\
= \\
= \\
= \\
= \\
=\end{array}$ & 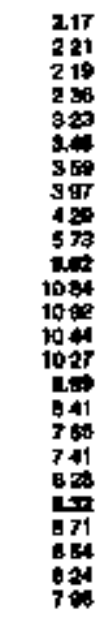 & 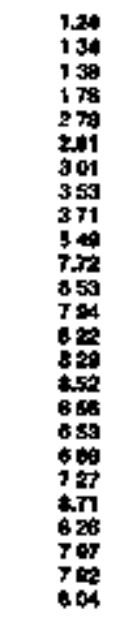 & 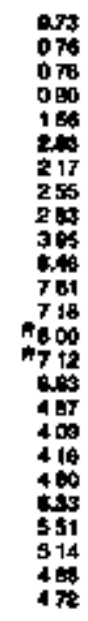 & 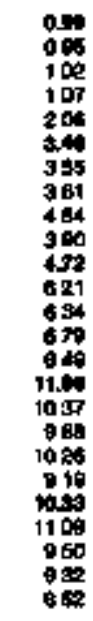 & 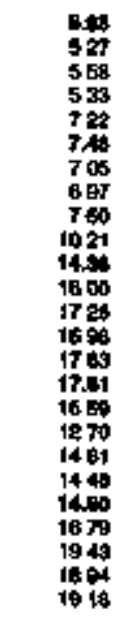 & 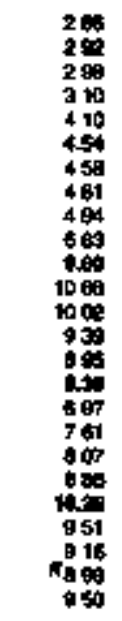 & 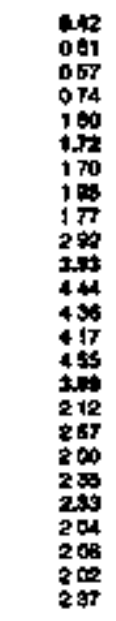 & 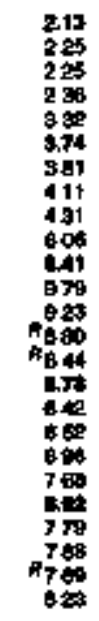 & 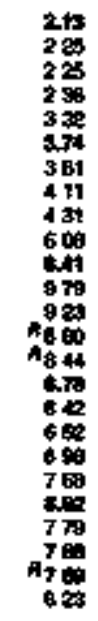 & $\begin{array}{l}= \\
= \\
= \\
= \\
= \\
= \\
= \\
= \\
= \\
= \\
= \\
= \\
= \\
= \\
=\end{array}$ & 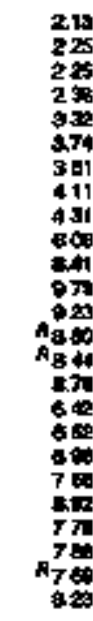 \\
\hline & \multicolumn{13}{|c|}{ 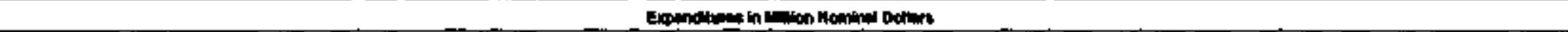 } \\
\hline 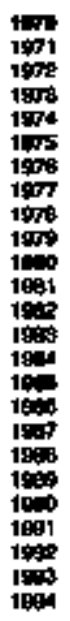 & 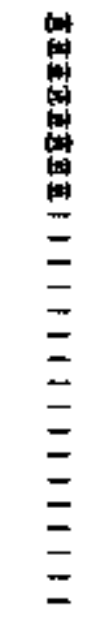 & $\begin{array}{l}= \\
= \\
= \\
= \\
= \\
= \\
= \\
= \\
= \\
= \\
= \\
= \\
=\end{array}$ & 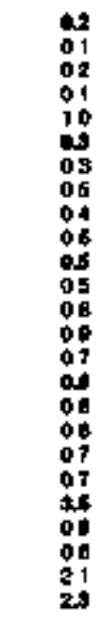 & 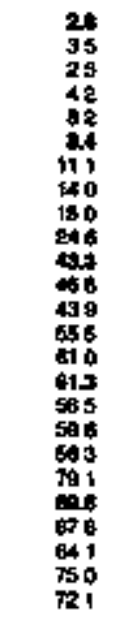 & 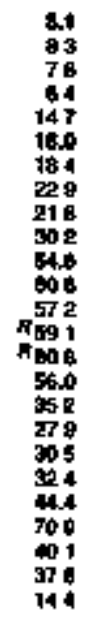 & $\begin{array}{l}01 \\
01 \\
01 \\
01 \\
02 \\
08 \\
05 \\
05 \\
10 \\
02 \\
02 \\
02 \\
02 \\
03 \\
02 \\
02 \\
17 \\
01 \\
02 \\
02 \\
02 \\
03 \\
02 \\
02 \\
02 \\
02\end{array}$ & $\begin{array}{l}21 \\
23 \\
28 \\
91 \\
27 \\
28 \\
14 \\
27 \\
31 \\
44 \\
55 \\
57 \\
68 \\
60 \\
64 \\
48 \\
54 \\
40 \\
55 \\
68 \\
58 \\
50 \\
70 \\
70 \\
74\end{array}$ & 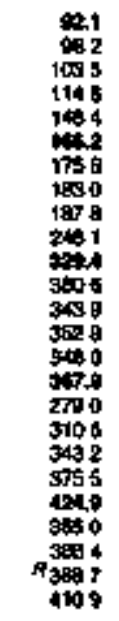 & $\begin{array}{r}18 \\
19 \\
33 \\
47 \\
197 \\
194 \\
110 \\
103 \\
98 \\
69 \\
201 \\
61 \\
55 \\
137 \\
193 \\
50 \\
78 \\
194 \\
110 \\
131 \\
134 \\
160 \\
194 \\
145 \\
189\end{array}$ & 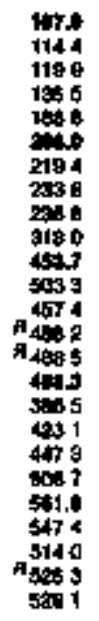 & 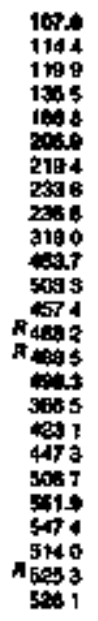 & $\begin{array}{l}= \\
= \\
= \\
= \\
= \\
= \\
= \\
= \\
= \\
= \\
= \\
= \\
= \\
= \\
=\end{array}$ & 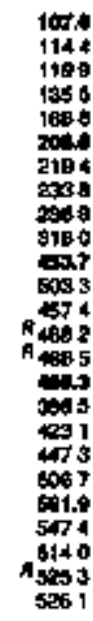 \\
\hline
\end{tabular}




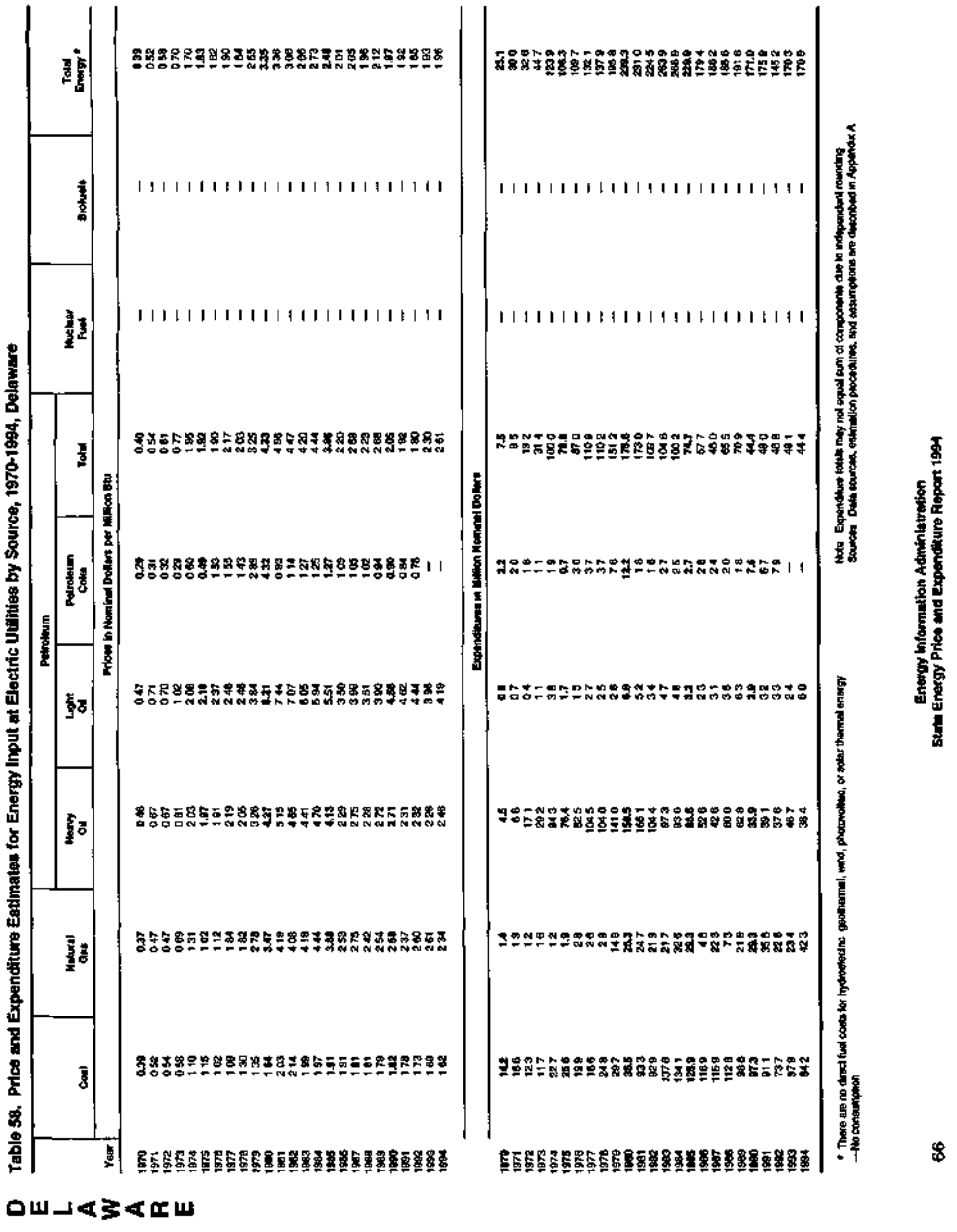


Tablo so. Energy Price and Expendlture Estimates by 8ource, 1970-1994, Dladret of Columble

\begin{tabular}{|c|c|c|c|c|c|c|c|c|c|c|c|c|c|c|c|c|c|}
\hline & \multicolumn{14}{|c|}{ Priney tentory } & \multirow{3}{*}{ White } & \multirow{3}{*}{ 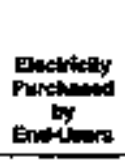 } & \multirow[b]{3}{*}{ trotal } \\
\hline & \multicolumn{3}{|c|}{ Cow } & \multirow[b]{2}{*}{ Mntherl } & \multicolumn{7}{|c|}{ 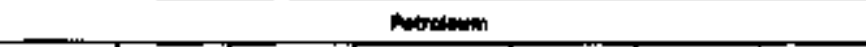 } & \multirow[b]{2}{*}{ Musiner } & \multirow[b]{2}{*}{ Elotumb } & \multirow[b]{2}{*}{ Toter } & & & \\
\hline & $\begin{array}{l}\text { cotong } \\
\text { cots }\end{array}$ & Somen & Ton & & 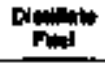 & Fued & cpas & 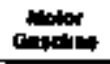 & $\begin{array}{l}\text { Racled } \\
\text { Fund }\end{array}$ & Ones & Fotal & & & & & & \\
\hline$\gamma=$ & \multicolumn{17}{|c|}{ 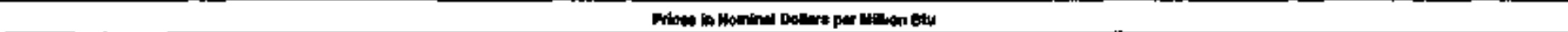 } \\
\hline \multirow[t]{2}{*}{ 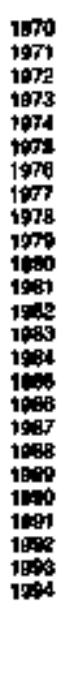 } & $\begin{array}{l}= \\
= \\
= \\
z \\
= \\
= \\
= \\
= \\
= \\
= \\
= \\
= \\
= \\
= \\
= \\
=\end{array}$ & 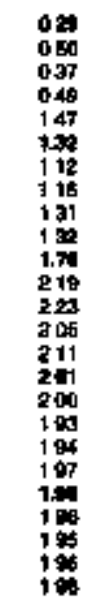 & 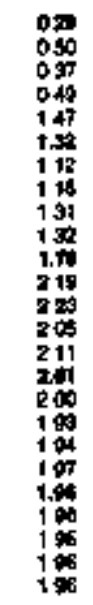 & 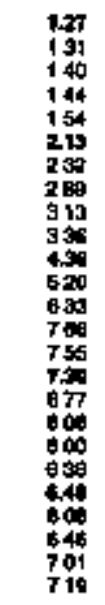 & 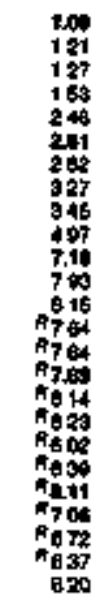 & 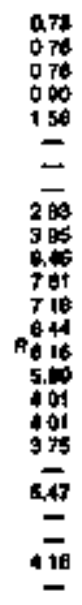 & 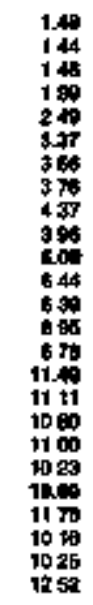 & 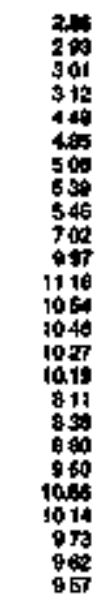 & 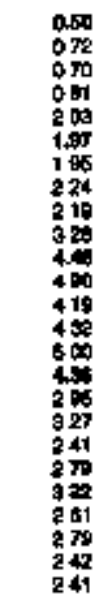 & 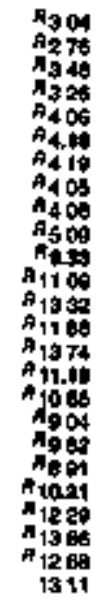 & 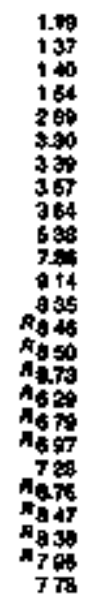 & $\begin{array}{l}= \\
= \\
= \\
= \\
= \\
= \\
= \\
= \\
= \\
= \\
= \\
= \\
=\end{array}$ & $\begin{array}{l}= \\
= \\
z \\
= \\
= \\
= \\
= \\
= \\
= \\
= \\
= \\
= \\
425 \\
393 \\
353 \\
353 \\
353\end{array}$ & 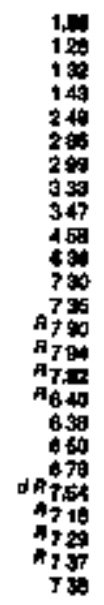 & 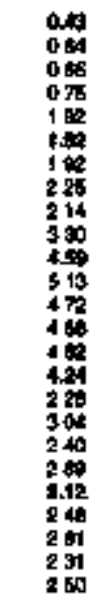 & 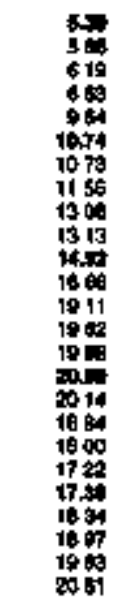 & 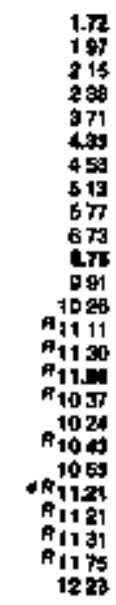 \\
\hline & \multicolumn{17}{|c|}{ 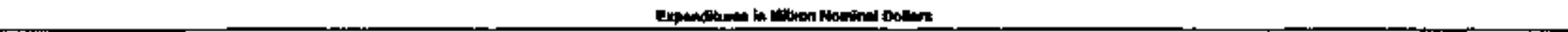 } \\
\hline 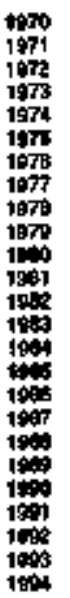 & $\begin{array}{l}= \\
= \\
= \\
= \\
= \\
= \\
= \\
= \\
= \\
= \\
= \\
= \\
= \\
=\end{array}$ & $\begin{array}{l}14 \\
70 \\
46 \\
58 \\
190 \\
134 \\
86 \\
46 \\
26 \\
36 \\
51 \\
53 \\
56 \\
62 \\
62 \\
70 \\
27 \\
34 \\
15 \\
28 \\
34 \\
32 \\
24 \\
26 \\
25\end{array}$ & 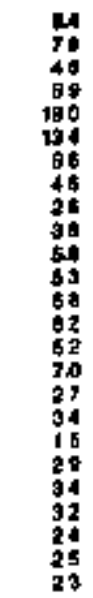 & 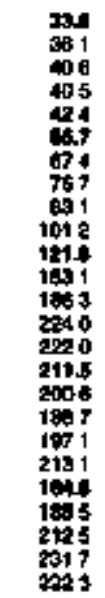 & 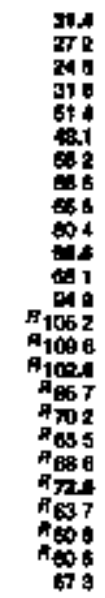 & 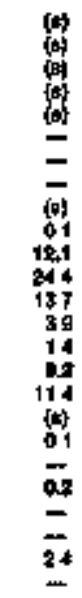 & $\begin{array}{l}\text { fy } \\
0 \\
0 y \\
0 \\
0 \\
01 \\
01 \\
01 \\
01 \\
0.1 \\
01 \\
01 \\
01 \\
01 \\
02 \\
02 \\
01 \\
02 \\
02 \\
02 \\
02 \\
02 \\
03 \\
02 \\
03\end{array}$ & 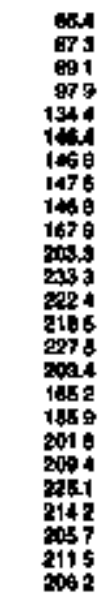 & 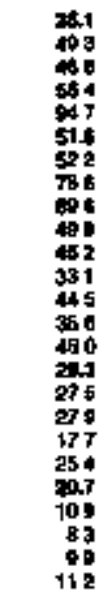 & 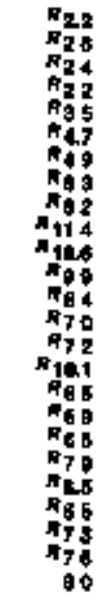 & 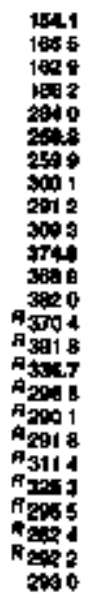 & $\begin{array}{l}= \\
= \\
= \\
= \\
= \\
= \\
= \\
= \\
= \\
= \\
= \\
= \\
=\end{array}$ & $\begin{array}{l}= \\
= \\
= \\
= \\
= \\
= \\
= \\
= \\
= \\
= \\
= \\
=21 \\
22 \\
23 \\
25\end{array}$ & 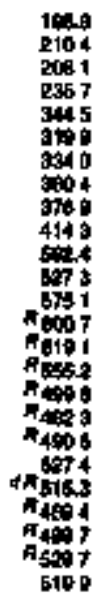 & 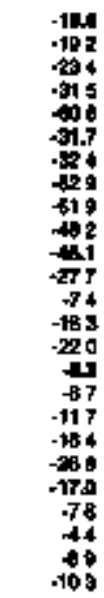 & 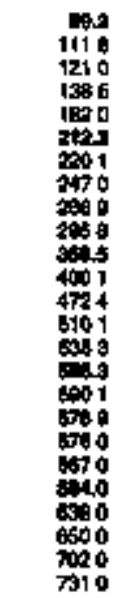 & 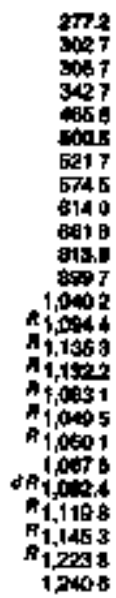 \\
\hline
\end{tabular}

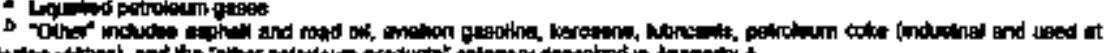

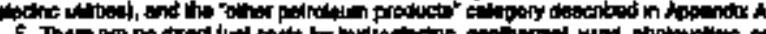

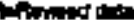

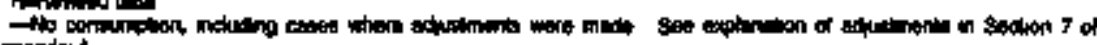
Aporidox $A$.

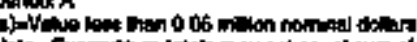

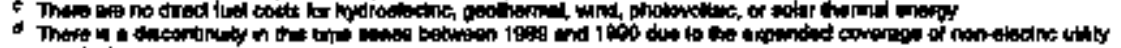

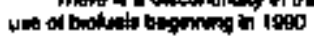

作

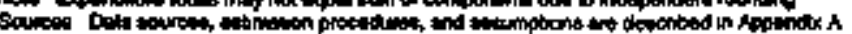




\begin{tabular}{|c|c|c|c|c|c|c|c|c|c|c|}
\hline \multirow[b]{4}{*}{ Tew } & \multicolumn{8}{|c|}{ 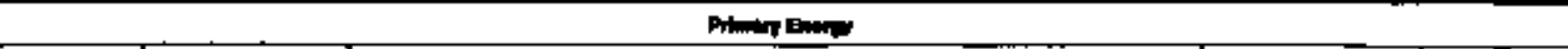 } & \multirow[b]{3}{*}{ 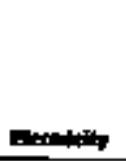 } & \multirow[b]{3}{*}{. } \\
\hline & \multirow[b]{2}{*}{ וat } & \multirow[b]{2}{*}{ mater } & \multicolumn{4}{|c|}{ nim } & \multirow[b]{2}{*}{ nanme: } & \multirow[b]{2}{*}{ Tre } & & \\
\hline & & & 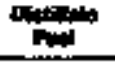 & mans & use. & $\pi$ & & & & \\
\hline & \multicolumn{10}{|c|}{ 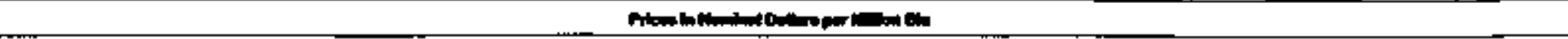 } \\
\hline \multirow[t]{2}{*}{ 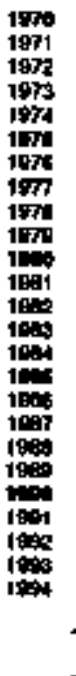 } & 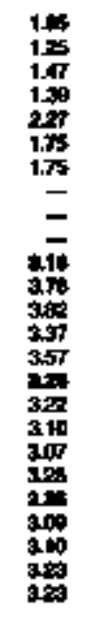 & 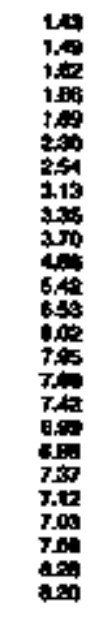 & 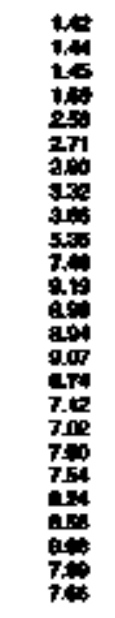 & 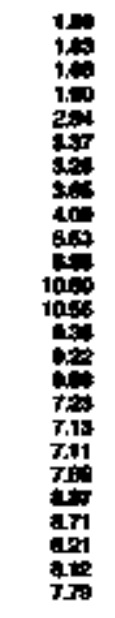 & 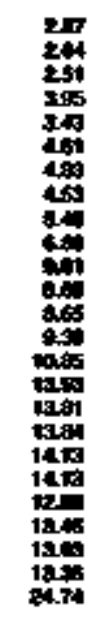 & 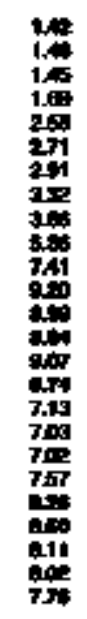 & 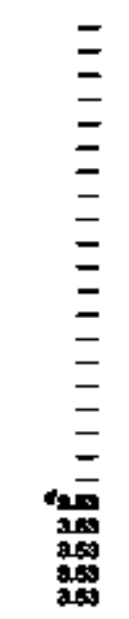 & 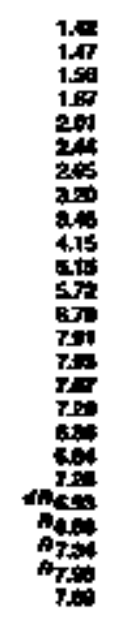 & 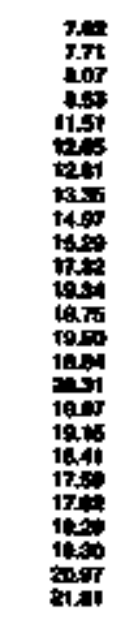 & 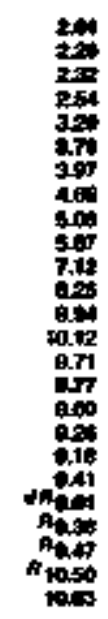 \\
\hline & \multicolumn{10}{|c|}{ | } \\
\hline 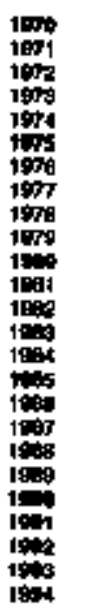 & 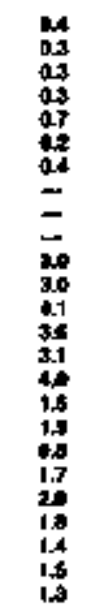 & 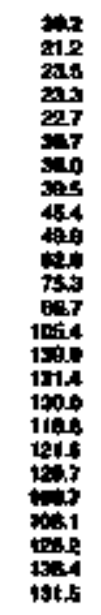 & 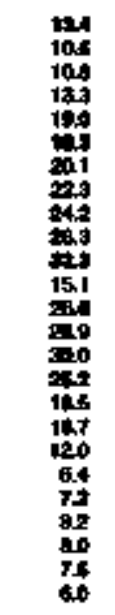 & 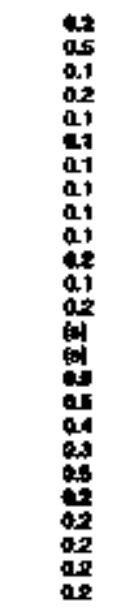 & 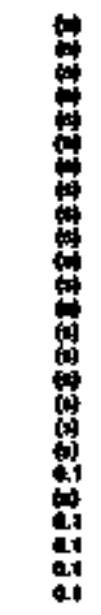 & 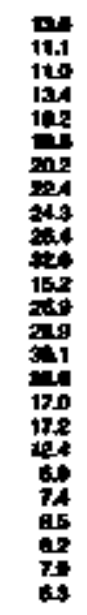 & $\begin{array}{l}= \\
z \\
z \\
z \\
z \\
z \\
z \\
\bar{z} \\
z \\
\bar{z} \\
\bar{z} \\
21 \\
22 \\
23 \\
24\end{array}$ & 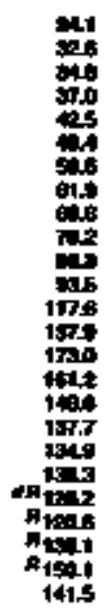 & 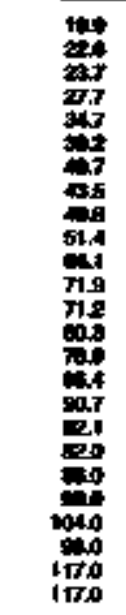 & 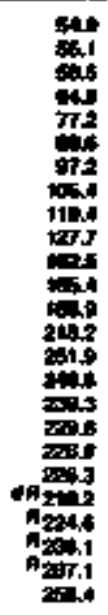 \\
\hline
\end{tabular}




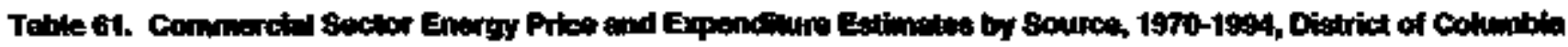

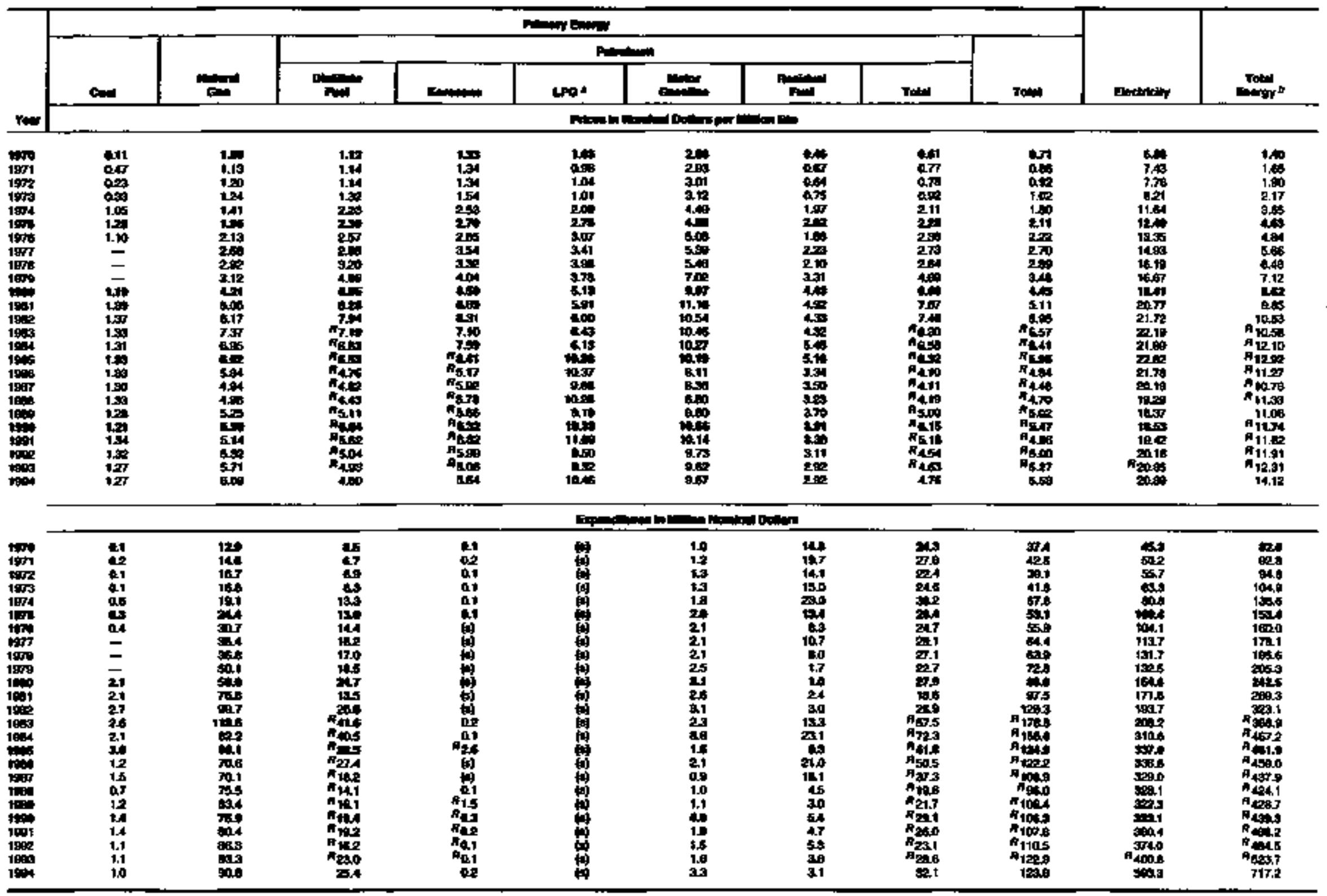




\begin{tabular}{|c|c|c|c|c|c|c|c|c|c|c|c|c|c|c|c|c|c|}
\hline \multirow[b]{4}{*}{ nerr } & \multicolumn{15}{|c|}{ 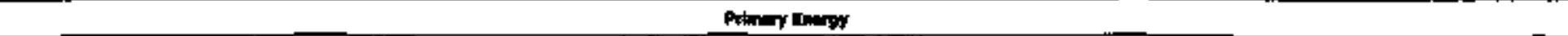 } & \multirow[b]{3}{*}{ Enctindir } & \multirow[b]{3}{*}{$\lim _{\lim } 0$} \\
\hline & \multicolumn{3}{|c|}{ com } & \multirow[b]{2}{*}{ ( } & \multicolumn{9}{|c|}{ Awrohen } & \multirow[b]{2}{*}{ Oatuelo: } & \multirow[b]{2}{*}{ 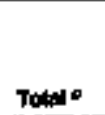 } & & \\
\hline & cotong & $\cos$ & Tots & & 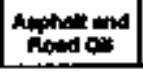 & 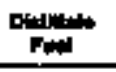 & 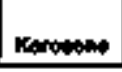 & 40. & Lubiome & Wolor & 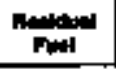 & onther: & Tolat & & & & \\
\hline & \multicolumn{17}{|c|}{ 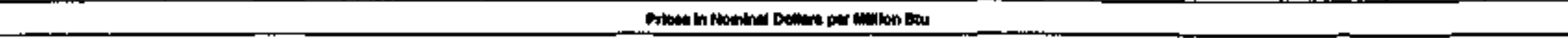 } \\
\hline \multirow[t]{2}{*}{ 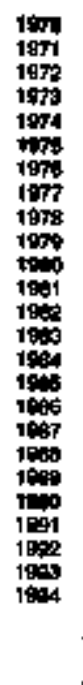 } & 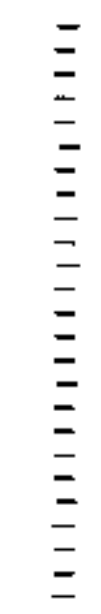 & 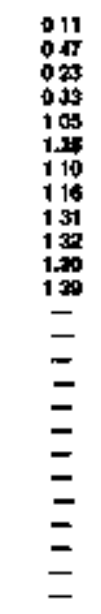 & $\begin{array}{l}911 \\
047 \\
023 \\
023 \\
039 \\
105 \\
131 \\
120 \\
116 \\
131 \\
132 \\
132 \\
139 \\
= \\
= \\
= \\
= \\
= \\
= \\
= \\
= \\
=\end{array}$ & $\begin{array}{l}007 \\
071 \\
077 \\
074 \\
077 \\
117 \\
19 \\
180 \\
218 \\
2199 \\
210 \\
240 \\
297 \\
= \\
= \\
= \\
= \\
= \\
= \\
= \\
= \\
=\end{array}$ & 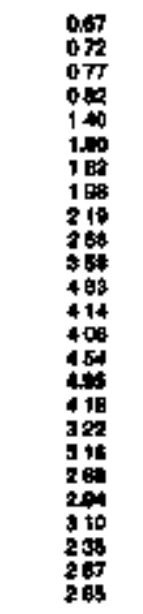 & 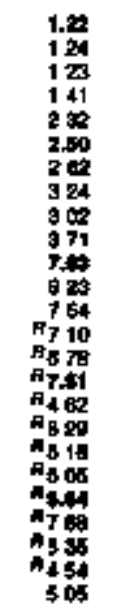 & 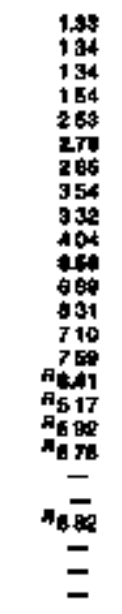 & 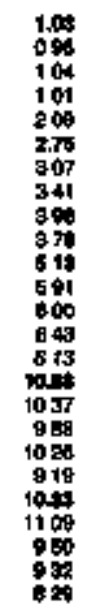 & 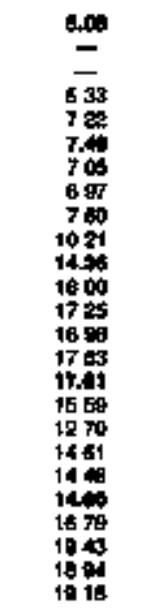 & 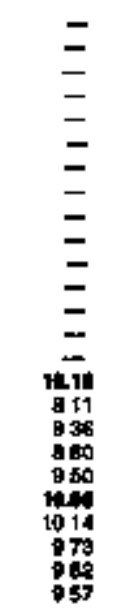 & 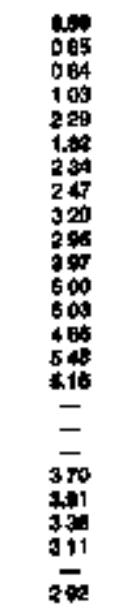 & $\begin{array}{l}z \\
= \\
z \\
z \\
= \\
z \\
z \\
z \\
= \\
= \\
z \\
z \\
z\end{array}$ & 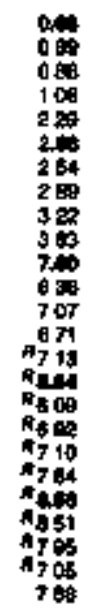 & $\begin{array}{l}z \\
z \\
z \\
z \\
z \\
z \\
z \\
z \\
= \\
= \\
z \\
z\end{array}$ & 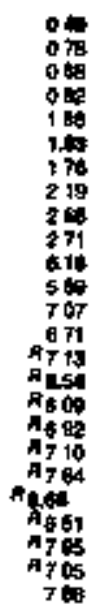 & 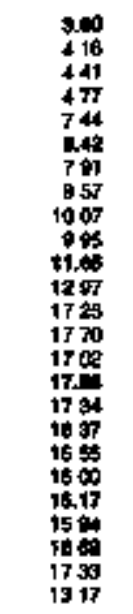 & 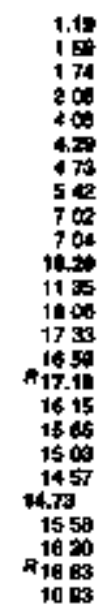 \\
\hline & \multicolumn{17}{|c|}{ 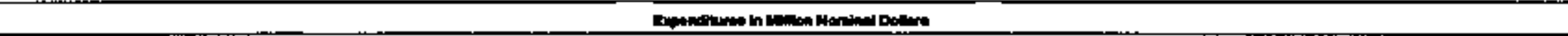 } \\
\hline 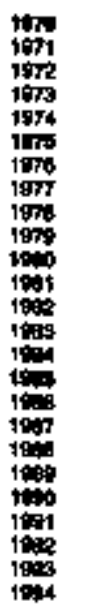 & $\begin{array}{l}z \\
z \\
z \\
z \\
z \\
z \\
z \\
z \\
z \\
z \\
z \\
z\end{array}$ & 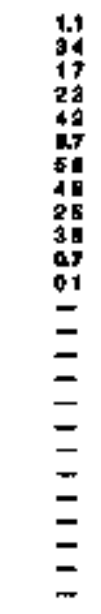 & 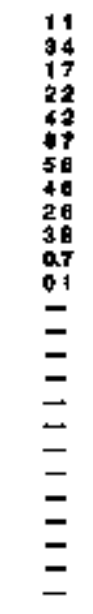 & 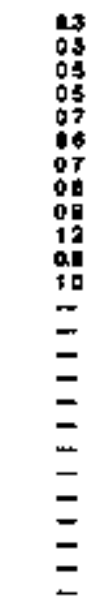 & 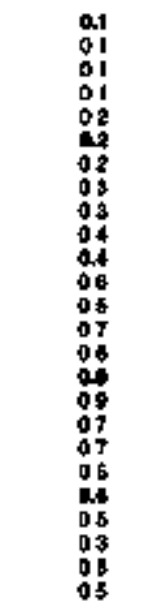 & 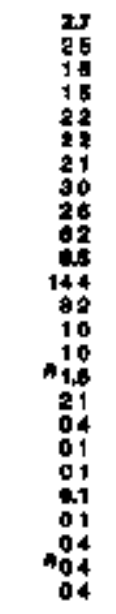 & 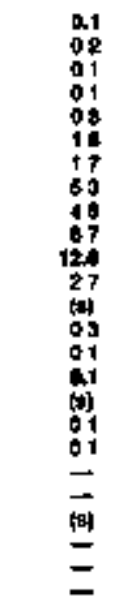 & 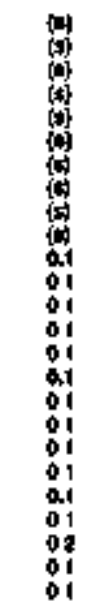 & 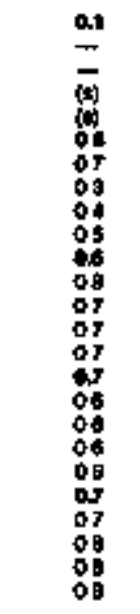 & $\begin{array}{l}z \\
z \\
z \\
z \\
z \\
z \\
z \\
z \\
= \\
z \\
21 \\
27 \\
35 \\
37 \\
31 \\
31 \\
39 \\
35\end{array}$ & 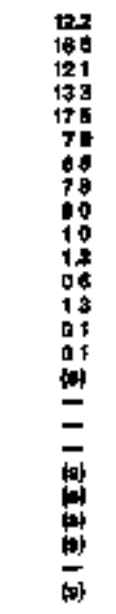 & $\begin{array}{l}= \\
= \\
z \\
z \\
z \\
z \\
= \\
= \\
= \\
= \\
= \\
z \\
z\end{array}$ & 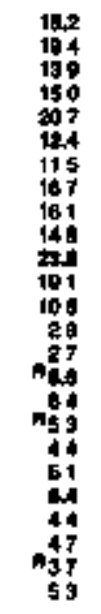 & $\begin{array}{l}z \\
z \\
z \\
z \\
z \\
z \\
z \\
z \\
= \\
= \\
z \\
z \\
z\end{array}$ & 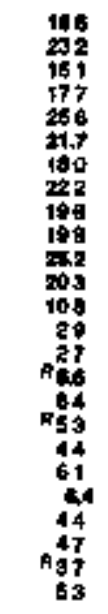 & 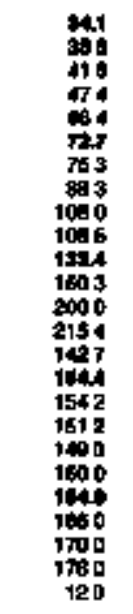 & 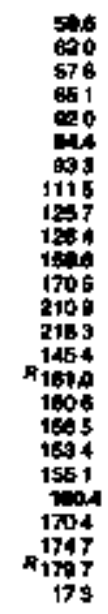 \\
\hline
\end{tabular}


Table 63. Transportailon Sector Energy Price and Expenditure Estimales by Sotrce, 1870-1994, Diathlet of Columbla

\begin{tabular}{|c|c|c|c|c|c|c|c|c|c|c|c|c|c|}
\hline \multirow[b]{4}{*}{$r=\pi$} & \multicolumn{11}{|c|}{ 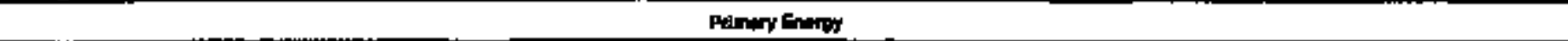 } & \multirow[b]{3}{*}{ 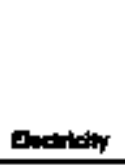 } & \multirow[b]{3}{*}{ Totid } \\
\hline & & \multirow[b]{2}{*}{ mant } & \multicolumn{8}{|c|}{ 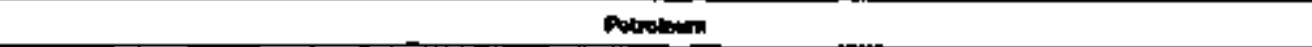 } & \multirow[b]{2}{*}{ Jot:d } & & \\
\hline & canter & & 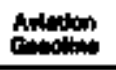 & 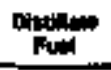 & 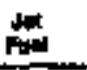 & Lega & 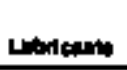 & mator & Find & Tout & & & \\
\hline & \multicolumn{13}{|c|}{ 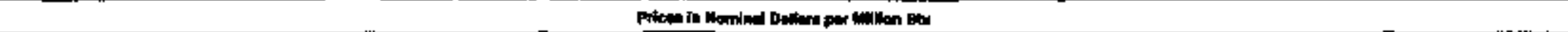 } \\
\hline 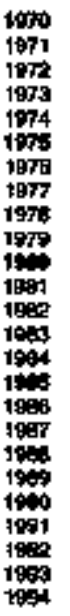 & $\begin{array}{l}1.11 \\
047 \\
023 \\
033 \\
105 \\
103 \\
110 \\
116 \\
= \\
= \\
= \\
= \\
= \\
= \\
= \\
= \\
= \\
=\end{array}$ & $\begin{array}{l}= \\
= \\
= \\
= \\
= \\
= \\
= \\
= \\
= \\
= \\
= \\
= \\
= \\
=\end{array}$ & $\begin{array}{l}= \\
= \\
= \\
= \\
= \\
= \\
= \\
= \\
= \\
= \\
= \\
= \\
= \\
=24\end{array}$ & 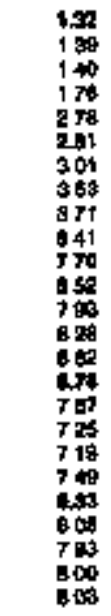 & 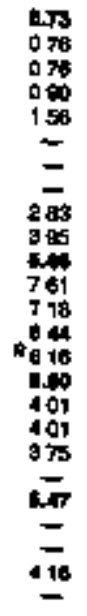 & 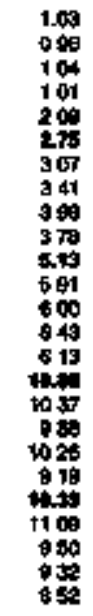 & 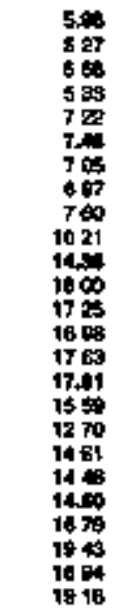 & 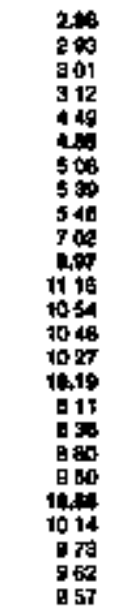 & 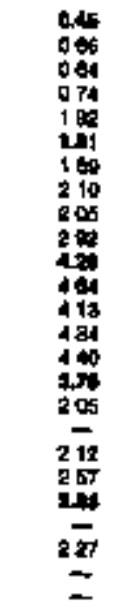 & 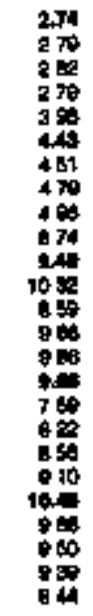 & 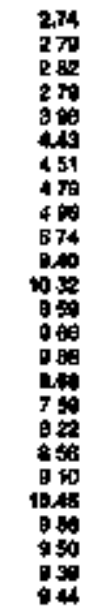 & 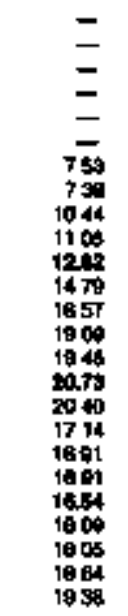 & 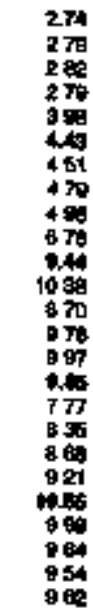 \\
\hline 1999 & \multicolumn{13}{|c|}{ 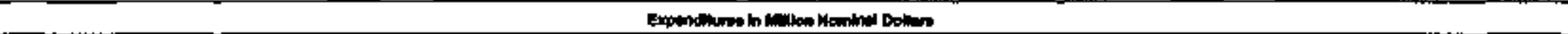 } \\
\hline 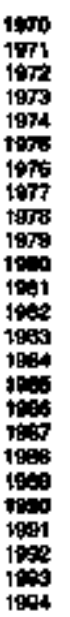 & 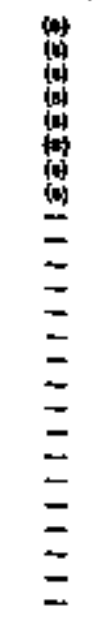 & $\begin{array}{l}= \\
= \\
= \\
= \\
= \\
= \\
= \\
= \\
= \\
= \\
= \\
= \\
= \\
=\end{array}$ & $\begin{array}{l}= \\
= \\
= \\
= \\
= \\
= \\
= \\
= \\
= \\
= \\
= \\
= \\
= \\
= \\
01 \\
01\end{array}$ & 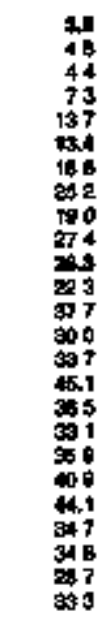 & 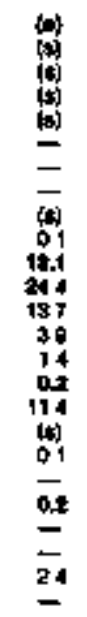 & 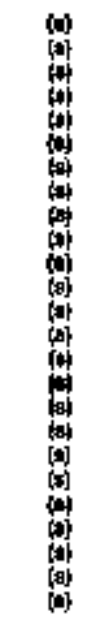 & $\begin{array}{l}14 \\
17 \\
20 \\
17 \\
22 \\
21 \\
29 \\
23 \\
87 \\
97 \\
47 \\
56 \\
48 \\
51 \\
58 \\
45 \\
48 \\
46 \\
17 \\
49 \\
60 \\
59 \\
58 \\
42\end{array}$ & 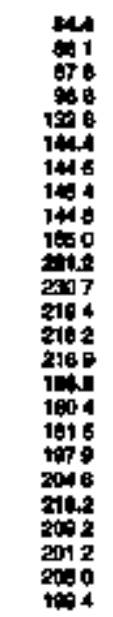 & 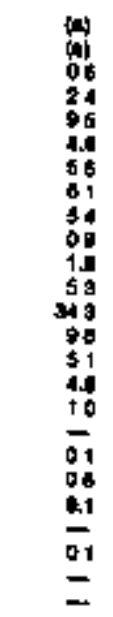 & 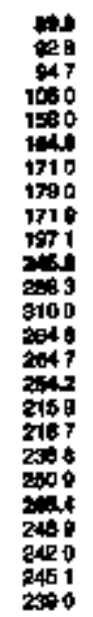 & 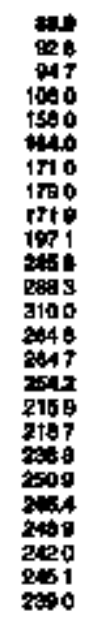 & $\begin{array}{l}= \\
= \\
= \\
= \\
01 \\
65 \\
14 \\
34 \\
44 \\
62 \\
74 \\
61 \\
61 \\
64 \\
63 \\
66 \\
69 \\
67 \\
01 \\
76 \\
60 \\
62 \\
17\end{array}$ & 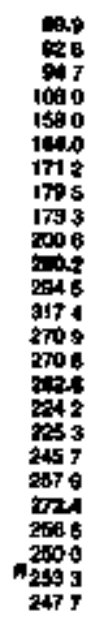 \\
\hline
\end{tabular}




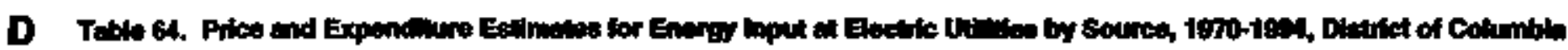

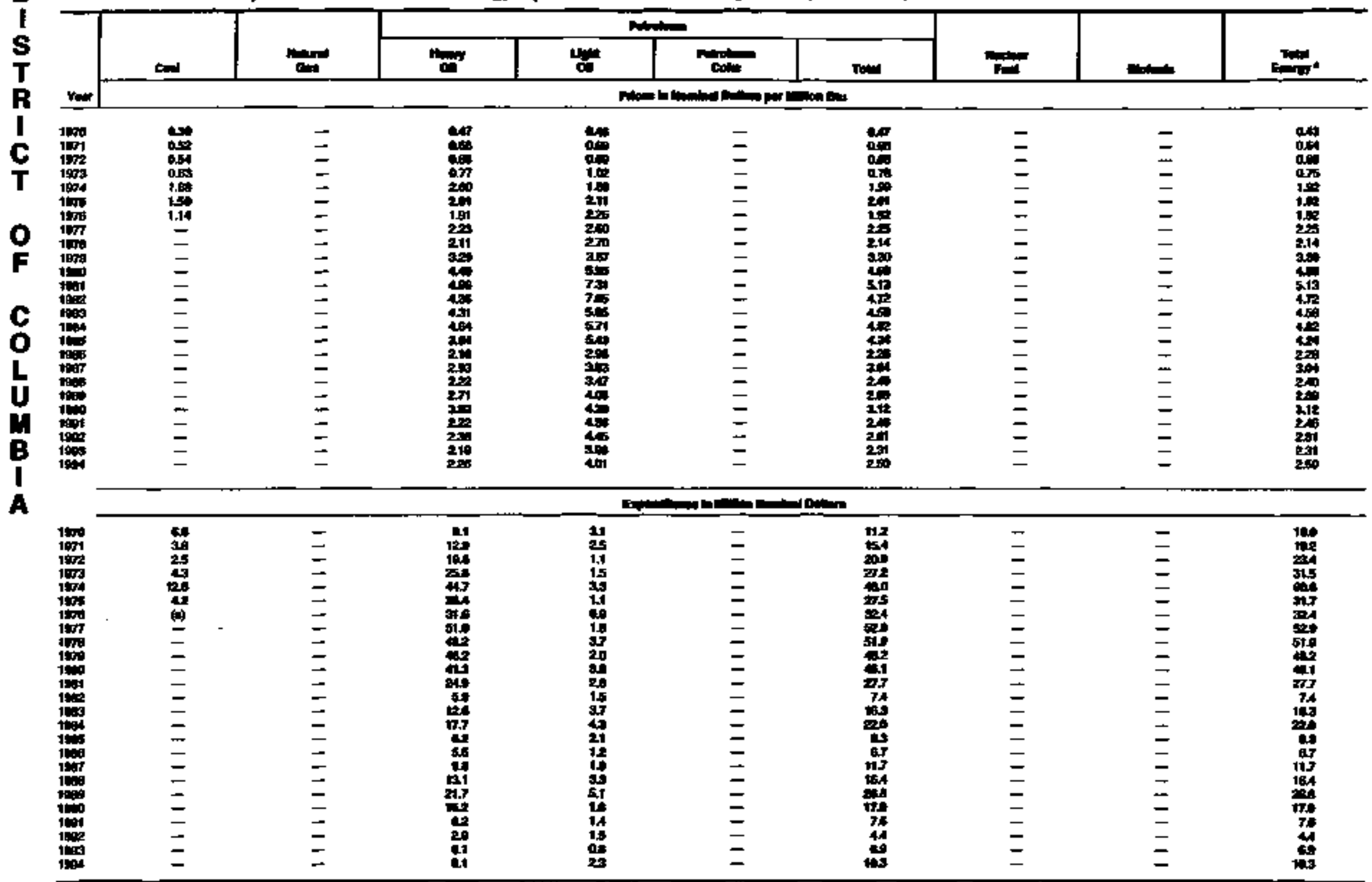

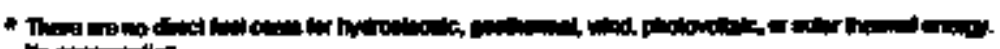

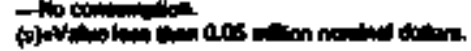




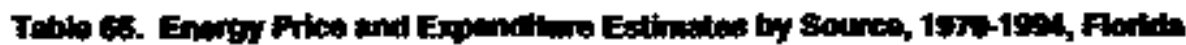

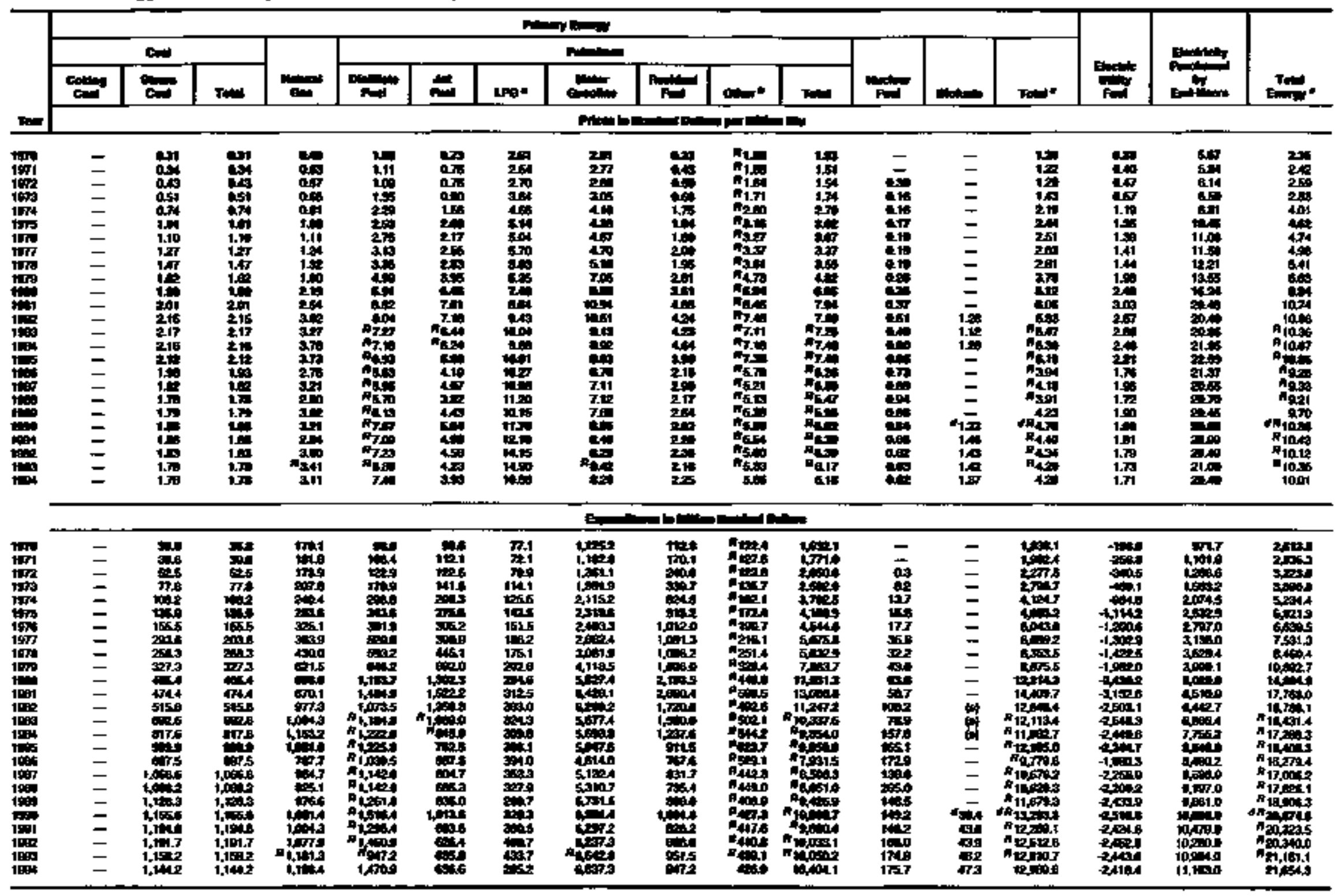

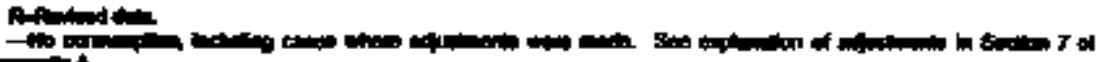

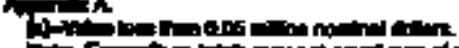

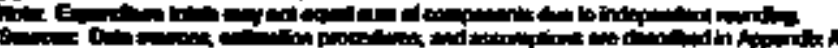




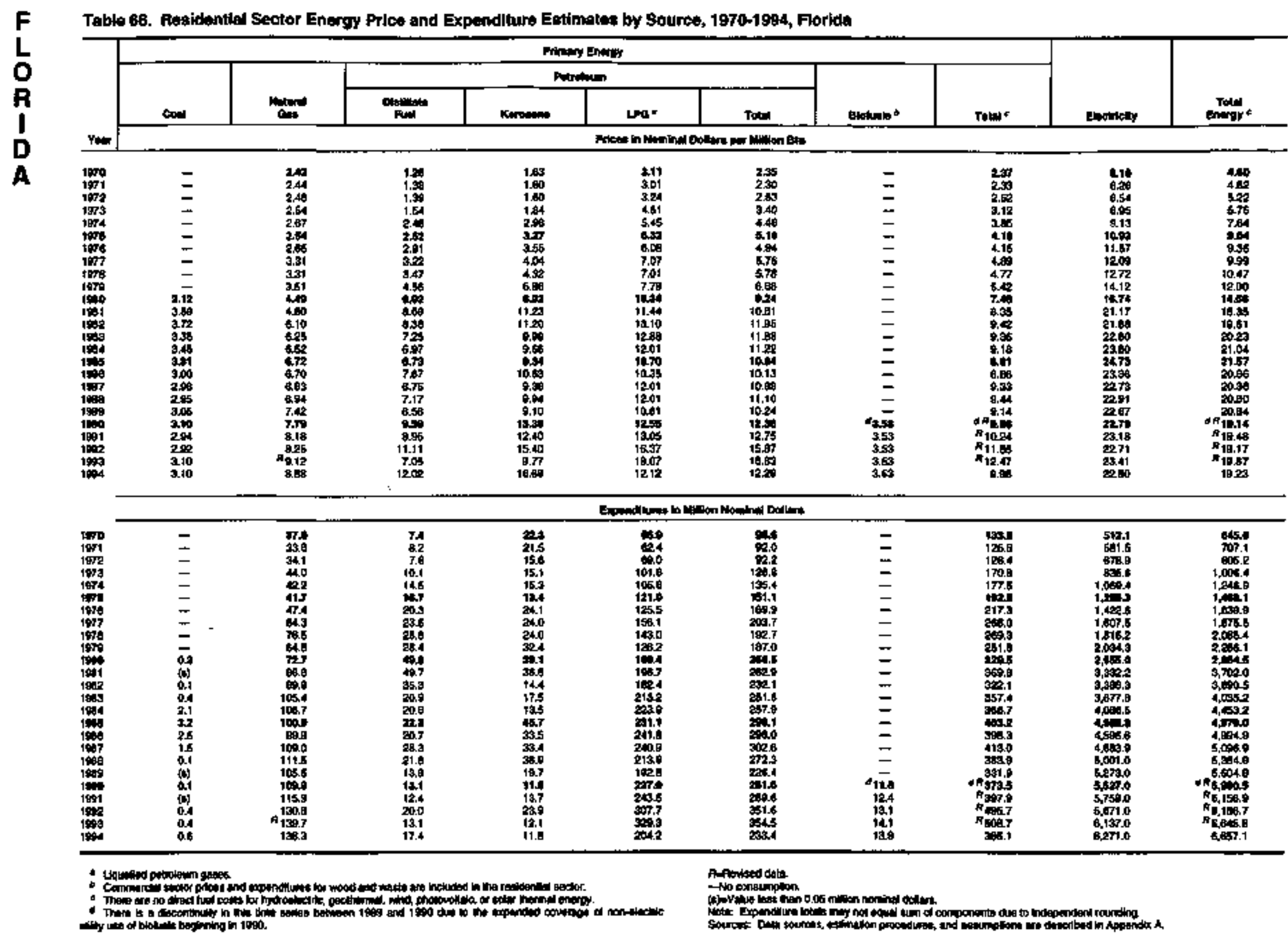


Table 67. Commercial Seotor Energy Price and Expendkure Estkmates by Soures, 1970-1994, Forida

\begin{tabular}{|c|c|c|c|c|c|c|c|c|c|c|c|}
\hline \multirow[b]{4}{*}{ vem } & \multicolumn{9}{|c|}{ Others enimg } & \multirow[b]{3}{*}{ Enctriattr } & \multirow[b]{3}{*}{ 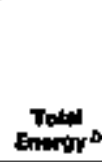 } \\
\hline & \multirow[b]{2}{*}{ Cod } & \multirow[b]{2}{*}{ 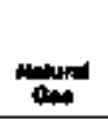 } & \multicolumn{6}{|c|}{ Putrolom } & \multirow[b]{2}{*}{ row } & & \\
\hline & & & 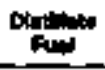 & Kanowine & Lea & molis & Anstuder & Tate & & & \\
\hline & \multicolumn{11}{|c|}{ 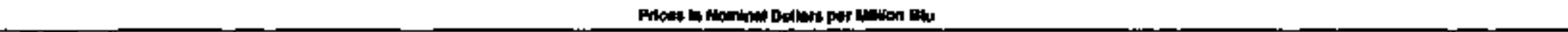 } \\
\hline \multirow[t]{2}{*}{ 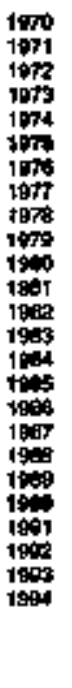 } & 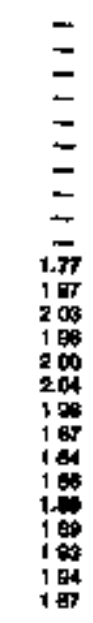 & 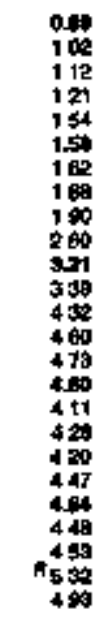 & 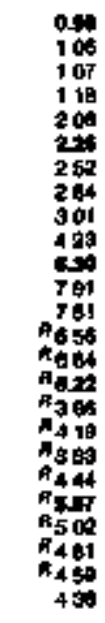 & 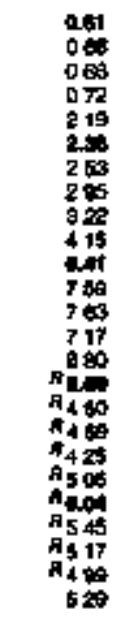 & 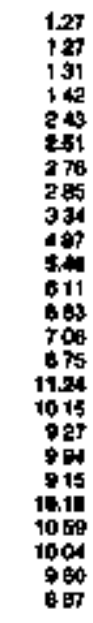 & 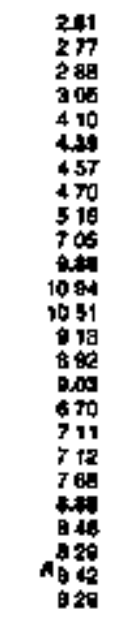 & 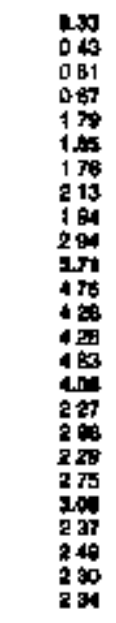 & 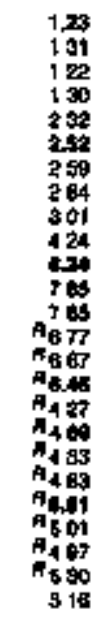 & 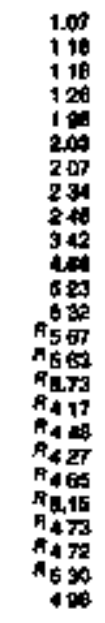 & 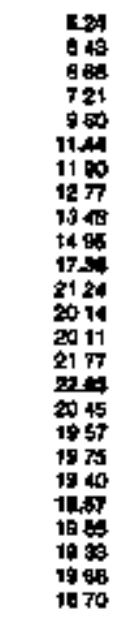 & 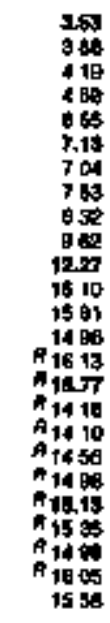 \\
\hline & \multicolumn{11}{|c|}{ 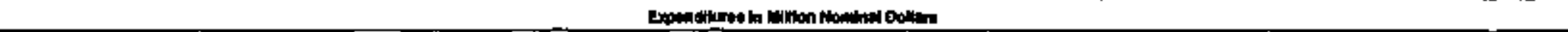 } \\
\hline 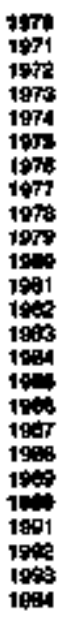 & 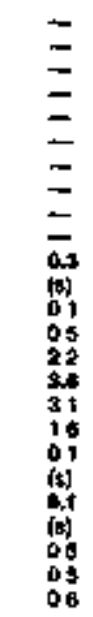 & 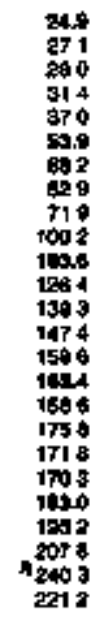 & 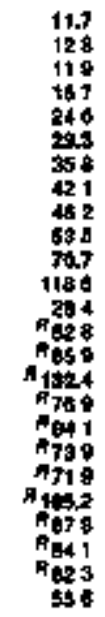 & 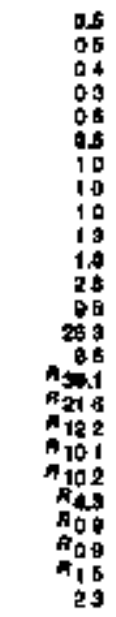 & 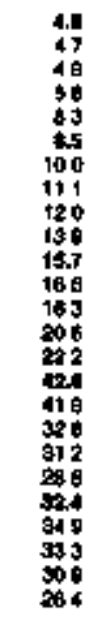 & 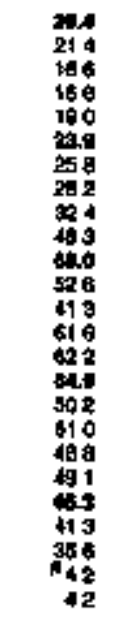 & 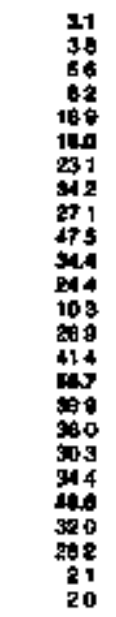 & 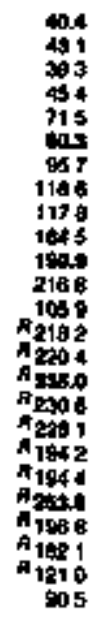 & 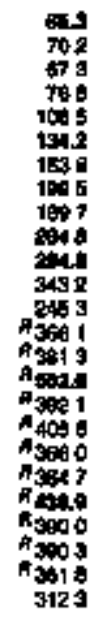 & 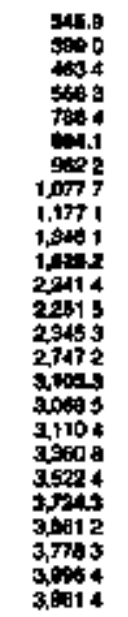 & 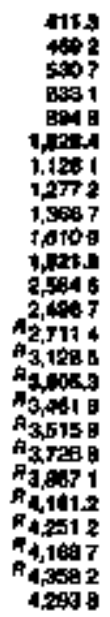 \\
\hline
\end{tabular}

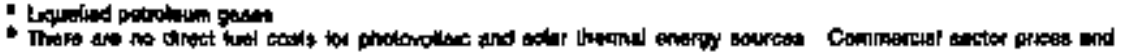

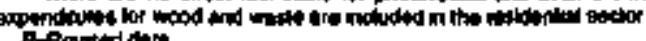

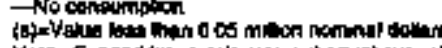

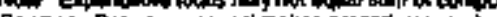




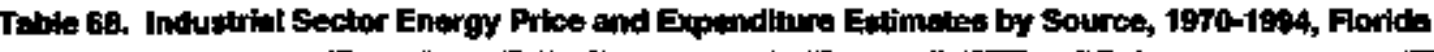

\begin{tabular}{|c|c|c|c|c|c|c|c|c|c|c|c|c|c|c|c|c|c|}
\hline & \multicolumn{15}{|c|}{ mimm thenty } & \multirow[b]{3}{*}{ Enctiking } & \multirow[b]{3}{*}{ 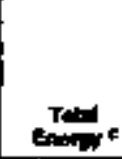 } \\
\hline & \multicolumn{3}{|c|}{ Cont } & \multirow[b]{2}{*}{ 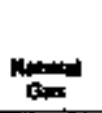 } & \multicolumn{9}{|c|}{ 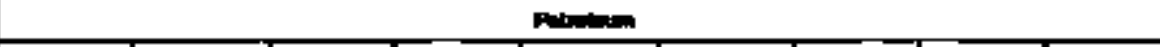 } & \multirow[b]{2}{*}{ 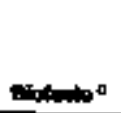 } & \multirow[b]{2}{*}{ Totos" } & & \\
\hline & cons & coll & Tom & & 1 & nim & Horomat: & LP: & untiont: & 年 & Intel & Dinet & Tatit & & & & \\
\hline$T_{i \rightarrow m}$ & \multicolumn{17}{|c|}{ 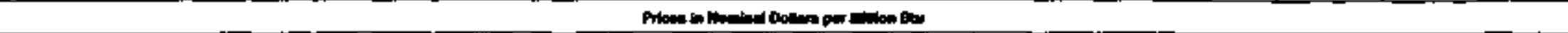 } \\
\hline \multirow[t]{2}{*}{ 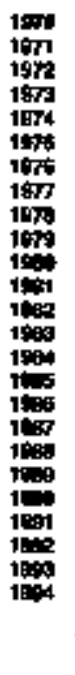 } & $\begin{array}{l}= \\
= \\
= \\
= \\
= \\
= \\
= \\
= \\
= \\
= \\
= \\
= \\
=\end{array}$ & 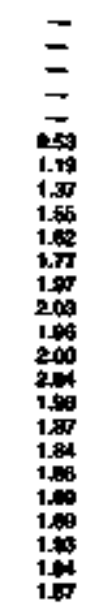 & 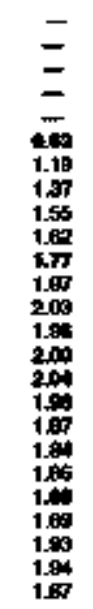 & 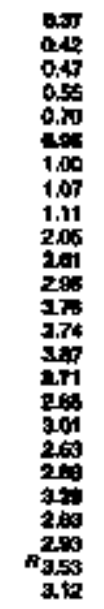 & 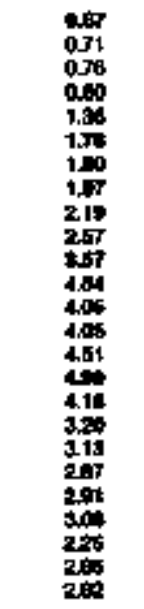 & 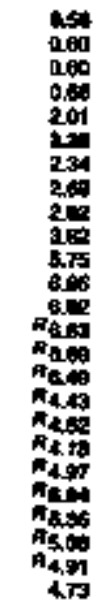 & 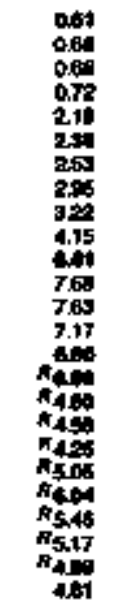 & 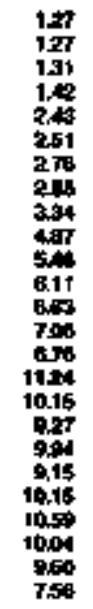 & 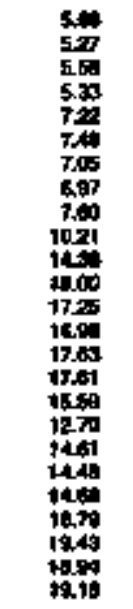 & 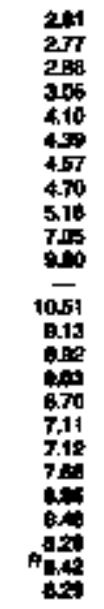 & 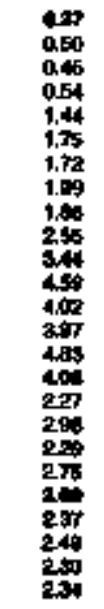 & 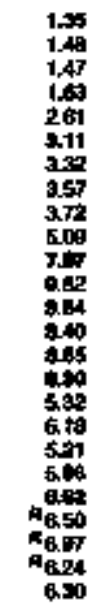 & 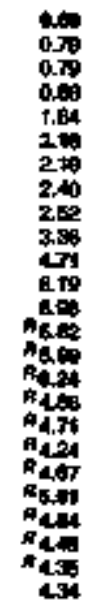 & $\begin{array}{l}= \\
= \\
= \\
= \\
= \\
= \\
= \\
= \\
= \\
= \\
= \\
\bar{a} \\
0.04 \\
1.14 \\
1.13\end{array}$ & 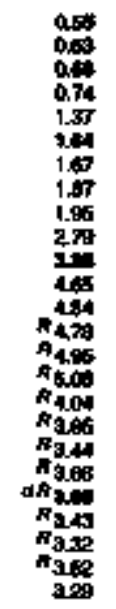 & 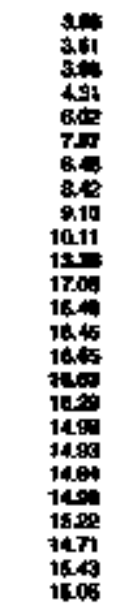 & 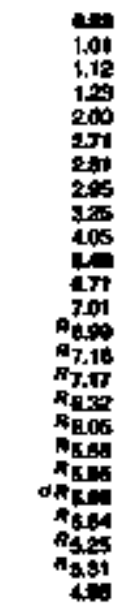 \\
\hline & \multicolumn{17}{|c|}{ 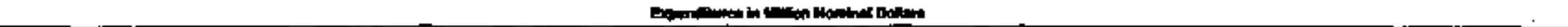 } \\
\hline 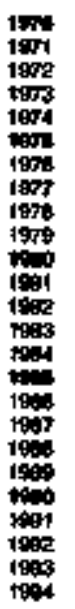 & $\begin{array}{l}= \\
z \\
= \\
= \\
= \\
= \\
= \\
= \\
= \\
= \\
= \\
= \\
= \\
= \\
=\end{array}$ & 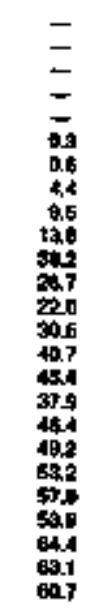 & 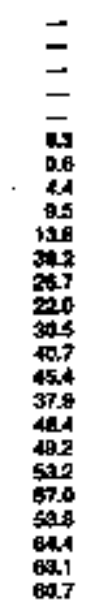 & 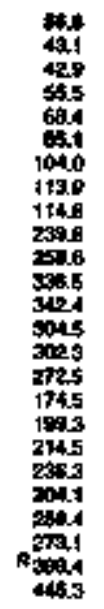 & 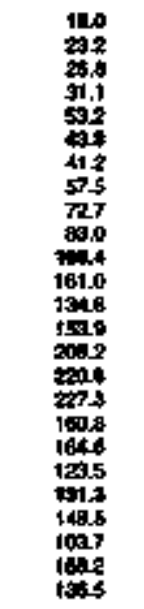 & 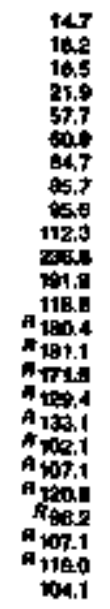 & 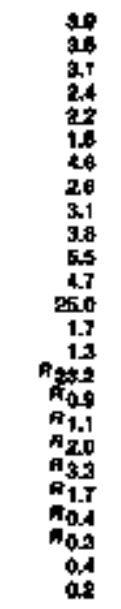 & 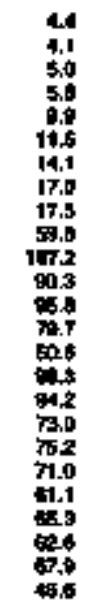 & 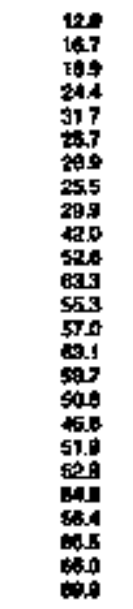 & 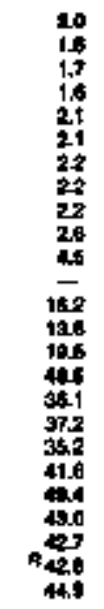 & 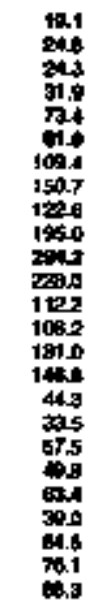 & 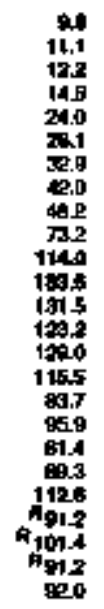 & 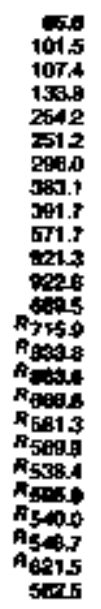 & $\begin{array}{l}= \\
= \\
= \\
= \\
= \\
= \\
= \\
= \\
= \\
= \\
= \\
d=6 \\
30.1 \\
306 \\
301\end{array}$ & 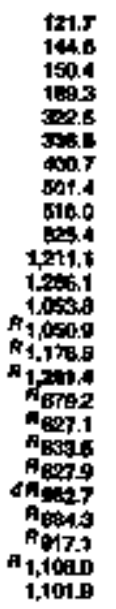 & 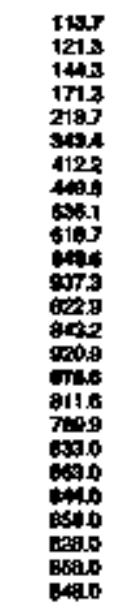 & 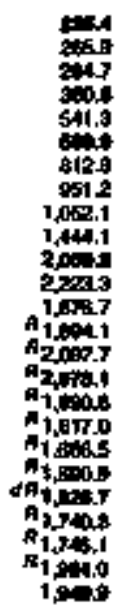 \\
\hline
\end{tabular}

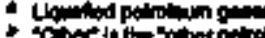

Antitad and

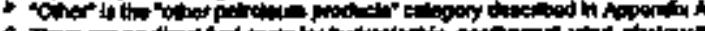

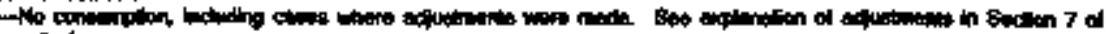

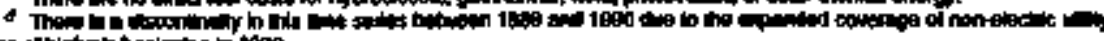
Appendex

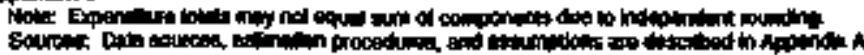

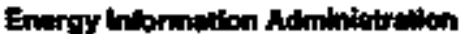




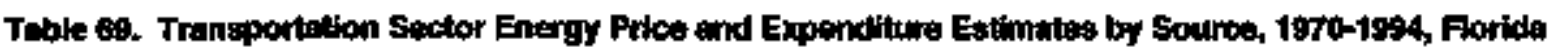

\begin{tabular}{|c|c|c|c|c|c|c|c|c|c|c|c|c|c|}
\hline & \multicolumn{11}{|c|}{ Mantry Eary } & \multirow[b]{3}{*}{ Enctiving } & \multirow[b]{3}{*}{ Toter } \\
\hline & & \multirow[b]{2}{*}{ 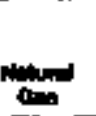 } & \multicolumn{8}{|c|}{ Finsm } & \multirow[b]{2}{*}{ Jove } & & \\
\hline & 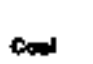 & & 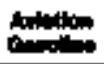 & 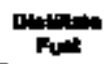 & . & LPOE" & 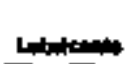 & thes & moldid & 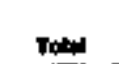 & & & \\
\hline ran & \multicolumn{13}{|c|}{ 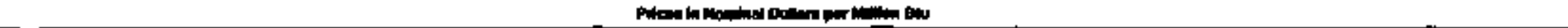 } \\
\hline 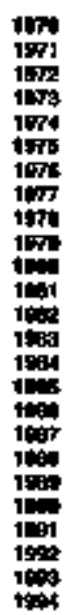 & 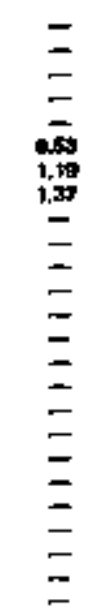 & $\begin{array}{l}= \\
= \\
= \\
= \\
= \\
= \\
= \\
= \\
= \\
= \\
= \\
= \\
25 \\
404 \\
406\end{array}$ & 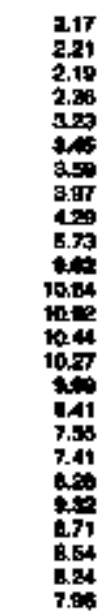 & 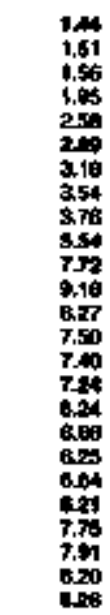 & 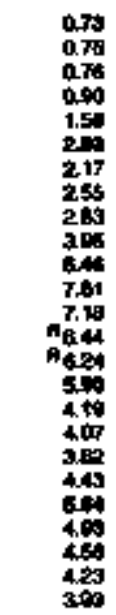 & 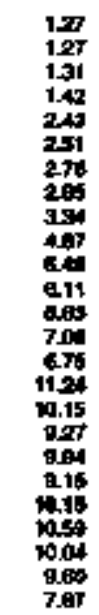 & 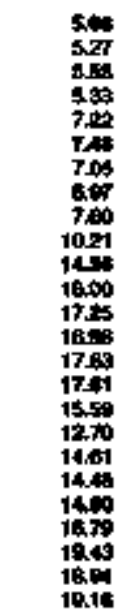 & 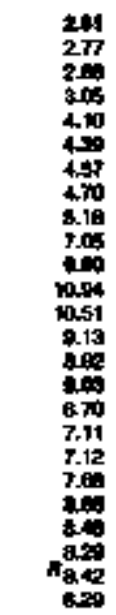 & 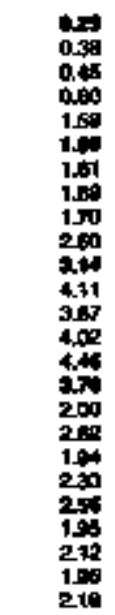 & 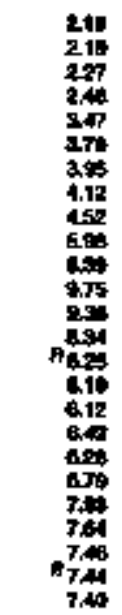 & 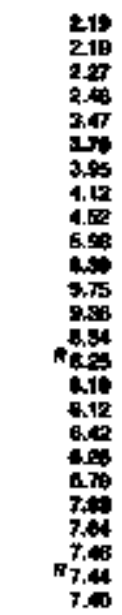 & 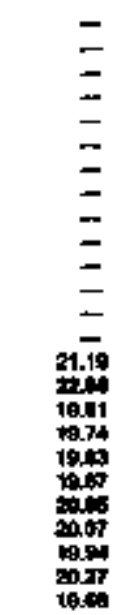 & 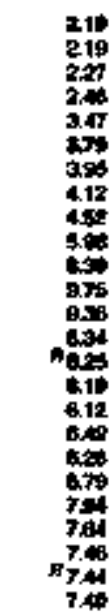 \\
\hline & \multicolumn{13}{|c|}{ 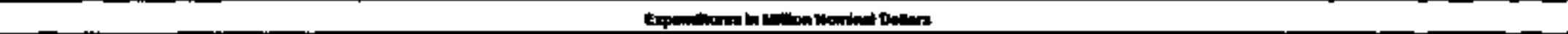 } \\
\hline 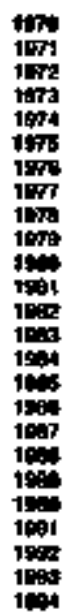 & $\begin{array}{l}= \\
= \\
= \\
= \\
= \\
= \\
= \\
= \\
= \\
= \\
= \\
= \\
=\end{array}$ & $\begin{array}{l}= \\
= \\
= \\
= \\
= \\
= \\
= \\
= \\
= \\
= \\
= \\
= \\
00 \\
60 \\
001\end{array}$ & 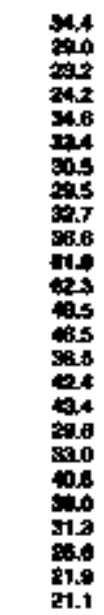 & 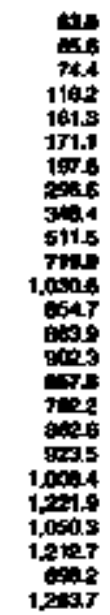 & 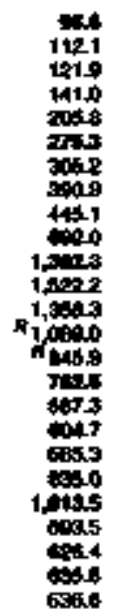 & 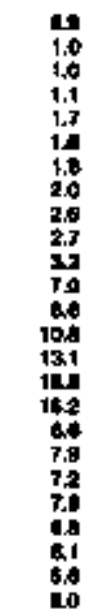 & 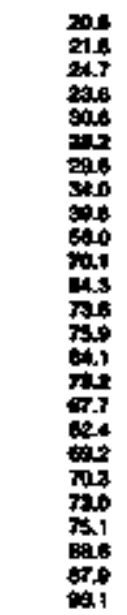 & 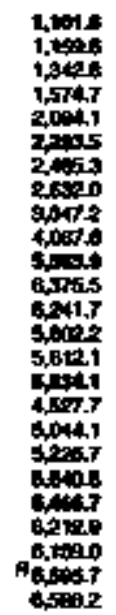 & 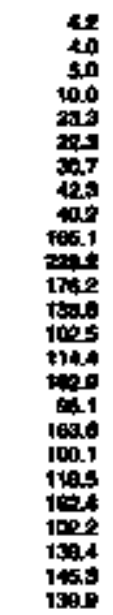 & 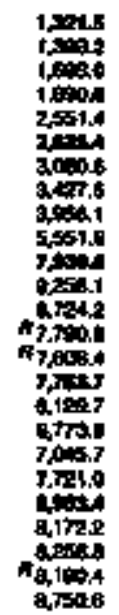 & 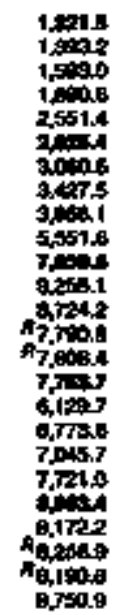 & $\begin{array}{l}= \\
= \\
= \\
= \\
= \\
= \\
= \\
= \\
= \\
0.5 \\
14 \\
2.5 \\
2.2 \\
2.8 \\
23 \\
2.3 \\
2.3 \\
2.0 \\
2.6\end{array}$ & 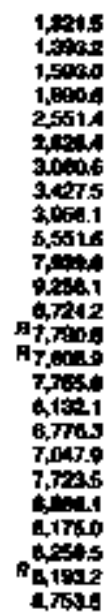 \\
\hline
\end{tabular}


F Table 70. Price and Expendlure Entimates for Enorgy inpurt nt Electric Utilitles by Source, 1970-1994, Florida

\begin{tabular}{|c|c|c|c|c|c|c|c|c|c|}
\hline \multirow[b]{3}{*}{$\mathrm{ven}$} & & \multirow[b]{2}{*}{ thithord } & \multicolumn{4}{|c|}{ Petrolanim } & \multirow[b]{2}{*}{ 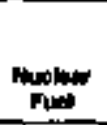 } & \multirow[b]{2}{*}{ Elopuns: } & \multirow[b]{2}{*}{ Fober. } \\
\hline & con & & Howy & on & Prome & Total & & & \\
\hline & \multicolumn{9}{|c|}{ 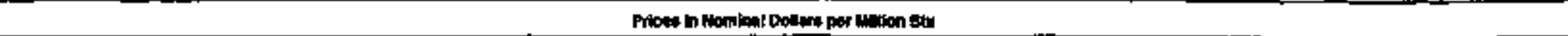 } \\
\hline \multirow[t]{2}{*}{ 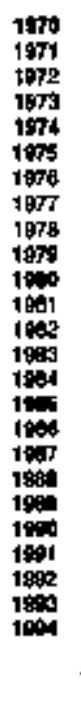 } & 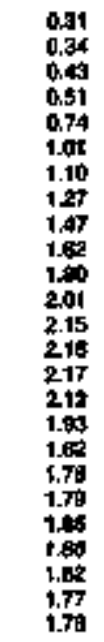 & 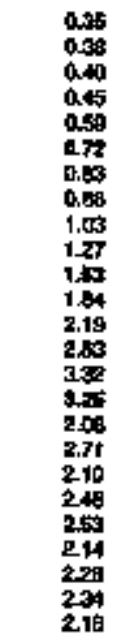 & 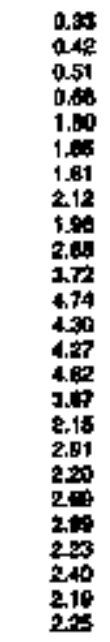 & 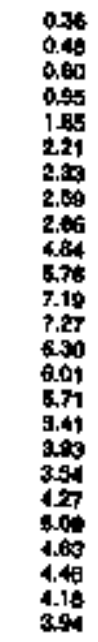 & $\begin{array}{l}= \\
= \\
= \\
= \\
= \\
= \\
= \\
= \\
= \\
= \\
= \\
= \\
=\end{array}$ & 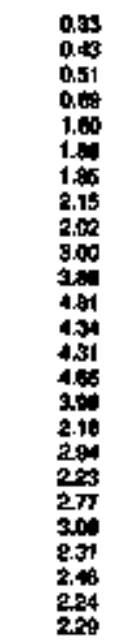 & 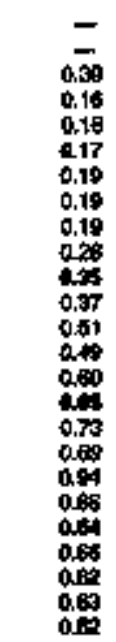 & $\begin{array}{l}= \\
= \\
= \\
= \\
= \\
= \\
= \\
120 \\
120 \\
= \\
= \\
= \\
= \\
= \\
=\end{array}$ & 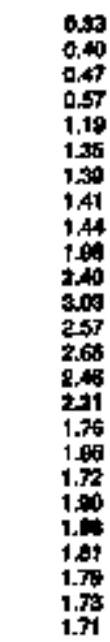 \\
\hline & \multicolumn{9}{|c|}{ 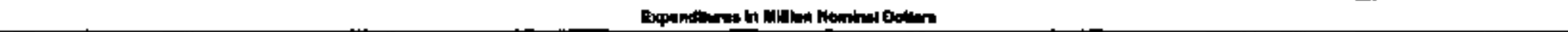 } \\
\hline 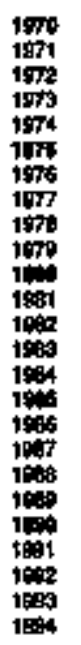 & 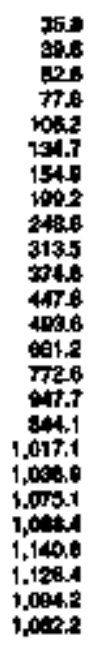 & 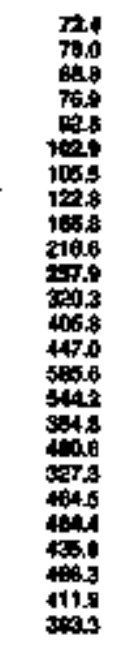 & 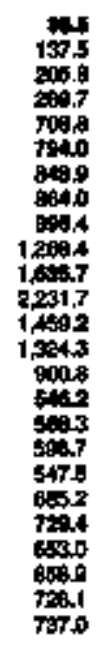 & 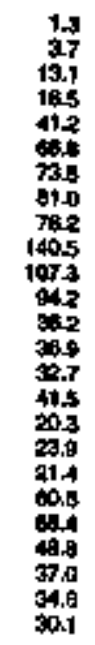 & $\begin{array}{l}= \\
= \\
= \\
= \\
= \\
= \\
= \\
= \\
= \\
= \\
= \\
= \\
= \\
=\end{array}$ & 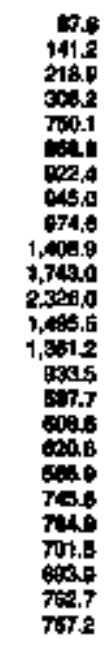 & 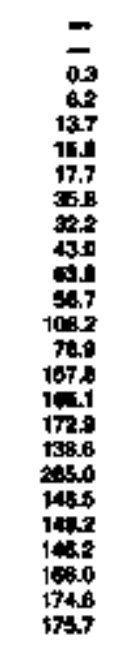 & $\begin{array}{l}= \\
= \\
= \\
= \\
= \\
= \\
= \\
(0) \\
= \\
= \\
= \\
= \\
= \\
=\end{array}$ & 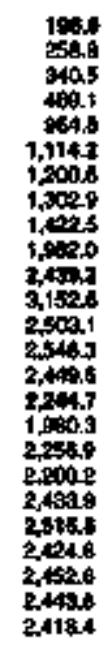 \\
\hline
\end{tabular}

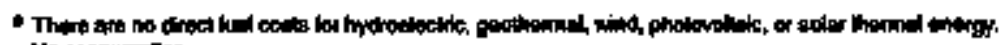
Tho canevinpton. 


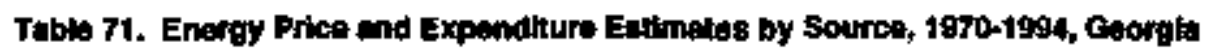

\begin{tabular}{|c|c|c|c|c|c|c|c|c|c|c|c|c|c|c|c|c|c|}
\hline & \multicolumn{14}{|c|}{ 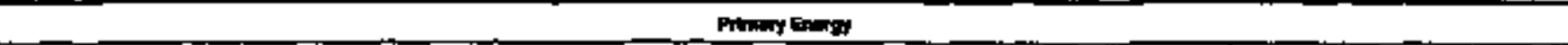 } & \multirow{3}{*}{ Elenghs } & \multirow{3}{*}{ istim } & \multirow[b]{3}{*}{ Tot } \\
\hline & \multicolumn{3}{|c|}{ tavi } & \multirow[b]{2}{*}{ Intion } & \multicolumn{7}{|c|}{ Perolon } & \multirow[b]{2}{*}{ Nentin } & \multirow[b]{2}{*}{ Elotum } & \multirow[b]{2}{*}{ Towite } & & & \\
\hline & conth & تئ日) & fole & & 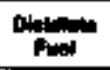 & 告 & LPA* & שה & 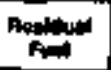 & Ohes & $T=0$ & & & & & & \\
\hline Yer. & \multicolumn{17}{|c|}{ 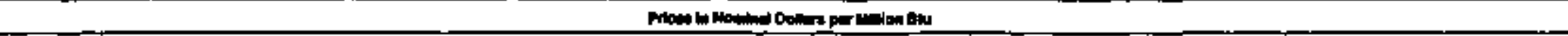 } \\
\hline \multirow[t]{2}{*}{ 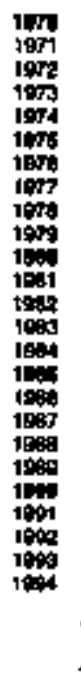 } & $\begin{array}{l}= \\
= \\
= \\
z \\
= \\
= \\
= \\
= \\
= \\
= \\
= \\
= \\
= \\
=\end{array}$ & 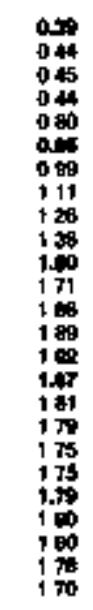 & 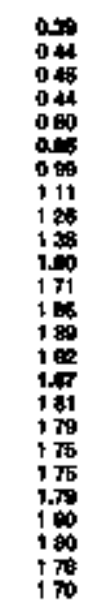 & 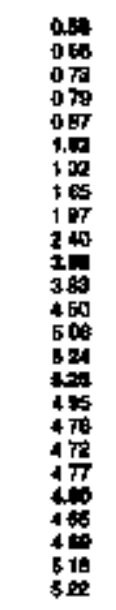 & 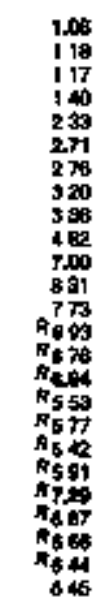 & 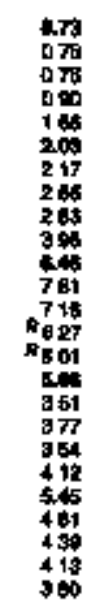 & 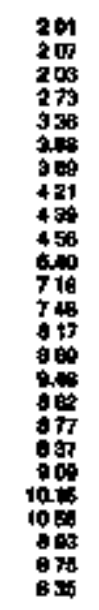 & 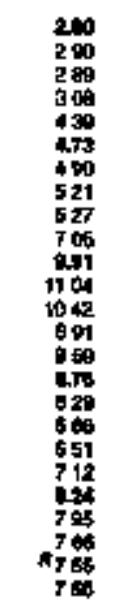 & 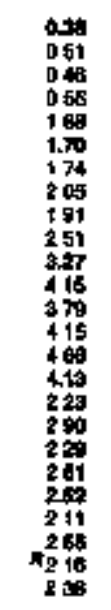 & 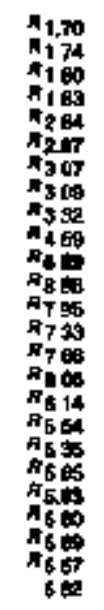 & 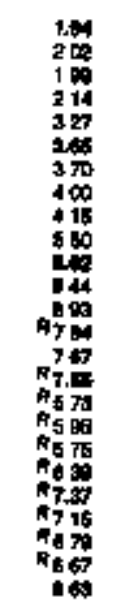 & 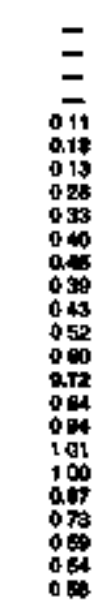 & $\begin{array}{l}= \\
= \\
= \\
= \\
= \\
= \\
= \\
= \\
= \\
= \\
= \\
10 \\
10\end{array}$ & 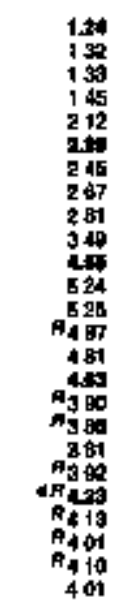 & 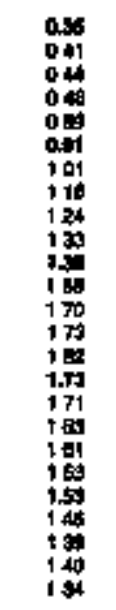 & 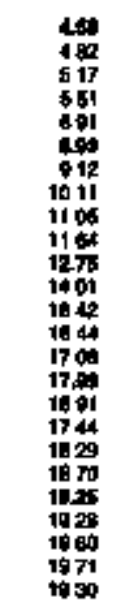 & 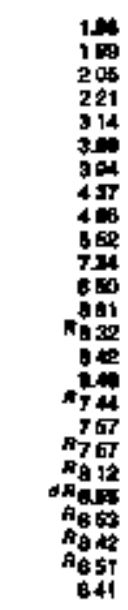 \\
\hline & \multicolumn{17}{|c|}{ 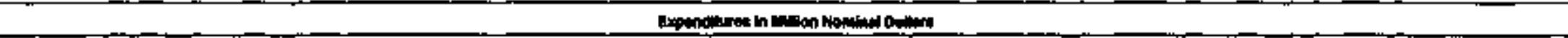 } \\
\hline 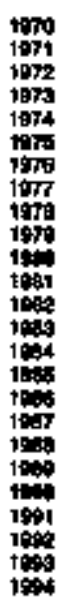 & $\begin{array}{l}= \\
z \\
z \\
= \\
= \\
= \\
= \\
= \\
= \\
= \\
= \\
= \\
= \\
= \\
=\end{array}$ & 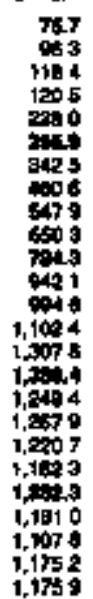 & 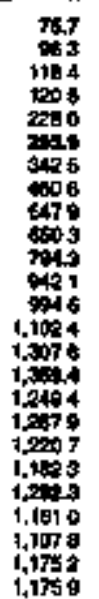 & 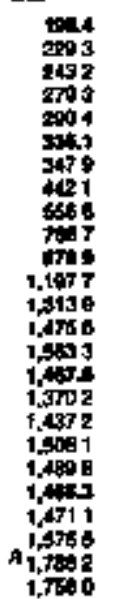 & 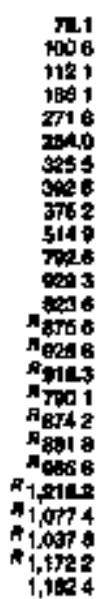 & 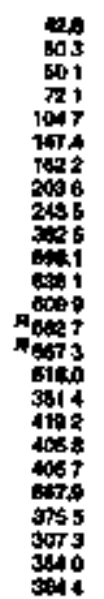 & 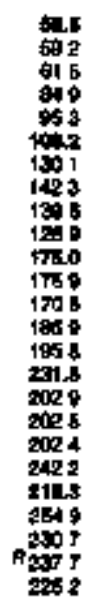 & 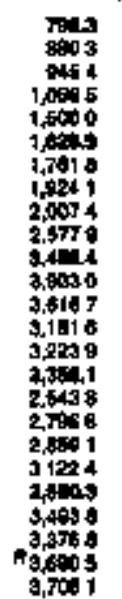 & 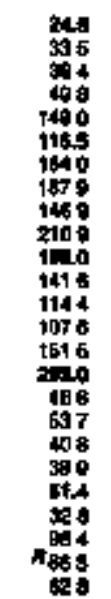 & 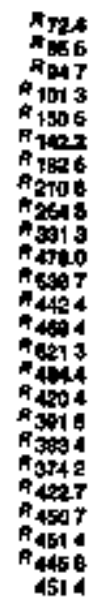 & 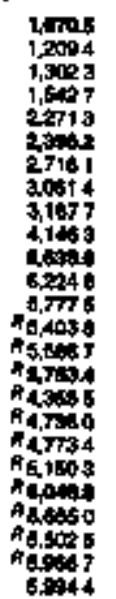 & 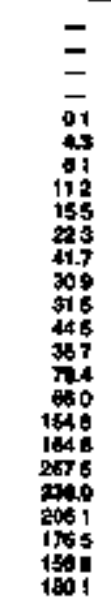 & $\begin{array}{l}= \\
= \\
= \\
= \\
= \\
= \\
= \\
= \\
= \\
= \\
= \\
= \\
\overrightarrow{019} \\
073 \\
7020 \\
750\end{array}$ & 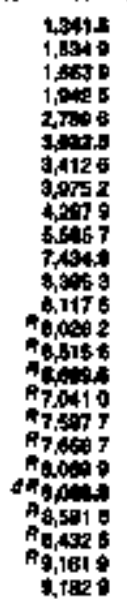 & 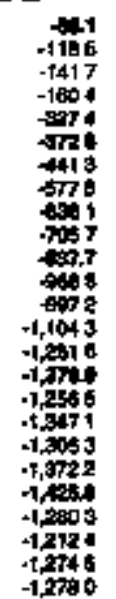 & 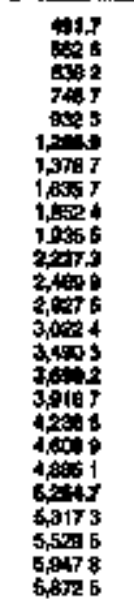 & 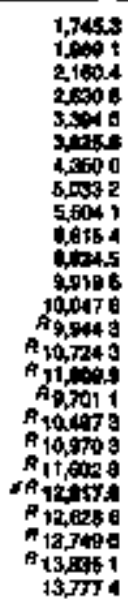 \\
\hline
\end{tabular}

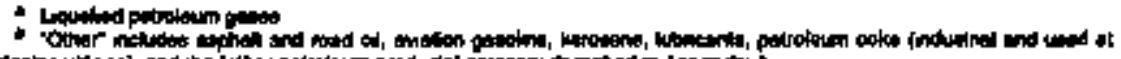

Aarimatem

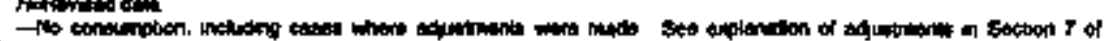

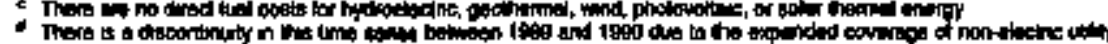
Aparenowich

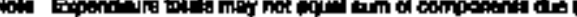

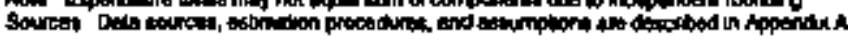
wa or enchers beponing on 1000 


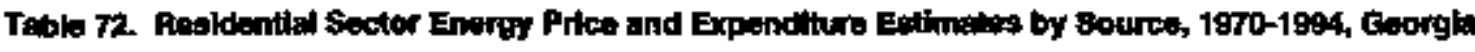

\begin{tabular}{|c|c|c|c|c|c|c|c|c|c|c|}
\hline \multirow[b]{4}{*}{$r=$} & \multicolumn{8}{|c|}{ Mmey Ensy } & \multirow[b]{3}{*}{ Dentrits } & \multirow[b]{3}{*}{ " } \\
\hline & \multirow[b]{2}{*}{ Cond } & \multirow[b]{2}{*}{ Menure } & \multicolumn{4}{|c|}{ Antrobnem } & \multirow[b]{2}{*}{ " } & \multirow[b]{2}{*}{ Teter } & & \\
\hline & & & 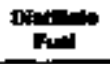 & Kand & $\mathbf{L P a}$ & البه & & & & \\
\hline & \multicolumn{10}{|c|}{ Plos } \\
\hline \multirow[t]{2}{*}{ 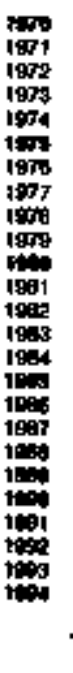 } & 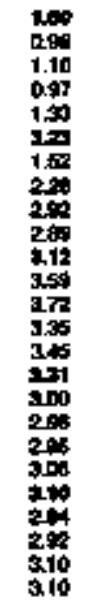 & 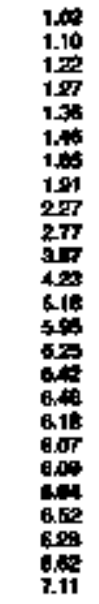 & 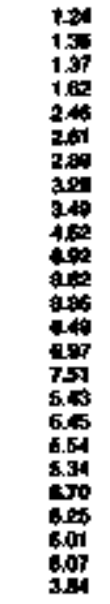 & 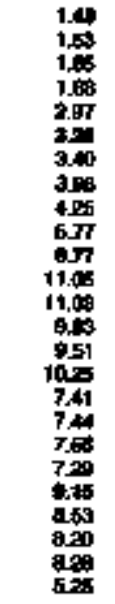 & 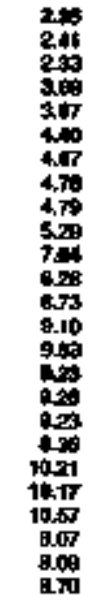 & 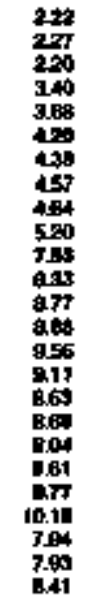 & $\begin{array}{l}= \\
= \\
= \\
= \\
= \\
= \\
= \\
= \\
= \\
= \\
= \\
= \\
\text { a } \\
\operatorname{s.53} \\
3.53 \\
3.53 \\
3.53\end{array}$ & 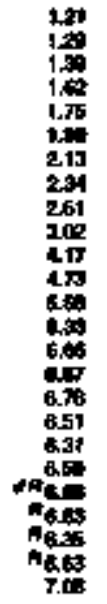 & 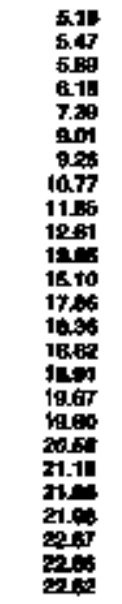 & 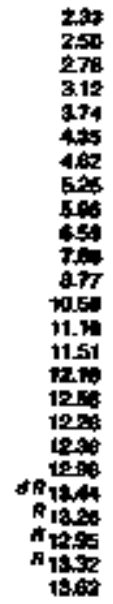 \\
\hline & \multicolumn{10}{|c|}{ Ela } \\
\hline 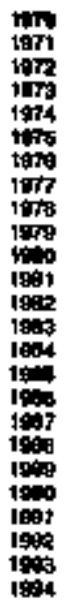 & 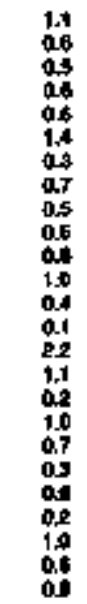 & 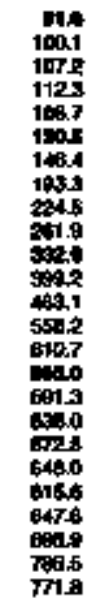 & 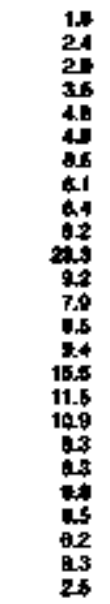 & 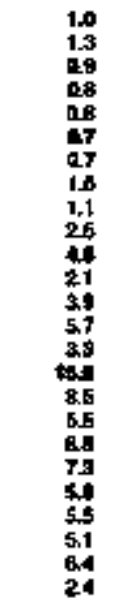 & 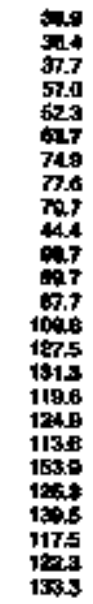 & 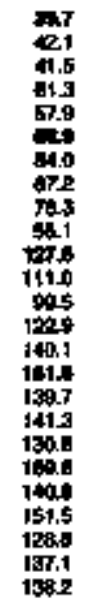 & 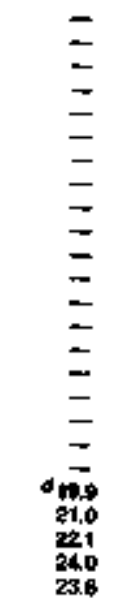 & 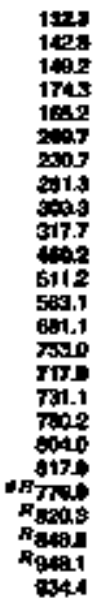 & 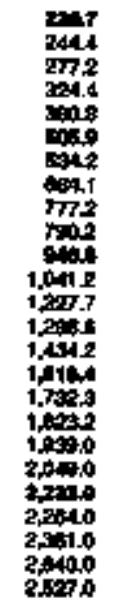 & 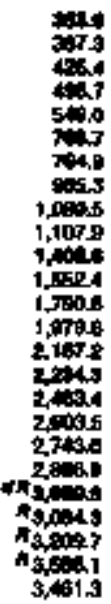 \\
\hline
\end{tabular}

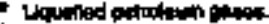

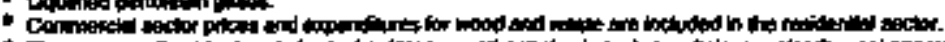

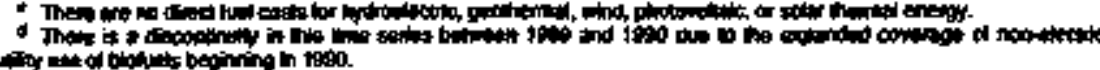

AFinceoddes

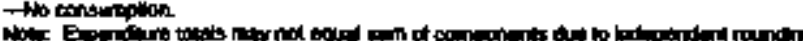

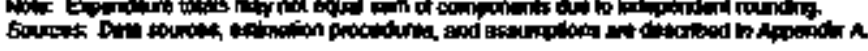




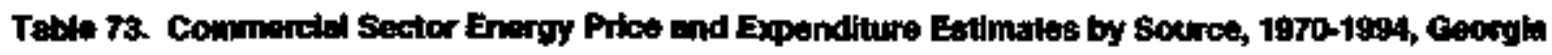

\begin{tabular}{|c|c|c|c|c|c|c|c|c|c|c|c|}
\hline \multirow[b]{4}{*}{ Tex } & \multicolumn{9}{|c|}{ Primang Ening } & \multirow[b]{3}{*}{ enoticlys: } & \multirow[b]{3}{*}{ 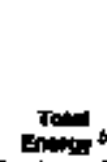 } \\
\hline & \multirow[b]{2}{*}{ (الهيn } & \multirow[b]{2}{*}{ 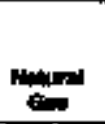 } & \multicolumn{6}{|c|}{ 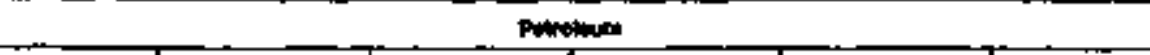 } & \multirow[b]{2}{*}{ Totid } & & \\
\hline & & & onimin & Kepospons & La: & constim & Fition & \multirow[t]{2}{*}{ Vots } & & & \\
\hline & \multicolumn{10}{|c|}{ 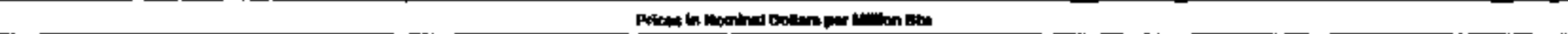 } & \\
\hline \multirow[t]{2}{*}{ 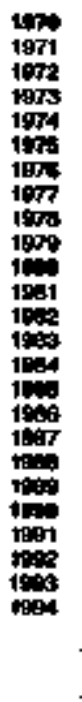 } & 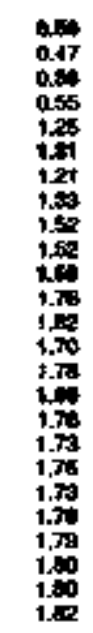 & 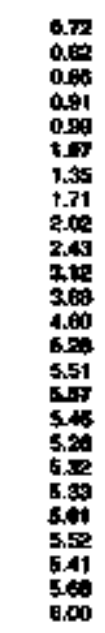 & 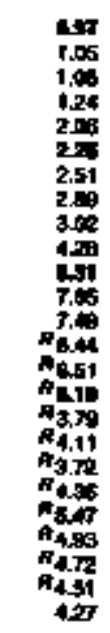 & 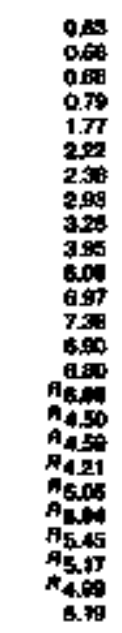 & 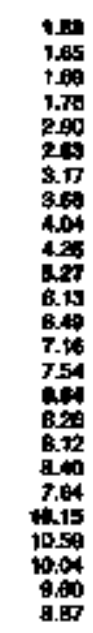 & 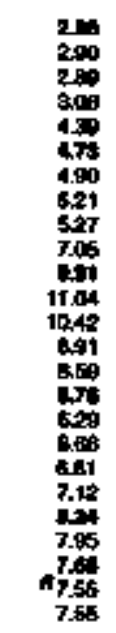 & 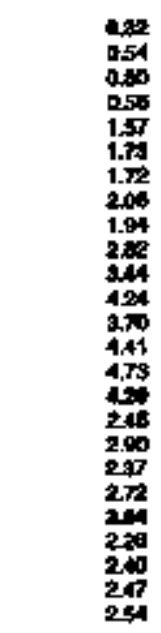 & 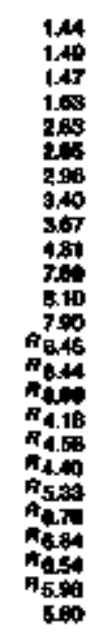 & 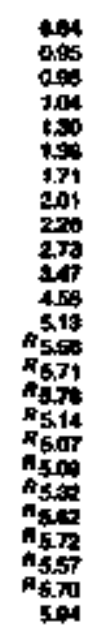 & 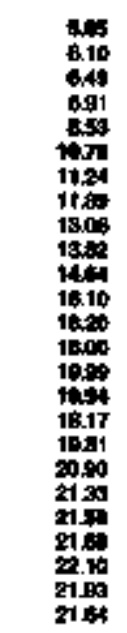 & 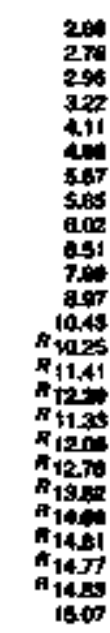 \\
\hline & \multicolumn{11}{|c|}{ 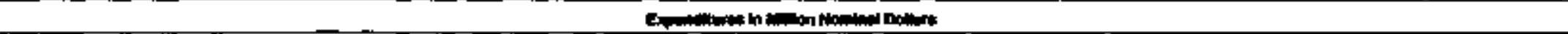 } \\
\hline 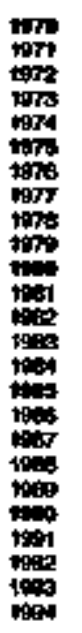 & 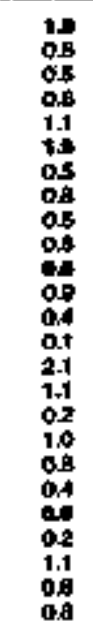 & 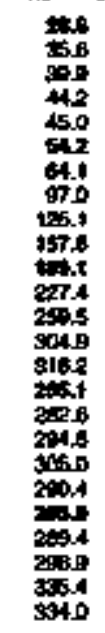 & 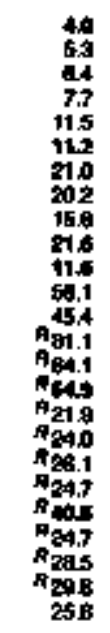 & 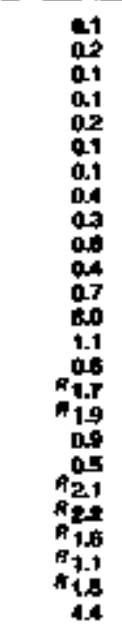 & 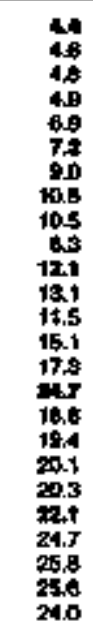 & 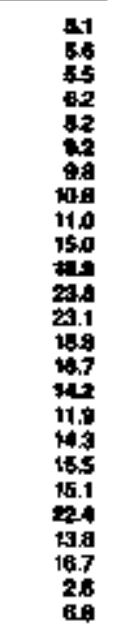 & 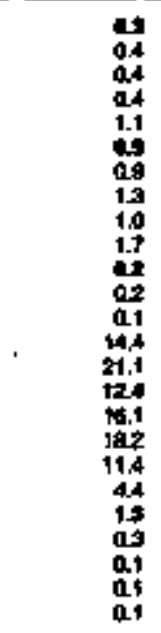 & 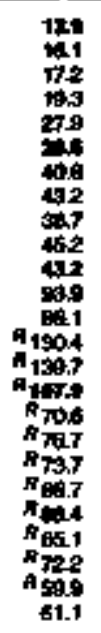 & 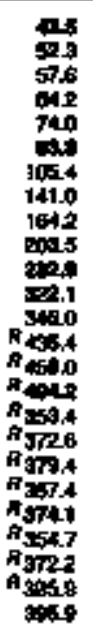 & 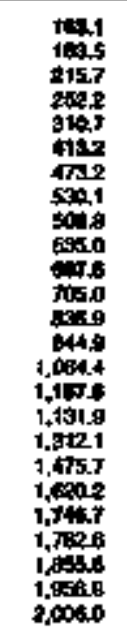 & 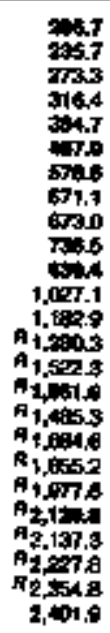 \\
\hline
\end{tabular}

Inined 
Table 74. Inchesirien Sactor Energy Price and Expenditure Eallmatas by Source, 1970-19a4, Goorgia

\begin{tabular}{|c|c|c|c|c|c|c|c|c|c|c|c|c|c|c|c|c|c|}
\hline & \multicolumn{15}{|c|}{ PMary enaty } & \multirow[b]{3}{*}{ Encoluterly } & \multirow[b]{3}{*}{ S } \\
\hline & \multicolumn{3}{|c|}{ cood } & \multirow[b]{2}{*}{ Malmed } & \multicolumn{9}{|c|}{ 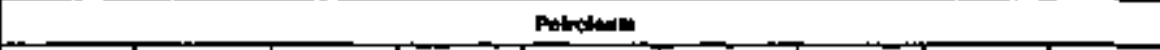 } & \multirow[b]{2}{*}{ Elonol: } & \multirow[b]{2}{*}{ Tolar ' } & & \\
\hline & $\begin{array}{l}\text { Colving } \\
\text { cosid }\end{array}$ & som & Tata & & moned and & Pentw & koromenes & und & ubriesntas & Molor & Mandid & Ortan ${ }^{6}$ & Tores & & & & \\
\hline Y* & \multicolumn{17}{|c|}{ 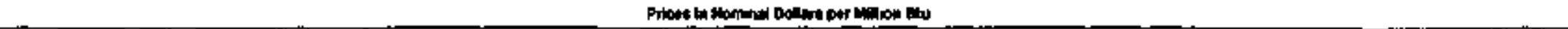 } \\
\hline \multirow[t]{2}{*}{ 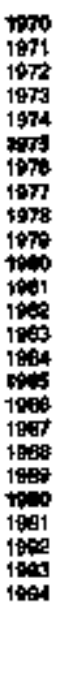 } & $\begin{array}{l}= \\
= \\
= \\
= \\
= \\
= \\
= \\
= \\
= \\
= \\
z \\
= \\
z \\
=\end{array}$ & 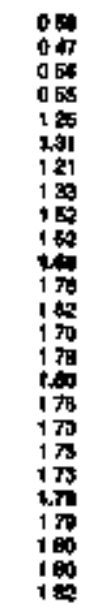 & 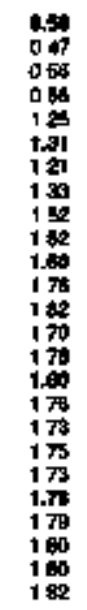 & 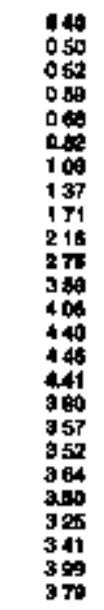 & 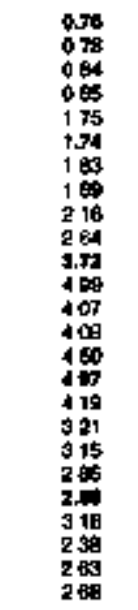 & 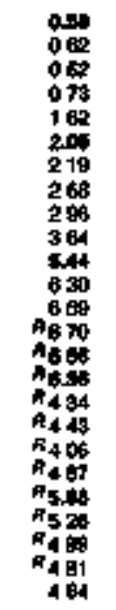 & 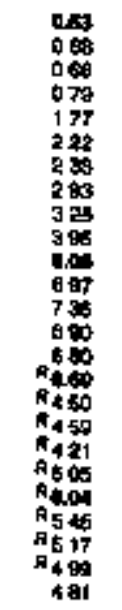 & 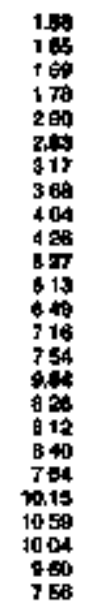 & 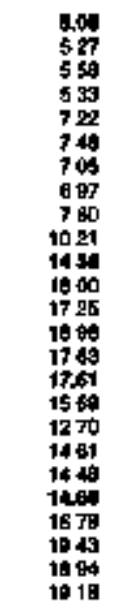 & 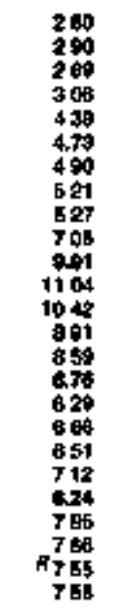 & $\begin{array}{l}640 \\
051 \\
045 \\
056 \\
176 \\
209 \\
174 \\
207 \\
191 \\
243 \\
244 \\
170 \\
421 \\
110 \\
173 \\
120 \\
246 \\
280 \\
237 \\
272 \\
304 \\
226 \\
249 \\
247 \\
264\end{array}$ & 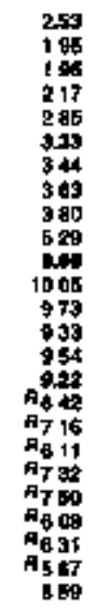 & 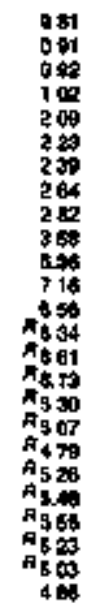 & 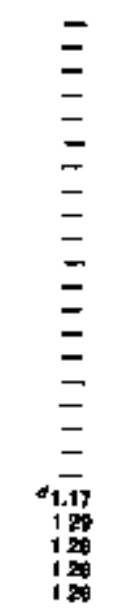 & 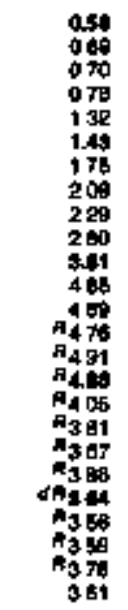 & 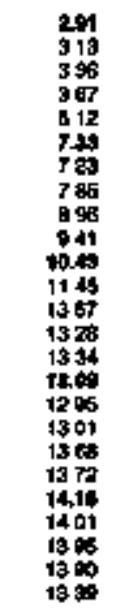 & 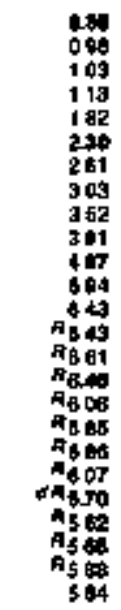 \\
\hline & \multicolumn{17}{|c|}{ 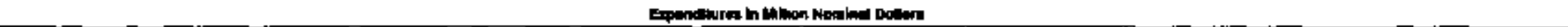 } \\
\hline 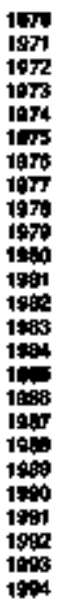 & $\begin{array}{l}= \\
= \\
= \\
= \\
= \\
= \\
= \\
= \\
= \\
= \\
= \\
= \\
= \\
=\end{array}$ & 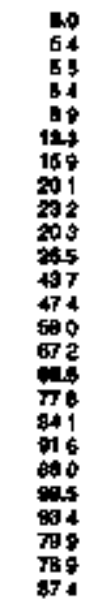 & 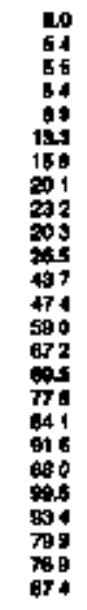 & 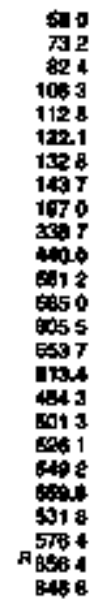 & 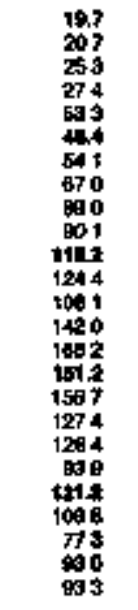 & 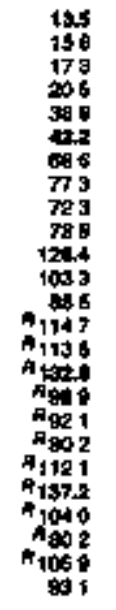 & 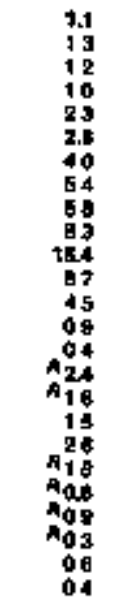 & 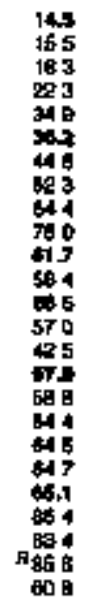 & 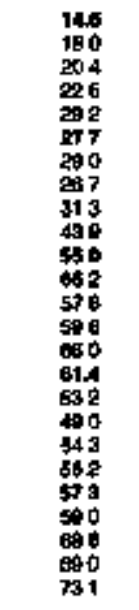 & 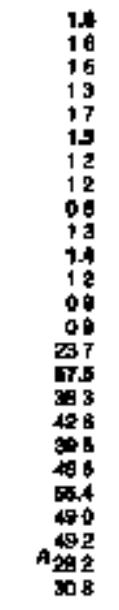 & 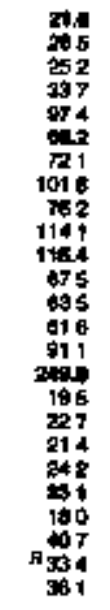 & 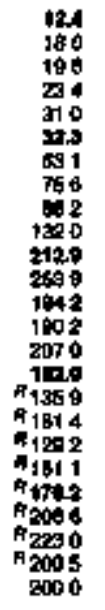 & 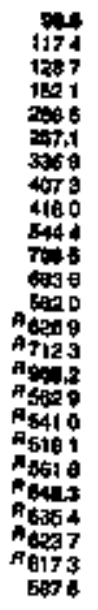 & $\begin{array}{l}= \\
= \\
= \\
= \\
= \\
= \\
= \\
= \\
= \\
= \\
= \\
=41.4 \\
493 \\
000 \\
510\end{array}$ & 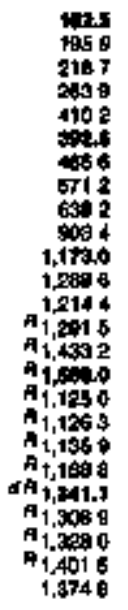 & 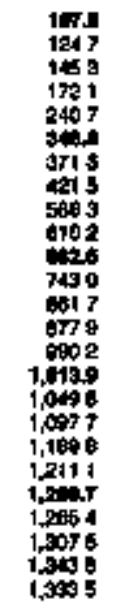 & 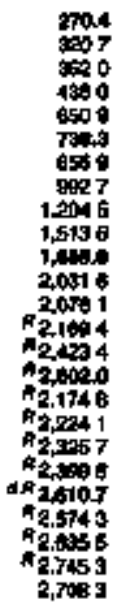 \\
\hline
\end{tabular}

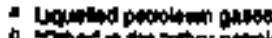

Anparaged div

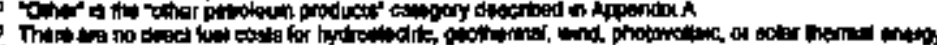

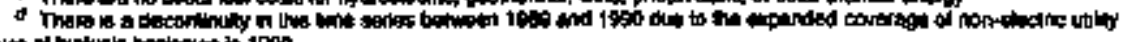
Appention 

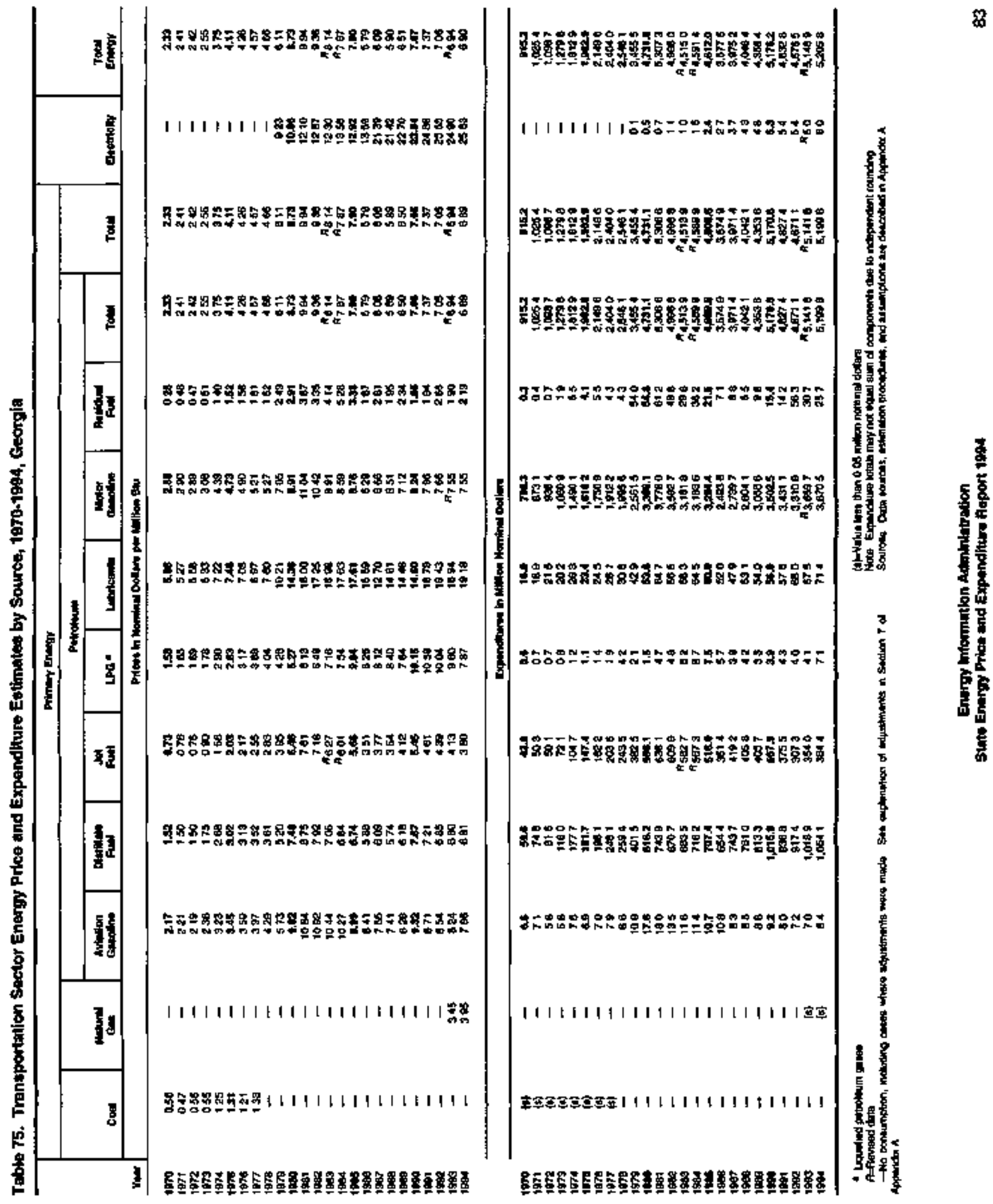


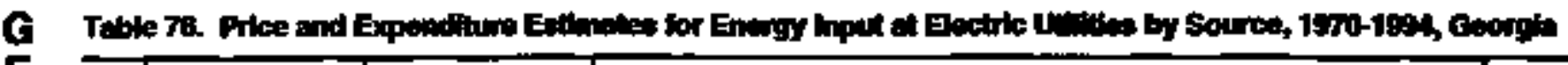

\begin{tabular}{|c|c|c|c|c|c|c|c|c|c|}
\hline \multirow[b]{3}{*}{$\mathbf{x}=$} & \multirow[b]{2}{*}{ coll } & \multirow[b]{2}{*}{ 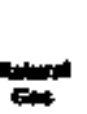 } & \multicolumn{4}{|c|}{ 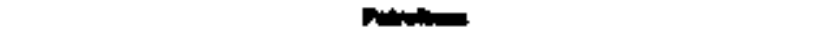 } & \multirow[b]{2}{*}{ Dand } & \multirow[b]{2}{*}{ 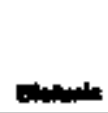 } & \multirow[b]{2}{*}{ 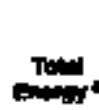 } \\
\hline & & & of & (1) & سم & 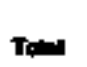 & & & \\
\hline & \multicolumn{9}{|c|}{ 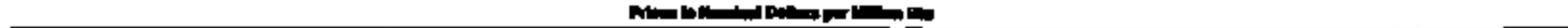 } \\
\hline \multirow[t]{2}{*}{ 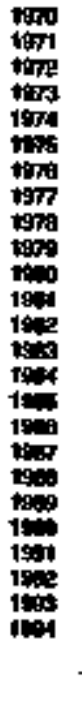 } & 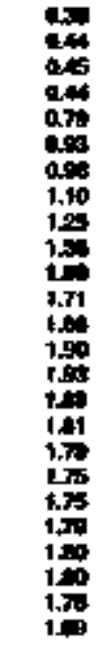 & 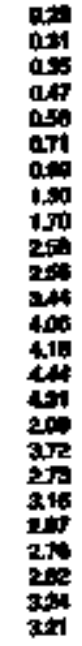 & 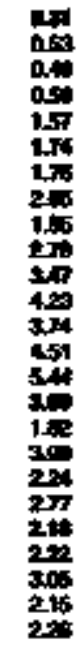 & 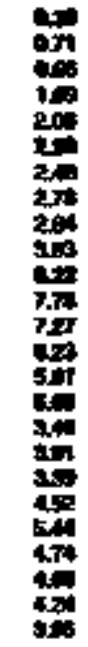 & $\begin{array}{l}= \\
= \\
= \\
= \\
= \\
= \\
= \\
= \\
= \\
= \\
= \\
= \\
=\end{array}$ & 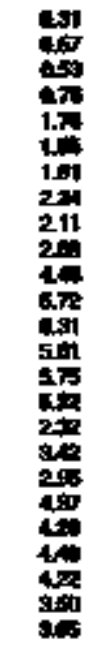 & 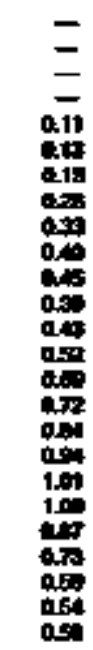 & $\begin{array}{l}= \\
z \\
z \\
z \\
= \\
= \\
= \\
= \\
= \\
= \\
= \\
= \\
= \\
=\end{array}$ & 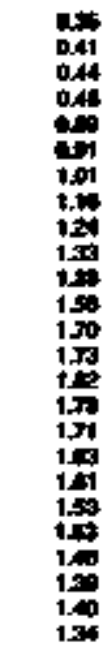 \\
\hline & \multicolumn{9}{|c|}{ 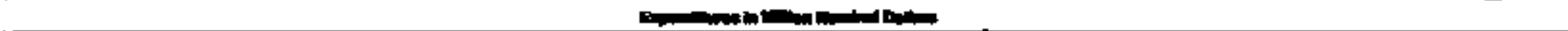 } \\
\hline 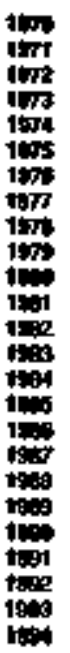 & 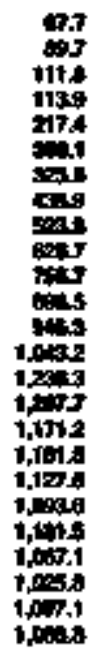 & 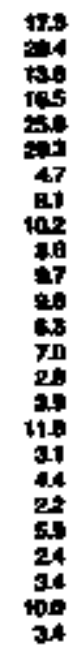 & 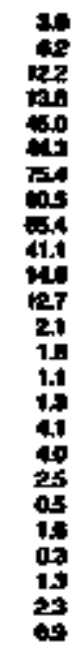 & 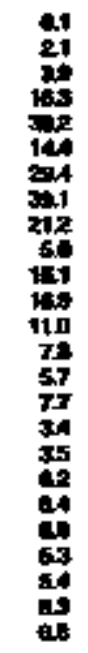 & $\begin{array}{l}= \\
= \\
= \\
= \\
= \\
= \\
= \\
= \\
= \\
= \\
= \\
= \\
= \\
=\end{array}$ & 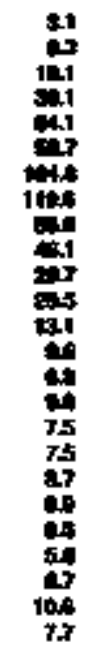 & 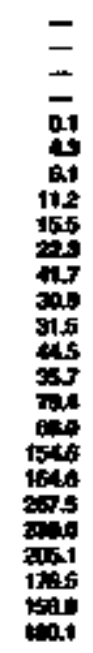 & $\begin{array}{l}= \\
= \\
= \\
= \\
= \\
= \\
= \\
= \\
= \\
= \\
= \\
= \\
= \\
=\end{array}$ & 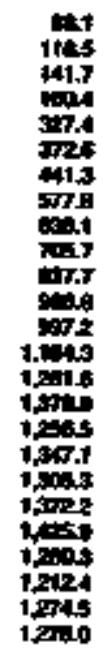 \\
\hline
\end{tabular}

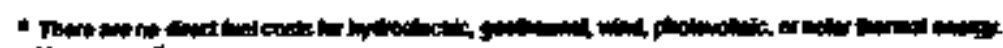

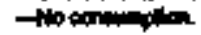

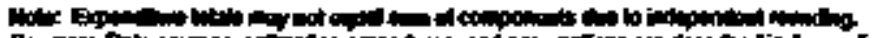

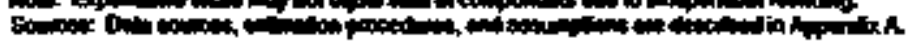




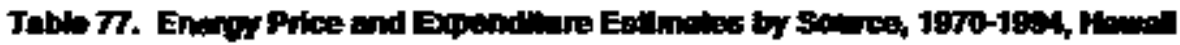

\begin{tabular}{|c|c|c|c|c|c|c|c|c|c|c|c|c|c|c|c|c|c|}
\hline \multirow[b]{4}{*}{$r=$} & \multicolumn{14}{|c|}{ nementer } & \multirow{3}{*}{ : } & \multirow{3}{*}{ 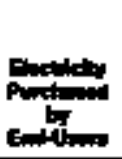 } & \multirow[b]{3}{*}{ 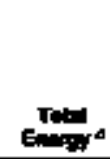 } \\
\hline & \multicolumn{3}{|c|}{$\infty$} & & \multicolumn{7}{|c|}{ 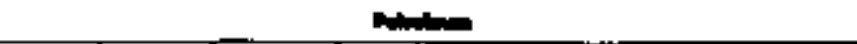 } & \multirow[b]{2}{*}{ "ה } & \multirow[b]{2}{*}{ 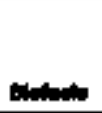 } & \multirow[b]{2}{*}{$T=0$} & & & \\
\hline & 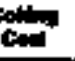 & היח & Tim & and & Font & it & use & & 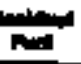 & & Tent & & & & & & \\
\hline & \multicolumn{17}{|c|}{ 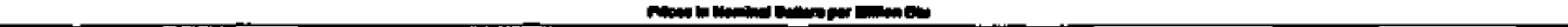 } \\
\hline 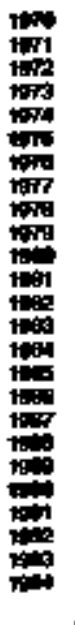 & $\begin{array}{l}= \\
= \\
= \\
= \\
= \\
= \\
= \\
= \\
= \\
= \\
= \\
= \\
= \\
= \\
=\end{array}$ & 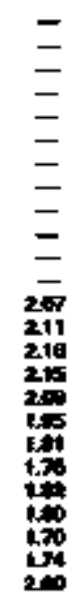 & 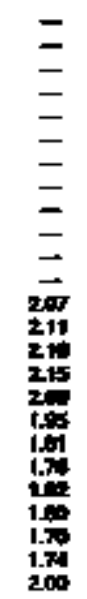 & 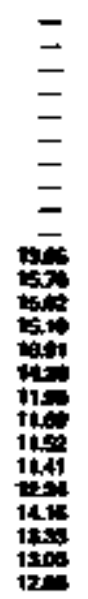 & 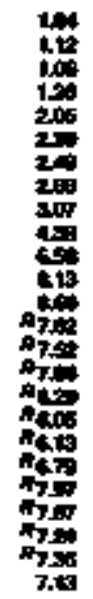 & 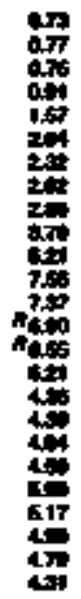 & 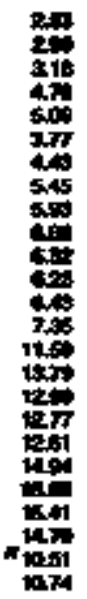 & 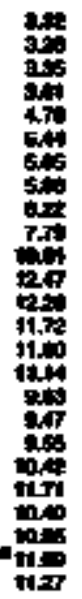 & 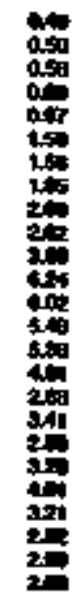 & 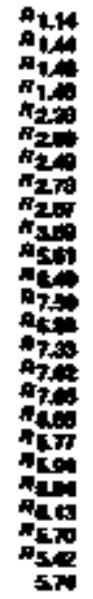 & 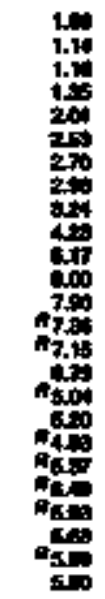 & $\begin{array}{l}= \\
= \\
= \\
= \\
= \\
= \\
= \\
= \\
= \\
= \\
= \\
= \\
= \\
= \\
=\end{array}$ & 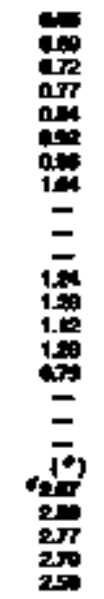 & 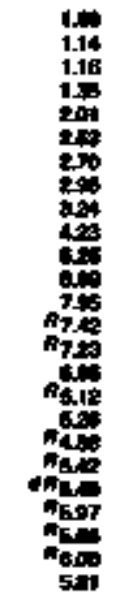 & 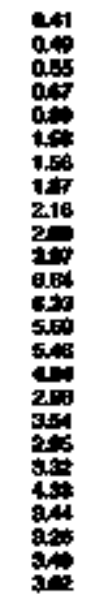 & 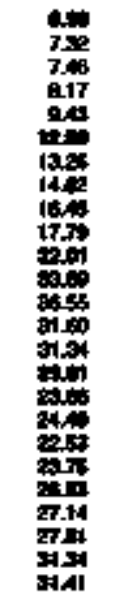 & 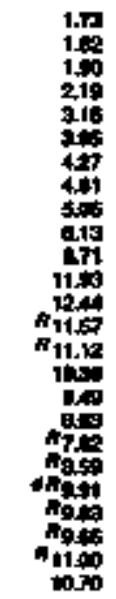 \\
\hline & \multicolumn{17}{|c|}{ rom } \\
\hline 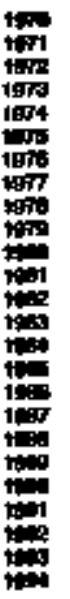 & $\begin{array}{l}= \\
= \\
= \\
= \\
= \\
= \\
= \\
= \\
= \\
= \\
= \\
= \\
= \\
= \\
=\end{array}$ & $\begin{array}{l}= \\
= \\
= \\
= \\
= \\
= \\
= \\
24 \\
22 \\
20 \\
21 \\
81 \\
31 \\
12 \\
14 \\
1.7 \\
20 \\
31 \\
37\end{array}$ & $\begin{array}{l}= \\
= \\
= \\
= \\
= \\
= \\
= \\
= \\
24 \\
22 \\
24 \\
19 \\
11 \\
21 \\
14 \\
17 \\
17 \\
29 \\
21 \\
31\end{array}$ & 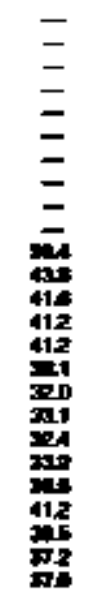 & 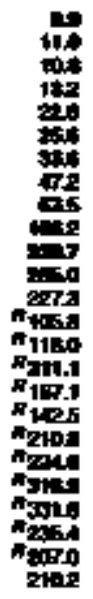 & 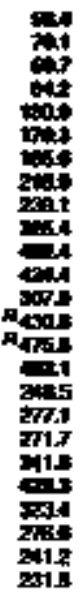 & 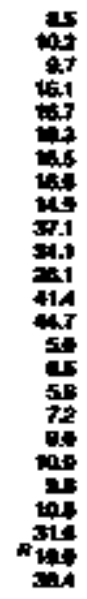 & 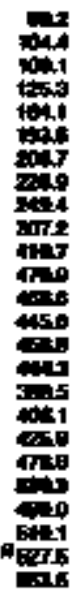 & 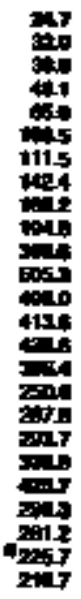 & 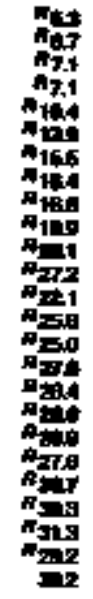 & 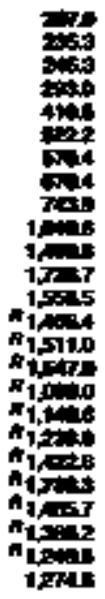 & $\begin{array}{l}= \\
= \\
= \\
= \\
= \\
= \\
= \\
= \\
= \\
= \\
= \\
=\end{array}$ & 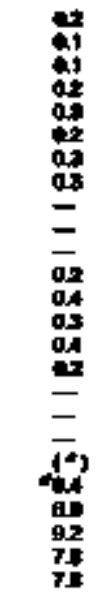 & 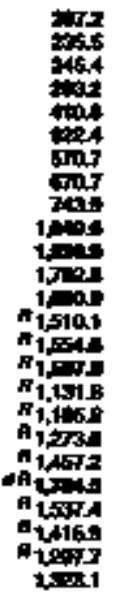 & 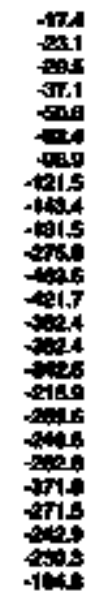 & 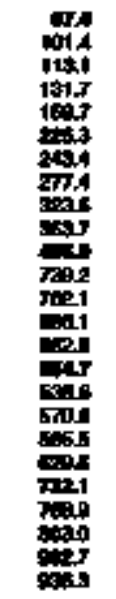 & 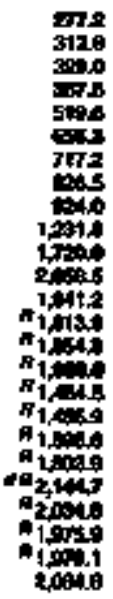 \\
\hline
\end{tabular}

-

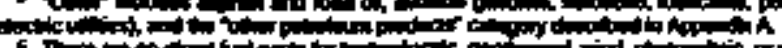

(1)

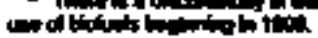

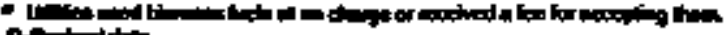

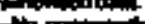


Tabile 78, Reeldential Sector Energy Price and Expenditure Eetiminges by Soures, 1970-1994, Hawal

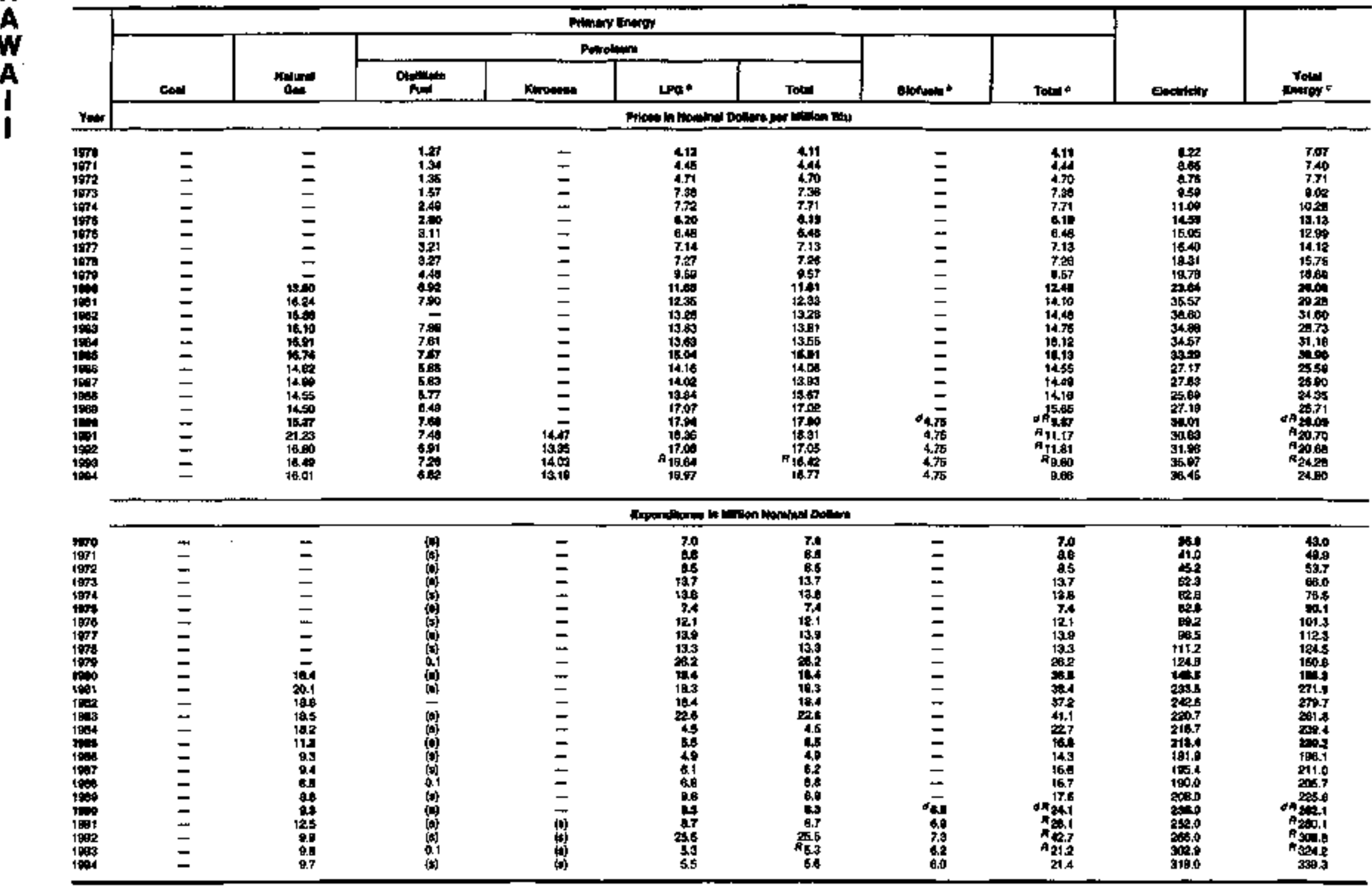

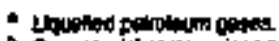

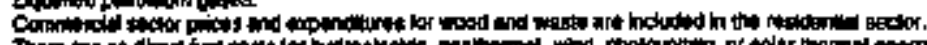

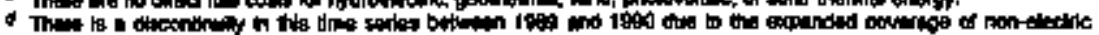

Paprinad dian.

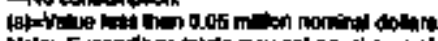

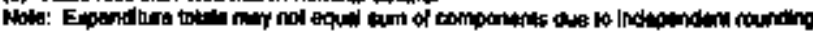

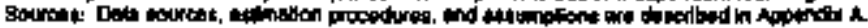




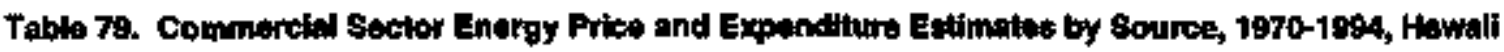

\begin{tabular}{|c|c|c|c|c|c|c|c|c|c|c|c|}
\hline \multirow[b]{4}{*}{ Yer } & \multicolumn{9}{|c|}{ Prinery linertr } & \multirow[b]{3}{*}{ Encinlety } & \multirow[b]{3}{*}{ Fontill } \\
\hline & \multirow[b]{2}{*}{ Co:t } & \multirow[b]{2}{*}{ Non } & \multicolumn{6}{|c|}{ Atroleum } & \multirow[b]{2}{*}{ Tot: } & & \\
\hline & & & Fintion & remonese & Let: & Alastor & int & $T a t$ & & & \\
\hline & \multicolumn{11}{|c|}{ 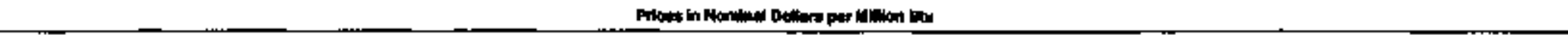 } \\
\hline \multirow[t]{2}{*}{ 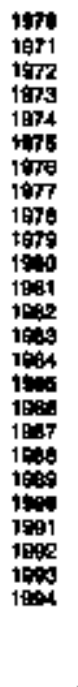 } & $\begin{array}{l}= \\
= \\
= \\
= \\
= \\
= \\
= \\
= \\
= \\
= \\
= \\
= \\
= \\
=\end{array}$ & 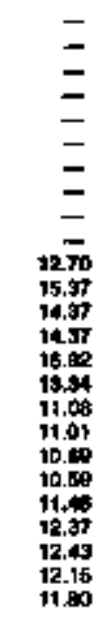 & 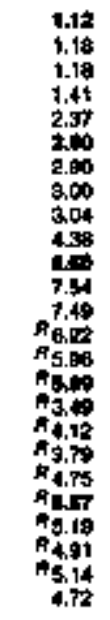 & 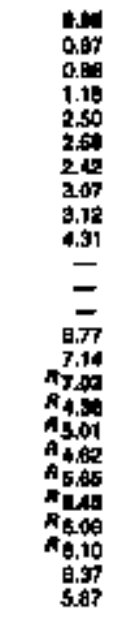 & 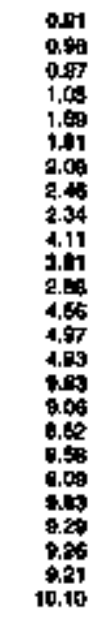 & 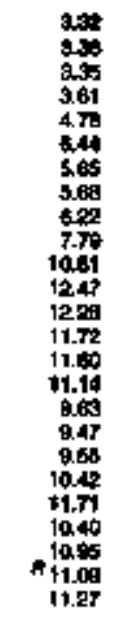 & 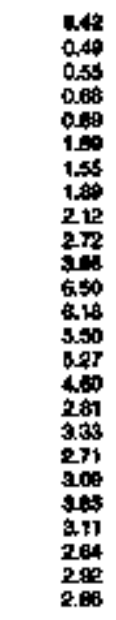 & 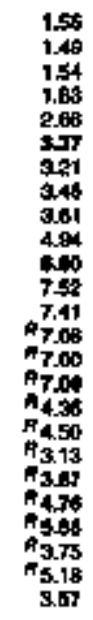 & 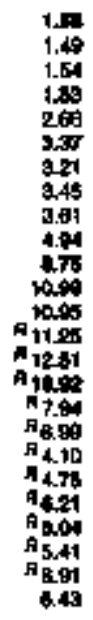 & 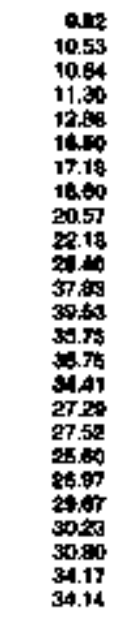 & 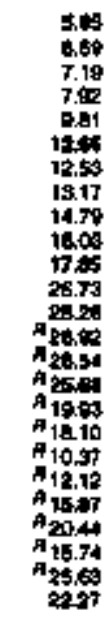 \\
\hline & \multicolumn{11}{|c|}{ 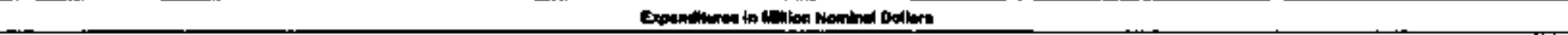 } \\
\hline 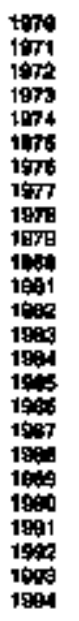 & $\begin{array}{l}= \\
= \\
= \\
= \\
= \\
= \\
= \\
= \\
= \\
= \\
= \\
= \\
= \\
= \\
=\end{array}$ & $\begin{array}{l}= \\
= \\
= \\
= \\
= \\
= \\
= \\
218 \\
227 \\
226 \\
226 \\
210 \\
218 \\
227 \\
297 \\
236 \\
244 \\
272 \\
297 \\
206 \\
27.4 \\
273\end{array}$ & 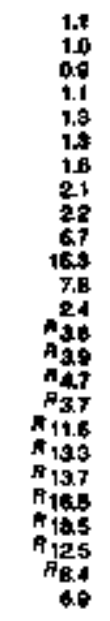 & 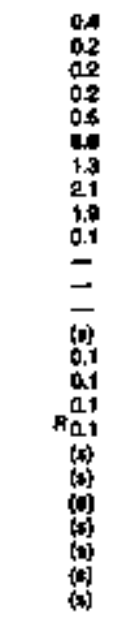 & $\begin{array}{l}0.3 \\
0.3 \\
0.9 \\
0.9 \\
0.6 \\
0.4 \\
0.7 \\
0.8 \\
0.0 \\
2.0 \\
.1 \\
0.9 \\
1.1 \\
1.4 \\
0.3 \\
0.0 \\
0.8 \\
0.7 \\
0.7 \\
0.7 \\
0.7 \\
0.0 \\
2.0 \\
0.5 \\
0.0\end{array}$ & $\begin{array}{l}2.3 \\
1.4 \\
1.5 \\
1.8 \\
1.8 \\
2.8 \\
2.8 \\
2.0 \\
3.3 \\
4.1 \\
4.1 \\
3.8 \\
3.8 \\
3.0 \\
2.6 \\
2.8 \\
2.3 \\
2.2 \\
2.7 \\
2.8 \\
3.4 \\
2.7 \\
2.8 \\
0.6 \\
0.8\end{array}$ & 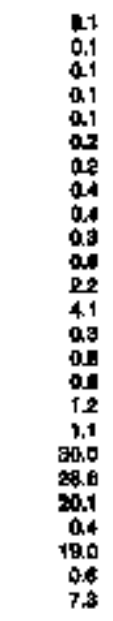 & 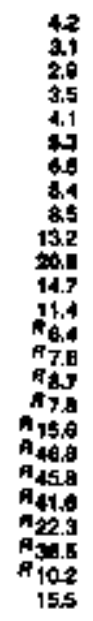 & 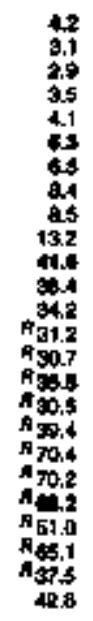 & 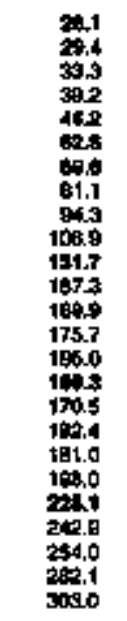 & 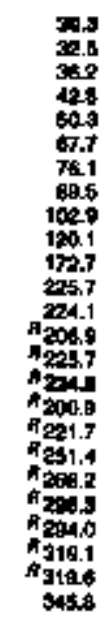 \\
\hline
\end{tabular}

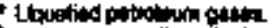

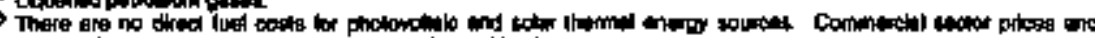

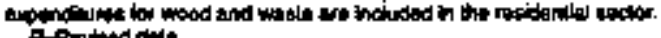

Hoconompon.

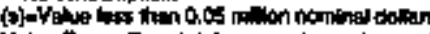

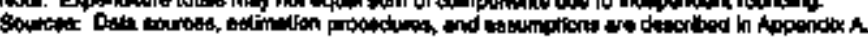




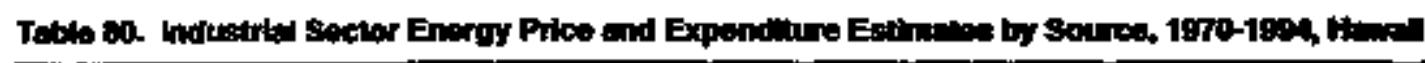

\begin{tabular}{|c|c|c|c|c|c|c|c|c|c|c|c|c|c|c|c|c|c|}
\hline & \multicolumn{15}{|c|}{ N } & \multirow{2}{*}{ nty } & \multirow{2}{*}{ 둘 } \\
\hline & \multicolumn{3}{|c|}{ and } & & \multicolumn{9}{|c|}{ Pans } & 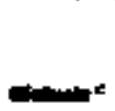 & The & & \\
\hline reor & \multicolumn{17}{|c|}{ 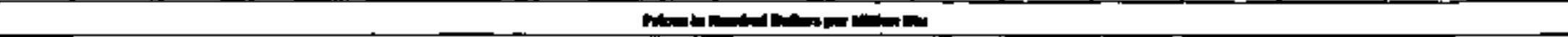 } \\
\hline 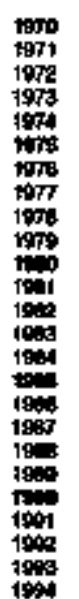 & $\begin{array}{l}= \\
= \\
= \\
= \\
= \\
= \\
= \\
= \\
= \\
= \\
= \\
= \\
=\end{array}$ & 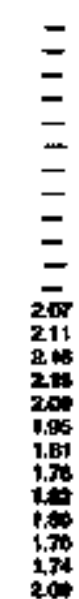 & 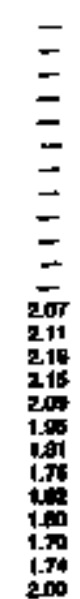 & $\begin{array}{l}= \\
= \\
= \\
= \\
= \\
= \\
= \\
= \\
= \\
= \\
= \\
=\end{array}$ & 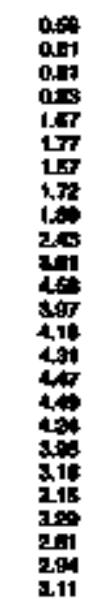 & 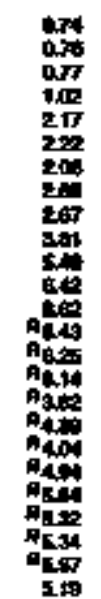 & 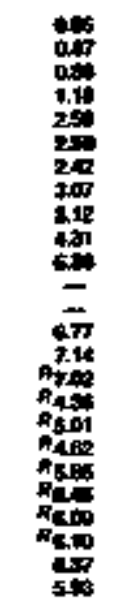 & 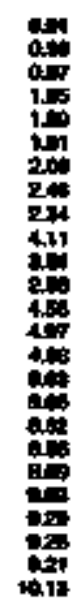 & 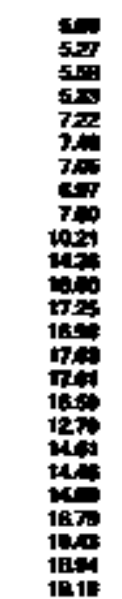 & 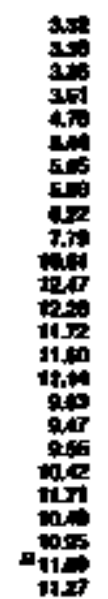 & 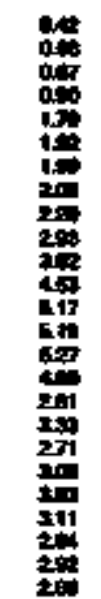 & 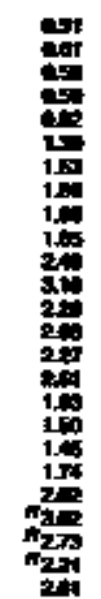 & 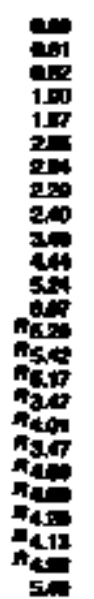 & 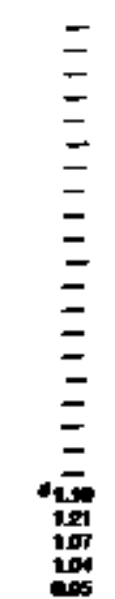 & 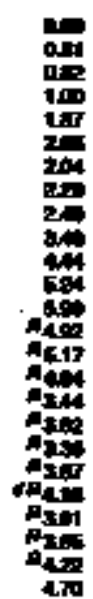 & 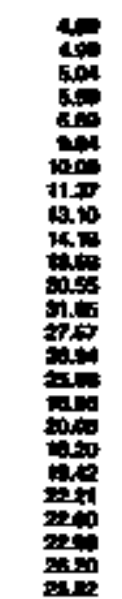 & 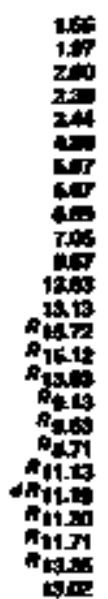 \\
\hline
\end{tabular}

\begin{tabular}{|c|c|c|c|c|c|c|c|c|c|c|c|c|c|c|c|c|c|}
\hline 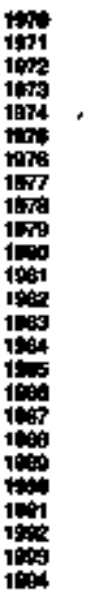 & $\begin{array}{l}= \\
z \\
z \\
= \\
z \\
z \\
= \\
= \\
= \\
= \\
= \\
= \\
=\end{array}$ & 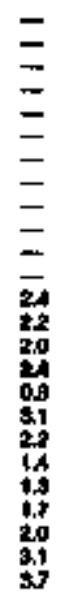 & 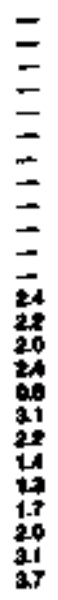 & $\begin{array}{l}z \\
z \\
z \\
z \\
z \\
z \\
z \\
= \\
= \\
= \\
= \\
= \\
=\end{array}$ & 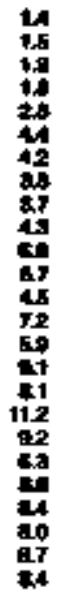 & 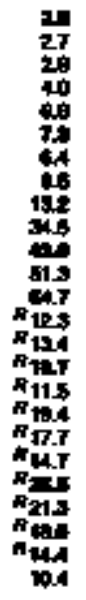 & 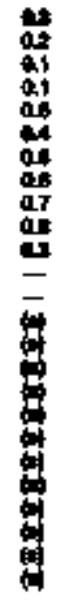 & 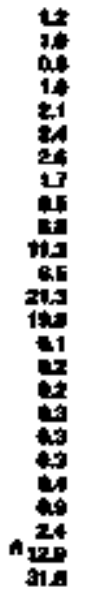 & 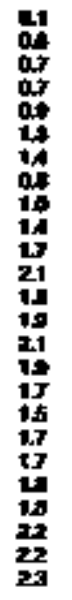 & 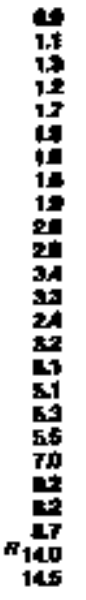 & 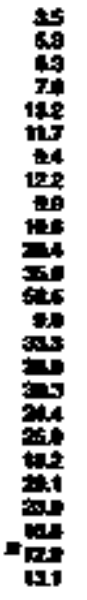 & 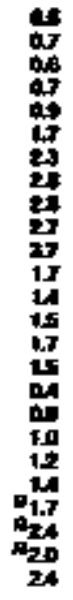 & 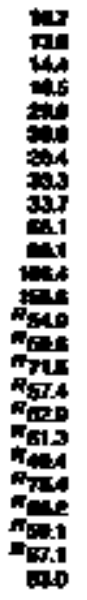 & 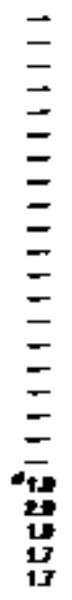 & 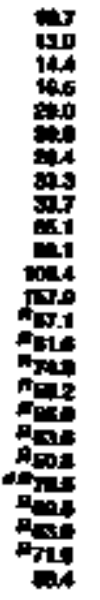 & 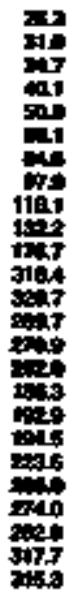 & 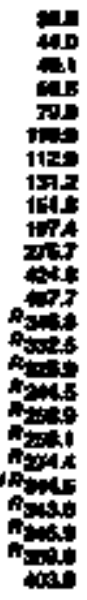 \\
\hline
\end{tabular}

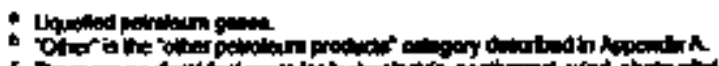

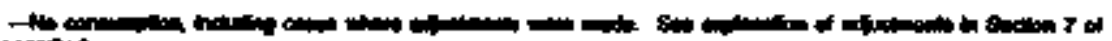

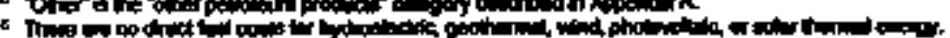

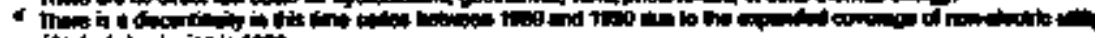

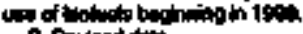

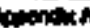

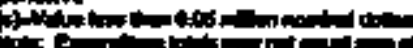

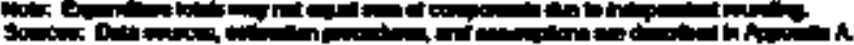




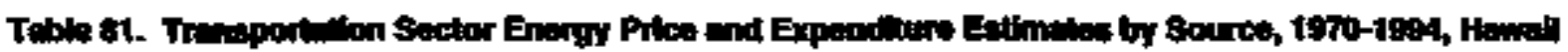

\begin{tabular}{|c|c|c|c|c|c|c|c|c|c|c|c|c|c|}
\hline & \multicolumn{11}{|c|}{ Anmenter } & \multirow[b]{3}{*}{ Enotichy } & \multirow[b]{3}{*}{ 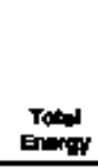 } \\
\hline & \multicolumn{10}{|c|}{ 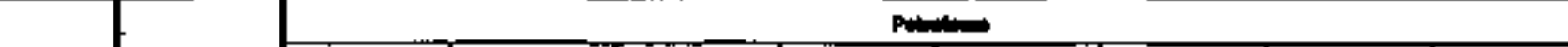 } & \multirow[b]{2}{*}{ 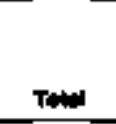 } & & \\
\hline & Den & 달 & 술 & for & in & $190=$ & 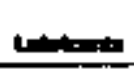 & ה & Rath & Bom & & & \\
\hline m & \multicolumn{13}{|c|}{ 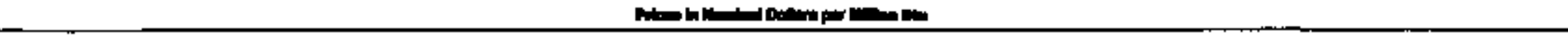 } \\
\hline 19 & $\begin{array}{l}= \\
= \\
= \\
= \\
= \\
= \\
= \\
= \\
= \\
= \\
= \\
= \\
= \\
=\end{array}$ & $\begin{array}{l}= \\
= \\
= \\
= \\
= \\
= \\
= \\
= \\
= \\
= \\
= \\
= \\
= \\
= \\
=\end{array}$ & 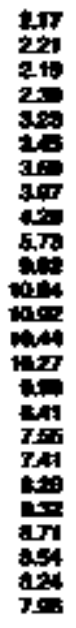 & 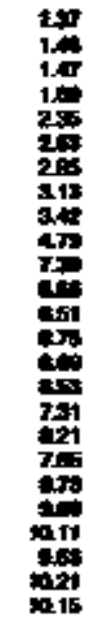 & 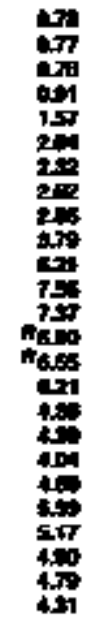 & 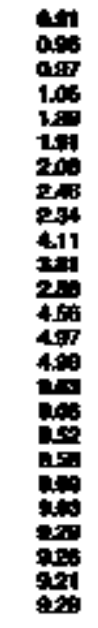 & 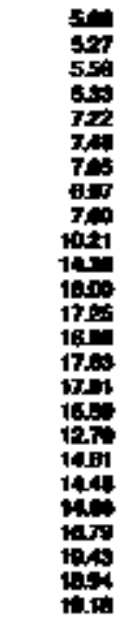 & 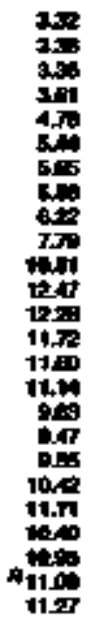 & 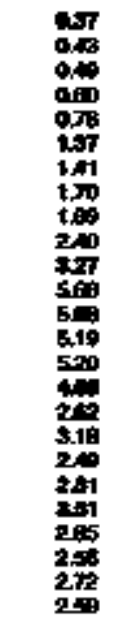 & 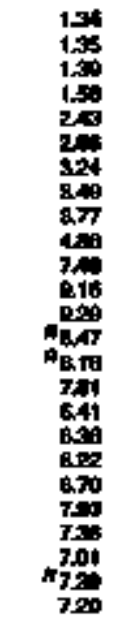 & 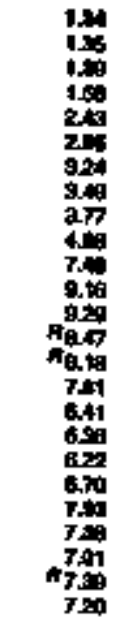 & $\begin{array}{l}= \\
= \\
= \\
= \\
= \\
= \\
= \\
= \\
= \\
= \\
= \\
= \\
= \\
=\end{array}$ & 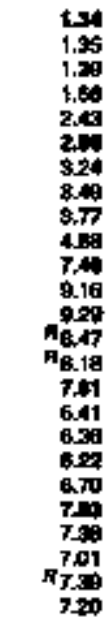 \\
\hline & \multicolumn{13}{|c|}{ 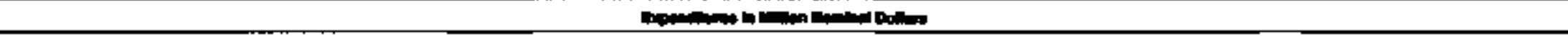 } \\
\hline 政 & $\begin{array}{l}= \\
= \\
= \\
= \\
= \\
= \\
= \\
= \\
= \\
= \\
= \\
= \\
= \\
=\end{array}$ & $\begin{array}{l}= \\
= \\
= \\
= \\
= \\
= \\
= \\
= \\
= \\
= \\
= \\
= \\
= \\
= \\
=\end{array}$ & 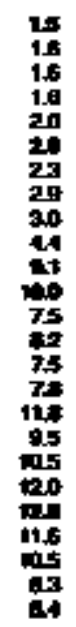 & 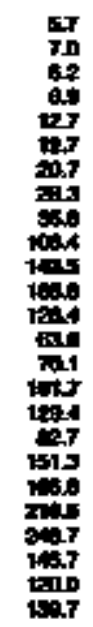 & 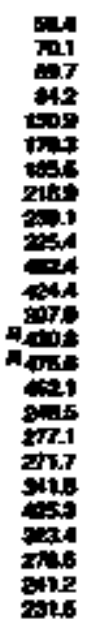 & 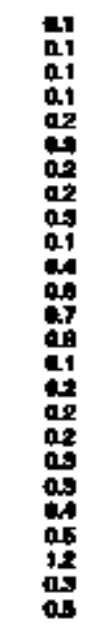 & 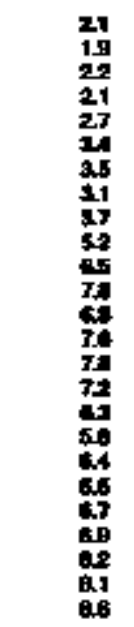 & 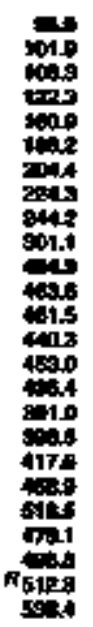 & 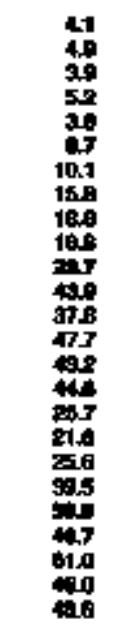 & 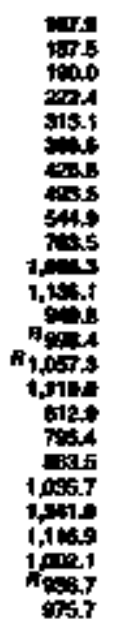 & 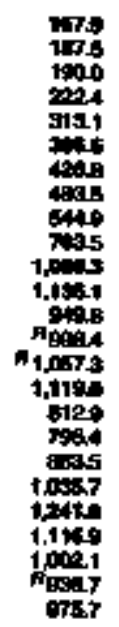 & $\begin{array}{l}= \\
= \\
= \\
= \\
= \\
= \\
= \\
= \\
= \\
= \\
= \\
= \\
= \\
=\end{array}$ & 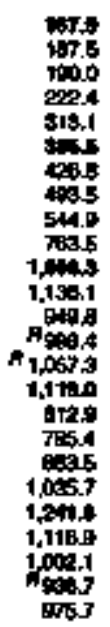 \\
\hline
\end{tabular}


H Tabłe 82. Price and Expenditure Estimates for Energy input at Electrlc Utiditod by Sourca, 1970-1994, Hawell

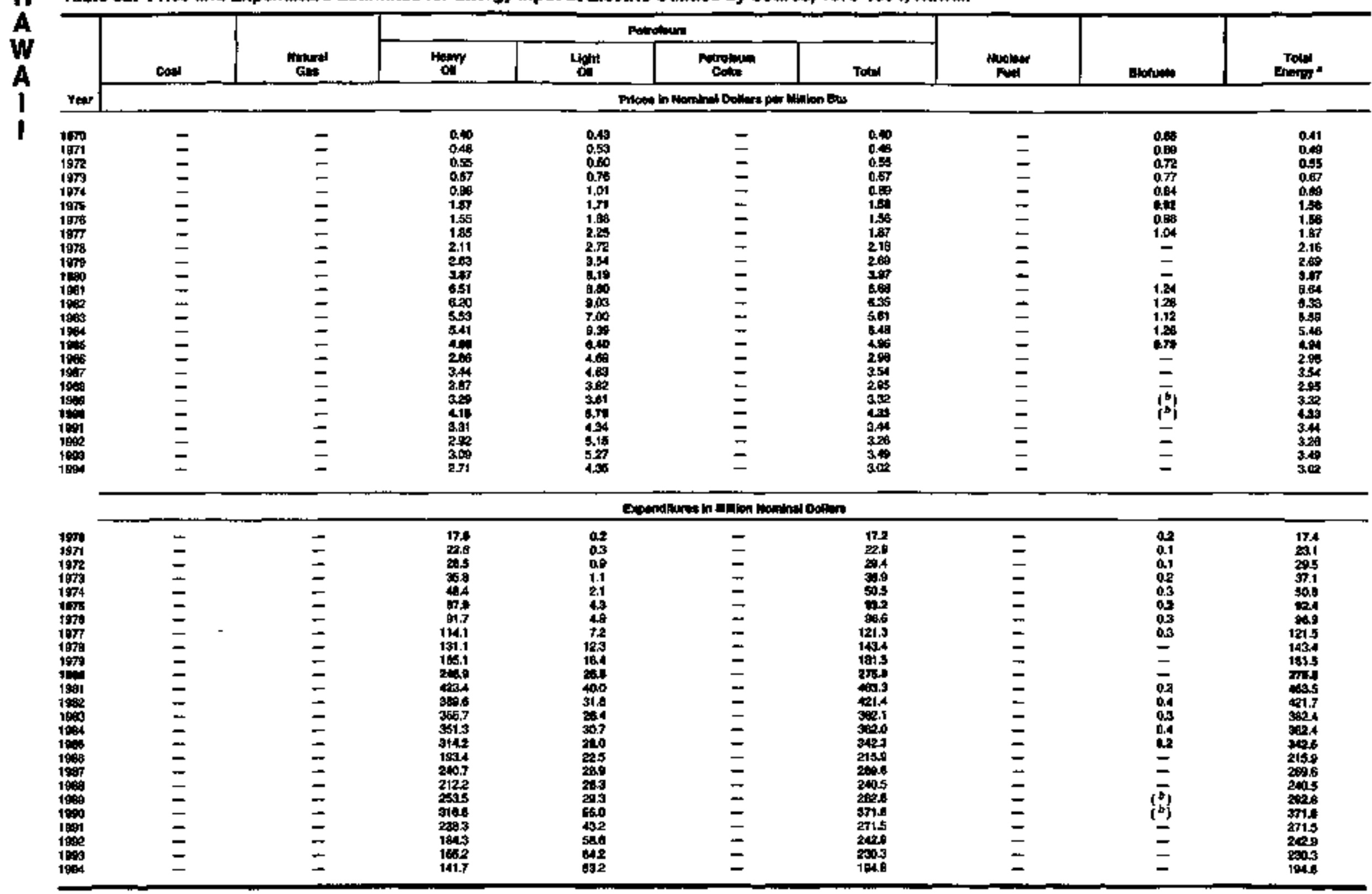

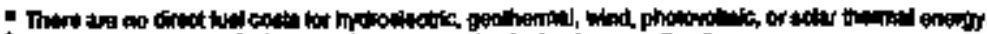

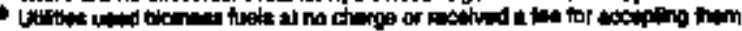
ntie eonewispilon.

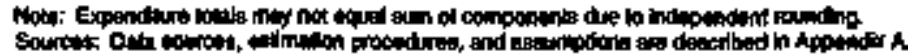




\begin{tabular}{|c|c|c|c|c|c|c|c|c|c|c|c|c|c|c|c|c|c|}
\hline \multirow[b]{4}{*}{$Y=$} & \multicolumn{14}{|c|}{ 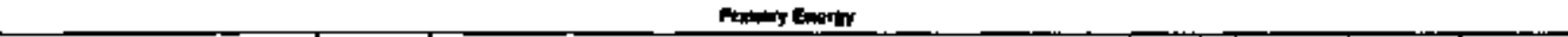 } & \multirow{3}{*}{ Eutins } & \multirow{3}{*}{ 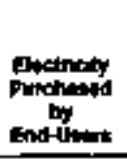 } & \multirow[b]{3}{*}{ 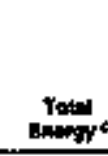 } \\
\hline & \multicolumn{3}{|c|}{ cotal } & \multirow[b]{2}{*}{ Noth } & \multicolumn{7}{|c|}{ Broothin } & \multirow[b]{2}{*}{ Fon } & \multirow[b]{2}{*}{ Bichum } & \multirow[b]{2}{*}{ Tht:le } & & & \\
\hline & conth & cand & Teth & & 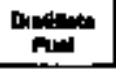 & Itot & LPQ: & Gompons & Pangution & Anes 4 & Toton & & & & & & \\
\hline & \multicolumn{17}{|c|}{ 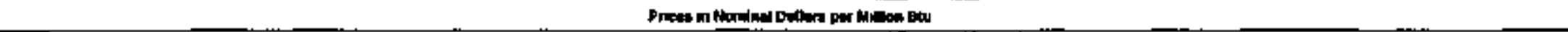 } \\
\hline \multirow[t]{2}{*}{ 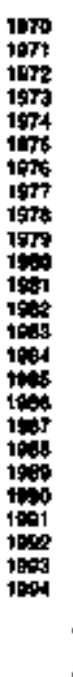 } & $\begin{array}{l}= \\
z \\
z \\
z \\
= \\
= \\
= \\
= \\
= \\
z \\
z \\
z \\
z \\
z \\
z\end{array}$ & 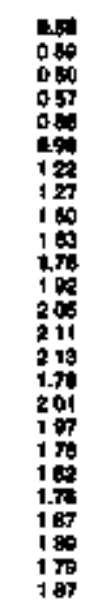 & 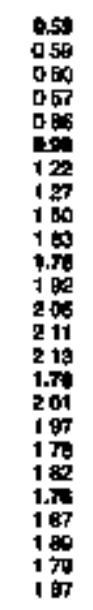 & 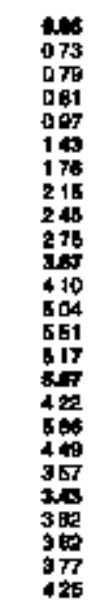 & 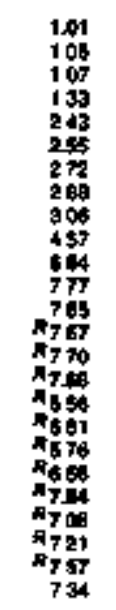 & 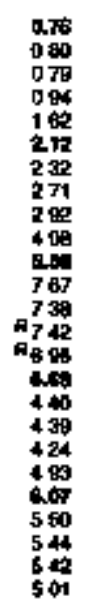 & 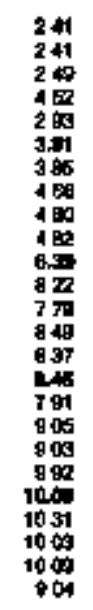 & 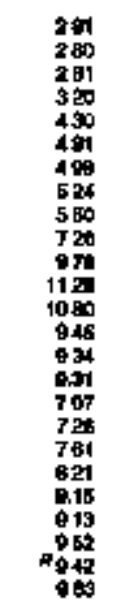 & 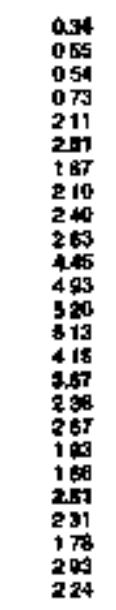 & 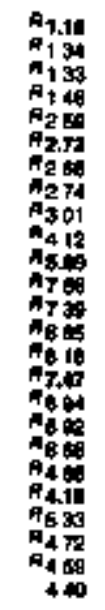 & 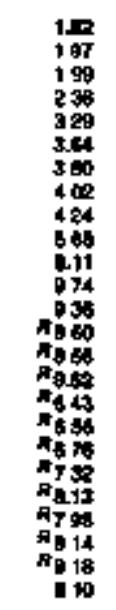 & $\begin{array}{l}= \\
= \\
z \\
= \\
= \\
= \\
= \\
= \\
= \\
= \\
= \\
= \\
= \\
= \\
= \\
=\end{array}$ & $\begin{array}{l}= \\
= \\
z \\
= \\
= \\
= \\
= \\
z \\
z \\
= \\
z \\
= \\
101 \\
160 \\
100\end{array}$ & 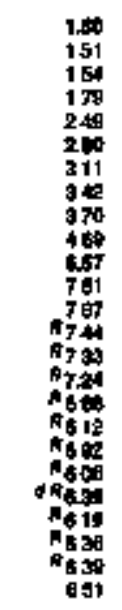 & 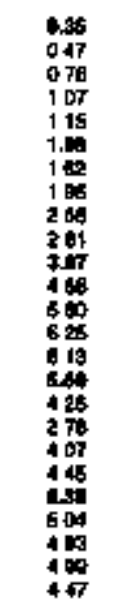 & 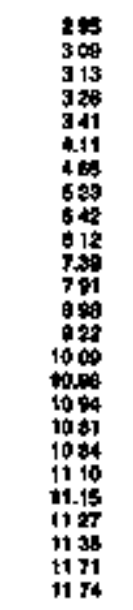 & 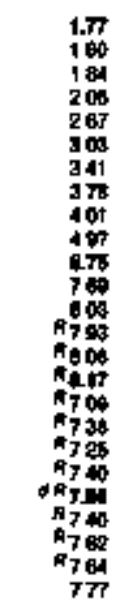 \\
\hline & \multicolumn{17}{|c|}{ 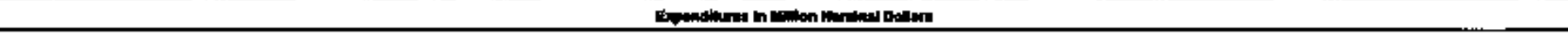 } \\
\hline 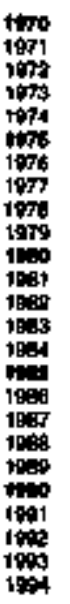 & $\begin{array}{l}= \\
= \\
= \\
= \\
= \\
= \\
= \\
= \\
= \\
= \\
= \\
= \\
= \\
=\end{array}$ & $\begin{array}{l}47 \\
70 \\
64 \\
61 \\
78 \\
13.1 \\
194 \\
164 \\
171 \\
217 \\
17.1 \\
189 \\
219 \\
180 \\
192 \\
191 \\
173 \\
174 \\
173 \\
177 \\
175 \\
229 \\
191 \\
175 \\
181\end{array}$ & 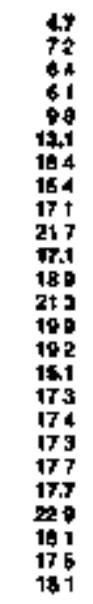 & 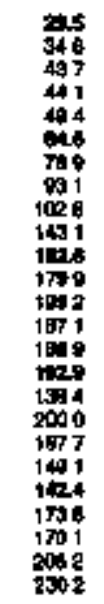 & 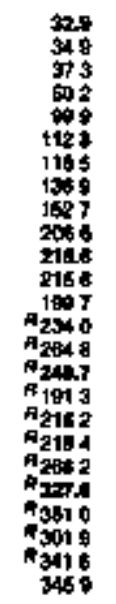 & 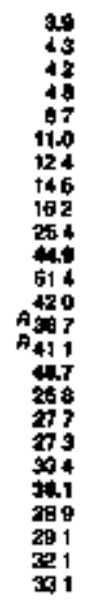 & 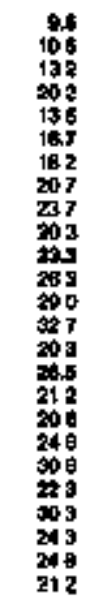 & 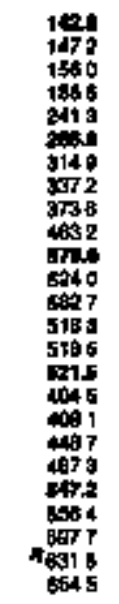 & 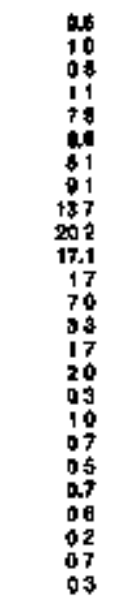 & 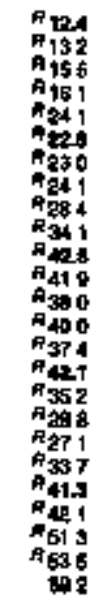 & 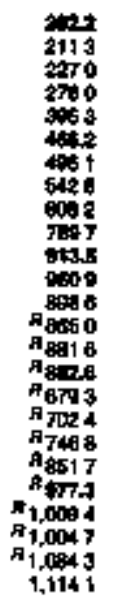 & $\begin{array}{l}= \\
z \\
z \\
z \\
= \\
= \\
= \\
= \\
= \\
= \\
= \\
= \\
= \\
= \\
=\end{array}$ & $\begin{array}{l}= \\
= \\
= \\
= \\
= \\
= \\
= \\
= \\
= \\
= \\
= \\
= \\
0 \\
17,5 \\
179 \\
177 \\
177\end{array}$ & 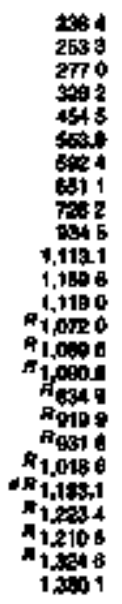 & 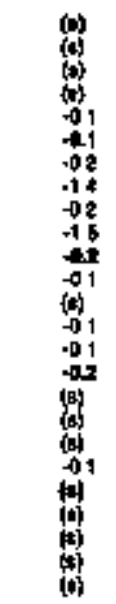 & 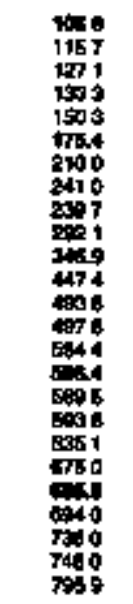 & 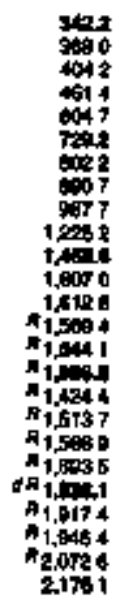 \\
\hline
\end{tabular}

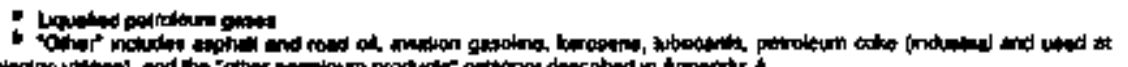

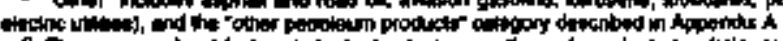

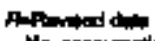

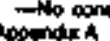

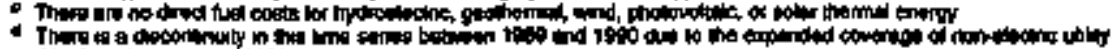

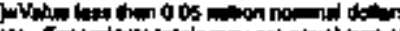

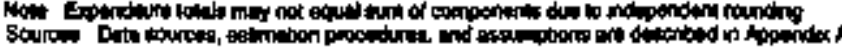


Table 84, Realdential Secter Endry Prlcs and Exponditure Eslimates by Source, 1970-1924, laho

$\underset{\mathbf{O}}{\mathbf{A}}$

\begin{tabular}{|c|c|c|c|c|c|c|c|c|c|c|}
\hline \multirow[b]{4}{*}{ Yex } & \multicolumn{8}{|c|}{ Promentering } & \multirow[b]{3}{*}{ Evetrents } & \multirow[b]{3}{*}{ 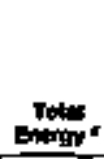 } \\
\hline & & \multirow[b]{2}{*}{ Notur } & \multicolumn{4}{|c|}{ 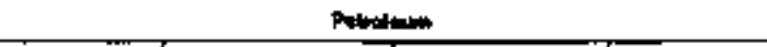 } & \multirow[b]{2}{*}{ Eonoter } & \multirow[b]{2}{*}{ Trind } & & \\
\hline & cons & & An & Kentatine & 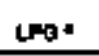 & Joted & & & & \\
\hline & \multicolumn{10}{|c|}{ 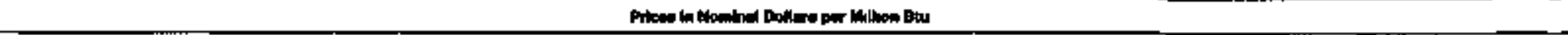 } \\
\hline 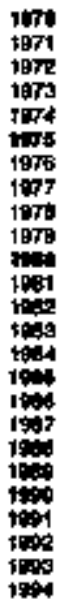 & 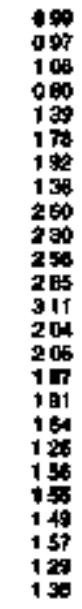 & 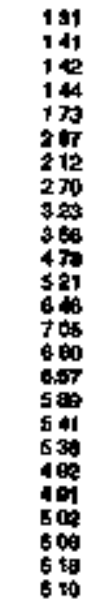 & 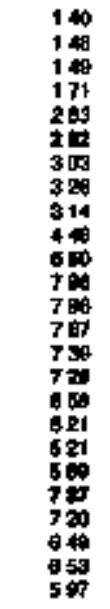 & 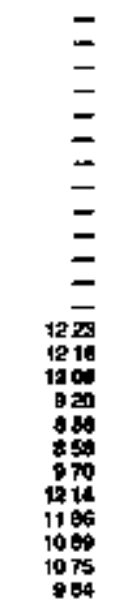 & 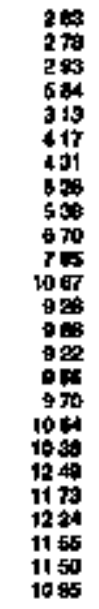 & 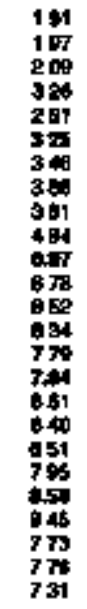 & $\begin{array}{l}= \\
= \\
= \\
= \\
= \\
= \\
= \\
= \\
= \\
= \\
= \\
= \\
4 \frac{75}{57} \\
475\end{array}$ & 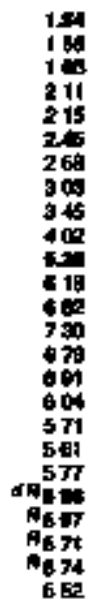 & 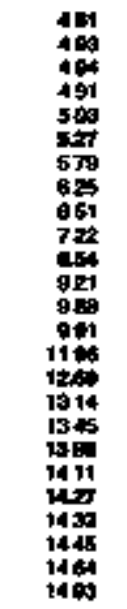 & 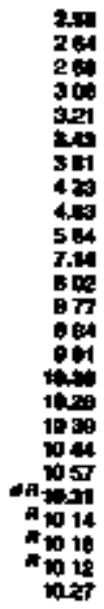 \\
\hline
\end{tabular}

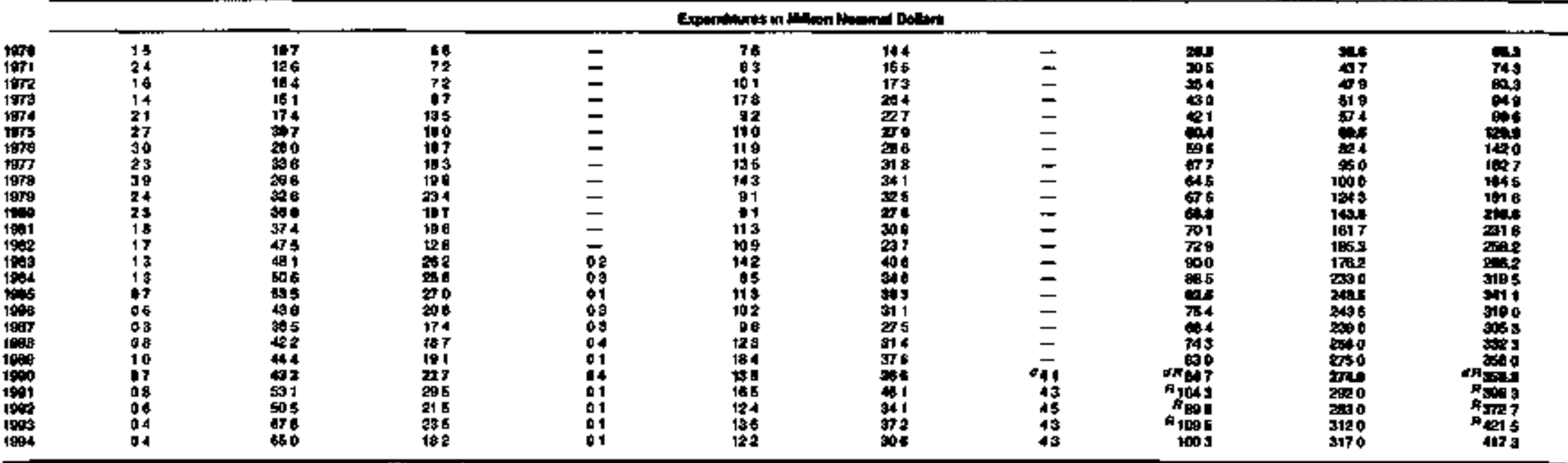

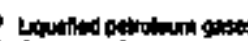

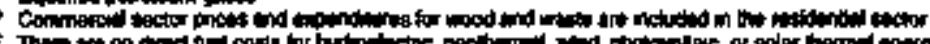

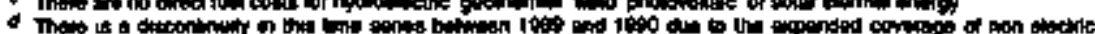

Fantadedin

7 consulapinom

Selo

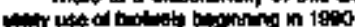


Twile 65. Cowmineflat Soctor Energy Price and Expenditure Eatimated by Source, 1970-1894, bdaho

\begin{tabular}{|c|c|c|c|c|c|c|c|c|c|c|c|}
\hline \multirow[b]{4}{*}{$x_{n=1}$} & \multicolumn{9}{|c|}{ Minner Ener } & \multirow[b]{3}{*}{ 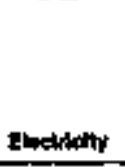 } & \multirow[b]{3}{*}{ Bons: } \\
\hline & \multirow[b]{2}{*}{$\infty$} & \multirow[b]{2}{*}{ an: } & \multicolumn{6}{|c|}{ Pulpoling } & \multirow[b]{2}{*}{ Totat } & & \\
\hline & & & 10 & Xeromente & LPD & 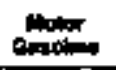 & Pontidud & الس & & & \\
\hline & \multicolumn{11}{|c|}{ 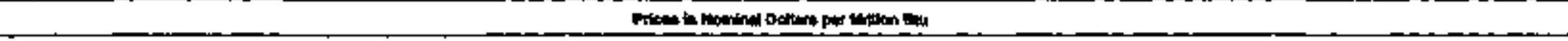 } \\
\hline \multirow[t]{2}{*}{ 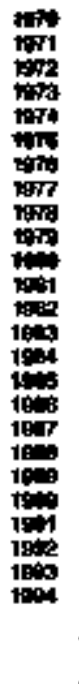 } & 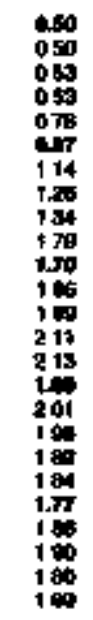 & 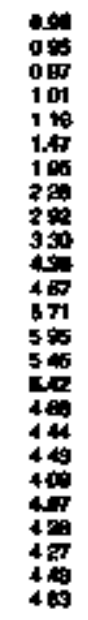 & 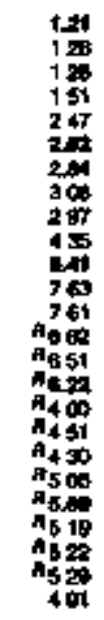 & 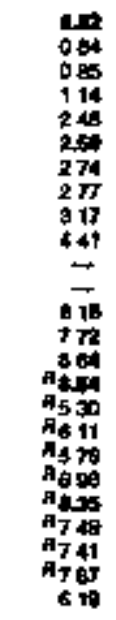 & 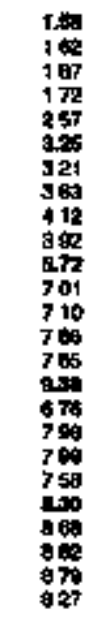 & 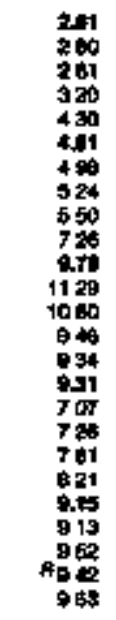 & 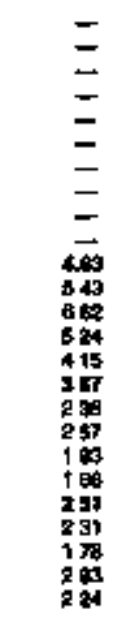 & 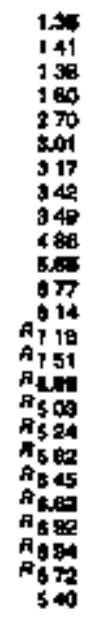 & 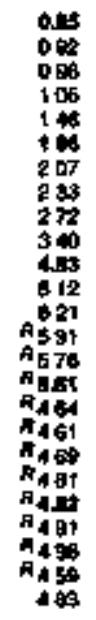 & 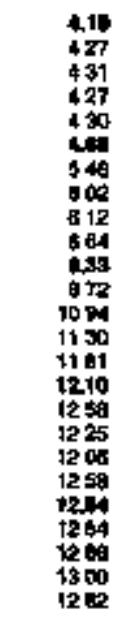 & 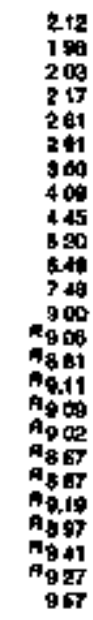 \\
\hline & \multicolumn{11}{|c|}{ 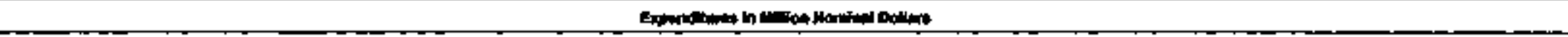 } \\
\hline 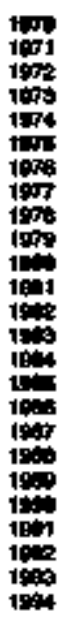 & $\begin{array}{l}14 \\
23 \\
35 \\
47 \\
22 \\
24 \\
33 \\
39 \\
38 \\
95 \\
20 \\
22 \\
20 \\
25 \\
26 \\
12 \\
11 \\
07 \\
20 \\
21 \\
18 \\
18 \\
14 \\
31 \\
14\end{array}$ & 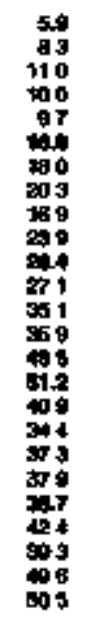 & 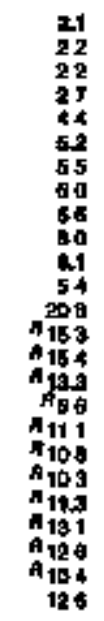 & 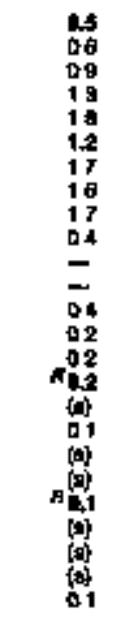 & $\begin{array}{l}17 \\
09 \\
10 \\
08 \\
13 \\
13 \\
16 \\
17 \\
19 \\
09 \\
19 \\
13 \\
13 \\
19 \\
13 \\
20 \\
13 \\
19 \\
17 \\
20 \\
77 \\
21 \\
17 \\
18 \\
18\end{array}$ & $\begin{array}{l}1.0 \\
10 \\
10 \\
12 \\
16 \\
29 \\
24 \\
26 \\
26 \\
37 \\
41 \\
70 \\
74 \\
59 \\
130 \\
65 \\
50 \\
54 \\
151 \\
154 \\
71 \\
165 \\
156 \\
19 \\
10\end{array}$ & $\begin{array}{l}= \\
= \\
= \\
= \\
= \\
= \\
\bar{z} \\
142 \\
10 \\
07 \\
08 \\
03 \\
04 \\
601 \\
02 \\
01 \\
03 \\
09 \\
10 \\
02 \\
02 \\
01\end{array}$ & 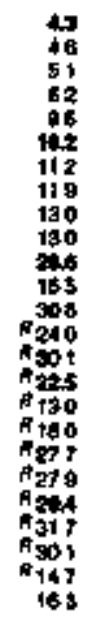 & 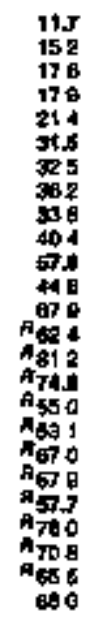 & 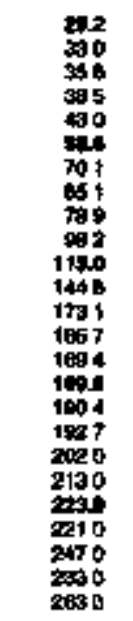 & 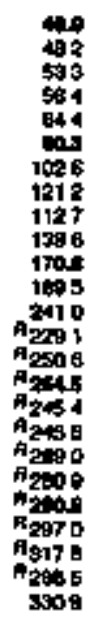 \\
\hline
\end{tabular}


Table 86. Induetrial Sector Energy Priks and Expenditure Eetimates by Source, 1970-1994, ldaho

A

H

\begin{tabular}{|c|c|c|c|c|c|c|c|c|c|c|c|c|c|c|c|c|c|}
\hline \multirow[b]{4}{*}{ vear } & \multicolumn{15}{|c|}{ Brining Ench } & \multirow[b]{3}{*}{ enountery } & \multirow[b]{3}{*}{ Emind } \\
\hline & \multicolumn{3}{|c|}{ dent } & \multirow[b]{2}{*}{ (4) } & \multicolumn{9}{|c|}{ 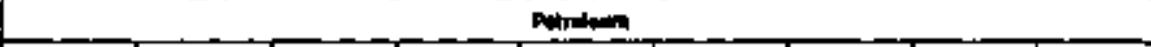 } & \multirow[b]{2}{*}{ 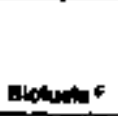 } & \multirow[b]{2}{*}{$\operatorname{Tr} \mathbb{A}^{\circ}$} & & \\
\hline & coltary & cont & Toton & & 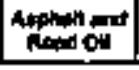 & 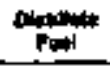 & Kinotion & Lra* & 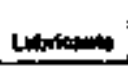 & 坚 & 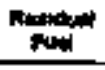 & otims & Ton & & & & \\
\hline & \multicolumn{17}{|c|}{ 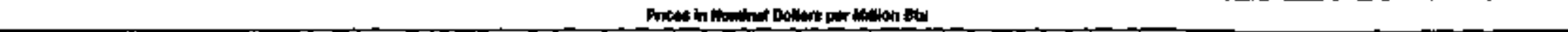 } \\
\hline 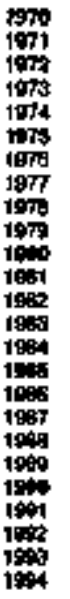 & $\begin{array}{l}= \\
= \\
= \\
= \\
= \\
= \\
= \\
= \\
= \\
= \\
= \\
= \\
=\end{array}$ & 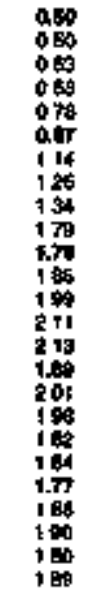 & 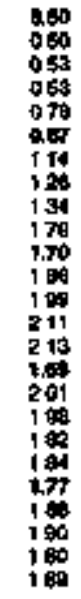 & 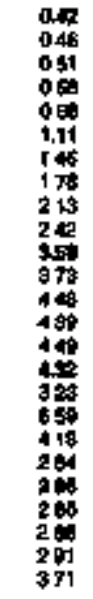 & 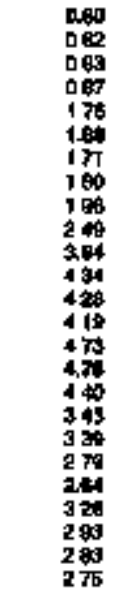 & 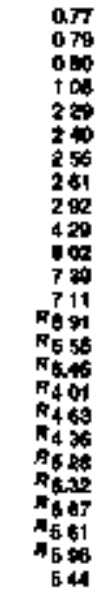 & 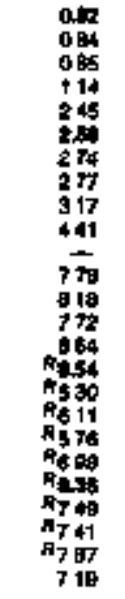 & 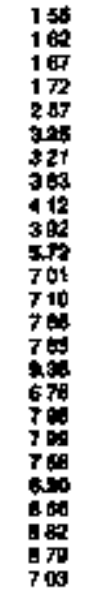 & 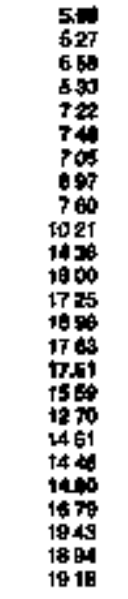 & 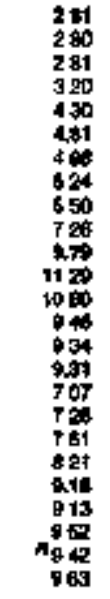 & 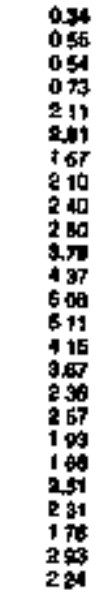 & $\begin{array}{r}114 \\
= \\
= \\
= \\
= \\
= \\
= \\
= \\
= \\
= \\
= \\
= \\
= \\
= \\
= \\
=10\end{array}$ & 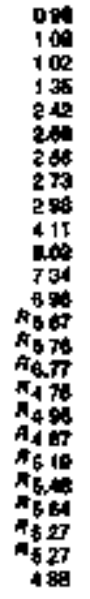 & $\begin{array}{l}= \\
= \\
= \\
= \\
= \\
= \\
= \\
= \\
= \\
= \\
\bar{z} \\
130 \\
139 \\
137 \\
137\end{array}$ & 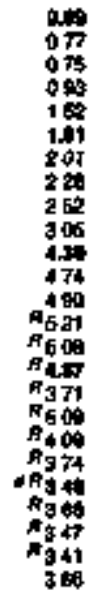 & 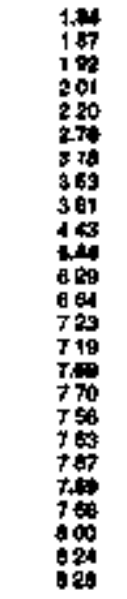 & 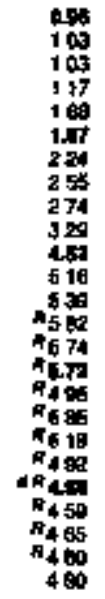 \\
\hline
\end{tabular}

\begin{tabular}{|c|c|c|c|c|c|c|c|c|}
\hline 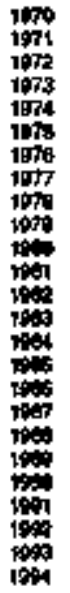 & $\begin{array}{l}= \\
= \\
= \\
= \\
= \\
= \\
= \\
= \\
= \\
= \\
= \\
= \\
=\end{array}$ & 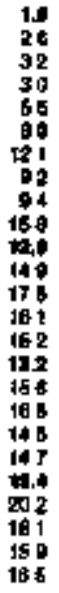 & 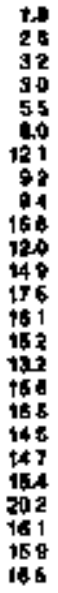 & 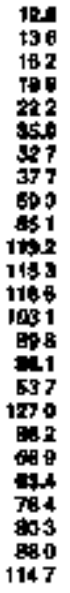 & 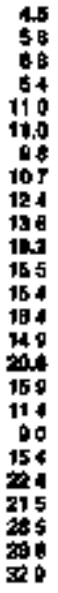 & 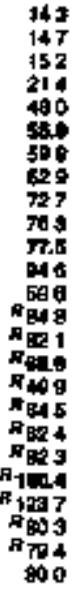 & 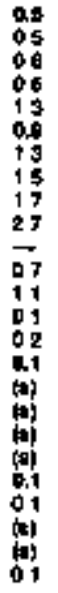 & 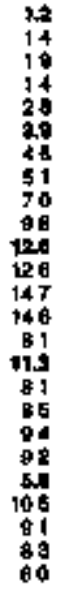 \\
\hline
\end{tabular}

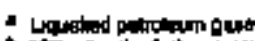

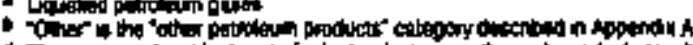

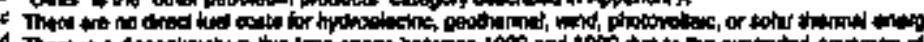

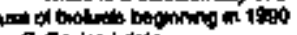

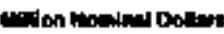

\begin{tabular}{|c|c|c|c|c|c|c|c|c|}
\hline $\begin{array}{l}10 \\
10 \\
12 \\
12 \\
16 \\
26 \\
21 \\
19 \\
82 \\
41 \\
21 \\
46 \\
40 \\
41 \\
46 \\
43 \\
37 \\
34 \\
38 \\
48 \\
40 \\
41 \\
40 \\
48 \\
61\end{array}$ & 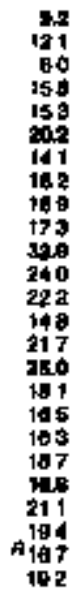 & 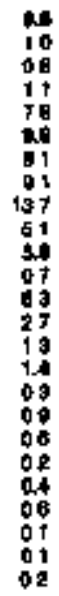 & $\begin{array}{l}a \\
= \\
= \\
= \\
= \\
= \\
= \\
= \\
= \\
= \\
= \\
= \\
= \\
10 \\
026 \\
022 \\
26\end{array}$ & 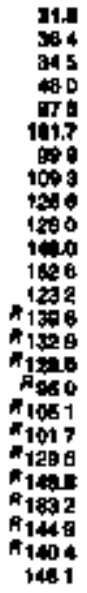 & $\begin{array}{l}= \\
= \\
= \\
= \\
= \\
= \\
= \\
= \\
= \\
= \\
= \\
\overline{119} \\
190 \\
150 \\
135 \\
194\end{array}$ & 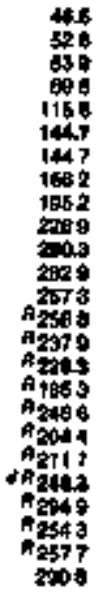 & 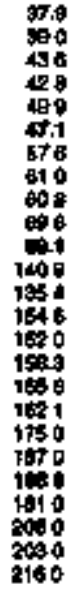 & 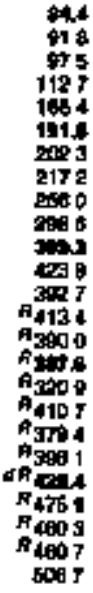 \\
\hline
\end{tabular}

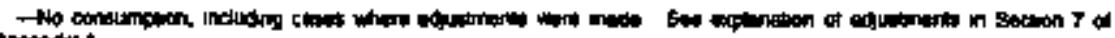

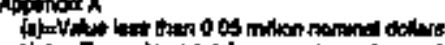

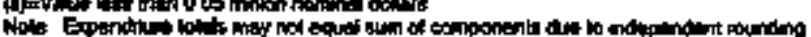
Sow 
Teble 87. Trantportation sector Eneroy Prics and Expenditure Estimates by Source, 1970-1994, ldaho

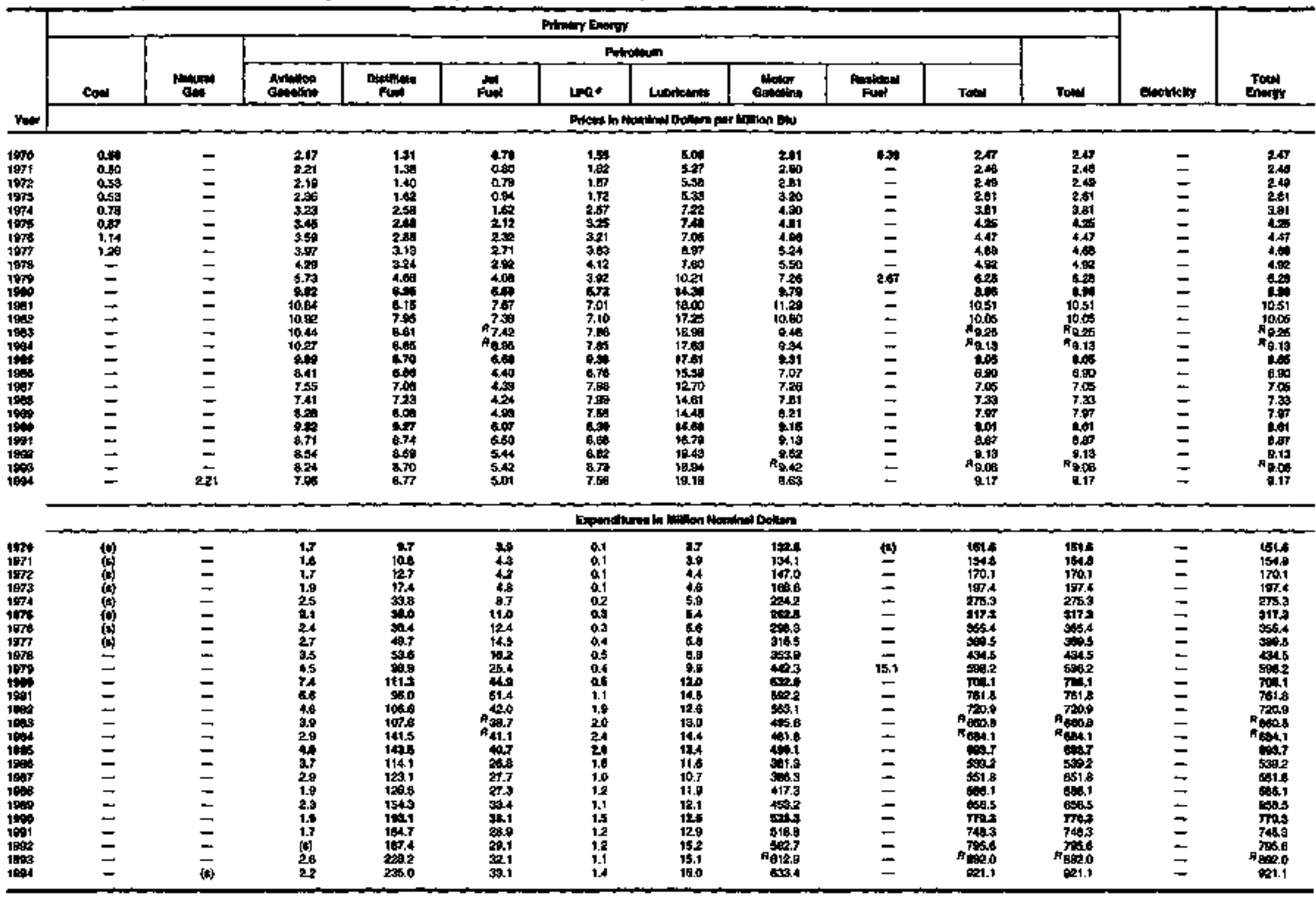


1 Table 8B. Price and Expenditure Estimates for Energy inpet et Electic Utilitlea by source, 1970-1994, ldaho

\begin{tabular}{|c|c|c|c|c|c|c|c|c|c|}
\hline \multirow[b]{3}{*}{ You } & & \multirow[b]{2}{*}{$\begin{array}{c}\text { Mesunner } \\
\text { and }\end{array}$} & \multicolumn{4}{|c|}{ Pentoineso } & \multirow[b]{2}{*}{ ment } & \multirow[b]{2}{*}{ 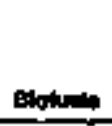 } & \multirow[b]{2}{*}{ "4t: } \\
\hline & cont & & onst & 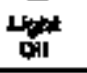 & 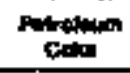 & Total & & & \\
\hline & \multicolumn{9}{|c|}{ 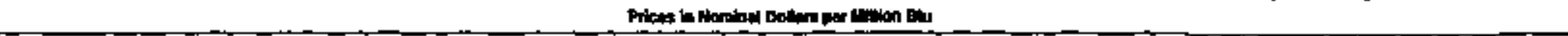 } \\
\hline \multirow{18}{*}{ 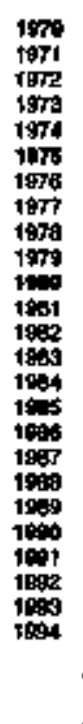 } & - & - & - & a.ss & - & 0.5 & - & - & .0 .53 \\
\hline & $\bar{z}$ & $=$ & $\bar{z}$ & $a n$ & & $\Delta \rightarrow$ & $=$ & - & 0.45 \\
\hline & $=$ & 5 & $\bar{z}$ & $\begin{array}{l}0.78 \\
1.07\end{array}$ & $\overline{-}$ & $\begin{array}{l}0.07 \\
1.07\end{array}$ & $=$ & $=$ & $i_{1,00}^{\infty}$ \\
\hline & $=$ & 1.52 & $=$ & $\begin{array}{l}2.10 \\
2.20\end{array}$ & $=$ & 210 & $=$ & $=$ & 1.15 \\
\hline & $=$ & 1.50 & $=$ & 2,4 & - & 2,4 & $=$ & $=$ & $\underset{1+2}{4}$ \\
\hline & $\bar{z}$ & 104 & $=$ & 20 & - & 289 & - & $=$ & 10 \\
\hline & $\bar{z}$ & $\underset{2,12}{2,12}$ & $=$ & 3.24 & $=$ & 324 & $=$ & $=$ & 258 \\
\hline & $=$ & 20 & $=$ & 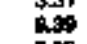 & $=$ & \& & $\bar{z}$ & $=$ & in \\
\hline & $z$ & $\begin{array}{l}121 \\
5.34\end{array}$ & $=$ & $7, x 3$ & $z$ & $\operatorname{lng}_{6 \times 4}$ & $=$ & $=$ & 4.90 \\
\hline & $=$ & 6.28 & $\bar{z}$ & 5.24 & $=$ & 621 & $\overline{-}$ & $=$ & 500 \\
\hline & $z$ & 4.12 & $=$ & $\begin{array}{l}\text { 8.14 } \\
\text { coft }\end{array}$ & $=$ & $\begin{array}{l}6.14 \\
6.07\end{array}$ & $=$ & $=$ & ata \\
\hline & $z$ & $4, \pi 9$ & $=$ & 374 & $z$ & 374 & 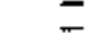 & $=$ & 4 \\
\hline & $=$ & 2.31 & $\bar{z}$ & 40 & $=$ & 407 & 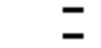 & $=$ & $\underset{107}{2 \pi}$ \\
\hline & $z$ & $=$ & $\bar{z}+x \cdot r \cdot r \cdot r \cdot r$ & 1.58 & $=$ & 急 & 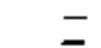 & $=$ & to \\
\hline & $=$ & $=$ & $=$ & 5.004 & $\bar{z}$ & s.04 & $=$ & $=$ & 5.04 \\
\hline & $=$ & $=$ & $\Xi$ & 40 & $=$ & $\begin{array}{l}400 \\
400\end{array}$ & $=$ & $=$ & 48 \\
\hline & - & - & - & 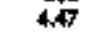 & - & 4.47 & ב & $=$ & in \\
\hline & \multicolumn{9}{|c|}{ 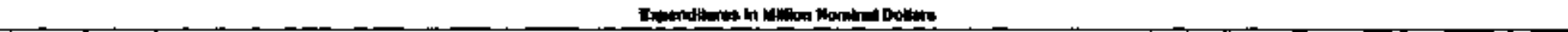 } \\
\hline Irti & $=$ & $=$ & $=$ & (1) & $=$ & (a) & $\bar{z}+1+x+10$ & $=$ & g) \\
\hline 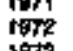 & $=$ & $\bar{z}$ & $\bar{z}$ & 6 & $=$ & (1) & $\bar{z}$ & $=$ & 9 \\
\hline 1973 & $\bar{z}$ & $\overline{0}$ & $=$ & a) & $=$ & (c) & $\bar{z}$ & $=$ & $\mathrm{a}_{1}$ \\
\hline 197 & $\bar{z}$ & (1) & 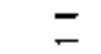 & $\frac{0.9}{9}$ & $\bar{z}$ & a. & $\bar{z}$ & $=$ & aid \\
\hline ign & $=$ & 14 & $=$ & 6 & \pm & 8 & $\bar{z}$ & $=$ & 14 \\
\hline 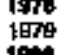 & $\bar{z}$ & 1.4 & $=$ & 0.1 & & a & $z$ & $\bar{z}$ & ${ }_{18}^{02}$ \\
\hline 100 & z & $\begin{array}{l}0.2 \\
0.1\end{array}$ & $=$ & 盟 & $z$ & 急 & $z$ & $=$ & $\frac{12}{41}$ \\
\hline $\begin{array}{l}1982 \\
1908\end{array}$ & $\overline{-}$ & (a) & $=$ & (1) & $=$ & 要 & $=$ & $=$ & 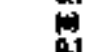 \\
\hline $\begin{array}{l}1884 \\
1104\end{array}$ & 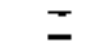 & (0) & $\bar{z}$ & 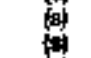 & $=$ & 留 & $\bar{z}$ & $=$ & E1 \\
\hline 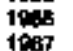 & $=$ & (6) & $=$ & 8 & $=$ & 唯 & $\bar{z}$ & $=$ & $(x)$ \\
\hline 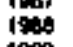 & $=$ & 믄 & $=$ & 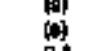 & $=$ & 相 & $\bar{z}$ & $=$ & 9 \\
\hline 1929 & $\bar{z}$ & $=$ & $\bar{z}+r+1+x$ & (i) & $=$ & a1 & $=$ & $=$ & 0.5 \\
\hline 1901 & & 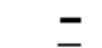 & 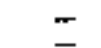 & 0 & $=$ & 8 & 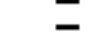 & $=$ & 6 \\
\hline 19020 & $=$ & 二 & $\bar{z}$ & (5) & 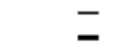 & (⿻) & $=$ & $=$ & 8 \\
\hline 1894 & - & - & - & (⿻日木一) & - & ณ & - & $=$ & 6 \\
\hline
\end{tabular}

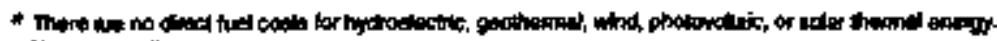

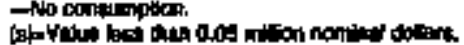

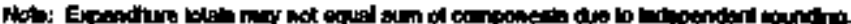

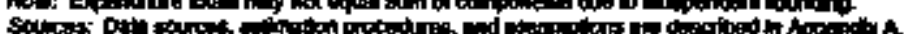




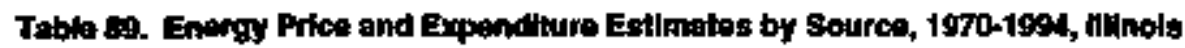

\begin{tabular}{|c|c|c|c|c|c|c|c|c|c|c|c|c|c|c|c|c|c|}
\hline & \multicolumn{14}{|c|}{ 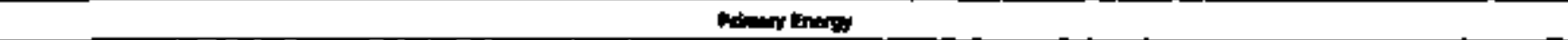 } & \multirow{3}{*}{ 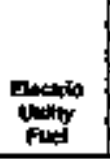 } & \multirow{3}{*}{ 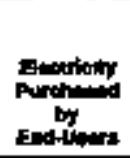 } & \multirow[b]{3}{*}{ Tot: } \\
\hline & \multicolumn{3}{|c|}{ cen } & \multirow[b]{2}{*}{ mand } & \multicolumn{7}{|c|}{ 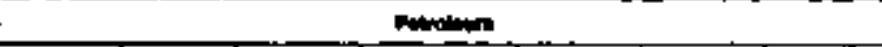 } & \multirow[b]{2}{*}{ Finger } & \multirow[b]{2}{*}{ Bdolman } & \multirow[b]{2}{*}{ Totent } & & & \\
\hline & Coling & יוה & Tw & & Pust & A & un: & 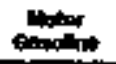 & 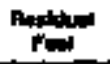 & Othere & Tot & & & & & & \\
\hline $\mathrm{rem}$ & \multicolumn{17}{|c|}{ 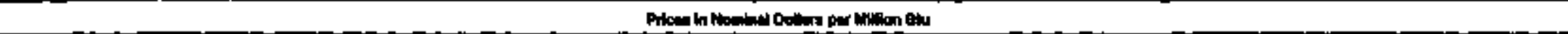 } \\
\hline \multirow[t]{2}{*}{ 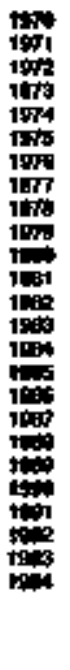 } & 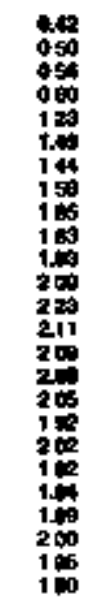 & 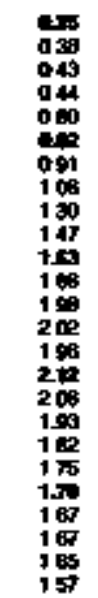 & 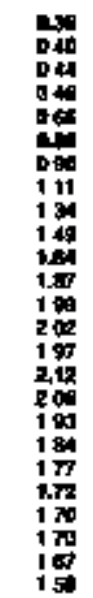 & 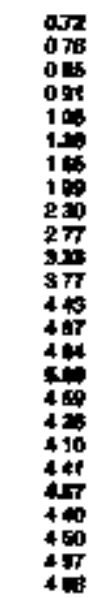 & 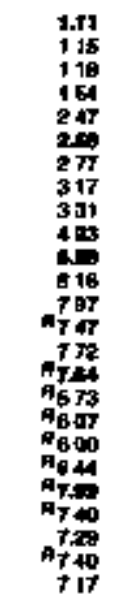 & 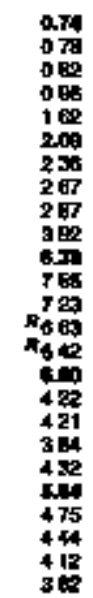 & 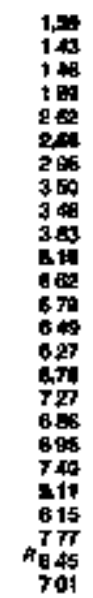 & 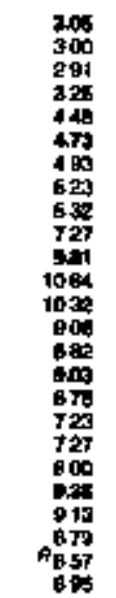 & 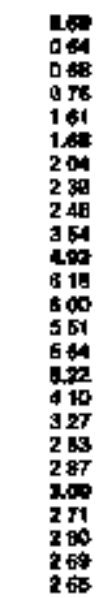 & 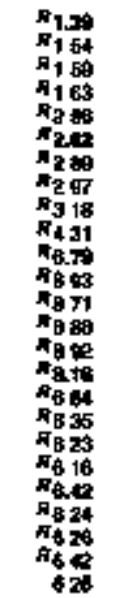 & 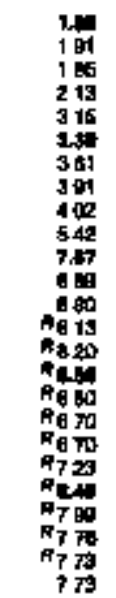 & 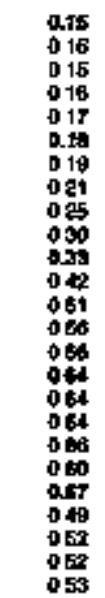 & $\begin{array}{l}06 \\
= \\
= \\
= \\
= \\
= \\
= \\
= \\
= \\
= \\
= \\
= \\
= \\
5 \times 12 \\
306 \\
312 \\
286 \\
370\end{array}$ & 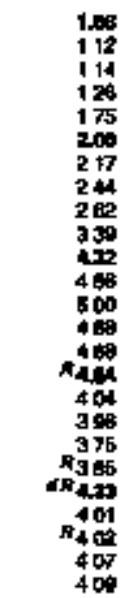 & 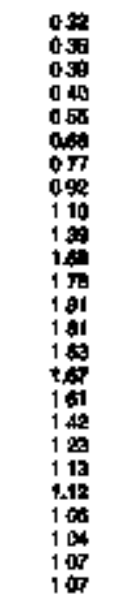 & 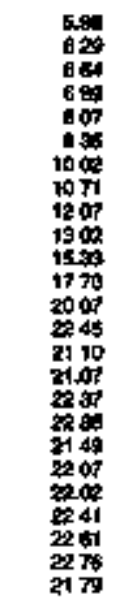 & 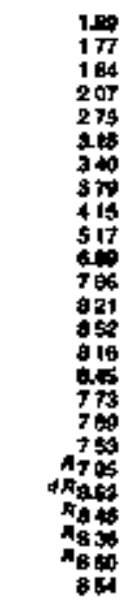 \\
\hline & \multicolumn{17}{|c|}{ 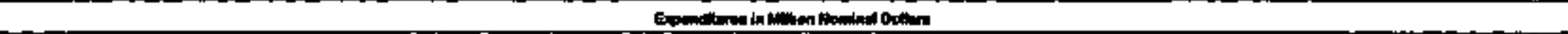 } \\
\hline 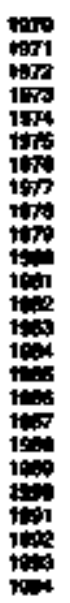 & 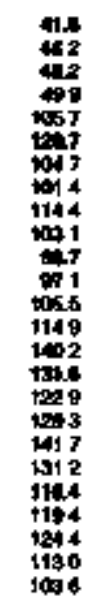 & 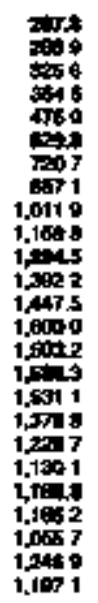 & 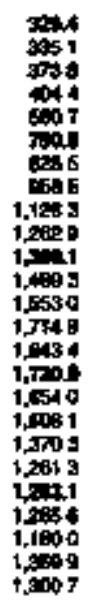 & 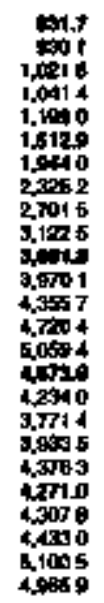 & 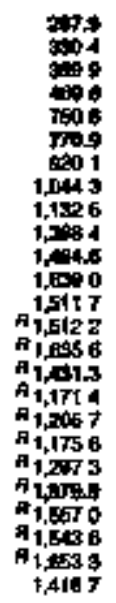 & 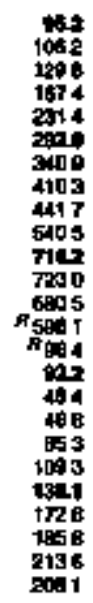 & 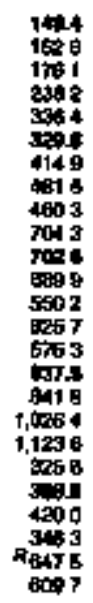 & 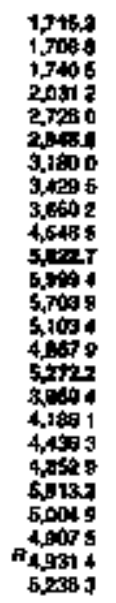 & 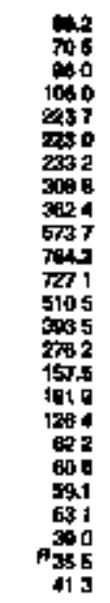 & 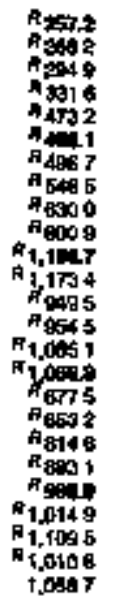 & 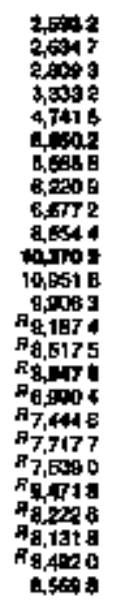 & 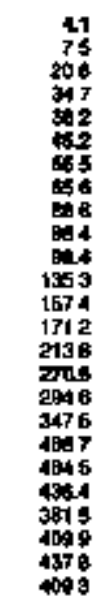 & 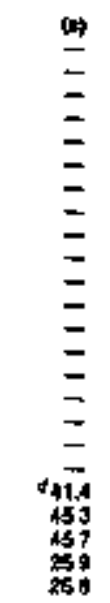 & 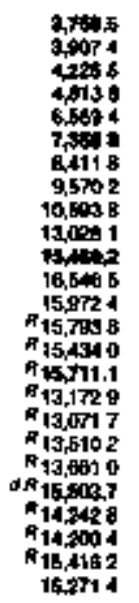 & 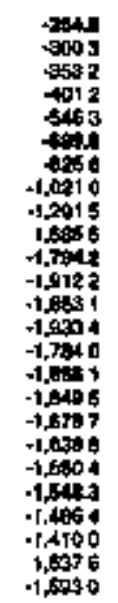 & 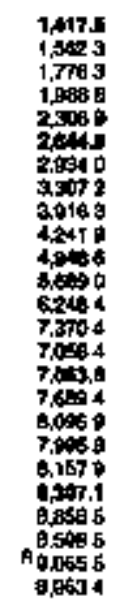 & 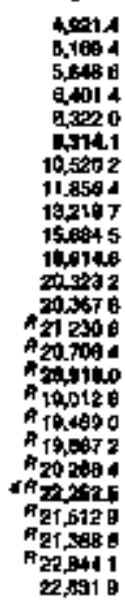 \\
\hline
\end{tabular}

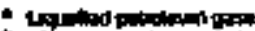

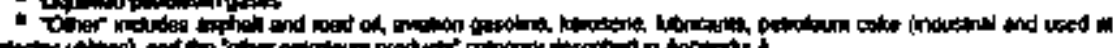

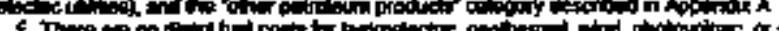

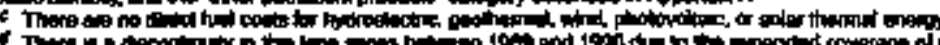

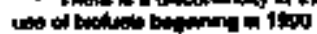

Amplimending

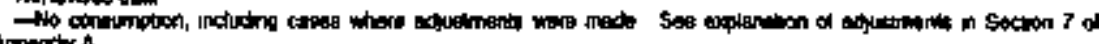

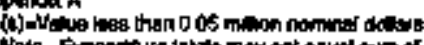

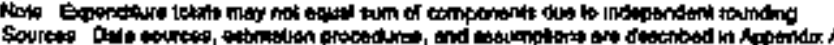


Table 90. Reoidenlial Sector Energy Price and Expencture Estimated by Sourca, 1970-1094, Illinols

\begin{tabular}{|c|c|c|c|c|c|c|c|c|c|c|}
\hline \multirow[b]{4}{*}{ Yes } & \multicolumn{8}{|c|}{ Phomery enory } & \multirow[b]{3}{*}{ Ampinting } & \multirow[b]{3}{*}{ Totid } \\
\hline & \multirow[b]{2}{*}{ cow } & \multirow[b]{2}{*}{ math } & \multicolumn{4}{|c|}{ Provolonit } & \multirow[b]{2}{*}{ Dowuels b } & \multirow[b]{2}{*}{ 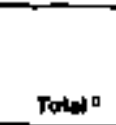 } & & \\
\hline & & & 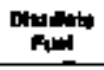 & Knimons & LPS: & Toket & & & & \\
\hline & \multicolumn{9}{|c|}{ 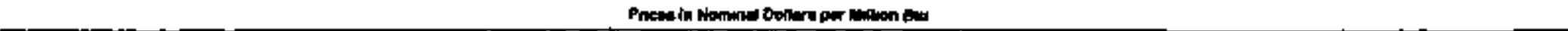 } & \\
\hline \multirow[t]{2}{*}{ 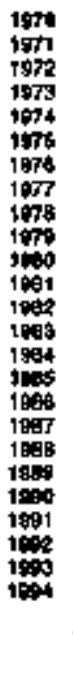 } & 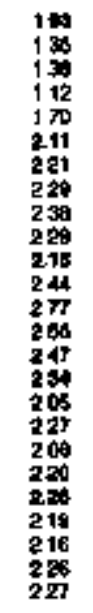 & 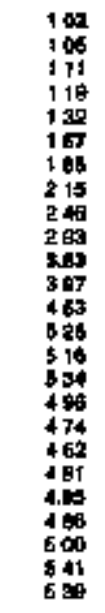 & 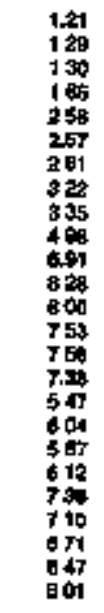 & 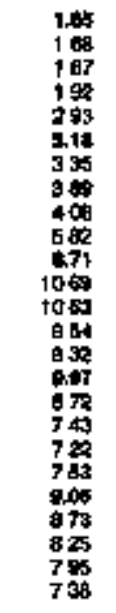 & 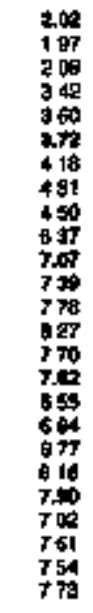 & 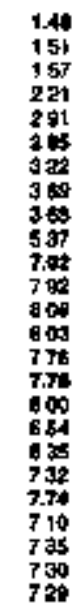 & 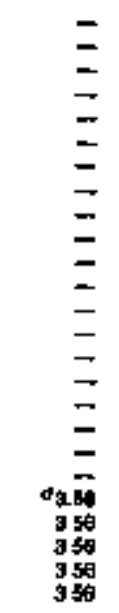 & 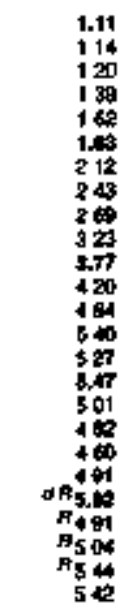 & 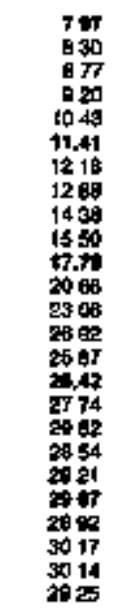 & 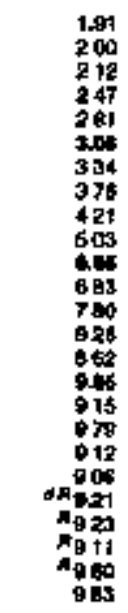 \\
\hline & \multicolumn{10}{|c|}{ 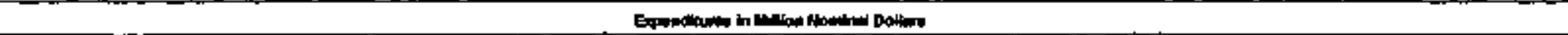 } \\
\hline 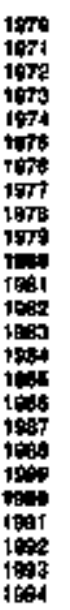 & $\begin{array}{l}192 \\
244 \\
150 \\
94 \\
140 \\
4.7 \\
181 \\
142 \\
154 \\
54 \\
41 \\
49 \\
75 \\
93 \\
87 \\
46 \\
44 \\
52 \\
44 \\
54 \\
47 \\
45 \\
49 \\
47 \\
48\end{array}$ & 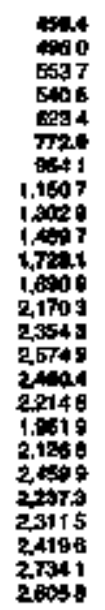 & 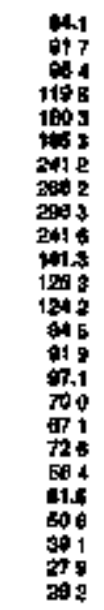 & 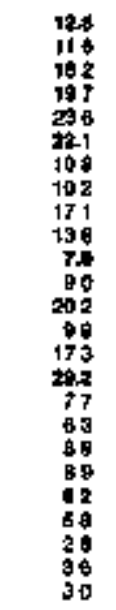 & 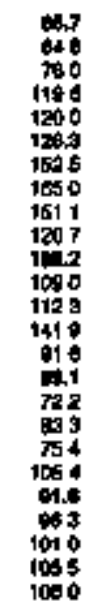 & 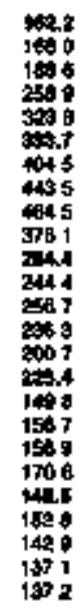 & $\begin{array}{l}= \\
= \\
= \\
= \\
= \\
= \\
= \\
= \\
= \\
= \\
= \\
402 \\
302 \\
404 \\
000\end{array}$ & 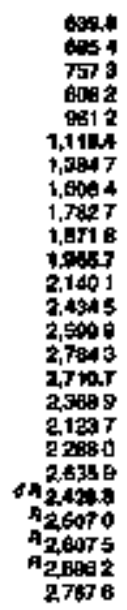 & 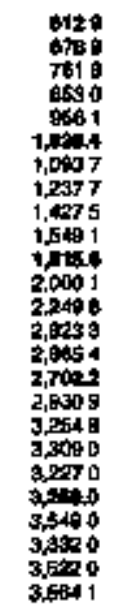 & 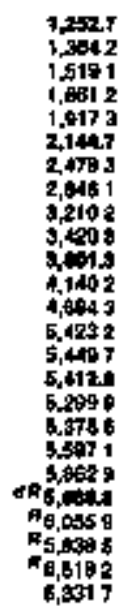 \\
\hline
\end{tabular}

- Lavilied patrolotem papos

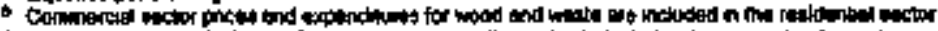

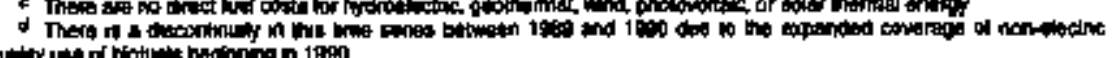
ARTomiand dit

to somantorion

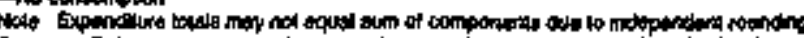
bedinna in 1990

Energy intormation Adininlatration 
Table 91. Commercial Sector Enargy Price and Expenditure Estimates by Solarce, 1970-1984, Llinois

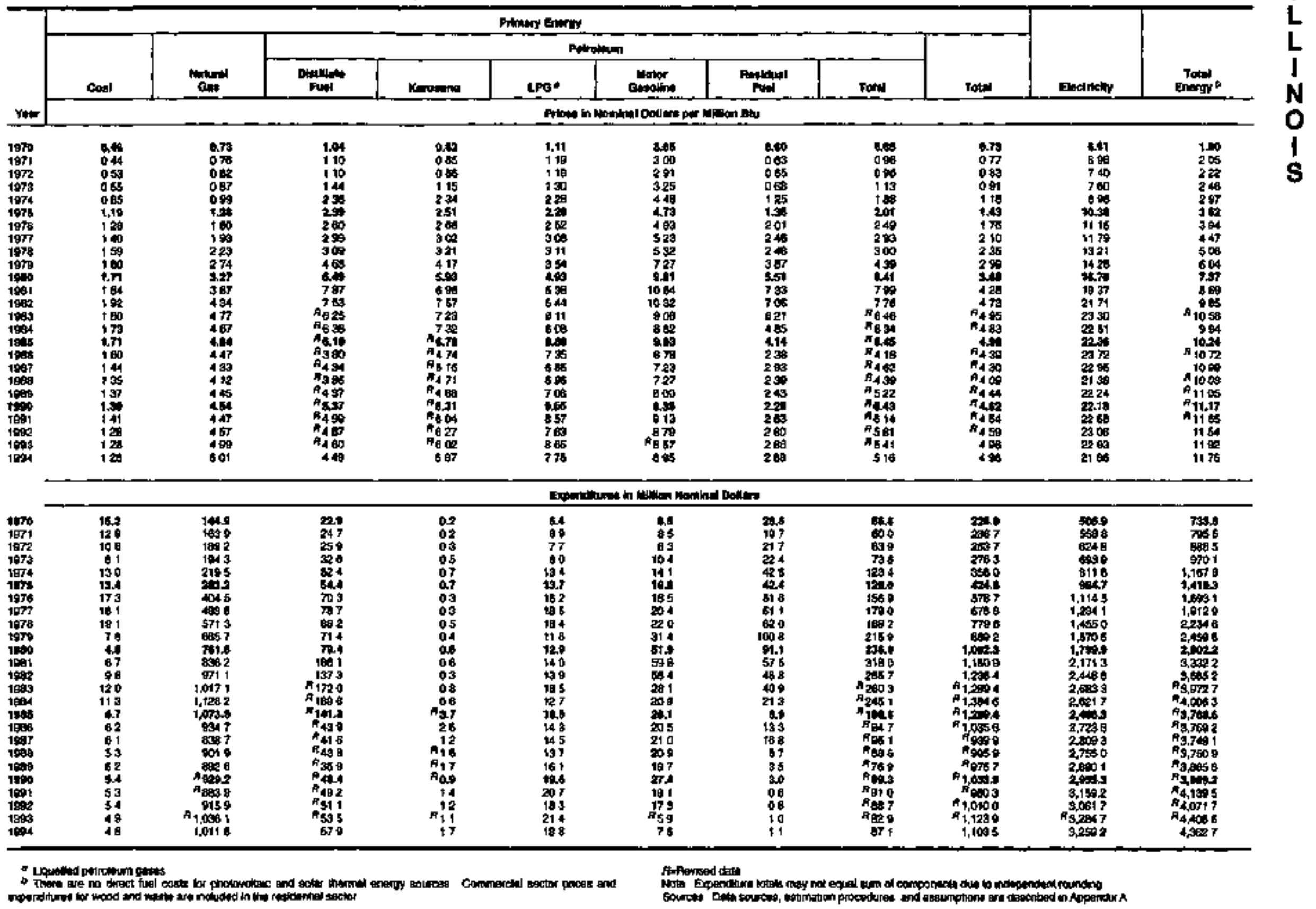




\begin{tabular}{|c|c|c|c|c|c|c|c|c|c|c|c|c|c|c|c|c|c|}
\hline \multirow[b]{4}{*}{$\mathrm{ven}$} & \multicolumn{15}{|c|}{$n_{1}$} & \multirow[b]{3}{*}{ 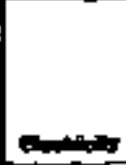 } & \multirow[b]{3}{*}{ ד. } \\
\hline & \multicolumn{3}{|c|}{$\infty$} & \multirow[b]{2}{*}{ 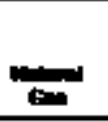 } & \multicolumn{9}{|c|}{ 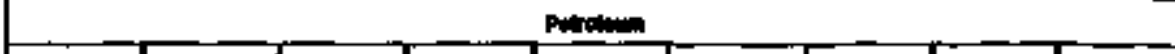 } & \multirow[b]{2}{*}{ s } & \multirow[b]{2}{*}{ שוחה } & & \\
\hline & 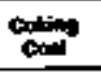 & 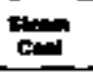 & Intal & & 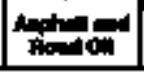 & "NA & Ensm & LWE & 느네 & 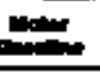 & 每 & 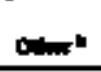 & $\pi$ & & & & \\
\hline & \multicolumn{17}{|c|}{ 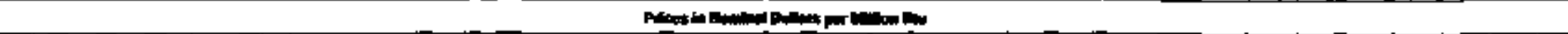 } \\
\hline \multirow[t]{2}{*}{ 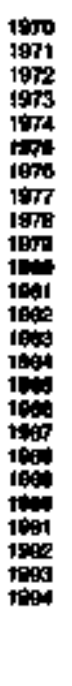 } & 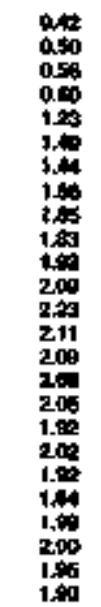 & 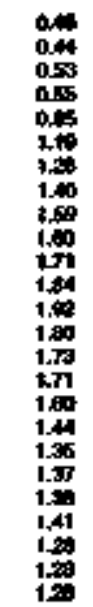 & 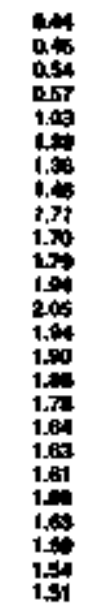 & 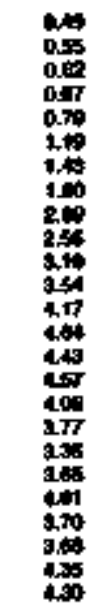 & 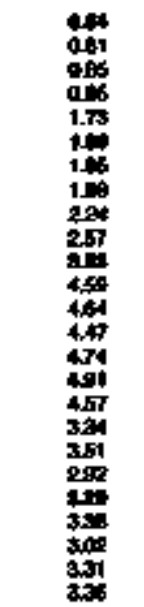 & 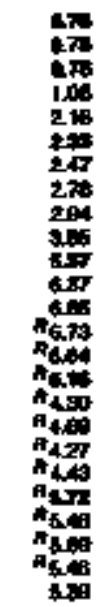 & 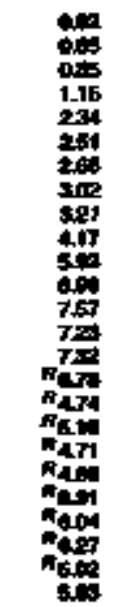 & 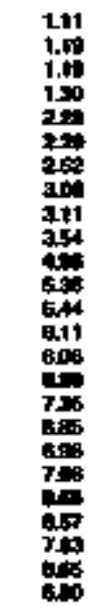 & 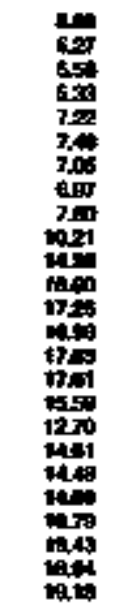 & 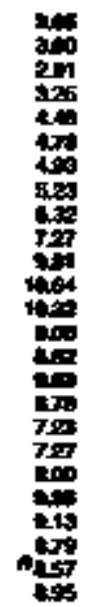 & 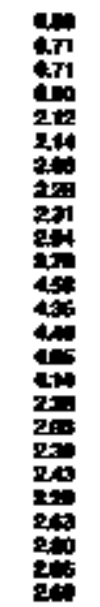 & 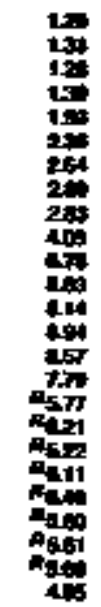 & 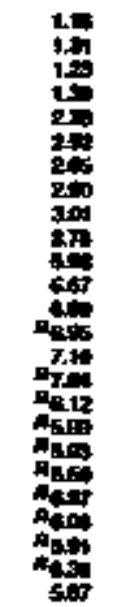 & $\begin{array}{l}= \\
= \\
= \\
= \\
= \\
= \\
= \\
= \\
= \\
= \\
\bar{z} \\
10 \\
10\end{array}$ & 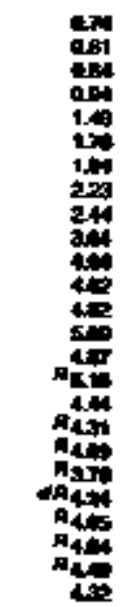 & 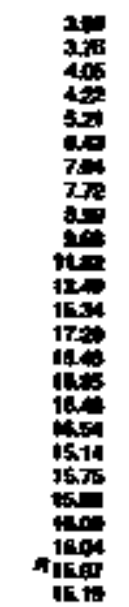 & 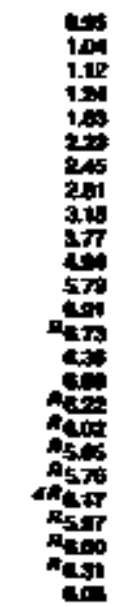 \\
\hline & \multicolumn{17}{|c|}{ 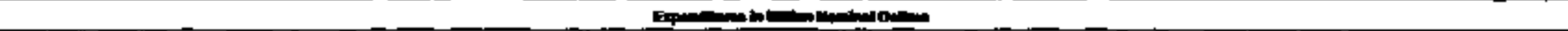 } \\
\hline 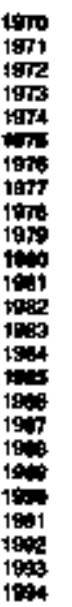 & 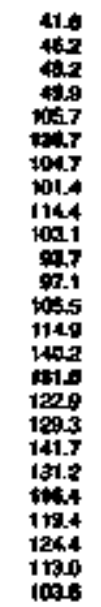 & 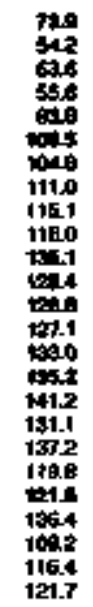 & 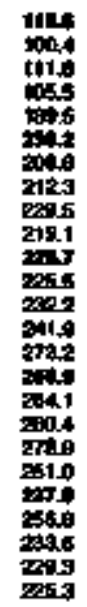 & 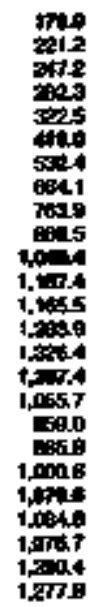 & 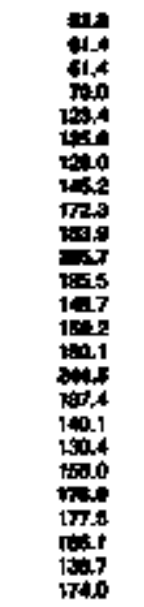 & 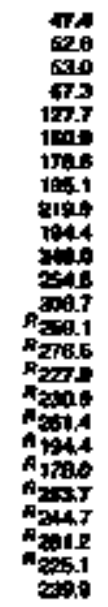 & 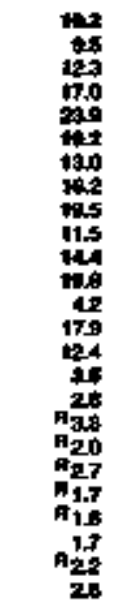 & 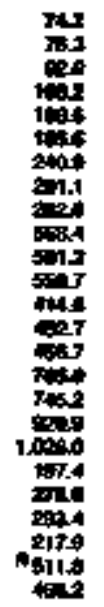 & 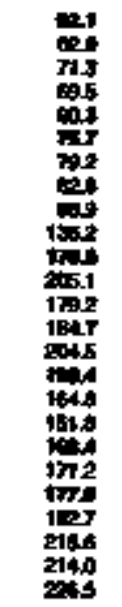 & 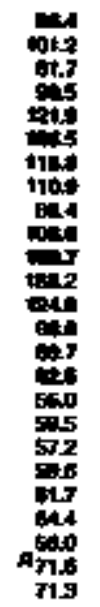 & 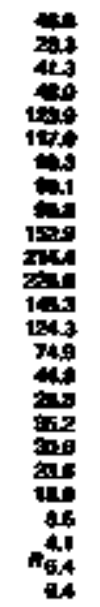 & 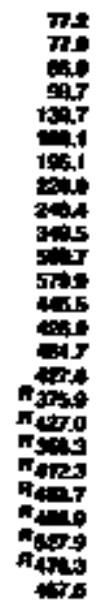 & 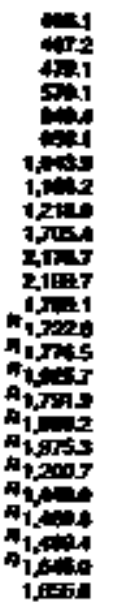 & 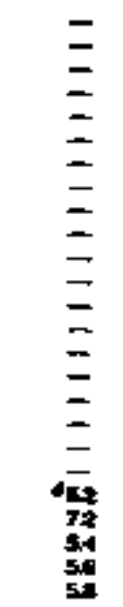 & 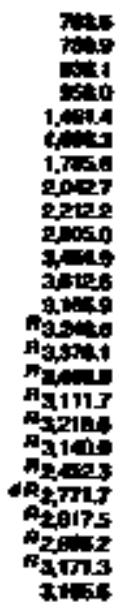 & 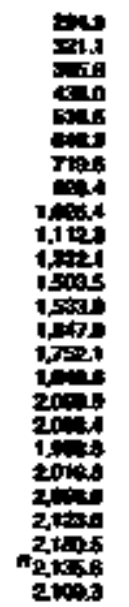 & 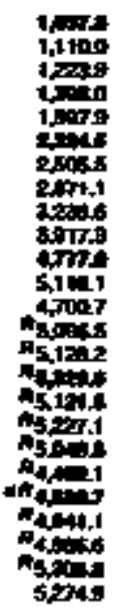 \\
\hline
\end{tabular}

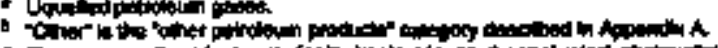

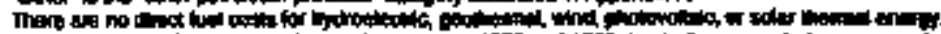

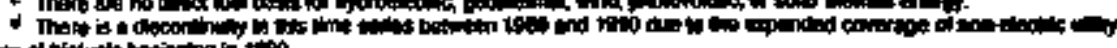

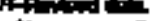

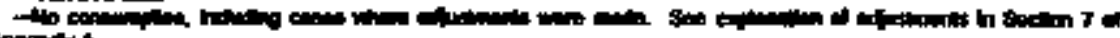

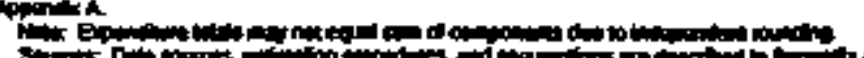

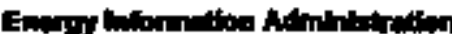




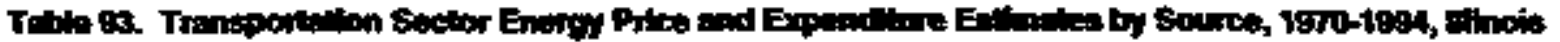

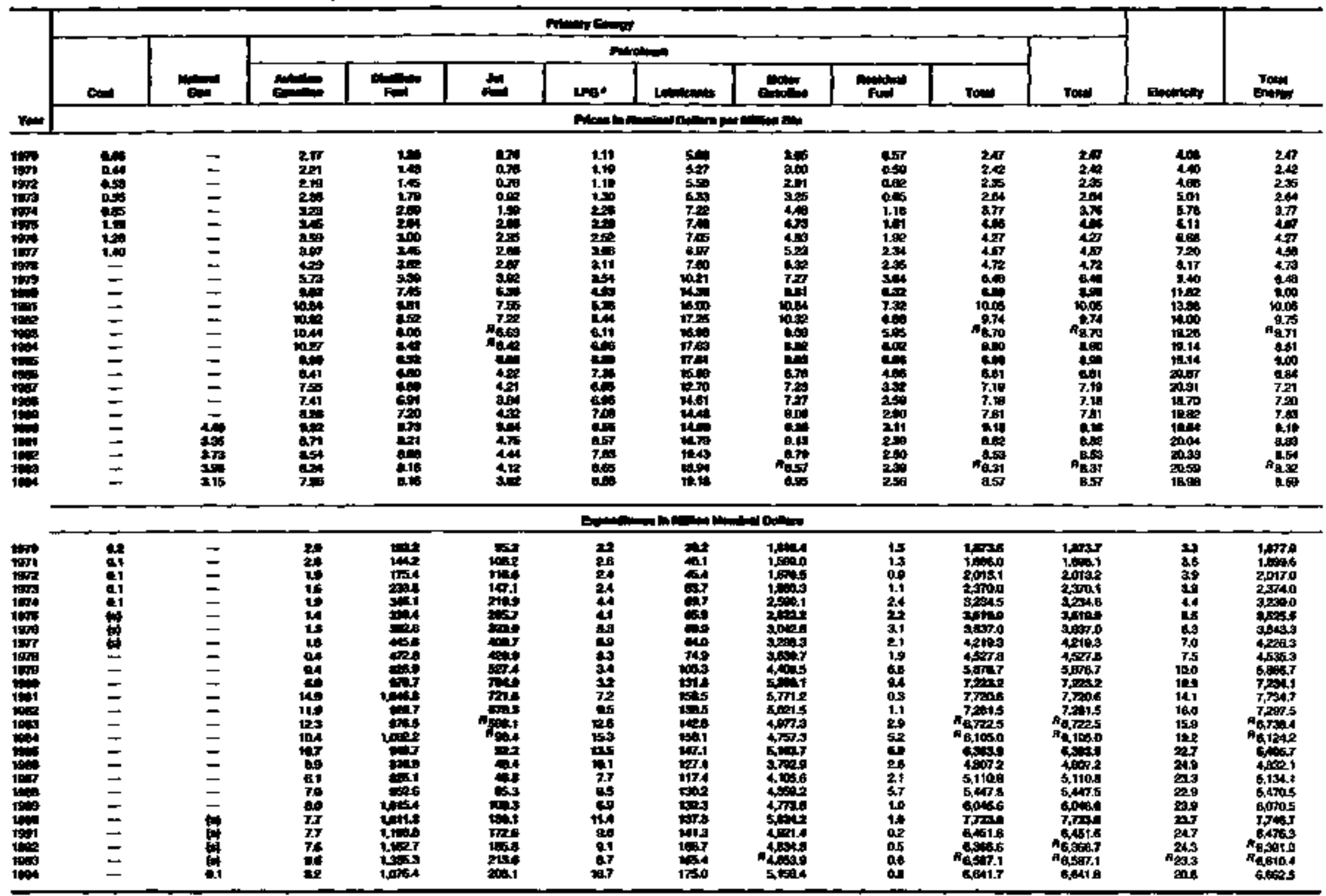


Table 94. Price and Expendluure Eetimatus for Eneroy mput at Electric Utilites by Source, 1970-1994, Inlinois

\begin{tabular}{|c|c|c|c|c|c|c|c|c|c|}
\hline \multirow[b]{3}{*}{ row } & \multirow[b]{2}{*}{ tont } & \multirow[b]{2}{*}{$\min _{0 \rightarrow 0}$} & \multicolumn{4}{|c|}{ Putrolevin } & \multirow[b]{2}{*}{ Pacionis } & \multirow[b]{2}{*}{ 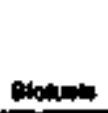 } & \multirow[b]{2}{*}{ Thited } \\
\hline & & & tintery & 에 & 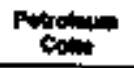 & Totid & & & \\
\hline & \multicolumn{9}{|c|}{ 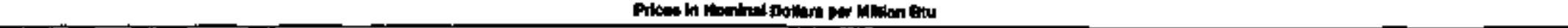 } \\
\hline \multirow[t]{2}{*}{ 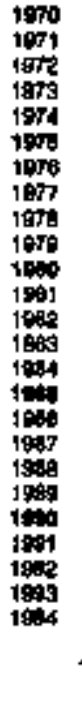 } & 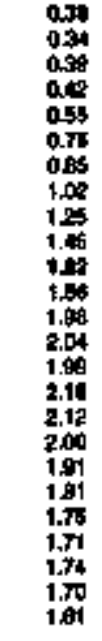 & 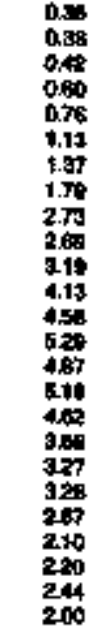 & 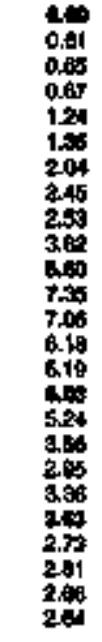 & 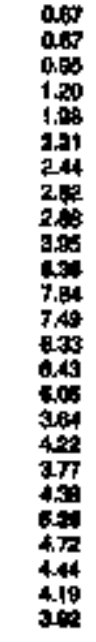 & $\begin{array}{l}= \\
= \\
= \\
= \\
= \\
= \\
= \\
= \\
= \\
= \\
= \\
= \\
=\end{array}$ & 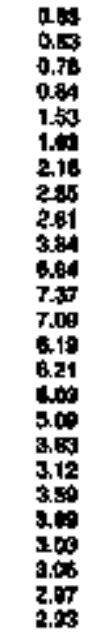 & 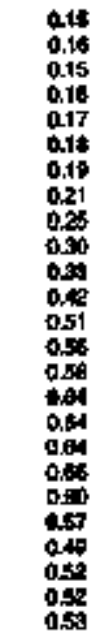 & $\begin{array}{l}0.5 \\
= \\
= \\
= \\
= \\
= \\
= \\
= \\
= \\
= \\
= \\
= \\
1.11 \\
=\end{array}$ & 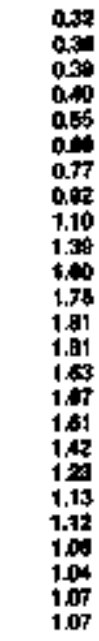 \\
\hline & \multicolumn{9}{|c|}{ 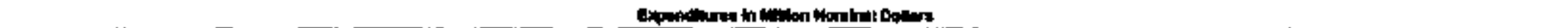 } \\
\hline 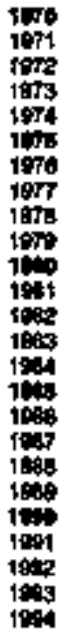 & 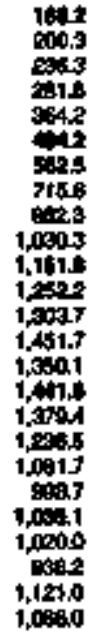 & 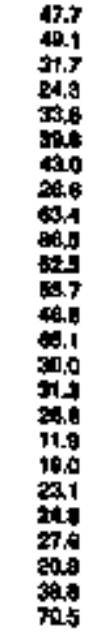 & 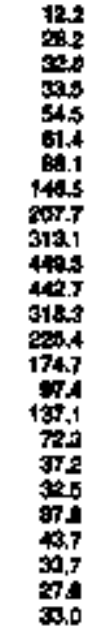 & 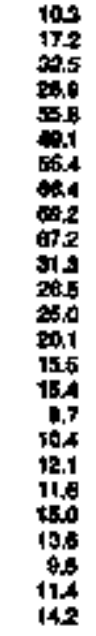 & $\begin{array}{l}= \\
z \\
z \\
= \\
z \\
= \\
= \\
= \\
= \\
= \\
= \\
= \\
= \\
=\end{array}$ & 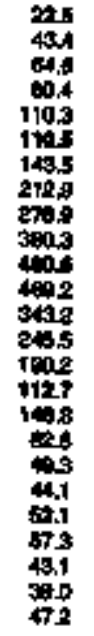 & 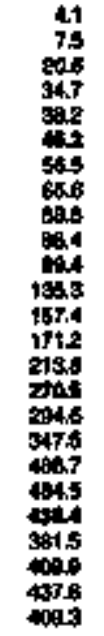 & $\begin{array}{l}= \\
= \\
= \\
= \\
= \\
= \\
= \\
= \\
= \\
= \\
= \\
= \\
= \\
=\end{array}$ & 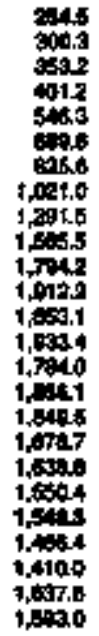 \\
\hline
\end{tabular}

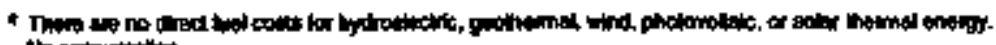

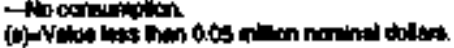

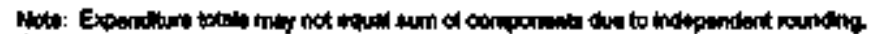

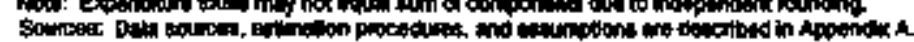


Table 9s, Enorgy Price and Expendlure Esthmates by soures, 1970-1994, incliant

\begin{tabular}{|c|c|c|c|c|c|c|c|c|c|c|c|c|c|c|c|c|c|}
\hline & \multicolumn{14}{|c|}{ Pringy Ementer } & \multirow{3}{*}{ Find } & \multirow{3}{*}{ 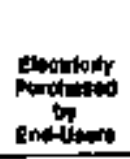 } & \multirow[b]{3}{*}{ Thats } \\
\hline & \multicolumn{3}{|c|}{ cosin } & \multirow[b]{2}{*}{ 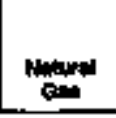 } & \multicolumn{7}{|c|}{ Atortan } & \multirow[b]{2}{*}{$\begin{array}{l}\text { Mucker } \\
\text { Fuel }\end{array}$} & \multirow[b]{2}{*}{ Efothati: } & \multirow[b]{2}{*}{ Tot $x$} & & & \\
\hline & Condne & stim & Totein & & Fin: & Furof & LOQ & 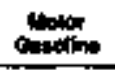 & $\begin{array}{c}\text { Aneldide } \\
\text { Five }\end{array}$ & Onwer " & Totes & & & & & & \\
\hline Yan & \multicolumn{17}{|c|}{ 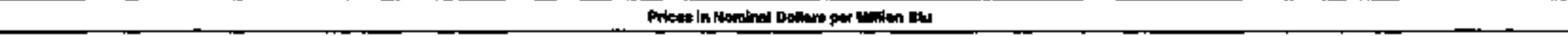 } \\
\hline \multirow[t]{2}{*}{ 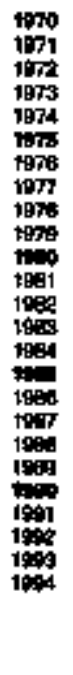 } & 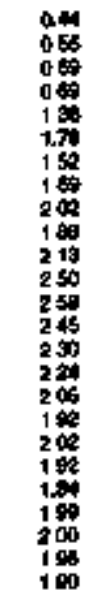 & 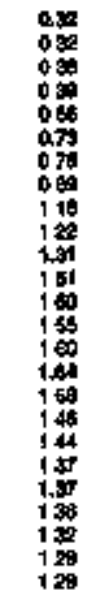 & 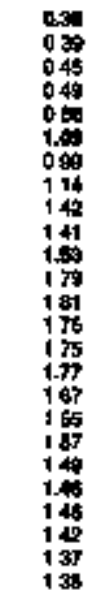 & 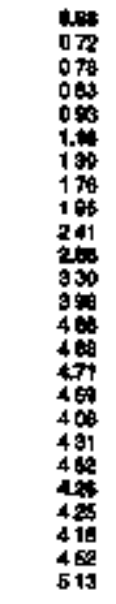 & 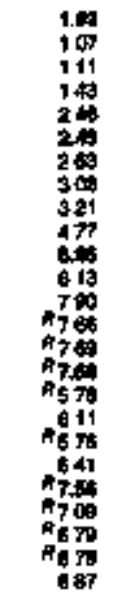 & 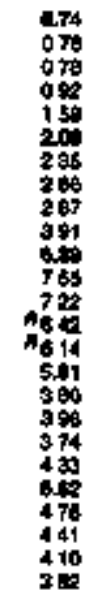 & 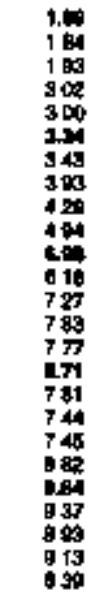 & 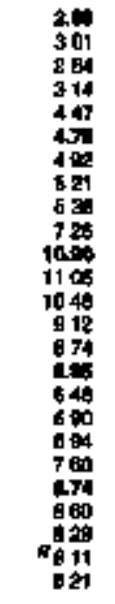 & 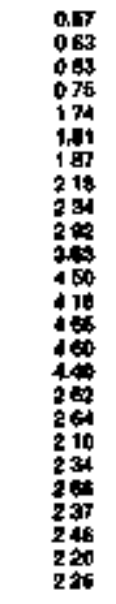 & 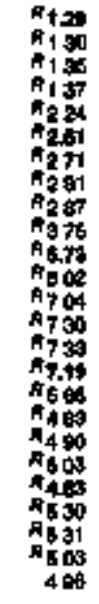 & 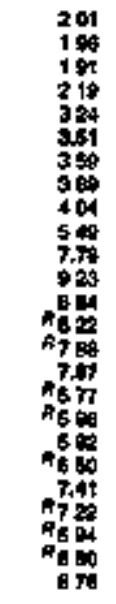 & $\begin{array}{l}= \\
= \\
= \\
= \\
= \\
= \\
= \\
= \\
= \\
= \\
= \\
= \\
= \\
=\end{array}$ & 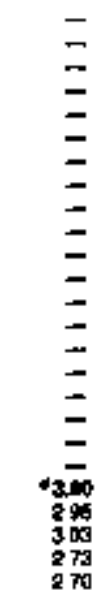 & 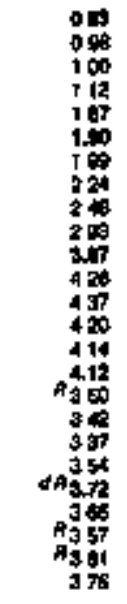 & 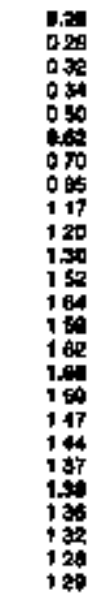 & 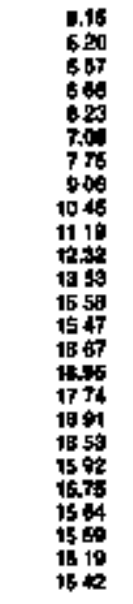 & 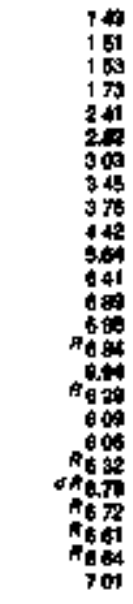 \\
\hline & \multicolumn{17}{|c|}{ 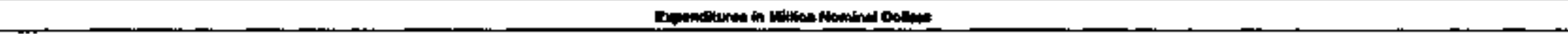 } \\
\hline 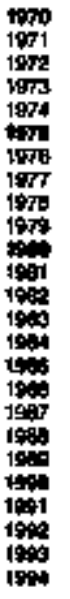 & 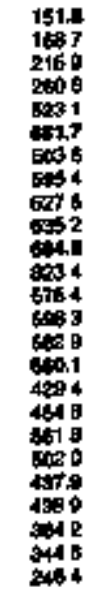 & 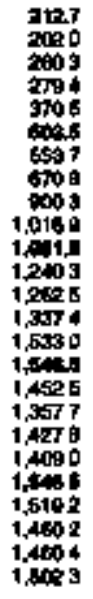 & 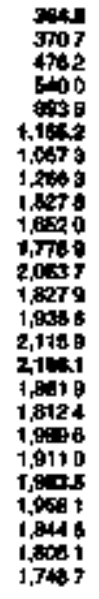 & 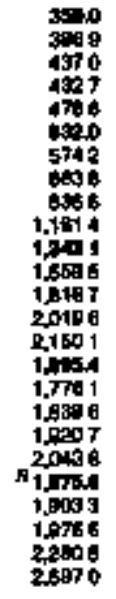 & 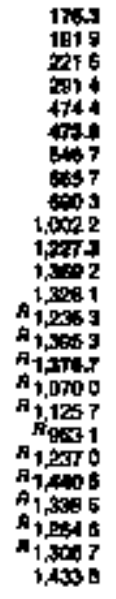 & 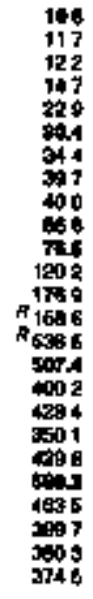 & 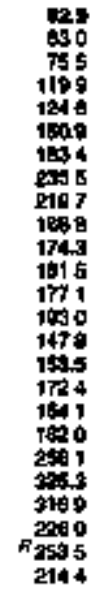 & 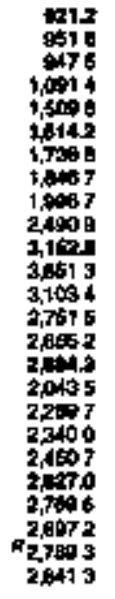 & 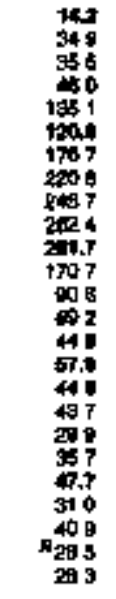 & 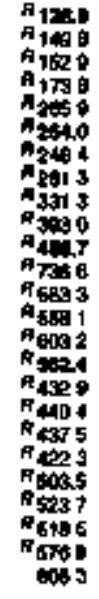 & 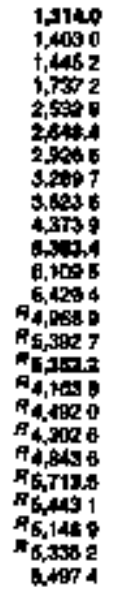 & $\begin{array}{l}= \\
= \\
= \\
= \\
= \\
= \\
= \\
= \\
= \\
= \\
= \\
= \\
= \\
=\end{array}$ & $\begin{array}{l}= \\
= \\
= \\
= \\
= \\
= \\
= \\
= \\
= \\
= \\
= \\
0.15 \\
234 \\
210 \\
140 \\
140\end{array}$ & 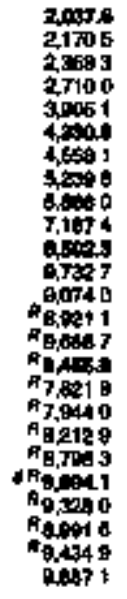 & 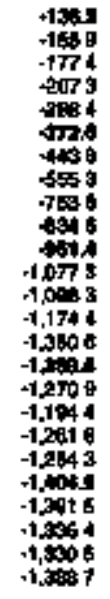 & 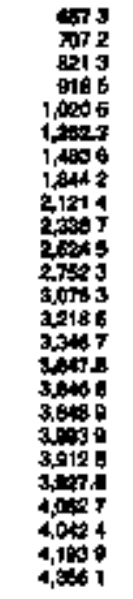 & 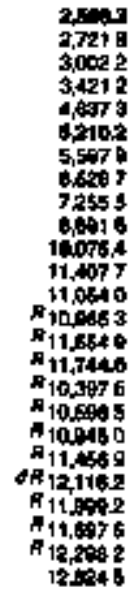 \\
\hline
\end{tabular}

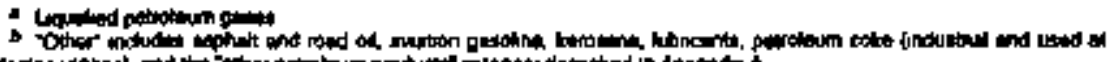

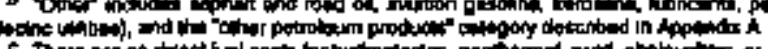

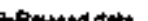

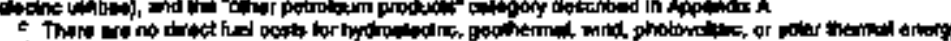

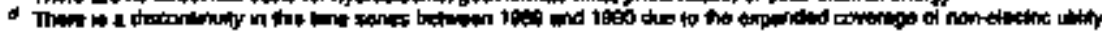

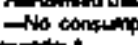

iponte

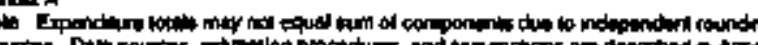

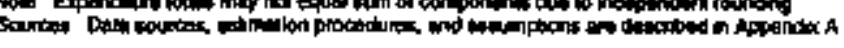




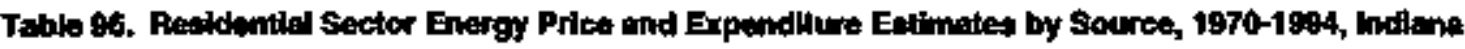

\begin{tabular}{|c|c|c|c|c|c|c|c|c|c|c|}
\hline \multirow{4}{*}{$\mathbf{p e r}$} & \multicolumn{8}{|c|}{ maney Enorgy } & \multirow[b]{3}{*}{ Evedritily } & \multirow[b]{3}{*}{ There } \\
\hline & \multirow[b]{2}{*}{ and } & \multirow[b]{2}{*}{ 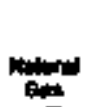 } & \multicolumn{4}{|c|}{ Puinuman } & \multirow[b]{2}{*}{ Obtwoing" } & \multirow[b]{2}{*}{ Tनыс } & & \\
\hline & & & finiming & 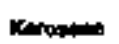 & LPa" & Tyent & & & & \\
\hline & \multicolumn{9}{|c|}{ 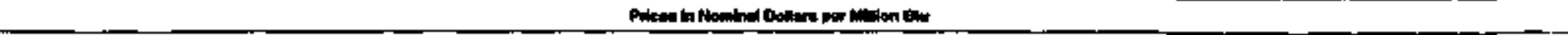 } & \\
\hline \multirow[t]{2}{*}{ 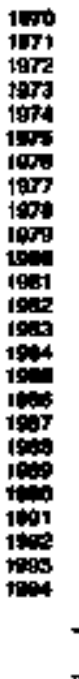 } & 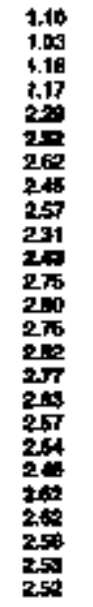 & 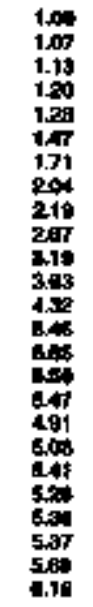 & 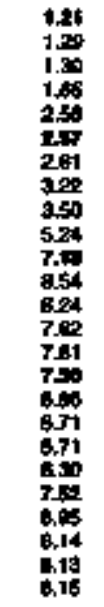 & 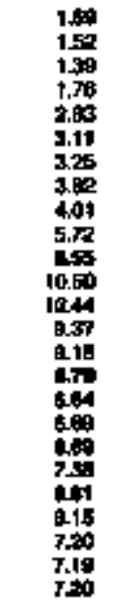 & 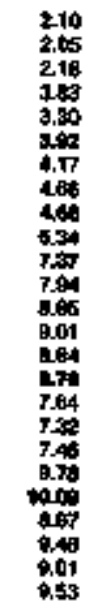 & 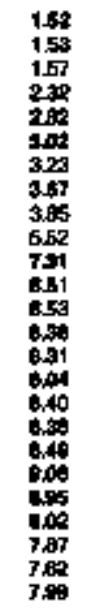 & $\begin{array}{r}= \\
= \\
= \\
= \\
= \\
= \\
= \\
= \\
= \\
= \\
= \\
= \\
0.0 \\
3.50 \\
3.56 \\
3.50 \\
3.50\end{array}$ & 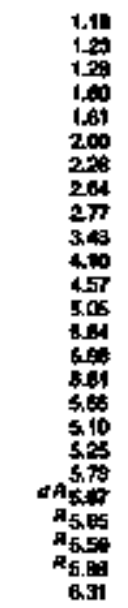 & 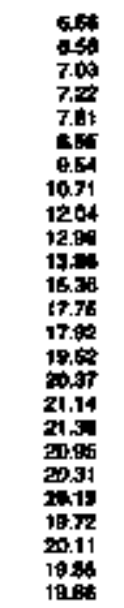 & 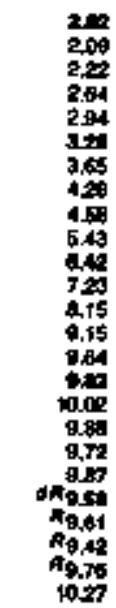 \\
\hline & \multicolumn{10}{|c|}{ 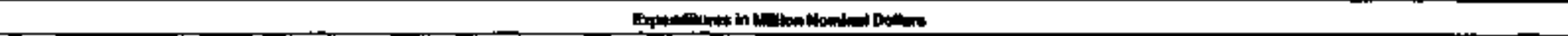 } \\
\hline 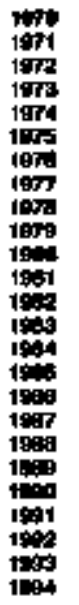 & 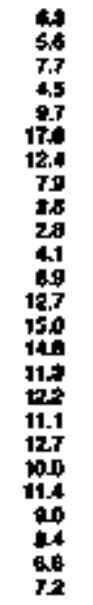 & 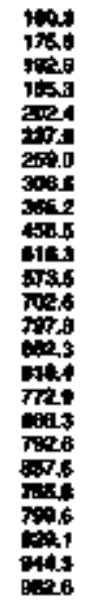 & 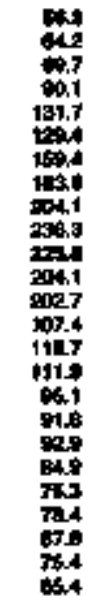 & 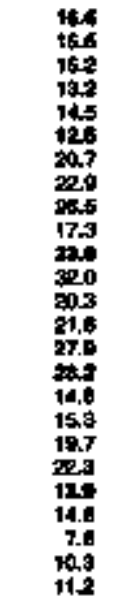 & 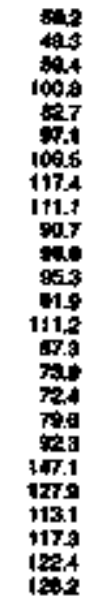 & 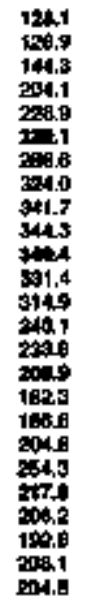 & $\begin{array}{l}= \\
= \\
= \\
= \\
= \\
= \\
= \\
= \\
= \\
= \\
= \\
= \\
10.1 \\
190 \\
0.0 \\
1030 \\
10.1\end{array}$ & 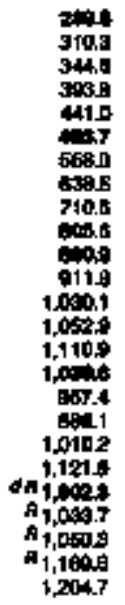 & 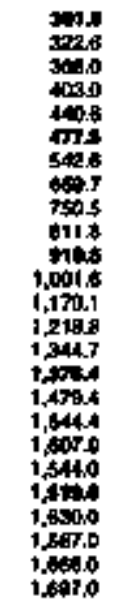 & 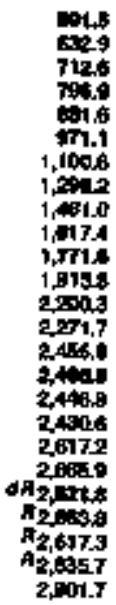 \\
\hline
\end{tabular}

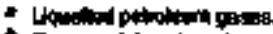

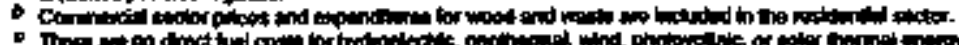

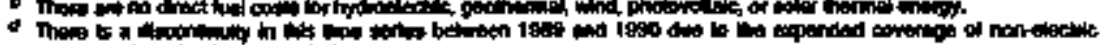

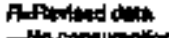

The consurpotion.

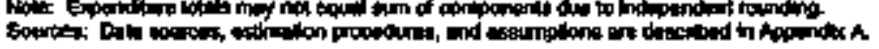

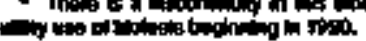


Table 97. Commercial Sector Energy Pilce and Expenditure Extimutes by Soerce, 1970-1994, Indiana

\begin{tabular}{|c|c|c|c|c|c|c|c|c|c|c|c|}
\hline \multirow[b]{4}{*}{ Yetr } & \multicolumn{9}{|c|}{ 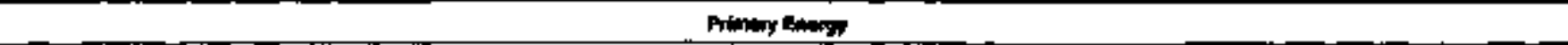 } & \multirow[b]{3}{*}{ Euctitely } & \multirow[b]{3}{*}{ 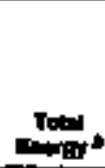 } \\
\hline & \multirow[b]{2}{*}{ on } & \multirow[b]{2}{*}{ 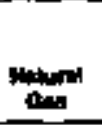 } & \multicolumn{6}{|c|}{ 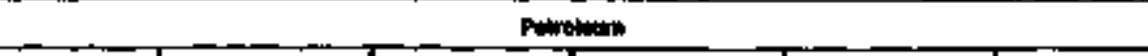 } & \multirow[b]{2}{*}{ Totes } & & \\
\hline & & & 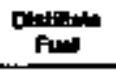 & 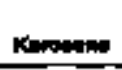 & LPQ* & 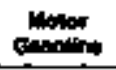 & Fonfleted & toter & & & \\
\hline & \multicolumn{11}{|c|}{ 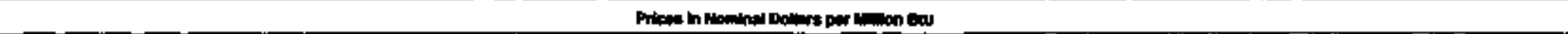 } \\
\hline \multirow[t]{2}{*}{ 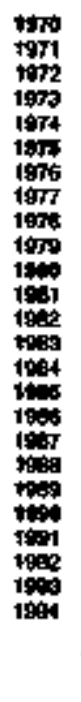 } & 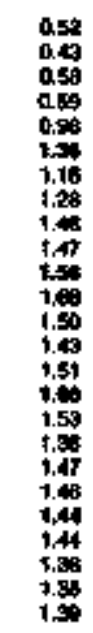 & 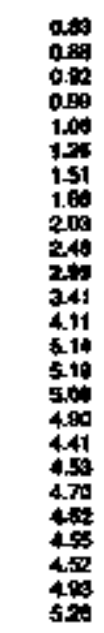 & 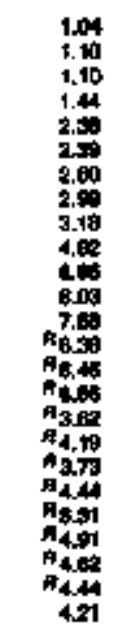 & 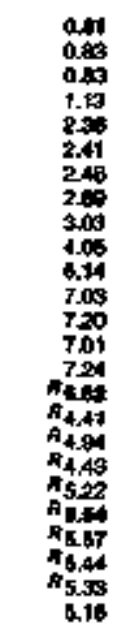 & 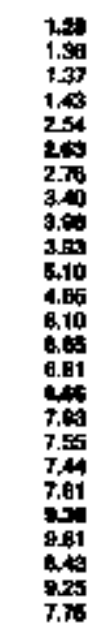 & 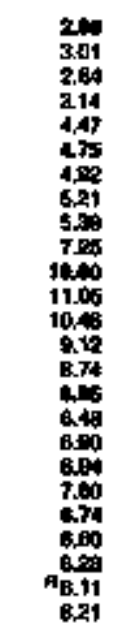 & 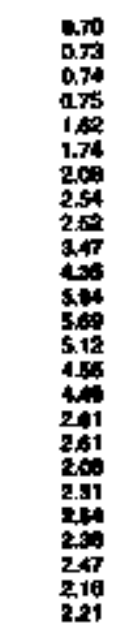 & 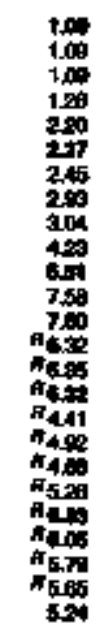 & 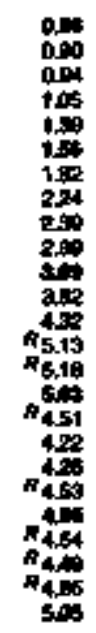 & 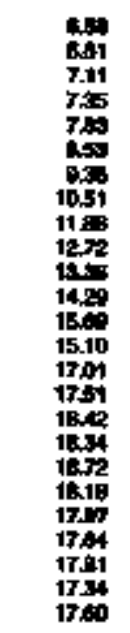 & 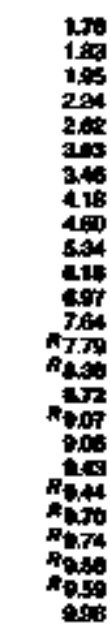 \\
\hline & \multicolumn{11}{|c|}{ 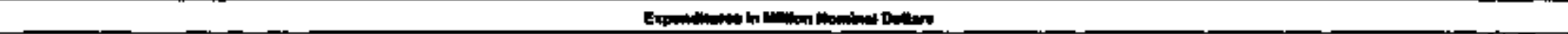 } \\
\hline 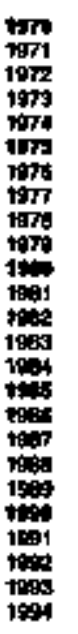 & 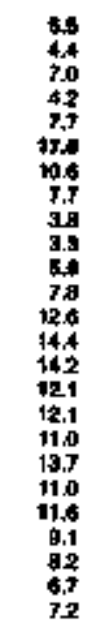 & 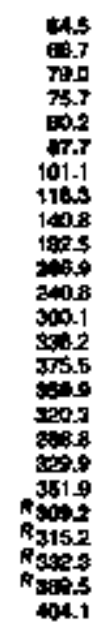 & 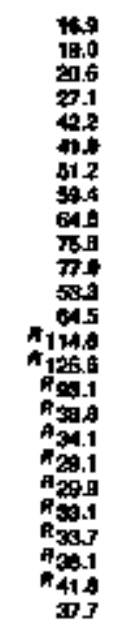 & 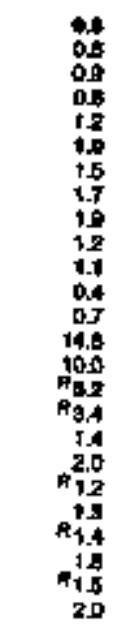 & 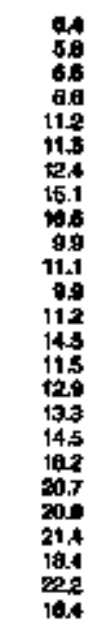 & 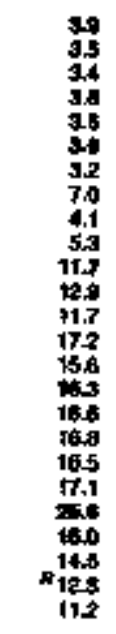 & 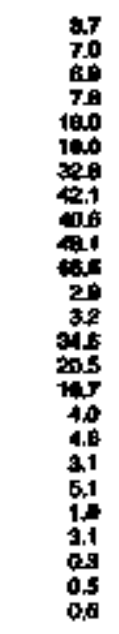 & 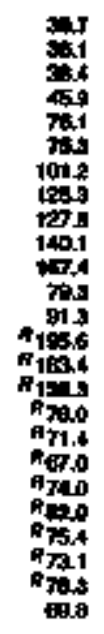 & 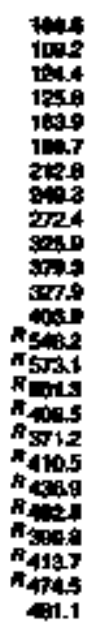 & 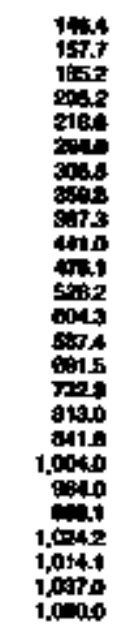 & 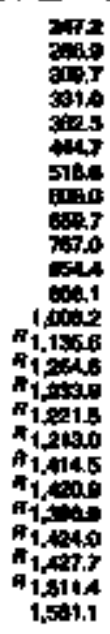 \\
\hline
\end{tabular}

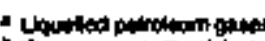

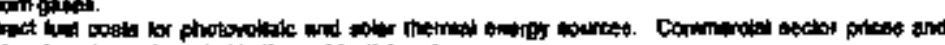

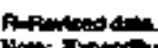

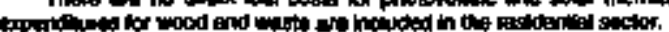

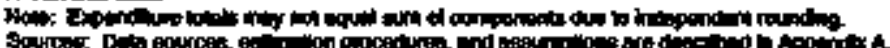


Toble 48. Industried Sector Energy Price and Expenciture Estimatea by Source, 1970-1994, Indiane

\begin{tabular}{|c|c|c|c|c|c|c|c|c|c|c|c|c|c|c|c|c|c|}
\hline & \multicolumn{15}{|c|}{ minny Bant } & \multirow[b]{3}{*}{ Endresty } & \multirow[b]{3}{*}{ Emaryo } \\
\hline & \multicolumn{3}{|c|}{$\infty$} & \multirow[b]{2}{*}{ ansinent } & \multicolumn{9}{|c|}{ Potromenten } & \multirow[b]{2}{*}{ Bolveng" } & \multirow[b]{2}{*}{$\operatorname{Tat}^{0}$} & & \\
\hline & colose & $\mathrm{Con}$ & $7+\infty$ & & 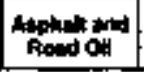 & 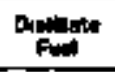 & Kwoments & $\angle 00 \%$ & 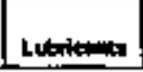 & mations & nentions & Oumer t & Fom & & & & \\
\hline Venr & \multicolumn{17}{|c|}{ 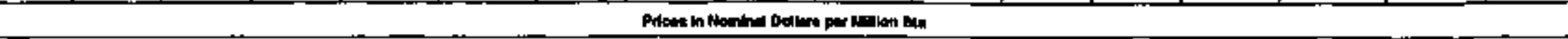 } \\
\hline \multirow[t]{2}{*}{ 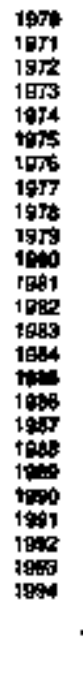 } & 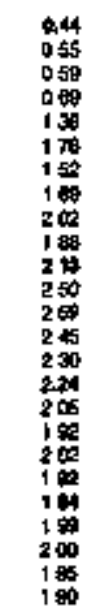 & 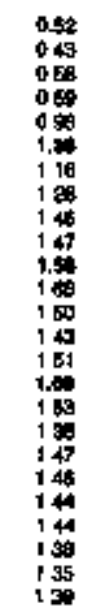 & 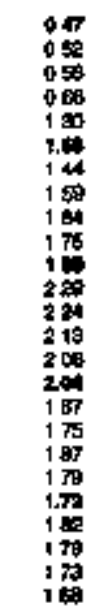 & 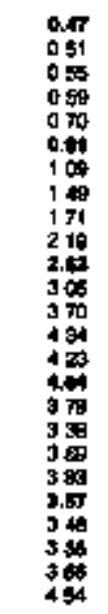 & 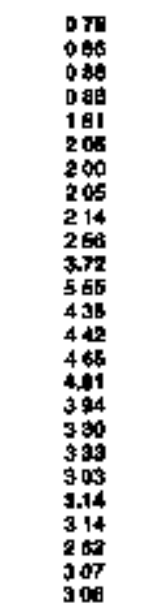 & 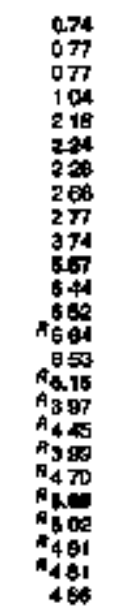 & 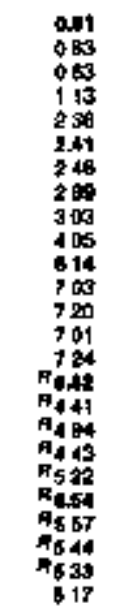 & 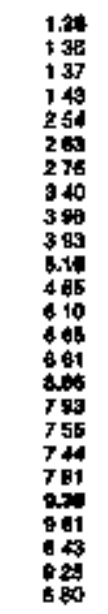 & 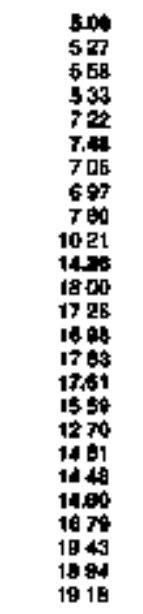 & 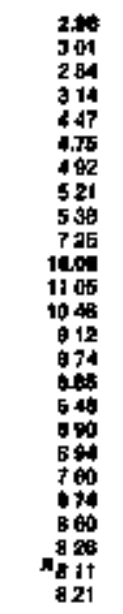 & 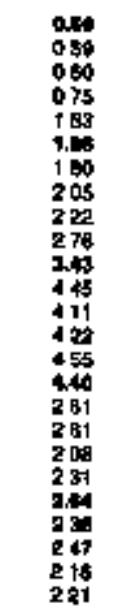 & 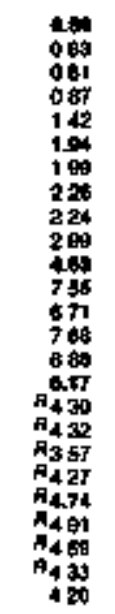 & 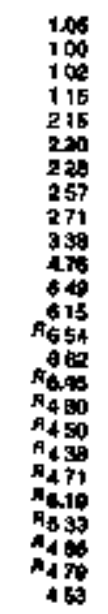 & 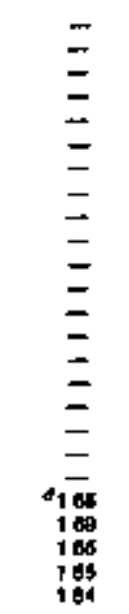 & 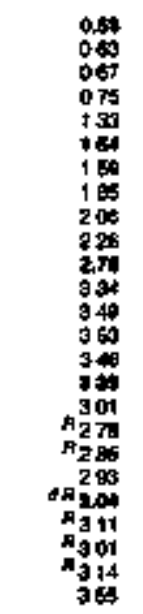 & 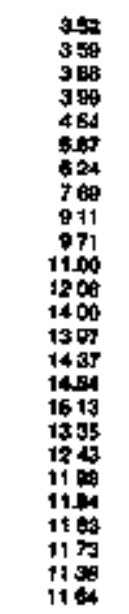 & 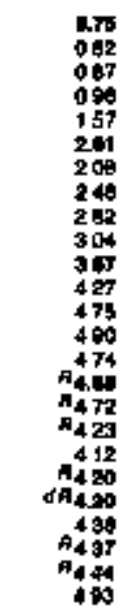 \\
\hline & \multicolumn{17}{|c|}{ 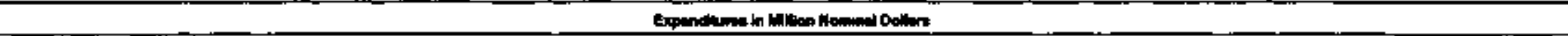 } \\
\hline 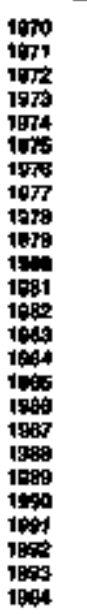 & 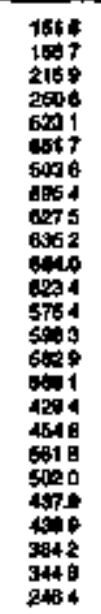 & 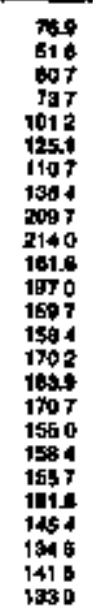 & 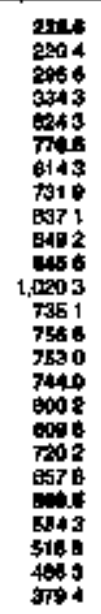 & 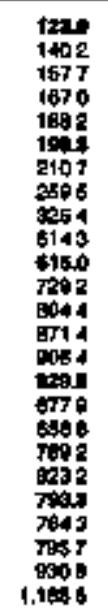 & 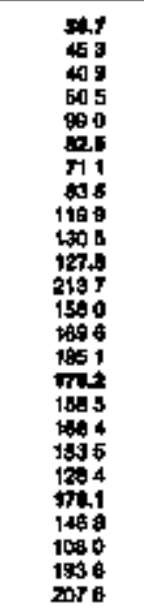 & 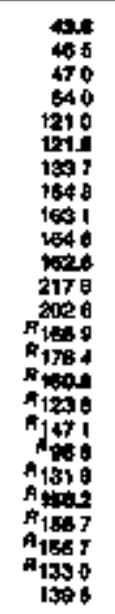 & 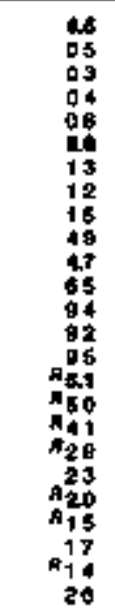 & 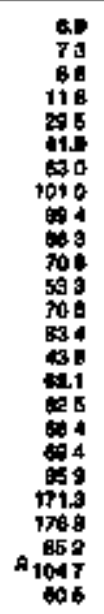 & 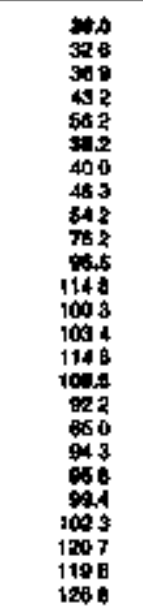 & 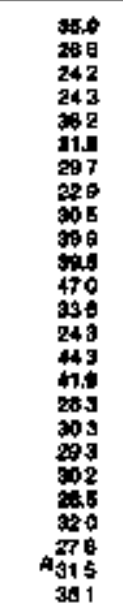 & 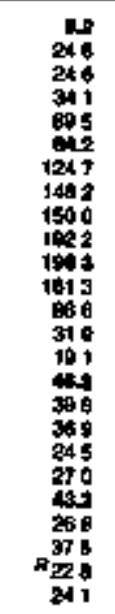 & 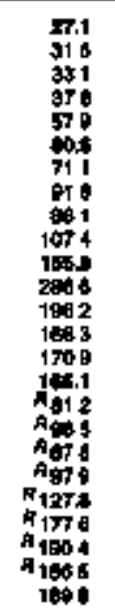 & 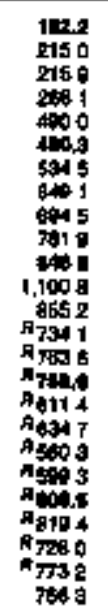 & 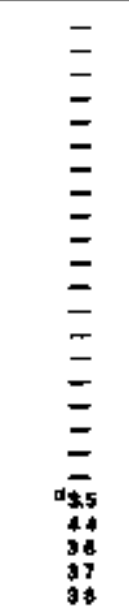 & 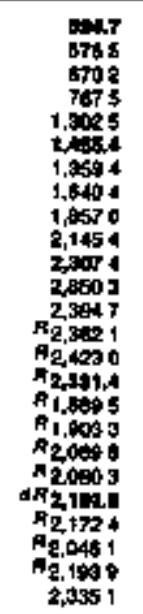 & 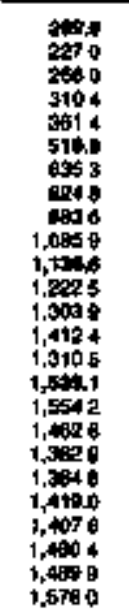 & 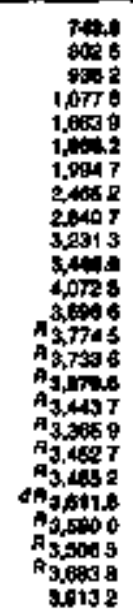 \\
\hline
\end{tabular}

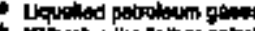

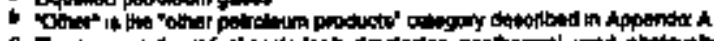

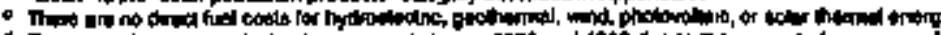

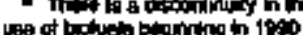

A-Nowled dat

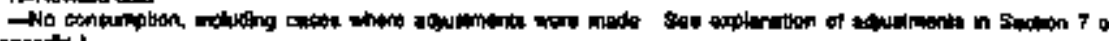
Nopentis A

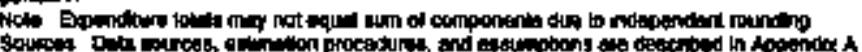

Energy Information Admintetration 


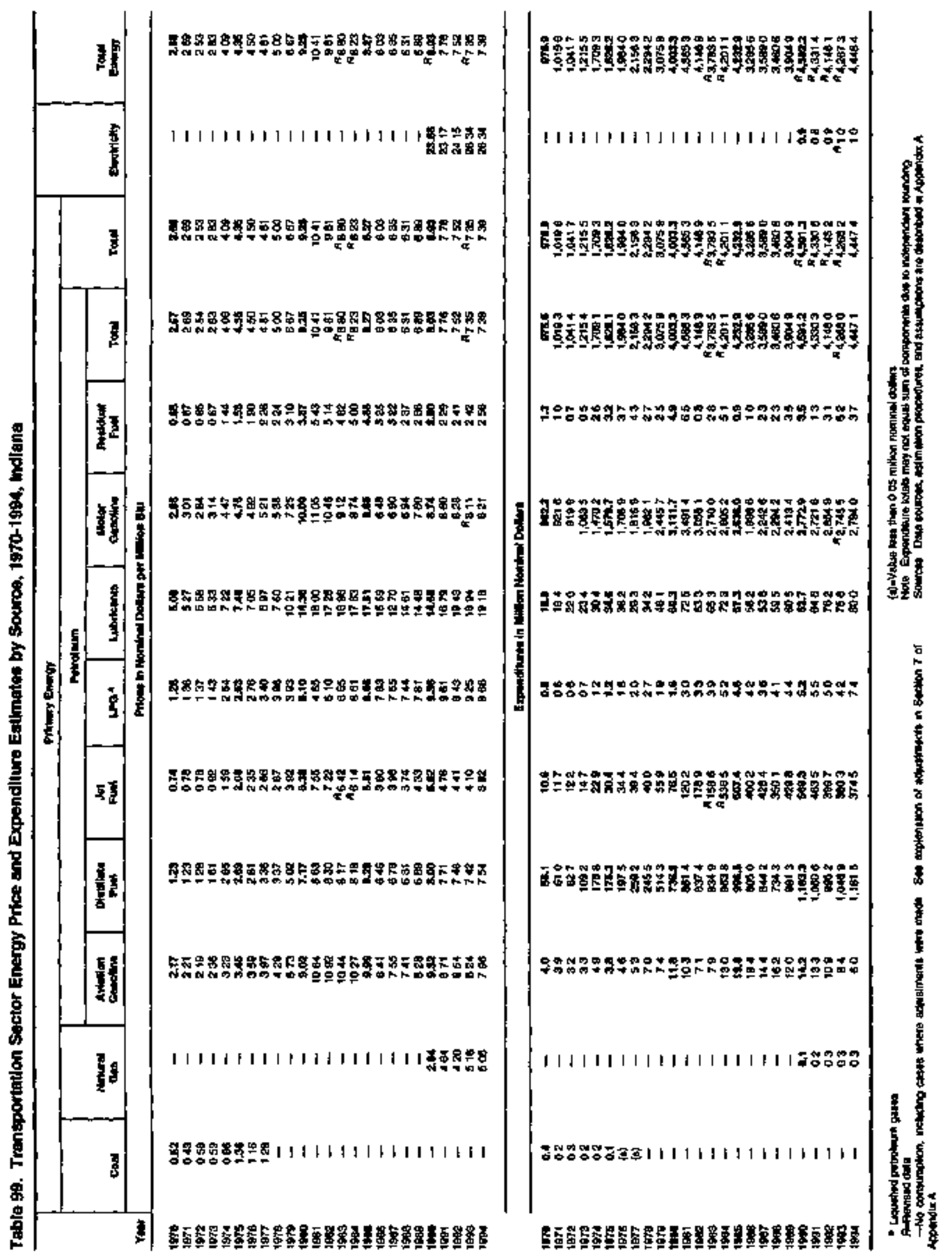




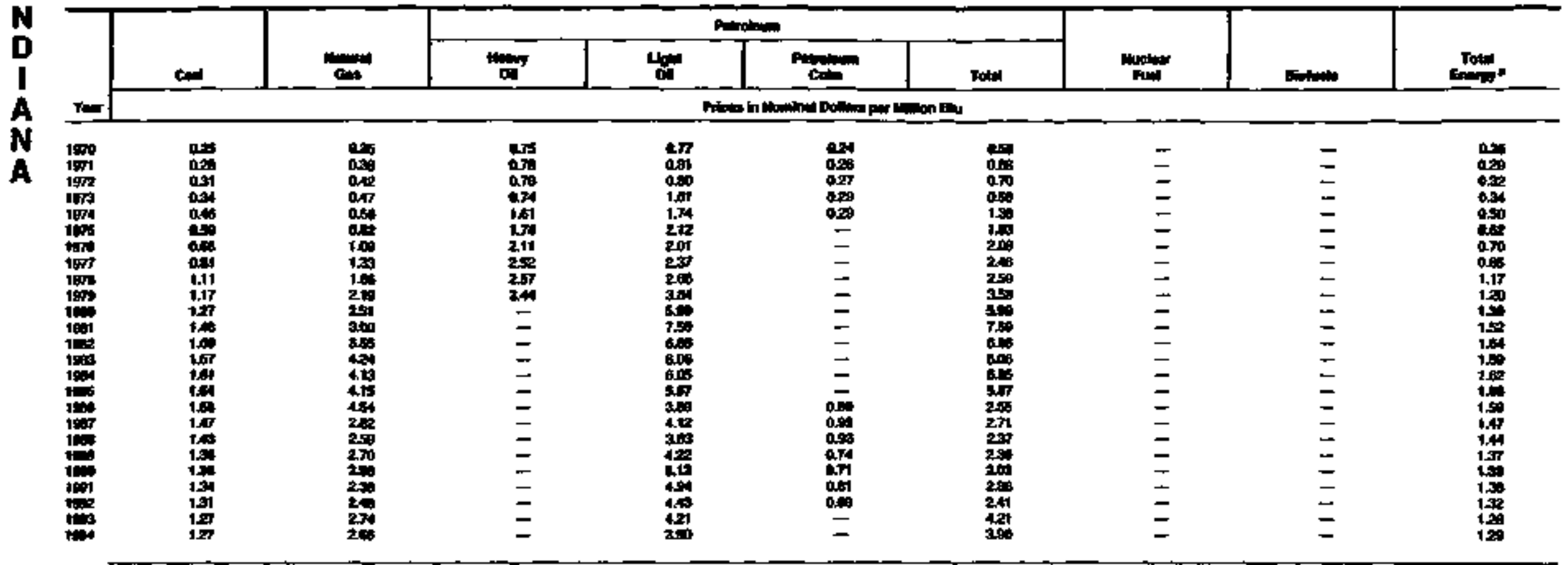

\begin{tabular}{|c|c|c|c|c|c|c|c|c|c|}
\hline \multirow[b]{2}{*}{ 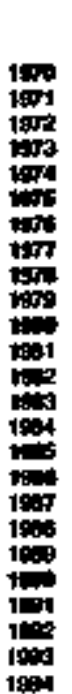 } & \multicolumn{9}{|c|}{ 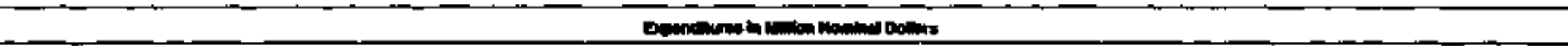 } \\
\hline & 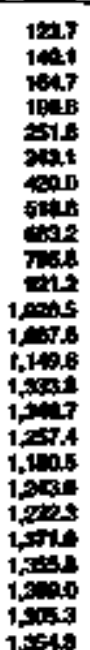 & 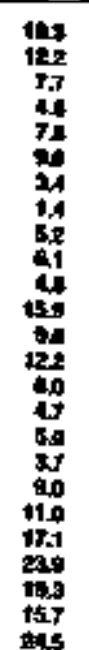 & 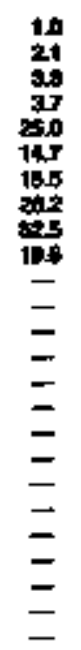 & 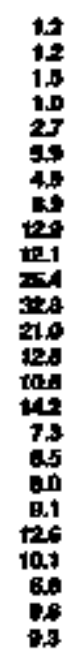 & 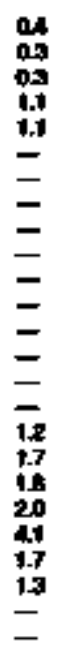 & 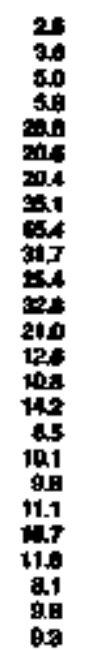 & $\begin{array}{l}z \\
z \\
z \\
z \\
z \\
z \\
z \\
z \\
z \\
z \\
z \\
z \\
z\end{array}$ & $\begin{array}{l}z \\
z \\
z \\
z \\
\bar{z} \\
\bar{z} \\
\vdots \\
\bar{z} \\
\vdots \\
\vdots \\
z \\
\vdots \\
z\end{array}$ & 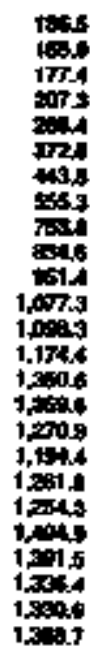 \\
\hline
\end{tabular}




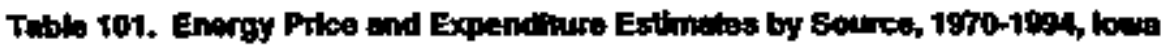

\begin{tabular}{|c|c|c|c|c|c|c|c|c|c|c|c|c|c|c|c|c|c|}
\hline & \multicolumn{14}{|c|}{ 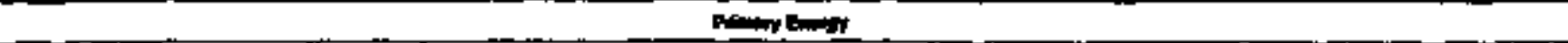 } & \multirow{3}{*}{ 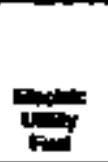 } & \multirow{3}{*}{ 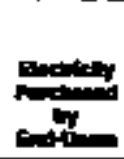 } & \multirow[b]{3}{*}{ (1) } \\
\hline & \multicolumn{3}{|c|}{ Con } & & \multicolumn{7}{|c|}{ 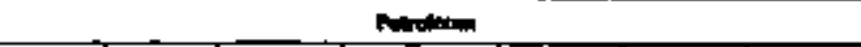 } & \multirow[b]{2}{*}{ 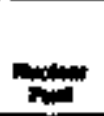 } & \multirow[b]{2}{*}{ 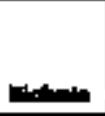 } & \multirow[b]{2}{*}{ thenc } & & & \\
\hline & 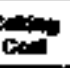 & تمش & Tat & 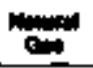 & ons & H & Lيe" & & 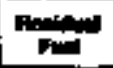 & tene" & Tom & & & & & & \\
\hline Yom & \multicolumn{17}{|c|}{ 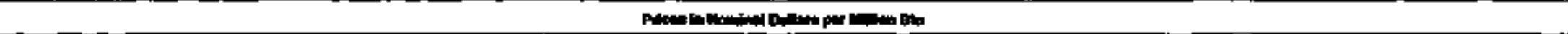 } \\
\hline \multirow[t]{2}{*}{ 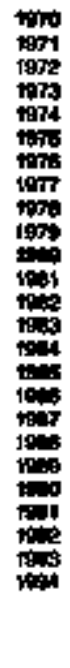 } & $\begin{array}{l}= \\
= \\
= \\
= \\
= \\
= \\
= \\
= \\
= \\
= \\
= \\
= \\
= \\
=\end{array}$ & 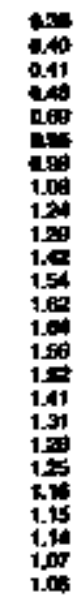 & 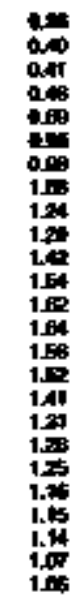 & 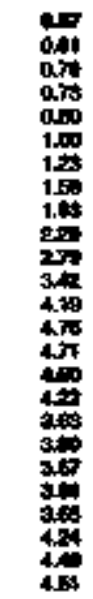 & 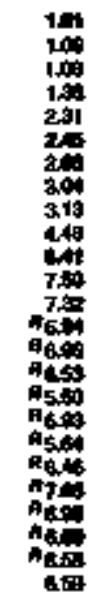 & 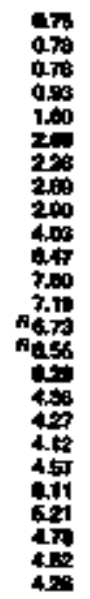 & 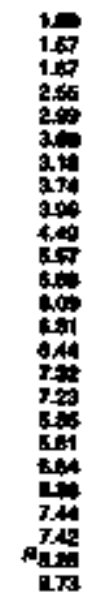 & 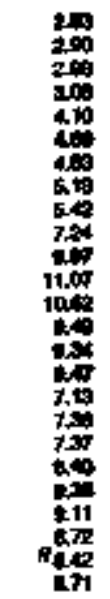 & 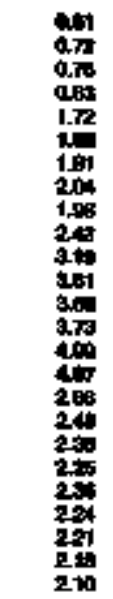 & 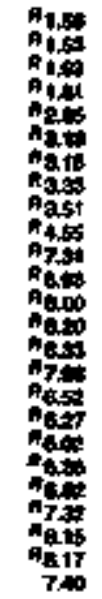 & 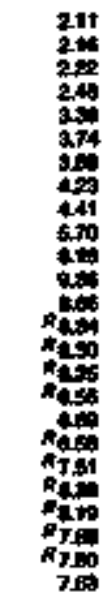 & 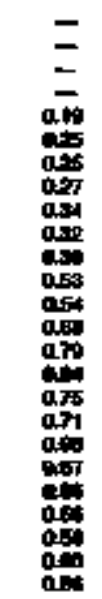 & 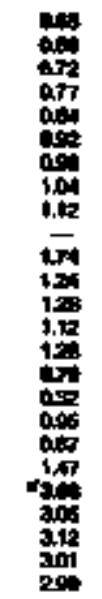 & 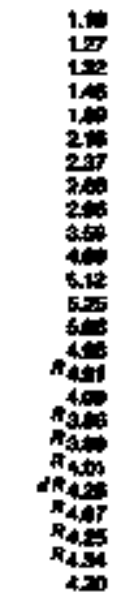 & 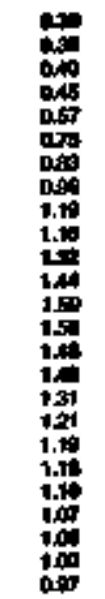 & 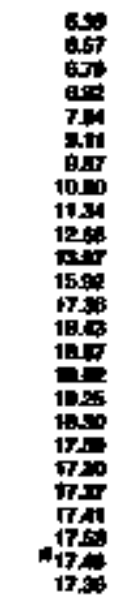 & 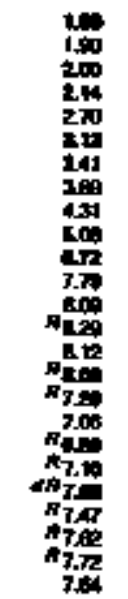 \\
\hline & \multicolumn{17}{|c|}{ 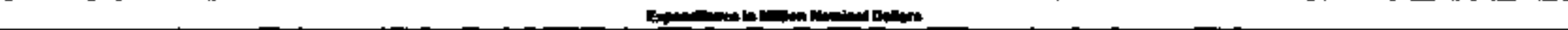 } \\
\hline 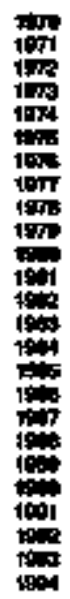 & $\begin{array}{l}= \\
= \\
= \\
= \\
= \\
= \\
= \\
= \\
= \\
= \\
= \\
= \\
=\end{array}$ & 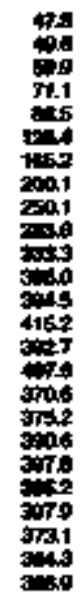 & 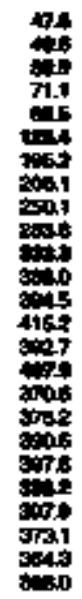 & 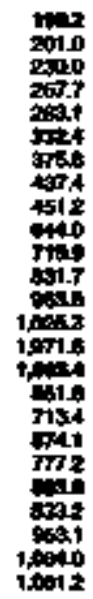 & 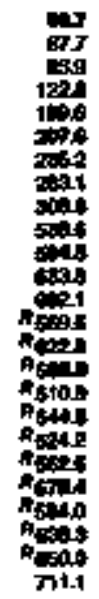 & 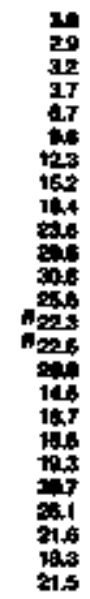 & 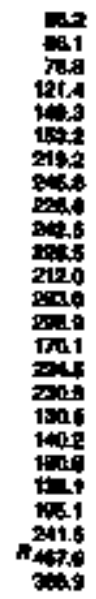 & 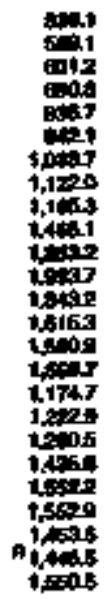 & 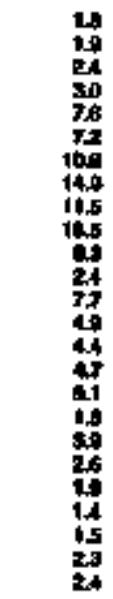 & 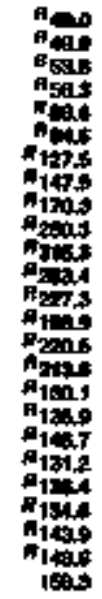 & 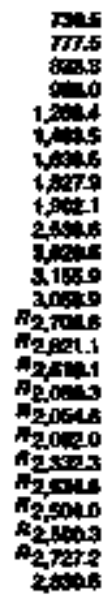 & 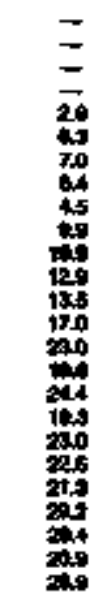 & 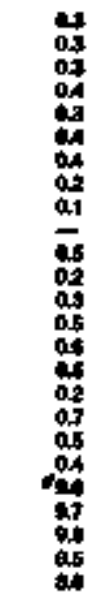 & 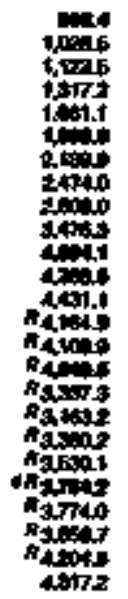 & 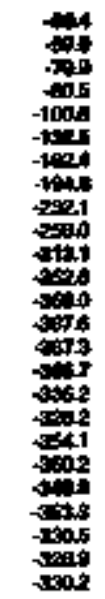 & 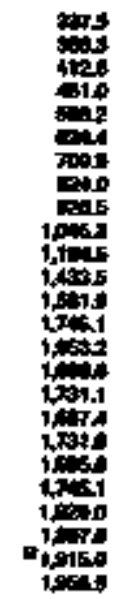 & 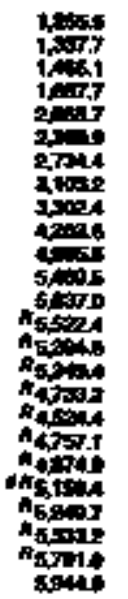 \\
\hline
\end{tabular}




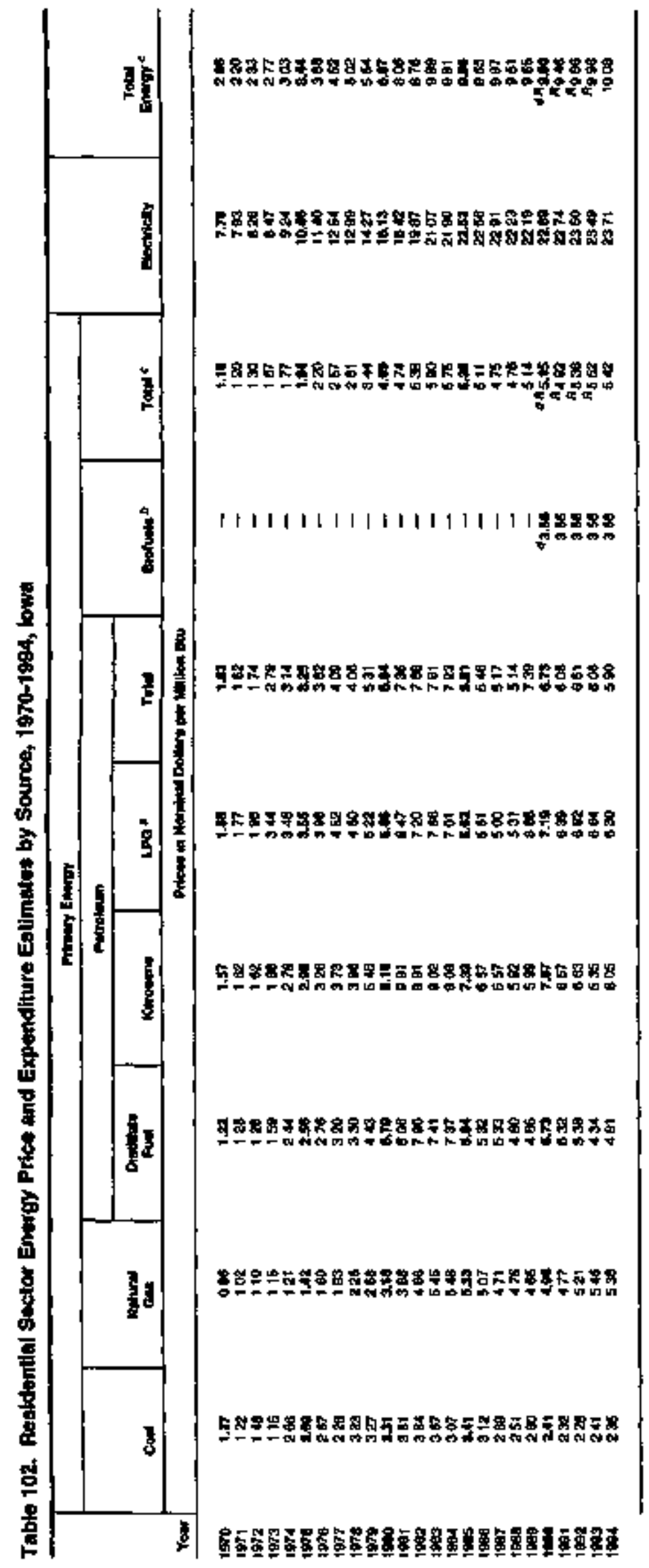

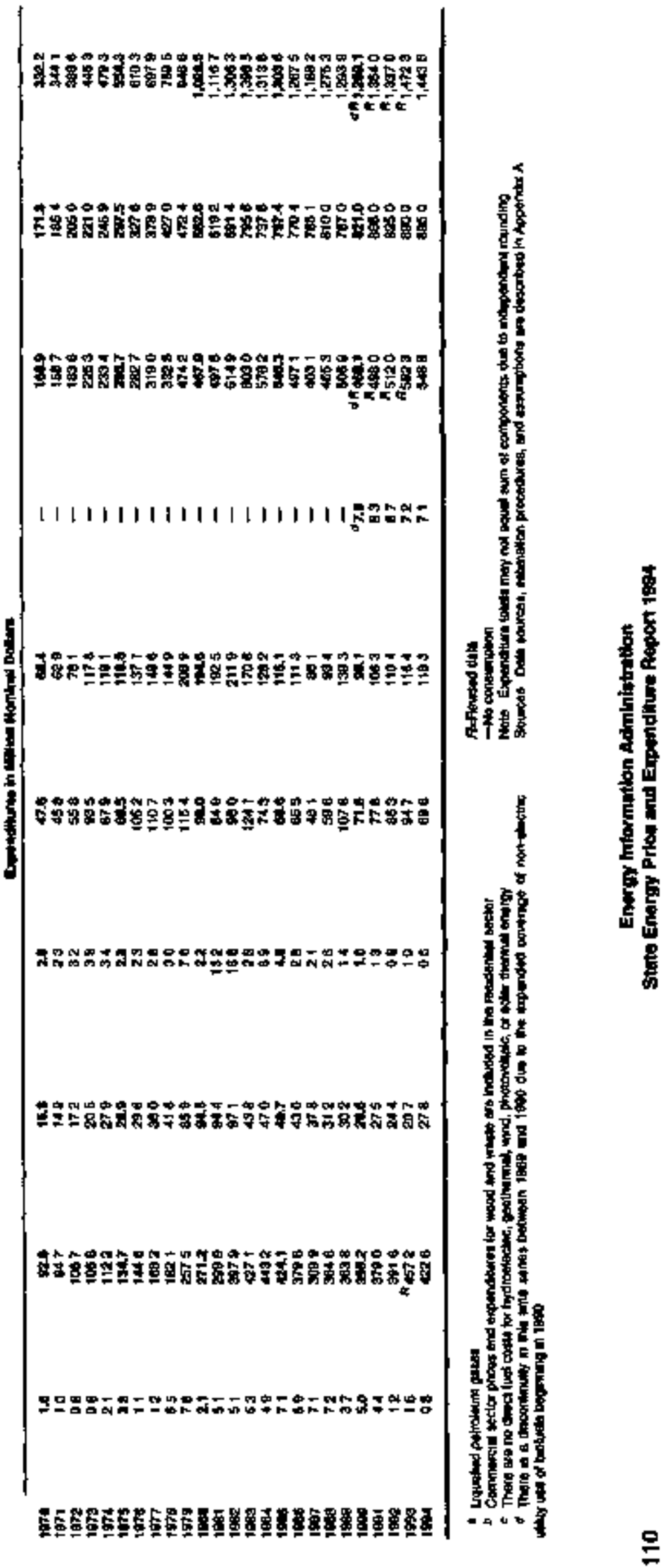




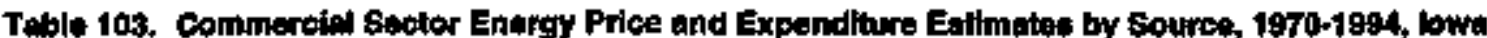

\begin{tabular}{|c|c|c|c|c|c|c|c|c|c|c|c|}
\hline \multirow[b]{4}{*}{ Yur } & \multicolumn{9}{|c|}{ Prtoney Enary } & \multirow[b]{3}{*}{ Evetionty } & \multirow[b]{3}{*}{ Iody. } \\
\hline & \multirow[b]{2}{*}{ Cond } & \multirow[b]{2}{*}{ Gente } & \multicolumn{6}{|c|}{ Potrolanim } & \multirow[b]{2}{*}{ Tot } & & \\
\hline & & & Dinger & 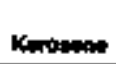 & Lo: & 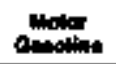 & Aneldyen & Totat & & & \\
\hline & \multicolumn{11}{|c|}{ 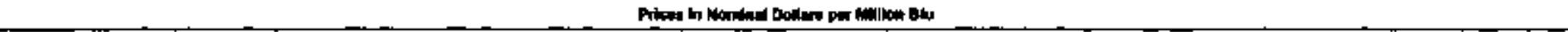 } \\
\hline \multirow[t]{2}{*}{ 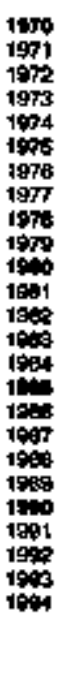 } & 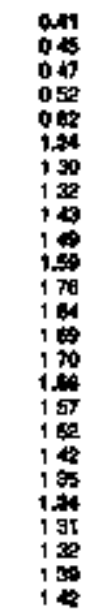 & 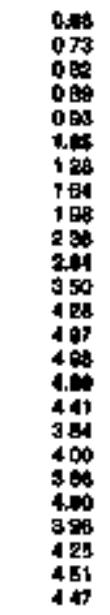 & 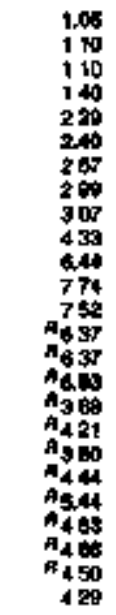 & 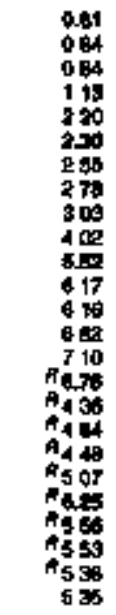 & 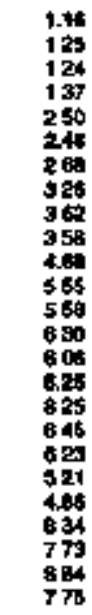 & 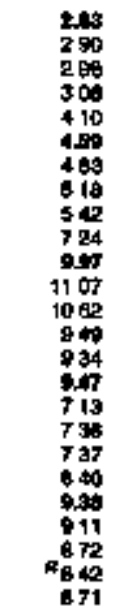 & 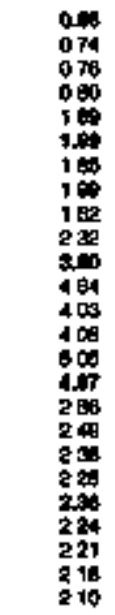 & 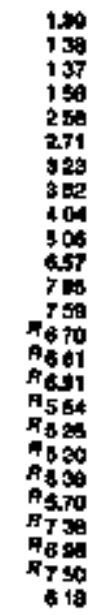 & 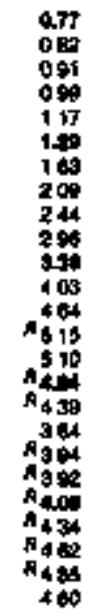 & 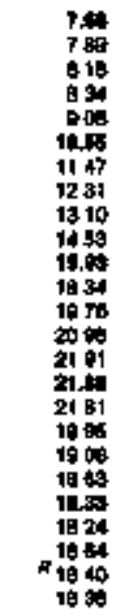 & 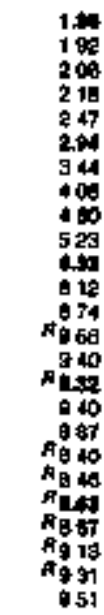 \\
\hline & \multicolumn{11}{|c|}{ 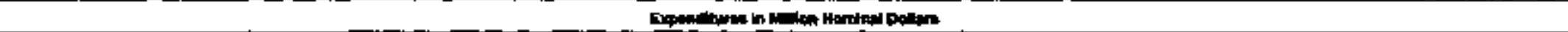 } \\
\hline 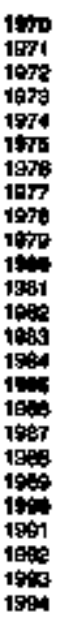 & $\begin{array}{l}10 \\
07 \\
06 \\
08 \\
12 \\
20 \\
10 \\
14 \\
48 \\
68 \\
19 \\
17 \\
44 \\
46 \\
40 \\
64 \\
65 \\
69 \\
72 \\
35 \\
91 \\
48 \\
13 \\
14 \\
09\end{array}$ & 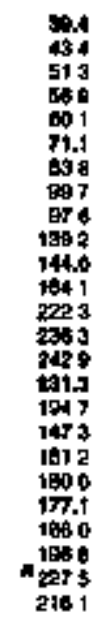 & 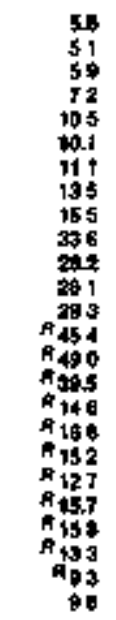 & 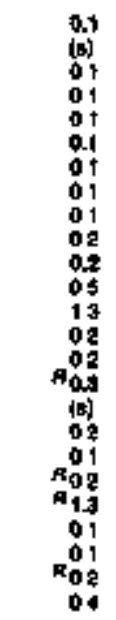 & 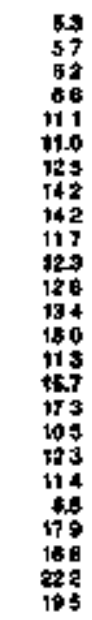 & 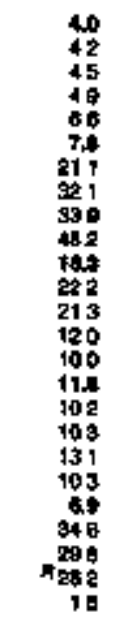 & $\begin{array}{l}43 \\
03 \\
03 \\
08 \\
18 \\
12 \\
17 \\
25 \\
18 \\
35 \\
19 \\
08 \\
07 \\
01 \\
01 \\
69 \\
07 \\
03 \\
03 \\
05 \\
05 \\
01 \\
05 \\
01 \\
101\end{array}$ & 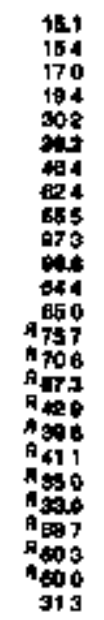 & 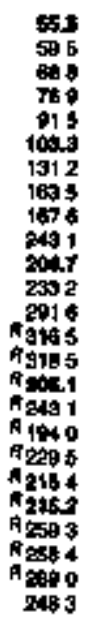 & 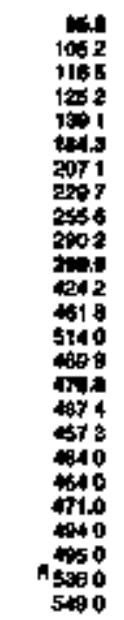 & 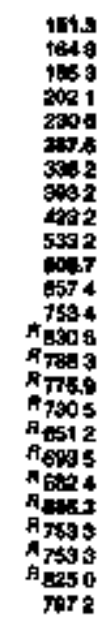 \\
\hline
\end{tabular}

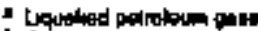

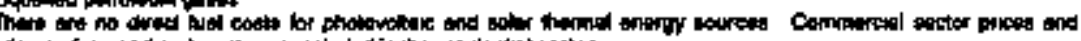

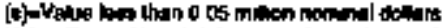

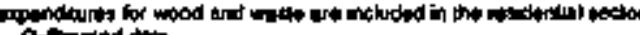

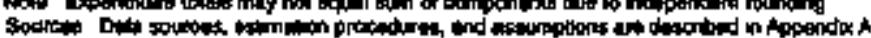




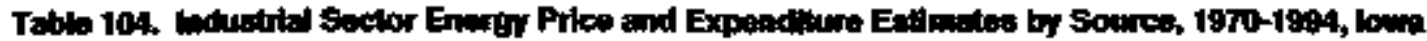

\begin{tabular}{|c|c|c|c|c|c|c|c|c|c|c|c|c|c|c|c|c|c|}
\hline & \multicolumn{15}{|c|}{ Anmytener } & \multirow[b]{3}{*}{ 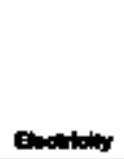 } & \multirow[b]{3}{*}{ thent } \\
\hline & \multicolumn{3}{|c|}{ Conil } & \multirow[b]{2}{*}{ 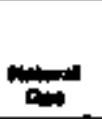 } & \multicolumn{9}{|c|}{ 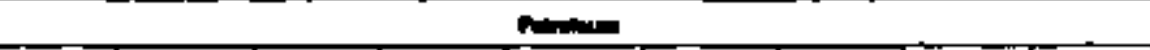 } & \multirow[b]{2}{*}{ 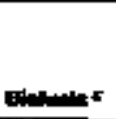 } & \multirow[b]{2}{*}{ Tated } & & \\
\hline & Cante & coll & the & & ה & Dinget & 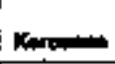 & Lat & Linnowa & & | & ont: & nen- & & & & \\
\hline Vin & \multicolumn{17}{|c|}{ 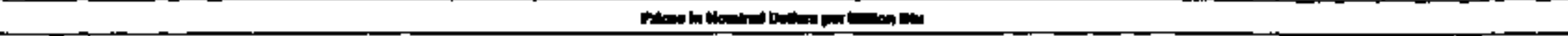 } \\
\hline \multirow[t]{2}{*}{ 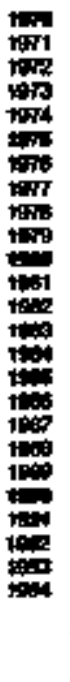 } & $\begin{array}{l}= \\
= \\
= \\
= \\
= \\
= \\
= \\
= \\
= \\
= \\
= \\
= \\
= \\
=\end{array}$ & 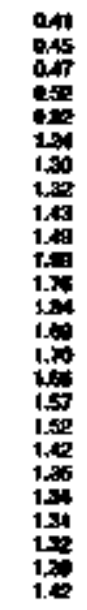 & 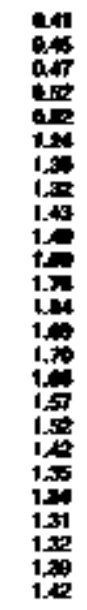 & 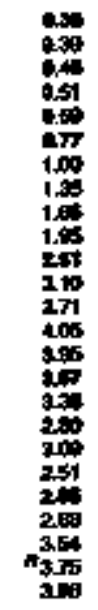 & 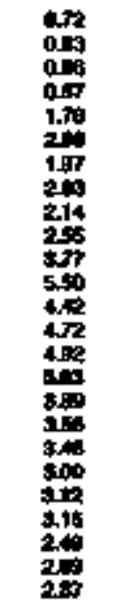 & 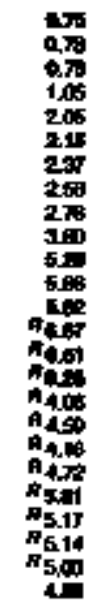 & 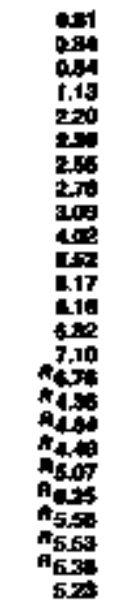 & 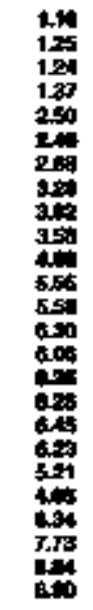 & 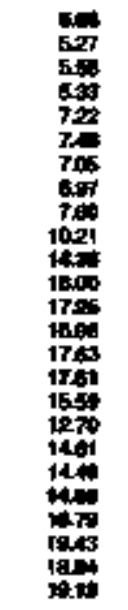 & 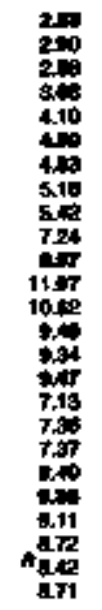 & 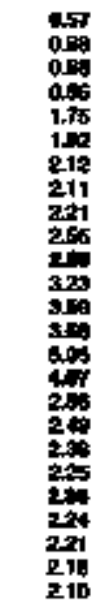 & 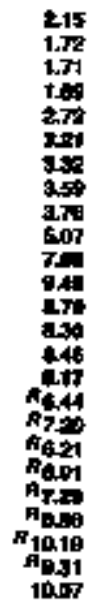 & 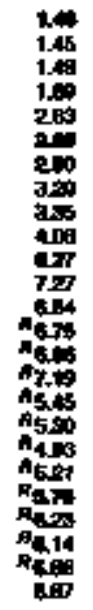 & $\begin{array}{l}\bar{z} \\
= \\
= \\
= \\
= \\
= \\
= \\
= \\
= \\
= \\
= \\
\bar{z} \\
1.68 \\
105 \\
104\end{array}$ & 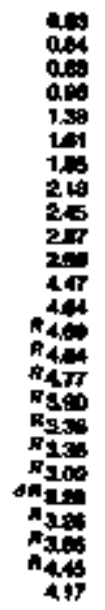 & 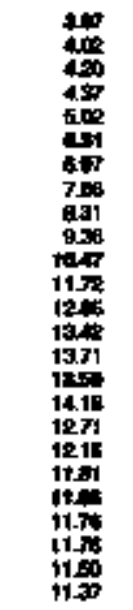 & 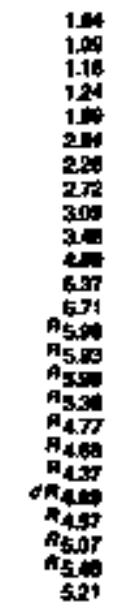 \\
\hline & \multicolumn{17}{|c|}{ 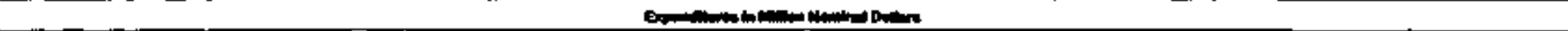 } \\
\hline 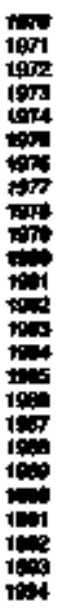 & $\begin{array}{l}= \\
= \\
= \\
= \\
= \\
= \\
= \\
= \\
= \\
= \\
= \\
= \\
= \\
=\end{array}$ & 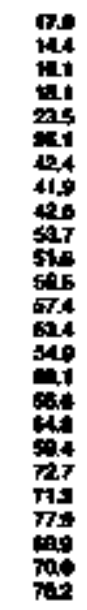 & 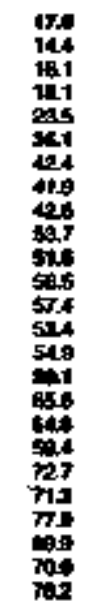 & 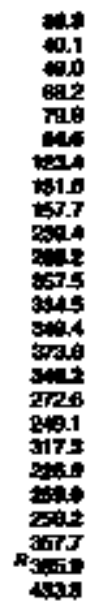 & 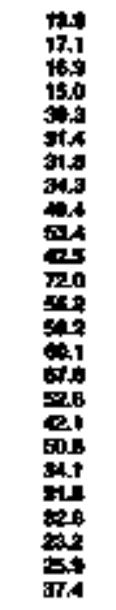 & 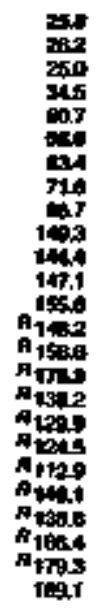 & 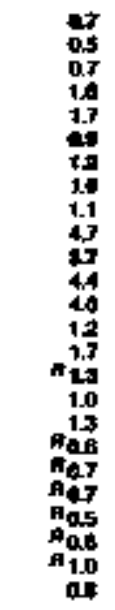 & 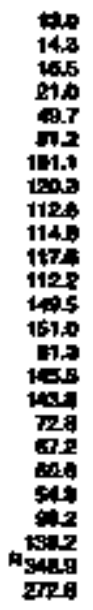 & 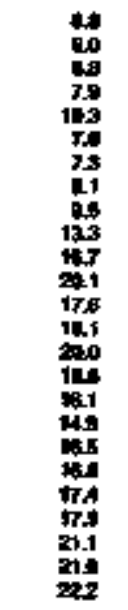 & 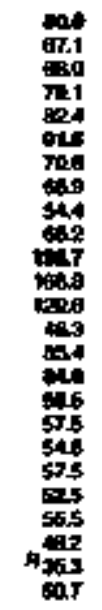 & 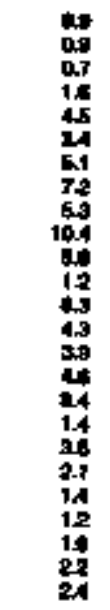 & 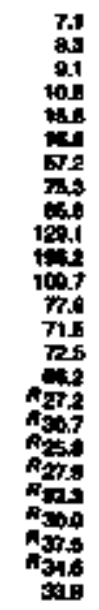 & 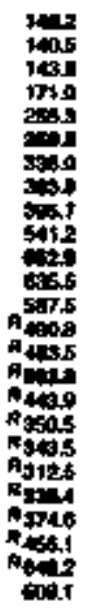 & $\begin{array}{l}= \\
= \\
= \\
= \\
= \\
= \\
= \\
= \\
= \\
= \\
= \\
= \\
0.9 \\
1.1 \\
1.0 \\
1.1\end{array}$ & 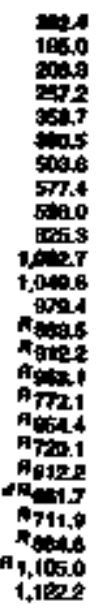 & 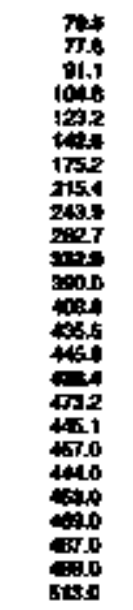 & 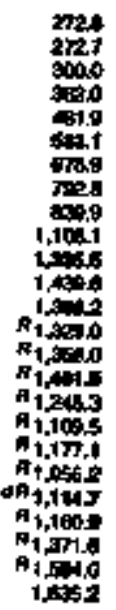 \\
\hline
\end{tabular}

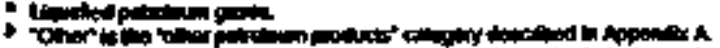

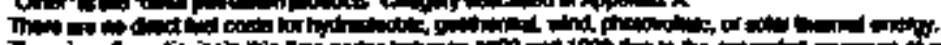

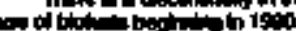

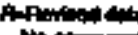

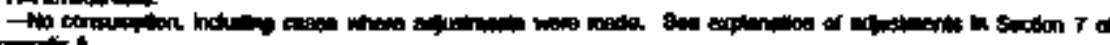
(n)

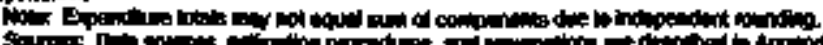

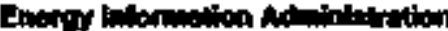




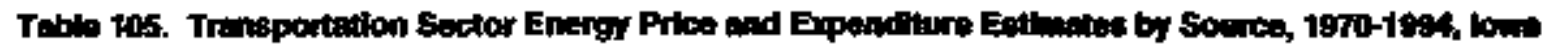

\begin{tabular}{|c|c|c|c|c|c|c|c|c|c|c|c|c|c|}
\hline \multirow[b]{4}{*}{ nim } & \multicolumn{11}{|c|}{ Prtmer Er } & \multirow[b]{3}{*}{ Extory } & \multirow[b]{3}{*}{ This } \\
\hline & & \multirow[b]{2}{*}{ 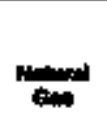 } & \multicolumn{8}{|c|}{ 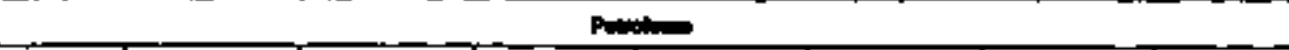 } & \multirow[b]{2}{*}{ Th } & & \\
\hline & con & & 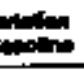 & 1. & in & 1pe: & Intren & chin & R' & rom & & & \\
\hline & \multicolumn{13}{|c|}{ ה" } \\
\hline \multirow[t]{2}{*}{ 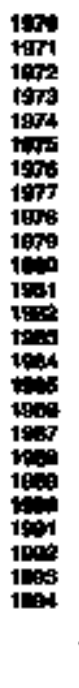 } & $\begin{array}{l}041 \\
046 \\
0.45 \\
0.58 \\
0.8 \\
1,21 \\
130 \\
1: 2 \\
= \\
= \\
= \\
= \\
= \\
= \\
= \\
= \\
= \\
= \\
=\end{array}$ & 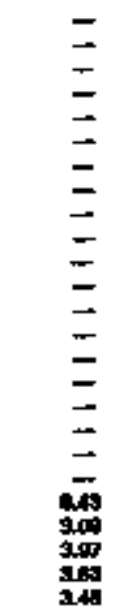 & 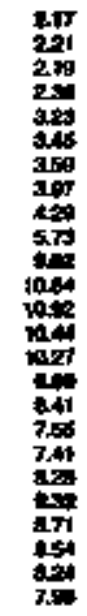 & 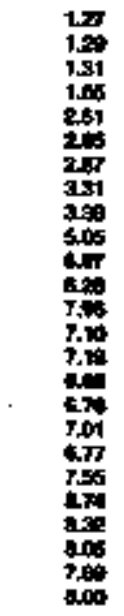 & 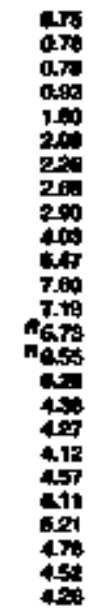 & 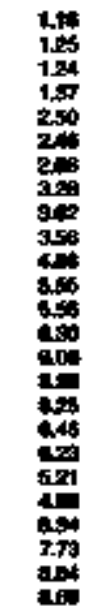 & 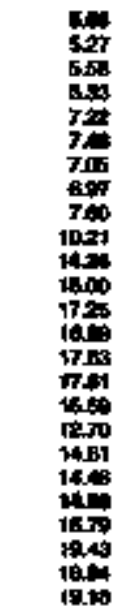 & 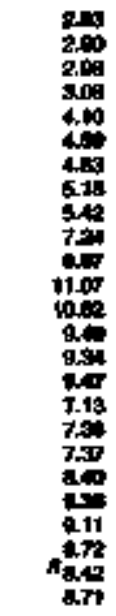 & 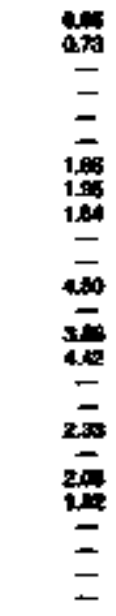 & 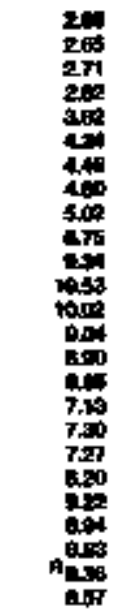 & 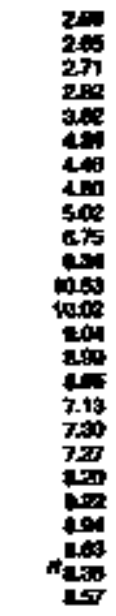 & $\begin{array}{l}= \\
z \\
z \\
= \\
= \\
= \\
= \\
= \\
= \\
= \\
= \\
= \\
z \\
= \\
= \\
z\end{array}$ & 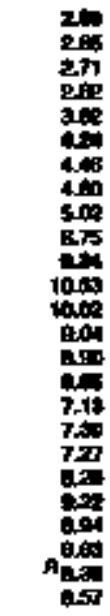 \\
\hline & \multicolumn{13}{|c|}{ 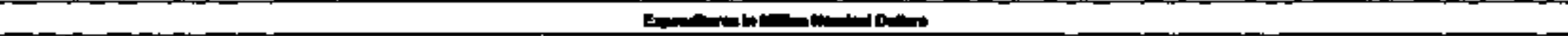 } \\
\hline 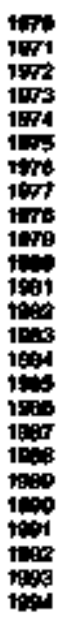 & 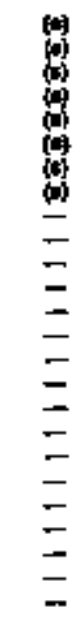 & $\begin{array}{l}= \\
= \\
= \\
= \\
= \\
= \\
= \\
= \\
= \\
= \\
= \\
= \\
9 \\
98\end{array}$ & 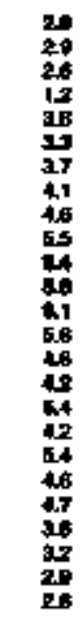 & 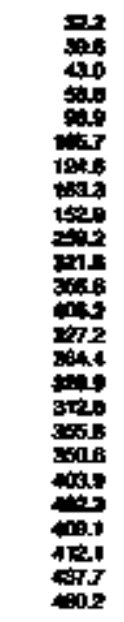 & 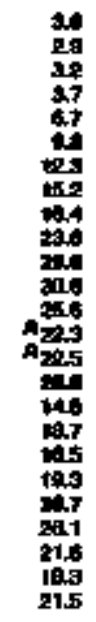 & 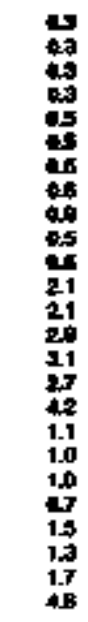 & 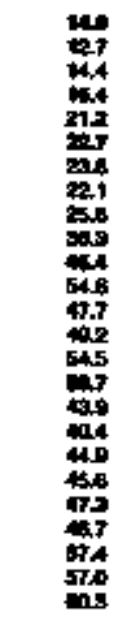 & o. & 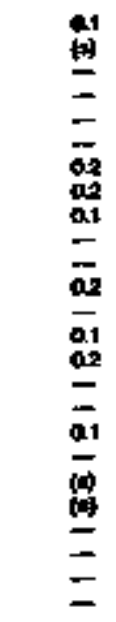 & 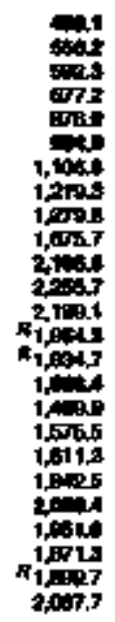 & 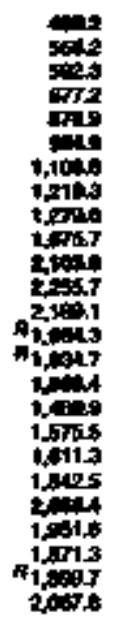 & $\begin{array}{l}= \\
= \\
= \\
= \\
= \\
= \\
= \\
= \\
= \\
= \\
= \\
= \\
=\end{array}$ & 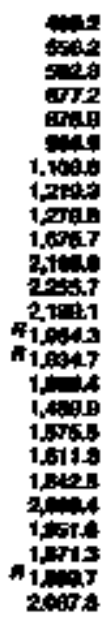 \\
\hline
\end{tabular}




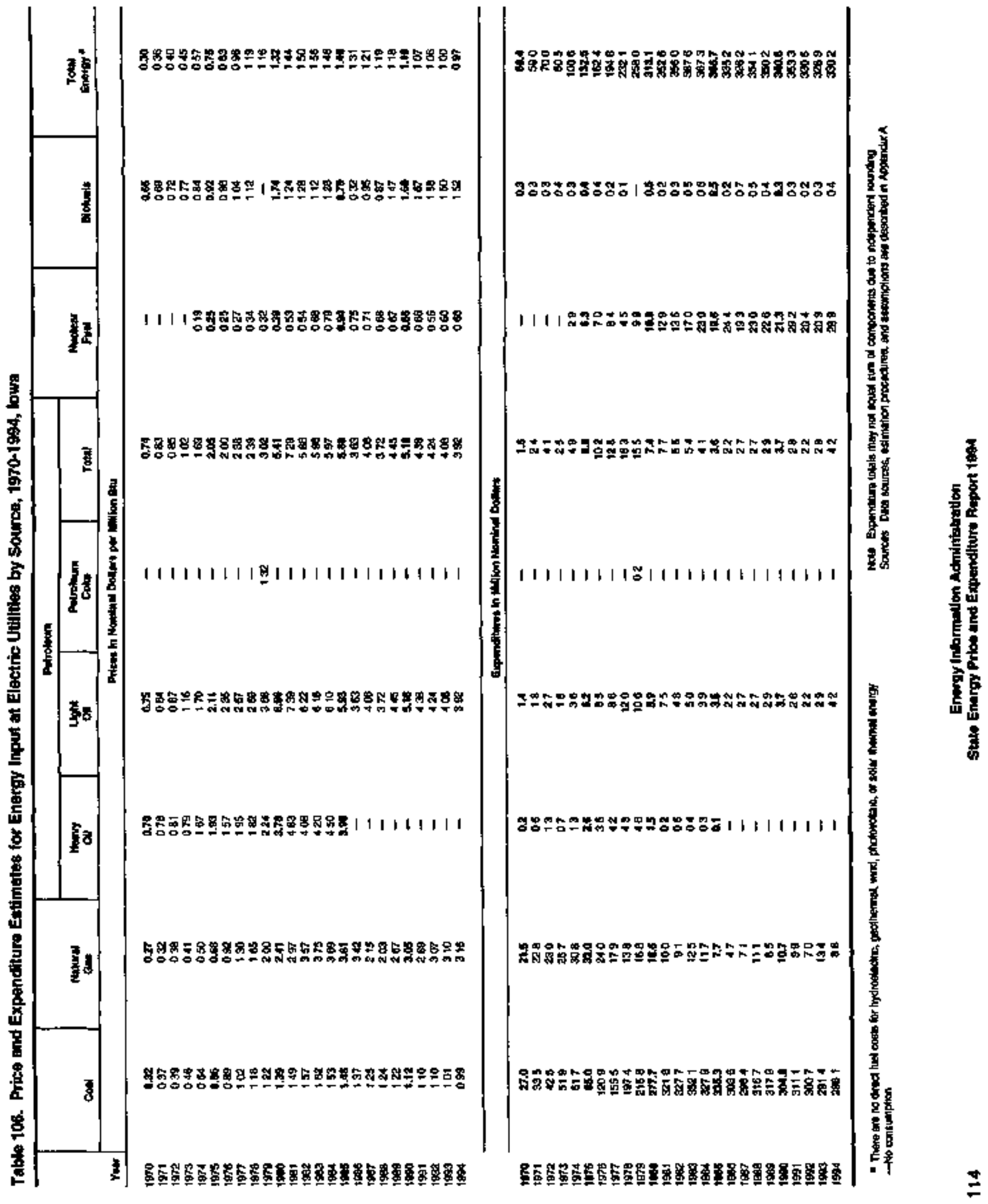




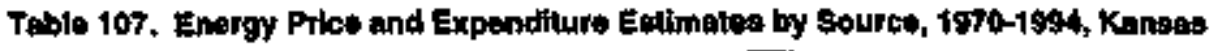

\begin{tabular}{|c|c|c|c|c|c|c|c|c|c|c|c|c|c|c|c|c|c|}
\hline & \multicolumn{14}{|c|}{ 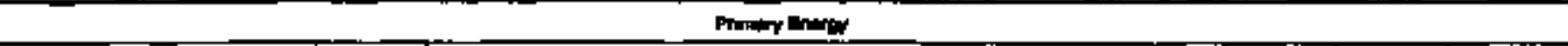 } & \multirow{2}{*}{ 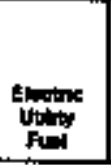 } & \multirow{2}{*}{ 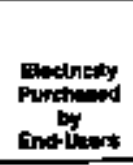 } & \multirow{2}{*}{$E^{T+\infty}$} \\
\hline & \multicolumn{3}{|c|}{ coen } & marm & \multicolumn{7}{|c|}{ 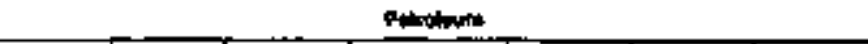 } & mosiken & Borvent & romo & & & \\
\hline 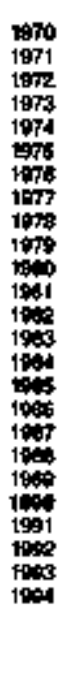 & $\begin{array}{l}= \\
z \\
= \\
= \\
= \\
= \\
= \\
= \\
= \\
= \\
= \\
= \\
=\end{array}$ & 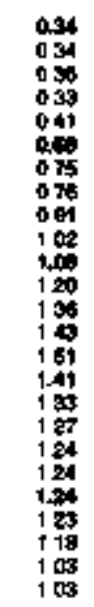 & 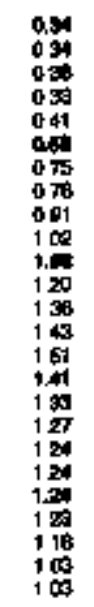 & 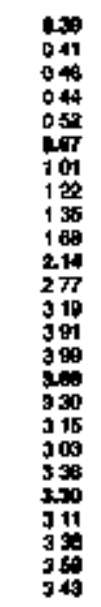 & 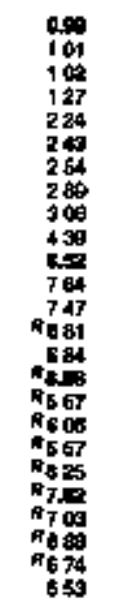 & 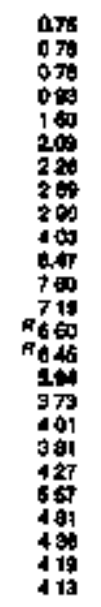 & 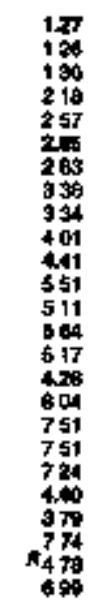 & 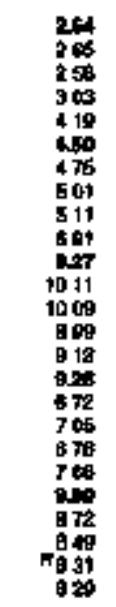 & 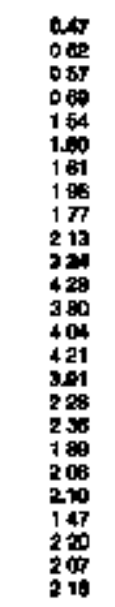 & 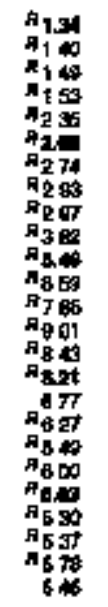 & 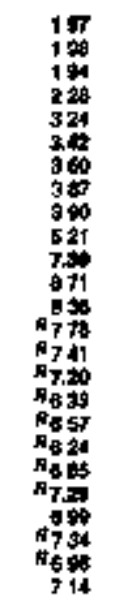 & $\begin{array}{l}= \\
= \\
= \\
z \\
= \\
= \\
= \\
= \\
= \\
\bar{z} \\
0.4 \\
063 \\
051 \\
045 \\
039 \\
090 \\
0.31 \\
093 \\
039 \\
0.35\end{array}$ & $\begin{array}{l}= \\
= \\
= \\
= \\
= \\
= \\
= \\
= \\
= \\
= \\
= \\
\bar{z} \\
3+1 \\
337 \\
342 \\
339\end{array}$ & 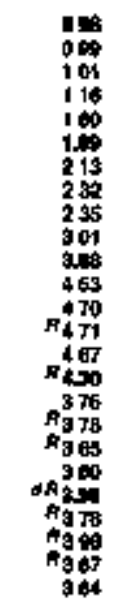 & 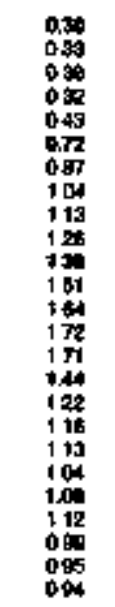 & 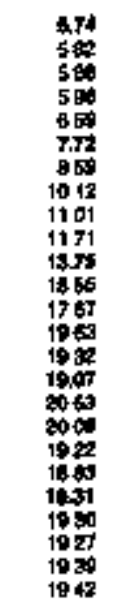 & 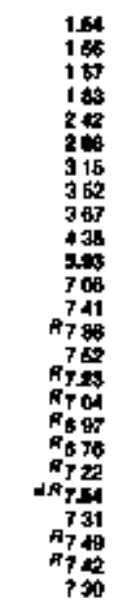 \\
\hline 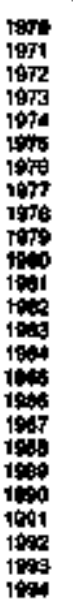 & $\begin{array}{l}= \\
= \\
= \\
= \\
= \\
= \\
= \\
= \\
= \\
= \\
= \\
=\end{array}$ & 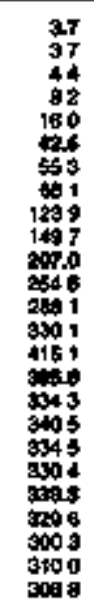 & 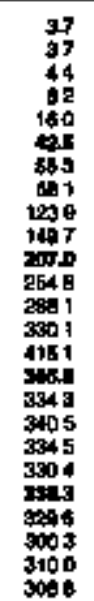 & 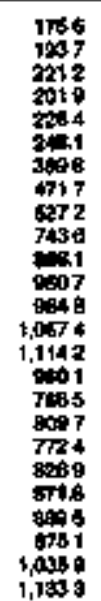 & 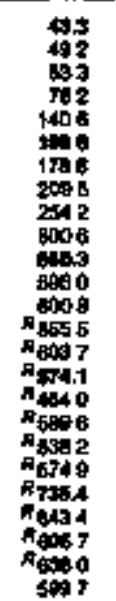 & 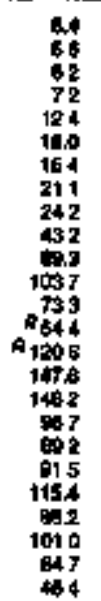 & 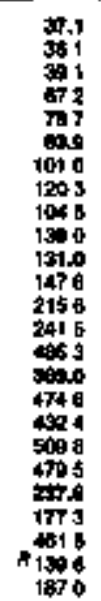 & 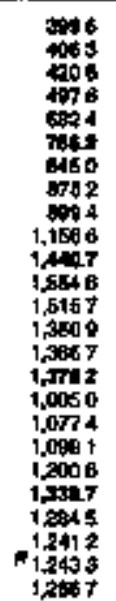 & 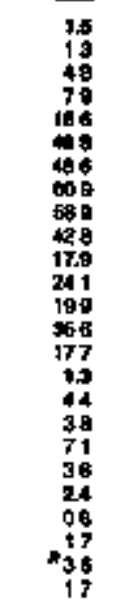 & 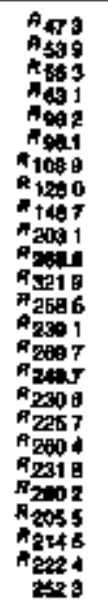 & 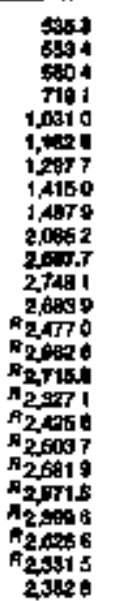 & 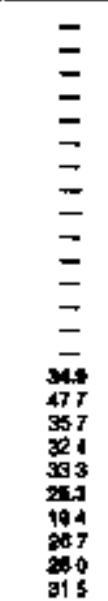 & 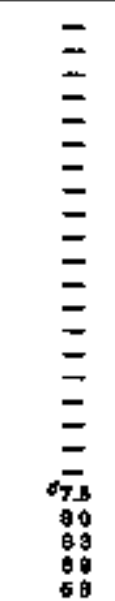 & 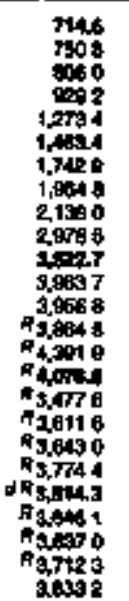 & 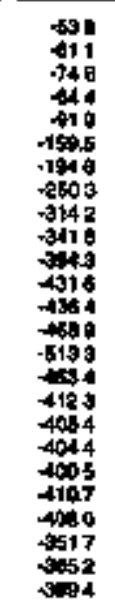 & 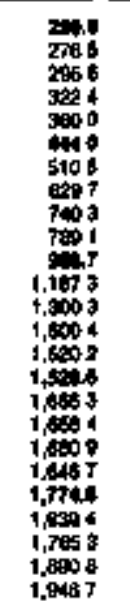 & 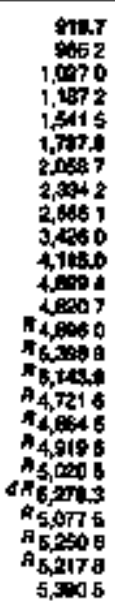 \\
\hline
\end{tabular}

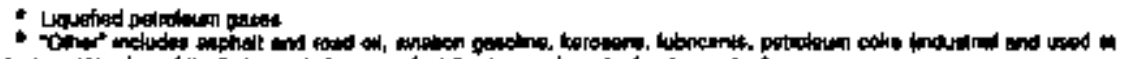

Anpimased dien

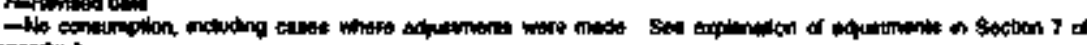

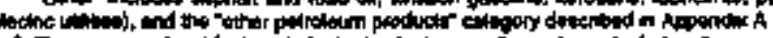

Appencter $A$.

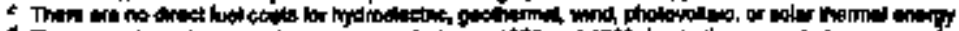

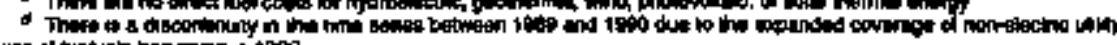

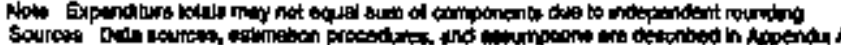




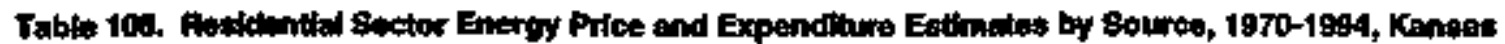

\begin{tabular}{|c|c|c|c|c|c|c|c|c|c|c|}
\hline \multirow[b]{4}{*}{$\mathrm{rem}$} & \multicolumn{8}{|c|}{ Nhmmy enoris: } & \multirow[b]{3}{*}{ 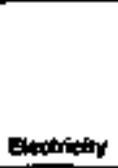 } & \multirow[b]{3}{*}{ 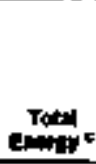 } \\
\hline & & \multirow[b]{2}{*}{ 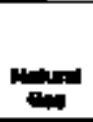 } & \multicolumn{4}{|c|}{ 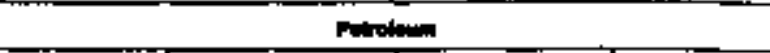 } & \multirow[b]{2}{*}{ 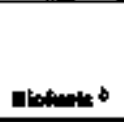 } & \multirow[b]{2}{*}{$T$} & & \\
\hline & coll & & Din & wament: & LPOA & Toter & & & & \\
\hline & \multicolumn{10}{|c|}{ 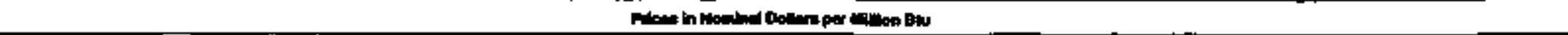 } \\
\hline 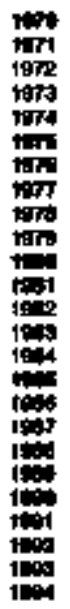 & 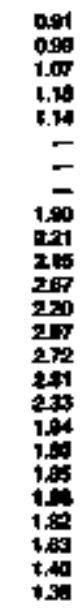 & 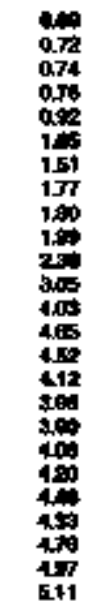 & 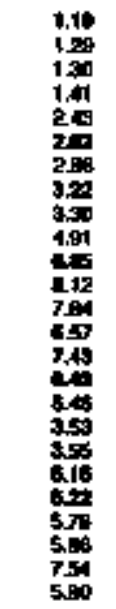 & 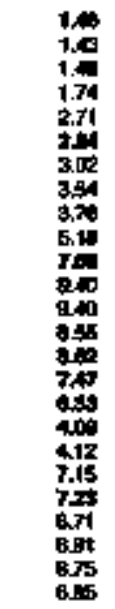 & 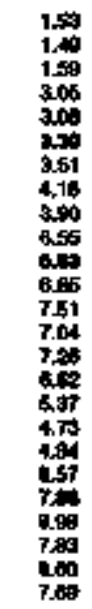 & 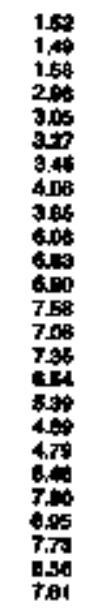 & $\begin{array}{l}= \\
= \\
= \\
= \\
= \\
= \\
= \\
= \\
= \\
= \\
= \\
= \\
0.50 \\
3.68 \\
3.56 \\
3.60\end{array}$ & 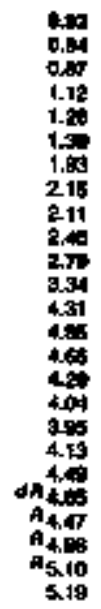 & 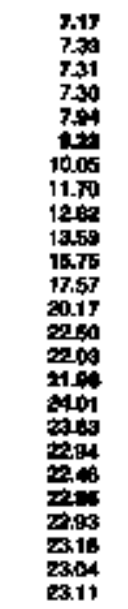 & 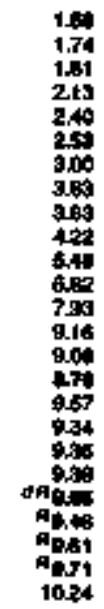 \\
\hline
\end{tabular}

\begin{tabular}{|c|c|c|c|c|c|}
\hline 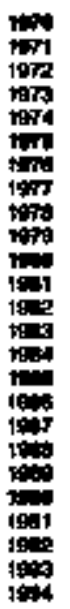 & 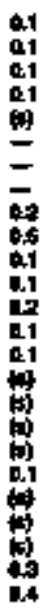 & 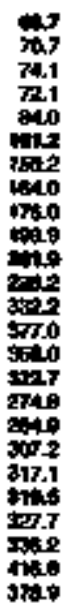 & 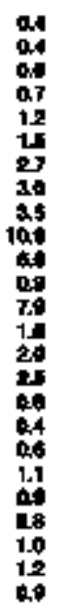 & 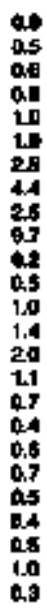 & 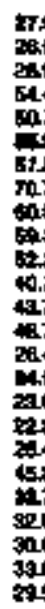 \\
\hline
\end{tabular}

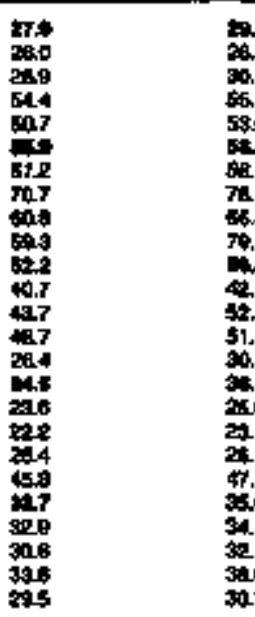

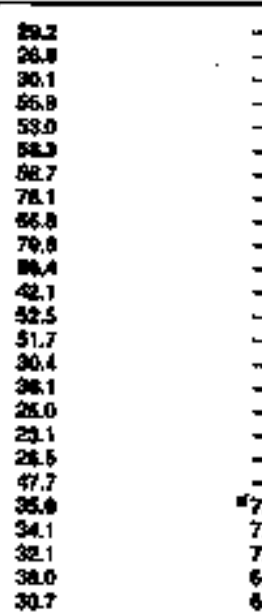

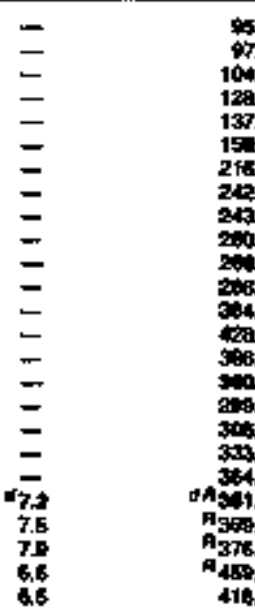

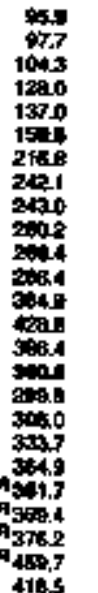

\begin{tabular}{|c|c|}
\hline 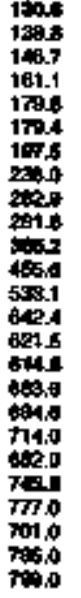 & 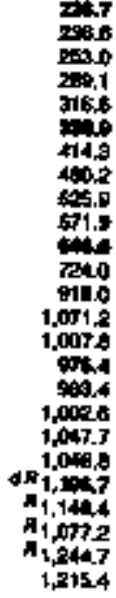 \\
\hline
\end{tabular}

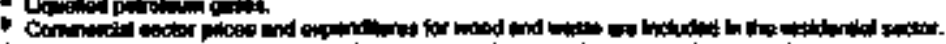

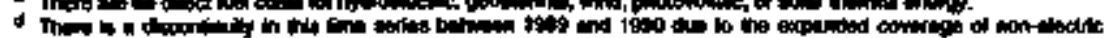

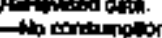

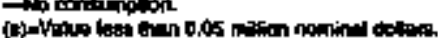

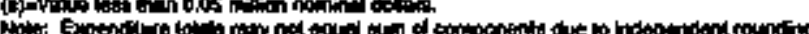

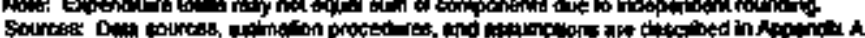


Table 109. Commercied Sector Energy Price and Expendture Estimates by Sourct, 1970-1984, Kansas

\begin{tabular}{|c|c|c|c|c|c|c|c|c|c|c|c|}
\hline \multirow[b]{4}{*}{$\mathbf{V}=$} & \multicolumn{9}{|c|}{ Pimpry } & \multirow[b]{3}{*}{ Enctidity } & \multirow[b]{3}{*}{$\operatorname{tom}_{\cos }$} \\
\hline & & \multirow[b]{2}{*}{ 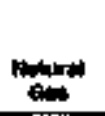 } & \multicolumn{6}{|c|}{ Putrinan } & \multirow[b]{2}{*}{ Than } & & \\
\hline & Co:4 & & Fation & Narowane & Lete & enter & Find & Toth & & & \\
\hline & \multicolumn{11}{|c|}{ Pnots h h Rom } \\
\hline \multirow[t]{2}{*}{ 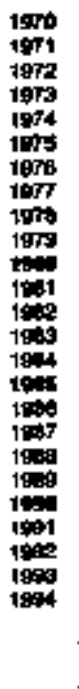 } & 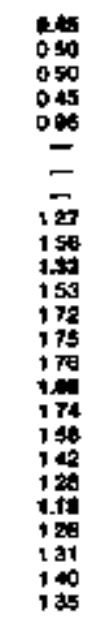 & 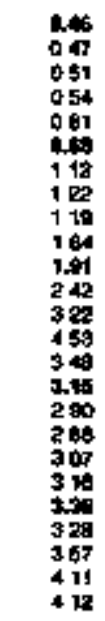 & 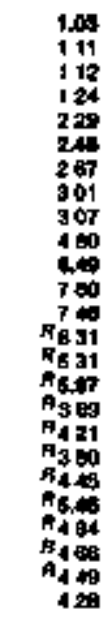 & 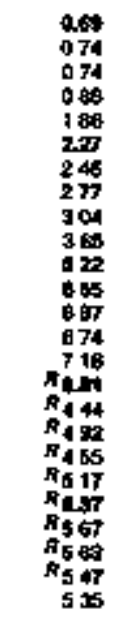 & 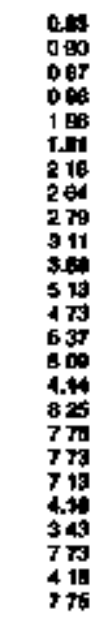 & 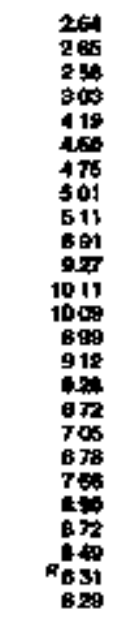 & 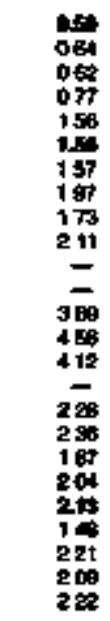 & 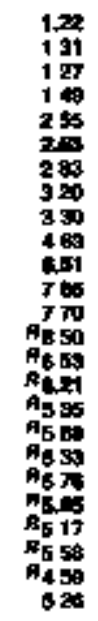 & 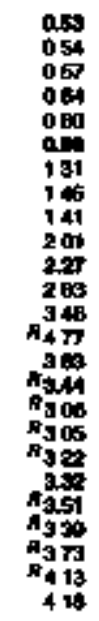 & 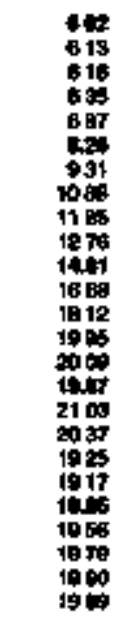 & 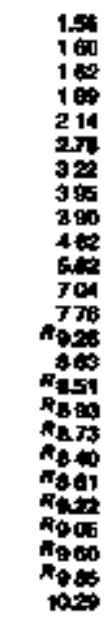 \\
\hline & \multicolumn{11}{|c|}{ 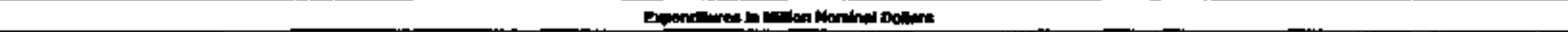 } \\
\hline 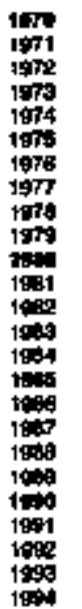 & 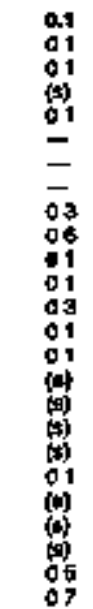 & 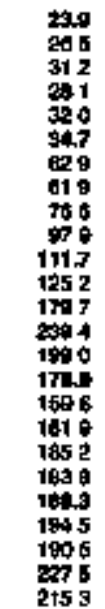 & 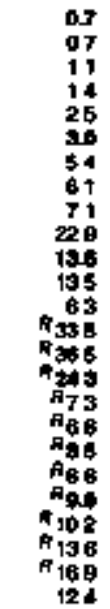 & $\begin{array}{c}01 \\
01 \\
01 \\
01 \\
01 \\
02 \\
02 \\
08 \\
10 \\
08 \\
10 \\
03 \\
02 \\
03 \\
04 \\
04 \\
04 \\
02 \\
02 \\
04 \\
02 \\
05 \\
02 \\
01 \\
01 \\
02 \\
01\end{array}$ & $\begin{array}{l}27 \\
27 \\
28 \\
31 \\
59 \\
57 \\
67 \\
89 \\
77 \\
50 \\
40 \\
54 \\
49 \\
66 \\
39 \\
39 \\
84 \\
84 \\
71 \\
87 \\
31 \\
29 \\
53 \\
29 \\
52\end{array}$ & 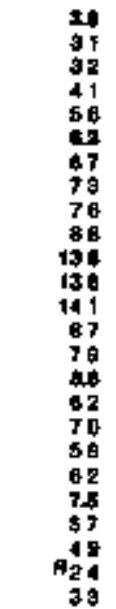 & 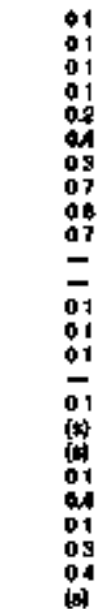 & 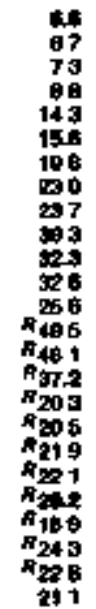 & 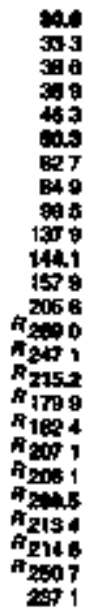 & 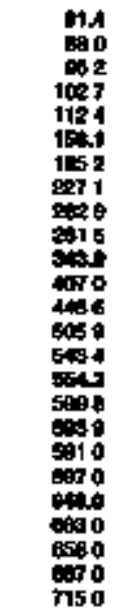 & 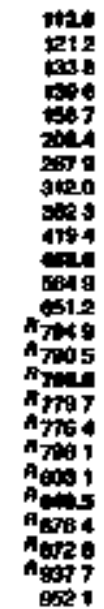 \\
\hline
\end{tabular}

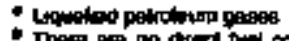

管

$\rightarrow$ Ho copounions

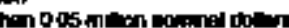

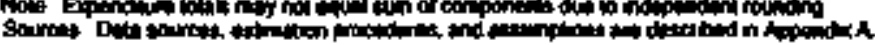


Table 110. Indueblal Bector Enorgy Price and Expenditure Eetlmates by Source, 1970-1994, Kanad.

\begin{tabular}{|c|c|c|c|c|c|c|c|c|c|c|c|c|c|c|c|c|c|}
\hline & \multicolumn{15}{|c|}{ Printris En } & \multirow[b]{3}{*}{ Enetricily } & \multirow[b]{3}{*}{ notery. } \\
\hline & \multicolumn{3}{|c|}{ Cont } & \multirow[b]{2}{*}{ inising } & \multicolumn{9}{|c|}{ Purrolum } & \multirow[b]{2}{*}{ Blofuntio } & \multirow[b]{2}{*}{ Tome } & & \\
\hline & conlen & Com & Toll & & 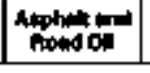 & Dow & 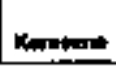 & LaA. & Iubrionts & moter & Anedow & onto ${ }^{\circ}$ & Toted & & & & \\
\hline $\mathrm{rem}$ & \multicolumn{17}{|c|}{ 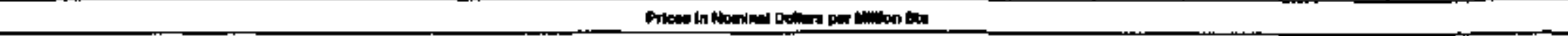 } \\
\hline \multirow[t]{2}{*}{ 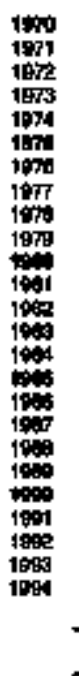 } & $\begin{array}{l}= \\
z \\
= \\
= \\
z \\
= \\
= \\
= \\
= \\
= \\
= \\
= \\
= \\
= \\
=\end{array}$ & 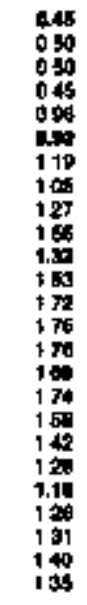 & 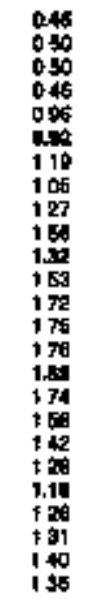 & 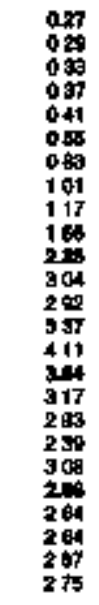 & 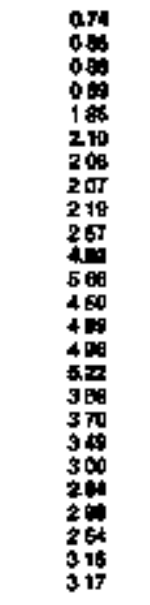 & 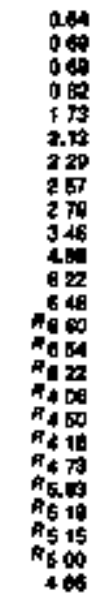 & 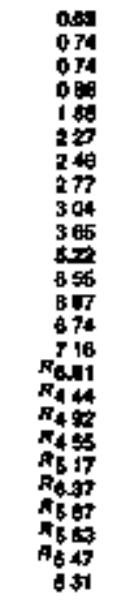 & 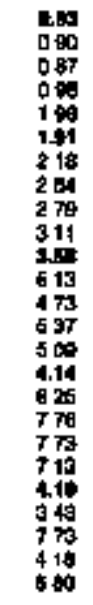 & 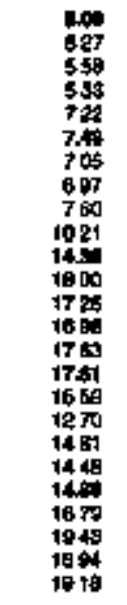 & 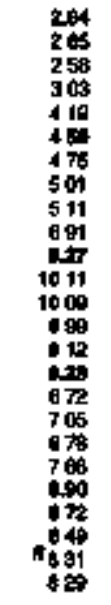 & 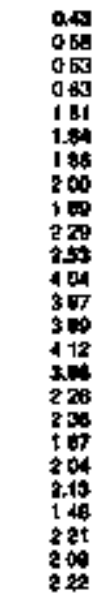 & 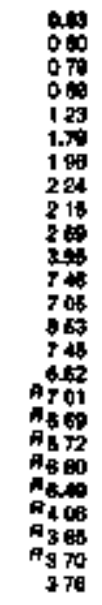 & 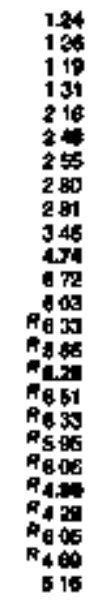 & $\begin{array}{l}= \\
= \\
= \\
= \\
= \\
= \\
= \\
= \\
= \\
= \\
= \\
= \\
= \\
0.71 \\
175 \\
171 \\
179 \\
179\end{array}$ & 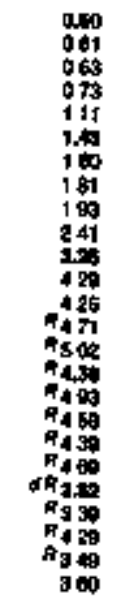 & 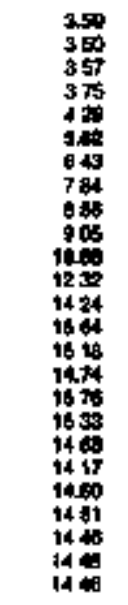 & 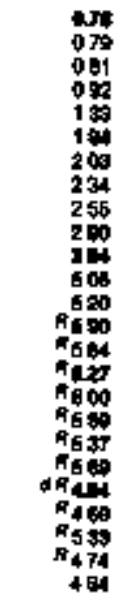 \\
\hline & \multicolumn{17}{|c|}{ 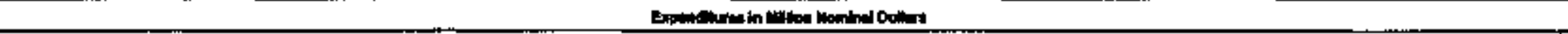 } \\
\hline 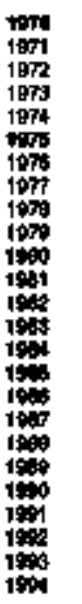 & $\begin{array}{l}= \\
= \\
z \\
= \\
= \\
= \\
= \\
= \\
= \\
= \\
= \\
= \\
= \\
z\end{array}$ & 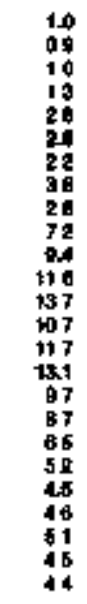 & 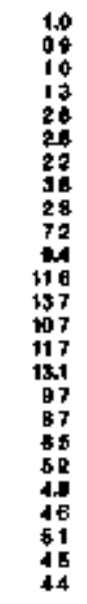 & 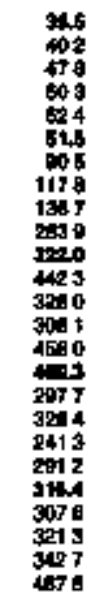 & 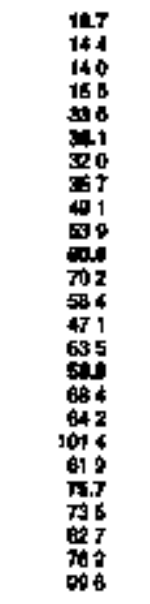 & 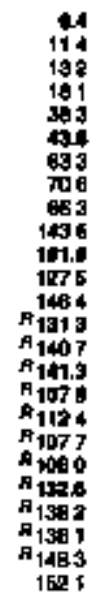 & 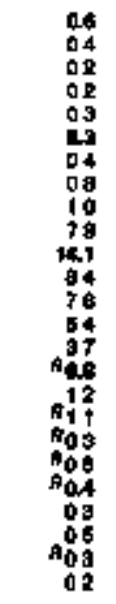 & 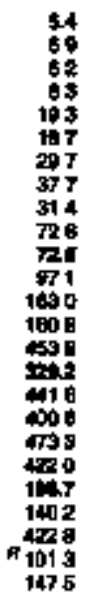 & 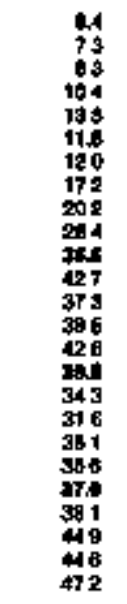 & 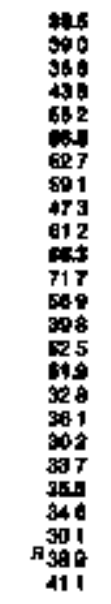 & 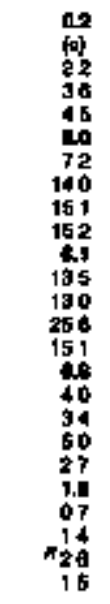 & 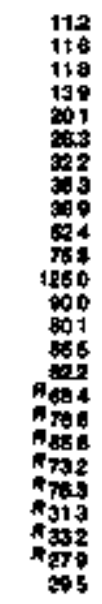 & 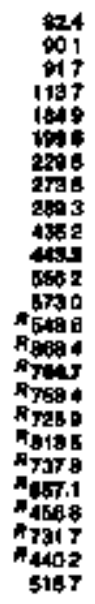 & $\begin{array}{l}= \\
= \\
= \\
= \\
= \\
= \\
= \\
= \\
= \\
= \\
= \\
03 \\
05 \\
03 \\
03\end{array}$ & 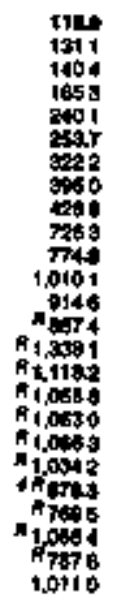 & 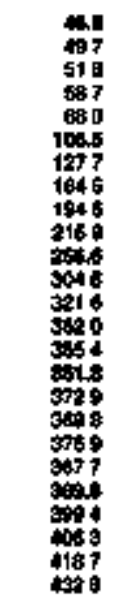 & 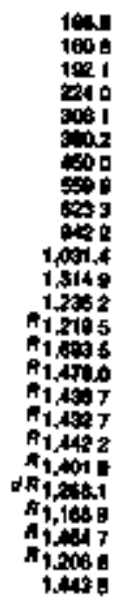 \\
\hline
\end{tabular}

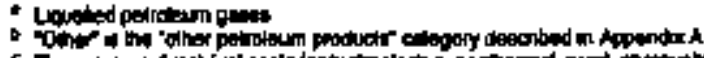

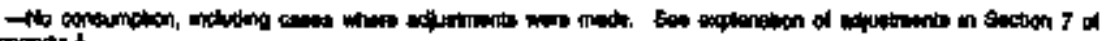

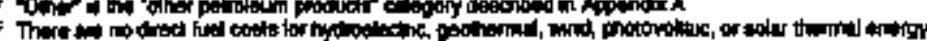

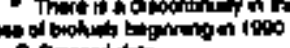

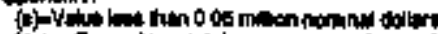

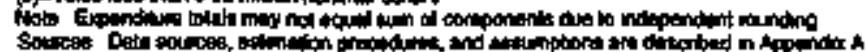
fifinepod doto 
Teble 111. Trangportation Sector Enordy Prlot and Expenditure Estimales by Source, 1970-1984, Kanags

\begin{tabular}{|c|c|c|c|c|c|c|c|c|c|c|c|c|c|}
\hline \multirow[b]{4}{*}{ ver } & \multicolumn{11}{|c|}{ 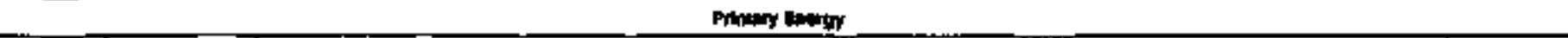 } & \multirow[b]{3}{*}{ 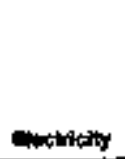 } & \multirow[b]{3}{*}{ Entiph } \\
\hline & \multirow[b]{2}{*}{ Cod } & \multirow[b]{2}{*}{ thend } & \multicolumn{8}{|c|}{ Putrolece } & \multirow[b]{2}{*}{ Telle } & & \\
\hline & & & colvalion & Diman & foll & $\operatorname{tand}$ & Itustomte & anding & 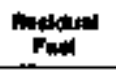 & Fit & & & \\
\hline & \multicolumn{13}{|c|}{ 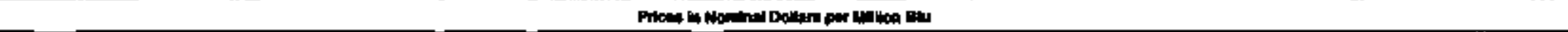 } \\
\hline \multirow[t]{2}{*}{ 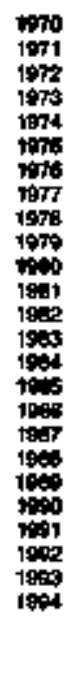 } & $\begin{array}{l}045 \\
050 \\
050 \\
045 \\
000 \\
000 \\
110 \\
106 \\
= \\
= \\
= \\
= \\
= \\
= \\
= \\
= \\
= \\
= \\
=\end{array}$ & $\begin{array}{l}= \\
= \\
= \\
= \\
= \\
= \\
= \\
= \\
= \\
= \\
= \\
= \\
\mathbf{z} \text {. }\end{array}$ & 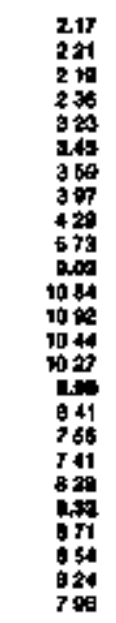 & 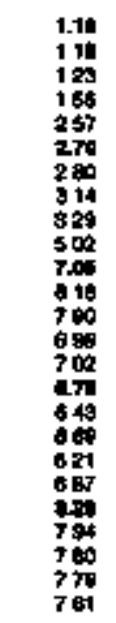 & 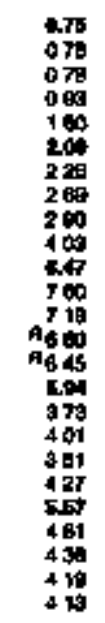 & 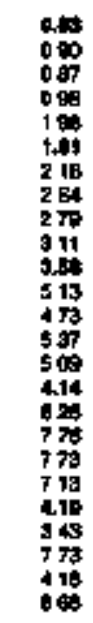 & 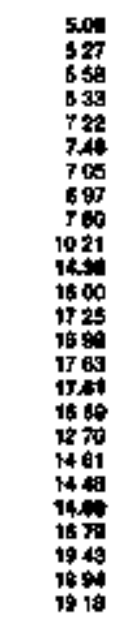 & 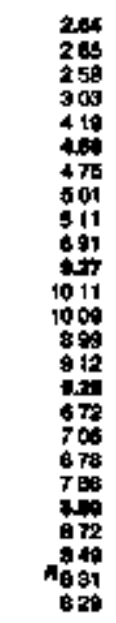 & 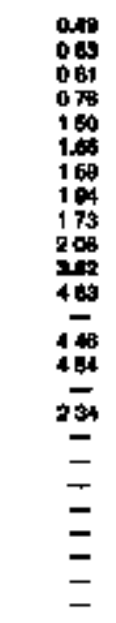 & 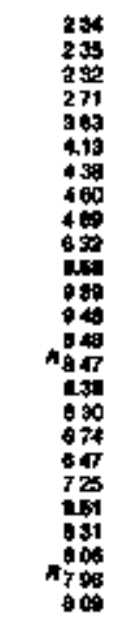 & 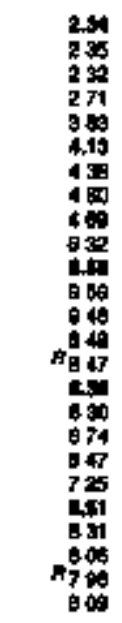 & $\begin{array}{l}= \\
= \\
= \\
= \\
= \\
= \\
= \\
= \\
= \\
= \\
= \\
= \\
= \\
=\end{array}$ & 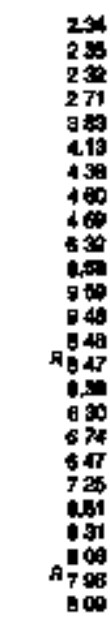 \\
\hline & \multicolumn{13}{|c|}{ 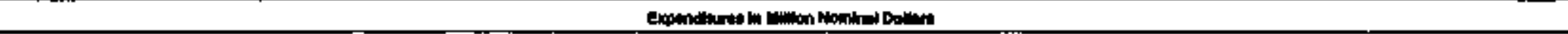 } \\
\hline 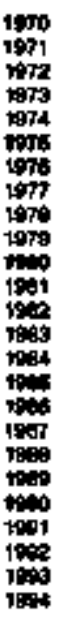 & $\begin{array}{l}0 \\
0 \\
000 \\
00 \\
0 \\
05 \\
= \\
= \\
= \\
= \\
= \\
= \\
= \\
= \\
= \\
=\end{array}$ & 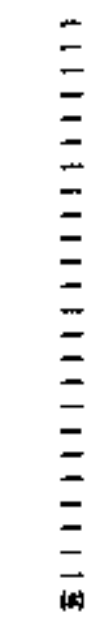 & 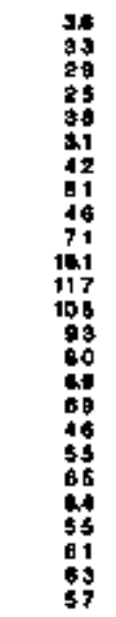 & 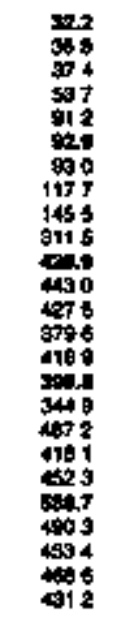 & 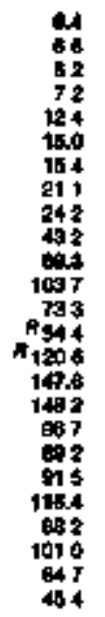 & 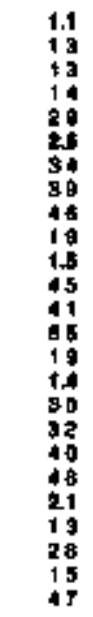 & 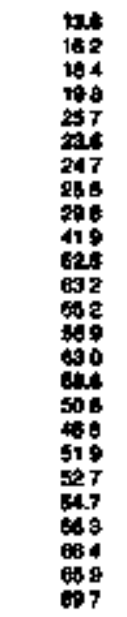 & 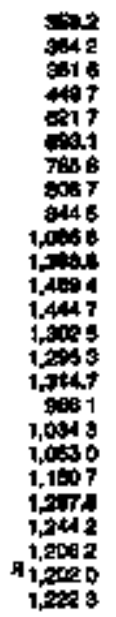 & 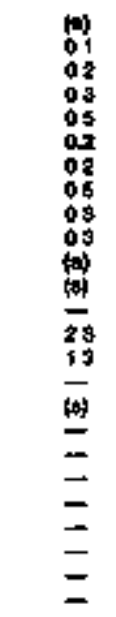 & 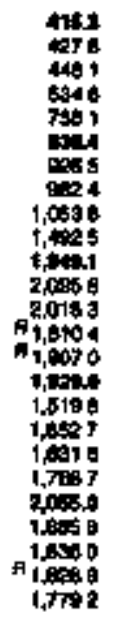 & 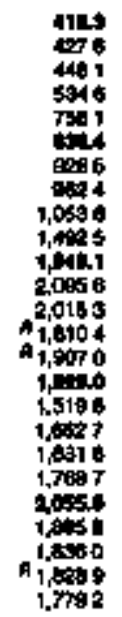 & $\begin{array}{l}= \\
= \\
= \\
= \\
= \\
= \\
= \\
= \\
= \\
= \\
= \\
= \\
=\end{array}$ & 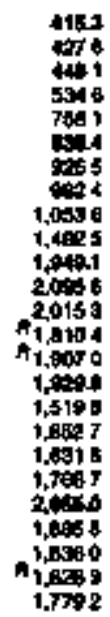 \\
\hline
\end{tabular}

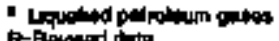

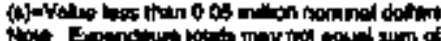

$K$

R= revaxd

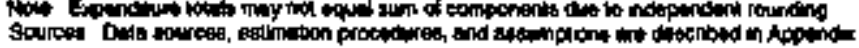
andin 


\begin{tabular}{|c|c|c|c|c|c|c|c|c|c|}
\hline \multirow[b]{3}{*}{$\mathrm{r}=$} & \multirow[b]{2}{*}{$\infty$} & \multirow[b]{2}{*}{ Gent } & \multicolumn{4}{|c|}{ Racoling } & \multirow[b]{2}{*}{ Pan: } & \multirow[b]{2}{*}{ 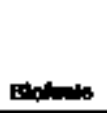 } & \multirow[b]{2}{*}{ 1 } \\
\hline & & & ad & 남해 & 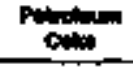 & rom & & & \\
\hline & \multicolumn{9}{|c|}{ 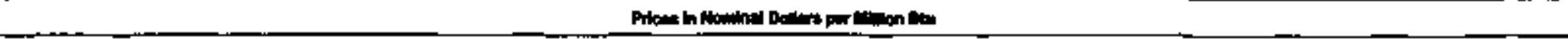 } \\
\hline \multirow[t]{2}{*}{ 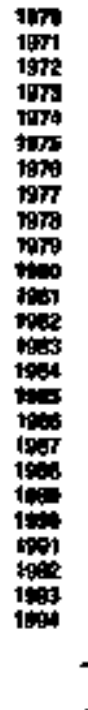 } & 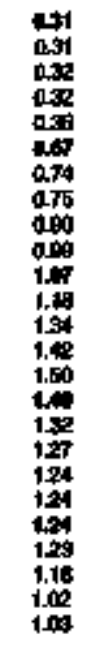 & 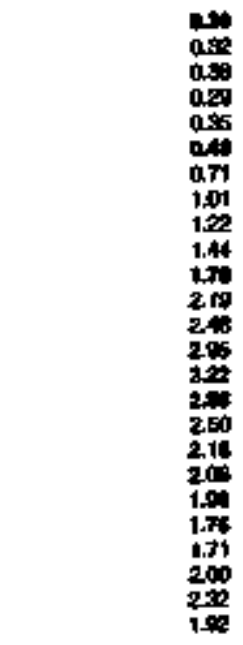 & 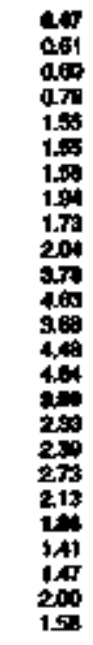 & 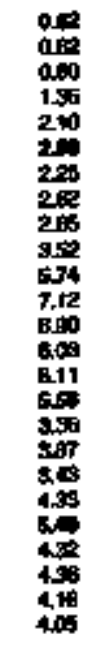 & $\begin{array}{l}\bar{z} \\
\bar{z} \\
\bar{z} \\
=\overline{=} \\
= \\
= \\
= \\
= \\
= \\
= \\
= \\
=\end{array}$ & 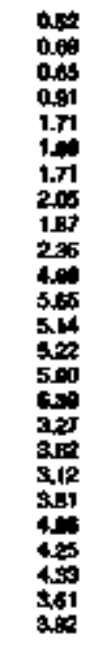 & 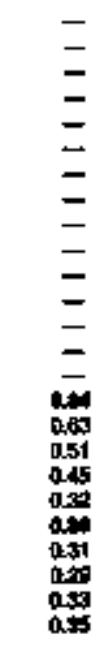 & $\begin{array}{l}= \\
= \\
= \\
= \\
= \\
= \\
= \\
= \\
= \\
= \\
= \\
= \\
= \\
=\end{array}$ & 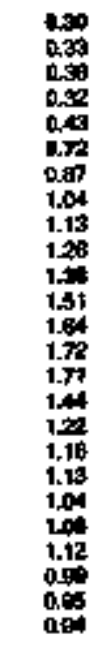 \\
\hline & \multicolumn{9}{|c|}{ E } \\
\hline 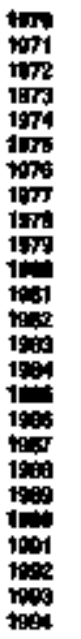 & 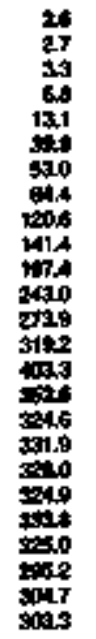 & 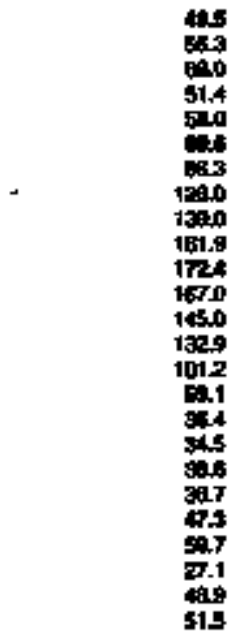 & 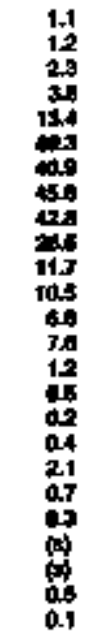 & 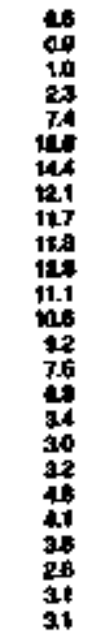 & $\begin{array}{l}= \\
\bar{z} \\
\bar{a} \\
\bar{a} \\
= \\
= \\
= \\
= \\
= \\
= \\
= \\
= \\
= \\
=\end{array}$ & 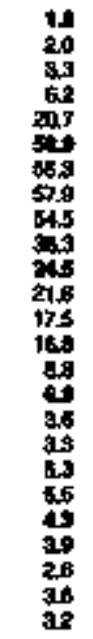 & $\begin{array}{l}= \\
= \\
= \\
= \\
= \\
= \\
= \\
= \\
= \\
= \\
345 \\
477 \\
357 \\
304 \\
303 \\
203 \\
307 \\
207 \\
315\end{array}$ & $\begin{array}{l}= \\
= \\
= \\
= \\
= \\
= \\
= \\
= \\
= \\
= \\
= \\
= \\
= \\
=\end{array}$ & 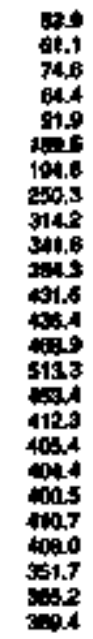 \\
\hline
\end{tabular}

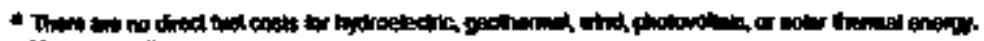

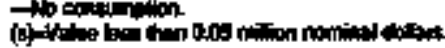




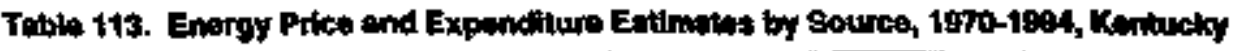

\begin{tabular}{|c|c|c|c|c|c|c|c|c|c|c|c|c|c|c|c|c|c|}
\hline & \multicolumn{14}{|c|}{ Puthery } & \multirow{3}{*}{ 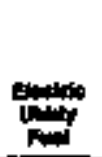 } & \multirow{3}{*}{ 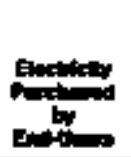 } & \multirow[b]{3}{*}{ 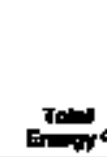 } \\
\hline & \multicolumn{3}{|c|}{ and } & \multirow[b]{2}{*}{ max } & \multicolumn{7}{|c|}{ 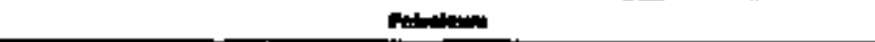 } & \multirow[b]{2}{*}{ 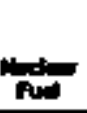 } & \multirow[b]{2}{*}{ ה } & \multirow[b]{2}{*}{ Toted" } & & & \\
\hline & Coling & היקט & Tan & & 口: & 新 & LP6* & 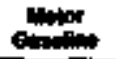 & flat & atm & Twe & & & & & & \\
\hline $\mathrm{ren}$ & \multicolumn{17}{|c|}{ 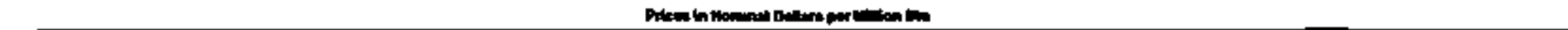 } \\
\hline \multirow[t]{2}{*}{ 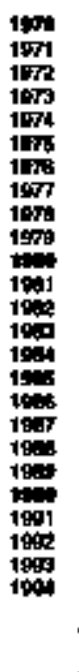 } & 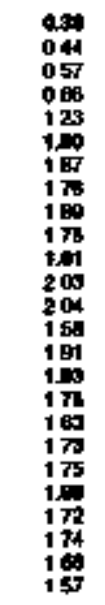 & 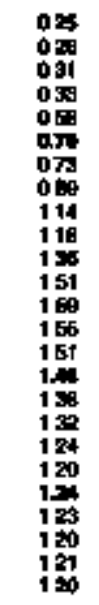 & 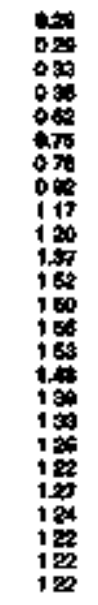 & 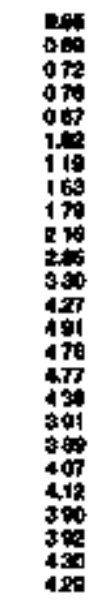 & 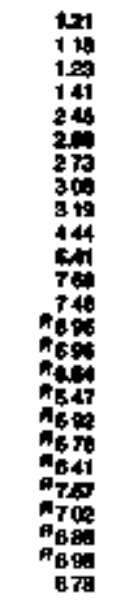 & 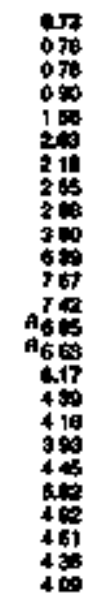 & 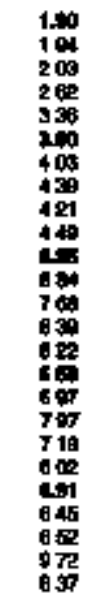 & 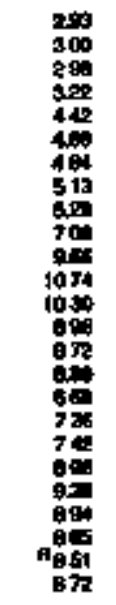 & 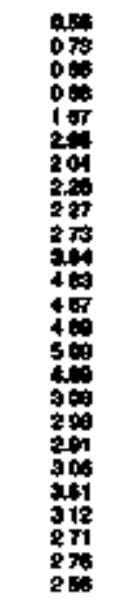 & 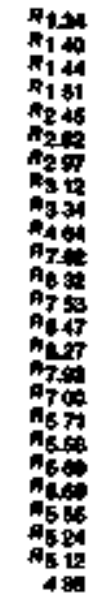 & 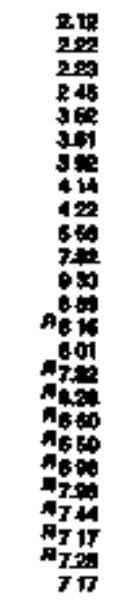 & $\begin{array}{l}= \\
= \\
= \\
= \\
= \\
= \\
= \\
= \\
= \\
= \\
= \\
= \\
= \\
=\end{array}$ & $\begin{array}{l}= \\
= \\
= \\
= \\
= \\
= \\
= \\
= \\
= \\
= \\
= \\
\bar{z} \\
2= \\
24 \\
27\end{array}$ & 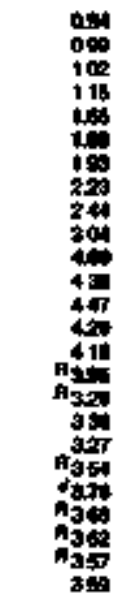 & 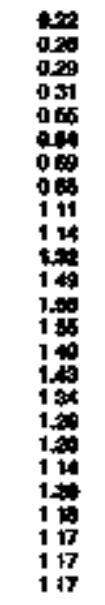 & 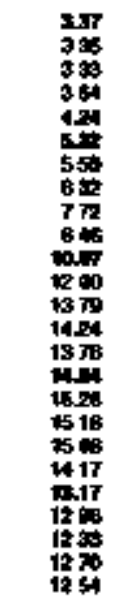 & 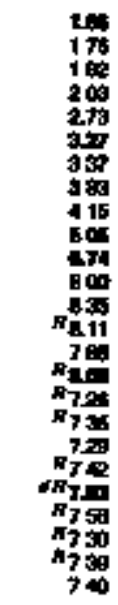 \\
\hline & \multicolumn{17}{|c|}{ 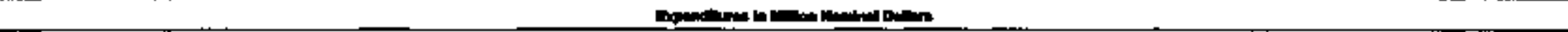 } \\
\hline 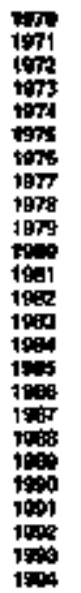 & 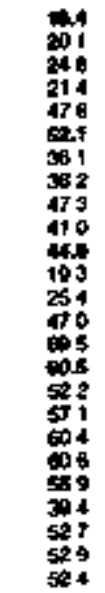 & 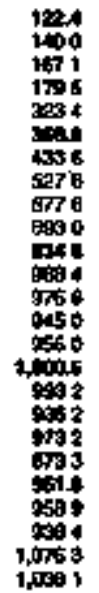 & 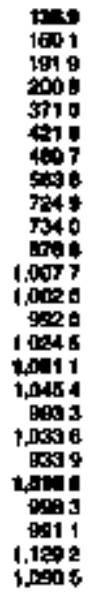 & 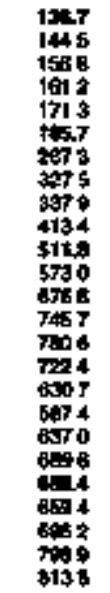 & 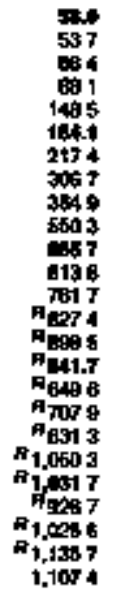 & 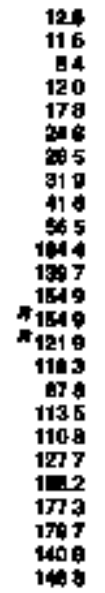 & 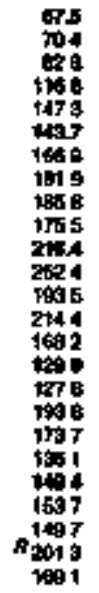 & 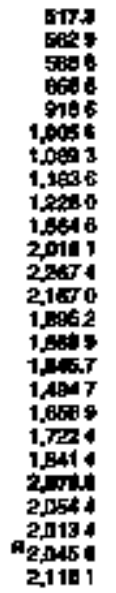 & 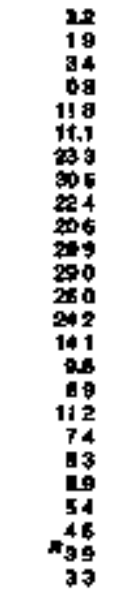 & 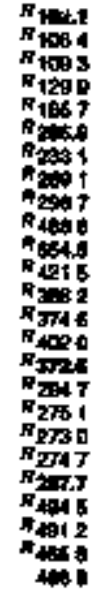 & 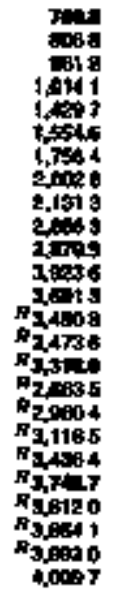 & $\begin{array}{l}= \\
= \\
= \\
= \\
= \\
= \\
= \\
= \\
= \\
= \\
= \\
= \\
= \\
=\end{array}$ & $\begin{array}{l}= \\
= \\
= \\
= \\
= \\
= \\
= \\
= \\
= \\
= \\
= \\
= \\
280 \\
204 \\
210 \\
200\end{array}$ & 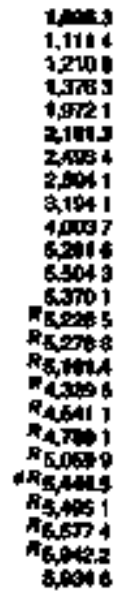 & 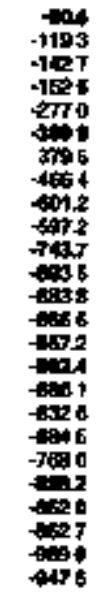 & 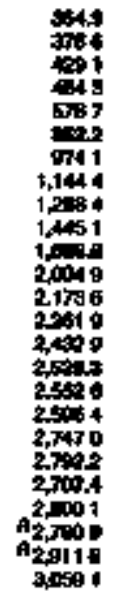 & 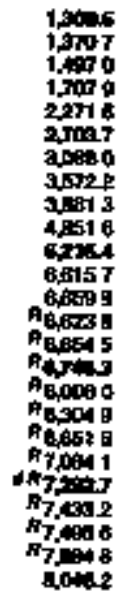 \\
\hline
\end{tabular}

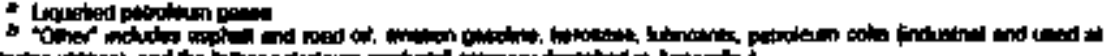

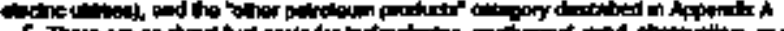

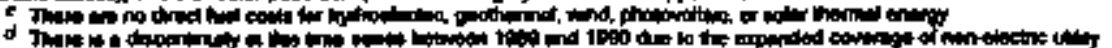

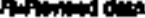

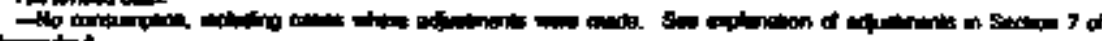

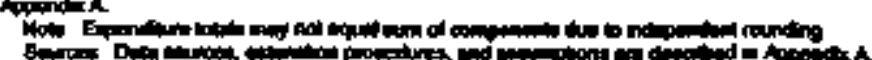
(a) 
Table 144. Residmbial Sector Energy Price and Expendituro Eetimatos by Source, 1970-1994, Kentucky

\begin{tabular}{|c|c|c|c|c|c|c|c|c|c|c|}
\hline \multirow[b]{4}{*}{ Verm } & \multicolumn{8}{|c|}{ Plimary Enwysy } & \multirow[b]{3}{*}{ Enetrileiny } & \multirow[b]{3}{*}{ Totd } \\
\hline & & \multirow[b]{2}{*}{ Mcluest } & \multicolumn{4}{|c|}{ Promoinam. } & \multirow[b]{2}{*}{ Eqonate: } & \multirow[b]{2}{*}{ Tow" } & & \\
\hline & Cod & & Otiallow & Keroweine & Laq. & $T=1$ & & & & \\
\hline & \multicolumn{10}{|c|}{ 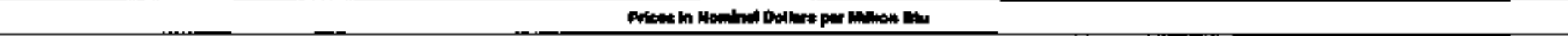 } \\
\hline \multirow[t]{2}{*}{ 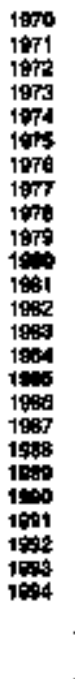 } & 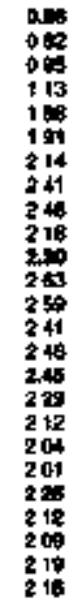 & 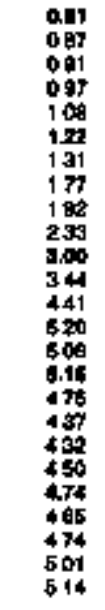 & 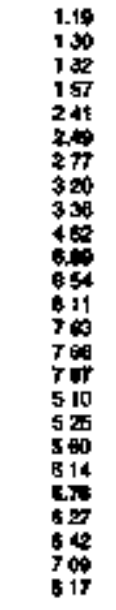 & 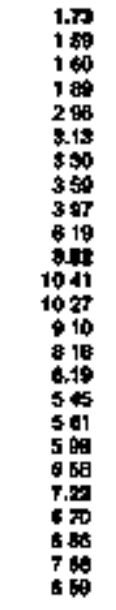 & 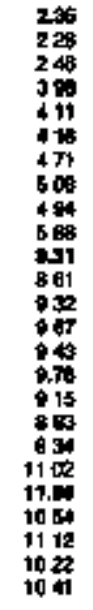 & 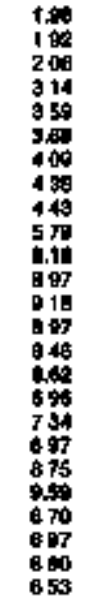 & $\begin{array}{l}= \\
= \\
= \\
= \\
= \\
= \\
= \\
= \\
= \\
= \\
= \\
= \\
2.5 \\
35 \\
36 \\
360\end{array}$ & 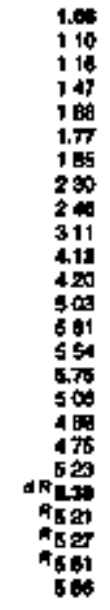 & 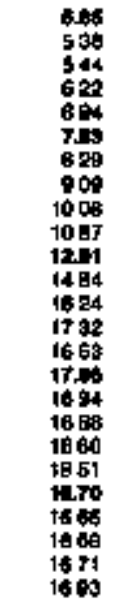 & 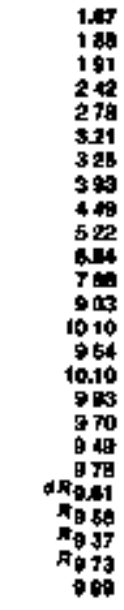 \\
\hline & \multicolumn{10}{|c|}{ 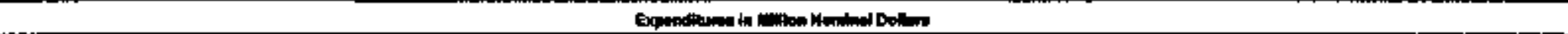 } \\
\hline 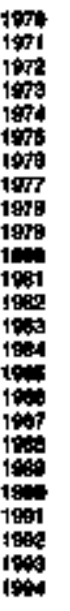 & 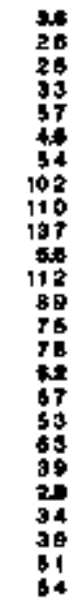 & 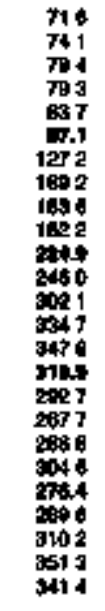 & 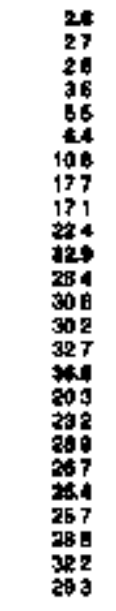 & $\begin{array}{l}244 \\
170 \\
126 \\
141 \\
218 \\
110 \\
157 \\
162 \\
105 \\
720 \\
046 \\
200 \\
307 \\
470 \\
396 \\
917 \\
207 \\
142 \\
210 \\
217 \\
124 \\
144 \\
142 \\
198 \\
140\end{array}$ & 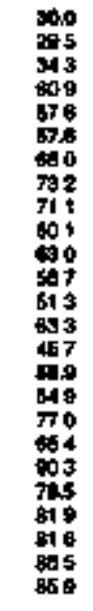 & 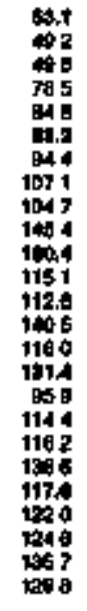 & $\begin{array}{l}= \\
= \\
= \\
= \\
= \\
= \\
= \\
= \\
= \\
= \\
= \\
0190 \\
1908 \\
060\end{array}$ & 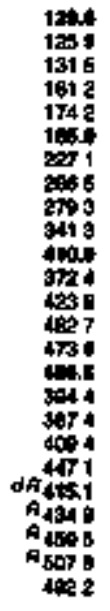 & 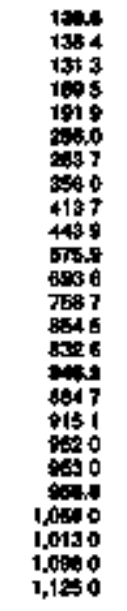 & 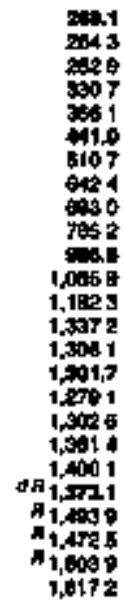 \\
\hline
\end{tabular}

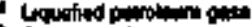

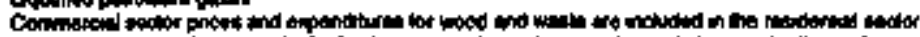

A-Revided dista

Alo contiumptorn

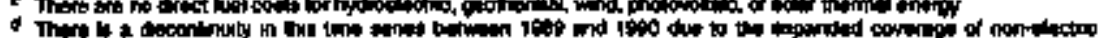

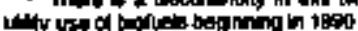


Table 115. Commerclel sector Energy Price and Expendifure Esilmates by Source, 1970-1994, Kentuchy

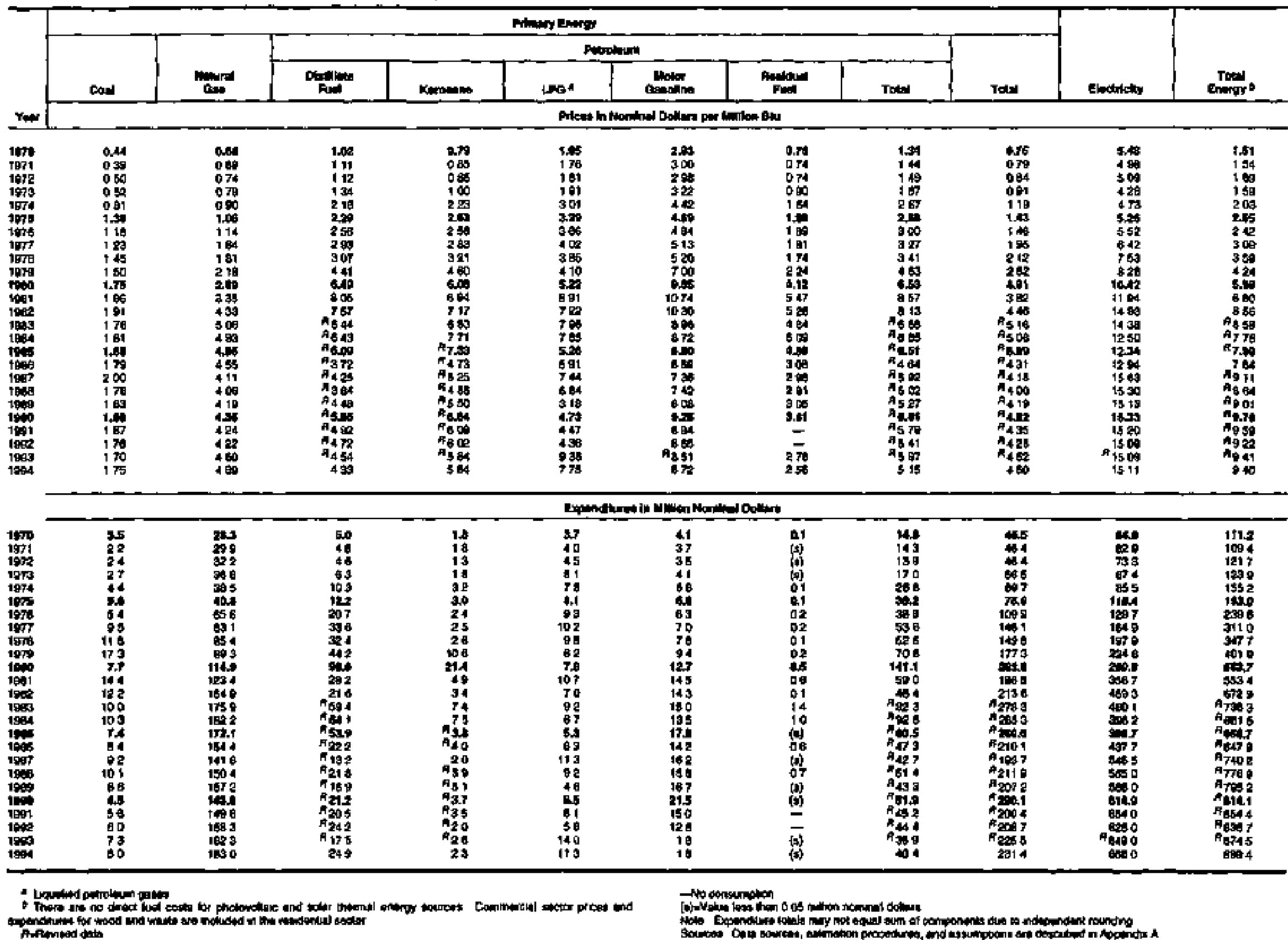




\begin{tabular}{|c|c|c|c|c|c|c|c|c|c|c|c|c|c|c|c|c|c|}
\hline & \multicolumn{15}{|c|}{$\min 20$} & \multirow[b]{3}{*}{ Enction } & \multirow[b]{3}{*}{ Tours: } \\
\hline & \multicolumn{3}{|c|}{ Low. } & \multirow[b]{2}{*}{$\mathrm{am}$} & \multicolumn{9}{|c|}{ - } & \multirow[b]{2}{*}{ 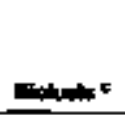 } & \multirow[b]{2}{*}{ Twal< } & & \\
\hline & conts & 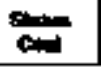 & 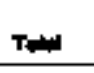 & & | & Fill & 年 & ceses & 至 & (6) & 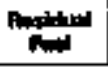 & ons & בומד & & & & \\
\hline rear & \multicolumn{17}{|c|}{ 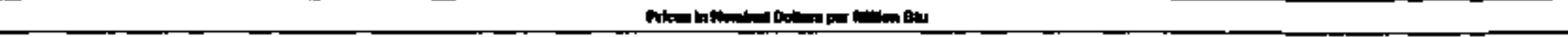 } \\
\hline \multirow[t]{2}{*}{ 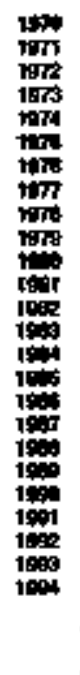 } & 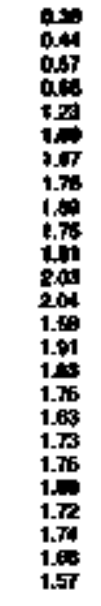 & 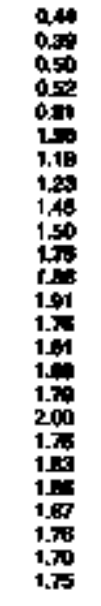 & 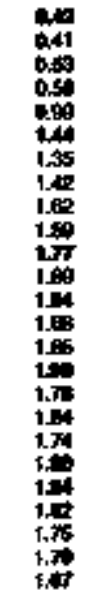 & 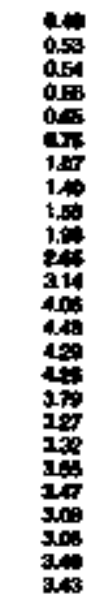 & 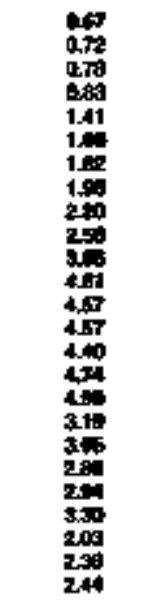 & 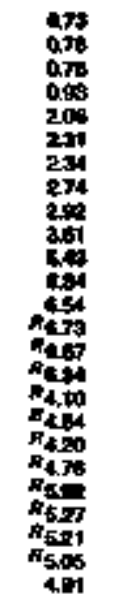 & 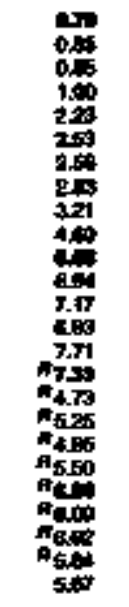 & 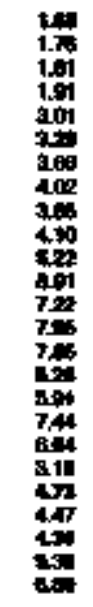 & 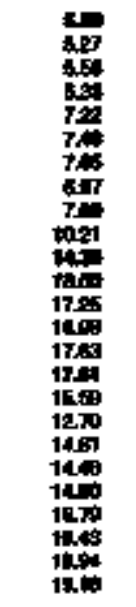 & 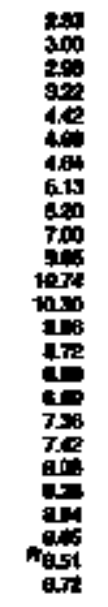 & 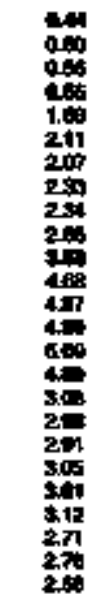 & 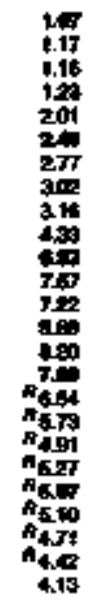 & 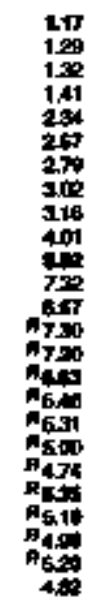 & $\begin{array}{l}= \\
= \\
= \\
= \\
= \\
= \\
= \\
= \\
= \\
= \\
= \\
= \\
100 \\
100 \\
100\end{array}$ & 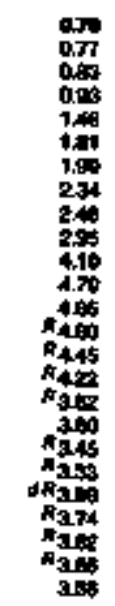 & 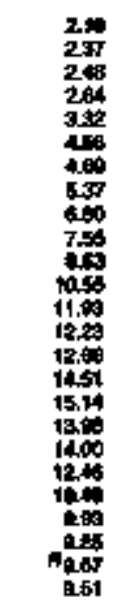 & 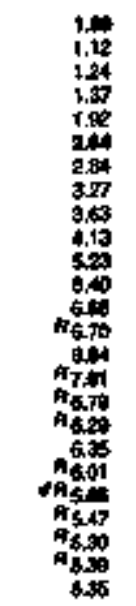 \\
\hline & \multicolumn{17}{|c|}{ 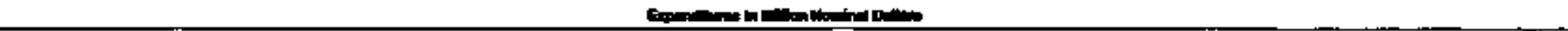 } \\
\hline 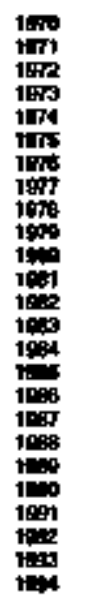 & 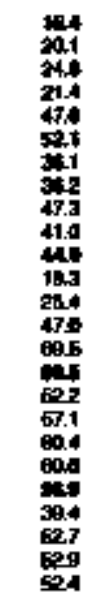 & 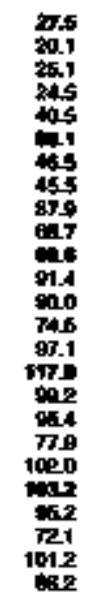 & 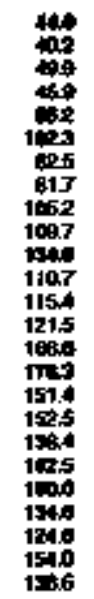 & 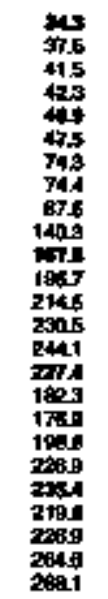 & 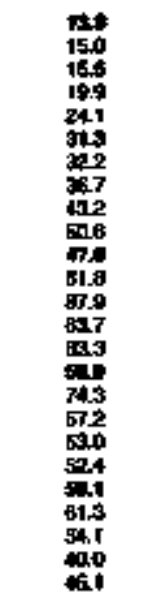 & 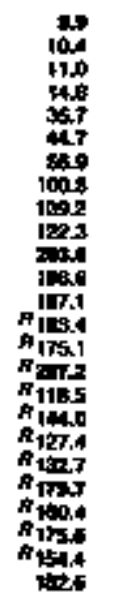 & 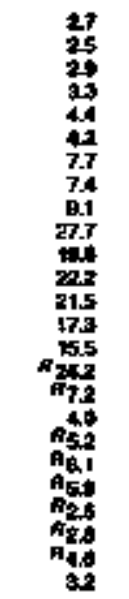 & 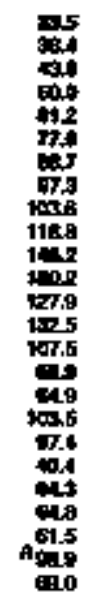 & 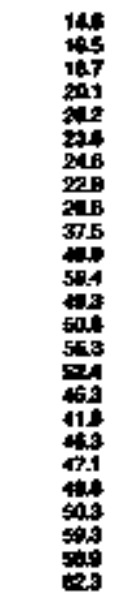 & 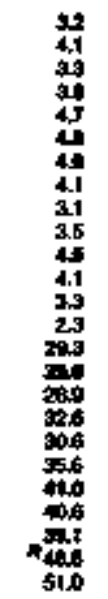 & 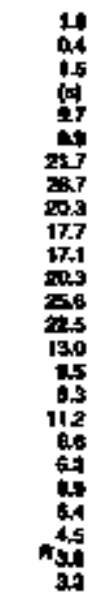 & 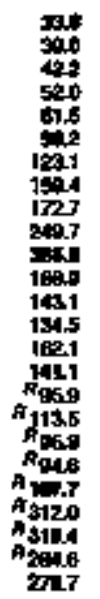 & 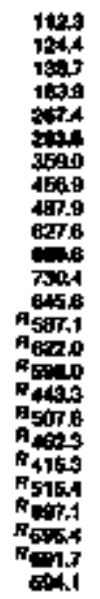 & 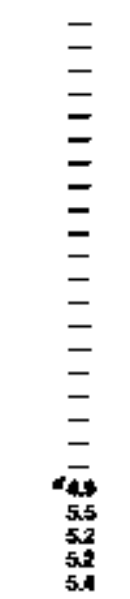 & 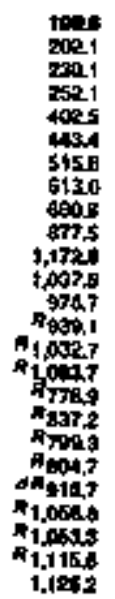 & 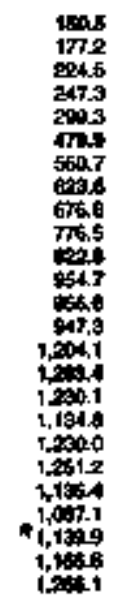 & 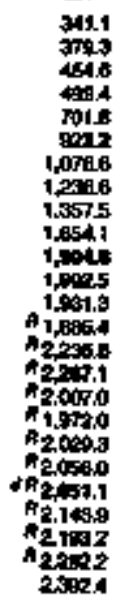 \\
\hline
\end{tabular}




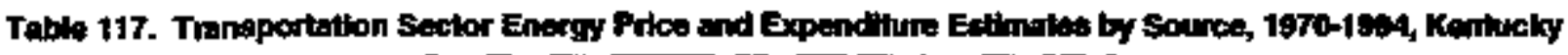

\begin{tabular}{|c|c|c|c|c|c|c|c|c|c|c|c|c|c|}
\hline \multirow[b]{4}{*}{ Tam } & \multicolumn{11}{|c|}{ Mments } & \multirow[b]{3}{*}{ Emplingy } & \multirow[b]{3}{*}{ 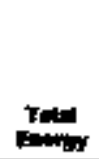 } \\
\hline & & & \multicolumn{8}{|c|}{ 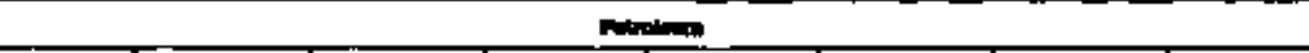 } & \multirow[b]{2}{*}{ Totid } & & \\
\hline & cod & 14 & 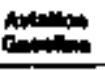 & Fum & F & Let: & 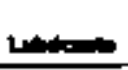 & 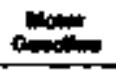 & 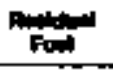 & $T=$ & & & \\
\hline & \multicolumn{13}{|c|}{ 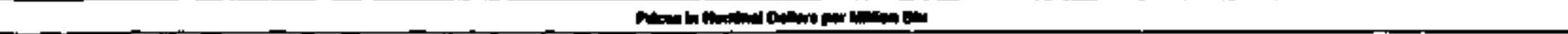 } \\
\hline 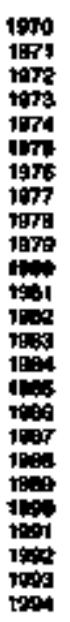 & $\begin{array}{l}04 \\
0.50 \\
0.50 \\
0.80 \\
0.87 \\
1.16 \\
1.23 \\
= \\
= \\
= \\
= \\
= \\
= \\
= \\
= \\
= \\
=\end{array}$ & $\begin{array}{l}= \\
= \\
= \\
= \\
= \\
= \\
= \\
= \\
= \\
= \\
= \\
= \\
= \\
35 \\
405\end{array}$ & 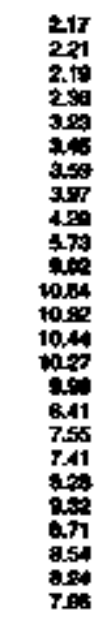 & 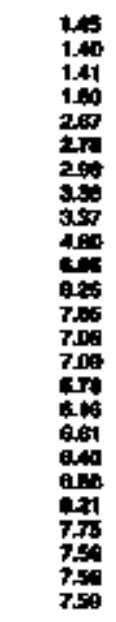 & 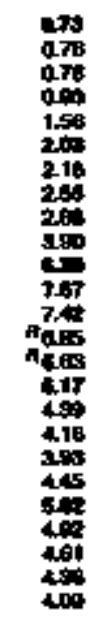 & 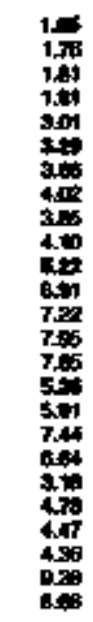 & 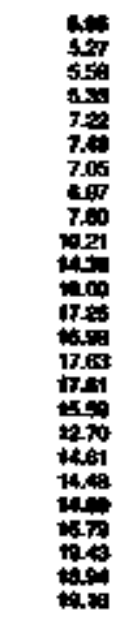 & 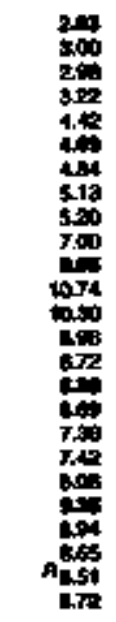 & 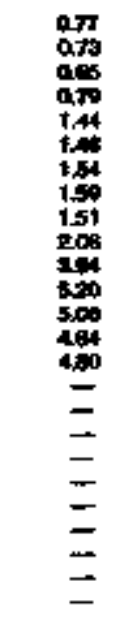 & 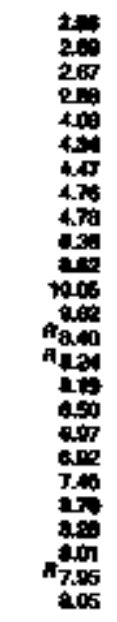 & 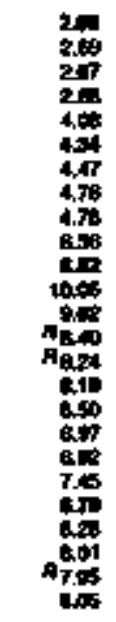 & $\begin{array}{l}= \\
= \\
= \\
= \\
= \\
= \\
= \\
= \\
= \\
= \\
= \\
= \\
=\end{array}$ & 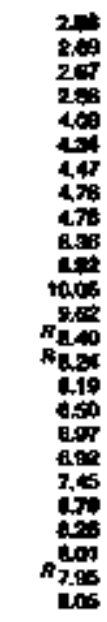 \\
\hline \multicolumn{14}{|c|}{ : } \\
\hline 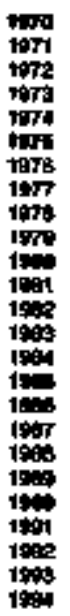 & $\begin{array}{l}0.1 \\
0 y \\
0 \\
0 \\
0 \\
= \\
= \\
= \\
= \\
= \\
= \\
= \\
= \\
= \\
=\end{array}$ & 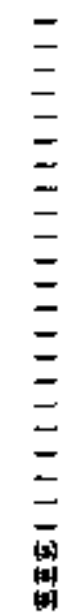 & 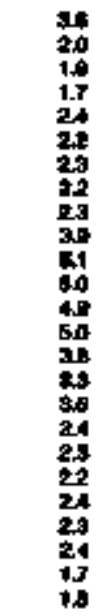 & 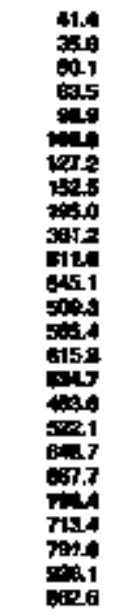 & 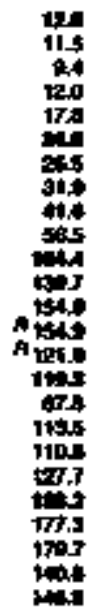 & 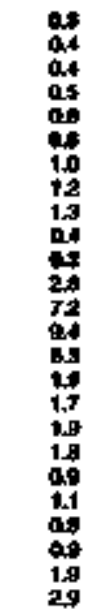 & 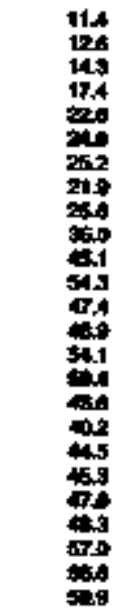 & 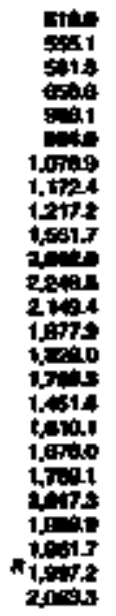 & 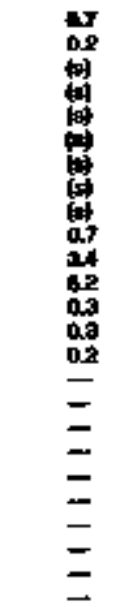 & 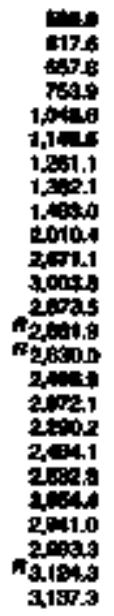 & 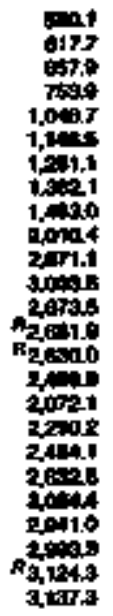 & $\begin{array}{l}= \\
= \\
= \\
= \\
= \\
= \\
= \\
= \\
= \\
= \\
= \\
=\end{array}$ & 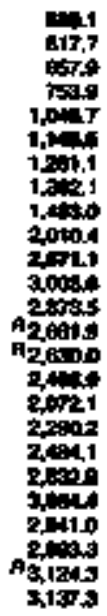 \\
\hline
\end{tabular}

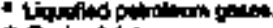

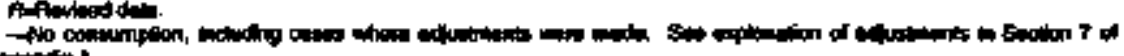

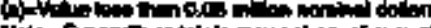

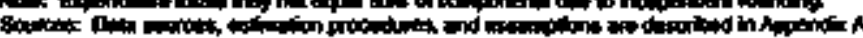




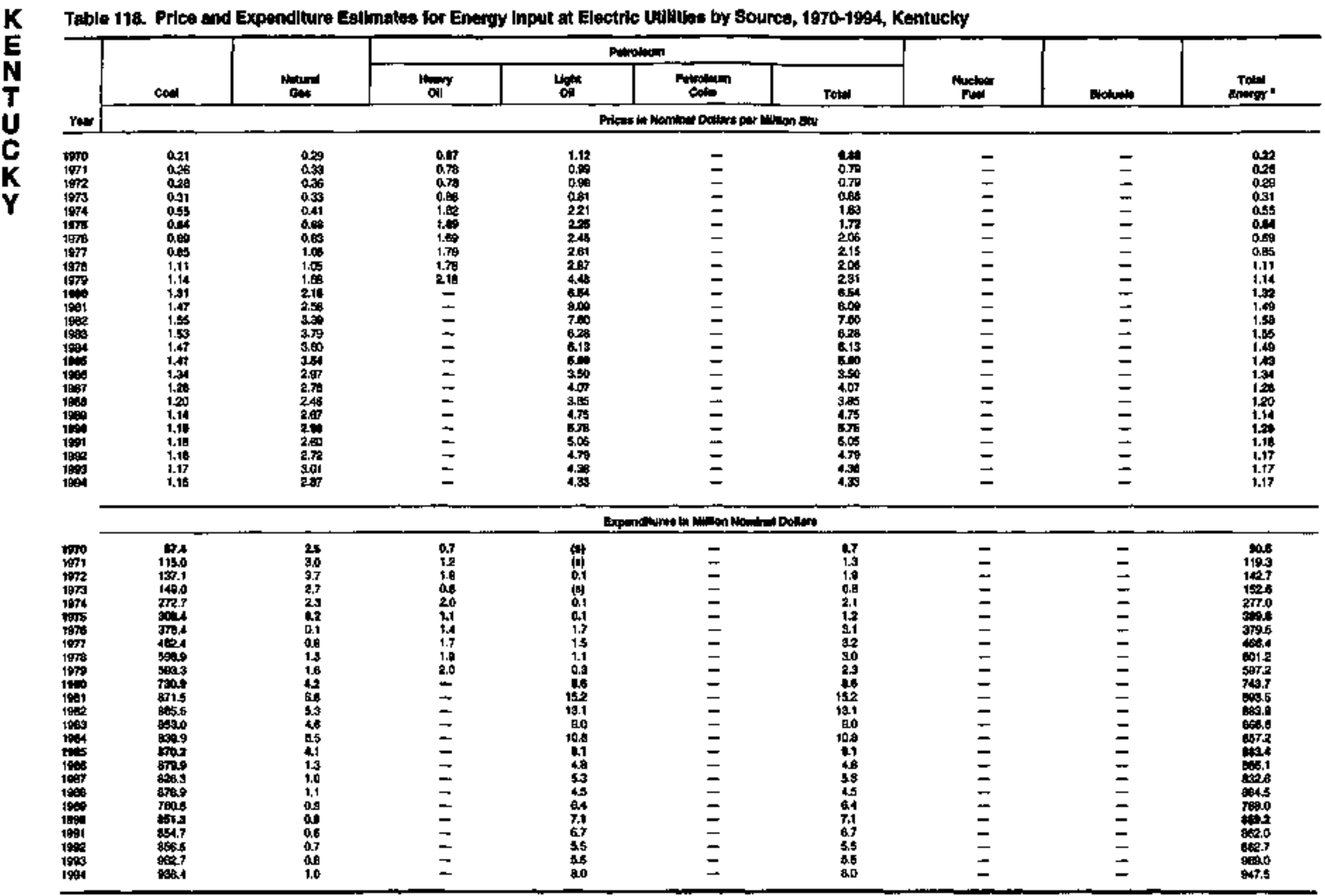

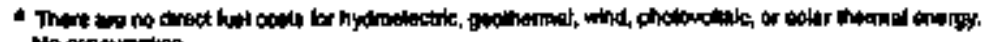
Tho crontumpion.

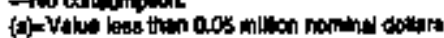

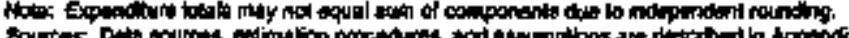

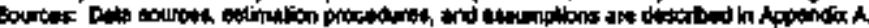


Toble 119. Energy Prles and Expendlum Ealimales by Source, 1970-1994, Loulsiana

\begin{tabular}{|c|c|c|c|c|c|c|c|c|c|c|c|c|c|c|c|c|c|}
\hline & \multicolumn{14}{|c|}{ Minery tingry } & \multirow{3}{*}{ Fullo } & \multirow{3}{*}{ 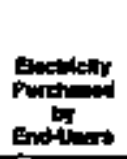 } & \multirow[b]{3}{*}{ Tom, } \\
\hline & \multicolumn{3}{|c|}{ coll } & \multirow[b]{2}{*}{ mencest. } & \multicolumn{7}{|c|}{ Putrome } & \multirow[b]{2}{*}{ meley } & \multirow[b]{2}{*}{ Elofue } & \multirow[b]{2}{*}{$T=0$} & & & \\
\hline & cond & con & 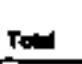 & & 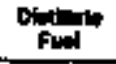 & fin & $\mathbf{L m}$ & motor. & minatid & Other" & Toted & & & & & & \\
\hline$r$ & \multicolumn{17}{|c|}{ 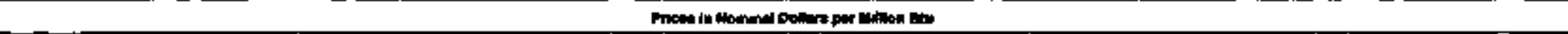 } \\
\hline \multirow[t]{2}{*}{ 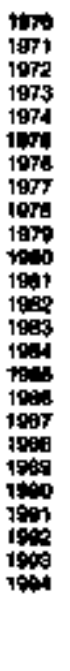 } & $\begin{array}{l}z \\
z \\
z \\
z \\
z \\
z \\
z \\
= \\
= \\
= \\
= \\
z \\
z \\
z \\
z\end{array}$ & 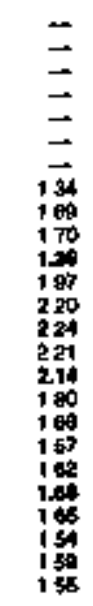 & 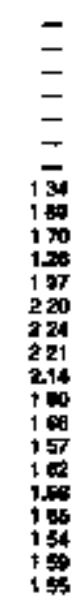 & 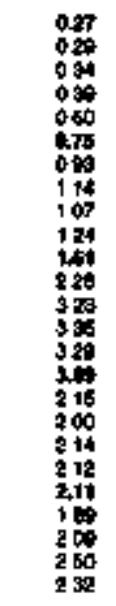 & 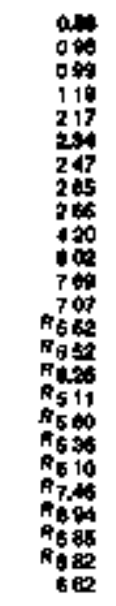 & 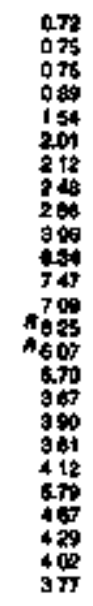 & 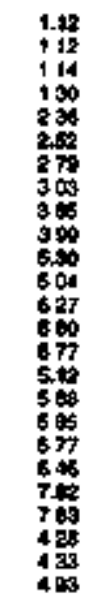 & 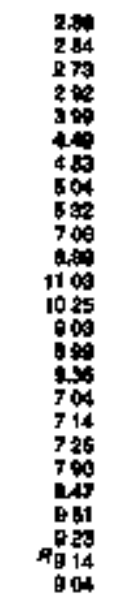 & 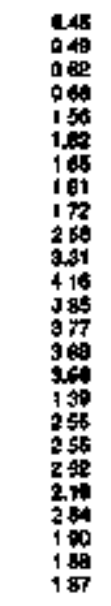 & 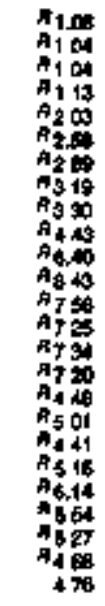 & 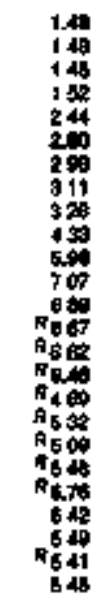 & 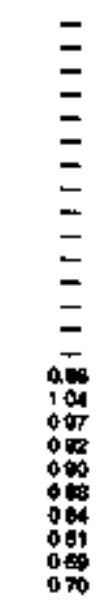 & $\begin{array}{l}= \\
= \\
= \\
= \\
= \\
= \\
= \\
= \\
= \\
= \\
1.4 \\
14 \\
14 \\
14 \\
14\end{array}$ & 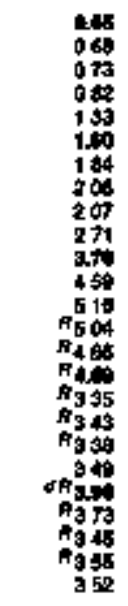 & 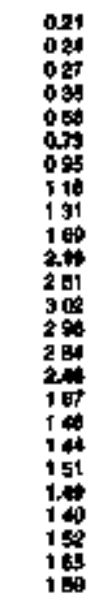 & 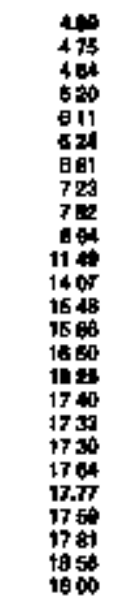 & 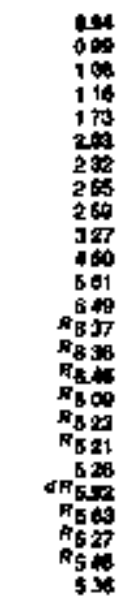 \\
\hline & \multicolumn{17}{|c|}{ 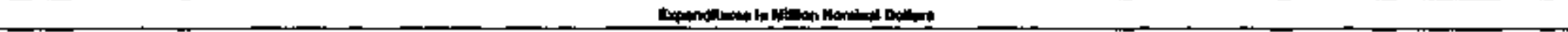 } \\
\hline 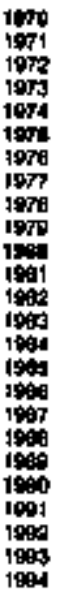 & $\begin{array}{l}= \\
= \\
= \\
= \\
= \\
= \\
= \\
= \\
= \\
= \\
= \\
= \\
= \\
=\end{array}$ & 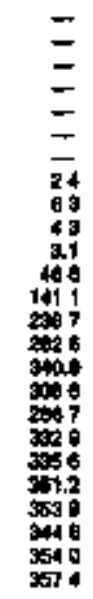 & 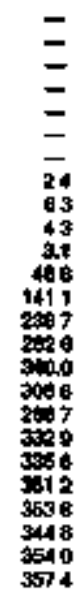 & 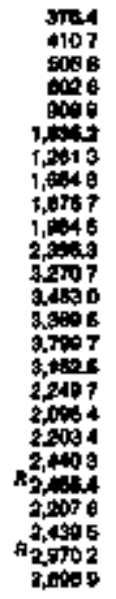 & 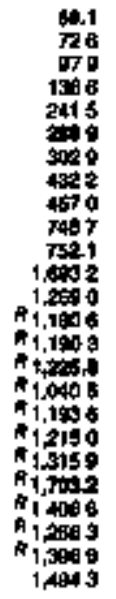 & 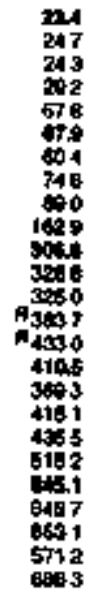 & 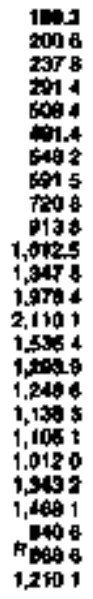 & 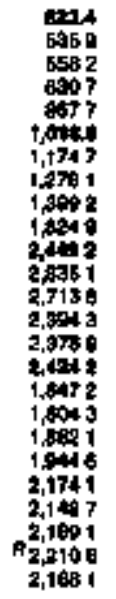 & 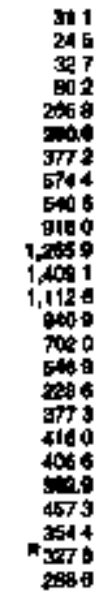 & 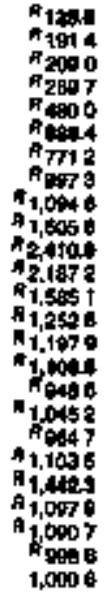 & 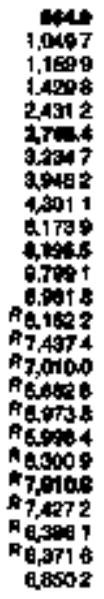 & $\begin{array}{l}= \\
= \\
= \\
= \\
= \\
= \\
= \\
= \\
= \\
275 \\
194 \\
1305 \\
1357 \\
198 \\
1354 \\
902 \\
1055 \\
901\end{array}$ & $\begin{array}{l}= \\
= \\
= \\
= \\
= \\
= \\
= \\
= \\
= \\
= \\
50 \\
85 \\
806 \\
860\end{array}$ & 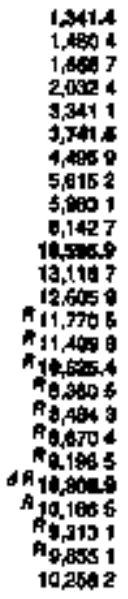 & 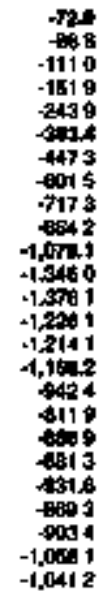 & 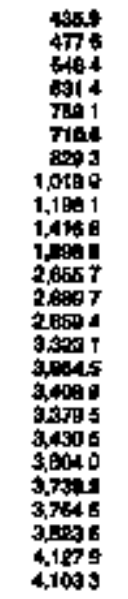 & 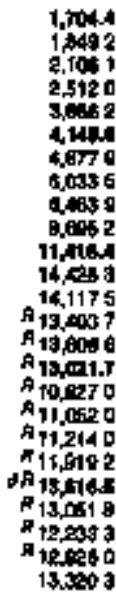 \\
\hline
\end{tabular}

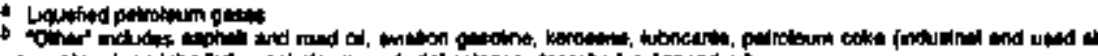

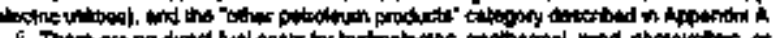

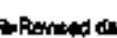

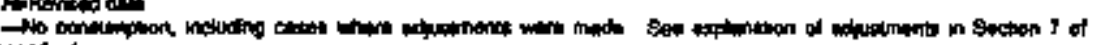

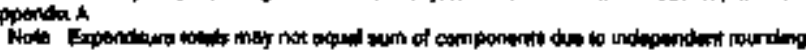

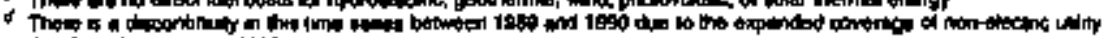

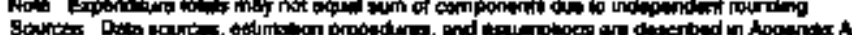




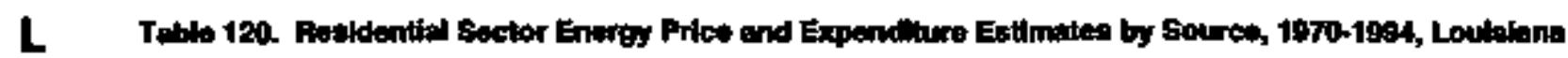

\begin{tabular}{|c|c|c|c|c|c|c|c|c|c|c|}
\hline \multirow[b]{4}{*}{$n$} & \multicolumn{8}{|c|}{ 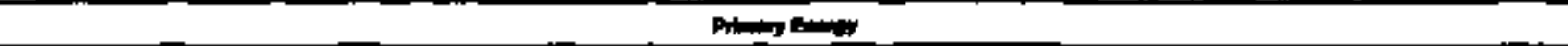 } & \multirow[b]{3}{*}{ 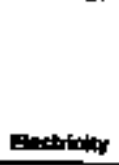 } & \multirow[b]{3}{*}{ 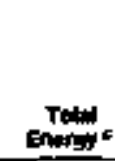 } \\
\hline & \multirow[b]{2}{*}{ can } & \multirow[b]{2}{*}{ Nen } & \multicolumn{4}{|c|}{ mann } & \multirow[b]{2}{*}{ Emanent } & \multirow[b]{2}{*}{ Tand: } & & \\
\hline & & & 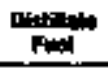 & Fon & Lo* & In: & & & & \\
\hline & \multicolumn{10}{|c|}{ 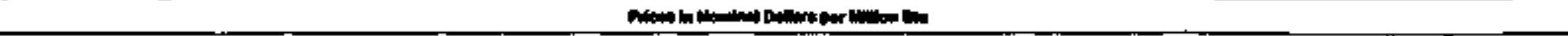 } \\
\hline \multirow[t]{2}{*}{ 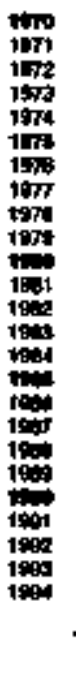 } & 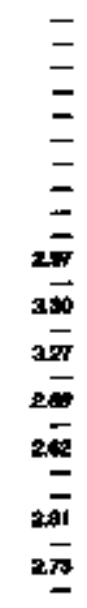 & 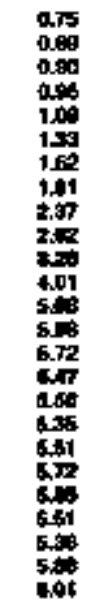 & 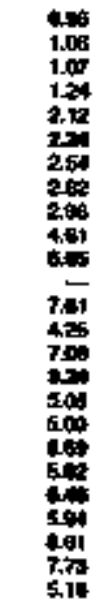 & 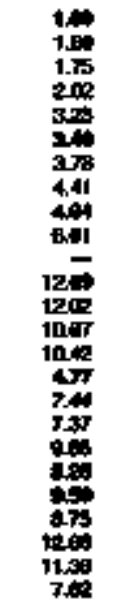 & 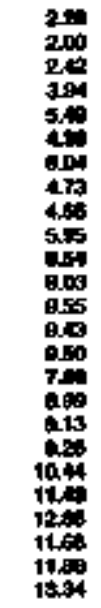 & 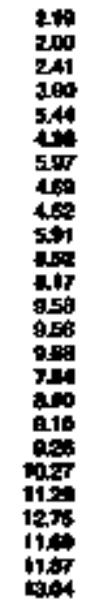 & 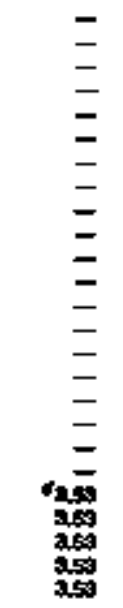 & 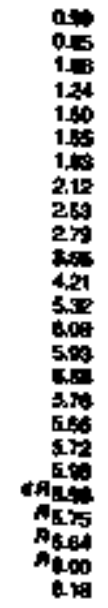 & 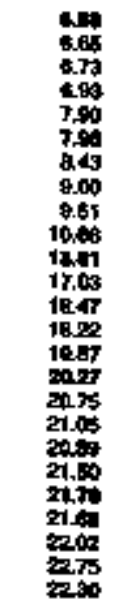 & 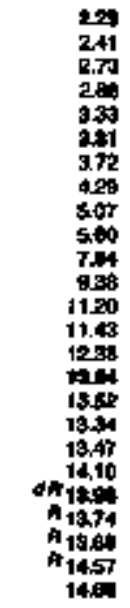 \\
\hline & \multicolumn{10}{|c|}{ 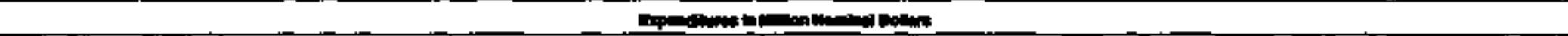 } \\
\hline 我 & 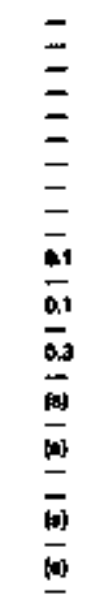 & 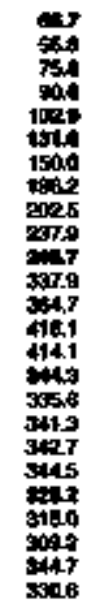 & 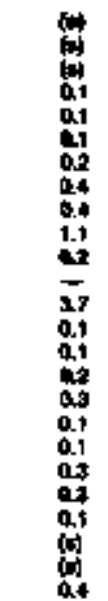 & 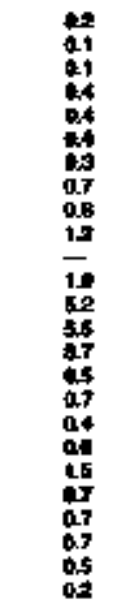 & 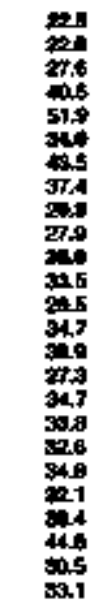 & 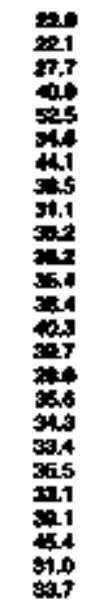 & $\begin{array}{l}= \\
= \\
= \\
= \\
= \\
= \\
= \\
= \\
= \\
= \\
= \\
11.6 \\
129 \\
129 \\
11.0\end{array}$ & 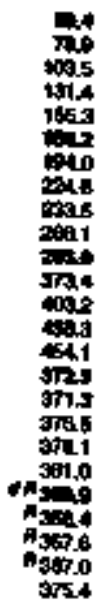 & 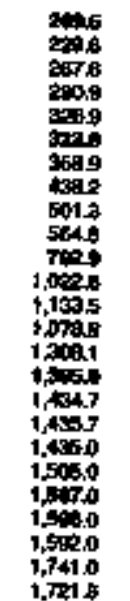 & 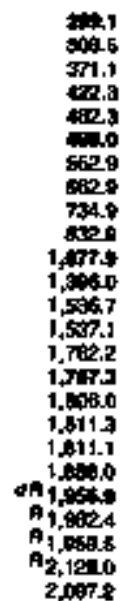 \\
\hline
\end{tabular}

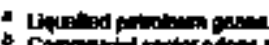

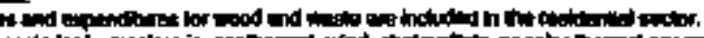

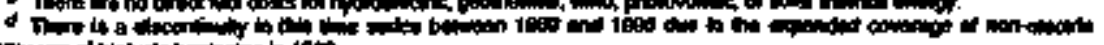

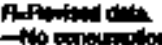

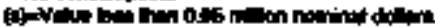

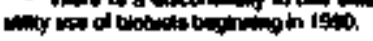

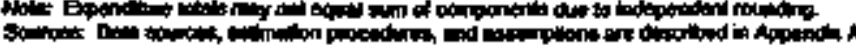




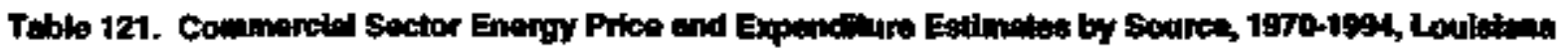

\begin{tabular}{|c|c|c|c|c|c|c|c|c|c|c|c|}
\hline \multirow[b]{4}{*}{$\boldsymbol{n}$} & \multicolumn{9}{|c|}{ Mary } & \multirow[b]{3}{*}{ Enctaty } & \multirow[b]{3}{*}{ 대에 } \\
\hline & \multirow[b]{2}{*}{ Cand } & \multirow[b]{2}{*}{ rats } & \multicolumn{6}{|c|}{ مصفمسn } & \multirow[b]{2}{*}{ Tot } & & \\
\hline & & & Fim & 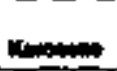 & $\mathbf{L P O}$ & 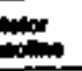 & Rall & Totow & & & \\
\hline & \multicolumn{11}{|c|}{ 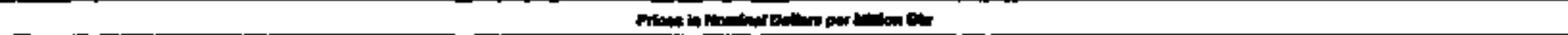 } \\
\hline \multirow[t]{2}{*}{ 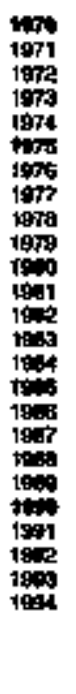 } & 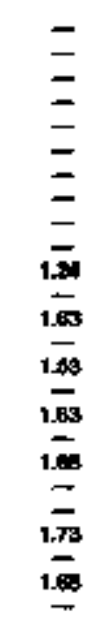 & 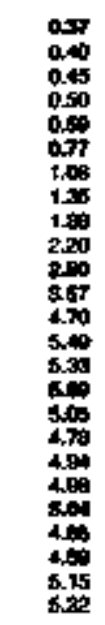 & 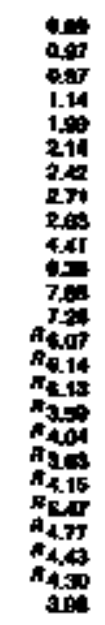 & 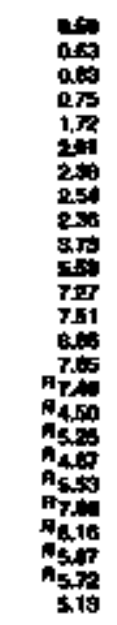 & 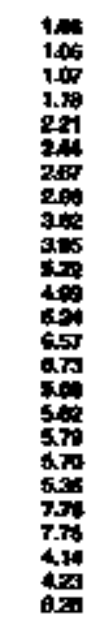 & 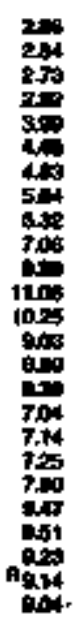 & 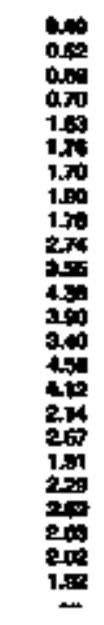 & 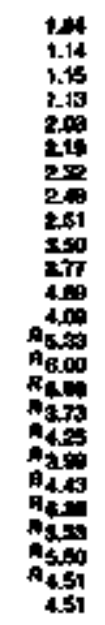 & 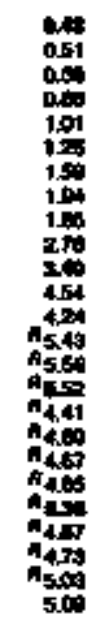 & 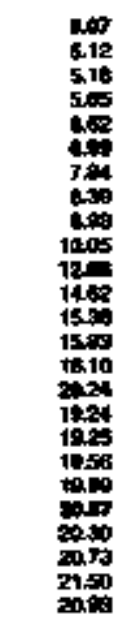 & 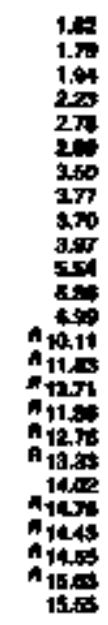 \\
\hline & \multicolumn{11}{|c|}{ 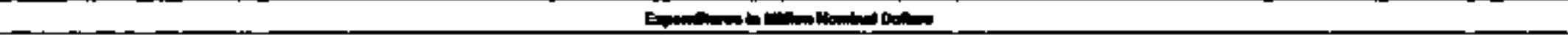 } \\
\hline 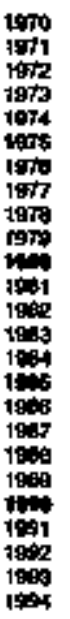 & 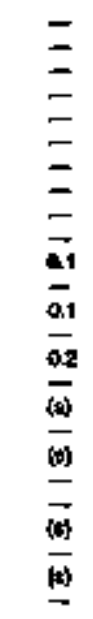 & 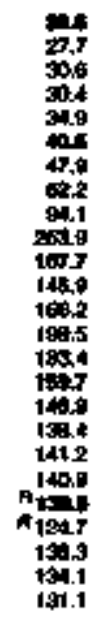 & 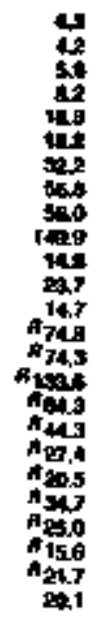 & 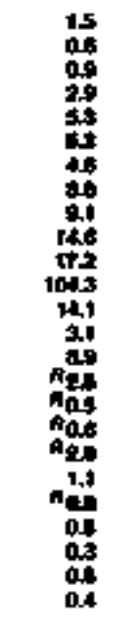 & 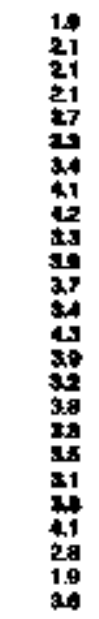 & 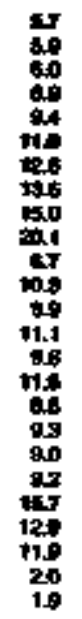 & 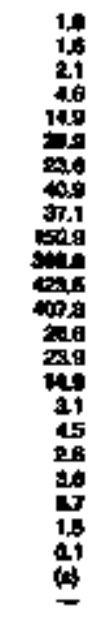 & 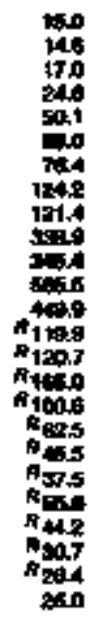 & 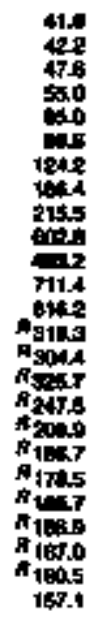 & 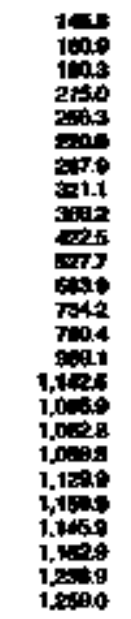 & 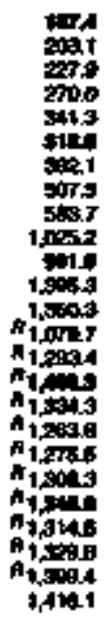 \\
\hline
\end{tabular}


L Table 122 Induatrial Sector Energy Prlca and Expenditure Estimates by Source, 1970-1994, Loulsana

\begin{tabular}{|c|c|c|c|c|c|c|c|c|c|c|c|c|c|c|c|c|c|}
\hline \multirow[b]{4}{*}{$\mathrm{rer}$} & \multicolumn{15}{|c|}{ 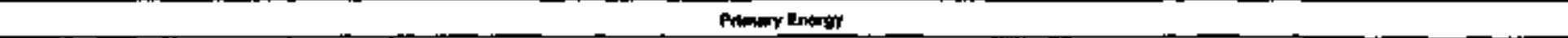 } & \multirow[b]{3}{*}{ Dmowant } & \multirow[b]{3}{*}{ 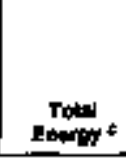 } \\
\hline & \multicolumn{3}{|c|}{ coed. } & \multirow[b]{2}{*}{ Fatit } & \multicolumn{9}{|c|}{ 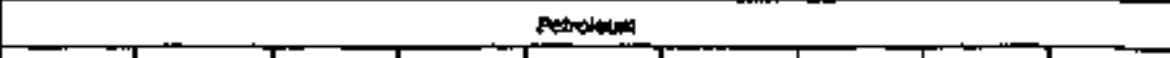 } & \multirow[b]{2}{*}{0} & \multirow[b]{2}{*}{ Town } & & \\
\hline & calpos & coot & $T_{0}$ & & 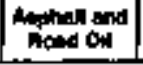 & Denthe & 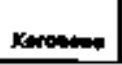 & Leo: & Lenteant & 然 & 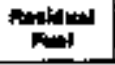 & Onner" & Toted & & & & \\
\hline & \multicolumn{17}{|c|}{ 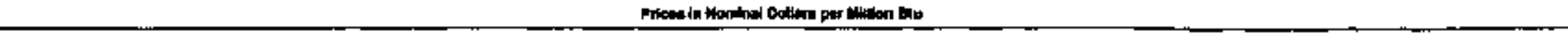 } \\
\hline \multirow[t]{2}{*}{ 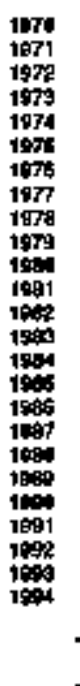 } & $\begin{array}{l}= \\
z \\
z \\
= \\
z \\
z \\
= \\
= \\
= \\
= \\
= \\
= \\
=\end{array}$ & 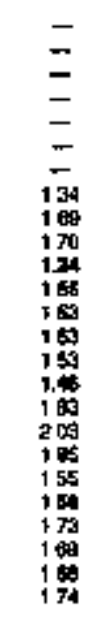 & 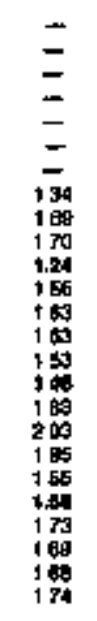 & 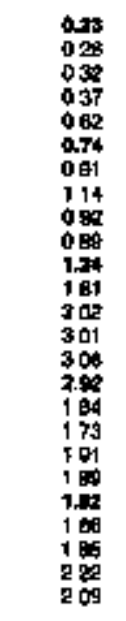 & 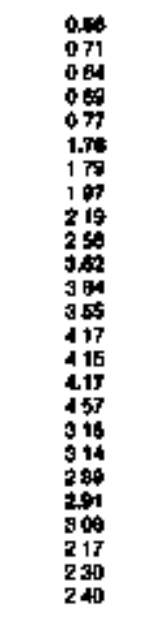 & 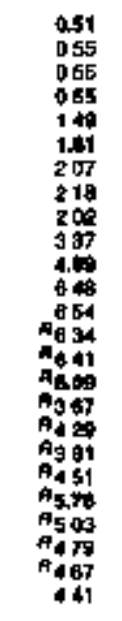 & 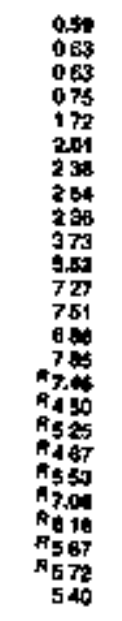 & 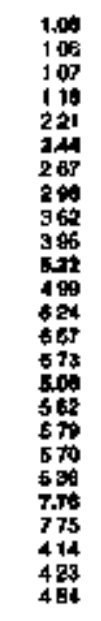 & 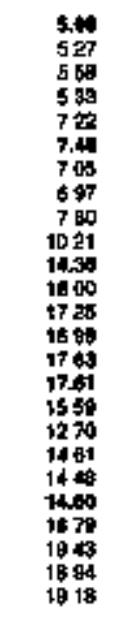 & 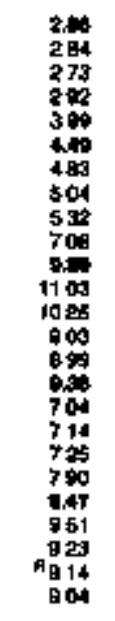 & 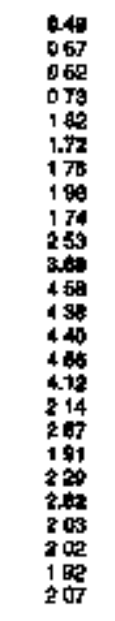 & 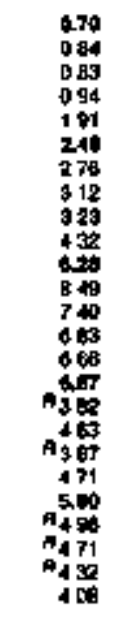 & 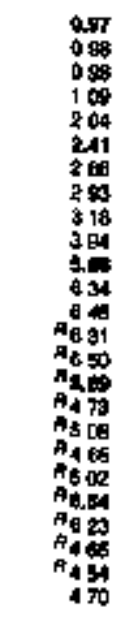 & 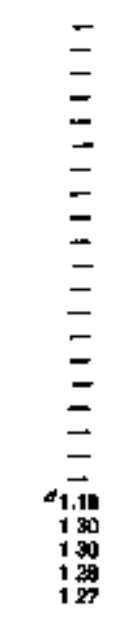 & 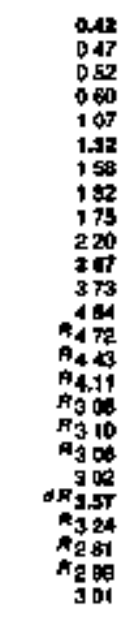 & 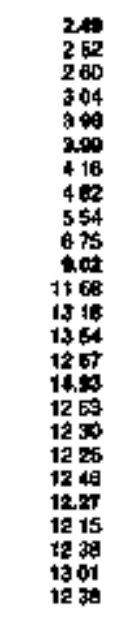 & 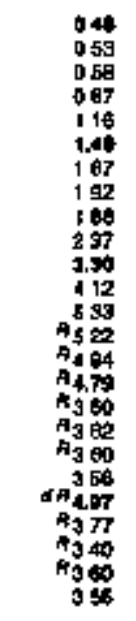 \\
\hline & \multicolumn{17}{|c|}{ 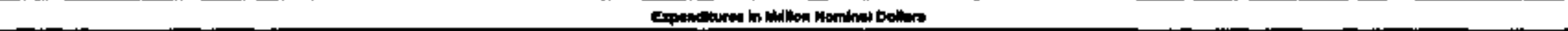 } \\
\hline 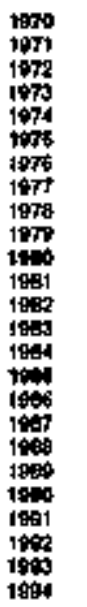 & $\begin{array}{l} \pm \\
= \\
= \\
= \\
= \\
= \\
= \\
= \\
= \\
= \\
= \\
= \\
=\end{array}$ & 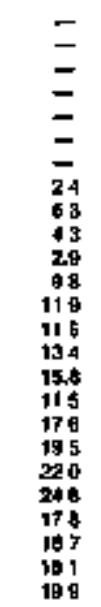 & 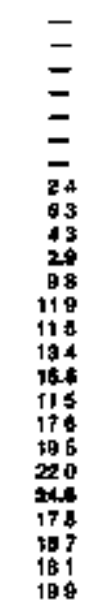 & 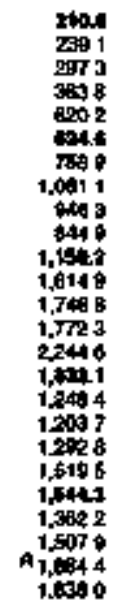 & 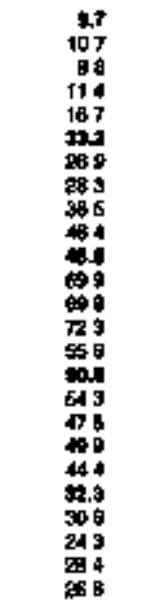 & 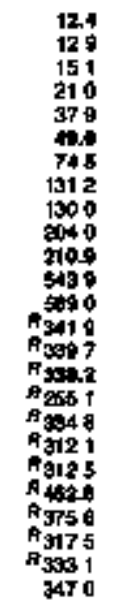 & 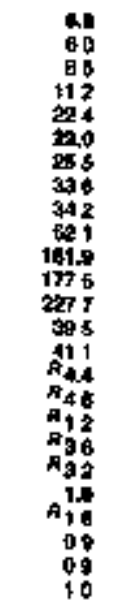 & 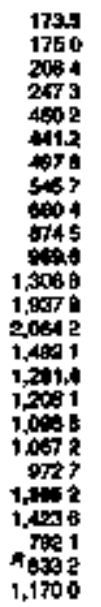 & 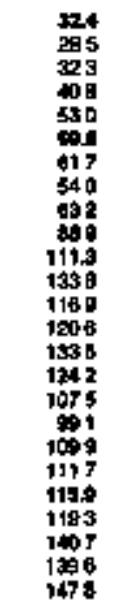 & 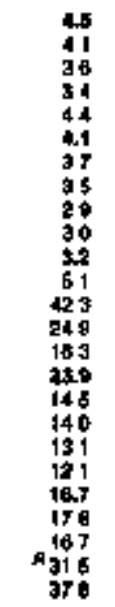 & 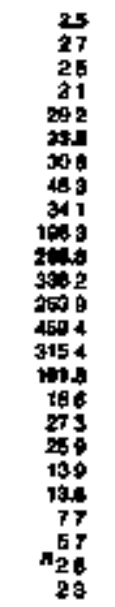 & 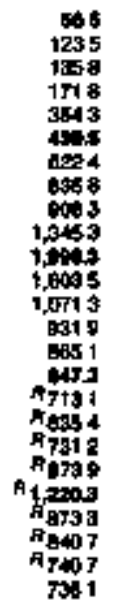 & 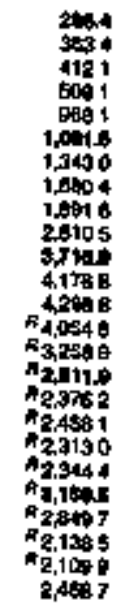 & 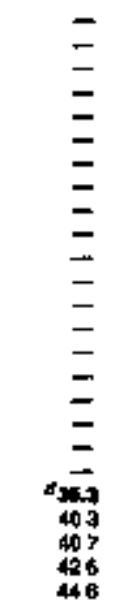 & 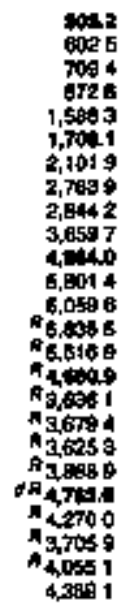 & 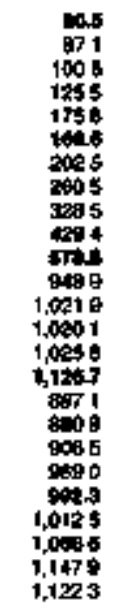 & 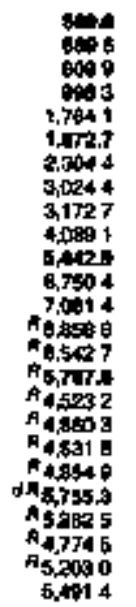 \\
\hline
\end{tabular}




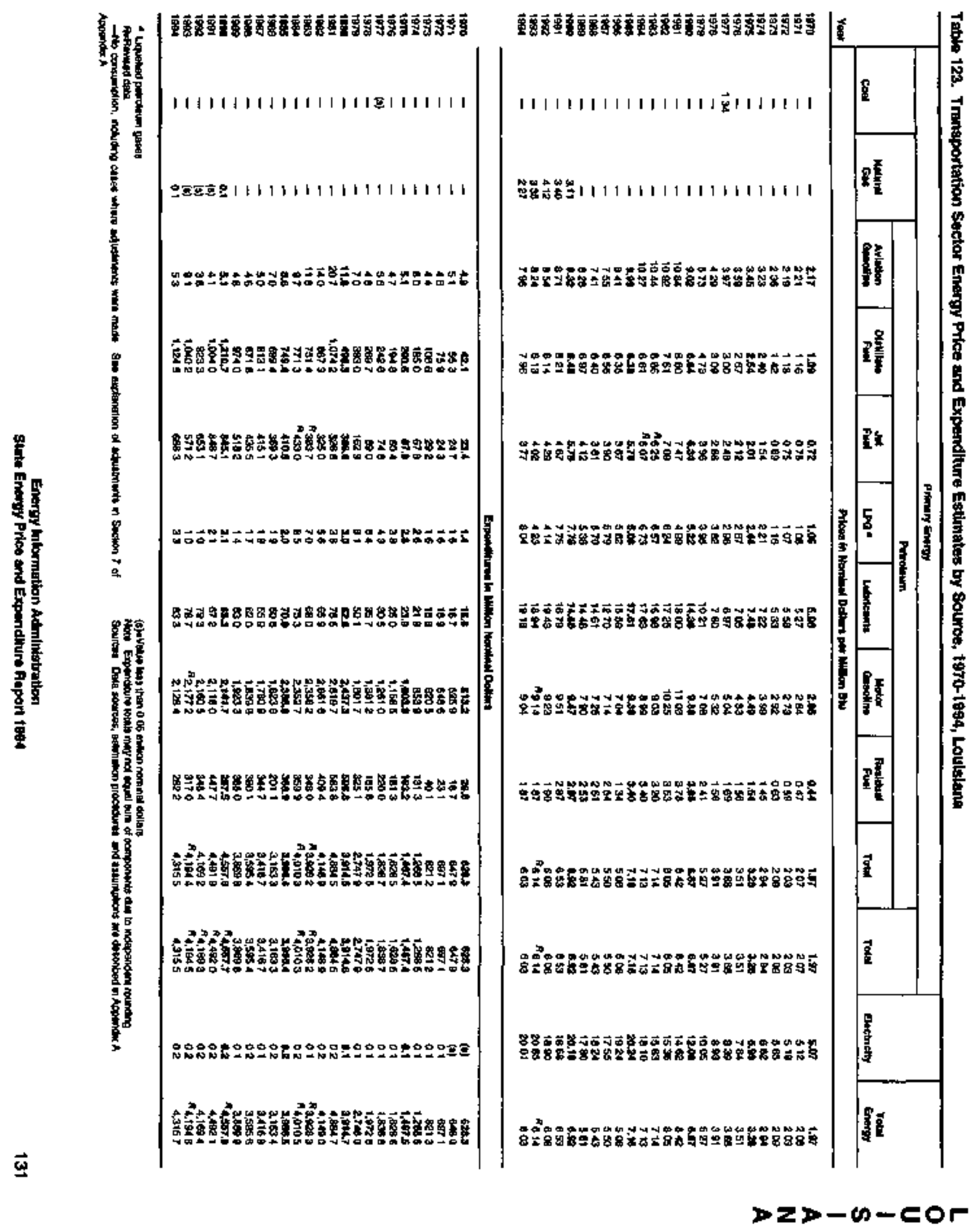




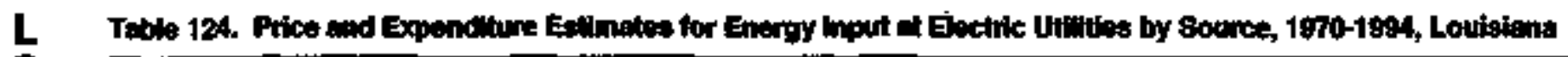

\begin{tabular}{|c|c|c|c|c|c|c|c|c|c|}
\hline \multirow[b]{3}{*}{ Yow } & \multirow[b]{2}{*}{ Cod } & \multirow[b]{2}{*}{ not } & \multicolumn{4}{|c|}{ Fenomingen } & \multirow[b]{2}{*}{ funder } & \multirow[b]{2}{*}{ 1. } & \multirow[b]{2}{*}{ Tots } \\
\hline & & & Hit & 10 & הומה: & Tot & & & \\
\hline & \multicolumn{9}{|c|}{ 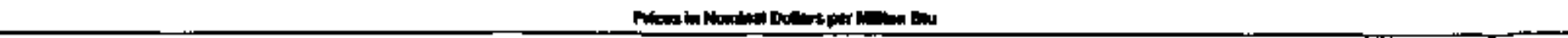 } \\
\hline \multirow[t]{2}{*}{ 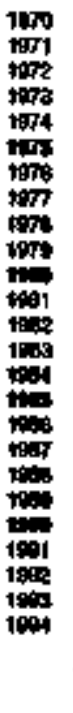 } & 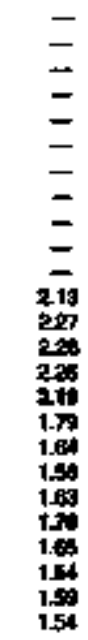 & 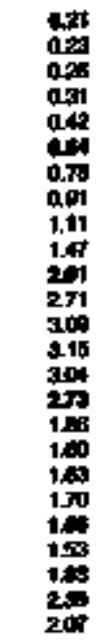 & 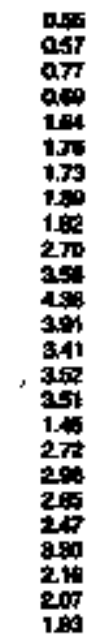 & 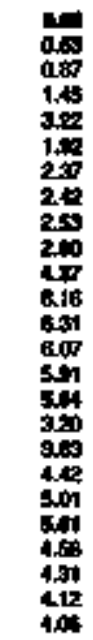 & $\begin{array}{l}= \\
= \\
= \\
= \\
= \\
= \\
= \\
= \\
= \\
= \\
= \\
5\end{array}$ & 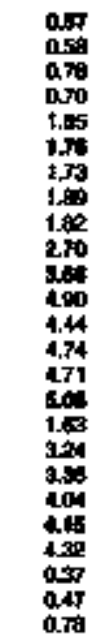 & 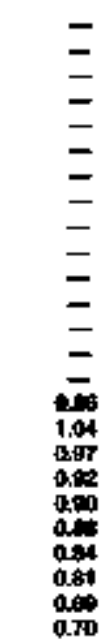 & $\begin{array}{l}= \\
= \\
= \\
= \\
= \\
= \\
= \\
= \\
= \\
= \\
= \\
= \\
= \\
= \\
=\end{array}$ & 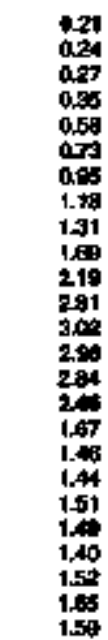 \\
\hline & \multicolumn{9}{|c|}{ ع } \\
\hline 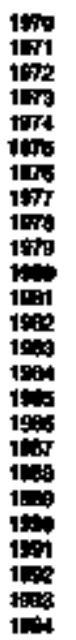 & 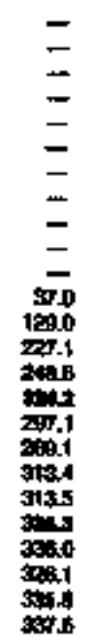 & 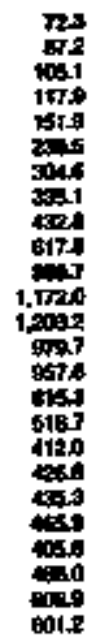 & 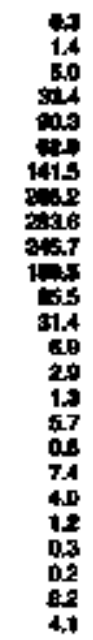 & 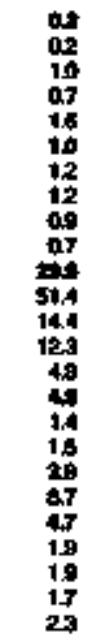 & $\begin{array}{l}= \\
= \\
= \\
= \\
= \\
= \\
= \\
= \\
= \\
= \\
= \\
= \\
5\end{array}$ & 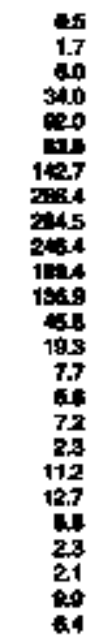 & 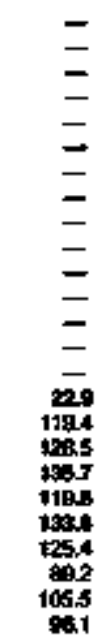 & $\begin{array}{l}= \\
= \\
= \\
= \\
= \\
= \\
= \\
= \\
= \\
= \\
= \\
= \\
= \\
=\end{array}$ & 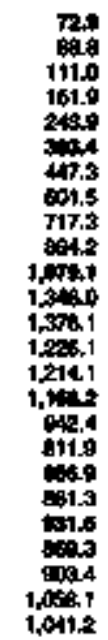 \\
\hline
\end{tabular}

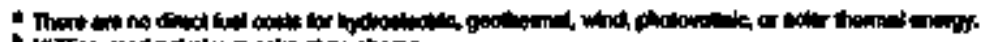

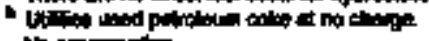

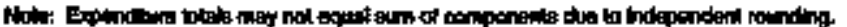

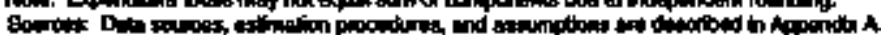


Tabto 125. Enewry Prico and Expenditure Eatimatos by 30urce, 1970-1994, Meino

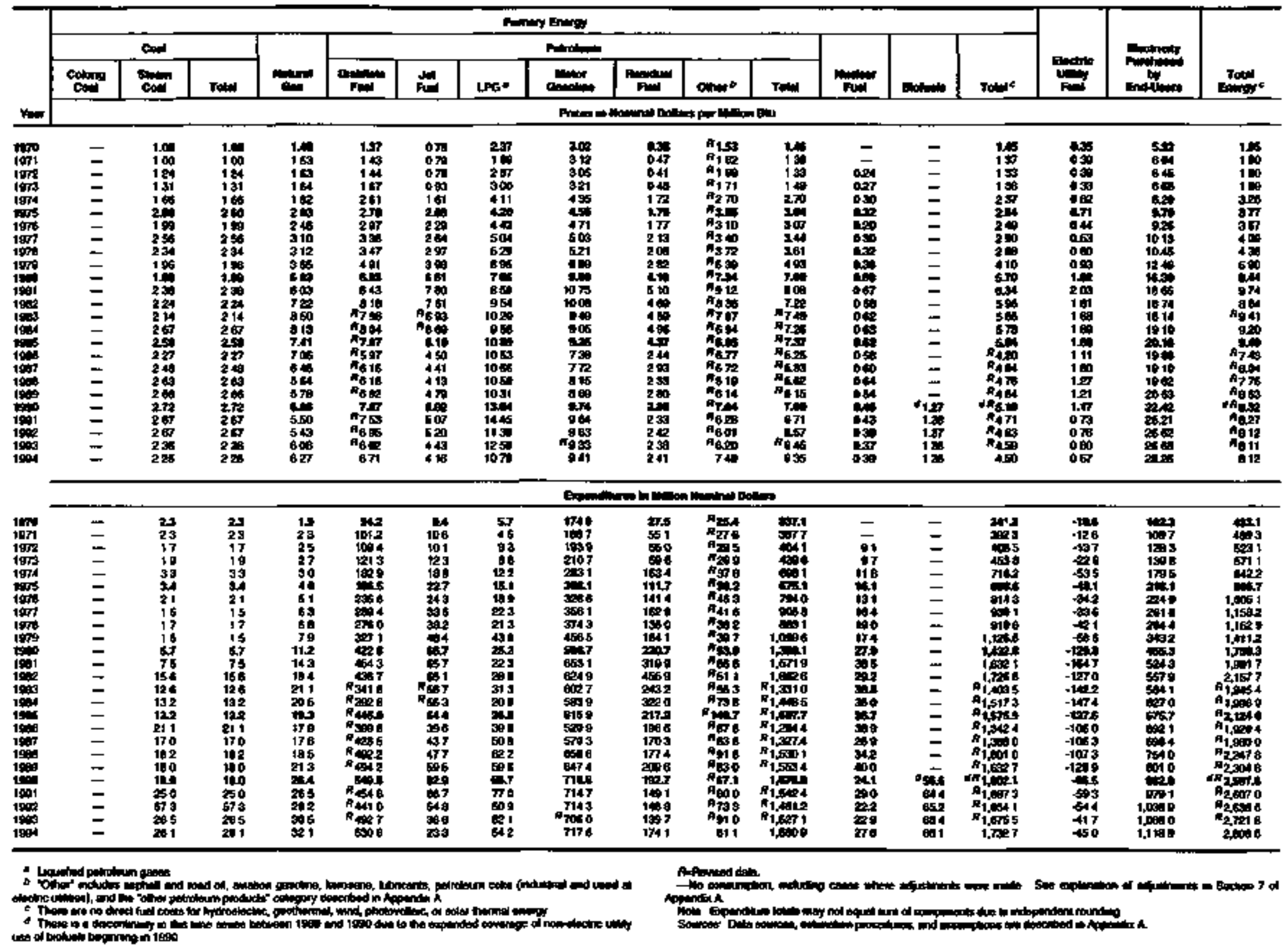



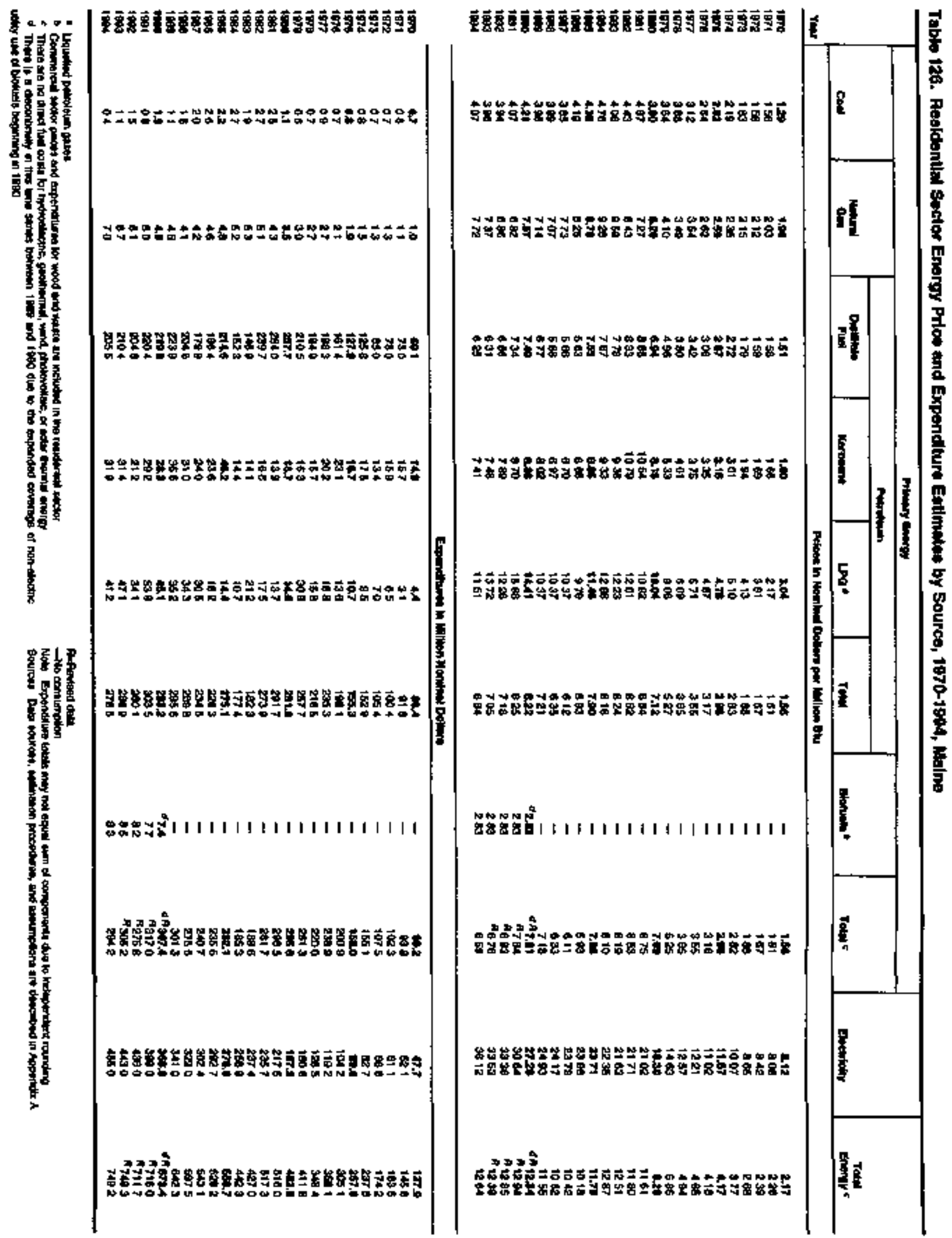

置

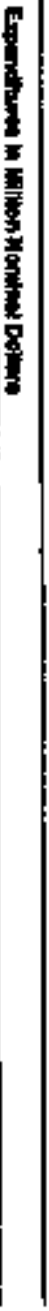

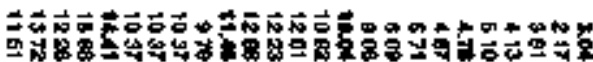

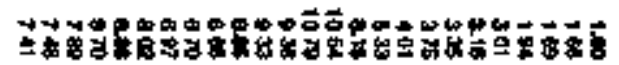

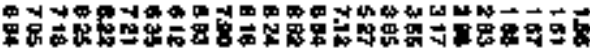

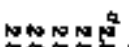

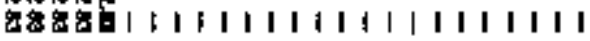

2.xis

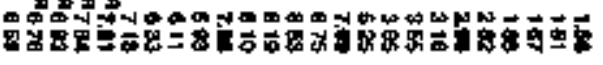

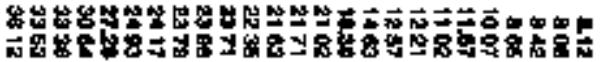

$\rightarrow 30$

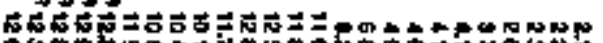

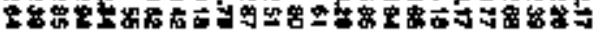

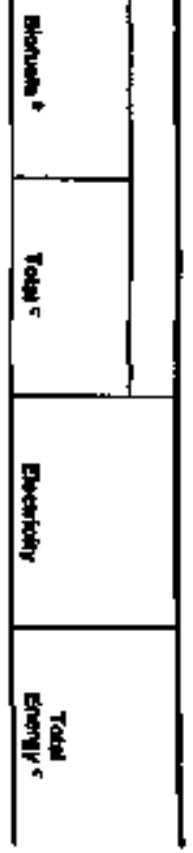




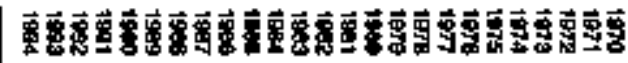

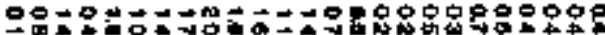

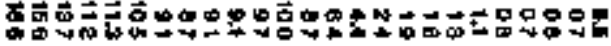

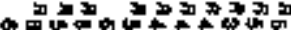

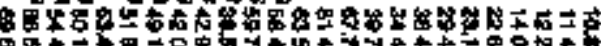
(1)

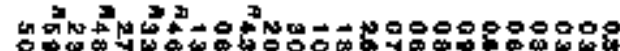

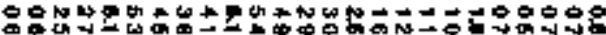

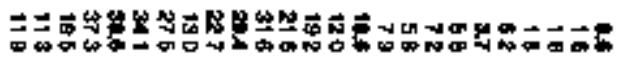

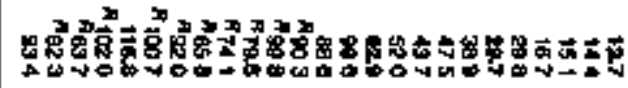

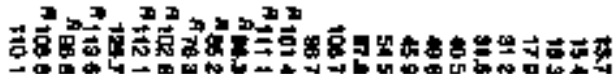

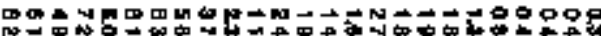

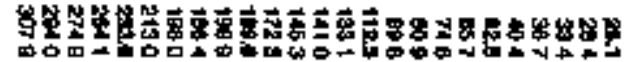

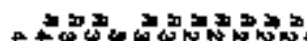

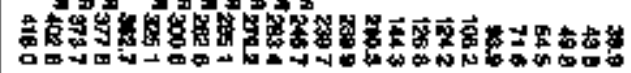

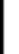

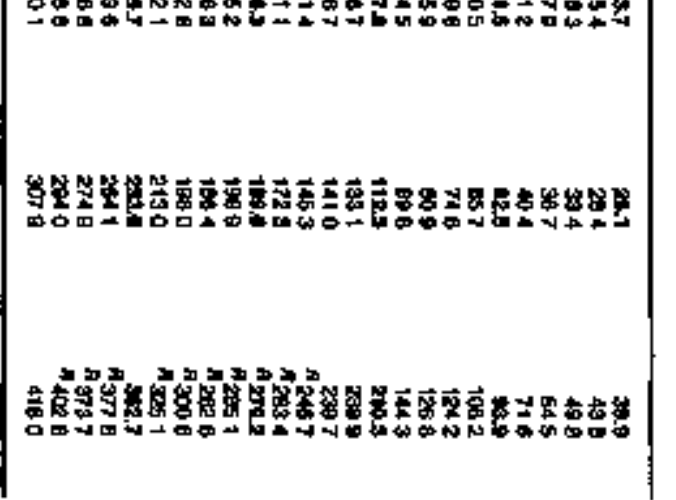

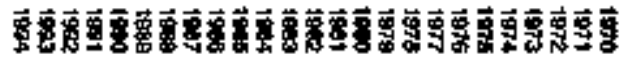

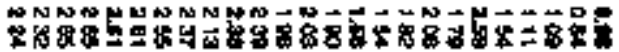

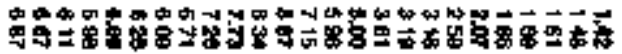

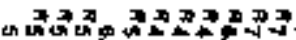

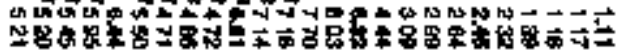

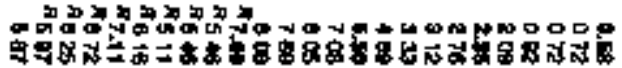

9R

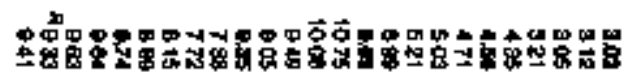

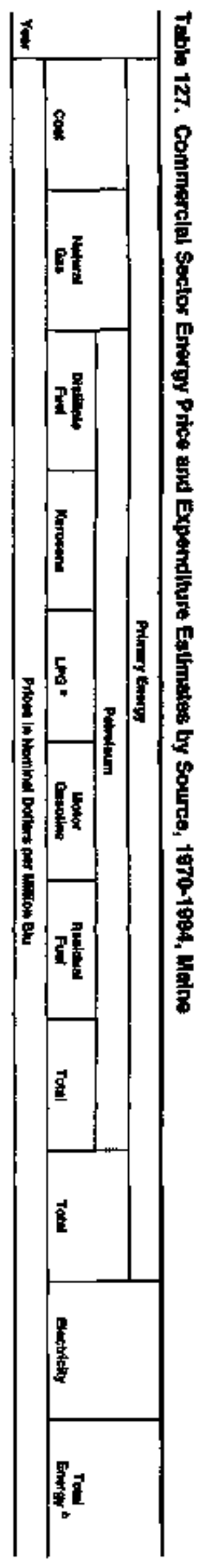

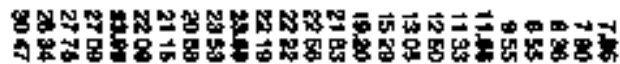

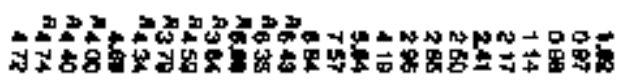

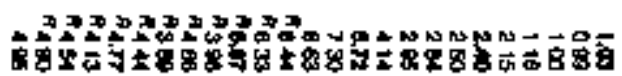

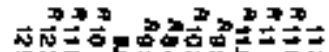

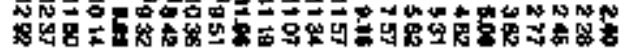

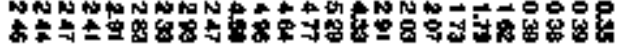

$m z-\geq z$ 


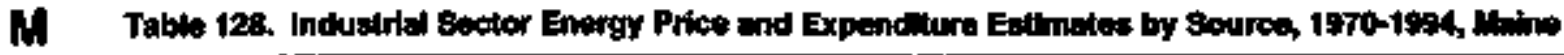

$\mathbf{A}$
$\mathbf{N}$
$\mathbf{E}$

\begin{tabular}{|c|c|c|c|c|c|c|c|c|c|c|c|c|c|c|c|c|c|}
\hline & \multicolumn{15}{|c|}{ 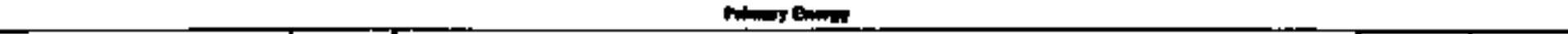 } & \multirow[b]{3}{*}{ 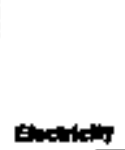 } & \multirow[b]{3}{*}{ Exise } \\
\hline & \multicolumn{3}{|c|}{ cont } & \multirow[b]{2}{*}{ Mnting } & \multicolumn{9}{|c|}{ 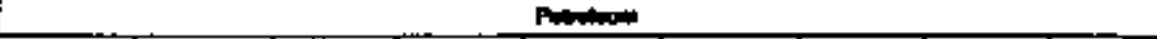 } & \multirow[b]{2}{*}{ e } & \multirow[b]{2}{*}{ Tome } & & \\
\hline & Cod & cond & Tot & & 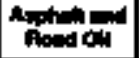 & 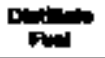 & 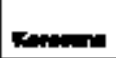 & $\mathbf{L} \mathbf{0}^{*}$ & Lulomin & & fintin & ${ }^{2}$ & $T=4$ & & & & \\
\hline $\mathbf{r}=\mathbf{E}$ & \multicolumn{17}{|c|}{ 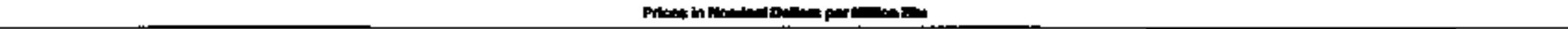 } \\
\hline 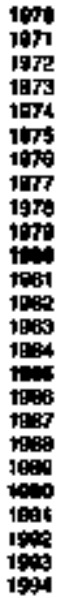 & $\begin{array}{l}= \\
= \\
= \\
= \\
= \\
= \\
= \\
= \\
= \\
= \\
= \\
= \\
=\end{array}$ & 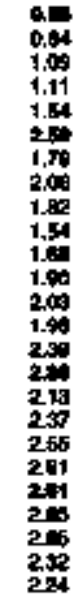 & 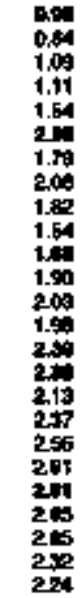 & 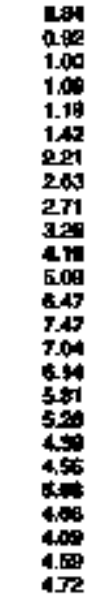 & 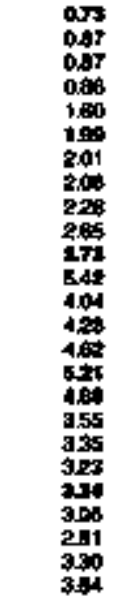 & 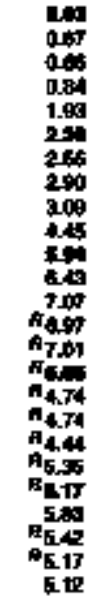 & 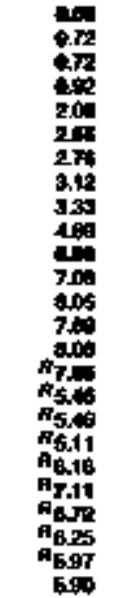 & 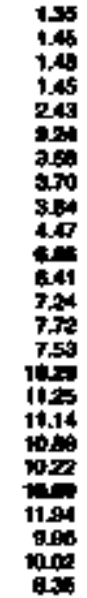 & 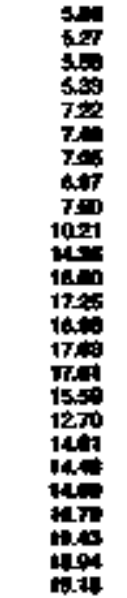 & 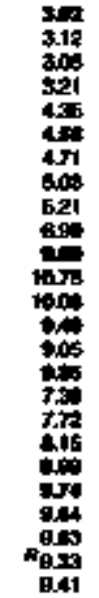 & 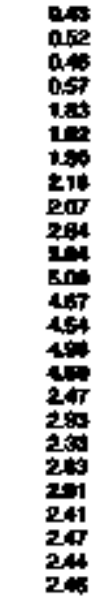 & 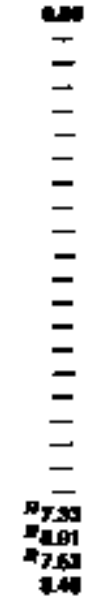 & 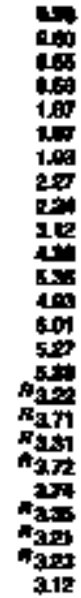 & $\begin{array}{l}= \\
= \\
= \\
= \\
= \\
= \\
= \\
= \\
= \\
= \\
= \\
\bar{z} \\
1.77 \\
1.29 \\
1.98 \\
127\end{array}$ & 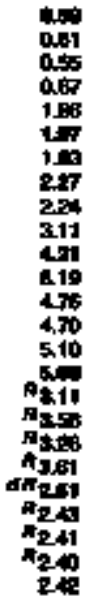 & 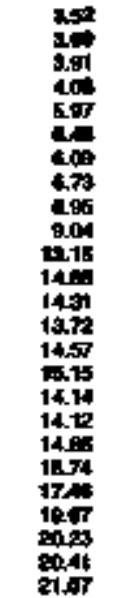 & 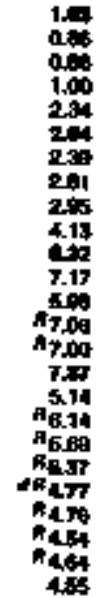 \\
\hline
\end{tabular}

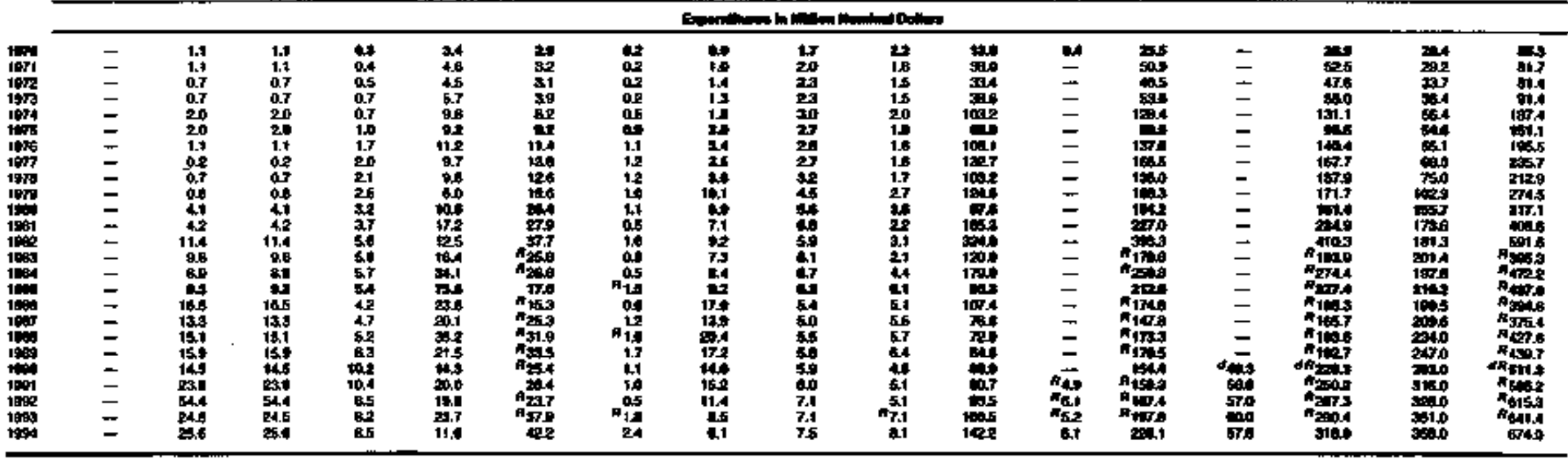

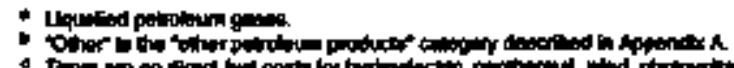

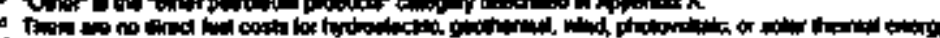

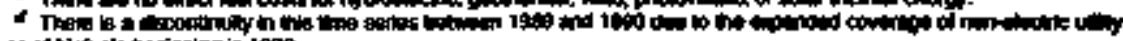

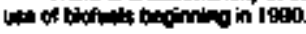

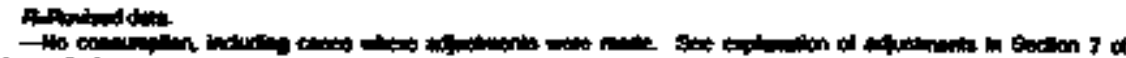

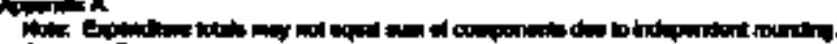




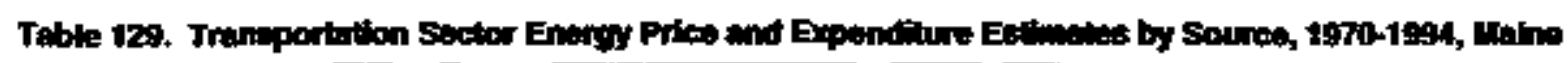

\begin{tabular}{|c|c|c|c|c|c|c|c|c|c|c|c|c|c|}
\hline \multirow[b]{4}{*}{ tom } & \multicolumn{11}{|c|}{ 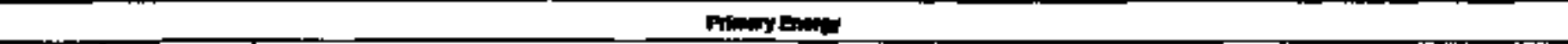 } & \multirow[b]{3}{*}{ Bucticty } & \multirow[b]{3}{*}{ rate: } \\
\hline & \multirow[b]{2}{*}{ 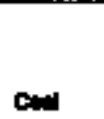 } & \multirow[b]{2}{*}{ 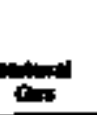 } & \multicolumn{8}{|c|}{ 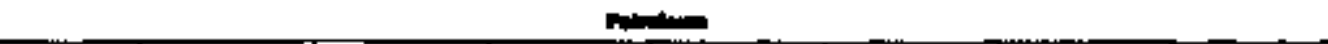 } & \multirow[b]{2}{*}{$T$} & & \\
\hline & & & ה & 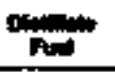 & 흘 & Lo:" & 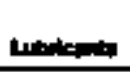 & 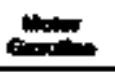 & "ה & Toter & & & \\
\hline & \multicolumn{13}{|c|}{ 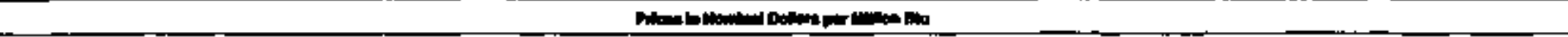 } \\
\hline \multirow[t]{2}{*}{ 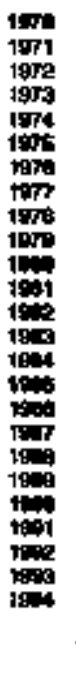 } & 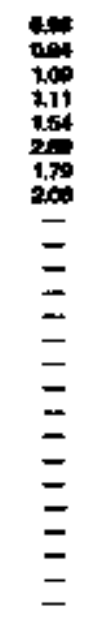 & $\begin{array}{l}= \\
= \\
= \\
= \\
= \\
= \\
= \\
= \\
= \\
= \\
= \\
z \\
z\end{array}$ & 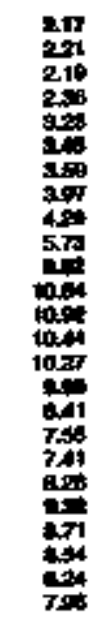 & 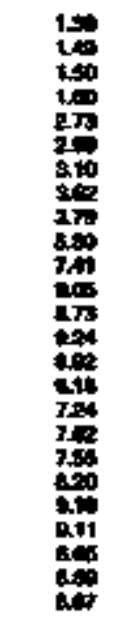 & 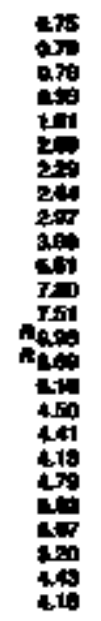 & 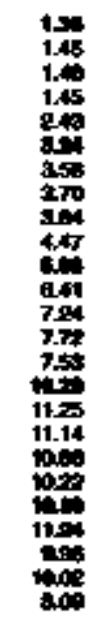 & 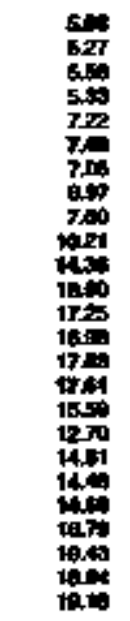 & 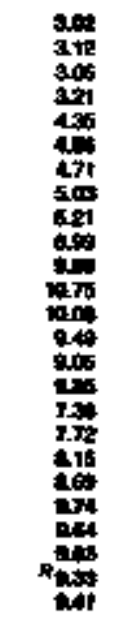 & 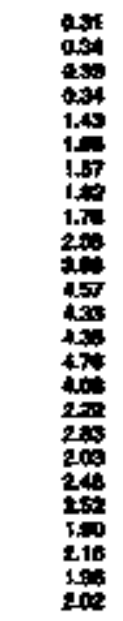 & 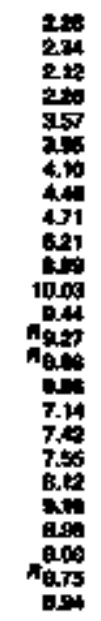 & 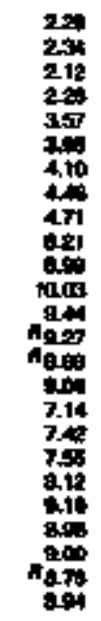 & $\begin{array}{l}\bar{z} \\
\bar{z} \\
\bar{z} \\
\bar{z} \\
= \\
= \\
= \\
= \\
= \\
= \\
z \\
=\end{array}$ & 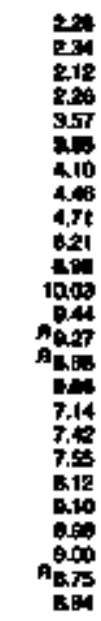 \\
\hline & \multicolumn{13}{|c|}{ 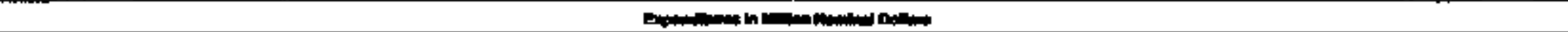 } \\
\hline 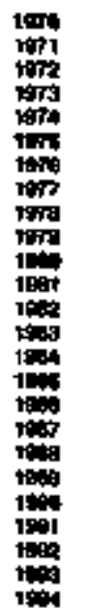 & $\begin{array}{l}8 \\
0 \\
0 \\
= \\
= \\
= \\
= \\
= \\
= \\
= \\
= \\
= \\
= \\
=\end{array}$ & $\begin{array}{l}= \\
z \\
z \\
= \\
z \\
= \\
= \\
z \\
= \\
= \\
= \\
= \\
= \\
=\end{array}$ & 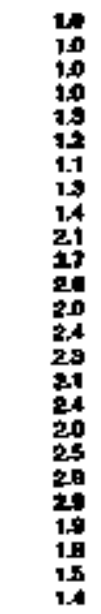 & 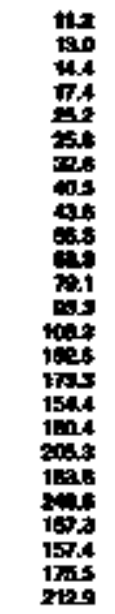 & 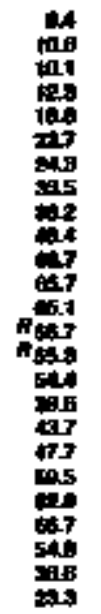 & 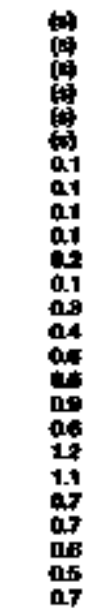 & 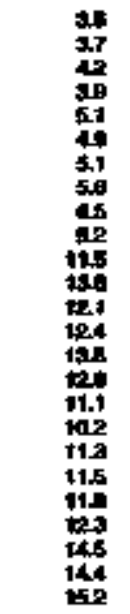 & 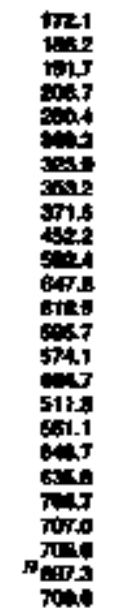 & 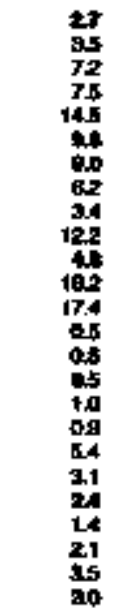 & 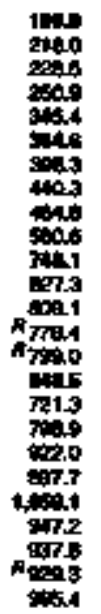 & 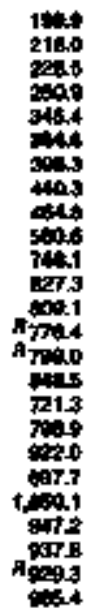 & $\begin{array}{l}= \\
= \\
= \\
= \\
z \\
= \\
= \\
= \\
= \\
= \\
= \\
= \\
=\end{array}$ & 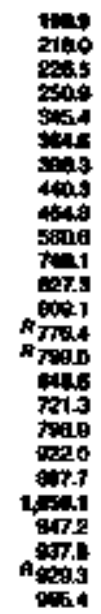 \\
\hline
\end{tabular}



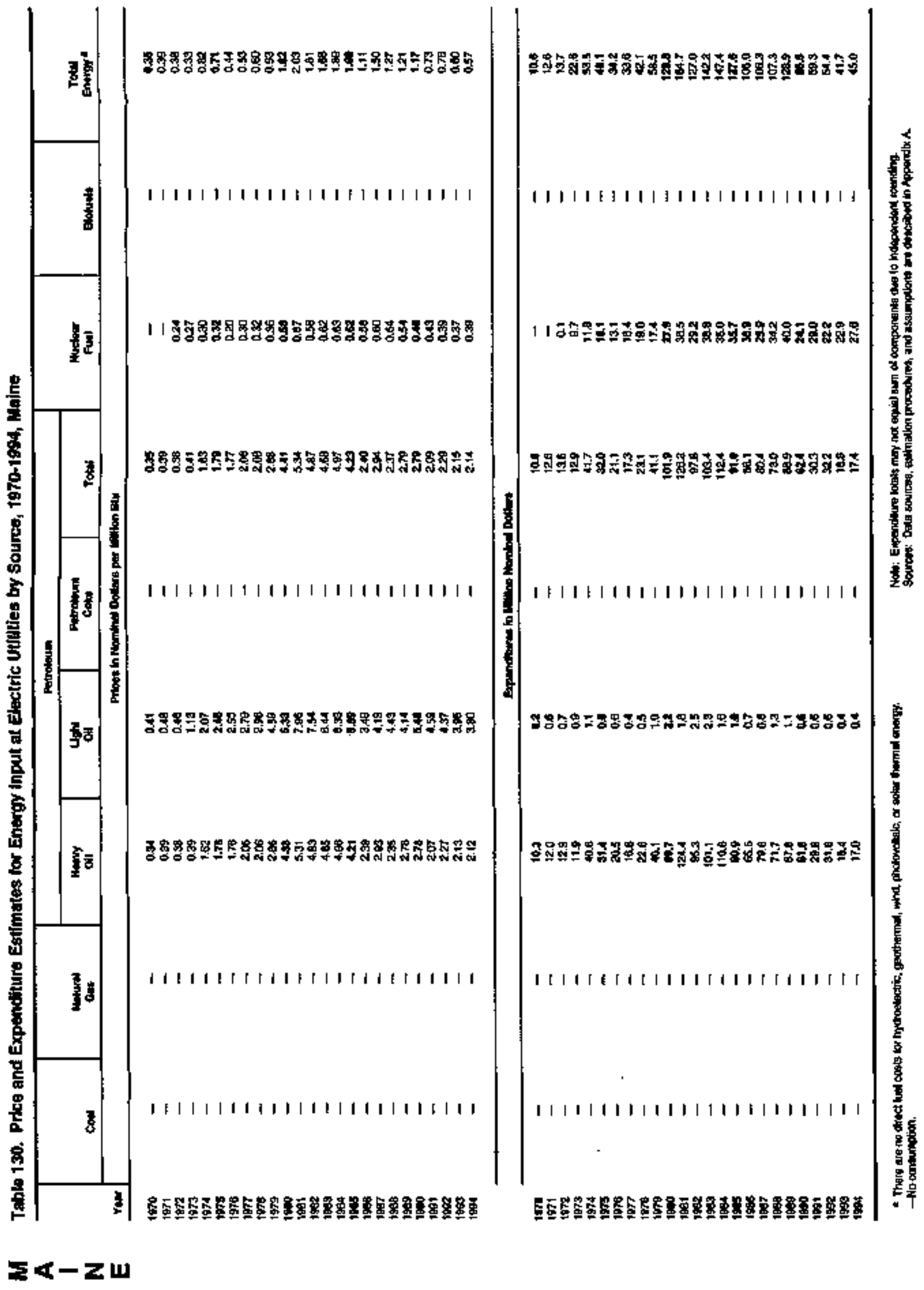
Table 1at. Energy Price and Expendlsure Eatimates by Soures, 1970-1994, Maryland

\begin{tabular}{|c|c|c|c|c|c|c|c|c|c|c|c|c|c|c|c|c|c|}
\hline \multirow[b]{4}{*}{$\mathbf{Y} \mathbf{Y}$} & \multicolumn{14}{|c|}{ 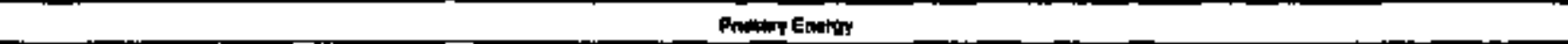 } & \multirow{3}{*}{ Pont } & \multirow{3}{*}{ 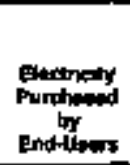 } & \multirow[b]{3}{*}{ Fots } \\
\hline & \multicolumn{3}{|c|}{ Cens } & \multirow[b]{2}{*}{ גוm } & \multicolumn{7}{|c|}{ Danmosam } & \multirow[b]{2}{*}{$\begin{array}{l}\text { Mwolewer } \\
\text { Fued }\end{array}$} & \multirow[b]{2}{*}{ Boding } & \multirow[b]{2}{*}{ Totec } & & & \\
\hline & Colting & cind & Tots & & 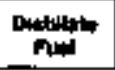 & for & LPG: & 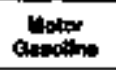 & Fatipand & othe" & Totw & & & & & & \\
\hline & \multicolumn{17}{|c|}{ 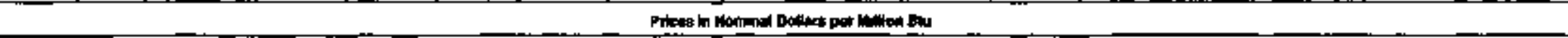 } \\
\hline \multirow[t]{2}{*}{ 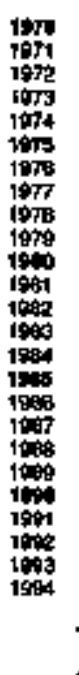 } & 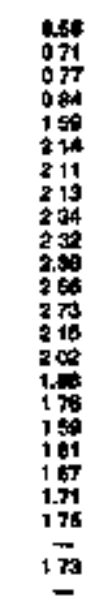 & 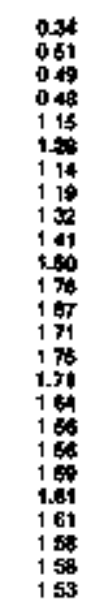 & 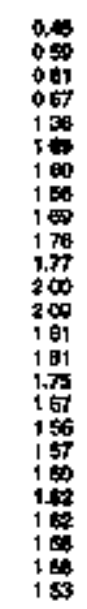 & 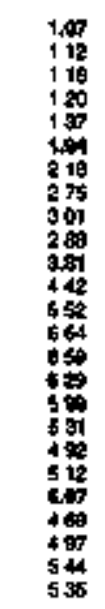 & 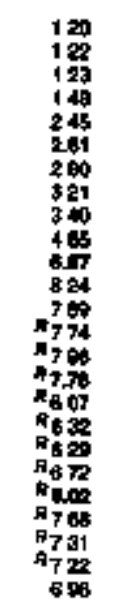 & 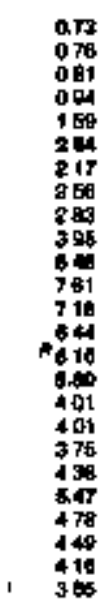 & 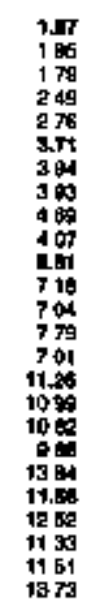 & 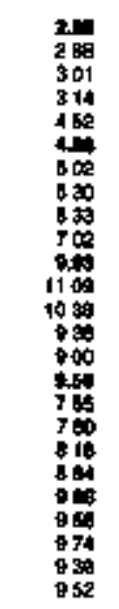 & 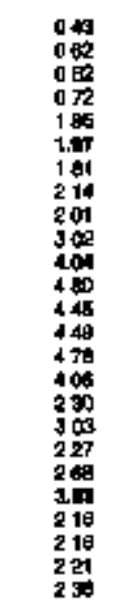 & 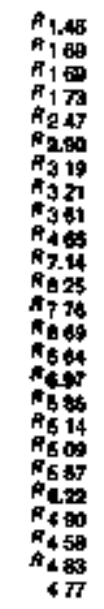 & 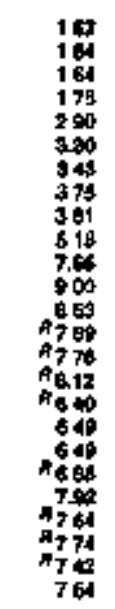 & 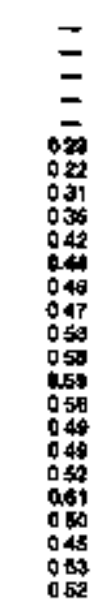 & 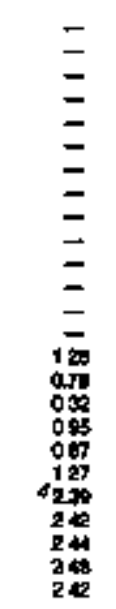 & 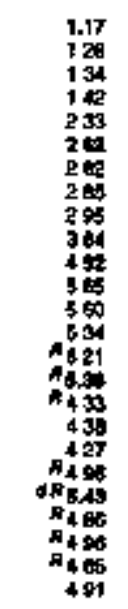 & 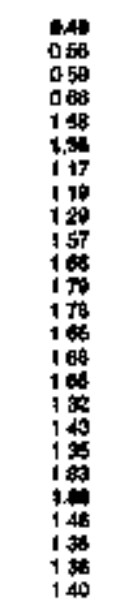 & 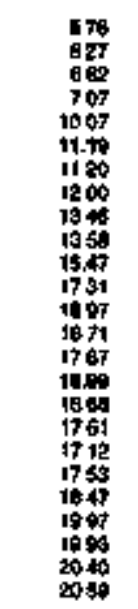 & 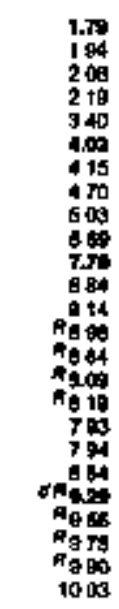 \\
\hline & \multicolumn{17}{|c|}{ 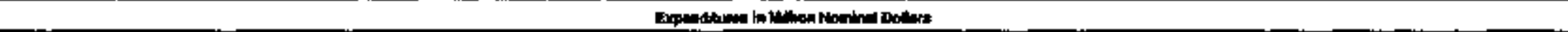 } \\
\hline 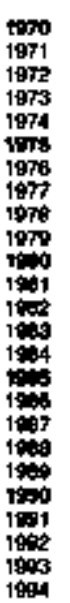 & 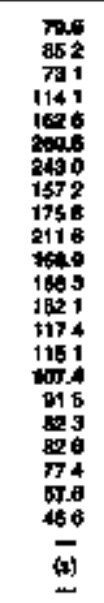 & 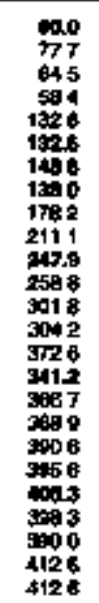 & 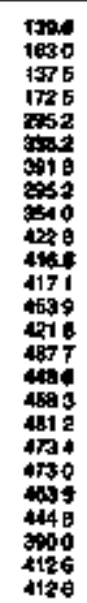 & 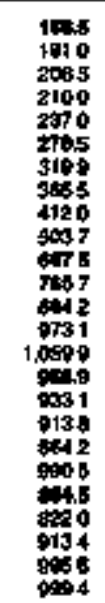 & 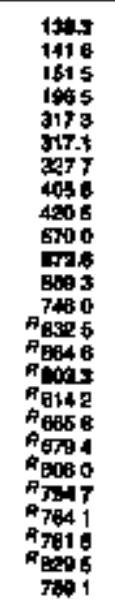 & 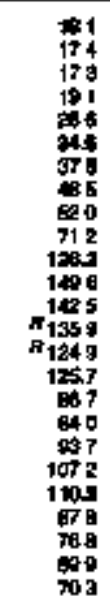 & 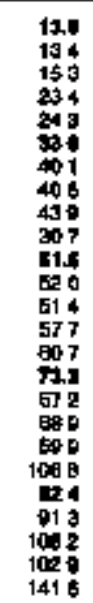 & 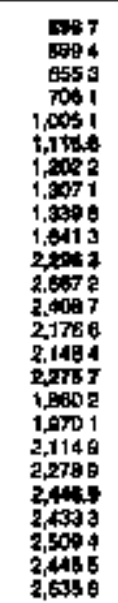 & 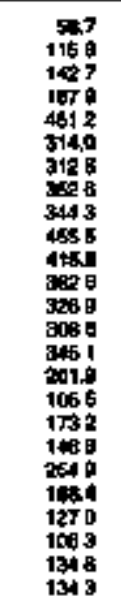 & 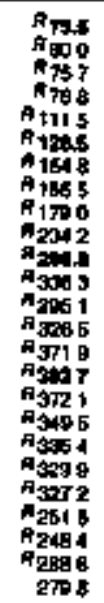 & 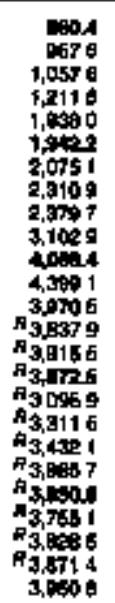 & 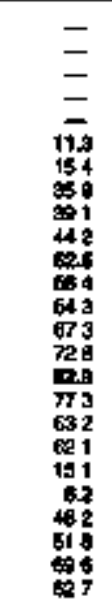 & $\begin{array}{l}= \\
= \\
= \\
= \\
= \\
= \\
= \\
= \\
= \\
08 \\
01 \\
01 \\
06 \\
02 \\
201 \\
224 \\
226 \\
209 \\
207\end{array}$ & 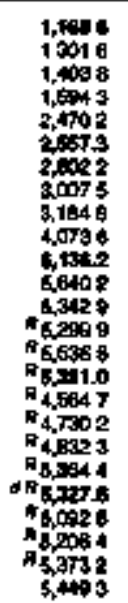 & 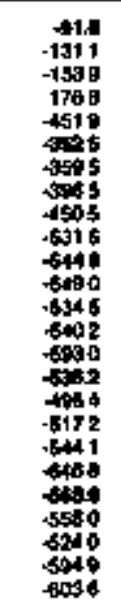 & 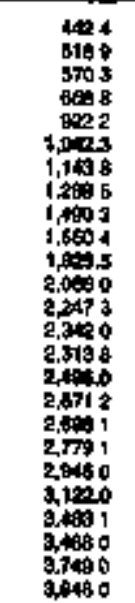 & 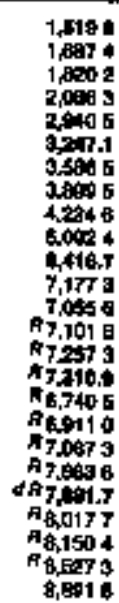 \\
\hline
\end{tabular}

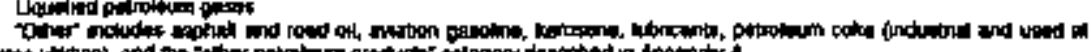

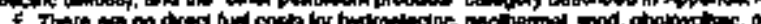

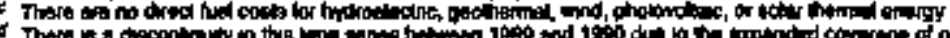

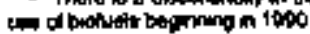

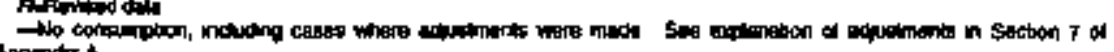

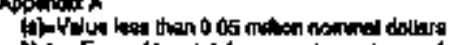

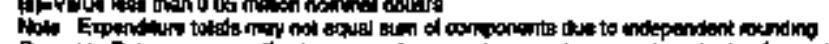




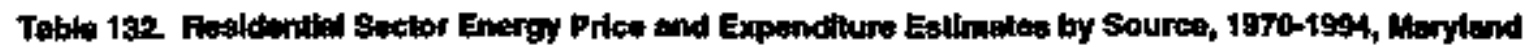

$\mathbf{A}$
$\mathbf{R}$
$\mathbf{L}$
$\mathbf{A}$
$\mathbf{N}$
$\mathbf{D}$

\begin{tabular}{|c|c|c|c|c|c|c|c|c|c|c|}
\hline \multirow[b]{4}{*}{ re } & \multicolumn{8}{|c|}{ Alloreters } & \multirow[b]{3}{*}{ 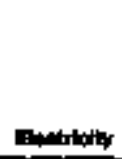 } & \multirow{3}{*}{ Thation } \\
\hline & \multirow[b]{2}{*}{ cen } & \multirow{2}{*}{ 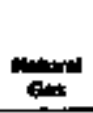 } & \multicolumn{4}{|c|}{ 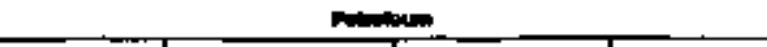 } & \multirow[b]{2}{*}{ cosine } & \multirow[b]{2}{*}{ Tonde } & & \\
\hline & & & Fis & (1) & Len:* & Toter & & & & \\
\hline & \multicolumn{10}{|c|}{ 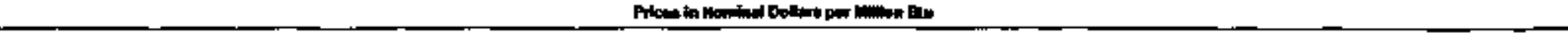 } \\
\hline \multirow[t]{2}{*}{ 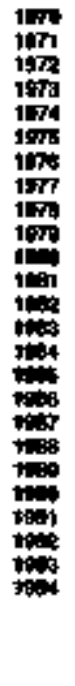 } & 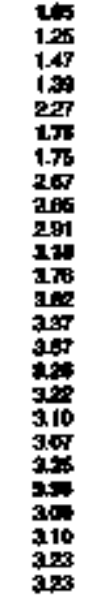 & 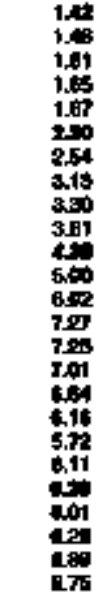 & 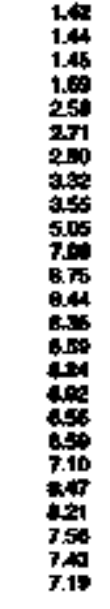 & 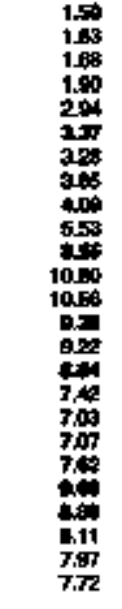 & 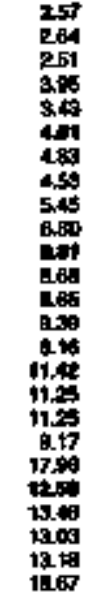 & 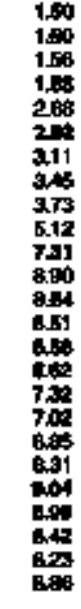 & $\begin{array}{l}= \\
= \\
= \\
= \\
= \\
= \\
= \\
= \\
= \\
= \\
= \\
z \\
0 \\
3.53 \\
3.59 \\
3.59 \\
3.59\end{array}$ & 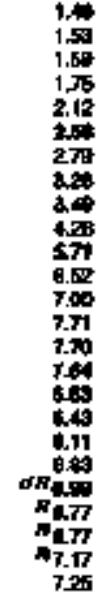 & 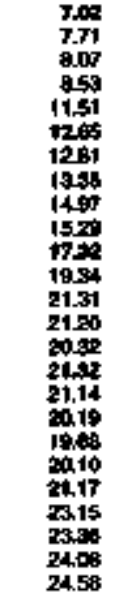 & 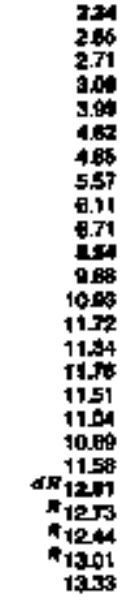 \\
\hline & \multicolumn{10}{|c|}{ 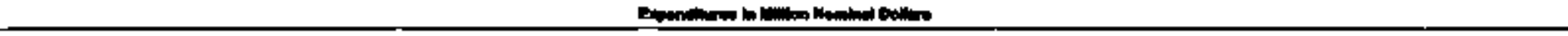 } \\
\hline 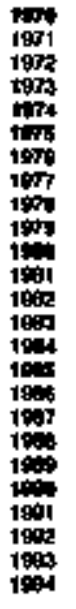 & 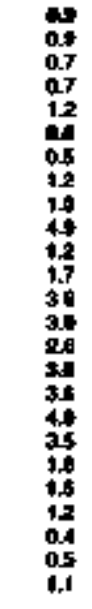 & 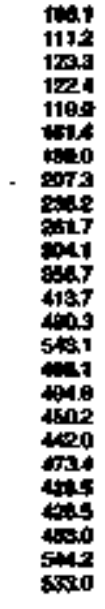 & 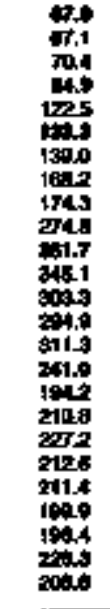 & 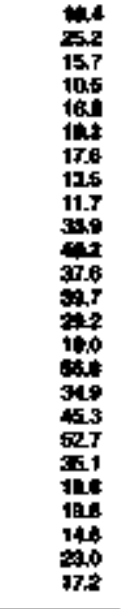 & 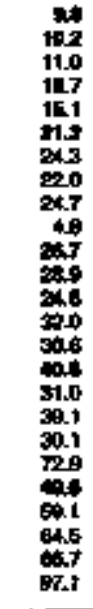 & 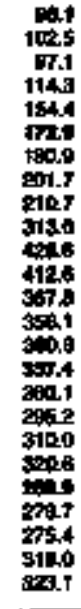 & $\begin{array}{l}= \\
= \\
= \\
= \\
= \\
= \\
= \\
= \\
= \\
= \\
= \\
= \\
= \\
150 \\
151 \\
17.4 \\
16.8\end{array}$ & 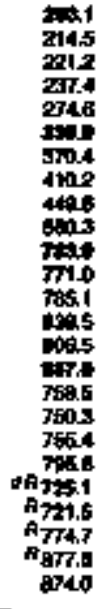 & 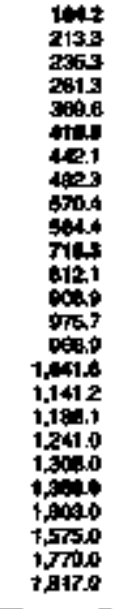 & 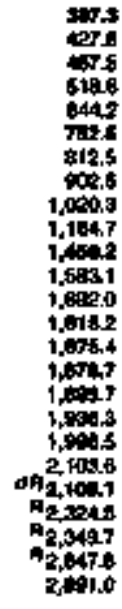 \\
\hline
\end{tabular}

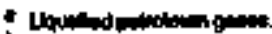

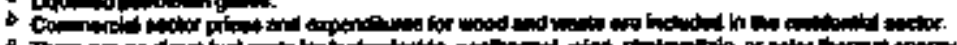

Anfinoddet

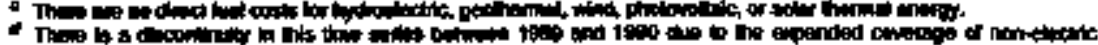

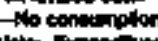

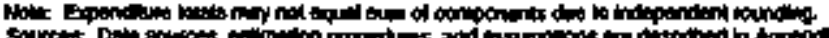

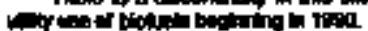


Table 133. Commercid Sector Energy Price and Expendlure Eatimatea by Source, 1970-1894, Warylend

\begin{tabular}{|c|c|c|c|c|c|c|c|c|c|c|c|}
\hline \multirow[b]{4}{*}{$x$} & \multicolumn{9}{|c|}{ Pather Enery } & \multirow[b]{3}{*}{ 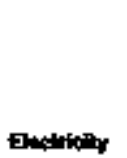 } & \multirow[b]{3}{*}{ Tots } \\
\hline & \multirow[b]{2}{*}{$\cos$} & \multirow[b]{2}{*}{ tama } & \multicolumn{6}{|c|}{ Fringum } & \multirow[b]{2}{*}{$T+1$} & & \\
\hline & & & fintur & Karease & LE* & 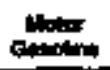 & 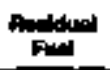 & $T+4$ & & & \\
\hline & \multicolumn{11}{|c|}{ 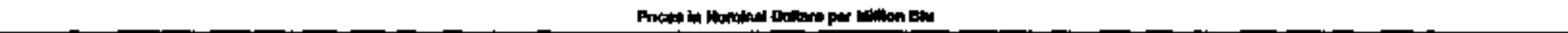 } \\
\hline \multirow[t]{2}{*}{ 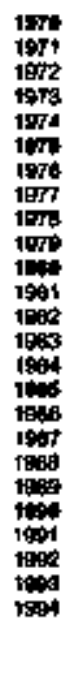 } & 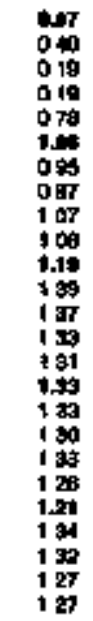 & 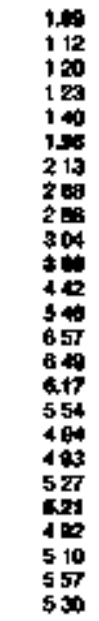 & 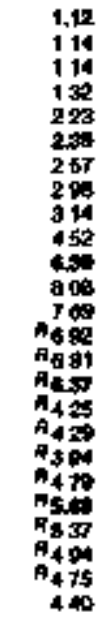 & 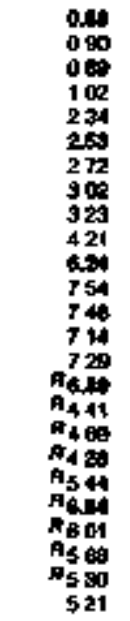 & 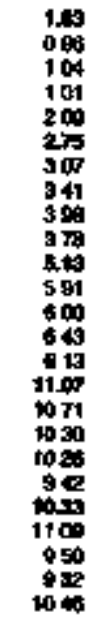 & 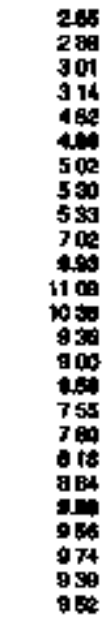 & 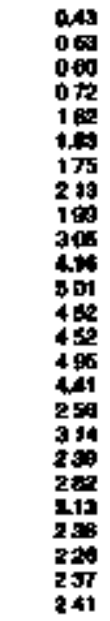 & 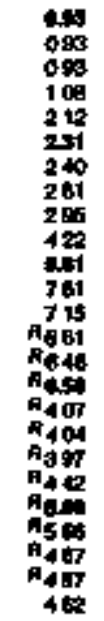 & 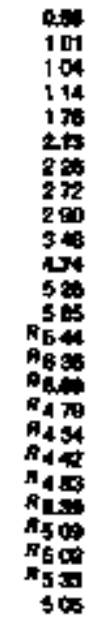 & 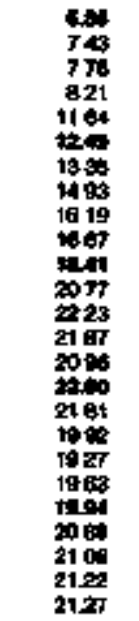 & 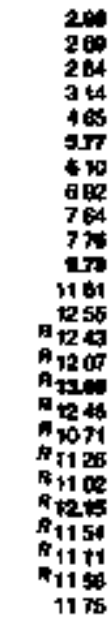 \\
\hline & \multicolumn{11}{|c|}{ 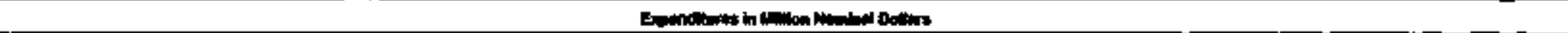 } \\
\hline 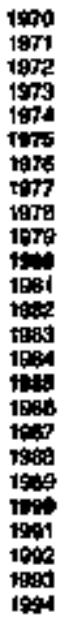 & $\begin{array}{l}01 \\
04 \\
09 \\
01 \\
08 \\
05 \\
03 \\
06 \\
05 \\
33 \\
01 \\
07 \\
27 \\
21 \\
16 \\
25 \\
27 \\
37 \\
26 \\
11 \\
09 \\
09 \\
03 \\
03 \\
07\end{array}$ & 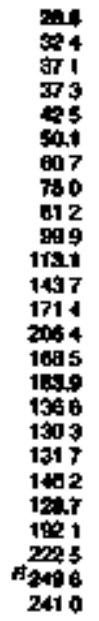 & 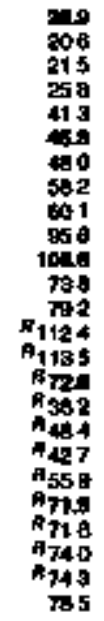 & 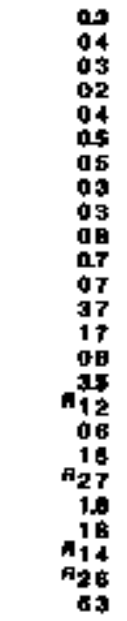 & 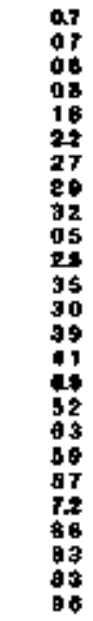 & $\begin{array}{l}18 \\
16 \\
17 \\
18 \\
26 \\
41 \\
23 \\
31 \\
95 \\
46 \\
45 \\
10 \\
74 \\
41 \\
71 \\
48 \\
69 \\
74 \\
73 \\
92 \\
114 \\
59 \\
53 \\
15 \\
16\end{array}$ & 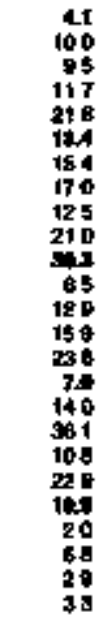 & 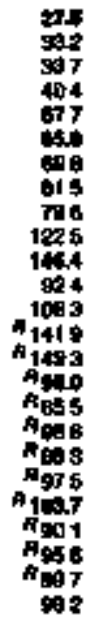 & 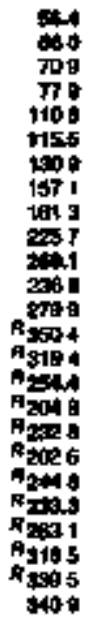 & 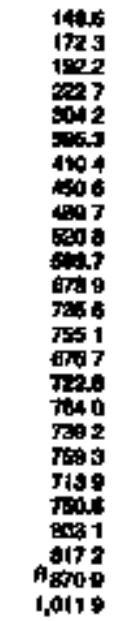 & 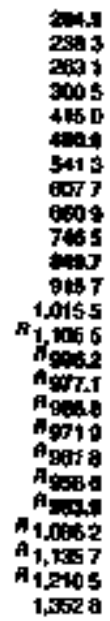 \\
\hline
\end{tabular}


Table 134. industrlel Sector Energy Price and Expenditum Estimetes by Source, 1970-1994, Maryland

$\mathbf{A}$
$\mathbf{R}$
$\mathbf{Y}$
$\mathbf{L}$
$\mathbf{A}$
$\mathbf{N}$
$\mathbf{D}$

\begin{tabular}{|c|c|c|c|c|c|c|c|c|c|c|c|c|c|c|c|c|c|}
\hline \multirow[b]{3}{*}{ ran } & \multicolumn{15}{|c|}{ 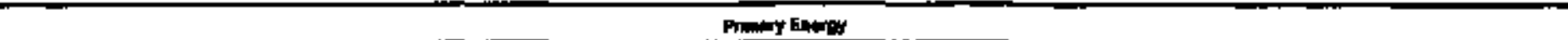 } & \multirow{2}{*}{ Exitedy } & \multirow{2}{*}{$\operatorname{Tim}_{\sin 2}$} \\
\hline & \multicolumn{3}{|c|}{$\cos$} & "דומה & \multicolumn{9}{|c|}{ Pomolenar } & Monume & Totols $c$ & & \\
\hline & \multicolumn{17}{|c|}{ 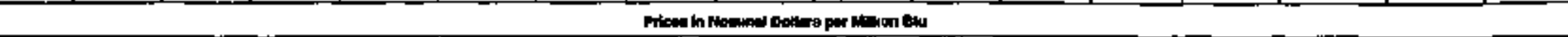 } \\
\hline 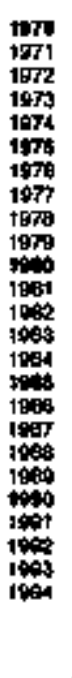 & 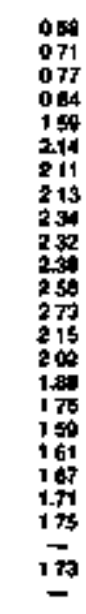 & 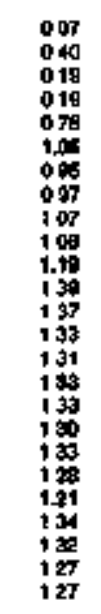 & 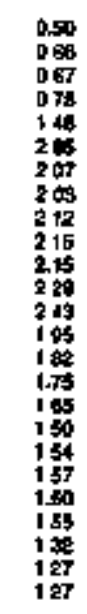 & 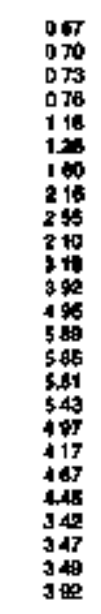 & 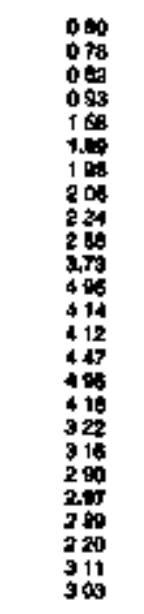 & 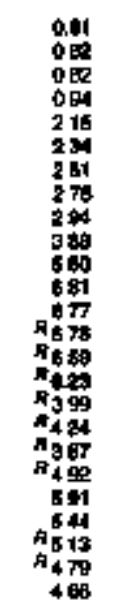 & 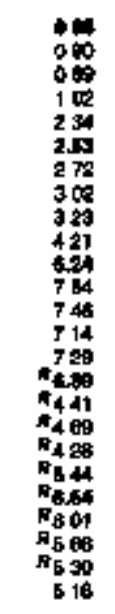 & 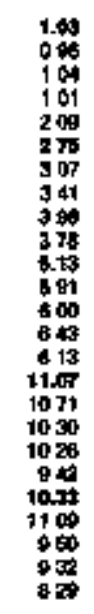 & 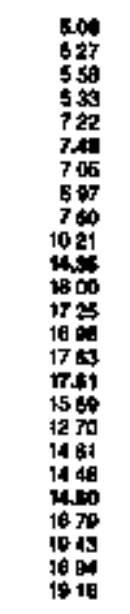 & 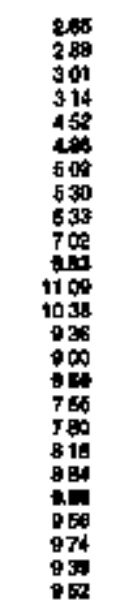 & 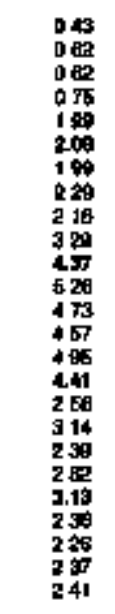 & 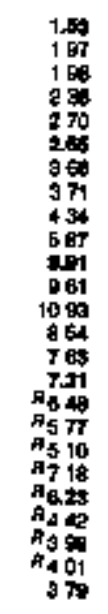 & 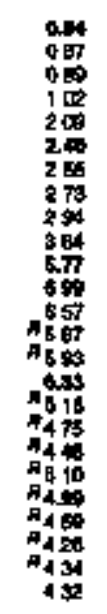 & 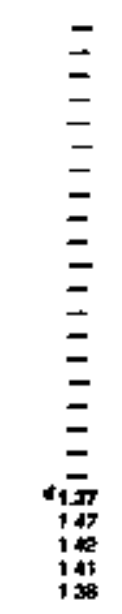 & 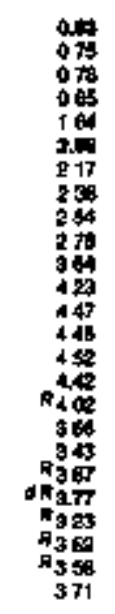 & 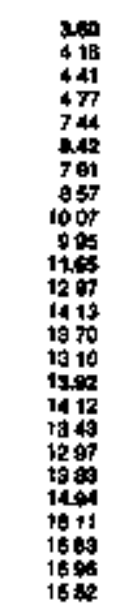 & 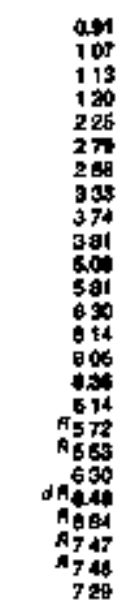 \\
\hline 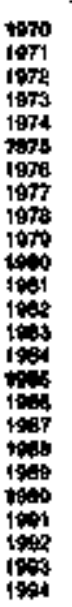 & 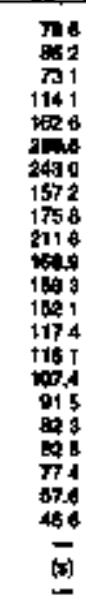 & 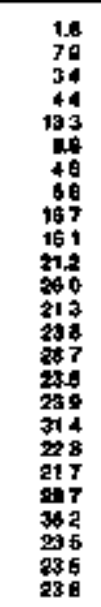 & 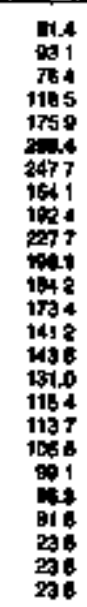 & 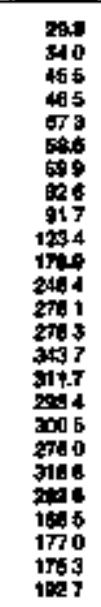 & 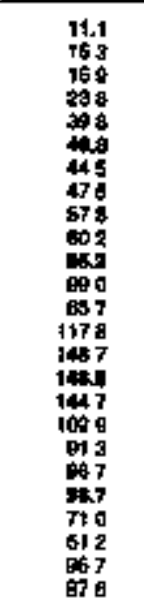 & 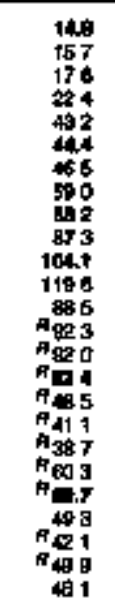 & 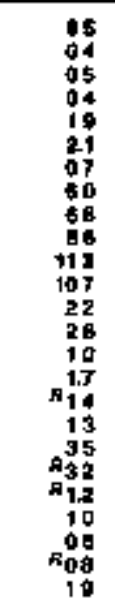 & 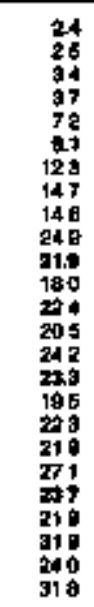 & 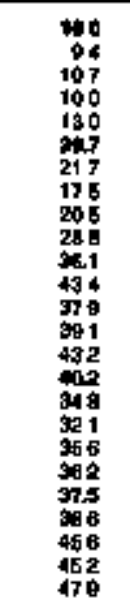 & 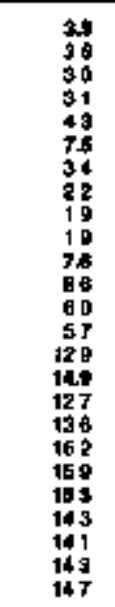 & 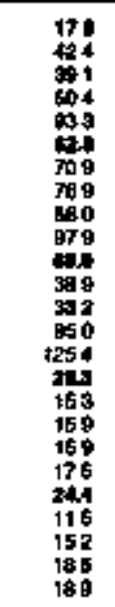 & 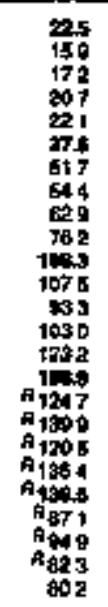 & 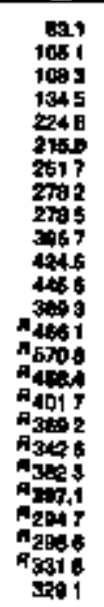 & 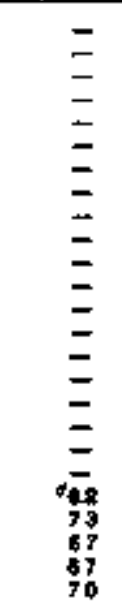 & 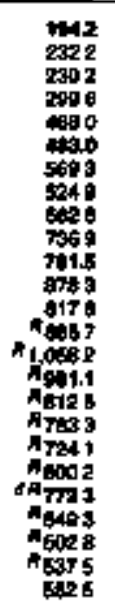 & 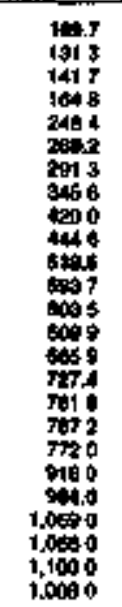 & 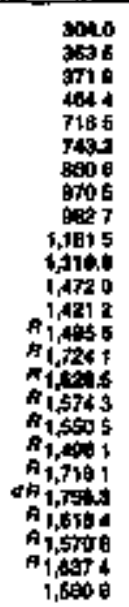 \\
\hline
\end{tabular}

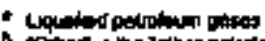

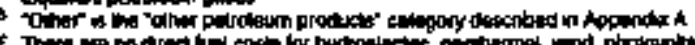

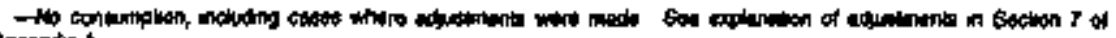

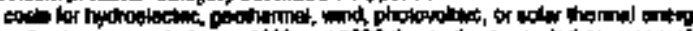

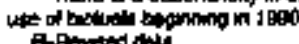

Appendin $A$

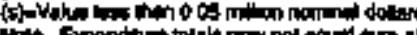

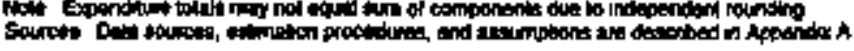




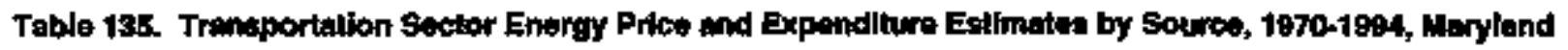

\begin{tabular}{|c|c|c|c|c|c|c|c|c|c|c|c|c|c|}
\hline \multirow[b]{4}{*}{ Wh } & \multicolumn{11}{|c|}{ Pinner Einery } & \multirow[b]{3}{*}{ Enotiletin } & \multirow[b]{3}{*}{$\underset{\operatorname{tin}}{\operatorname{Tot}}$} \\
\hline & \multirow[b]{2}{*}{ cod } & \multirow[b]{2}{*}{ Netsols } & \multicolumn{8}{|c|}{ Nutrolanis. } & \multirow[b]{2}{*}{ Talv } & & \\
\hline & & & מon & 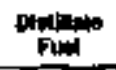 & Pix & LPQ: & Limican & IIt) & $\begin{array}{l}\text { Reldugol } \\
\text { Fuil }\end{array}$ & Tow & & & \\
\hline & \multicolumn{13}{|c|}{ 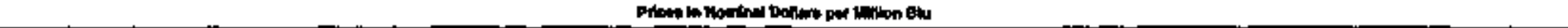 } \\
\hline \multirow[t]{2}{*}{ 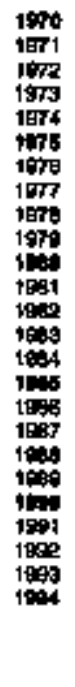 } & $\begin{array}{l}000 \\
040 \\
019 \\
018 \\
078 \\
0.88 \\
0 \% 8 \\
007 \\
= \\
= \\
= \\
= \\
= \\
= \\
= \\
= \\
= \\
= \\
= \\
=\end{array}$ & $\begin{array}{l}= \\
= \\
= \\
= \\
= \\
= \\
= \\
= \\
= \\
= \\
= \\
= \\
345\end{array}$ & 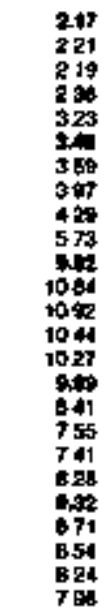 & 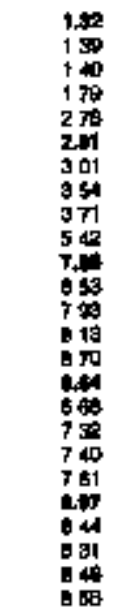 & 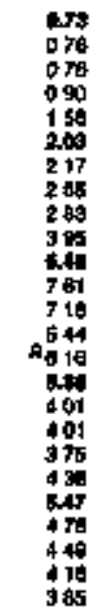 & 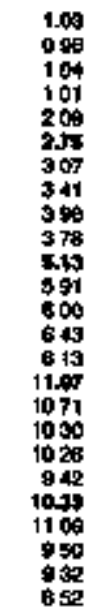 & 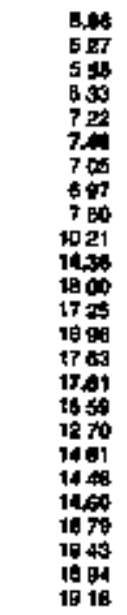 & 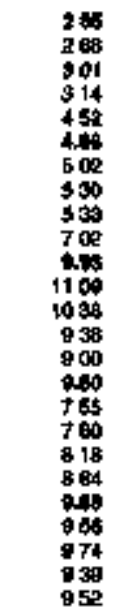 & 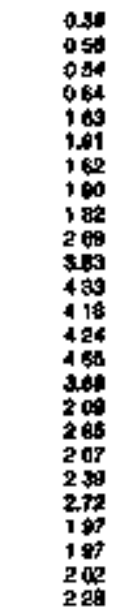 & 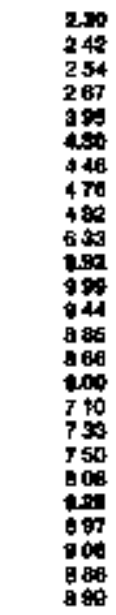 & 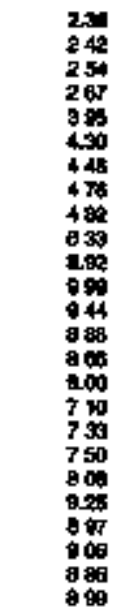 & 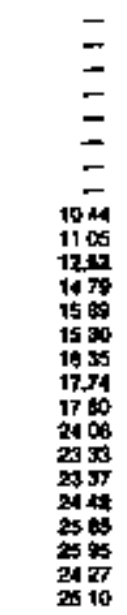 & 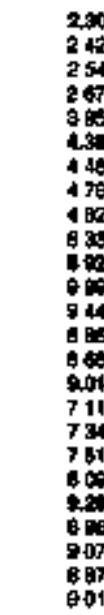 \\
\hline & \multicolumn{13}{|c|}{ 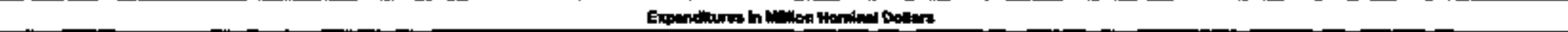 } \\
\hline 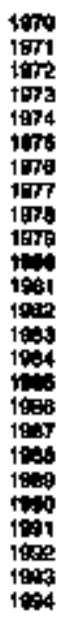 & 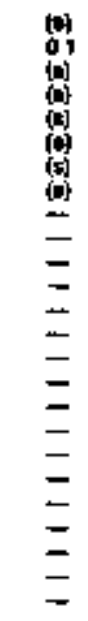 & $\begin{array}{l}= \\
= \\
= \\
= \\
= \\
= \\
= \\
= \\
= \\
= \\
= \\
= \\
= \\
01\end{array}$ & $\begin{array}{l}34 \\
34 \\
30 \\
31 \\
44 \\
94 \\
30 \\
41 \\
39 \\
42 \\
75 \\
76 \\
11 \\
30 \\
35 \\
30 \\
13 \\
33 \\
35 \\
35 \\
31 \\
39 \\
11 \\
49 \\
30\end{array}$ & 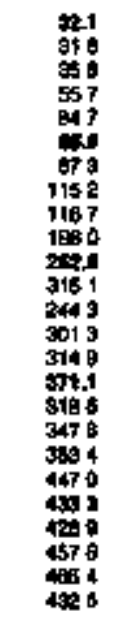 & 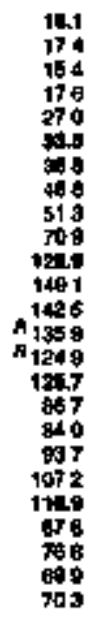 & $\begin{array}{l}11 \\
02 \\
02 \\
02 \\
04 \\
0.5 \\
07 \\
09 \\
13 \\
01 \\
18 \\
18 \\
11 \\
13 \\
19 \\
24 \\
11 \\
12 \\
21 \\
20 \\
19 \\
17 \\
95 \\
38 \\
90\end{array}$ & 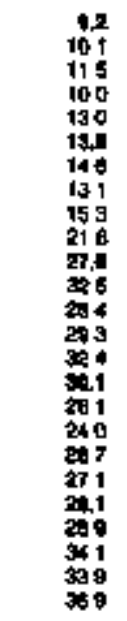 & 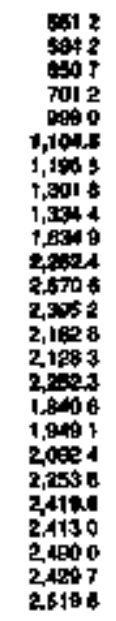 & 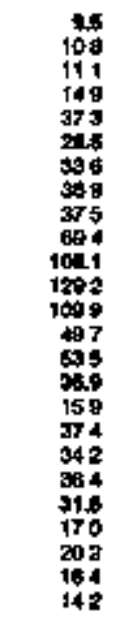 & 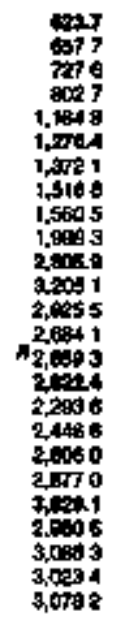 & 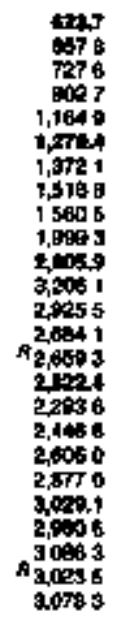 & $\begin{array}{l}= \\
= \\
= \\
= \\
= \\
02 \\
05 \\
01 \\
13 \\
13 \\
12 \\
24 \\
41 \\
42 \\
57 \\
60 \\
02 \\
74 \\
79 \\
78 \\
00 \\
01\end{array}$ & 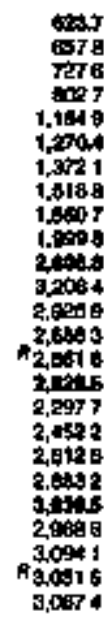 \\
\hline
\end{tabular}




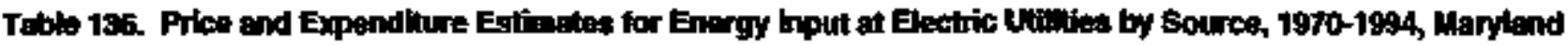

\begin{tabular}{|c|c|c|c|c|c|c|c|c|c|}
\hline \multirow[b]{3}{*}{$Y=$} & \multirow[b]{2}{*}{ cont } & \multirow[b]{2}{*}{$\ln$} & \multicolumn{4}{|c|}{ nutromen } & \multirow[b]{2}{*}{ menter } & \multirow[b]{2}{*}{ Emand: } & \multirow[b]{2}{*}{ 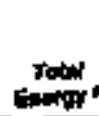 } \\
\hline & & & ox & 管 & 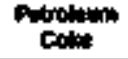 & $T$ & & & \\
\hline & \multicolumn{9}{|c|}{ A } \\
\hline \multirow{21}{*}{ 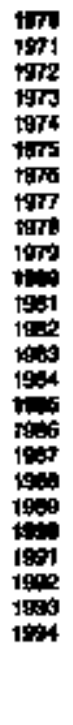 } & 27 & $0 \pm 2$ & 14 & at: & ـ & M4 & - & - & ENo \\
\hline & 0.52 & a.s3 & 0.68 & and & $\bar{z}$ & 200 & $=$ & - & a56 \\
\hline & 056 & 00 & 0.73 & 0.20 & $\Xi$ & abs & $\bar{z}$ & $=$ & a.5n \\
\hline & 1.21 & o.dit & 1.84 & 1.0 & - & 1.85 & - & $=$ & 1.56 \\
\hline & 130 & 0.10 & 105 & 20 & - & 1.6 & 020 & - & 1.30 \\
\hline & 1.8 & 7.02 & 1.80 & 2.31 & - & 1,62 & 0.22 & - & 1.17 \\
\hline & 1.20 & 201 & 2.4 & 267 & - & 215 & 0.31 & - & 1.19 \\
\hline & 1.5 & $\begin{array}{l}1.45 \\
1.76\end{array}$ & $\begin{array}{l}200 \\
3.02\end{array}$ & $\begin{array}{l}2.7 \\
117\end{array}$ & & $\underset{300}{209}$ & a.36 & $=$ & 129 \\
\hline & 154 & 26 & 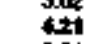 & 17 & 二 & 400 & Let & $=$ & 1,37 \\
\hline & 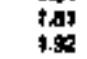 & 276 & $\begin{array}{l}3.04 \\
4.50\end{array}$ & 7.06 & $\bar{z}$ & 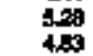 & 0.47 & $\bar{z}$ & 1.79 \\
\hline & iss & 140 & 4.50 & 5 & $=$ & 4.71 & 0,53 & 二 & $1 \%$ \\
\hline & $\operatorname{lig}_{13}$ & $\frac{1.53}{278}$ & 471 & 505 & $\bar{z}$ & 4 & 056 & $1 \%$ & 1.89 \\
\hline & 0,07 & 274 & 2.25 & 330 & - & $24 t$ & $0 \leq 0$ & axe & 02 \\
\hline & 1.0 & 269 & 3.09 & 19a & - & 317 & a.to & 280 & 9.49 \\
\hline & 2.60 & 200 & $2 * 3$ & 24 & - & $2 \%$ & 048 & 08 & 1.35 \\
\hline & 1.61 & 200 & 272 & 412 & - & 203 & ass & 10 & J.83 \\
\hline & 160 & 20 & $\begin{array}{l}310 \\
2,17\end{array}$ & 57 & $\bar{z}$ & $\frac{1374}{232}$ & 101 & $\bar{z}$ & 1.48 \\
\hline & 100 & 258 & 2.19 & 40 & - & 2.37 & a.45 & $=$ & 1.35 \\
\hline & 1,60 & 260 & 2.21 & 39 & - & 23 & $a s s$ & - & 1.38 \\
\hline & $1 * 0$ & 29 & 2.77 & 304 & - & 256 & 0.58 & - & 1,40 \\
\hline & \multicolumn{9}{|c|}{ 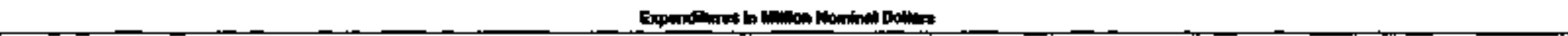 } \\
\hline $10 \mathrm{tr}$ & \$N & 3* & EA & 20 & - & sete & $\rightarrow$ & - & 0100 \\
\hline 1971 & Gas & 33 & s.7 & 6.5 & - & 52 & - & - & 131.1 \\
\hline $18 \pi$ & $\infty 2$ & 26 & so & a. & - & 91,1 & - & הـ & 15398 \\
\hline 1993 & 81 & $3 \mathbf{E}$ & two: & 2.1 & - & 1200 & - & - & $17 \% 6$ \\
\hline 1994 & $\pi$ & a) & $\$ 0$ & 2.3 & - & 3 & - & - & 41.9 \\
\hline $10 \mathrm{~s}$ & the & as & 203 & $\$ 7$ & - & 2100 & 11.3 & - & $=05$ \\
\hline 1978 & 432 & a4 & tege & 7B & - & 200.6 & 15.4 & - & 50 \\
\hline 1907 & $\tan 3$ & 0.7 & 23.8 & 27 & - & 230.6 & 3.6 & - & 4 \\
\hline 1906 & 1601 & 10 & 2303 & 13.1 & - & $=0.3$ & ? 1 & - & 450.5 \\
\hline 1909 & $+\infty=0$ & 106 & $a+3$ & 146 & - & 2016 & 44.2 & - & ș1.5 \\
\hline till & 2ate & 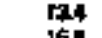 & $\operatorname{mis}_{5}$ & 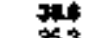 & $\overline{-}$ & काal & 52.5 & - & 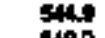 \\
\hline 1991 & 2305 & ${ }_{368}^{28}$ & 2000 & $\$ 3.3$ & $=$ & 2403 & sthe & $=$ & 54.0 \\
\hline $\begin{array}{l}1988 \\
12005\end{array}$ & $\begin{array}{l}27 \mathrm{RB} \\
2752\end{array}$ & $\begin{array}{ll}3.0 \\
\text { Q.1 }\end{array}$ & 150, & $\begin{array}{l}50.6 \\
31.8\end{array}$ & $\bar{z}$ & $\begin{array}{l}201.5 \\
206\end{array}$ & $\begin{array}{l}54.3 \\
67.3\end{array}$ & $\bar{z}$ & 5405 \\
\hline 1894 & 306 & 4.7 & 142 & 329 & - & $173 s$ & 72.0 & 0.6 & 583,0 \\
\hline איבו & 3114 & 57 & tat? & $* ?$ & $\bar{z}$ & Lot & 9t: & n1 & 50,2 \\
\hline 1908 & $\mathbf{3 2 4 B}$ & 6.4 & $\infty .3$ & 147 & $\bar{z}$ & $\begin{aligned} 75,1 \\
00+7\end{aligned}$ & 77.3 & Q.1 & $\operatorname{sis}$ \\
\hline 1987 & tols & 528 & 600 & 17.0 & & 00,7 & 592 & D.S & 5172 \\
\hline rise & $3 * 1.6$ & 14.4 & 60 & 17,4 & - & 100.3 & 21 & 0.5 & 5et4.1 \\
\hline tis & $3 \pi 12$ & 521 & $\begin{array}{l}171 \\
13 t 5\end{array}$ & 301 & $\bar{z}$ & 208 & $\begin{array}{r}15.1 \\
12\end{array}$ & $\underline{02}$ & stato \\
\hline 19 & 3615 & stis & 9.5 & 14.3 & - & 1100 & 42 & - & 500 \\
\hline $19 x$ & s.t. & sof & 60 & 11,5 & - & 753 & 51.6 & - & $\$ 20$ \\
\hline $\operatorname{tog}$ & 53.3 & aㅗ & $\theta 70$ & 126 & - & 100. & $\$ 0$ & - & $504 \mathrm{~F}$ \\
\hline+4 & 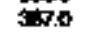 & $\overline{23}$ & F.9 & $\mathbf{2 3 . 3}$ & - & 1212 & 化7 & $\rightarrow$ & 603.6 \\
\hline
\end{tabular}




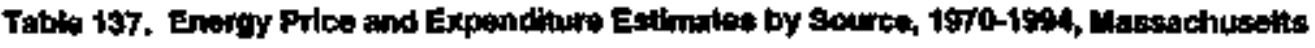

\begin{tabular}{|c|c|c|c|c|c|c|c|c|c|c|c|c|c|c|c|c|c|}
\hline & \multicolumn{14}{|c|}{ Funn Dasing } & \multirow{3}{*}{ 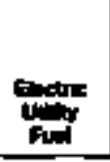 } & \multirow{3}{*}{ 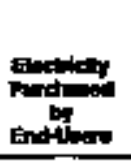 } & \multirow[b]{3}{*}{ Then } \\
\hline & \multicolumn{3}{|c|}{ البه: } & \multirow[b]{2}{*}{ manow } & \multicolumn{7}{|c|}{ 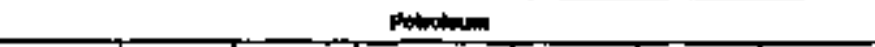 } & \multirow[b]{2}{*}{ Fintil } & \multirow[b]{2}{*}{ Mohuch } & \multirow[b]{2}{*}{$T=\mathbf{s}^{0}$} & & & \\
\hline & $\begin{array}{c}\text { Columy } \\
\text { Codel }\end{array}$ & (i) & $T$ & & Puil & (4) & Lea" & הירו" & Andond & Ones & Toter & & & & & & \\
\hline Yam & \multicolumn{17}{|c|}{ 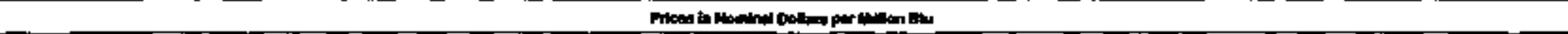 } \\
\hline \multirow[t]{2}{*}{ 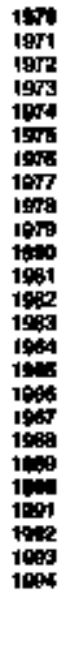 } & $\begin{array}{l}= \\
z \\
z \\
z \\
z \\
= \\
= \\
= \\
= \\
= \\
= \\
= \\
= \\
=\end{array}$ & 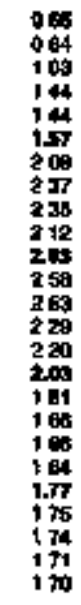 & 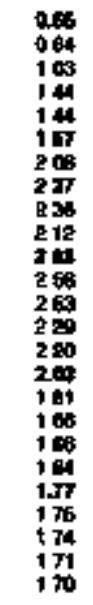 & 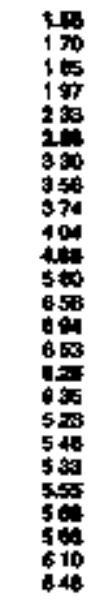 & 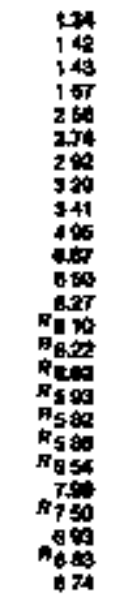 & 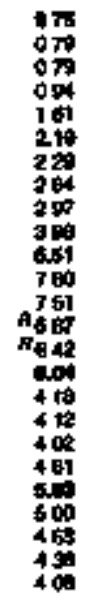 & 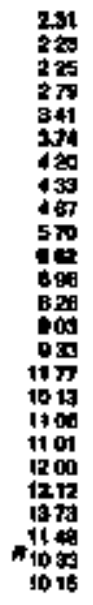 & 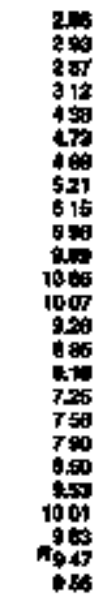 & 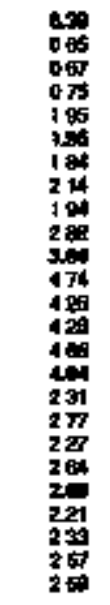 & 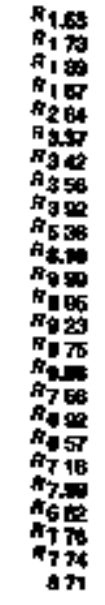 & 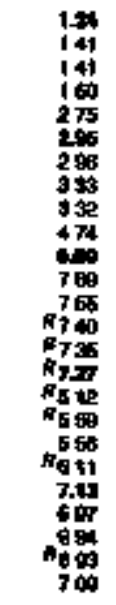 & 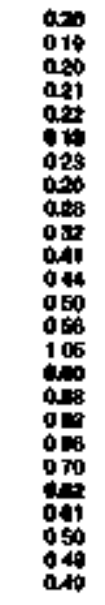 & 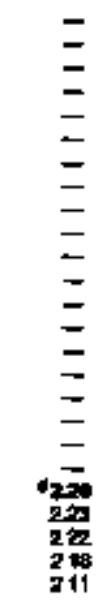 & 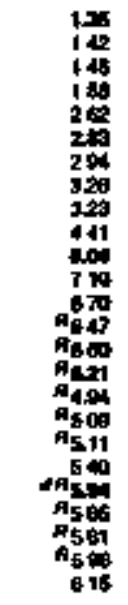 & 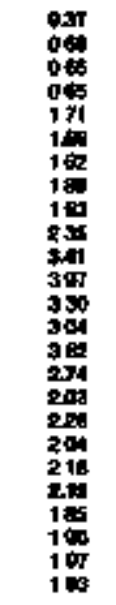 & 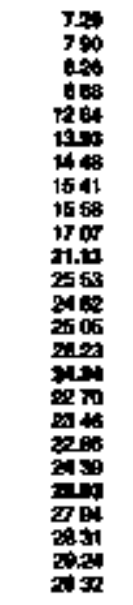 & 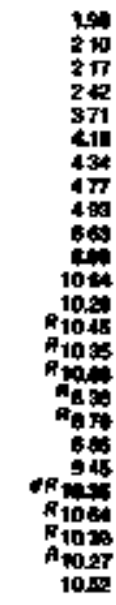 \\
\hline & \multicolumn{17}{|c|}{ 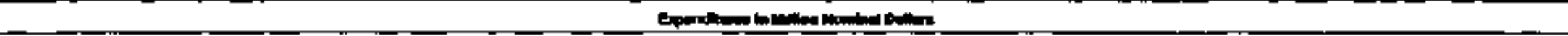 } \\
\hline 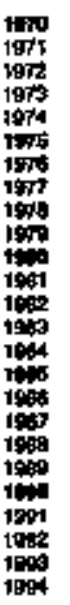 & $\begin{array}{l}= \\
= \\
= \\
= \\
= \\
= \\
= \\
= \\
= \\
= \\
= \\
= \\
= \\
=\end{array}$ & 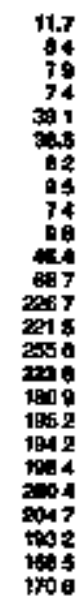 & 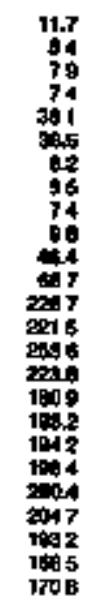 & 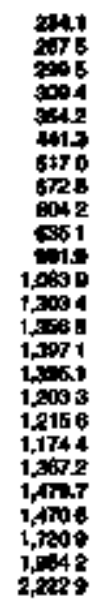 & 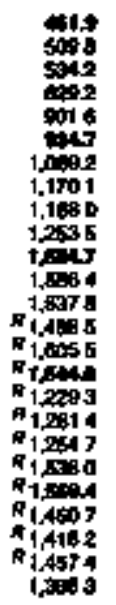 & 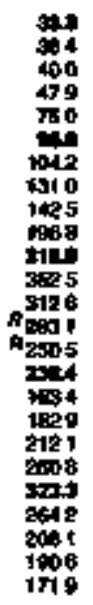 & 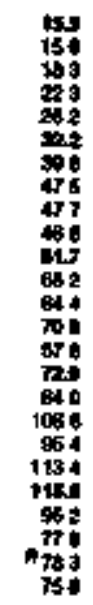 & 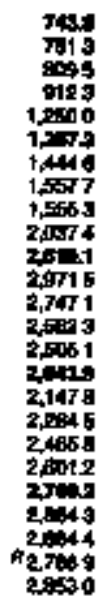 & 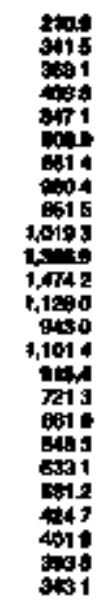 & 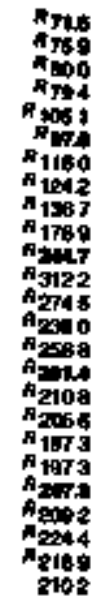 & 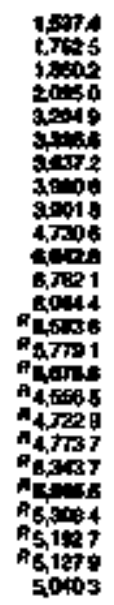 & 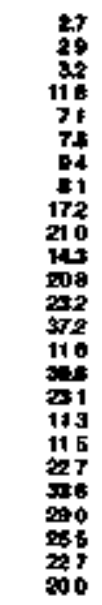 & $\begin{array}{l}= \\
= \\
= \\
= \\
= \\
= \\
= \\
= \\
= \\
= \\
= \\
40 \\
42 \\
\$ 0\end{array}$ & 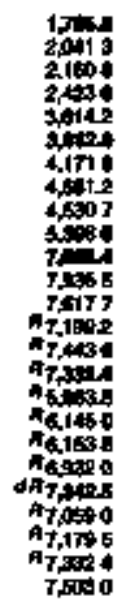 & 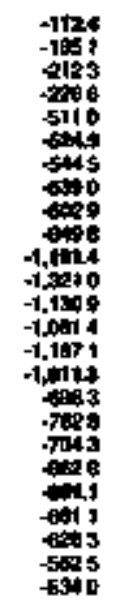 & 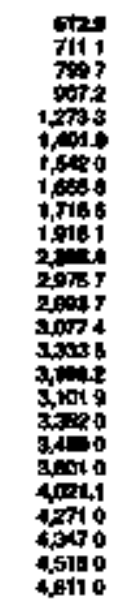 & 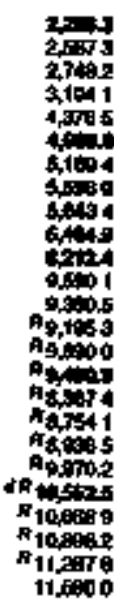 \\
\hline
\end{tabular}

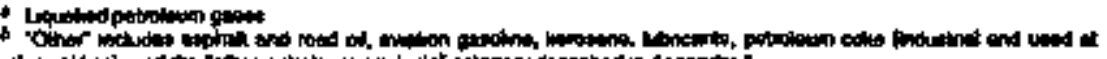

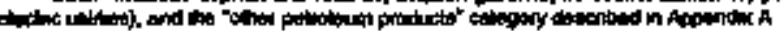

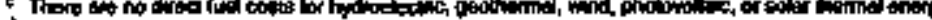

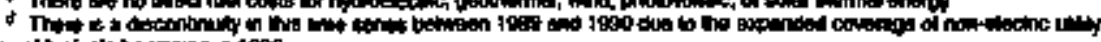

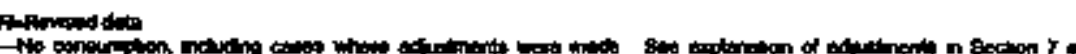
uption $A$

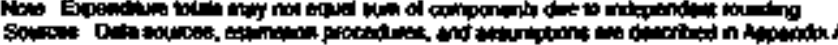




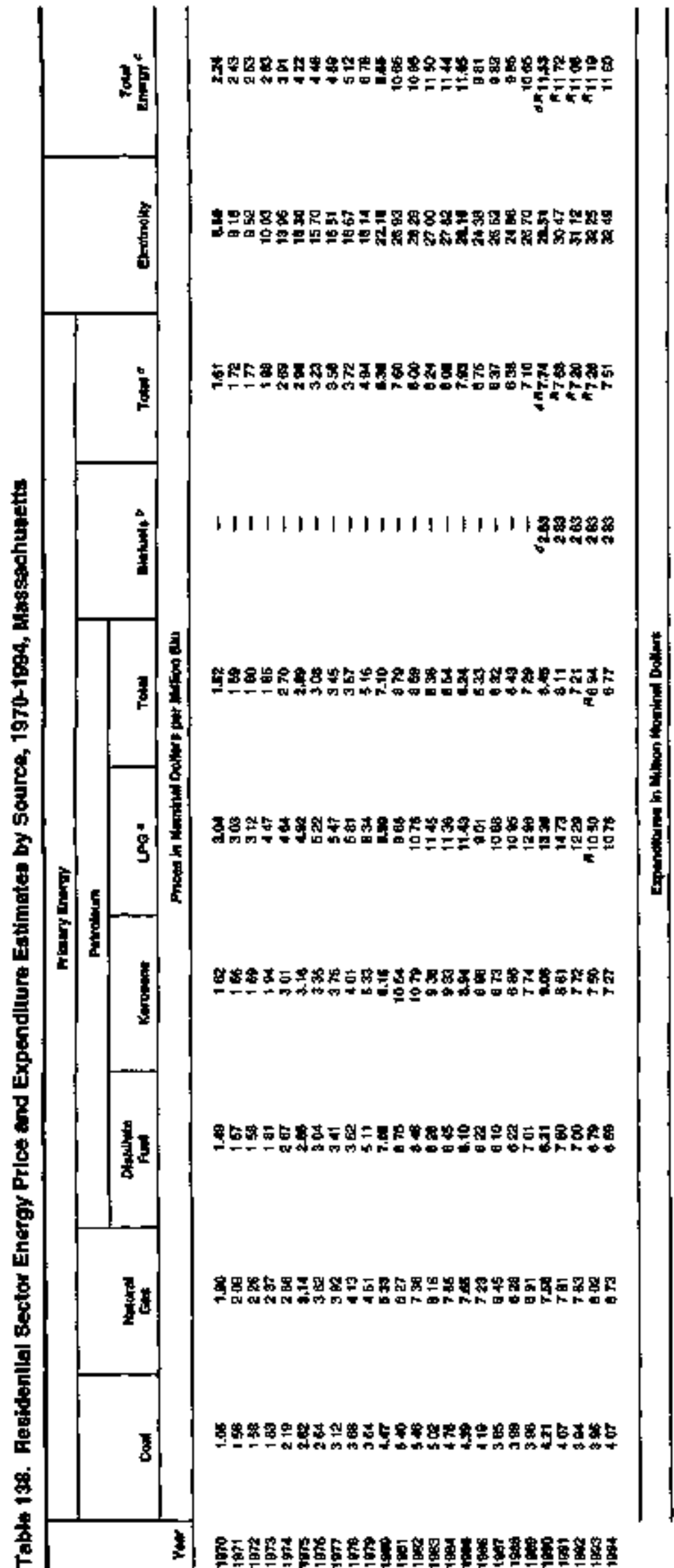

how

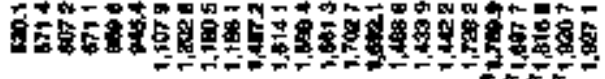

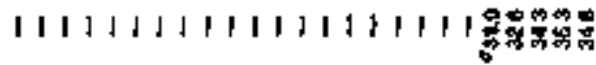

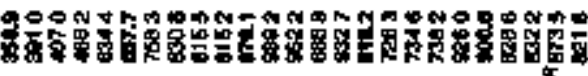

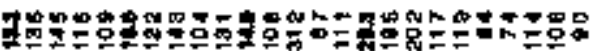

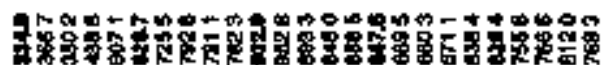

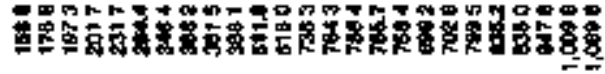

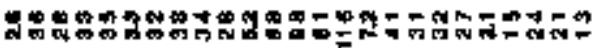

วั0ำ

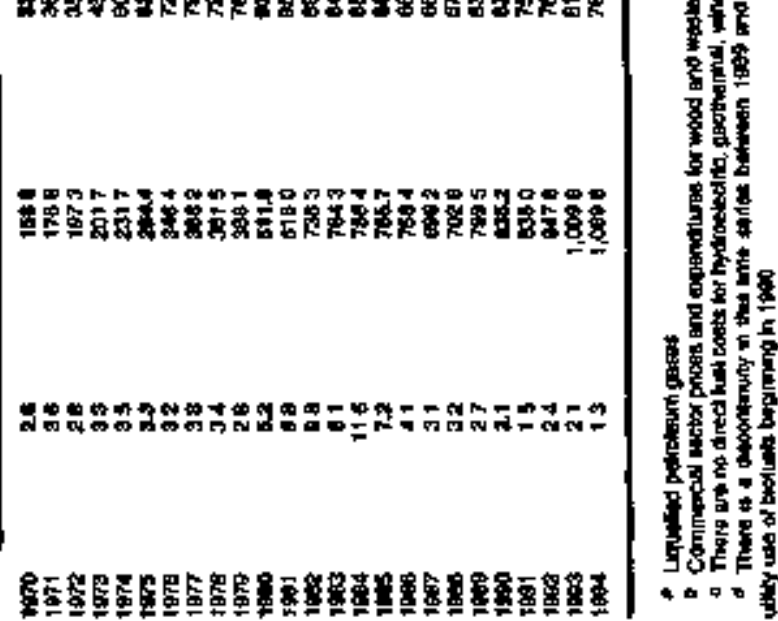




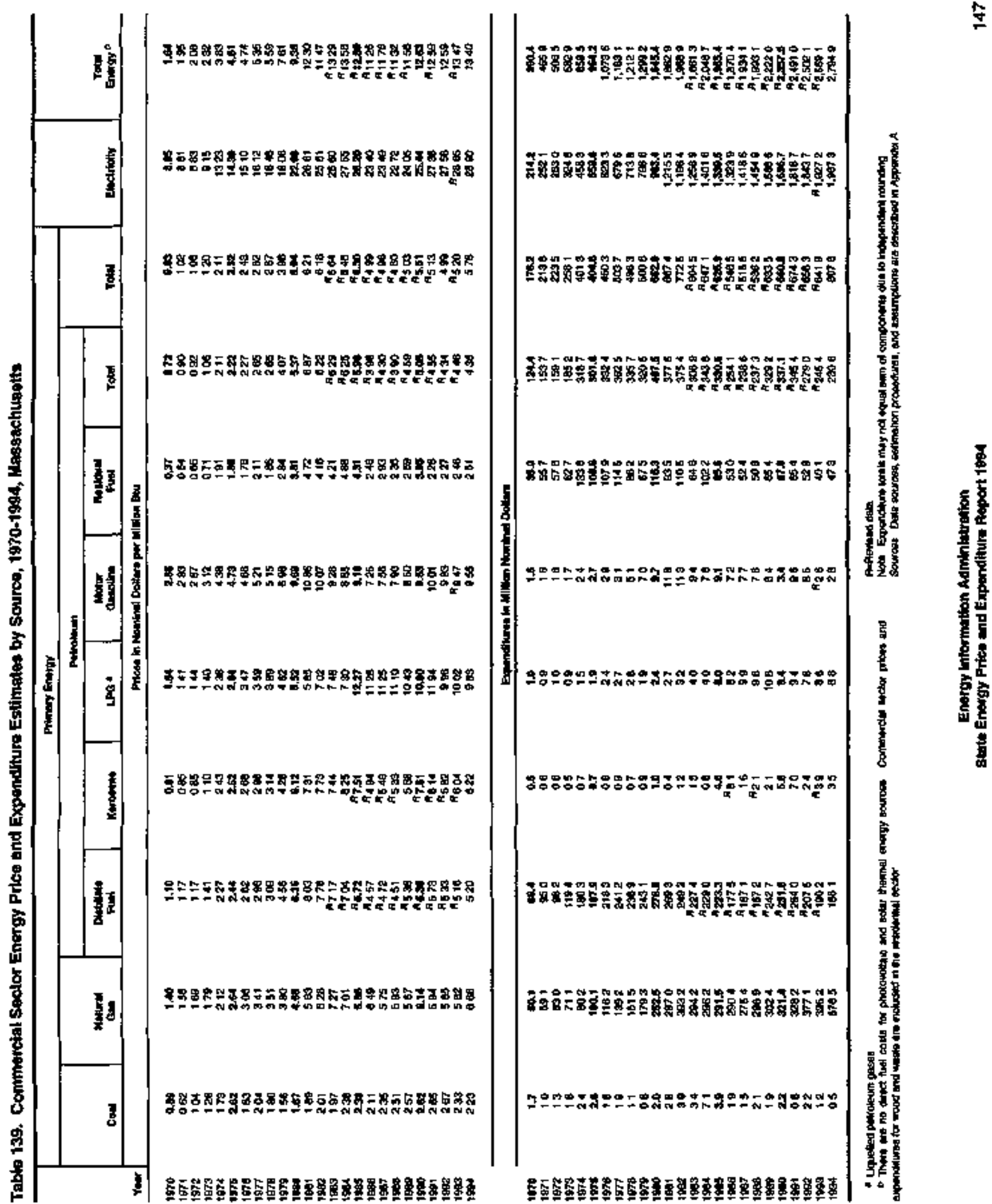




\begin{tabular}{|c|c|c|c|c|c|c|c|c|c|c|c|c|c|c|c|c|c|}
\hline \multirow[b]{4}{*}{$\max$} & \multicolumn{15}{|c|}{ ا } & \multirow[b]{3}{*}{ Enedvery } & \multirow[b]{3}{*}{ 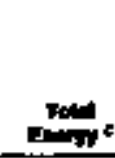 } \\
\hline & \multicolumn{3}{|c|}{ n. } & \multirow[b]{2}{*}{ mand } & \multicolumn{9}{|c|}{ 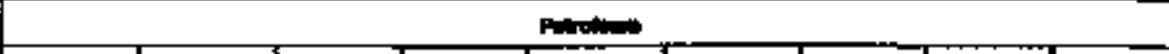 } & \multirow[b]{2}{*}{ Eloniste } & \multirow[b]{2}{*}{ Totent } & & \\
\hline & 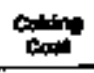 & $\cos$ & Toter & & Amponen & Find & (1) & Lad & كبعشي & m & Fint & 에으. & 데에 & & & & \\
\hline & \multicolumn{17}{|c|}{ 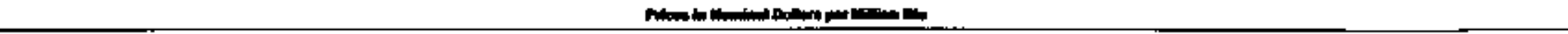 } \\
\hline \multirow[t]{2}{*}{ 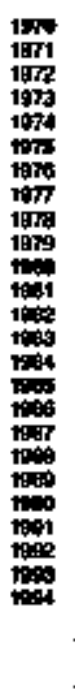 } & $\begin{array}{l}= \\
z \\
z \\
= \\
= \\
= \\
= \\
= \\
= \\
= \\
= \\
= \\
=\end{array}$ & 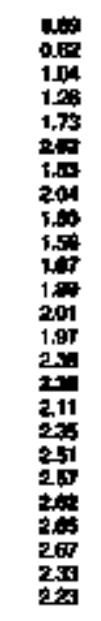 & 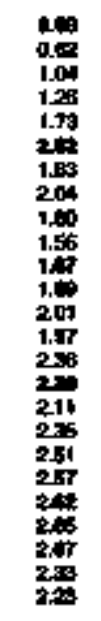 & 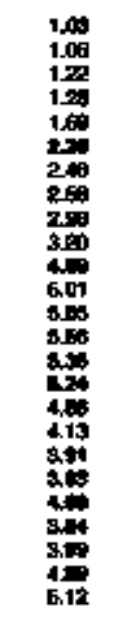 & 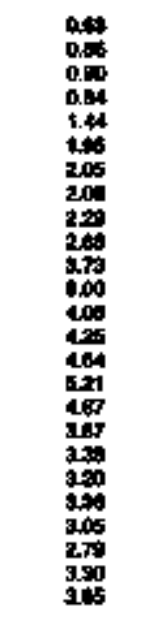 & 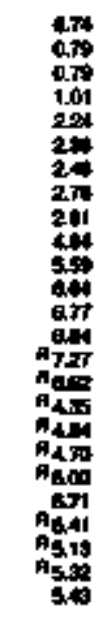 & 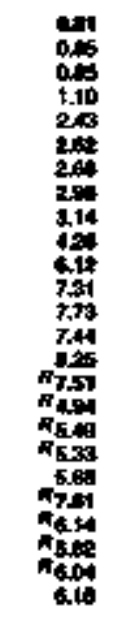 & 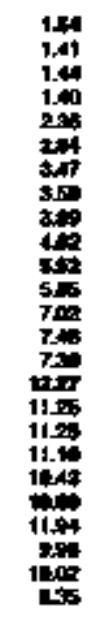 & 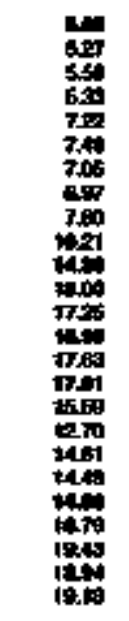 & 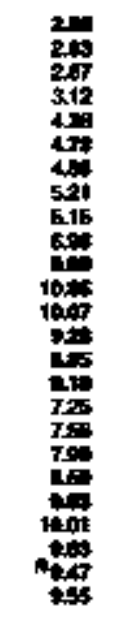 & 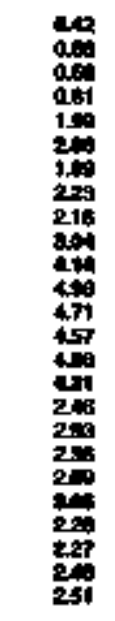 & 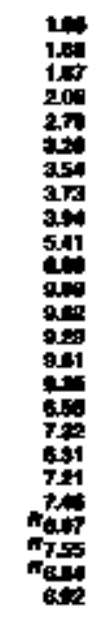 & 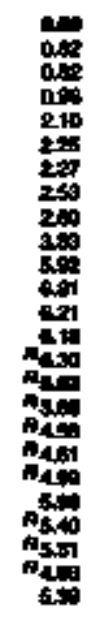 & 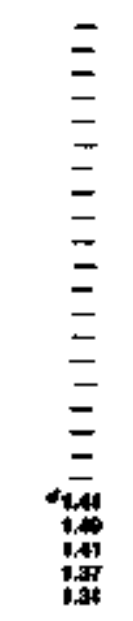 & 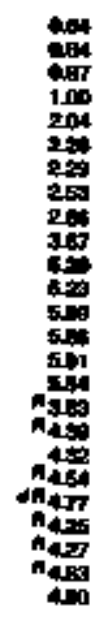 & 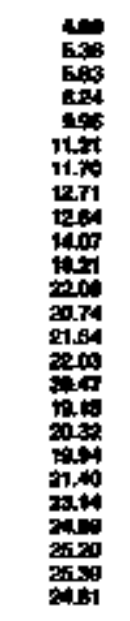 & 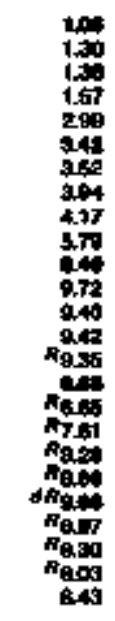 \\
\hline & \multicolumn{17}{|c|}{ 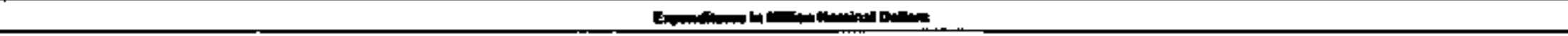 } \\
\hline 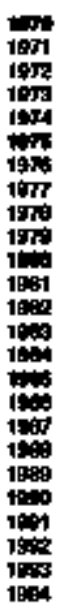 & $\begin{array}{l}z \\
= \\
z \\
= \\
z \\
= \\
= \\
= \\
= \\
= \\
z \\
z\end{array}$ & 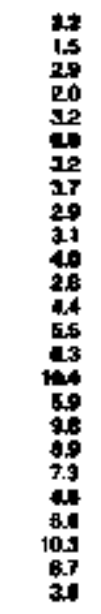 & 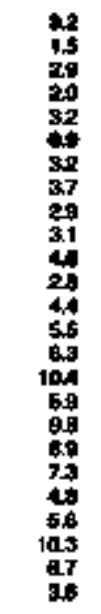 & 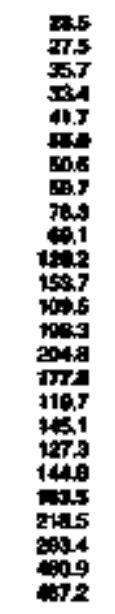 & 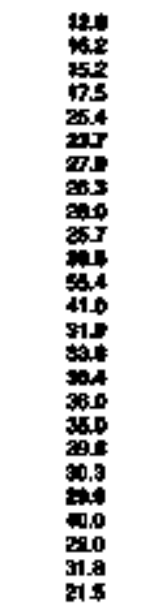 & 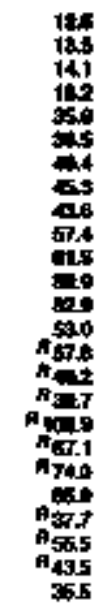 & 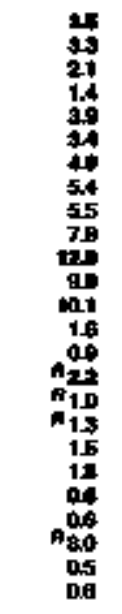 & 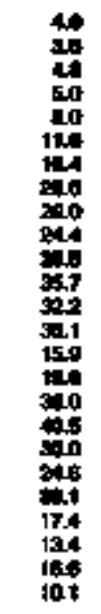 & 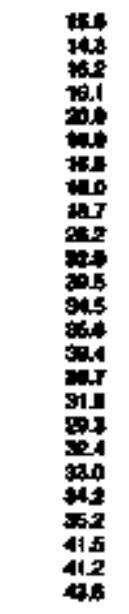 & 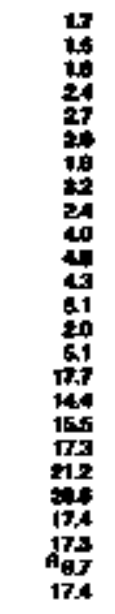 & 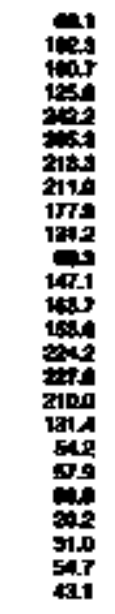 & 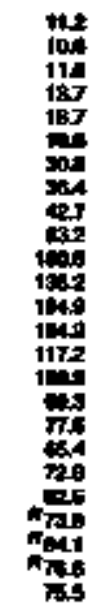 & 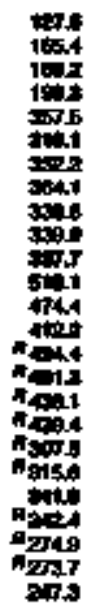 & 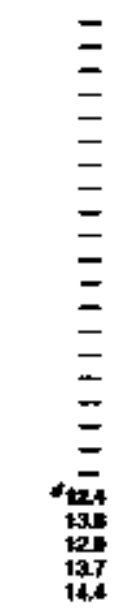 & 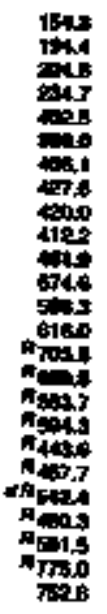 & 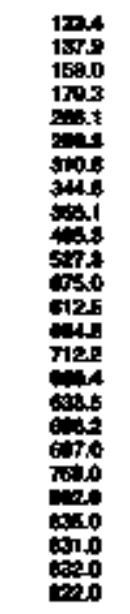 & 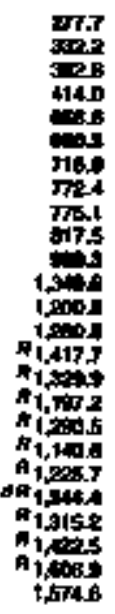 \\
\hline
\end{tabular}

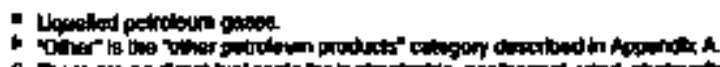

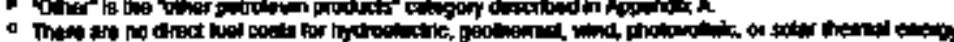

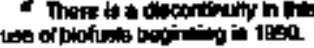

Antioding

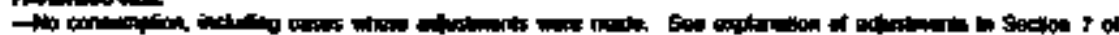

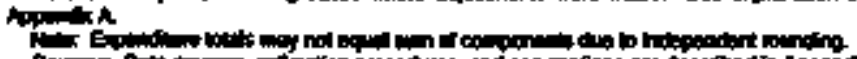

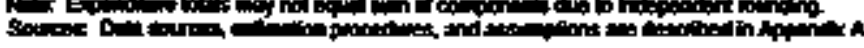




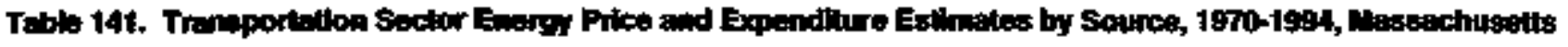

\begin{tabular}{|c|c|c|c|c|c|c|c|c|c|c|c|c|c|}
\hline \multirow[b]{4}{*}{ Yax } & \multicolumn{11}{|c|}{ 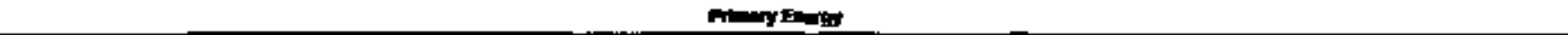 } & \multirow[b]{3}{*}{ Enctiolitr } & \multirow[b]{3}{*}{ Tom } \\
\hline & \multirow[b]{2}{*}{ con } & \multirow[b]{2}{*}{ ati } & \multicolumn{8}{|c|}{ 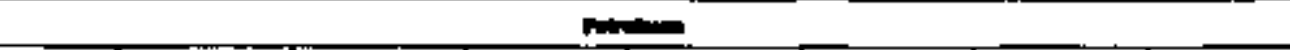 } & \multirow[b]{2}{*}{ Tot } & & \\
\hline & & & |cis & 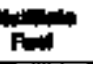 & A & LPa: & 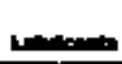 & . & 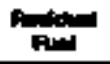 & Tom & & & \\
\hline & \multicolumn{13}{|c|}{ 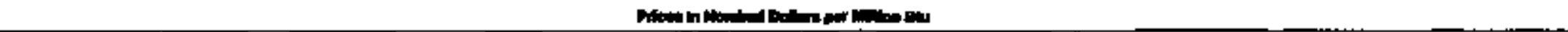 } \\
\hline \multirow[t]{2}{*}{ 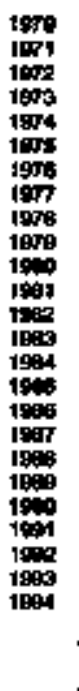 } & $\begin{array}{l}00 \\
008 \\
100 \\
1070 \\
170 \\
200 \\
100 \\
20 \\
= \\
= \\
= \\
= \\
= \\
= \\
= \\
= \\
= \\
=\end{array}$ & $\begin{array}{l}= \\
= \\
= \\
= \\
= \\
= \\
= \\
= \\
= \\
= \\
= \\
= \\
265 \\
370 \\
470 \\
270\end{array}$ & 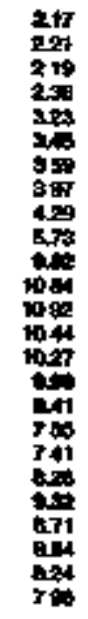 & 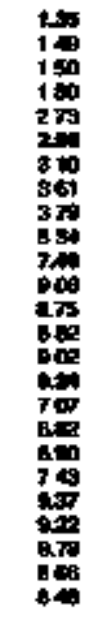 & 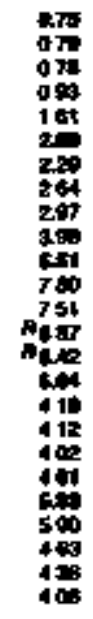 & 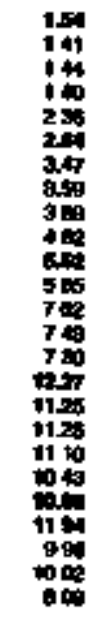 & 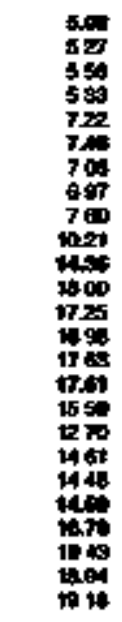 & 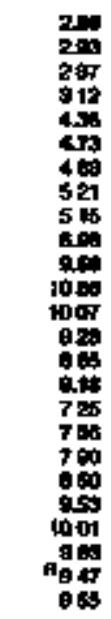 & 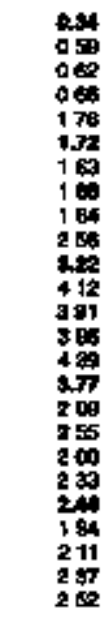 & 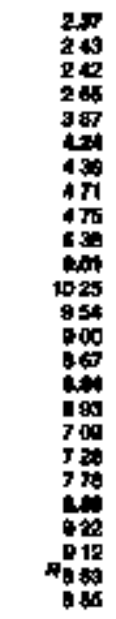 & 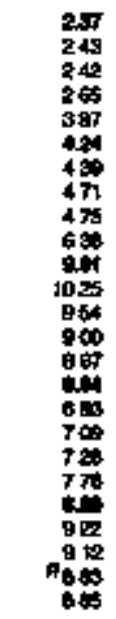 & 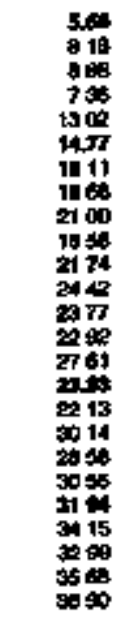 & 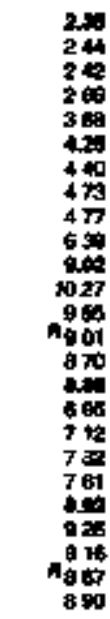 \\
\hline & \multicolumn{13}{|c|}{ E E } \\
\hline 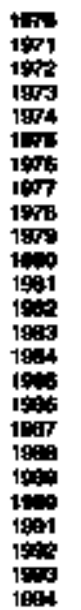 & $\begin{array}{l}\text { 监 } \\
\text { 露 } \\
= \\
= \\
= \\
= \\
= \\
= \\
= \\
= \\
= \\
=\end{array}$ & $\begin{array}{l}= \\
= \\
= \\
= \\
= \\
= \\
= \\
= \\
= \\
= \\
= \\
= \\
=\end{array}$ & 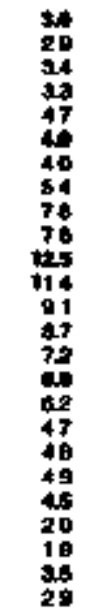 & 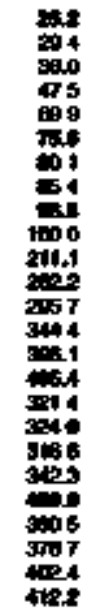 & 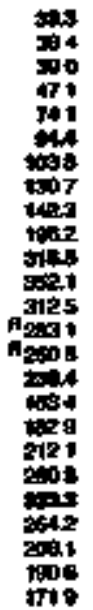 & 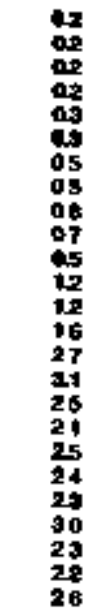 & 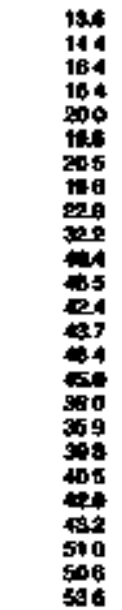 & 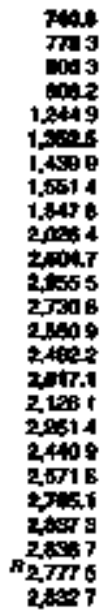 & 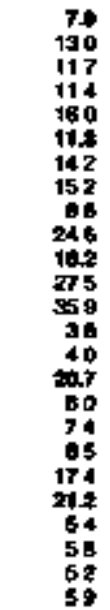 & 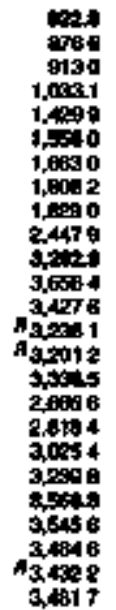 & 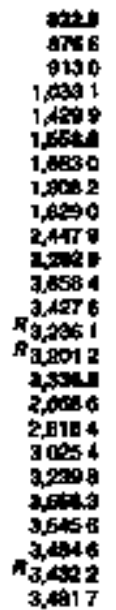 & 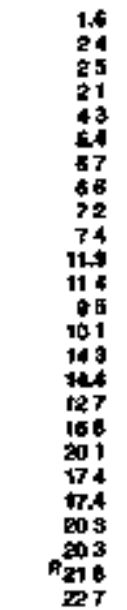 & 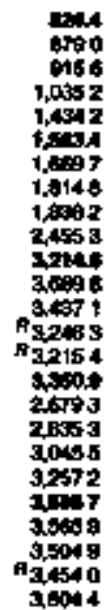 \\
\hline
\end{tabular}

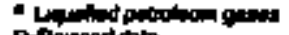
Nobintint

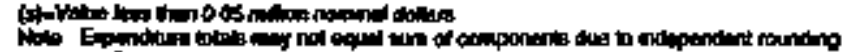

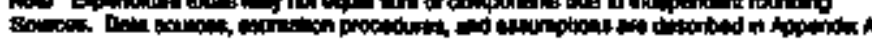



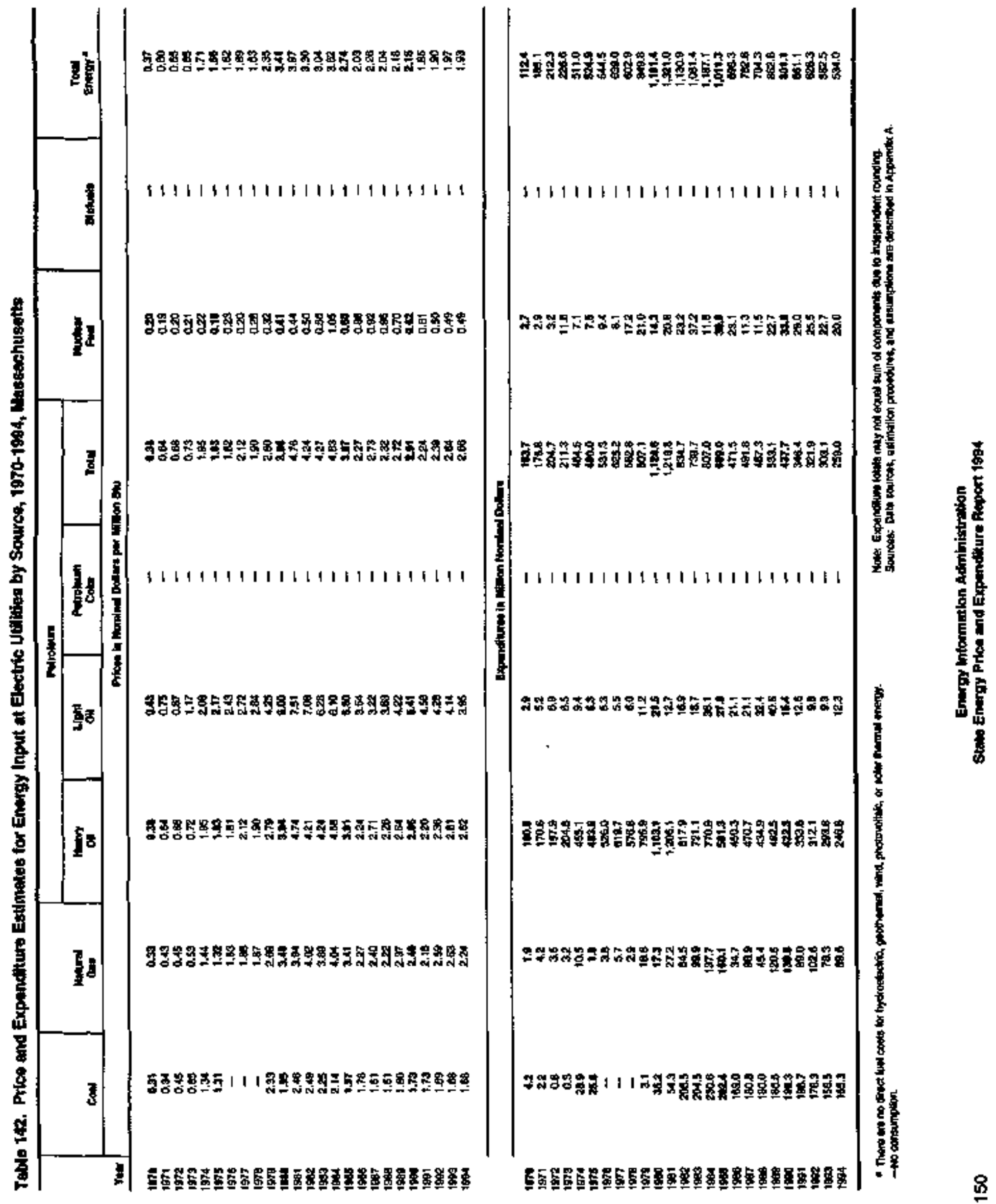

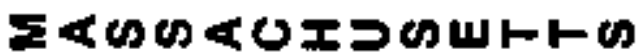


Tabts 143. Enwrey Price and Expendsture Estlmolea by Souros, 1970-1994, Mlahigan

\begin{tabular}{|c|c|c|c|c|c|c|c|c|c|c|c|c|c|c|c|c|c|}
\hline \multirow[b]{3}{*}{$r_{e r}$} & \multicolumn{14}{|c|}{ 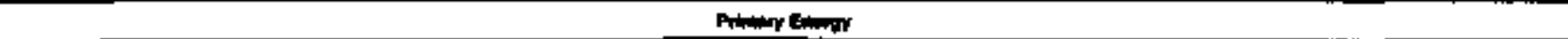 } & \multirow{2}{*}{ Fonde } & \multirow{2}{*}{ 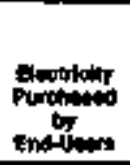 } & \multirow{2}{*}{ Toteng } \\
\hline & \multicolumn{3}{|c|}{ con } & Mond & \multicolumn{7}{|c|}{ niming } & moner & 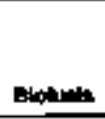 & T = & & & \\
\hline & \multicolumn{17}{|c|}{ 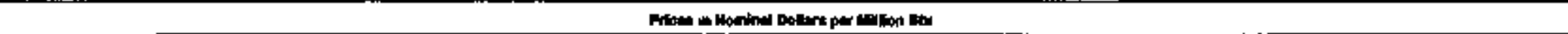 } \\
\hline 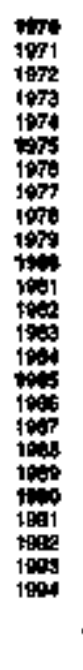 & 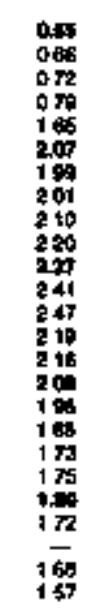 & 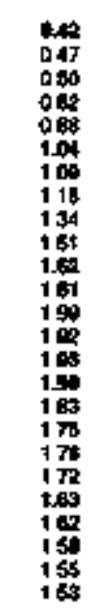 & 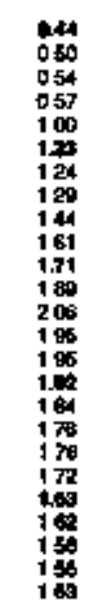 & 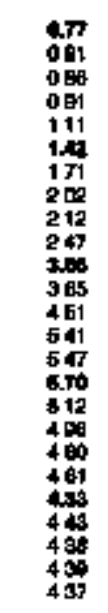 & 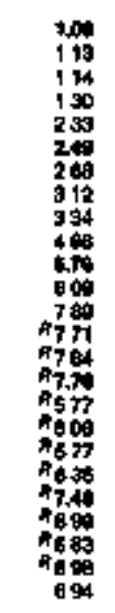 & 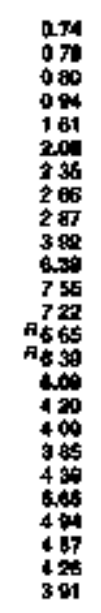 & 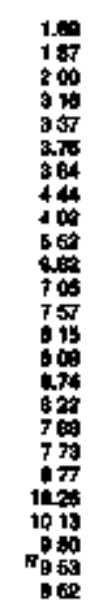 & 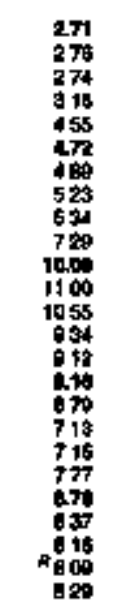 & 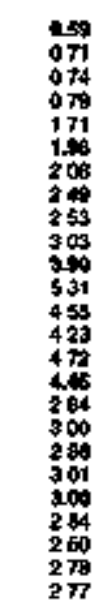 & 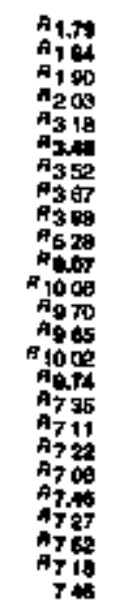 & 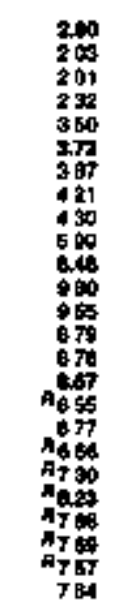 & 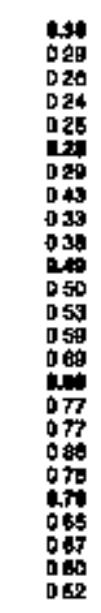 & 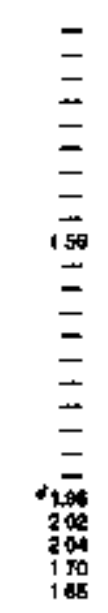 & 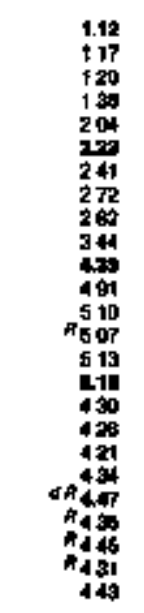 & 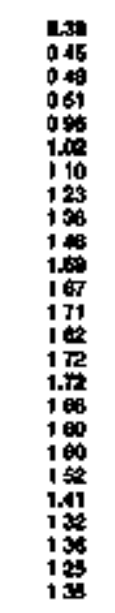 & 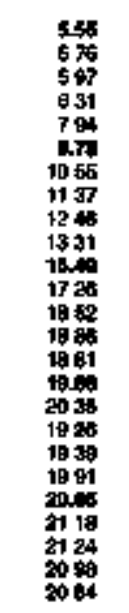 & 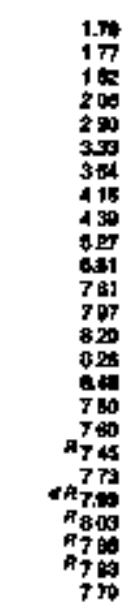 \\
\hline 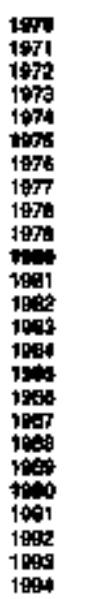 & 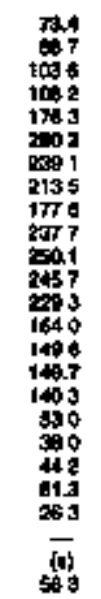 & 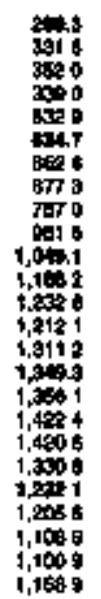 & 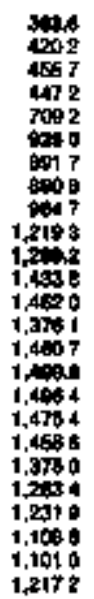 & 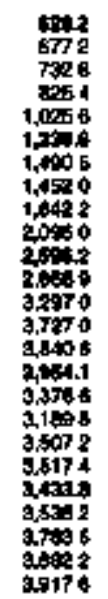 & 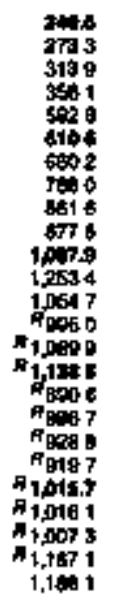 & 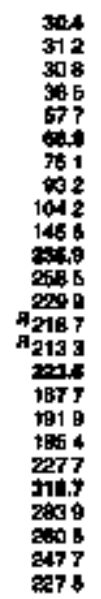 & 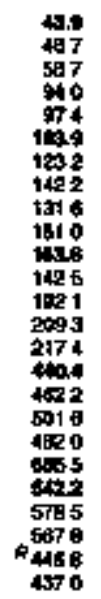 & 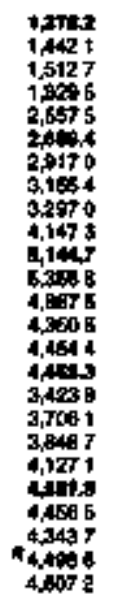 & 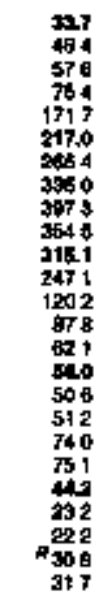 & 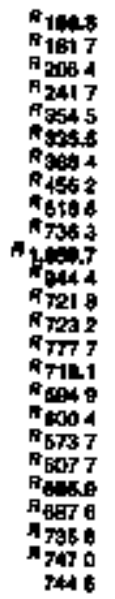 & 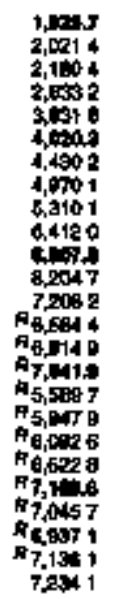 & 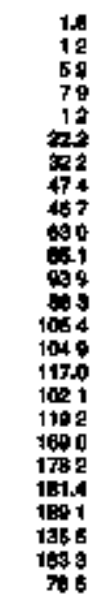 & 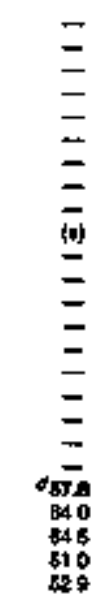 & 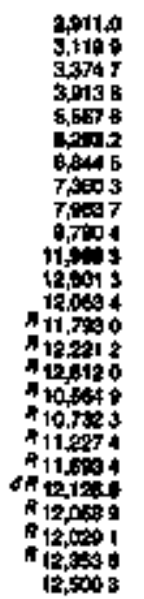 & 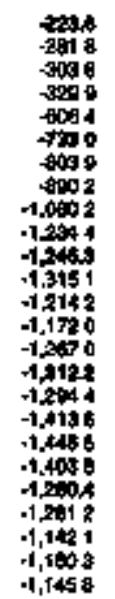 & 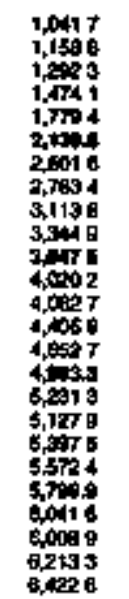 & 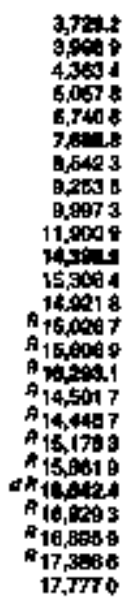 \\
\hline
\end{tabular}

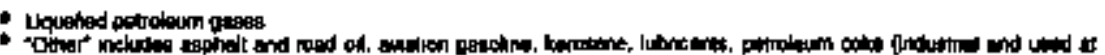

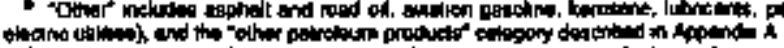

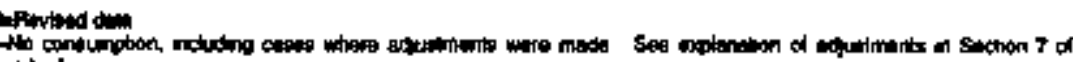

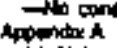

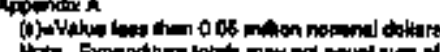

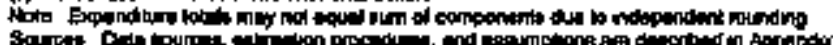

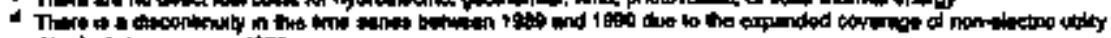

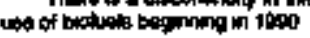

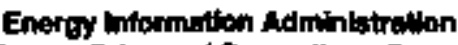




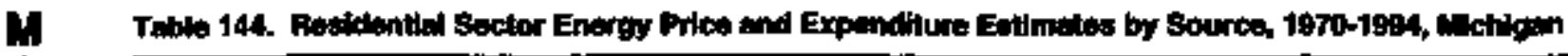

\begin{tabular}{|c|c|c|c|c|c|c|c|c|c|c|}
\hline \multirow[b]{4}{*}{ Yer } & \multicolumn{8}{|c|}{ 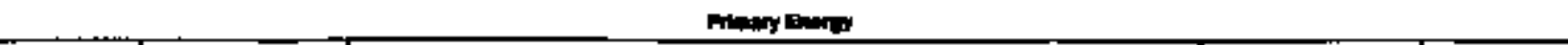 } & \multirow[b]{3}{*}{ Eutinlely } & \multirow{3}{*}{ 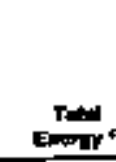 } \\
\hline & & & \multicolumn{4}{|c|}{ Pantime. } & \multirow[b]{2}{*}{ E|ct } & \multirow[b]{2}{*}{ Tot:= } & & \\
\hline & con & fine & Find & 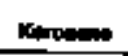 & 19: & Toted & & & & \\
\hline & \multicolumn{10}{|c|}{ 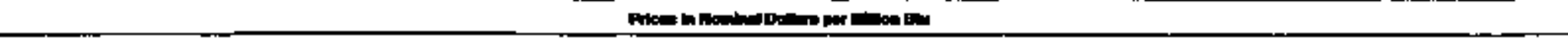 } \\
\hline \multirow[t]{2}{*}{ 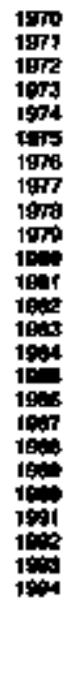 } & 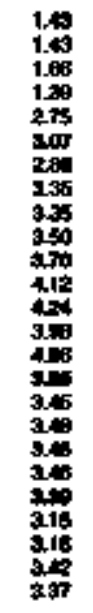 & 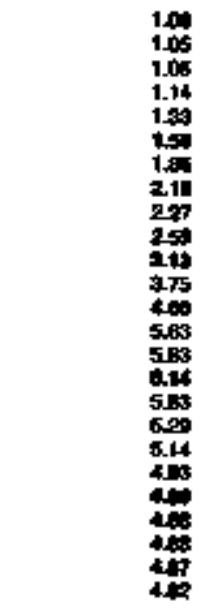 & 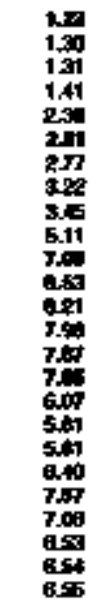 & 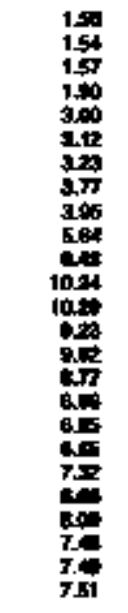 & 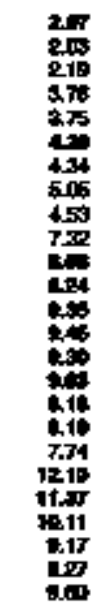 & 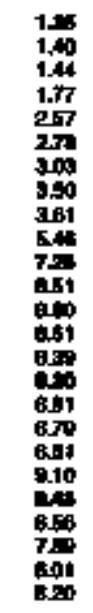 & 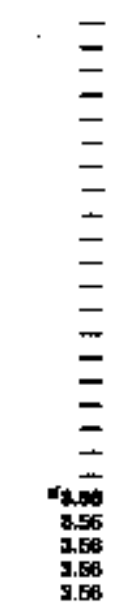 & 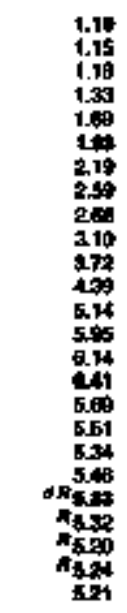 & 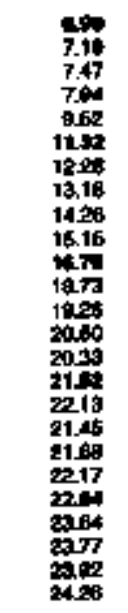 & 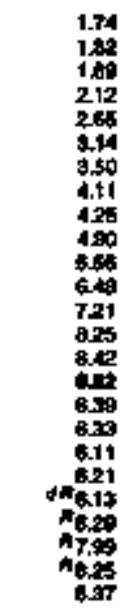 \\
\hline & \multicolumn{10}{|c|}{ 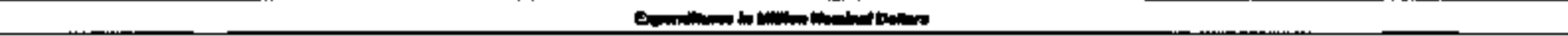 } \\
\hline 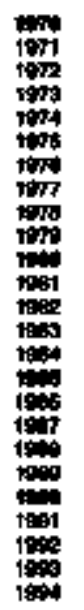 & 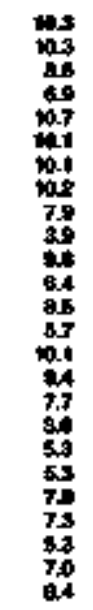 & 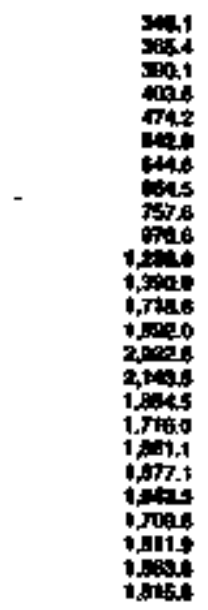 & 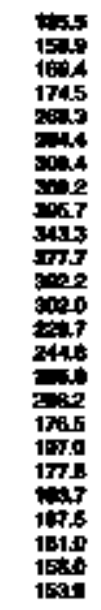 & 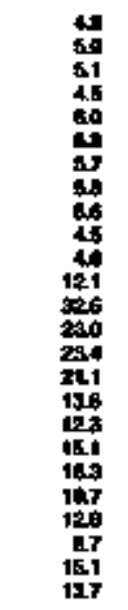 & 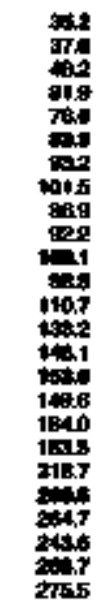 & 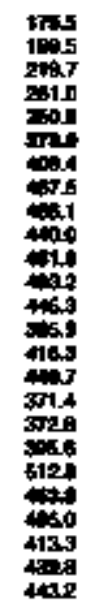 & 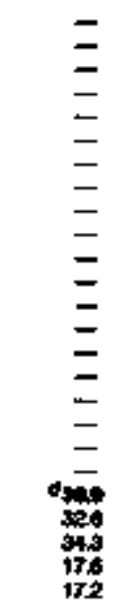 & 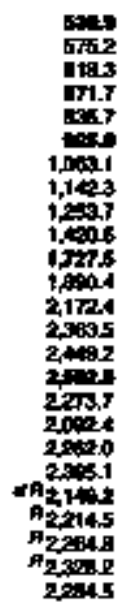 & 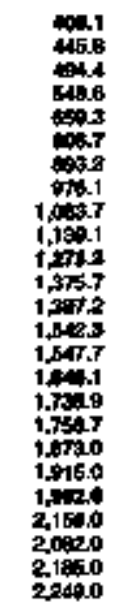 & 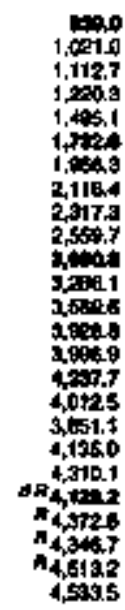 \\
\hline
\end{tabular}

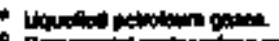

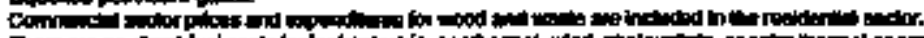

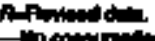

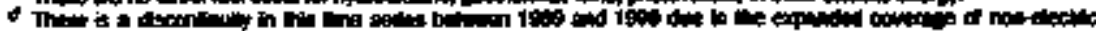

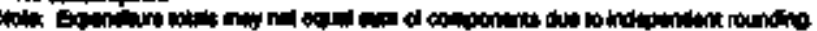

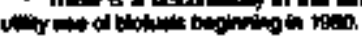




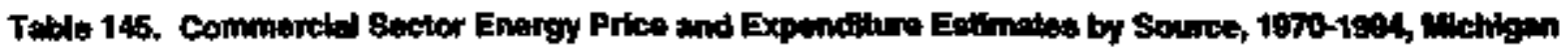

\begin{tabular}{|c|c|c|c|c|c|c|c|c|c|c|c|}
\hline \multirow[b]{4}{*}{$\mathrm{rm}$} & \multicolumn{9}{|c|}{ Pant } & \multirow[b]{3}{*}{ Enomich } & \multirow[b]{3}{*}{ 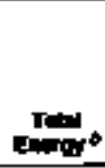 } \\
\hline & & \multirow[b]{2}{*}{ mated } & \multicolumn{6}{|c|}{ 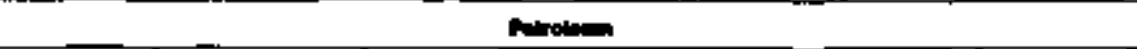 } & \multirow[b]{2}{*}{ Tolid } & & \\
\hline & ord & & Pow & Cominose & INo $=$ & ה & 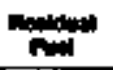 & Tot & & & \\
\hline & \multicolumn{11}{|c|}{ 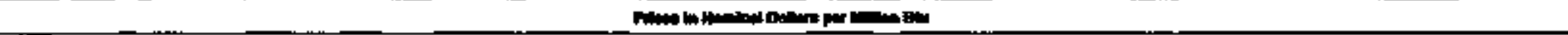 } \\
\hline \multirow[t]{2}{*}{ 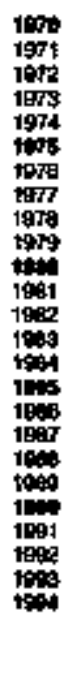 } & 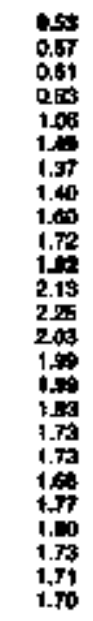 & 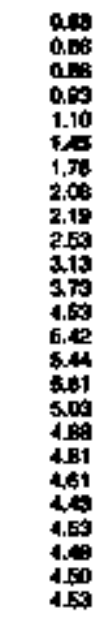 & 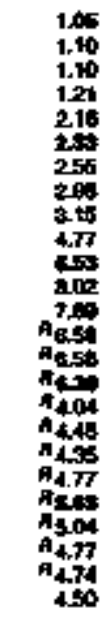 & 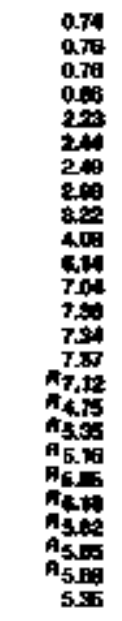 & 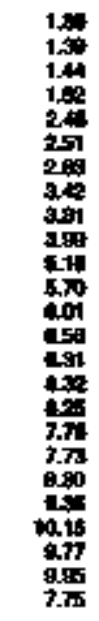 & 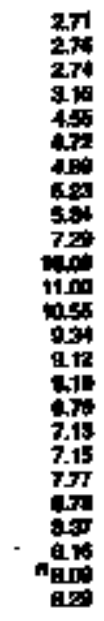 & 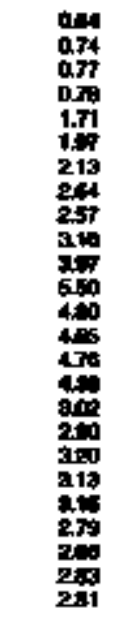 & 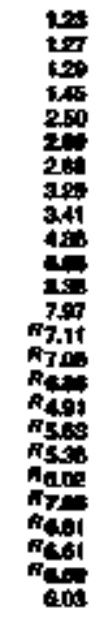 & 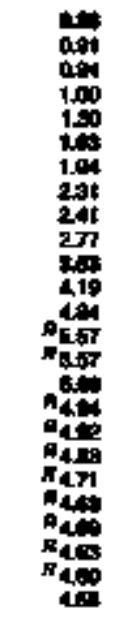 & 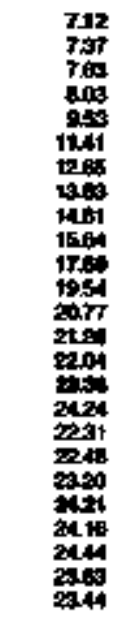 & 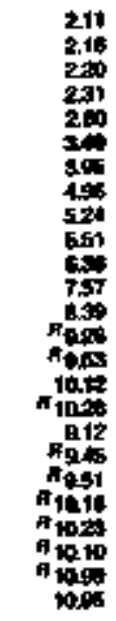 \\
\hline & \multicolumn{11}{|c|}{ 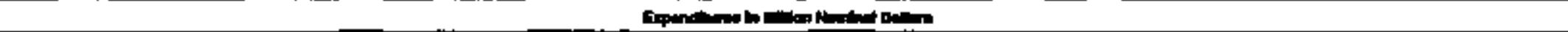 } \\
\hline 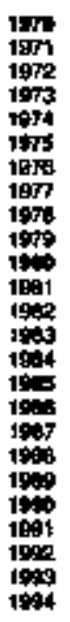 & 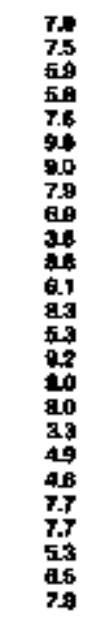 & 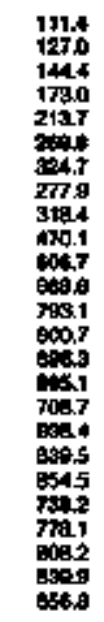 & 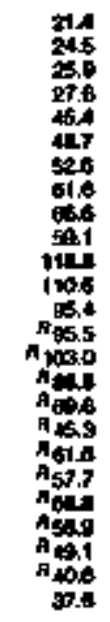 & 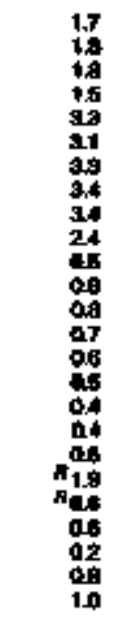 & 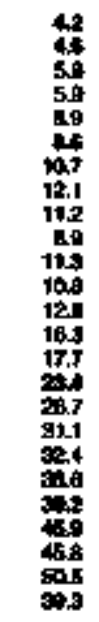 & 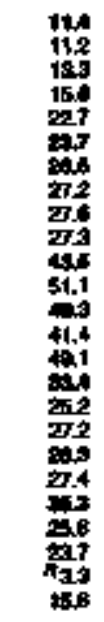 & 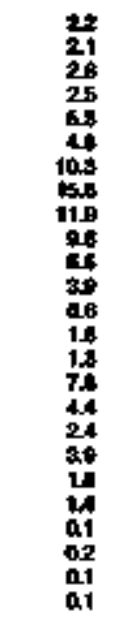 & 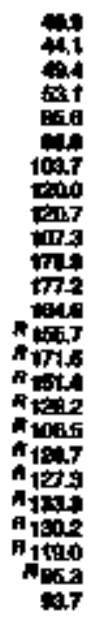 & 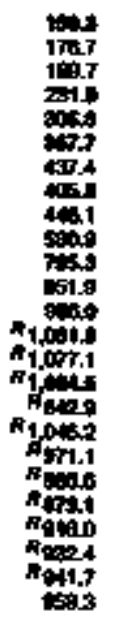 & 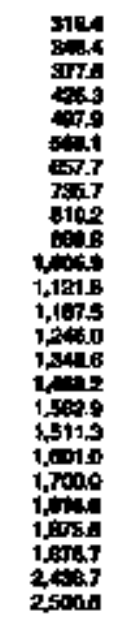 & 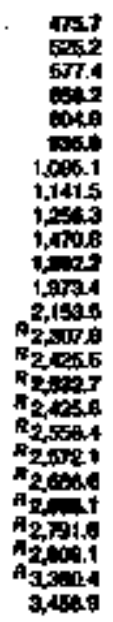 \\
\hline
\end{tabular}

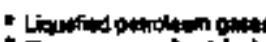

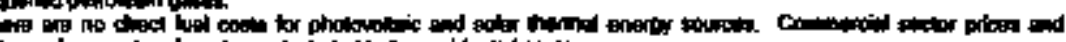

Antions

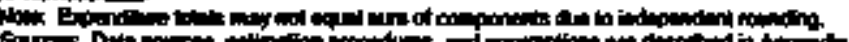

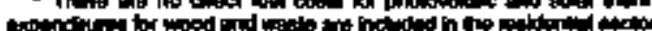




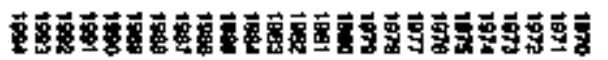

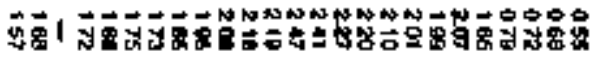

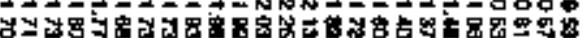

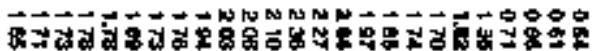

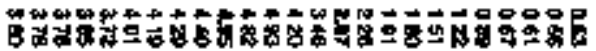

NNNNLN L L L

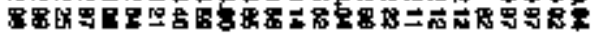

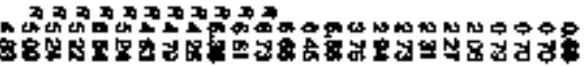

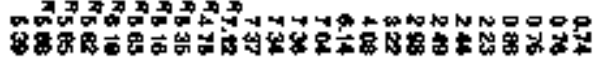

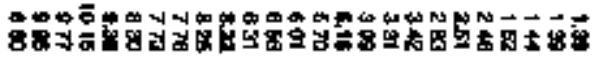

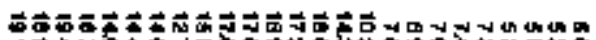

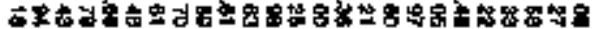

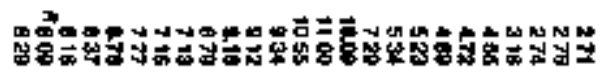

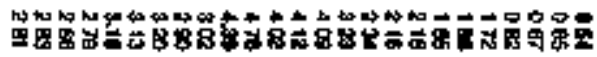

บn

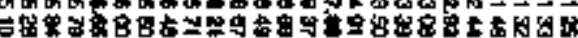

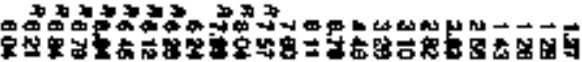

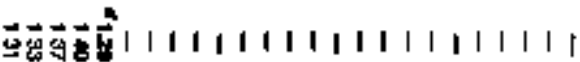

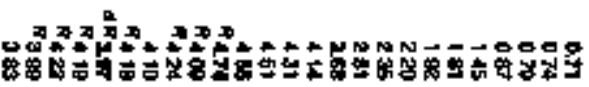

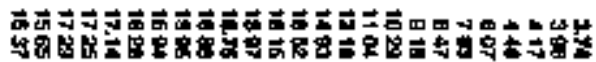

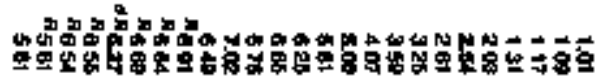

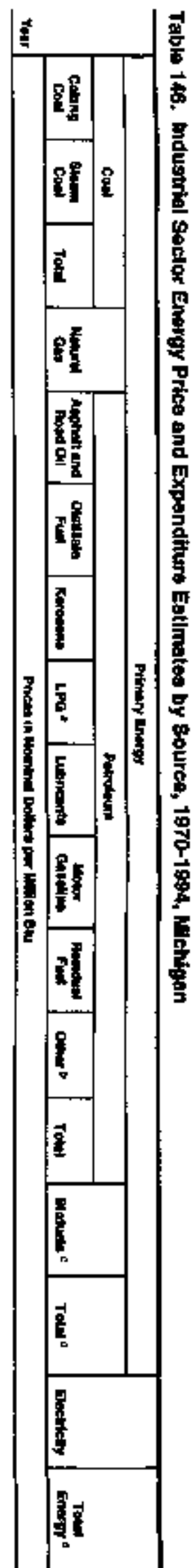


Table 147. Trensportation Sector Energy Price and Expenditure Ectimatos by Souroe, 1970-1904, wichlgan

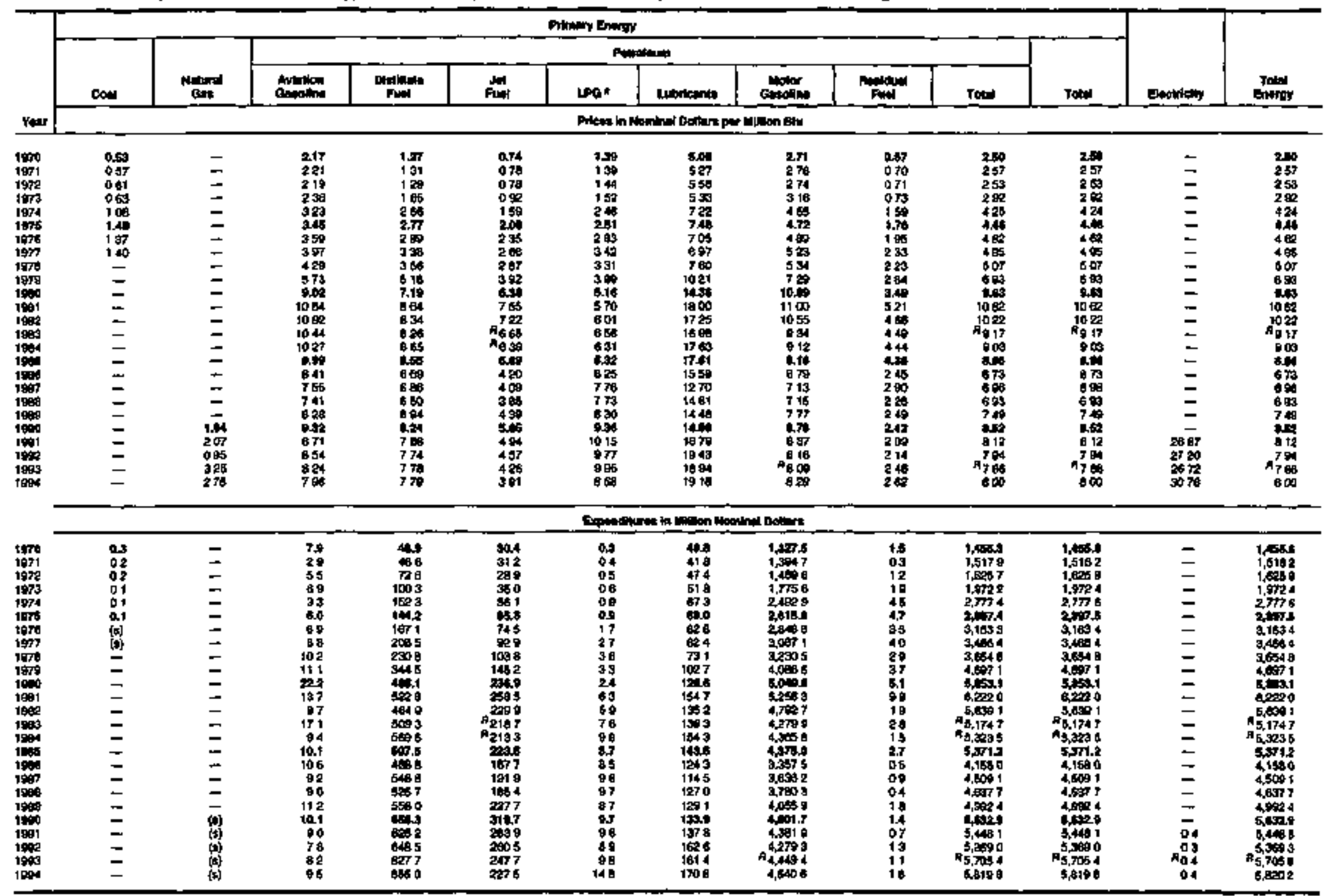




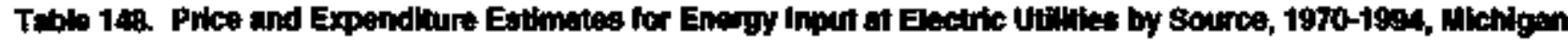

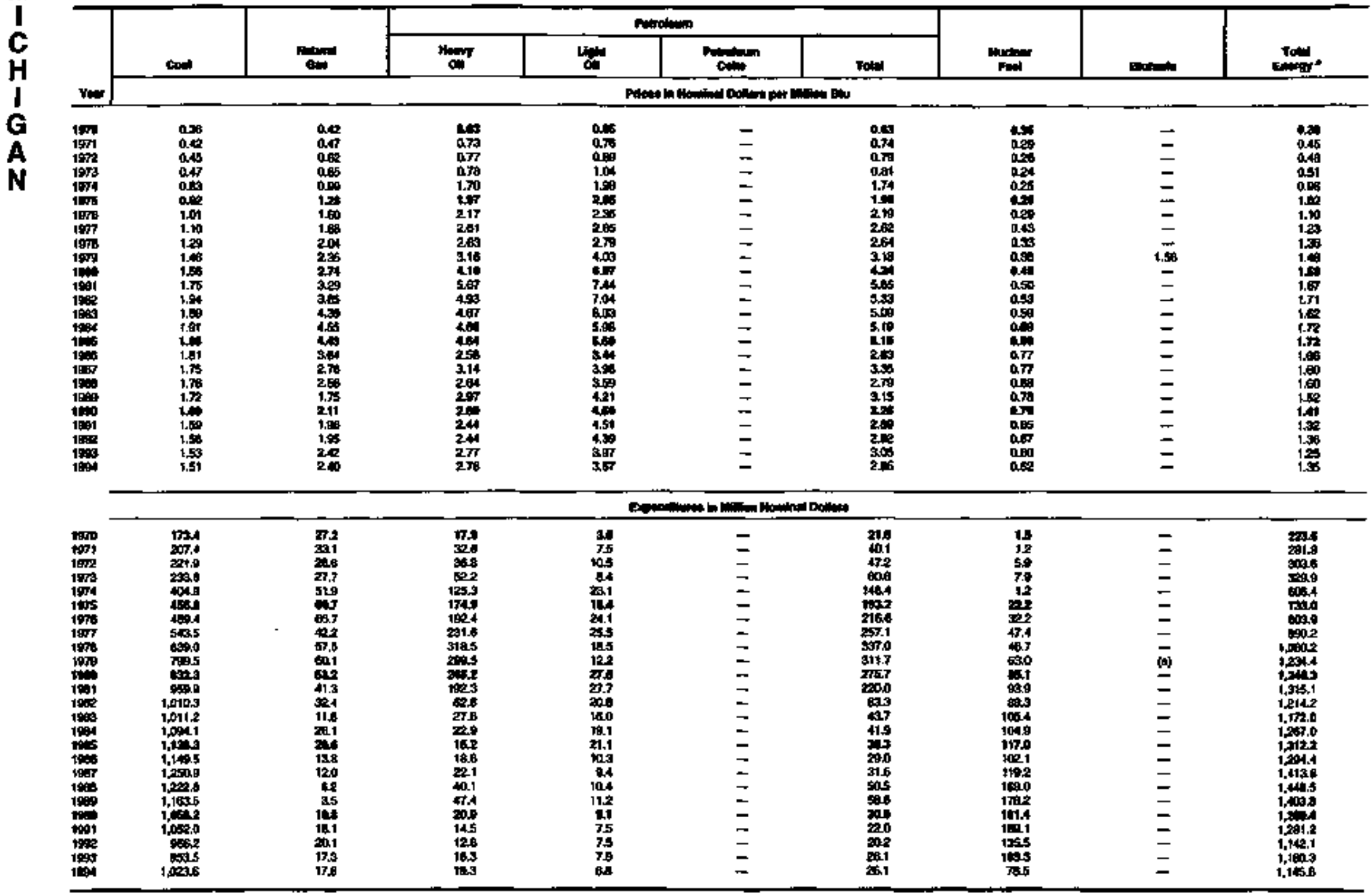

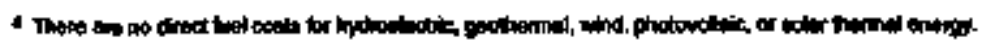

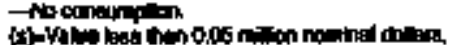

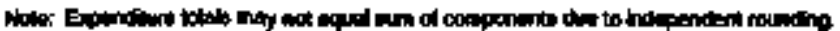
burome to 
Tablo 149. Enargy Prloe and Expenditure Estimates by Source, 1970-1994, Mnnosota

\begin{tabular}{|c|c|c|c|c|c|c|c|c|c|c|c|c|c|c|c|c|c|}
\hline \multirow[b]{4}{*}{ Nat } & \multicolumn{14}{|c|}{ Prtiment tingy } & \multirow{3}{*}{ 年 } & \multirow{3}{*}{ 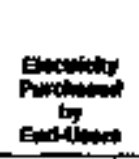 } & \multirow[b]{3}{*}{ Tets } \\
\hline & \multicolumn{3}{|c|}{ Con } & \multirow[b]{2}{*}{ ה: } & \multicolumn{7}{|c|}{ המאויمشת } & \multirow[b]{2}{*}{ Finger } & \multirow[b]{2}{*}{ 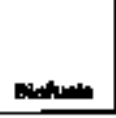 } & \multirow[b]{2}{*}{ TAtc } & & & \\
\hline & toming & ind & الוt? & & 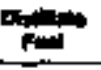 & fw & tone & 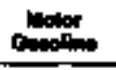 & fon & Onmb' & $T$ & & & & & & \\
\hline & \multicolumn{17}{|c|}{ 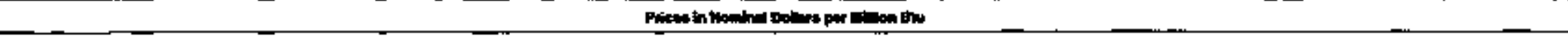 } \\
\hline \multirow[t]{2}{*}{ 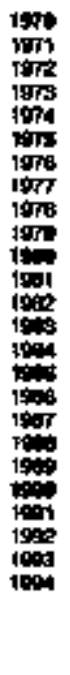 } & 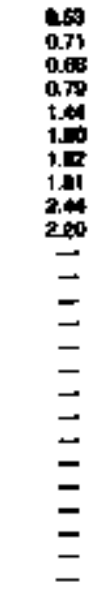 & 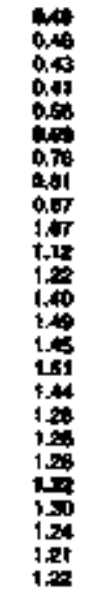 & 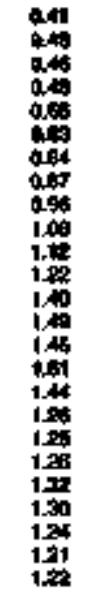 & 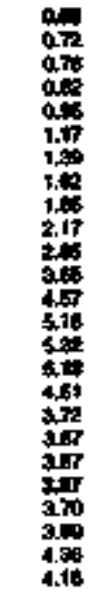 & 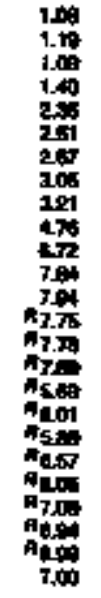 & 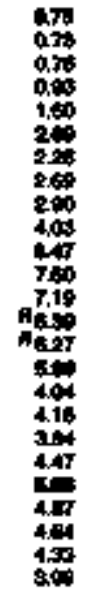 & 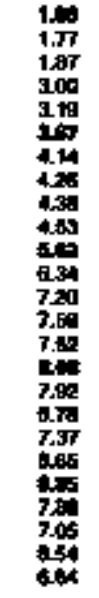 & 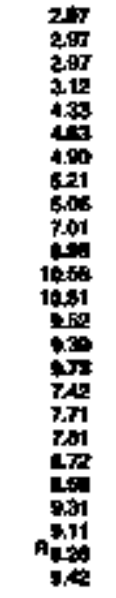 & 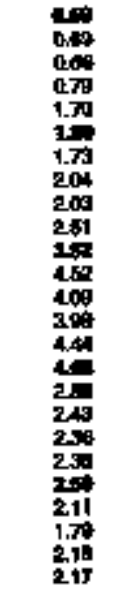 & 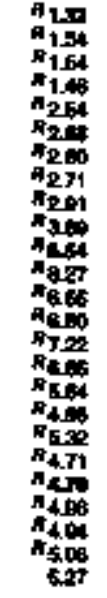 & 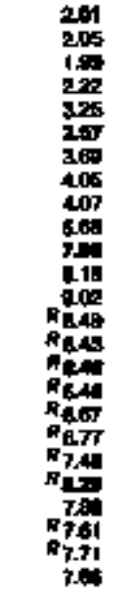 & 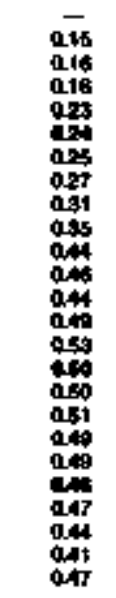 & 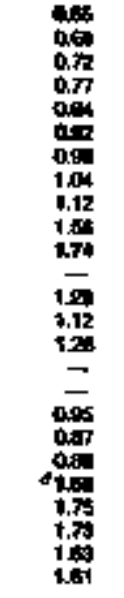 & 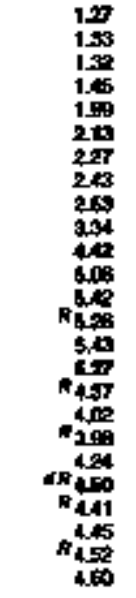 & 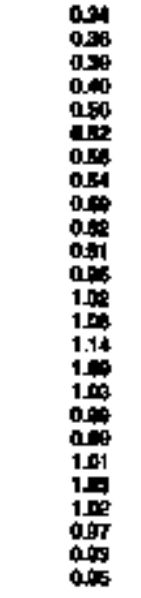 & 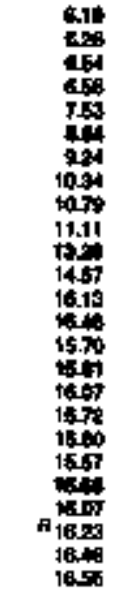 & 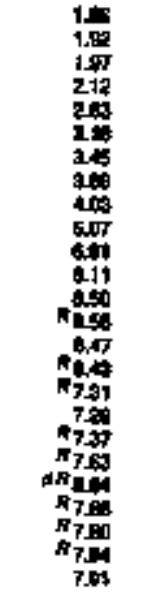 \\
\hline & \multicolumn{17}{|c|}{ 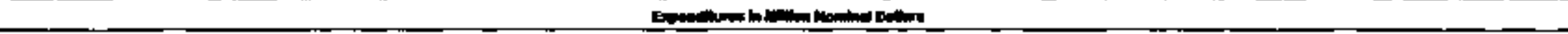 } \\
\hline 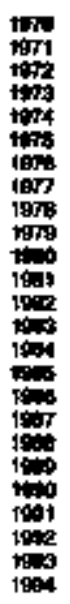 & 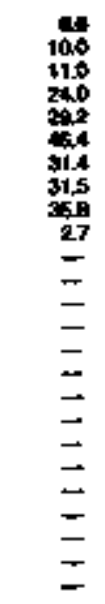 & 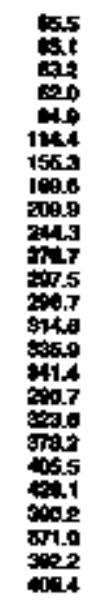 & 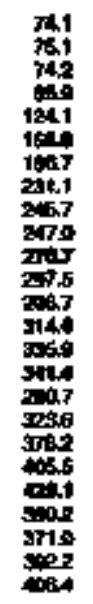 & 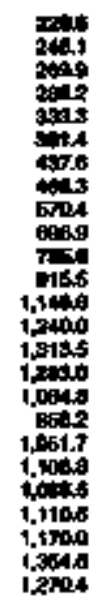 & 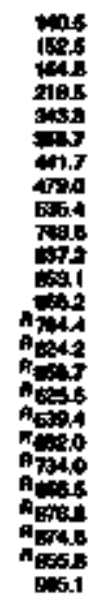 & 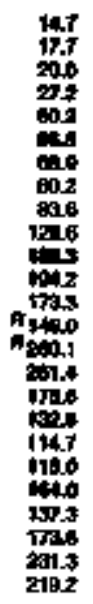 & 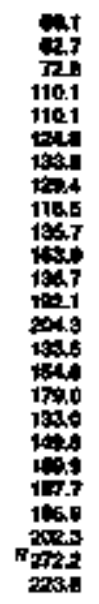 & 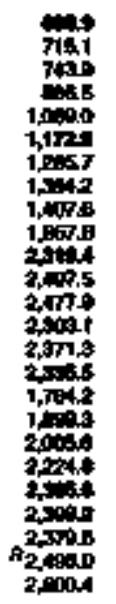 & 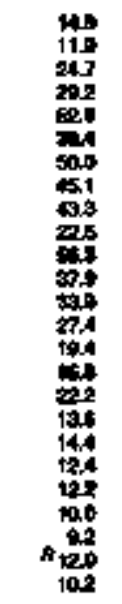 & 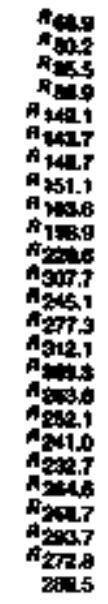 & 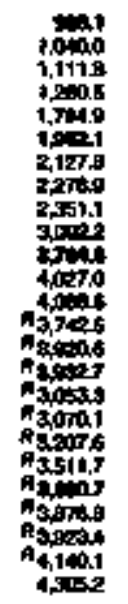 & 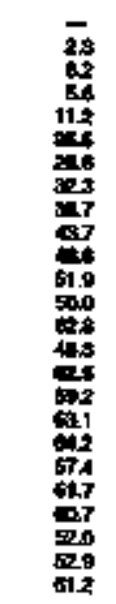 & 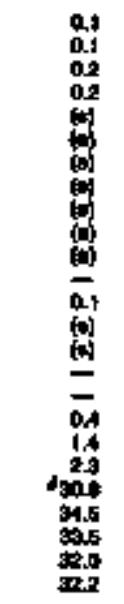 & 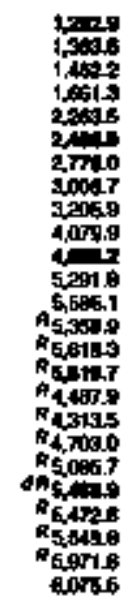 & 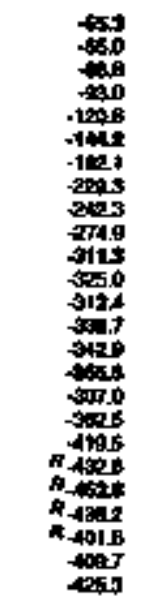 & 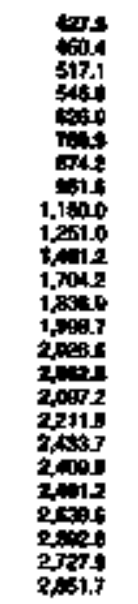 & 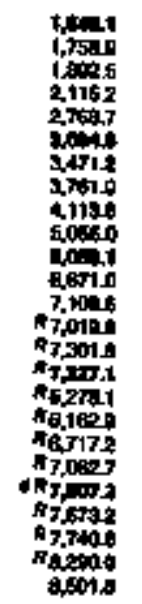 \\
\hline
\end{tabular}




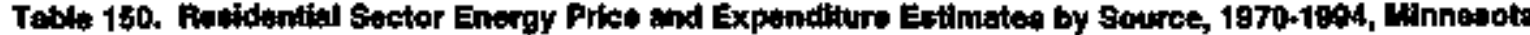

\begin{tabular}{|c|c|c|c|c|c|c|c|c|c|c|}
\hline \multirow{4}{*}{ rem } & \multicolumn{8}{|c|}{ Prilater Energy } & \multirow[b]{3}{*}{ Encticts } & \multirow[b]{3}{*}{ tom } \\
\hline & \multirow[b]{2}{*}{$\infty$} & \multirow[b]{2}{*}{ Bation } & \multicolumn{4}{|c|}{ Porrobevin } & \multirow[b]{2}{*}{ Bonemind } & \multirow[b]{2}{*}{ Towe } & & \\
\hline & & & onsusen & Kanoseses & $4 \mathrm{mg} *$ & Tatid & & & & \\
\hline & \multicolumn{10}{|c|}{ 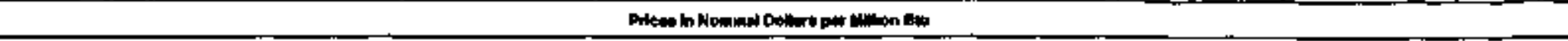 } \\
\hline \multirow[t]{2}{*}{ 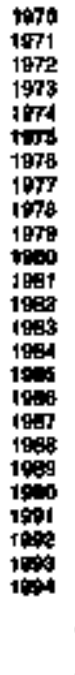 } & 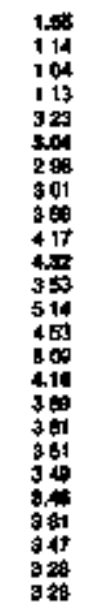 & 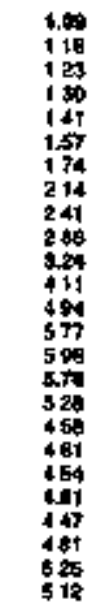 & 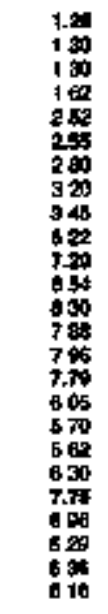 & 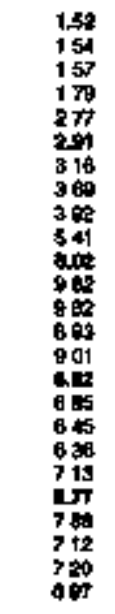 & 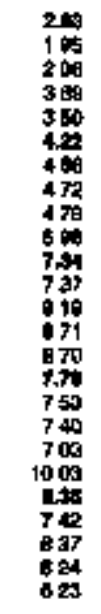 & 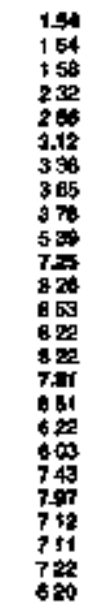 & 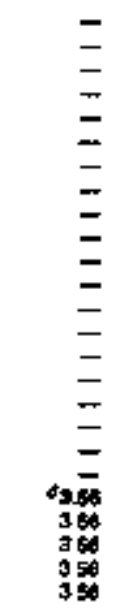 & 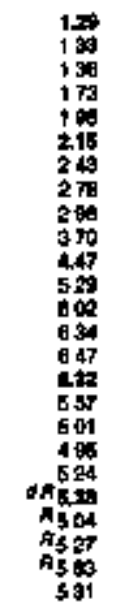 & 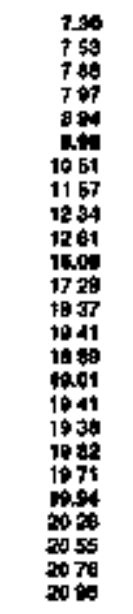 & 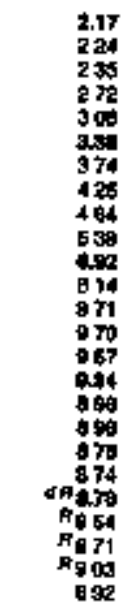 \\
\hline & \multicolumn{10}{|c|}{ 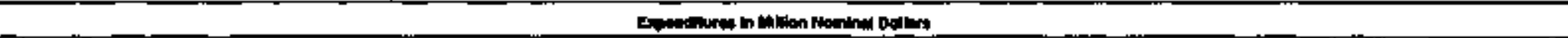 } \\
\hline 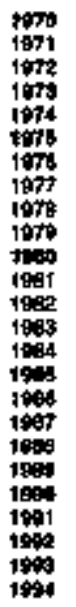 & $\begin{array}{l}14 \\
41 \\
22 \\
23 \\
74 \\
40 \\
38 \\
28 \\
19 \\
02 \\
13 \\
30 \\
83 \\
54 \\
78 \\
68 \\
60 \\
41 \\
52 \\
58 \\
38 \\
22 \\
03 \\
23 \\
52\end{array}$ & 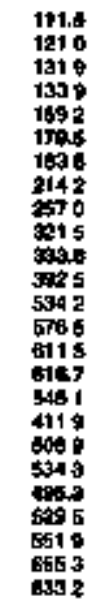 & 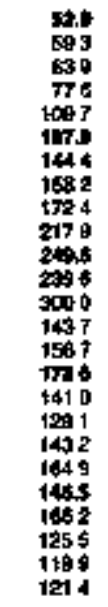 & 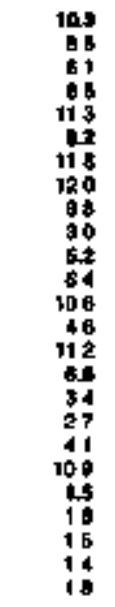 & 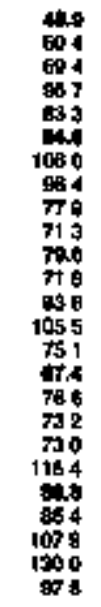 & 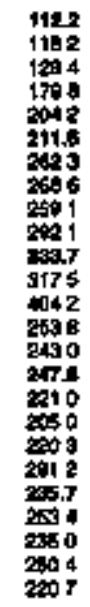 & $\begin{array}{l}\bar{z} \\
\bar{z} \\
\bar{z} \\
\bar{z} \\
\bar{z} \\
\bar{z} \\
= \\
\bar{z} \\
\bar{z} \\
\bar{z} \\
1+27 \\
193 \\
140 \\
118 \\
116\end{array}$ & 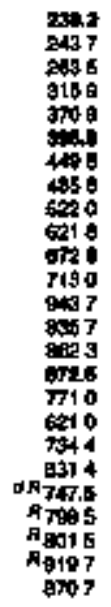 & 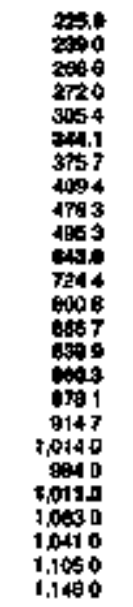 & 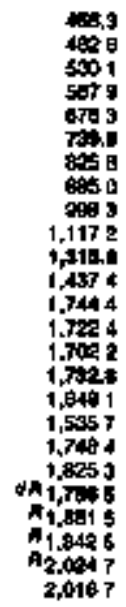 \\
\hline
\end{tabular}

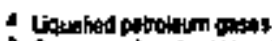

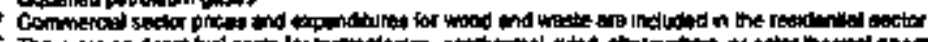

Apposand di:t

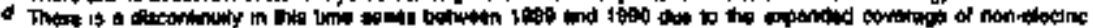

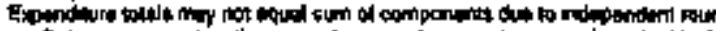

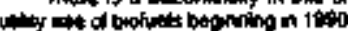




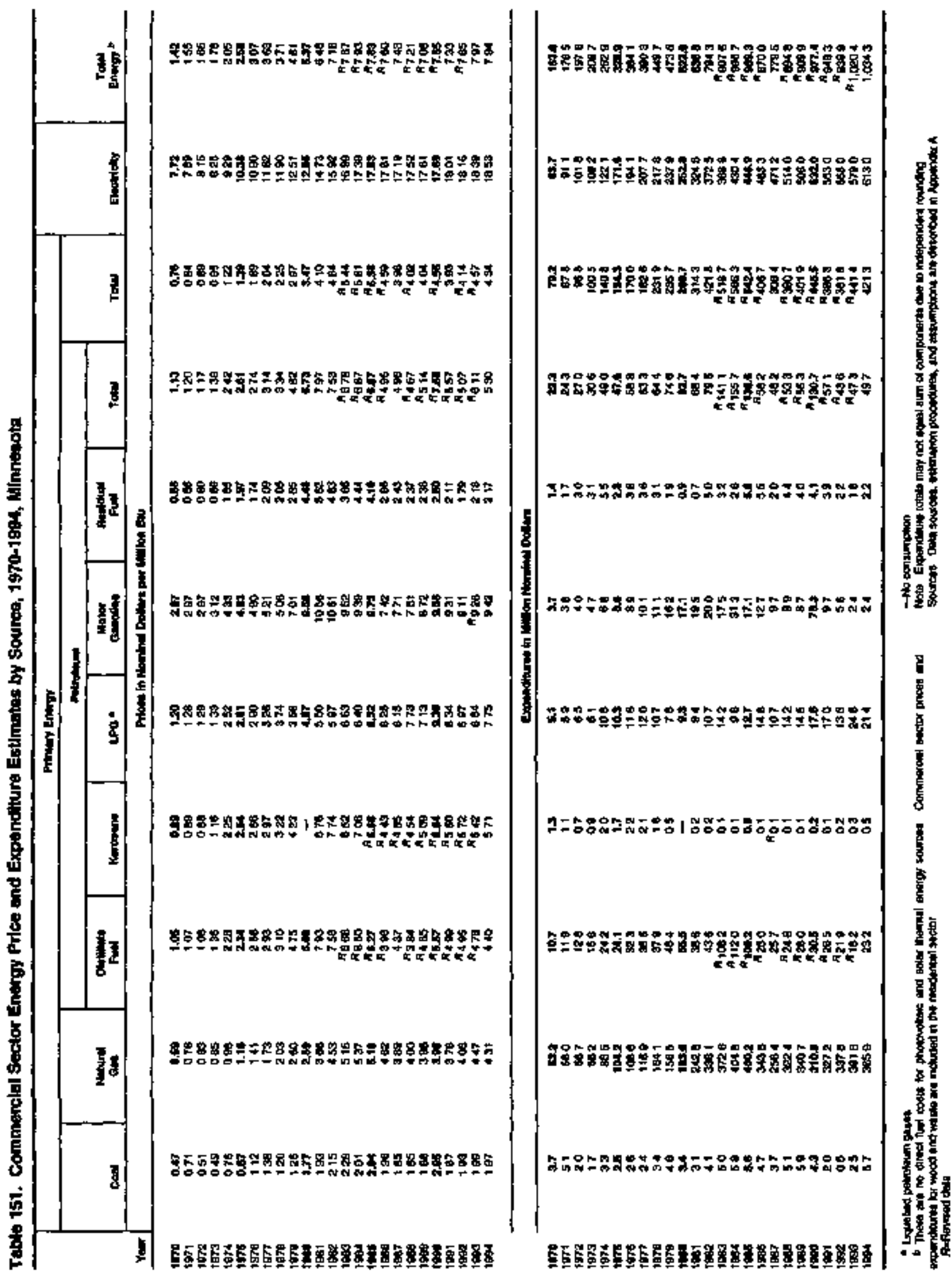




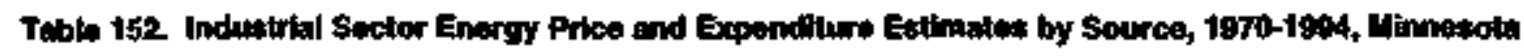

\begin{tabular}{|c|c|c|c|c|c|c|c|c|c|c|c|c|c|c|c|c|c|}
\hline \multirow[b]{4}{*}{$\underline{\mathbf{m}}$} & \multicolumn{15}{|c|}{ 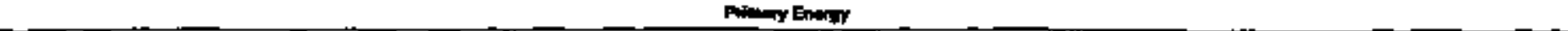 } & \multirow[b]{3}{*}{ 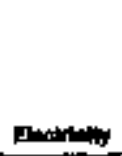 } & \multirow[b]{3}{*}{ 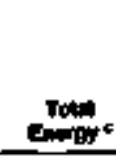 } \\
\hline & \multicolumn{3}{|c|}{ Cod } & \multirow[b]{2}{*}{ Nand } & \multicolumn{9}{|c|}{ Antroluns } & \multirow[b]{2}{*}{ 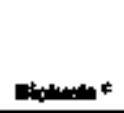 } & \multirow[b]{2}{*}{ Told t t } & & \\
\hline & Dowid & sone & Told & & Ampingand and & Din & 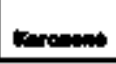 & $\mathbf{m a}=$ & Lotionint & 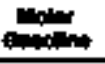 & and & oting & Told & & & & \\
\hline & \multicolumn{17}{|c|}{ 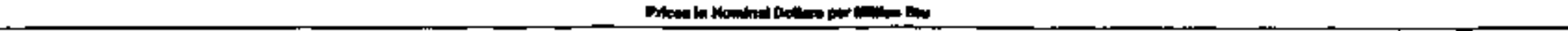 } \\
\hline \multirow[t]{2}{*}{ 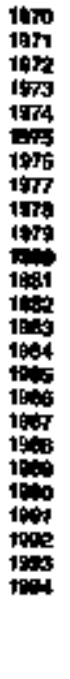 } & $\begin{array}{l}050 \\
071 \\
069 \\
070 \\
10 \\
100 \\
190 \\
100 \\
240 \\
20 \\
= \\
= \\
= \\
= \\
= \\
= \\
= \\
= \\
= \\
=\end{array}$ & 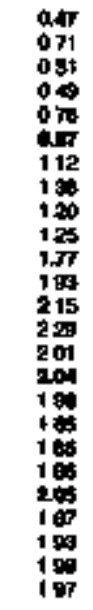 & 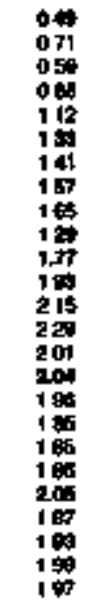 & 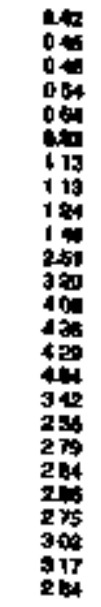 & 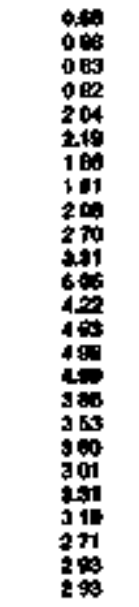 & 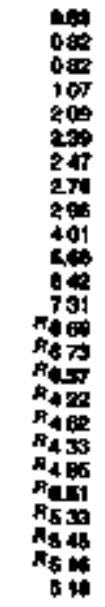 & 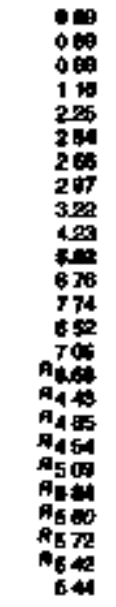 & 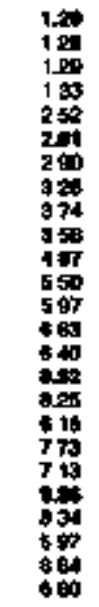 & 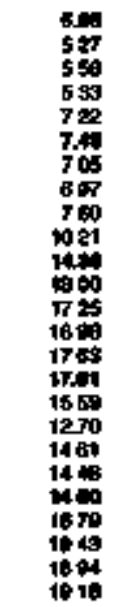 & 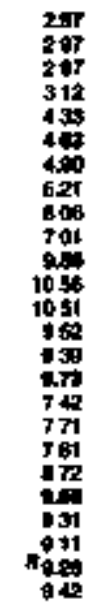 & 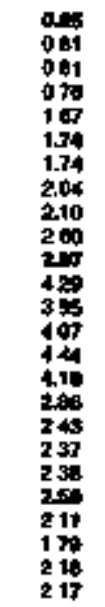 & 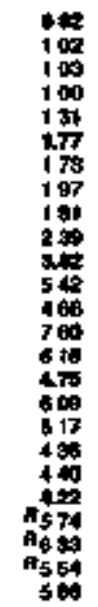 & 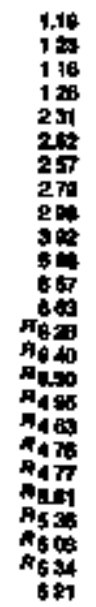 & $\begin{array}{l}= \\
= \\
= \\
= \\
= \\
= \\
= \\
= \\
= \\
= \\
= \\
1=8 \\
184 \\
181 \\
10\end{array}$ & 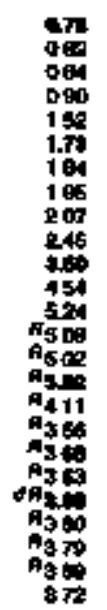 & 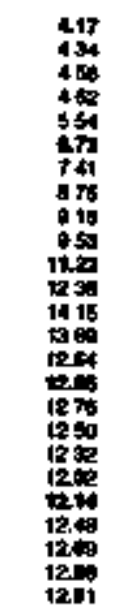 & 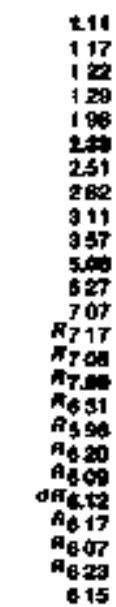 \\
\hline & \multicolumn{17}{|c|}{ 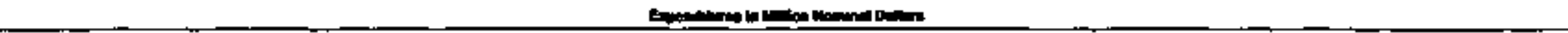 } \\
\hline 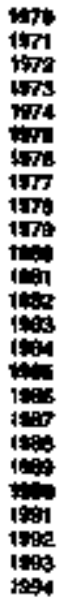 & $\begin{array}{l}100 \\
110 \\
240 \\
292 \\
314 \\
315 \\
369 \\
287 \\
= \\
= \\
= \\
= \\
= \\
= \\
= \\
= \\
= \\
=\end{array}$ & 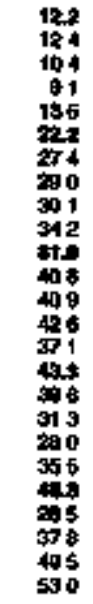 & 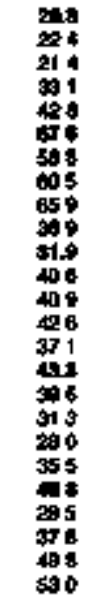 & 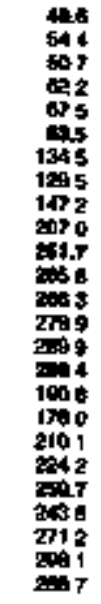 & 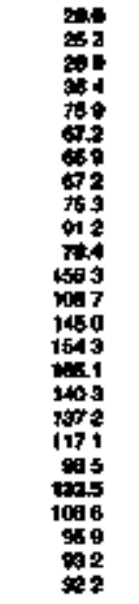 & 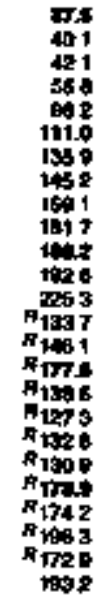 & $\begin{array}{l}10 \\
11 \\
07 \\
07 \\
23 \\
25 \\
38 \\
37 \\
36 \\
24 \\
93 \\
11 \\
18 \\
16 \\
20 \\
49 \\
08 \\
03 \\
09 \\
814 \\
09 \\
03 \\
03 \\
05 \\
23\end{array}$ & 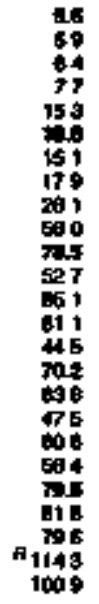 & 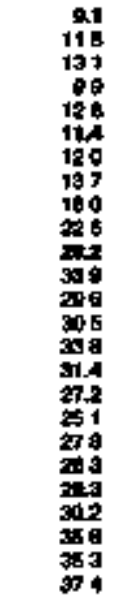 & 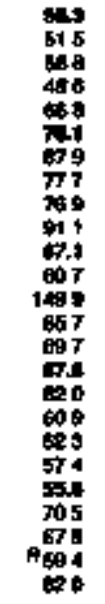 & 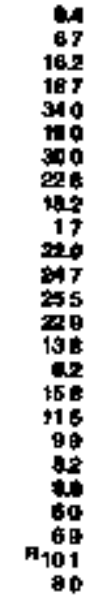 & 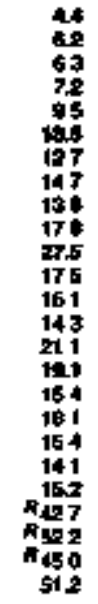 & 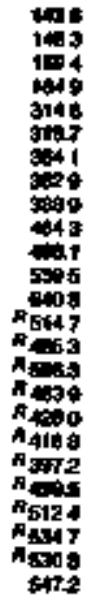 & $\begin{array}{l}= \\
= \\
= \\
= \\
= \\
= \\
= \\
= \\
= \\
= \\
= \\
= \\
0150 \\
192 \\
160 \\
170 \\
104\end{array}$ & 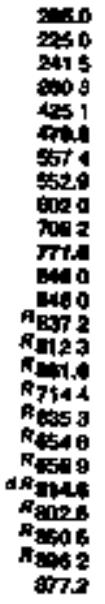 & 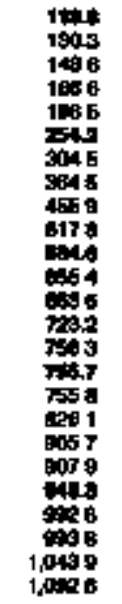 & 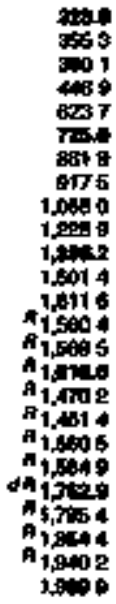 \\
\hline
\end{tabular}

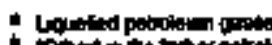

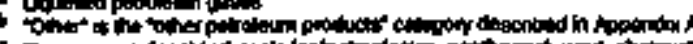

Aflevnow data

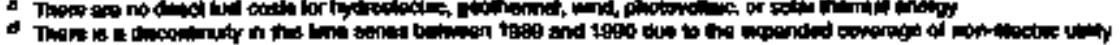
$\rightarrow \lim _{\lim }$

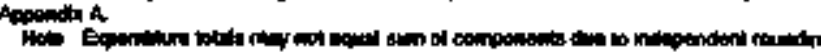

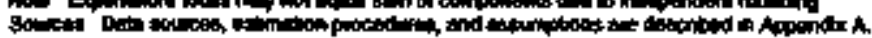




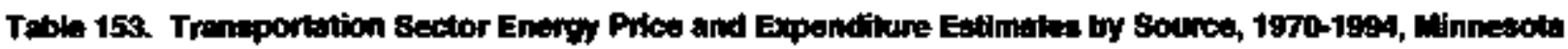

\begin{tabular}{|c|c|c|c|c|c|c|c|c|c|c|c|c|c|}
\hline \multirow[b]{4}{*}{ Yar } & \multicolumn{11}{|c|}{ Prom ENory } & \multirow[b]{3}{*}{ 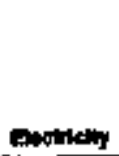 } & \multirow[b]{3}{*}{ 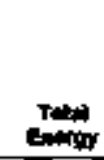 } \\
\hline & & \multirow[b]{2}{*}{ 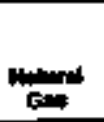 } & \multicolumn{8}{|c|}{ Purater } & \multirow[b]{2}{*}{ Totem } & & \\
\hline & Con & & ה: & 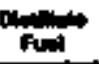 & m & بs. & 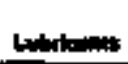 & 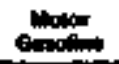 & 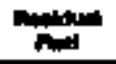 & rotat & & & \\
\hline & \multicolumn{13}{|c|}{ 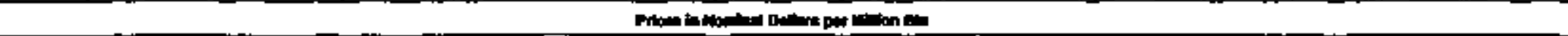 } \\
\hline \multirow[t]{2}{*}{ 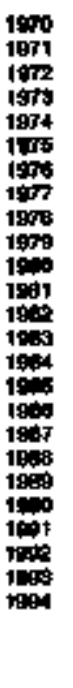 } & $\begin{array}{l}0.47 \\
0.71 \\
0.51 \\
0.45 \\
0.78 \\
0.7 \\
2.18 \\
1.37 \\
= \\
= \\
= \\
= \\
= \\
= \\
= \\
= \\
= \\
=\end{array}$ & $\begin{array}{l}= \\
= \\
= \\
= \\
= \\
= \\
= \\
= \\
= \\
= \\
= \\
= \\
0.00\end{array}$ & 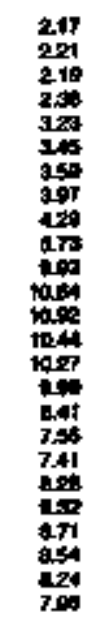 & 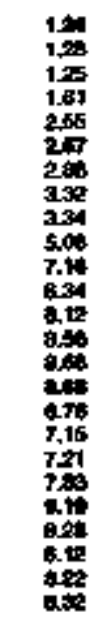 & 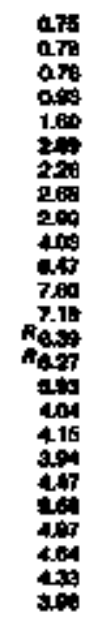 & 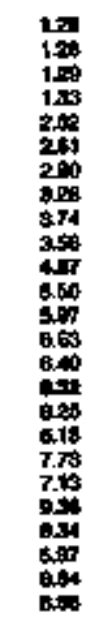 & 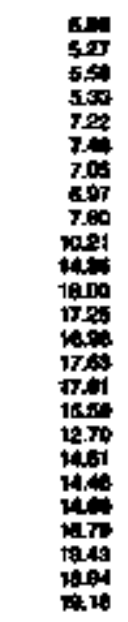 & 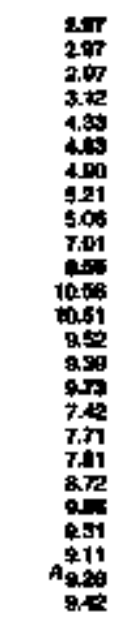 & 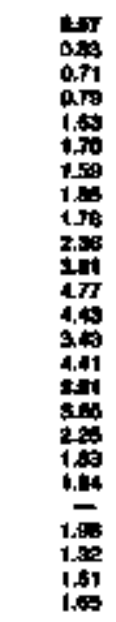 & 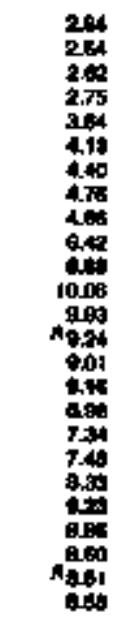 & 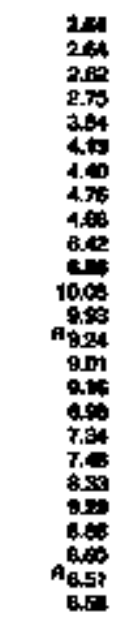 & $\begin{array}{l}= \\
= \\
= \\
= \\
= \\
= \\
= \\
= \\
= \\
= \\
= \\
= \\
= \\
=\end{array}$ & 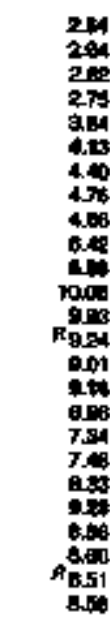 \\
\hline & \multicolumn{13}{|c|}{ Ect } \\
\hline 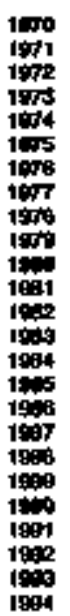 & $\begin{array}{l}0 \\
0 \\
0 \\
0 \\
0 \\
= \\
= \\
= \\
= \\
= \\
= \\
= \\
= \\
= \\
= \\
=\end{array}$ & $\begin{array}{l}= \\
= \\
= \\
= \\
= \\
= \\
= \\
= \\
= \\
= \\
= \\
= \\
= \\
= \\
=\end{array}$ & 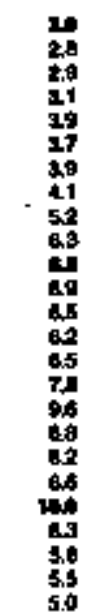 & 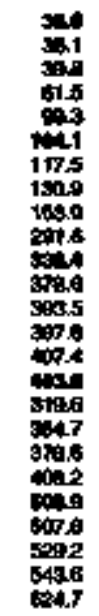 & 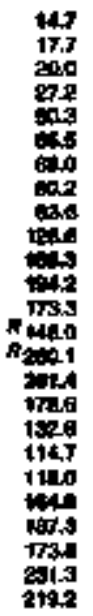 & 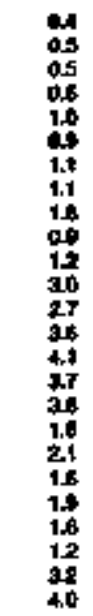 & 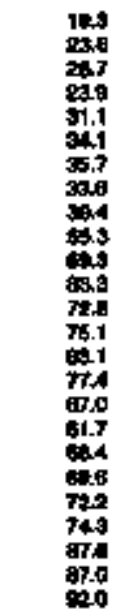 & 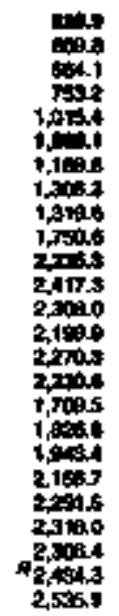 & 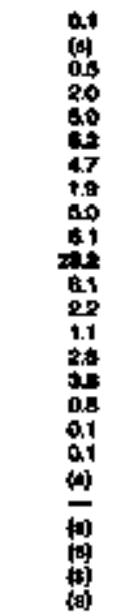 & 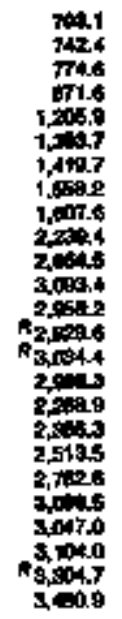 & 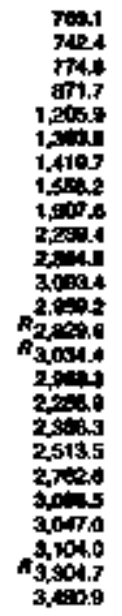 & $\begin{array}{l}= \\
= \\
= \\
= \\
= \\
= \\
= \\
= \\
= \\
= \\
= \\
= \\
= \\
=\end{array}$ & 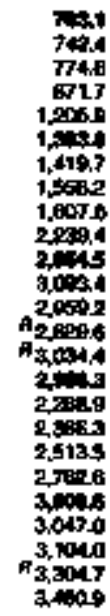 \\
\hline
\end{tabular}

- Lapaned potrobum gasal

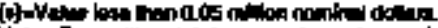

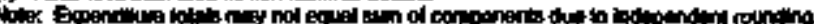

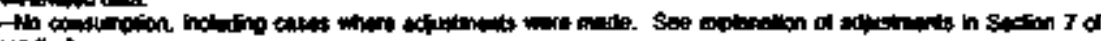

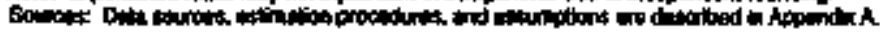

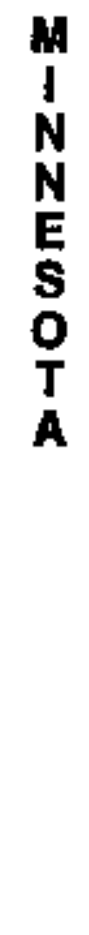




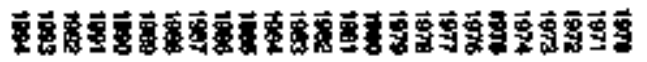

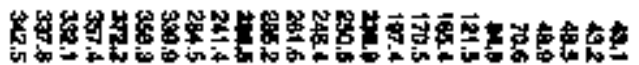

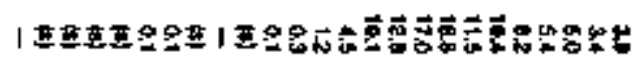

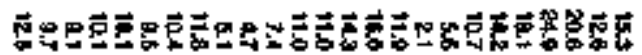

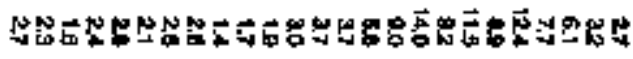

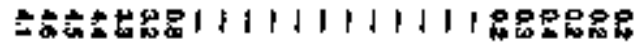

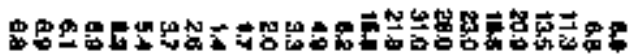

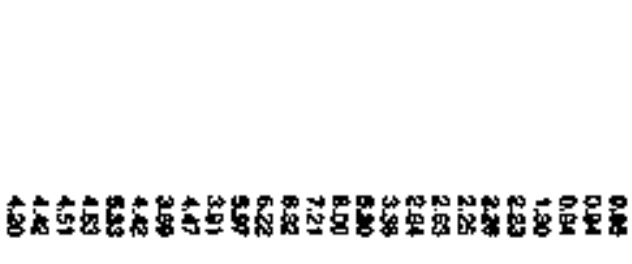

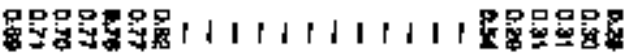

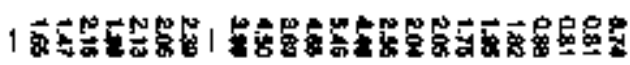

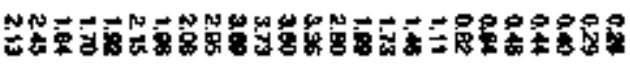

15

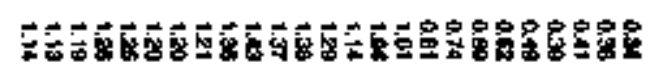

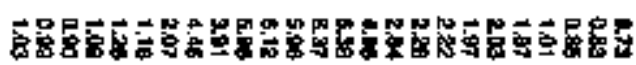

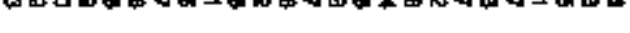

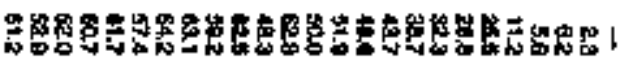

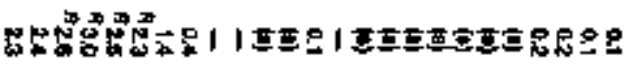

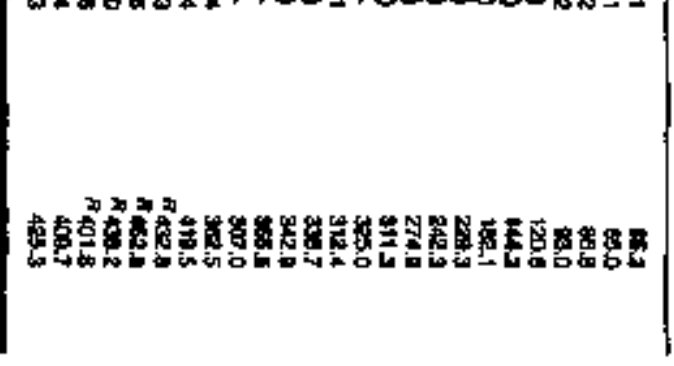

$$
\text { \% }
$$

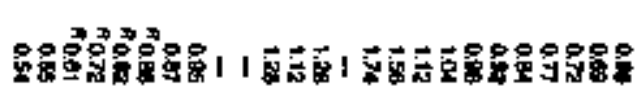

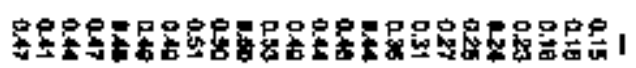
(1)

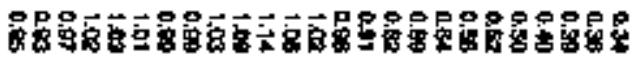


Table 155. Energy Price and Expendthure Estimateo by Source, 1970-1934, Misalasippi

\begin{tabular}{|c|c|c|c|c|c|c|c|c|c|c|c|c|c|c|c|c|c|}
\hline \multirow{3}{*}{ 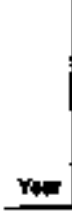 } & \multicolumn{14}{|c|}{ Primery linesp } & \multirow{3}{*}{ 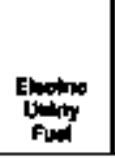 } & \multirow{3}{*}{ 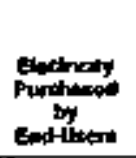 } & \multirow[b]{3}{*}{ Town: } \\
\hline & \multicolumn{3}{|c|}{ Conl } & \multirow[b]{2}{*}{ Mand } & \multicolumn{7}{|c|}{ Perovanem } & \multirow[b]{2}{*}{ Fucter } & \multirow[b]{2}{*}{ 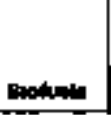 } & \multirow[b]{2}{*}{ Toded } & & & \\
\hline & $\begin{array}{c}\text { coltomp } \\
\text { cosel }\end{array}$ & . & Towat & & 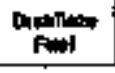 & 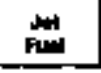 & $100^{*}$ & Hots & Pancell & ovens" & Tow & & & & & & \\
\hline 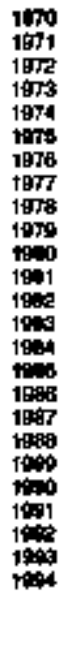 & $\begin{array}{l}z \\
= \\
= \\
= \\
= \\
z \\
z \\
= \\
= \\
= \\
= \\
= \\
z \\
z\end{array}$ & 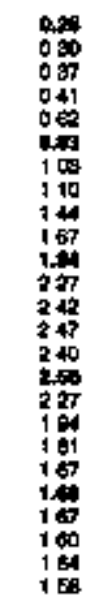 & 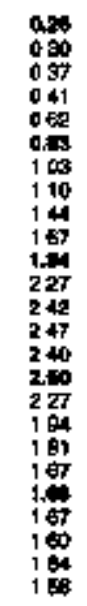 & 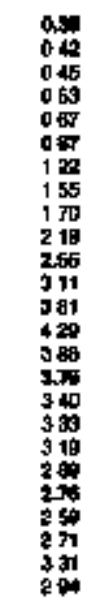 & 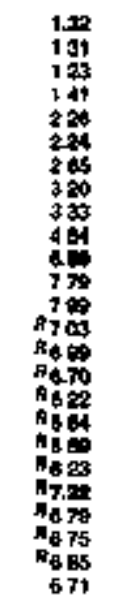 & 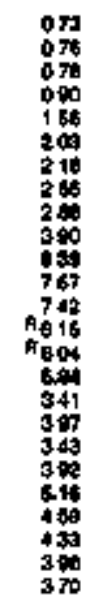 & 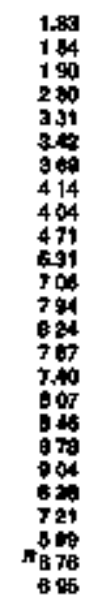 & 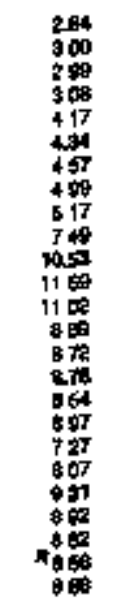 & 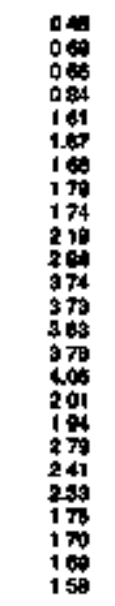 & 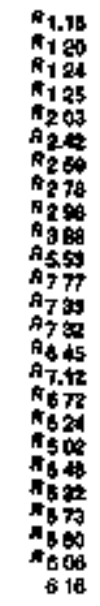 & 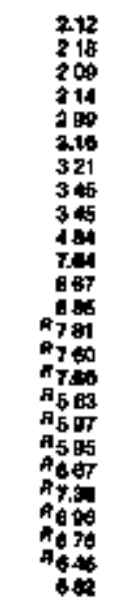 & 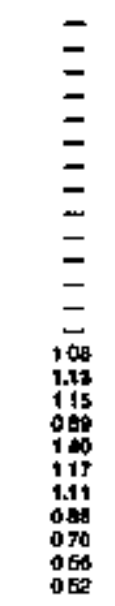 & $\begin{array}{l}\bar{z} \\
\bar{z} \\
\bar{z} \\
\bar{z} \\
\bar{z} \\
\bar{z} \\
\bar{z} \\
\bar{z} \\
\bar{z} \\
100 \\
100 \\
100 \\
102 \\
102\end{array}$ & 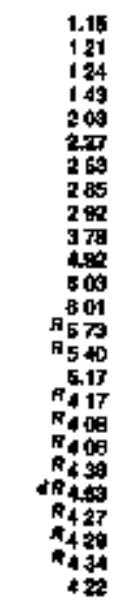 & 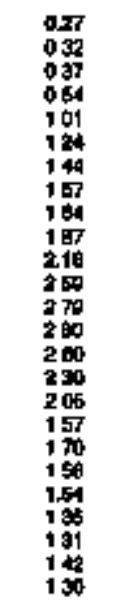 & 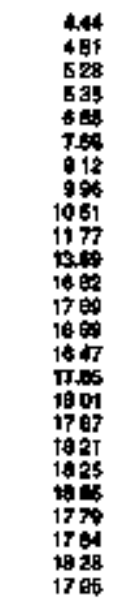 & 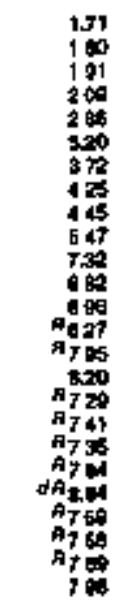 \\
\hline 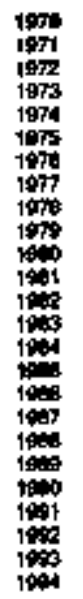 & $\begin{array}{l}= \\
= \\
= \\
= \\
= \\
= \\
= \\
= \\
= \\
= \\
= \\
= \\
=\end{array}$ & 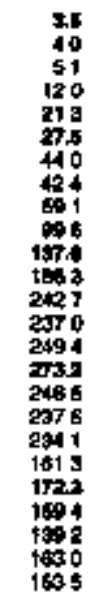 & 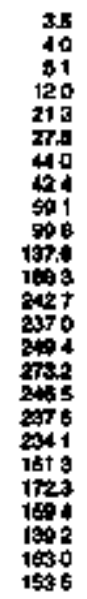 & 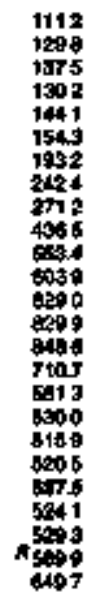 & 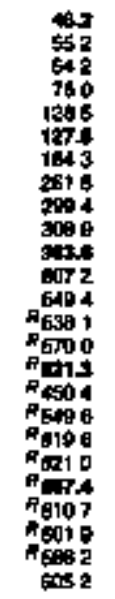 & 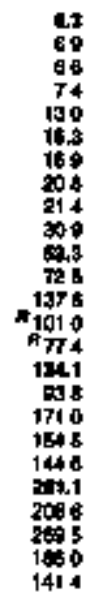 & 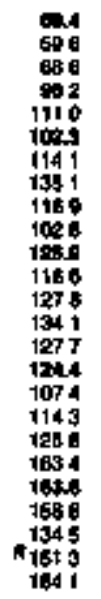 & 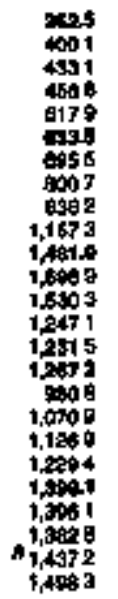 & 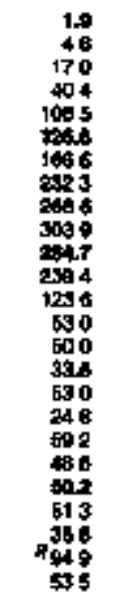 & 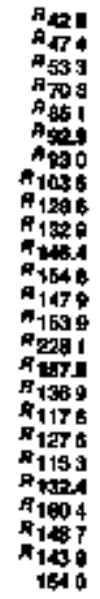 & 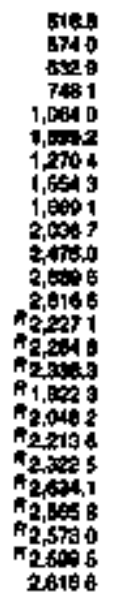 & 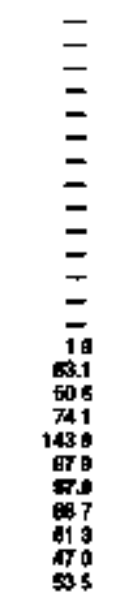 & $\begin{array}{l}= \\
= \\
= \\
= \\
= \\
= \\
= \\
= \\
= \\
= \\
= \\
497 \\
476 \\
465 \\
459\end{array}$ & 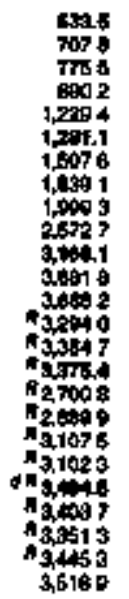 & 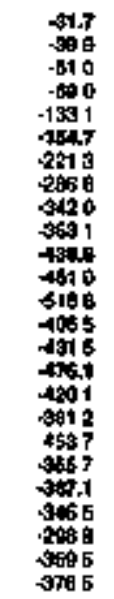 & 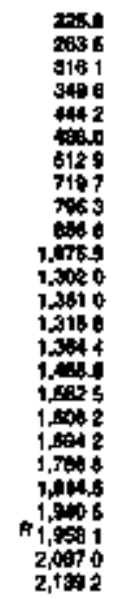 & 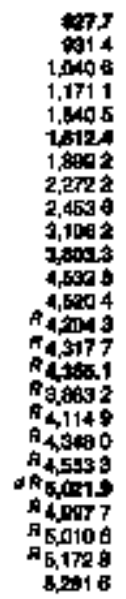 \\
\hline
\end{tabular}

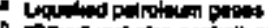

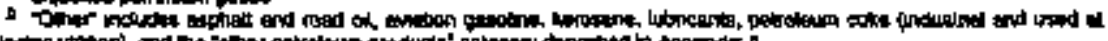

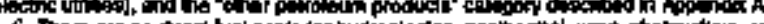

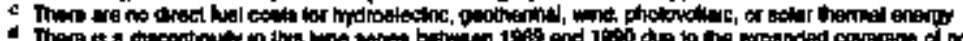

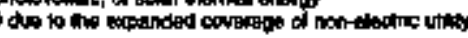

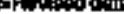

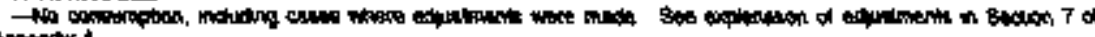
Appondix $\lambda$

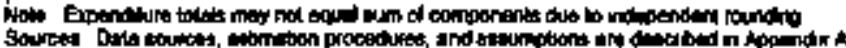




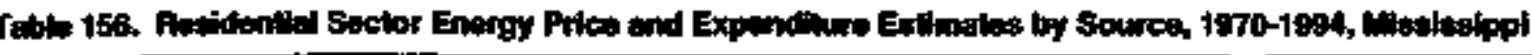

\begin{tabular}{|c|c|c|c|c|c|c|c|c|c|c|}
\hline & \multicolumn{8}{|c|}{$M$} & \multirow[b]{3}{*}{ Eicinioln } & \multirow[b]{3}{*}{ 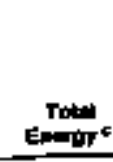 } \\
\hline & & & \multicolumn{4}{|c|}{ 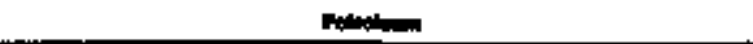 } & \multirow{2}{*}{. } & \multirow[b]{2}{*}{ Totent } & & \\
\hline & Den & $\theta=1$ & forl & (1) & Lan" & Tot & & & & \\
\hline $\operatorname{rim}$ & \multicolumn{10}{|c|}{ 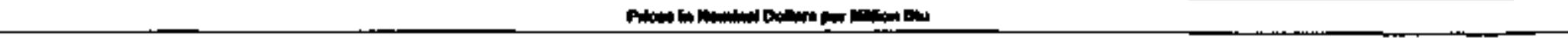 } \\
\hline 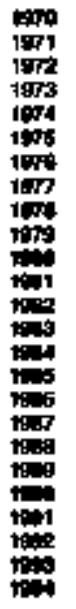 & 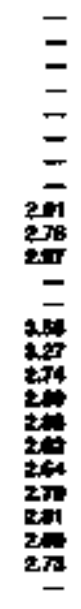 & 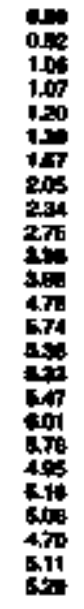 & 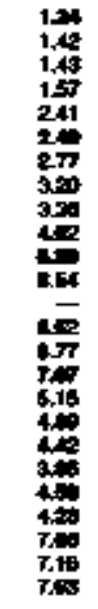 & 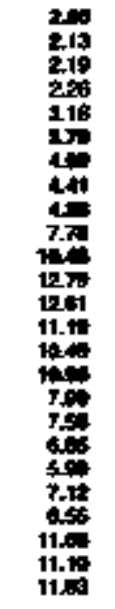 & 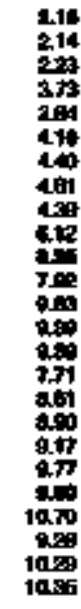 & 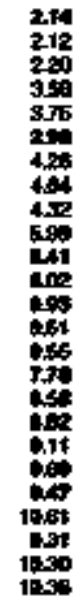 & $\begin{array}{l}= \\
= \\
= \\
= \\
= \\
= \\
= \\
= \\
= \\
= \\
= \\
3= \\
353 \\
353 \\
353\end{array}$ & 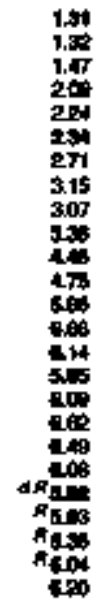 & 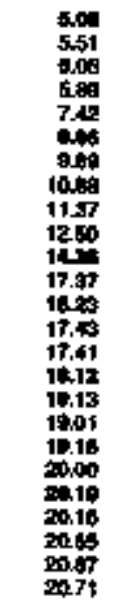 & 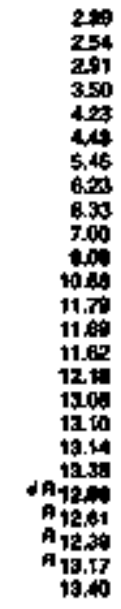 \\
\hline \multicolumn{11}{|c|}{ 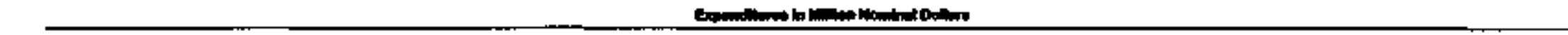 } \\
\hline 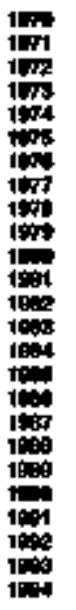 & $\begin{array}{l}= \\
= \\
= \\
= \\
\bar{z} \\
01 \\
01 \\
0 . \\
\overline{0} \\
0.1 \\
09 \\
01 \\
01 \\
03 \\
0 \\
0 \\
0 \\
0\end{array}$ & 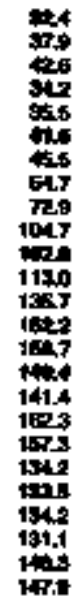 & 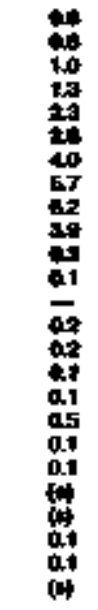 & 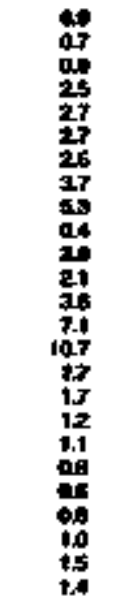 & 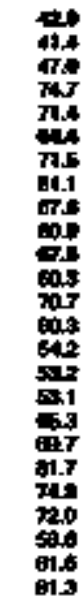 & 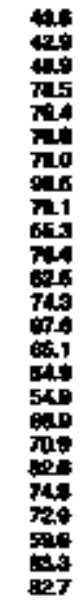 & $\begin{array}{l}= \\
= \\
= \\
= \\
= \\
= \\
= \\
= \\
= \\
= \\
\bar{z} \\
0126 \\
103 \\
105 \\
103\end{array}$ & 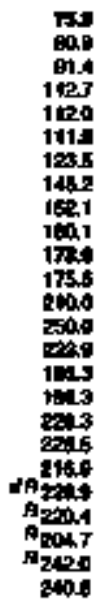 & 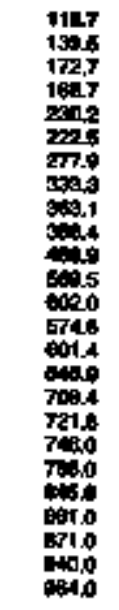 & 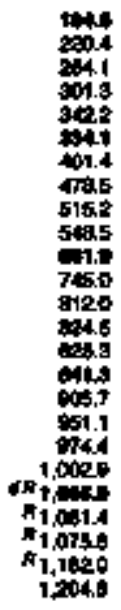 \\
\hline
\end{tabular}

1)

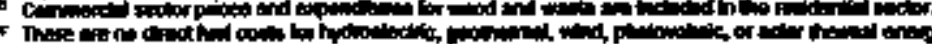

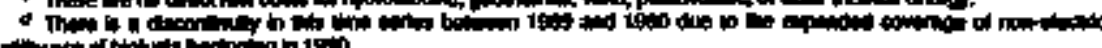

An:

(1)

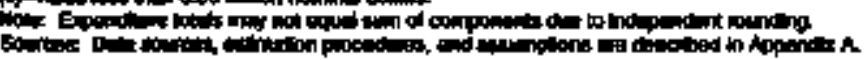

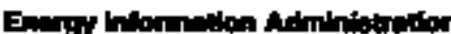




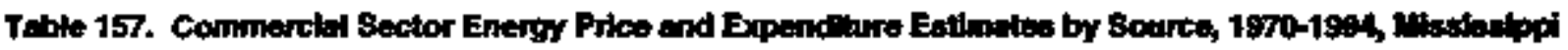

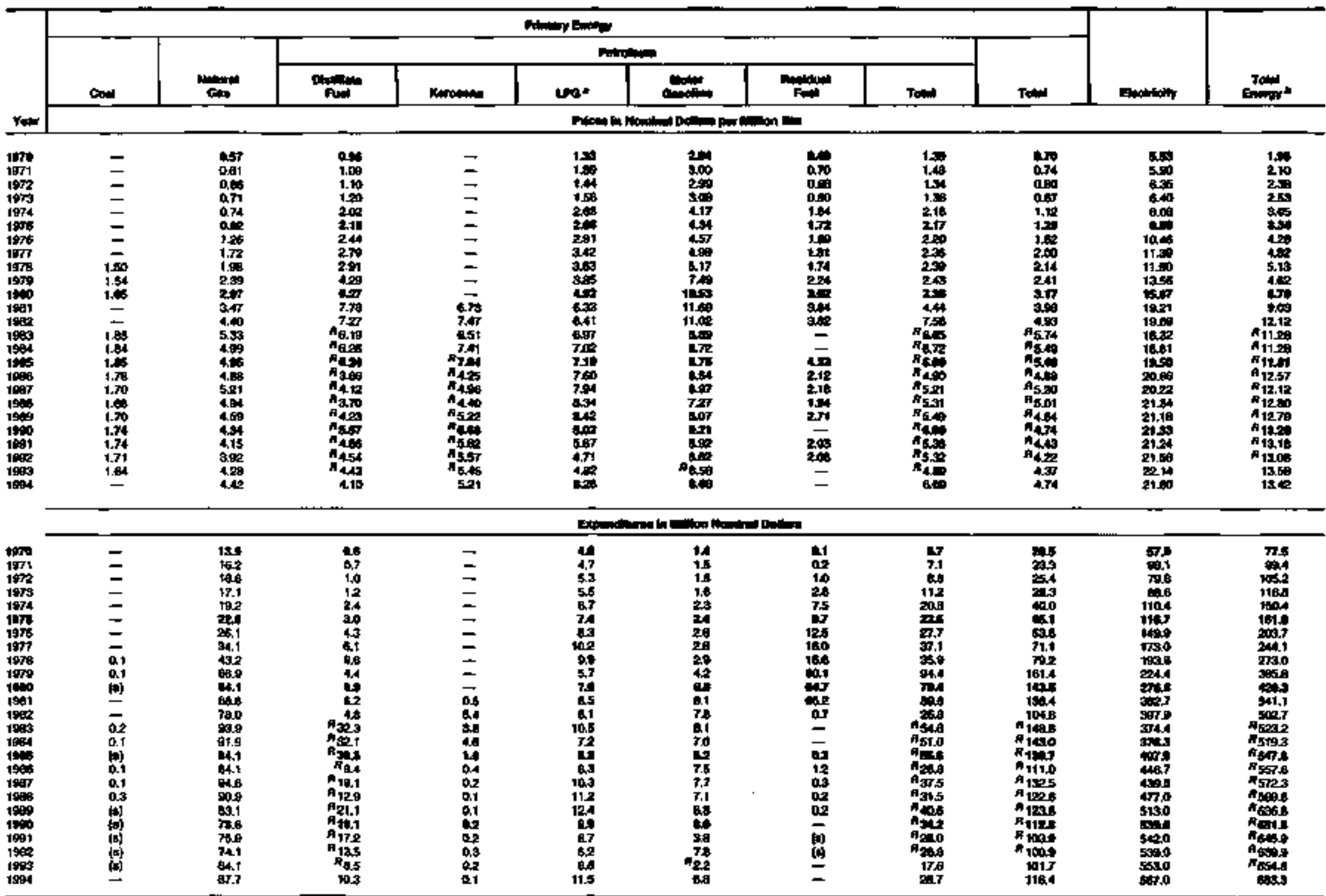

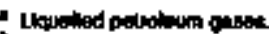

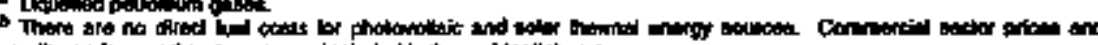

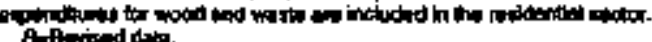

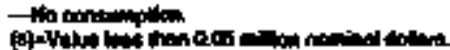

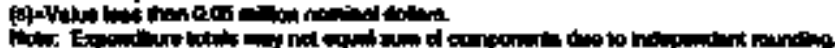

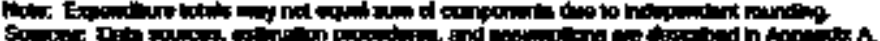




\begin{tabular}{|c|c|c|c|c|c|c|c|c|c|c|c|c|c|c|c|c|c|}
\hline & \multicolumn{15}{|c|}{ Anes Enmy } & \multirow[b]{3}{*}{ endiletis } & \multirow[b]{3}{*}{ Told, } \\
\hline & \multicolumn{3}{|c|}{ cond } & \multirow[b]{2}{*}{ لה: } & \multicolumn{9}{|c|}{ Putrolum } & \multirow[b]{2}{*}{ Bnolome } & \multirow[b]{2}{*}{ Town } & & \\
\hline & $\begin{array}{c}\text { coltind } \\
\text { coll }\end{array}$ & con & Tow & & Mand & Dinto & 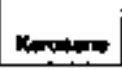 & LPQ* & Lutionsts & motion & Fuidid & $\operatorname{Antan}{ }^{\circ}$ & Totw & & & & \\
\hline Yaer & \multicolumn{17}{|c|}{ 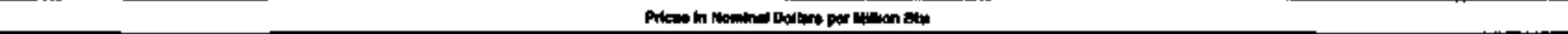 } \\
\hline \multirow[t]{2}{*}{ 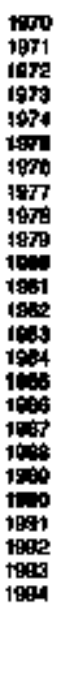 } & $\begin{array}{l}= \\
= \\
= \\
= \\
= \\
= \\
= \\
= \\
= \\
= \\
= \\
= \\
=\end{array}$ & 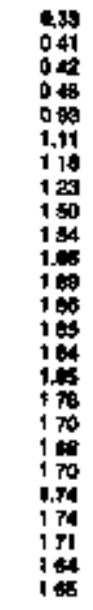 & 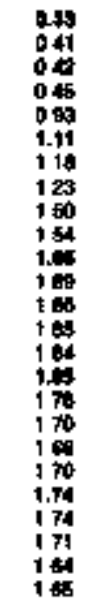 & 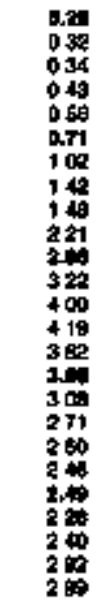 & 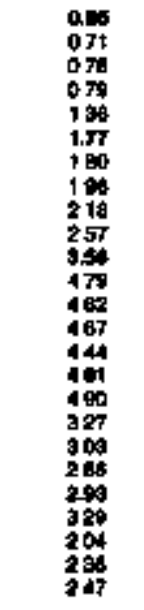 & 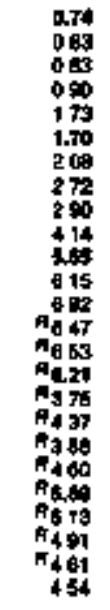 & 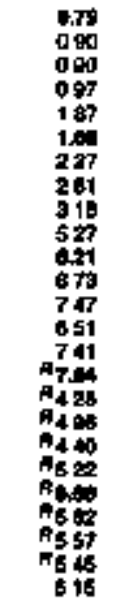 & 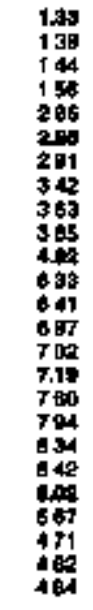 & 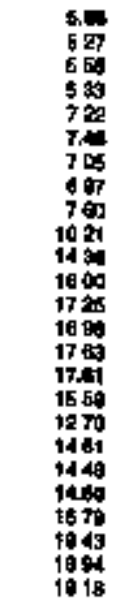 & 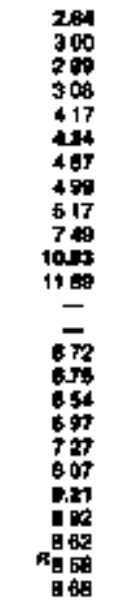 & 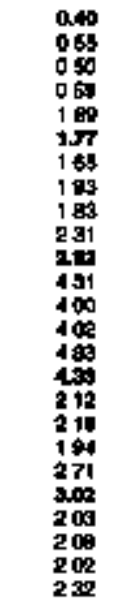 & 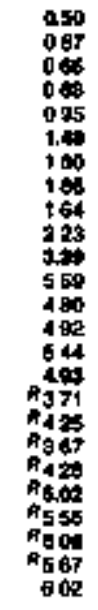 & 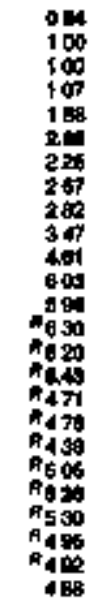 & $\begin{array}{l}= \\
= \\
= \\
= \\
= \\
= \\
= \\
= \\
= \\
= \\
= \\
= \\
= \\
\bar{z} \\
0,21 \\
132 \\
131 \\
130 \\
130\end{array}$ & 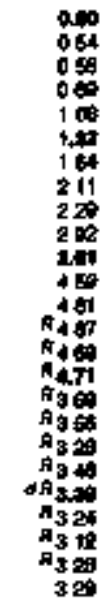 & 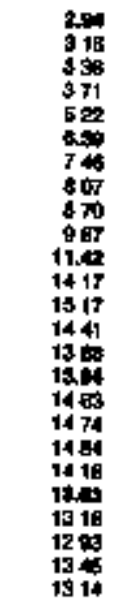 & 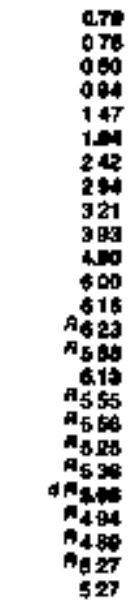 \\
\hline & \multicolumn{17}{|c|}{ 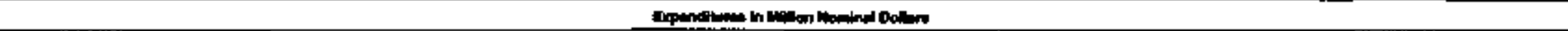 } \\
\hline 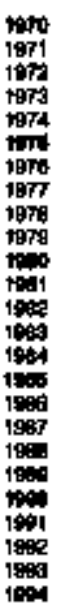 & $\begin{array}{l}= \\
= \\
= \\
= \\
= \\
= \\
= \\
= \\
= \\
= \\
= \\
= \\
= \\
=\end{array}$ & 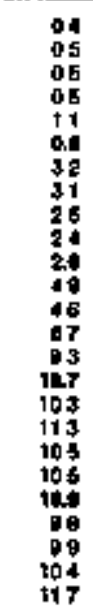 & $\begin{array}{c}01 \\
05 \\
05 \\
06 \\
11 \\
04 \\
32 \\
31 \\
86 \\
84 \\
24 \\
10 \\
46 \\
67 \\
93 \\
117 \\
103 \\
113 \\
105 \\
108 \\
108 \\
188 \\
89 \\
104 \\
117\end{array}$ & 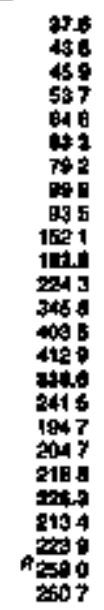 & 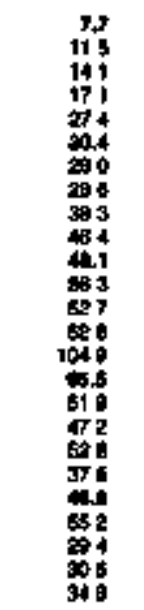 & 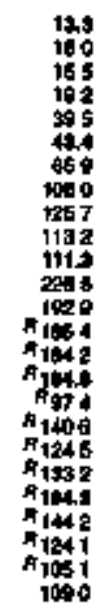 & 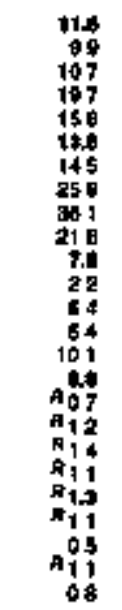 & 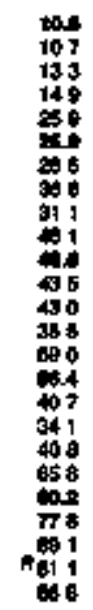 & 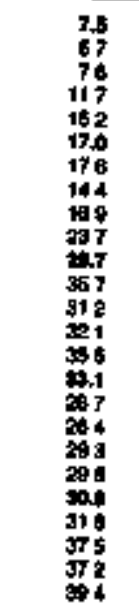 & 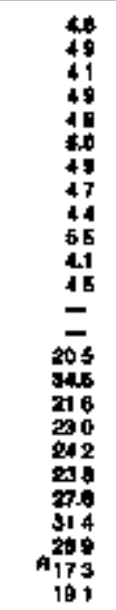 & 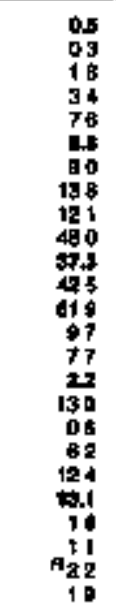 & 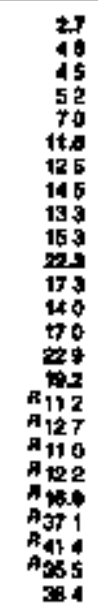 & 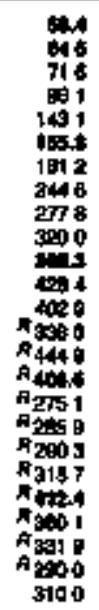 & $\begin{array}{l}= \\
= \\
= \\
= \\
= \\
= \\
= \\
= \\
= \\
= \\
= \\
= \\
= \\
401 \\
343 \\
345 \\
355 \\
356\end{array}$ & 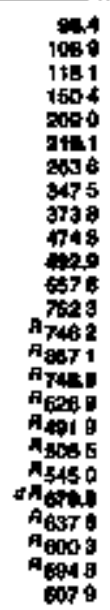 & 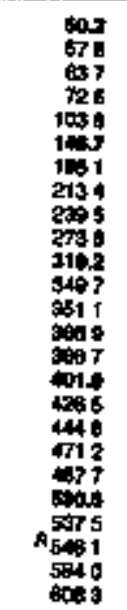 & 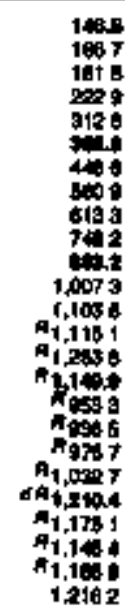 \\
\hline
\end{tabular}

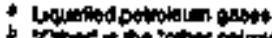

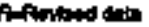

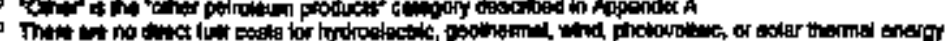

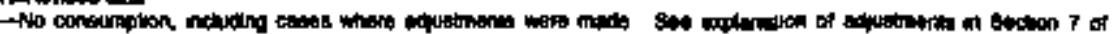

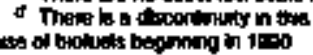

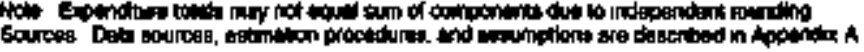


Teble 159. Transportation Sector Energy Price end Expenditure Estlmates by Source, 1970-1994, Mlealsalppi

\begin{tabular}{|c|c|c|c|c|c|c|c|c|c|c|c|c|c|}
\hline \multirow[b]{4}{*}{$\operatorname{Ver}$} & \multicolumn{11}{|c|}{ 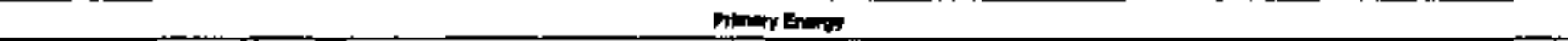 } & \multirow[b]{3}{*}{ elactolkely } & \multirow[b]{3}{*}{ Tot: } \\
\hline & \multirow[b]{2}{*}{ conel } & \multirow[b]{2}{*}{ rention } & \multicolumn{8}{|c|}{ 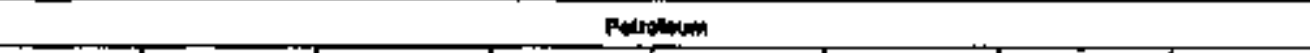 } & \multirow[b]{2}{*}{ Tow } & & \\
\hline & & & Antion & 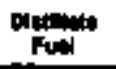 & chan & LFe* & سل & Motor & $\begin{array}{l}\text { Remiduat } \\
\text { Funf }\end{array}$ & Total & & & \\
\hline & \multicolumn{13}{|c|}{ 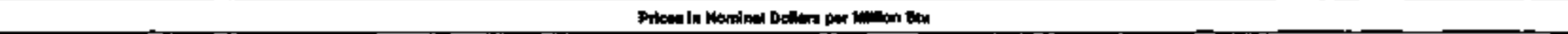 } \\
\hline \multirow[t]{2}{*}{ 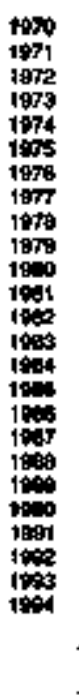 } & $\begin{array}{l}00 \\
0411 \\
040 \\
045 \\
090 \\
091 \\
119 \\
100 \\
= \\
= \\
= \\
= \\
= \\
= \\
= \\
= \\
= \\
=\end{array}$ & $\begin{array}{l}= \\
= \\
z \\
z \\
= \\
= \\
= \\
= \\
= \\
= \\
= \\
= \\
2 \overline{73}\end{array}$ & 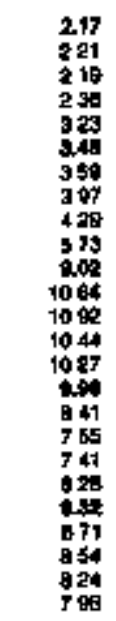 & 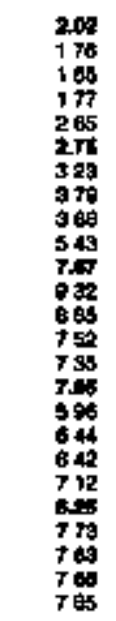 & 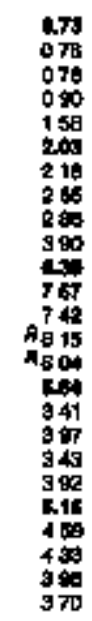 & 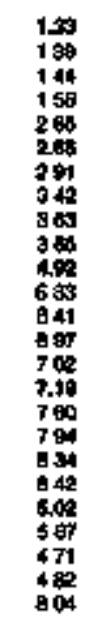 & 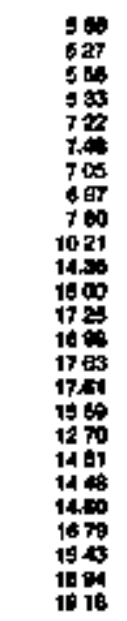 & 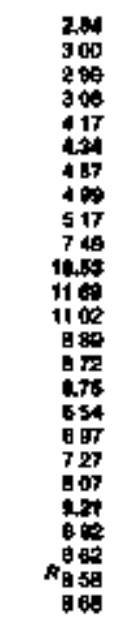 & 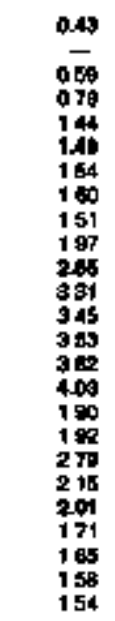 & 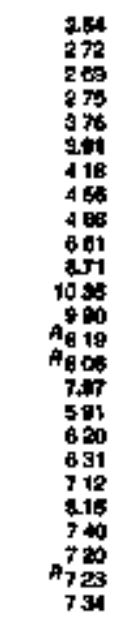 & 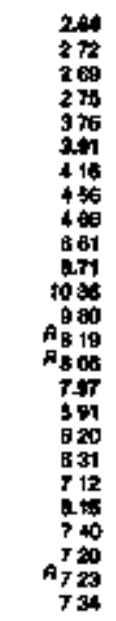 & $\begin{array}{l}= \\
= \\
= \\
= \\
= \\
= \\
= \\
= \\
= \\
= \\
= \\
= \\
= \\
= \\
=\end{array}$ & 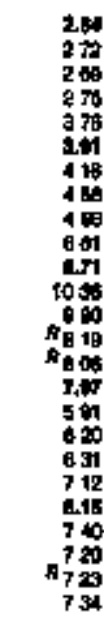 \\
\hline & \multicolumn{13}{|c|}{ 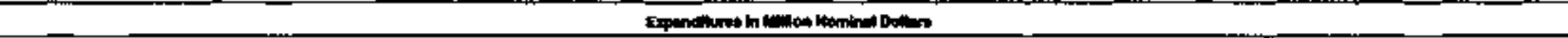 } \\
\hline 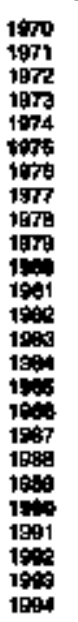 & $\begin{array}{l}00 \\
000 \\
= \\
= \\
= \\
= \\
= \\
= \\
= \\
= \\
= \\
= \\
= \\
=\end{array}$ & $\begin{array}{l}= \\
= \\
= \\
= \\
= \\
= \\
= \\
= \\
= \\
= \\
= \\
= \\
= \\
= \\
\bar{n}\end{array}$ & $\begin{array}{l}35 \\
37 \\
37 \\
41 \\
39 \\
95 \\
31 \\
32 \\
32 \\
39 \\
04 \\
76 \\
68 \\
60 \\
93 \\
54 \\
58 \\
43 \\
46 \\
64 \\
64 \\
46 \\
41 \\
35 \\
29\end{array}$ & 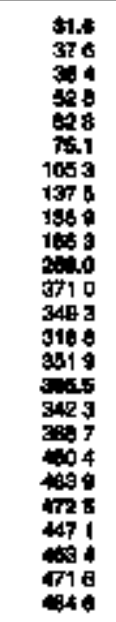 & 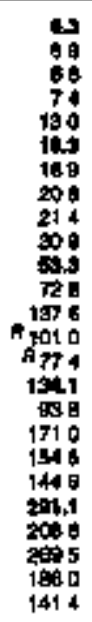 & $\begin{array}{l}24 \\
24 \\
30 \\
31 \\
50 \\
46 \\
58 \\
72 \\
83 \\
01 \\
27 \\
42 \\
40 \\
77 \\
73 \\
40 \\
53 \\
45 \\
11 \\
35 \\
24 \\
212 \\
18 \\
18 \\
48\end{array}$ & 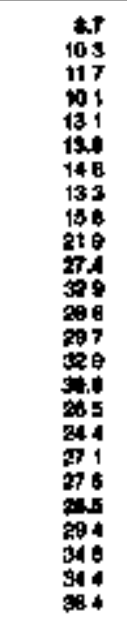 & 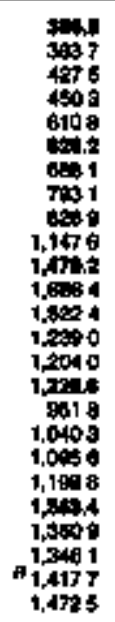 & 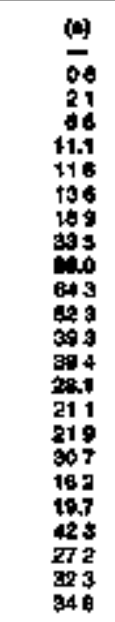 & 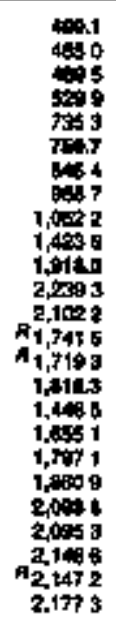 & 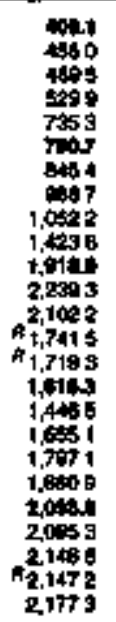 & $\begin{array}{l}= \\
= \\
= \\
= \\
= \\
= \\
= \\
= \\
= \\
= \\
= \\
= \\
= \\
=\end{array}$ & 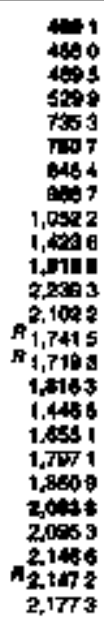 \\
\hline
\end{tabular}


Table 160. Price and Expendliure Extmates for Energy Inpul at Electric Utilities by Soumbe, 1970-1994, Misslosippi

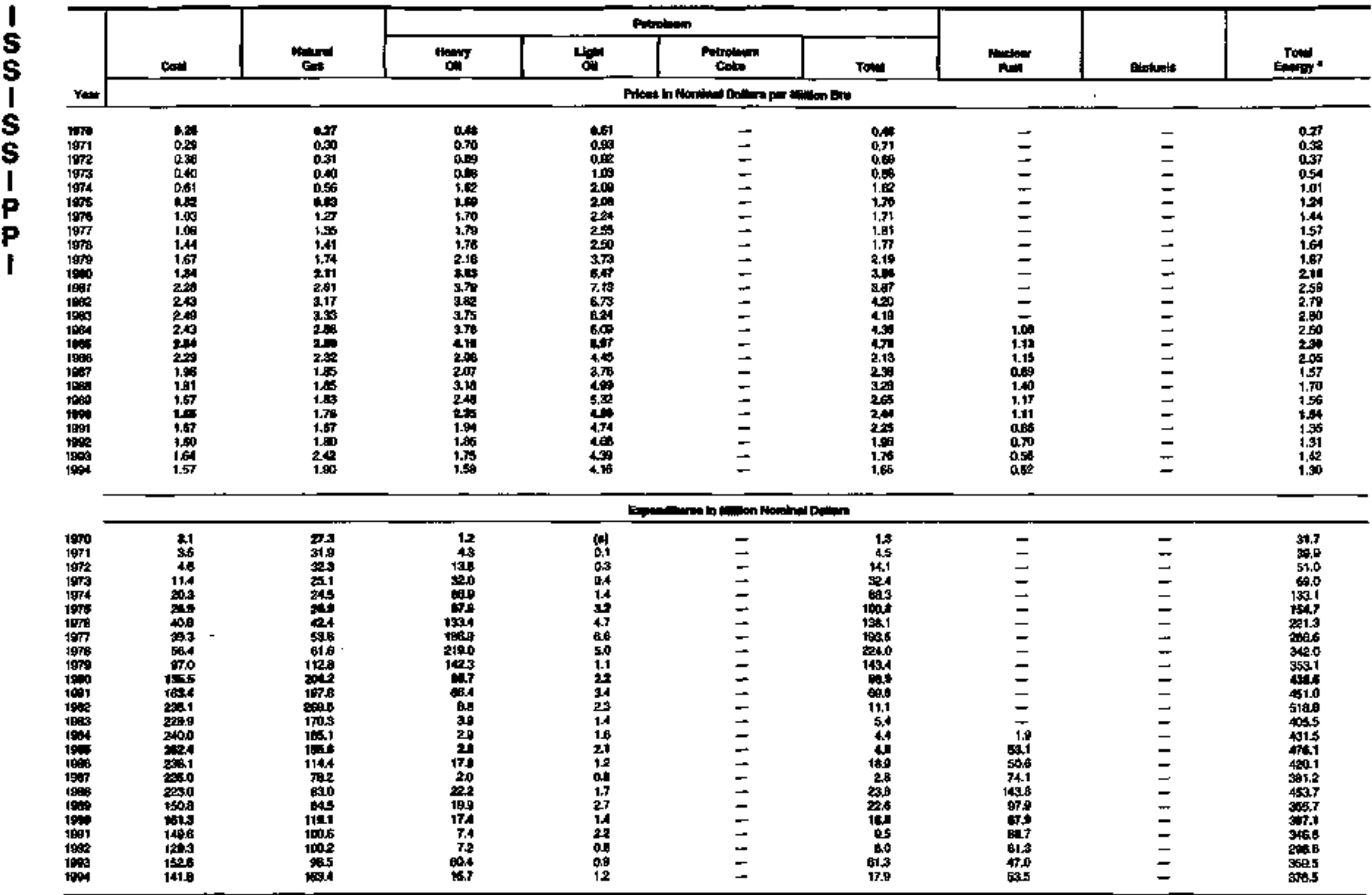

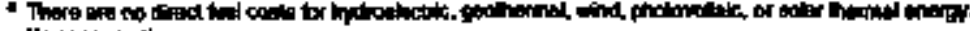

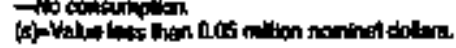

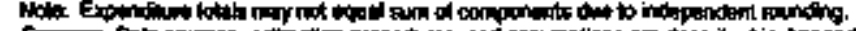

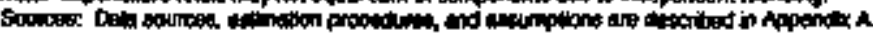


Table 161. Energy Plice and Expendlube Estimetes by source, 1970-1594, Mlseouri

\begin{tabular}{|c|c|c|c|c|c|c|c|c|c|c|c|c|c|c|c|c|c|}
\hline \multirow[b]{4}{*}{$\mathrm{Ven}$} & \multicolumn{14}{|c|}{ Fint } & \multirow{3}{*}{ 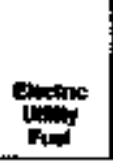 } & \multirow{3}{*}{ 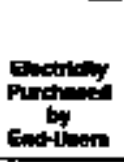 } & \multirow[b]{3}{*}{ Enereste } \\
\hline & \multicolumn{3}{|c|}{ Con } & \multirow[b]{2}{*}{ minrd } & \multicolumn{7}{|c|}{ 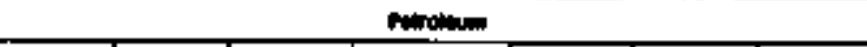 } & \multirow[b]{2}{*}{ fuct } & \multirow[b]{2}{*}{ Banat: } & \multirow[b]{2}{*}{ Totele } & & & \\
\hline & Colven & cons & Toltitn & & Full & 幽 & LPR' & 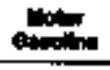 & 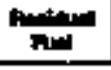 & other & Toted & & & & & & \\
\hline & \multicolumn{17}{|c|}{ 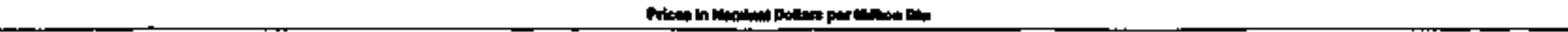 } \\
\hline \multirow[t]{2}{*}{ 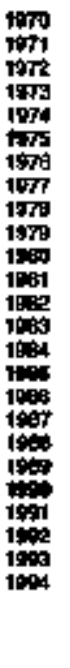 } & 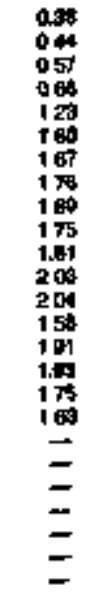 & 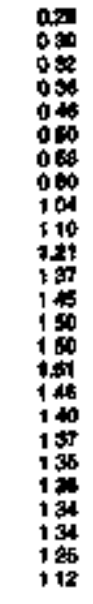 & 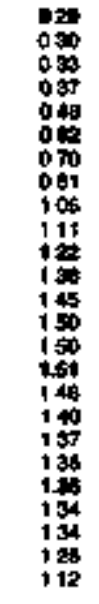 & 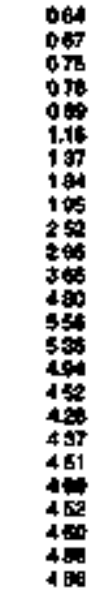 & 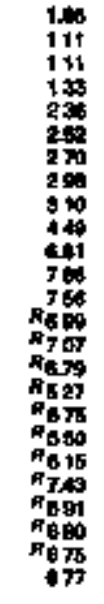 & 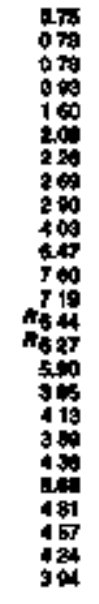 & 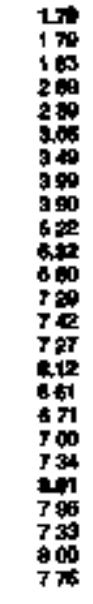 & 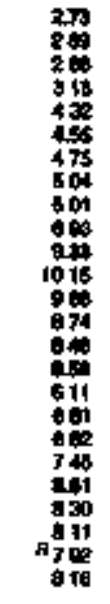 & 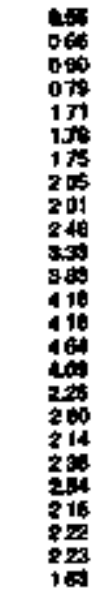 & 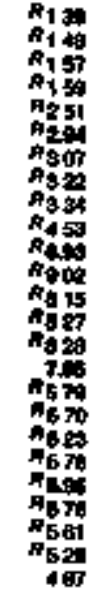 & 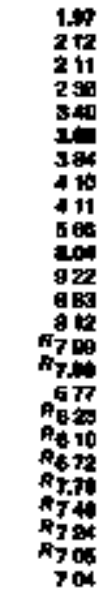 & 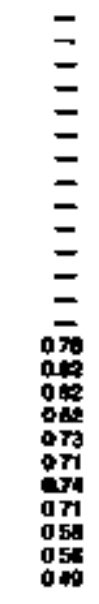 & $\begin{array}{l}= \\
= \\
= \\
= \\
= \\
= \\
= \\
= \\
= \\
= \\
= \\
\bar{z} \\
20 \\
304 \\
300 \\
200\end{array}$ & 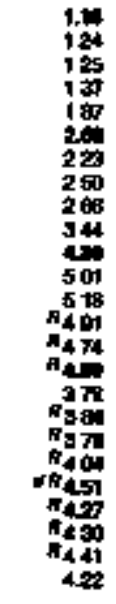 & 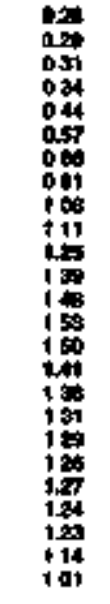 & 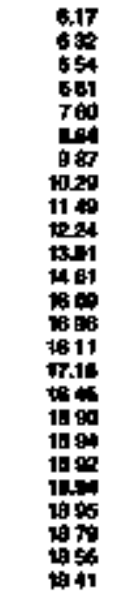 & 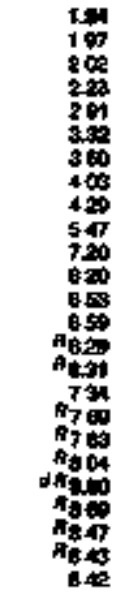 \\
\hline & \multicolumn{17}{|c|}{ 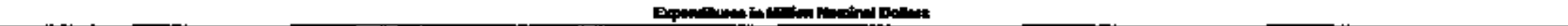 } \\
\hline 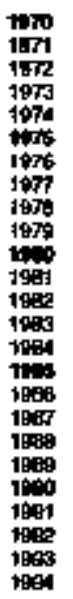 & $\begin{array}{c}31 \\
37 \\
49 \\
60 \\
161 \\
519 \\
131 \\
151 \\
102 \\
130 \\
68 \\
100 \\
81 \\
64 \\
54 \\
128 \\
108 \\
34 \\
= \\
= \\
= \\
= \\
= \\
=\end{array}$ & 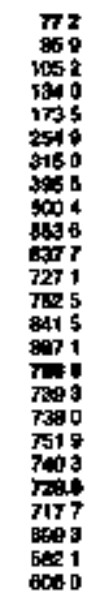 & 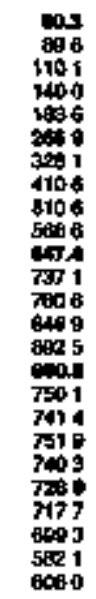 & 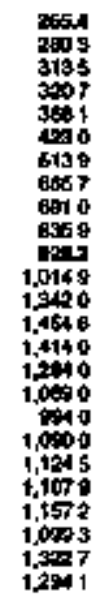 & 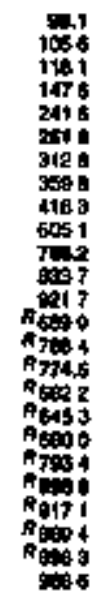 & 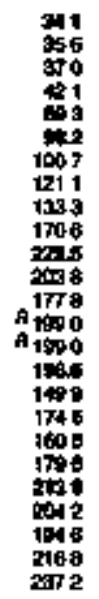 & 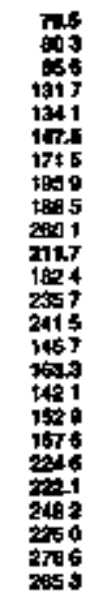 & 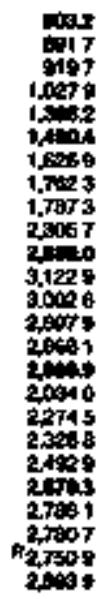 & 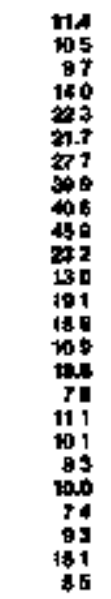 & 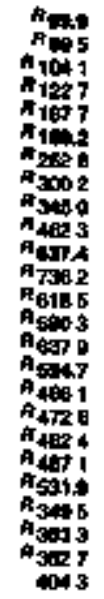 & 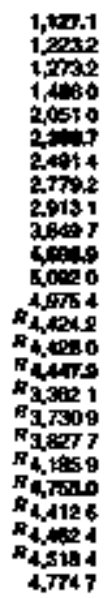 & $\begin{array}{l}= \\
= \\
= \\
= \\
= \\
= \\
= \\
= \\
78 \\
715 \\
601 \\
310 \\
703 \\
01 \\
06 \\
707 \\
501 \\
524\end{array}$ & $\begin{array}{l}= \\
= \\
= \\
= \\
= \\
= \\
= \\
= \\
= \\
= \\
= \\
= \\
= \\
175 \\
108 \\
090 \\
0.0\end{array}$ & 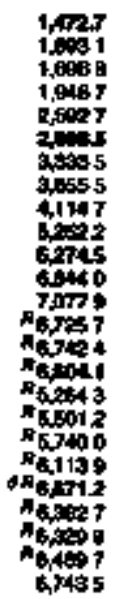 & 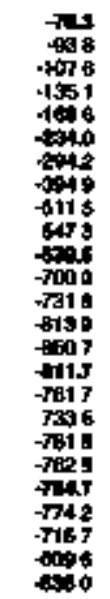 & 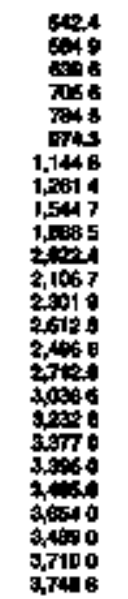 & 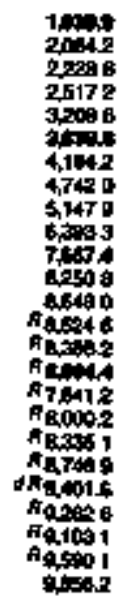 \\
\hline
\end{tabular}

Antomed dita

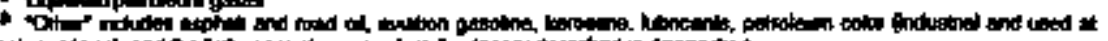

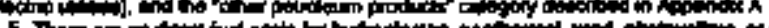

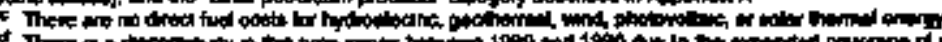

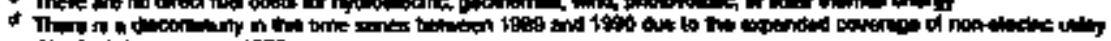

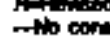

Appentix a

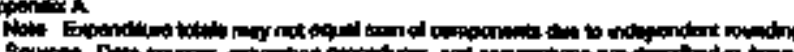

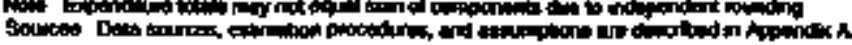

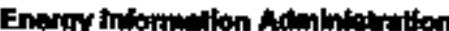


Table 162. Residentlat Sector Energy Prlce and Expendture Estimales by Soures, 1970-1994, Mls8ouri

\begin{tabular}{|c|c|c|c|c|c|c|c|c|c|c|}
\hline \multirow{3}{*}{ you } & \multicolumn{8}{|c|}{ Prorery Enengy, } & \multirow[b]{3}{*}{ enown } & \multirow[b]{3}{*}{ Tow } \\
\hline & \multirow[b]{2}{*}{$\mathrm{cos}$} & \multirow[b]{2}{*}{ mant } & \multicolumn{4}{|c|}{ Pestrolum } & \multirow[b]{2}{*}{ achmatet } & \multirow[b]{2}{*}{ Tatose } & & \\
\hline & & & ond & Kpostis) & LAG: & Tow & & & & \\
\hline 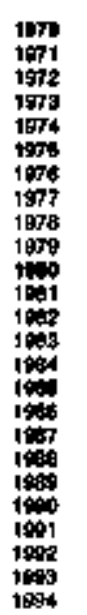 & 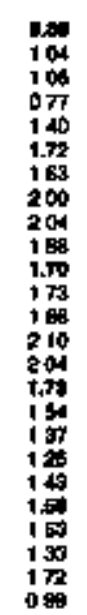 & 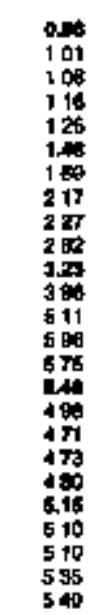 & 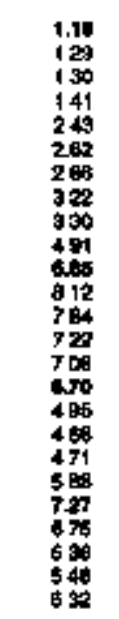 & 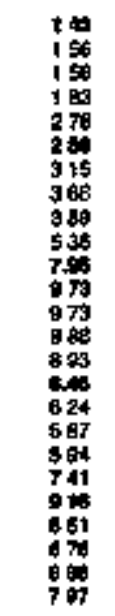 & 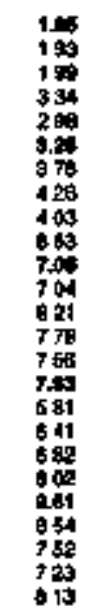 & 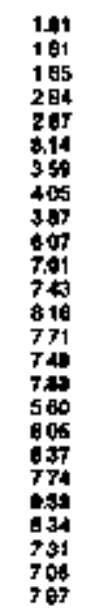 & 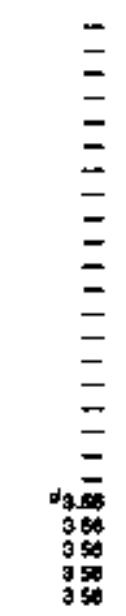 & 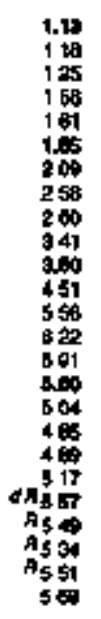 & 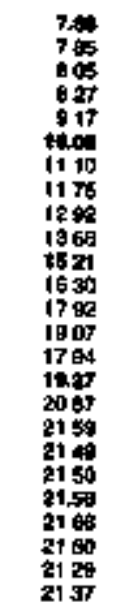 & 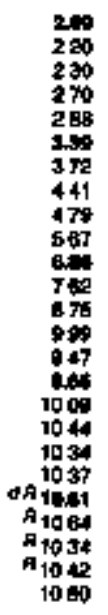 \\
\hline
\end{tabular}

\begin{tabular}{|c|c|c|c|c|c|c|c|c|c|c|}
\hline 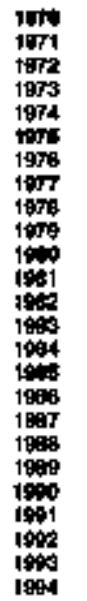 & $\begin{array}{l}44 \\
06 \\
97 \\
12 \\
23 \\
26 \\
22 \\
17 \\
98 \\
14 \\
1,1 \\
14 \\
11 \\
21 \\
20 \\
24 \\
13 \\
26 \\
21 \\
28 \\
34 \\
30 \\
23 \\
35 \\
17\end{array}$ & 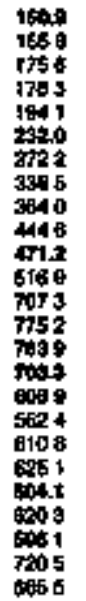 & 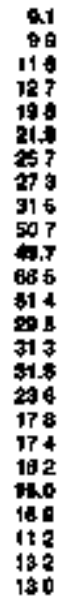 & $\begin{array}{l}04 \\
07 \\
64 \\
04 \\
06 \\
05 \\
06 \\
09 \\
69 \\
14 \\
29 \\
12 \\
68 \\
38 \\
81 \\
48 \\
20 \\
10 \\
20 \\
20 \\
19 \\
18 \\
08 \\
16 \\
11\end{array}$ & 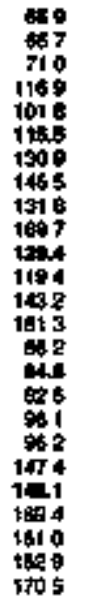 & 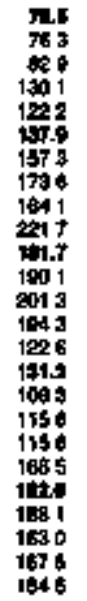 & $\begin{array}{l}z \\
\bar{z} \\
\bar{z} \\
\bar{z} \\
\bar{z} \\
\bar{z} \\
\bar{z} \\
\bar{z} \\
\bar{z} \\
\bar{z} \\
921 \\
189 \\
167 \\
199 \\
130\end{array}$ & 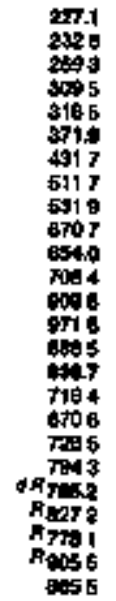 & 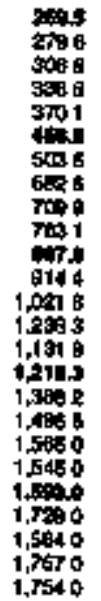 & 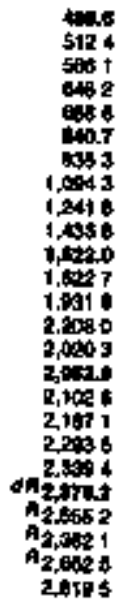 \\
\hline
\end{tabular}



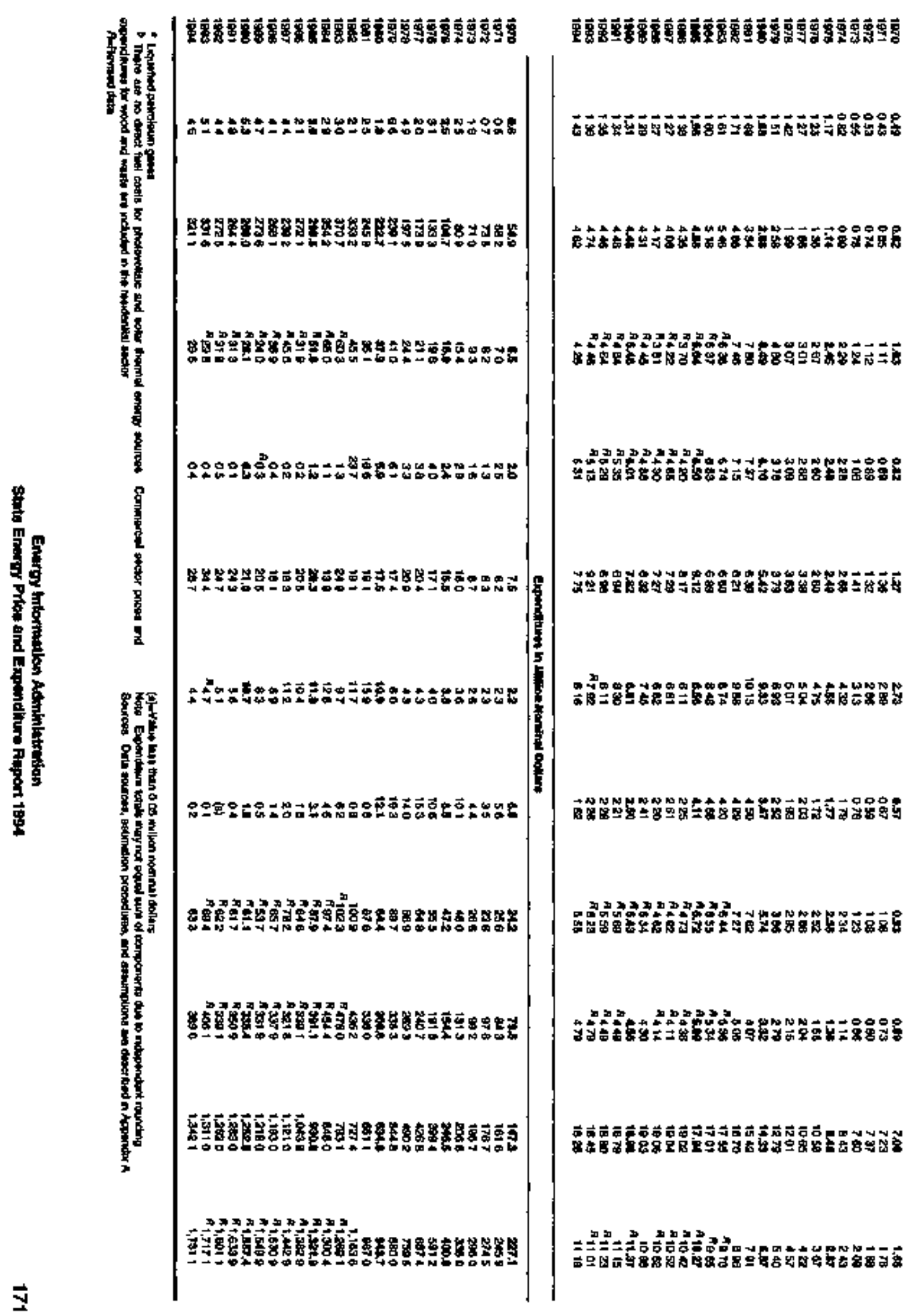

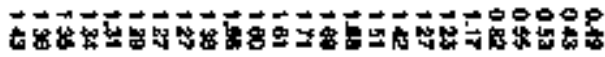

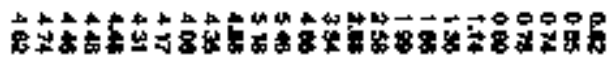

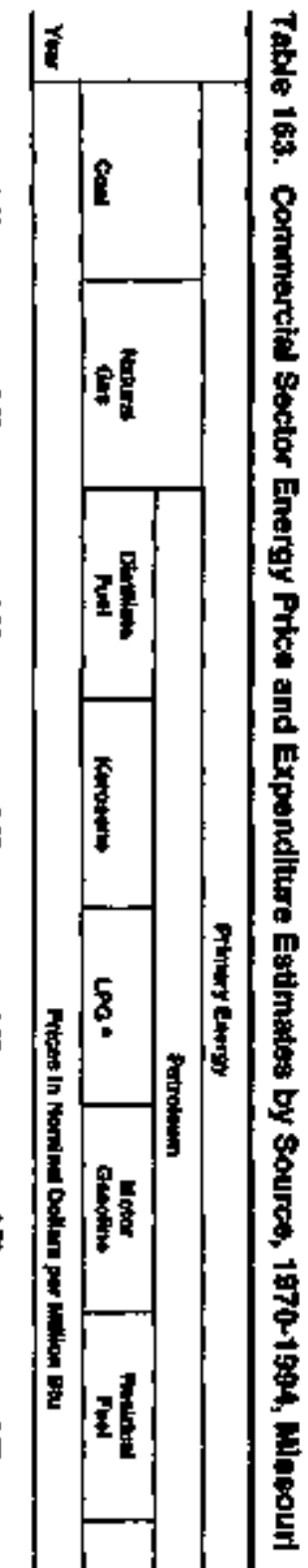

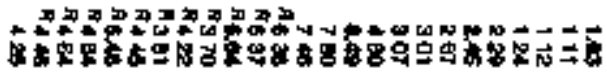

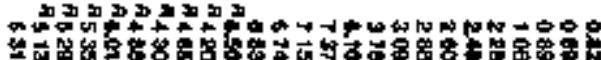

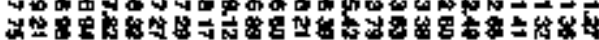

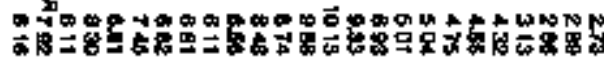

$\vec{B}$

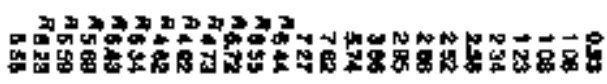

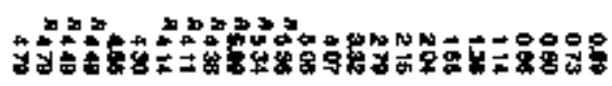

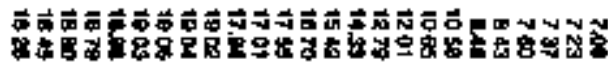

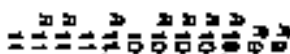

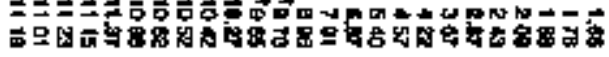

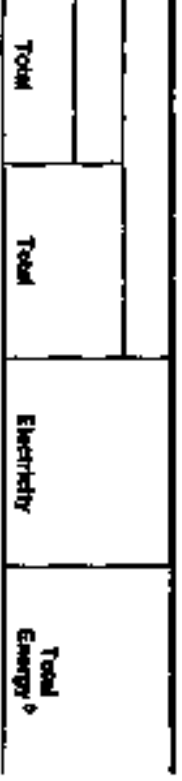




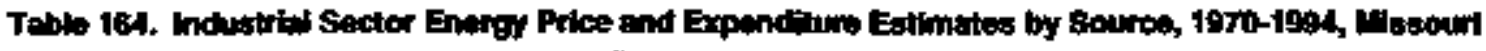

\begin{tabular}{|c|c|c|c|c|c|c|c|c|c|c|c|c|c|c|c|c|c|}
\hline \multirow[b]{4}{*}{$\mathrm{r}$} & \multicolumn{15}{|c|}{ 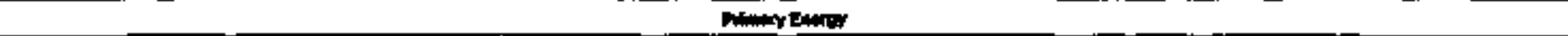 } & \multirow[b]{3}{*}{ Dectikly } & \multirow[b]{3}{*}{$\lim ^{2} \times$} \\
\hline & \multicolumn{3}{|c|}{ بn } & \multirow[b]{2}{*}{ and } & \multicolumn{9}{|c|}{ 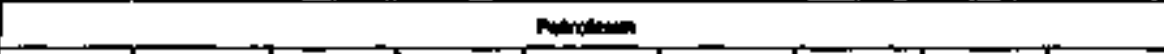 } & \multirow[b]{2}{*}{ antsos } & \multirow[b]{2}{*}{$T \operatorname{Tot}^{*}$} & & \\
\hline & 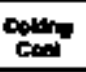 & inos & Tole & & 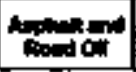 & Fut & (n) & Le* & Lutsingt: & mover & إن & Othes ${ }^{s}$ & Totel & & & & \\
\hline & \multicolumn{17}{|c|}{ 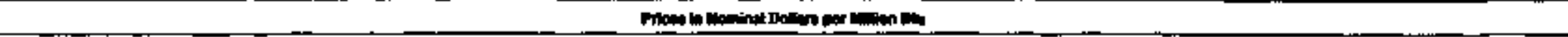 } \\
\hline \multirow[t]{2}{*}{ 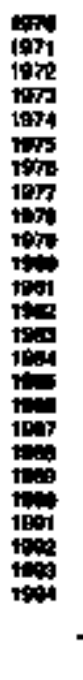 } & 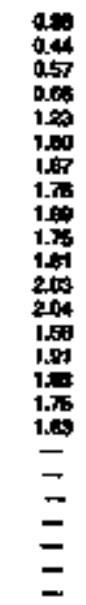 & 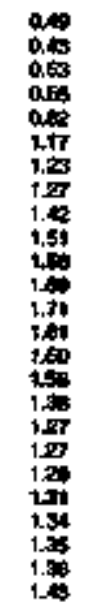 & 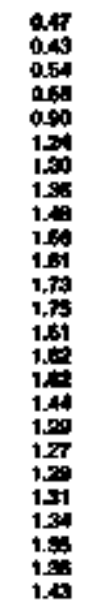 & 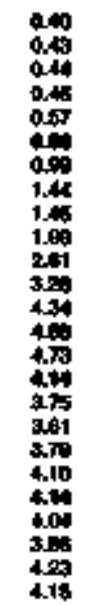 & 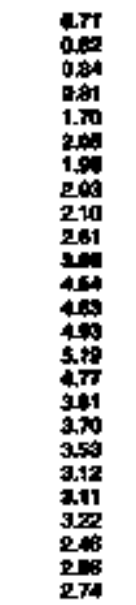 & 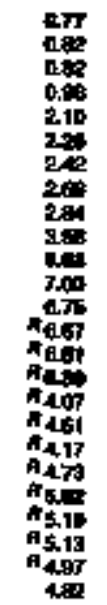 & 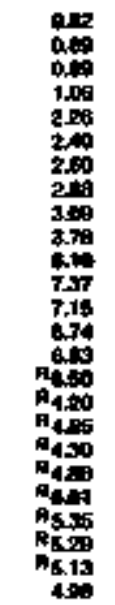 & 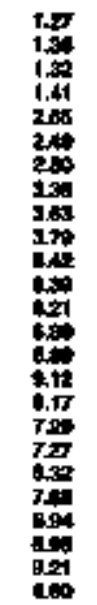 & 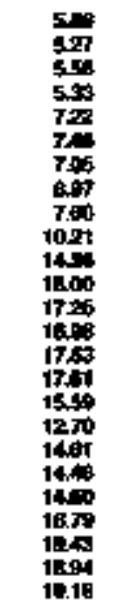 & 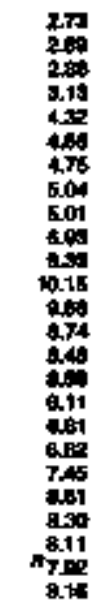 & 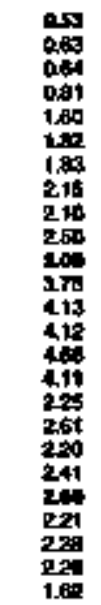 & 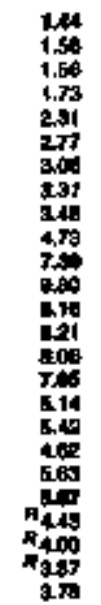 & 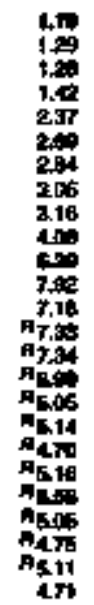 & 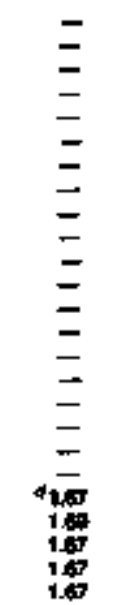 & 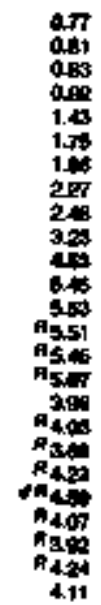 & 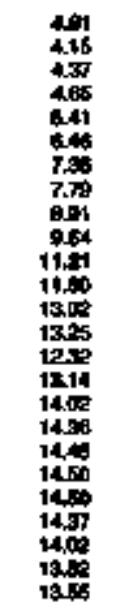 & 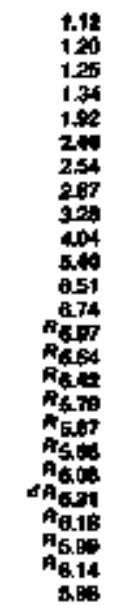 \\
\hline & \multicolumn{17}{|c|}{ 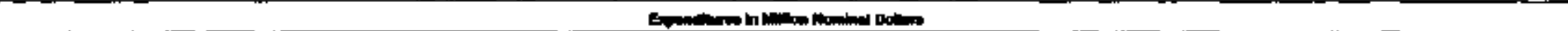 } \\
\hline 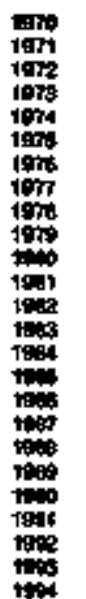 & 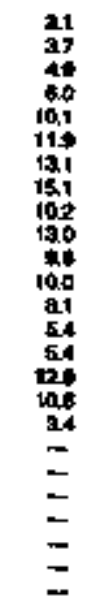 & 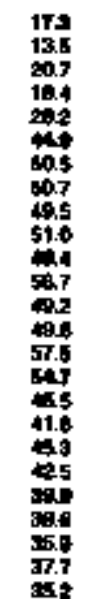 & 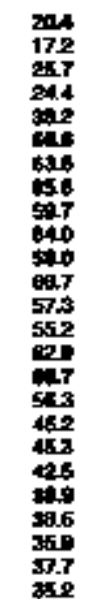 & 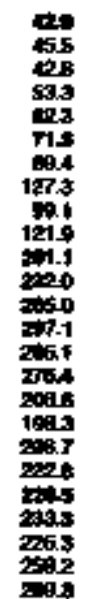 & 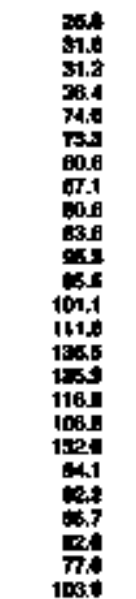 & 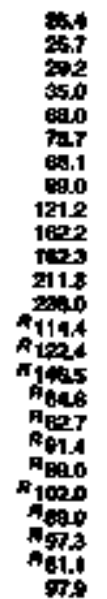 & 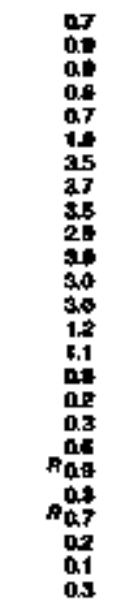 & 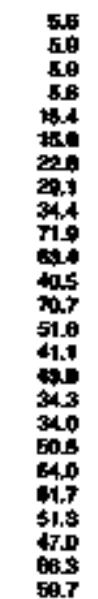 & 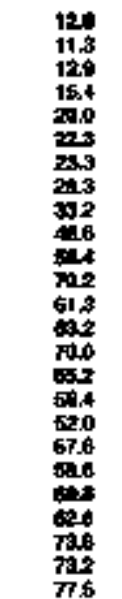 & 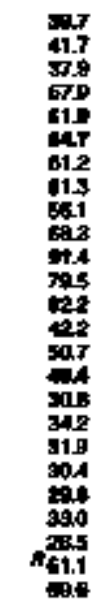 & 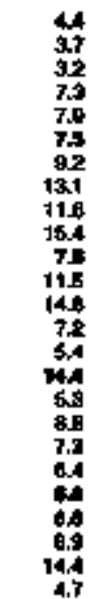 & 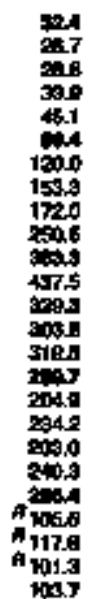 & 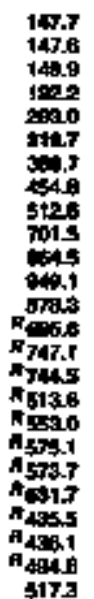 & 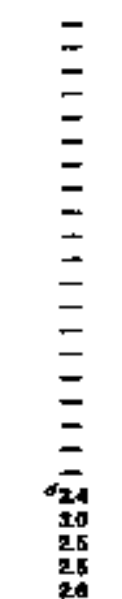 & 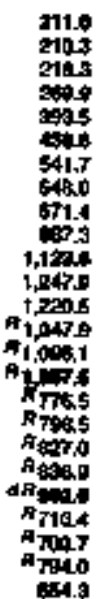 & 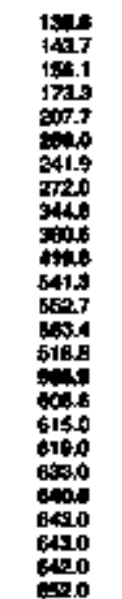 & 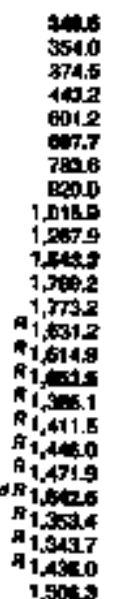 \\
\hline
\end{tabular}

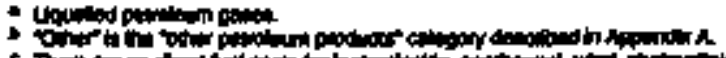

Plomind dath

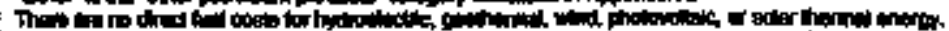

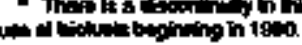

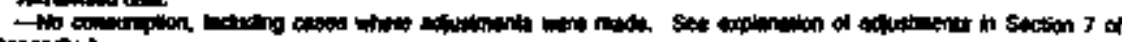
Appoint $\lambda$

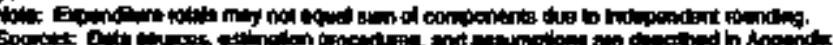




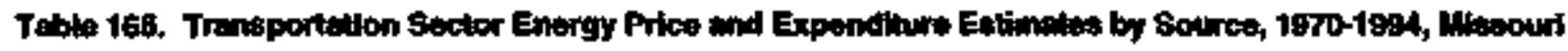

\begin{tabular}{|c|c|c|c|c|c|c|c|c|c|c|c|c|c|}
\hline \multirow[b]{4}{*}{ Yum } & \multicolumn{11}{|c|}{ 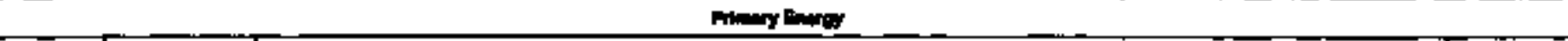 } & \multirow[b]{3}{*}{ Encheng } & \multirow[b]{3}{*}{ 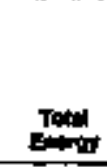 } \\
\hline & & & \multicolumn{8}{|c|}{ Ptroinen } & \multirow[b]{2}{*}{ Tim } & & \\
\hline & Con & 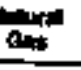 & Mition & إت & it & 10* & Lيف: & 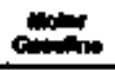 & Raldent & Toted & & & \\
\hline & \multicolumn{13}{|c|}{ Puta } \\
\hline \multirow[t]{2}{*}{ 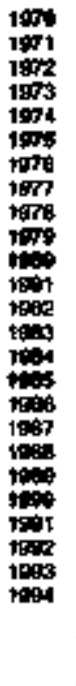 } & $\begin{array}{l}0.4 \\
0.43 \\
0.53 \\
0.55 \\
0.92 \\
193 \\
120 \\
= \\
= \\
= \\
= \\
= \\
= \\
= \\
= \\
= \\
= \\
=\end{array}$ & $\begin{array}{l}= \\
= \\
= \\
= \\
= \\
= \\
= \\
= \\
= \\
= \\
= \\
= \\
=\end{array}$ & 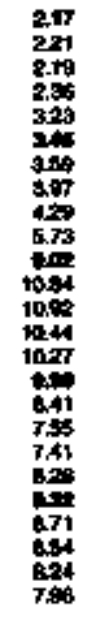 & 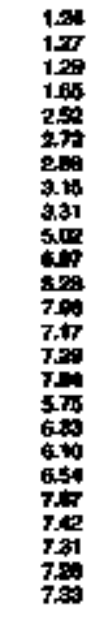 & 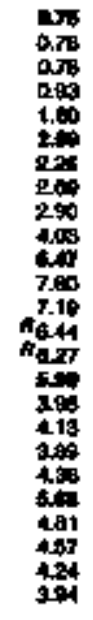 & 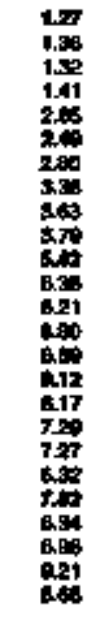 & 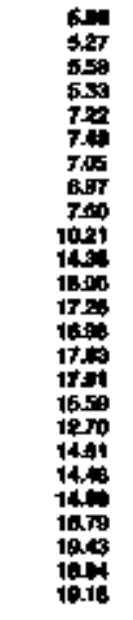 & 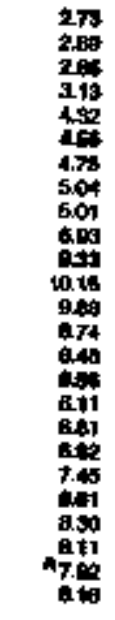 & 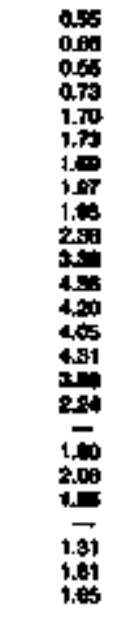 & 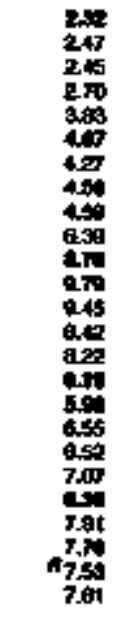 & 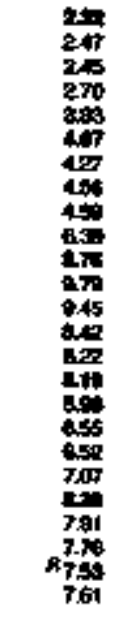 & $\begin{array}{l}= \\
= \\
= \\
= \\
= \\
= \\
= \\
= \\
= \\
= \\
= \\
= \\
=0\end{array}$ & 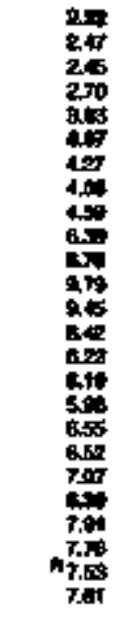 \\
\hline & \multicolumn{13}{|c|}{ F } \\
\hline 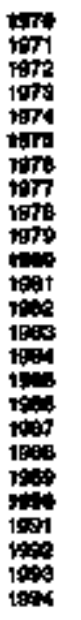 & $\begin{array}{l}6 \\
60 \\
0 \\
0 \\
= \\
= \\
= \\
= \\
= \\
= \\
= \\
= \\
= \\
=\end{array}$ & $\begin{array}{l}= \\
= \\
= \\
= \\
= \\
= \\
= \\
= \\
= \\
= \\
= \\
= \\
= \\
\text { (c) }\end{array}$ & 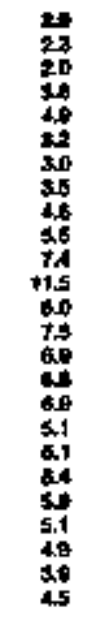 & 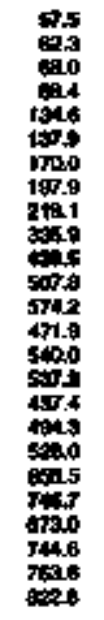 & 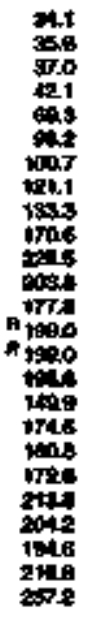 & 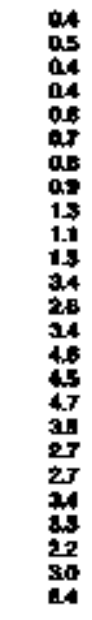 & 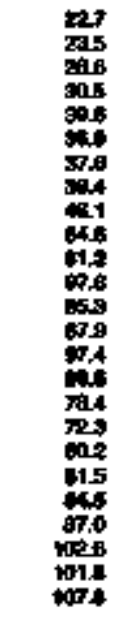 & 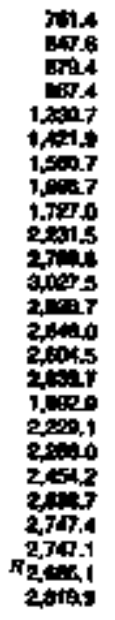 & 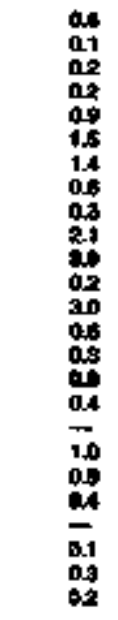 & 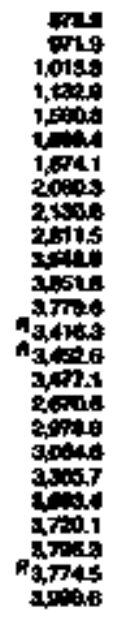 & 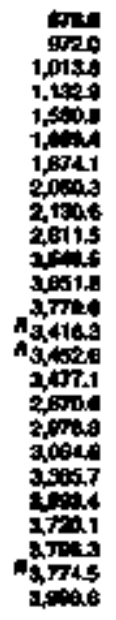 & $\begin{array}{l}= \\
= \\
= \\
= \\
= \\
= \\
= \\
= \\
= \\
= \\
= \\
= \\
= \\
0.7\end{array}$ & 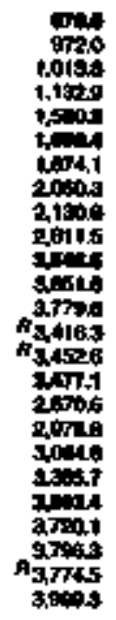 \\
\hline
\end{tabular}




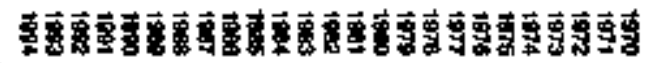

4.

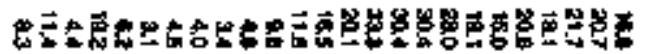

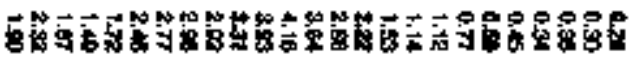

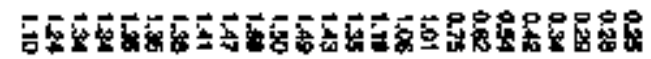

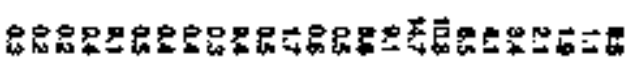

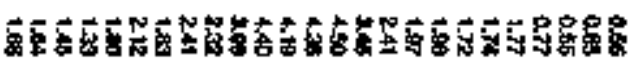

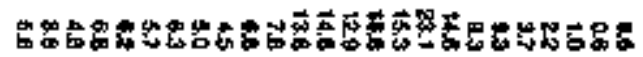

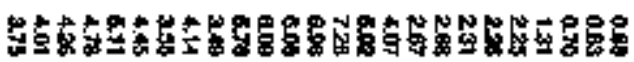

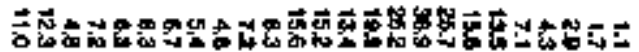

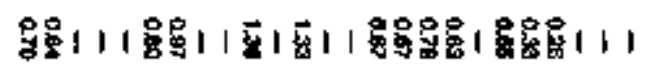

1

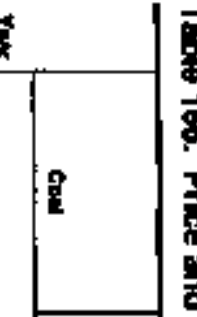

if

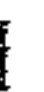

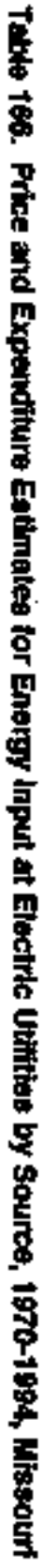

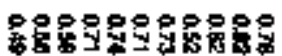

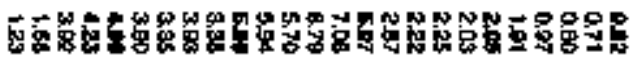

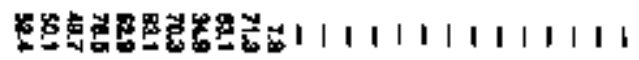

.

쪼픈||||||||||||||||||||小|

8 운

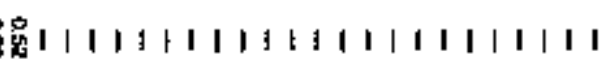

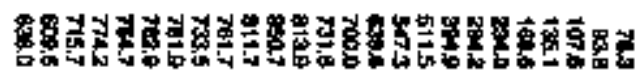


Toblo 187. Energy Price and Exponilture Eetimated by Source, 1970-1984, Moniand

\begin{tabular}{|c|c|c|c|c|c|c|c|c|c|c|c|c|c|c|c|c|c|}
\hline \multirow[b]{4}{*}{ Yex: } & \multicolumn{14}{|c|}{ 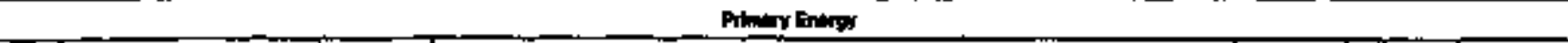 } & \multirow{3}{*}{ Futs } & \multirow{3}{*}{ 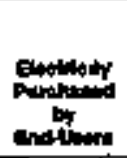 } & \multirow[b]{3}{*}{ Tolve } \\
\hline & \multicolumn{3}{|c|}{ con } & \multirow[b]{2}{*}{ Moturil } & \multicolumn{7}{|c|}{ Pronimen } & \multirow[b]{2}{*}{ Futiol } & \multirow[b]{2}{*}{ molnes } & \multirow[b]{2}{*}{ Toles: } & & & \\
\hline & 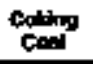 & Shent & $\operatorname{Tim}$ & & 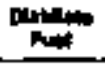 & 些 & Lat & 萑 & mondul & Othen to & Tot & & & & & & \\
\hline & \multicolumn{17}{|c|}{ 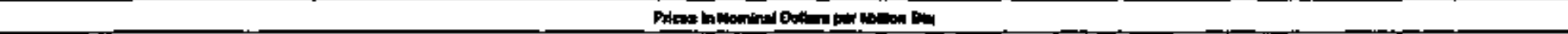 } \\
\hline \multirow[t]{2}{*}{ 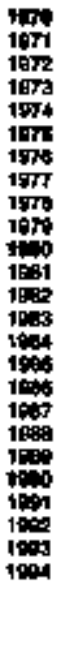 } & $\begin{array}{l}= \\
= \\
= \\
= \\
= \\
= \\
= \\
= \\
= \\
= \\
= \\
= \\
= \\
=\end{array}$ & 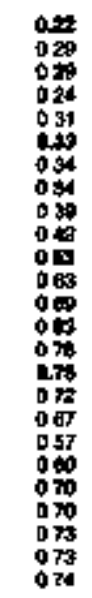 & 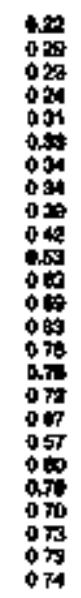 & 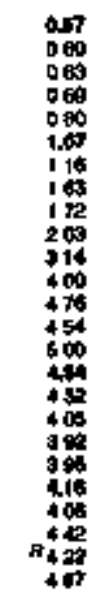 & 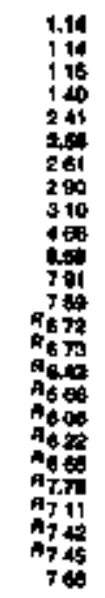 & 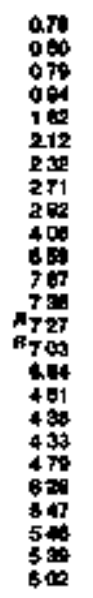 & 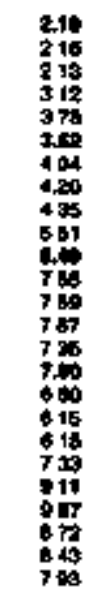 & 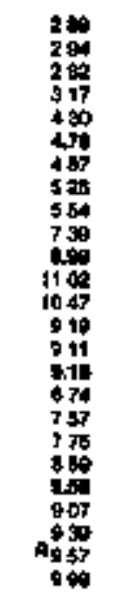 & 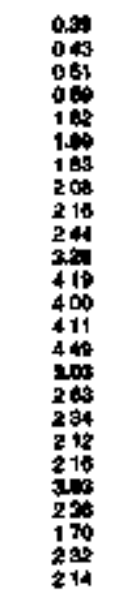 & 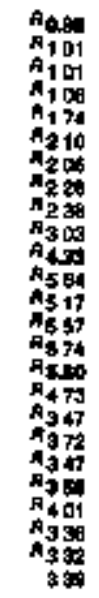 & 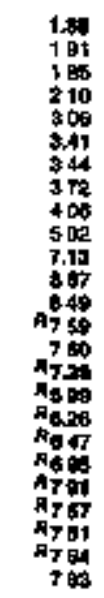 & $\begin{array}{l}= \\
= \\
= \\
= \\
= \\
= \\
= \\
= \\
= \\
= \\
= \\
= \\
=\end{array}$ & 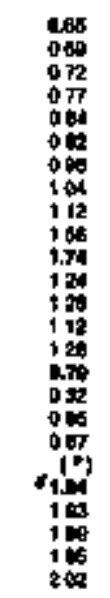 & 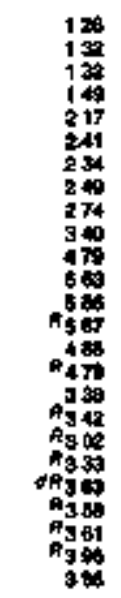 & 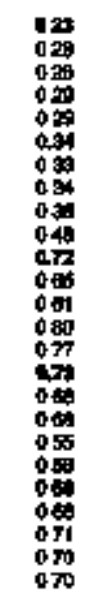 & 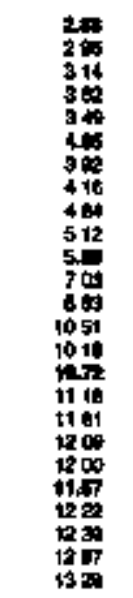 & 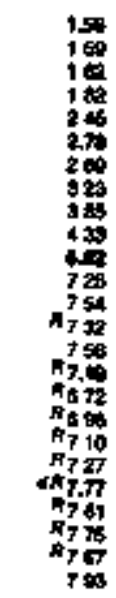 \\
\hline & \multicolumn{17}{|c|}{ 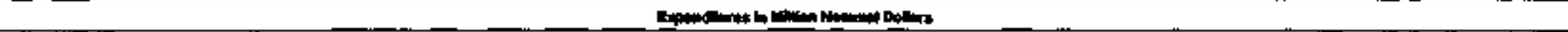 } \\
\hline 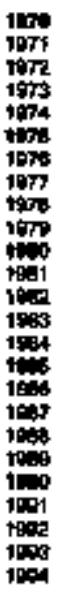 & $\begin{array}{l}= \\
= \\
= \\
= \\
= \\
= \\
= \\
= \\
= \\
= \\
= \\
= \\
= \\
=\end{array}$ & 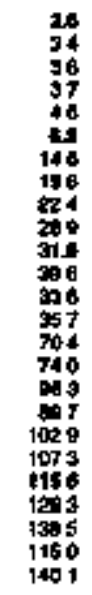 & 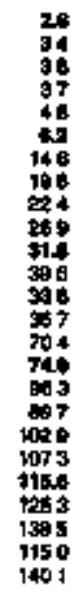 & 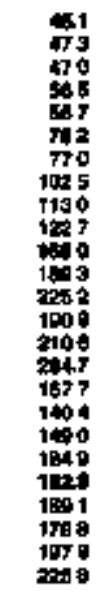 & 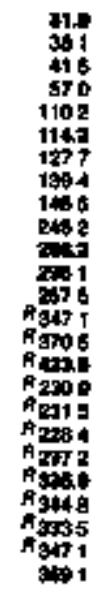 & 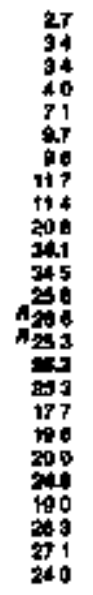 & 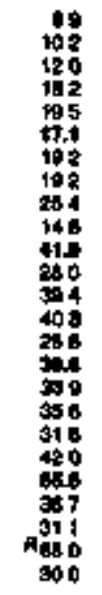 & 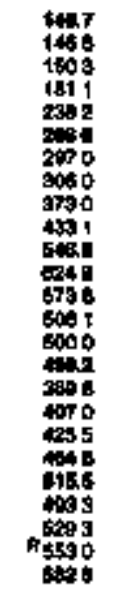 & 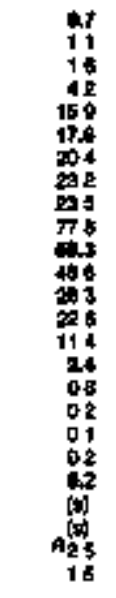 & 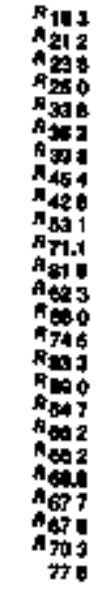 & 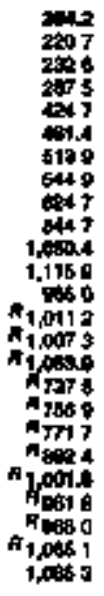 & $\begin{array}{l}= \\
= \\
= \\
= \\
= \\
= \\
= \\
= \\
= \\
= \\
= \\
= \\
=\end{array}$ & 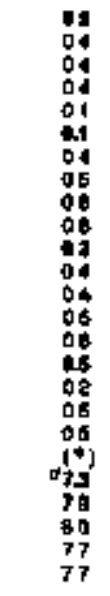 & 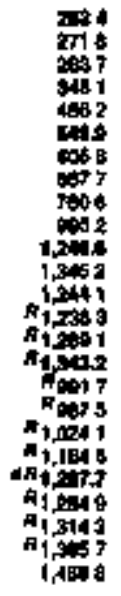 & 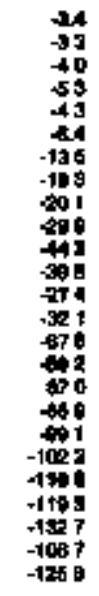 & 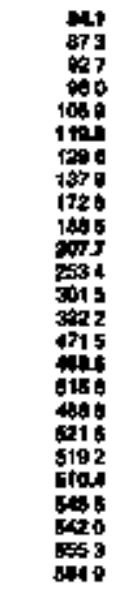 & 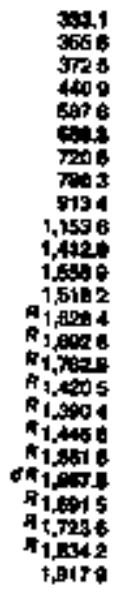 \\
\hline
\end{tabular}

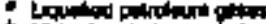

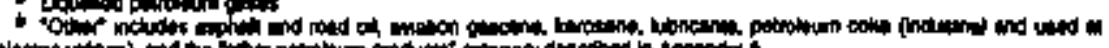
Antion

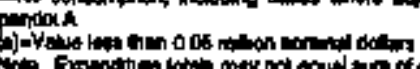

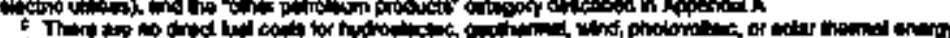

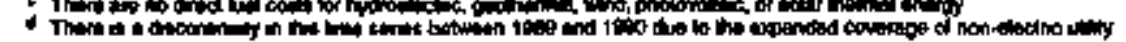

(1)

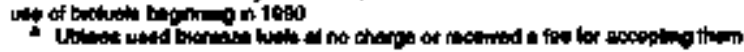

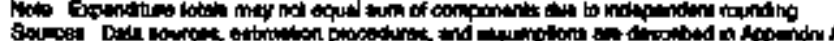

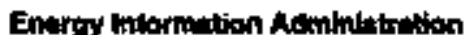

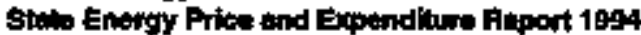




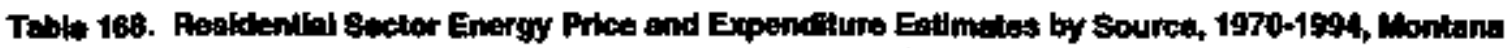

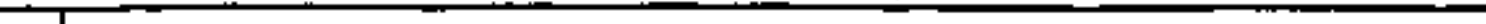

\begin{tabular}{|c|c|c|c|c|c|c|c|c|c|c|}
\hline \multirow[b]{4}{*}{ Kar } & \multicolumn{8}{|c|}{ PYhmem } & \multirow[b]{3}{*}{ Shotidity } & \multirow[b]{3}{*}{ 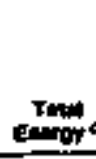 } \\
\hline & \multirow[b]{2}{*}{ cin } & \multirow{2}{*}{$t=$} & \multicolumn{4}{|c|}{ ntim } & \multirow[b]{2}{*}{ Ahtume } & \multirow[b]{2}{*}{ Totale } & & \\
\hline & & & Fud & Fromen & un: & Tw: & & & & \\
\hline & \multicolumn{9}{|c|}{ 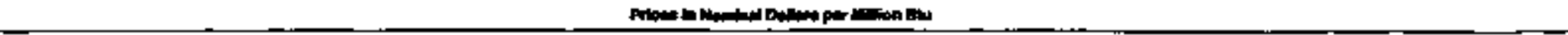 } & \\
\hline \multirow[t]{2}{*}{ 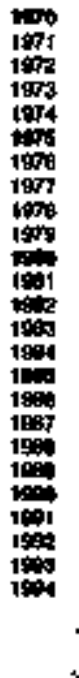 } & 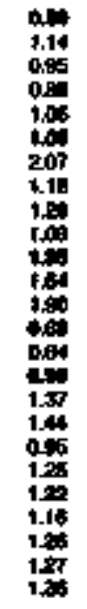 & 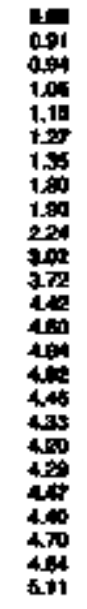 & 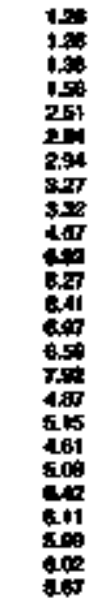 & 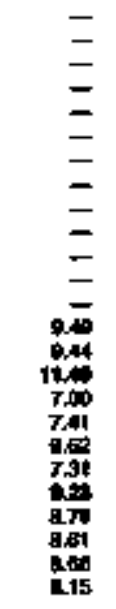 & 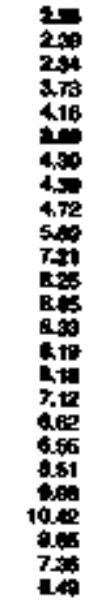 & 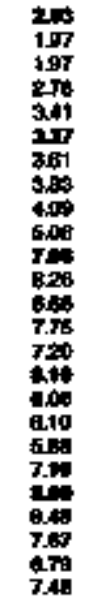 & 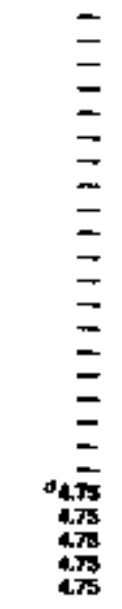 & 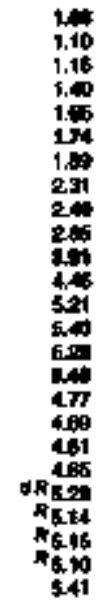 & 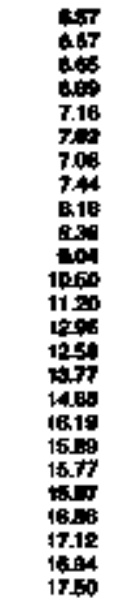 & 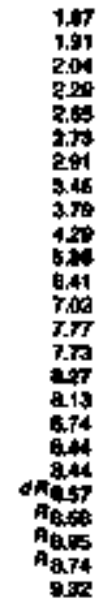 \\
\hline & \multicolumn{10}{|c|}{ 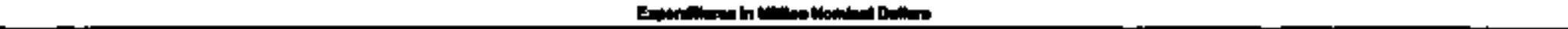 } \\
\hline 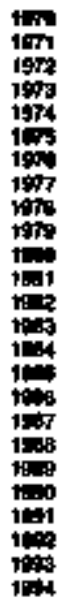 & $\begin{array}{l}01 \\
0.2 \\
0.1 \\
0.1 \\
0.1 \\
01 \\
0.1 \\
0.1 \\
0.1 \\
0.1 \\
0.1 \\
0.1 \\
0.1 \\
01 \\
0.0 \\
0.0 \\
0.2 \\
0.1 \\
0.1 \\
0.5 \\
0.5 \\
0.3 \\
0.2 \\
0.1 \\
0.1\end{array}$ & 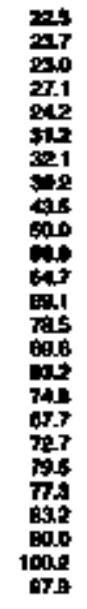 & 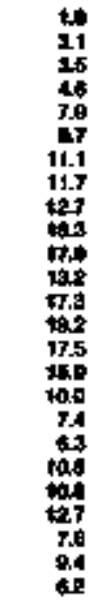 & $\begin{array}{l}= \\
= \\
= \\
= \\
= \\
= \\
= \\
= \\
= \\
0.0 \\
00 \\
00 \\
01 \\
01 \\
01 \\
0=1 \\
01 \\
01 \\
0 j \\
03 \\
0.9\end{array}$ & 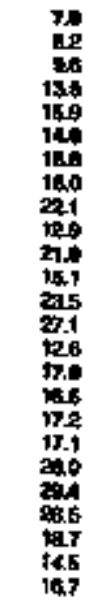 & 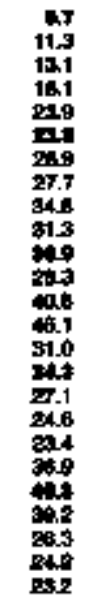 & $\begin{array}{l}= \\
= \\
= \\
= \\
= \\
= \\
= \\
= \\
= \\
= \\
= \\
= \\
536 \\
30 \\
40 \\
30\end{array}$ & 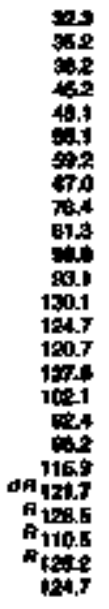 & 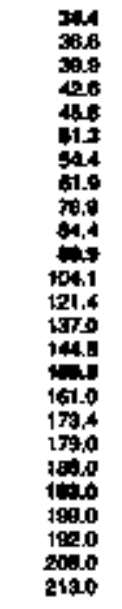 & 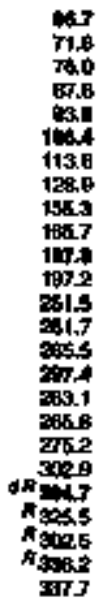 \\
\hline
\end{tabular}

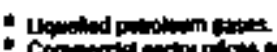

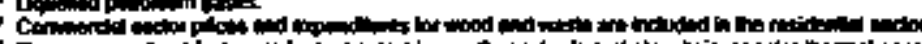

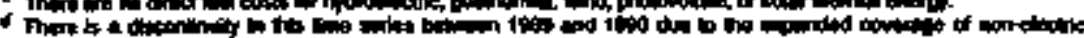

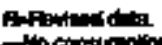

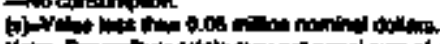

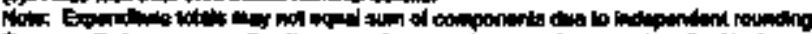

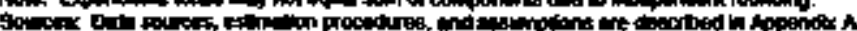

Energy litormiation Adminiotration 


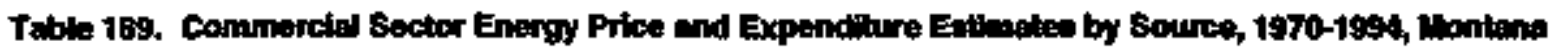

\begin{tabular}{|c|c|c|c|c|c|c|c|c|c|c|c|}
\hline \multirow[b]{4}{*}{ Ver } & \multicolumn{9}{|c|}{ 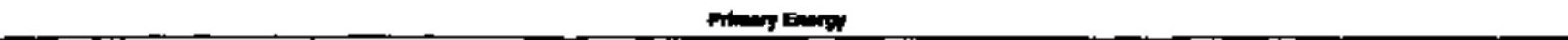 } & \multirow[b]{3}{*}{ Enctisthy } & \multirow[b]{3}{*}{ 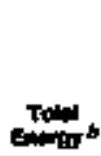 } \\
\hline & \multirow[b]{2}{*}{ con } & \multirow[b]{2}{*}{ 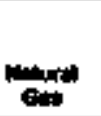 } & \multicolumn{6}{|c|}{ Finiman } & \multirow[b]{2}{*}{ Int } & & \\
\hline & & & 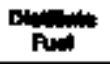 & החטה:" & $\mathbf{t n t}$ & 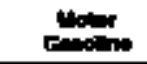 & 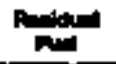 & Tas & & & \\
\hline & \multicolumn{11}{|c|}{ 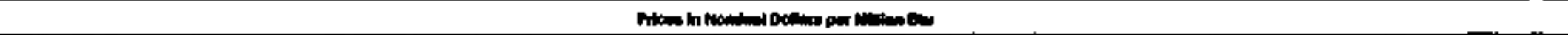 } \\
\hline \multirow[t]{2}{*}{ 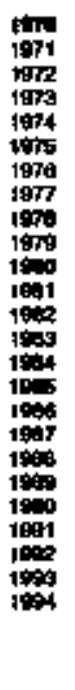 } & 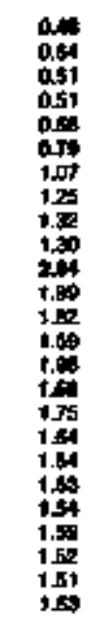 & 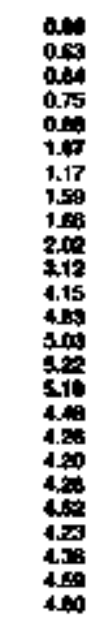 & 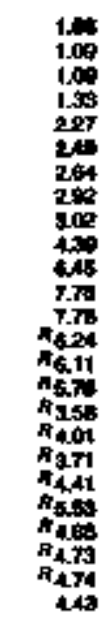 & 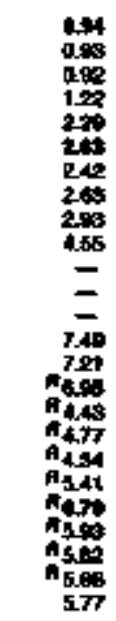 & 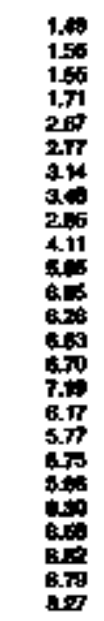 & 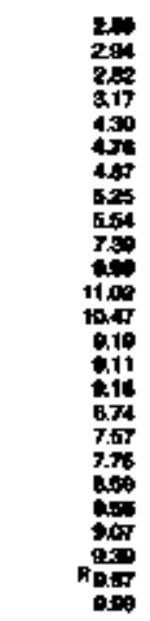 & 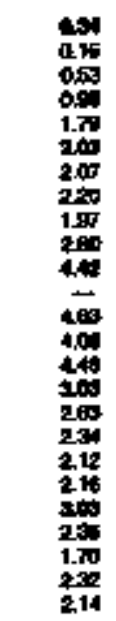 & 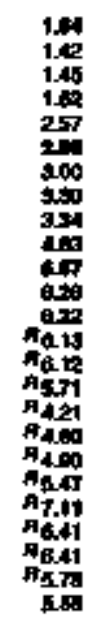 & 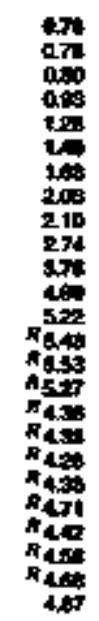 & 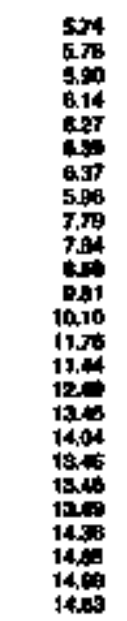 & 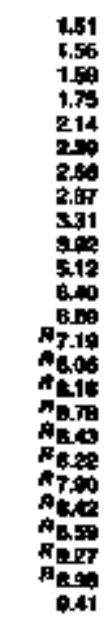 \\
\hline & \multicolumn{11}{|c|}{ 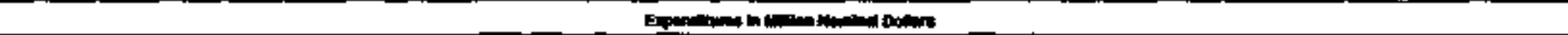 } \\
\hline 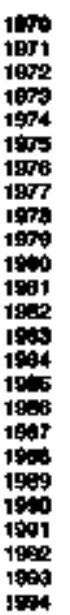 & $\begin{array}{l}0.1 \\
0.2 \\
0.1 \\
0.1 \\
0.1 \\
0.1 \\
0.1 \\
0.1 \\
0.3 \\
02 \\
0.4 \\
0.2 \\
02 \\
0.2 \\
0.1 \\
0.2 \\
0.4 \\
0.1 \\
02 \\
1.0 \\
1.1 \\
0.5 \\
0.4 \\
0.2 \\
0.1\end{array}$ & 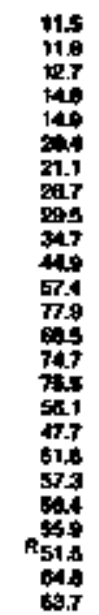 & 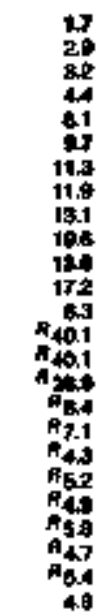 & 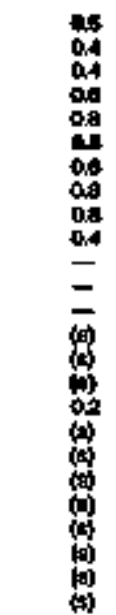 & 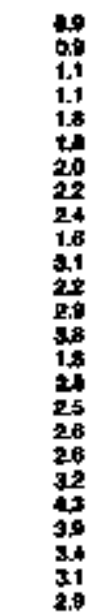 & 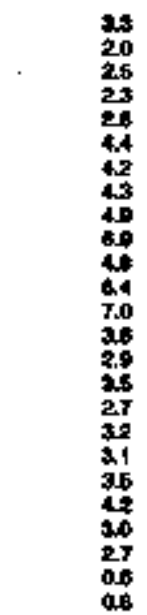 & 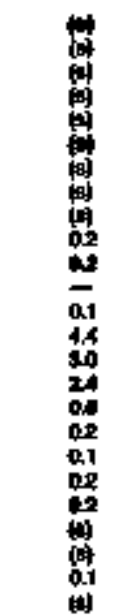 & 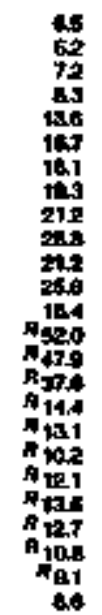 & 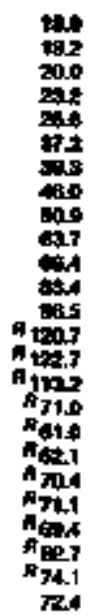 & 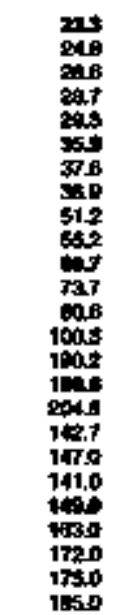 & 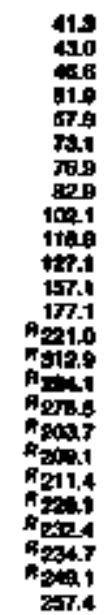 \\
\hline
\end{tabular}

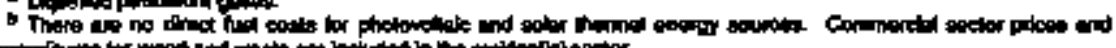

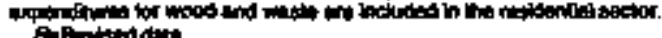

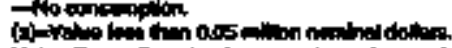

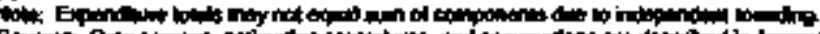

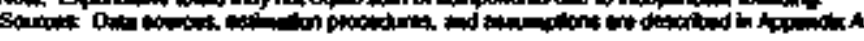


Table 170. udustrial Sector Energy Prce and Expendlume Estimaten by Source, 1970-1994, Montana

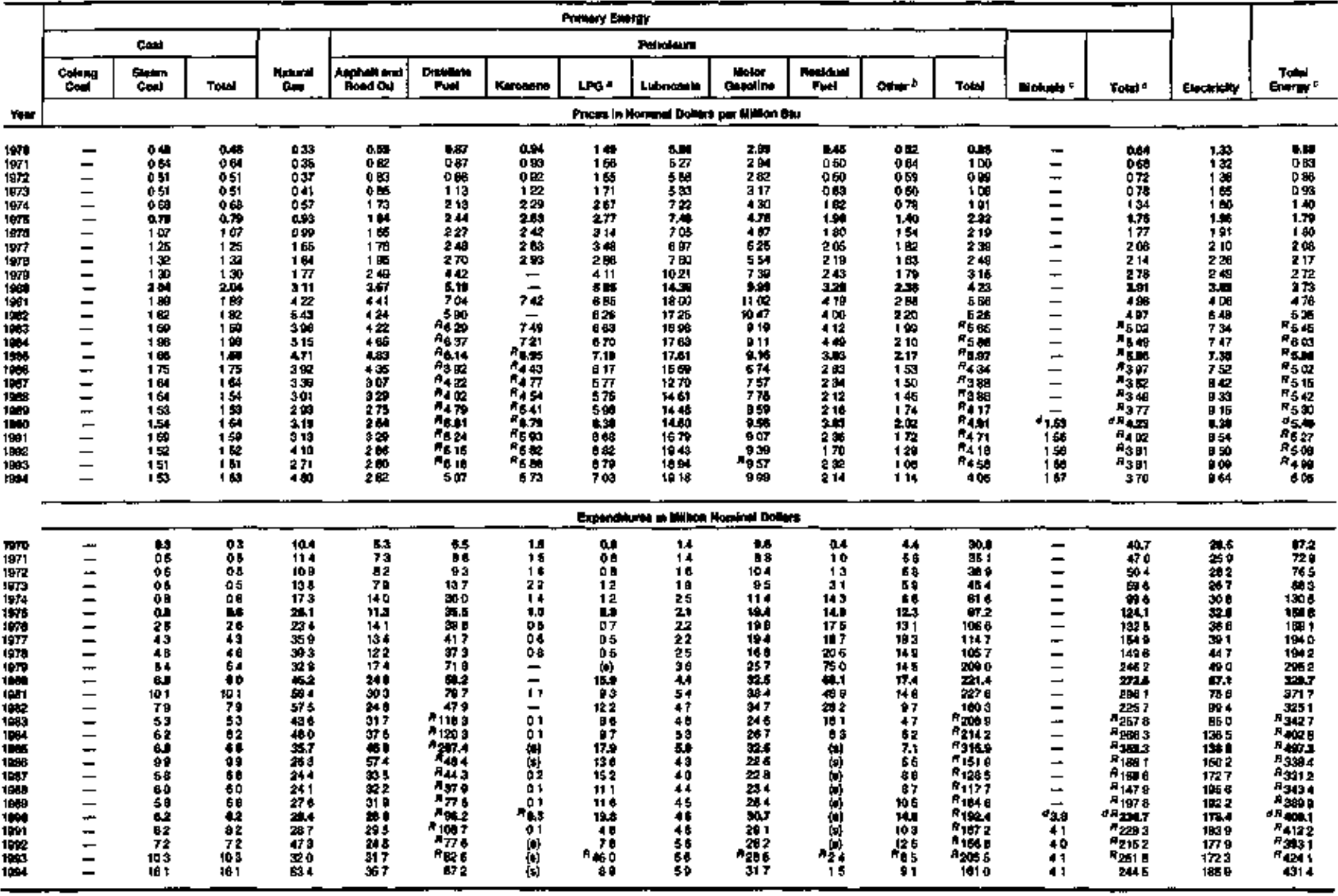

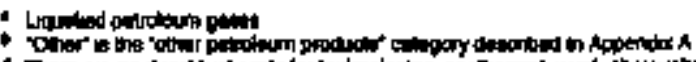

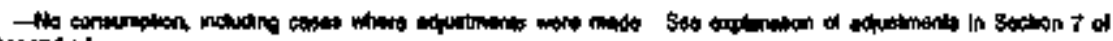

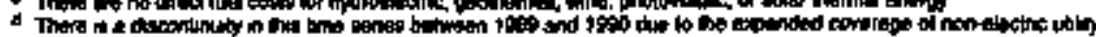

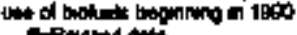

(n)

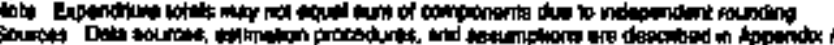
mimion 
Table 171. Tranaportation Seetor Enargy Price and Expenditure Estmales by Source, 1970-1994, Montana

\begin{tabular}{|c|c|c|c|c|c|c|c|c|c|c|c|c|c|}
\hline \multirow[b]{4}{*}{$\mathrm{rem}$} & \multicolumn{11}{|c|}{ Patrey landy } & \multirow[b]{3}{*}{ Eloolinaty } & \multirow[b]{3}{*}{$\begin{array}{l}\text { Tatol } \\
\text { Gaterger }\end{array}$} \\
\hline & \multirow[b]{2}{*}{ Cost } & \multirow[b]{2}{*}{ mound } & \multicolumn{8}{|c|}{ Purnatern } & \multirow[b]{2}{*}{ Tot } & & \\
\hline & & & Auterion & Dintiont & Imint & HQ⿻ & Lutik=ants & motor & 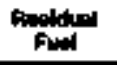 & $T=1$ & & & \\
\hline & \multicolumn{13}{|c|}{ 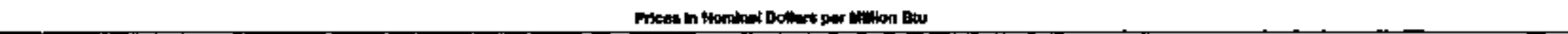 } \\
\hline \multirow[t]{2}{*}{ 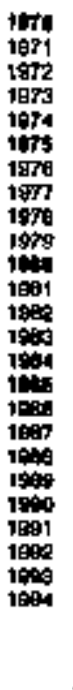 } & $\begin{array}{l}0.40 \\
064 \\
051 \\
061 \\
060 \\
0.70 \\
107 \\
126 \\
= \\
= \\
= \\
= \\
= \\
= \\
= \\
= \\
= \\
= \\
=\end{array}$ & 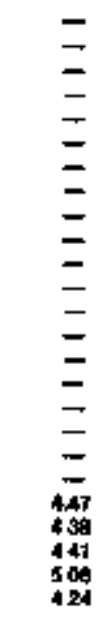 & 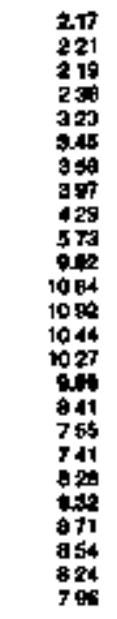 & 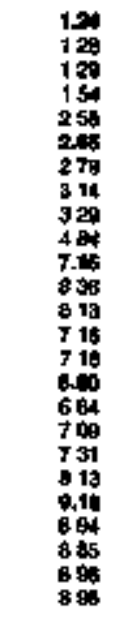 & 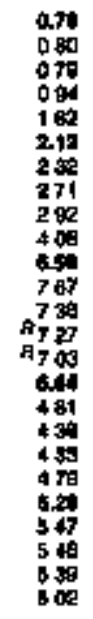 & 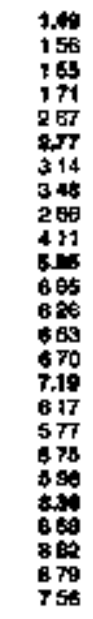 & 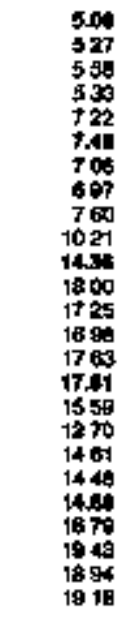 & 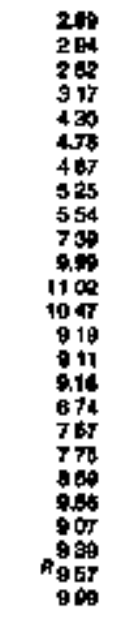 & 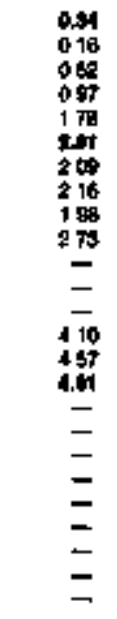 & 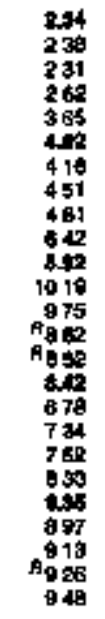 & 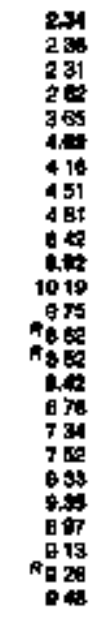 & $\begin{array}{l}= \\
= \\
= \\
= \\
= \\
= \\
= \\
= \\
= \\
= \\
= \\
= \\
=\end{array}$ & 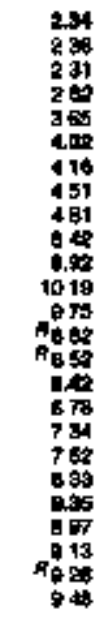 \\
\hline & \multicolumn{13}{|c|}{ 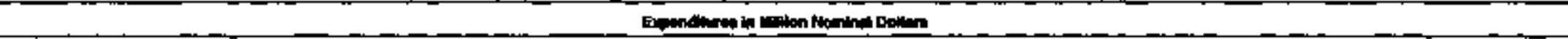 } \\
\hline 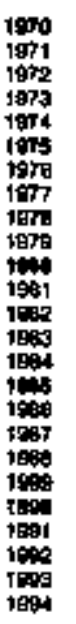 & $\begin{array}{l}5 \\
= \\
= \\
= \\
= \\
= \\
= \\
= \\
= \\
= \\
= \\
=\end{array}$ & 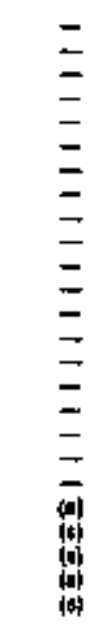 & $\begin{array}{l}18 \\
65 \\
10 \\
15 \\
17 \\
14 \\
17 \\
19 \\
19 \\
25 \\
79 \\
67 \\
51 \\
54 \\
40 \\
46 \\
44 \\
31 \\
40 \\
40 \\
52 \\
48 \\
39 \\
20 \\
30\end{array}$ & 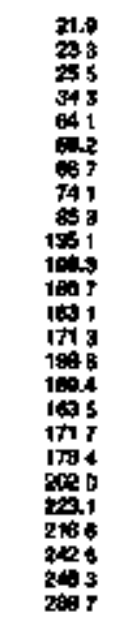 & 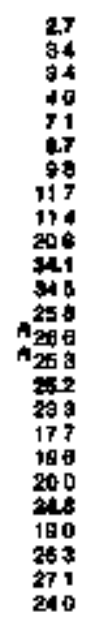 & $\begin{array}{l}12 \\
03 \\
05 \\
04 \\
05 \\
05 \\
05 \\
05 \\
05 \\
03 \\
10 \\
13 \\
06 \\
13 \\
17 \\
13 \\
12 \\
06 \\
10 \\
12 \\
20 \\
15 \\
11 \\
14 \\
16\end{array}$ & $\begin{array}{l}47 \\
46 \\
52 \\
51 \\
67 \\
73 \\
77 \\
63 \\
97 \\
136 \\
771 \\
206 \\
190 \\
185 \\
205 \\
121 \\
155 \\
152 \\
199 \\
172 \\
784 \\
183 \\
218 \\
214 \\
227\end{array}$ & 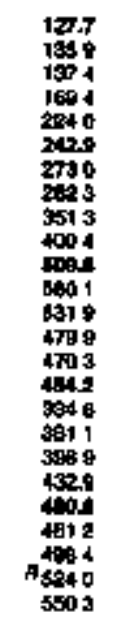 & $\begin{array}{l}05 \\
01 \\
02 \\
09 \\
10 \\
28 \\
10 \\
10 \\
17 \\
04 \\
= \\
= \\
01 \\
194 \\
= \\
= \\
= \\
= \\
= \\
= \\
=\end{array}$ & 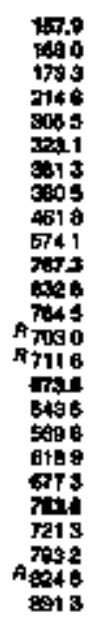 & 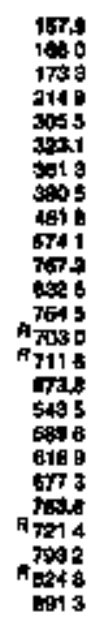 & $\begin{array}{l}= \\
= \\
z \\
= \\
= \\
= \\
= \\
= \\
= \\
= \\
= \\
= \\
=\end{array}$ & 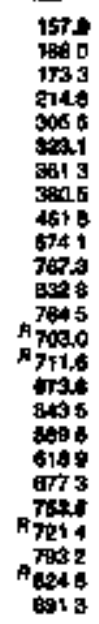 \\
\hline
\end{tabular}




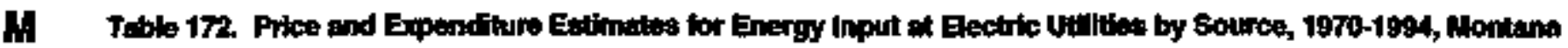

\begin{tabular}{|c|c|c|c|c|c|c|c|c|c|}
\hline \multirow[b]{3}{*}{$t=$} & \multirow[b]{2}{*}{ Cons } & \multirow[b]{2}{*}{ 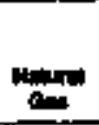 } & \multicolumn{4}{|c|}{ Pandioner } & \multirow[b]{2}{*}{ 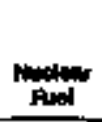 } & \multirow[b]{2}{*}{ Elotukt: } & \multirow[b]{2}{*}{ Tots. } \\
\hline & & & of & L & constim & 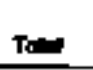 & & & \\
\hline & \multicolumn{9}{|c|}{ 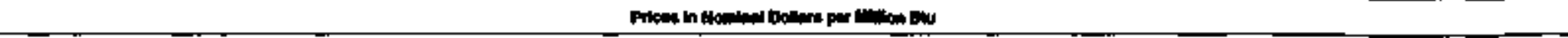 } \\
\hline \multirow[t]{2}{*}{ 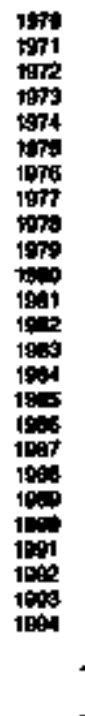 } & 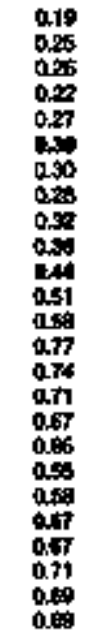 & 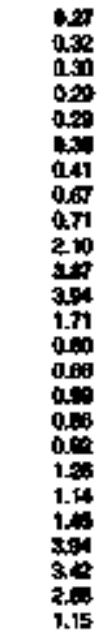 & $\begin{array}{l}0.10 \\
0.18 \\
0.54 \\
0.97 \\
1.79 \\
1.90 \\
2.11 \\
2.10 \\
2.00 \\
2.74 \\
= \\
= \\
= \\
= \\
= \\
= \\
= \\
= \\
=\end{array}$ & 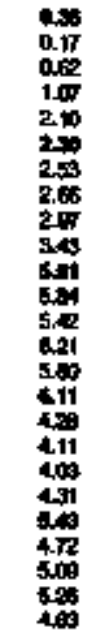 & $\begin{array}{l}= \\
= \\
= \\
= \\
= \\
= \\
= \\
= \\
= \\
= \\
= \\
= \\
=\end{array}$ & 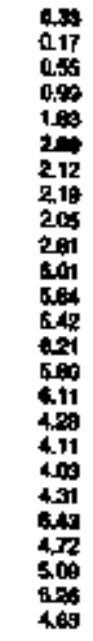 & $\begin{array}{l}= \\
= \\
= \\
= \\
= \\
= \\
= \\
= \\
= \\
= \\
= \\
= \\
= \\
=\end{array}$ & 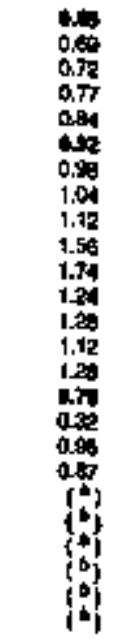 & 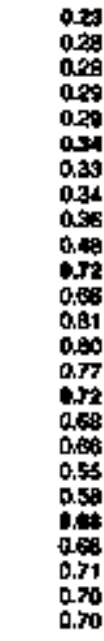 \\
\hline & \multicolumn{9}{|c|}{ 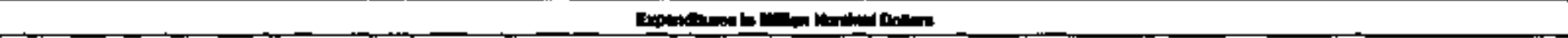 } \\
\hline 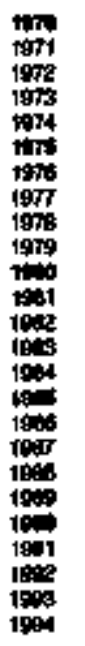 & 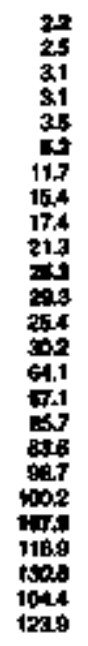 & 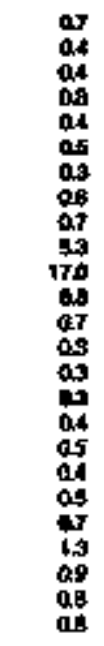 & $\begin{array}{l}10 \\
09 \\
09 \\
01 \\
07 \\
1.0 \\
20 \\
1.2 \\
20 \\
= \\
= \\
= \\
= \\
= \\
= \\
= \\
= \\
= \\
=\end{array}$ & 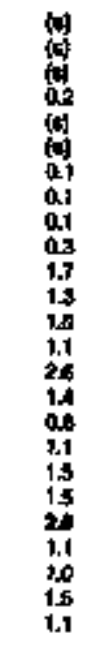 & $\begin{array}{l}= \\
= \\
= \\
= \\
= \\
= \\
= \\
= \\
= \\
= \\
= \\
= \\
= \\
= \\
=\end{array}$ & $\begin{array}{l}0.1 \\
0.1 \\
0.1 \\
1.0 \\
0.2 \\
0.7 \\
1.5 \\
2.7 \\
1.3 \\
2.6 \\
1.5 \\
1.5 \\
1.0 \\
1.1 \\
2.8 \\
1.4 \\
0.8 \\
1.1 \\
1.5 \\
1.5 \\
2.4 \\
1.1 \\
1.0 \\
1.5 \\
1.1\end{array}$ & $\begin{array}{l}= \\
= \\
= \\
= \\
= \\
= \\
= \\
= \\
= \\
= \\
= \\
= \\
= \\
=\end{array}$ & 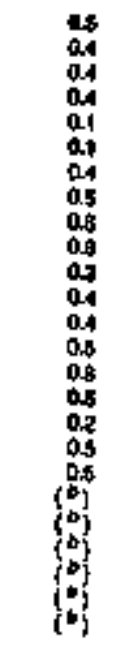 & 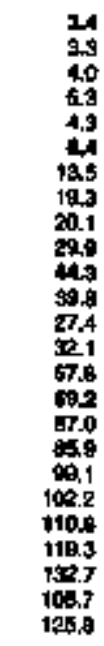 \\
\hline
\end{tabular}

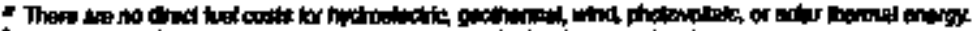

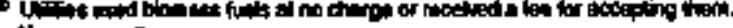

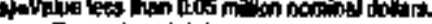

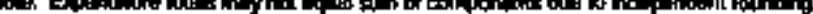

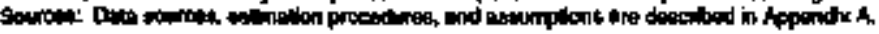


Table 173. Enorgy Prtce and Expenditure Estimates by Source, 1970-1994, Nobrayte

\begin{tabular}{|c|c|c|c|c|c|c|c|c|c|c|c|c|c|c|c|c|c|}
\hline \multirow[b]{4}{*}{ rati } & \multicolumn{14}{|c|}{ 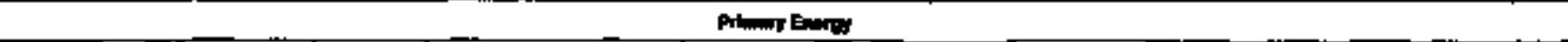 } & \multirow{3}{*}{ 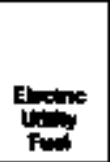 } & \multirow{3}{*}{ 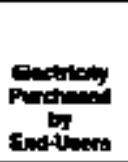 } & \multirow[b]{3}{*}{ Tinter } \\
\hline & \multicolumn{3}{|c|}{ con } & \multirow[b]{2}{*}{ din } & \multicolumn{7}{|c|}{ Patron } & \multirow[b]{2}{*}{ Min } & \multirow[b]{2}{*}{ 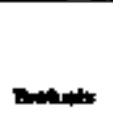 } & \multirow[b]{2}{*}{ Tomb" } & & & \\
\hline & Cotas & conen & Tow & & 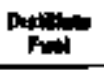 & 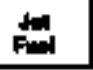 & Len: & . & ה-مسה: & Oher & Toten & & & & & & \\
\hline & \multicolumn{17}{|c|}{ 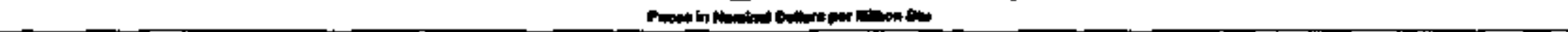 } \\
\hline \multirow[t]{2}{*}{ 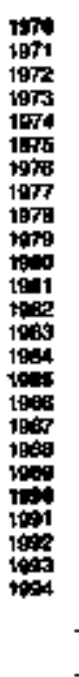 } & $\begin{array}{l}z \\
z \\
z \\
z \\
z \\
z \\
= \\
z \\
z \\
= \\
z \\
z \\
z \\
z \\
z\end{array}$ & 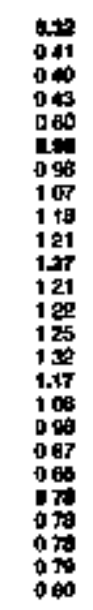 & 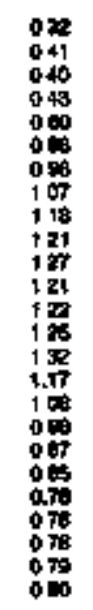 & 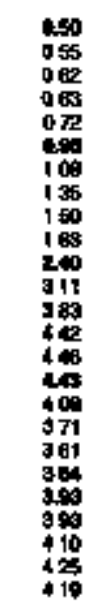 & 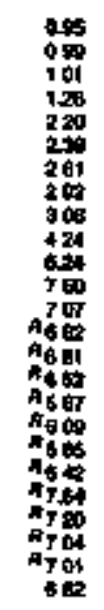 & 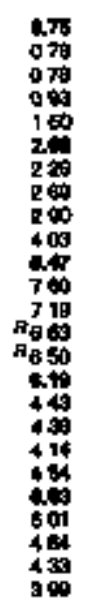 & 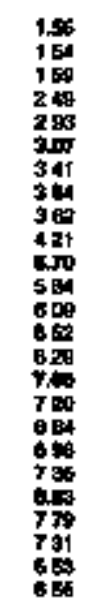 & 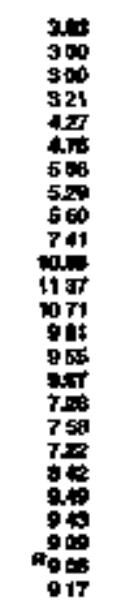 & 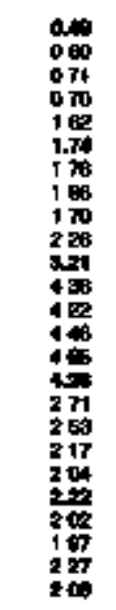 & 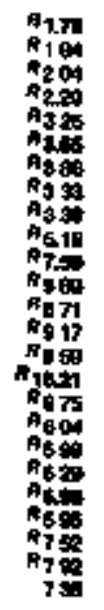 & 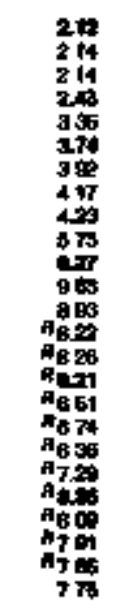 & 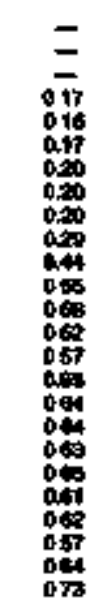 & $\begin{array}{l}= \\
= \\
= \\
= \\
= \\
= \\
= \\
= \\
= \\
= \\
= \\
=3 \\
35 \\
350 \\
350\end{array}$ & 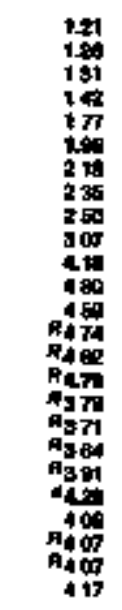 & 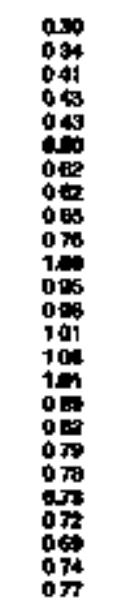 & 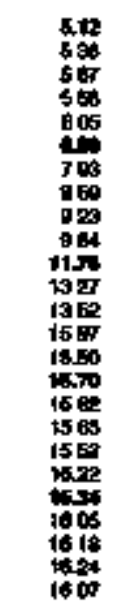 & 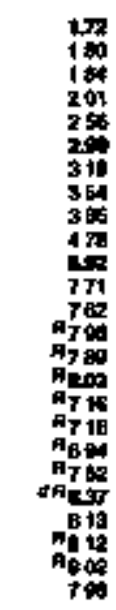 \\
\hline & \multicolumn{17}{|c|}{ Bis } \\
\hline 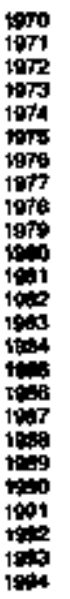 & $\begin{array}{l}= \\
= \\
= \\
= \\
= \\
= \\
= \\
= \\
= \\
= \\
= \\
= \\
= \\
=\end{array}$ & 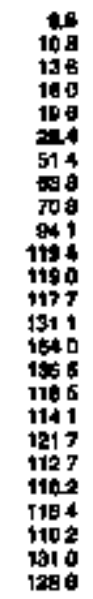 & 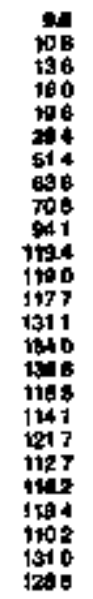 & 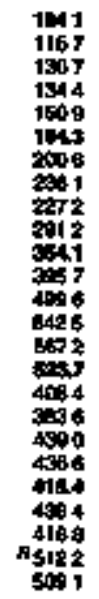 & 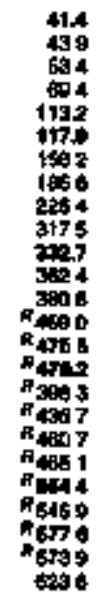 & 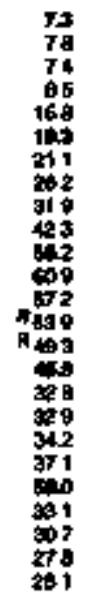 & 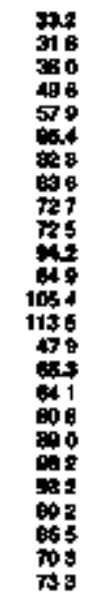 & 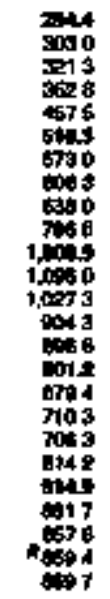 & 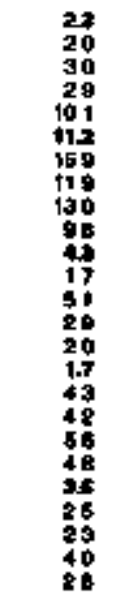 & 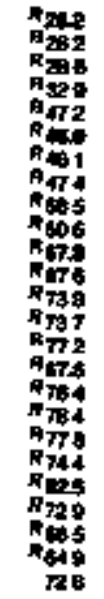 & 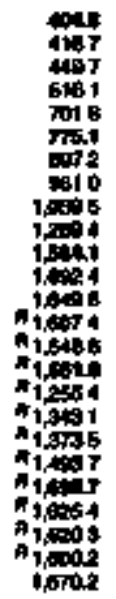 & 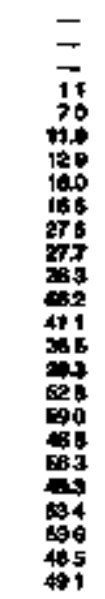 & $\begin{array}{l}= \\
= \\
= \\
= \\
= \\
= \\
= \\
= \\
= \\
= \\
= \\
= \\
54 \\
54 \\
45 \\
45\end{array}$ & 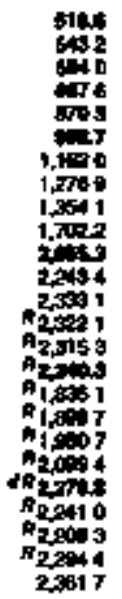 & 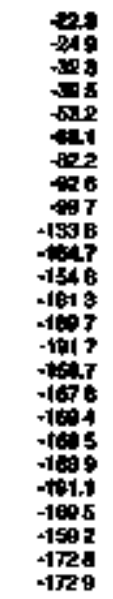 & 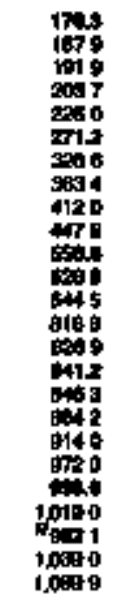 & 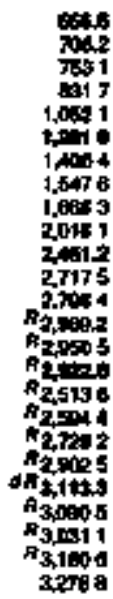 \\
\hline
\end{tabular}

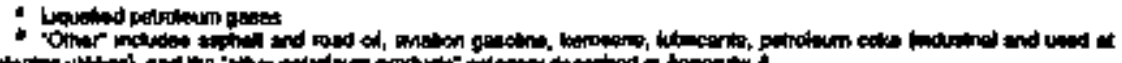

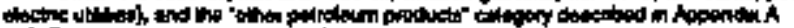

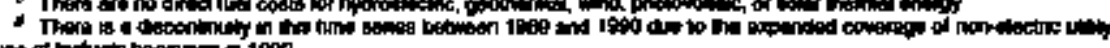

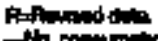

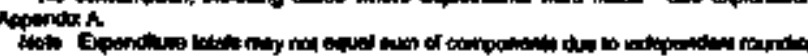

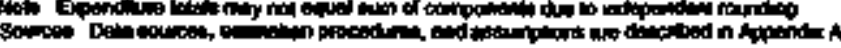




\begin{tabular}{|c|c|c|c|c|c|c|c|c|c|c|}
\hline & \multicolumn{8}{|c|}{ 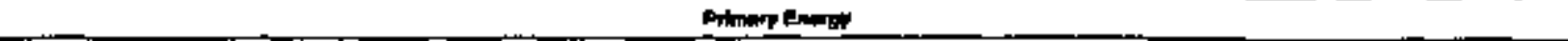 } & \multirow[b]{3}{*}{ Exancly } & \multirow[b]{3}{*}{ Tlow" } \\
\hline & \multirow[b]{2}{*}{$\cos 1$} & \multirow[b]{2}{*}{ 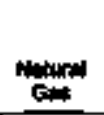 } & \multicolumn{4}{|c|}{ menomim } & \multirow[b]{2}{*}{ moams: } & \multirow[b]{2}{*}{ Tane } & & \\
\hline & & & 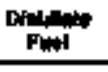 & Korpemen & LPE* & Totw & & & & \\
\hline yeer & \multicolumn{10}{|c|}{ 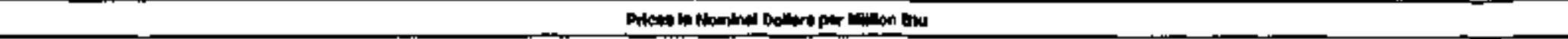 } \\
\hline \multirow[t]{2}{*}{ 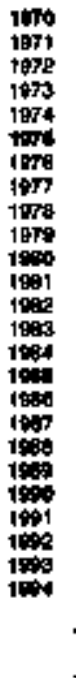 } & 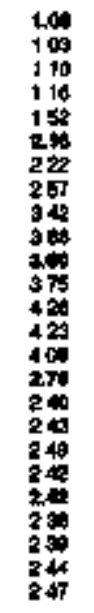 & 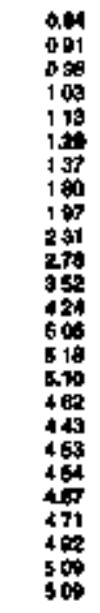 & 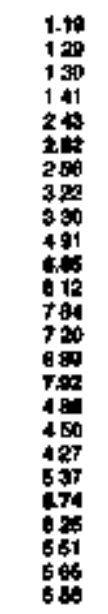 & 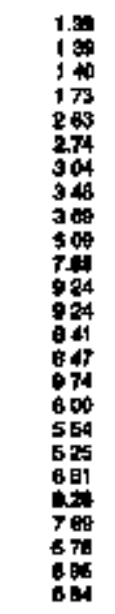 & 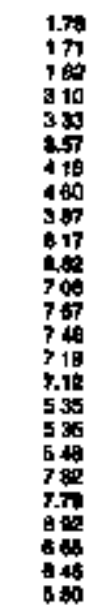 & 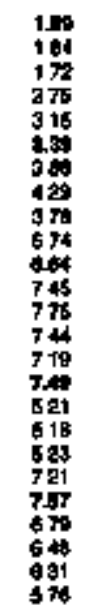 & 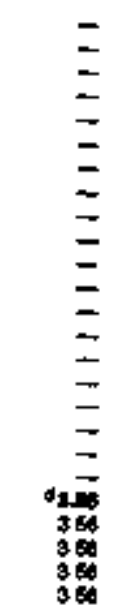 & 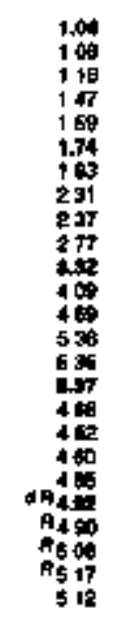 & 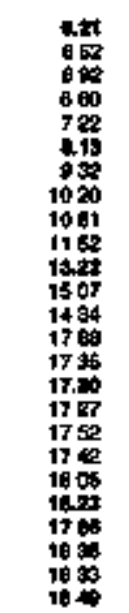 & 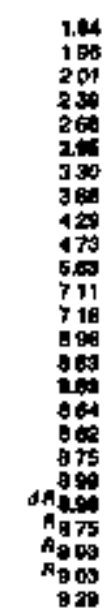 \\
\hline & \multicolumn{10}{|c|}{ 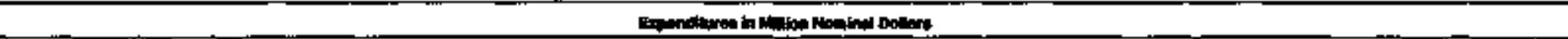 } \\
\hline 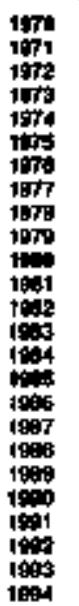 & $\begin{array}{l}09 \\
02 \\
03 \\
02 \\
02 \\
01 \\
01 \\
01 \\
09 \\
09 \\
04 \\
04 \\
04 \\
09 \\
18 \\
18 \\
28 \\
04 \\
04 \\
01 \\
01 \\
07 \\
01 \\
01 \\
03 \\
01 \\
01 \\
01 \\
01\end{array}$ & 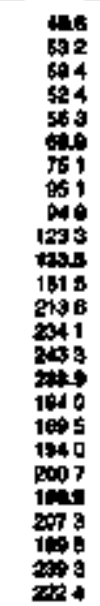 & 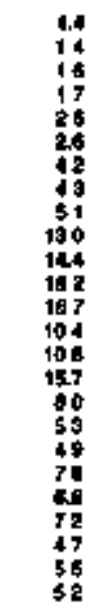 & 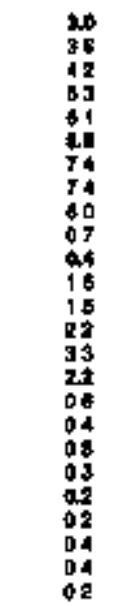 & 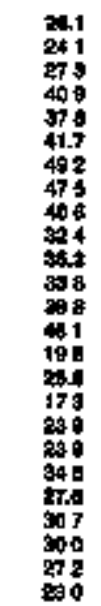 & 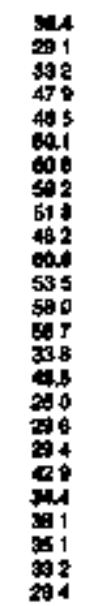 & $\begin{array}{l}= \\
= \\
= \\
= \\
= \\
= \\
= \\
z \\
= \\
= \\
= \\
04 \\
40 \\
40 \\
41\end{array}$ & 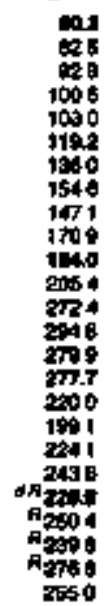 & 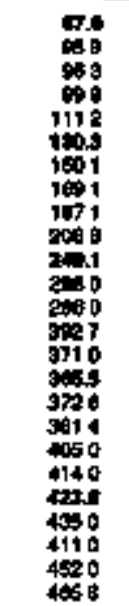 & 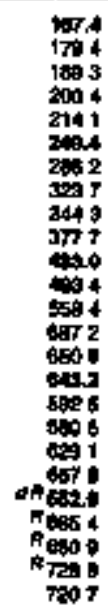 \\
\hline
\end{tabular}




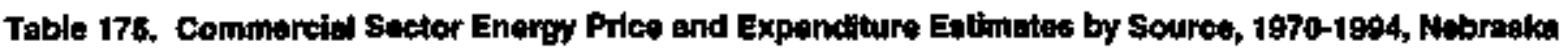

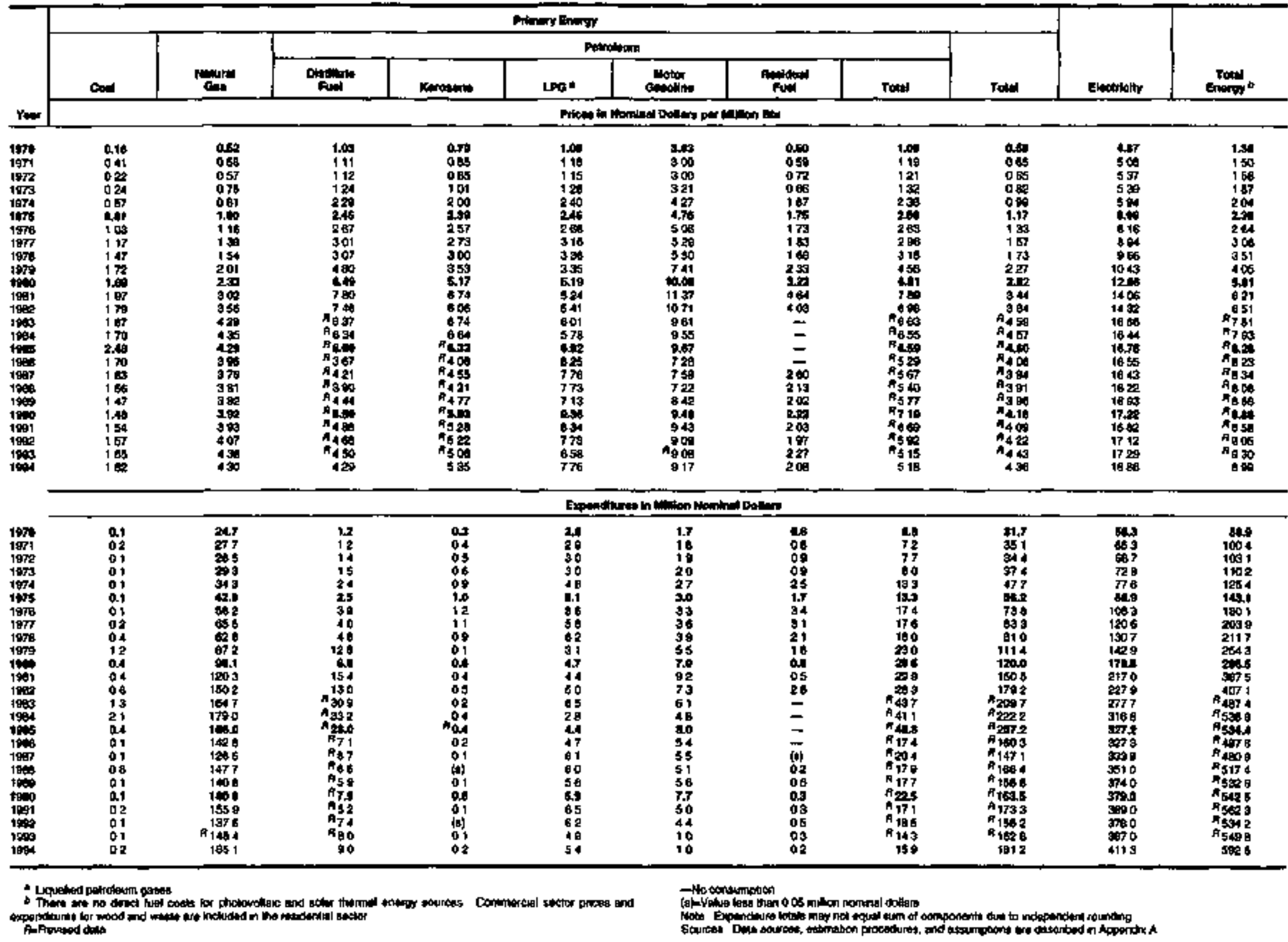




\begin{tabular}{|c|c|c|c|c|c|c|c|c|c|c|c|c|c|c|c|c|c|}
\hline & \multicolumn{15}{|c|}{ 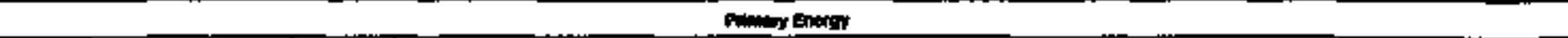 } & \multirow[b]{3}{*}{ 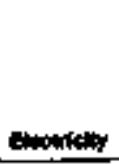 } & \multirow[b]{3}{*}{ 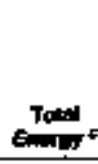 } \\
\hline & \multicolumn{3}{|c|}{ Cnd } & \multirow[b]{2}{*}{ ind } & \multicolumn{9}{|c|}{ 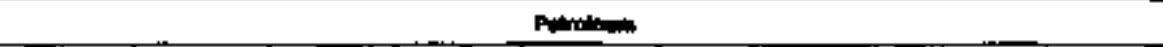 } & \multirow[b]{2}{*}{ Elonumet } & \multirow[b]{2}{*}{ Totatc } & & \\
\hline & chent & con & Tolet & & 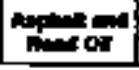 & 10. & Hawown & LQC. & trutingats & 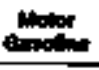 & Fint & $a \cos ^{\circ}$ & Tot & & & & \\
\hline $\mathrm{rem}$ & \multicolumn{17}{|c|}{ 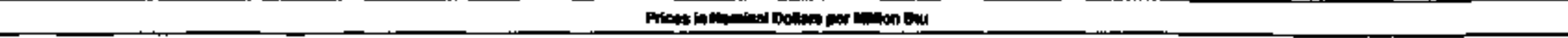 } \\
\hline \multirow[t]{2}{*}{ 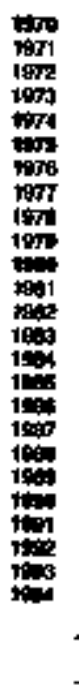 } & $\begin{array}{l}= \\
= \\
= \\
= \\
= \\
= \\
= \\
= \\
= \\
= \\
= \\
= \\
=\end{array}$ & 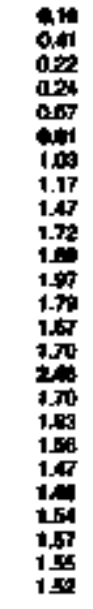 & 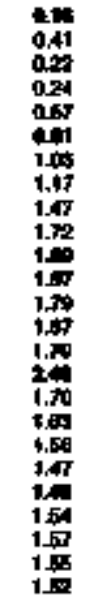 & 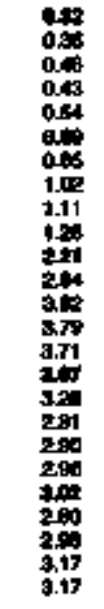 & 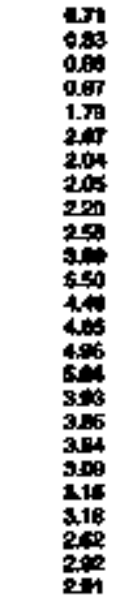 & 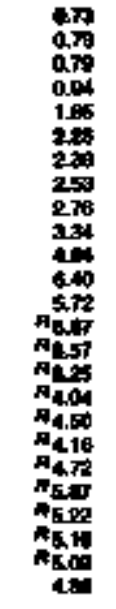 & 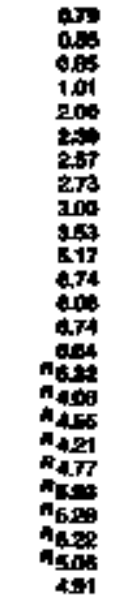 & 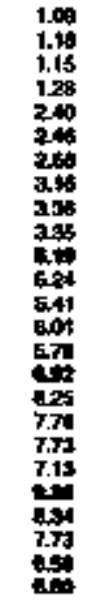 & 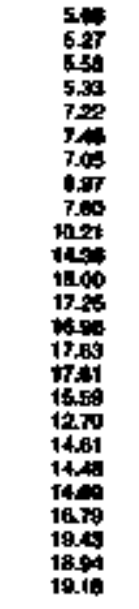 & 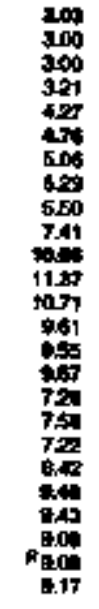 & 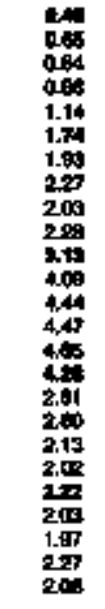 & 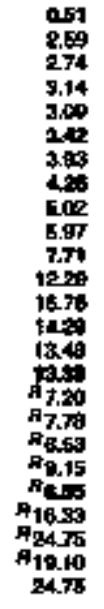 & 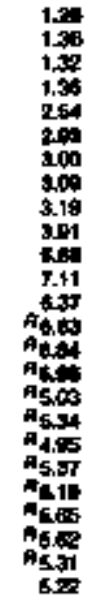 & 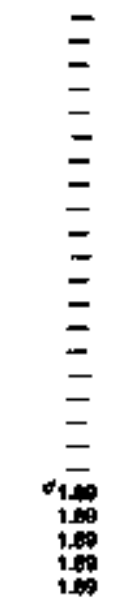 & 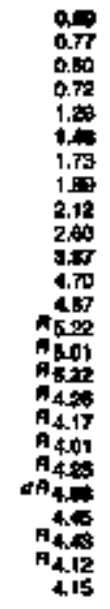 & 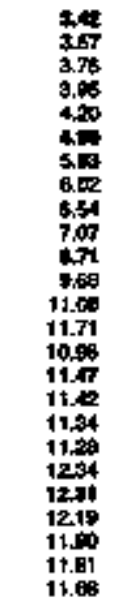 & 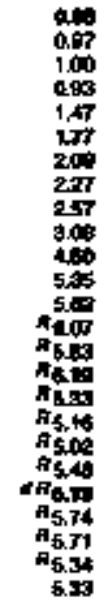 \\
\hline & \multicolumn{17}{|c|}{ 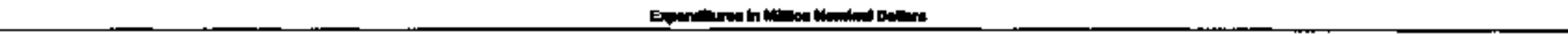 } \\
\hline 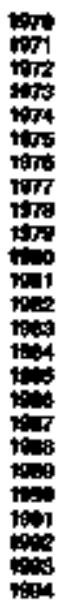 & $\begin{array}{l}= \\
= \\
= \\
= \\
= \\
= \\
= \\
= \\
= \\
= \\
= \\
= \\
= \\
=\end{array}$ & 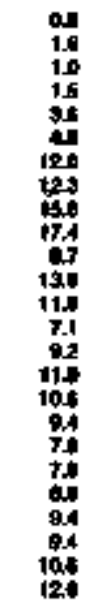 & 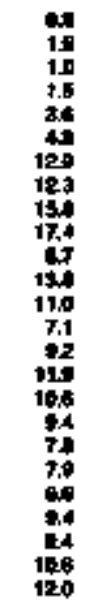 & 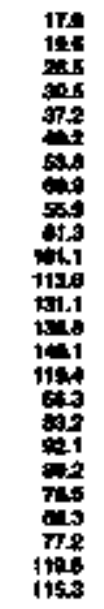 & 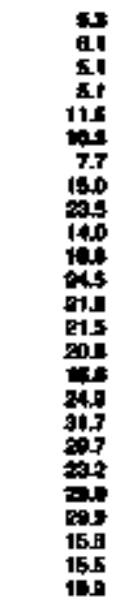 & 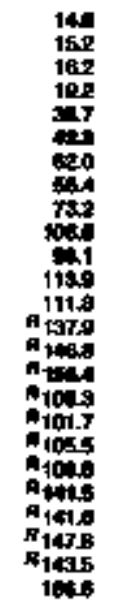 & 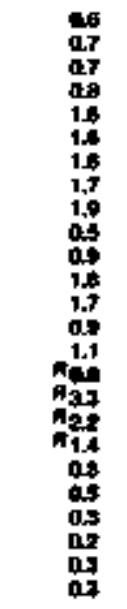 & 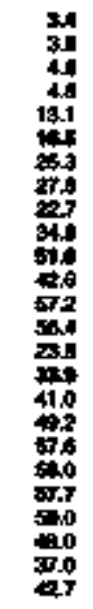 & 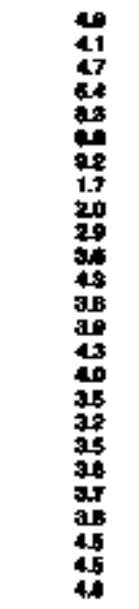 & 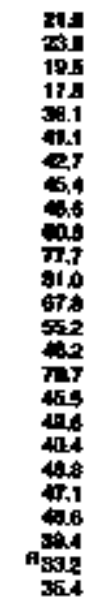 & 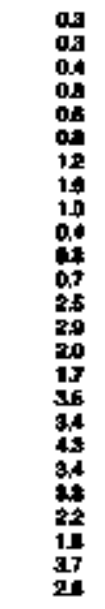 & 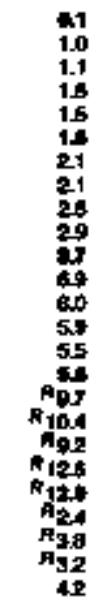 & 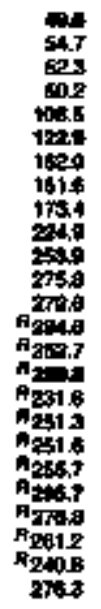 & $\begin{array}{l}= \\
= \\
= \\
= \\
= \\
= \\
= \\
= \\
= \\
= \\
= \\
= \\
= \\
0.3 \\
0.9 \\
0.3 \\
0.3\end{array}$ & 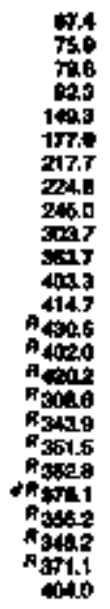 & 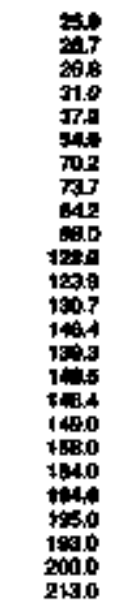 & 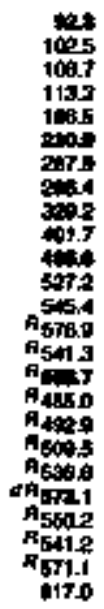 \\
\hline
\end{tabular}

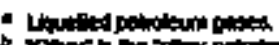

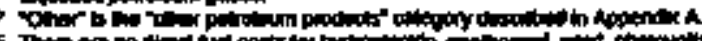

I Die

Amatom then

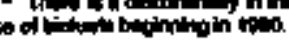

$\rightarrow$

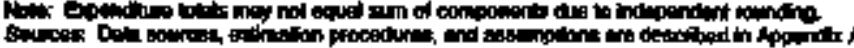




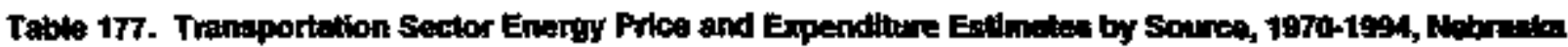

\begin{tabular}{|c|c|c|c|c|c|c|c|c|c|c|c|c|c|}
\hline \multirow[b]{4}{*}{ Yeer } & \multicolumn{11}{|c|}{ Phtr t En } & \multirow[b]{3}{*}{ Enotitexp } & \multirow[b]{3}{*}{ tont } \\
\hline & \multirow[b]{2}{*}{ cont } & \multirow[b]{2}{*}{ cimil } & \multicolumn{8}{|c|}{ 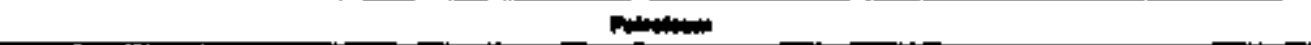 } & \multirow[b]{2}{*}{ Tot } & & \\
\hline & & & Averion & Pin & الוk & $\cot$ & Wirkatet & 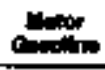 & Fint & Fot & & & \\
\hline & \multicolumn{13}{|c|}{ 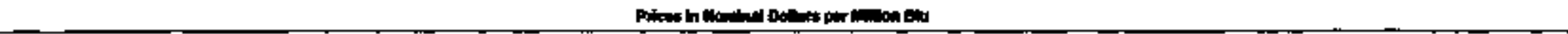 } \\
\hline \multirow[t]{2}{*}{ 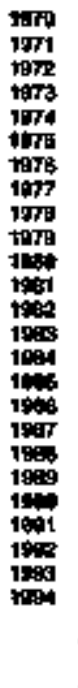 } & $\begin{array}{l}0.51 \\
0.11 \\
0.28 \\
0.20 \\
0.57 \\
0.51 \\
1.00 \\
1.17 \\
= \\
= \\
= \\
= \\
= \\
= \\
= \\
= \\
= \\
= \\
=\end{array}$ & $\begin{array}{l}= \\
= \\
= \\
= \\
= \\
= \\
= \\
= \\
= \\
= \\
= \\
= \\
= \\
17 \\
474\end{array}$ & 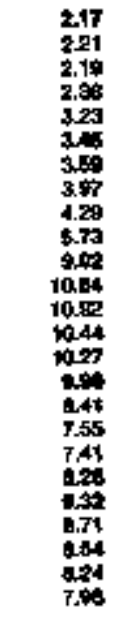 & 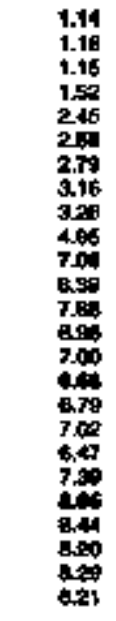 & 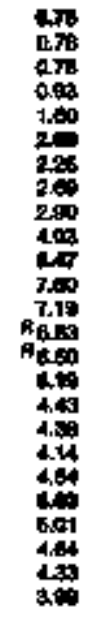 & 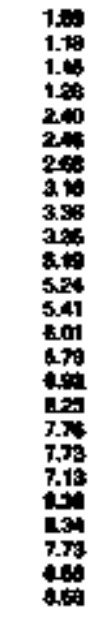 & 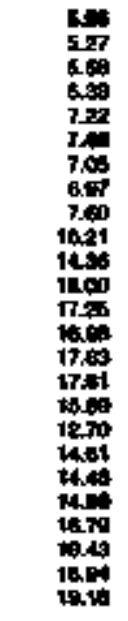 & 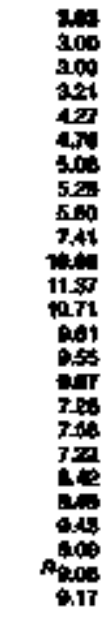 & 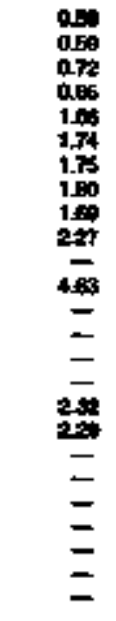 & 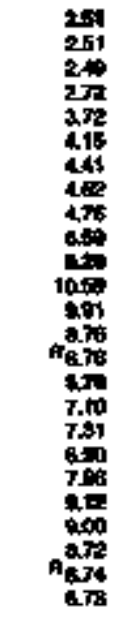 & 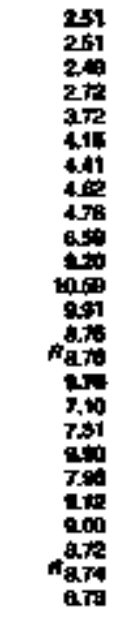 & $\begin{array}{l}= \\
= \\
= \\
= \\
= \\
= \\
= \\
= \\
= \\
= \\
= \\
= \\
= \\
=\end{array}$ & 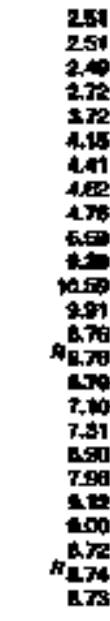 \\
\hline & \multicolumn{13}{|c|}{ 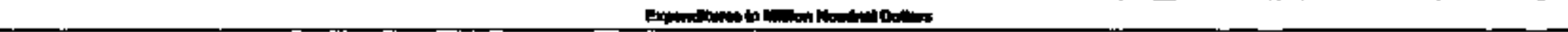 } \\
\hline 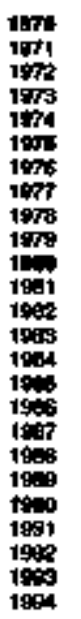 & 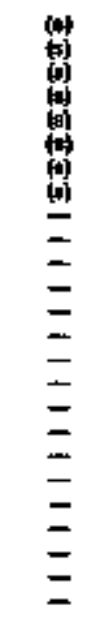 & $\begin{array}{l}= \\
= \\
= \\
= \\
= \\
= \\
= \\
= \\
= \\
= \\
= \\
= \\
= \\
\\
0\end{array}$ & 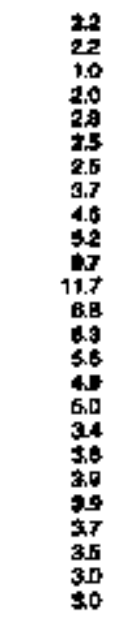 & 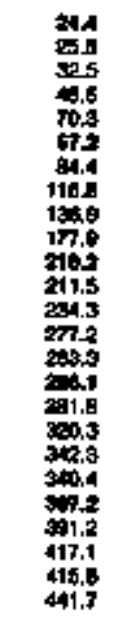 & 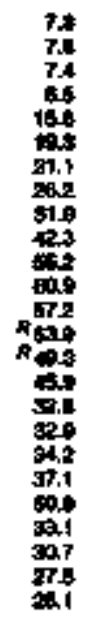 & $\begin{array}{l}1.1 \\
1.0 \\
1.1 \\
2.1 \\
2.1 \\
2.7 \\
2.8 \\
41 \\
2.2 \\
.45 \\
4.0 \\
3.4 \\
4.5 \\
4.5 \\
1.1 \\
1.4 \\
1.4 \\
1.7 \\
21 \\
1.4 \\
1.3 \\
1.1 \\
2.5\end{array}$ & 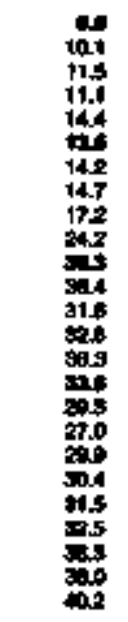 & 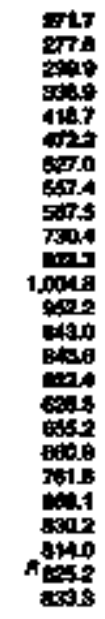 & $\begin{array}{l}a 7 \\
06 \\
00 \\
0.7 \\
18 \\
18 \\
04 \\
0.9 \\
0.2 \\
\frac{a 1}{a 1} \\
= \\
= \\
= \\
0 \\
= \\
= \\
= \\
= \\
=\end{array}$ & 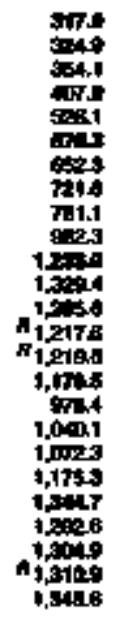 & 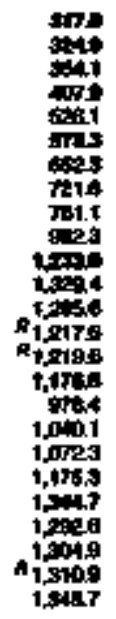 & $\begin{array}{l}= \\
= \\
= \\
= \\
= \\
= \\
= \\
= \\
= \\
= \\
= \\
= \\
= \\
=\end{array}$ & 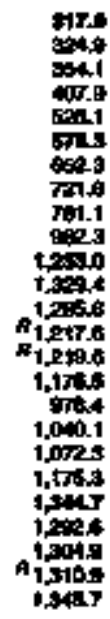 \\
\hline
\end{tabular}




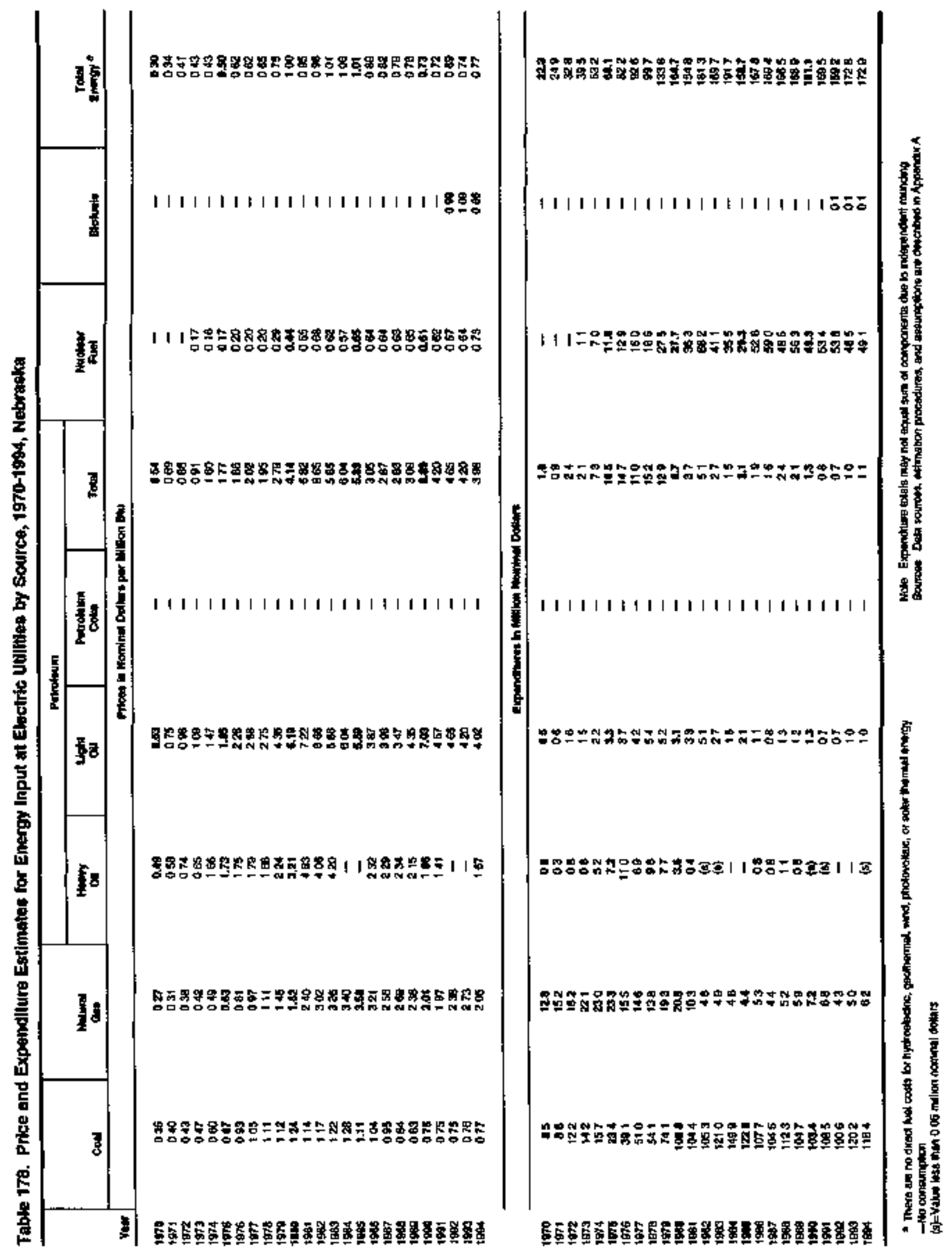


Table 179. Energy Price and Expendfure Ethmolis by Source, 1970-1994, Nevado

\begin{tabular}{|c|c|c|c|c|c|c|c|c|c|c|c|c|c|c|c|c|c|}
\hline \multirow[b]{4}{*}{ var } & \multicolumn{14}{|c|}{ Priansy Encingr } & \multirow{3}{*}{ 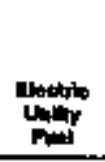 } & \multirow{3}{*}{ 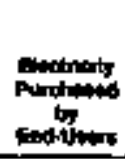 } & \multirow[b]{3}{*}{ 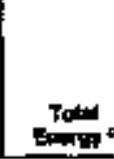 } \\
\hline & \multicolumn{3}{|c|}{ Co:l } & \multirow[b]{2}{*}{ Monst } & \multicolumn{7}{|c|}{ Puratiman } & \multirow[b]{2}{*}{ mingeis } & \multirow[b]{2}{*}{ Potuste } & \multirow[b]{2}{*}{ Total $F$} & & & \\
\hline & Colten & sintin & Tatal & & Otom & Avt & LPa & & 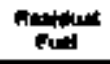 & Otwer * & Tand & & & & & & \\
\hline & \multicolumn{17}{|c|}{ 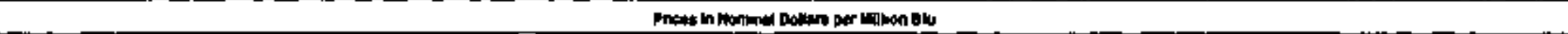 } \\
\hline \multirow[t]{2}{*}{ 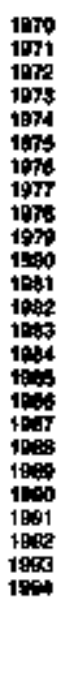 } & $\begin{array}{l}= \\
= \\
= \\
= \\
= \\
= \\
= \\
= \\
= \\
= \\
= \\
= \\
=\end{array}$ & 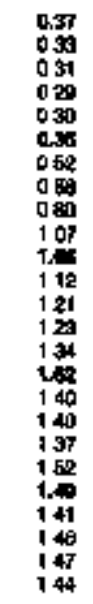 & 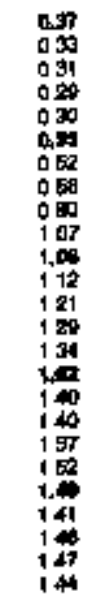 & 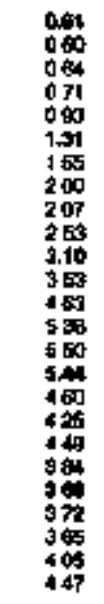 & 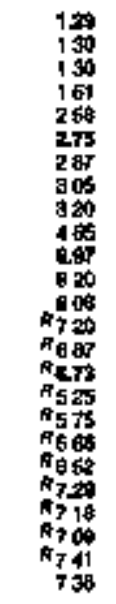 & 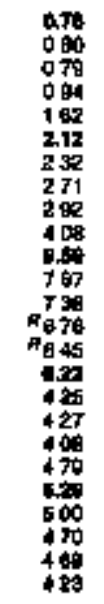 & 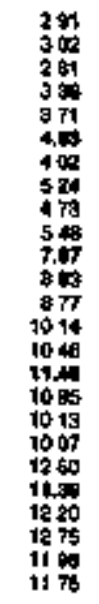 & 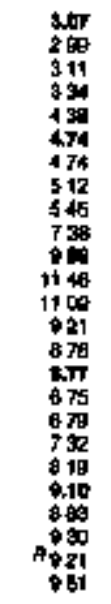 & 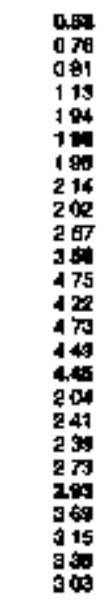 & 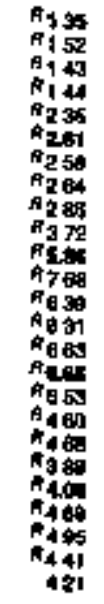 & 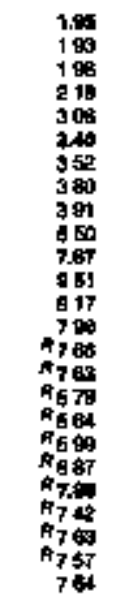 & $\begin{array}{l}= \\
= \\
= \\
= \\
= \\
= \\
= \\
= \\
= \\
= \\
= \\
= \\
= \\
=\end{array}$ & $\begin{array}{l}= \\
= \\
= \\
= \\
= \\
= \\
= \\
= \\
= \\
= \\
= \\
403 \\
464 \\
464\end{array}$ & 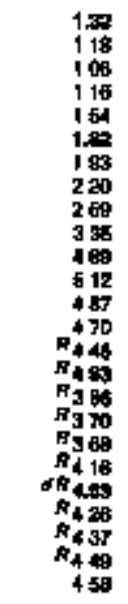 & 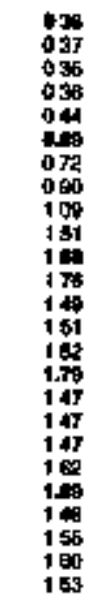 & 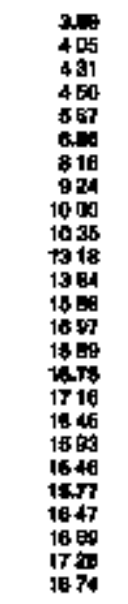 & 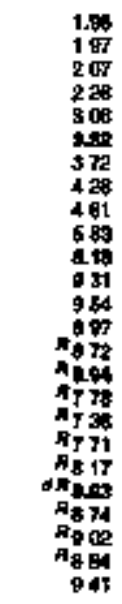 \\
\hline & \multicolumn{17}{|c|}{ 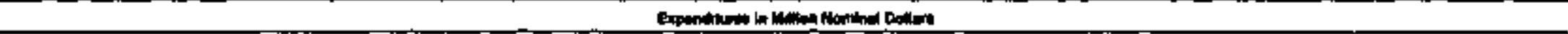 } \\
\hline 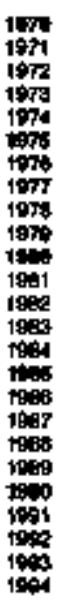 & $\begin{array}{l}= \\
z \\
= \\
= \\
= \\
= \\
= \\
= \\
= \\
= \\
= \\
= \\
= \\
=\end{array}$ & 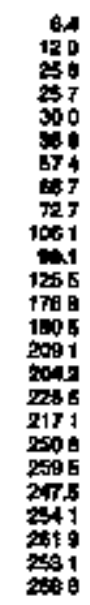 & 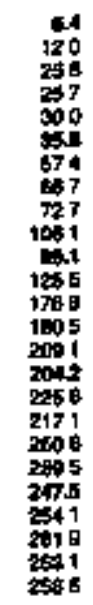 & 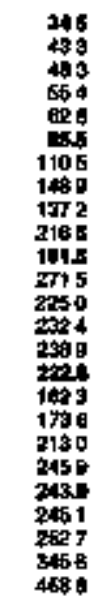 & 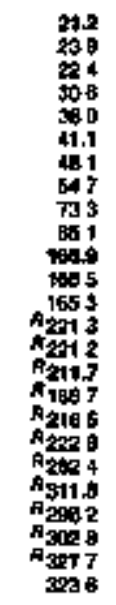 & 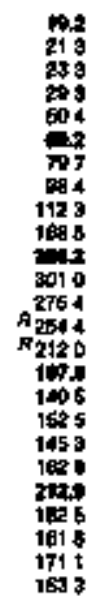 & 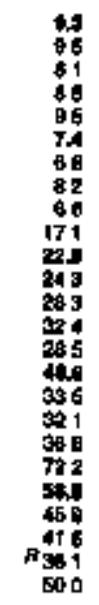 & 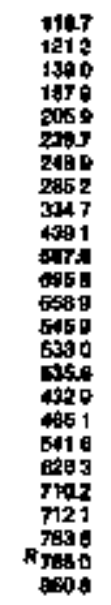 & 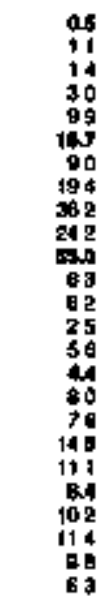 & 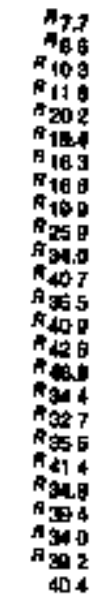 & 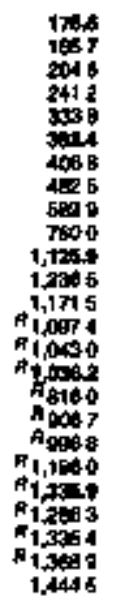 & $\begin{array}{l}= \\
= \\
= \\
= \\
= \\
= \\
= \\
= \\
= \\
= \\
= \\
= \\
=\end{array}$ & $\begin{array}{l}= \\
= \\
= \\
= \\
= \\
= \\
= \\
= \\
= \\
= \\
= \\
=5.2 \\
68 \\
68 \\
60 \\
6.8\end{array}$ & 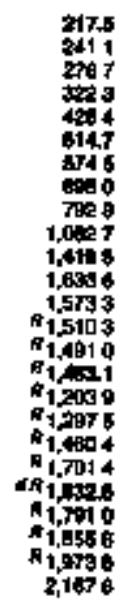 & 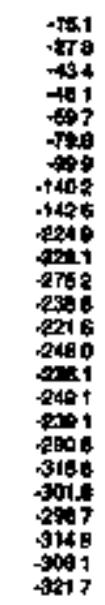 & 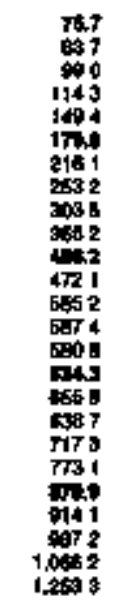 & 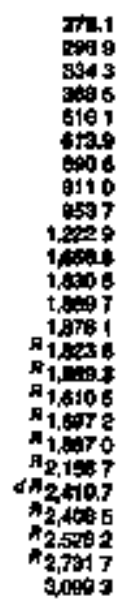 \\
\hline
\end{tabular}

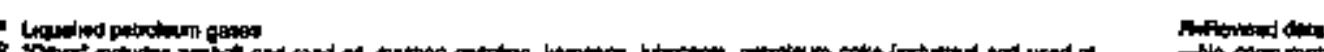

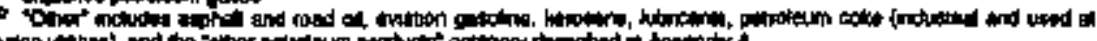

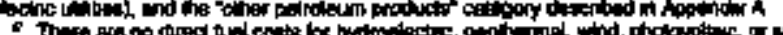

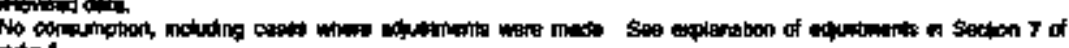

Thent

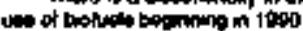

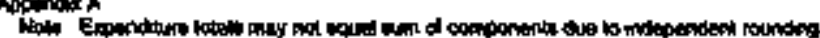

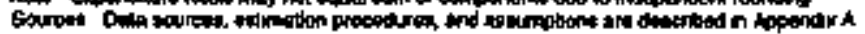




\begin{tabular}{|c|c|c|c|c|c|c|c|c|c|c|}
\hline & \multicolumn{8}{|c|}{ 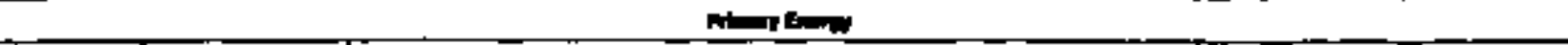 } & \multirow[b]{3}{*}{ enoutels } & \multirow{3}{*}{ Tow } \\
\hline & \multirow[b]{2}{*}{ EN } & \multirow{2}{*}{ s. } & \multicolumn{4}{|c|}{ (1) } & \multirow[b]{2}{*}{ (2) } & \multirow[b]{2}{*}{ 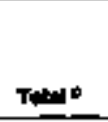 } & & \\
\hline & & & in & $x=0$ & IFa* & N & & & & \\
\hline$\underline{\text { v. }}$ & \multicolumn{10}{|c|}{ 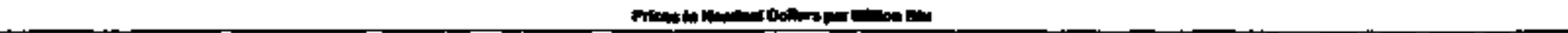 } \\
\hline \multirow[t]{2}{*}{ 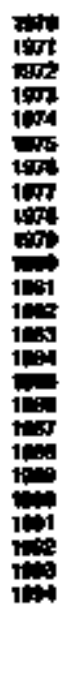 } & 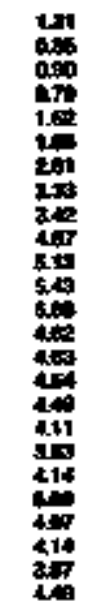 & 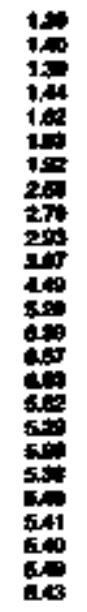 & 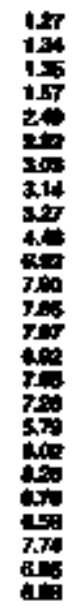 & 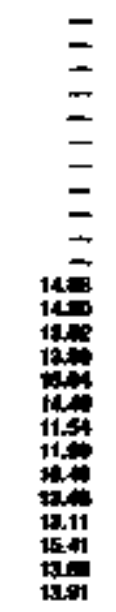 & 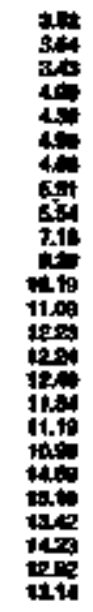 & 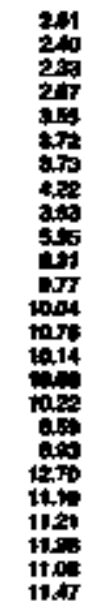 & 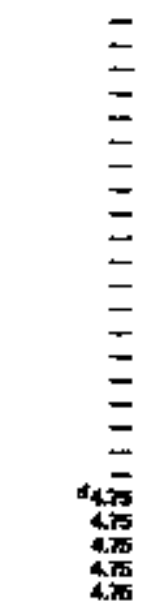 & 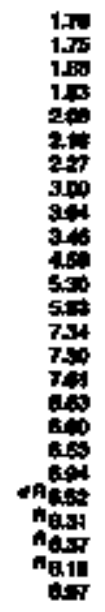 & 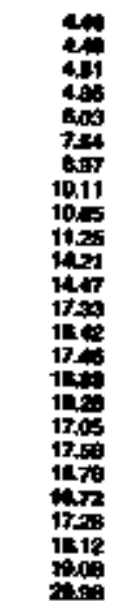 & 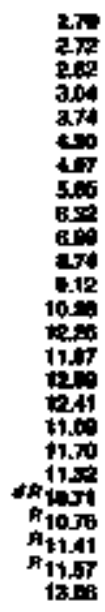 \\
\hline & \multicolumn{10}{|c|}{ 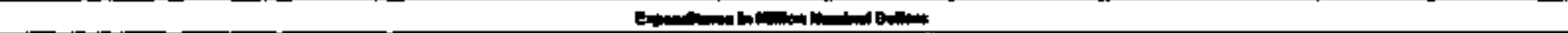 } \\
\hline 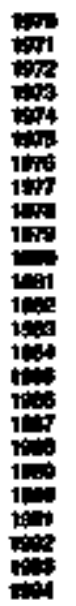 & 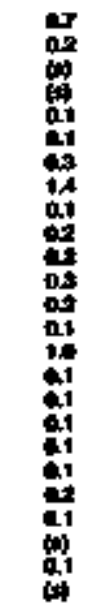 & 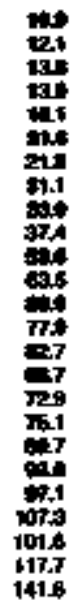 & 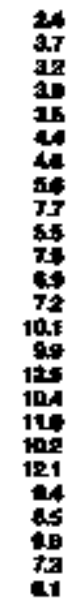 & 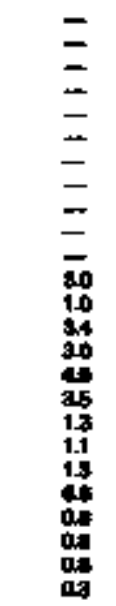 & 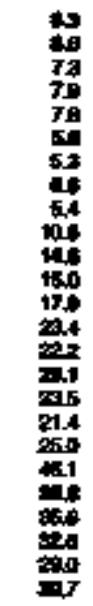 & 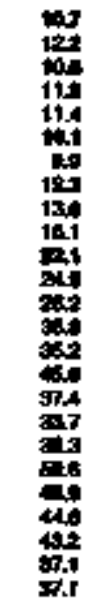 & $\begin{array}{l}= \\
= \\
= \\
= \\
= \\
= \\
= \\
= \\
= \\
= \\
= \\
5 \\
54 \\
59 \\
59\end{array}$ & 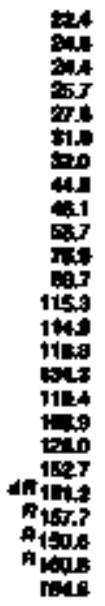 & 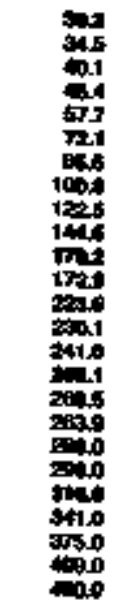 & 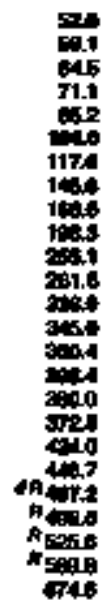 \\
\hline
\end{tabular}




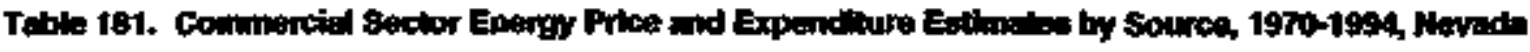

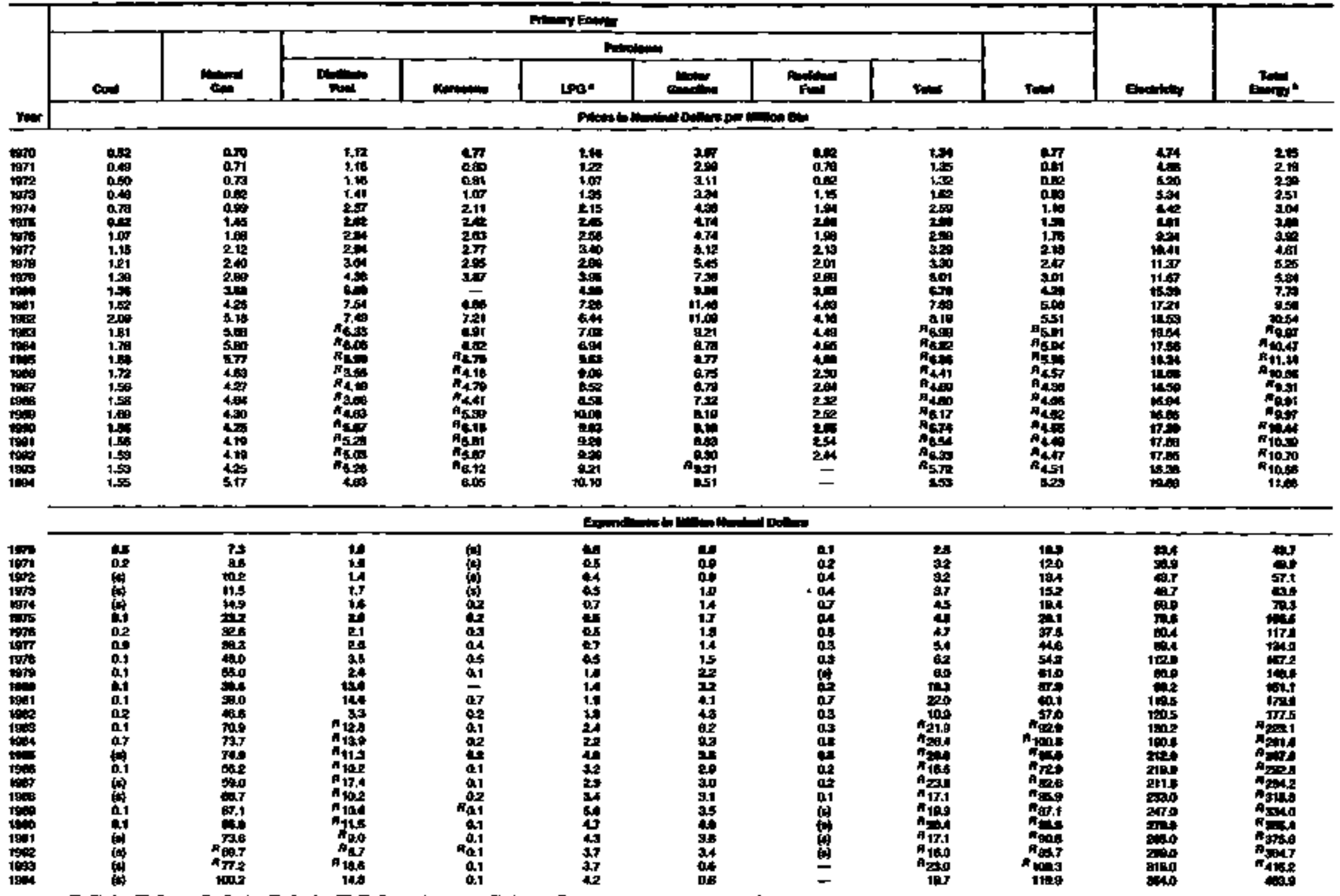


Table 182. Induetrled Soctor Energy Price and Expendilum Eytimetes by Source, 1970-1994, Noveda

\begin{tabular}{|c|c|c|c|c|c|c|c|c|c|c|c|c|c|c|c|c|c|}
\hline \multirow{2}{*}{ res } & \multicolumn{15}{|c|}{ 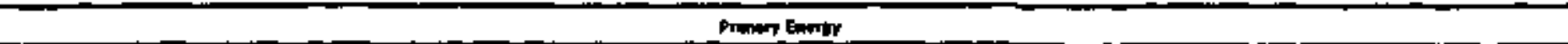 } & \multirow{2}{*}{ Ewertory } & \multirow{2}{*}{ 1. } \\
\hline & \multicolumn{3}{|c|}{$\infty$} & 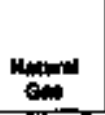 & \multicolumn{9}{|c|}{ Purolvers } & Ensonge & Town: & & \\
\hline 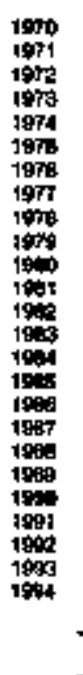 & $\begin{array}{l}= \\
= \\
= \\
z \\
= \\
z \\
z \\
= \\
= \\
= \\
z \\
z\end{array}$ & 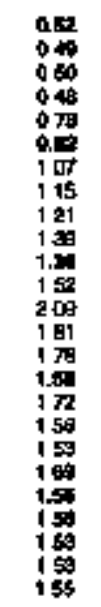 & 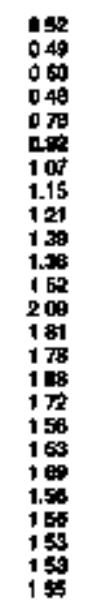 & 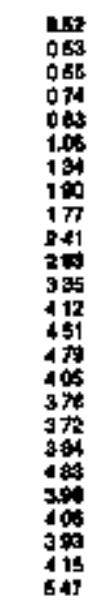 & 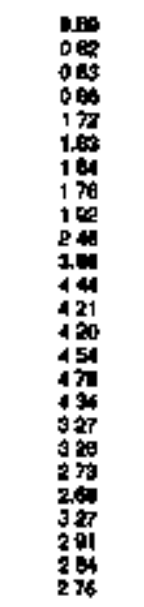 & 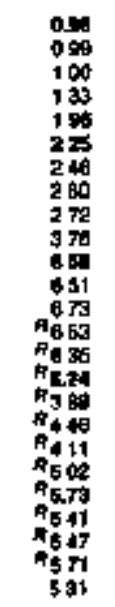 & 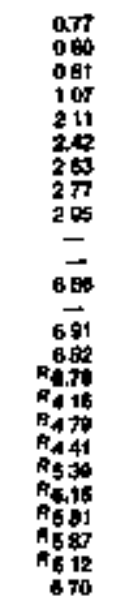 & 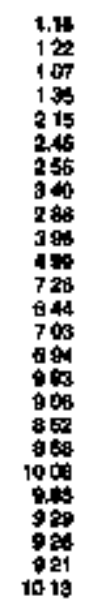 & 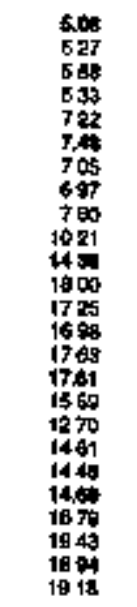 & 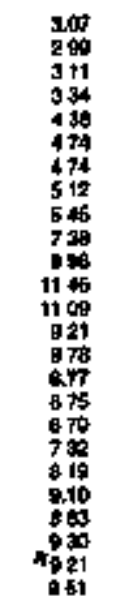 & 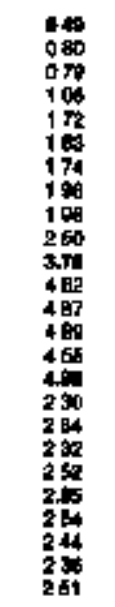 & 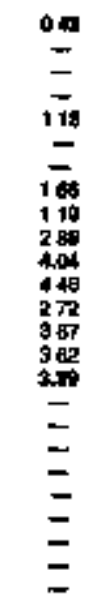 & 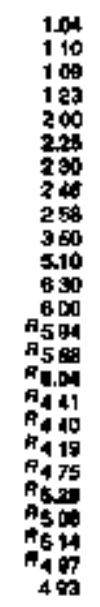 & $\begin{array}{l}= \\
= \\
= \\
= \\
= \\
= \\
= \\
= \\
= \\
= \\
= \\
\bar{z} \\
16 \\
180 \\
100\end{array}$ & 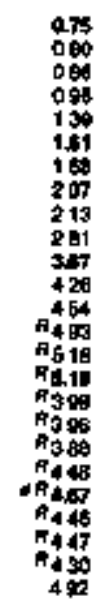 & 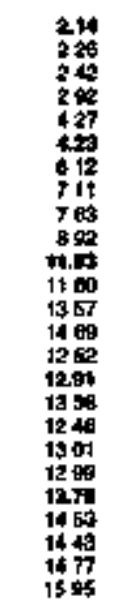 & 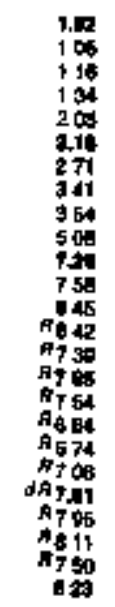 \\
\hline 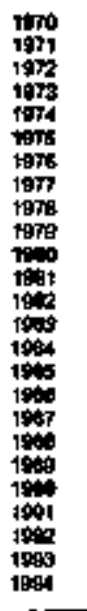 & $\begin{array}{l}= \\
= \\
z \\
z \\
z \\
z \\
= \\
= \\
= \\
= \\
= \\
= \\
=\end{array}$ & $\begin{array}{l}04 \\
14 \\
19 \\
18 \\
24 \\
14 \\
34 \\
34 \\
24 \\
34 \\
4 \\
198 \\
104 \\
71 \\
46 \\
41 \\
43 \\
41 \\
43 \\
64 \\
6.1 \\
71 \\
61 \\
70 \\
70\end{array}$ & 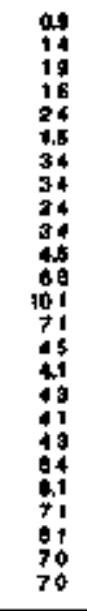 & 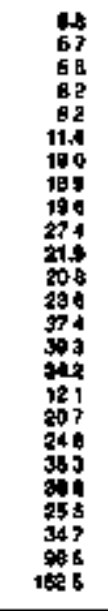 & 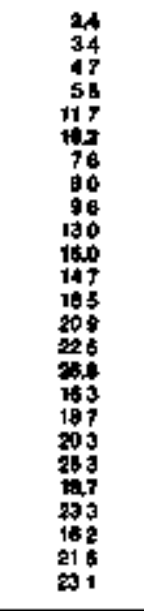 & 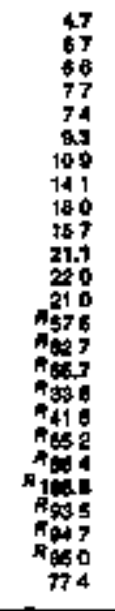 & 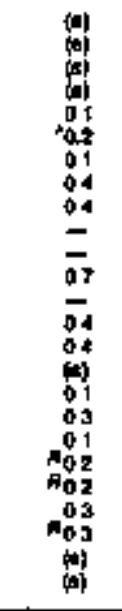 & 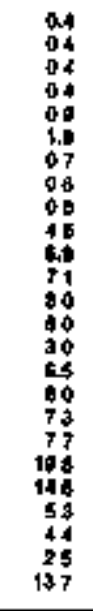 & $\begin{array}{l}99 \\
07 \\
99 \\
09 \\
10 \\
13 \\
19 \\
11 \\
12 \\
17 \\
27 \\
26 \\
23 \\
24 \\
20 \\
24 \\
21 \\
19 \\
22 \\
32 \\
23 \\
23 \\
29 \\
27 \\
20\end{array}$ & 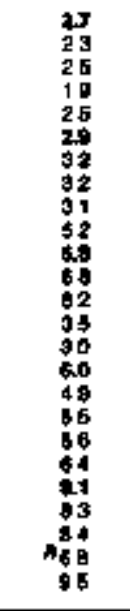 & 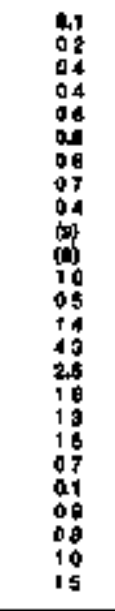 & 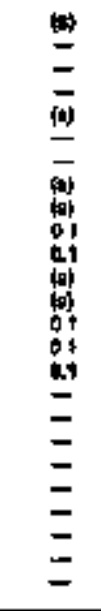 & 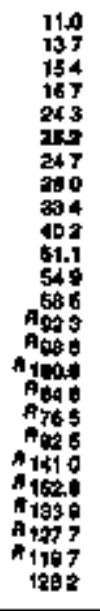 & 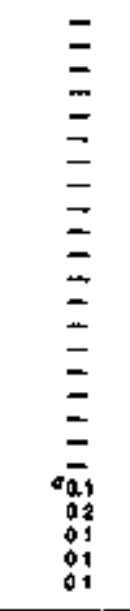 & 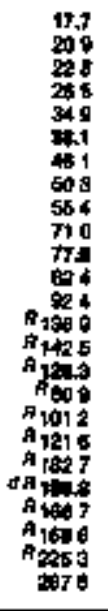 & 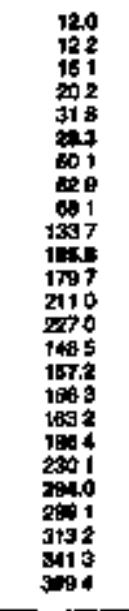 & 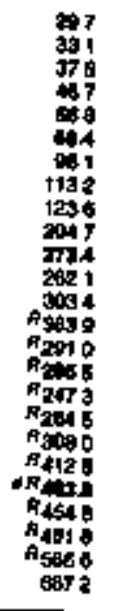 \\
\hline
\end{tabular}

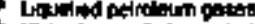

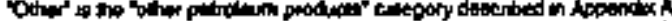

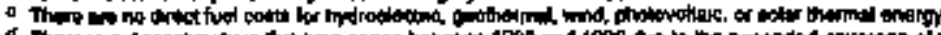

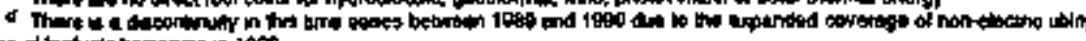

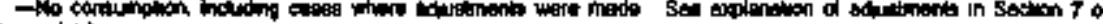
Apeendor $\lambda$

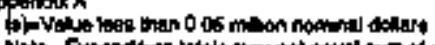

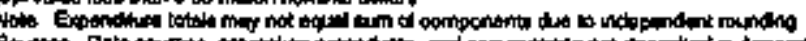

Afomerod detie

Enters intomition Admuliotration 


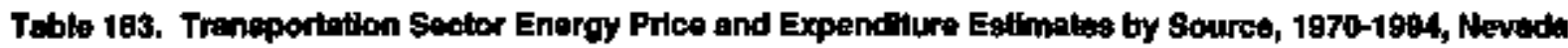

\begin{tabular}{|c|c|c|c|c|c|c|c|c|c|c|c|c|c|}
\hline \multirow[b]{4}{*}{$Y=$} & \multicolumn{11}{|c|}{ PNnoing Pinergy } & \multirow[b]{3}{*}{ Evolingly } & \multirow[b]{3}{*}{ Fotisy } \\
\hline & \multirow[b]{2}{*}{ call } & \multirow[b]{2}{*}{ 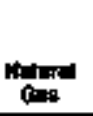 } & \multicolumn{8}{|c|}{ Putrialen } & \multirow[b]{2}{*}{ Told } & & \\
\hline & & & andination & 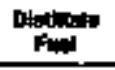 & Fin & $\mathbf{L u t h}^{\mathbf{4}}$ & Lovicants & monor & Powet & Tot & & & \\
\hline & \multicolumn{13}{|c|}{ 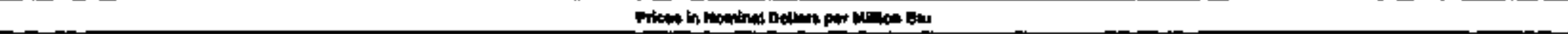 } \\
\hline \multirow[t]{2}{*}{ 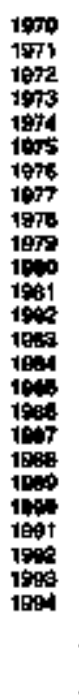 } & $\begin{array}{l}050 \\
049 \\
050 \\
040 \\
070 \\
002 \\
107 \\
115 \\
= \\
= \\
= \\
= \\
= \\
= \\
= \\
= \\
= \\
=\end{array}$ & $\begin{array}{l}= \\
= \\
= \\
= \\
= \\
= \\
= \\
= \\
= \\
= \\
= \\
= \\
399 \\
334 \\
392 \\
300\end{array}$ & 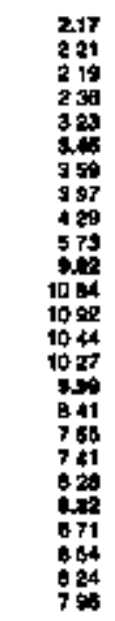 & 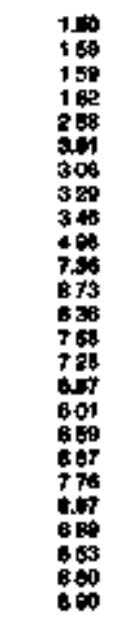 & 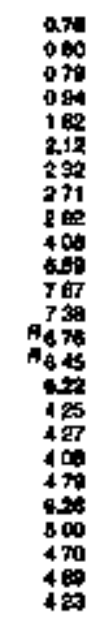 & 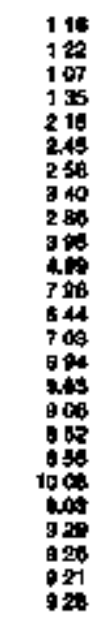 & 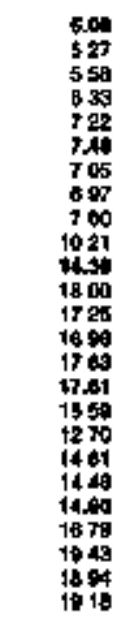 & 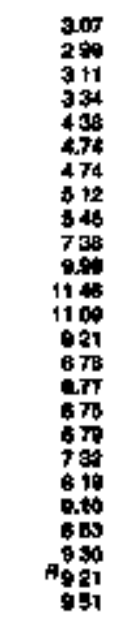 & $\begin{array}{l}100 \\
07 \\
011 \\
107 \\
230 \\
1404 \\
240 \\
= \\
= \\
= \\
40 \\
= \\
= \\
= \\
= \\
= \\
=\end{array}$ & 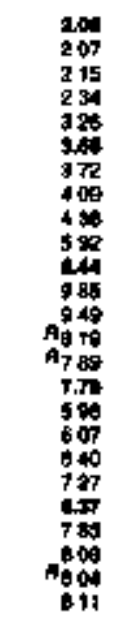 & 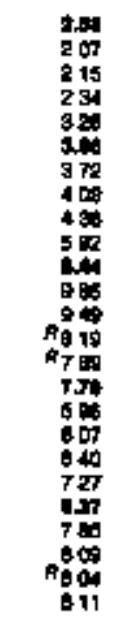 & $\begin{array}{l}= \\
= \\
= \\
= \\
z \\
= \\
= \\
= \\
= \\
= \\
= \\
= \\
= \\
= \\
=\end{array}$ & 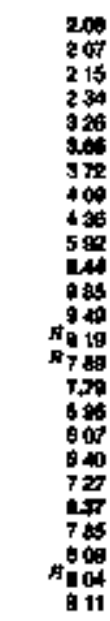 \\
\hline & \multicolumn{13}{|c|}{ 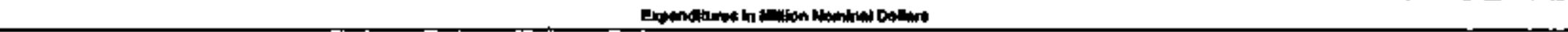 } \\
\hline 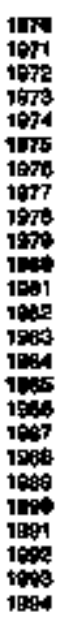 & $\begin{array}{l}09 \\
0 \\
0 \\
0 \\
= \\
= \\
= \\
= \\
= \\
= \\
= \\
= \\
= \\
= \\
= \\
=\end{array}$ & $\begin{array}{l}= \\
= \\
= \\
= \\
= \\
= \\
= \\
= \\
= \\
= \\
= \\
= \\
= \\
01 \\
00 \\
01 \\
01\end{array}$ & 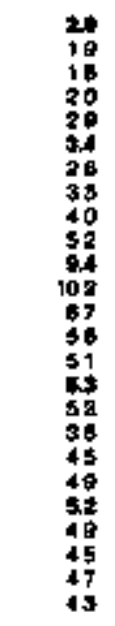 & 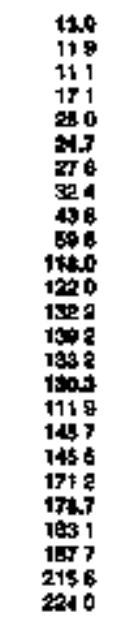 & 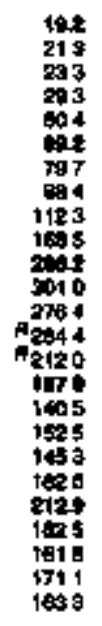 & 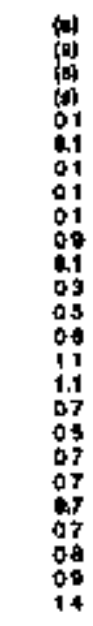 & $\begin{array}{l}26 \\
26 \\
30 \\
33 \\
43 \\
42 \\
44 \\
35 \\
41 \\
38 \\
73 \\
97 \\
78 \\
79 \\
97 \\
97 \\
70 \\
65 \\
72 \\
73 \\
74 \\
78 \\
92 \\
01 \\
96\end{array}$ & 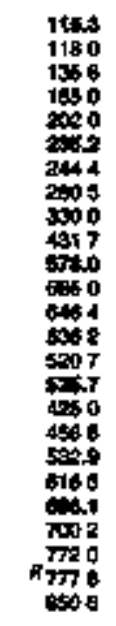 & $\begin{array}{l}\frac{10}{2} \\
{[a b]} \\
00] \\
001 \\
0.1 \\
01 \\
01 \\
{[0]} \\
= \\
= \\
= \\
01 \\
015] \\
= \\
= \\
= \\
= \\
= \\
= \\
=\end{array}$ & 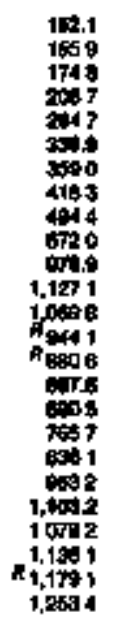 & 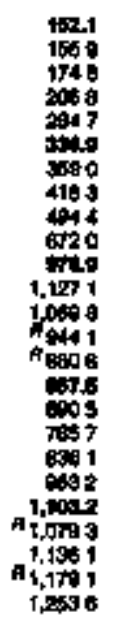 & $\begin{array}{l}= \\
= \\
= \\
= \\
= \\
= \\
= \\
= \\
= \\
= \\
= \\
= \\
=\end{array}$ & 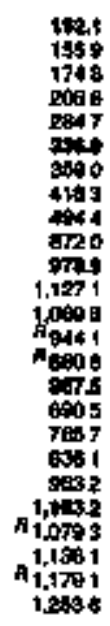 \\
\hline
\end{tabular}




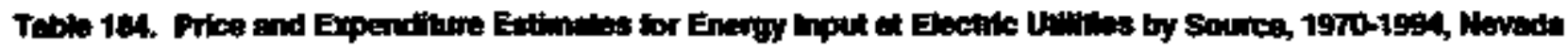

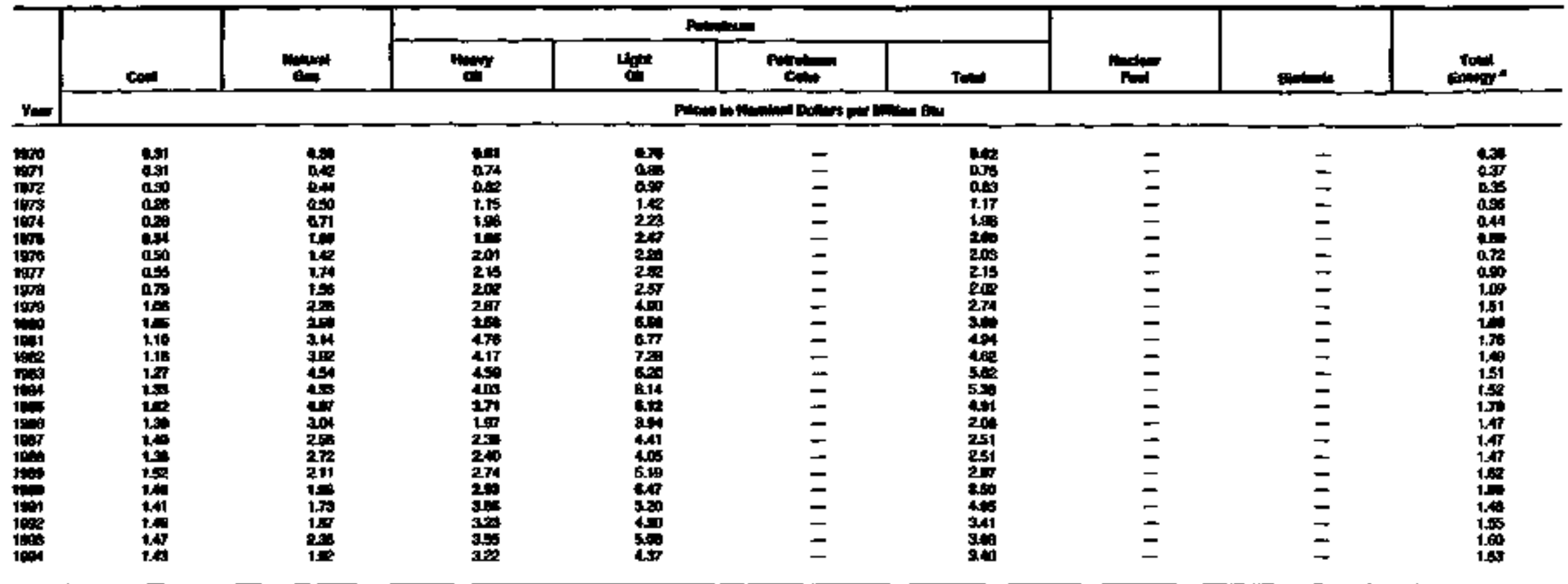

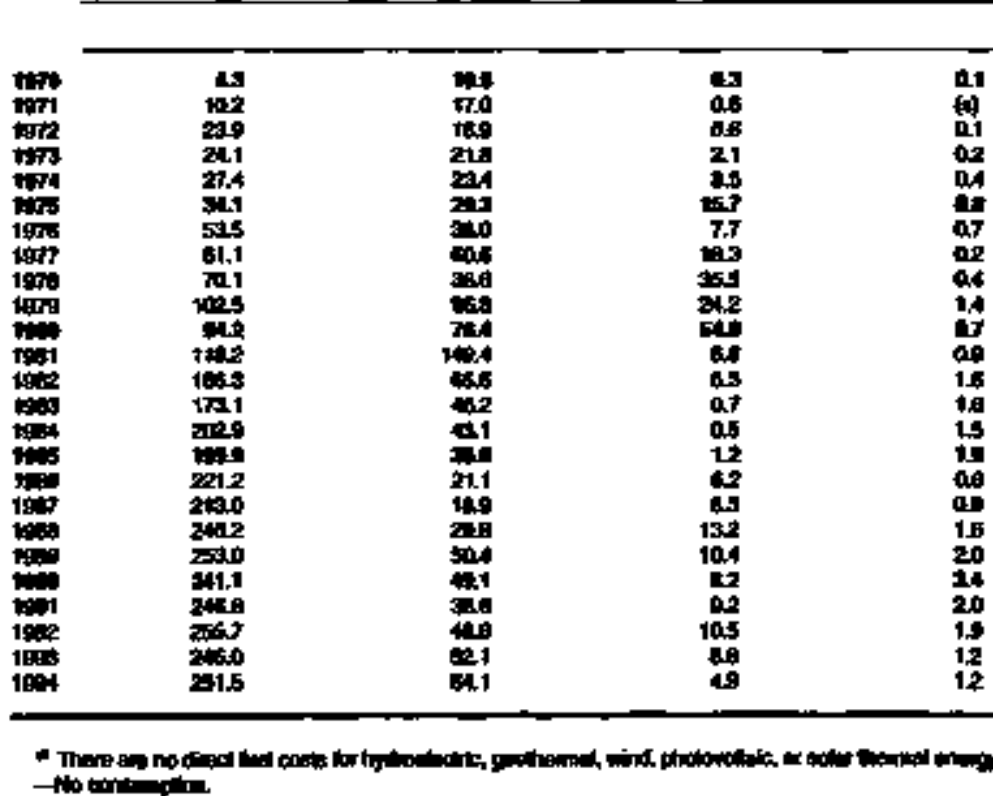

Ey

=

of

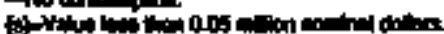

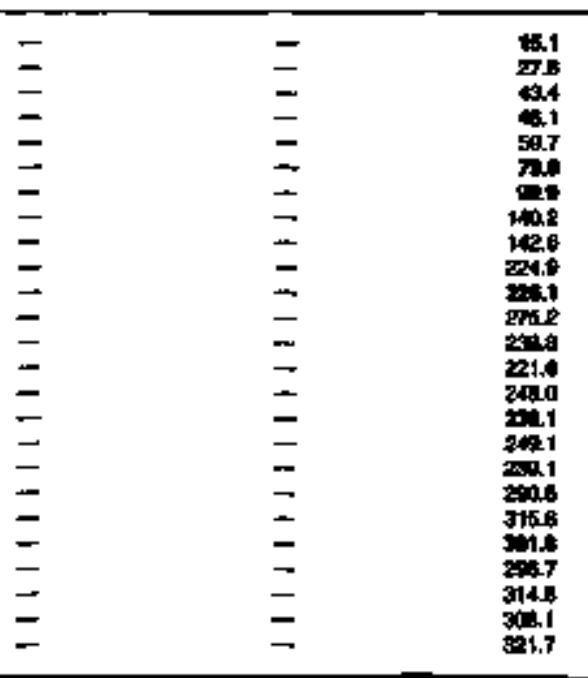




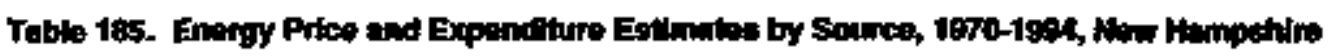

\begin{tabular}{|c|c|c|c|c|c|c|c|c|c|c|c|c|c|c|c|c|c|}
\hline & \multicolumn{13}{|c|}{ nimbers } & \multirow[b]{2}{*}{ Tnt } & \multirow{2}{*}{\multicolumn{2}{|c|}{ 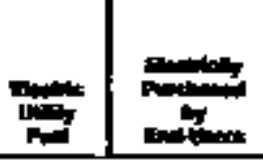 }} & \multirow[b]{2}{*}{ ז } \\
\hline & catho & تبv & $T=$ & r & مו1 & An & Le* & & 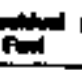 & 0 & $T=1$ & Fin & 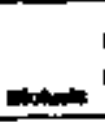 & & & & \\
\hline $\mathrm{V}=$ & \multicolumn{17}{|c|}{ 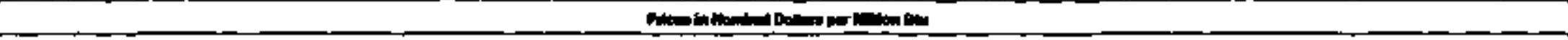 } \\
\hline \multirow[t]{2}{*}{ 番 } & $\begin{array}{l}= \\
= \\
= \\
= \\
= \\
= \\
= \\
= \\
= \\
= \\
=\end{array}$ & 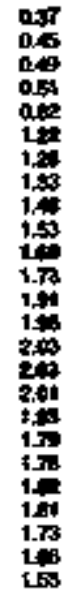 & 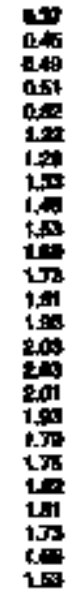 & 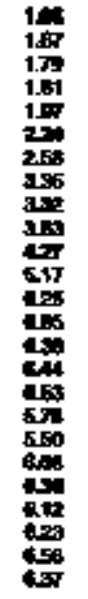 & 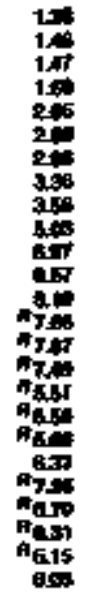 & 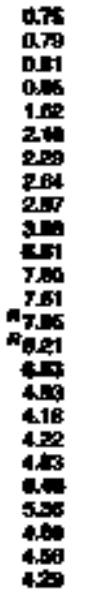 & 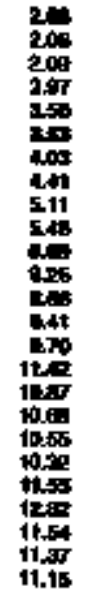 & 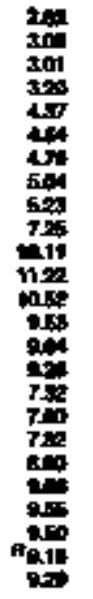 & 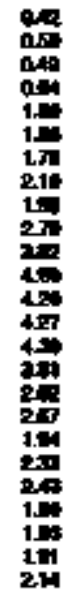 & 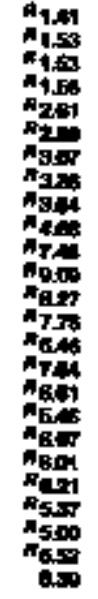 & 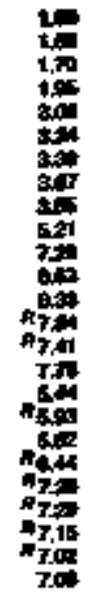 & $\begin{array}{l}= \\
= \\
= \\
= \\
= \\
= \\
= \\
= \\
= \\
= \\
= \\
=0 \\
000\end{array}$ & $\begin{array}{l}= \\
= \\
= \\
= \\
= \\
= \\
= \\
= \\
= \\
= \\
150 \\
158 \\
151\end{array}$ & 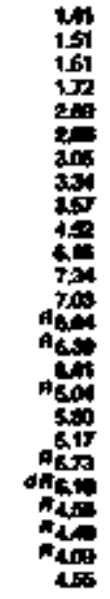 & 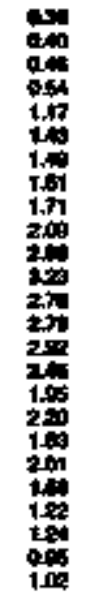 & 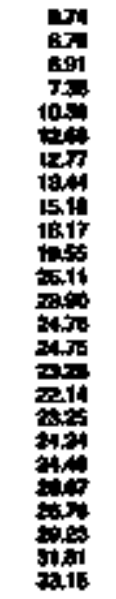 & 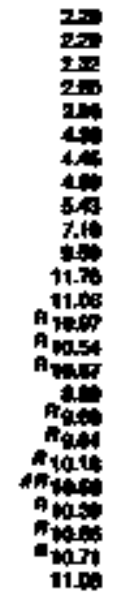 \\
\hline & \multicolumn{17}{|c|}{ 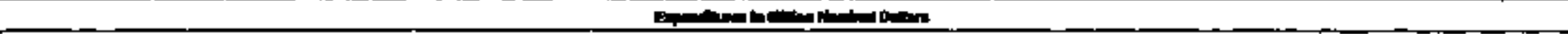 } \\
\hline . & $\begin{array}{l}= \\
= \\
= \\
= \\
= \\
= \\
= \\
= \\
= \\
= \\
= \\
= \\
=\end{array}$ & 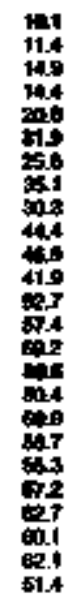 & 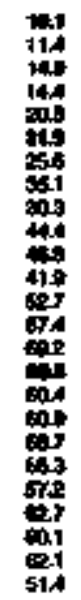 & 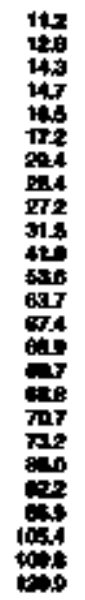 & 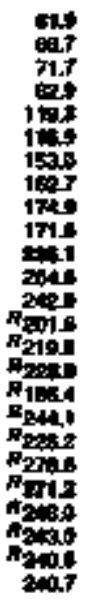 & 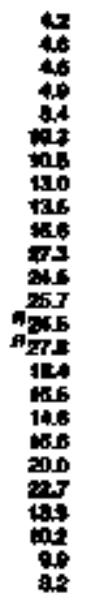 & 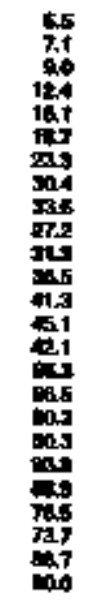 & 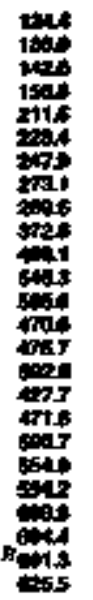 & 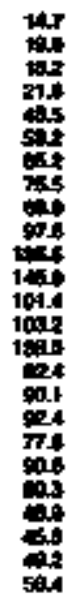 & 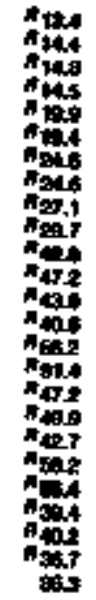 & 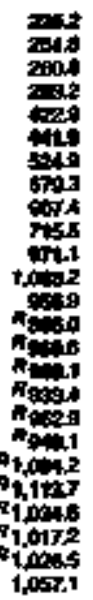 & $\begin{array}{l}= \\
= \\
= \\
= \\
= \\
= \\
= \\
= \\
= \\
= \\
= \\
= \\
010 \\
010 \\
050\end{array}$ & $\begin{array}{l}= \\
= \\
= \\
= \\
= \\
= \\
= \\
= \\
= \\
= \\
= \\
=0 \\
21.1 \\
215 \\
21.5\end{array}$ & 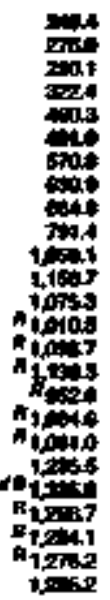 & 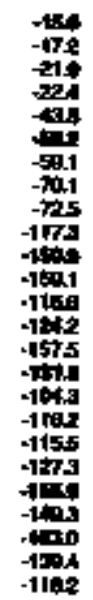 & 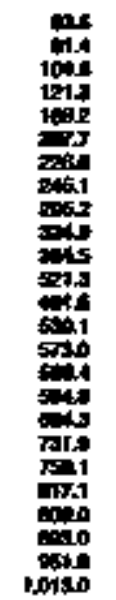 & 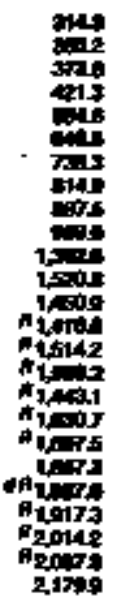 \\
\hline
\end{tabular}

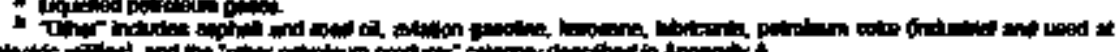

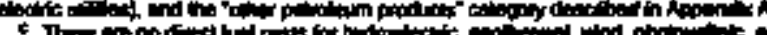

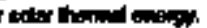

Artiond th

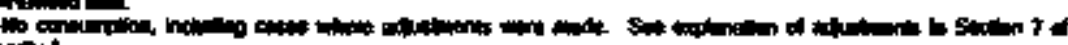
Anter

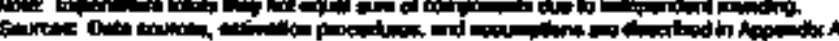

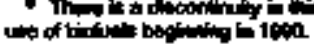




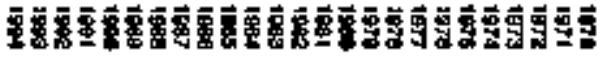

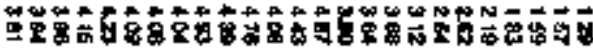

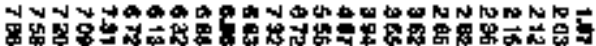

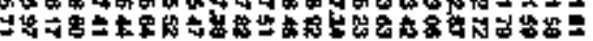

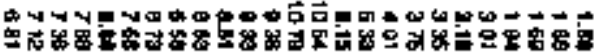

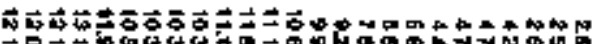

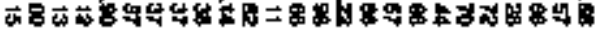

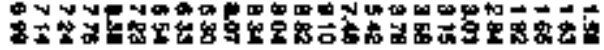

$\cos 20$

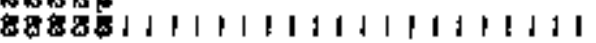

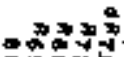

\$\&\$

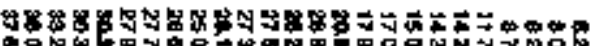

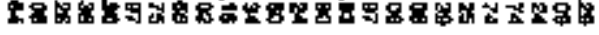

$\Rightarrow x=5$

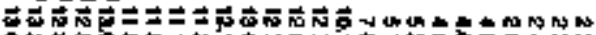

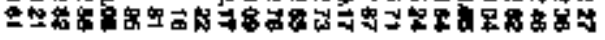

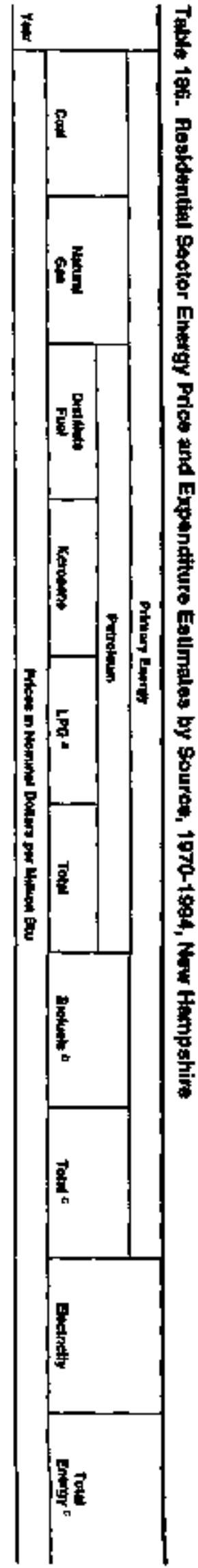


Table 187. Cormmetcial Sector Energy Plce and Expenditure Estimalieq by Solure, 1971-1994, Now Hampshire

\begin{tabular}{|c|c|c|c|c|c|c|c|c|c|c|c|}
\hline \multirow[b]{4}{*}{ Yow } & \multicolumn{9}{|c|}{ 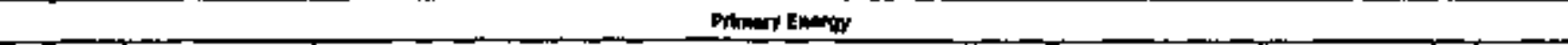 } & \multirow[b]{3}{*}{ Electuleity } & \multirow[b]{3}{*}{ Endots } \\
\hline & & \multirow[b]{2}{*}{ minged } & \multicolumn{6}{|c|}{ Palroliving } & \multirow[b]{2}{*}{ Told } & & \\
\hline & Cod & & $\begin{array}{l}\text { Diptiont } \\
\text { Fump }\end{array}$ & 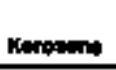 & EPG* & 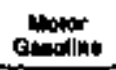 & 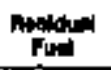 & Total & & & \\
\hline & \multicolumn{11}{|c|}{ 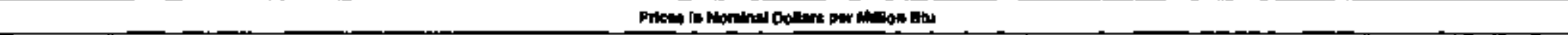 } \\
\hline \multirow[t]{2}{*}{ 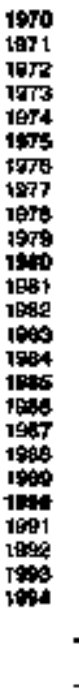 } & 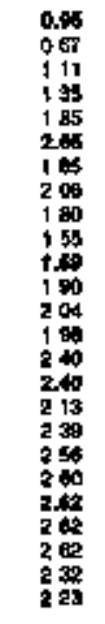 & 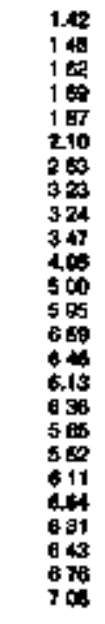 & 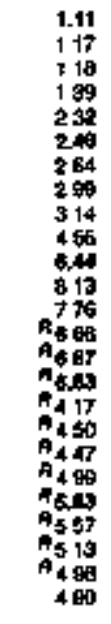 & 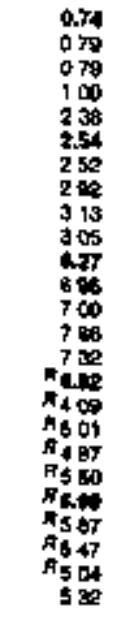 & 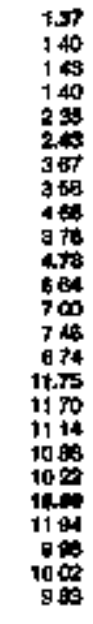 & 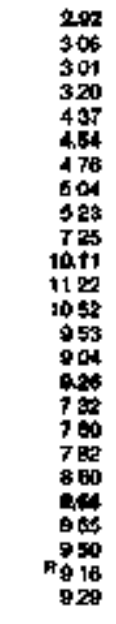 & 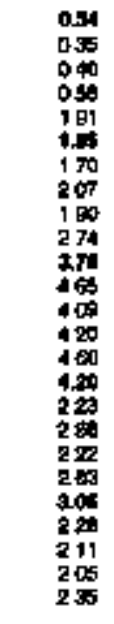 & 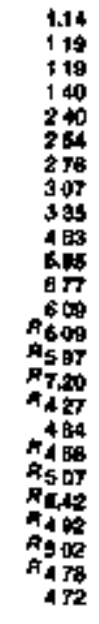 & 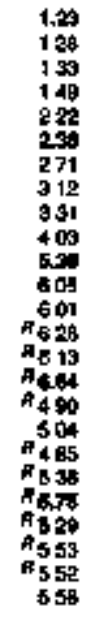 & 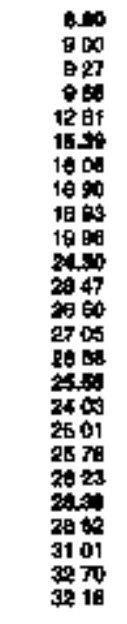 & 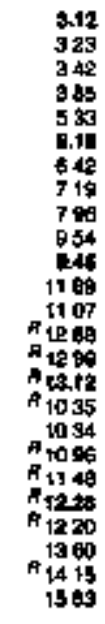 \\
\hline & \multicolumn{11}{|c|}{ 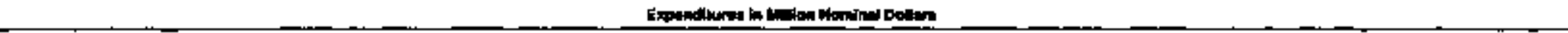 } \\
\hline 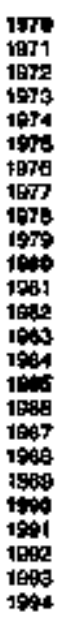 & 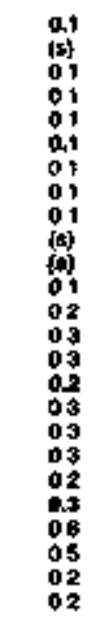 & 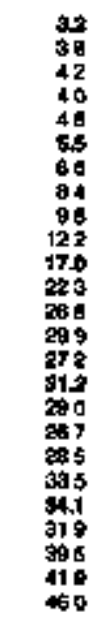 & 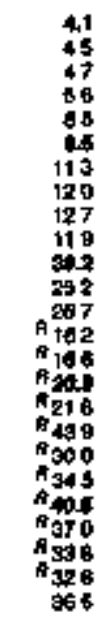 & $\begin{array}{l}0.1 \\
01 \\
01 \\
01 \\
02 \\
02 \\
02 \\
02 \\
02 \\
01 \\
09 \\
02 \\
03 \\
02 \\
02 \\
02 \\
4.6 \\
015 \\
10 \\
012 \\
017 \\
A_{10} \\
07 \\
07 \\
10 \\
12\end{array}$ & 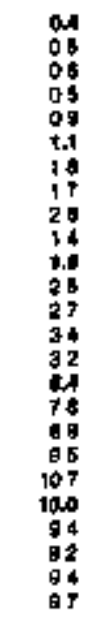 & 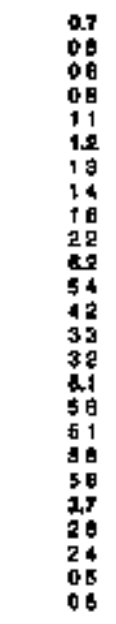 & $\begin{array}{c}02 \\
02 \\
02 \\
03 \\
07 \\
09 \\
09 \\
09 \\
08 \\
03 \\
09 \\
137 \\
181 \\
02 \\
122 \\
29 \\
73 \\
81 \\
68 \\
78 \\
128 \\
96 \\
43 \\
49 \\
67\end{array}$ & 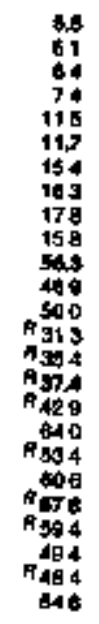 & 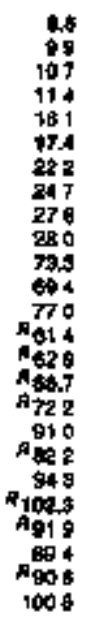 & 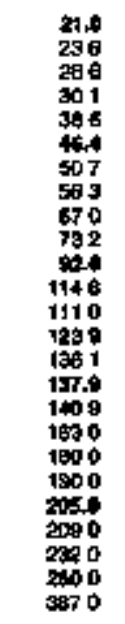 & 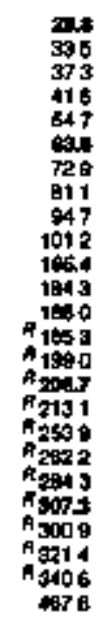 \\
\hline
\end{tabular}

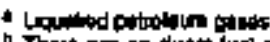

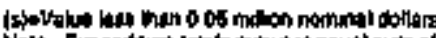

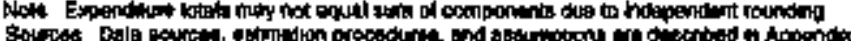




\begin{tabular}{|c|c|c|c|c|c|c|c|c|c|c|c|c|c|c|c|c|c|}
\hline & \multicolumn{15}{|c|}{ m Em } & \multirow[b]{3}{*}{ Expitehty } & \multirow[b]{3}{*}{ tronger } \\
\hline & \multicolumn{3}{|c|}{ וns } & \multirow[b]{2}{*}{ "مسف } & \multicolumn{9}{|c|}{ החומה } & \multirow[b]{2}{*}{ Bhentos" } & \multirow[b]{2}{*}{$r=0$} & & \\
\hline & Cand & 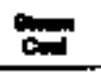 & $T=$ & & 19: & intin & - & LPa= & 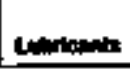 & 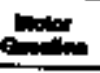 & Givitid & 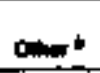 & 1ר: & & & & \\
\hline 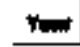 & \multicolumn{17}{|c|}{ 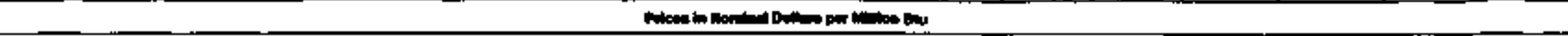 } \\
\hline \multirow[t]{2}{*}{ 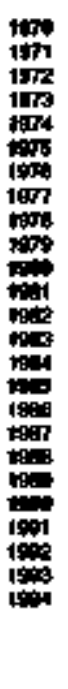 } & $\begin{array}{l}= \\
= \\
= \\
= \\
= \\
= \\
= \\
= \\
= \\
= \\
= \\
= \\
=\end{array}$ & 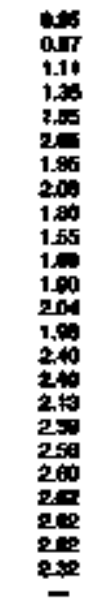 & 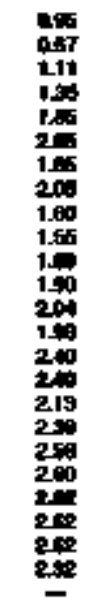 & 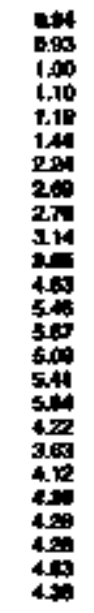 & 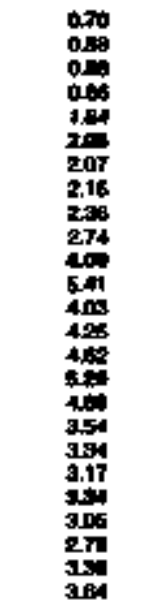 & 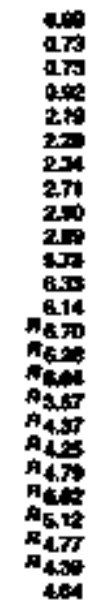 & 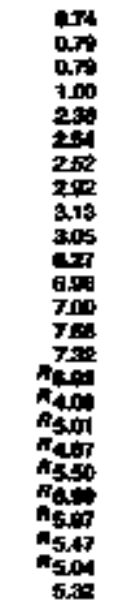 & 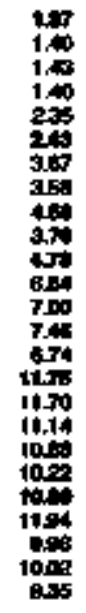 & 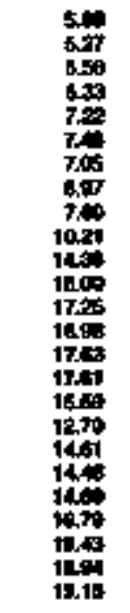 & 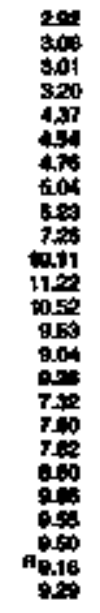 & 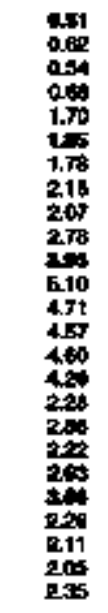 & 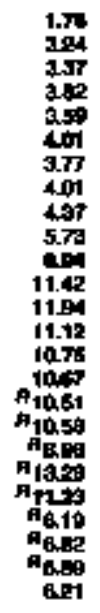 & 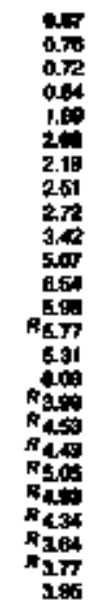 & 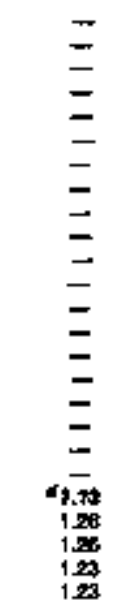 & 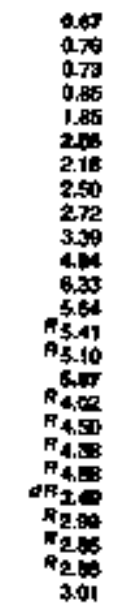 & 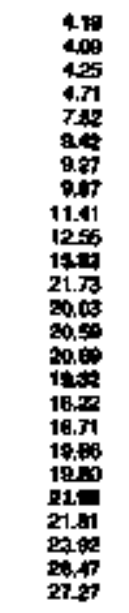 & 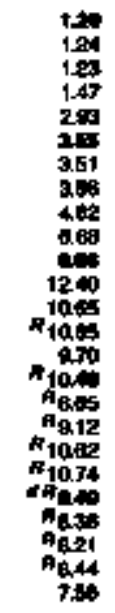 \\
\hline & \multicolumn{17}{|c|}{ 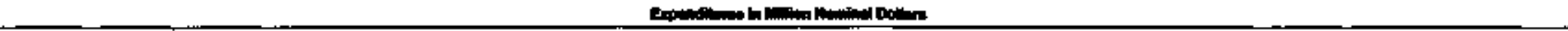 } \\
\hline 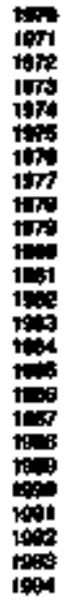 & $\begin{array}{l}= \\
= \\
= \\
= \\
= \\
= \\
= \\
= \\
= \\
= \\
= \\
= \\
=\end{array}$ & 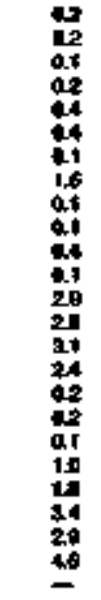 & 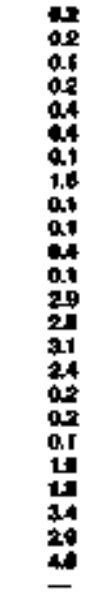 & 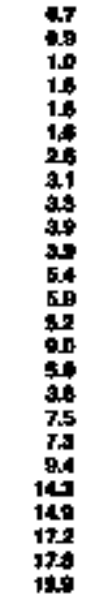 & 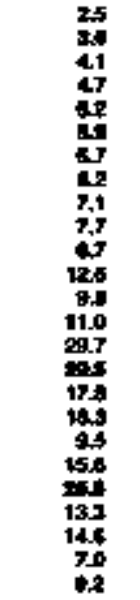 & 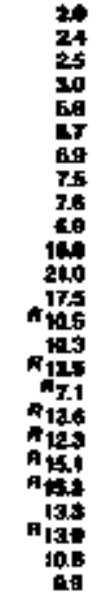 & 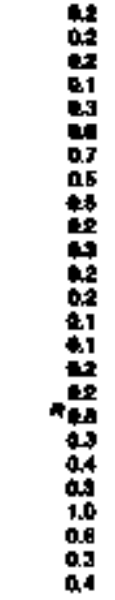 & 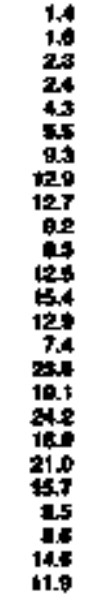 & 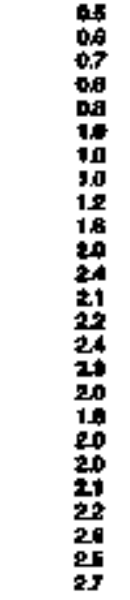 & 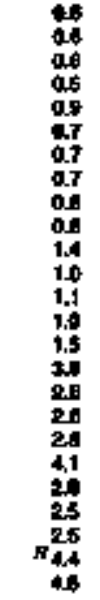 & 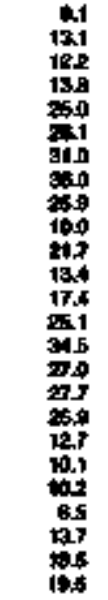 & 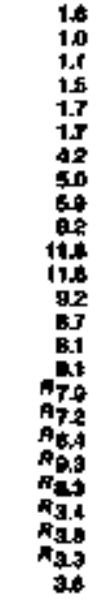 & 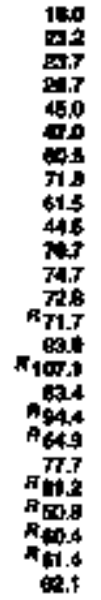 & 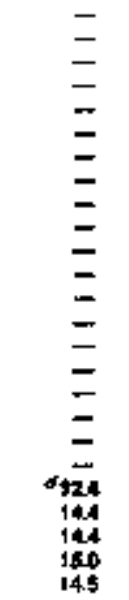 & 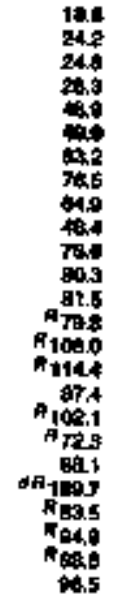 & 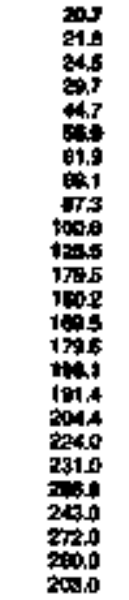 & 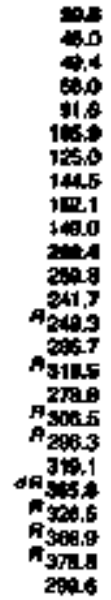 \\
\hline
\end{tabular}

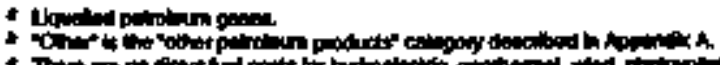

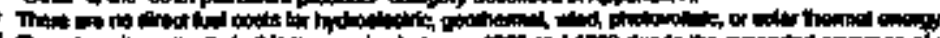

Co

Anowed ath.

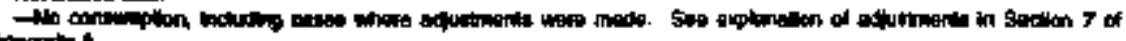

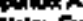

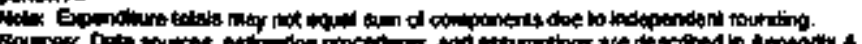

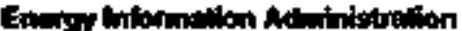

Stip Encigy Prito and Ey 


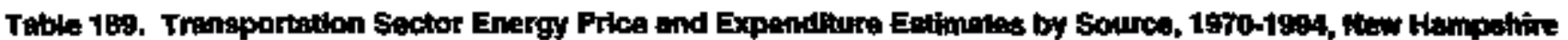

\begin{tabular}{|c|c|c|c|c|c|c|c|c|c|c|c|c|c|}
\hline \multirow[b]{4}{*}{$r$} & \multicolumn{11}{|c|}{ 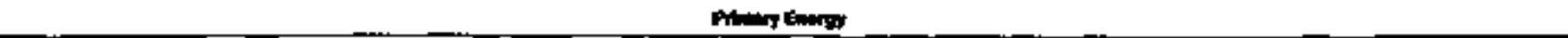 } & \multirow[b]{3}{*}{ 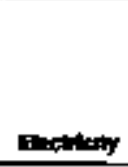 } & \multirow[b]{3}{*}{ 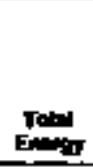 } \\
\hline & \multirow[b]{2}{*}{ Cod } & \multirow[b]{2}{*}{ mand } & \multicolumn{8}{|c|}{ Punolimera } & \multirow[b]{2}{*}{ Ipted } & & \\
\hline & & & ontion & Fin & 岾 & 4n:* & Intcm & تئبn & Fintert & $\operatorname{Tr}$ & & & \\
\hline & \multicolumn{13}{|c|}{ 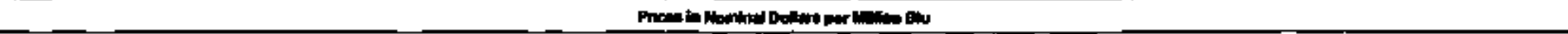 } \\
\hline \multirow[t]{2}{*}{ 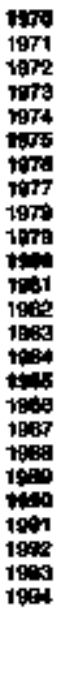 } & $\begin{array}{l}085 \\
067 \\
111 \\
135 \\
185 \\
205 \\
20 \\
= \\
= \\
= \\
= \\
= \\
= \\
= \\
= \\
= \\
=\end{array}$ & $\begin{array}{l}= \\
= \\
= \\
= \\
= \\
= \\
= \\
= \\
= \\
= \\
= \\
= \\
013\end{array}$ & 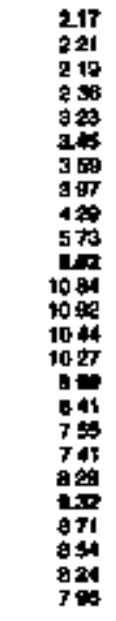 & 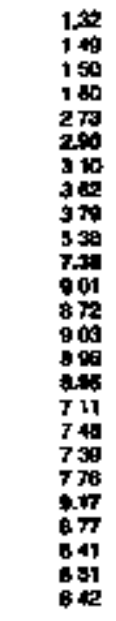 & 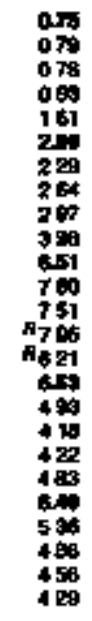 & 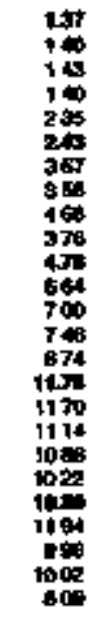 & 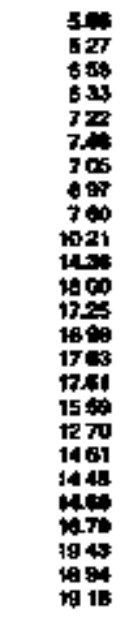 & 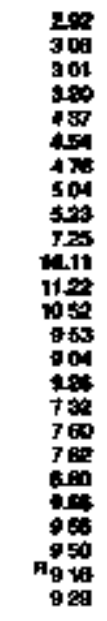 & 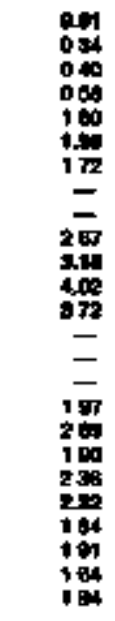 & 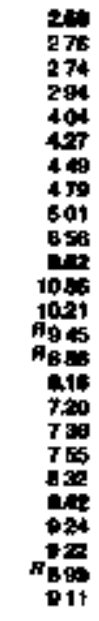 & 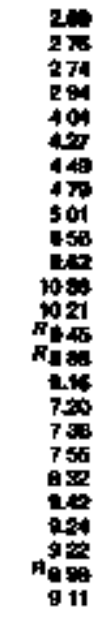 & $\begin{array}{l}= \\
= \\
= \\
= \\
= \\
= \\
= \\
= \\
= \\
= \\
= \\
= \\
= \\
=\end{array}$ & 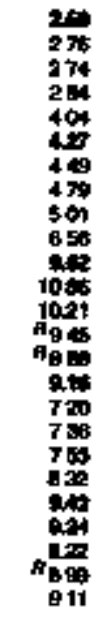 \\
\hline & \multicolumn{13}{|c|}{ E, } \\
\hline 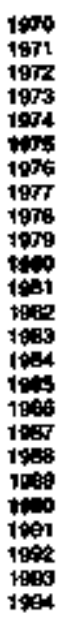 & 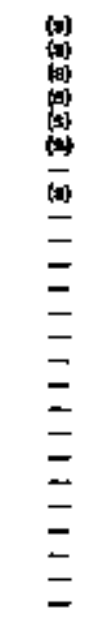 & $\begin{array}{l}= \\
= \\
= \\
= \\
= \\
= \\
= \\
= \\
= \\
= \\
= \\
= \\
= \\
=0\end{array}$ & $\begin{array}{l}04 \\
08 \\
08 \\
05 \\
07 \\
09 \\
05 \\
07 \\
09 \\
12 \\
11 \\
18 \\
14 \\
11 \\
10 \\
12 \\
10 \\
11 \\
10 \\
14 \\
01 \\
11 \\
09 \\
10 \\
10\end{array}$ & 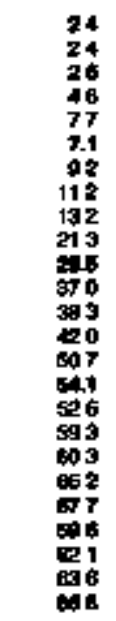 & 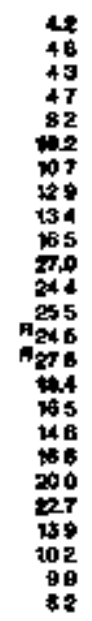 & 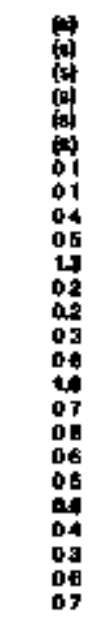 & 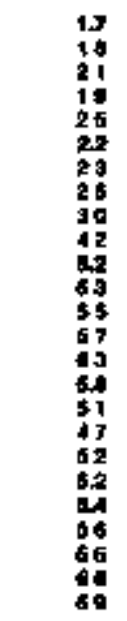 & 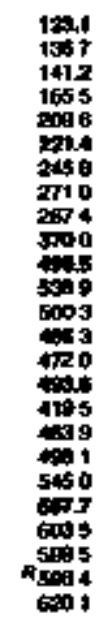 & $\begin{array}{l}44 \\
01 \\
01 \\
01 \\
09 \\
01 \\
01 \\
= \\
145 \\
10 \\
12 \\
07 \\
= \\
= \\
05 \\
38 \\
17 \\
03 \\
14 \\
83 \\
15 \\
04 \\
01\end{array}$ & 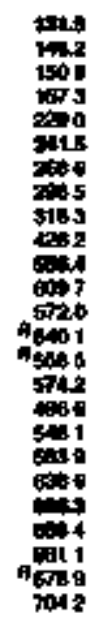 & 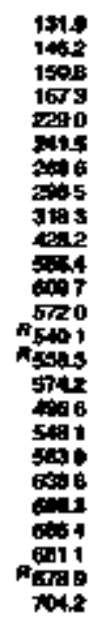 & $\begin{array}{l}= \\
= \\
= \\
= \\
= \\
= \\
= \\
= \\
= \\
= \\
= \\
= \\
=\end{array}$ & 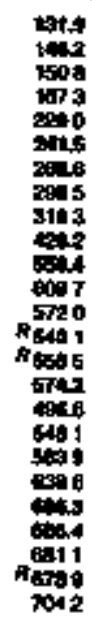 \\
\hline
\end{tabular}




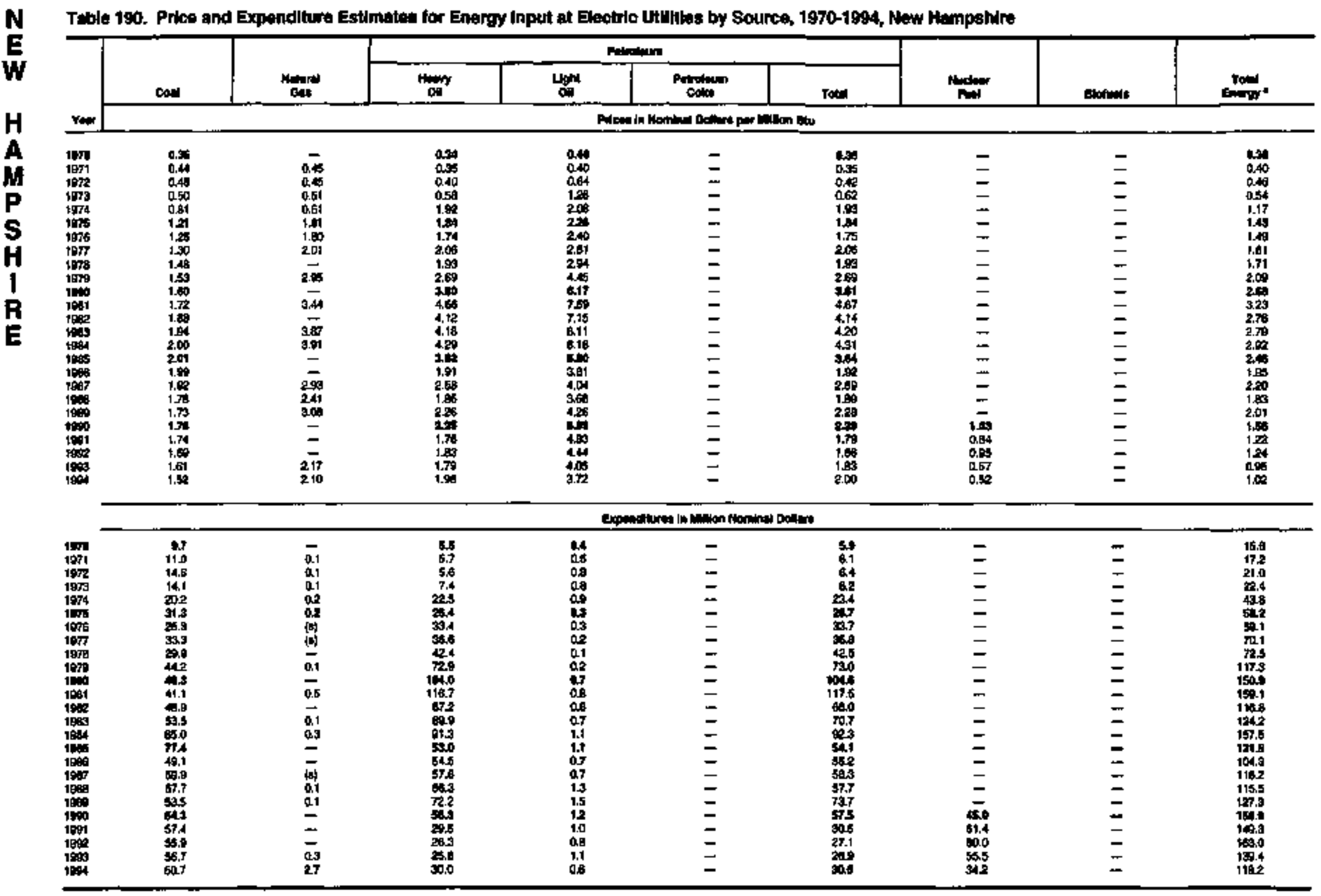

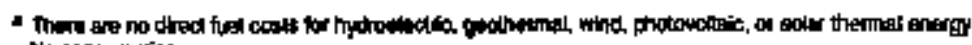

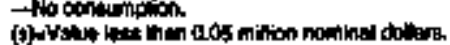


Table 191. Erofgy Price enth Expendilure Eotimates by Sounce, 1970-1994, New Jartoy

\begin{tabular}{|c|c|c|c|c|c|c|c|c|c|c|c|c|c|c|c|c|c|}
\hline \multirow[b]{4}{*}{ Yem } & \multicolumn{14}{|c|}{ Prinimy E:anty } & \multirow{3}{*}{ (1) } & \multirow{3}{*}{ 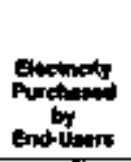 } & \multirow[b]{3}{*}{ Tober } \\
\hline & \multicolumn{3}{|c|}{ con } & \multirow[b]{2}{*}{ (I) } & \multicolumn{7}{|c|}{ Antrointin } & \multirow[b]{2}{*}{ Muctorer } & \multirow[b]{2}{*}{ Ond } & \multirow[b]{2}{*}{ Totite } & & & \\
\hline & $\begin{array}{l}\text { Colving } \\
\text { Cond }\end{array}$ & $\begin{array}{l}\text { gnen } \\
\text { cent }\end{array}$ & Tot: & & Olland & A & Lma: & Motors & $\begin{array}{l}\text { Arodicud } \\
\text { Fuer }\end{array}$ & Other" & Toter & & & & & & \\
\hline & \multicolumn{17}{|c|}{ 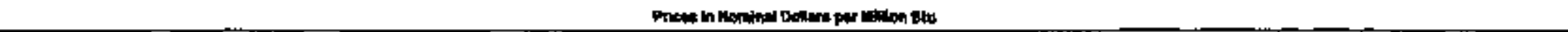 } \\
\hline \multirow[t]{2}{*}{ 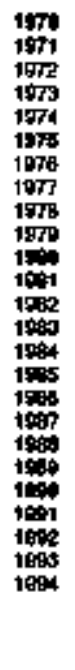 } & $\begin{array}{l}\text { as } \\
= \\
= \\
= \\
= \\
= \\
= \\
= \\
= \\
= \\
= \\
= \\
= \\
=\end{array}$ & 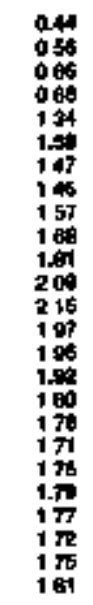 & 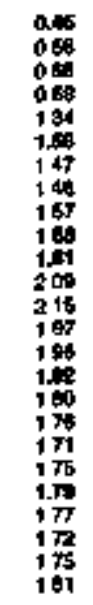 & 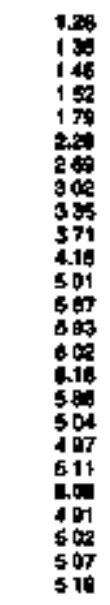 & 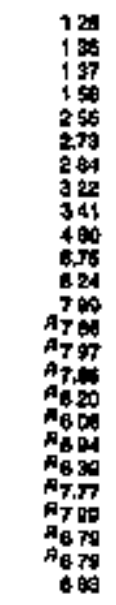 & 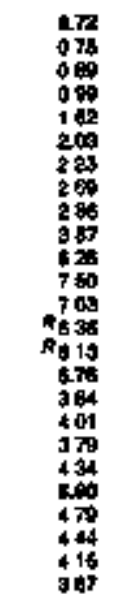 & 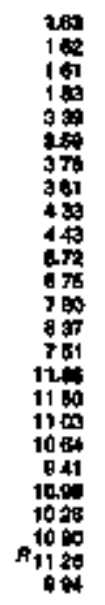 & 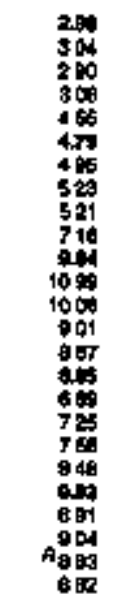 & 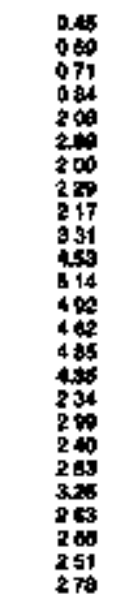 & 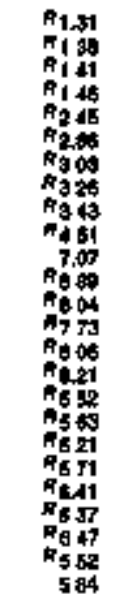 & 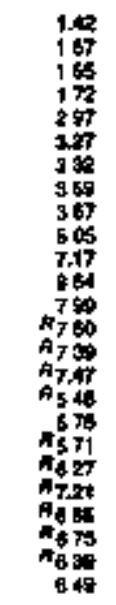 & 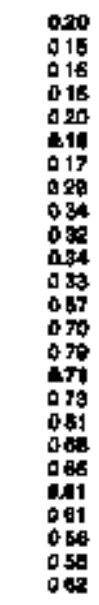 & $\begin{array}{l}= \\
z \\
= \\
= \\
= \\
= \\
= \\
= \\
= \\
= \\
= \\
= \\
23 \\
133 \\
233 \\
238\end{array}$ & 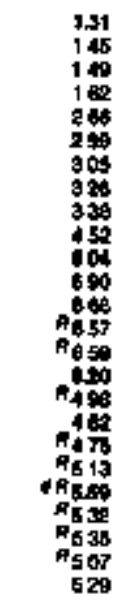 & 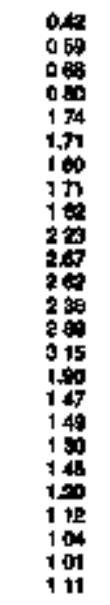 & 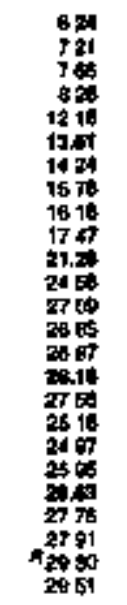 & 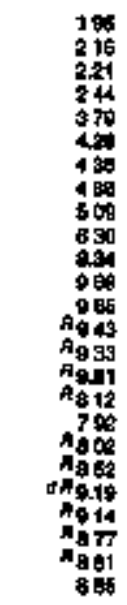 \\
\hline & \multicolumn{17}{|c|}{ 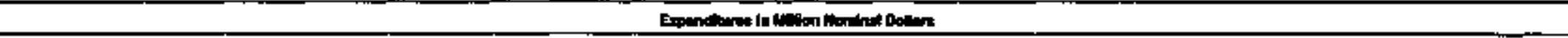 } \\
\hline 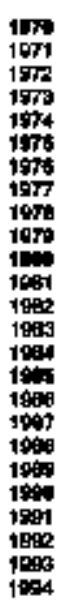 & $\begin{array}{l}= \\
= \\
= \\
= \\
= \\
= \\
= \\
= \\
= \\
= \\
= \\
= \\
= \\
=\end{array}$ & 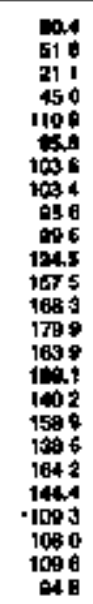 & 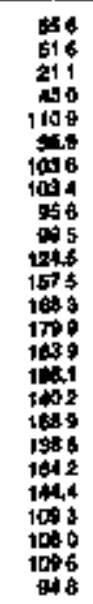 & 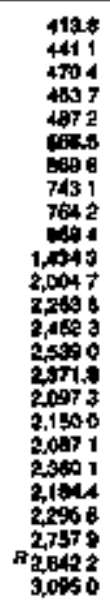 & 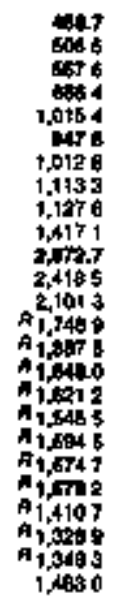 & 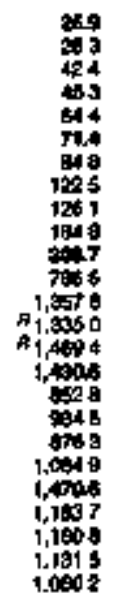 & 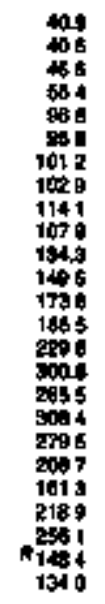 & 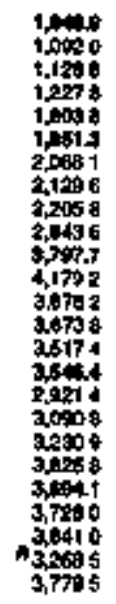 & 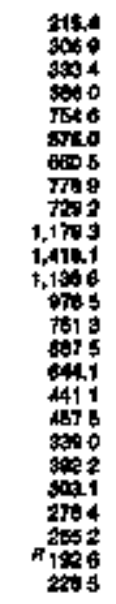 & 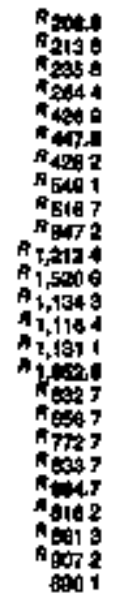 & 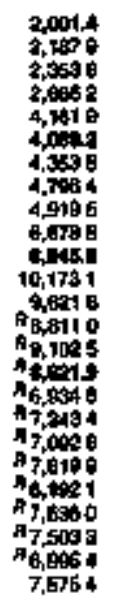 & 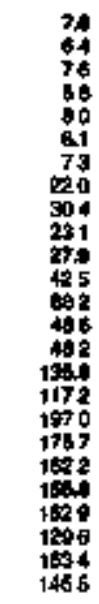 & $\begin{array}{l}= \\
= \\
= \\
= \\
= \\
= \\
= \\
= \\
= \\
= \\
= \\
= \\
5206 \\
313 \\
314 \\
330 \\
332\end{array}$ & 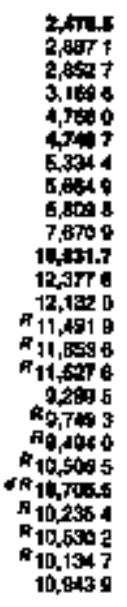 & 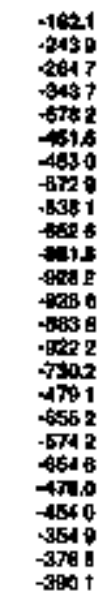 & 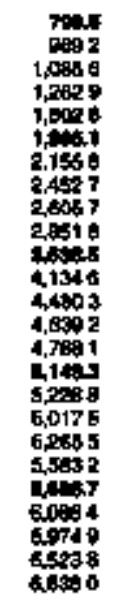 & 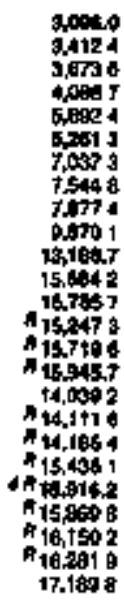 \\
\hline
\end{tabular}

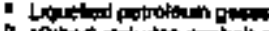

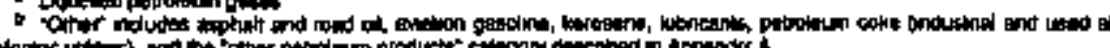

Afinverd bats $\operatorname{lomax}$

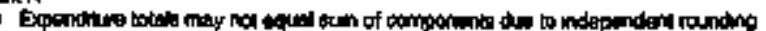

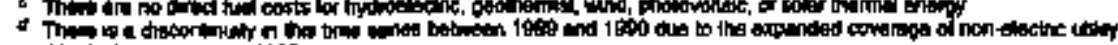

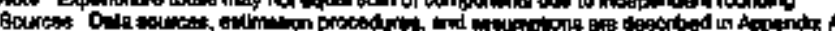




\begin{tabular}{|c|c|c|c|c|c|c|c|c|c|c|}
\hline \multirow[b]{4}{*}{ 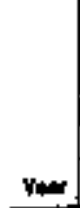 } & \multicolumn{8}{|c|}{ 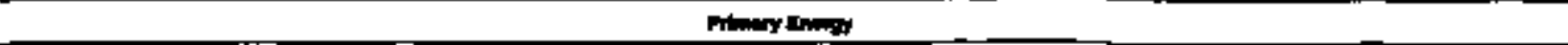 } & \multirow[b]{3}{*}{ Buctotity } & \multirow[b]{3}{*}{$e^{70 m}$} \\
\hline & \multirow[b]{2}{*}{ cont } & \multirow[b]{2}{*}{ 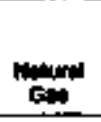 } & \multicolumn{4}{|c|}{ המוסדות } & \multirow[b]{2}{*}{ " } & \multirow[b]{2}{*}{ Tolita 0} & & \\
\hline & & & Din & Karom & LPa: & rota & & & & \\
\hline & \multicolumn{10}{|c|}{ 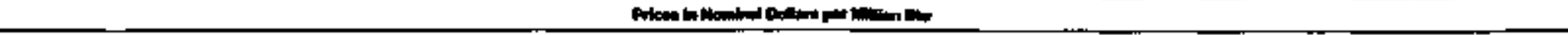 } \\
\hline \multirow[t]{2}{*}{ 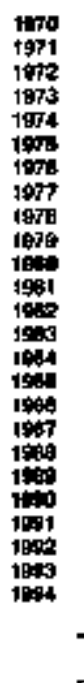 } & 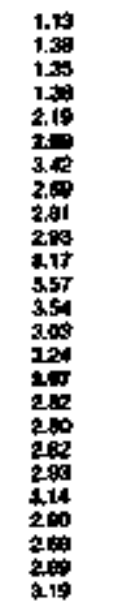 & 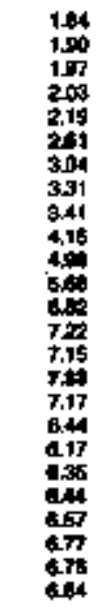 & 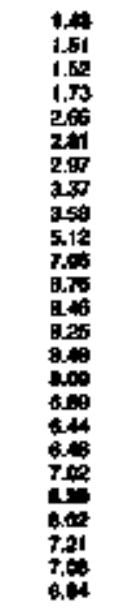 & 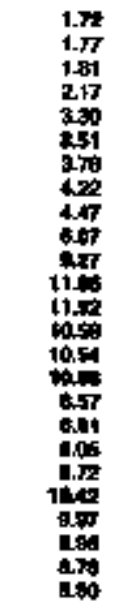 & 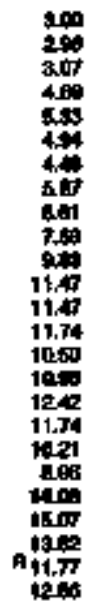 & 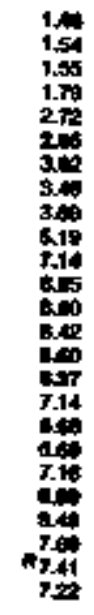 & 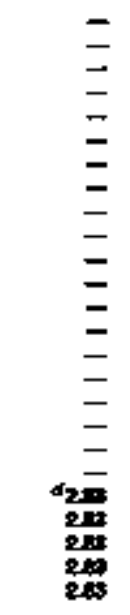 & 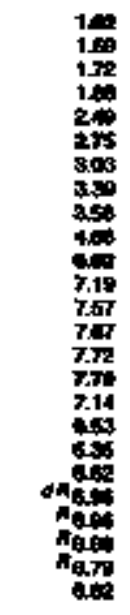 & 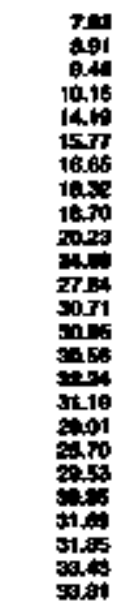 & 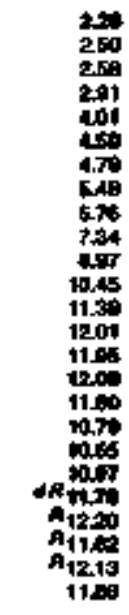 \\
\hline & \multicolumn{10}{|c|}{ 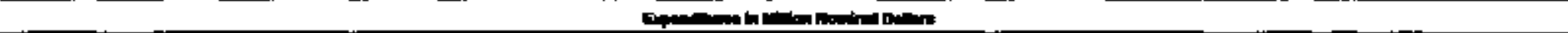 } \\
\hline 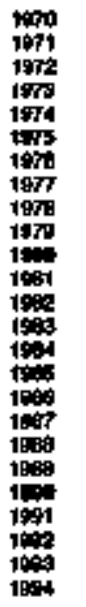 & 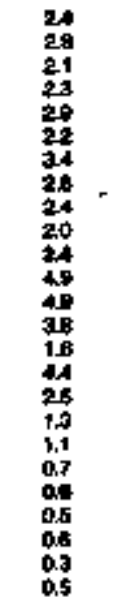 & 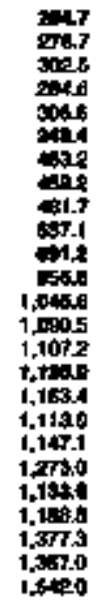 & 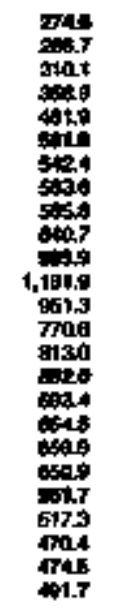 & 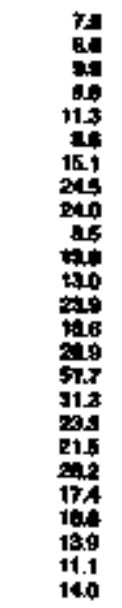 & 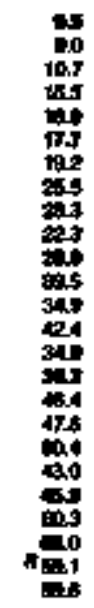 & 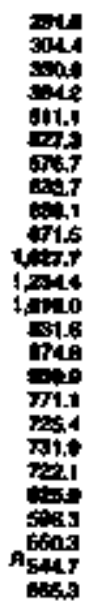 & 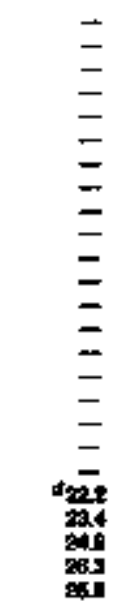 & 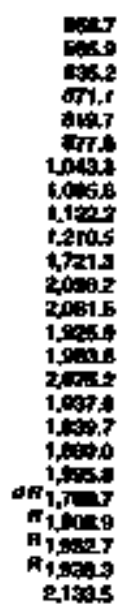 & 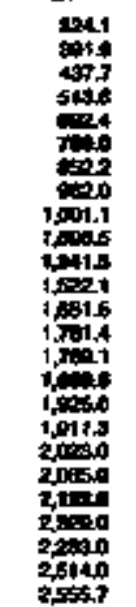 & 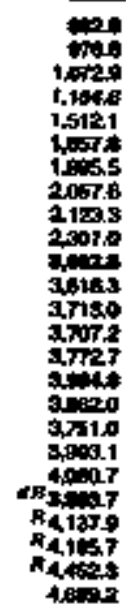 \\
\hline
\end{tabular}




\begin{tabular}{|c|c|c|c|c|c|c|c|c|c|c|c|}
\hline \multirow[b]{4}{*}{ Vom } & \multicolumn{9}{|c|}{ nimengen } & \multirow[b]{3}{*}{ enowitay } & \multirow[b]{3}{*}{ Satid } \\
\hline & & \multirow[b]{2}{*}{ Ain. } & \multicolumn{6}{|c|}{ Buntom } & \multirow[b]{2}{*}{ Tos } & & \\
\hline & coll & & Pin & Drown & Lب: & 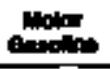 & Ratave & Tots & & & \\
\hline & \multicolumn{11}{|c|}{ 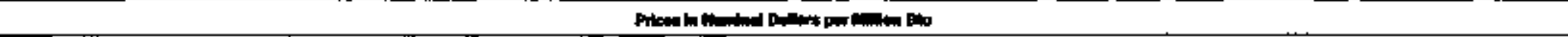 } \\
\hline \multirow[t]{2}{*}{ 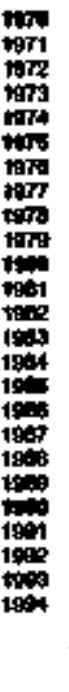 } & 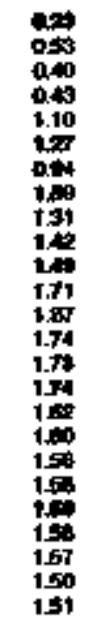 & 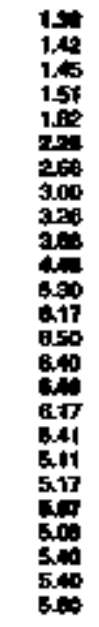 & 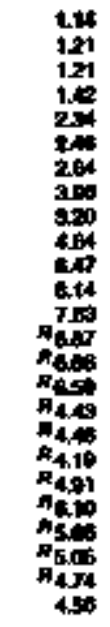 & 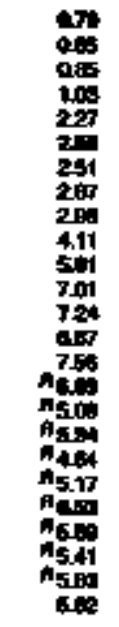 & 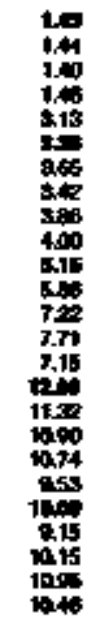 & 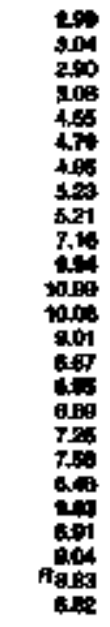 & 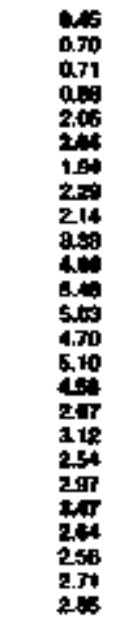 & 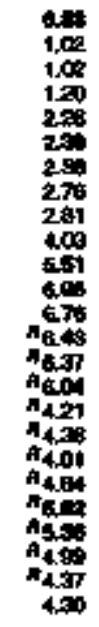 & 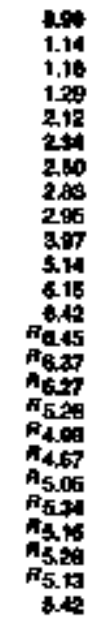 & 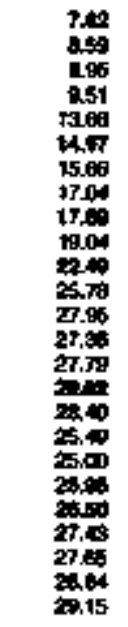 & 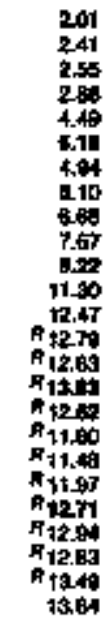 \\
\hline & \multicolumn{11}{|c|}{ 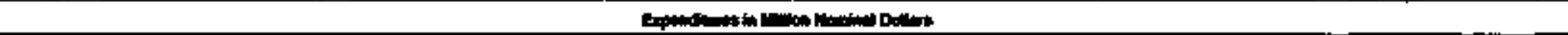 } \\
\hline 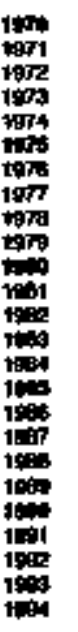 & 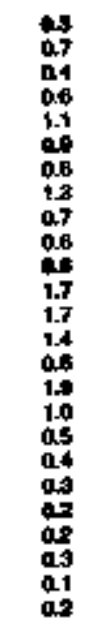 & 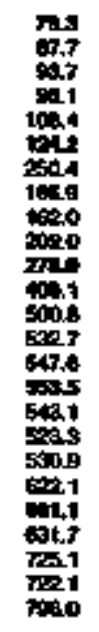 & 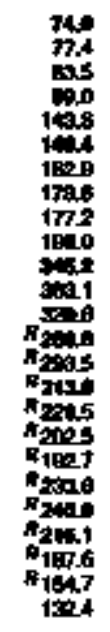 & 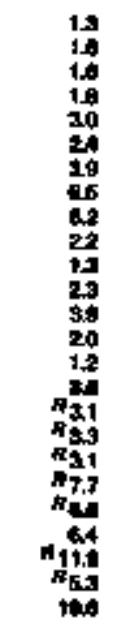 & 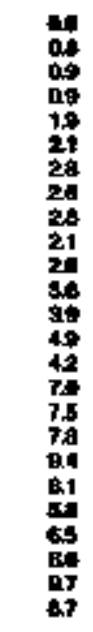 & 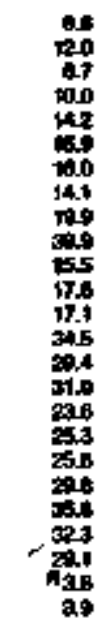 & 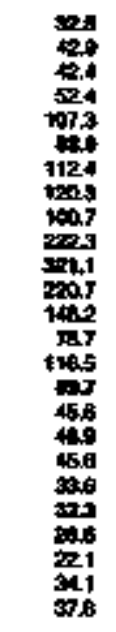 & 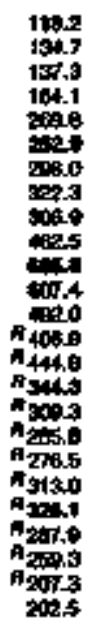 & 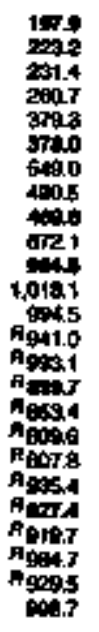 & 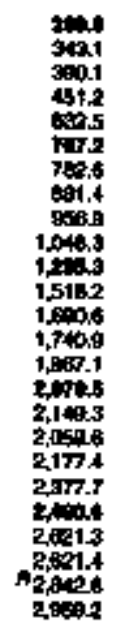 & 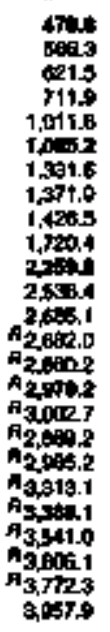 \\
\hline
\end{tabular}

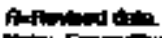

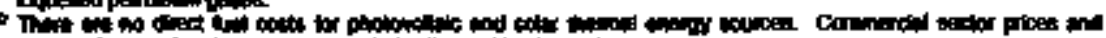

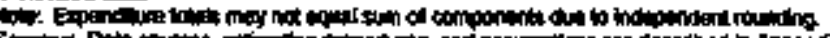

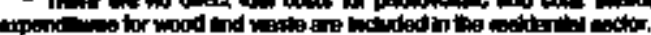




\begin{tabular}{|c|c|c|c|c|c|c|c|c|c|c|c|c|c|c|c|c|c|}
\hline \multirow[b]{4}{*}{ xerr } & \multicolumn{15}{|c|}{ Prominy Enarigr } & \multirow[b]{3}{*}{ Enotnolyy } & \multirow[b]{3}{*}{$\operatorname{Tan}$} \\
\hline & \multicolumn{3}{|c|}{ cont } & \multirow[b]{2}{*}{ (1) } & \multicolumn{9}{|c|}{ Protoming } & \multirow[b]{2}{*}{ Bonuenef } & \multirow[b]{2}{*}{ Te } & & \\
\hline & contures & sioen & Totat & & Anphes and & 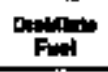 & Kanowe & Let & Lwongen & mosir & Angadur & anters & Toted & & & & \\
\hline & \multicolumn{17}{|c|}{ 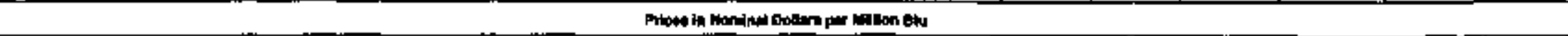 } \\
\hline \multirow[t]{2}{*}{ 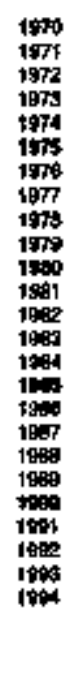 } & $\begin{array}{l}=0 \\
= \\
= \\
= \\
= \\
= \\
= \\
= \\
= \\
= \\
= \\
= \\
= \\
z\end{array}$ & 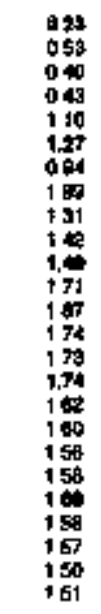 & 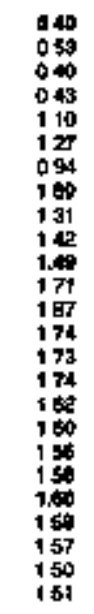 & 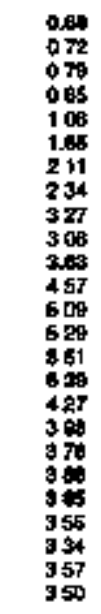 & 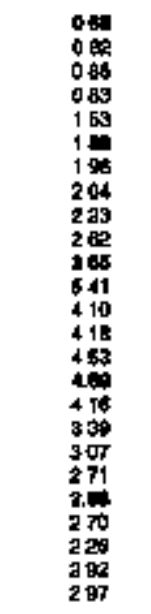 & 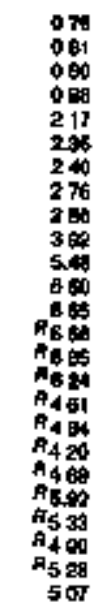 & 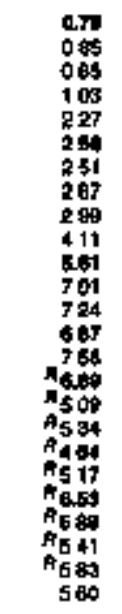 & 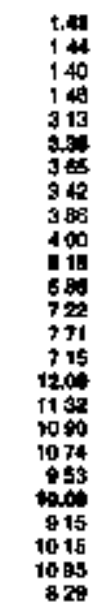 & 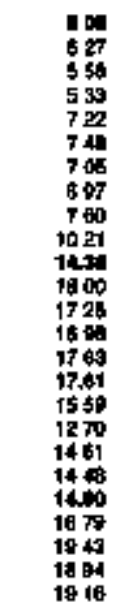 & 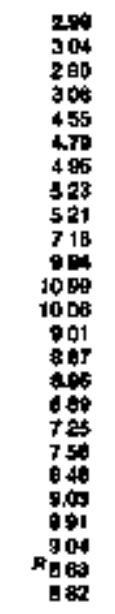 & 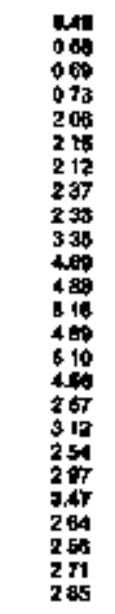 & 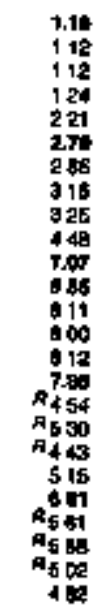 & 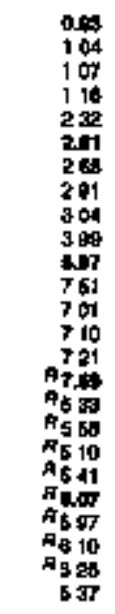 & 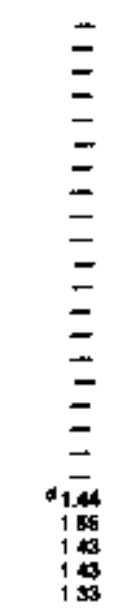 & 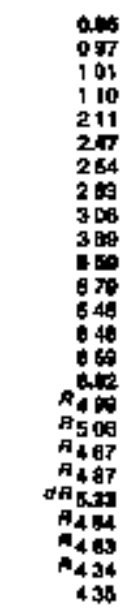 & 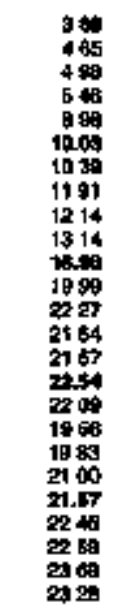 & 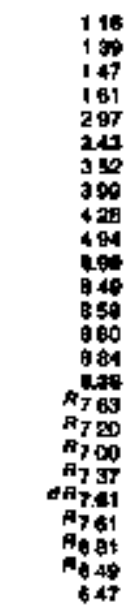 \\
\hline & \multicolumn{17}{|c|}{ 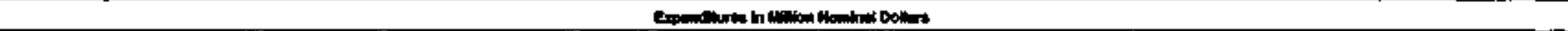 } \\
\hline 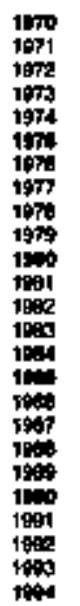 & $\begin{array}{l}53 \\
= \\
= \\
= \\
= \\
= \\
= \\
= \\
= \\
= \\
= \\
= \\
= \\
= \\
=\end{array}$ & 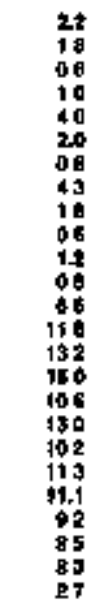 & 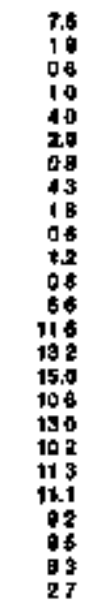 & 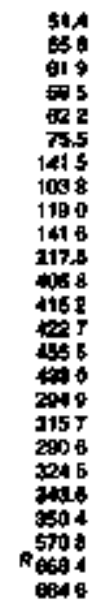 & 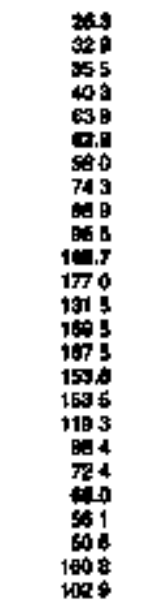 & 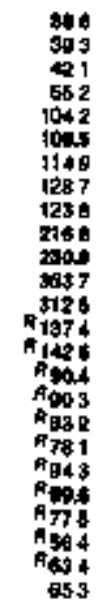 & 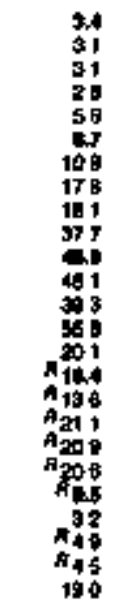 & 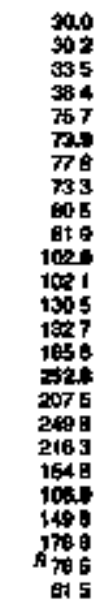 & 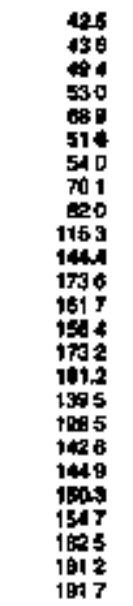 & 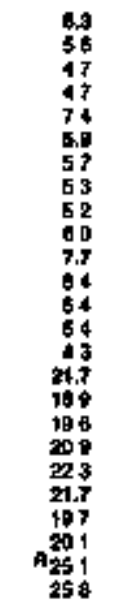 & 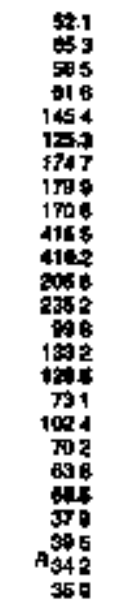 & 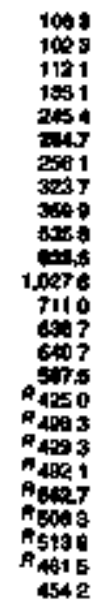 & 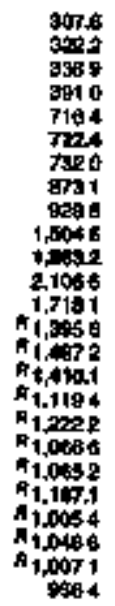 & $\begin{array}{l}= \\
= \\
= \\
= \\
= \\
= \\
= \\
= \\
= \\
= \\
= \\
\bar{z} \\
60 \\
60 \\
68 \\
67\end{array}$ & 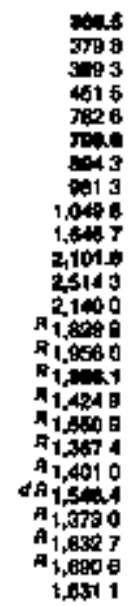 & 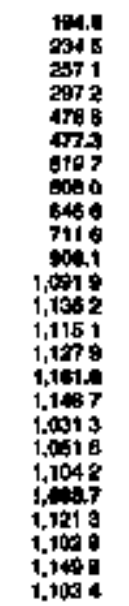 & 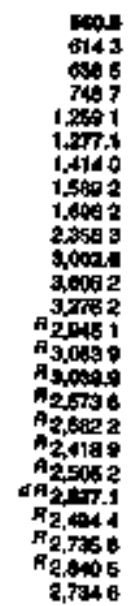 \\
\hline
\end{tabular}



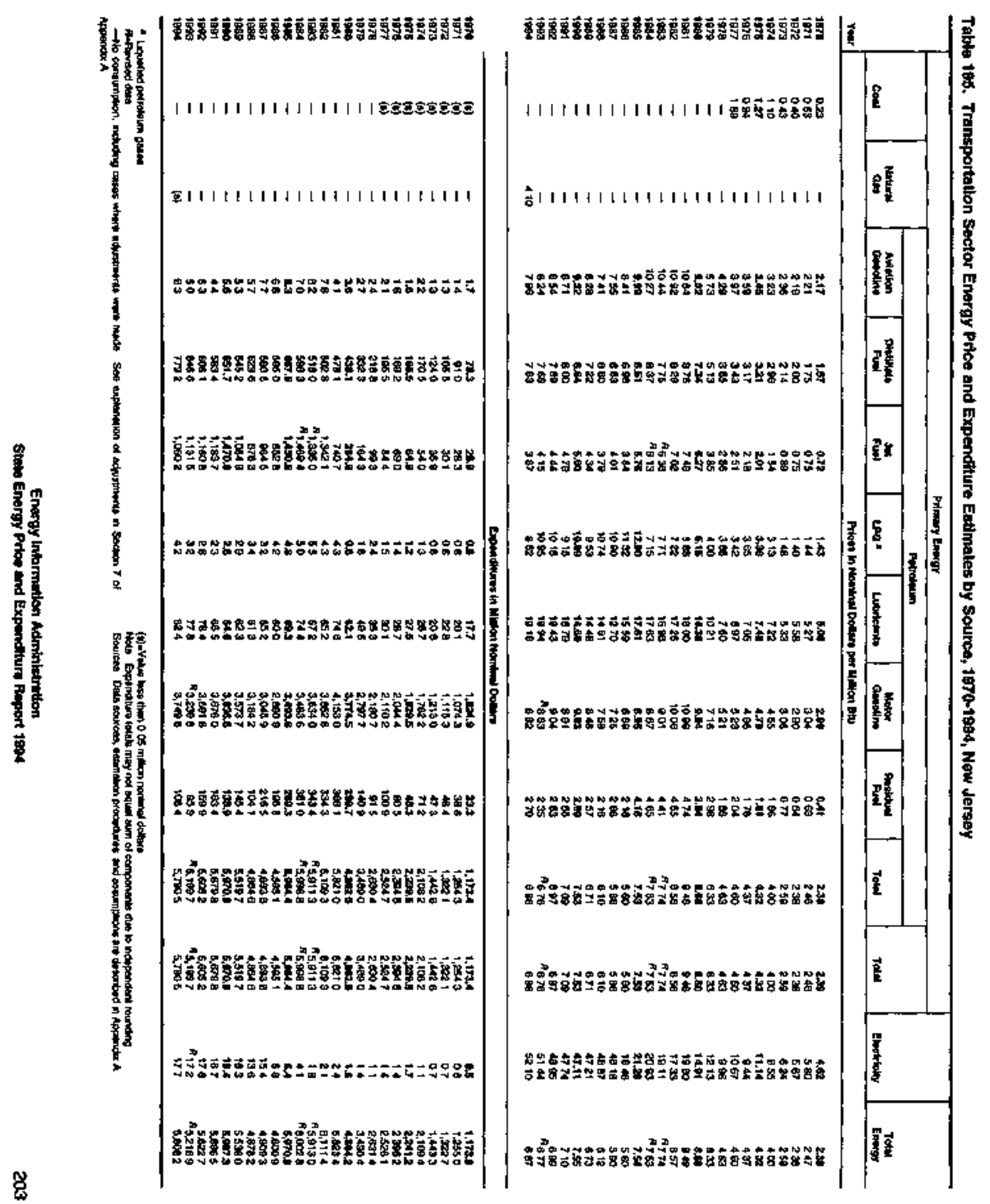


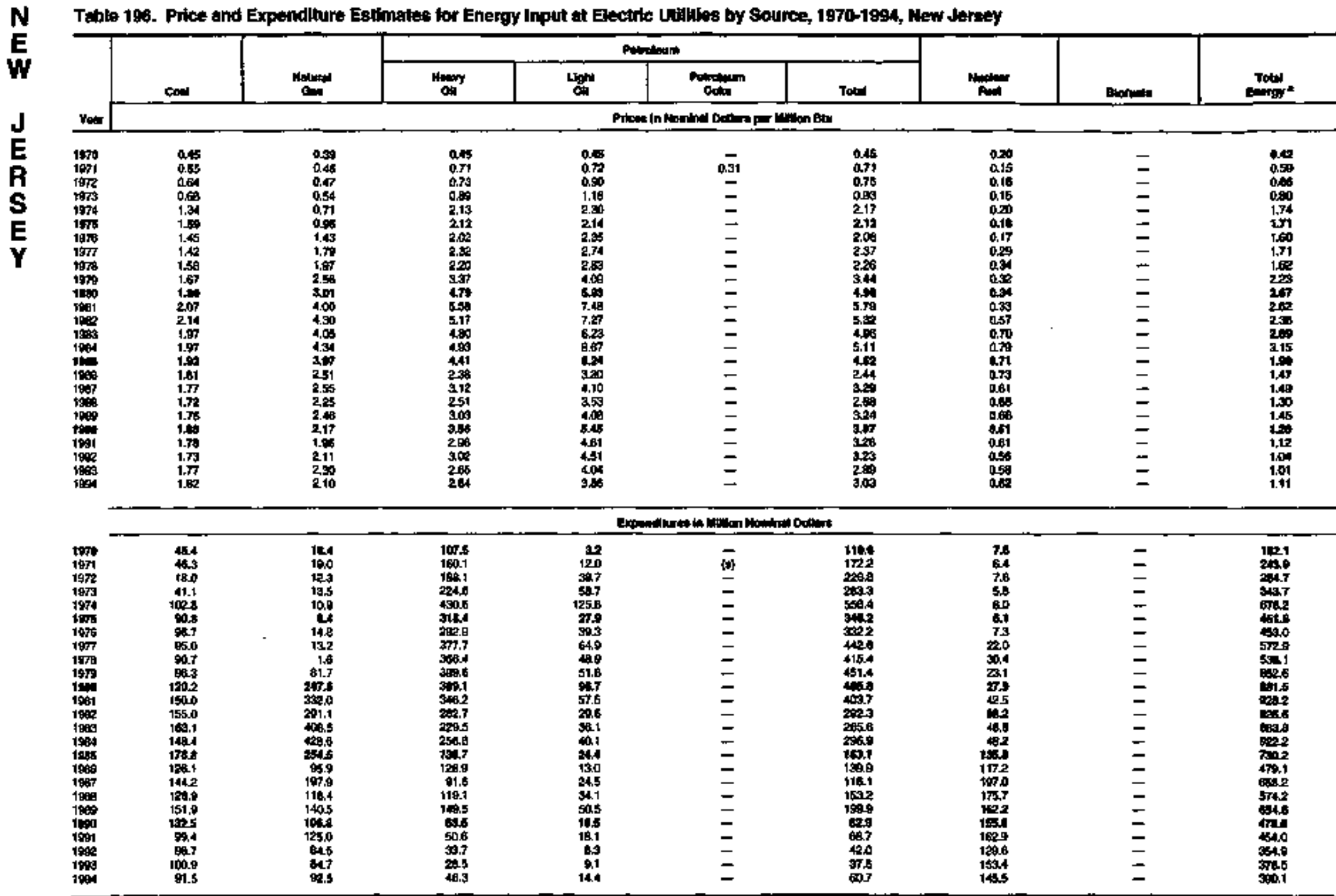

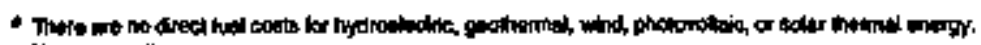

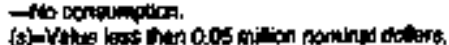

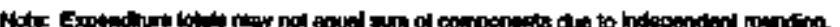

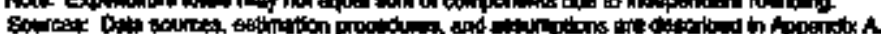


Table 197. Energy Price and Expondthure Eatlmatos by Source, 1970-1694, Now Hexlco

\begin{tabular}{|c|c|c|c|c|c|c|c|c|c|c|c|c|c|c|c|c|c|}
\hline & \multicolumn{14}{|c|}{ 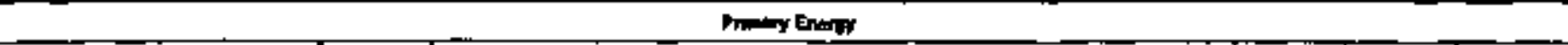 } & \multirow{3}{*}{ 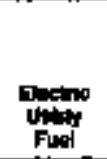 } & \multirow{3}{*}{ 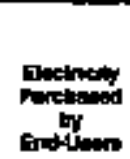 } & \multirow[b]{3}{*}{ Totel } \\
\hline & \multicolumn{3}{|c|}{ col } & \multirow[b]{2}{*}{$\min _{0 \rightarrow+5}$} & \multicolumn{7}{|c|}{ Putrobare } & \multirow[b]{2}{*}{ Mydor } & \multirow[b]{2}{*}{ motint: } & \multirow[b]{2}{*}{ Totold a } & & & \\
\hline & cons & Cod & Total & & שה: & Fit & LPo: & Dor & 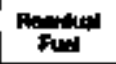 & Otwer" & Totes & & & & & & \\
\hline $\mathrm{ram}$ & \multicolumn{17}{|c|}{ 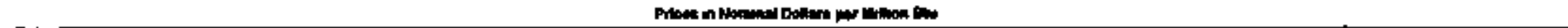 } \\
\hline \multirow[t]{2}{*}{ 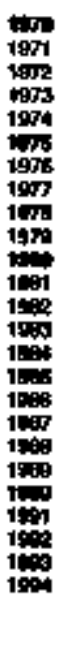 } & $\begin{array}{l}= \\
= \\
= \\
= \\
= \\
= \\
= \\
= \\
= \\
= \\
= \\
= \\
= \\
= \\
=\end{array}$ & 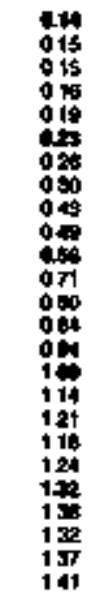 & 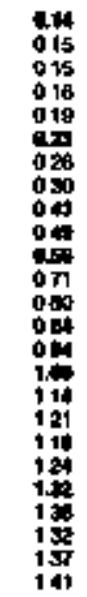 & 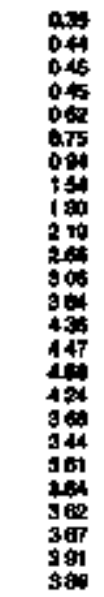 & 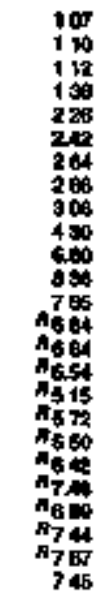 & 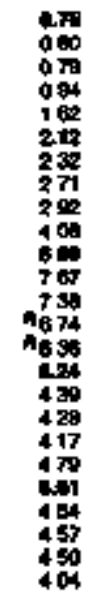 & 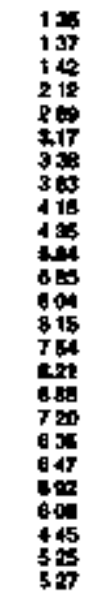 & 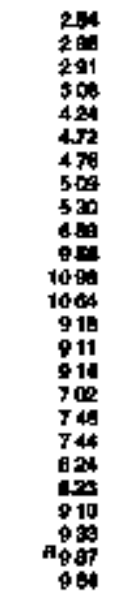 & 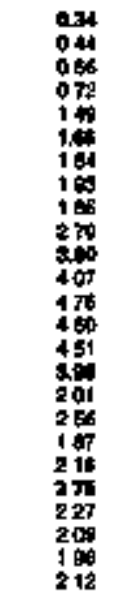 & 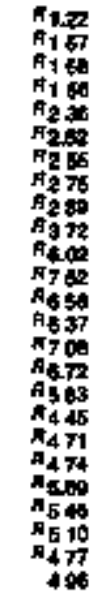 & 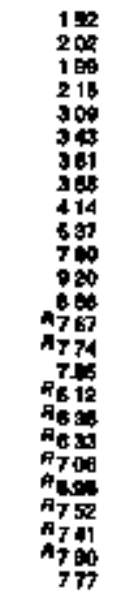 & $\begin{array}{l}= \\
= \\
= \\
= \\
= \\
= \\
= \\
= \\
= \\
= \\
= \\
= \\
= \\
=\end{array}$ & $\begin{array}{l}= \\
= \\
= \\
= \\
= \\
= \\
= \\
= \\
= \\
= \\
= \\
= \\
= \\
459 \\
372 \\
377 \\
373 \\
399\end{array}$ & 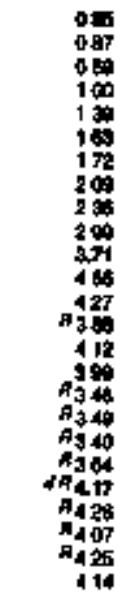 & 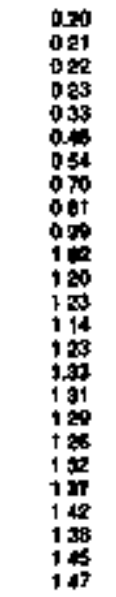 & 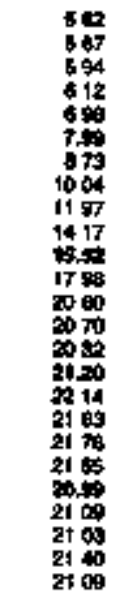 & 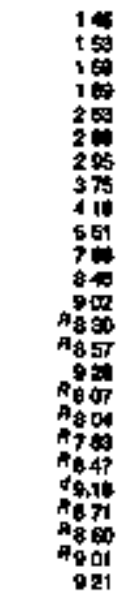 \\
\hline & \multicolumn{17}{|c|}{ 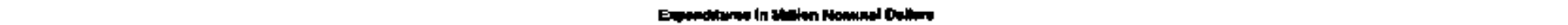 } \\
\hline 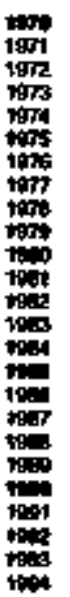 & $\begin{array}{l}= \\
= \\
= \\
= \\
= \\
= \\
= \\
= \\
= \\
= \\
= \\
= \\
= \\
=\end{array}$ & 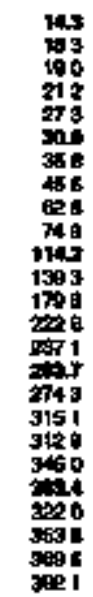 & 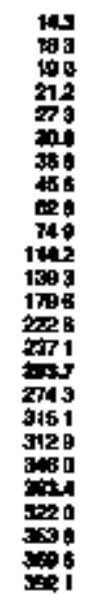 & 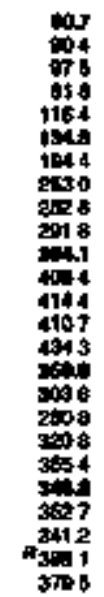 & 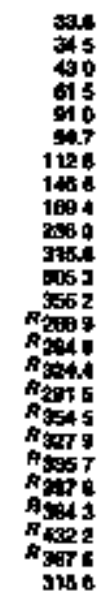 & 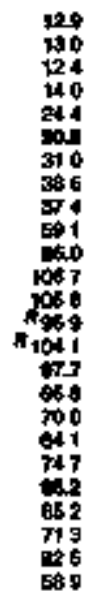 & 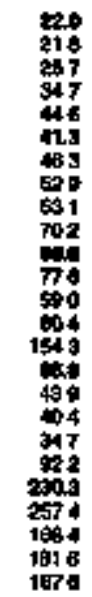 & 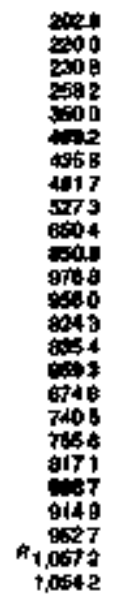 & 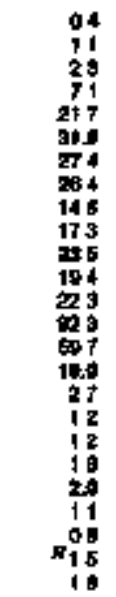 & 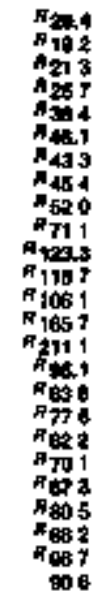 & 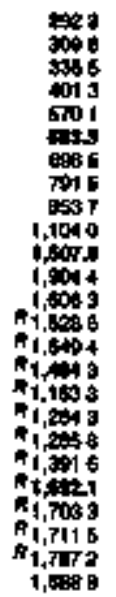 & $\begin{array}{l}= \\
= \\
= \\
= \\
= \\
= \\
= \\
= \\
= \\
= \\
= \\
= \\
=\end{array}$ & $\begin{array}{l}= \\
= \\
= \\
= \\
= \\
= \\
= \\
= \\
= \\
= \\
= \\
47 \\
75 \\
70 \\
78\end{array}$ & 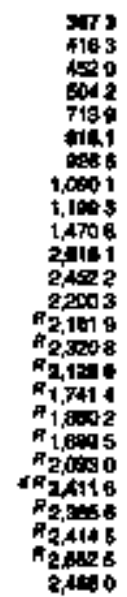 & 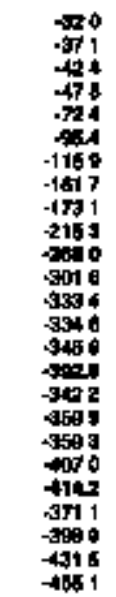 & 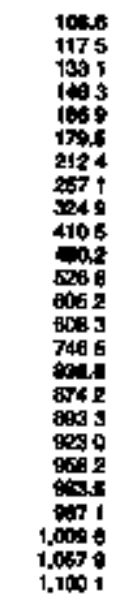 & 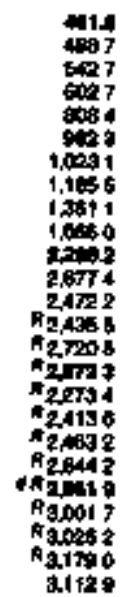 \\
\hline
\end{tabular}

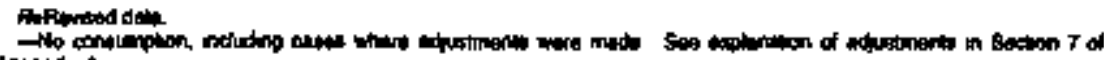

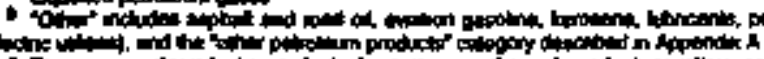
spondixis

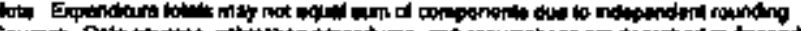

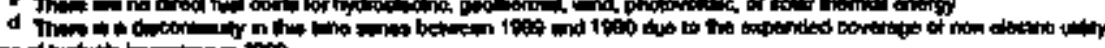

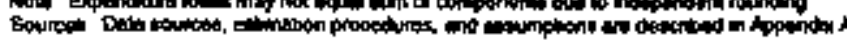




\begin{tabular}{|c|c|c|c|c|c|c|c|c|c|c|}
\hline \multirow[b]{4}{*}{ Yom } & \multicolumn{8}{|c|}{ Atwomer rongy } & \multirow[b]{3}{*}{ Evalinaty } & \multirow[b]{3}{*}{ 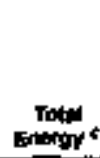 } \\
\hline & \multirow[b]{2}{*}{ coed } & \multirow[b]{2}{*}{ 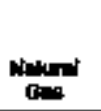 } & \multicolumn{4}{|c|}{ Aatrolem } & \multirow[b]{2}{*}{ Butan: } & \multirow[b]{2}{*}{ Tatal: } & & \\
\hline & & & $\begin{array}{l}\text { Dangition } \\
\text { Fud }\end{array}$ & Kerrement & Lea: & Tow & & & & \\
\hline & \multicolumn{10}{|c|}{ 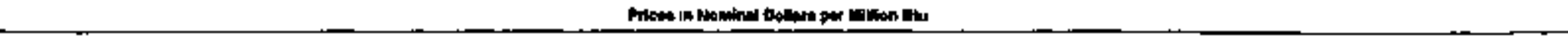 } \\
\hline \multirow[t]{2}{*}{ 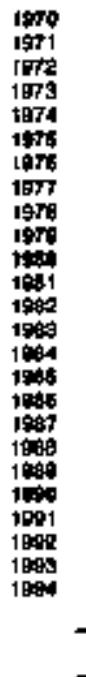 } & 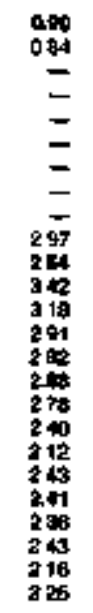 & 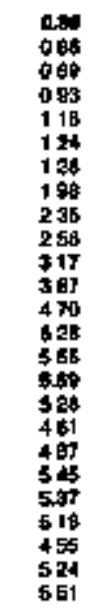 & 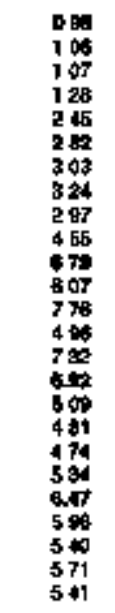 & 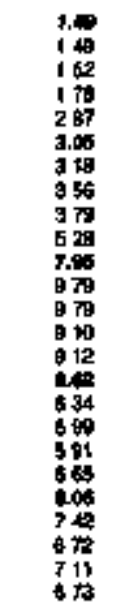 & 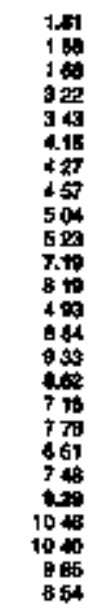 & 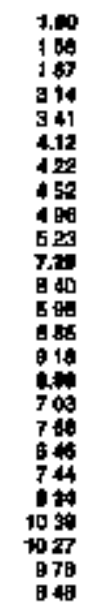 & $\begin{array}{l}= \\
z \\
z \\
= \\
= \\
z \\
z \\
= \\
= \\
= \\
= \\
= \\
4475 \\
475 \\
475 \\
475\end{array}$ & 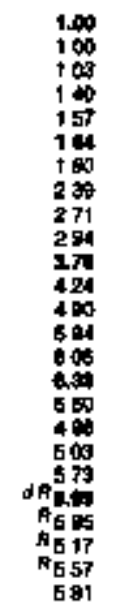 & 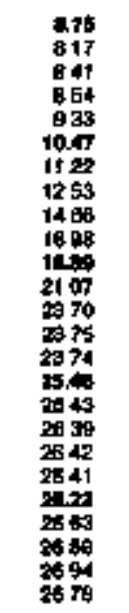 & 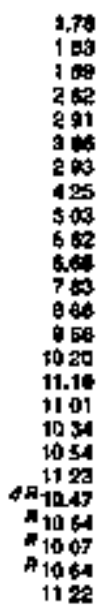 \\
\hline & \multicolumn{10}{|c|}{ 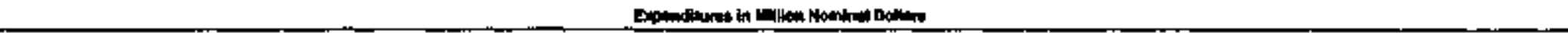 } \\
\hline 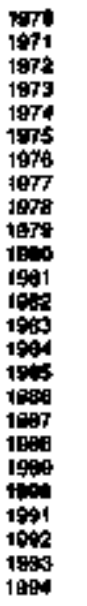 & $\begin{array}{l}9 \\
02 \\
= \\
= \\
= \\
= \\
7 \\
01 \\
01 \\
01 \\
02 \\
02 \\
02 \\
02 \\
01 \\
01 \\
08 \\
02 \\
01 \\
01 \\
02 \\
02 \\
01\end{array}$ & 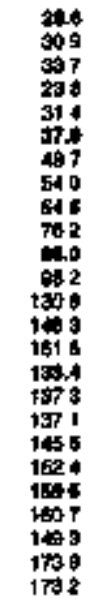 & 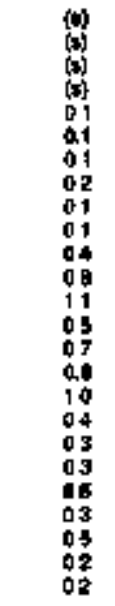 & 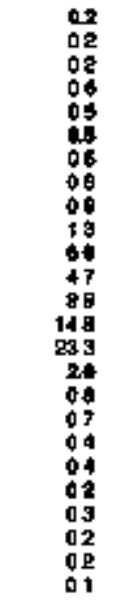 & 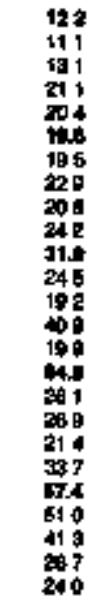 & 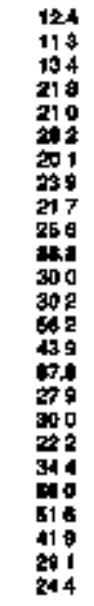 & $\begin{array}{l}z \\
z \\
z \\
z \\
z \\
z \\
z \\
z \\
z \\
z \\
z \\
\bar{Z} \\
68 \\
68 \\
64\end{array}$ & 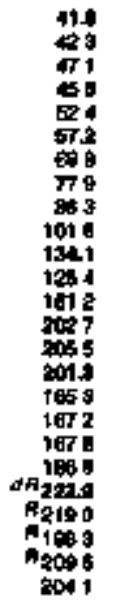 & 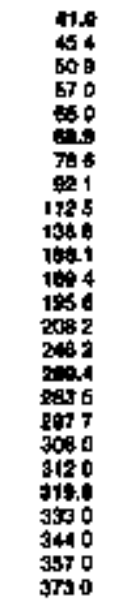 & 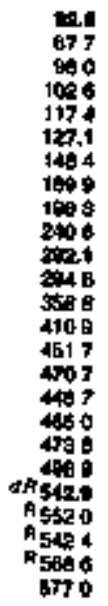 \\
\hline
\end{tabular}

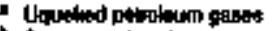

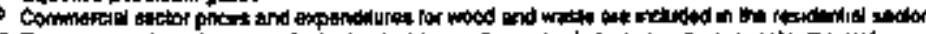

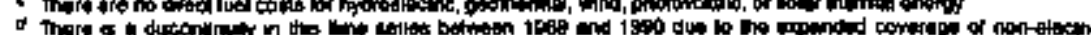

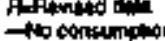

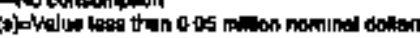


Teble 199. Commerclet Sector Energy Price and Expenditure Eetimatas by soture, 1970-1994, New 140xico

\begin{tabular}{|c|c|c|c|c|c|c|c|c|c|c|c|}
\hline \multirow[b]{4}{*}{ Yer } & \multicolumn{9}{|c|}{ Powny thingy } & \multirow[b]{3}{*}{ 由ectivioin } & \multirow[b]{3}{*}{ Towil. } \\
\hline & \multirow[b]{2}{*}{ Cont } & \multirow[b]{2}{*}{ inds: } & \multicolumn{6}{|c|}{ Panm } & \multirow[b]{2}{*}{ Tortin } & & \\
\hline & & & 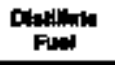 & Keromene & $\mathbf{u n}$ & 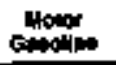 & nowation & Totd & & & \\
\hline & \multicolumn{11}{|c|}{ 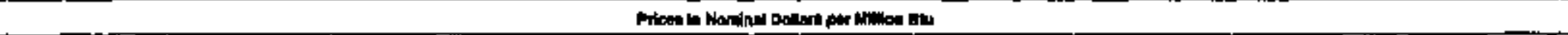 } \\
\hline \multirow[t]{2}{*}{ 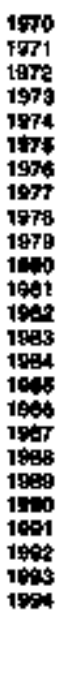 } & 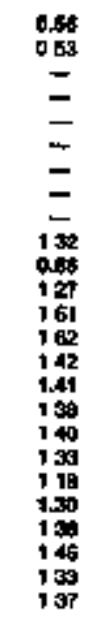 & 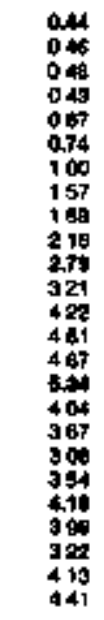 & 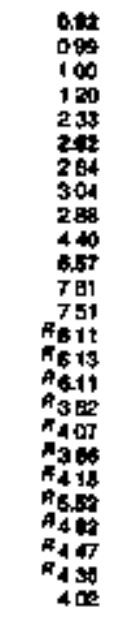 & 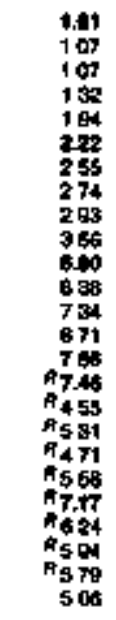 & 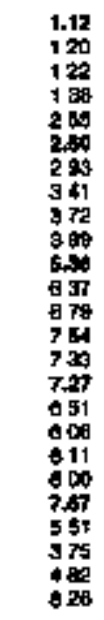 & 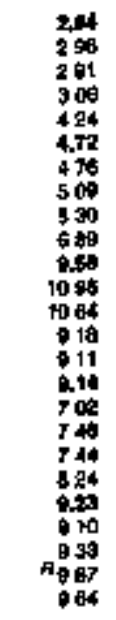 & $\begin{array}{l}= \\
= \\
= \\
= \\
= \\
= \\
= \\
400 \\
451 \\
400 \\
= \\
= \\
= \\
= \\
=\end{array}$ & 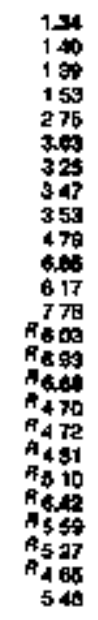 & 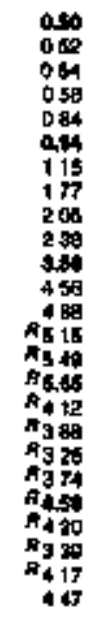 & 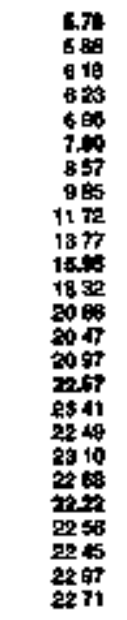 & 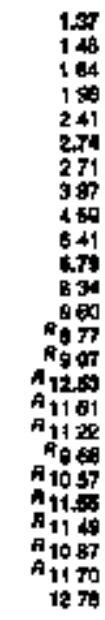 \\
\hline & \multicolumn{11}{|c|}{ 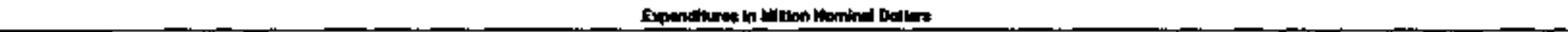 } \\
\hline 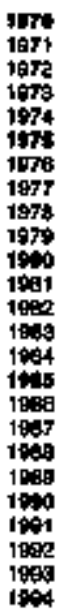 & $\begin{array}{l}\text { (a) } \\
\text { (a) } \\
= \\
= \\
= \\
= \\
= \\
\overline{0}-1 \\
0.5 \\
01 \\
01 \\
02 \\
01 \\
01 \\
01 \\
01 \\
01 \\
01 \\
01 \\
01 \\
01 \\
02 \\
02 \\
02\end{array}$ & 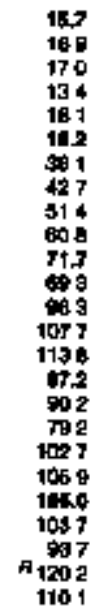 & 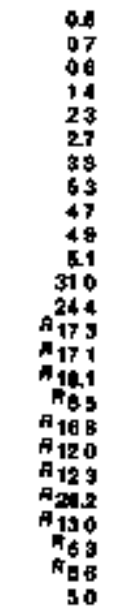 & 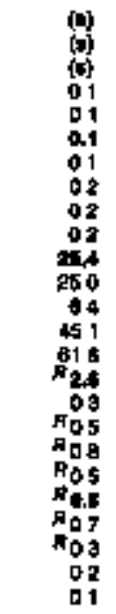 & $\begin{array}{l}11 \\
75 \\
17 \\
16 \\
97 \\
87 \\
24 \\
30 \\
27 \\
38 \\
47 \\
34 \\
47 \\
62 \\
28 \\
07 \\
42 \\
40 \\
36 \\
48 \\
44 \\
47 \\
26 \\
25 \\
41\end{array}$ & $\begin{array}{l}1.1 \\
11 \\
11 \\
12 \\
19 \\
23 \\
24 \\
28 \\
08 \\
36 \\
5.5 \\
69 \\
64 \\
51 \\
40 \\
54 \\
43 \\
10 \\
10 \\
52 \\
01 \\
54 \\
10 \\
09 \\
09\end{array}$ & $\begin{array}{l}= \\
= \\
= \\
= \\
= \\
= \\
= \\
= \\
157 \\
0.1 \\
= \\
= \\
= \\
= \\
=\end{array}$ & 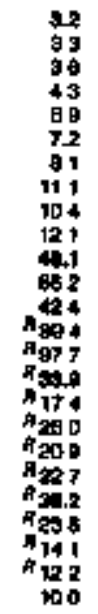 & 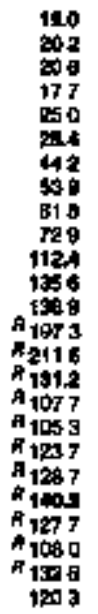 & 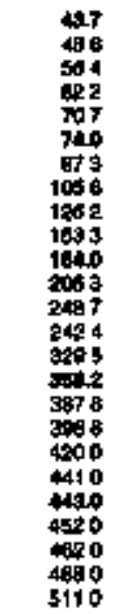 & 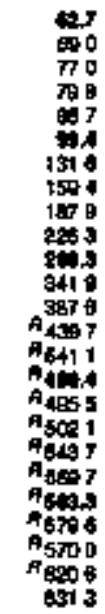 \\
\hline
\end{tabular}




\begin{tabular}{|c|c|c|c|c|c|c|c|c|c|c|c|c|c|c|c|c|c|}
\hline & \multicolumn{15}{|c|}{ 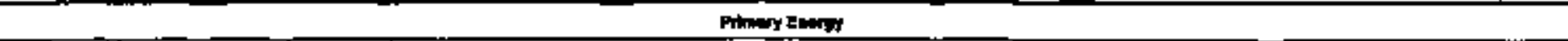 } & \multirow[b]{3}{*}{ encikitis } & \multirow[b]{3}{*}{ 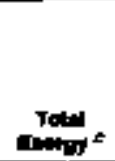 } \\
\hline & \multicolumn{3}{|c|}{ con } & \multirow[b]{2}{*}{ ment } & \multicolumn{9}{|c|}{ 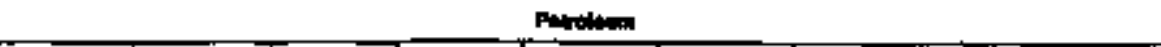 } & \multirow[b]{2}{*}{ E } & \multirow[b]{2}{*}{ Trow } & & \\
\hline & Coth & $\sin$ & Toen & & 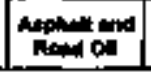 & 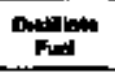 & 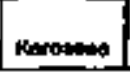 & Leo: & Lemanas & and & mingen & Oners ${ }^{2}$ & $T=$ & & & & \\
\hline$x=$ & \multicolumn{17}{|c|}{ 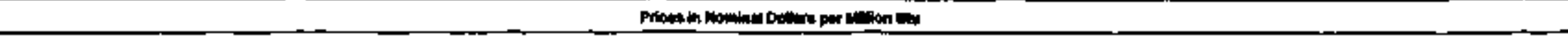 } \\
\hline \multirow[t]{2}{*}{ 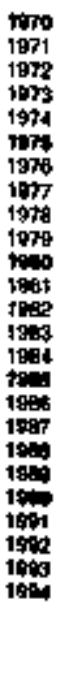 } & $\begin{array}{l}= \\
= \\
z \\
z \\
= \\
z \\
= \\
= \\
= \\
= \\
= \\
= \\
=\end{array}$ & 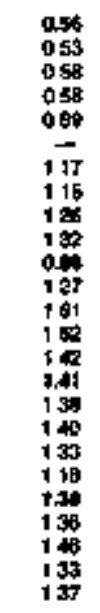 & 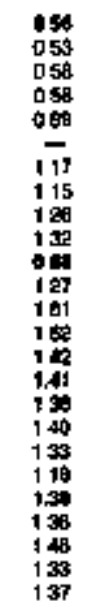 & 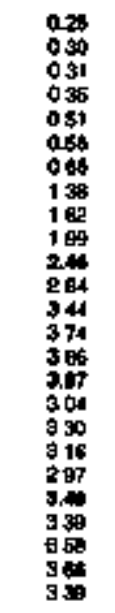 & 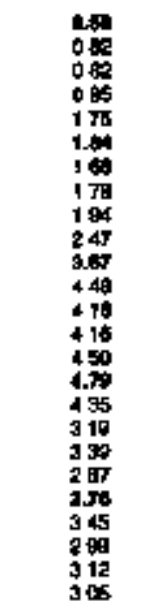 & 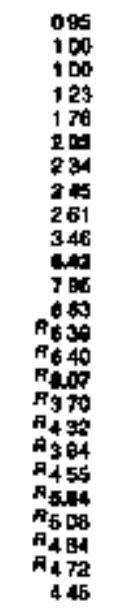 & 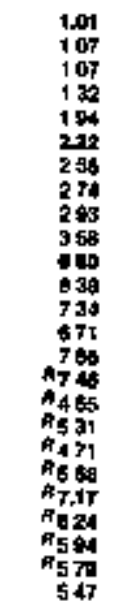 & 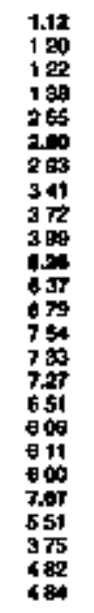 & 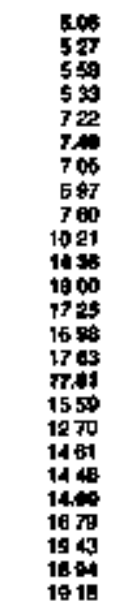 & 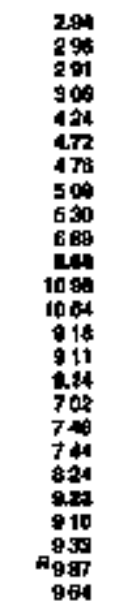 & 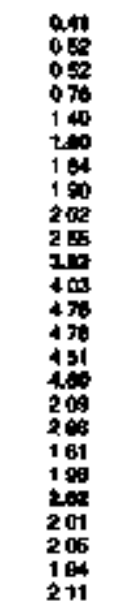 & 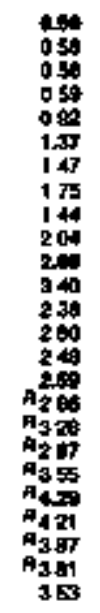 & 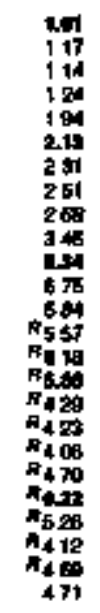 & $\begin{array}{l}= \\
= \\
= \\
= \\
= \\
= \\
= \\
= \\
= \\
= \\
= \\
\bar{z} \\
194 \\
190 \\
150 \\
18\end{array}$ & 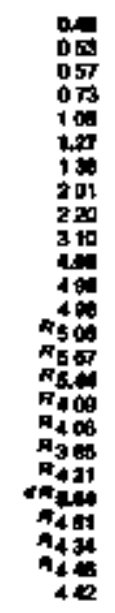 & 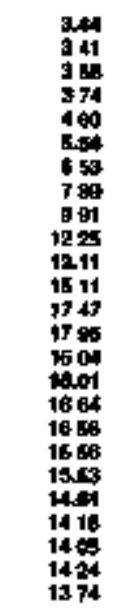 & 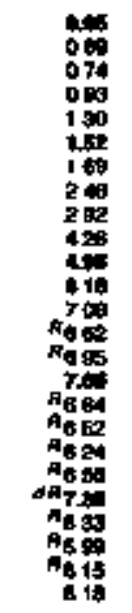 \\
\hline & \multicolumn{17}{|c|}{ 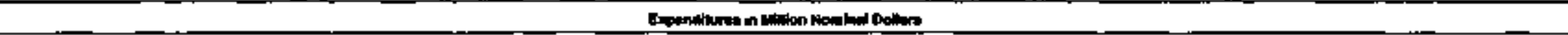 } \\
\hline 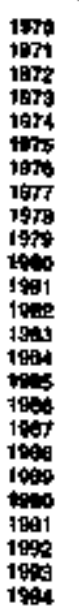 & $\begin{array}{l}= \\
= \\
= \\
= \\
= \\
= \\
= \\
= \\
= \\
= \\
= \\
=\end{array}$ & $\begin{array}{l}01 \\
01 \\
01 \\
01 \\
02 \\
01 \\
01 \\
03 \\
04 \\
04 \\
22 \\
27 \\
02 \\
34 \\
44 \\
34 \\
29 \\
29 \\
24 \\
15 \\
18 \\
11 \\
11 \\
12 \\
14 \\
17 \\
20\end{array}$ & $\begin{array}{l}01 \\
01 \\
01 \\
01 \\
02 \\
01 \\
-12 \\
02 \\
04 \\
22 \\
27 \\
02 \\
34 \\
44 \\
34 \\
23 \\
25 \\
28 \\
15 \\
15 \\
15 \\
11 \\
11 \\
12 \\
14 \\
17 \\
20 \\
20\end{array}$ & 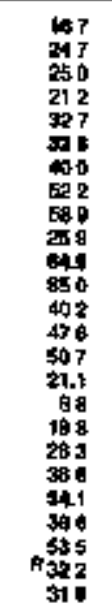 & 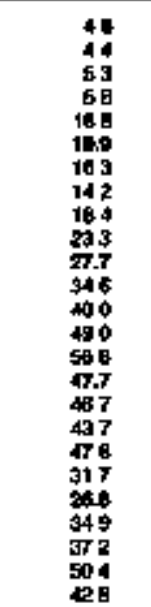 & 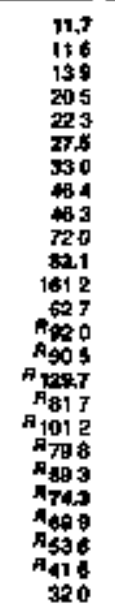 & 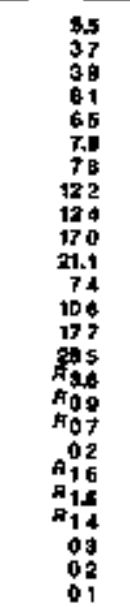 & 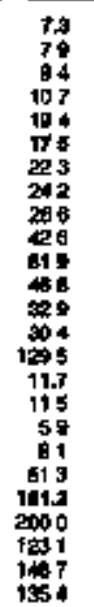 & 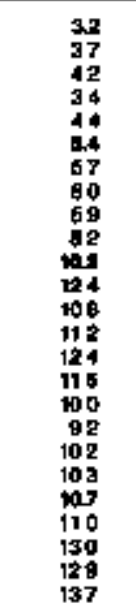 & 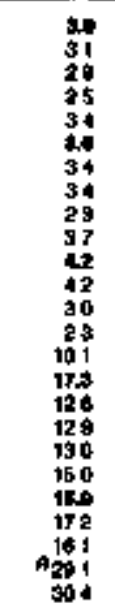 & 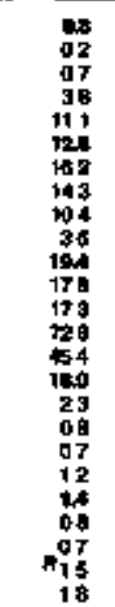 & 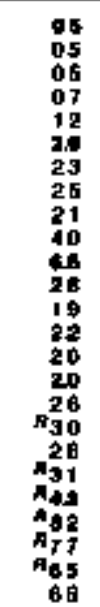 & 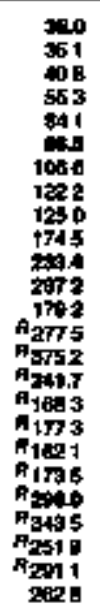 & 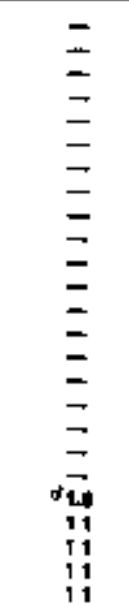 & 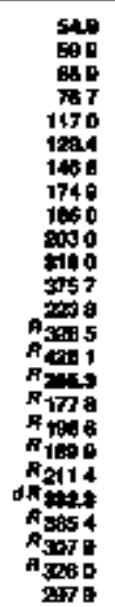 & 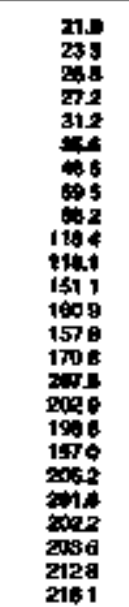 & 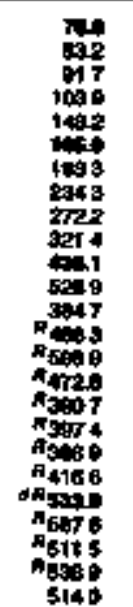 \\
\hline
\end{tabular}


Tabie 201. Transportation Spetor Enargy Price and Expondlitre Eatimatea by Source, 1970-1994, Now Moxico

\begin{tabular}{|c|c|c|c|c|c|c|c|c|c|c|c|c|c|}
\hline & \multicolumn{11}{|c|}{ Phiner Eimgr } & \multirow[b]{3}{*}{ Encotileky } & \multirow[b]{3}{*}{ 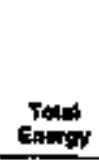 } \\
\hline & \multirow[b]{2}{*}{ Cod } & \multirow[b]{2}{*}{ 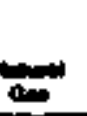 } & \multicolumn{8}{|c|}{ rowowen. } & \multirow[b]{2}{*}{ Totest } & & \\
\hline & & & 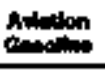 & Din & int & $\underline{\mathbf{L n}}$ & Ltwkantip. & monts & Fantand & Toted & & & \\
\hline ven & \multicolumn{13}{|c|}{ 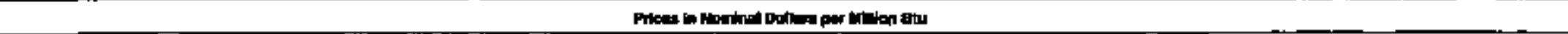 } \\
\hline 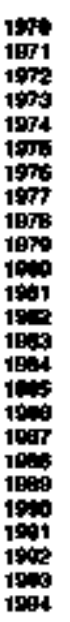 & $\begin{array}{l}05 \\
050 \\
05 \\
050 \\
00 \\
117 \\
115 \\
= \\
= \\
= \\
= \\
= \\
= \\
= \\
= \\
=\end{array}$ & $\begin{array}{l}= \\
= \\
= \\
= \\
= \\
= \\
= \\
= \\
= \\
= \\
= \\
= \\
309\end{array}$ & 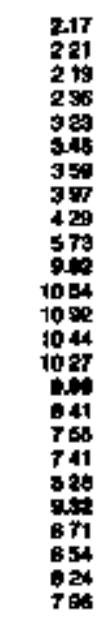 & 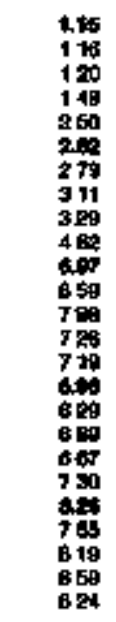 & 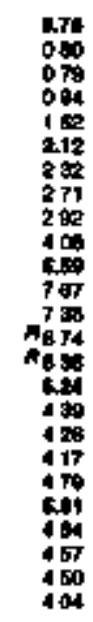 & 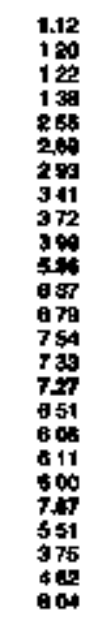 & 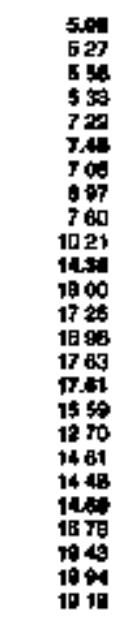 & 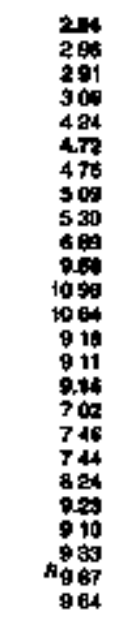 & $\begin{array}{l}0.0 \\
= \\
= \\
= \\
= \\
= \\
= \\
= \\
453 \\
= \\
= \\
= \\
= \\
= \\
=\end{array}$ & 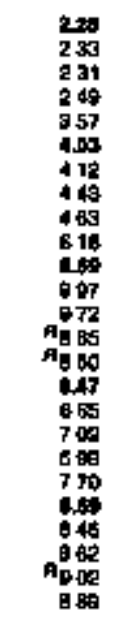 & 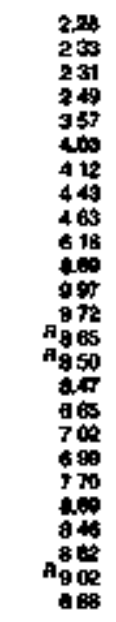 & $\begin{array}{l}= \\
= \\
z \\
= \\
= \\
= \\
= \\
= \\
= \\
= \\
= \\
= \\
= \\
= \\
=\end{array}$ & 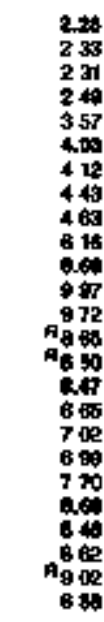 \\
\hline & \multicolumn{13}{|c|}{ 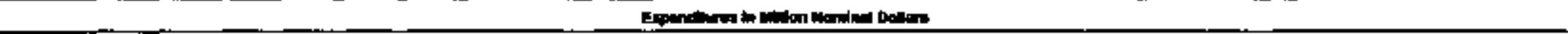 } \\
\hline 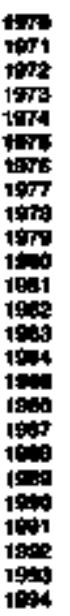 & $\begin{array}{l}0=0 \\
000 \\
0=0 \\
0=0 \\
= \\
= \\
= \\
= \\
= \\
= \\
= \\
= \\
= \\
=\end{array}$ & $\begin{array}{l}= \\
= \\
= \\
= \\
= \\
= \\
= \\
= \\
= \\
= \\
= \\
= \\
=01 \\
01\end{array}$ & $\begin{array}{l}19 \\
13 \\
12 \\
10 \\
17 \\
14 \\
14 \\
10 \\
11 \\
23 \\
74 \\
74 \\
71 \\
58 \\
43 \\
49 \\
14 \\
93 \\
21 \\
10 \\
14 \\
41 \\
40 \\
29 \\
25\end{array}$ & 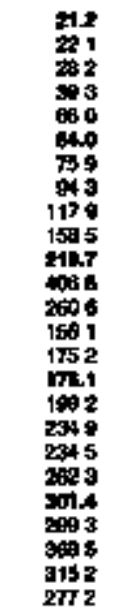 & 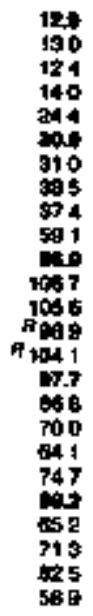 & $\begin{array}{l}10 \\
13 \\
15 \\
12 \\
21 \\
20 \\
22 \\
29 \\
30 \\
01 \\
14 \\
29 \\
32 \\
29 \\
22 \\
28 \\
22 \\
10 \\
16 \\
34 \\
13 \\
18 \\
11 \\
17 \\
42\end{array}$ & 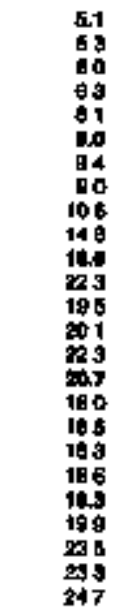 & 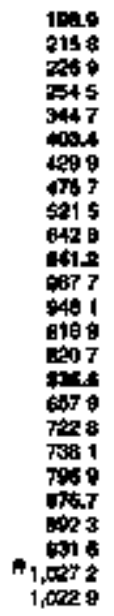 & $\begin{array}{l}= \\
= \\
= \\
= \\
= \\
= \\
= \\
= \\
= \\
= \\
= \\
= \\
= \\
= \\
=\end{array}$ & 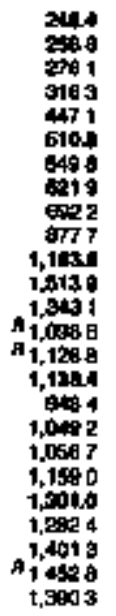 & 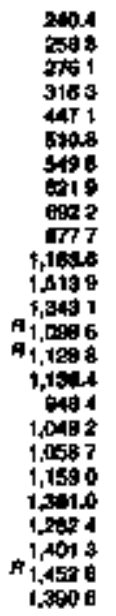 & $\begin{array}{l}= \\
= \\
= \\
= \\
= \\
= \\
= \\
= \\
= \\
= \\
= \\
= \\
= \\
=\end{array}$ & 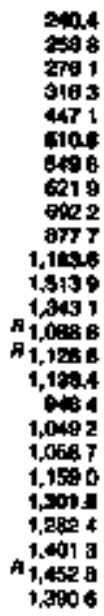 \\
\hline
\end{tabular}

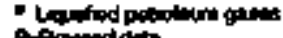

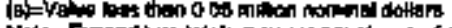

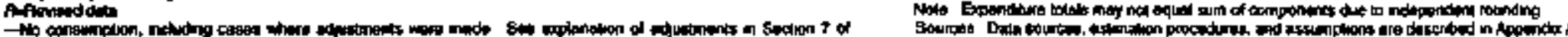

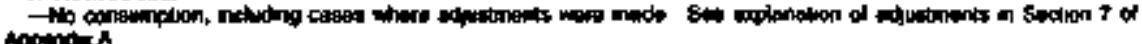




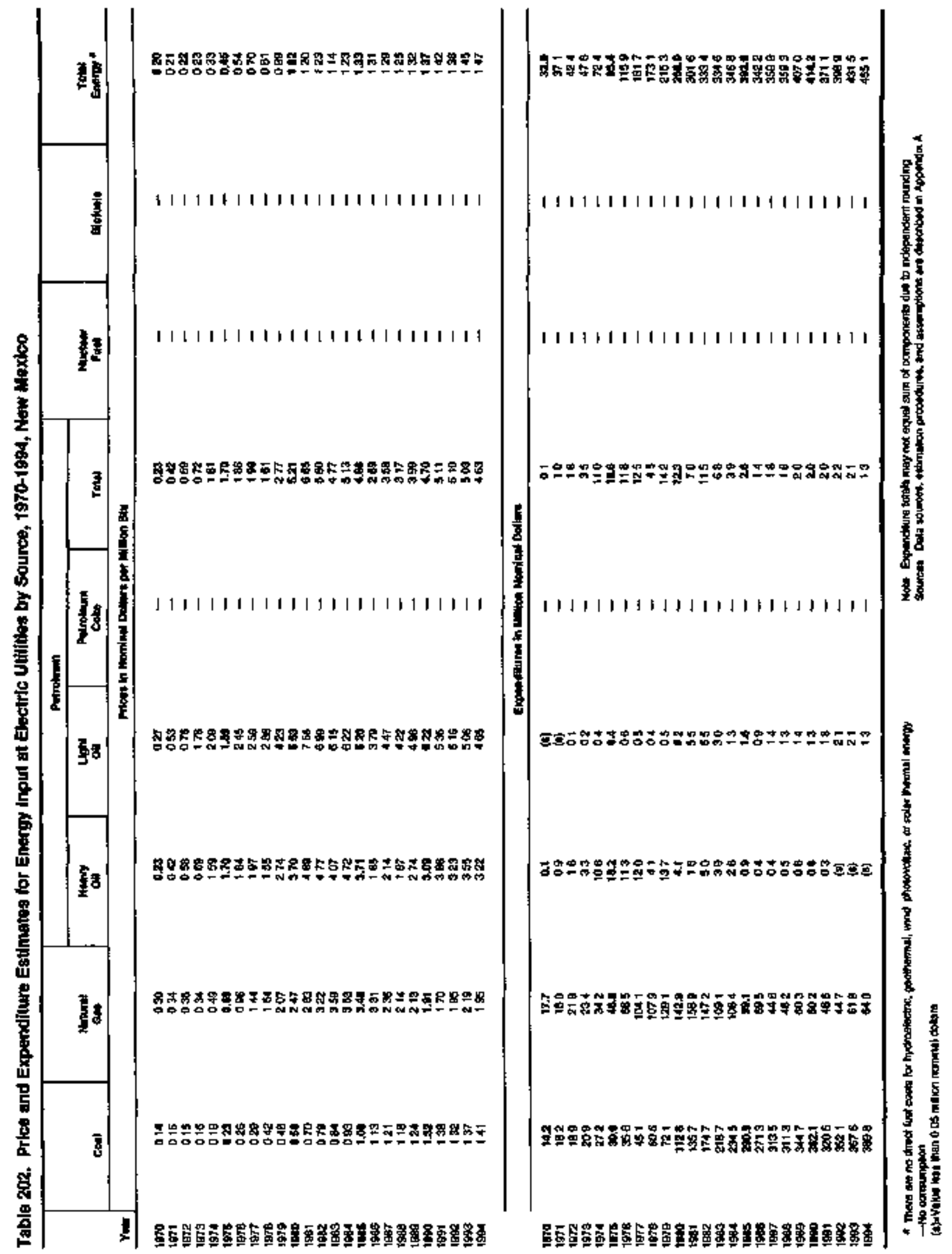


Table 203. Energy Price and Expenditure Eutmales by Sourcs, 1970-1994, Now York

\begin{tabular}{|c|c|c|c|c|c|c|c|c|c|c|c|c|c|c|c|c|c|}
\hline \multirow[b]{4}{*}{ Yom: } & \multicolumn{14}{|c|}{ Pindery Enoty } & \multirow{3}{*}{ 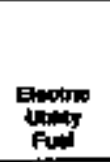 } & \multirow{3}{*}{ Enototy } & \multirow[b]{3}{*}{ Tow } \\
\hline & \multicolumn{3}{|c|}{ cen } & \multirow[b]{2}{*}{ Notull } & \multicolumn{7}{|c|}{ Putralum } & \multirow[b]{2}{*}{ 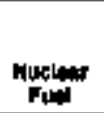 } & \multirow[b]{2}{*}{ Dhoheris } & \multirow[b]{2}{*}{ Totid $=$} & & & \\
\hline & $\begin{array}{l}\text { Cotang } \\
\text { Cond }\end{array}$ & 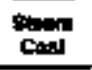 & Ton & & Din & A th & LPE. & Motom & 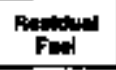 & other: & Total & & & & & & \\
\hline & \multicolumn{17}{|c|}{ 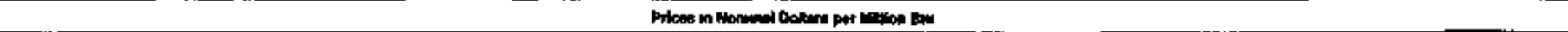 } \\
\hline \multirow[t]{2}{*}{ 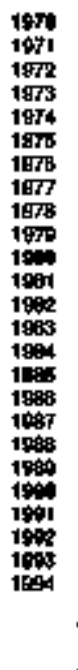 } & 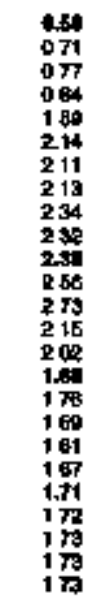 & 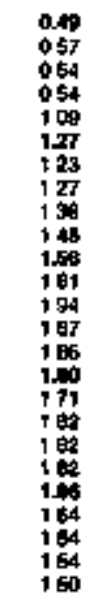 & 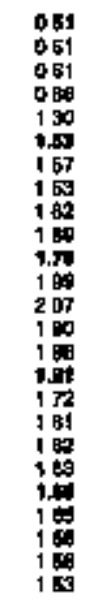 & 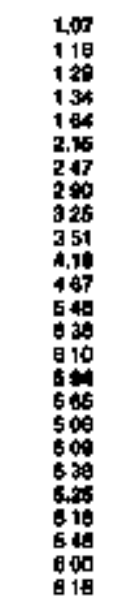 & 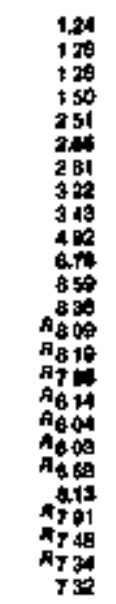 & 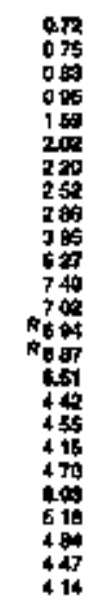 & 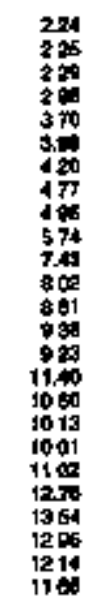 & 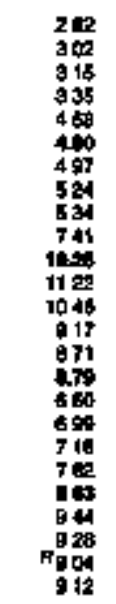 & 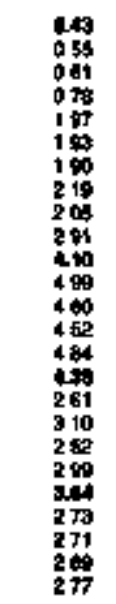 & 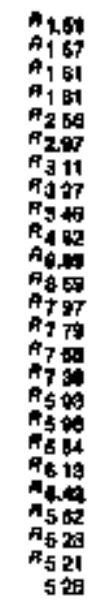 & 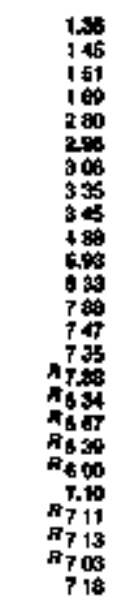 & 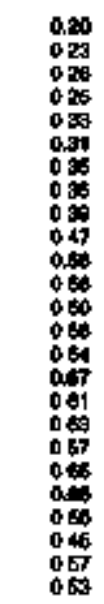 & 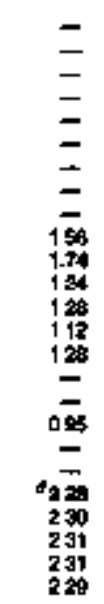 & 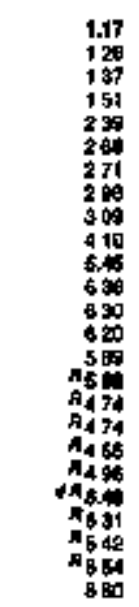 & 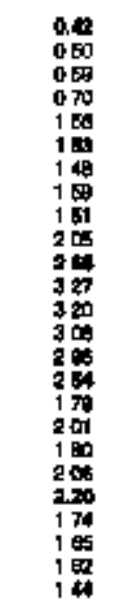 & 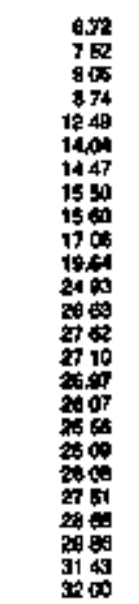 & 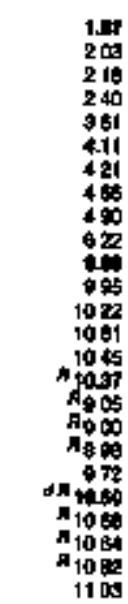 \\
\hline & \multicolumn{17}{|c|}{ 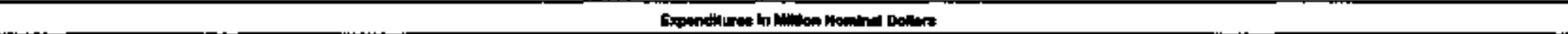 } \\
\hline 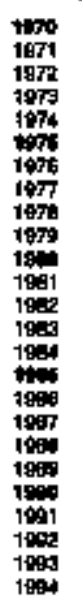 & 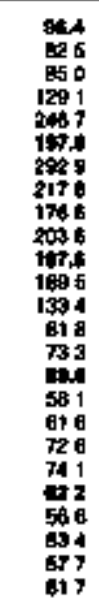 & 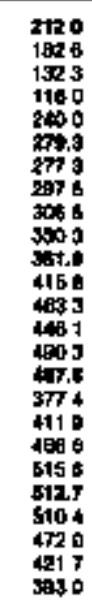 & 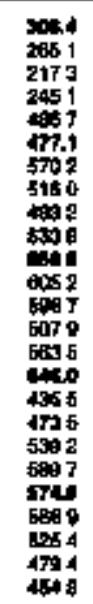 & 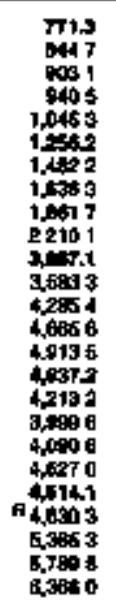 & 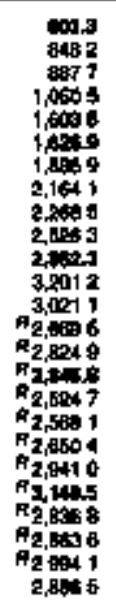 & 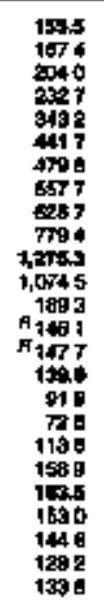 & 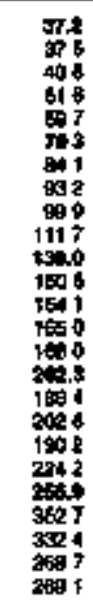 & 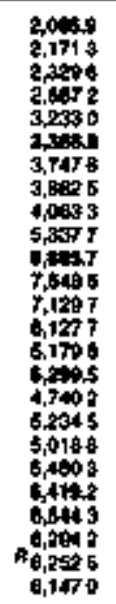 & 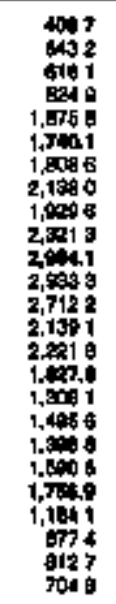 & 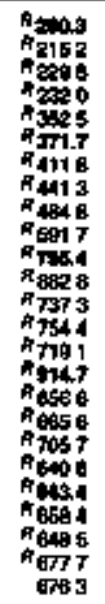 & 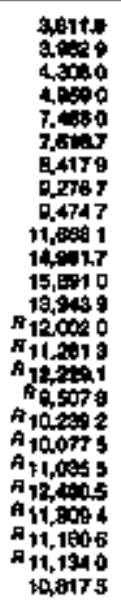 & 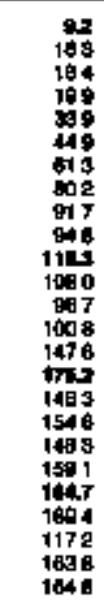 & $\begin{array}{l}= \\
= \\
= \\
= \\
= \\
= \\
11 \\
02 \\
011 \\
01 \\
02 \\
01 \\
= \\
=103 \\
= \\
1042 \\
1130 \\
1147 \\
1219 \\
1204\end{array}$ & 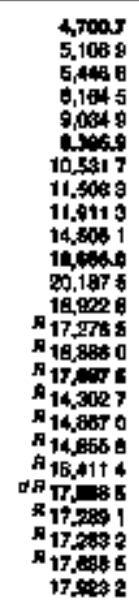 & 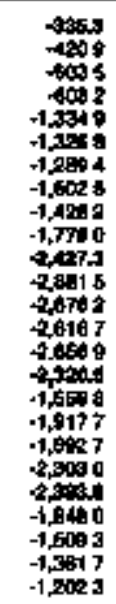 & 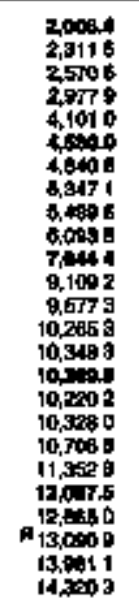 & 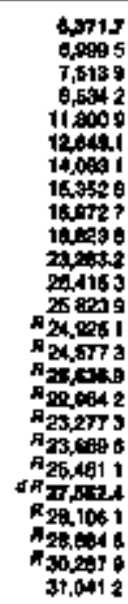 \\
\hline
\end{tabular}

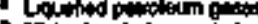

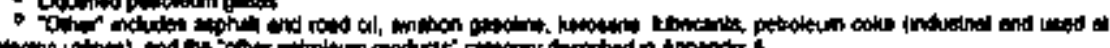

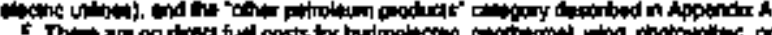

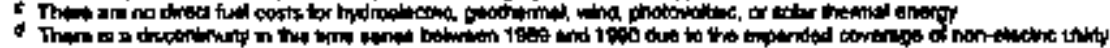

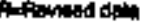

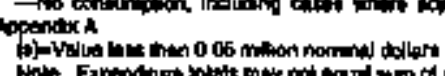

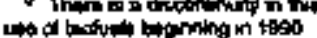

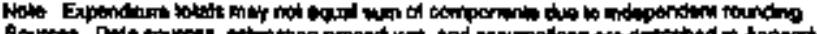

Eneroy intormation Adininistrition 


\begin{tabular}{|c|c|c|c|c|c|c|c|c|c|c|}
\hline \multirow[b]{4}{*}{ Yeer } & \multicolumn{8}{|c|}{ Ant } & \multirow[b]{3}{*}{ Enclow } & \multirow[b]{3}{*}{ 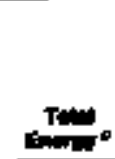 } \\
\hline & \multirow[b]{2}{*}{ بent } & \multirow[b]{2}{*}{ יודיה } & \multicolumn{4}{|c|}{ 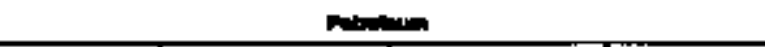 } & \multirow[b]{2}{*}{ EAm: } & \multirow[b]{2}{*}{ Then } & & \\
\hline & & & Dim & 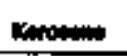 & LO" & Towell & & & & \\
\hline & \multicolumn{10}{|c|}{ 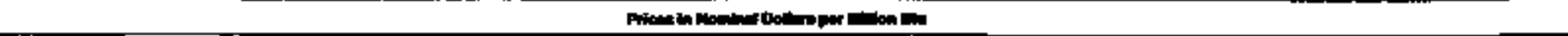 } \\
\hline \multirow[t]{2}{*}{ 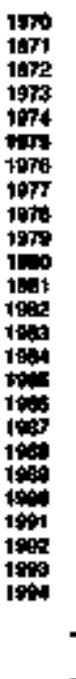 } & 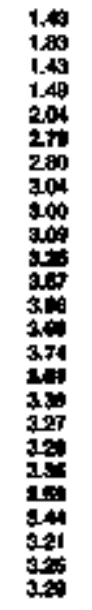 & 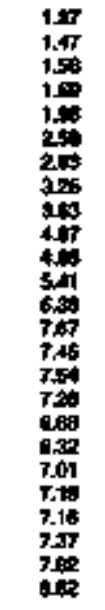 & 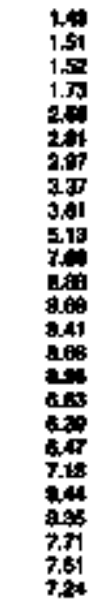 & 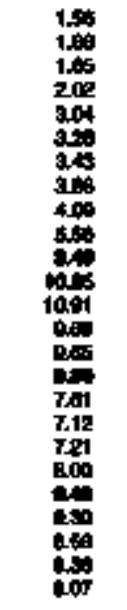 & 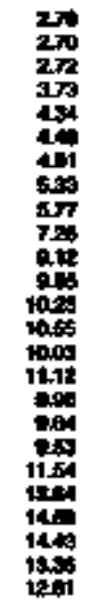 & 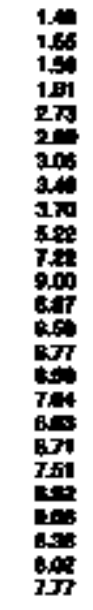 & $\begin{array}{l}= \\
= \\
= \\
= \\
= \\
= \\
= \\
= \\
= \\
= \\
= \\
\bar{z} \\
20 \\
20\end{array}$ & 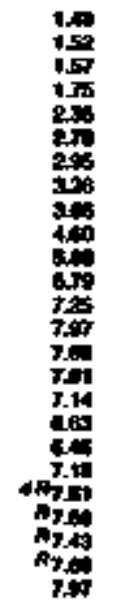 & 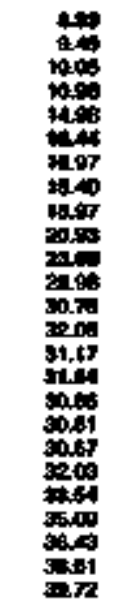 & 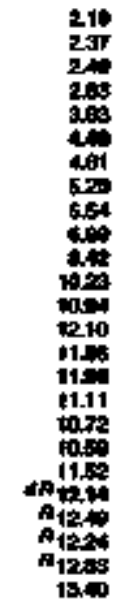 \\
\hline & \multicolumn{10}{|c|}{ ת } \\
\hline 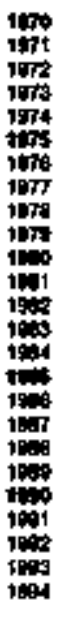 & 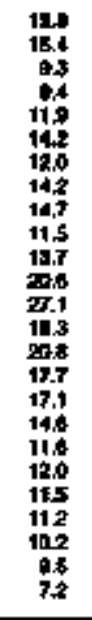 & 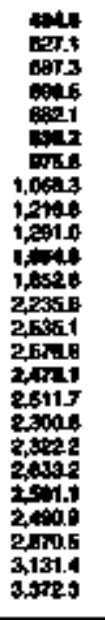 & 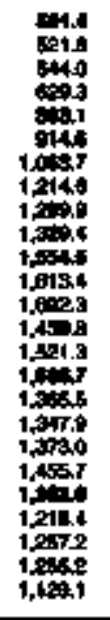 & 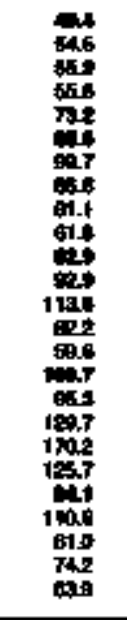 & 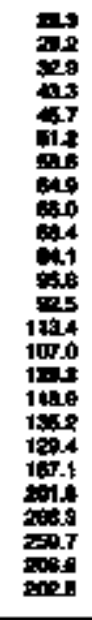 & 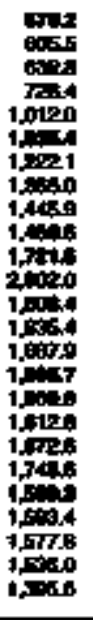 & $\begin{array}{l}= \\
= \\
= \\
= \\
= \\
= \\
= \\
= \\
= \\
= \\
= \\
= \\
z \\
\text { and }\end{array}$ & 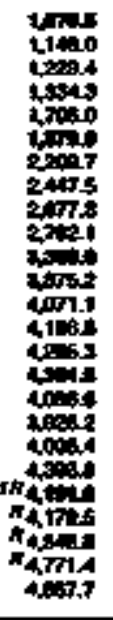 & 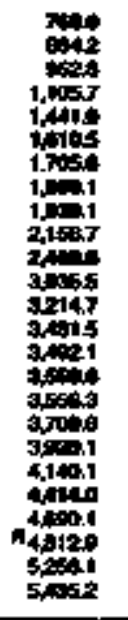 & 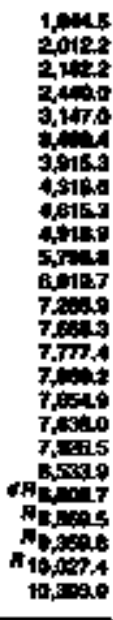 \\
\hline
\end{tabular}

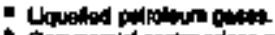

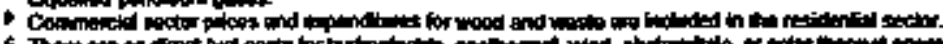

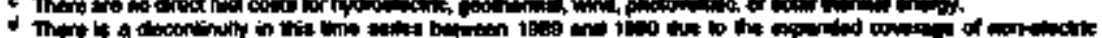

Actiond

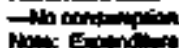

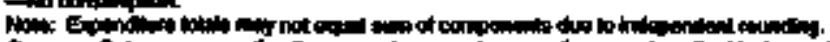

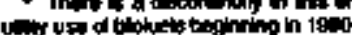




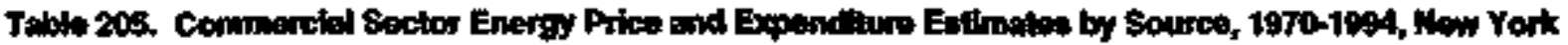

\begin{tabular}{|c|c|c|c|c|c|c|c|c|c|c|c|}
\hline \multirow[b]{4}{*}{$r=$} & \multicolumn{9}{|c|}{ Anty } & \multirow[b]{3}{*}{ Goedrloty } & \multirow[b]{3}{*}{ Tamplat } \\
\hline & & \multirow[b]{2}{*}{ mis } & \multicolumn{6}{|c|}{ Putrats } & \multirow[b]{2}{*}{ Tond } & & \\
\hline & En & & "ה & 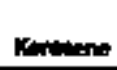 & con" & . & 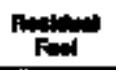 & Tat & & & \\
\hline & \multicolumn{11}{|c|}{ 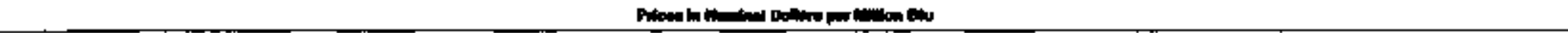 } \\
\hline \multirow[t]{2}{*}{ 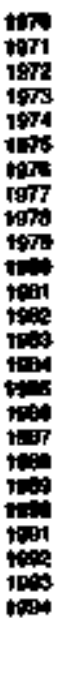 } & 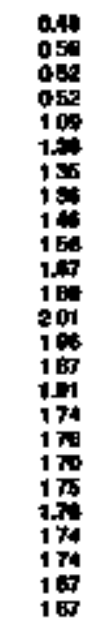 & 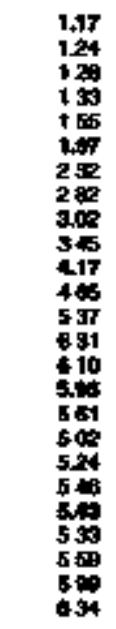 & 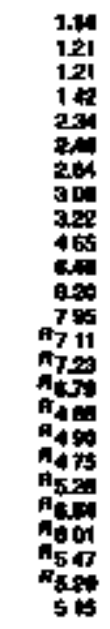 & 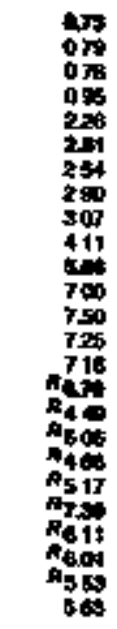 & 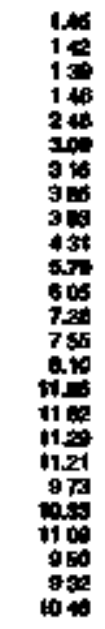 & 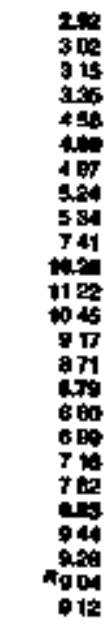 & 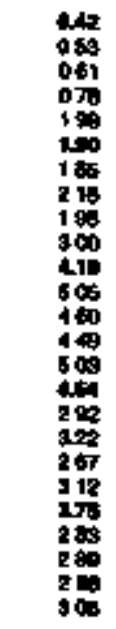 & 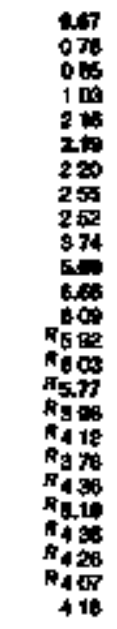 & 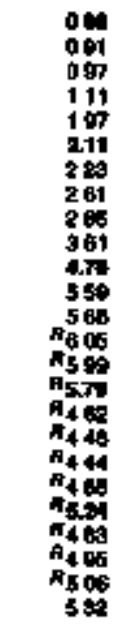 & 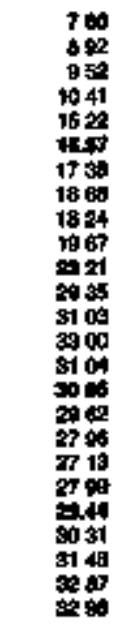 & 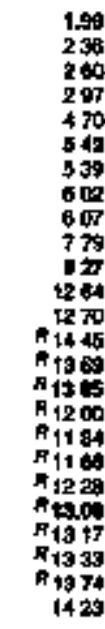 \\
\hline & \multicolumn{11}{|c|}{ 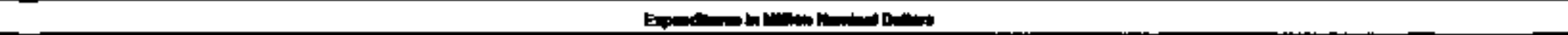 } \\
\hline 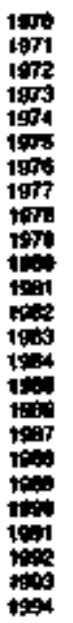 & 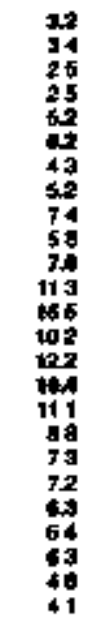 & 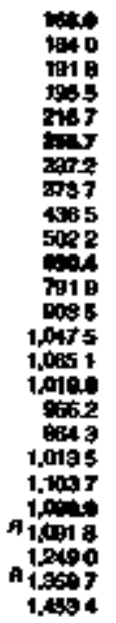 & 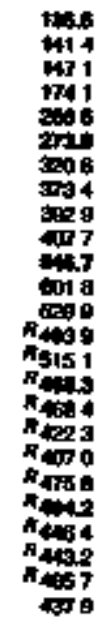 & 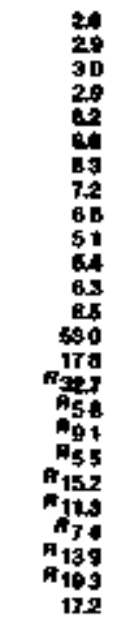 & 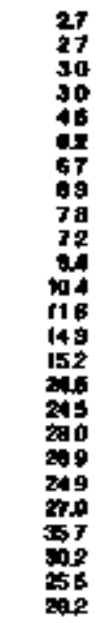 & 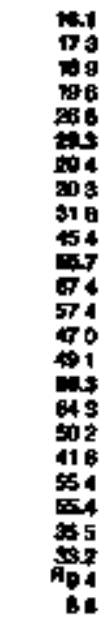 & 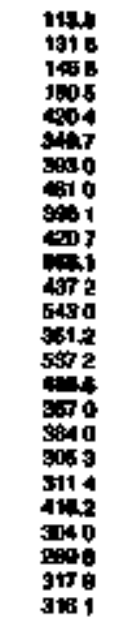 & 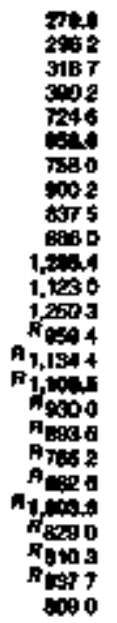 & 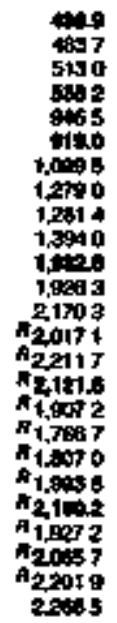 & 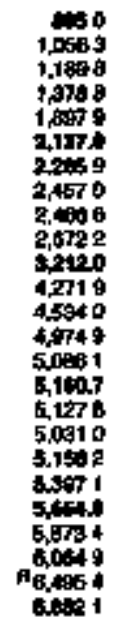 & 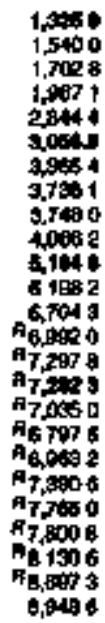 \\
\hline
\end{tabular}

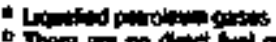

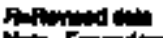

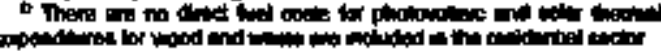

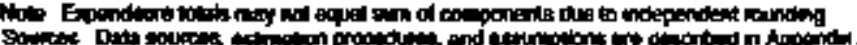


$\stackrel{N}{\mathbb{E}}$

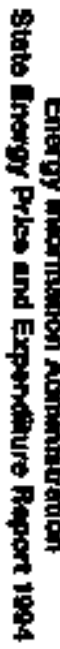

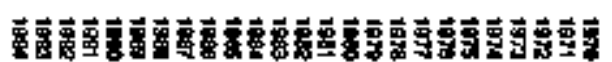

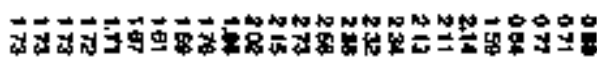

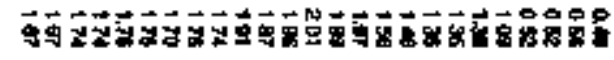

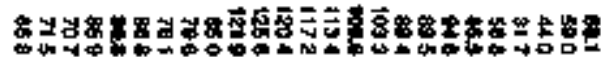

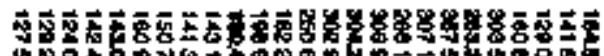

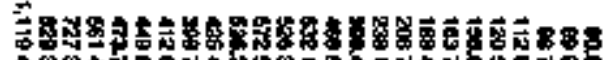

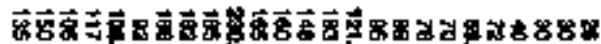

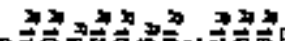

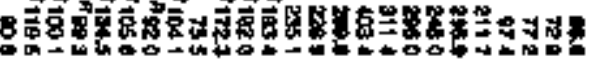

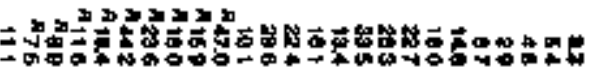

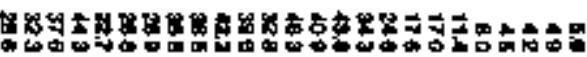

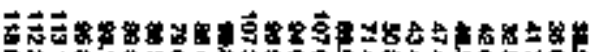

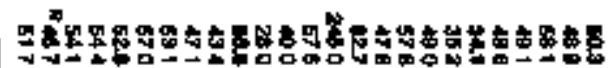

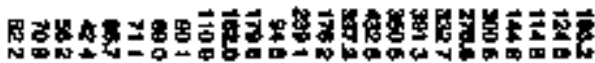

773.137x 3

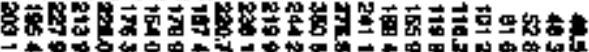

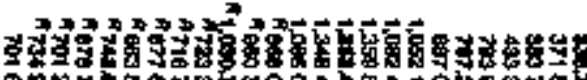

के

$4 \sin 5$

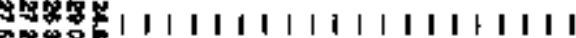

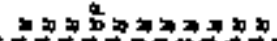

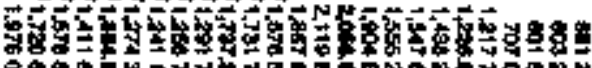

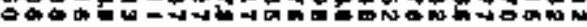

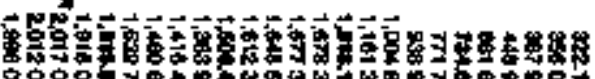

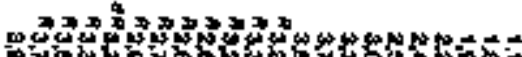

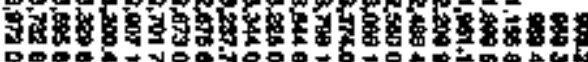

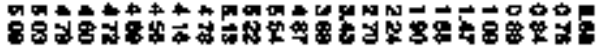

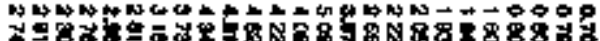

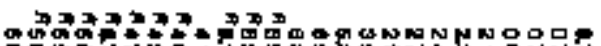

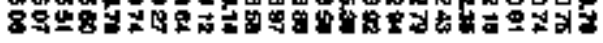

MUZZ3 г

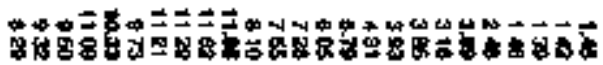

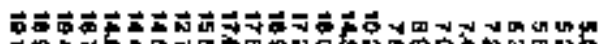

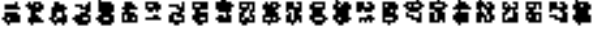

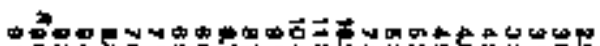

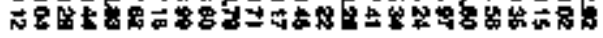

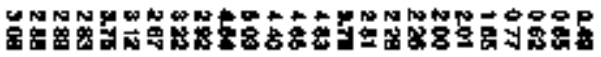

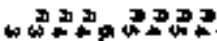

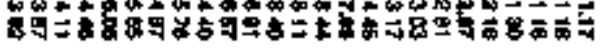

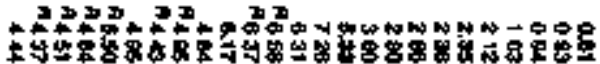

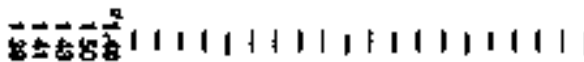

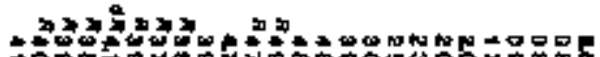

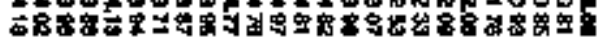

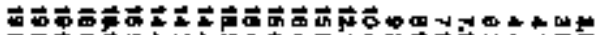

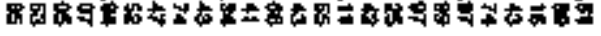

- 73.

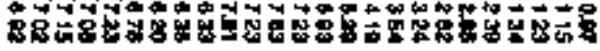

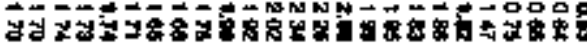

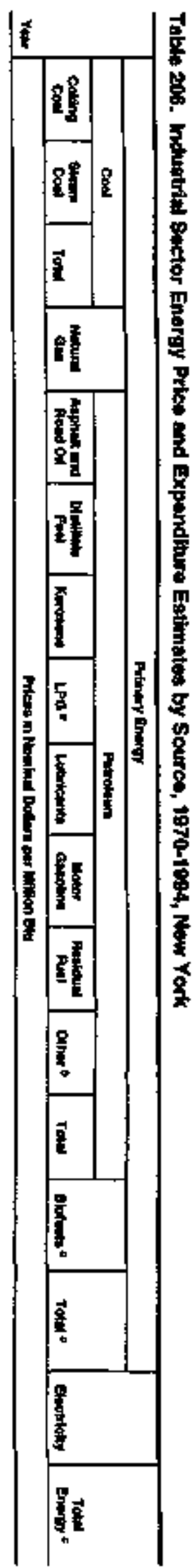


Table 207. Transportation Sector Enorgy Price and Expenditure Estimetes by Source, 1970-1994, New York

\begin{tabular}{|c|c|c|c|c|c|c|c|c|c|c|c|c|c|}
\hline \multirow[b]{4}{*}{ Y4: ? } & \multicolumn{11}{|c|}{ 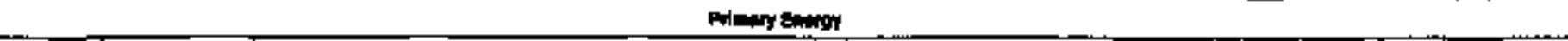 } & \multirow[b]{3}{*}{ 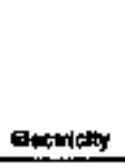 } & \multirow[b]{3}{*}{ 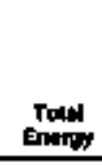 } \\
\hline & \multirow[b]{2}{*}{ Cont } & \multirow[b]{2}{*}{$\begin{array}{c}\text { Nolued } \\
\text { o.d }\end{array}$} & \multicolumn{8}{|c|}{ Probovedn } & \multirow[b]{2}{*}{ Tot:at } & & \\
\hline & & & 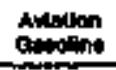 & $\begin{array}{c}\text { Desiand } \\
\text { Fum }\end{array}$ & int & LPG. & Lبtiminas: & 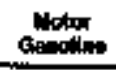 & Pandidid & Tofin & & & \\
\hline & \multicolumn{13}{|c|}{ 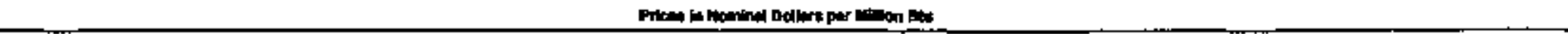 } \\
\hline \multirow[t]{2}{*}{ 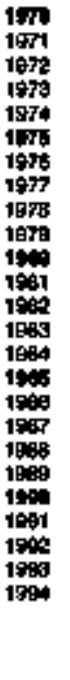 } & $\begin{array}{l}466 \\
056 \\
068 \\
068 \\
109 \\
138 \\
130 \\
= \\
= \\
= \\
= \\
= \\
= \\
= \\
= \\
= \\
= \\
=\end{array}$ & $\begin{array}{l}= \\
= \\
= \\
= \\
= \\
= \\
= \\
= \\
= \\
= \\
= \\
= \\
= \\
457 \\
458 \\
430 \\
904\end{array}$ & 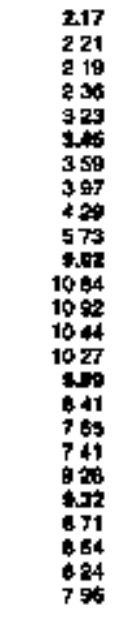 & 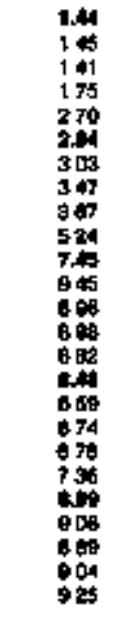 & 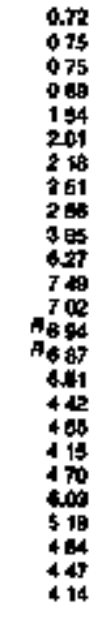 & 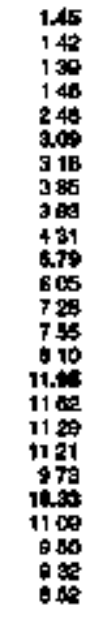 & 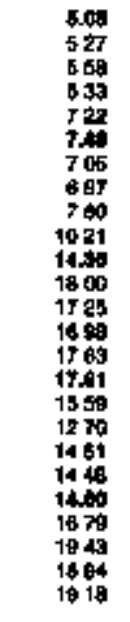 & 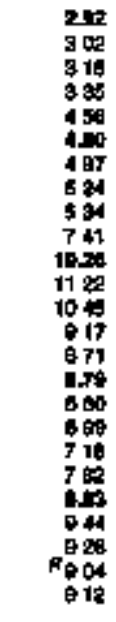 & 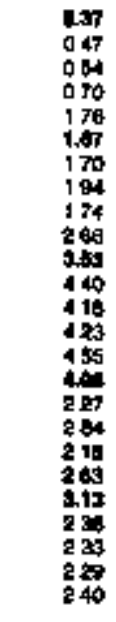 & 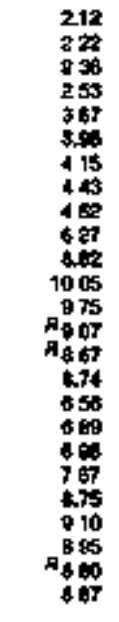 & 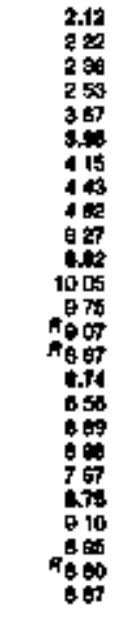 & 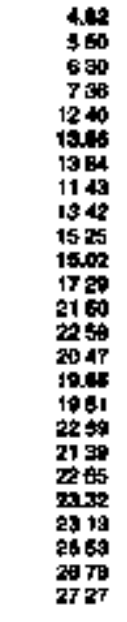 & 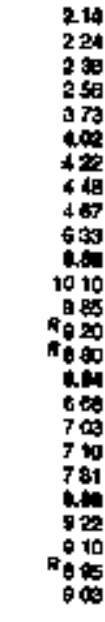 \\
\hline & \multicolumn{13}{|c|}{ 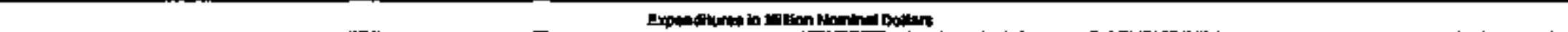 } \\
\hline 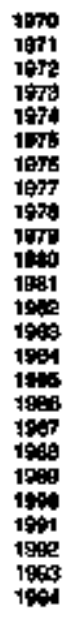 & $\begin{array}{l}0.2 \\
0.1 \\
0 \\
01 \\
01 \\
01 \\
04 \\
04 \\
=1 \\
= \\
= \\
= \\
= \\
= \\
= \\
= \\
= \\
= \\
= \\
= \\
=\end{array}$ & $\begin{array}{l}= \\
= \\
= \\
= \\
= \\
= \\
= \\
= \\
= \\
= \\
= \\
= \\
= \\
09 \\
01 \\
00 \\
01 \\
02\end{array}$ & $\begin{array}{r}27 \\
24 \\
20 \\
19 \\
35 \\
40 \\
46 \\
55 \\
60 \\
90 \\
146 \\
149 \\
109 \\
118 \\
86 \\
111 \\
109 \\
19 \\
39 \\
37 \\
18 \\
29 \\
32 \\
25 \\
40\end{array}$ & 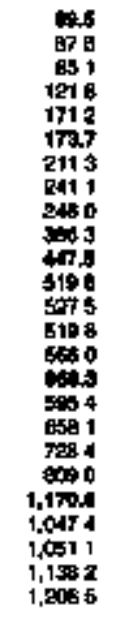 & 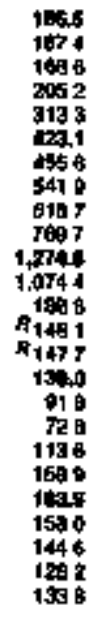 & 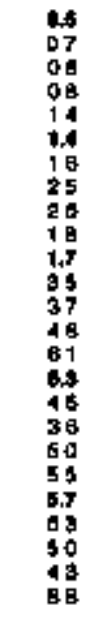 & 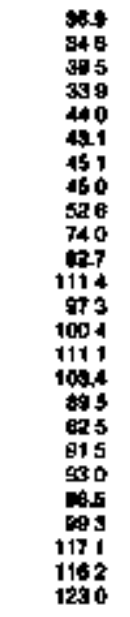 & 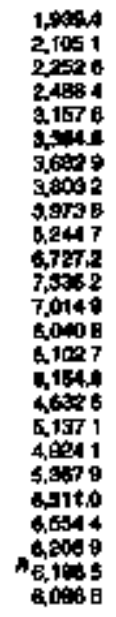 & 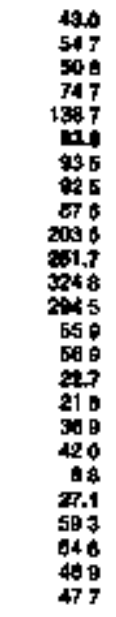 & 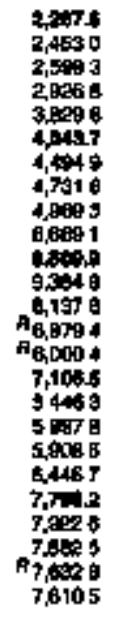 & 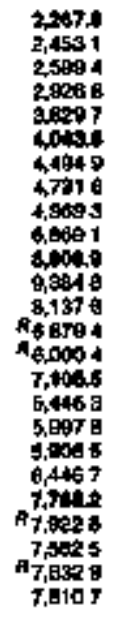 & 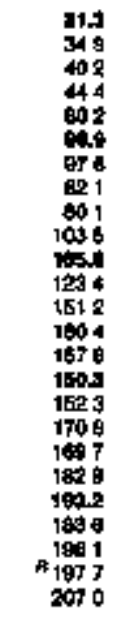 & 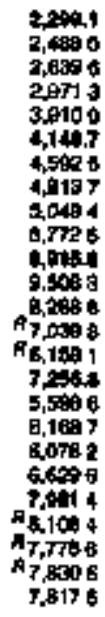 \\
\hline
\end{tabular}




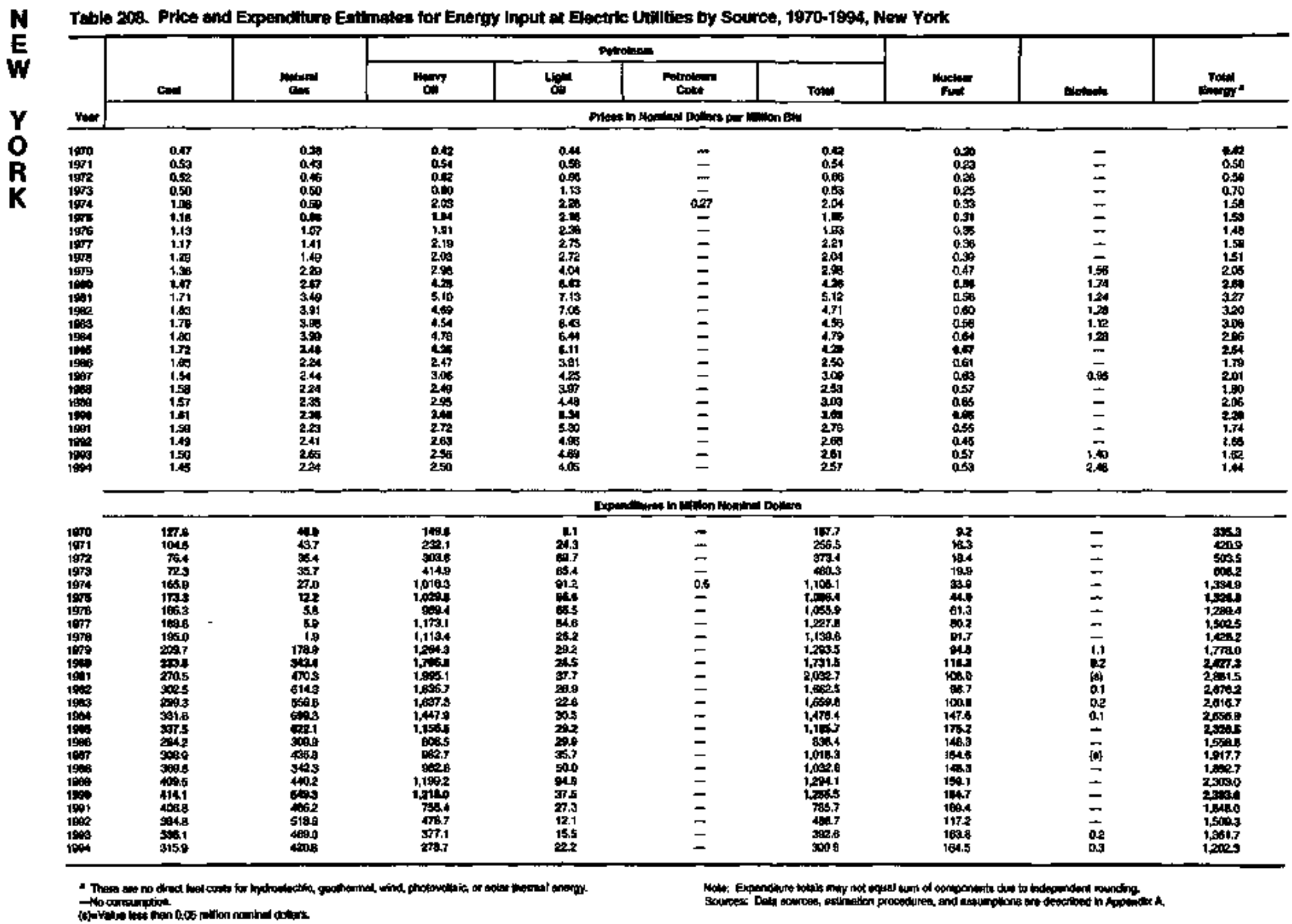


Tabla 209. Energy Price and Expenditure Ealinates by Source, 1970-1994, North Carolina

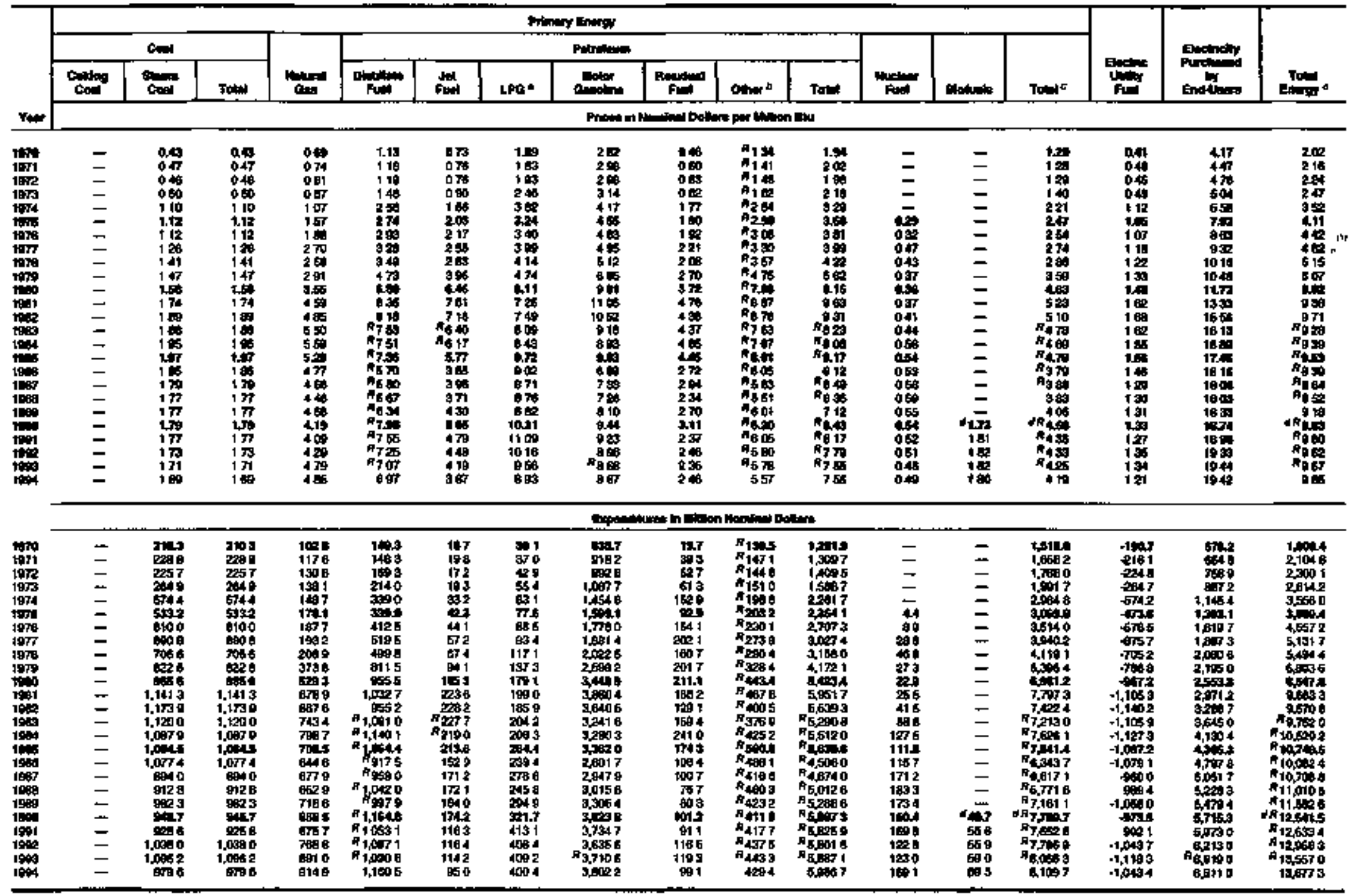

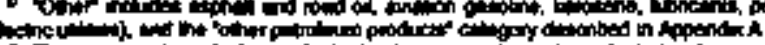

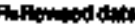
$\rightarrow x_{0}$ 
N Table 210. Aedidential Sattor Energy Prlce and Expendlture Estimates by Source, 1970-1904, Narth Carolina

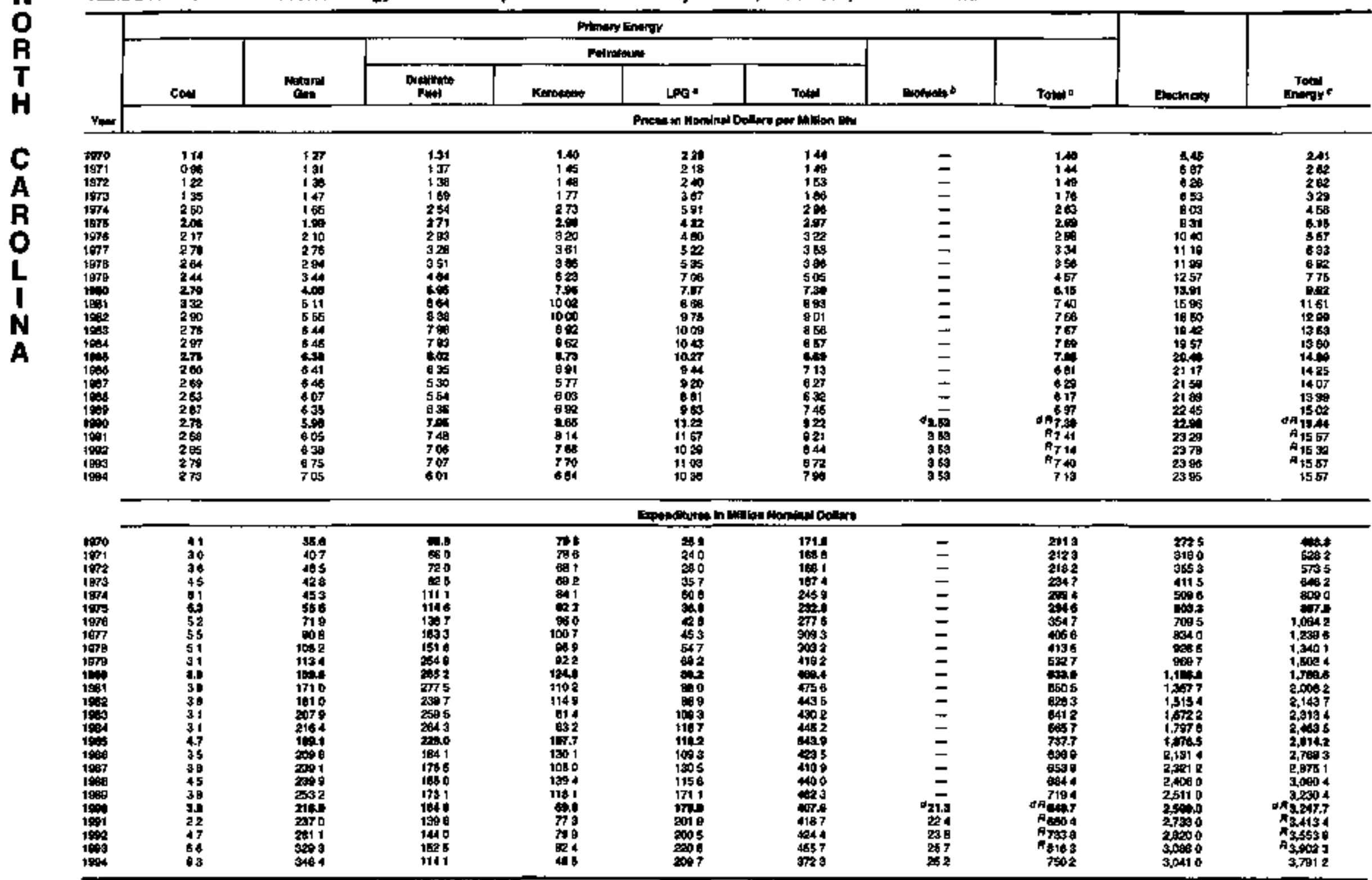

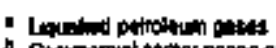

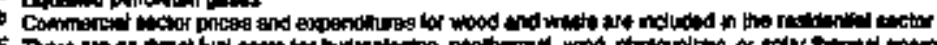

Antimated dat

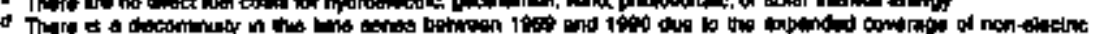
$\rightarrow 10$ conowation

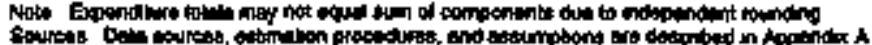

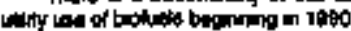




\begin{tabular}{|c|c|c|c|c|c|c|c|c|c|c|c|}
\hline \multirow[b]{4}{*}{ ver } & \multicolumn{9}{|c|}{ Putilaty energy } & \multirow[b]{3}{*}{ Elecinetikg } & \multirow[b]{3}{*}{ Toterl. } \\
\hline & \multirow[b]{2}{*}{$\infty$} & \multirow[b]{2}{*}{ Matid } & \multicolumn{6}{|c|}{ : } & \multirow[b]{2}{*}{ Tols } & & \\
\hline & & & 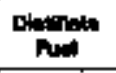 & Haromene & La: & crorer & Find & Tok: & & & \\
\hline & \multicolumn{11}{|c|}{ 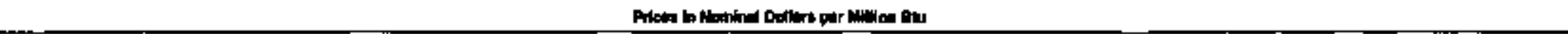 } \\
\hline \multirow[t]{2}{*}{ 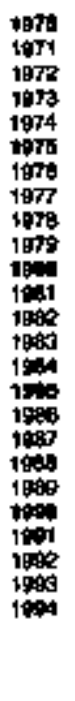 } & 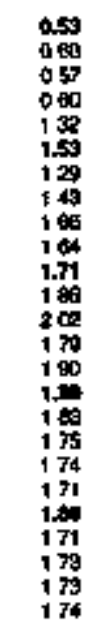 & 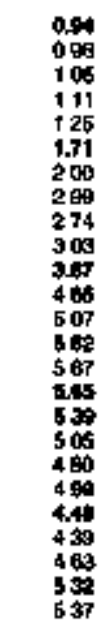 & 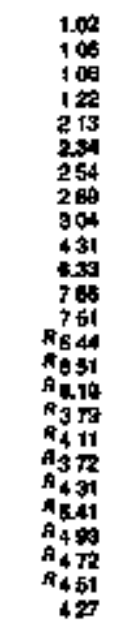 & 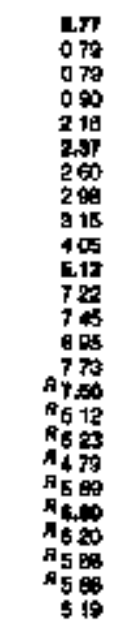 & 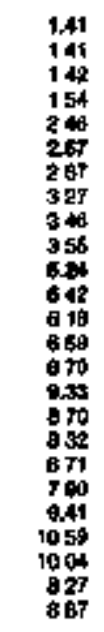 & 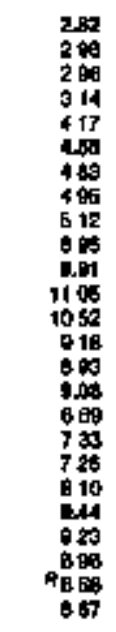 & 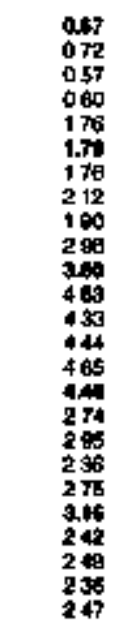 & 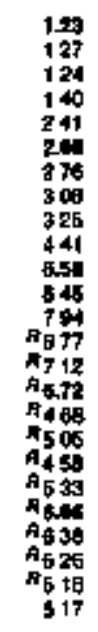 & 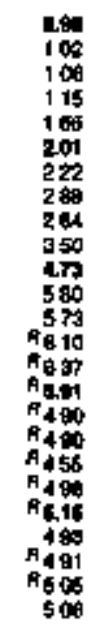 & 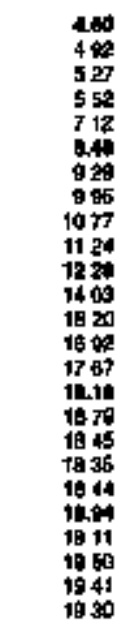 & 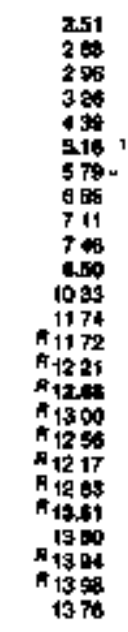 \\
\hline & \multicolumn{11}{|c|}{ 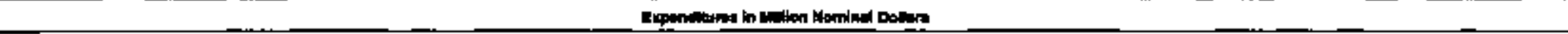 } \\
\hline 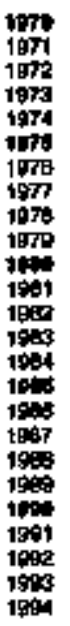 & $\begin{array}{l}34 \\
34 \\
31 \\
37 \\
79 \\
14 \\
50 \\
52 \\
60 \\
30 \\
45 \\
41 \\
50 \\
37 \\
98 \\
60 \\
40 \\
40 \\
50 \\
42 \\
14 \\
27 \\
57 \\
64 \\
74\end{array}$ & 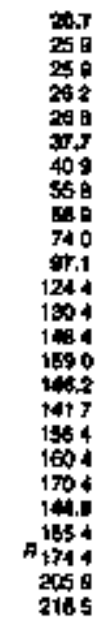 & 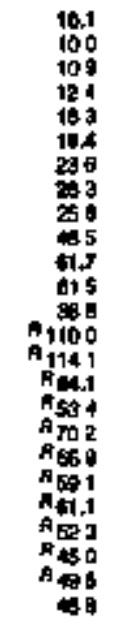 & 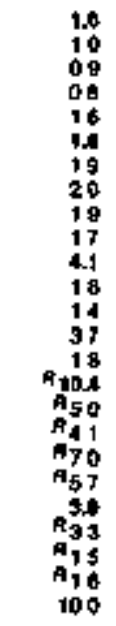 & 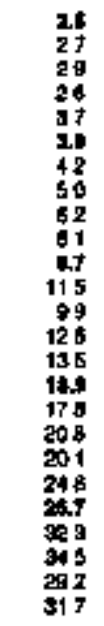 & 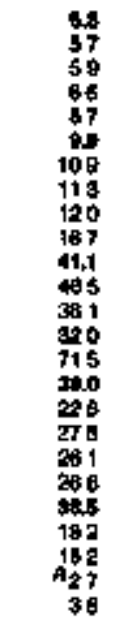 & $\begin{array}{l}0.4 \\
13 \\
13 \\
11 \\
31 \\
24 \\
42 \\
60 \\
41 \\
70 \\
117 \\
30 \\
50 \\
17 \\
70 \\
80 \\
11 \\
12 \\
42 \\
39 \\
45 \\
19 \\
18 \\
43 \\
12\end{array}$ & 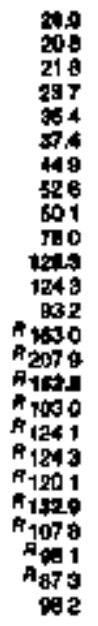 & 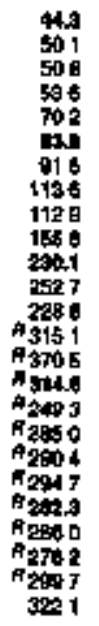 & 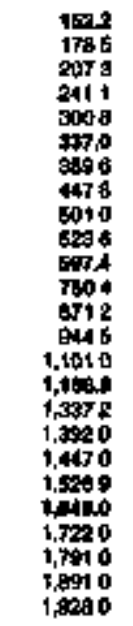 & 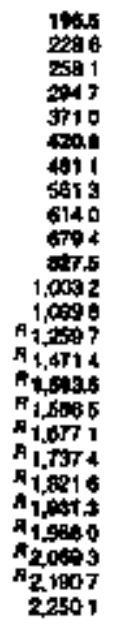 \\
\hline
\end{tabular}




\begin{tabular}{|c|c|c|c|c|c|c|c|c|c|c|c|c|c|c|c|c|c|}
\hline & \multicolumn{15}{|c|}{ Pampretwy } & \multirow[b]{3}{*}{ Exponsy } & \multirow[b]{3}{*}{ The } \\
\hline & \multicolumn{3}{|c|}{ an } & \multirow[b]{2}{*}{$\operatorname{man}$} & \multicolumn{9}{|c|}{ M } & \multirow[b]{2}{*}{0} & \multirow[b]{2}{*}{ Totes $F$} & & \\
\hline & coster & 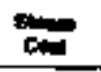 & tote & & 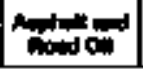 & in & nom & Let & 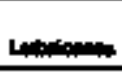 & ming & maid & oner. & 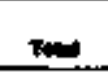 & & & & \\
\hline $\max$ & \multicolumn{17}{|c|}{ 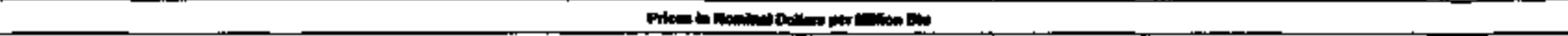 } \\
\hline \multirow[t]{2}{*}{ 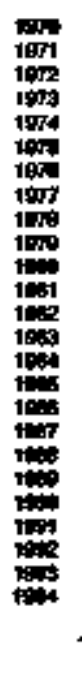 } & $\begin{array}{l}= \\
= \\
= \\
= \\
= \\
= \\
= \\
= \\
= \\
= \\
= \\
= \\
=\end{array}$ & 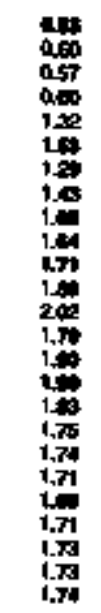 & 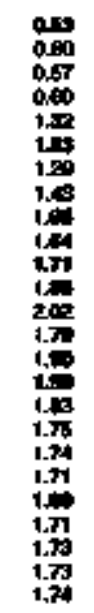 & 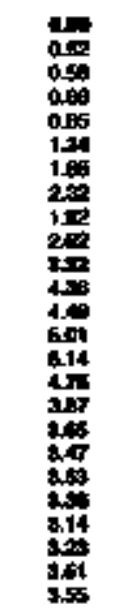 & 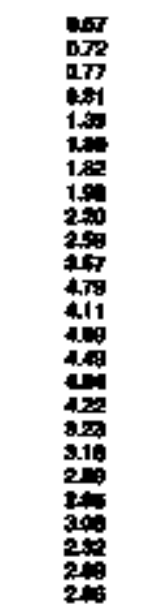 & 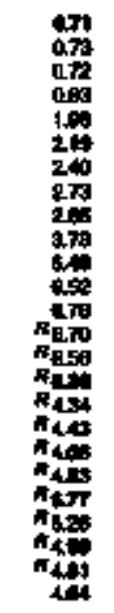 & 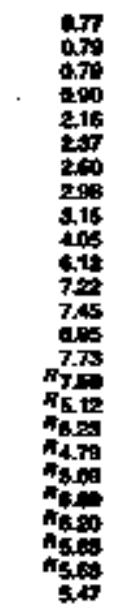 & 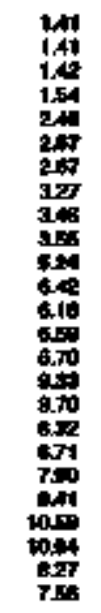 & 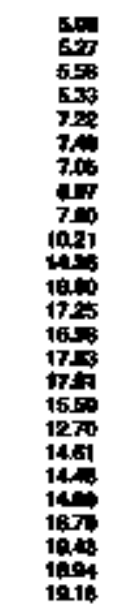 & 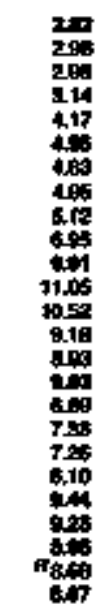 & 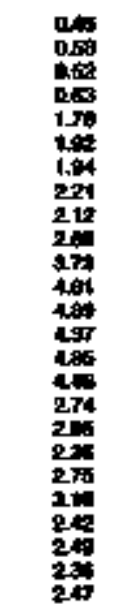 & 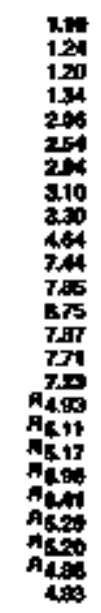 & 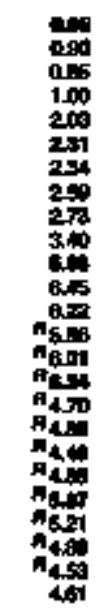 & 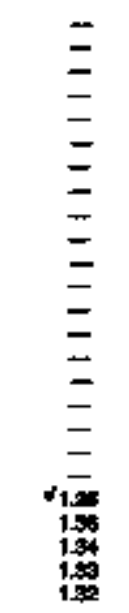 & 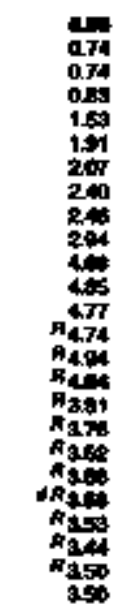 & 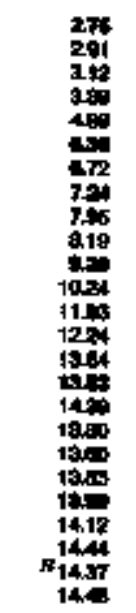 & 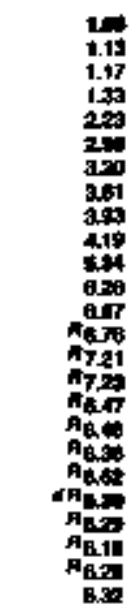 \\
\hline & \multicolumn{17}{|c|}{ 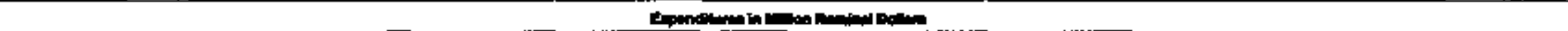 } \\
\hline 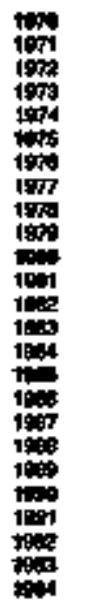 & $\begin{array}{l}= \\
= \\
= \\
= \\
= \\
= \\
= \\
= \\
= \\
= \\
= \\
= \\
=\end{array}$ & 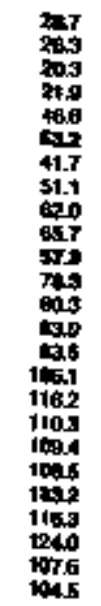 & 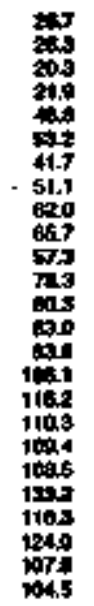 & 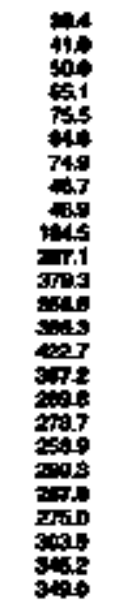 & 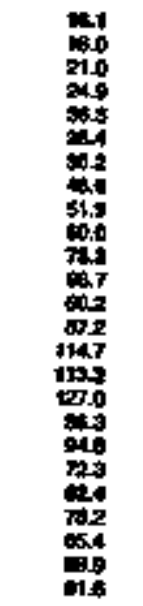 & 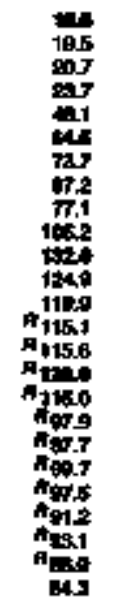 & 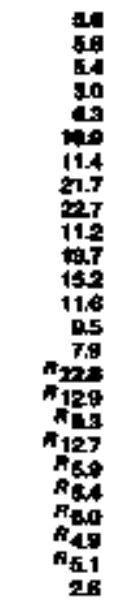 & 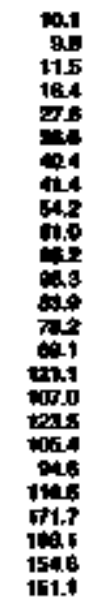 & 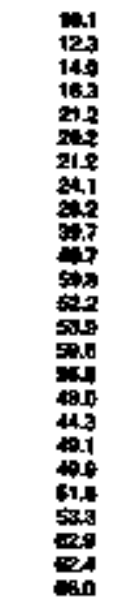 & 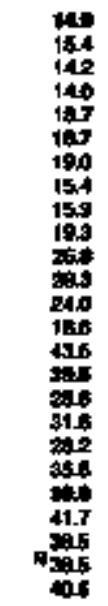 & 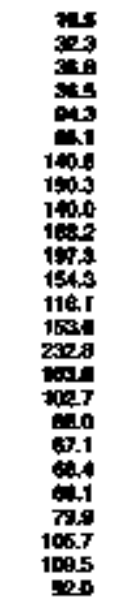 & 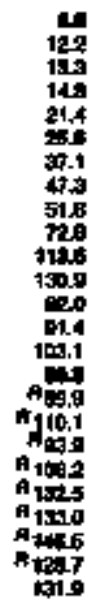 & 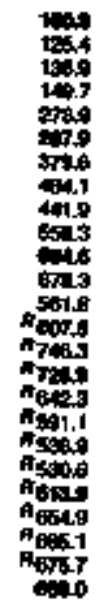 & 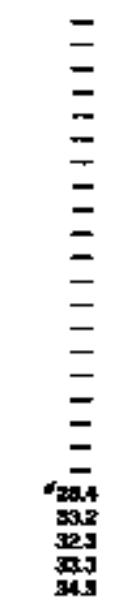 & 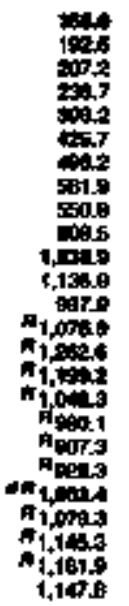 & 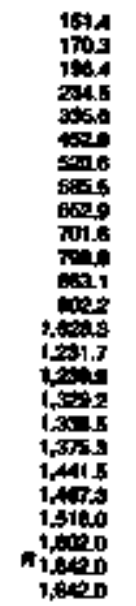 & 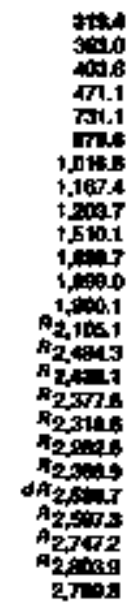 \\
\hline
\end{tabular}

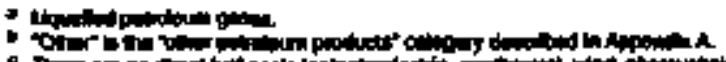

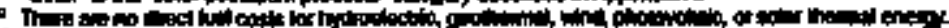

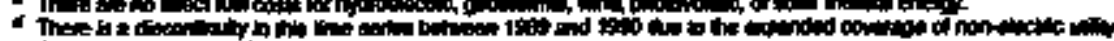

Antementim

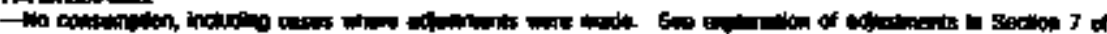

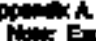

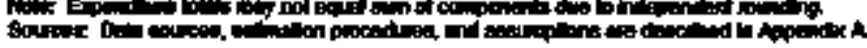


Tabla 213. Traneportution Sector Energy Pulce and Expandilure Eatinatea by Source, 1970-1994, North Ceroline

\begin{tabular}{|c|c|c|c|c|c|c|c|c|c|c|c|c|c|}
\hline \multirow{2}{*}{$\underline{v}$} & \multicolumn{11}{|c|}{ Dhereng } & \multirow{2}{*}{ Actioly } & \multirow{2}{*}{ Theor } \\
\hline & بs & 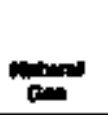 & \multicolumn{8}{|c|}{ ח } & tot & & \\
\hline 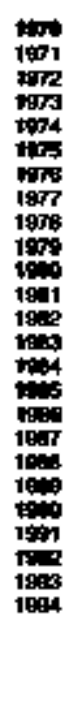 & 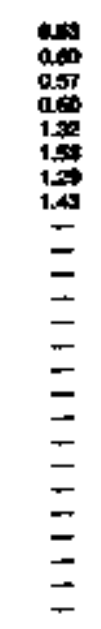 & $\begin{array}{l}= \\
= \\
= \\
= \\
= \\
= \\
= \\
= \\
= \\
= \\
= \\
z \\
40 \\
451 \\
491\end{array}$ & 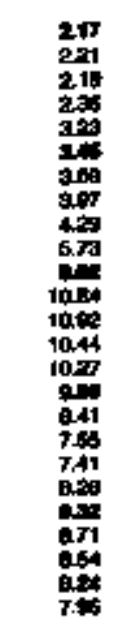 & 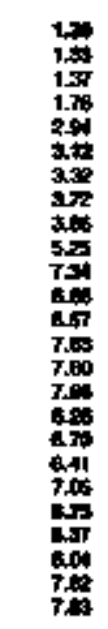 & 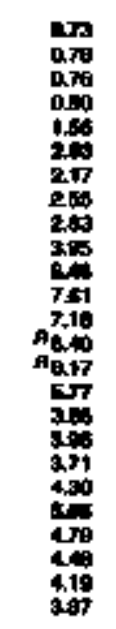 & 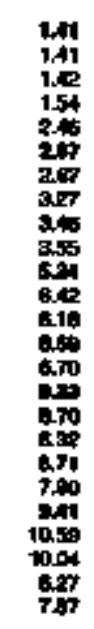 & 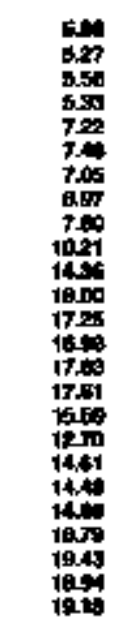 & 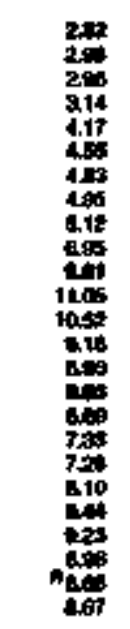 & 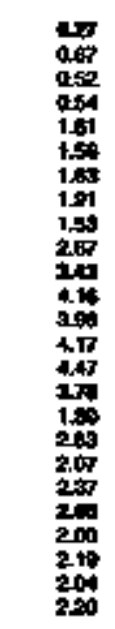 & 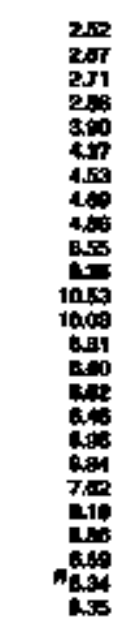 & 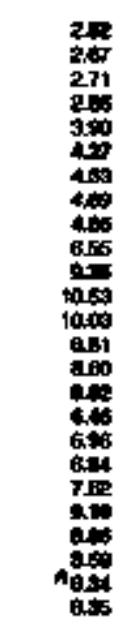 & $\begin{array}{l}= \\
z \\
z \\
z \\
z \\
z \\
= \\
= \\
= \\
z \\
z\end{array}$ & 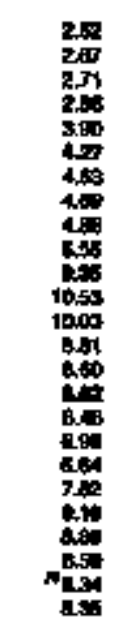 \\
\hline 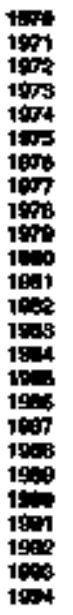 & $\begin{array}{l}8 \\
0 \\
0 \\
0 \\
0 \\
= \\
= \\
= \\
= \\
= \\
= \\
= \\
= \\
=\end{array}$ & $\begin{array}{l}= \\
= \\
= \\
= \\
= \\
= \\
= \\
= \\
= \\
= \\
= \\
9 \\
9 \\
6\end{array}$ & 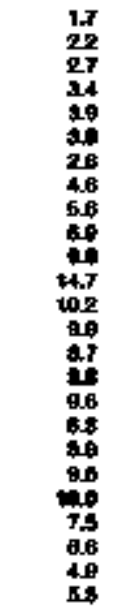 & 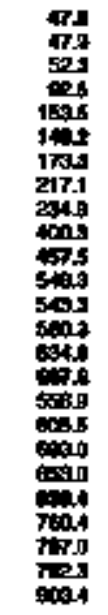 & 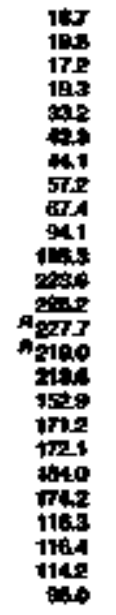 & 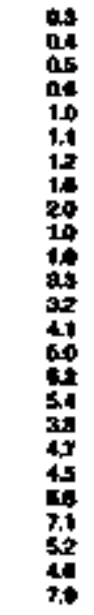 & 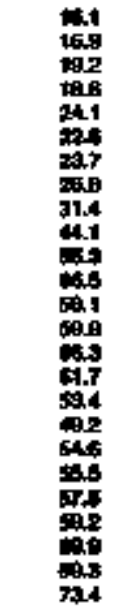 & 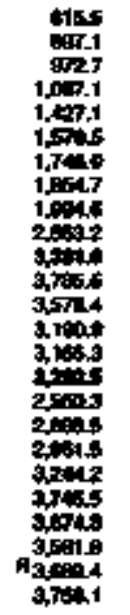 & 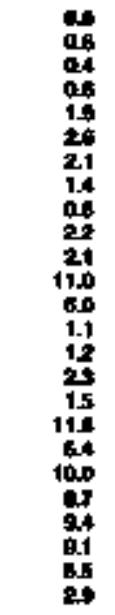 & 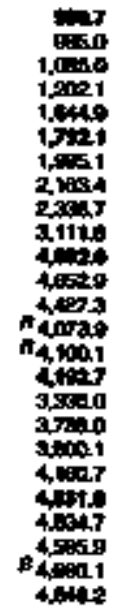 & 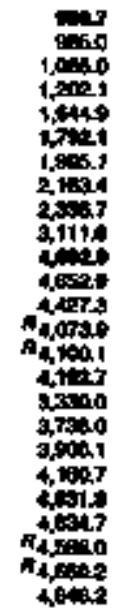 & $\begin{array}{l}= \\
= \\
= \\
= \\
= \\
= \\
= \\
= \\
= \\
= \\
=\end{array}$ & 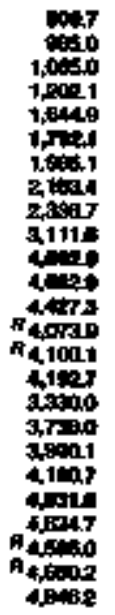 \\
\hline
\end{tabular}




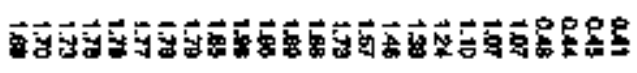

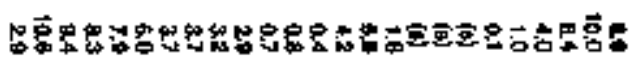

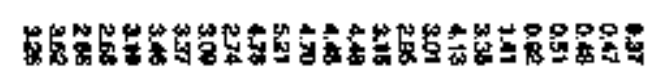

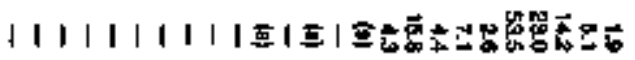

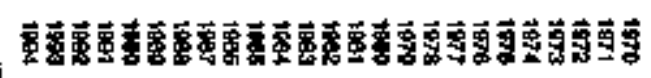

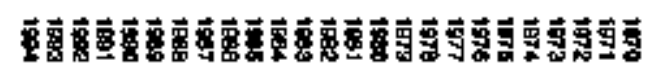

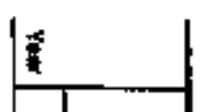

$+5$

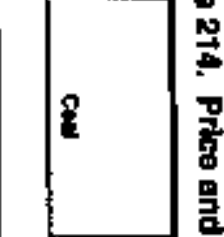
( (1)

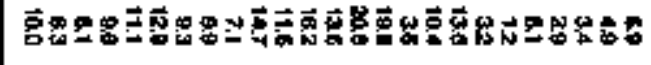

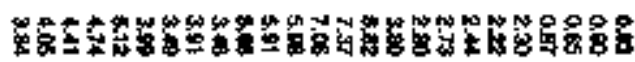

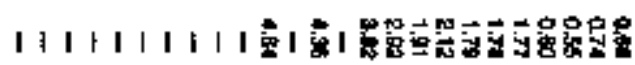

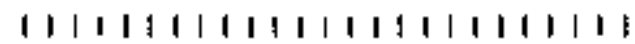

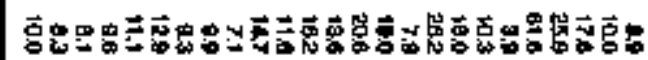

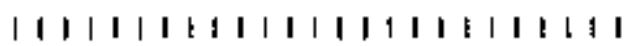

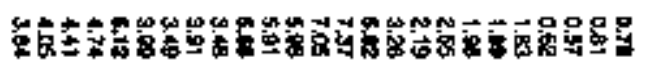

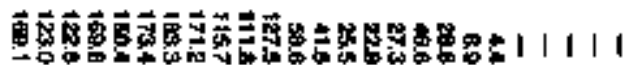

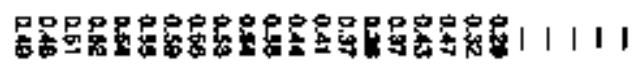


Table 215. Erergy Proe and Expondture Estumates by sourea, 1970-1994, North Dakeda

\begin{tabular}{|c|c|c|c|c|c|c|c|c|c|c|c|c|c|c|c|c|c|}
\hline \multirow[b]{4}{*}{ r } & \multicolumn{14}{|c|}{ Prinury enerey } & \multirow{3}{*}{ ind } & \multirow{3}{*}{ 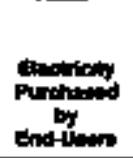 } & \multirow[b]{3}{*}{ البشה } \\
\hline & \multicolumn{3}{|c|}{ Cosen } & \multirow[b]{2}{*}{ 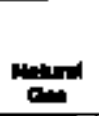 } & \multicolumn{7}{|c|}{ montom } & \multirow[b]{2}{*}{ mander } & \multirow[b]{2}{*}{ Not } & \multirow[b]{2}{*}{$T={ }^{e}$} & & & \\
\hline & Cotane & $\cos ^{2}$ & 7ot: & & Derillow & 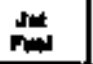 & Lo* & contion & renther & Cha & Tot & & & & & & \\
\hline & \multicolumn{17}{|c|}{ 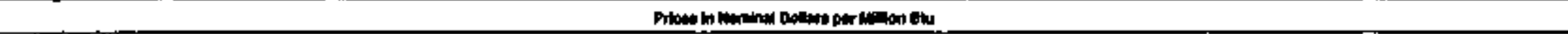 } \\
\hline \multirow[t]{2}{*}{ 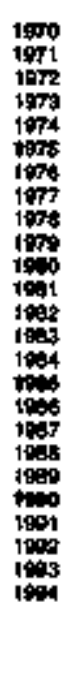 } & $\begin{array}{l}= \\
= \\
= \\
= \\
= \\
= \\
= \\
= \\
= \\
= \\
= \\
= \\
= \\
=\end{array}$ & 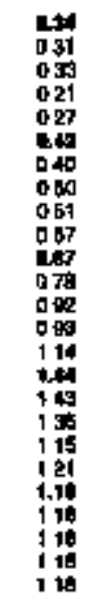 & 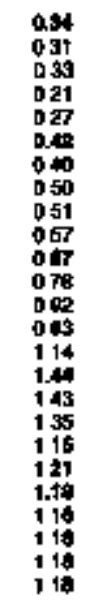 & 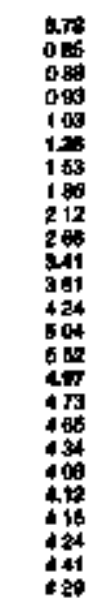 & 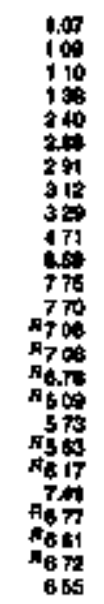 & 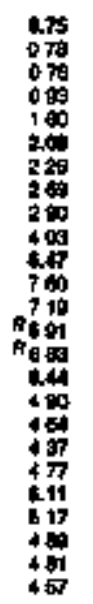 & 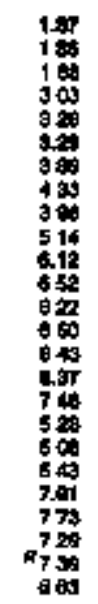 & 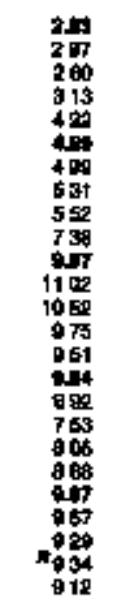 & 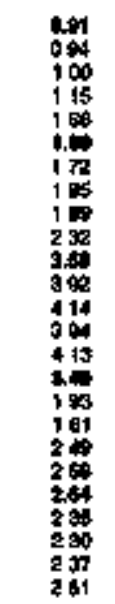 & 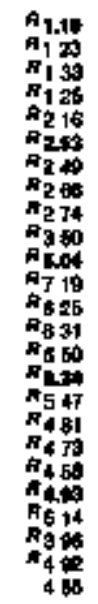 & 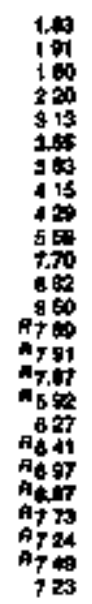 & $\begin{array}{l}= \\
= \\
= \\
= \\
= \\
= \\
= \\
= \\
= \\
= \\
= \\
= \\
= \\
=\end{array}$ & 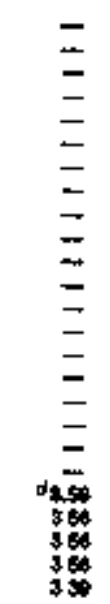 & 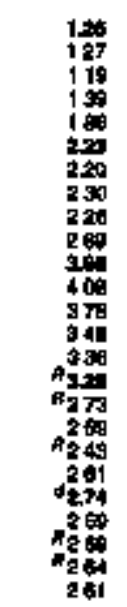 & 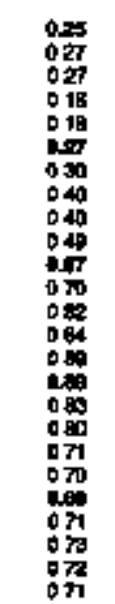 & 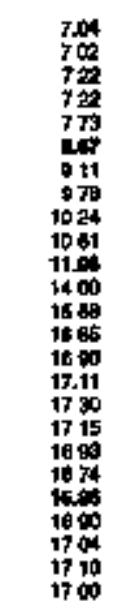 & 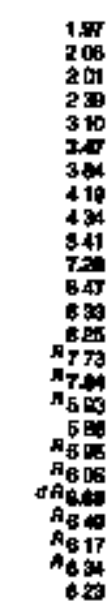 \\
\hline & \multicolumn{17}{|c|}{ 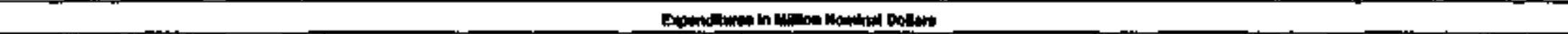 } \\
\hline 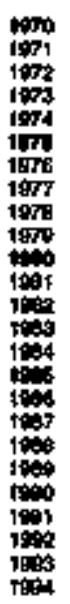 & $\begin{array}{l}= \\
= \\
= \\
= \\
= \\
= \\
= \\
= \\
= \\
= \\
= \\
= \\
=\end{array}$ & 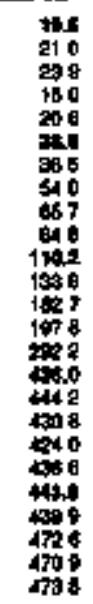 & 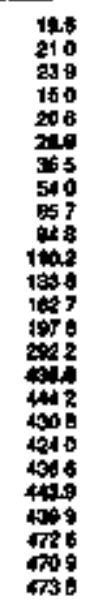 & 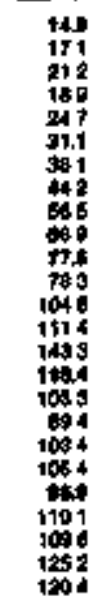 & 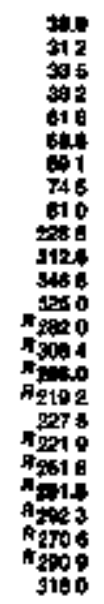 & 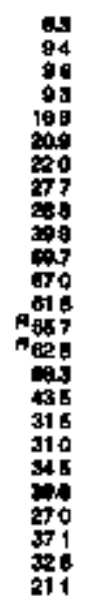 & 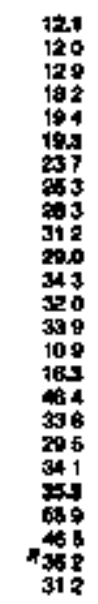 & 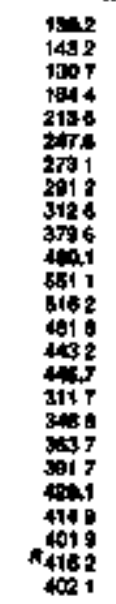 & 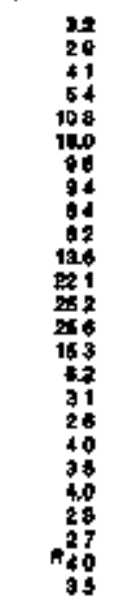 & 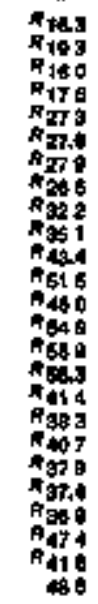 & 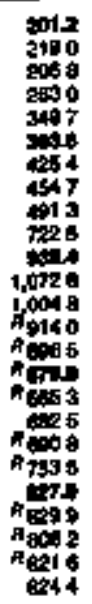 & $\begin{array}{l}= \\
= \\
= \\
= \\
= \\
= \\
= \\
= \\
= \\
= \\
= \\
= \\
=\end{array}$ & $\begin{array}{l}= \\
= \\
= \\
= \\
= \\
= \\
= \\
= \\
= \\
= \\
= \\
415 \\
20 \\
21 \\
17\end{array}$ & 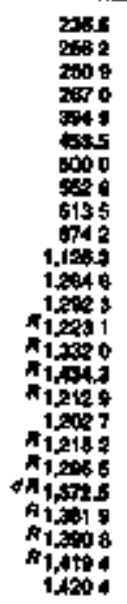 & 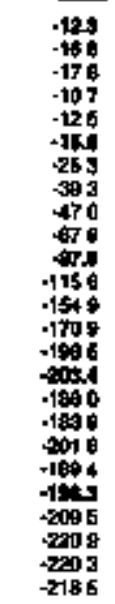 & 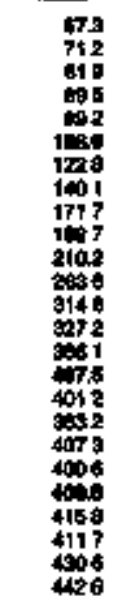 & 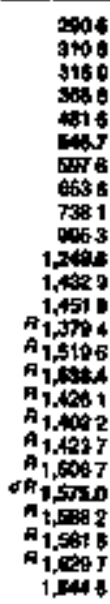 \\
\hline
\end{tabular}

- Lequetad potrolemm pates

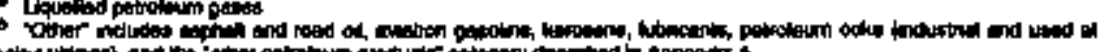

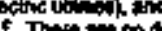

Arimad ding

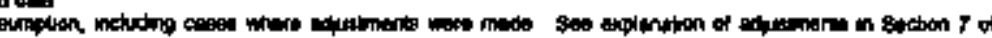

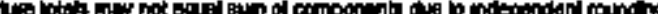

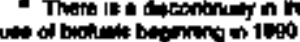


N Tablo 216. Residential Sockor Energy Price and Expendifuxe Eotimales by Source, 1970-1904, North Dakota

\begin{tabular}{|c|c|c|c|c|c|c|c|c|c|c|}
\hline \multirow[b]{4}{*}{ rem } & \multicolumn{8}{|c|}{ 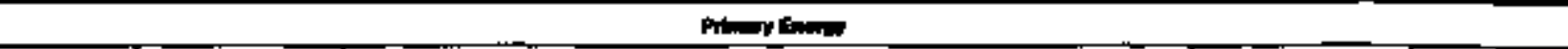 } & \multirow[b]{3}{*}{ anding } & \multirow[b]{3}{*}{ mos. } \\
\hline & \multirow[b]{2}{*}{$\cos$} & \multirow[b]{2}{*}{ 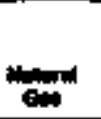 } & \multicolumn{4}{|c|}{ תוn } & \multirow[b]{2}{*}{ (n) } & \multirow[b]{2}{*}{ 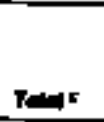 } & & \\
\hline & & & 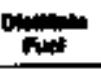 & (1) & LNa: & Tots & & & & \\
\hline & \multicolumn{10}{|c|}{ 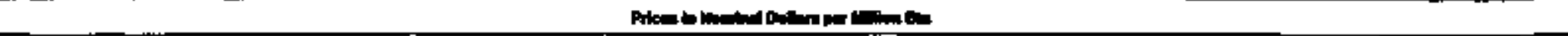 } \\
\hline \multirow[t]{2}{*}{ 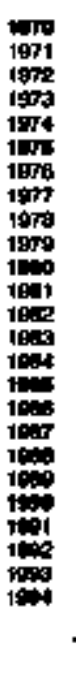 } & 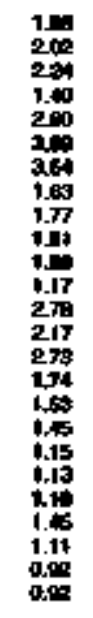 & 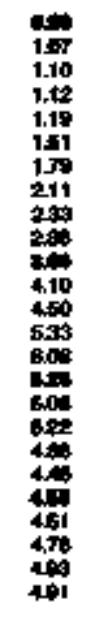 & 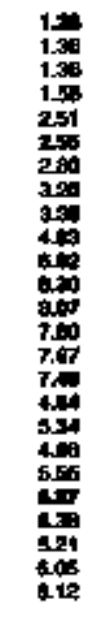 & 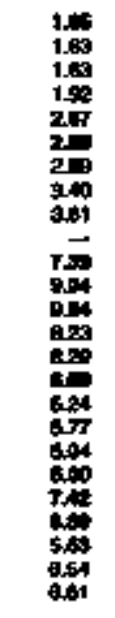 & 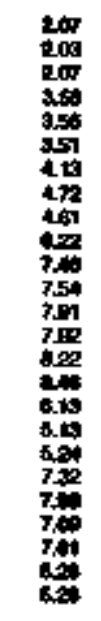 & 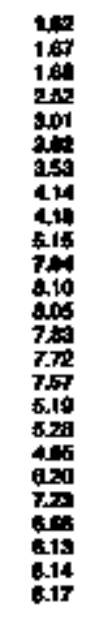 & 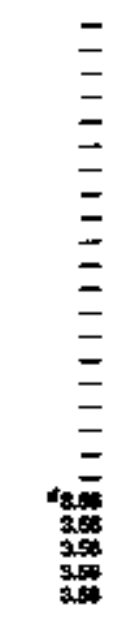 & 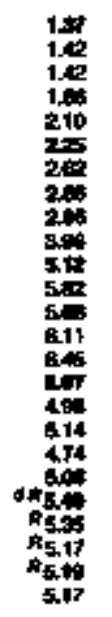 & 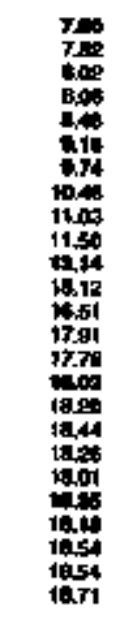 & 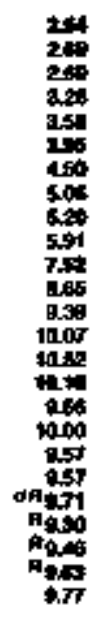 \\
\hline & \multicolumn{10}{|c|}{ 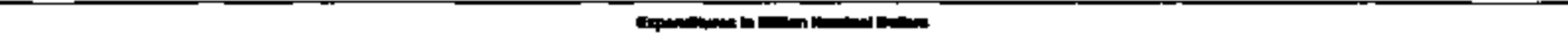 } \\
\hline 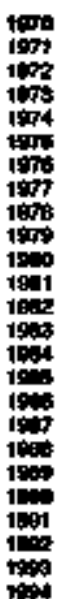 & 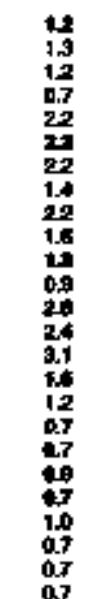 & 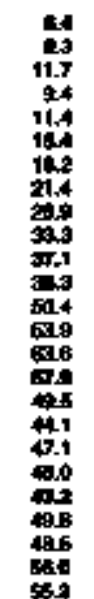 & 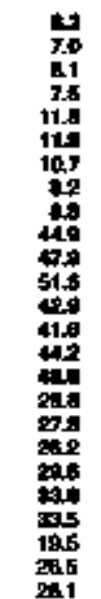 & 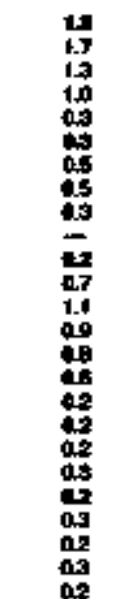 & 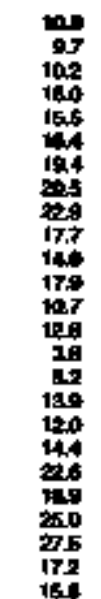 & 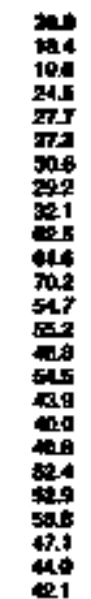 & 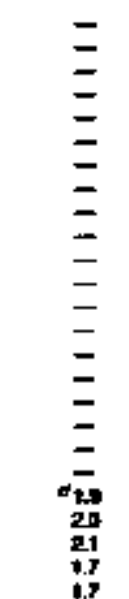 & 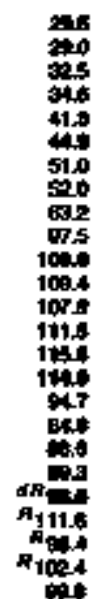 & 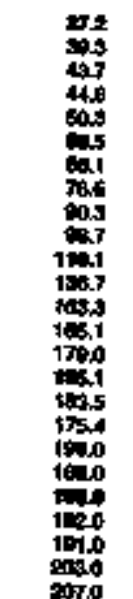 & 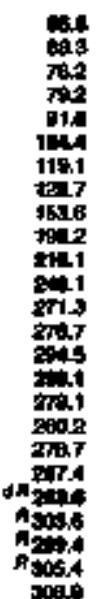 \\
\hline
\end{tabular}

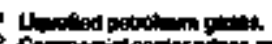

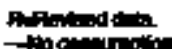

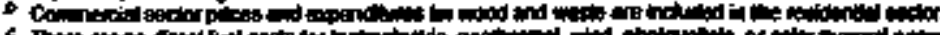

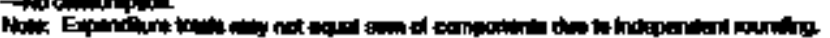

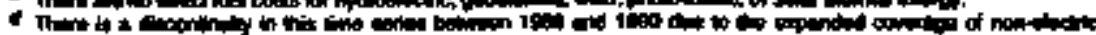

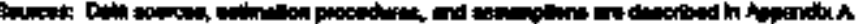




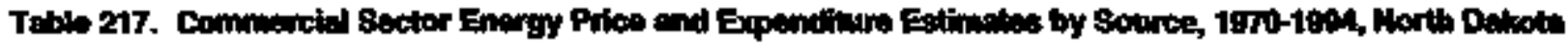

\begin{tabular}{|c|c|c|c|c|c|c|c|c|c|c|c|}
\hline & \multicolumn{9}{|c|}{ 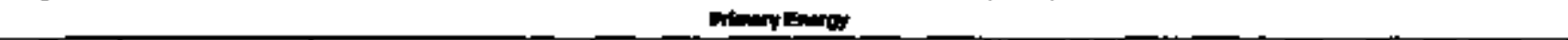 } & \multirow[b]{3}{*}{ 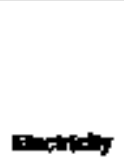 } & \multirow[b]{3}{*}{ E } \\
\hline & \multirow[b]{2}{*}{ n } & \multirow[b]{2}{*}{ 1) } & \multicolumn{6}{|c|}{ 1rishes } & \multirow[b]{2}{*}{ nil } & & \\
\hline & & & (5) & 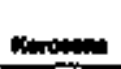 & Lo: & 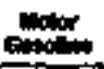 & 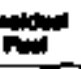 & roded & & & \\
\hline w & \multicolumn{11}{|c|}{ 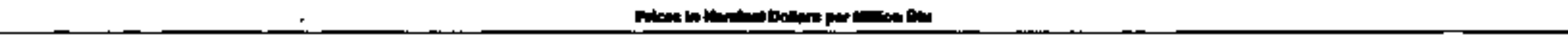 } \\
\hline \multirow[t]{2}{*}{ 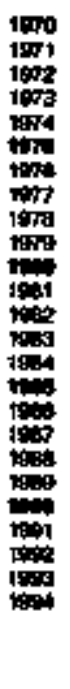 } & 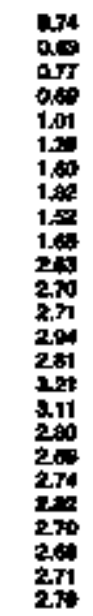 & 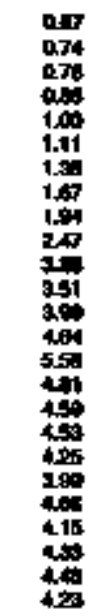 & 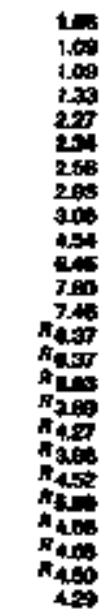 & 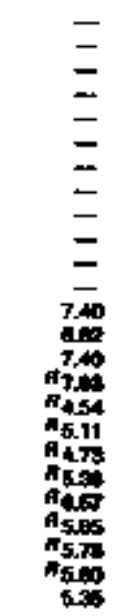 & 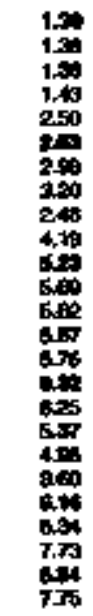 & 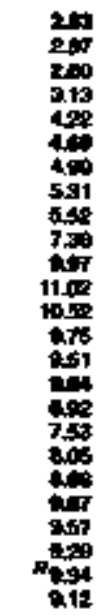 & 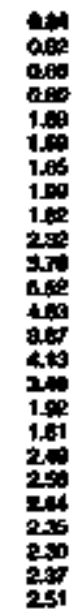 & 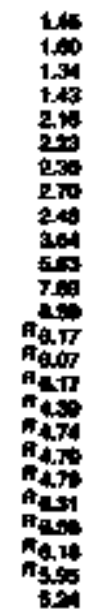 & 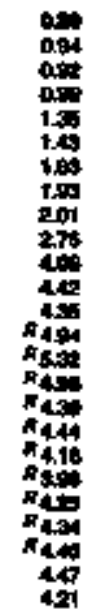 & 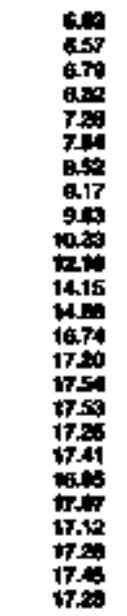 & 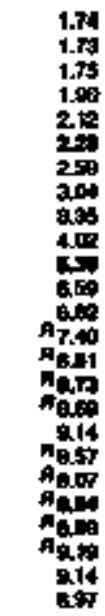 \\
\hline & \multicolumn{11}{|c|}{ E } \\
\hline 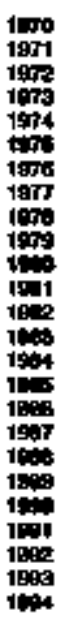 & 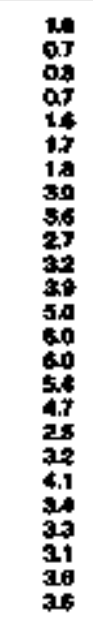 & 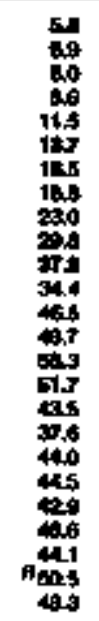 & 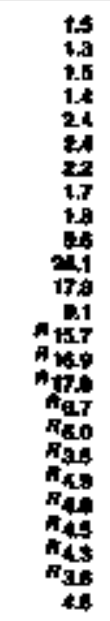 & $\begin{array}{l}= \\
\bar{z} \\
= \\
= \\
= \\
= \\
\bar{z} \\
0 \\
0 \\
0\end{array}$ & 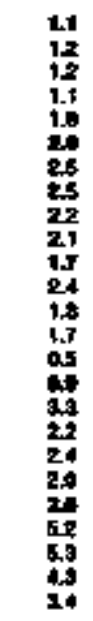 & 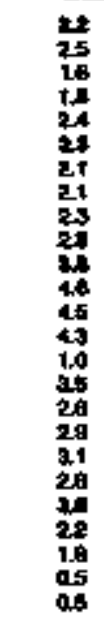 & 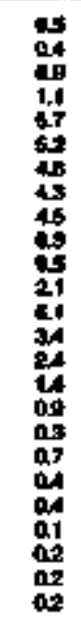 & 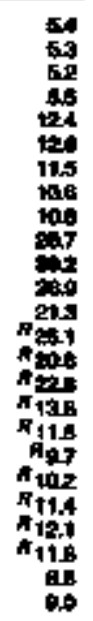 & 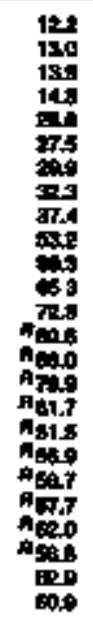 & 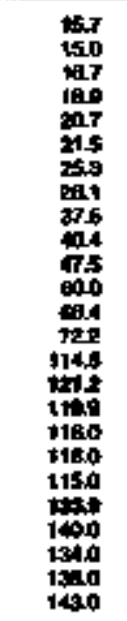 & 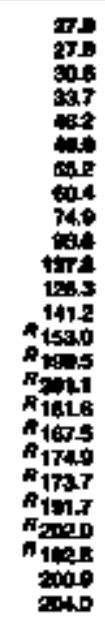 \\
\hline
\end{tabular}

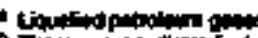

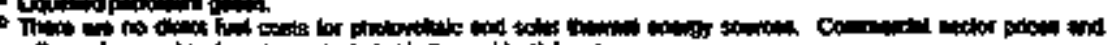

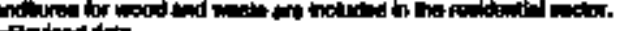

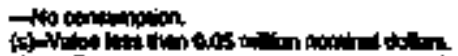

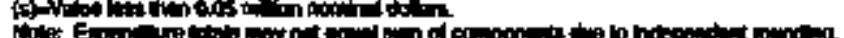

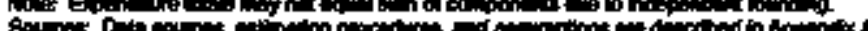




\begin{tabular}{|c|c|c|c|c|c|c|c|c|c|c|c|c|c|c|c|c|c|}
\hline \multirow[b]{4}{*}{ Vur } & \multicolumn{15}{|c|}{ Praney Enowy } & \multirow[b]{3}{*}{ Geednety } & \multirow[b]{3}{*}{ 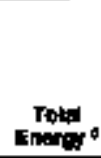 } \\
\hline & \multicolumn{3}{|c|}{ tom } & \multirow[b]{2}{*}{ manes } & \multicolumn{9}{|c|}{ Parrowen } & \multirow[b]{2}{*}{ motules } & \multirow[b]{2}{*}{ Totals } & & \\
\hline & 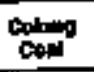 & coed & Tow & & 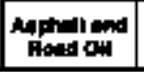 & ontallo & Kerosens & LPa: & Lorvementh & not & Fingerd & onmen & Toter & & & & \\
\hline & \multicolumn{17}{|c|}{ 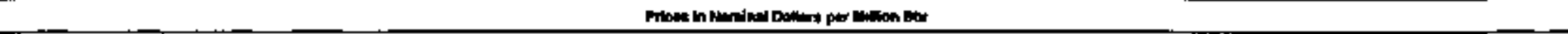 } \\
\hline \multirow[t]{2}{*}{ 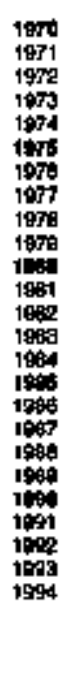 } & $\begin{array}{l}= \\
= \\
z \\
= \\
= \\
= \\
= \\
= \\
z \\
= \\
= \\
z \\
z \\
z\end{array}$ & 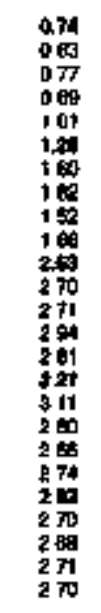 & 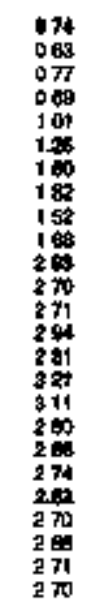 & 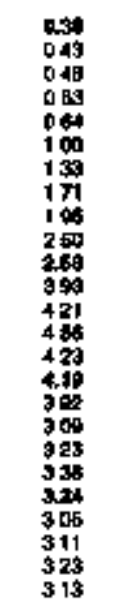 & 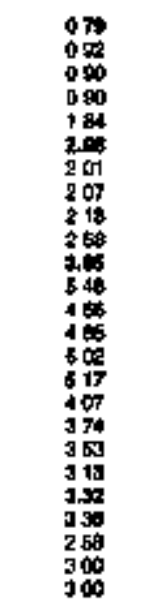 & 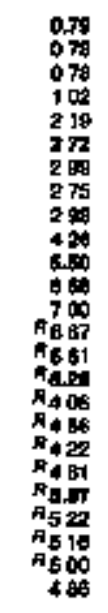 & 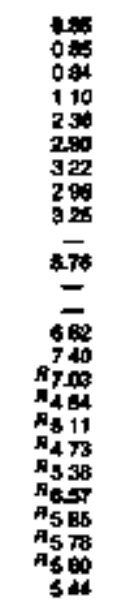 & 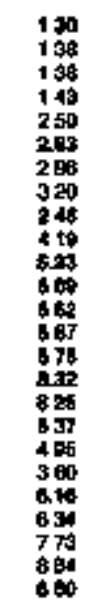 & 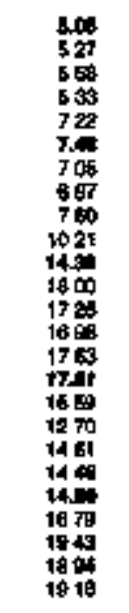 & 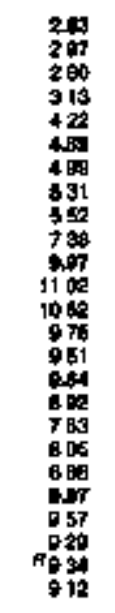 & 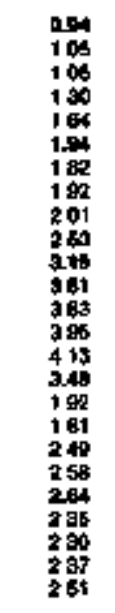 & 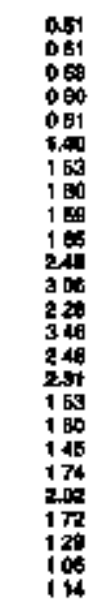 & 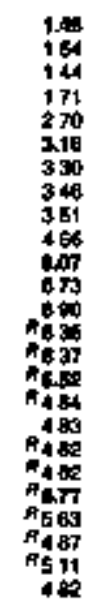 & $\begin{array}{l}\bar{z} \\
\bar{z} \\
z \\
z \\
z \\
z \\
z \\
z \\
\bar{z} \\
z \\
\bar{z} \\
\bar{z} \\
\overline{0}\end{array}$ & 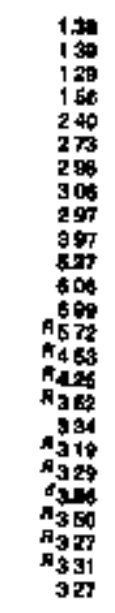 & 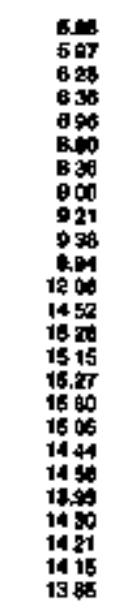 & 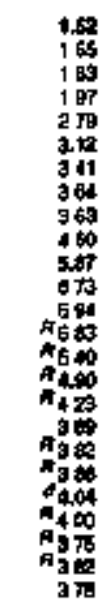 \\
\hline & \multicolumn{17}{|c|}{ 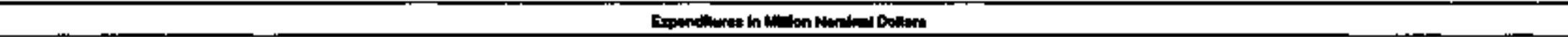 } \\
\hline 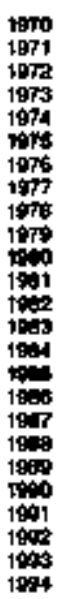 & $\begin{array}{l}= \\
= \\
= \\
= \\
= \\
= \\
= \\
= \\
= \\
= \\
= \\
=\end{array}$ & 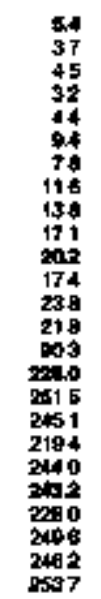 & 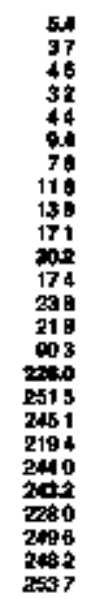 & 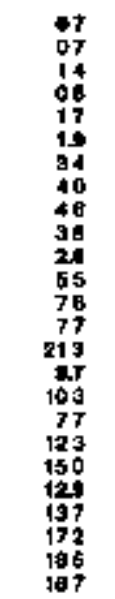 & 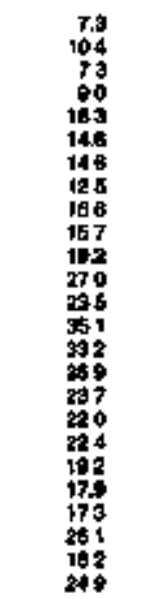 & 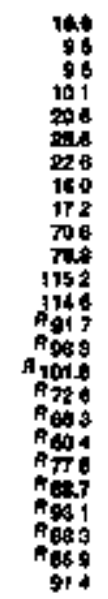 & 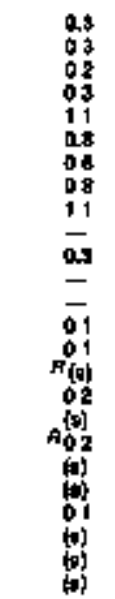 & 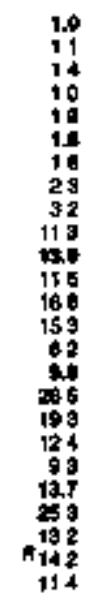 & 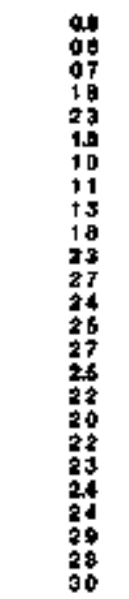 & 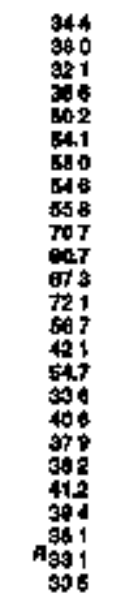 & 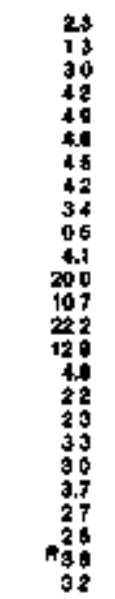 & 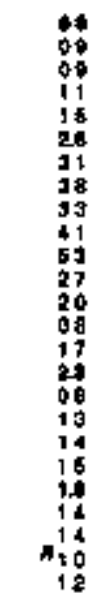 & 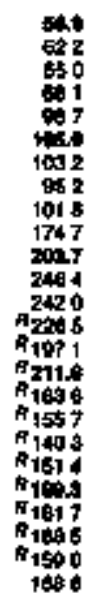 & $\begin{array}{l}= \\
\underline{z} \\
\bar{z} \\
\bar{z} \\
= \\
= \\
= \\
= \\
\bar{z} \\
\bar{z} \\
\bar{z} \\
\bar{z}\end{array}$ & 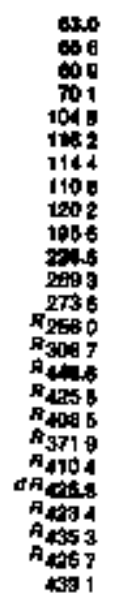 & 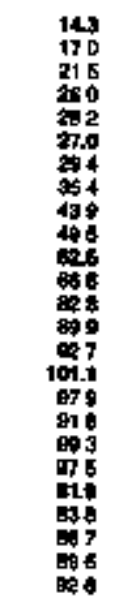 & 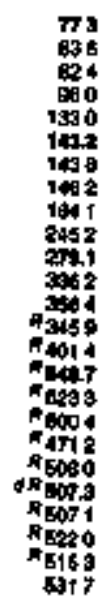 \\
\hline
\end{tabular}

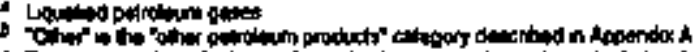

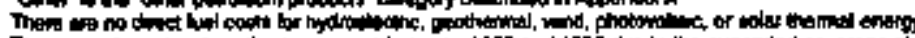

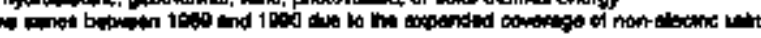

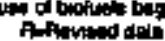

inperater

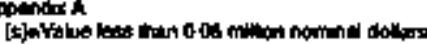

a 
Table 219. Trannportation Sector Energy Price and Expenditure Eutimatea by Sounes, 1970-1994, North Dakota

\begin{tabular}{|c|c|c|c|c|c|c|c|c|c|c|c|c|c|}
\hline \multirow[b]{4}{*}{$\mathrm{ren}$} & \multicolumn{11}{|c|}{ Prtanery Entory } & \multirow[b]{3}{*}{ Enctolstif } & \multirow[b]{3}{*}{ Energy } \\
\hline & & \multirow[b]{2}{*}{$\operatorname{mot}_{0.01}$} & \multicolumn{8}{|c|}{ Punoline } & \multirow[b]{2}{*}{ Tot } & & \\
\hline & Com & & Cotition & $\begin{array}{c}\text { Dinallate } \\
\text { Fuet }\end{array}$ & Fat & LPa* & Lutreanto & 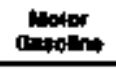 & 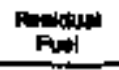 & Totat & & & \\
\hline & \multicolumn{13}{|c|}{ 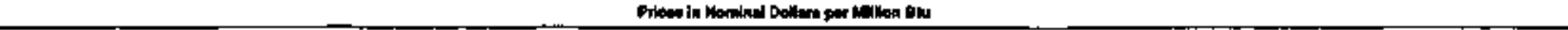 } \\
\hline \multirow[t]{2}{*}{ 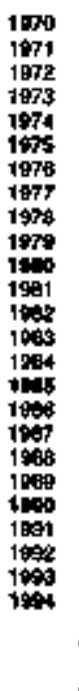 } & $\begin{array}{l}074 \\
000 \\
077 \\
060 \\
101 \\
1.70 \\
190 \\
180 \\
= \\
= \\
= \\
= \\
= \\
= \\
= \\
= \\
= \\
= \\
=\end{array}$ & $\begin{array}{l}= \\
= \\
= \\
= \\
= \\
= \\
= \\
= \\
= \\
= \\
= \\
= \\
310 \\
407\end{array}$ & 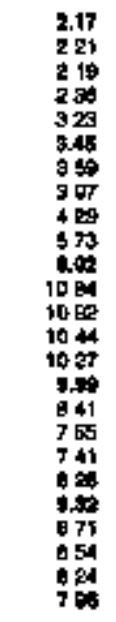 & 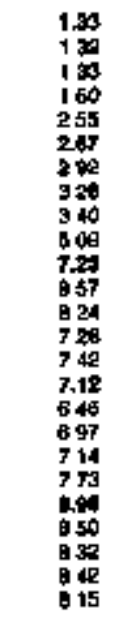 & 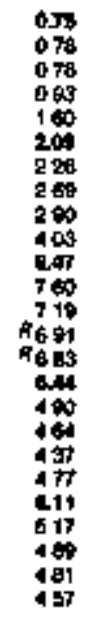 & 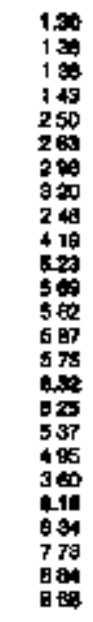 & 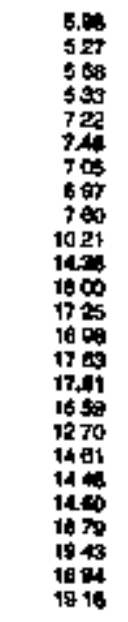 & 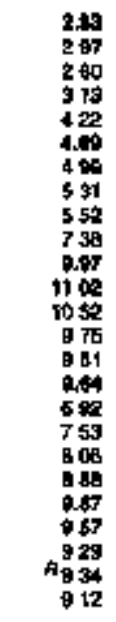 & $\begin{array}{l}0.00 \\
= \\
= \\
= \\
= \\
2=0 \\
= \\
420 \\
190 \\
234 \\
= \\
= \\
= \\
=\end{array}$ & 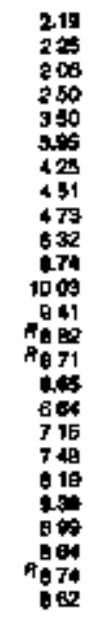 & 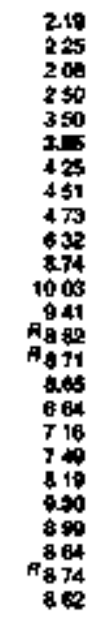 & $\begin{array}{l}= \\
= \\
= \\
= \\
= \\
= \\
= \\
= \\
= \\
= \\
= \\
= \\
= \\
=\end{array}$ & 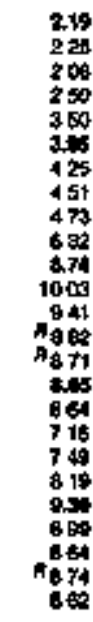 \\
\hline & \multicolumn{13}{|c|}{ 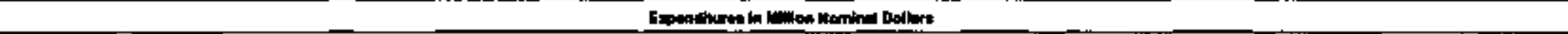 } \\
\hline 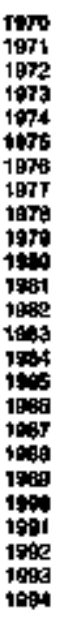 & 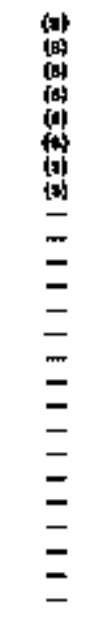 & $\begin{array}{l}= \\
= \\
= \\
= \\
= \\
= \\
= \\
= \\
= \\
= \\
= \\
(0) \\
(0) \\
(0)\end{array}$ & $\begin{array}{l}10 \\
13 \\
17 \\
11 \\
14 \\
15 \\
15 \\
16 \\
21 \\
29 \\
29 \\
27 \\
19 \\
13 \\
16 \\
02 \\
16 \\
11 \\
12 \\
13 \\
13 \\
12 \\
12 \\
28 \\
17\end{array}$ & 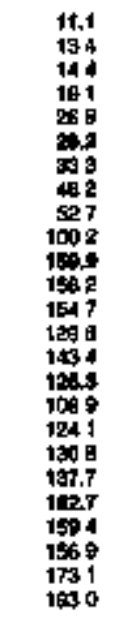 & 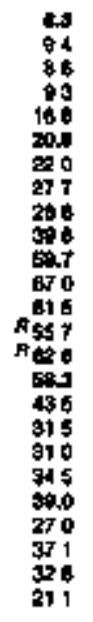 & 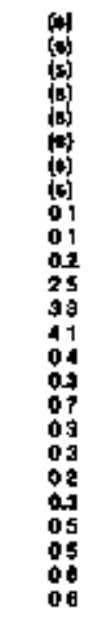 & 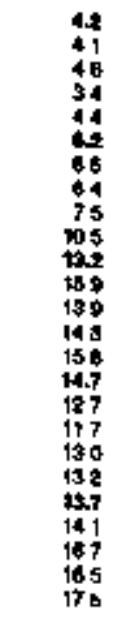 & 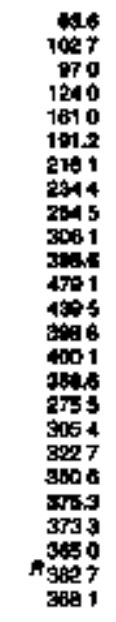 & 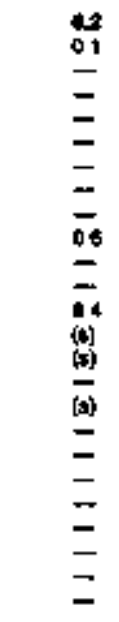 & 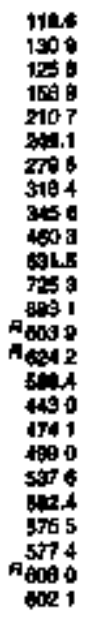 & 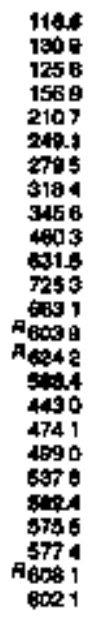 & $\begin{array}{l}= \\
= \\
= \\
= \\
= \\
= \\
= \\
= \\
= \\
= \\
= \\
= \\
= \\
= \\
=\end{array}$ & 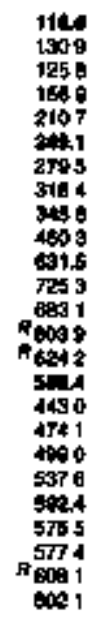 \\
\hline
\end{tabular}
anowhen $A$ 
N Teble 220. Price and Expenditure Estinatos for Eneryy hput at Eloctrio Utilibios by Source, 1970-1904, Morth Dekota

\begin{tabular}{|c|c|c|c|c|c|c|c|c|c|}
\hline \multirow[b]{3}{*}{ rem } & \multirow[b]{2}{*}{ con } & \multirow[b]{2}{*}{ des } & \multicolumn{4}{|c|}{ 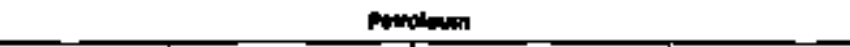 } & \multirow[b]{2}{*}{ Find } & \multirow[b]{2}{*}{ ototude } & \multirow[b]{2}{*}{ trats: } \\
\hline & & & tons & of & Pandon & Totod & & & \\
\hline & \multicolumn{9}{|c|}{ 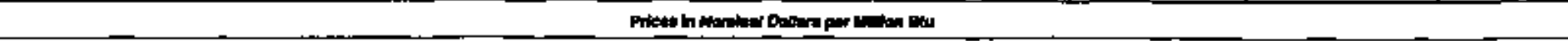 } \\
\hline \multirow[t]{2}{*}{ 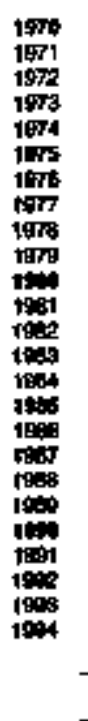 } & 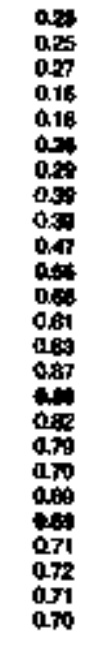 & 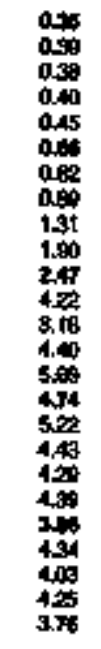 & 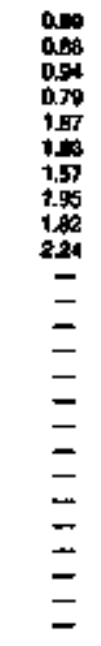 & 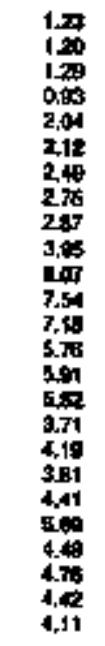 & 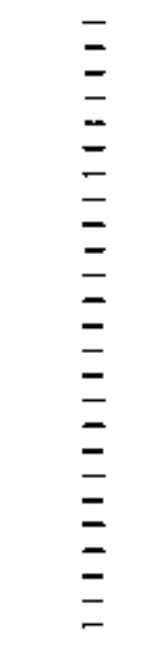 & 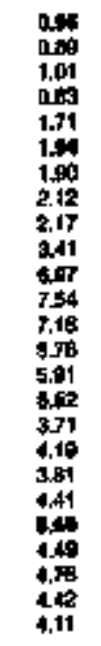 & $\begin{array}{l}= \\
z \\
z \\
z \\
= \\
z \\
= \\
= \\
= \\
= \\
= \\
= \\
z \\
z\end{array}$ & $\begin{array}{l}z \\
z \\
z \\
z \\
= \\
z \\
z \\
= \\
= \\
= \\
= \\
= \\
z \\
=\end{array}$ & 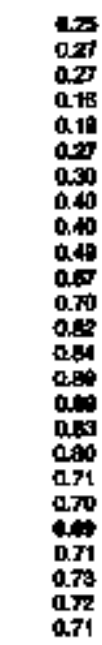 \\
\hline & \multicolumn{9}{|c|}{ 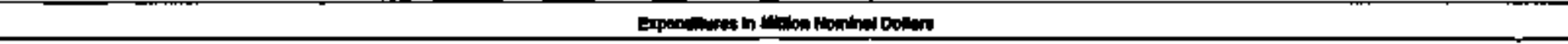 } \\
\hline 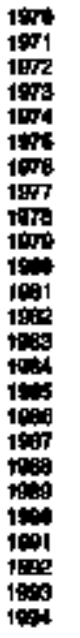 & 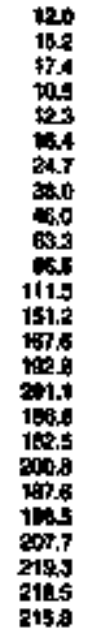 & 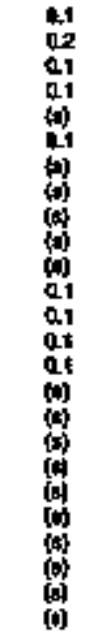 & 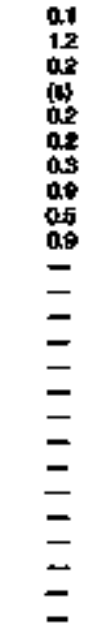 & 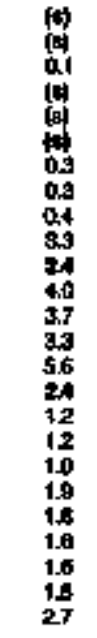 & $\begin{array}{l}z \\
z \\
z \\
z \\
z \\
z \\
z \\
z \\
z \\
z \\
z \\
z \\
z\end{array}$ & 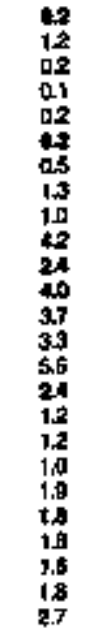 & $\begin{array}{l}z \\
= \\
= \\
= \\
= \\
= \\
z \\
= \\
= \\
= \\
= \\
= \\
z\end{array}$ & $\begin{array}{l}= \\
z \\
z \\
z \\
= \\
= \\
= \\
= \\
= \\
= \\
z \\
z\end{array}$ & 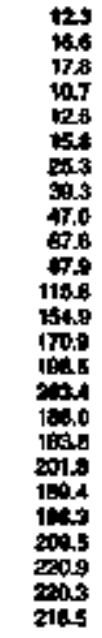 \\
\hline
\end{tabular}

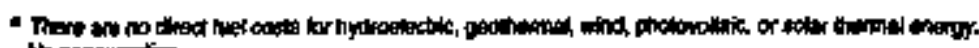

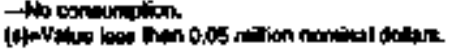

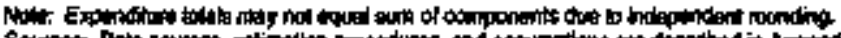

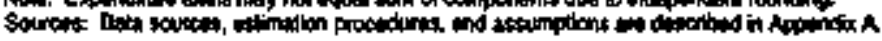




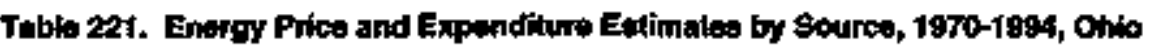

\begin{tabular}{|c|c|c|c|c|c|c|c|c|c|c|c|c|c|c|c|c|c|}
\hline \multirow[b]{4}{*}{ tow } & \multicolumn{14}{|c|}{ 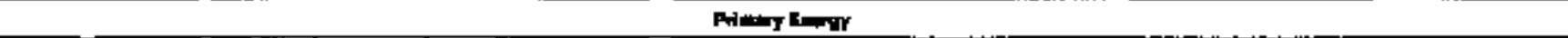 } & \multirow{3}{*}{ 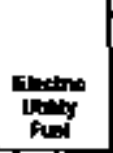 } & \multirow{3}{*}{ ניוה } & \multirow[b]{3}{*}{ Ther } \\
\hline & \multicolumn{3}{|c|}{ Eow } & \multirow[b]{2}{*}{ 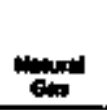 } & \multicolumn{7}{|c|}{ 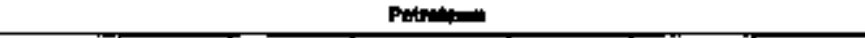 } & \multirow[b]{2}{*}{ Folimil } & \multirow[b]{2}{*}{ 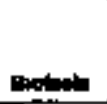 } & \multirow[b]{2}{*}{ Toten } & & & \\
\hline & Colved & consen & Tot. & & Full & tot & Lot. & 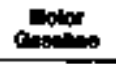 & Fulder & Oats & Totol & & & & & & \\
\hline & \multicolumn{17}{|c|}{ 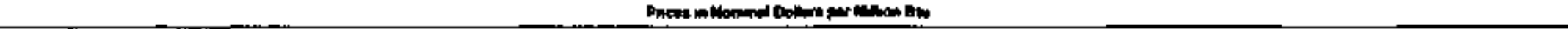 } \\
\hline \multirow[t]{2}{*}{ 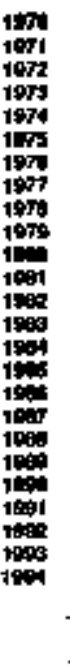 } & 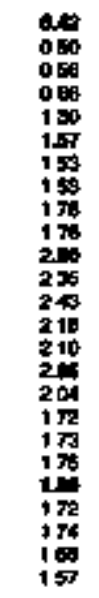 & 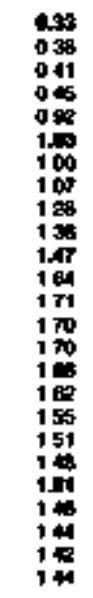 & 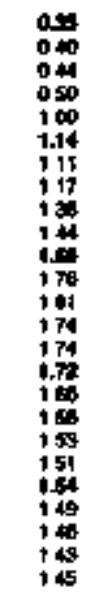 & 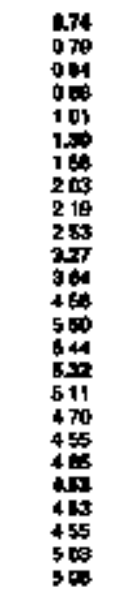 & 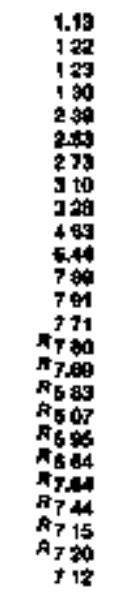 & 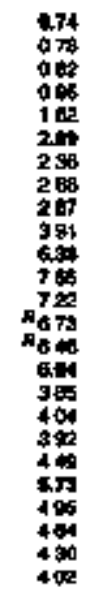 & 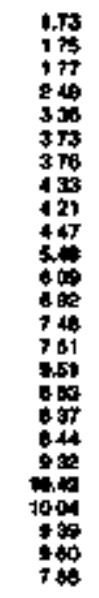 & 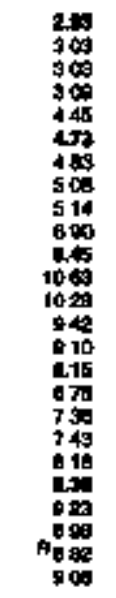 & 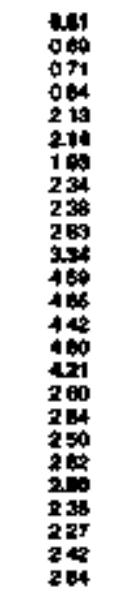 & 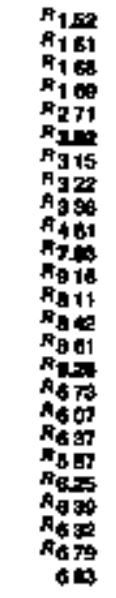 & 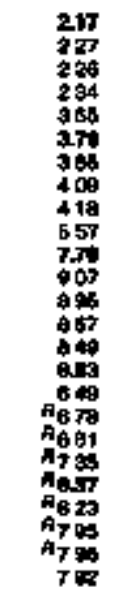 & 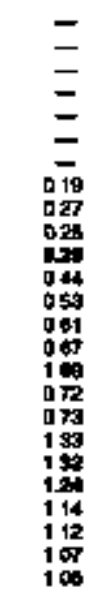 & 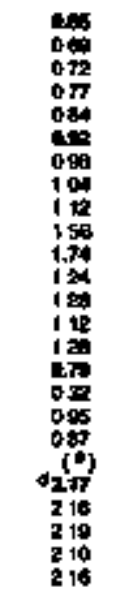 & 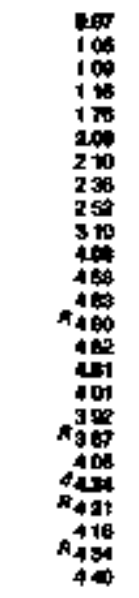 & 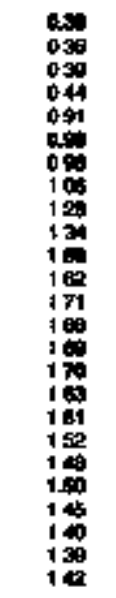 & 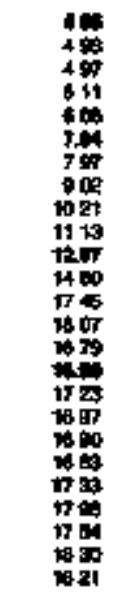 & 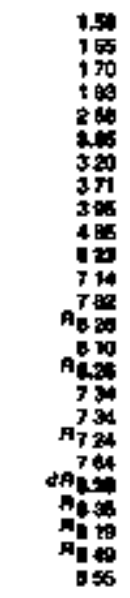 \\
\hline & \multicolumn{17}{|c|}{ 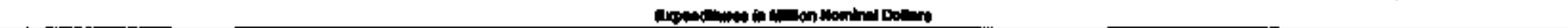 } \\
\hline 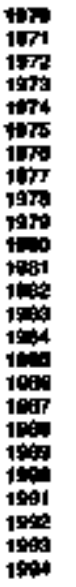 & 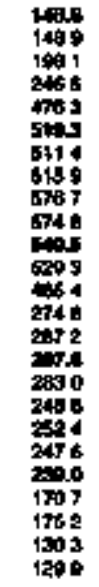 & 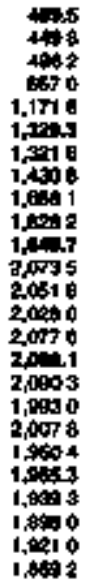 & 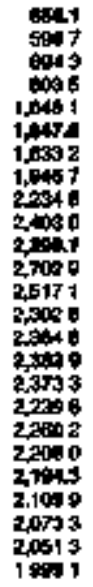 & 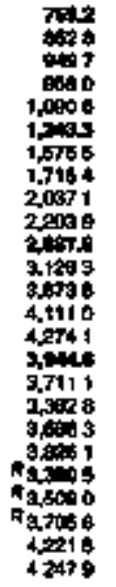 & 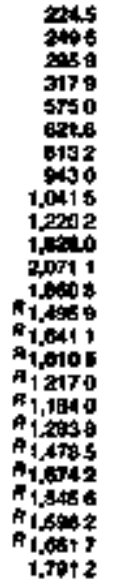 & 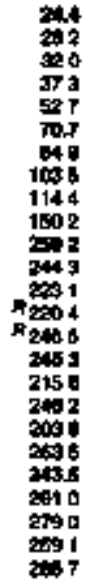 & 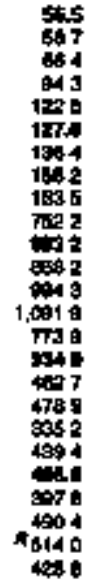 & 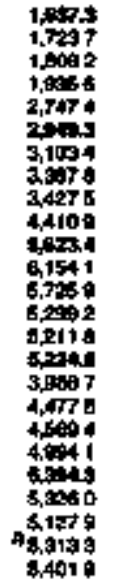 & 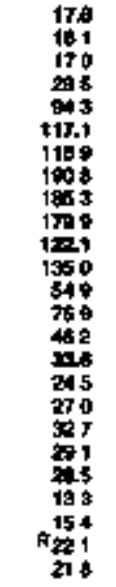 & 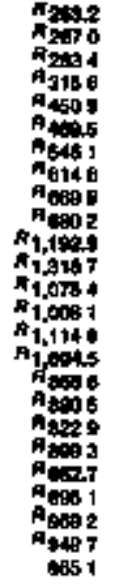 & 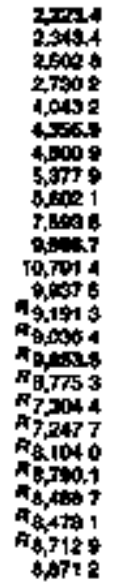 & 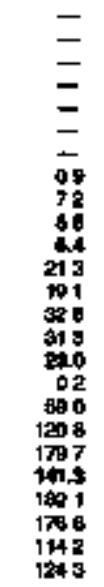 & 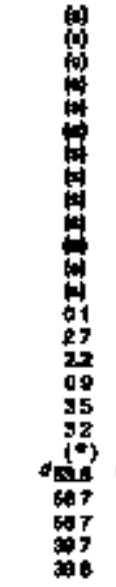 & 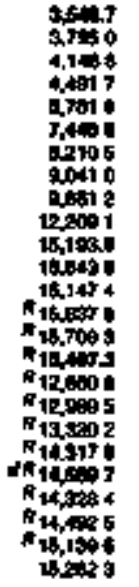 & 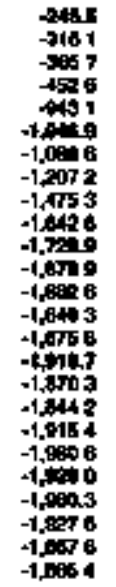 & 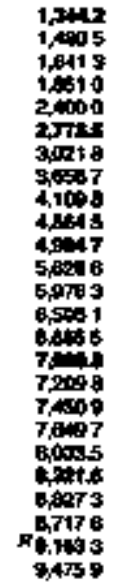 & 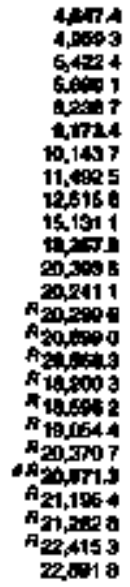 \\
\hline
\end{tabular}

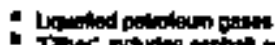

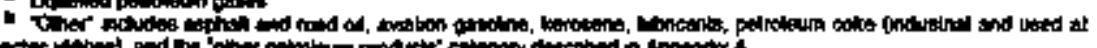

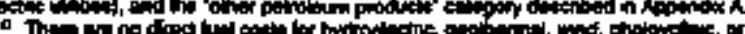

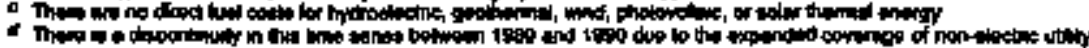
Cod

Afomedte.

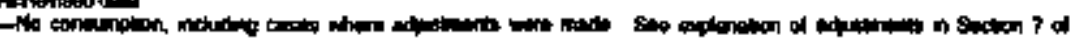
Apond $\times$ A

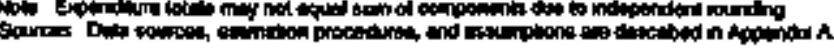


$\circ$
$H$
0

Table 222. Reoldentlal 8ector Energy Price and Expenditure Eallonatua by Source, 1970-1994, Onlo

\begin{tabular}{|c|c|c|c|c|c|c|c|c|c|c|}
\hline \multirow[b]{4}{*}{ Year } & \multicolumn{8}{|c|}{ 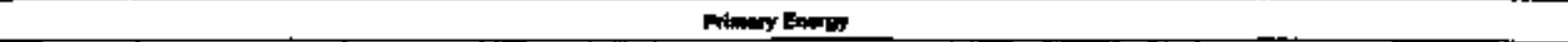 } & \multirow[b]{3}{*}{ Enotionf } & \multirow[b]{3}{*}{ Totats } \\
\hline & \multirow[b]{2}{*}{ Con } & \multirow[b]{2}{*}{ 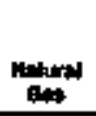 } & \multicolumn{4}{|c|}{ Putrolation } & \multirow[b]{2}{*}{ Bohing b" } & \multirow[b]{2}{*}{ Tot:- } & & \\
\hline & & & Pun & Krovene & LPQ $*$ & Tatell & & & & \\
\hline & \multicolumn{10}{|c|}{ 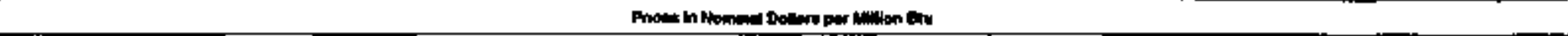 } \\
\hline \multirow[t]{2}{*}{ 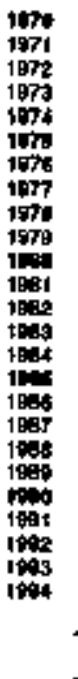 } & 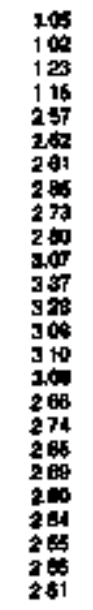 & 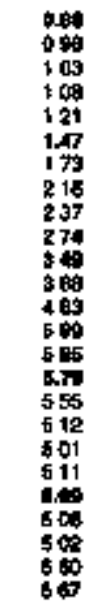 & 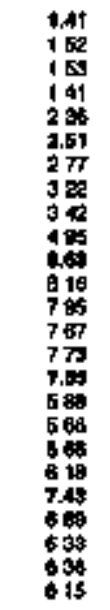 & 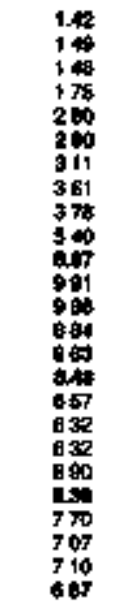 & 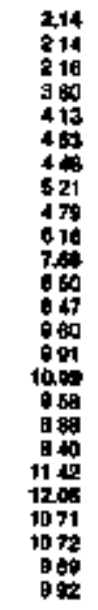 & 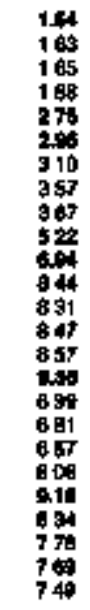 & $\begin{array}{l}= \\
= \\
= \\
= \\
= \\
= \\
= \\
= \\
= \\
= \\
= \\
= \\
30 \\
3.56 \\
36\end{array}$ & 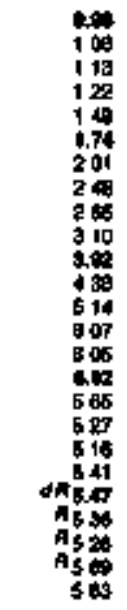 & 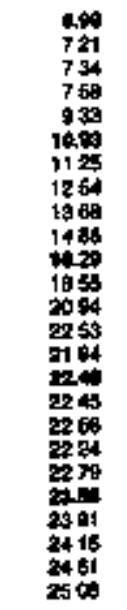 & 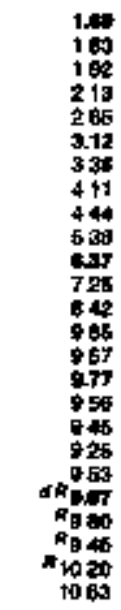 \\
\hline & \multicolumn{10}{|c|}{ 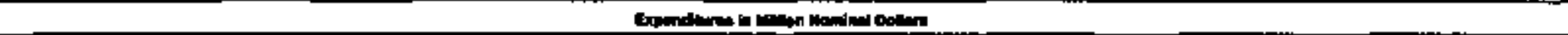 } \\
\hline 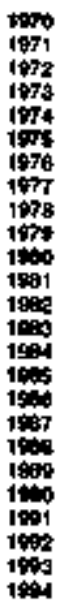 & 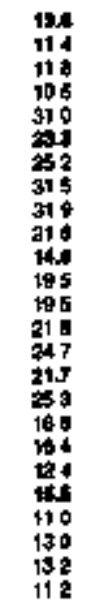 & 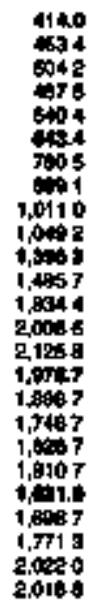 & 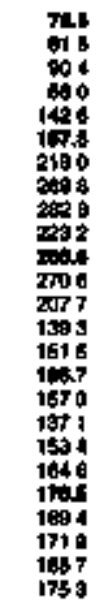 & 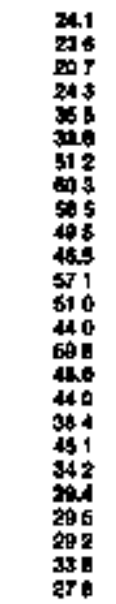 & 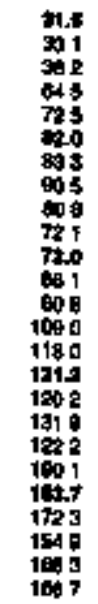 & 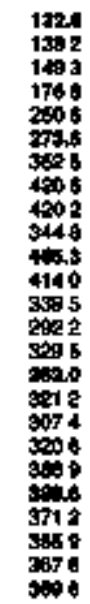 & $\begin{array}{l}= \\
= \\
= \\
= \\
= \\
= \\
= \\
= \\
= \\
= \\
= \\
\bar{z} \\
0 \\
0.1 \\
370 \\
300 \\
100 \\
190\end{array}$ & 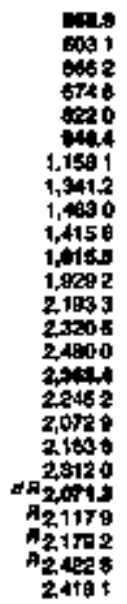 & 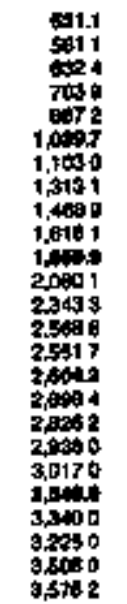 & 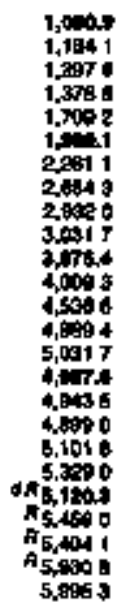 \\
\hline
\end{tabular}




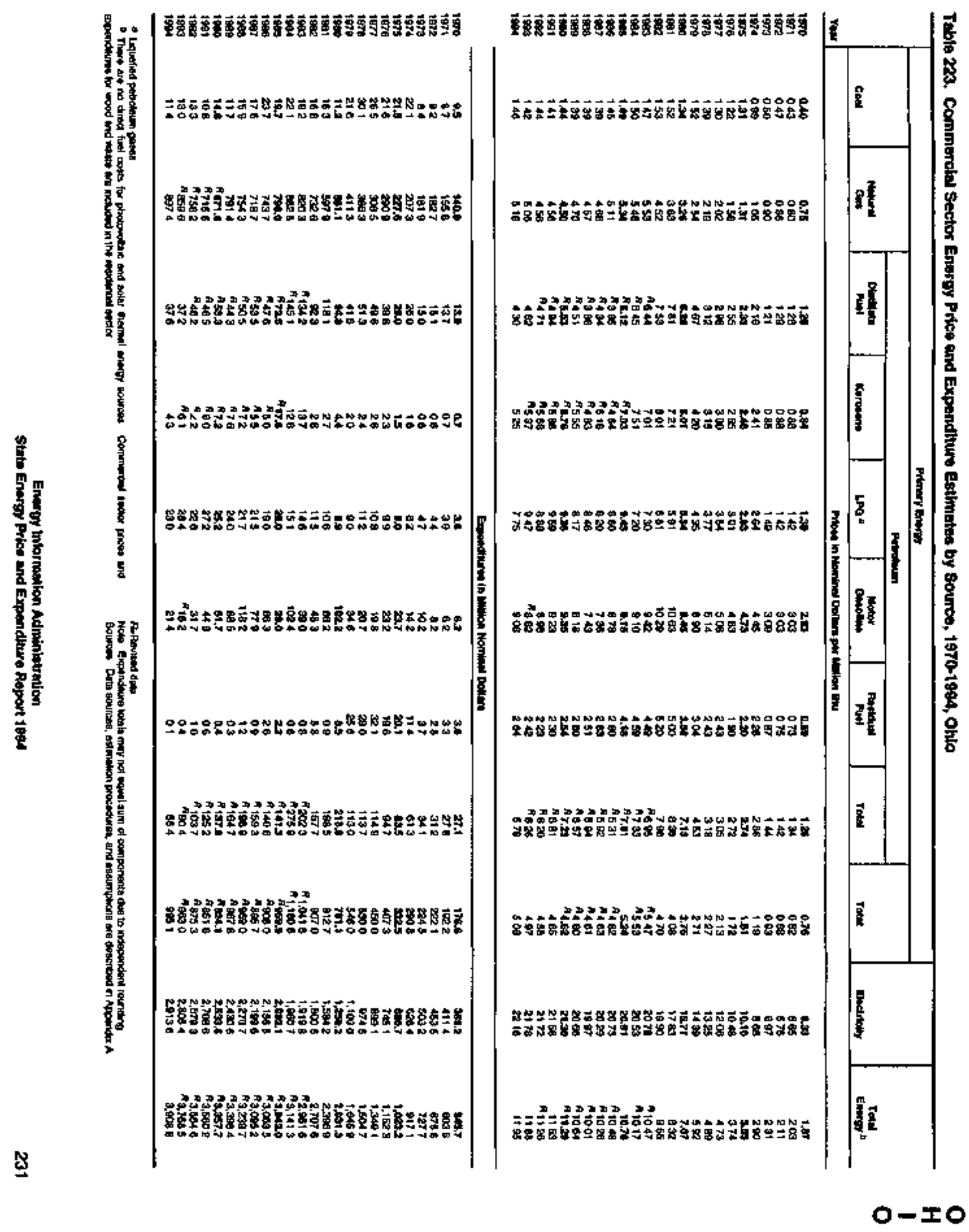




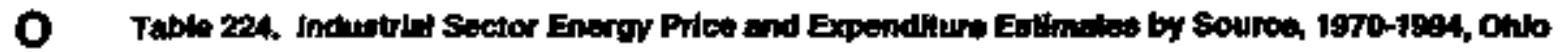

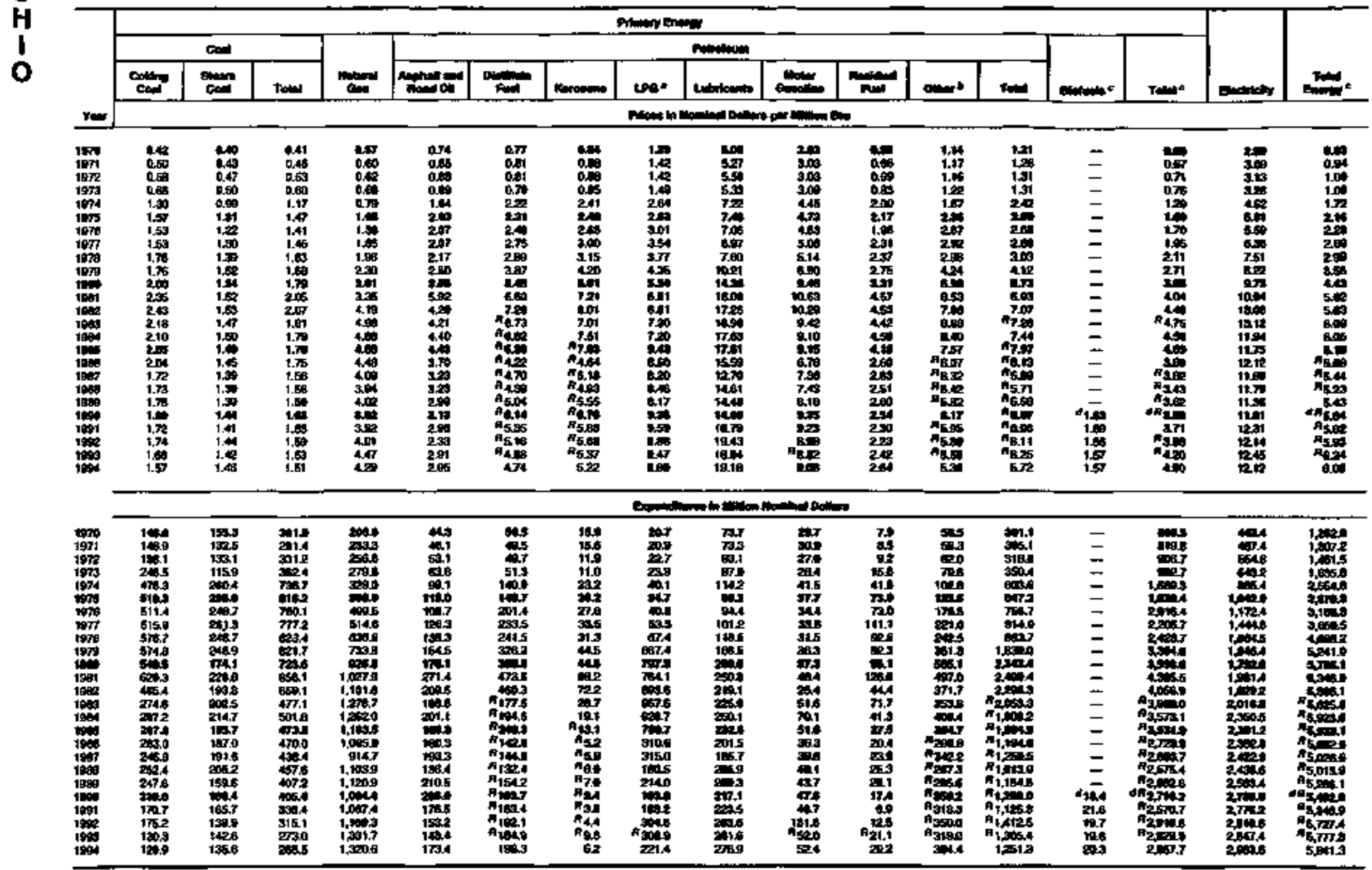

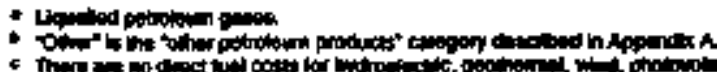

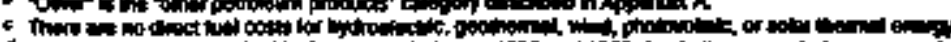

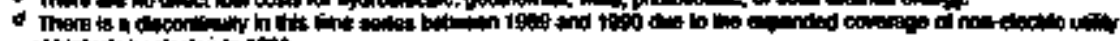

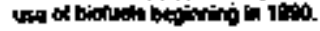

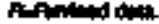

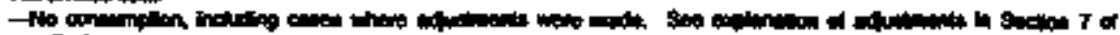
Apponima

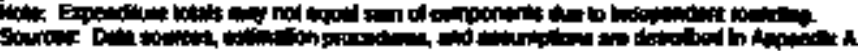




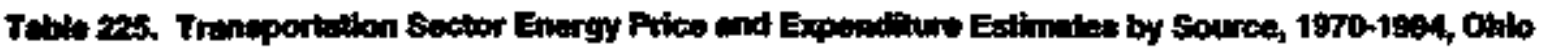

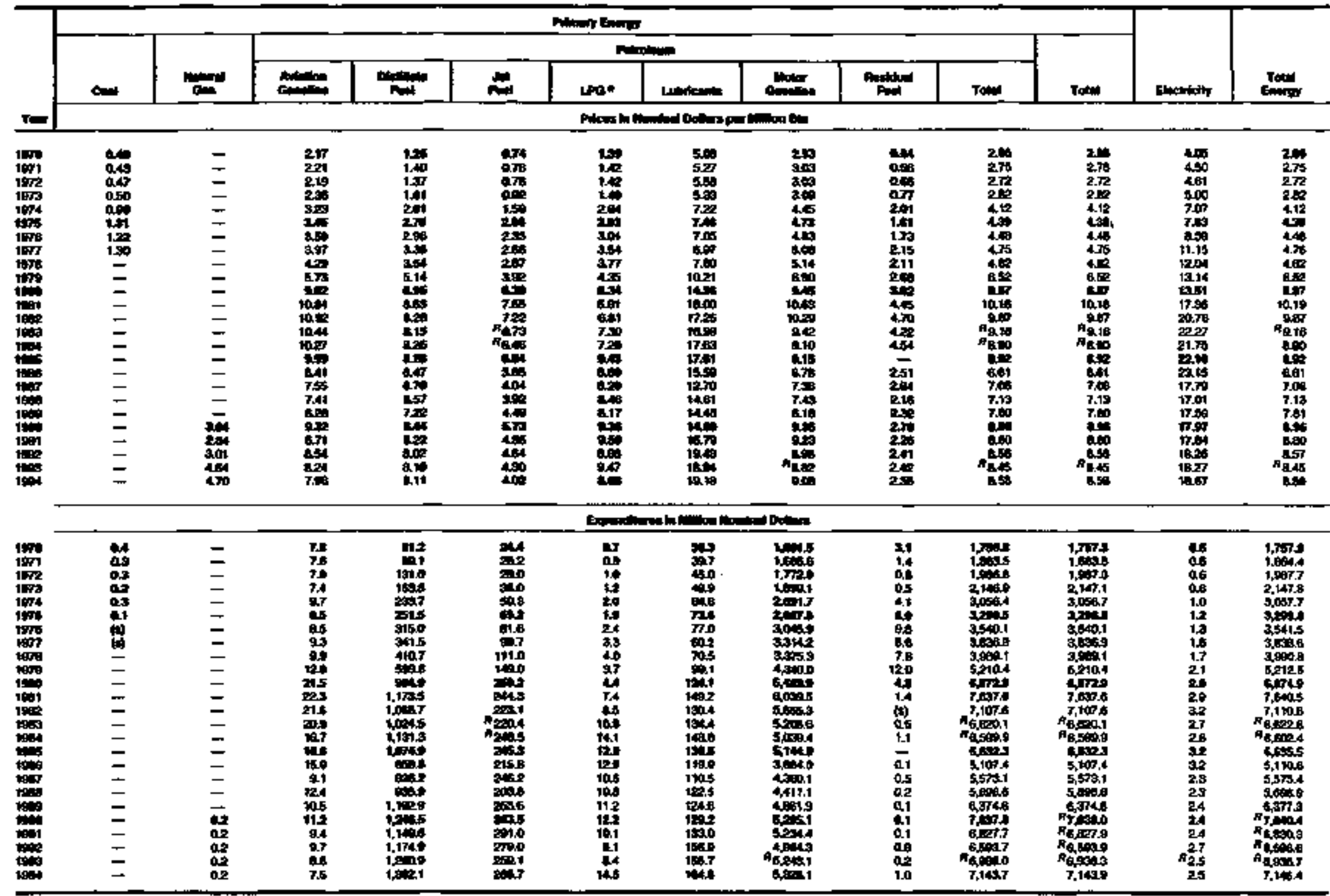

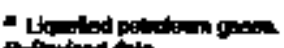

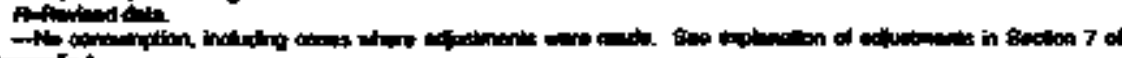

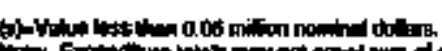

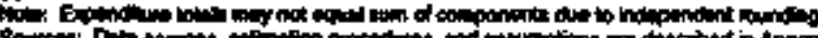




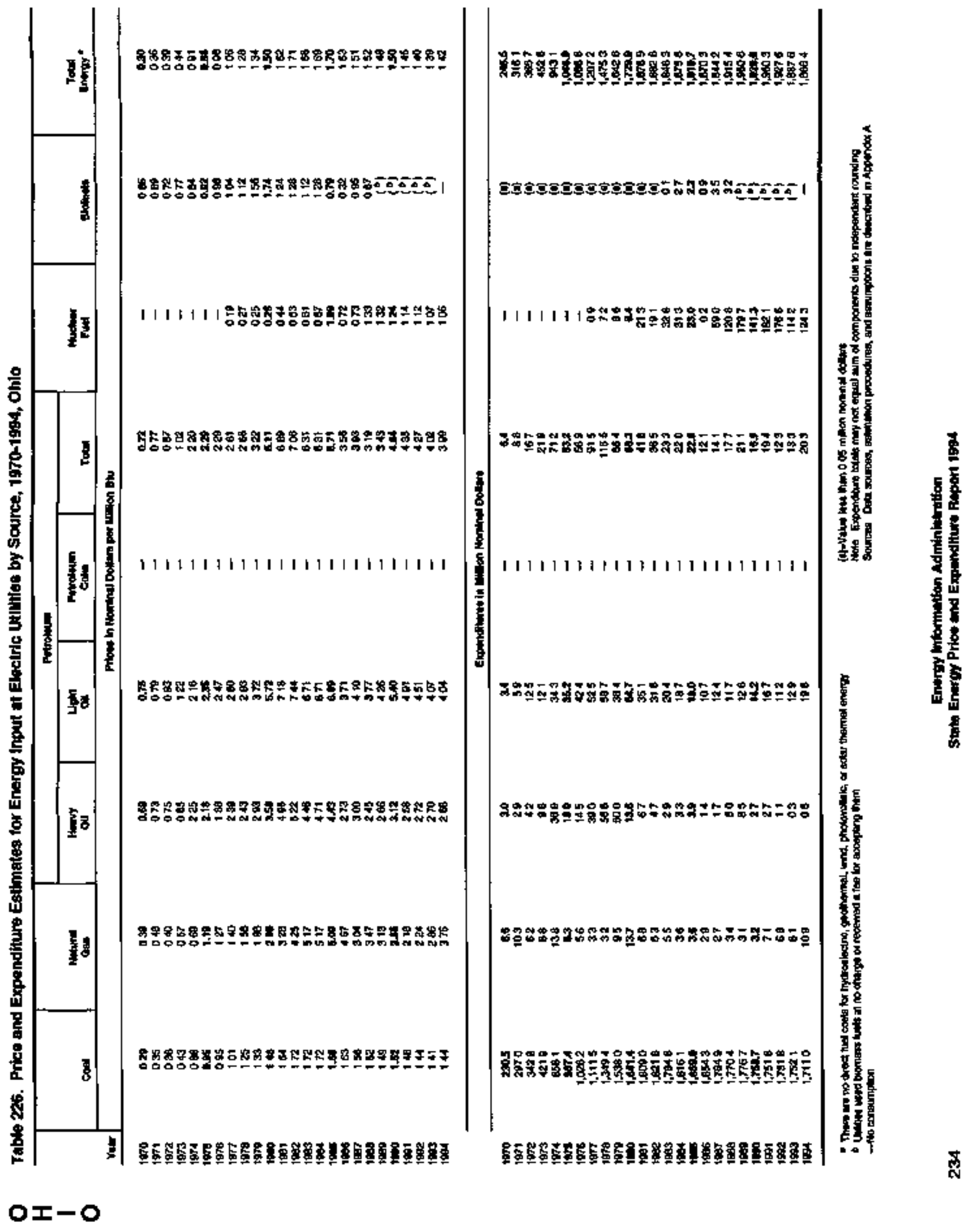


Toble 227. Energy Prite and Expenditure Ettimalwa by 8ourea, 1970-1994, Oklehome

\begin{tabular}{|c|c|c|c|c|c|c|c|c|c|c|c|c|c|c|c|c|c|}
\hline & \multicolumn{14}{|c|}{ Puntury Enogy } & \multirow{2}{*}{ Thentid } & \multirow{2}{*}{ 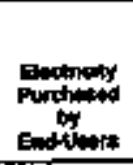 } & \multirow{2}{*}{ 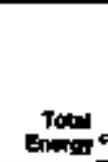 } \\
\hline & \multicolumn{3}{|c|}{ Con } & matex & \multicolumn{7}{|c|}{ Powothom } & Finer & wothe & Totale & & & \\
\hline$x=$ & \multicolumn{17}{|c|}{ 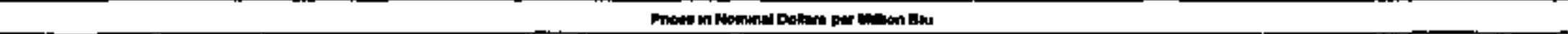 } \\
\hline 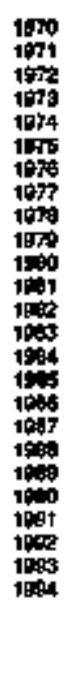 & $\begin{array}{l}= \\
\text { z } \\
= \\
= \\
\text { z } \\
= \\
= \\
= \\
= \\
= \\
= \\
= \\
= \\
=\end{array}$ & 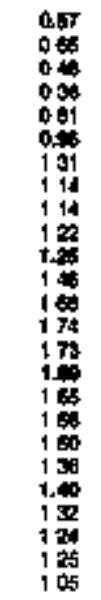 & 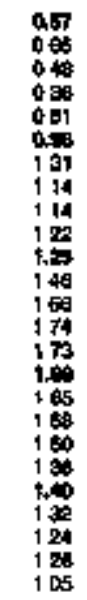 & 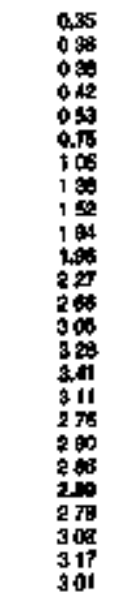 & 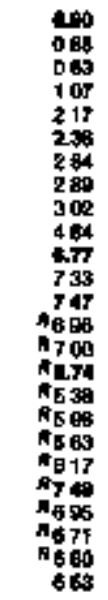 & 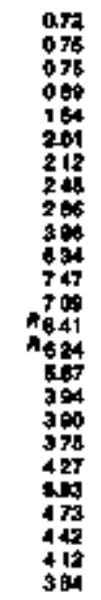 & 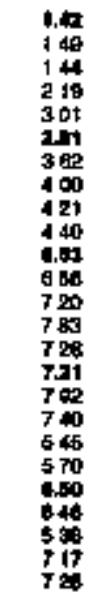 & 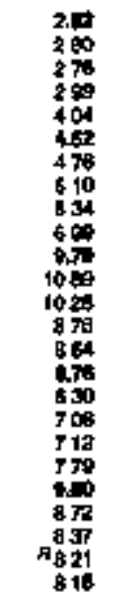 & 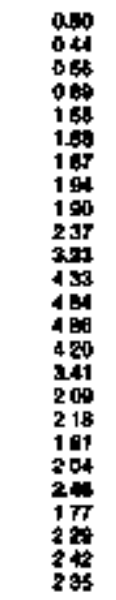 & 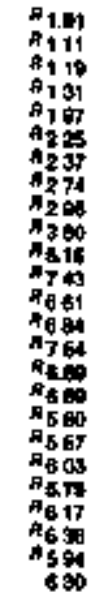 & 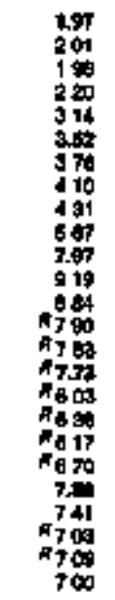 & $\begin{array}{l}= \\
= \\
= \\
= \\
= \\
= \\
= \\
= \\
= \\
= \\
= \\
= \\
=\end{array}$ & 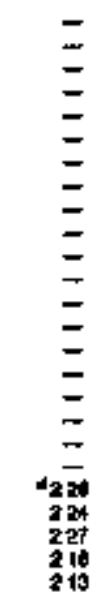 & 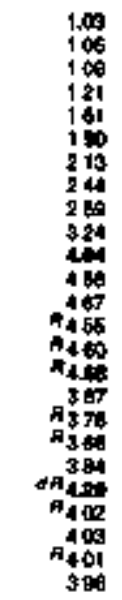 & 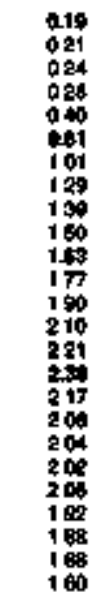 & 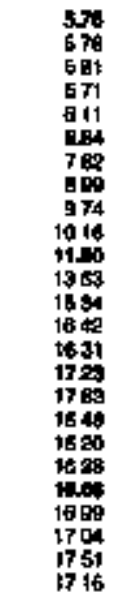 & 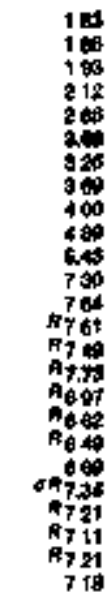 \\
\hline 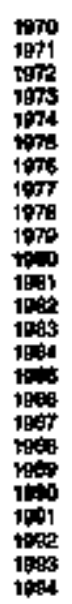 & $\begin{array}{l}\bar{Z} \\
\bar{z} \\
\bar{z} \\
\bar{z} \\
\bar{z} \\
\bar{z} \\
\bar{z} \\
\bar{z} \\
\bar{z} \\
\bar{z} \\
\bar{z}\end{array}$ & 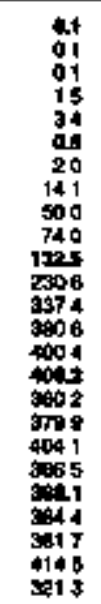 & 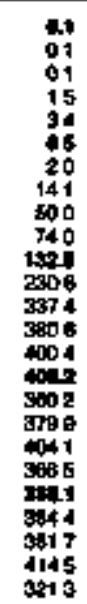 & 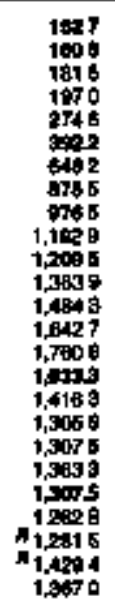 & 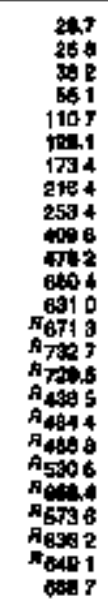 & 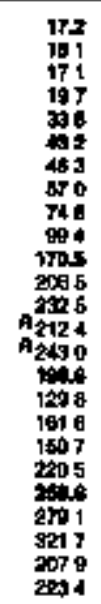 & 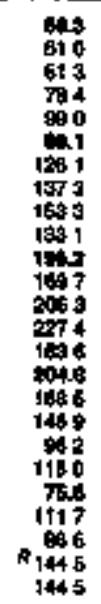 & 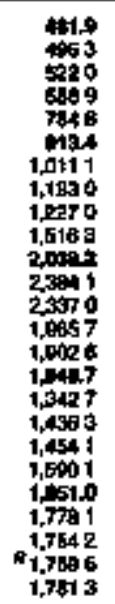 & 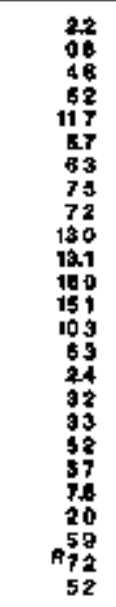 & 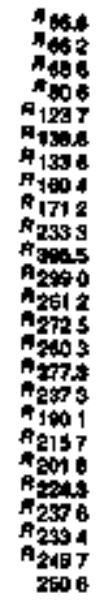 & 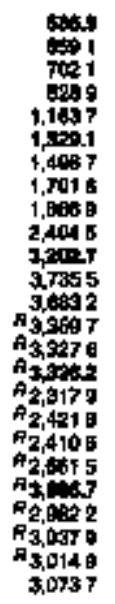 & $\begin{array}{l}\bar{z} \\
= \\
= \\
= \\
= \\
= \\
= \\
= \\
= \\
= \\
= \\
= \\
= \\
=\end{array}$ & 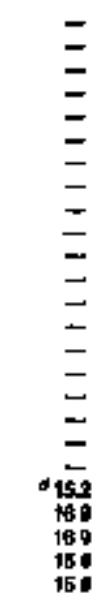 & 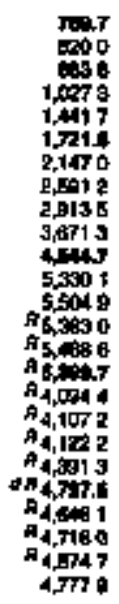 & 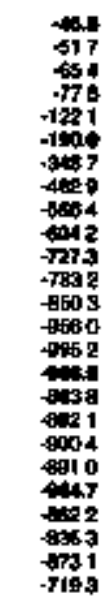 & 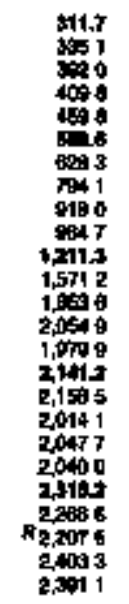 & 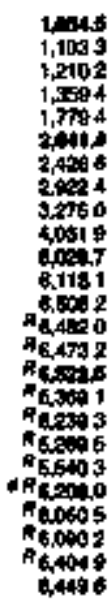 \\
\hline
\end{tabular}

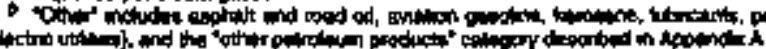

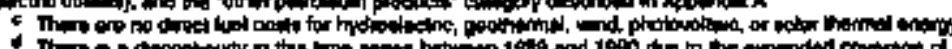

Anflenoted dum

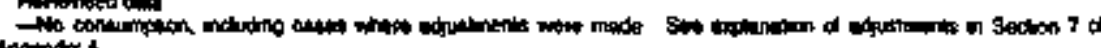
pandis A

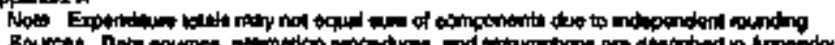

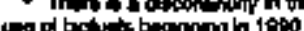


K

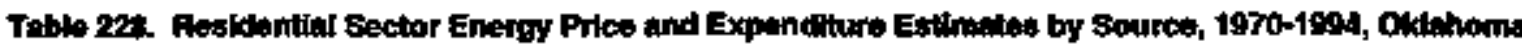

\begin{tabular}{|c|c|c|c|c|c|c|c|c|c|c|}
\hline \multirow[b]{4}{*}{ res } & \multicolumn{8}{|c|}{ Primany } & \multirow[b]{3}{*}{ ancinles: } & \multirow[b]{3}{*}{ Txid } \\
\hline & \multirow[b]{2}{*}{ and } & \multirow[b]{2}{*}{ 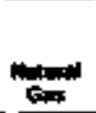 } & \multicolumn{4}{|c|}{ Onmingm } & \multirow[b]{2}{*}{ " } & \multirow[b]{2}{*}{ Tato" } & & \\
\hline & & & 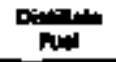 & 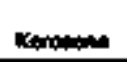 & 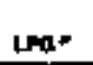 & Tow & & & & \\
\hline & \multicolumn{10}{|c|}{ 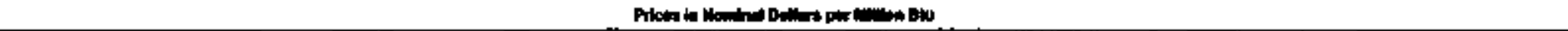 } \\
\hline \multirow[t]{2}{*}{ 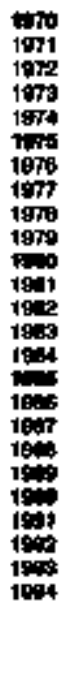 } & 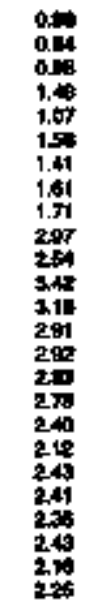 & 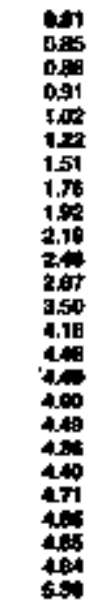 & 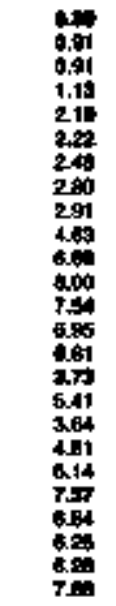 & 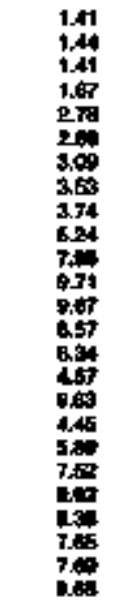 & 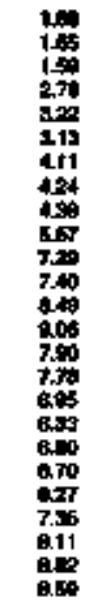 & 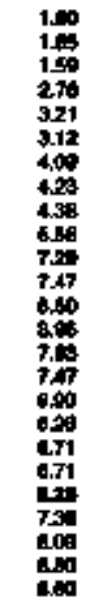 & $\begin{array}{l}= \\
= \\
= \\
= \\
= \\
= \\
= \\
= \\
= \\
= \\
= \\
= \\
35 \\
353 \\
3.53 \\
3.53\end{array}$ & 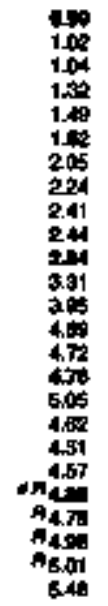 & 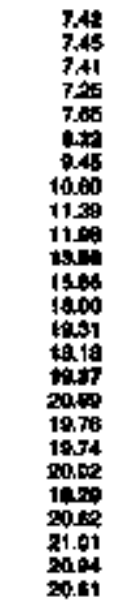 & 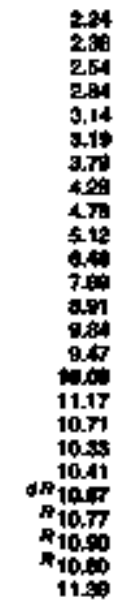 \\
\hline & \multicolumn{10}{|c|}{ 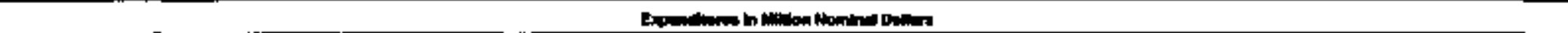 } \\
\hline 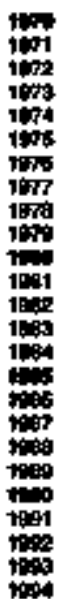 & 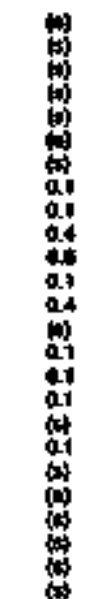 & 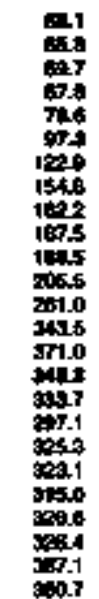 & 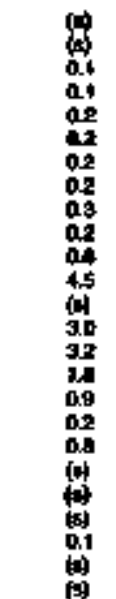 & 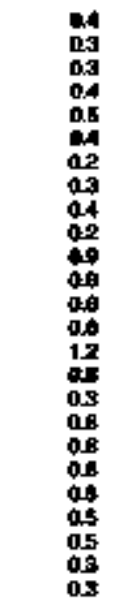 & 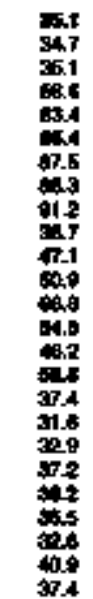 & 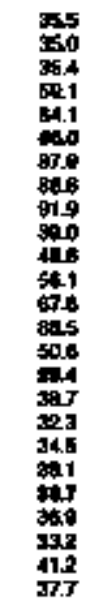 & $\begin{array}{l}= \\
= \\
= \\
= \\
= \\
= \\
= \\
= \\
= \\
= \\
= \\
\bar{z} \\
0.00 \\
0.0 \\
0.0 \\
0.2\end{array}$ & 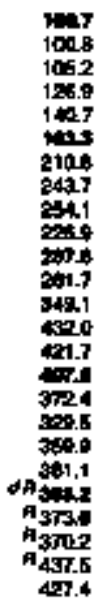 & 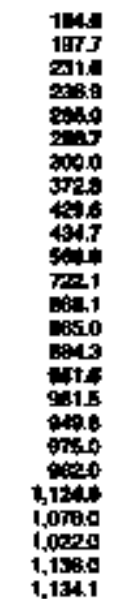 & 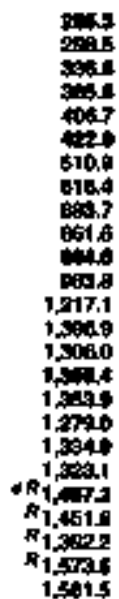 \\
\hline
\end{tabular}

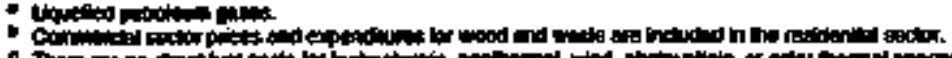

Alanosod dab

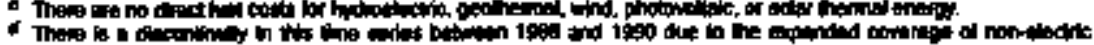

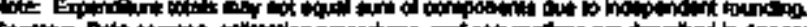

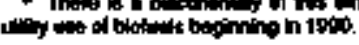

En.gy Intornintion Ndminlotration 
Tave 209. Commorctal Sector Energy Price and Expenditure Estlmates by Source, 1970-1994, Oktahoma

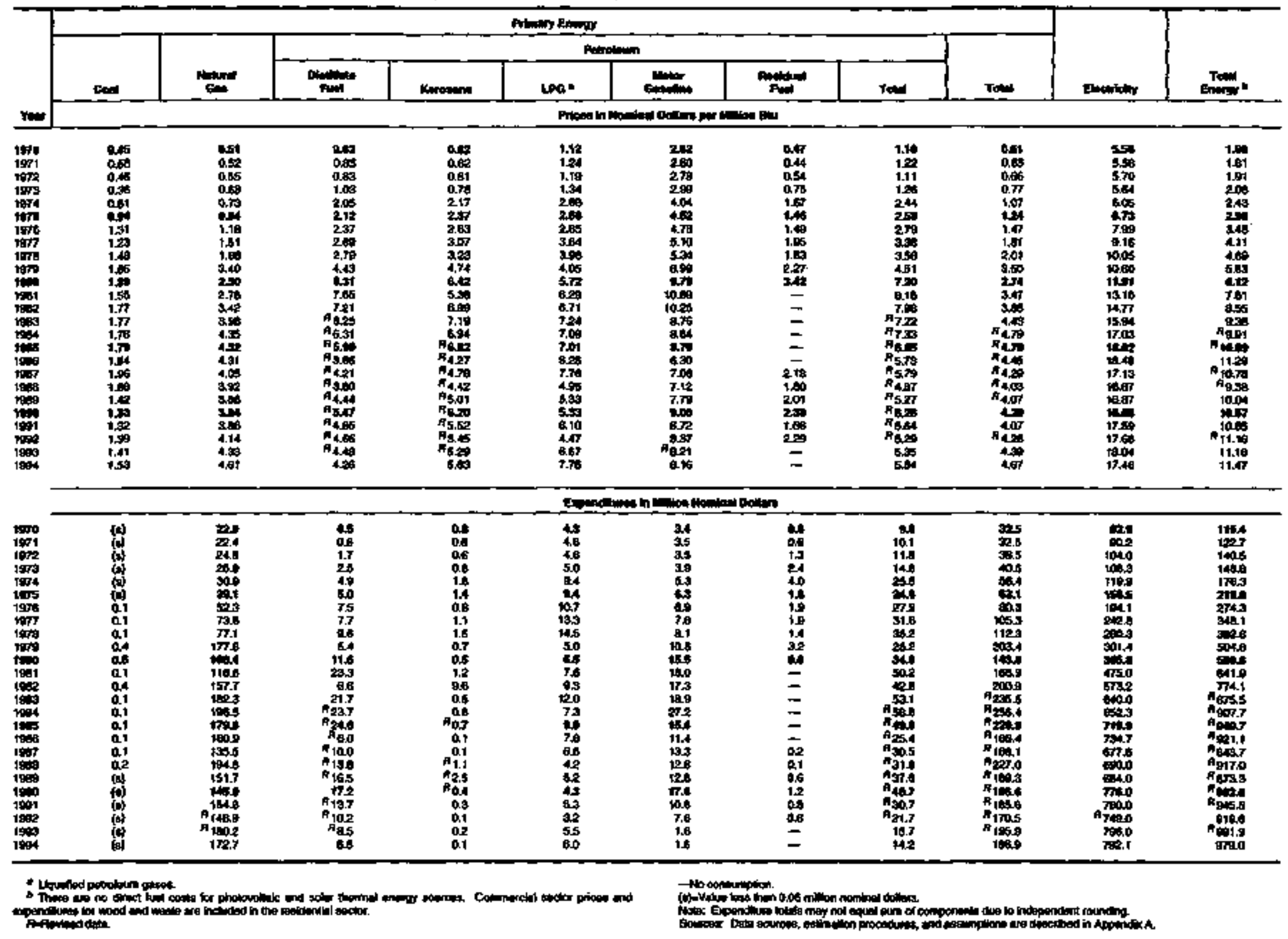




\begin{tabular}{|c|c|c|c|c|c|c|c|c|c|c|c|c|c|c|c|c|c|}
\hline & \multicolumn{15}{|c|}{ Prlming tenen } & \multirow[b]{3}{*}{ Einctulein } & \multirow[b]{3}{*}{ case } \\
\hline & \multicolumn{3}{|c|}{ cos } & \multirow[b]{2}{*}{ Nored } & \multicolumn{9}{|c|}{ Patrolewn } & \multirow[b]{2}{*}{ Blotude } & \multirow[b]{2}{*}{ Towit } & & \\
\hline & chem & ind & Tow & & rephend ond & 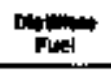 & Kenyeane & $\mathbf{u g} *$ & Lbatis & (1) & Mack & Dthes ${ }^{\circ}$ & Tit & & & & \\
\hline 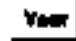 & \multicolumn{17}{|c|}{ 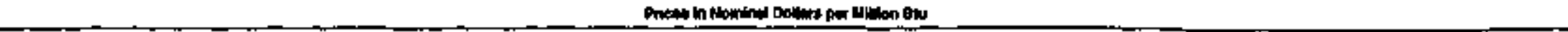 } \\
\hline \multirow[t]{2}{*}{ 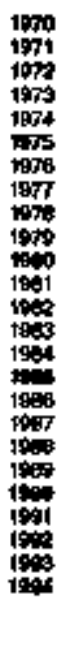 } & $\begin{array}{l}= \\
= \\
z \\
= \\
= \\
= \\
= \\
= \\
= \\
= \\
= \\
= \\
=\end{array}$ & 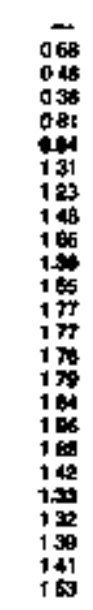 & 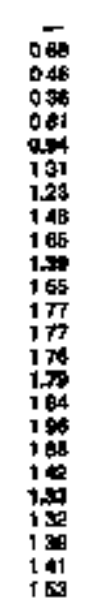 & 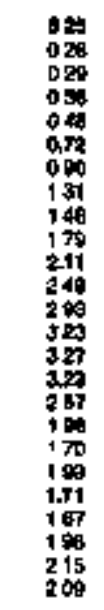 & 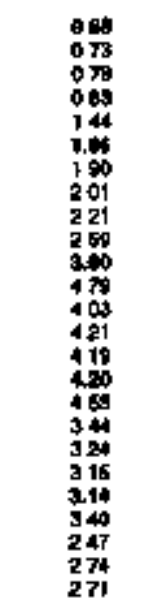 & 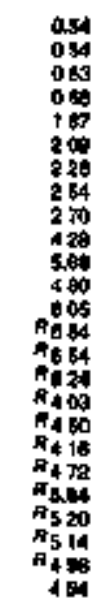 & 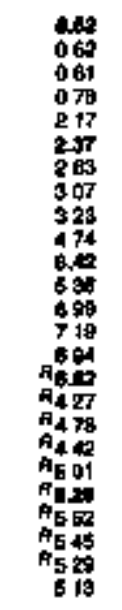 & 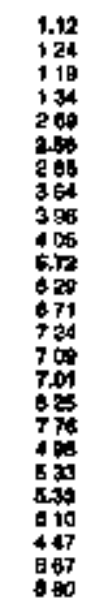 & 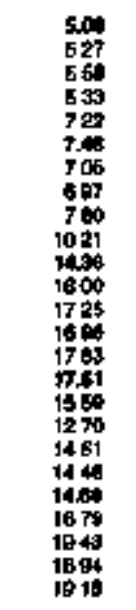 & 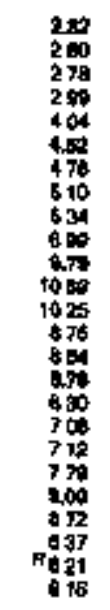 & 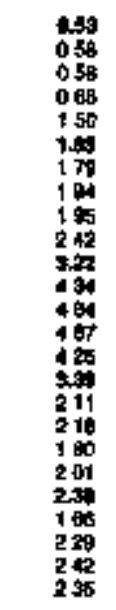 & 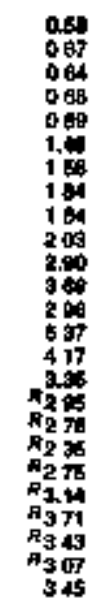 & 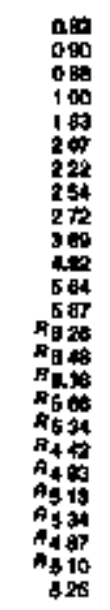 & $\begin{array}{l}= \\
= \\
= \\
= \\
= \\
= \\
= \\
= \\
= \\
= \\
= \\
= \\
= \\
143 \\
148 \\
142 \\
140\end{array}$ & 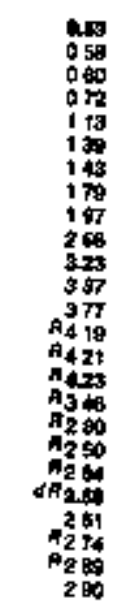 & 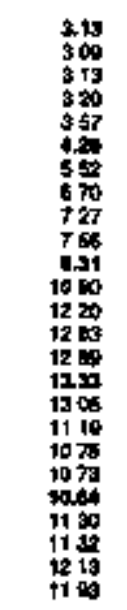 & 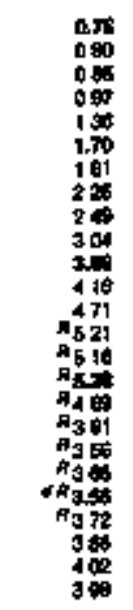 \\
\hline & \multicolumn{17}{|c|}{ 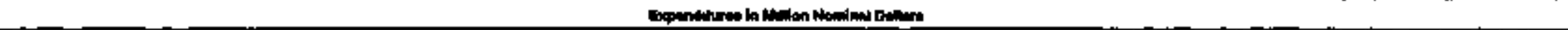 } \\
\hline 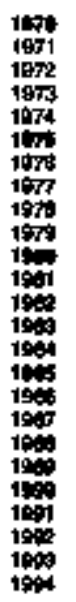 & $\begin{array}{l}= \\
= \\
= \\
z \\
z \\
= \\
= \\
= \\
= \\
= \\
= \\
= \\
= \\
=\end{array}$ & 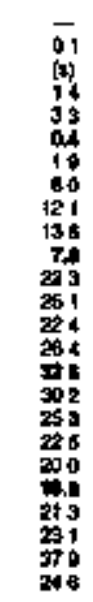 & 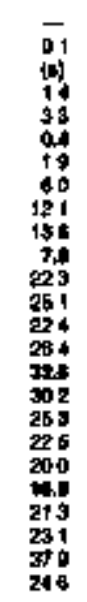 & 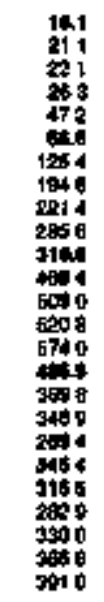 & 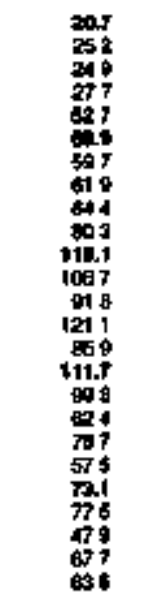 & 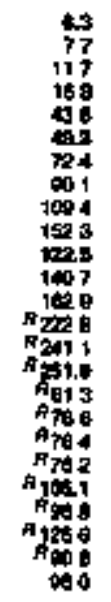 & 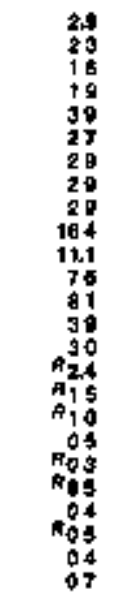 & 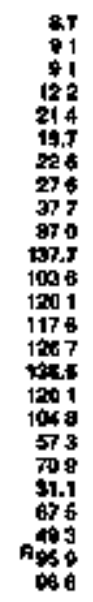 & 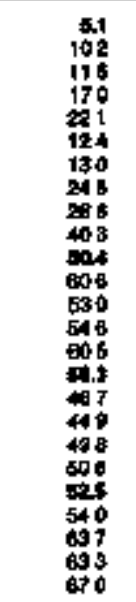 & 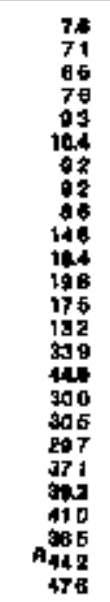 & $\begin{array}{r}12 \\
01 \\
27 \\
33 \\
57 \\
32 \\
40 \\
99 \\
43 \\
69 \\
128 \\
150 \\
151 \\
103 \\
50 \\
6.2 \\
31 \\
30 \\
50 \\
30 \\
5.3 \\
10 \\
61 \\
971 \\
51\end{array}$ & 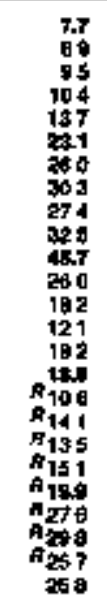 & 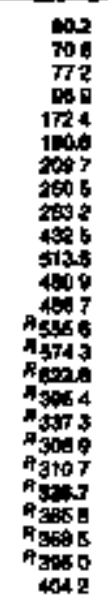 & $\begin{array}{l}= \\
= \\
= \\
= \\
= \\
= \\
= \\
= \\
= \\
= \\
\bar{z} \\
07 \\
68 \\
67 \\
68\end{array}$ & 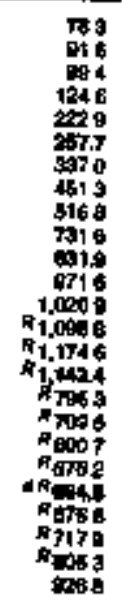 & 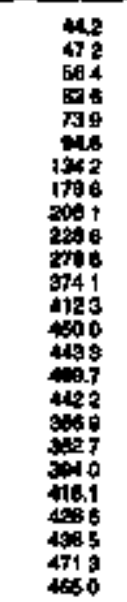 & 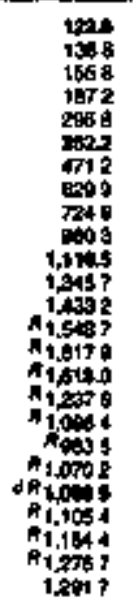 \\
\hline
\end{tabular}




\begin{tabular}{|c|c|c|c|c|c|c|c|c|c|c|c|c|c|}
\hline \multirow[b]{4}{*}{$x=$} & \multicolumn{11}{|c|}{ Ptiney renergy } & \multirow[b]{3}{*}{ Dintity } & \multirow[b]{3}{*}{ Tol } \\
\hline & & \multirow[b]{2}{*}{ mant } & \multicolumn{8}{|c|}{ Patrolem } & \multirow[b]{2}{*}{ Todel } & & \\
\hline & coll & & Antition & 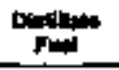 & Fin & La: & Lubienow & 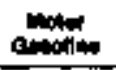 & Pallot & Tolat & & & \\
\hline & \multicolumn{13}{|c|}{ 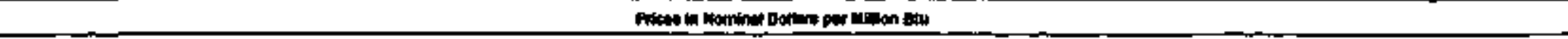 } \\
\hline 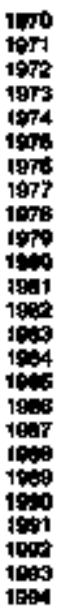 & $\begin{array}{l}0.0 \\
0.40 \\
0.38 \\
0.01 \\
0.31 \\
1.20 \\
= \\
= \\
= \\
= \\
= \\
= \\
= \\
= \\
= \\
=\end{array}$ & $\begin{array}{l}= \\
= \\
= \\
= \\
= \\
= \\
= \\
= \\
= \\
= \\
= \\
= \\
= \\
3.79 \\
2.00 \\
2.00\end{array}$ & 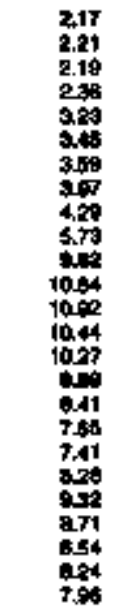 & 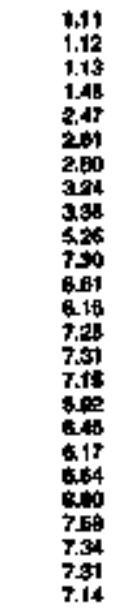 & 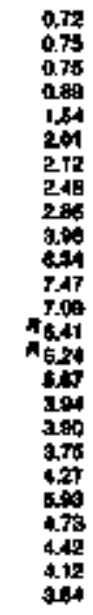 & 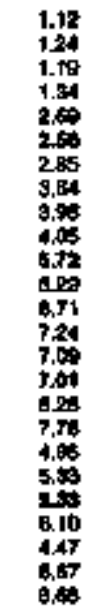 & 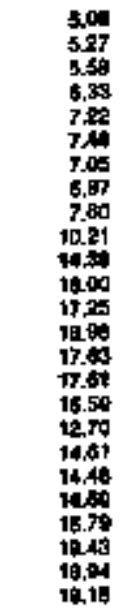 & 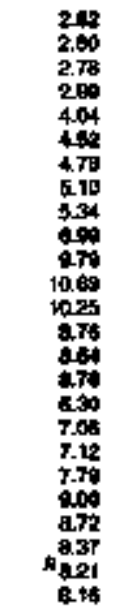 & $\begin{array}{l}0.45 \\
0.43 \\
0.69 \\
0.73 \\
1.73 \\
= \\
= \\
= \\
= \\
= \\
= \\
= \\
= \\
= \\
= \\
= \\
=\end{array}$ & 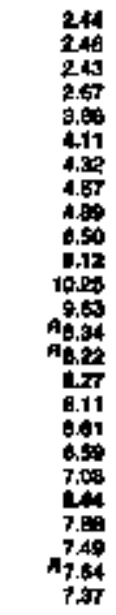 & 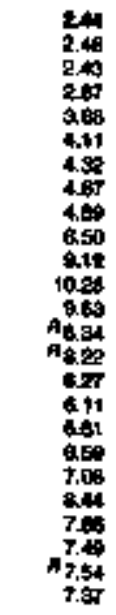 & $\begin{array}{l}= \\
= \\
= \\
= \\
= \\
= \\
= \\
= \\
= \\
= \\
= \\
= \\
=\end{array}$ & 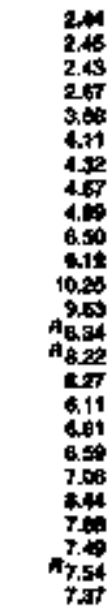 \\
\hline \multicolumn{14}{|c|}{ 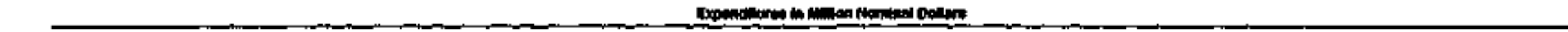 } \\
\hline 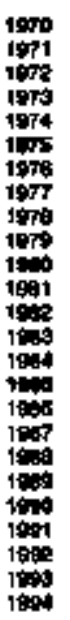 & $\begin{array}{l}\overline{6} \\
00 \\
00 \\
0 \\
= \\
= \\
= \\
= \\
= \\
= \\
= \\
= \\
= \\
=\end{array}$ & $\begin{array}{l}= \\
z \\
z \\
= \\
= \\
= \\
= \\
= \\
= \\
= \\
= \\
= \\
= \\
\frac{10}{0.1} \\
0.0\end{array}$ & 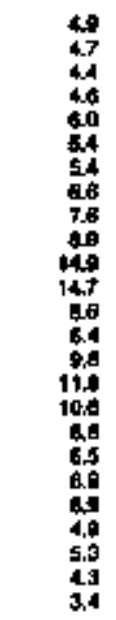 & 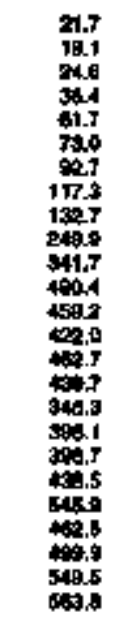 & 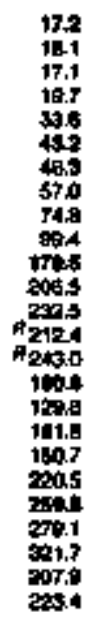 & 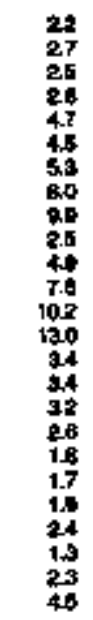 & 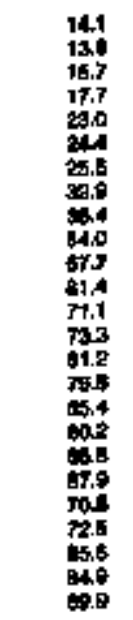 & 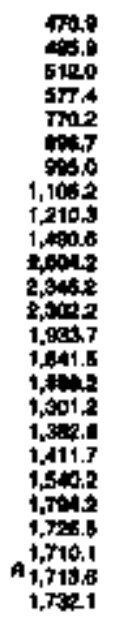 & $\begin{array}{l}02 \\
0.1 \\
100 \\
0.0 \\
0.2 \\
= \\
= \\
= \\
= \\
= \\
= \\
= \\
= \\
= \\
= \\
= \\
= \\
=\end{array}$ & 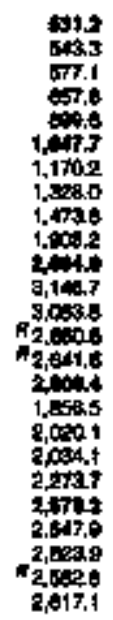 & 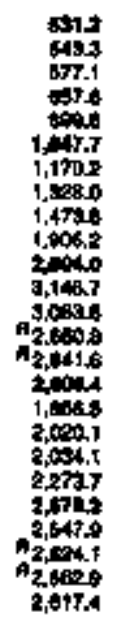 & $\begin{array}{l}= \\
= \\
= \\
= \\
= \\
= \\
= \\
= \\
= \\
= \\
= \\
= \\
= \\
=\end{array}$ & 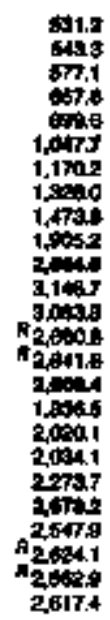 \\
\hline
\end{tabular}




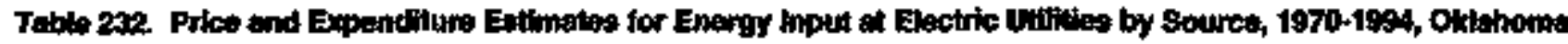

\begin{tabular}{|c|c|c|c|c|c|c|c|c|c|}
\hline \multirow[b]{3}{*}{$\underline{\mathbf{Y}}$} & \multirow[b]{2}{*}{ and } & \multirow[b]{2}{*}{ "int } & \multicolumn{4}{|c|}{ 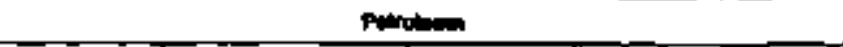 } & \multirow[b]{2}{*}{ mint } & \multirow[b]{2}{*}{ 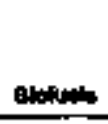 } & \multirow[b]{2}{*}{ thenty } \\
\hline & & & ons & Lin & 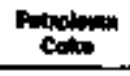 & Totid & & & \\
\hline & \multicolumn{9}{|c|}{ 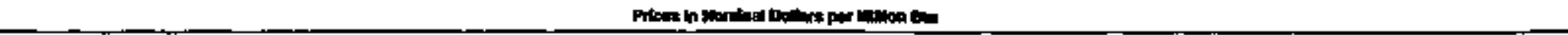 } \\
\hline 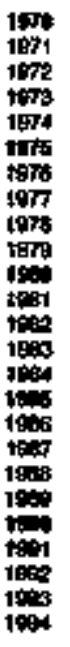 & 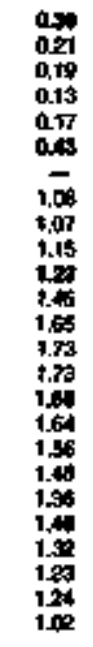 & 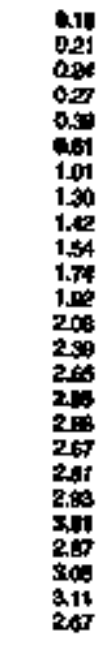 & 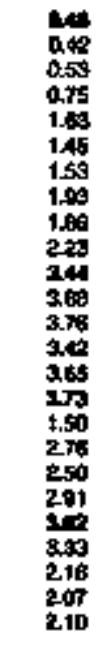 & 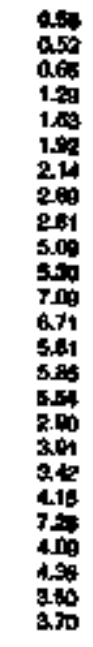 & $\begin{array}{l}= \\
= \\
= \\
= \\
= \\
= \\
= \\
= \\
= \\
= \\
= \\
= \\
=\end{array}$ & 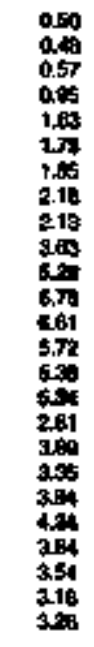 & $\begin{array}{l}= \\
= \\
= \\
= \\
= \\
= \\
= \\
= \\
= \\
= \\
= \\
= \\
= \\
=\end{array}$ & $\begin{array}{l}= \\
= \\
= \\
= \\
= \\
= \\
= \\
= \\
= \\
= \\
= \\
= \\
=\end{array}$ & 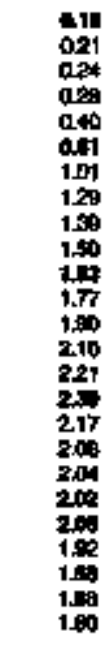 \\
\hline & \multicolumn{9}{|c|}{ 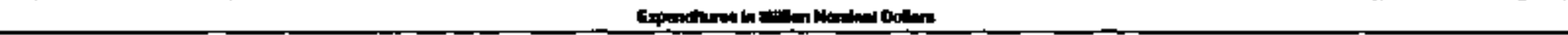 } \\
\hline 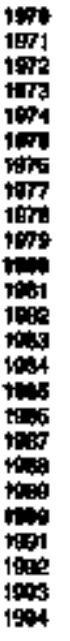 & 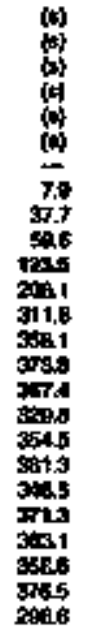 & 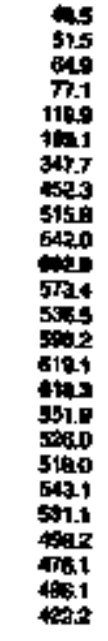 & 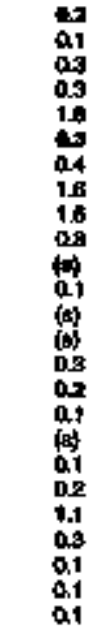 & $\begin{array}{l}0.2 \\
0.1 \\
0.2 \\
0.4 \\
0.4 \\
0.4 \\
1.1 \\
1.3 \\
1.4 \\
1.1 \\
1.5 \\
2.2 \\
1.7 \\
2.0 \\
2.5 \\
1.9 \\
1.5 \\
1.1 \\
1.3 \\
1.3 \\
0.5 \\
0.5 \\
0.4 \\
0.4\end{array}$ & $\begin{array}{l}= \\
= \\
= \\
= \\
= \\
= \\
= \\
= \\
= \\
= \\
= \\
= \\
= \\
=\end{array}$ & $\begin{array}{l}04 \\
0.2 \\
0.5 \\
0.7 \\
24 \\
0.0 \\
1.0 \\
2.8 \\
2.9 \\
2.6 \\
1.0 \\
1.6 \\
2.2 \\
1.7 \\
2.3 \\
2.7 \\
2.0 \\
1.6 \\
1.2 \\
1.5 \\
2.3 \\
9.0 \\
0.6 \\
0.5 \\
1.8\end{array}$ & $\begin{array}{l}= \\
= \\
= \\
= \\
= \\
= \\
= \\
= \\
= \\
= \\
= \\
= \\
= \\
=\end{array}$ & $\begin{array}{l}= \\
= \\
= \\
= \\
= \\
= \\
= \\
= \\
= \\
= \\
= \\
= \\
=\end{array}$ & 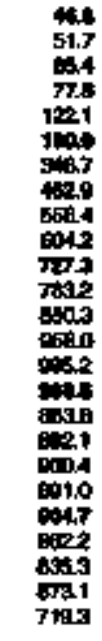 \\
\hline
\end{tabular}

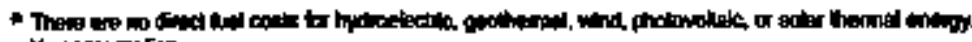

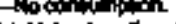


Table 2xa, Energy Price and Expendlune Eatimates by Source, 1970-1994, Crobon

\begin{tabular}{|c|c|c|c|c|c|c|c|c|c|c|c|c|c|c|c|c|c|}
\hline & \multicolumn{14}{|c|}{ 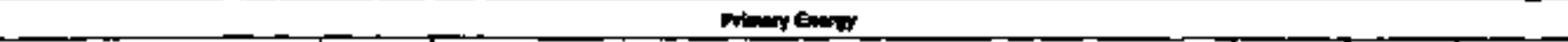 } & \multirow{3}{*}{ Focte } & \multirow{3}{*}{ גוty } & \multirow[b]{3}{*}{ Told } \\
\hline & \multicolumn{3}{|c|}{ and } & \multirow[b]{2}{*}{ 党 } & \multicolumn{7}{|c|}{ Patans } & \multirow[b]{2}{*}{ puret } & \multirow[b]{2}{*}{ 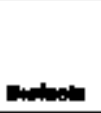 } & \multirow[b]{2}{*}{ Tolene } & & & \\
\hline & Codsh & cand & Tot & & pont & Pun & LPG. & 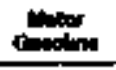 & Fent & 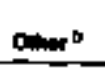 & Tow & & & & & & \\
\hline var. & \multicolumn{17}{|c|}{ 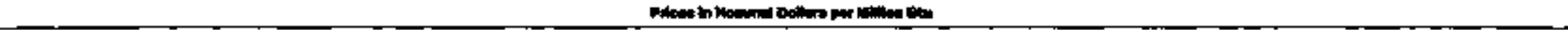 } \\
\hline \multirow[t]{2}{*}{ 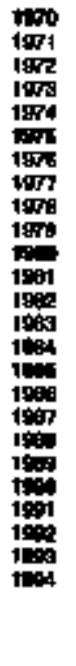 } & $\begin{array}{l}z \\
z \\
z \\
z \\
z \\
z \\
z \\
z \\
= \\
= \\
= \\
z \\
z \\
z \\
z \\
z\end{array}$ & 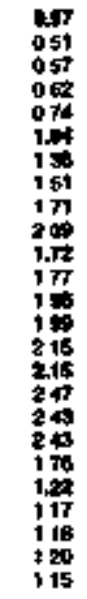 & 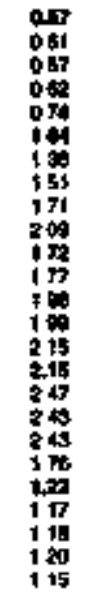 & 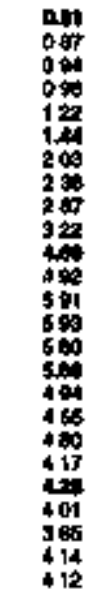 & 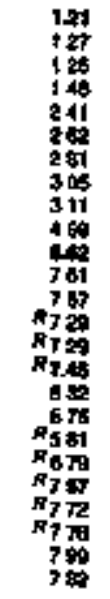 & 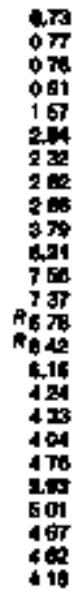 & 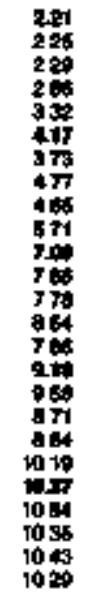 & 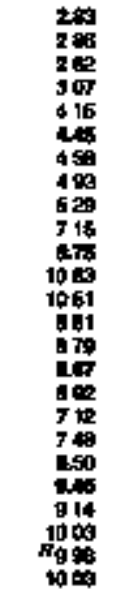 & 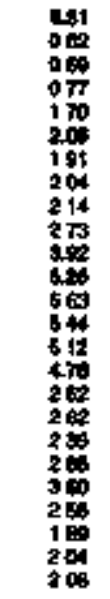 & 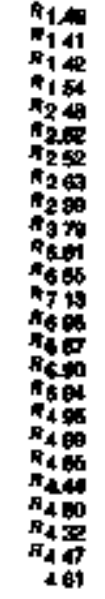 & 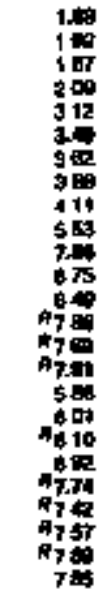 & 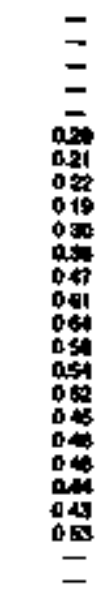 & 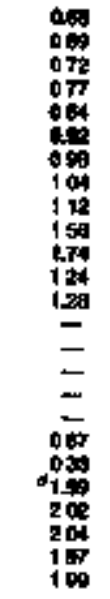 & 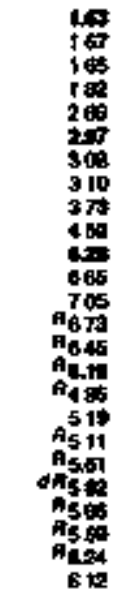 & 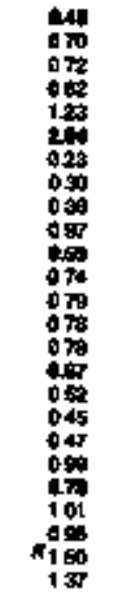 & 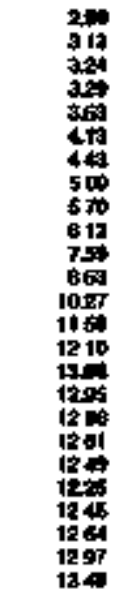 & 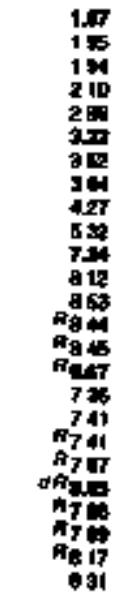 \\
\hline & \multicolumn{17}{|c|}{ 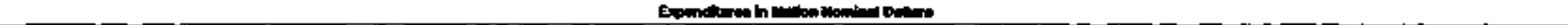 } \\
\hline 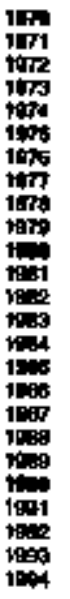 & $\begin{array}{l}= \\
= \\
= \\
= \\
= \\
z \\
= \\
= \\
= \\
= \\
= \\
= \\
= \\
= \\
=\end{array}$ & 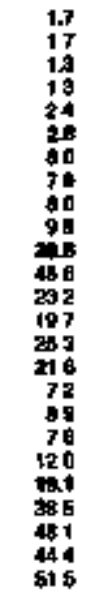 & 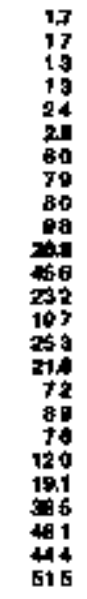 & 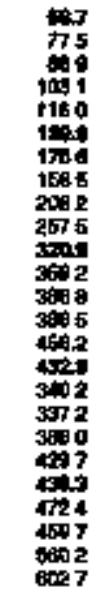 & 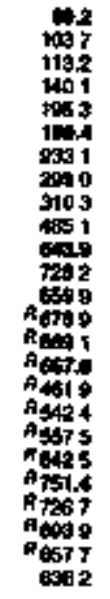 & 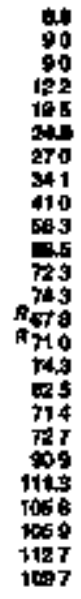 & 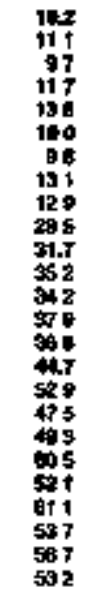 & 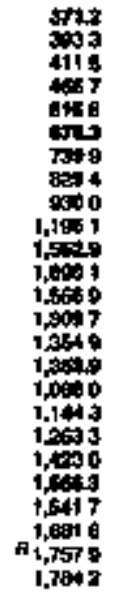 & 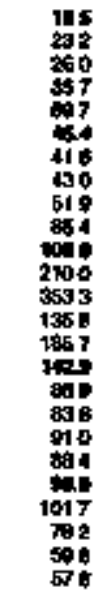 & 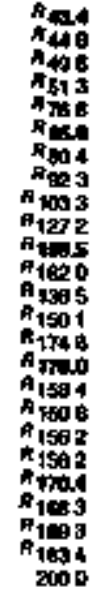 & 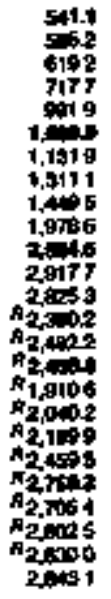 & 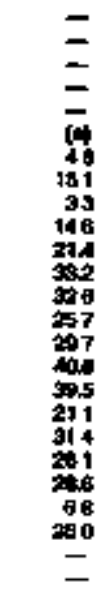 & 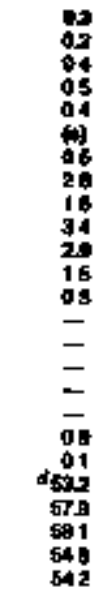 & 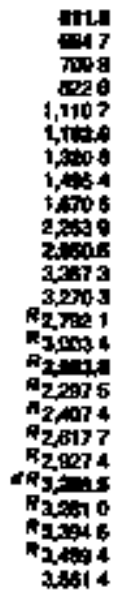 & 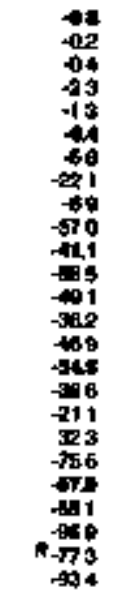 & 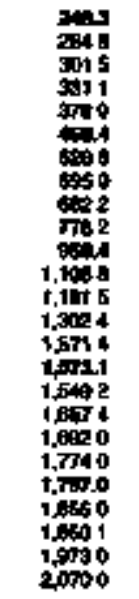 & 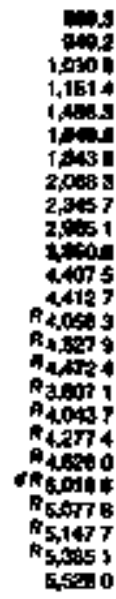 \\
\hline
\end{tabular}

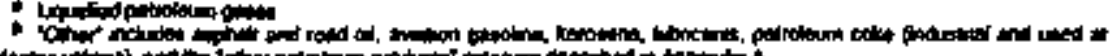

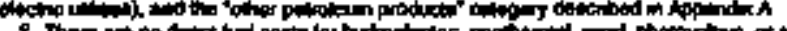

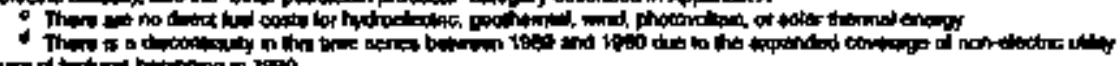

A the

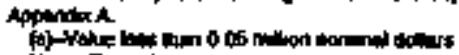

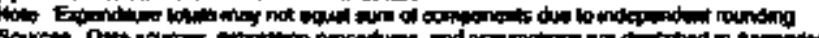


O

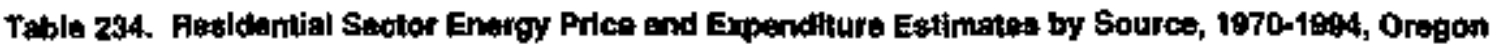

\begin{tabular}{|c|c|c|c|c|c|c|c|c|c|c|}
\hline \multirow[b]{4}{*}{ ras } & \multicolumn{8}{|c|}{ Prestrenengy } & \multirow[b]{3}{*}{ Emeriekty } & \multirow[b]{3}{*}{ raterese } \\
\hline & \multirow[b]{2}{*}{ coos } & \multirow[b]{2}{*}{ (1) } & \multicolumn{4}{|c|}{ Datroutm } & \multirow[b]{2}{*}{-0} & \multirow[b]{2}{*}{ Toun 5} & & \\
\hline & & & Dons & Keroesmen & La" & Tow & & & & \\
\hline & \multicolumn{10}{|c|}{ 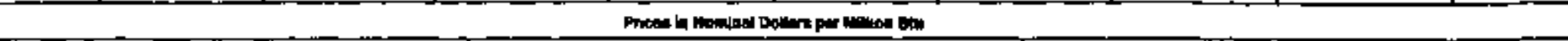 } \\
\hline \multirow[t]{2}{*}{ 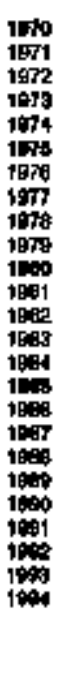 } & 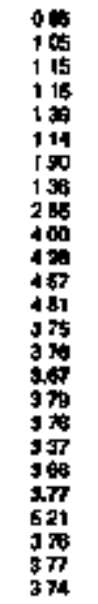 & 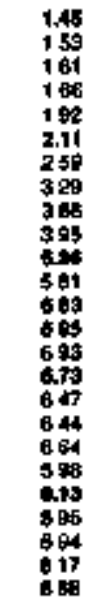 & 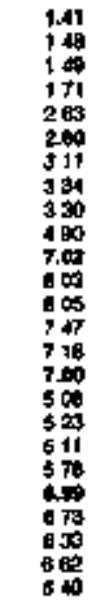 & 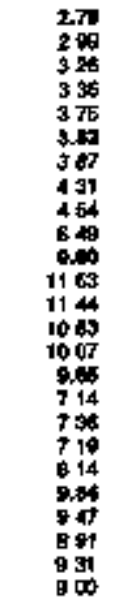 & 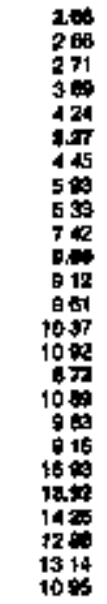 & 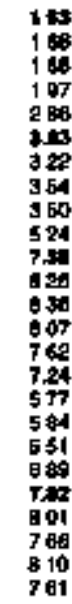 & 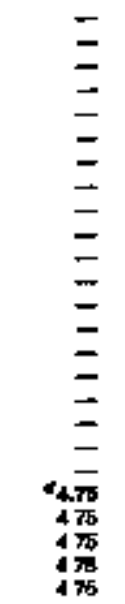 & 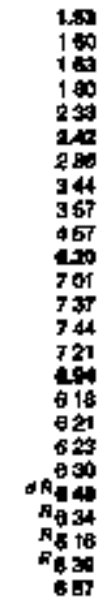 & 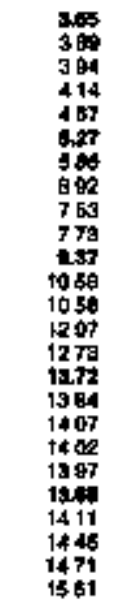 & 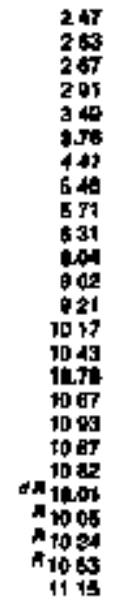 \\
\hline & \multicolumn{10}{|c|}{ 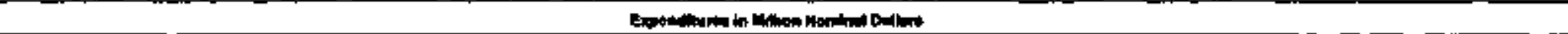 } \\
\hline 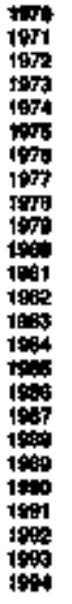 & 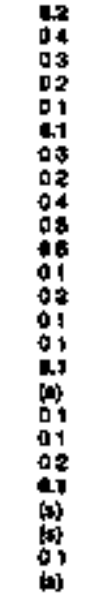 & 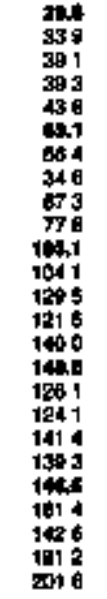 & 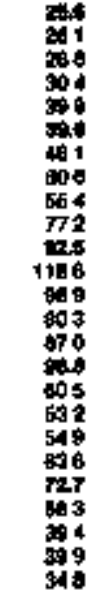 & 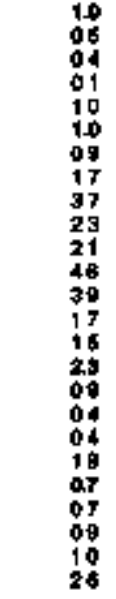 & 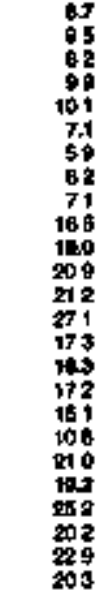 & 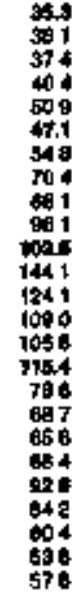 & 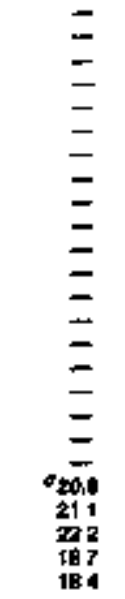 & 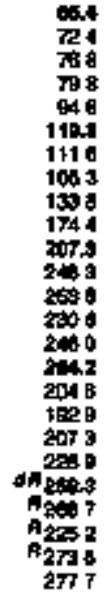 & 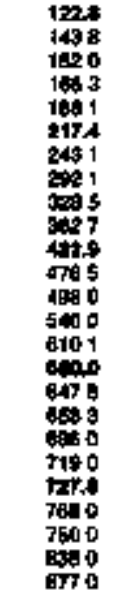 & 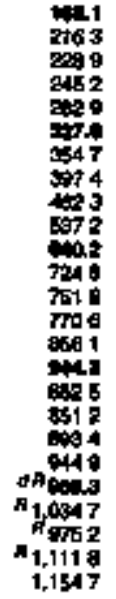 \\
\hline
\end{tabular}

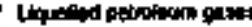

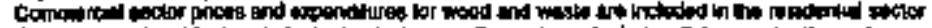

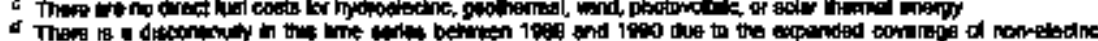

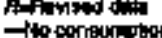

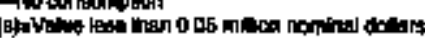

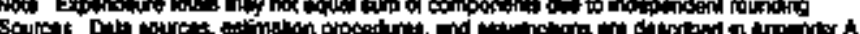

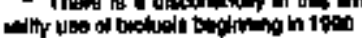

Enerty hilorphotion Adiminimbation 


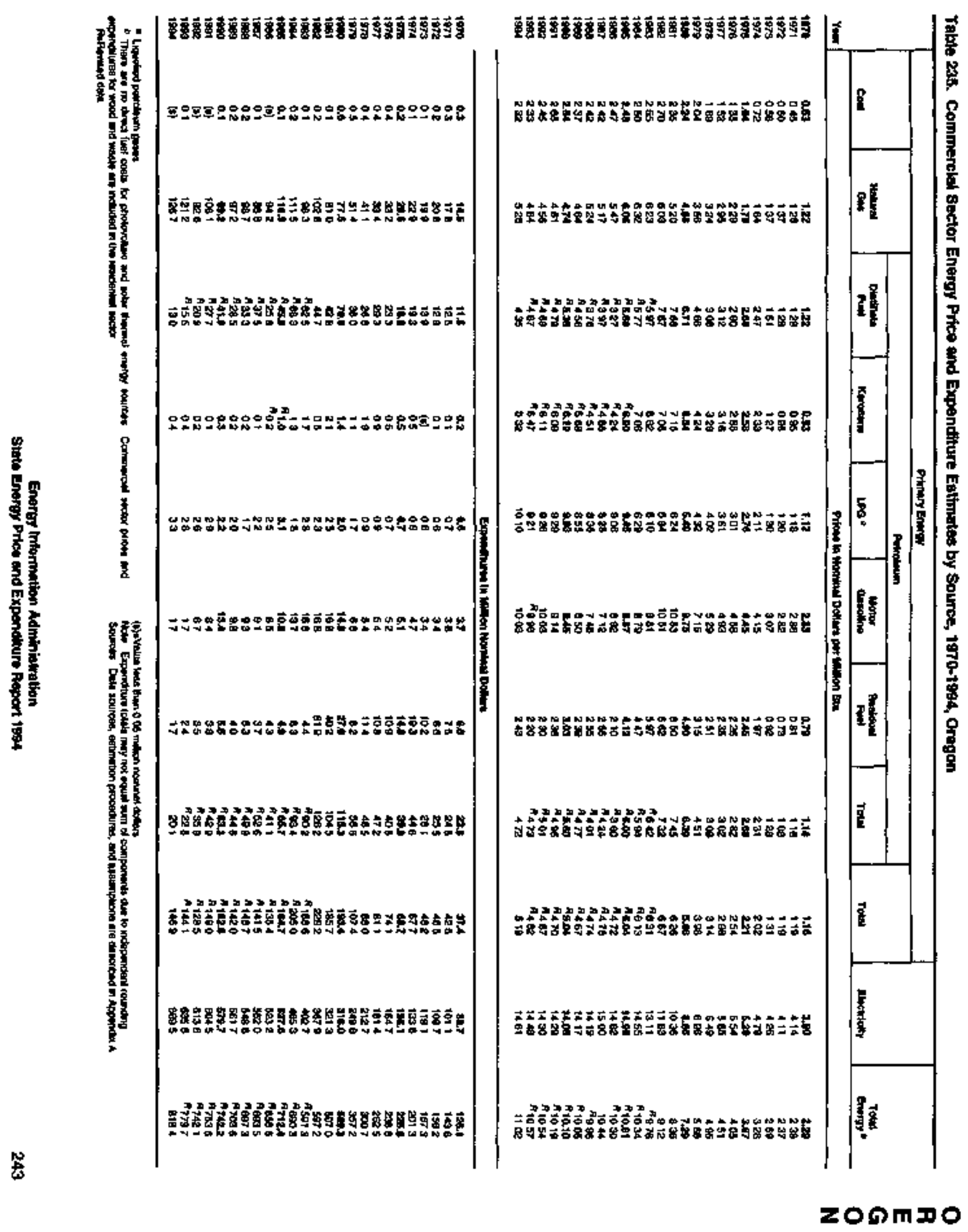




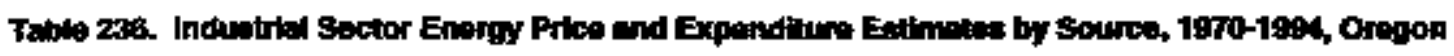

\begin{tabular}{|c|c|c|c|c|c|c|c|c|c|c|c|c|c|c|c|c|c|}
\hline & \multicolumn{15}{|c|}{ 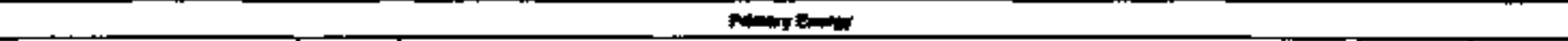 } & \multirow[b]{3}{*}{ E } & \multirow[b]{3}{*}{ " } \\
\hline & \multicolumn{3}{|c|}{ en } & \multirow[b]{2}{*}{ إن } & \multicolumn{9}{|c|}{ 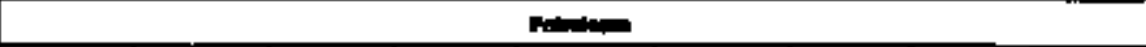 } & \multirow[b]{2}{*}{ 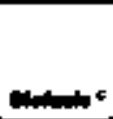 } & \multirow[b]{2}{*}{ Patri } & & \\
\hline & congen & sill & 7 & & S & 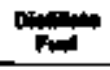 & 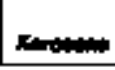 & mas & 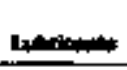 & & Int & onmer & Tet & & & & \\
\hline Yes & \multicolumn{17}{|c|}{ 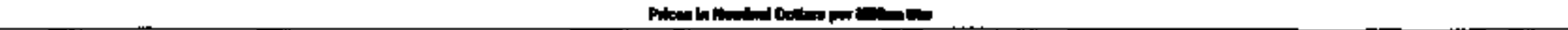 } \\
\hline \multirow[t]{2}{*}{ 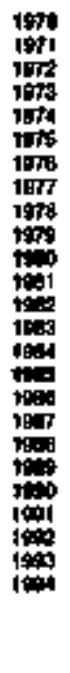 } & $\begin{array}{l}= \\
= \\
= \\
= \\
= \\
= \\
= \\
= \\
= \\
= \\
= \\
= \\
=\end{array}$ & 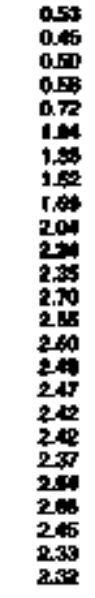 & 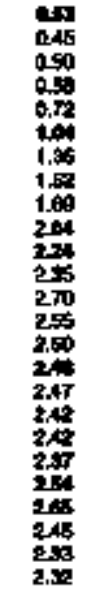 & 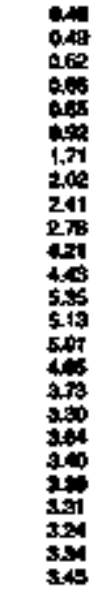 & 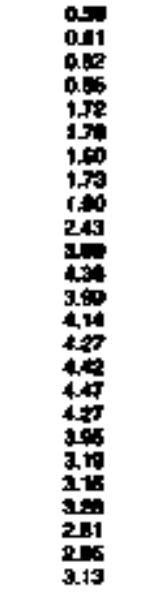 & 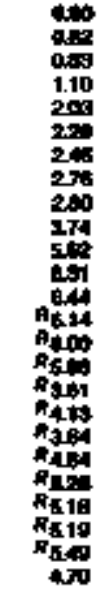 & 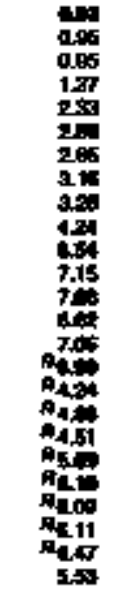 & 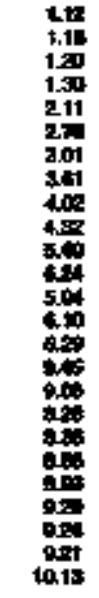 & 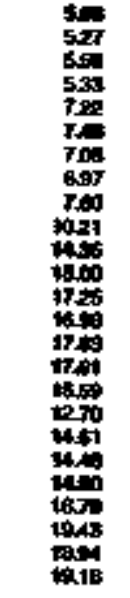 & 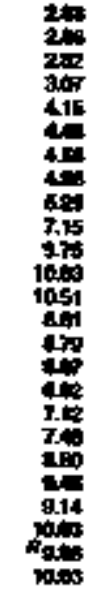 & 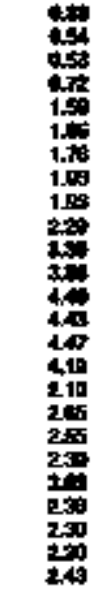 & 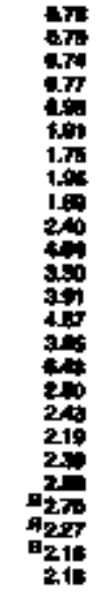 & 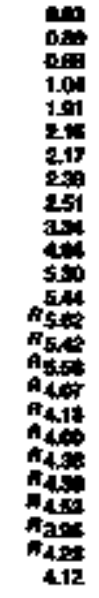 & 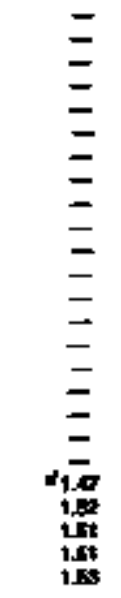 & 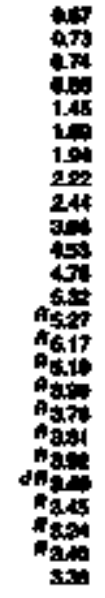 & 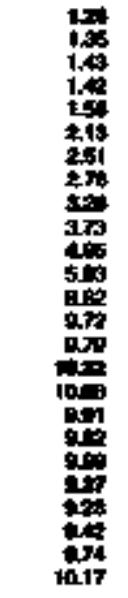 & 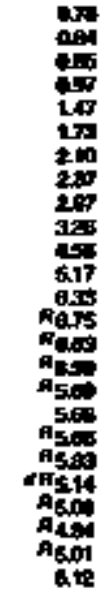 \\
\hline & \multicolumn{17}{|c|}{ 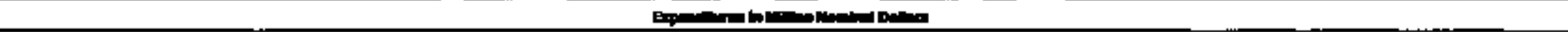 } \\
\hline 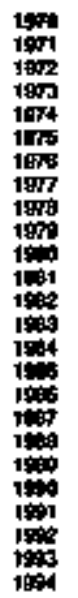 & $\begin{array}{l}= \\
= \\
= \\
= \\
= \\
= \\
= \\
= \\
= \\
= \\
= \\
= \\
= \\
=\end{array}$ & 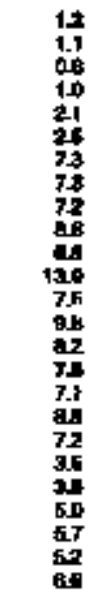 & 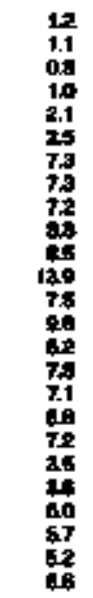 & 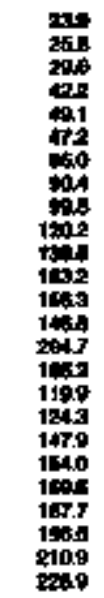 & 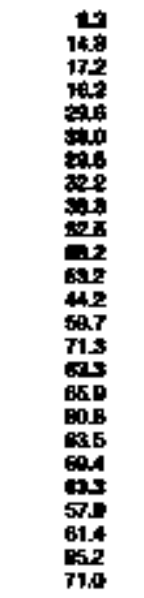 & 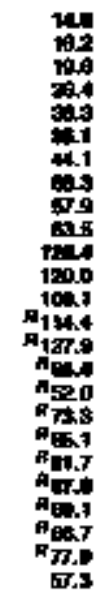 & 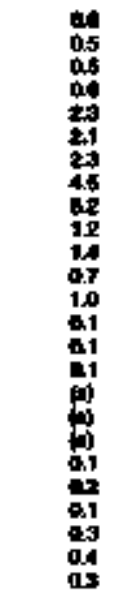 & 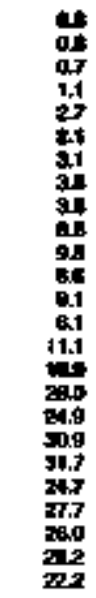 & 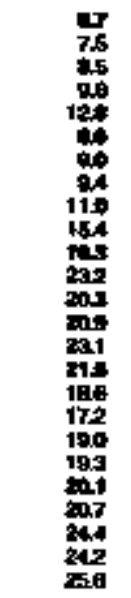 & 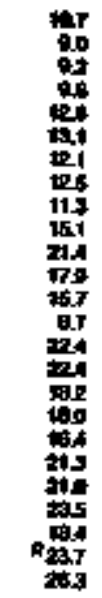 & 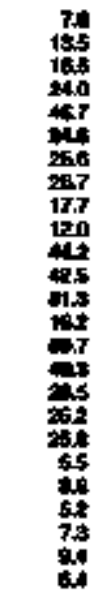 & 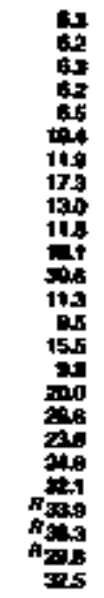 & 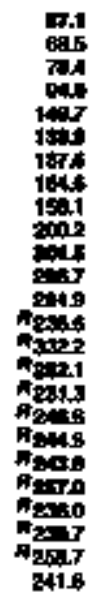 & 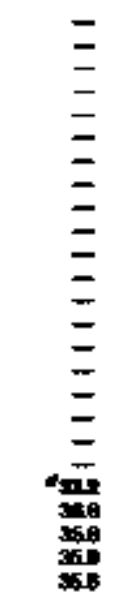 & 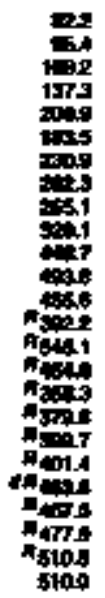 & 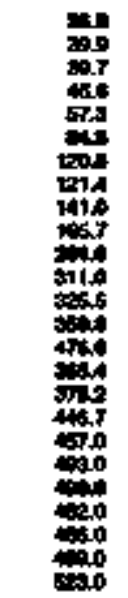 & 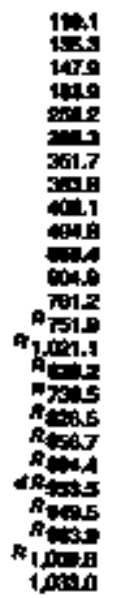 \\
\hline
\end{tabular}

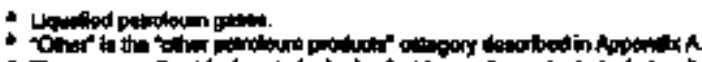

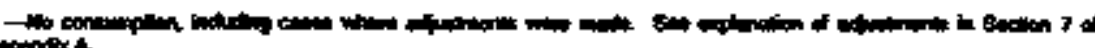

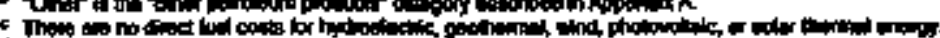

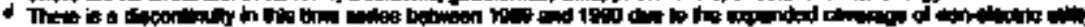

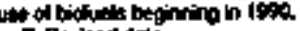

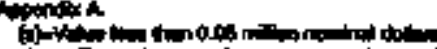

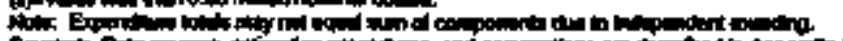

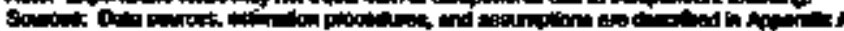




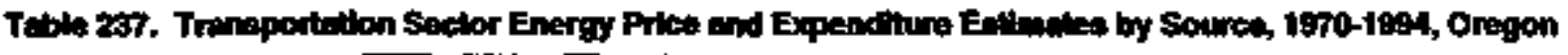

\begin{tabular}{|c|c|c|c|c|c|c|c|c|c|c|c|c|c|}
\hline \multirow[b]{4}{*}{$n=$} & \multicolumn{11}{|c|}{ Plintry } & \multirow[b]{3}{*}{ Foctichly } & \multirow[b]{3}{*}{ Tongry } \\
\hline & \multirow[b]{2}{*}{$\infty$} & \multirow[b]{2}{*}{ 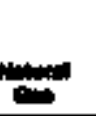 } & \multicolumn{8}{|c|}{ ה } & \multirow[b]{2}{*}{ Toted } & & \\
\hline & & & היומה & and & D. & soo & undenente & 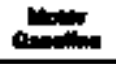 & Find & Ton & & & \\
\hline & \multicolumn{13}{|c|}{ 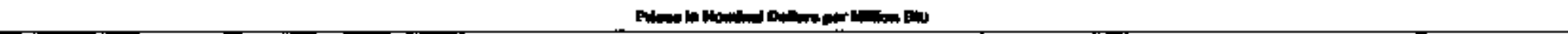 } \\
\hline \multirow[t]{2}{*}{ 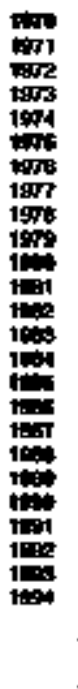 } & 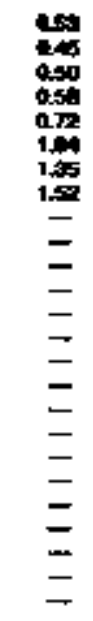 & $\begin{array}{l}= \\
= \\
= \\
= \\
= \\
= \\
= \\
= \\
= \\
= \\
= \\
20\end{array}$ & 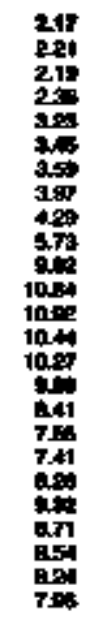 & 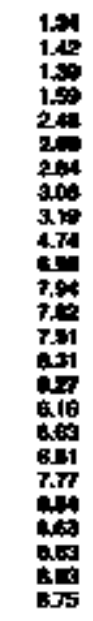 & 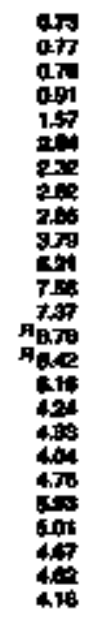 & 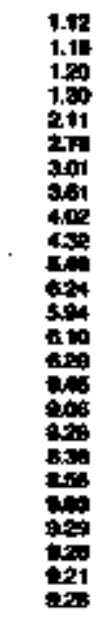 & 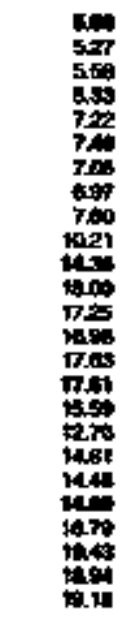 & 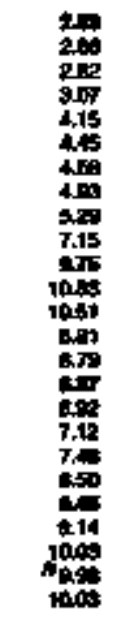 & 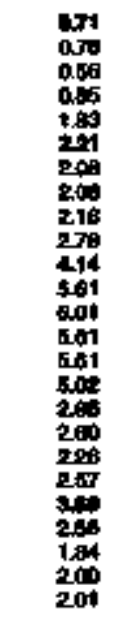 & 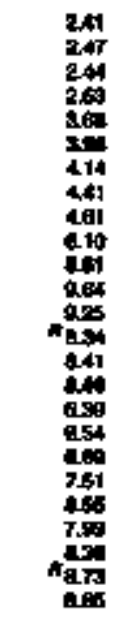 & 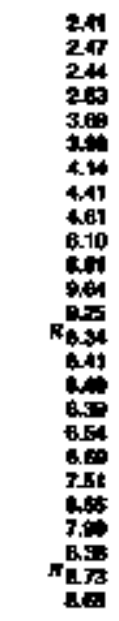 & 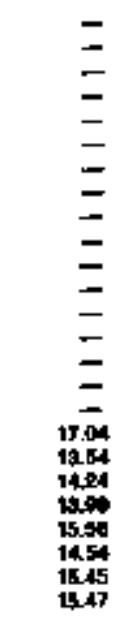 & 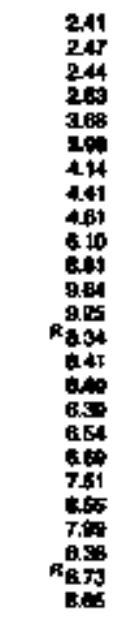 \\
\hline & \multicolumn{13}{|c|}{ 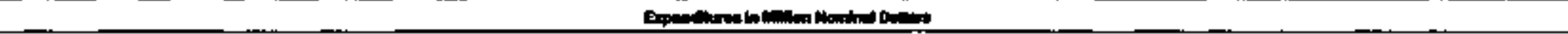 } \\
\hline 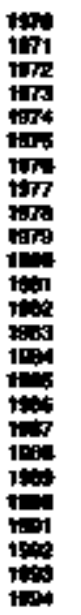 & 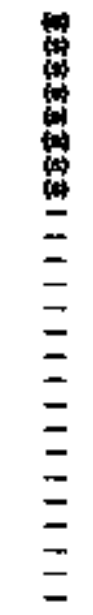 & $\begin{array}{l}\vec{z} \\
= \\
= \\
= \\
= \\
= \\
= \\
= \\
= \\
= \\
= \\
0 \\
0\end{array}$ & 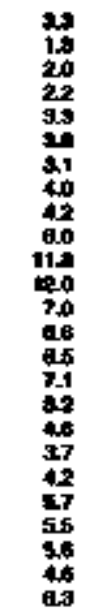 & 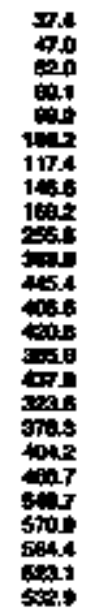 & 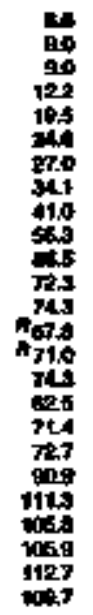 & 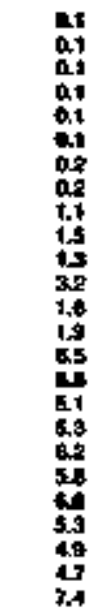 & 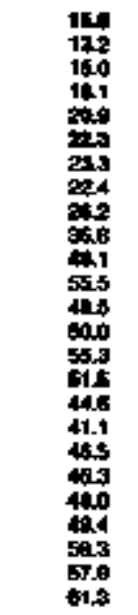 & 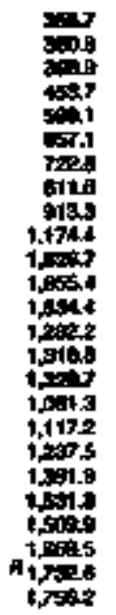 & 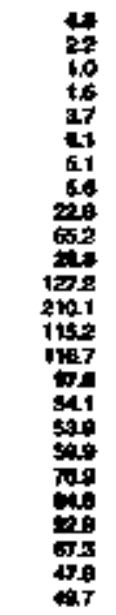 & 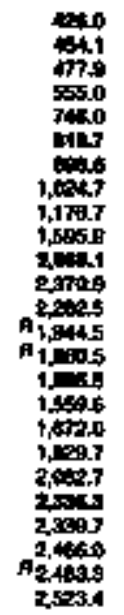 & 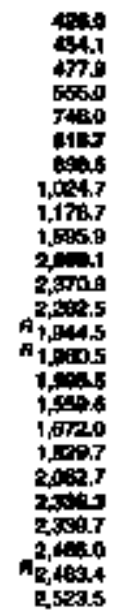 & $\begin{array}{l}= \\
= \\
= \\
= \\
= \\
= \\
= \\
= \\
= \\
= \\
= \\
0.4 \\
0.4 \\
0.4 \\
0.4 \\
0.5 \\
0.4 \\
0.4 \\
0.4\end{array}$ & 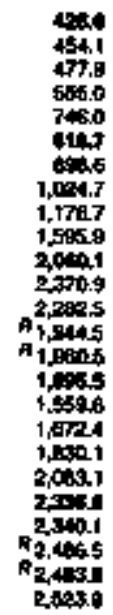 \\
\hline
\end{tabular}

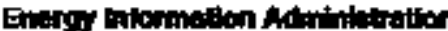


O Table 239. Price and Expenditure Estimates for Energy Input at Eleotric Utiltises by source, 1970-1994, Oregon

\begin{tabular}{|c|c|c|c|c|c|c|c|c|c|}
\hline \multirow[b]{3}{*}{ Yw: } & \multirow[b]{2}{*}{$\cos$} & \multirow[b]{2}{*}{ ind } & \multicolumn{4}{|c|}{ Datrolanth } & \multirow[b]{2}{*}{ Munele } & \multirow[b]{2}{*}{ cliofinte } & \multirow[b]{2}{*}{ Tow } \\
\hline & & & ons & 湈 & 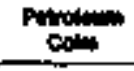 & Tolat & & & \\
\hline & \multicolumn{9}{|c|}{ 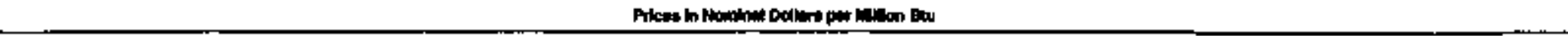 } \\
\hline 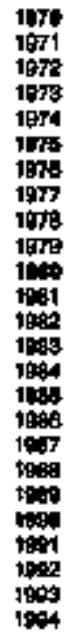 & 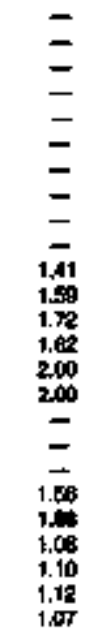 & 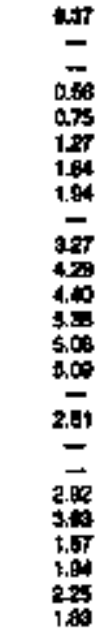 & $\begin{array}{l}0.0 \\
= \\
0.001 \\
=01 \\
= \\
= \\
= \\
= \\
= \\
= \\
= \\
= \\
= \\
= \\
=\end{array}$ & 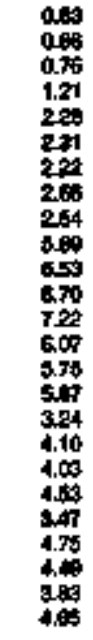 & $\begin{array}{l}= \\
= \\
= \\
= \\
= \\
= \\
= \\
= \\
= \\
= \\
z \\
= \\
=\end{array}$ & 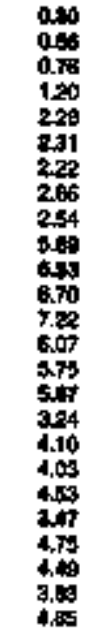 & 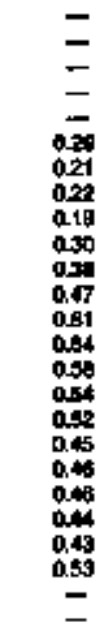 & 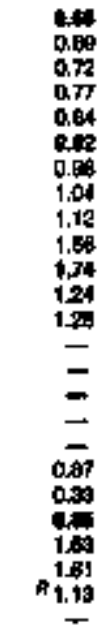 & 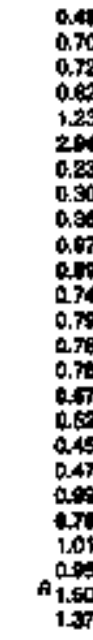 \\
\hline & \multicolumn{9}{|c|}{ 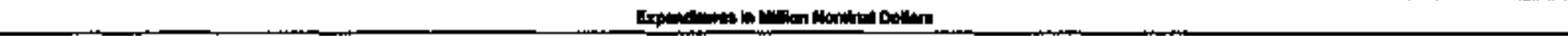 } \\
\hline 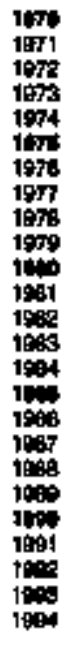 & 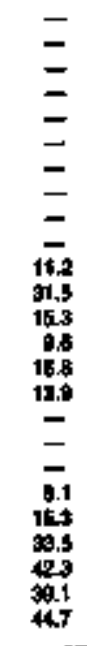 & 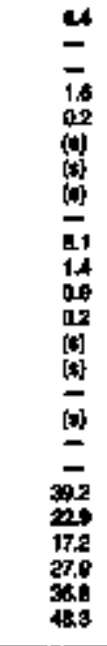 & $\begin{array}{l}0.1 \\
= \\
{[0]} \\
= \\
= \\
= \\
= \\
= \\
= \\
= \\
= \\
= \\
= \\
= \\
= \\
=\end{array}$ & 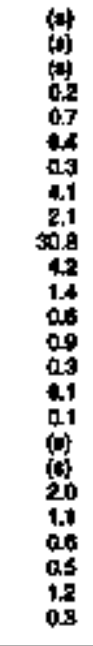 & $\begin{array}{l}\bar{z} \\
\bar{z} \\
z \\
z \\
z \\
z \\
z \\
z \\
z \\
z \\
z \\
z \\
z \\
z\end{array}$ & 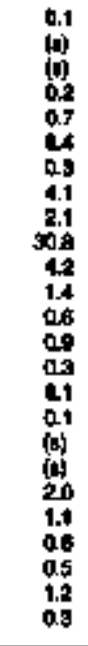 & 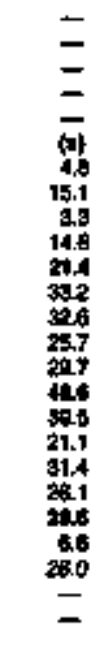 & 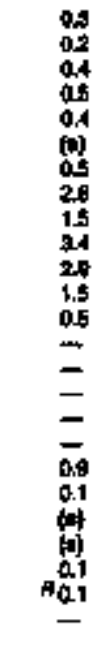 & 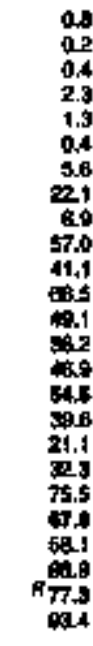 \\
\hline
\end{tabular}

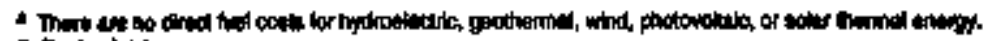

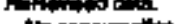

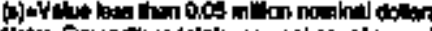


Jable 239. Enargy Price and Expondilure Estimales by 9ource, 1970-1994, Pannsylumbla

\begin{tabular}{|c|c|c|c|c|c|c|c|c|c|c|c|c|c|c|c|c|c|}
\hline & \multicolumn{14}{|c|}{ Prowerenty } & \multirow{3}{*}{ Fing } & \multirow{3}{*}{ Enstrity } & \multirow[b]{3}{*}{ Tated } \\
\hline & \multicolumn{3}{|c|}{ Com } & \multirow[b]{2}{*}{$\lim _{n=1}$} & \multicolumn{7}{|c|}{ Atroing } & \multirow[b]{2}{*}{ Munew } & \multirow[b]{2}{*}{ Enownts } & \multirow[b]{2}{*}{ Tatos } & & & \\
\hline & Conter & siman & Ton & & For & fort & LPQ: & Catorim & mestots & ather & Totw & & & & & & \\
\hline$v_{4}=$ & \multicolumn{17}{|c|}{ 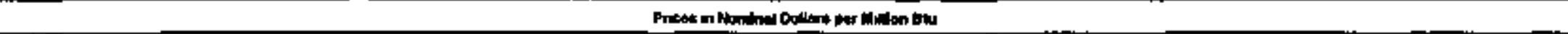 } \\
\hline \multirow[t]{2}{*}{ 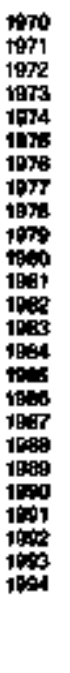 } & 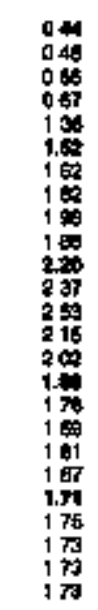 & 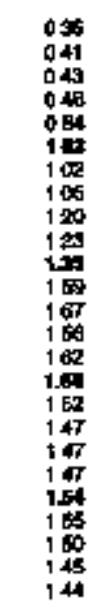 & 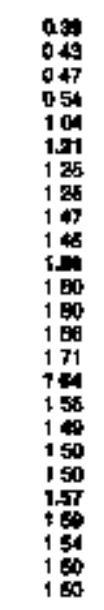 & 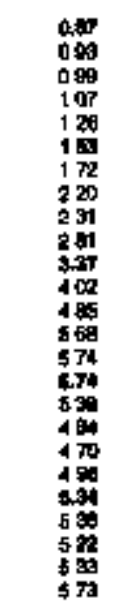 & 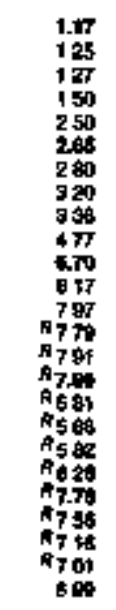 & 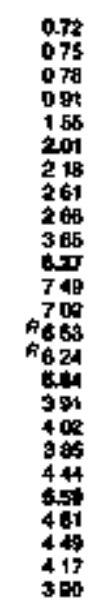 & 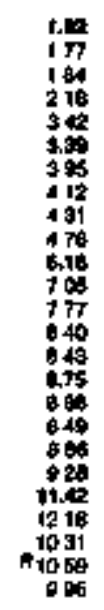 & 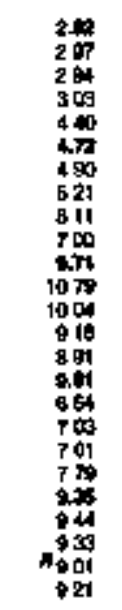 & 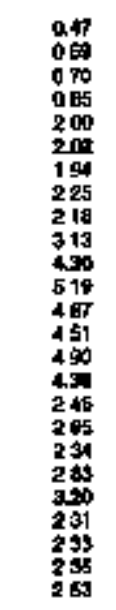 & 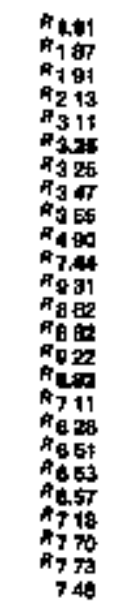 & 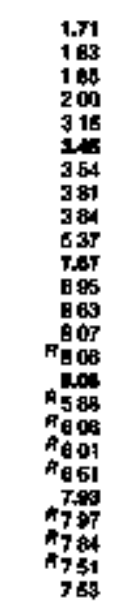 & 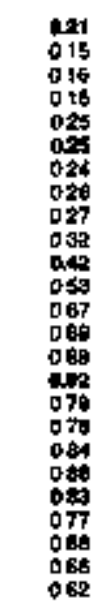 & 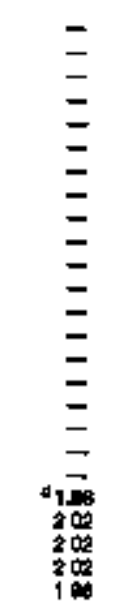 & 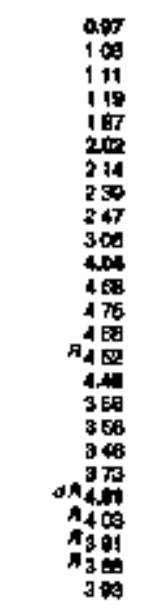 & 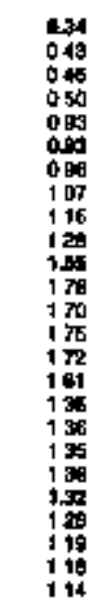 & 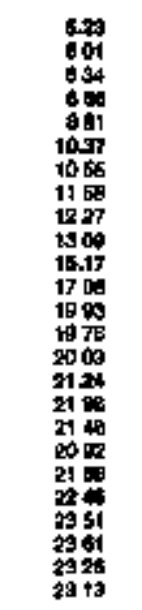 & 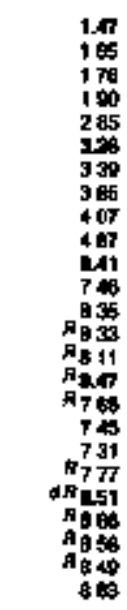 \\
\hline & \multicolumn{17}{|c|}{ 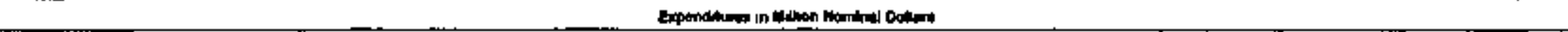 } \\
\hline 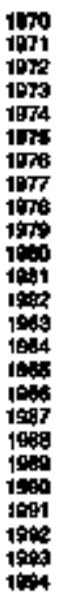 & 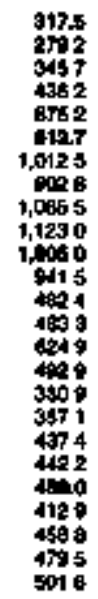 & 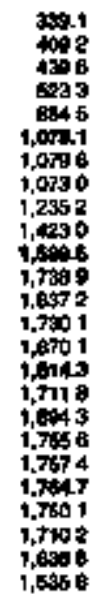 & 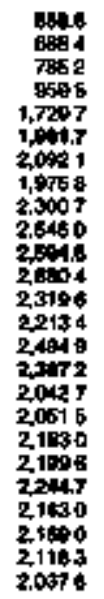 & 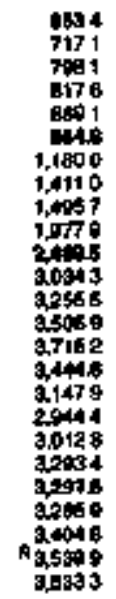 & 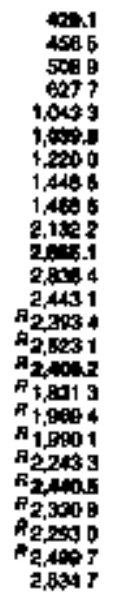 & 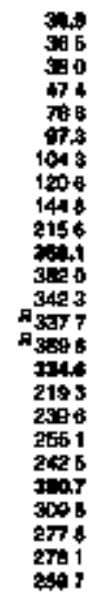 & 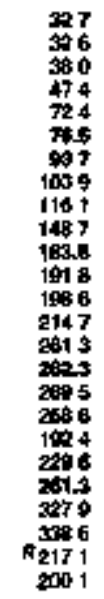 & 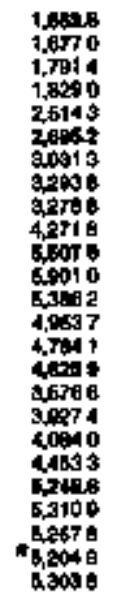 & 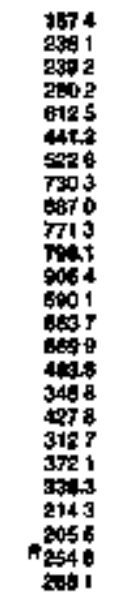 & 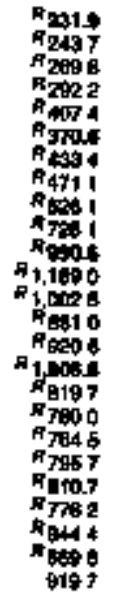 & 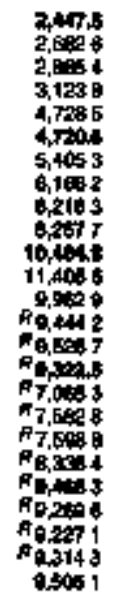 & 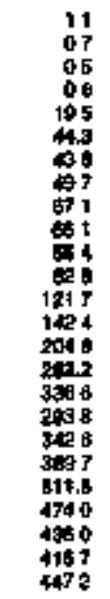 & $\begin{array}{l}= \\
= \\
= \\
= \\
= \\
= \\
= \\
= \\
= \\
= \\
= \\
= \\
619 \\
676 \\
071 \\
714 \\
725\end{array}$ & 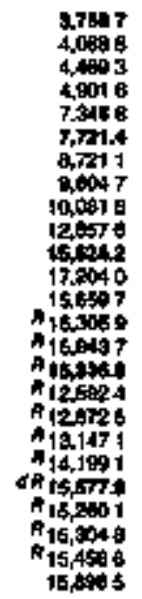 & 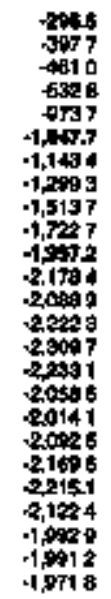 & 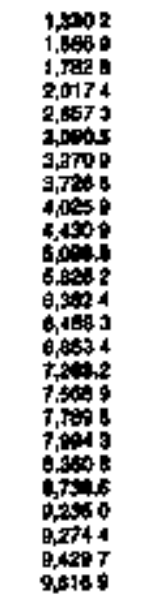 & 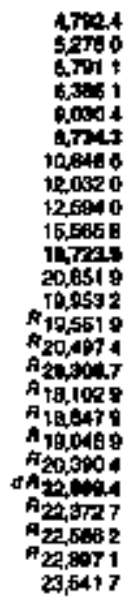 \\
\hline
\end{tabular}

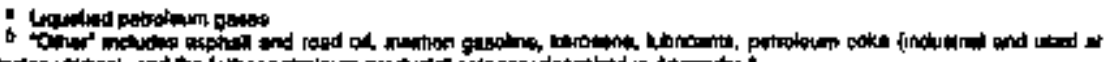

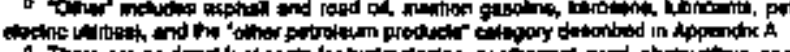

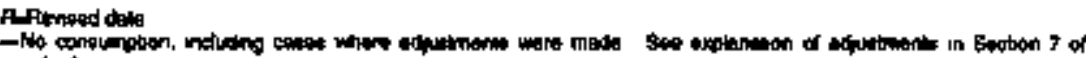

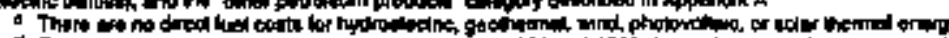
Apponder A

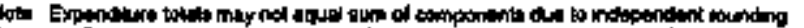

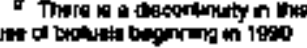




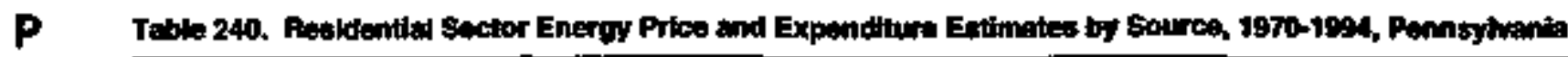

\begin{tabular}{|c|c|c|c|c|c|c|c|c|c|c|}
\hline \multirow[b]{4}{*}{$x=$} & \multicolumn{8}{|c|}{ mopenty } & \multirow[b]{3}{*}{ Enotidy } & \multirow[b]{3}{*}{. } \\
\hline & \multirow[b]{2}{*}{ - } & \multirow[b]{2}{*}{ " } & \multicolumn{4}{|c|}{ Pexinne } & \multirow[b]{2}{*}{ 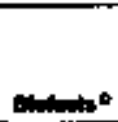 } & \multirow[b]{2}{*}{ nowe } & & \\
\hline & & & "ורוn: & $x=$ & $50 *$ & Toto & & & & \\
\hline & \multicolumn{10}{|c|}{ 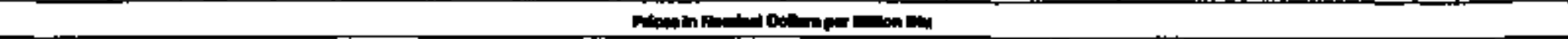 } \\
\hline \multirow[t]{2}{*}{ 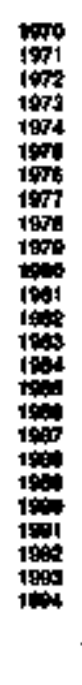 } & 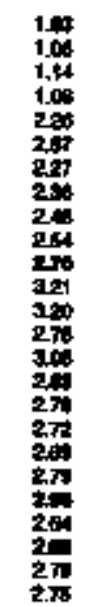 & 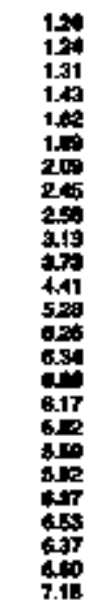 & 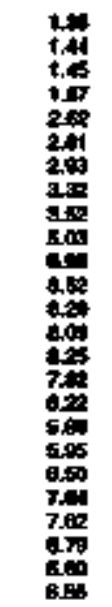 & 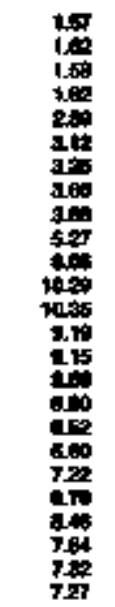 & 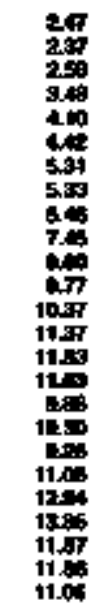 & 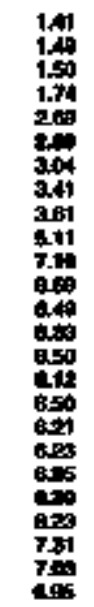 & $\begin{array}{l}\bar{z} \\
\bar{z} \\
z \\
z \\
\bar{z} \\
\bar{z} \\
\bar{z} \\
\bar{z} \\
\bar{z} \\
\bar{a} \\
200 \\
200 \\
200 \\
200\end{array}$ & 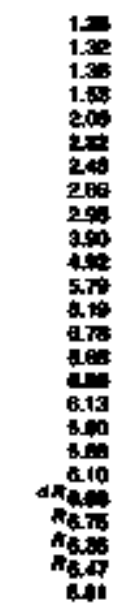 & 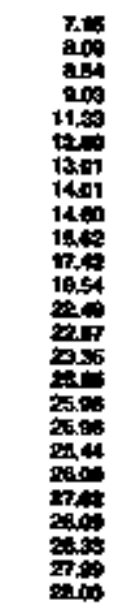 & 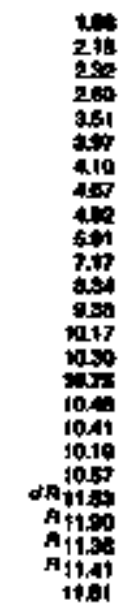 \\
\hline & \multicolumn{10}{|c|}{ ST } \\
\hline 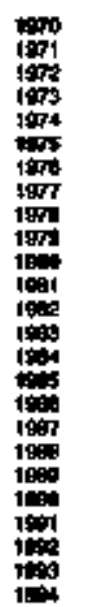 & 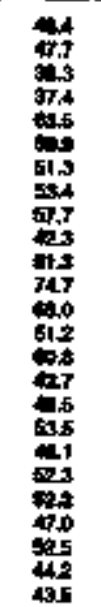 & 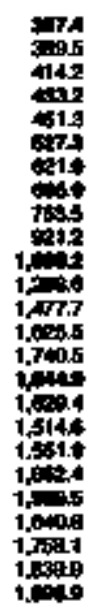 & 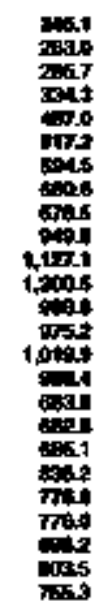 & 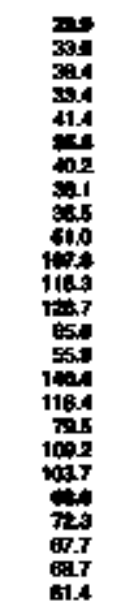 & 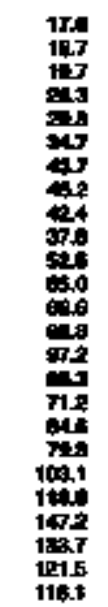 & 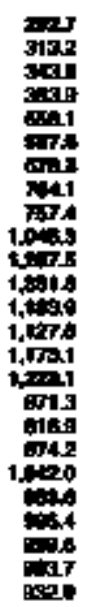 & 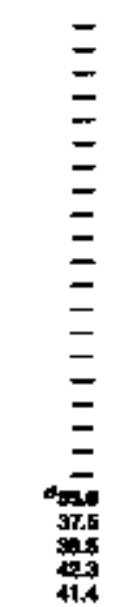 & 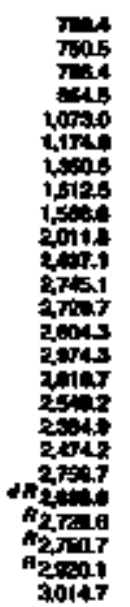 & 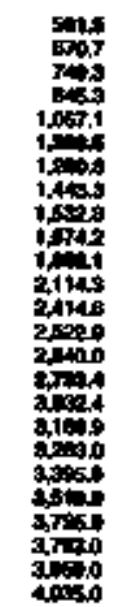 & 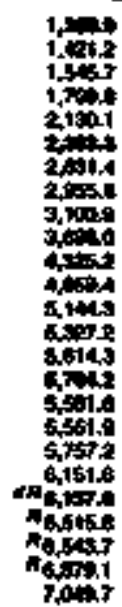 \\
\hline
\end{tabular}

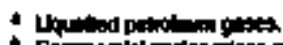

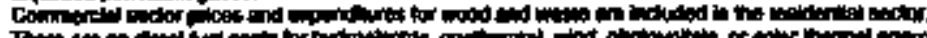

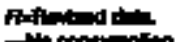

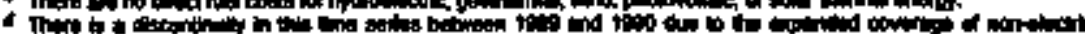

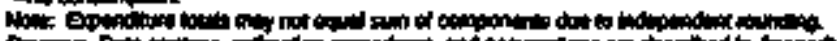

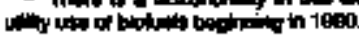




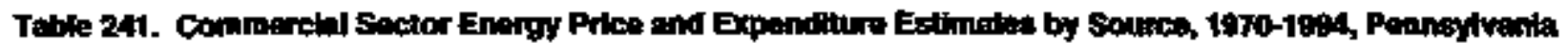

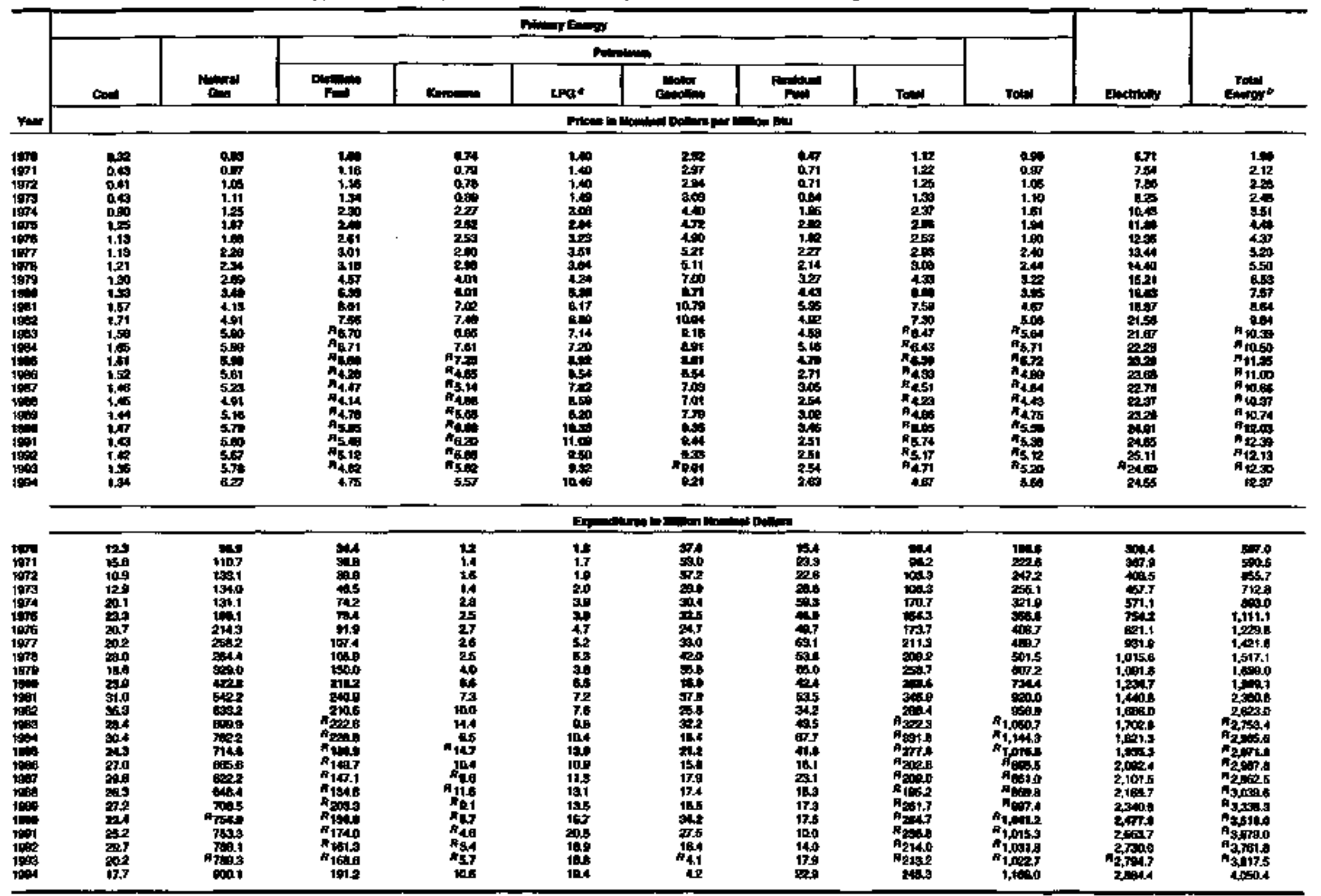




\begin{tabular}{|c|c|c|c|c|c|c|c|c|c|c|c|c|c|c|c|c|c|}
\hline \multirow[b]{4}{*}{ ] } & \multicolumn{15}{|c|}{ Primery teneror } & \multirow[b]{3}{*}{ Antonewy } & \multirow[b]{3}{*}{ Toted } \\
\hline & \multicolumn{3}{|c|}{$\operatorname{cod}$} & \multirow[b]{2}{*}{ menem } & \multicolumn{9}{|c|}{ Patrotom } & \multirow[b]{2}{*}{ Sidumen" } & \multirow[b]{2}{*}{$\operatorname{ros} 0$} & & \\
\hline & 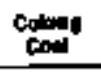 & Stom & Toled & & Mand and and & 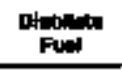 & Kenosens & LSG: & 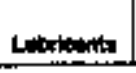 & mosede & Anedert & Othen & Tow & & & & \\
\hline & \multicolumn{17}{|c|}{ 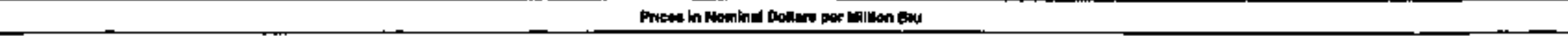 } \\
\hline \multirow[t]{2}{*}{ 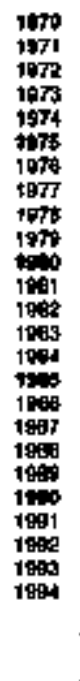 } & 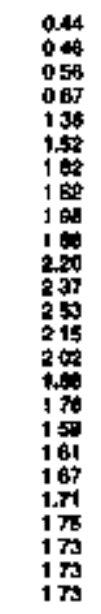 & 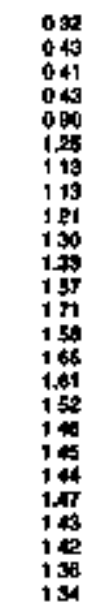 & 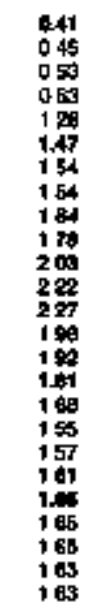 & 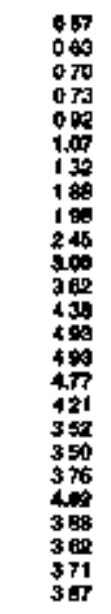 & 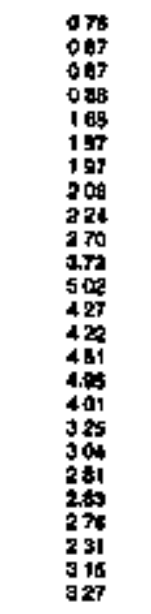 & 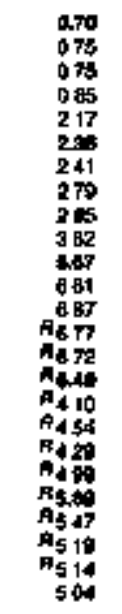 & 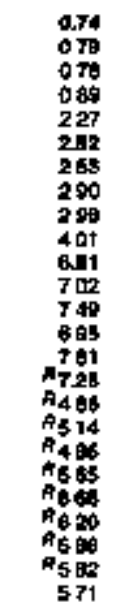 & 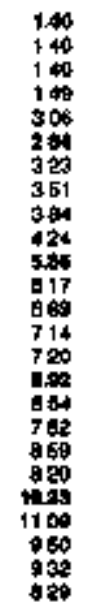 & 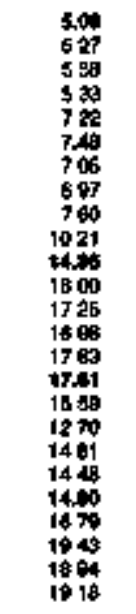 & 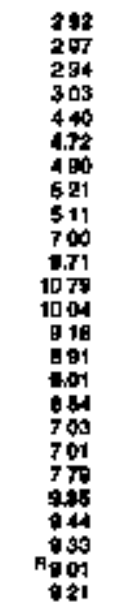 & 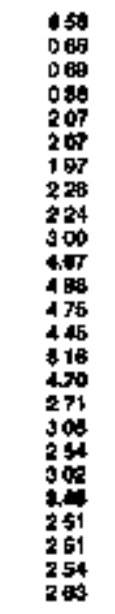 & 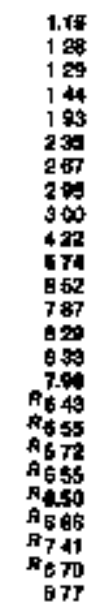 & 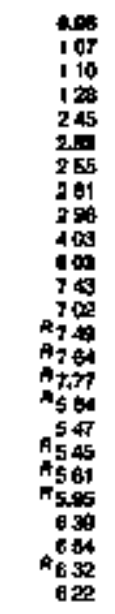 & 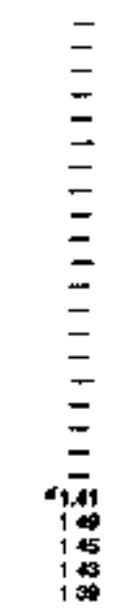 & 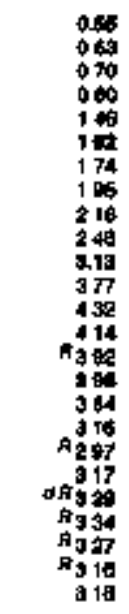 & 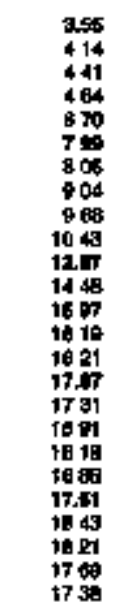 & 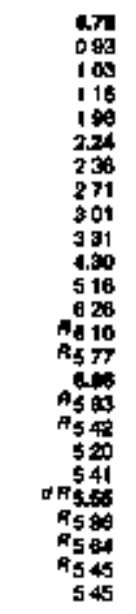 \\
\hline & \multicolumn{17}{|c|}{ 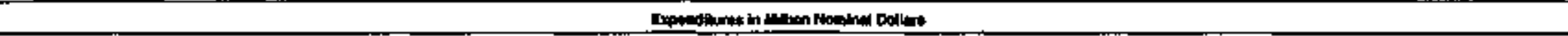 } \\
\hline 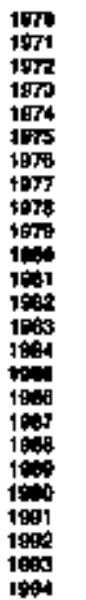 & 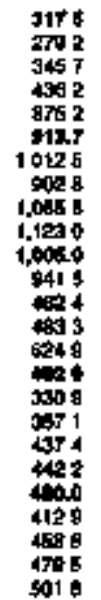 & 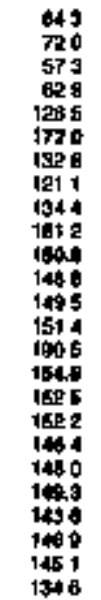 & 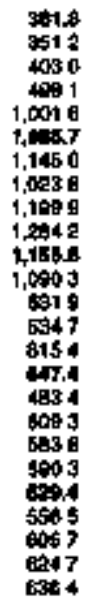 & 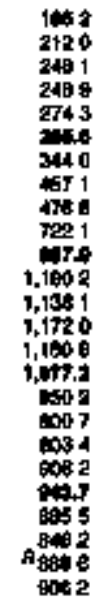 & 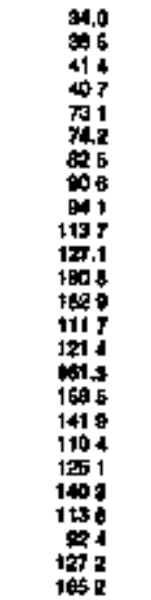 & 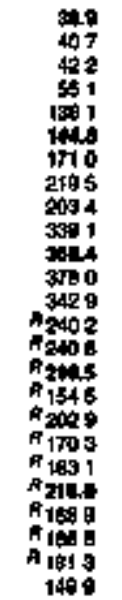 & 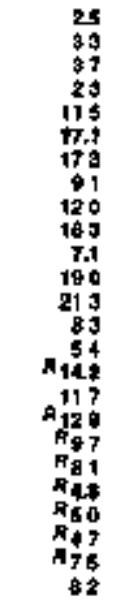 & 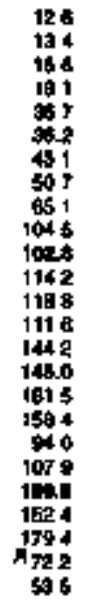 & 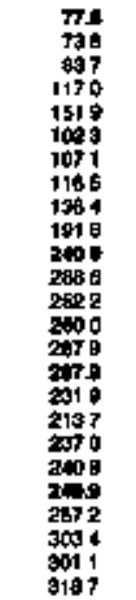 & 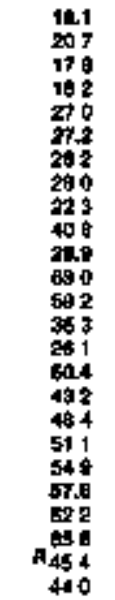 & 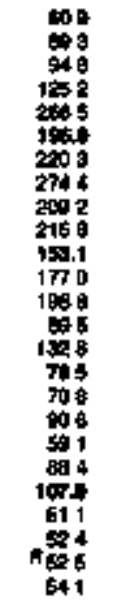 & 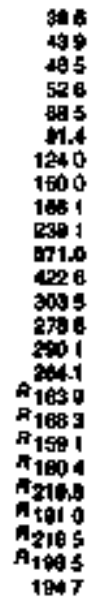 & 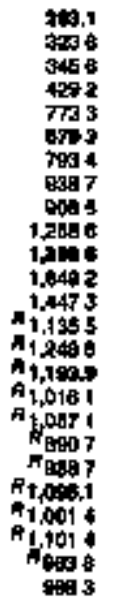 & 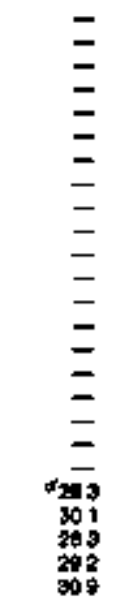 & 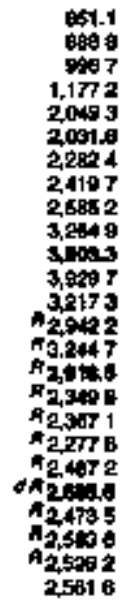 & 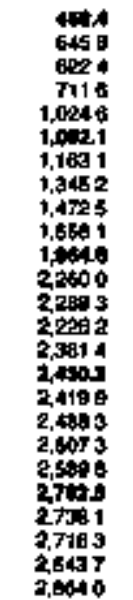 & 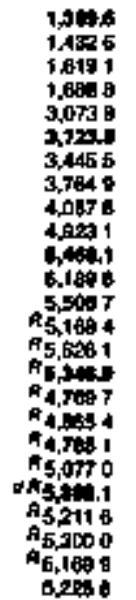 \\
\hline
\end{tabular}



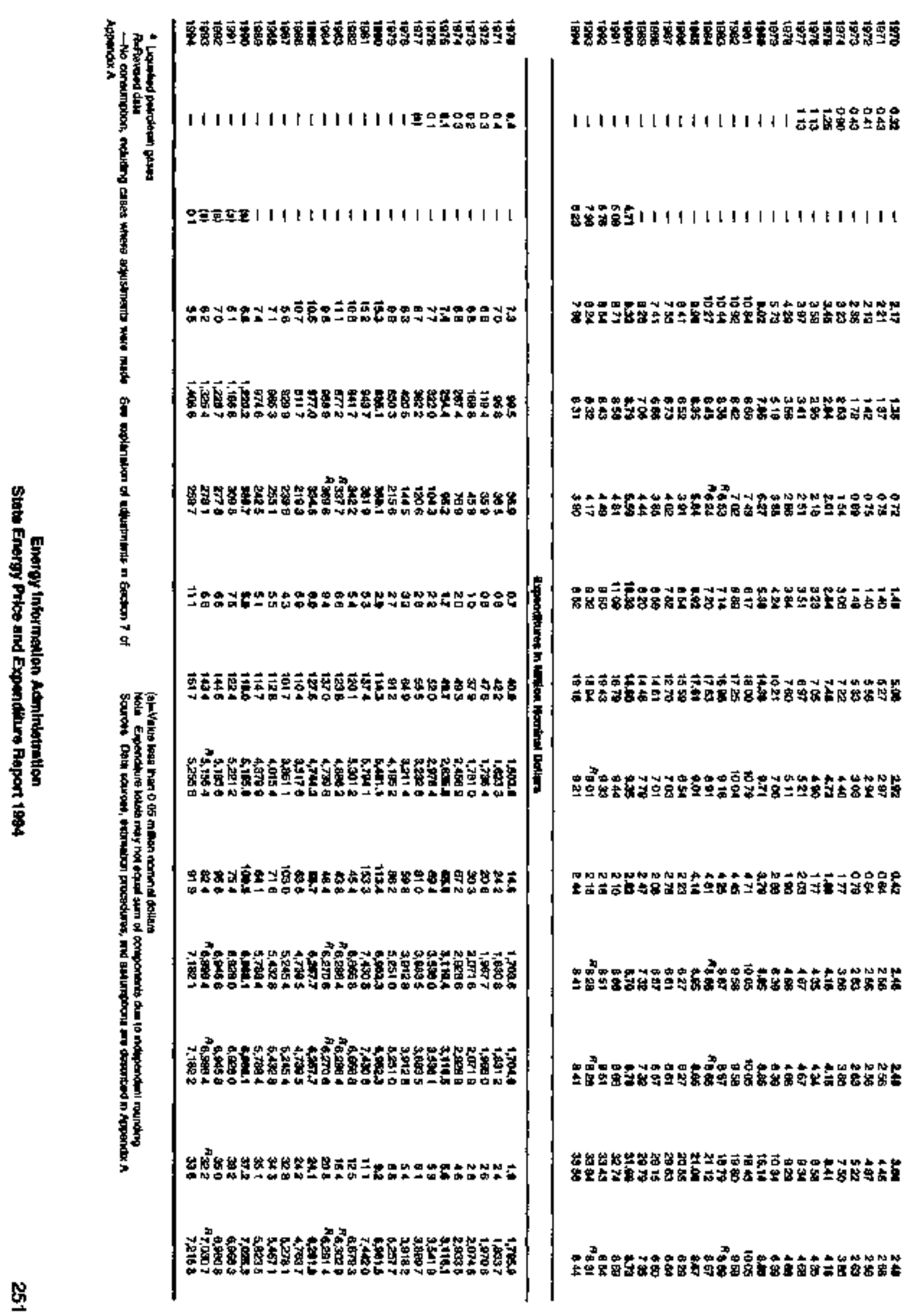

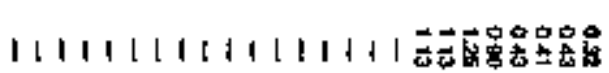

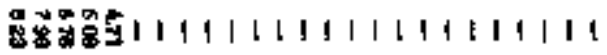

ข

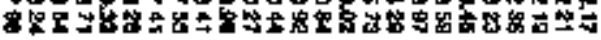

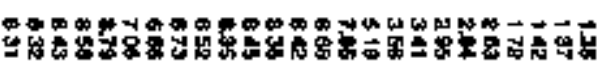

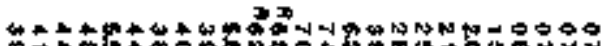

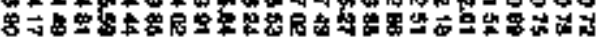

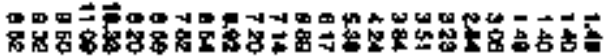

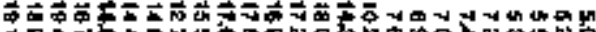

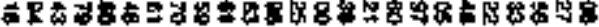

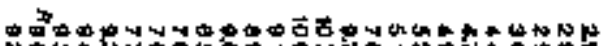

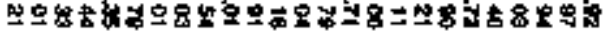

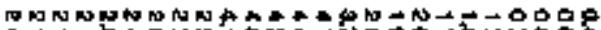

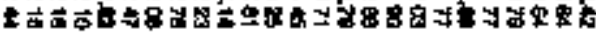

由

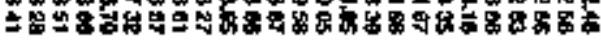

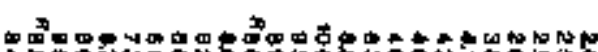

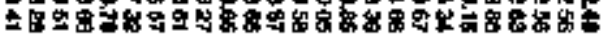

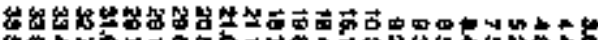

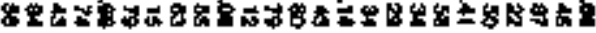

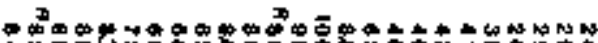

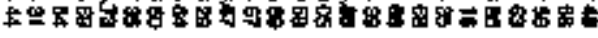

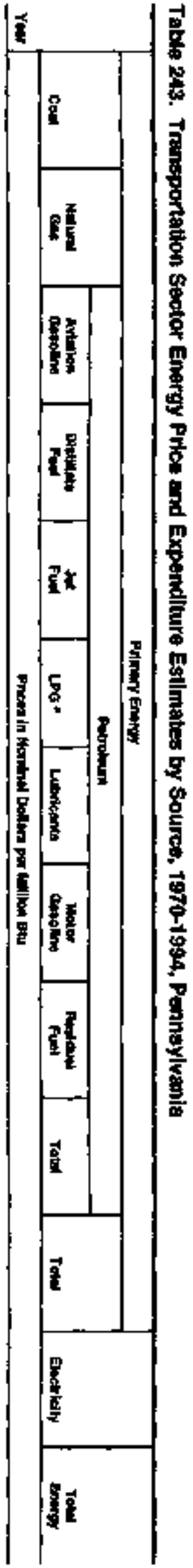




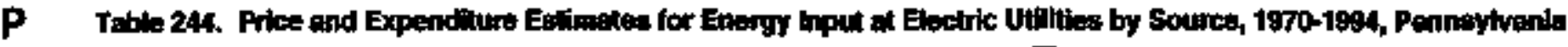

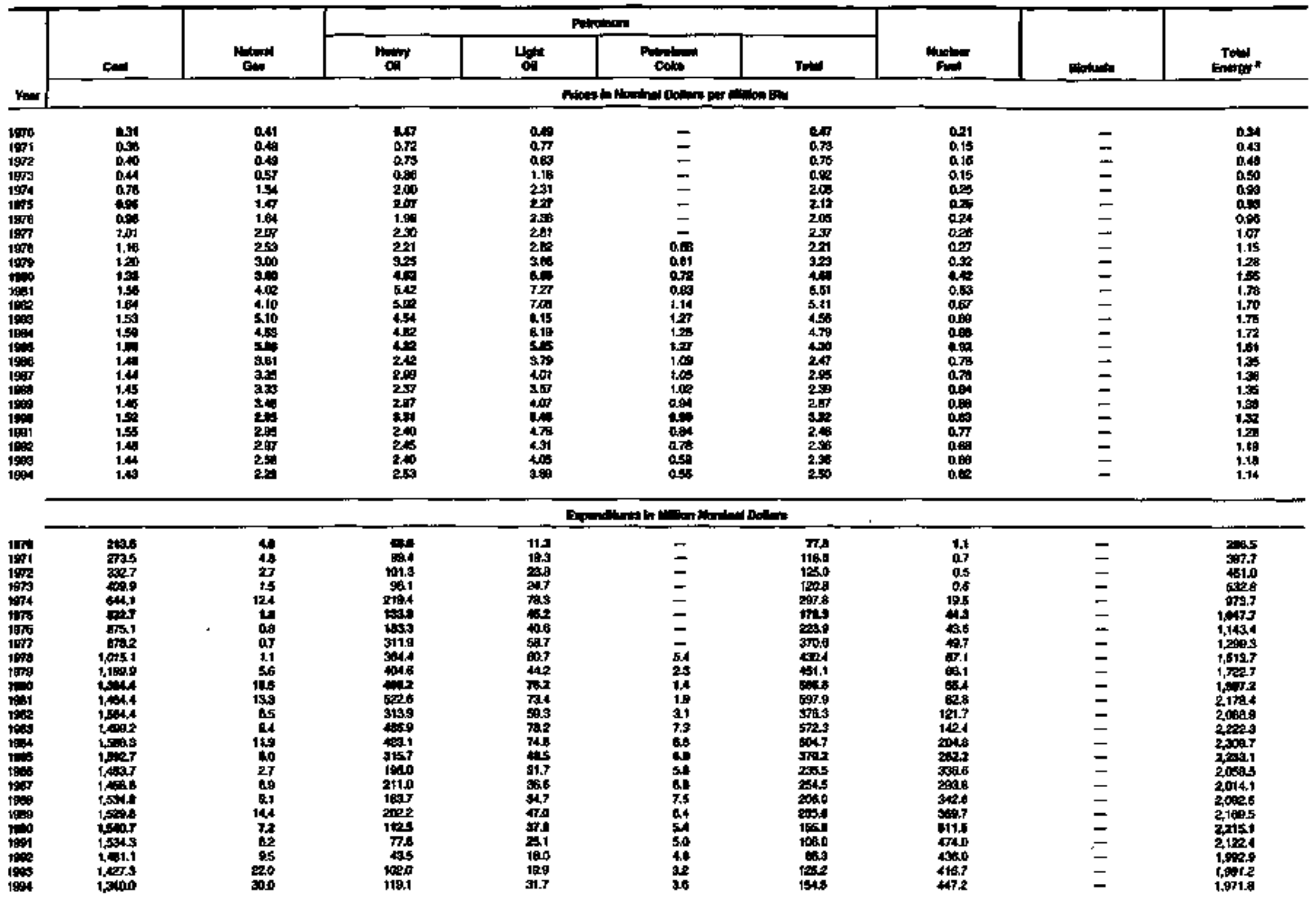




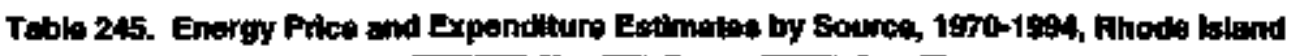

\begin{tabular}{|c|c|c|c|c|c|c|c|c|c|c|c|c|c|c|c|c|c|}
\hline & \multicolumn{14}{|c|}{ Atnor Eners } & \multirow{3}{*}{ מש: } & \multirow{3}{*}{ 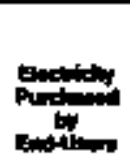 } & \multirow[b]{3}{*}{ TH: } \\
\hline & \multicolumn{3}{|c|}{ (1) } & \multirow[b]{2}{*}{ "dint } & \multicolumn{7}{|c|}{ Fotnolen } & \multirow[b]{2}{*}{ 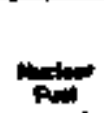 } & \multirow[b]{2}{*}{ 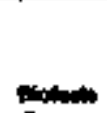 } & \multirow[b]{2}{*}{ TE: } & & & \\
\hline & קה & 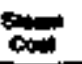 & Totol & & 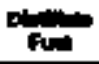 & fot & Ipa* & 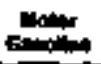 & 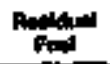 & and & Tw & & & & & & \\
\hline $\mathrm{Yw}$ & \multicolumn{17}{|c|}{ 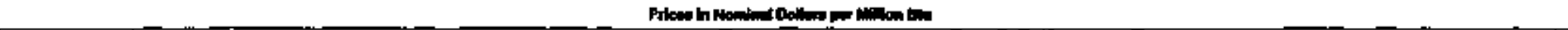 } \\
\hline \multirow[t]{2}{*}{ 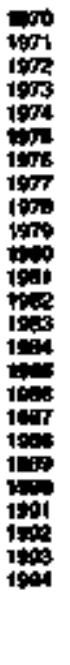 } & $\begin{array}{l}= \\
= \\
= \\
= \\
= \\
= \\
= \\
= \\
= \\
= \\
= \\
= \\
=\end{array}$ & 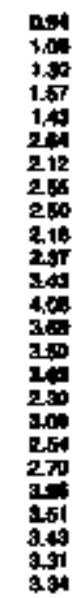 & 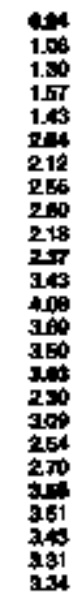 & 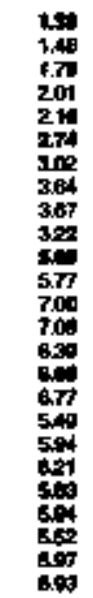 & 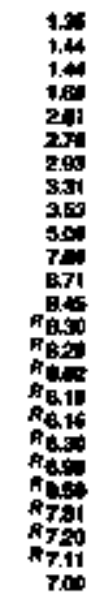 & 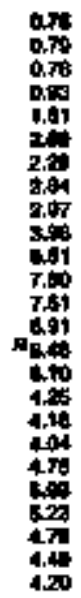 & 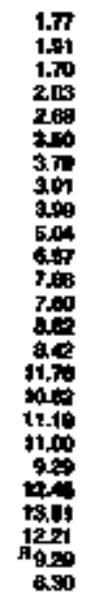 & 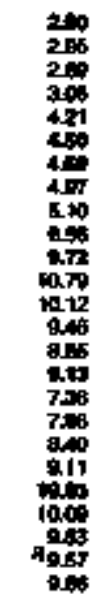 & 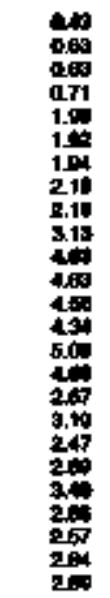 & 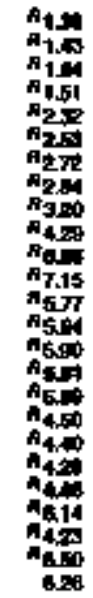 & 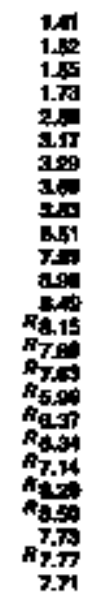 & $\begin{array}{l}= \\
= \\
= \\
= \\
= \\
= \\
= \\
= \\
= \\
= \\
= \\
= \\
=\end{array}$ & $\begin{array}{l}= \\
= \\
z \\
z \\
= \\
= \\
= \\
= \\
= \\
= \\
= \\
= \\
24 \\
24 \\
24\end{array}$ & 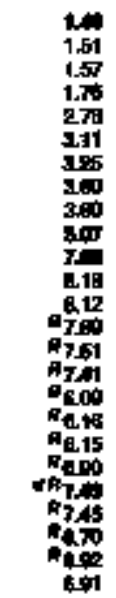 & 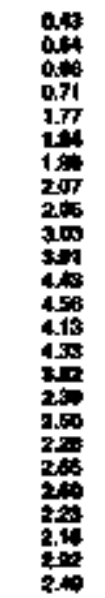 & 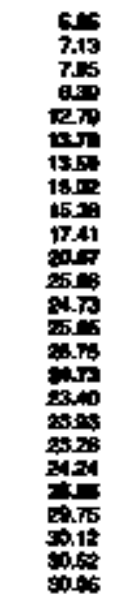 & 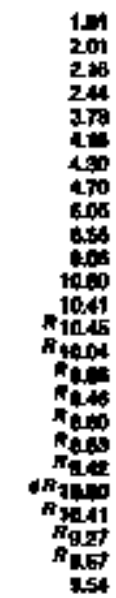 \\
\hline & \multicolumn{17}{|c|}{ 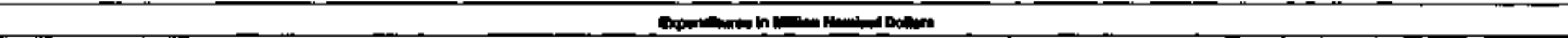 } \\
\hline 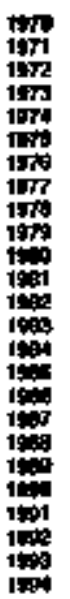 & $\begin{array}{l}= \\
= \\
= \\
= \\
= \\
= \\
= \\
= \\
= \\
= \\
= \\
= \\
=\end{array}$ & 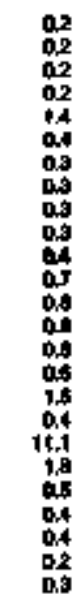 & 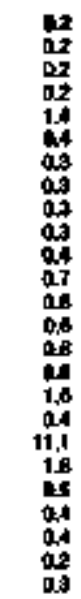 & 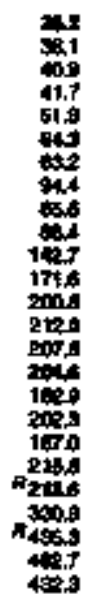 & 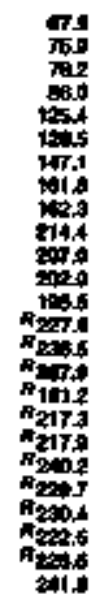 & 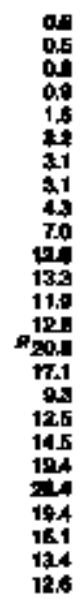 & 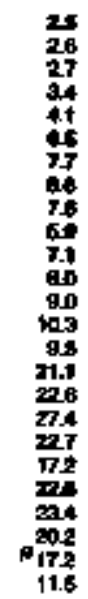 & 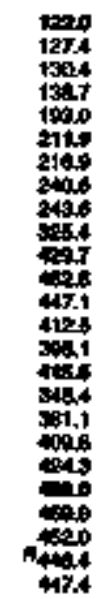 & 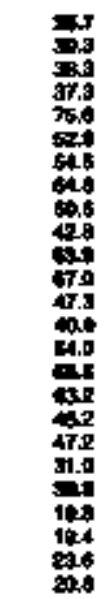 & 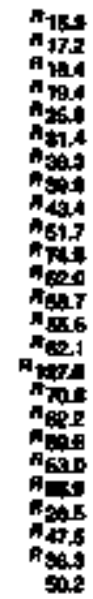 & 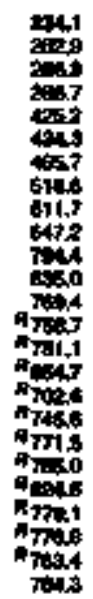 & $\begin{array}{l}= \\
= \\
= \\
= \\
= \\
= \\
= \\
= \\
= \\
= \\
= \\
= \\
=\end{array}$ & $\begin{array}{l}= \\
= \\
= \\
= \\
= \\
= \\
= \\
= \\
= \\
= \\
= \\
40 \\
04 \\
64\end{array}$ & 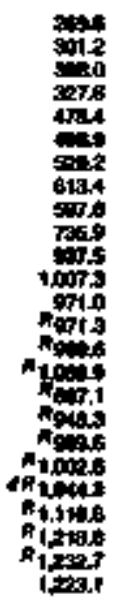 & 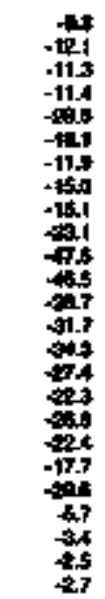 & 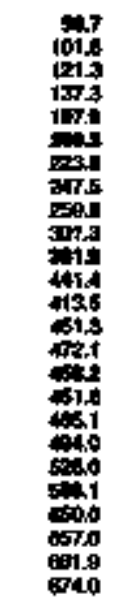 & 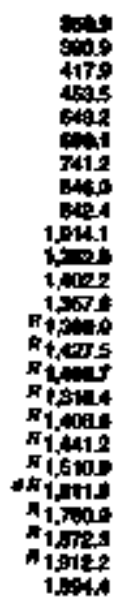 \\
\hline
\end{tabular}

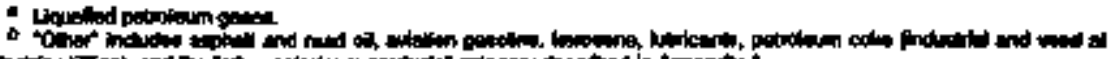

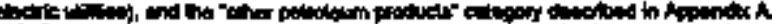

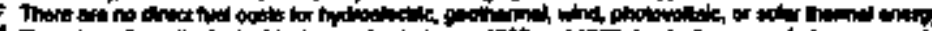

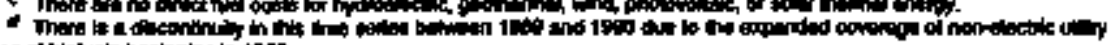

Arfinted dota

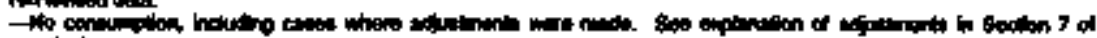

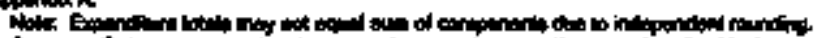

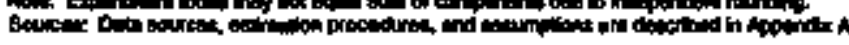

O

D 
Toble 246. Recldential Sector Entrigy Price and Expendlure Eotimates by Source, 1970-1994, Fhodo keland

$\underset{\mathbf{O}}{\mathbf{H}}$

\begin{tabular}{|c|c|c|c|c|c|c|c|c|c|c|}
\hline \multirow[b]{4}{*}{$\mathrm{V}=\mathrm{ur}$} & \multicolumn{8}{|c|}{ Priment Enom } & \multirow[b]{3}{*}{ Eherothy } & \multirow[b]{3}{*}{ 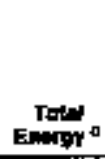 } \\
\hline & & \multirow[b]{2}{*}{ mons } & \multicolumn{4}{|c|}{ Puroinen } & \multirow[b]{2}{*}{ Buabuats o } & \multirow[b]{2}{*}{ Totwe c c } & & \\
\hline & Cont & & 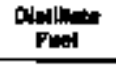 & 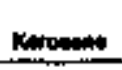 & LAG: & Tow & & & & \\
\hline & \multicolumn{10}{|c|}{ 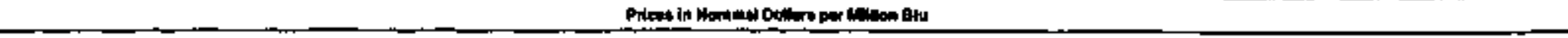 } \\
\hline \multirow[t]{2}{*}{ 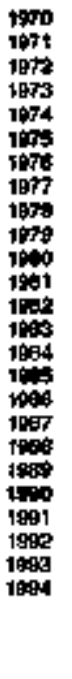 } & 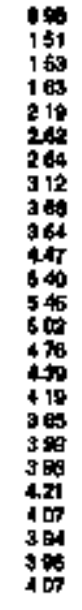 & 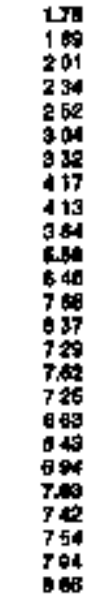 & 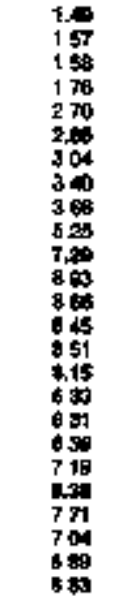 & 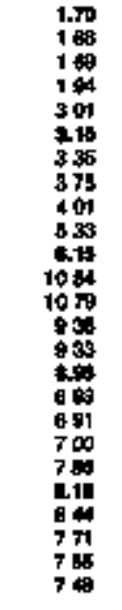 & 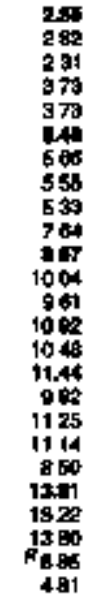 & 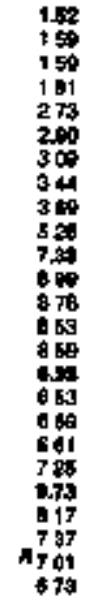 & $\begin{array}{r}= \\
= \\
= \\
= \\
= \\
= \\
= \\
= \\
= \\
= \\
= \\
= \\
= \\
24 \\
243 \\
243 \\
203\end{array}$ & 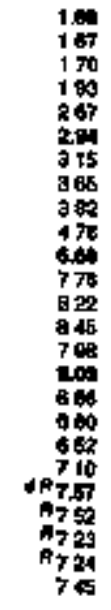 & 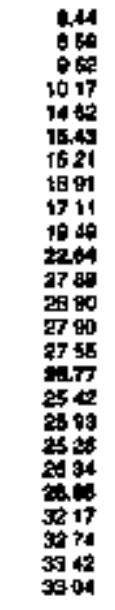 & 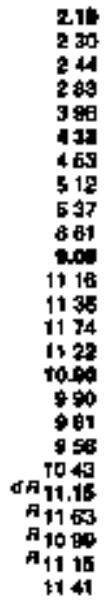 \\
\hline & \multicolumn{10}{|c|}{ 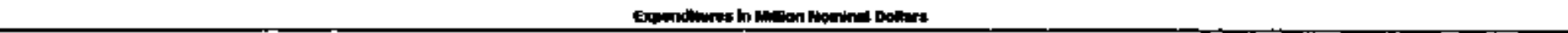 } \\
\hline 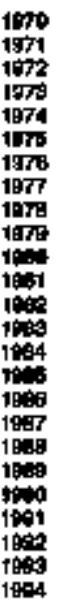 & $\begin{array}{l}01 \\
02 \\
01 \\
01 \\
01 \\
01 \\
01 \\
02 \\
02 \\
01 \\
02 \\
06 \\
00 \\
05 \\
05 \\
03 \\
03 \\
02 \\
02 \\
02 \\
00 \\
02 \\
03 \\
02 \\
02\end{array}$ & 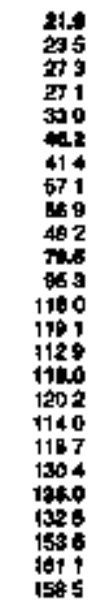 & 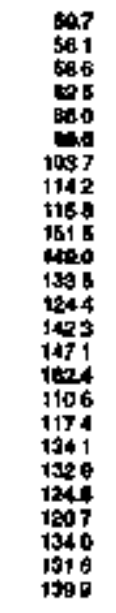 & $\begin{array}{l}22 \\
31 \\
28 \\
20 \\
21 \\
18 \\
22 \\
16 \\
13 \\
14 \\
28 \\
28 \\
75 \\
30 \\
19 \\
98 \\
84 \\
40 \\
98 \\
24 \\
28 \\
17 \\
10 \\
17 \\
19\end{array}$ & 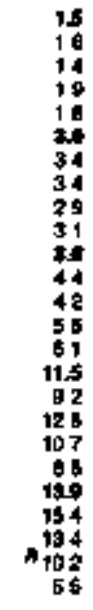 & 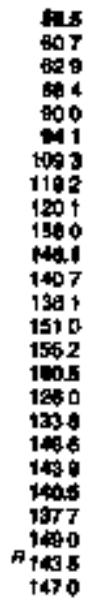 & $\begin{array}{l}= \\
= \\
= \\
= \\
= \\
= \\
= \\
= \\
= \\
= \\
= \\
=5 \\
055 \\
55 \\
58 \\
59\end{array}$ & 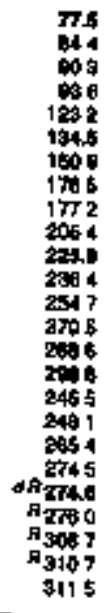 & 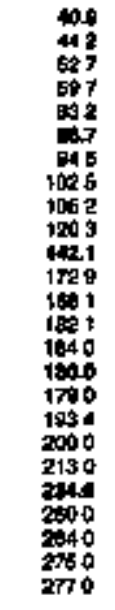 & 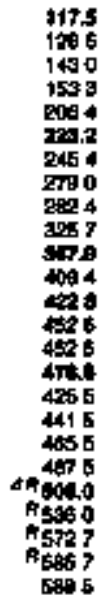 \\
\hline
\end{tabular}

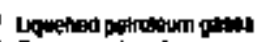

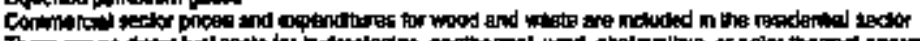

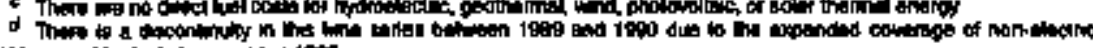

A-povesed dald

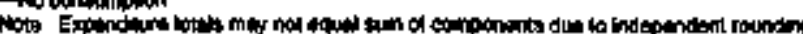

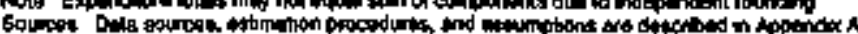


Tabla 247. Commeralal Sector Energy Prien and Expenditure Eatimates by Source, 1970-1994, Ahode baknd

\begin{tabular}{|c|c|c|c|c|c|c|c|c|c|c|c|}
\hline \multirow[b]{4}{*}{$Y=$} & \multicolumn{9}{|c|}{ 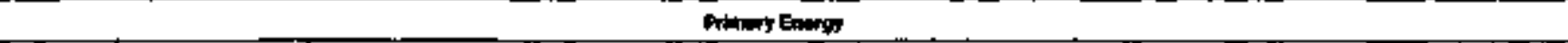 } & \multirow[b]{3}{*}{ Enotraling } & \multirow[b]{3}{*}{ 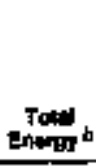 } \\
\hline & & \multirow[b]{2}{*}{ 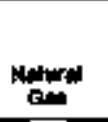 } & \multicolumn{6}{|c|}{ 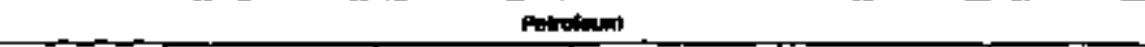 } & \multirow[b]{2}{*}{ Totil } & & \\
\hline & Cod & & 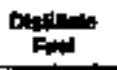 & tantant & Log 4 & mollow & Pand & Tatis & & & \\
\hline & \multicolumn{11}{|c|}{ 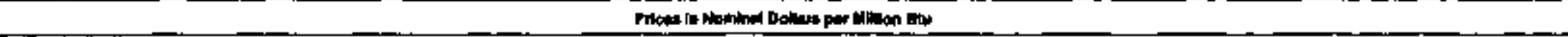 } \\
\hline \multirow[t]{2}{*}{ 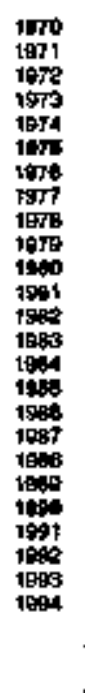 } & 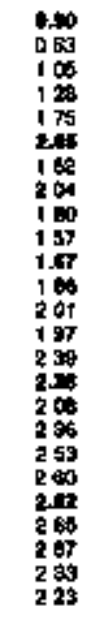 & 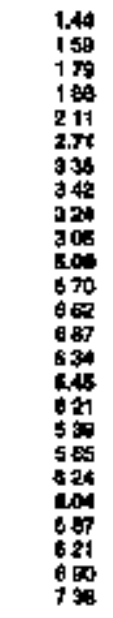 & 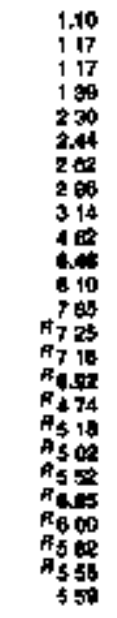 & 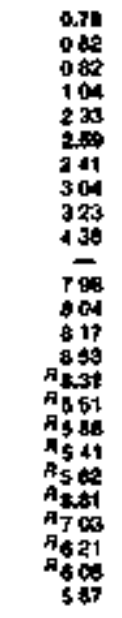 & 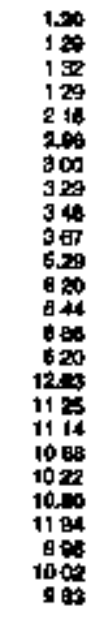 & 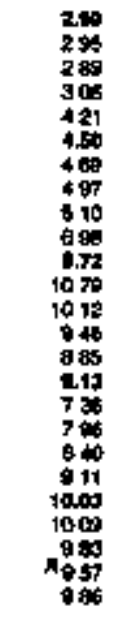 & 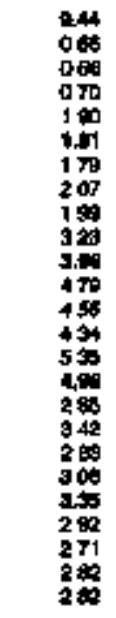 & 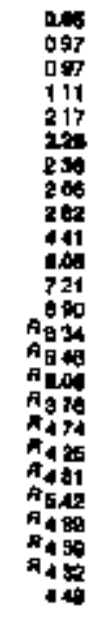 & 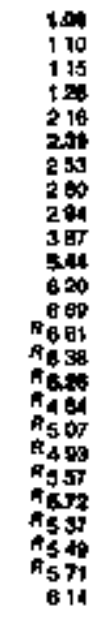 & 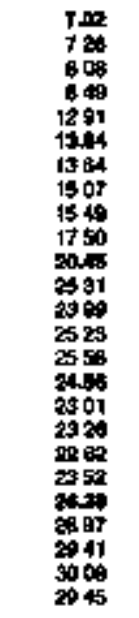 & 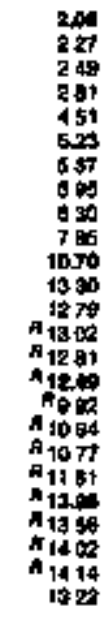 \\
\hline & \multicolumn{11}{|c|}{ 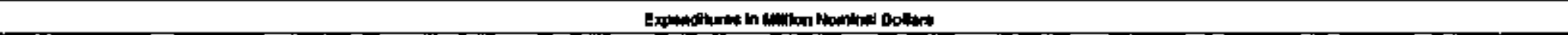 } \\
\hline 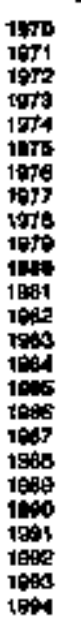 & $\begin{array}{l}0.1 \\
0101 \\
01 \\
01 \\
01 \\
01 \\
0.1 \\
01 \\
01 \\
01 \\
01 \\
05 \\
01 \\
01 \\
02 \\
01 \\
01 \\
0.1 \\
01 \\
01 \\
01 \\
01 \\
01 \\
0.1 \\
01 \\
01 \\
01 \\
01 \\
01\end{array}$ & 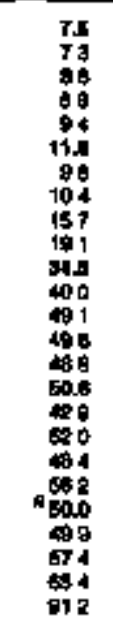 & 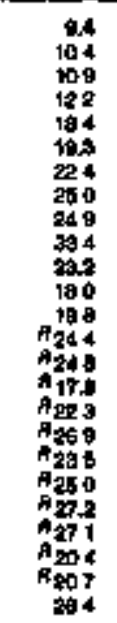 & 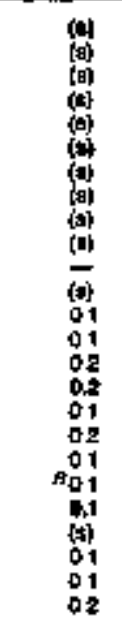 & $\begin{array}{l}0.1 \\
01 \\
01 \\
09 \\
02 \\
01 \\
03 \\
04 \\
03 \\
03 \\
04 \\
05 \\
05 \\
08 \\
08 \\
21 \\
18 \\
28 \\
18 \\
18 \\
13 \\
21 \\
17 \\
20 \\
20\end{array}$ & 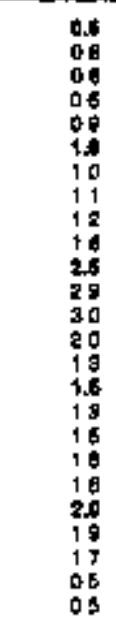 & 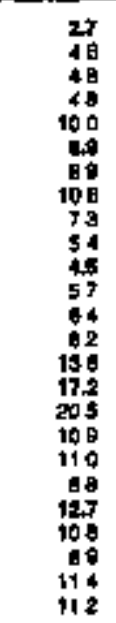 & 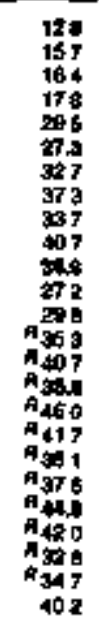 & 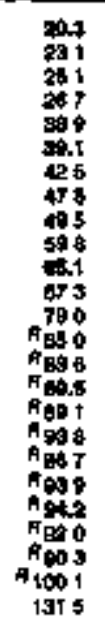 & 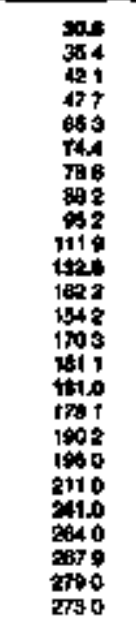 & 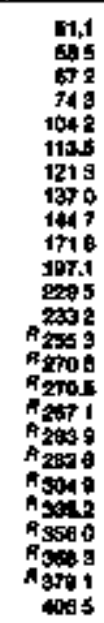 \\
\hline
\end{tabular}




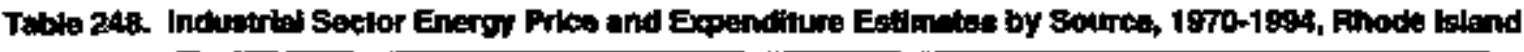

\begin{tabular}{|c|c|c|c|c|c|c|c|c|c|c|c|c|c|c|c|c|c|}
\hline & \multicolumn{15}{|c|}{ Mingringy } & \multirow[b]{3}{*}{ 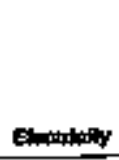 } & \multirow[b]{3}{*}{ S } \\
\hline & \multicolumn{3}{|c|}{ cis } & \multirow[b]{2}{*}{ 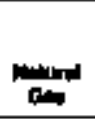 } & \multicolumn{9}{|c|}{ 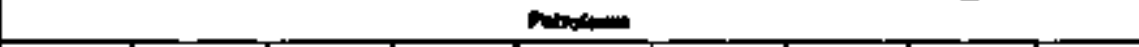 } & \multirow[b]{2}{*}{ " } & \multirow[b]{2}{*}{ Tated 0} & & \\
\hline & Colde & and & Toin & & 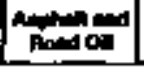 & Denter & monen & LPEA & 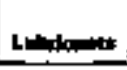 & . & 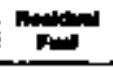 & 94m- & Totol & & & & \\
\hline Yoer & \multicolumn{17}{|c|}{ 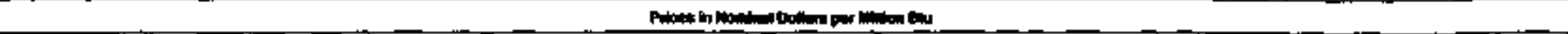 } \\
\hline \multirow[t]{2}{*}{ 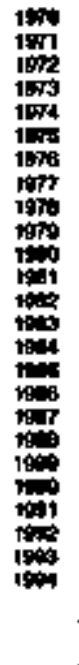 } & $\begin{array}{l}= \\
= \\
= \\
= \\
= \\
= \\
= \\
= \\
= \\
= \\
= \\
= \\
=\end{array}$ & 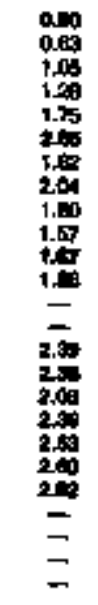 & 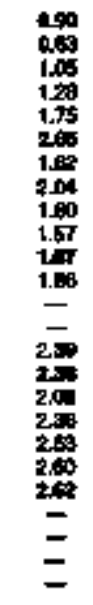 & 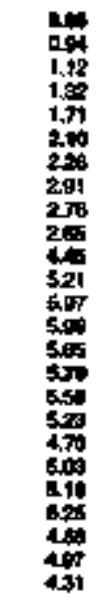 & 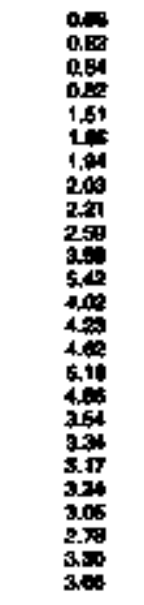 & 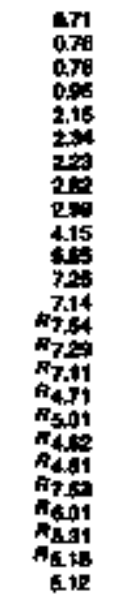 & 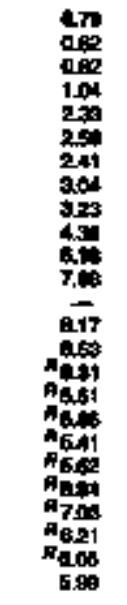 & 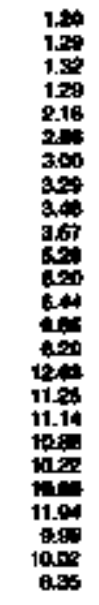 & 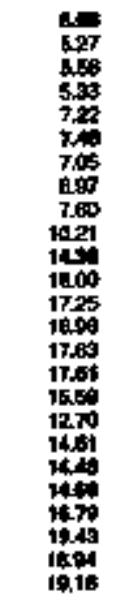 & 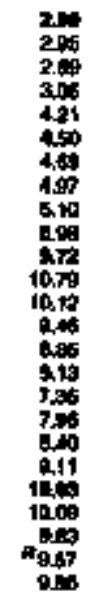 & 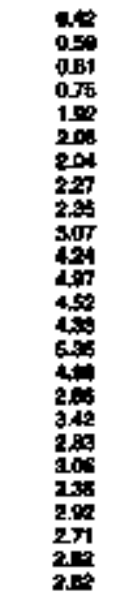 & 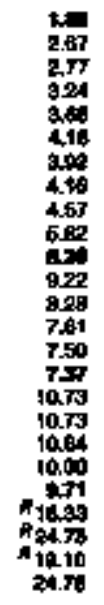 & 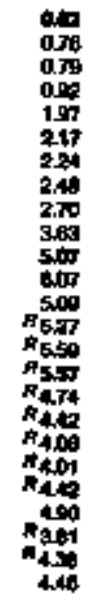 & 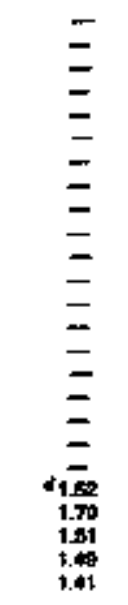 & 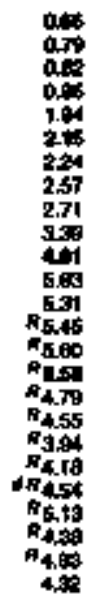 & 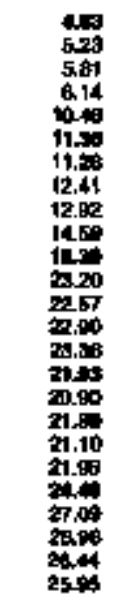 & 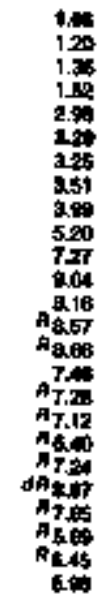 \\
\hline & \multicolumn{17}{|c|}{ EF } \\
\hline 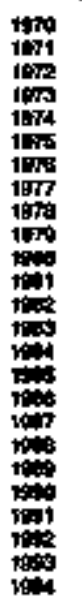 & $\begin{array}{l}= \\
= \\
= \\
= \\
= \\
= \\
= \\
= \\
= \\
= \\
= \\
= \\
=\end{array}$ & 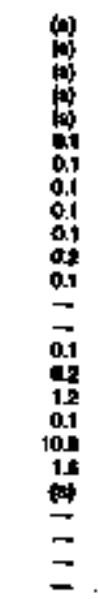 & 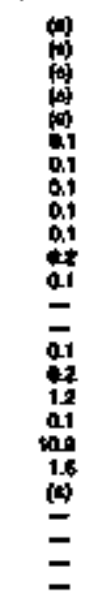 & 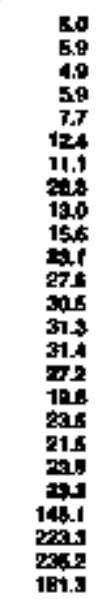 & 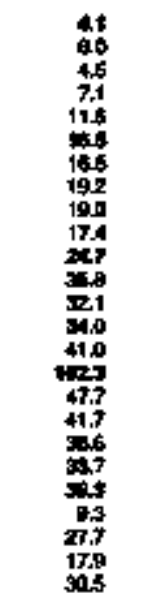 & 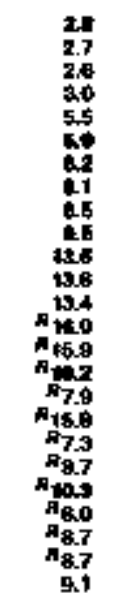 & 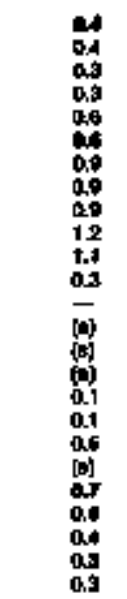 & 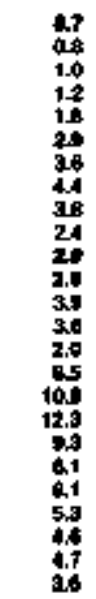 & 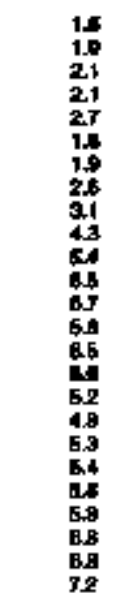 & $\begin{array}{l}9 \\
90 \\
04 \\
0.1 \\
0.1 \\
0.1 \\
0.1 \\
0.1 \\
0.1 \\
0.1 \\
0.1 \\
01 \\
01 \\
01 \\
0.6 \\
13 \\
12 \\
12 \\
1.6 \\
1.7 \\
1.4 \\
1.4 \\
25 \\
25\end{array}$ & 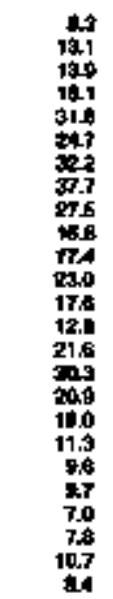 & 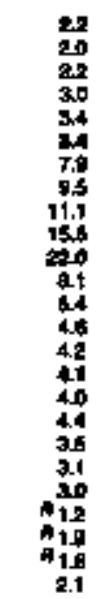 & 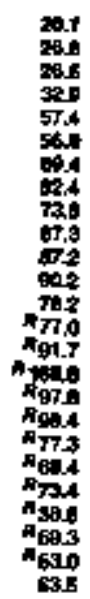 & $\begin{array}{l}= \\
= \\
= \\
= \\
= \\
= \\
= \\
= \\
= \\
= \\
= \\
=0 \\
0.0 \\
0.5 \\
0.5\end{array}$ & 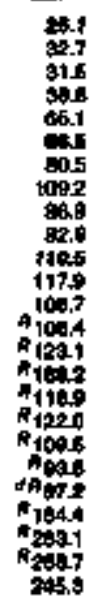 & 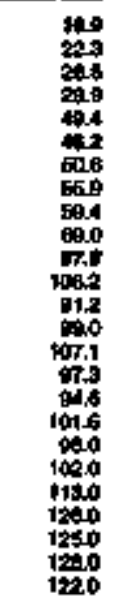 & 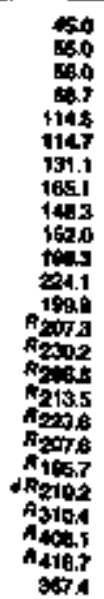 \\
\hline
\end{tabular}

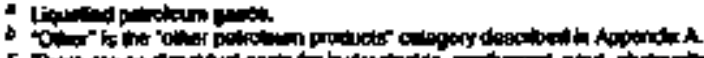

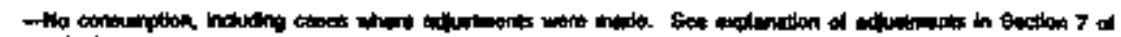

Nopendis

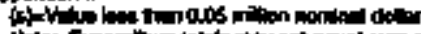

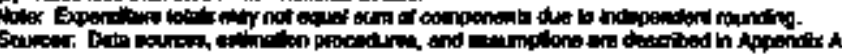

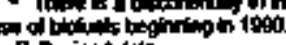




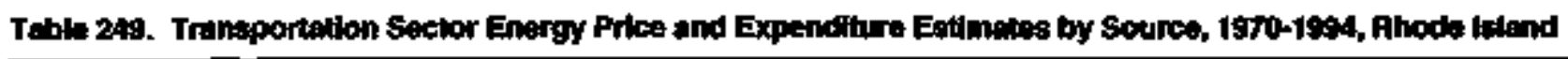

\begin{tabular}{|c|c|c|c|c|c|c|c|c|c|c|c|c|c|}
\hline \multirow[b]{4}{*}{ Yais } & \multicolumn{11}{|c|}{ Ptwnery Einegry } & \multirow[b]{3}{*}{ Enctetey } & \multirow[b]{3}{*}{$\operatorname{Tim}_{\text {thety }}$} \\
\hline & \multirow[b]{2}{*}{ Cont } & \multirow[b]{2}{*}{ 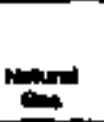 } & \multicolumn{8}{|c|}{ 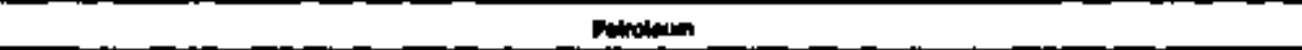 } & \multirow[b]{2}{*}{ Tota } & & \\
\hline & & & הורו & Pate & إن & ut* & 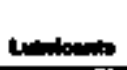 & For & Puld & $T=1$ & & & \\
\hline & \multicolumn{13}{|c|}{ 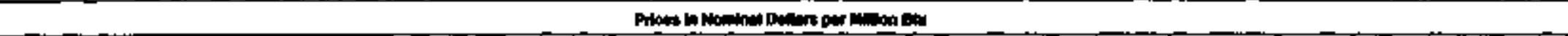 } \\
\hline \multirow[t]{2}{*}{ 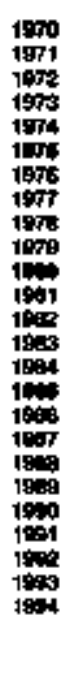 } & $\begin{array}{l}= \\
z \\
z= \\
= \\
= \\
= \\
= \\
= \\
= \\
= \\
= \\
= \\
=\end{array}$ & $\begin{array}{l}= \\
= \\
= \\
= \\
= \\
= \\
= \\
= \\
= \\
= \\
= \\
\overrightarrow{1 \pi} \\
301 \\
000\end{array}$ & 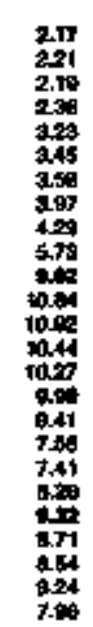 & 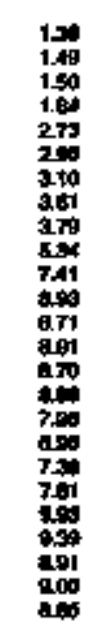 & 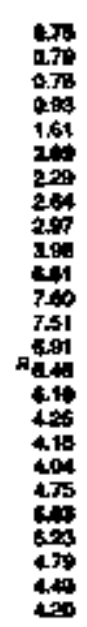 & 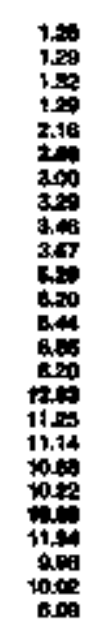 & 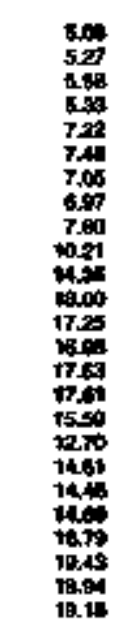 & 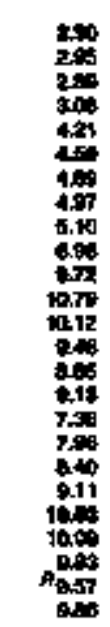 & 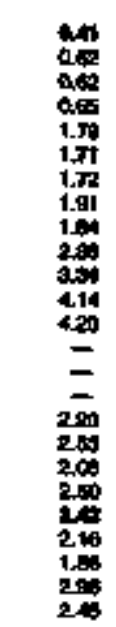 & 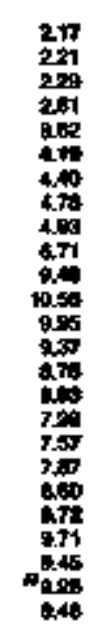 & 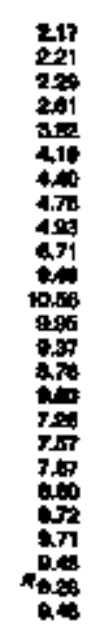 & $\begin{array}{l}= \\
= \\
= \\
= \\
= \\
= \\
= \\
= \\
= \\
= \\
= \\
= \\
= \\
=\end{array}$ & 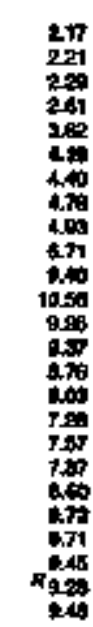 \\
\hline & \multicolumn{13}{|c|}{ Eppon } \\
\hline 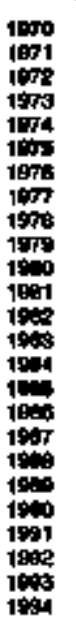 & $\begin{array}{l}= \\
z \\
= \\
\text { a } \\
= \\
z \\
= \\
= \\
= \\
= \\
= \\
= \\
= \\
= \\
=\end{array}$ & $\begin{array}{l}= \\
= \\
= \\
= \\
= \\
= \\
= \\
= \\
= \\
= \\
= \\
0\end{array}$ & 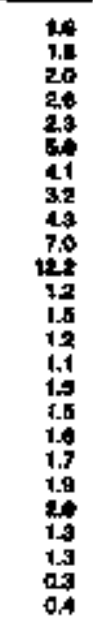 & 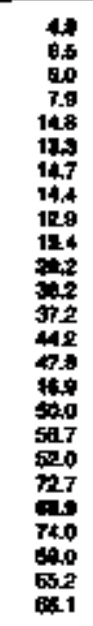 & 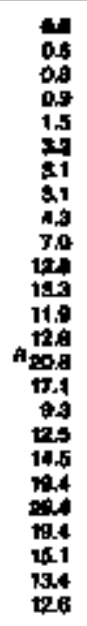 & 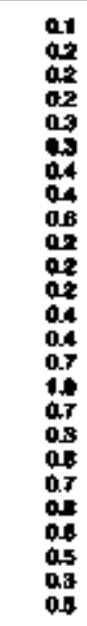 & 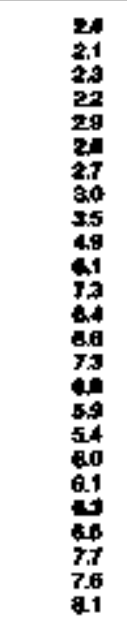 & 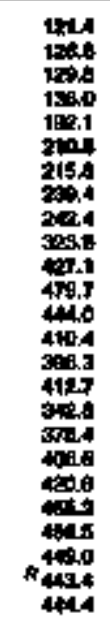 & 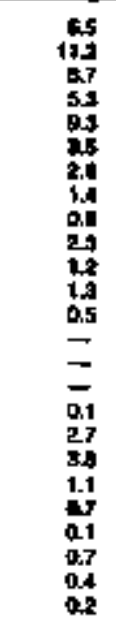 & 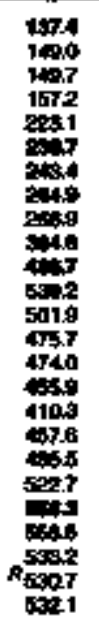 & 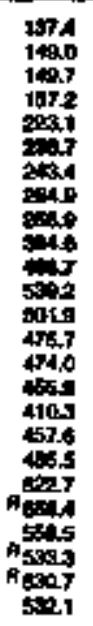 & $\begin{array}{l}= \\
= \\
= \\
= \\
= \\
= \\
= \\
= \\
= \\
= \\
= \\
= \\
= \\
=\end{array}$ & 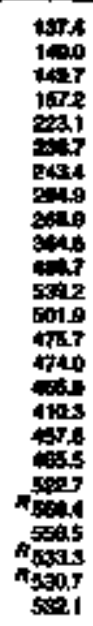 \\
\hline
\end{tabular}




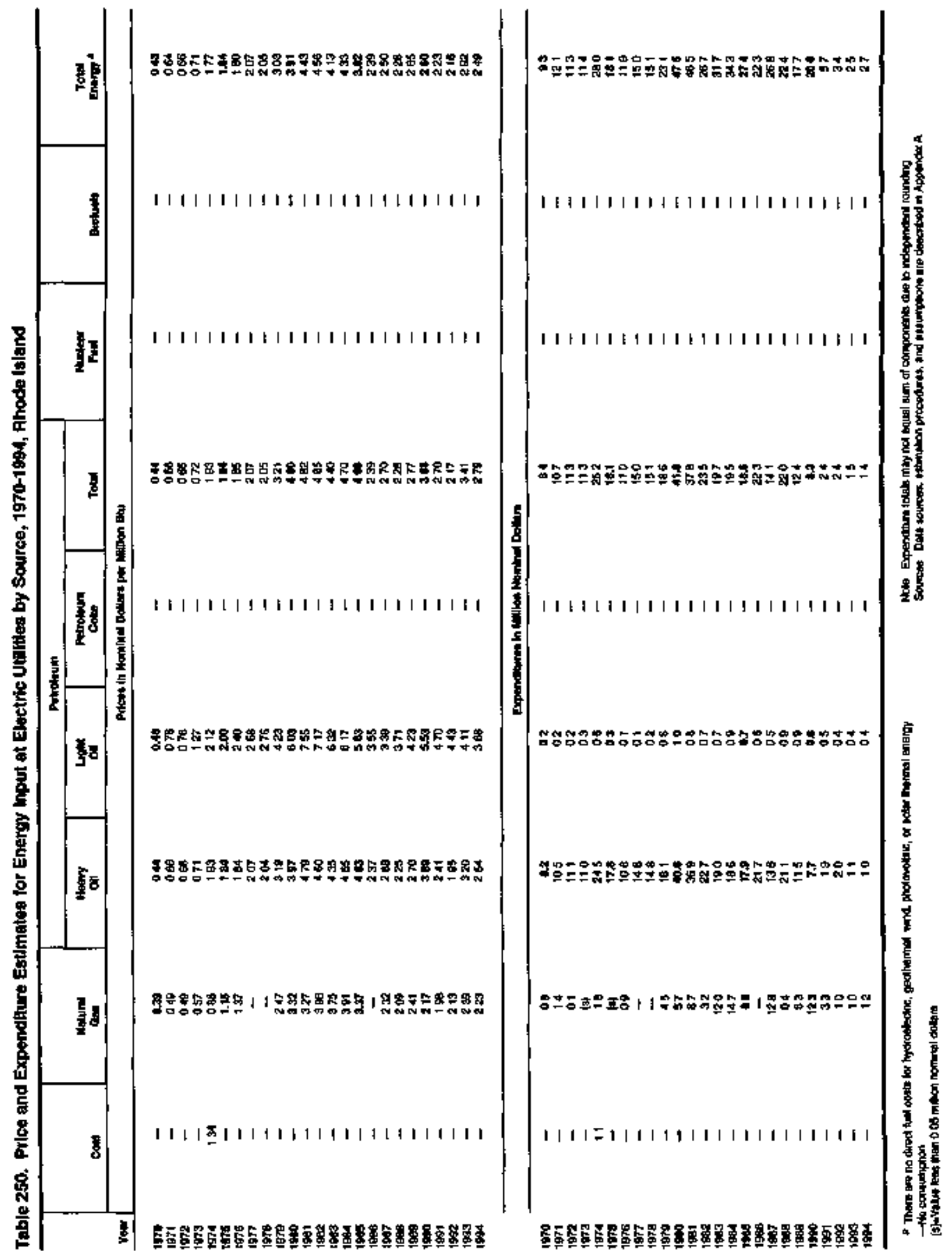


Table 251. Enthgy Price and Expenditure Estmates by Sourca, 1970-1994, South Caroling

\begin{tabular}{|c|c|c|c|c|c|c|c|c|c|c|c|c|c|c|c|c|c|}
\hline \multirow[b]{4}{*}{ Vasr } & \multicolumn{14}{|c|}{ Punery Enary } & \multirow{3}{*}{ 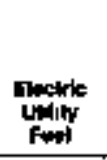 } & \multirow{3}{*}{ 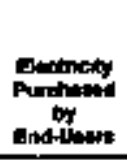 } & \multirow[b]{3}{*}{ Tow } \\
\hline & \multicolumn{3}{|c|}{ cont } & \multirow[b]{2}{*}{ Matur } & \multicolumn{7}{|c|}{ 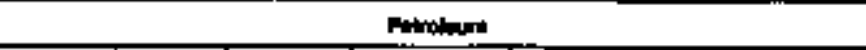 } & \multirow[b]{2}{*}{ Muckar } & \multirow[b]{2}{*}{ 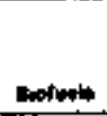 } & \multirow[b]{2}{*}{ Todes } & & & \\
\hline & costentin & gine: & Totim & & mint & Fon & Lma* & 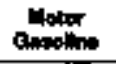 & Roelturew & Oth & Told & & & & & & \\
\hline & \multicolumn{17}{|c|}{ 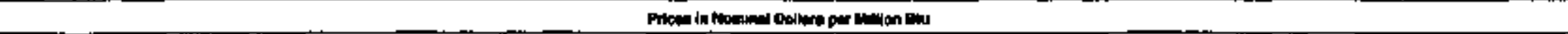 } \\
\hline \multirow[t]{2}{*}{ 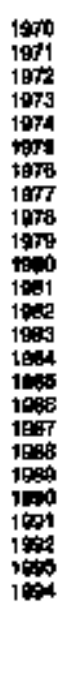 } & $\begin{array}{l}z \\
z \\
z \\
z \\
z \\
= \\
= \\
= \\
= \\
= \\
= \\
= \\
= \\
=\end{array}$ & 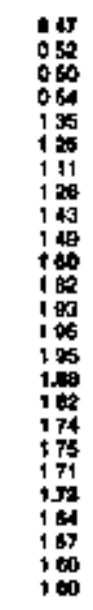 & 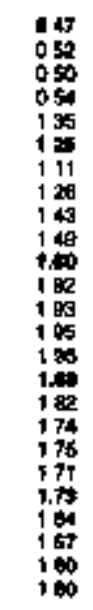 & 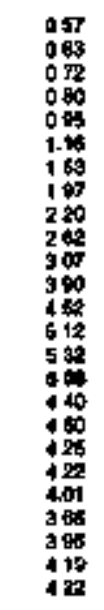 & 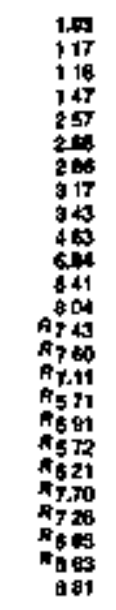 & 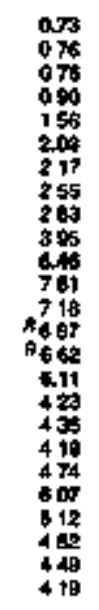 & 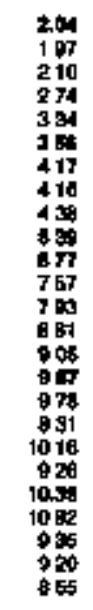 & 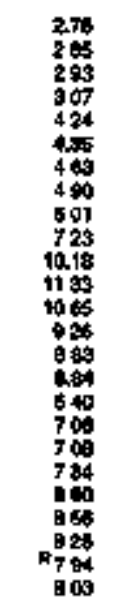 & 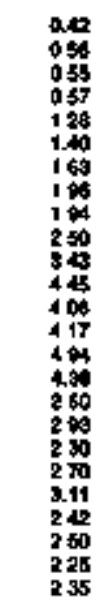 & 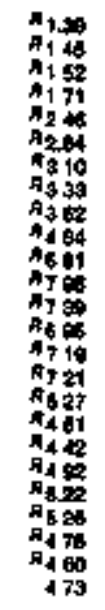 & 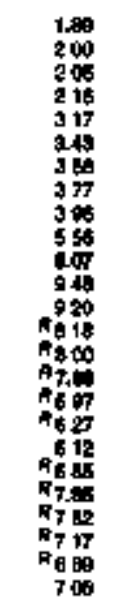 & 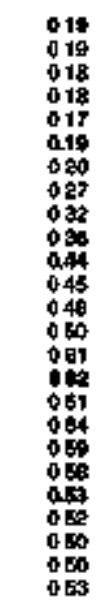 & $\begin{array}{l}= \\
= \\
= \\
= \\
= \\
= \\
= \\
= \\
= \\
= \\
= \\
= \\
\overline{0} \\
181 \\
160 \\
161 \\
183 \\
181\end{array}$ & 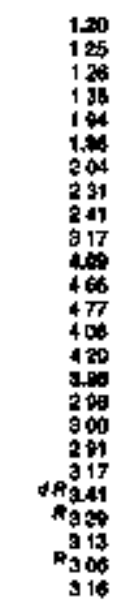 & 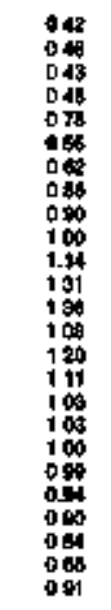 & 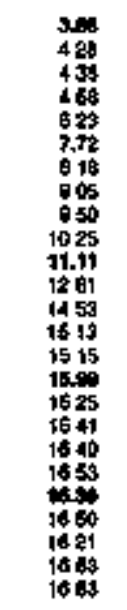 & 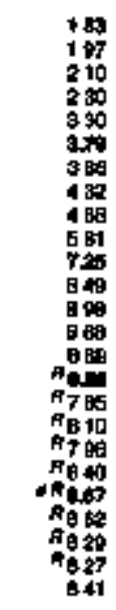 \\
\hline & \multicolumn{17}{|c|}{ 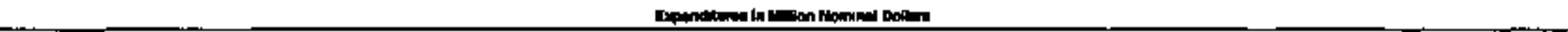 } \\
\hline 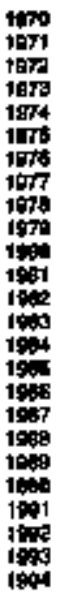 & $\begin{array}{l}= \\
= \\
= \\
= \\
= \\
= \\
= \\
= \\
= \\
= \\
= \\
= \\
= \\
=\end{array}$ & 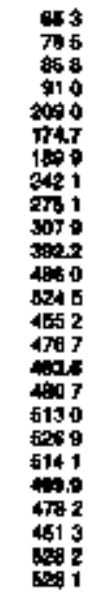 & 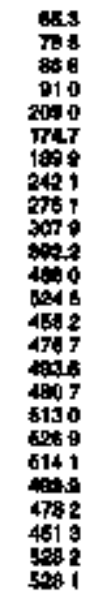 & 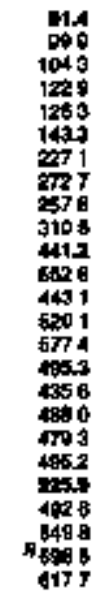 & 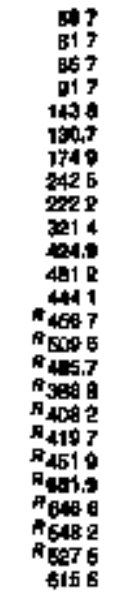 & 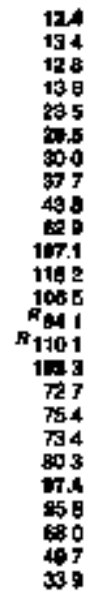 & 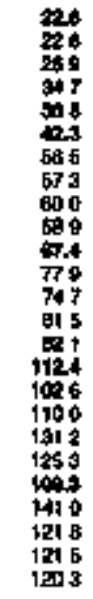 & 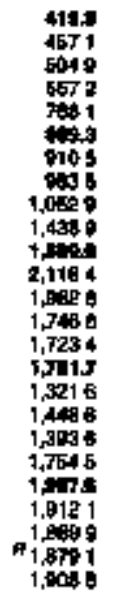 & 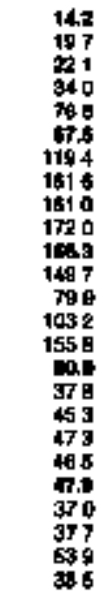 & 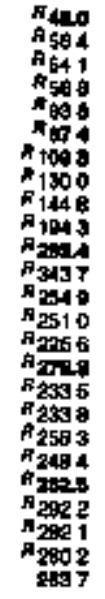 & 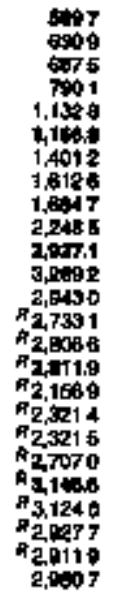 & 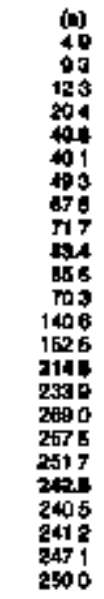 & $\begin{array}{l}= \\
= \\
= \\
= \\
= \\
= \\
= \\
= \\
= \\
= \\
= \\
= \\
402 \\
410 \\
410 \\
422\end{array}$ & 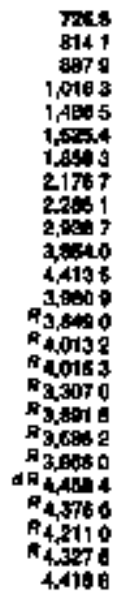 & 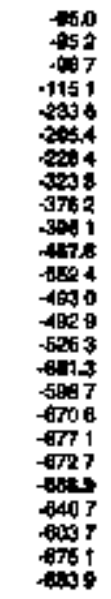 & 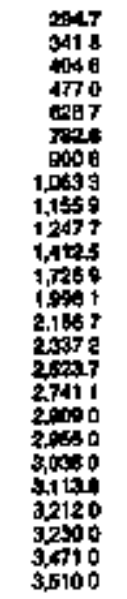 & 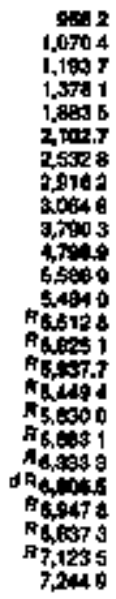 \\
\hline
\end{tabular}

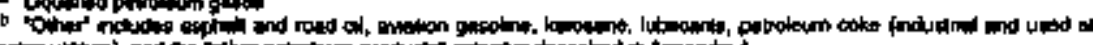

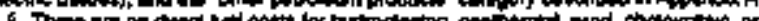

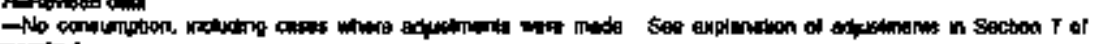

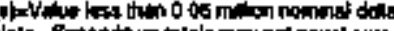




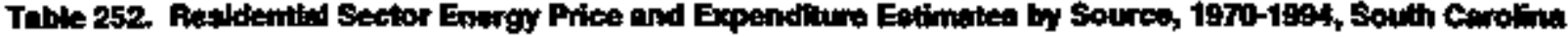

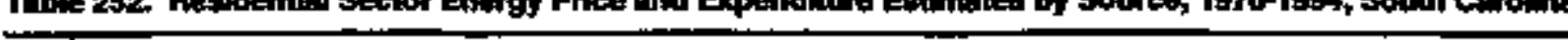

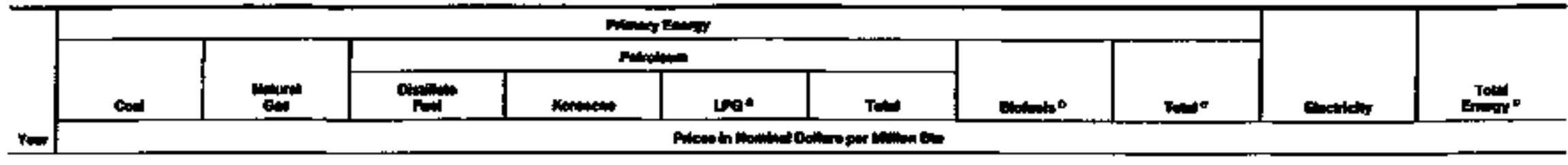

$\mathbf{C}$
$\mathbf{A}$
$\mathbf{R}$
$\mathbf{0}$
$\mathbf{1}$
$\mathbf{N}$

\begin{tabular}{|c|c|c|c|c|c|c|c|c|c|c|}
\hline 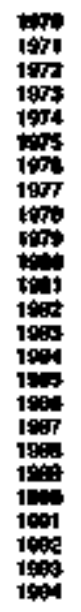 & 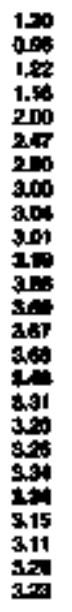 & 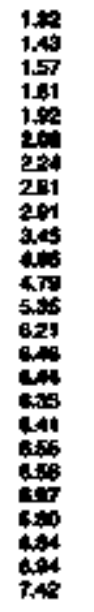 & 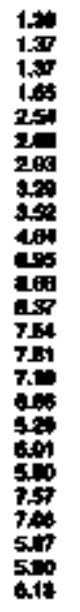 & 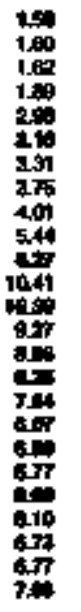 & 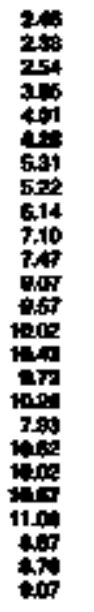 & 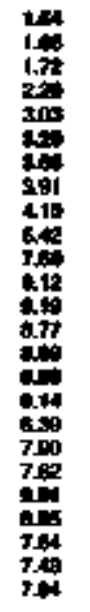 & 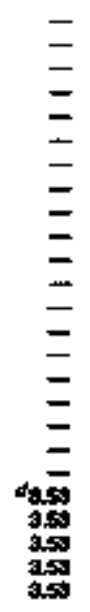 & 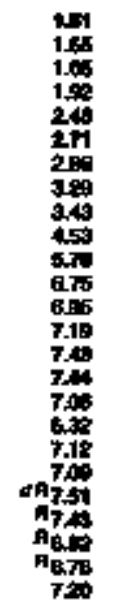 & 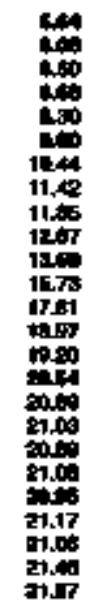 & 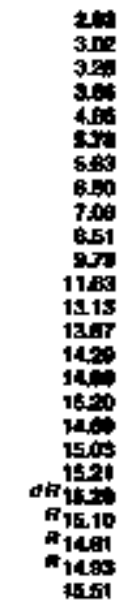 \\
\hline & \multicolumn{10}{|c|}{ monm- } \\
\hline 物 & 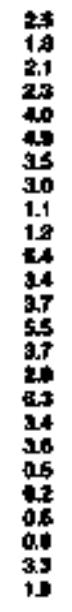 & 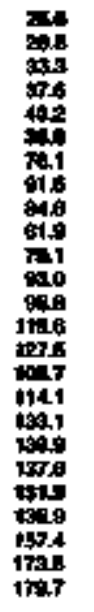 & 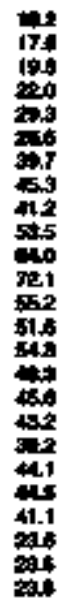 & 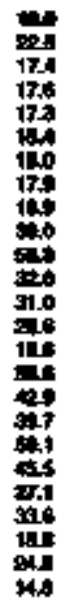 & 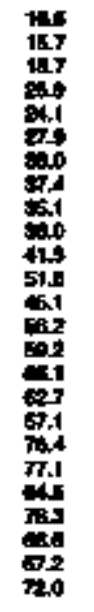 & 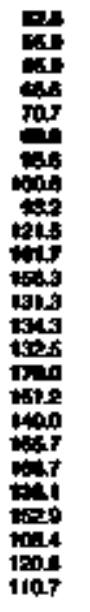 & 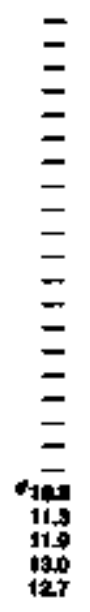 & 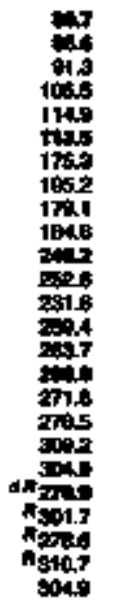 & 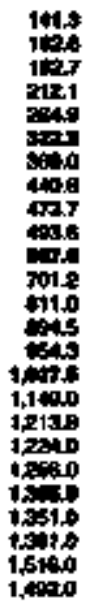 & 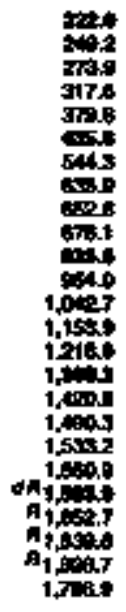 \\
\hline
\end{tabular}

- Liper pototion pasese

Do in

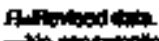

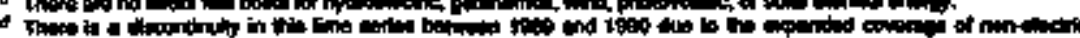

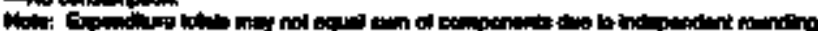

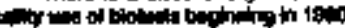

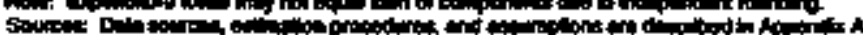




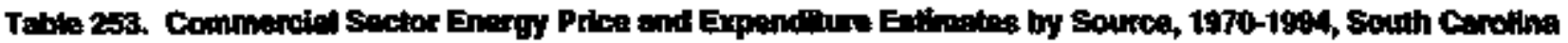

\begin{tabular}{|c|c|c|c|c|c|c|c|c|c|c|c|}
\hline \multirow[b]{4}{*}{$\mathbf{x}$} & \multicolumn{9}{|c|}{ Putrey Eann: } & \multirow[b]{3}{*}{ Eactiply } & \multirow[b]{3}{*}{ Exts } \\
\hline & \multirow[b]{2}{*}{ Dov } & \multirow[b]{2}{*}{ intint } & \multicolumn{6}{|c|}{ Pureinen } & \multirow[b]{2}{*}{ Tomin } & & \\
\hline & & & "Non & Crmonim & 1n: & 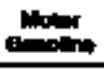 & Fand & To: & & & \\
\hline & \multicolumn{11}{|c|}{ 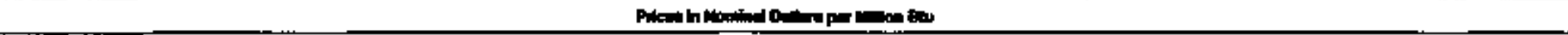 } \\
\hline \multirow[t]{2}{*}{ 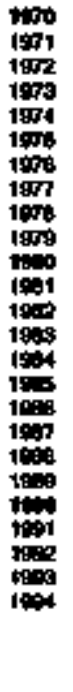 } & 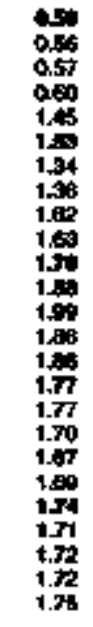 & 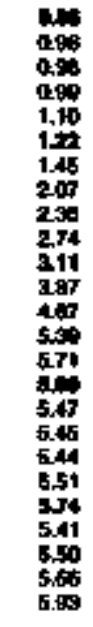 & 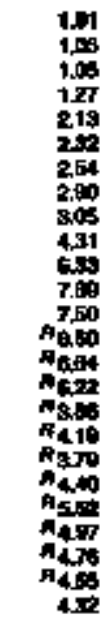 & 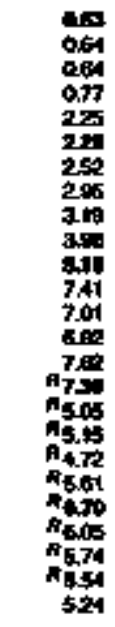 & 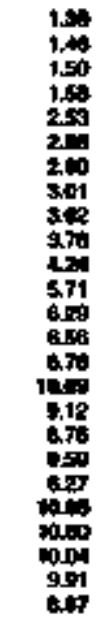 & 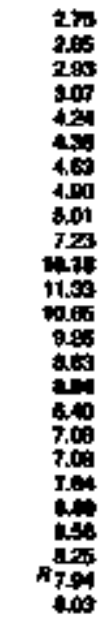 & 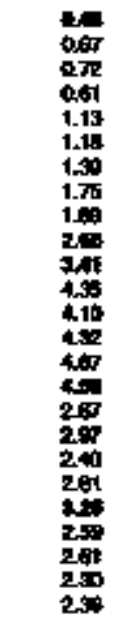 & 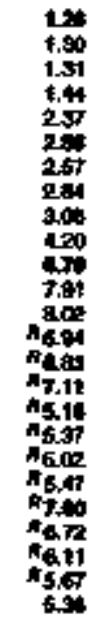 & 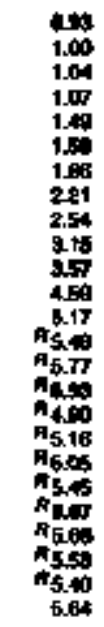 & 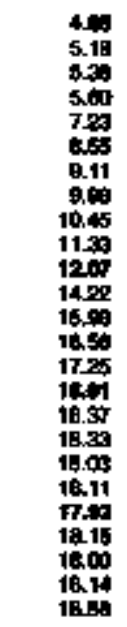 & 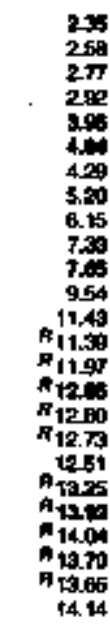 \\
\hline & \multicolumn{11}{|c|}{ C. } \\
\hline 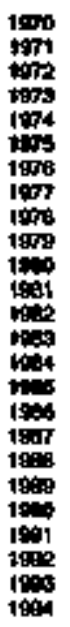 & 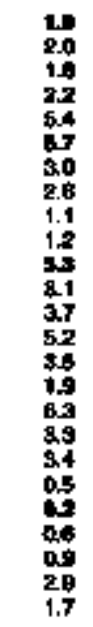 & 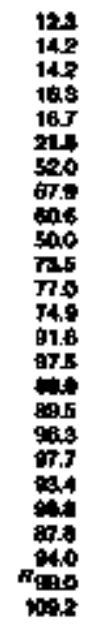 & 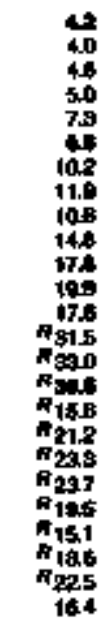 & 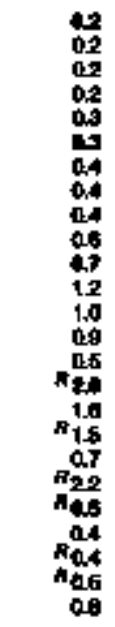 & 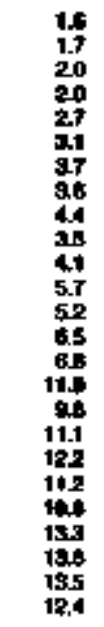 & 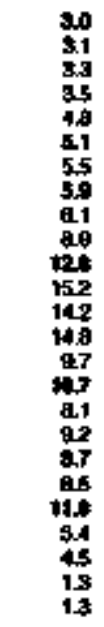 & 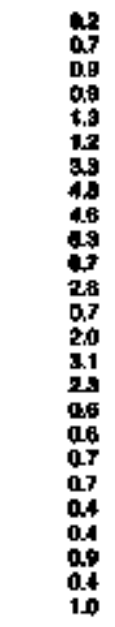 & 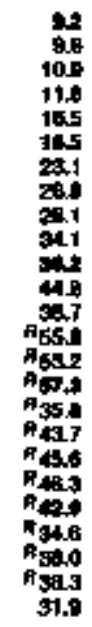 & 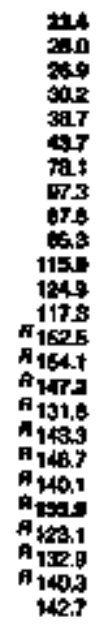 & 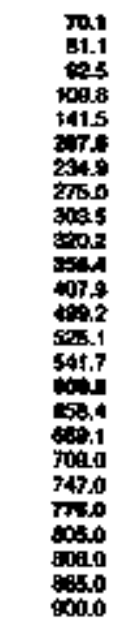 & 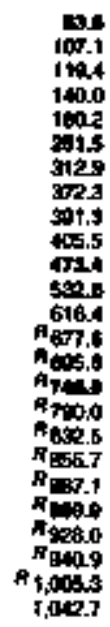 \\
\hline
\end{tabular}

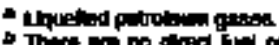

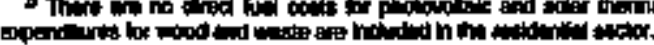

Afradite.

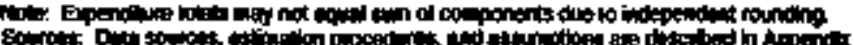


Toblo 254. indualrial Sector Enorgy Prites and Expenditure Eatimeles by Source, 1970-1994, south Ceroline

$\mathbf{T}$

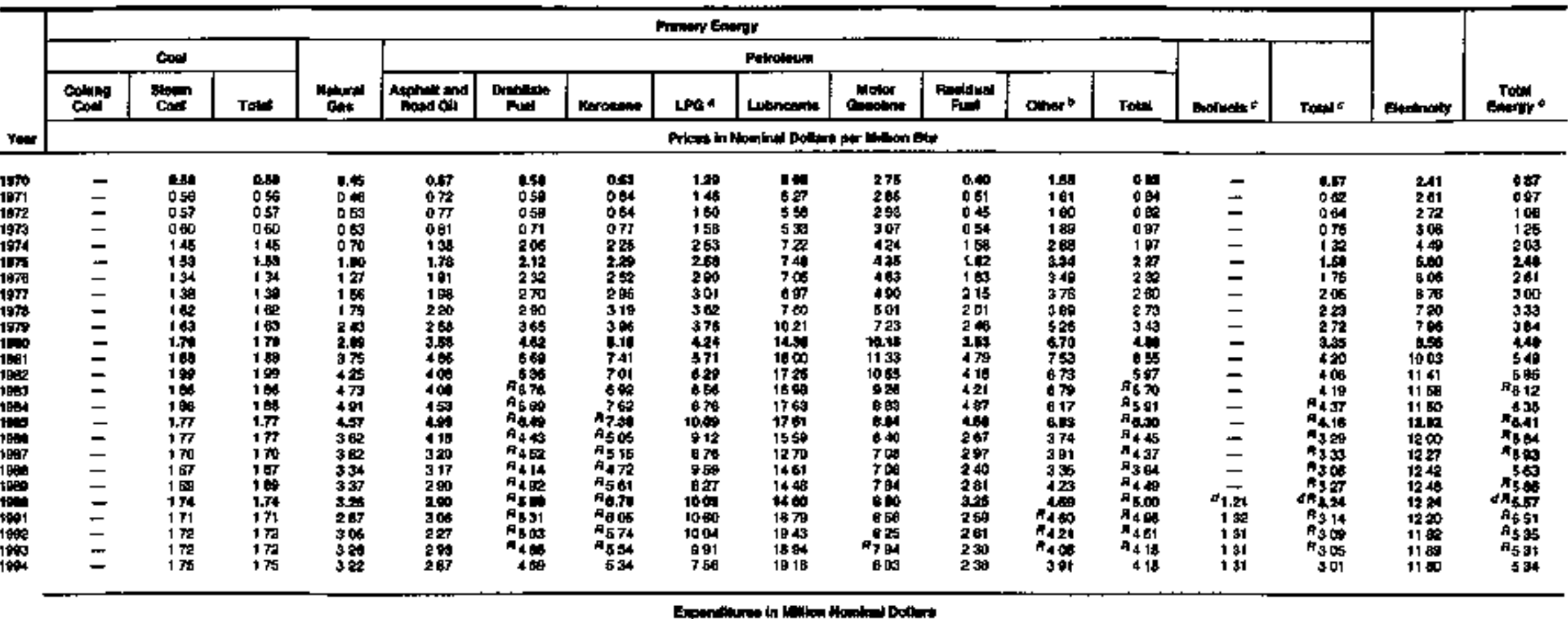

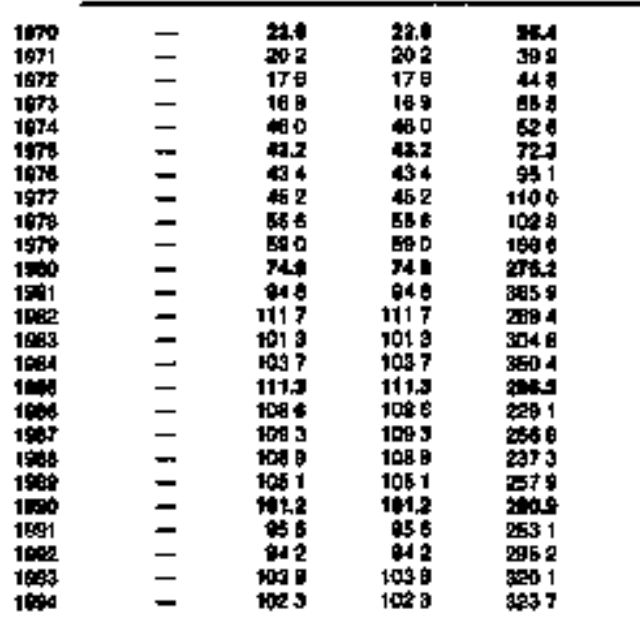

\begin{tabular}{|c|c|c|c|}
\hline 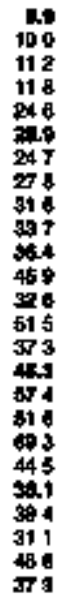 & 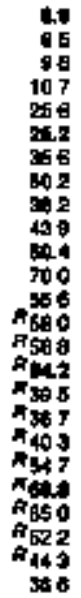 & 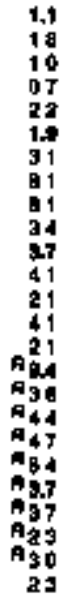 & 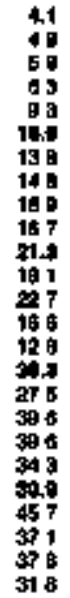 \\
\hline
\end{tabular}

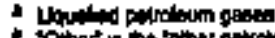

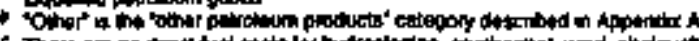

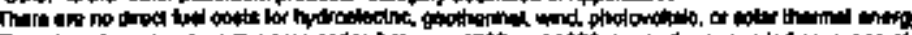

A A

\begin{tabular}{|c|c|c|c|c|c|c|c|c|}
\hline 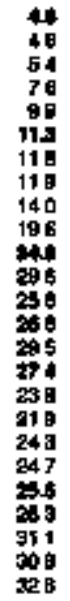 & $\begin{array}{r}40 \\
13 \\
32 \\
15 \\
53 \\
11 \\
11 \\
38 \\
38 \\
13 \\
11 \\
43 \\
30 \\
14 \\
138 \\
36 \\
213 \\
247 \\
298 \\
302 \\
303 \\
302 \\
310 \\
4191 \\
175\end{array}$ & 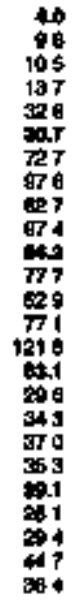 & 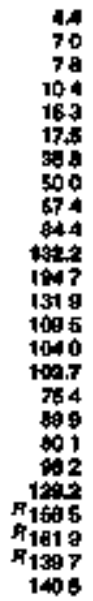 & 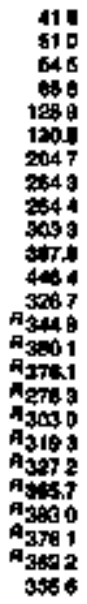 & $\begin{array}{l}= \\
= \\
= \\
= \\
= \\
= \\
= \\
= \\
= \\
= \\
= \\
= \\
=20 \\
202 \\
281 \\
208\end{array}$ & 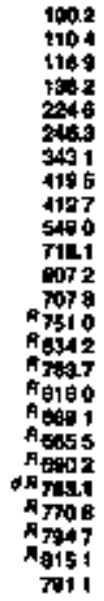 & 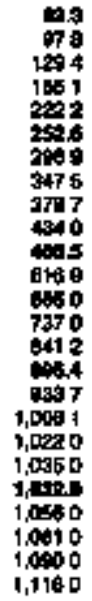 & 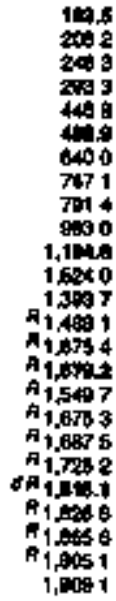 \\
\hline
\end{tabular}

Fintused ditit

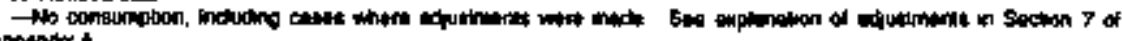

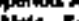

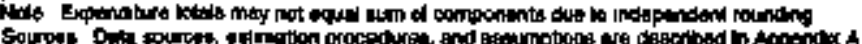

Eneroy Infocmation Adminlotration 
Tublo 255. Thankportution Sector Energy Price and Expendthure Estlmatee by Sounce, 1970-1994, Sorth Corolba

\begin{tabular}{|c|c|c|c|c|c|c|c|c|c|c|c|c|c|}
\hline \multirow[b]{4}{*}{ Verr } & \multicolumn{11}{|c|}{ Parmerem } & \multirow[b]{3}{*}{ Enetricky } & \multirow[b]{3}{*}{ Ty } \\
\hline & \multirow[b]{2}{*}{ Cons } & \multirow[b]{2}{*}{ mistis } & \multicolumn{8}{|c|}{ 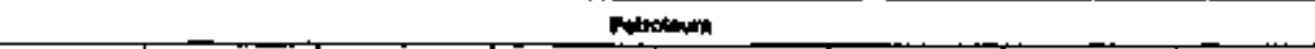 } & \multirow[b]{2}{*}{ Told } & & \\
\hline & & & Antation & Dollation & 네 & Len & Irbitchit & Motor & Fondent & Totr & & & \\
\hline & \multicolumn{13}{|c|}{ 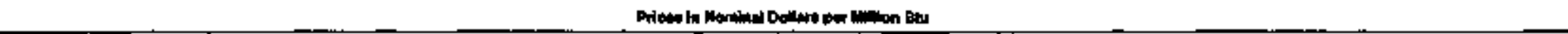 } \\
\hline \multirow[t]{2}{*}{ 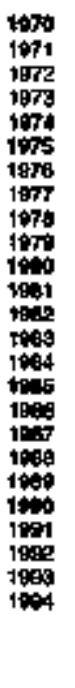 } & $\begin{array}{l}100 \\
086 \\
057 \\
050 \\
145 \\
190 \\
134 \\
130 \\
= \\
= \\
= \\
= \\
= \\
= \\
= \\
= \\
= \\
=\end{array}$ & $\begin{array}{l}= \\
= \\
z \\
= \\
= \\
= \\
= \\
= \\
= \\
= \\
= \\
= \\
= \\
\mathbf{s} \mathbf{z}\end{array}$ & 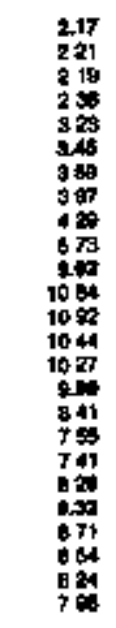 & 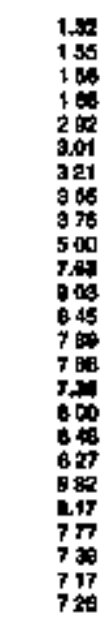 & 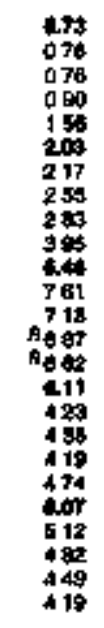 & 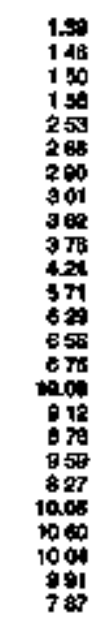 & 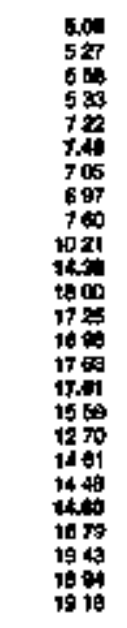 & 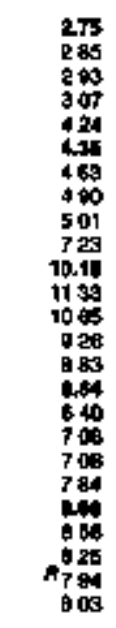 & 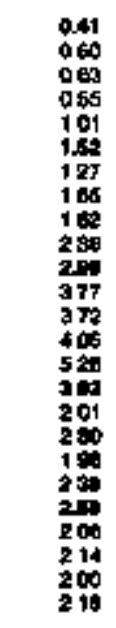 & 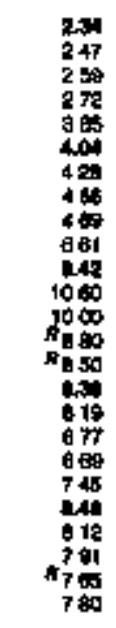 & 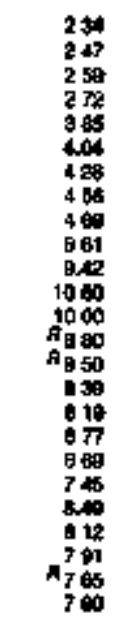 & $\begin{array}{l}= \\
= \\
= \\
= \\
= \\
= \\
= \\
= \\
= \\
= \\
= \\
= \\
= \\
= \\
=\end{array}$ & 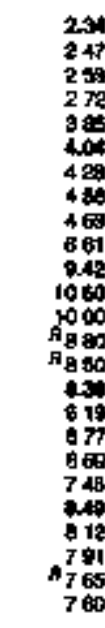 \\
\hline & \multicolumn{13}{|c|}{ 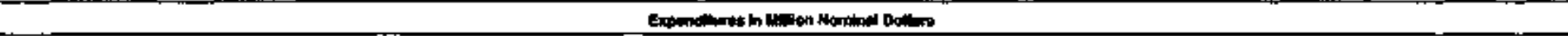 } \\
\hline 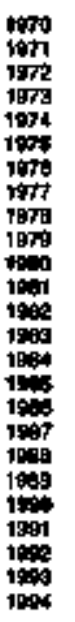 & $\begin{array}{l}\text { 梅 } \\
\text { 照 } \\
\text { 盟 } \\
= \\
= \\
= \\
= \\
= \\
= \\
= \\
= \\
= \\
= \\
=\end{array}$ & $\begin{array}{l}= \\
= \\
z \\
= \\
= \\
= \\
= \\
= \\
= \\
= \\
= \\
= \\
= \\
=\end{array}$ & $\begin{array}{l}24 \\
22 \\
25 \\
22 \\
24 \\
25 \\
30 \\
28 \\
95 \\
46 \\
64 \\
73 \\
96 \\
70 \\
63 \\
69 \\
68 \\
48 \\
47 \\
50 \\
48 \\
79 \\
07 \\
70 \\
46\end{array}$ & 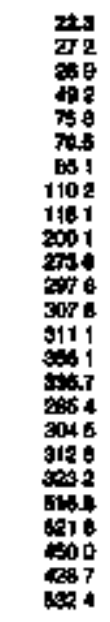 & 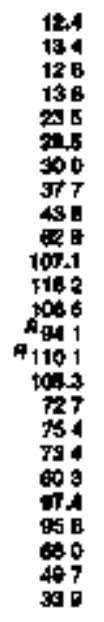 & 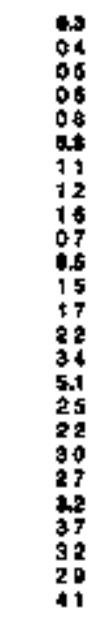 & 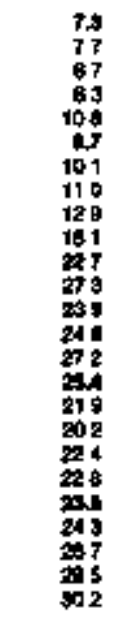 & 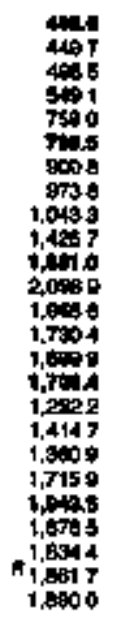 & 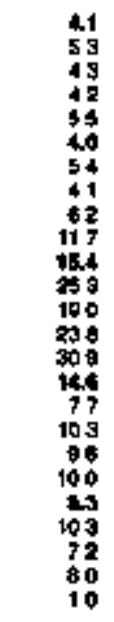 & 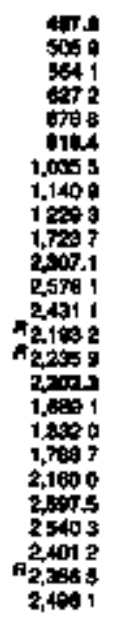 & 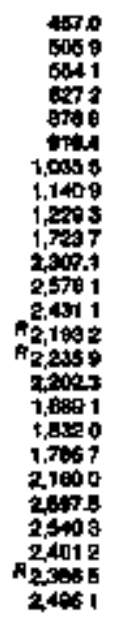 & $\begin{array}{l}= \\
= \\
= \\
= \\
= \\
= \\
= \\
= \\
= \\
= \\
= \\
= \\
= \\
=\end{array}$ & 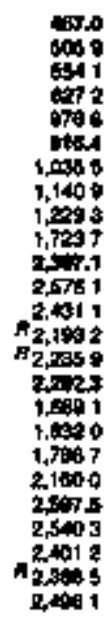 \\
\hline
\end{tabular}




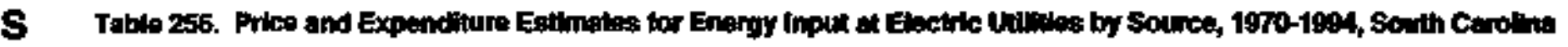

\begin{tabular}{|c|c|c|c|c|c|c|c|c|c|}
\hline \multirow[b]{3}{*}{$n=$} & & \multirow[b]{2}{*}{ mand } & \multicolumn{4}{|c|}{ 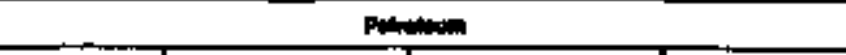 } & \multirow[b]{2}{*}{ "إn } & \multirow[b]{2}{*}{ ants } & \multirow[b]{2}{*}{ 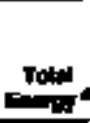 } \\
\hline & men & & then & c) & הומרזה & Fint & & & \\
\hline & \multicolumn{9}{|c|}{ 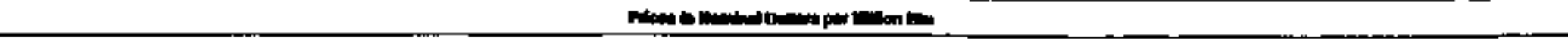 } \\
\hline \multirow[t]{2}{*}{ 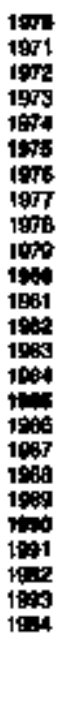 } & 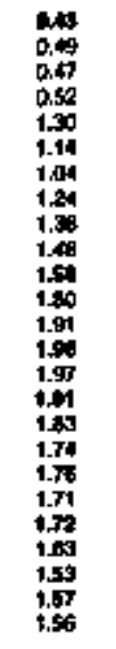 & 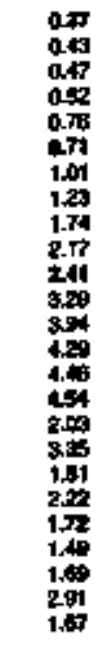 & 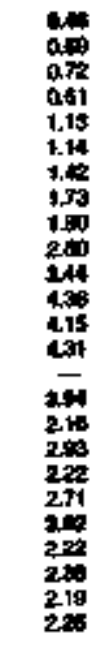 & 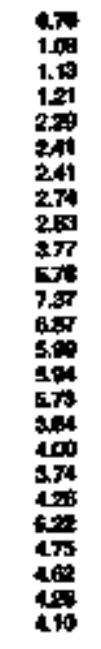 & $\begin{array}{l}= \\
= \\
= \\
= \\
= \\
= \\
= \\
= \\
= \\
= \\
= \\
=\end{array}$ & 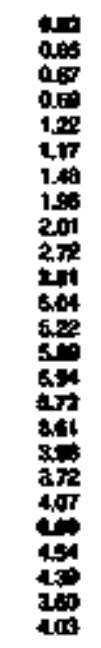 & 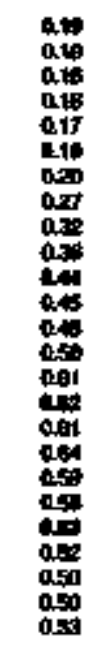 & $\begin{array}{l}= \\
z \\
z \\
z \\
= \\
= \\
= \\
= \\
= \\
= \\
z \\
z \\
z \\
z\end{array}$ & 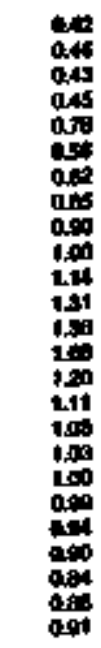 \\
\hline & \multicolumn{9}{|c|}{ Ex } \\
\hline 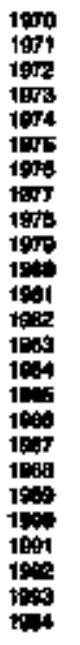 & 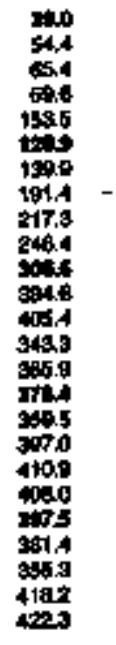 & 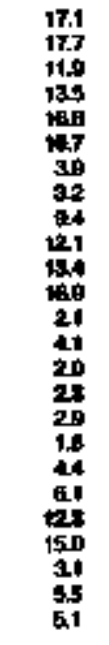 & 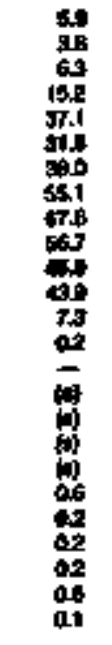 & 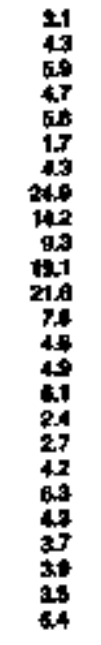 & $\begin{array}{l}= \\
= \\
= \\
= \\
= \\
= \\
= \\
= \\
= \\
= \\
= \\
z\end{array}$ & 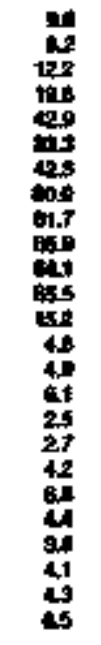 & 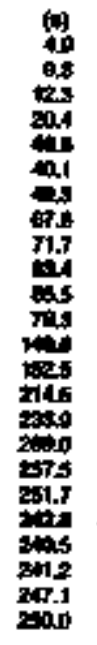 & $\begin{array}{l}= \\
= \\
z \\
= \\
z \\
= \\
= \\
= \\
= \\
= \\
= \\
= \\
=\end{array}$ & 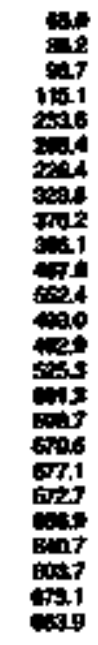 \\
\hline
\end{tabular}

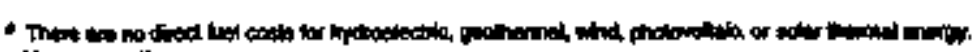

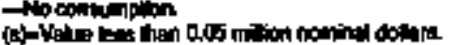




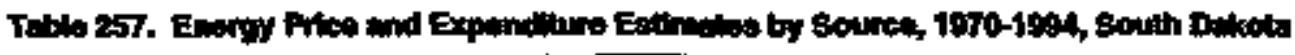

\begin{tabular}{|c|c|c|c|c|c|c|c|c|c|c|c|c|c|c|c|c|c|}
\hline & \multicolumn{14}{|c|}{ menters } & \multirow{3}{*}{ tive } & \multirow{3}{*}{ 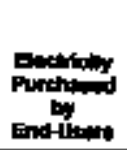 } & \multirow[b]{3}{*}{ Inters' } \\
\hline & \multicolumn{3}{|c|}{ 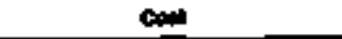 } & & \multicolumn{7}{|c|}{ 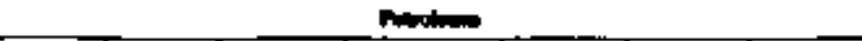 } & \multirow[b]{2}{*}{ דוئ } & \multirow[b]{2}{*}{ 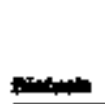 } & \multirow[b]{2}{*}{ T:A } & & & \\
\hline & קורים & "إنبة & Tallat & 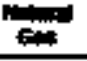 & For & tw & LM⿻上丨" & 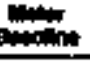 & Fid & Ontome & 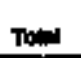 & & & & & & \\
\hline $\mathbf{n}$ & \multicolumn{17}{|c|}{ 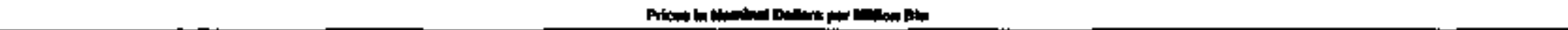 } \\
\hline 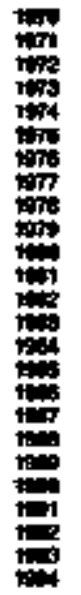 & $\begin{array}{l}= \\
= \\
= \\
= \\
= \\
= \\
= \\
= \\
= \\
= \\
= \\
= \\
= \\
=\end{array}$ & 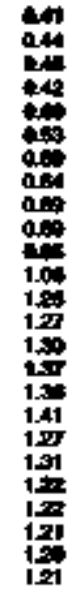 & 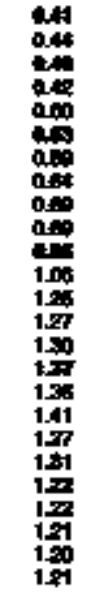 & 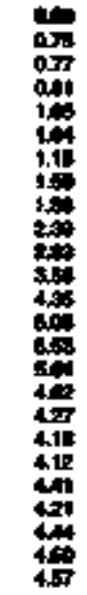 & 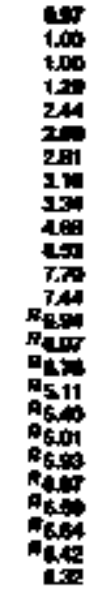 & 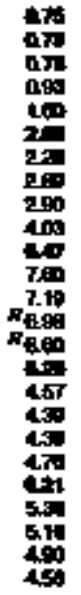 & 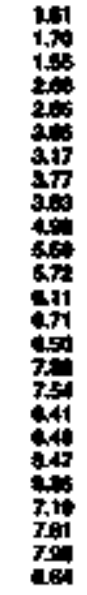 & 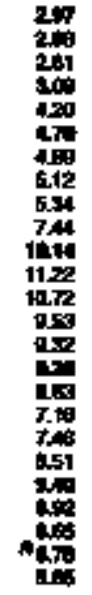 & 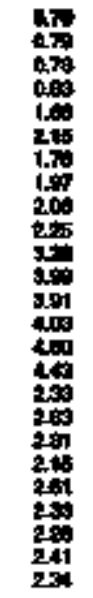 & 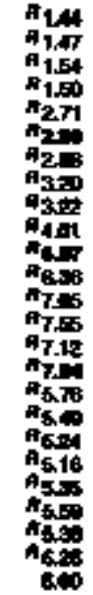 & 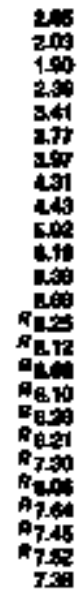 & $\begin{array}{l}= \\
= \\
= \\
= \\
= \\
= \\
= \\
= \\
= \\
= \\
= \\
= \\
= \\
= \\
=\end{array}$ & $\begin{array}{r}= \\
= \\
= \\
= \\
= \\
= \\
= \\
= \\
= \\
= \\
= \\
= \\
200 \\
200 \\
201\end{array}$ & 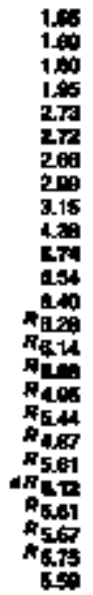 & 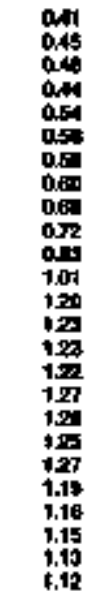 & 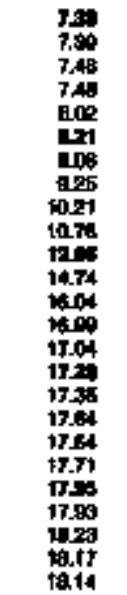 & 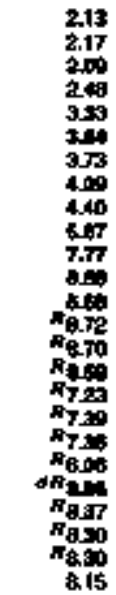 \\
\hline & \multicolumn{17}{|c|}{ 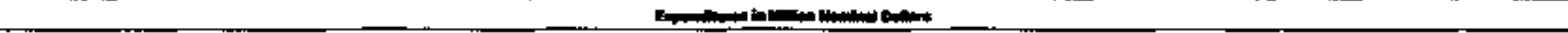 } \\
\hline 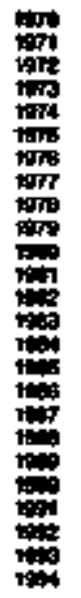 & $\begin{array}{l}= \\
= \\
= \\
= \\
= \\
= \\
= \\
= \\
= \\
= \\
= \\
= \\
=\end{array}$ & 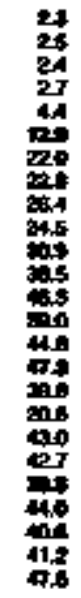 & 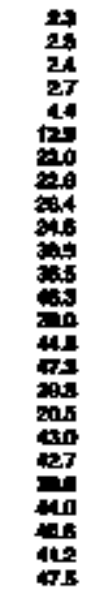 & 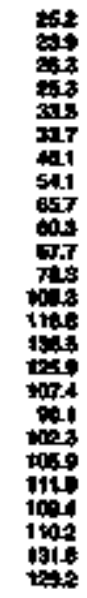 & 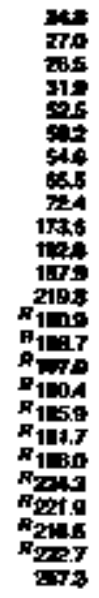 & 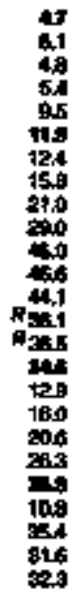 & 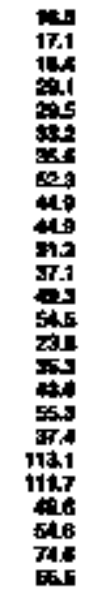 & 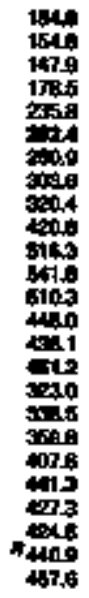 & 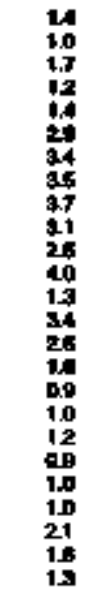 & 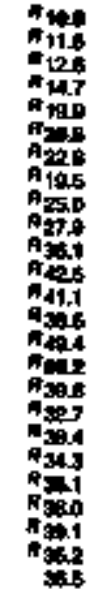 & 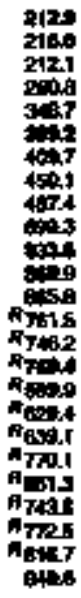 & $\begin{array}{l}= \\
= \\
= \\
= \\
= \\
= \\
= \\
= \\
= \\
= \\
= \\
= \\
=\end{array}$ & $\begin{array}{l}= \\
= \\
= \\
= \\
= \\
= \\
= \\
= \\
= \\
= \\
= \\
42, \\
20,0 \\
2.0\end{array}$ & 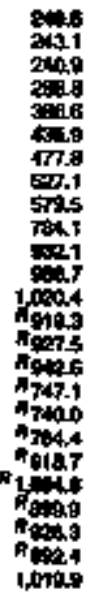 & 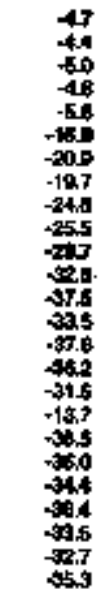 & 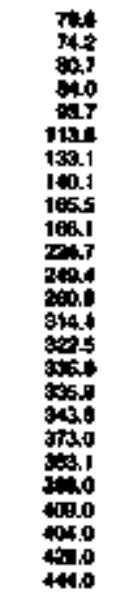 & 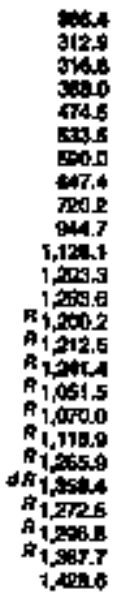 \\
\hline
\end{tabular}

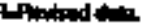

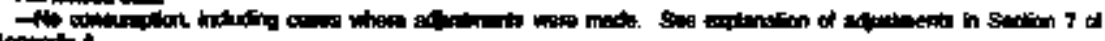

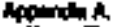


Table 259. Commarelal Sector Energy Price and Expenditure Estlmatea by Source, 1970-1994, South Dakoth

\begin{tabular}{|c|c|c|c|c|c|c|c|c|c|c|c|}
\hline \multirow[b]{4}{*}{ ran } & \multicolumn{9}{|c|}{ Potinery Enowy } & \multirow[b]{3}{*}{ Eeotikithr } & \multirow[b]{3}{*}{ Tatist } \\
\hline & \multirow[b]{2}{*}{ cost } & \multirow[b]{2}{*}{ matis } & \multicolumn{6}{|c|}{ Putrolominin } & \multirow[b]{2}{*}{ Toun } & & \\
\hline & & & 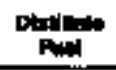 & Keropent & 4rat & 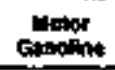 & Probous & \multirow[t]{2}{*}{ Totent } & & & \\
\hline & \multicolumn{10}{|c|}{ 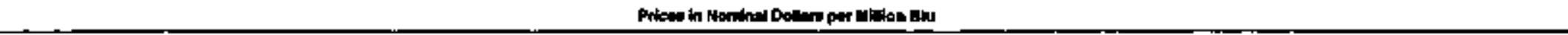 } & \\
\hline \multirow[t]{2}{*}{ 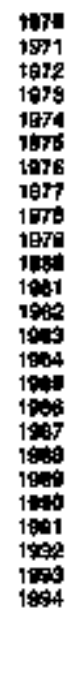 } & 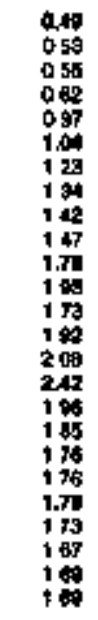 & 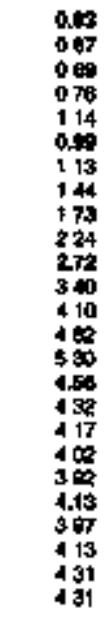 & 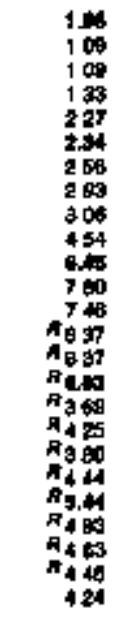 & 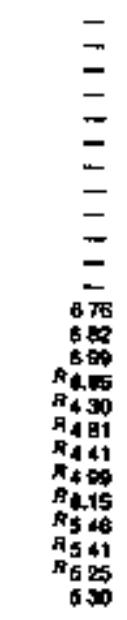 & 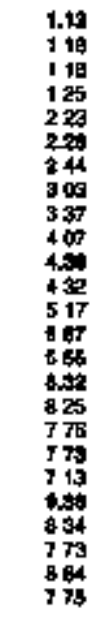 & 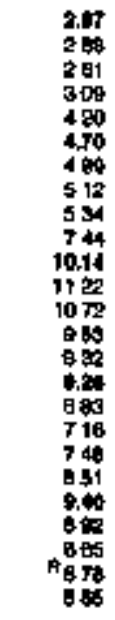 & 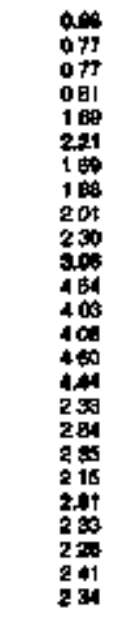 & 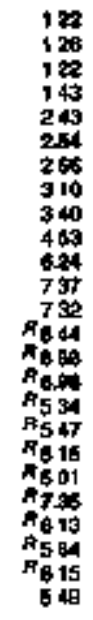 & 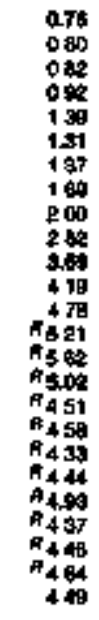 & 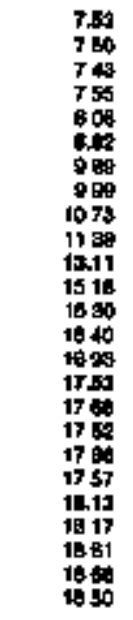 & 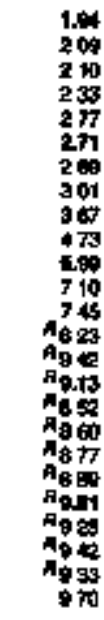 \\
\hline & \multicolumn{11}{|c|}{ 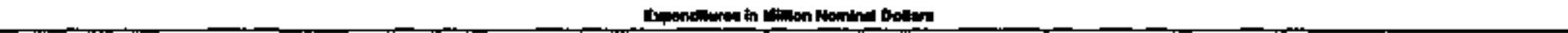 } \\
\hline 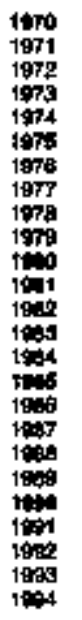 & $\begin{array}{l}02 \\
02 \\
02 \\
02 \\
04 \\
0.3 \\
01 \\
04 \\
02 \\
03 \\
43 \\
13 \\
04 \\
01 \\
02 \\
05 \\
00 \\
01 \\
01 \\
01 \\
01 \\
01 \\
015 \\
69 \\
03\end{array}$ & 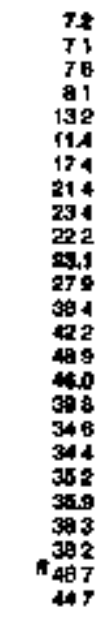 & 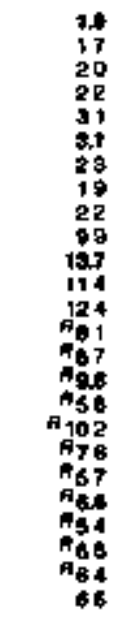 & 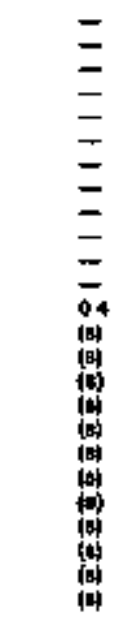 & $\begin{array}{l}18 \\
15 \\
18 \\
16 \\
27 \\
20 \\
34 \\
40 \\
46 \\
29 \\
13 \\
28 \\
31 \\
41 \\
21 \\
97 \\
45 \\
85 \\
47 \\
58 \\
104 \\
56 \\
50 \\
76 \\
84\end{array}$ & 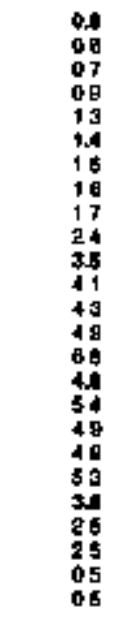 & 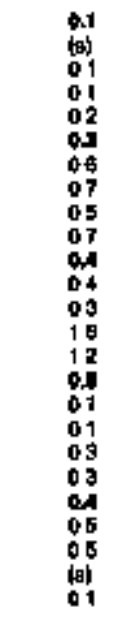 & 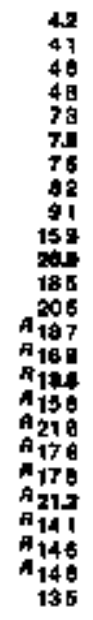 & 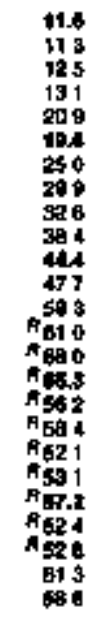 & 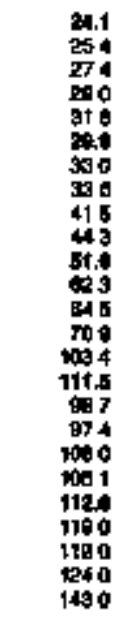 & 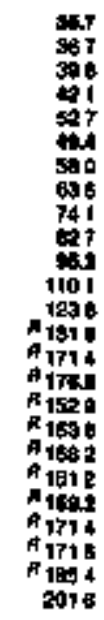 \\
\hline
\end{tabular}


Iahla 260. Industfial Sector Energy Price and Expendwure EAlimates by Fourta, 1970-1994, South Dakota

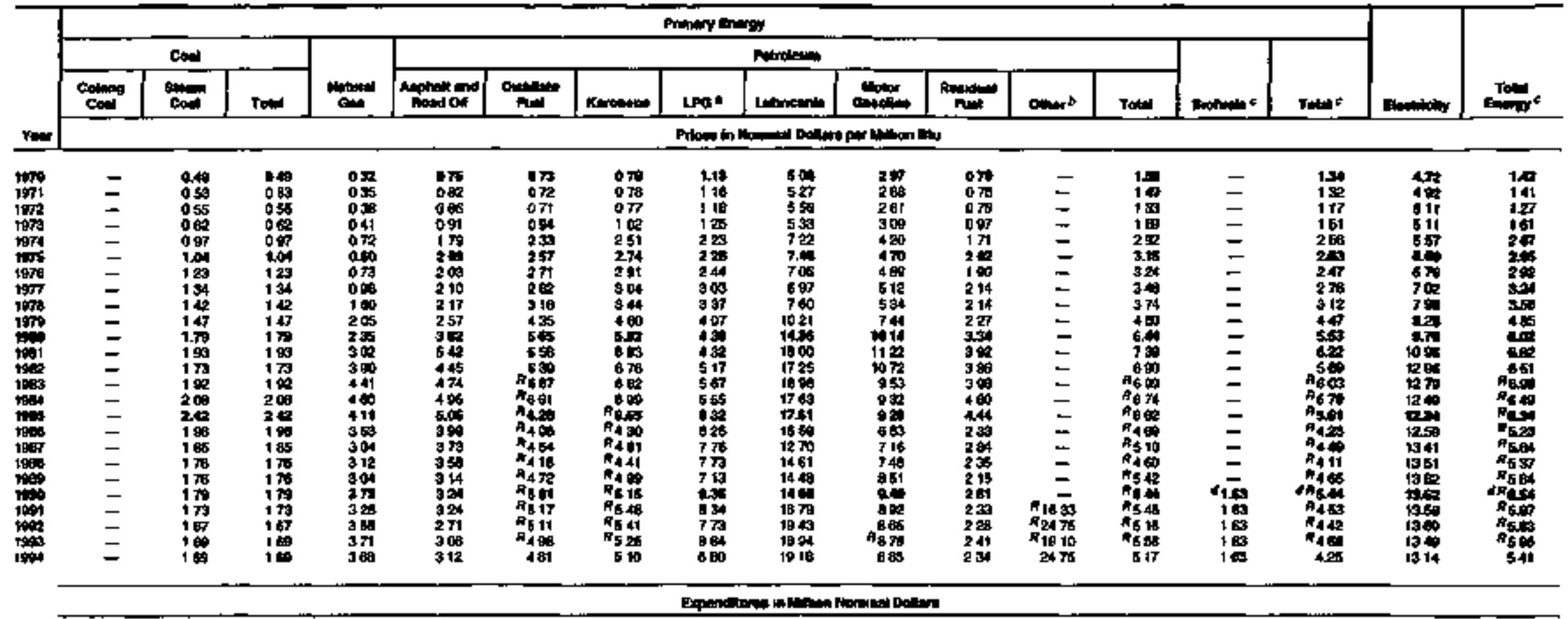

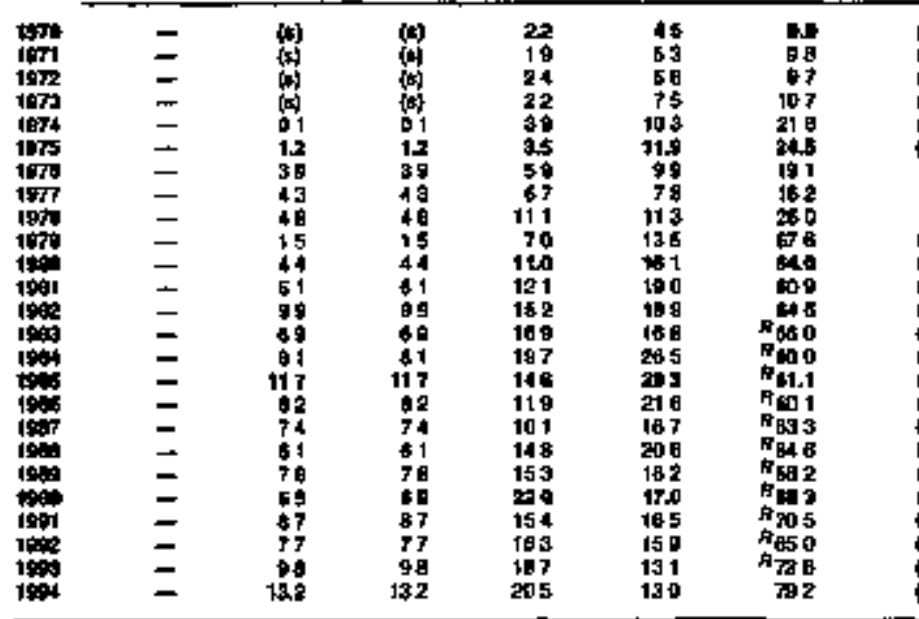

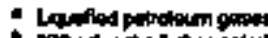

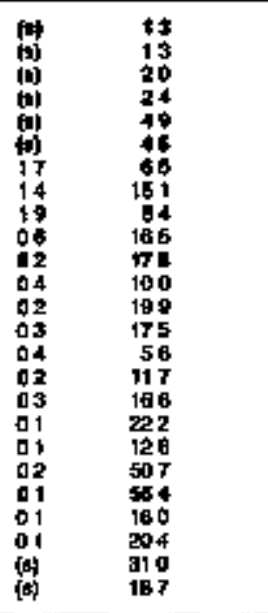

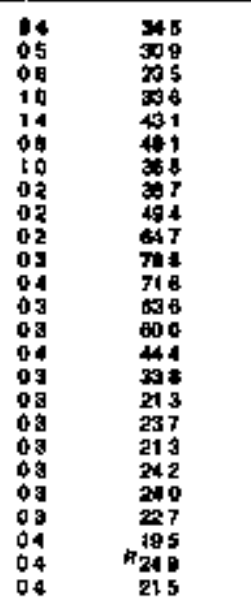

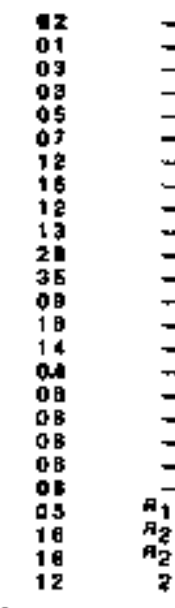

$\rightarrow+\infty \mathrm{cons}$

\begin{tabular}{|c|c|c|c|c|}
\hline 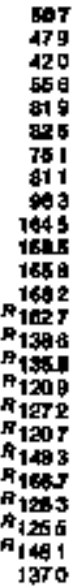 & $\begin{array}{l}= \\
= \\
= \\
= \\
= \\
= \\
= \\
= \\
= \\
= \\
= \\
= \\
067 \\
00 \\
08 \\
08\end{array}$ & 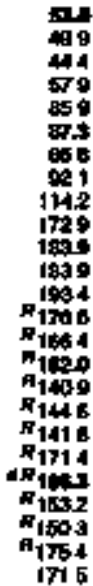 & 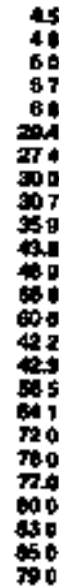 & 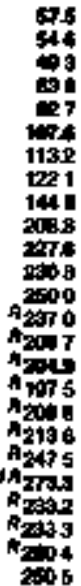 \\
\hline
\end{tabular}

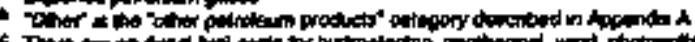

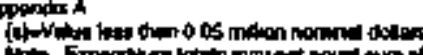

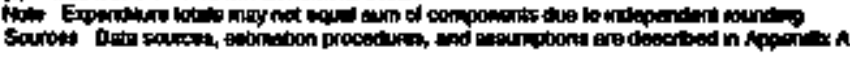
ate of botrel becting in 1990 
Tend 231. Trineportalion Sector Energy Price and Expenditure Estimuted by Sourea, 1970-1894, South Dakpta

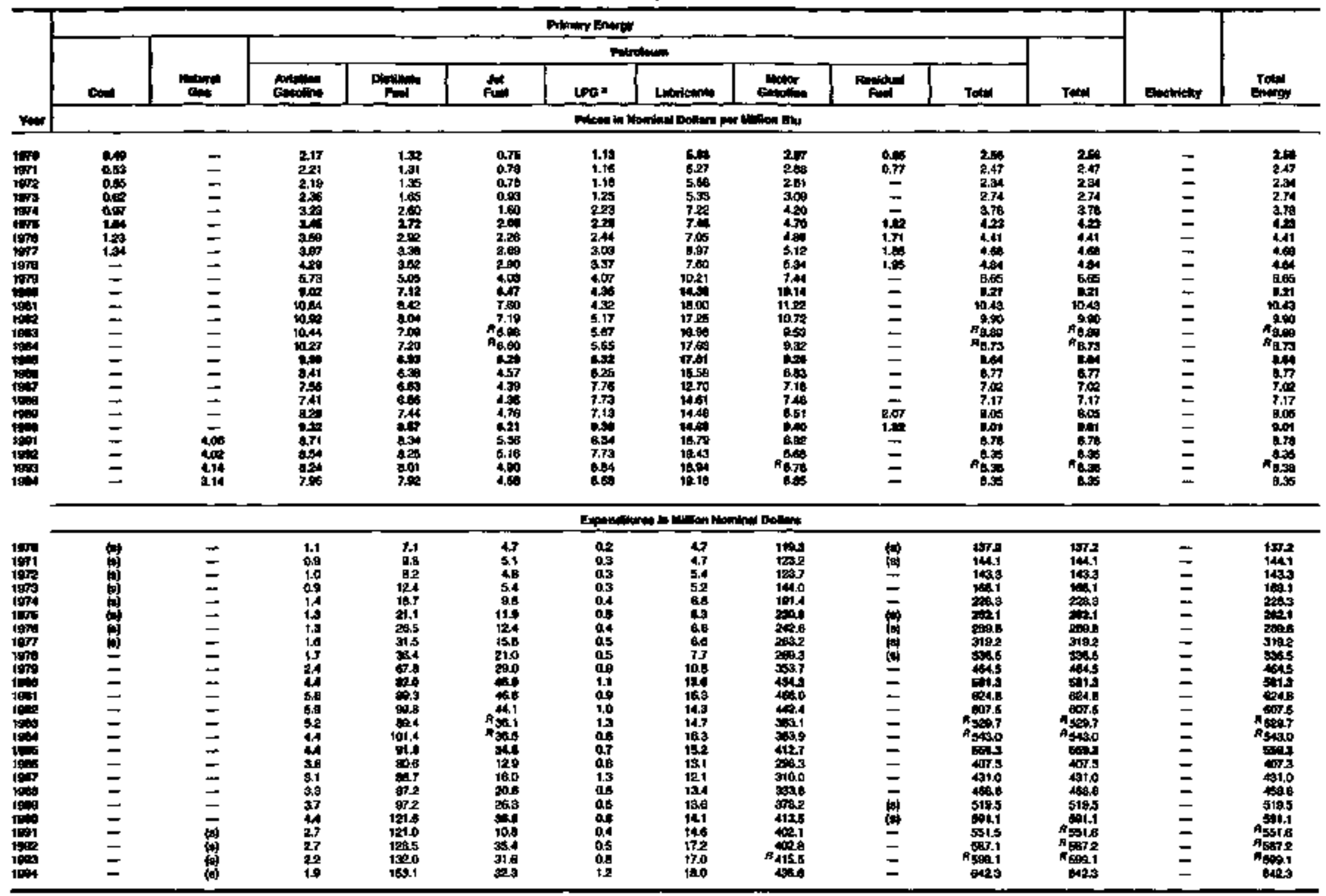

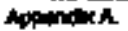


5 Table 252. Price und Expendlifure Estimates for Energy Inpurt al Electrlc Uhities by Bource, 1970-1994, South Dakota

\begin{tabular}{|c|c|c|c|c|c|c|c|c|c|}
\hline \multirow[b]{3}{*}{ Year } & & \multirow[b]{2}{*}{. } & \multicolumn{4}{|c|}{ Nonowern } & \multirow[b]{2}{*}{ Fin } & \multirow[b]{2}{*}{ Bionese } & \multirow[b]{2}{*}{ Enerd: } \\
\hline & cont & & menty & $\begin{array}{c}\text { Uon } \\
\text { on } \\
\end{array}$ & 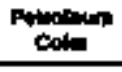 & Tation & & & \\
\hline & \multicolumn{9}{|c|}{ 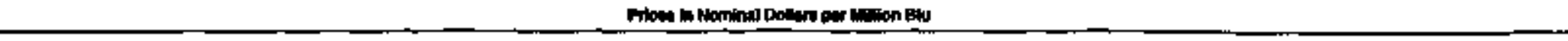 } \\
\hline 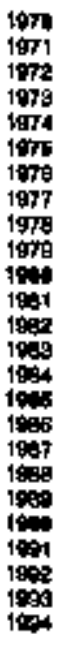 & 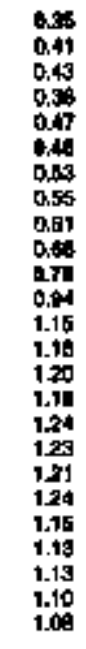 & 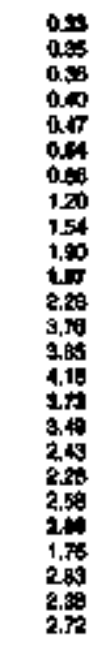 & 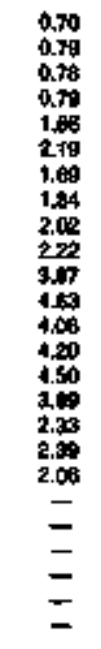 & 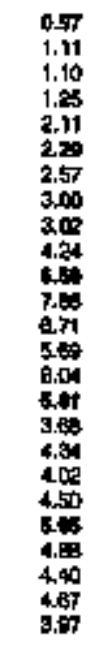 & $\begin{array}{l}z \\
z \\
z \\
z \\
z \\
z \\
z \\
z \\
z \\
z \\
z \\
z \\
z\end{array}$ & 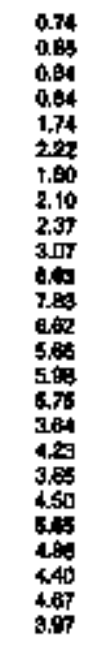 & $\begin{array}{l}z \\
z \\
z \\
z \\
z \\
z \\
z \\
z \\
z \\
= \\
z \\
z \\
z\end{array}$ & $\begin{array}{l}z \\
z \\
z \\
z \\
z \\
z \\
z \\
z \\
z \\
z \\
z \\
z \\
z\end{array}$ & 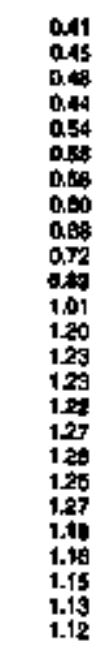 \\
\hline & \multicolumn{9}{|c|}{ 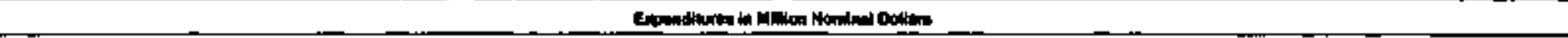 } \\
\hline 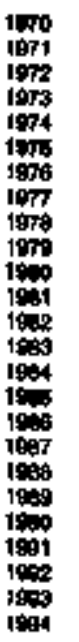 & 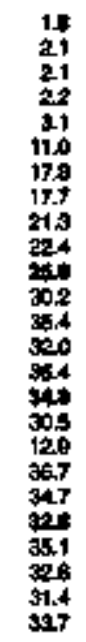 & 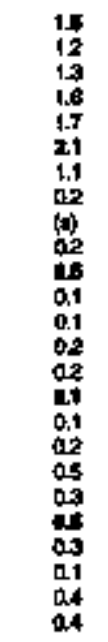 & 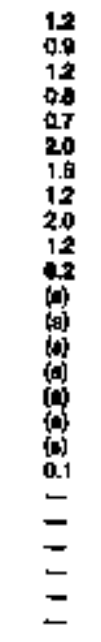 & 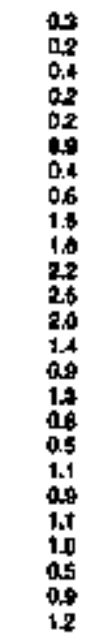 & $\begin{array}{l}z \\
z \\
z \\
z \\
z \\
z \\
z \\
z \\
z \\
z \\
z \\
z \\
z \\
z\end{array}$ & 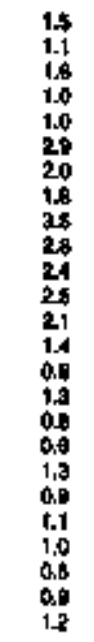 & $\begin{array}{l}z \\
z \\
z \\
z \\
z \\
z \\
z \\
z \\
z \\
z \\
z \\
z \\
z\end{array}$ & $\begin{array}{l}z \\
z \\
z \\
z \\
z \\
z \\
z \\
z \\
z \\
z \\
= \\
= \\
= \\
z\end{array}$ & 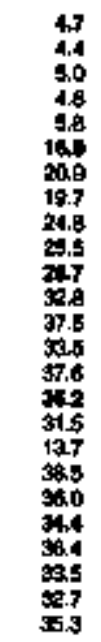 \\
\hline
\end{tabular}

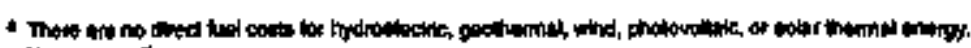

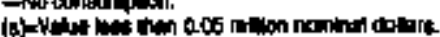

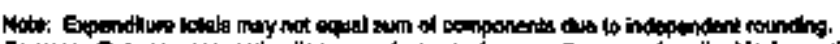

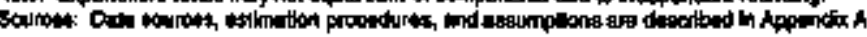


Toble 243. Energy Price and Experkiture Estimates by Source, 1970-1894, Tennessed

\begin{tabular}{|c|c|c|c|c|c|c|c|c|c|c|c|c|c|c|c|c|c|}
\hline \multirow[b]{4}{*}{ Yeat } & \multicolumn{14}{|c|}{ Prlandy Enerir } & \multirow{3}{*}{ 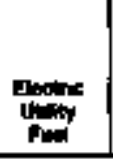 } & \multirow{3}{*}{ 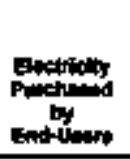 } & \multirow[b]{3}{*}{ Toror } \\
\hline & \multicolumn{3}{|c|}{ Con } & \multirow[b]{2}{*}{ יוm } & \multicolumn{7}{|c|}{ Prosian } & \multirow[b]{2}{*}{ moctorer } & \multirow[b]{2}{*}{ Evatum } & \multirow[b]{2}{*}{ Tok:e } & & & \\
\hline & Contin & (1) & $T=\mathbf{l}$ & & Onting & ה & Lman & Molor & Finguld & Dater & Toten & & & & & & \\
\hline & \multicolumn{17}{|c|}{ 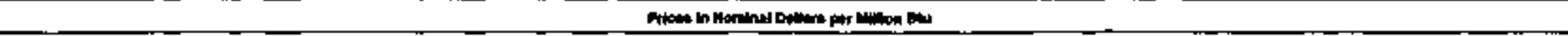 } \\
\hline \multirow[t]{2}{*}{ 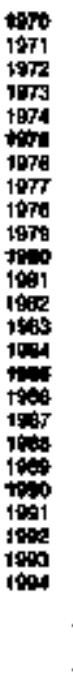 } & 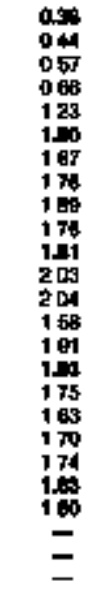 & 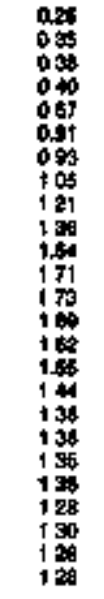 & 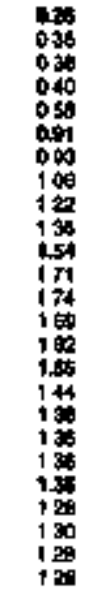 & 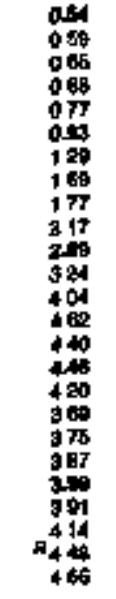 & 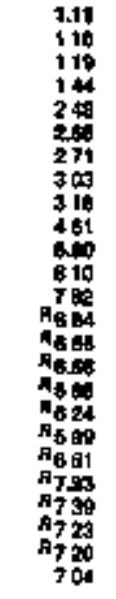 & 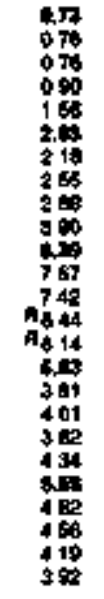 & 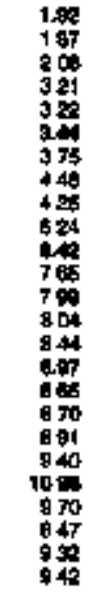 & 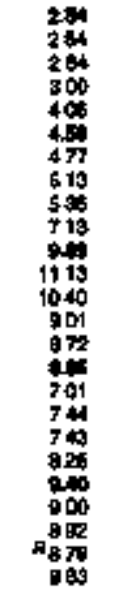 & 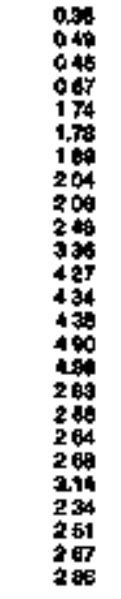 & 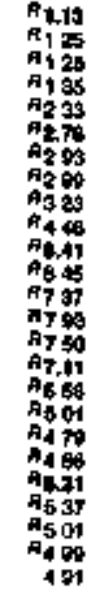 & 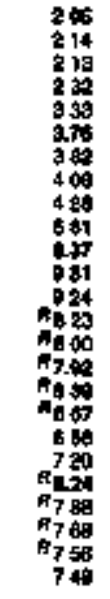 & 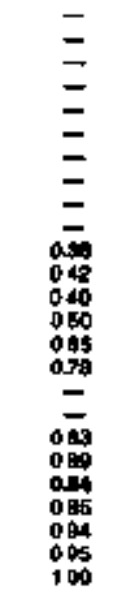 & 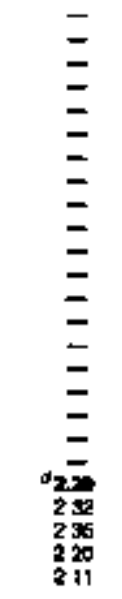 & 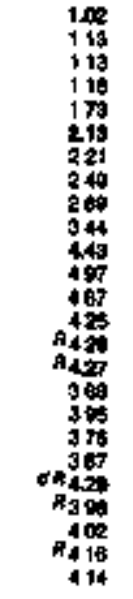 & 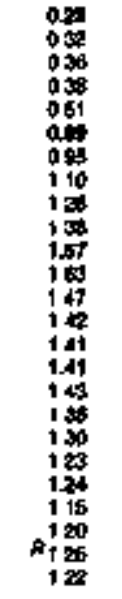 & 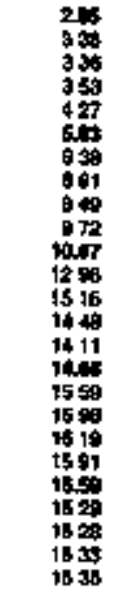 & 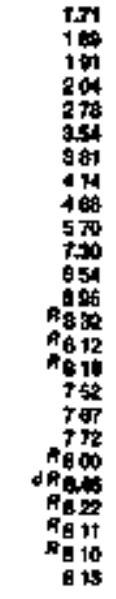 \\
\hline & \multicolumn{17}{|c|}{ 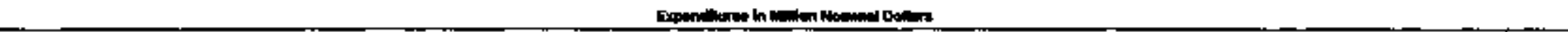 } \\
\hline 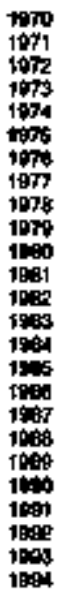 & 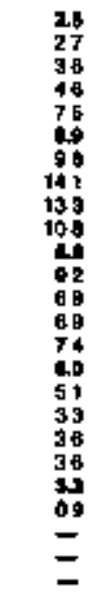 & 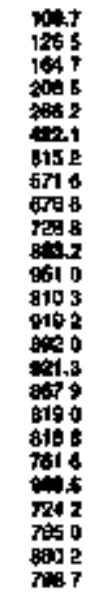 & 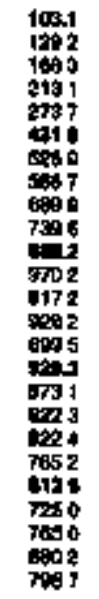 & 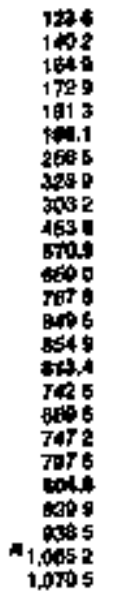 & 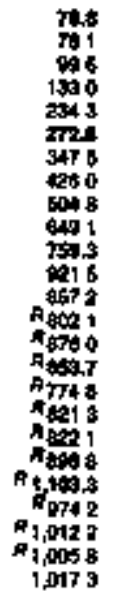 & 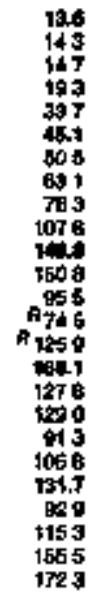 & 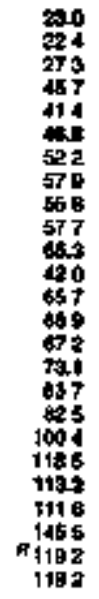 & 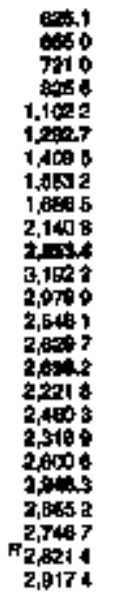 & $\begin{array}{r}1.1 \\
04 \\
60 \\
06 \\
51 \\
43 \\
282 \\
329 \\
186 \\
330 \\
292 \\
285 \\
153 \\
200 \\
128 \\
71 \\
71 \\
45 \\
55 \\
57 \\
46 \\
40 \\
45 \\
964 \\
84\end{array}$ & 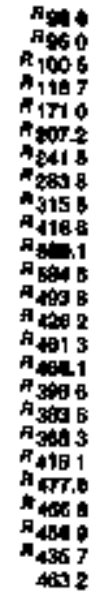 & 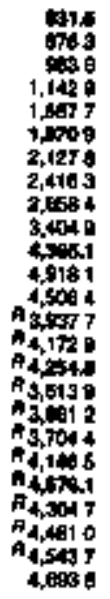 & $\begin{array}{l}= \\
= \\
= \\
= \\
= \\
= \\
21 \\
216 \\
416 \\
766 \\
697 \\
61.1 \\
= \\
349 \\
1492 \\
1350 \\
1511 \\
1564 \\
356 \\
1277\end{array}$ & $\begin{array}{l}= \\
= \\
= \\
= \\
= \\
= \\
= \\
= \\
= \\
= \\
= \\
40.1 \\
447 \\
379 \\
3 \neq 0\end{array}$ & 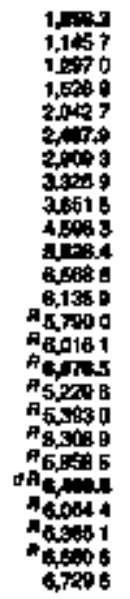 & 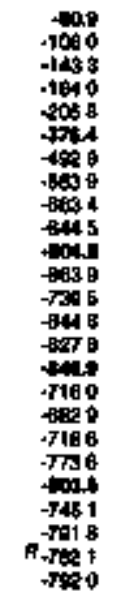 & 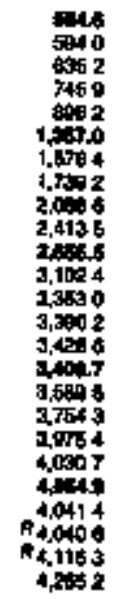 & 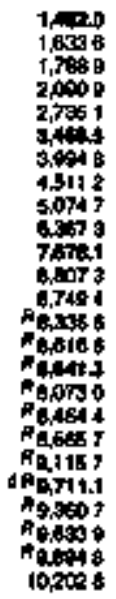 \\
\hline
\end{tabular}

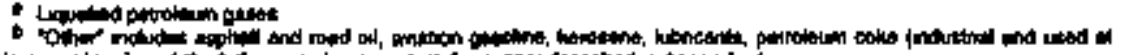

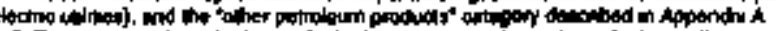

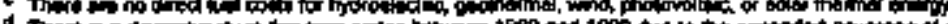

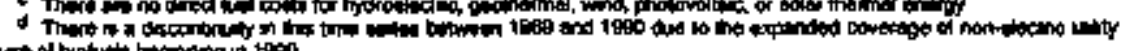

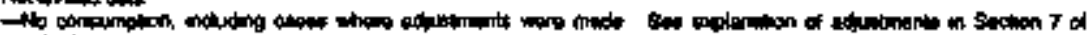

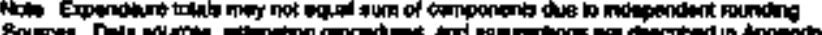




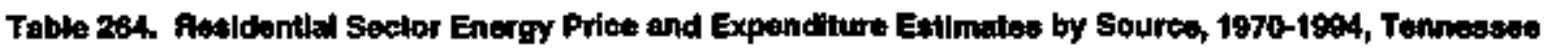

\begin{tabular}{|c|c|c|c|c|c|c|c|c|c|c|}
\hline \multirow[b]{4}{*}{$\mathrm{rm}$} & \multicolumn{8}{|c|}{ 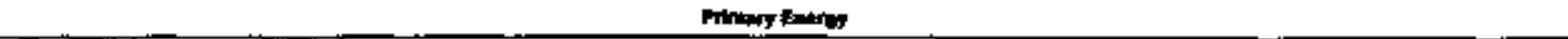 } & \multirow[b]{3}{*}{ Eantery } & \multirow{3}{*}{ " } \\
\hline & \multirow[b]{2}{*}{ cont } & \multirow{2}{*}{ 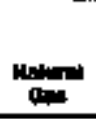 } & \multicolumn{4}{|c|}{ An: } & \multirow[b]{2}{*}{ 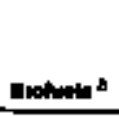 } & \multirow[b]{2}{*}{ Toter } & & \\
\hline & & & Fis & 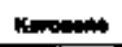 & uma* & Total & & & & \\
\hline & \multicolumn{9}{|c|}{ 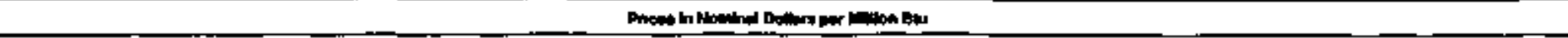 } & \\
\hline \multirow[t]{2}{*}{ 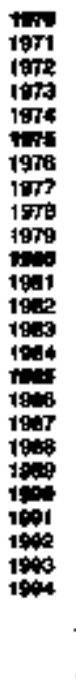 } & 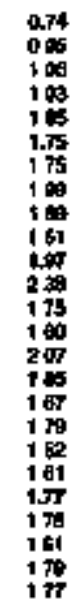 & 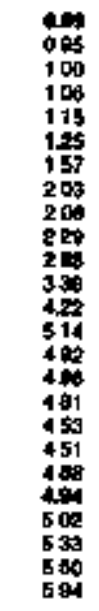 & 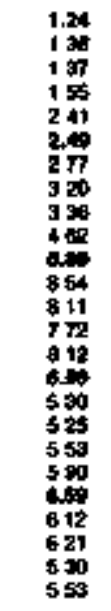 & 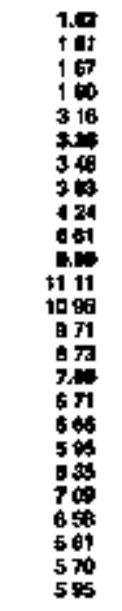 & 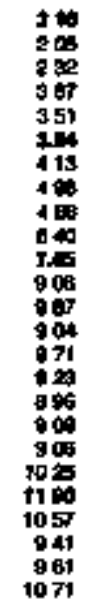 & 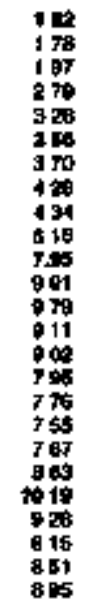 & 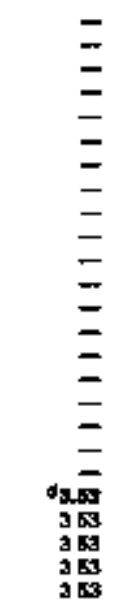 & 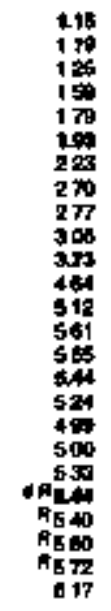 & 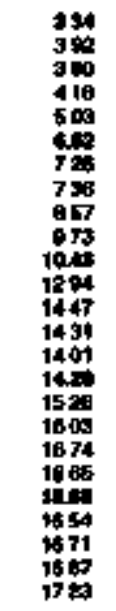 & 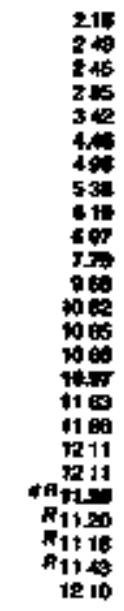 \\
\hline & \multicolumn{10}{|c|}{ 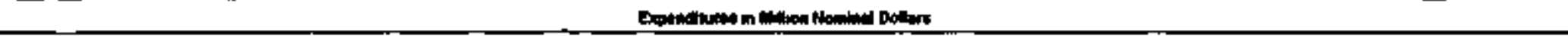 } \\
\hline 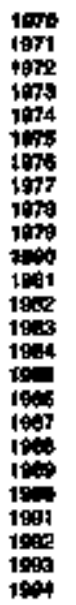 & 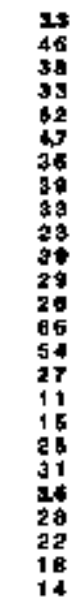 & 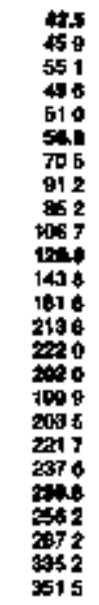 & $\begin{array}{l}12 \\
14 \\
17 \\
22 \\
31 \\
34 \\
69 \\
72 \\
75 \\
109 \\
124 \\
234 \\
160 \\
119 \\
134 \\
89 \\
51 \\
70 \\
73 \\
64 \\
11 \\
96 \\
79 \\
63 \\
07\end{array}$ & 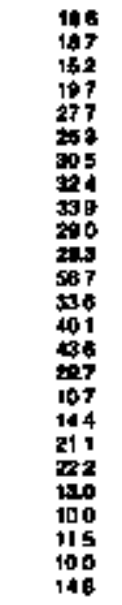 & 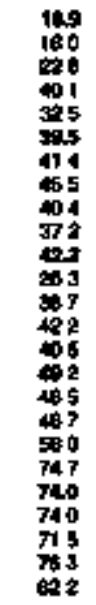 & 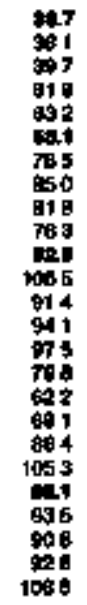 & 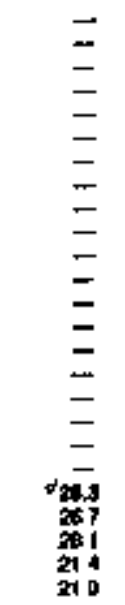 & 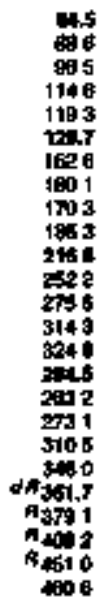 & 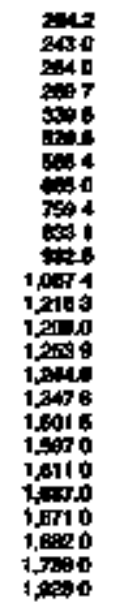 & 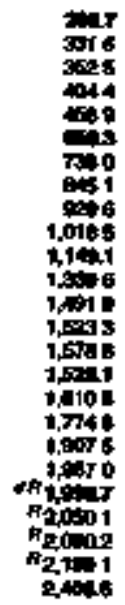 \\
\hline
\end{tabular}

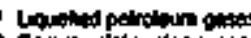

Antorsed deth

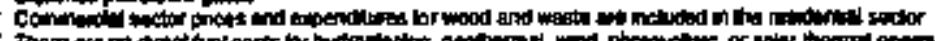

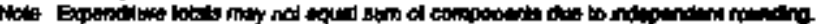

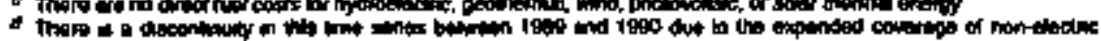

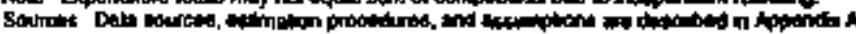




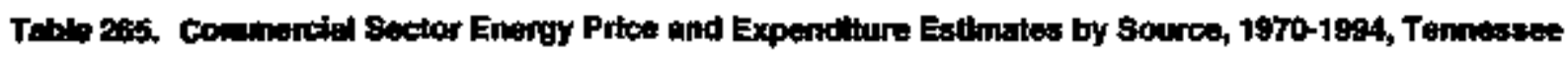

\begin{tabular}{|c|c|c|c|c|c|c|c|c|c|c|c|}
\hline & \multicolumn{9}{|c|}{ Miment Entier } & \multirow[b]{3}{*}{ Electriaty } & \multirow[b]{3}{*}{ Tats } \\
\hline & & \multirow[b]{2}{*}{ natid } & \multicolumn{6}{|c|}{ 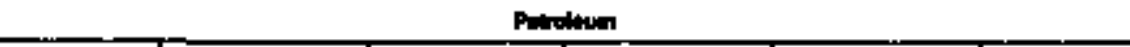 } & \multirow[b]{2}{*}{ السئ Tr } & & \\
\hline & cen & & Fin & Heresomen & LPGA & mater & fontur & Tot & & & \\
\hline $\mathbf{m}$ & \multicolumn{11}{|c|}{ 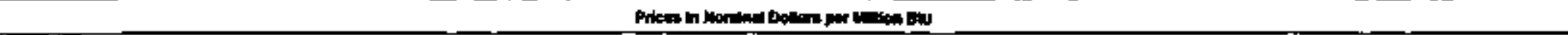 } \\
\hline \multirow[t]{2}{*}{ 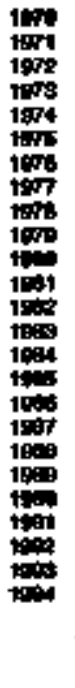 } & 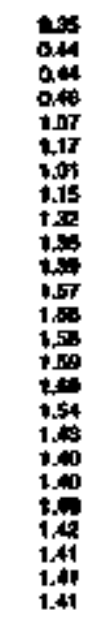 & 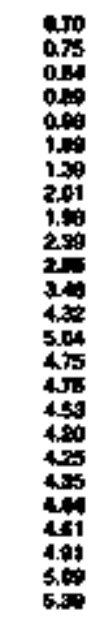 & 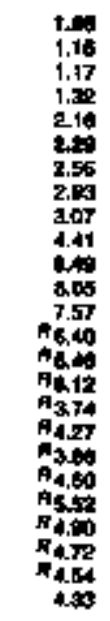 & 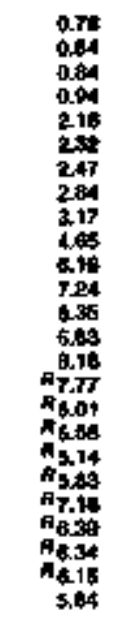 & 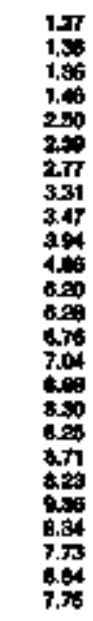 & 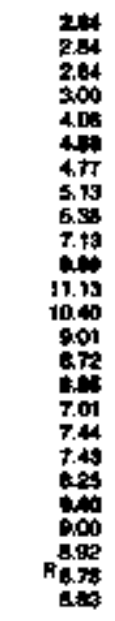 & 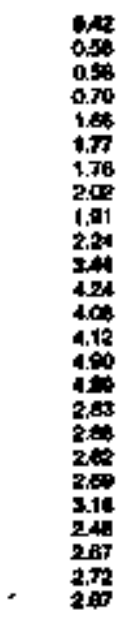 & 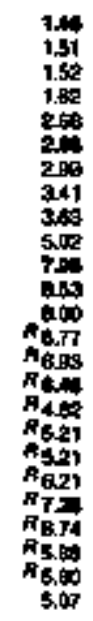 & 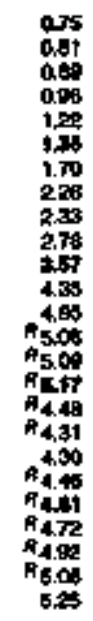 & 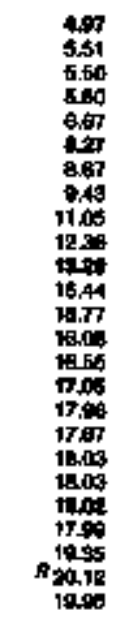 & 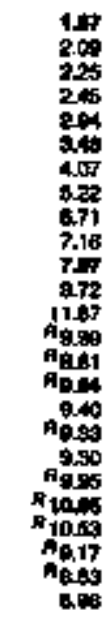 \\
\hline & \multicolumn{11}{|c|}{ 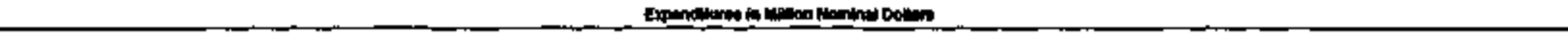 } \\
\hline 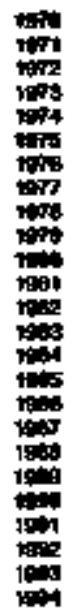 & 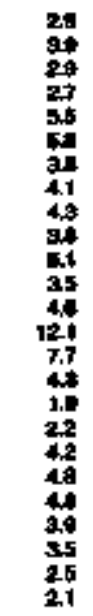 & 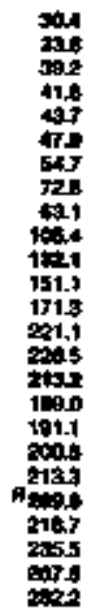 & 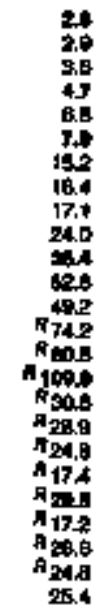 & 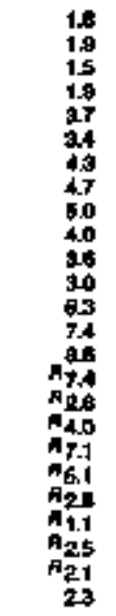 & 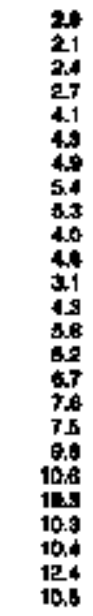 & 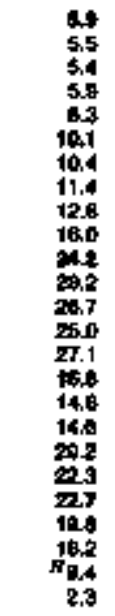 & 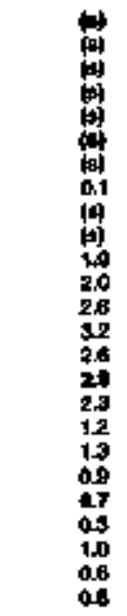 & 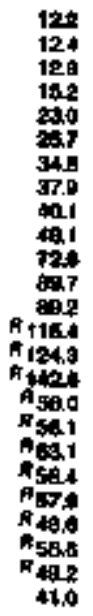 & 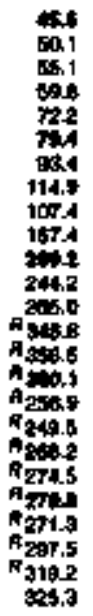 & 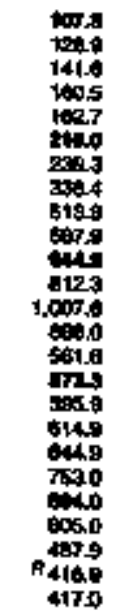 & 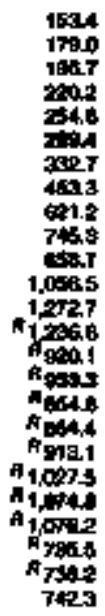 \\
\hline
\end{tabular}

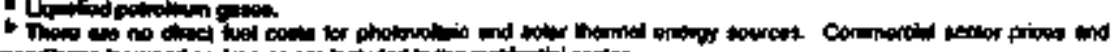

(C)

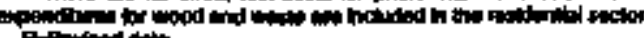




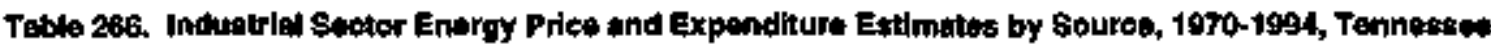

\begin{tabular}{|c|c|c|c|c|c|c|c|c|c|c|c|c|c|c|c|c|}
\hline \multicolumn{15}{|c|}{ Prinm EMany } & \multirow[b]{3}{*}{ lloctriety } & \multirow[b]{3}{*}{ 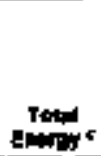 } \\
\hline \multicolumn{3}{|c|}{ cell } & \multirow[b]{2}{*}{ Intal } & \multicolumn{9}{|c|}{ Adrelanm } & \multirow[b]{2}{*}{ Olotuna } & \multirow[b]{2}{*}{ Totw } & & \\
\hline Cosing & Bar & Toll & & Aashand and & Dhallon & Karoment & LFB* & Luthents: & motor & Reviver & oners & Tot & & & & \\
\hline \multicolumn{17}{|c|}{ 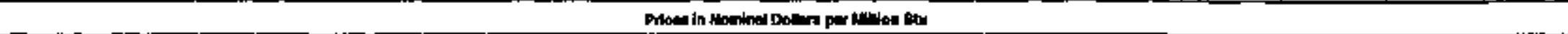 } \\
\hline 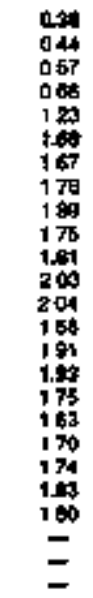 & 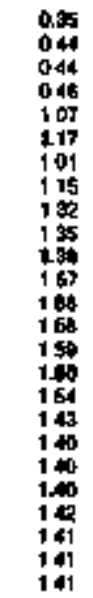 & 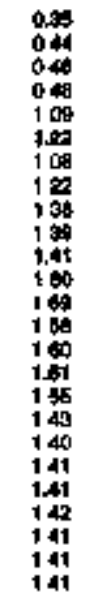 & 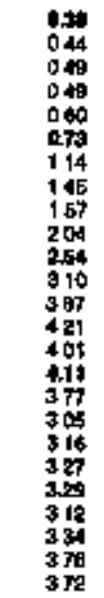 & 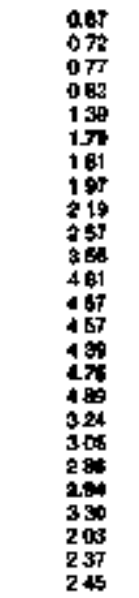 & 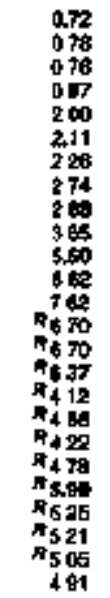 & 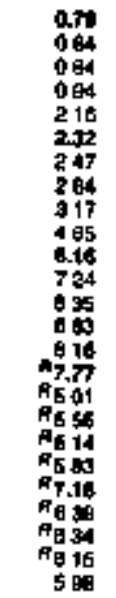 & 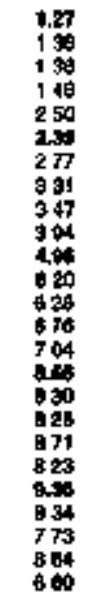 & 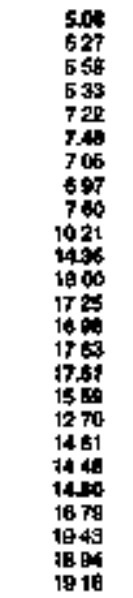 & 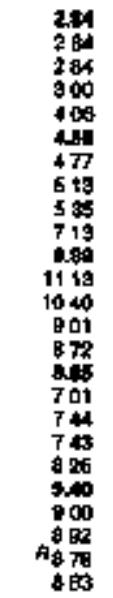 & 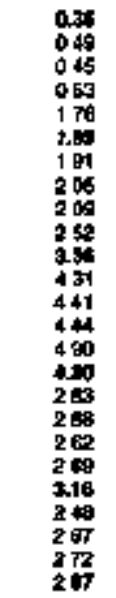 & 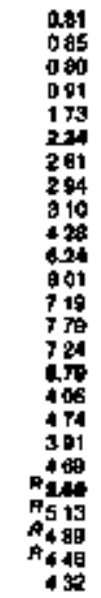 & 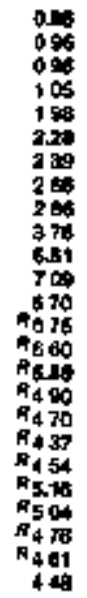 & $\begin{array}{l}= \\
= \\
= \\
= \\
= \\
= \\
= \\
= \\
= \\
= \\
= \\
0 \\
100 \\
148 \\
148 \\
144\end{array}$ & 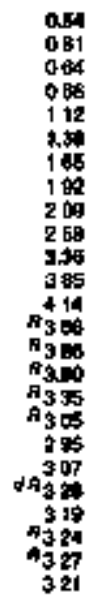 & 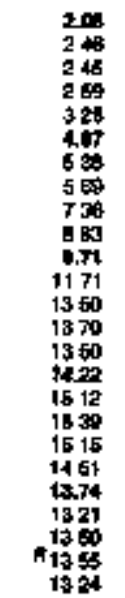 & 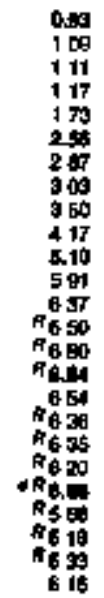 \\
\hline
\end{tabular}

\begin{tabular}{|c|c|c|c|c|c|c|c|c|c|c|c|c|c|c|c|c|c|}
\hline \multirow[b]{2}{*}{ 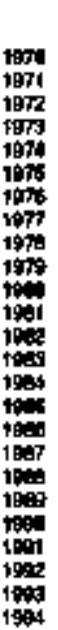 } & \multicolumn{17}{|c|}{ 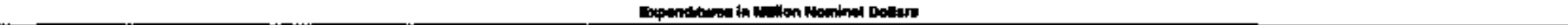 } \\
\hline & 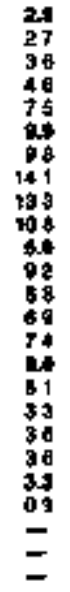 & 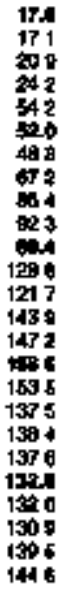 & 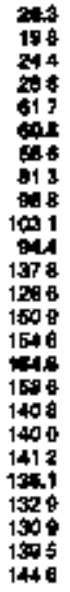 & 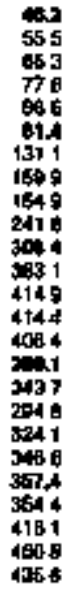 & 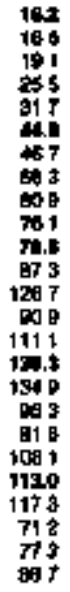 & 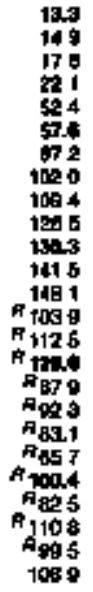 & 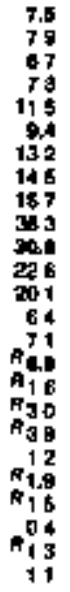 & 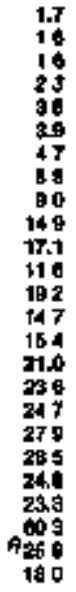 & 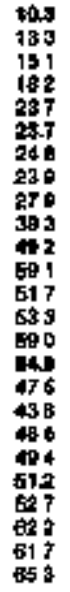 & 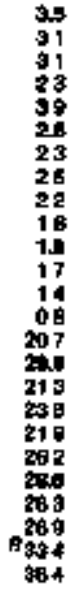 & 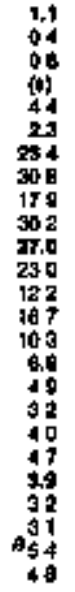 & 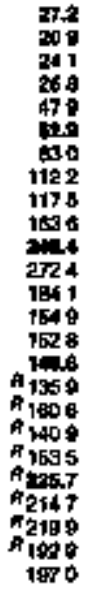 & 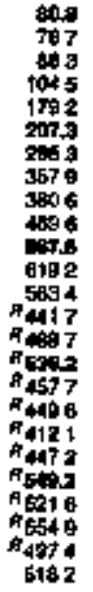 & $\begin{array}{l}= \\
= \\
= \\
= \\
= \\
= \\
= \\
= \\
= \\
= \\
= \\
= \\
1158 \\
170 \\
182 \\
186 \\
178\end{array}$ & 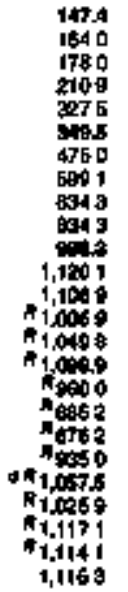 & 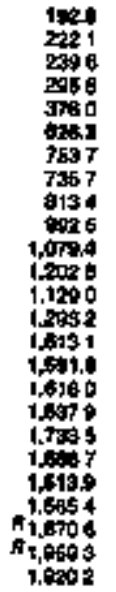 & 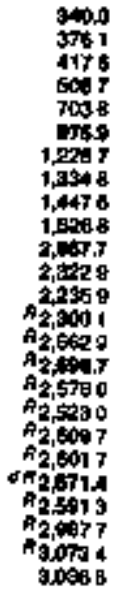 \\
\hline
\end{tabular}

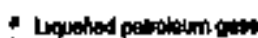

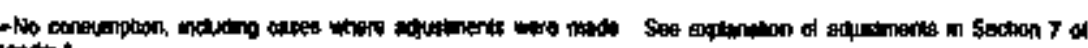

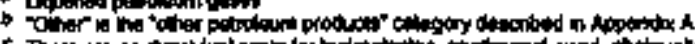

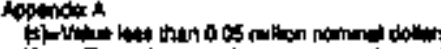

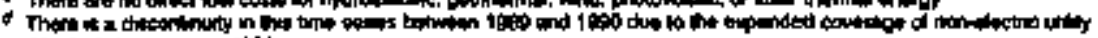

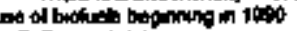

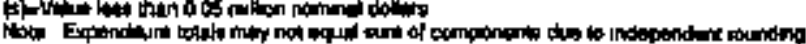

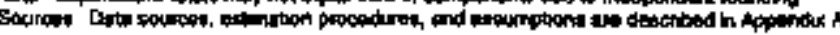


Table 267. Transportation Sector Energy Price and Expendivure Estintes by Scurce, 1970-1994, Tennessee

\begin{tabular}{|c|c|c|c|c|c|c|c|c|c|c|c|c|c|}
\hline \multirow[b]{4}{*}{ Yenr } & \multicolumn{11}{|c|}{ Primery Energy } & \multirow[b]{3}{*}{ Eectriatity } & \multirow[b]{3}{*}{ Toled } \\
\hline & & \multirow[b]{2}{*}{ 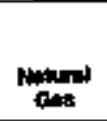 } & \multicolumn{8}{|c|}{ Purreintin } & \multirow[b]{2}{*}{ Tots } & & \\
\hline & conl & & 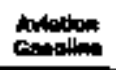 & Otorinem & fater & LPo: & Letrowits & Motom & Propictur & Total & & & \\
\hline & \multicolumn{13}{|c|}{ 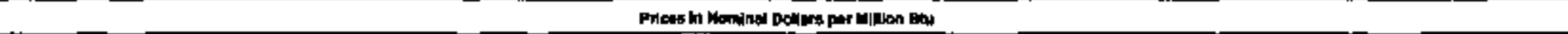 } \\
\hline \multirow[t]{2}{*}{ 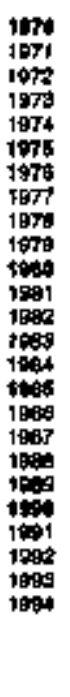 } & $\begin{array}{l}0.05 \\
0.40 \\
0.44 \\
0.46 \\
107 \\
1.17 \\
101 \\
1015 \\
= \\
= \\
= \\
= \\
= \\
= \\
= \\
= \\
= \\
= \\
=\end{array}$ & $\begin{array}{l}= \\
= \\
= \\
= \\
= \\
= \\
= \\
= \\
= \\
= \\
= \\
= \\
= \\
4.14 \\
300 \\
428 \\
451 \\
551\end{array}$ & 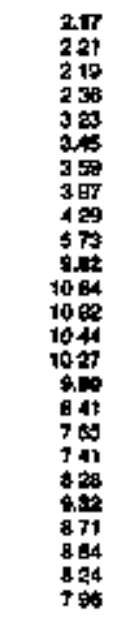 & 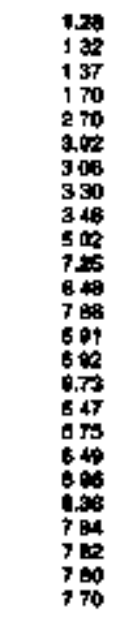 & 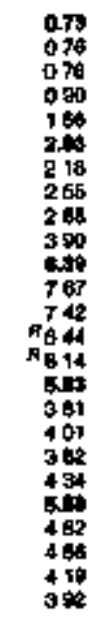 & 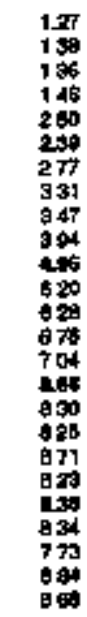 & 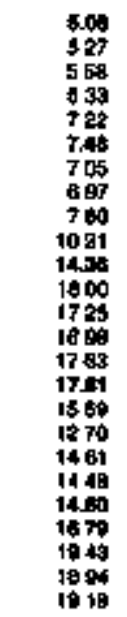 & 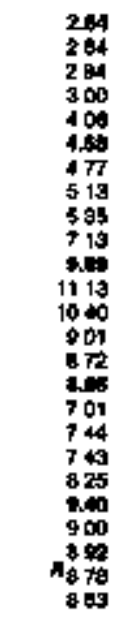 & 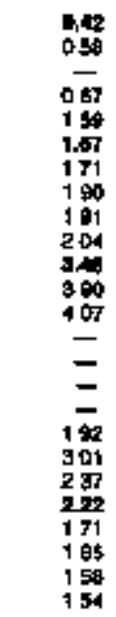 & 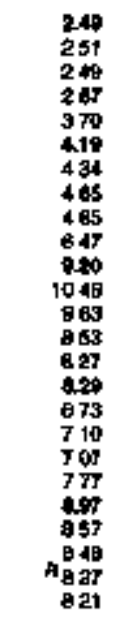 & 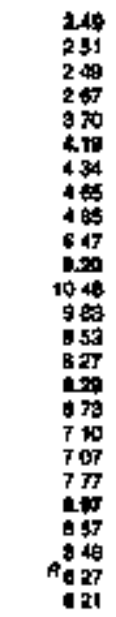 & 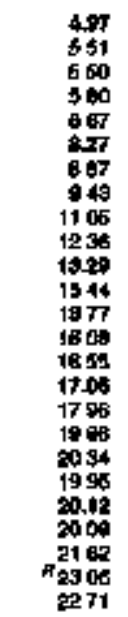 & 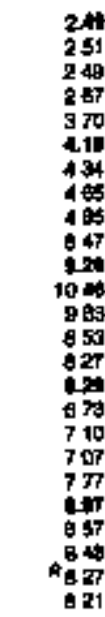 \\
\hline & \multicolumn{13}{|c|}{ 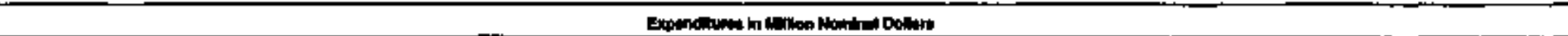 } \\
\hline 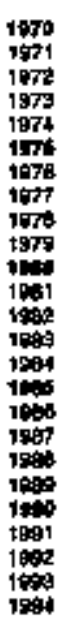 & $\begin{array}{l}10 \\
00 \\
0 \\
0 \\
0 \\
= \\
= \\
= \\
= \\
= \\
= \\
= \\
= \\
= \\
= \\
=\end{array}$ & 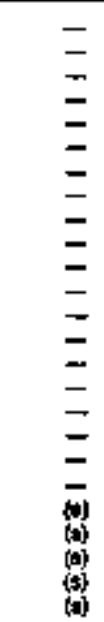 & 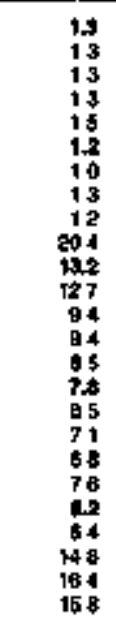 & 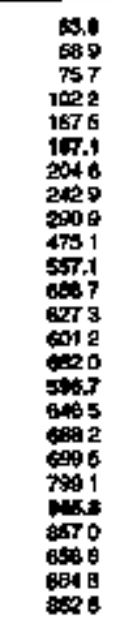 & 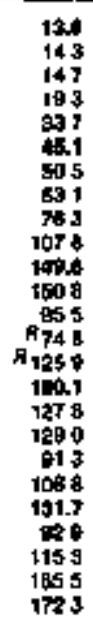 & $\begin{array}{l}0.5 \\
05 \\
05 \\
07 \\
11 \\
1.1 \\
18 \\
15 \\
21 \\
18 \\
1.1 \\
20 \\
35 \\
48 \\
01 \\
52 \\
61 \\
38 \\
47 \\
47 \\
47 \\
41 \\
94 \\
47 \\
78\end{array}$ & 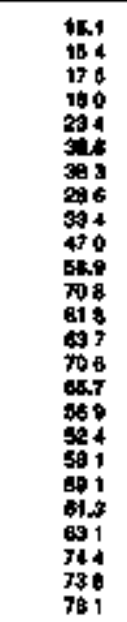 & 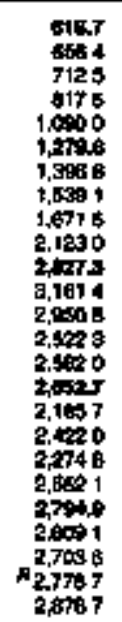 & 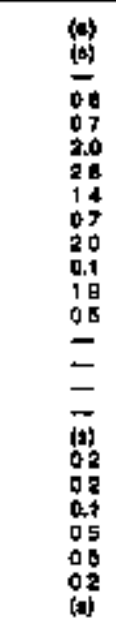 & 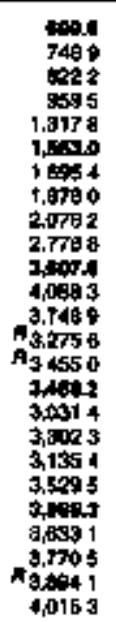 & 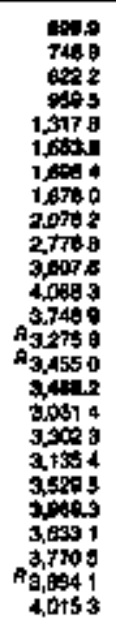 & 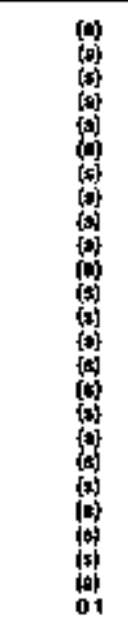 & 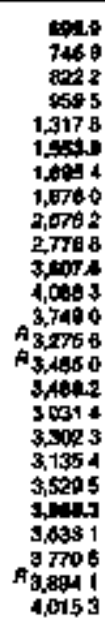 \\
\hline
\end{tabular}

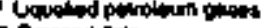

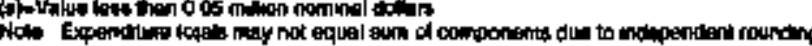

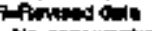
-No corting 


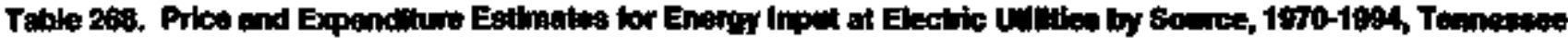

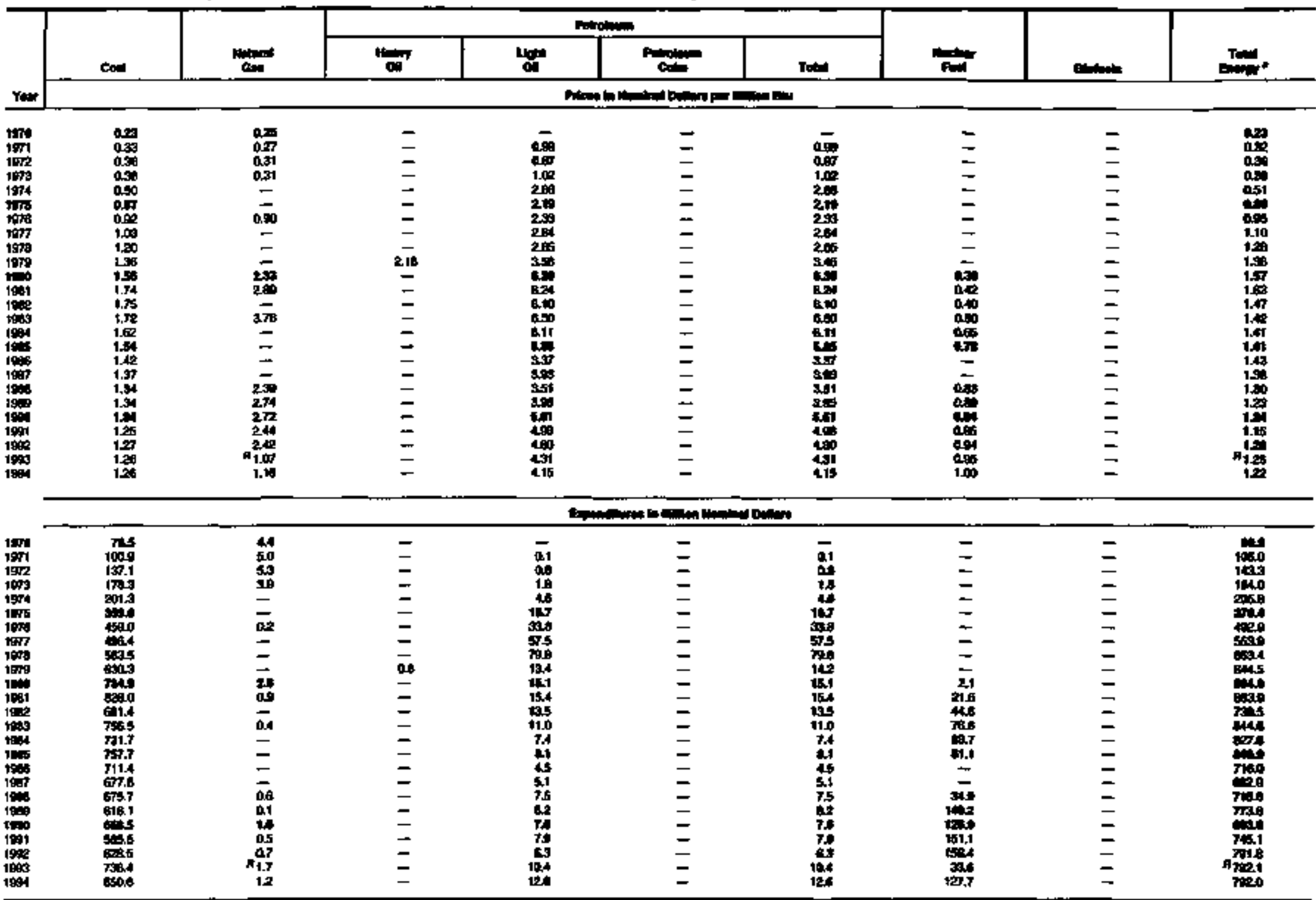

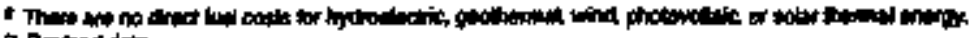
Antroed dis.

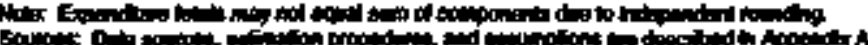

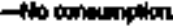




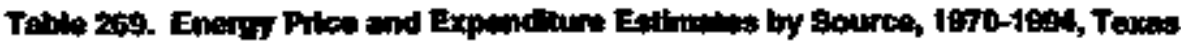

\begin{tabular}{|c|c|c|c|c|c|c|c|c|c|c|c|c|c|c|c|c|c|}
\hline \multirow[b]{3}{*}{ Nem } & \multicolumn{14}{|c|}{ 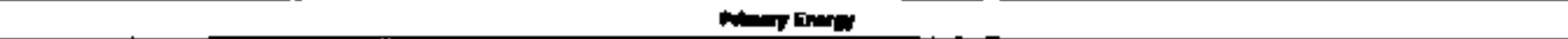 } & \multirow{2}{*}{ Pons } & \multirow{2}{*}{ 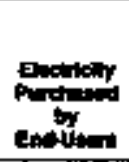 } & \multirow{2}{*}{ s } \\
\hline & \multicolumn{3}{|c|}{ ant } & דיi & \multicolumn{7}{|c|}{ Ninn } & nem & OMnom & $T=\|^{x}$ & & & \\
\hline & \multicolumn{17}{|c|}{ 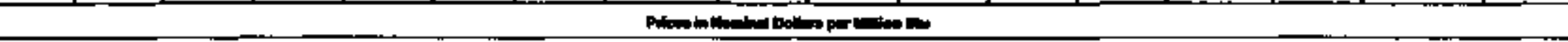 } \\
\hline 急 & 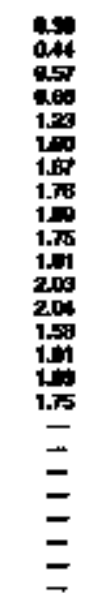 & 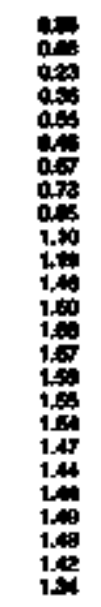 & 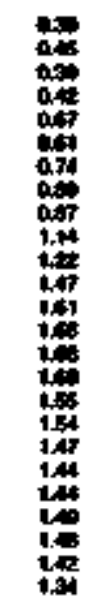 & 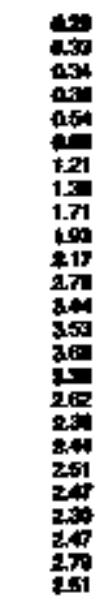 & 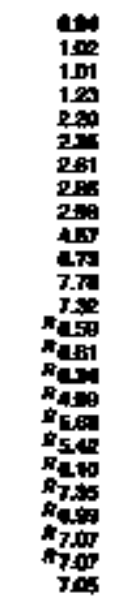 & 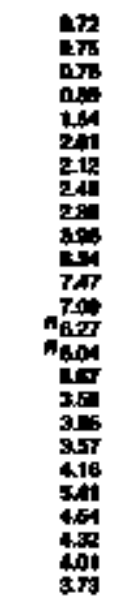 & 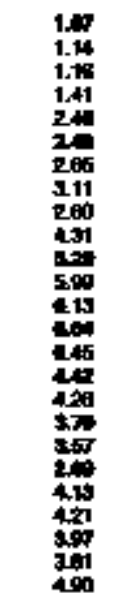 & 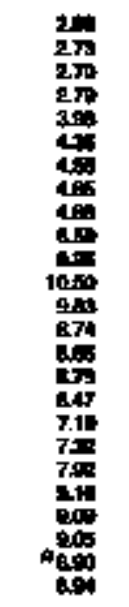 & 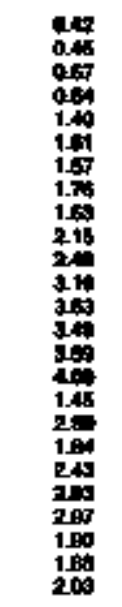 & 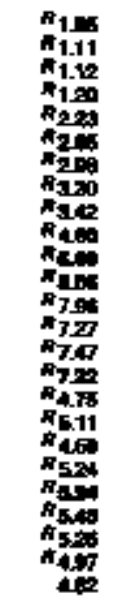 & 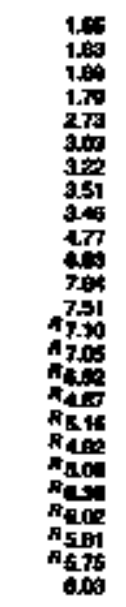 & 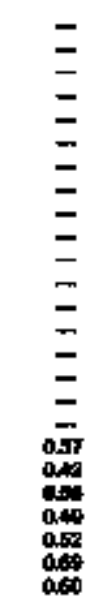 & 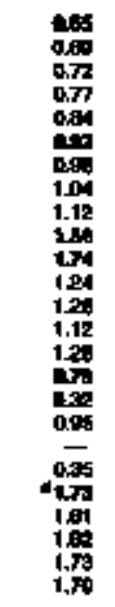 & 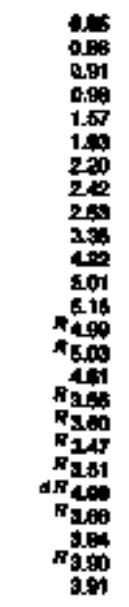 & 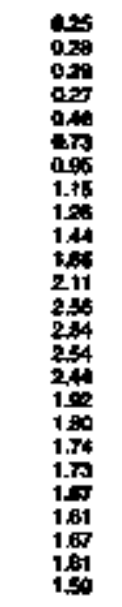 & 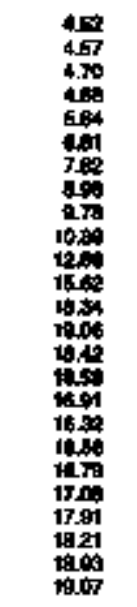 & 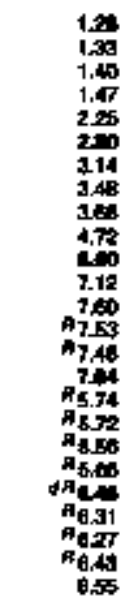 \\
\hline - & 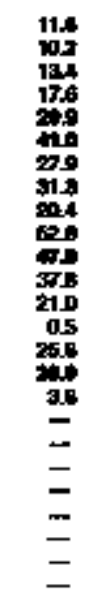 & 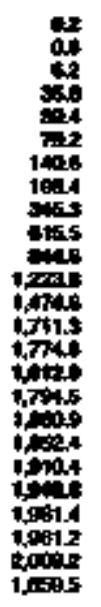 & 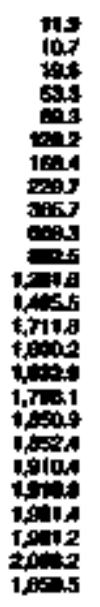 & 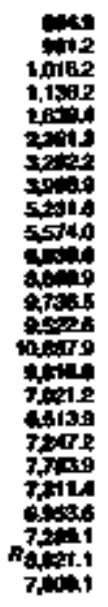 & 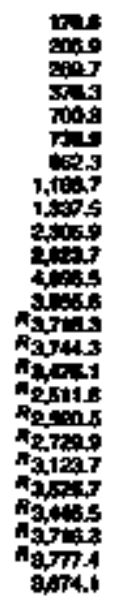 & 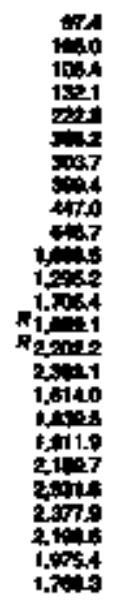 & 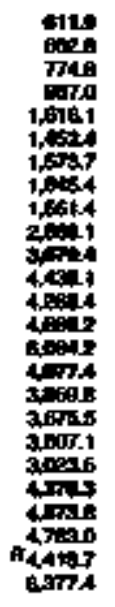 & 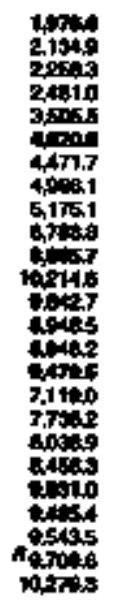 & 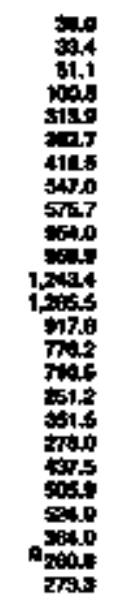 & 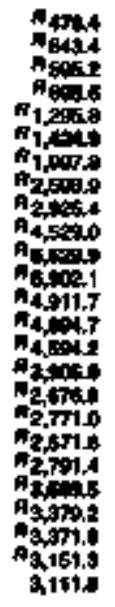 & 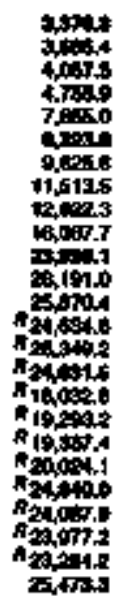 & $\begin{array}{l}= \\
z \\
= \\
= \\
= \\
= \\
= \\
= \\
= \\
\bar{z} \\
18.1 \\
416 \\
1093 \\
1050 \\
91.7\end{array}$ & 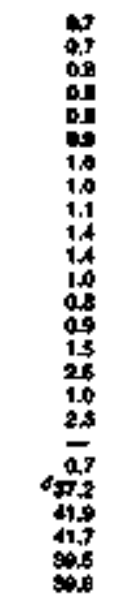 & 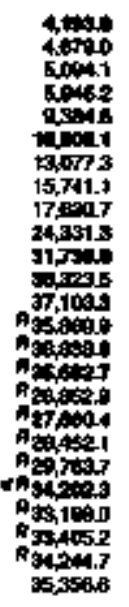 & 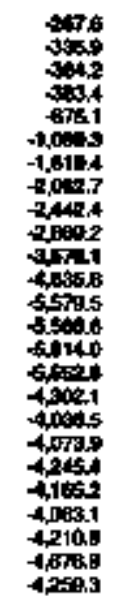 & 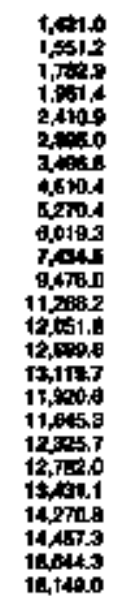 & 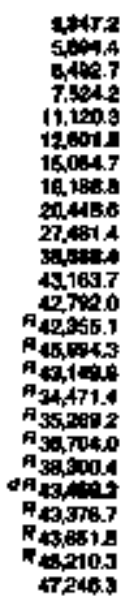 \\
\hline
\end{tabular}

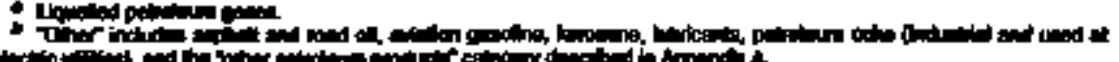

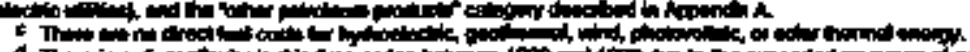

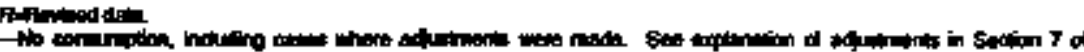
a The 
Tahle 270. Rosidentiol Sector Energy Prlce and Expenditure Esllmates by Source, 1970-1944, Texo:

\begin{tabular}{|c|c|c|c|c|c|c|c|c|c|c|}
\hline \multirow[b]{4}{*}{ rover } & \multicolumn{8}{|c|}{ Prisery Enegy } & \multirow[b]{3}{*}{ Enatenter } & \multirow[b]{3}{*}{ 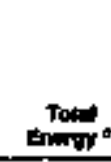 } \\
\hline & \multirow[b]{2}{*}{ cos } & \multirow[b]{2}{*}{ Matid } & \multicolumn{4}{|c|}{ Motrolusen } & \multirow[b]{2}{*}{ 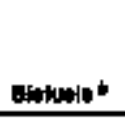 } & \multirow[b]{2}{*}{ Tot: } & & \\
\hline & & & 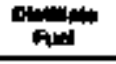 & Kerouma & 눙: & Tonat & & & & \\
\hline & \multicolumn{9}{|c|}{ 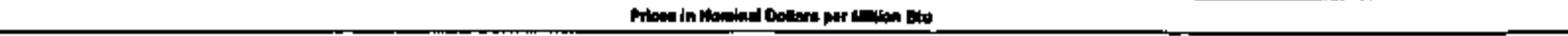 } & \\
\hline \multirow[t]{2}{*}{ 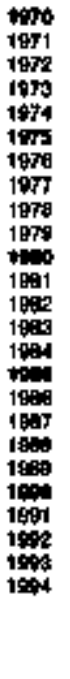 } & 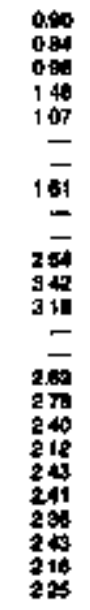 & 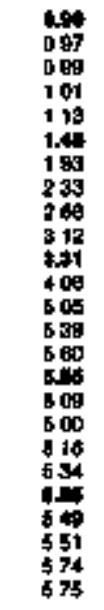 & 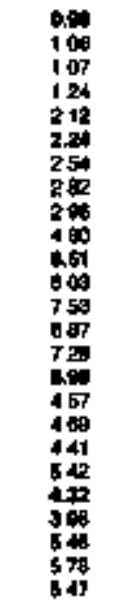 & 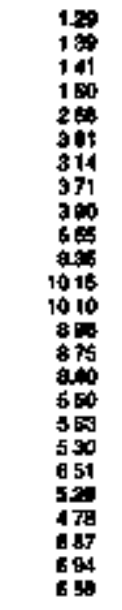 & 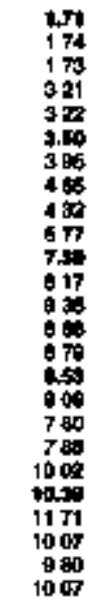 & 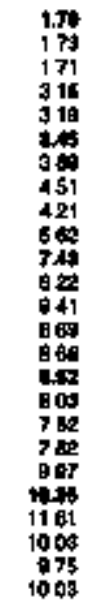 & $\begin{array}{l}= \\
= \\
= \\
= \\
= \\
= \\
= \\
= \\
= \\
= \\
= \\
= \\
450 \\
353 \\
353 \\
363\end{array}$ & 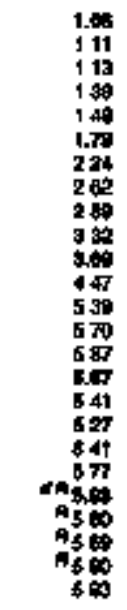 & 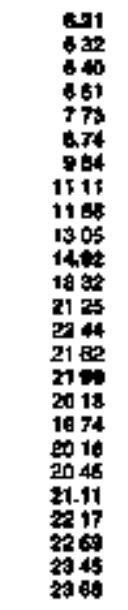 & 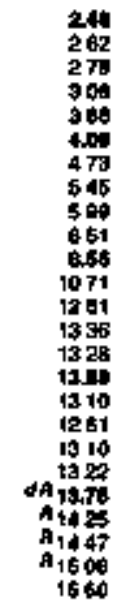 \\
\hline & \multicolumn{10}{|c|}{ 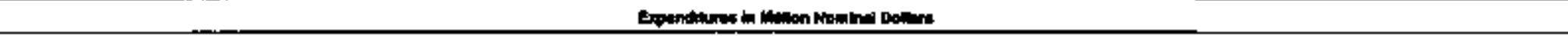 } \\
\hline 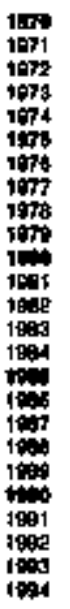 & 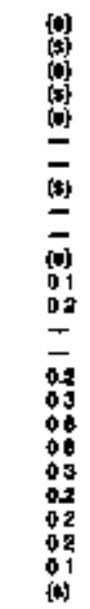 & 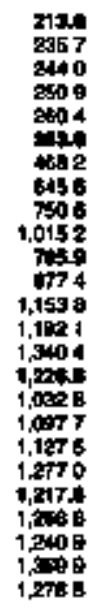 & 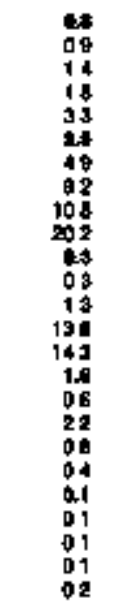 & $\begin{array}{l}82 \\
02 \\
03 \\
04 \\
08 \\
07 \\
05 \\
07 \\
09 \\
90 \\
14 \\
06 \\
04 \\
57 \\
57 \\
54 \\
14 \\
10 \\
18 \\
16 \\
60 \\
08 \\
06 \\
62 \\
07\end{array}$ & 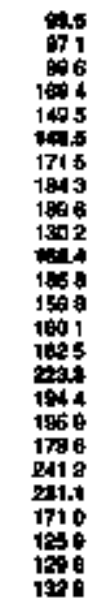 & 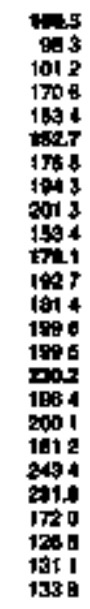 & $\begin{array}{l}z \\
= \\
= \\
= \\
= \\
= \\
= \\
= \\
= \\
= \\
= \\
= \\
= \\
200 \\
217 \\
20 \\
00 \\
190\end{array}$ & 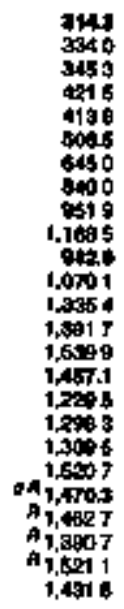 & 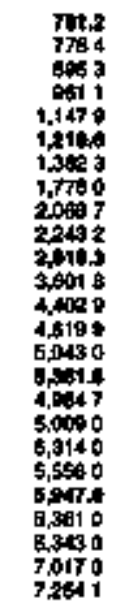 & 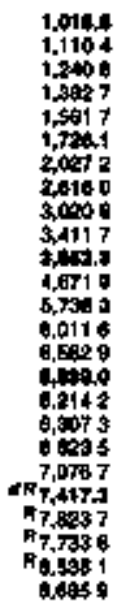 \\
\hline
\end{tabular}




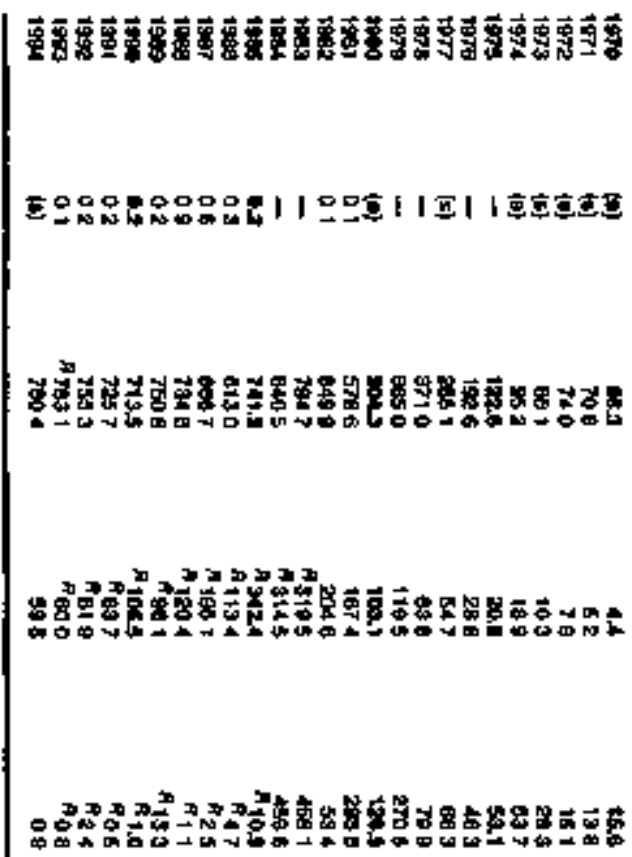

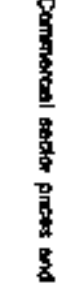

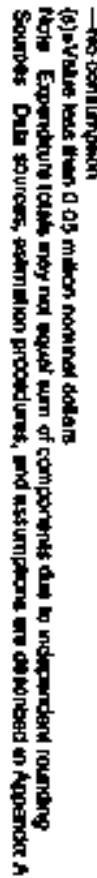

บิ

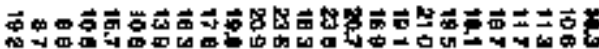

문

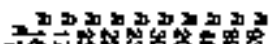

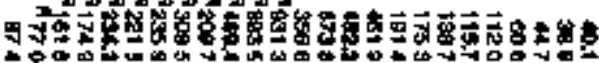

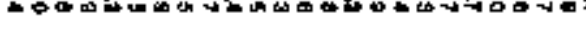

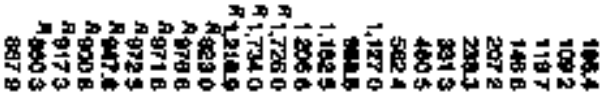

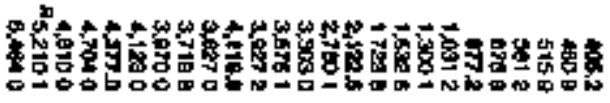

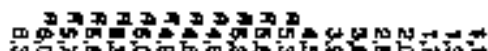
迸过 8

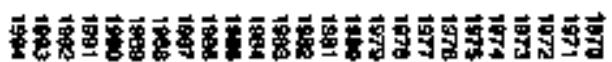

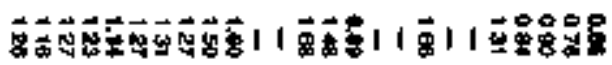

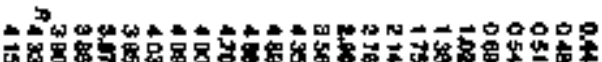

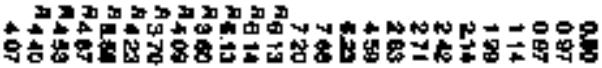

W

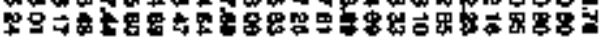

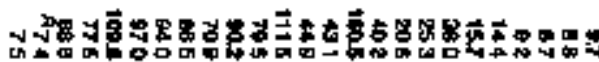

ח

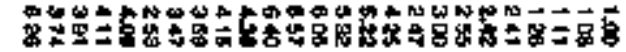

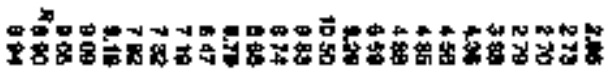

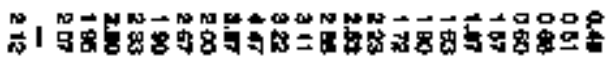

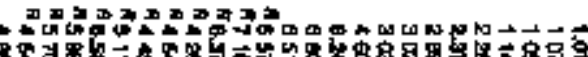

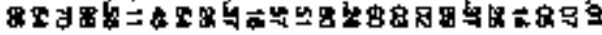

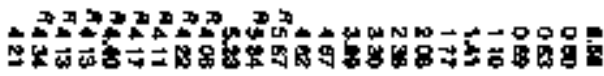

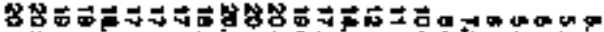
万人

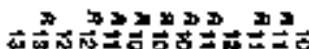

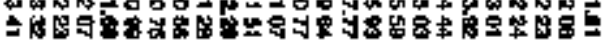

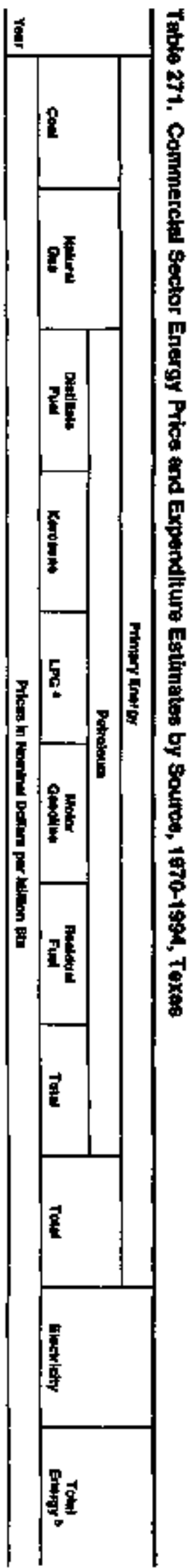

$\omega \times x m-1$ 


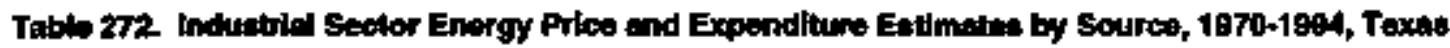

\begin{tabular}{|c|c|c|c|c|c|c|c|c|c|c|c|c|c|c|c|c|c|}
\hline & \multicolumn{15}{|c|}{ manoy anews } & \multirow[b]{3}{*}{ Exominir } & \multirow[b]{3}{*}{ Totist } \\
\hline & \multicolumn{3}{|c|}{ and } & \multirow[b]{2}{*}{ 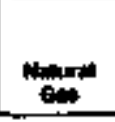 } & \multicolumn{9}{|c|}{ 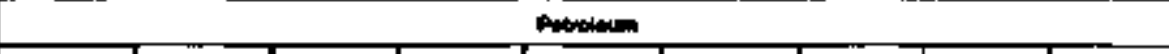 } & \multirow[b]{2}{*}{ Bontis } & \multirow[b]{2}{*}{ 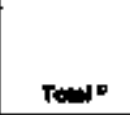 } & & \\
\hline & colct & id & Tow & & 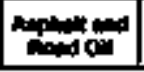 & oting & Noromene & $\mathbf{M a *}$ & Lampentis & inding & manterd & onts & Tow & & & & \\
\hline שמץ & \multicolumn{17}{|c|}{ 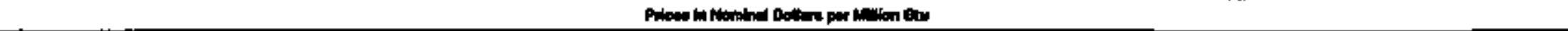 } \\
\hline \multirow[t]{2}{*}{ 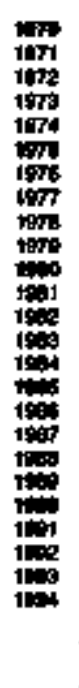 } & 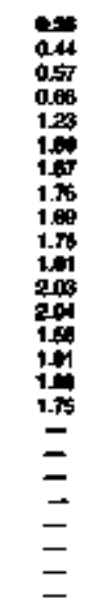 & 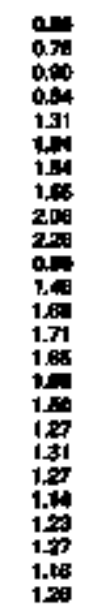 & 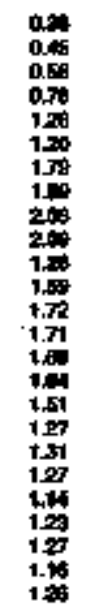 & 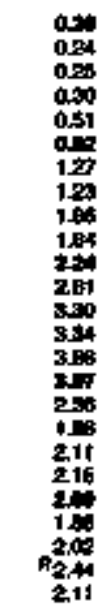 & 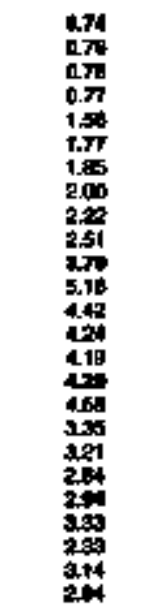 & 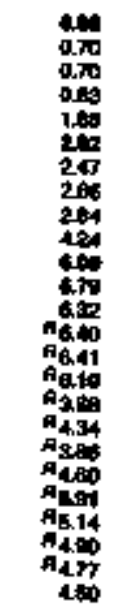 & 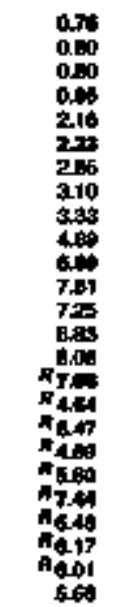 & 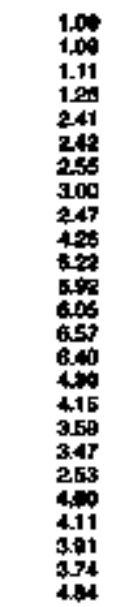 & 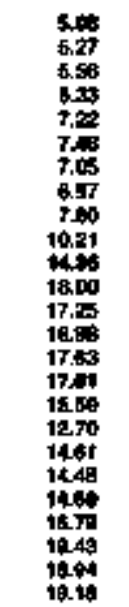 & 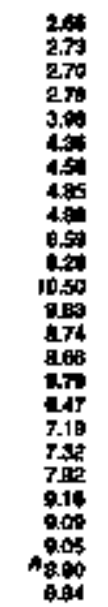 & 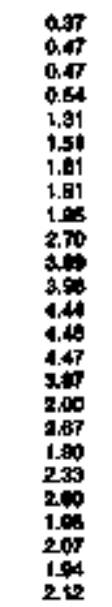 & 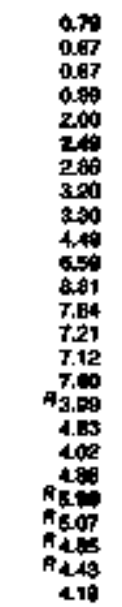 & 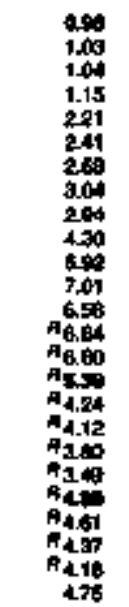 & 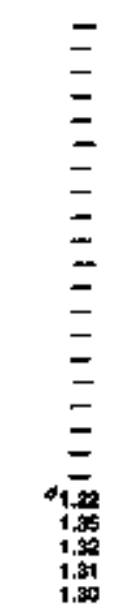 & 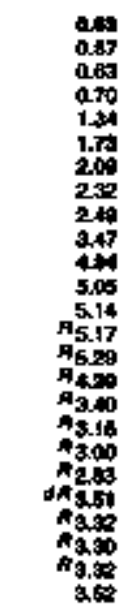 & 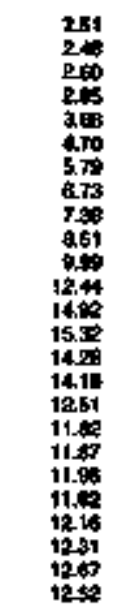 & 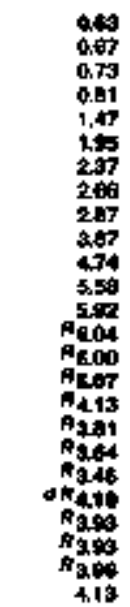 \\
\hline & \multicolumn{17}{|c|}{ 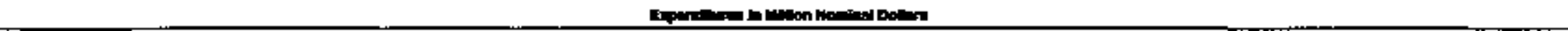 } \\
\hline 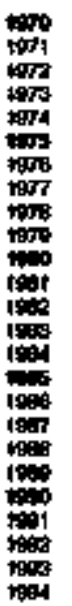 & 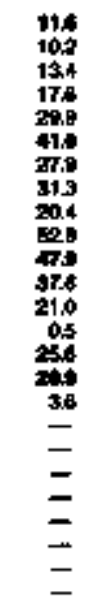 & 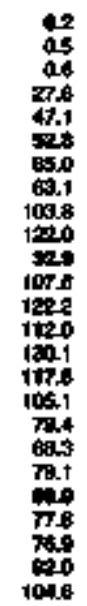 & 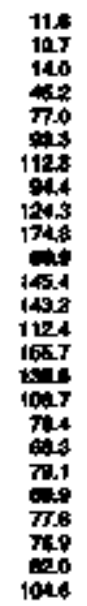 & 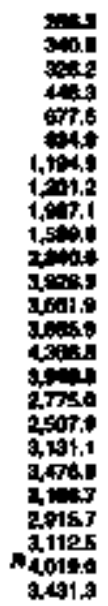 & 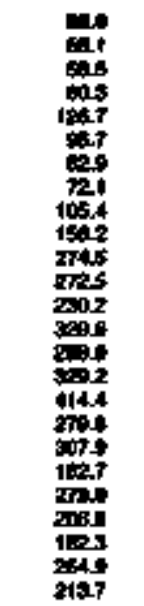 & 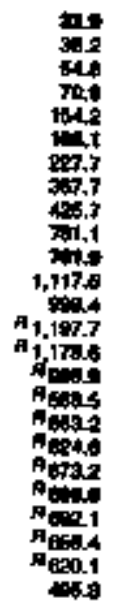 & 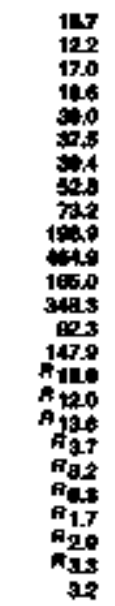 & 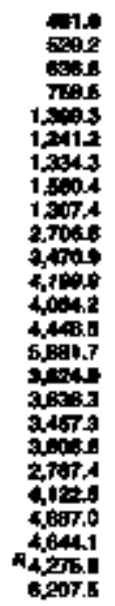 & 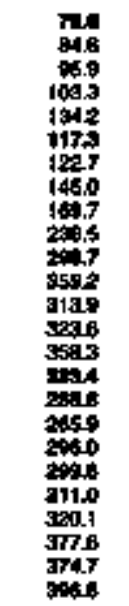 & 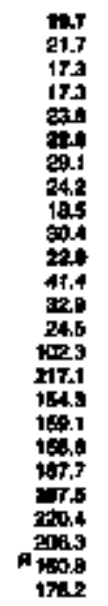 & 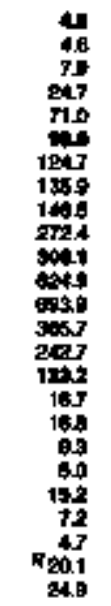 & 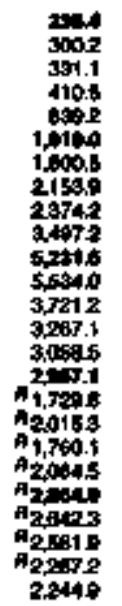 & 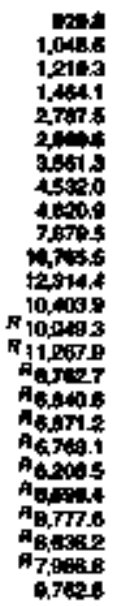 & 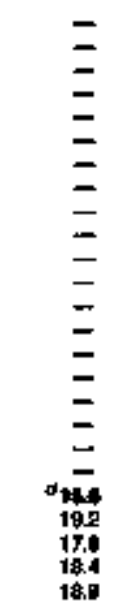 & 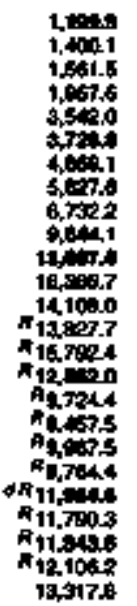 & 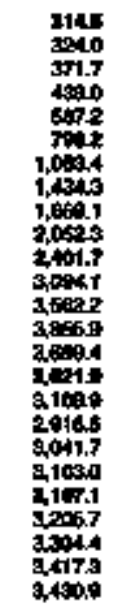 & 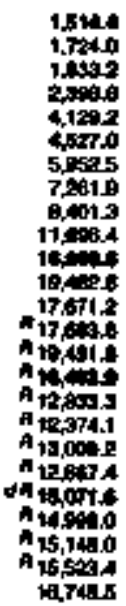 \\
\hline
\end{tabular}

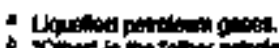

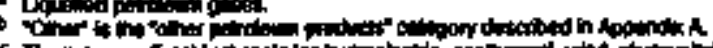

Anteped ing

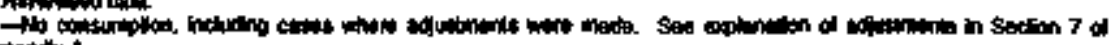

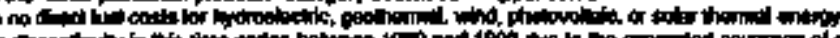

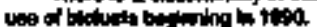

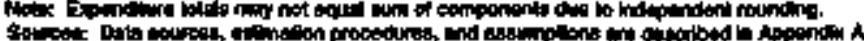




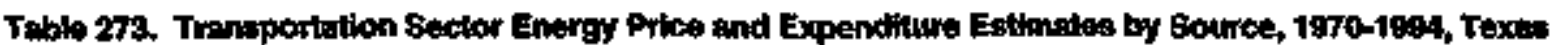

\begin{tabular}{|c|c|c|c|c|c|c|c|c|c|c|c|c|c|}
\hline \multirow[b]{4}{*}{ rer } & \multicolumn{11}{|c|}{ 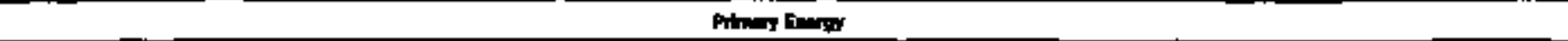 } & \multirow[b]{3}{*}{ Endy } & \multirow[b]{3}{*}{$\begin{array}{cc}T+0 \\
0\end{array}$} \\
\hline & & \multirow[b]{2}{*}{ 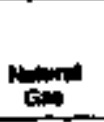 } & \multicolumn{8}{|c|}{ Patronsm } & \multirow[b]{2}{*}{ Tolit } & & \\
\hline & cod & & contellow & Find & int & LPa: & 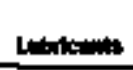 & 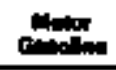 & Pand & It & & & \\
\hline & \multicolumn{13}{|c|}{ 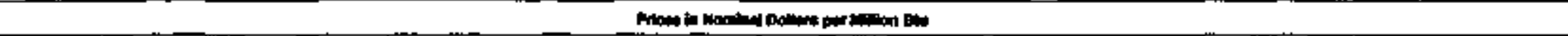 } \\
\hline \multirow[t]{2}{*}{ 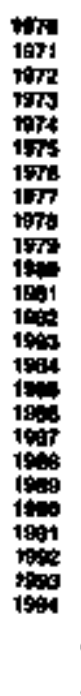 } & $\begin{array}{l}0.70 \\
0.00 \\
0.01 \\
1.01 \\
1.00 \\
= \\
= \\
= \\
= \\
= \\
= \\
= \\
= \\
= \\
=\end{array}$ & $\begin{array}{l}= \\
= \\
= \\
= \\
= \\
= \\
= \\
= \\
= \\
= \\
= \\
2 \overline{58} \\
498 \\
498\end{array}$ & 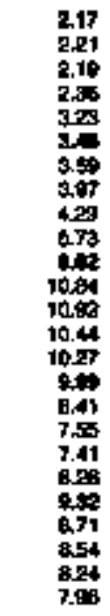 & 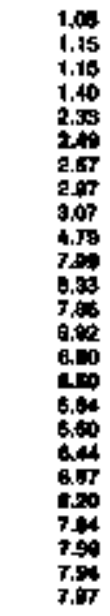 & 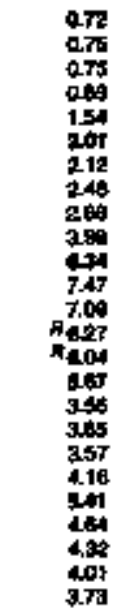 & 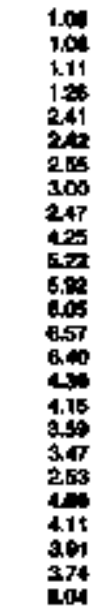 & 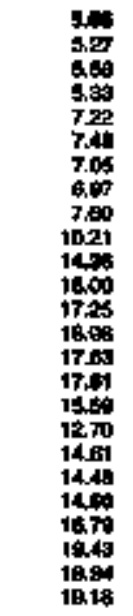 & 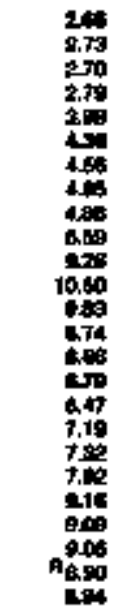 & 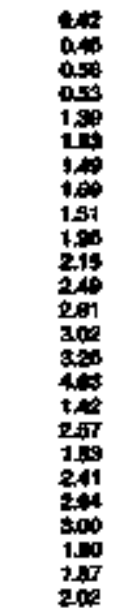 & 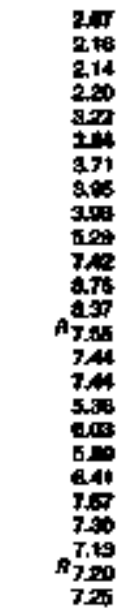 & 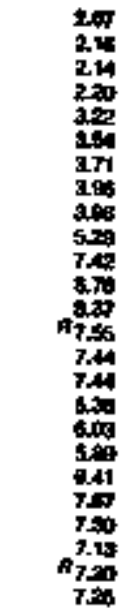 & $\begin{array}{l}= \\
= \\
= \\
= \\
= \\
= \\
= \\
= \\
= \\
= \\
= \\
= \\
=\end{array}$ & 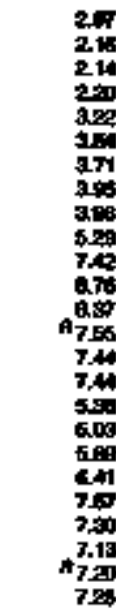 \\
\hline & \multicolumn{13}{|c|}{ 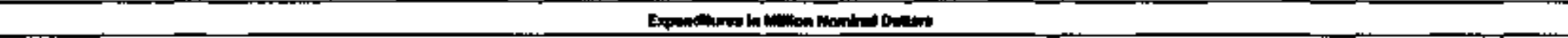 } \\
\hline 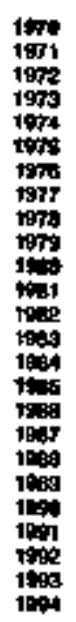 & 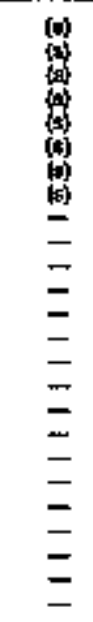 & 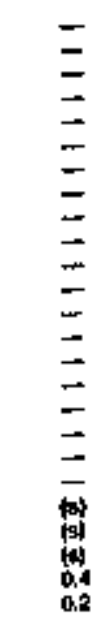 & 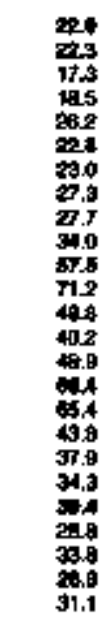 & 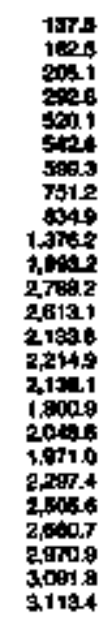 & 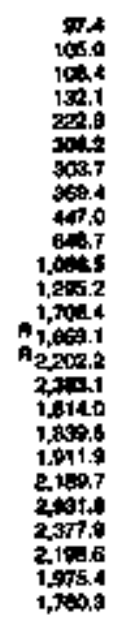 & 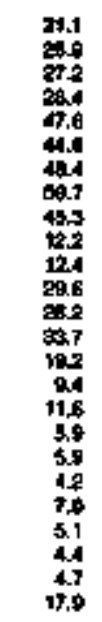 & 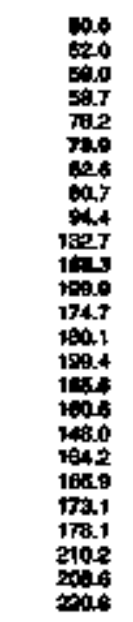 & 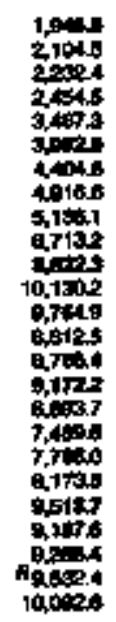 & 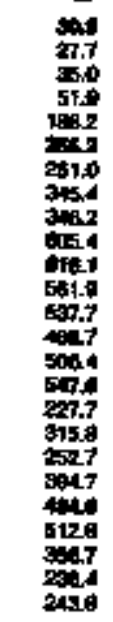 & 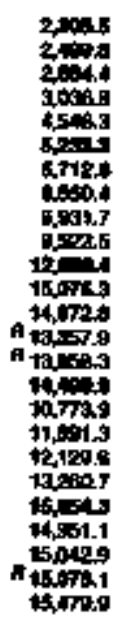 & 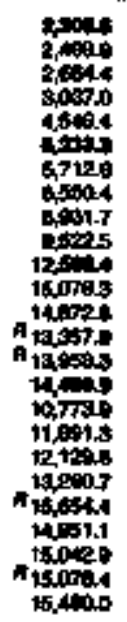 & $\begin{array}{l}= \\
= \\
= \\
= \\
= \\
= \\
= \\
= \\
= \\
= \\
= \\
\tilde{m}_{00}\end{array}$ & 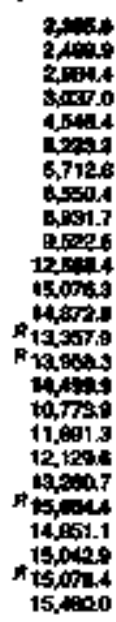 \\
\hline
\end{tabular}

Lqueted potratom gaver

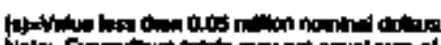

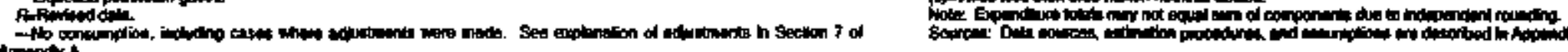



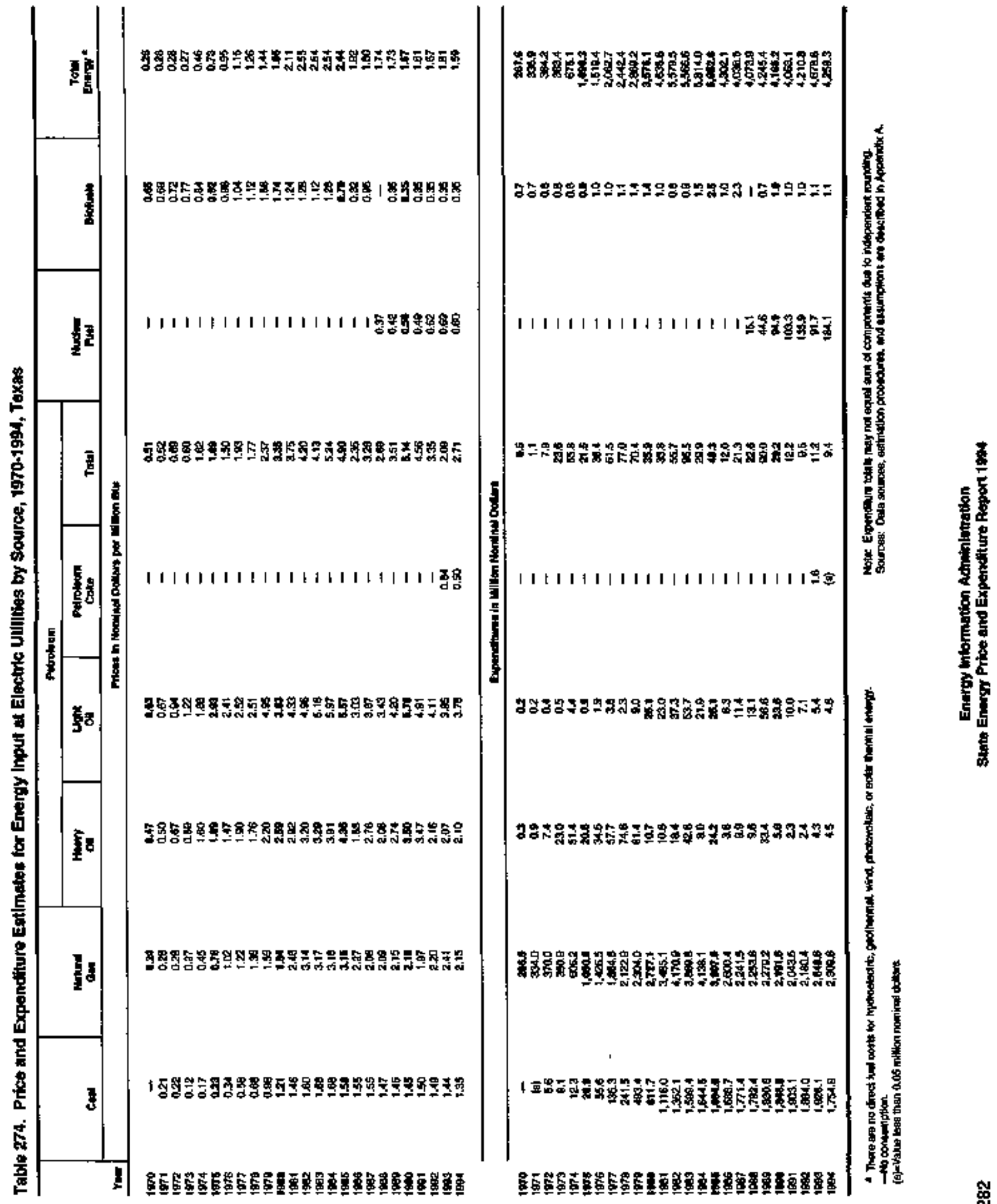
Tabfe 275. Energy Price and Expenditune Eslinates by Source, 1970-1994, Utah

\begin{tabular}{|c|c|c|c|c|c|c|c|c|c|c|c|c|c|c|c|c|c|}
\hline \multirow[b]{4}{*}{$\underline{n}$} & \multicolumn{14}{|c|}{ 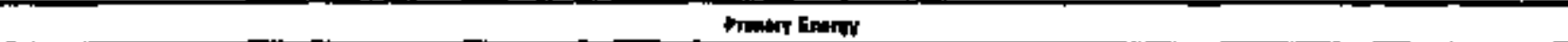 } & \multirow{3}{*}{ Evith } & \multirow{3}{*}{ endition } & \multirow[b]{3}{*}{ Tom } \\
\hline & \multicolumn{3}{|c|}{ المية } & \multirow[b]{2}{*}{ Mand } & \multicolumn{7}{|c|}{ Powominn } & \multirow[b]{2}{*}{ ristion } & \multirow[b]{2}{*}{ Anolumin } & \multirow[b]{2}{*}{ Tate } & & & \\
\hline & $\cos$ & Con & Foled & & Dectan & Fum & LPo* & 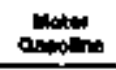 & d & Othen th & Total & & & & & & \\
\hline & \multicolumn{17}{|c|}{ 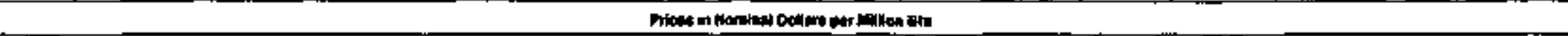 } \\
\hline \multirow[t]{2}{*}{ 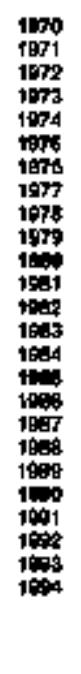 } & 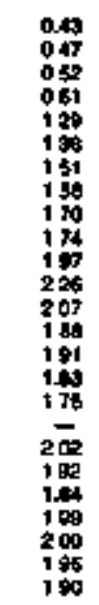 & 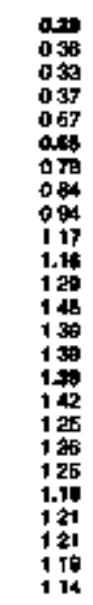 & 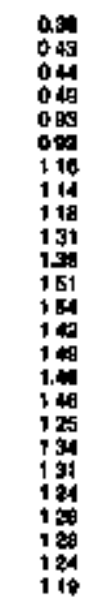 & 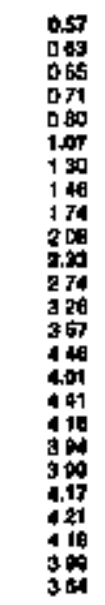 & 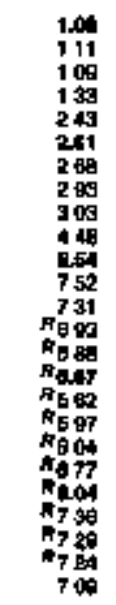 & 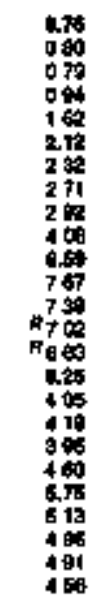 & 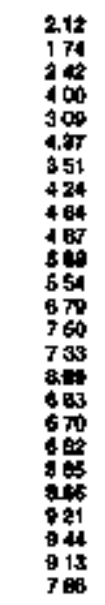 & 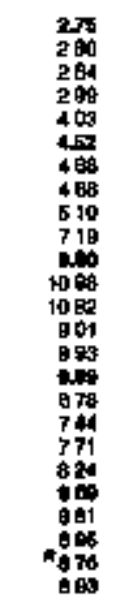 & 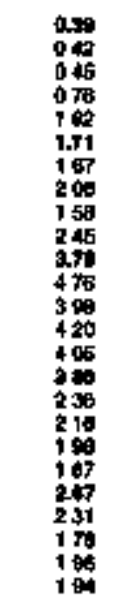 & 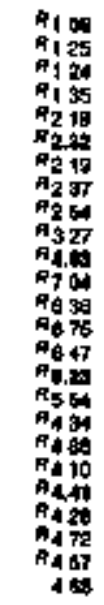 & 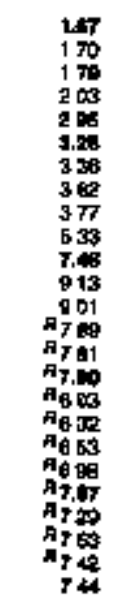 & $\begin{array}{l}= \\
= \\
= \\
= \\
= \\
= \\
= \\
= \\
= \\
= \\
= \\
= \\
= \\
= \\
=\end{array}$ & $\begin{array}{l}= \\
= \\
= \\
= \\
= \\
= \\
= \\
= \\
= \\
= \\
= \\
= \\
05 \\
355 \\
355 \\
351 \\
3 \pi\end{array}$ & 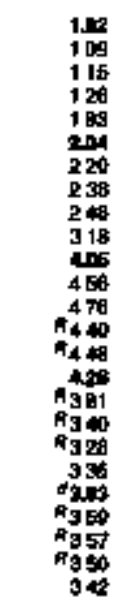 & 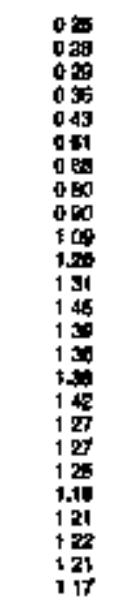 & 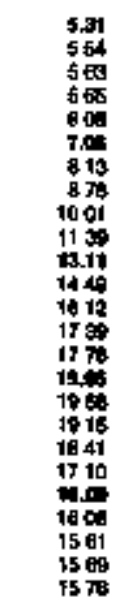 & 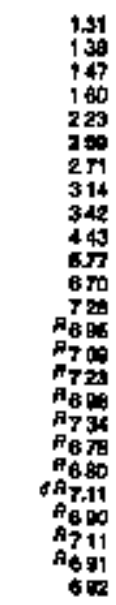 \\
\hline & \multicolumn{17}{|c|}{ 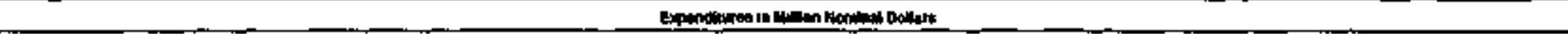 } \\
\hline 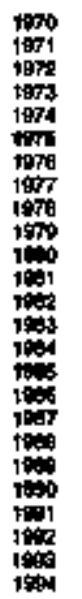 & 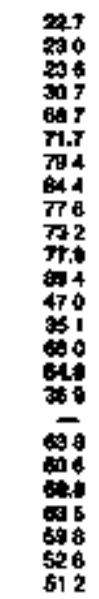 & 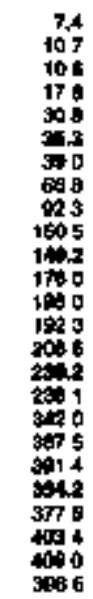 & 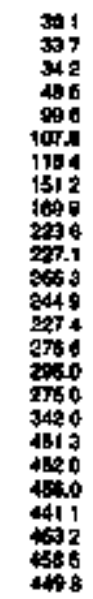 & 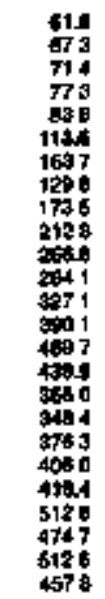 & 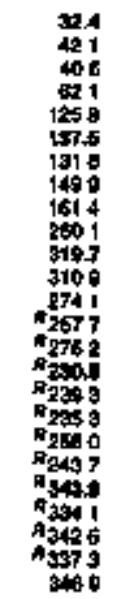 & 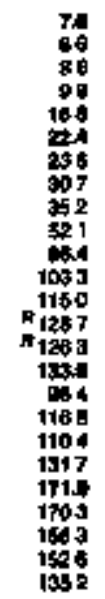 & $\begin{array}{r}67 \\
89 \\
98 \\
140 \\
112 \\
154 \\
729 \\
120 \\
108 \\
278 \\
298 \\
307 \\
370 \\
412 \\
349 \\
434 \\
380 \\
378 \\
392 \\
118 \\
724 \\
739 \\
797 \\
796 \\
219\end{array}$ & 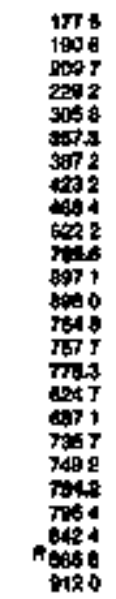 & 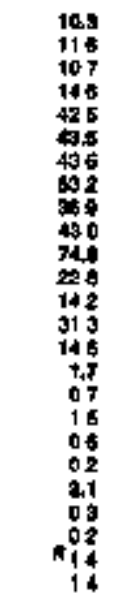 & 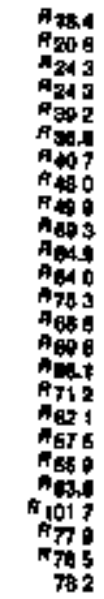 & 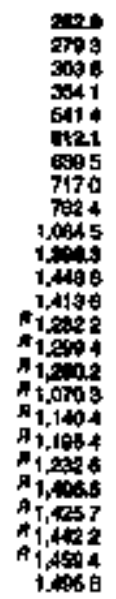 & $\begin{array}{l}= \\
= \\
= \\
= \\
= \\
= \\
= \\
= \\
= \\
= \\
= \\
= \\
= \\
=\end{array}$ & $\begin{array}{l}= \\
= \\
= \\
= \\
= \\
= \\
= \\
= \\
= \\
= \\
= \\
169 \\
75 \\
75 \\
72\end{array}$ & 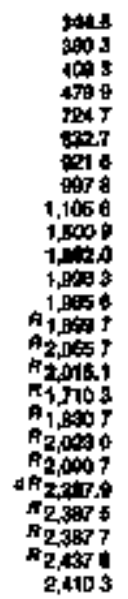 & 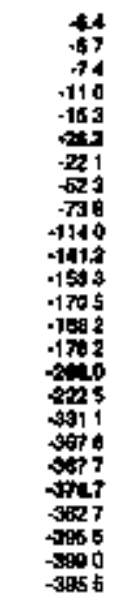 & 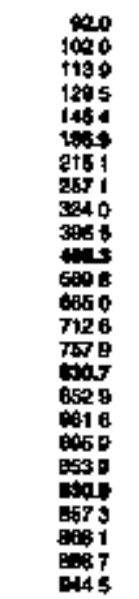 & 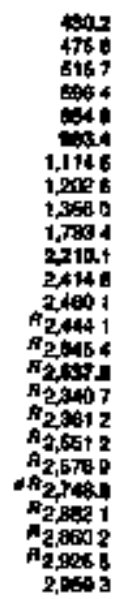 \\
\hline
\end{tabular}

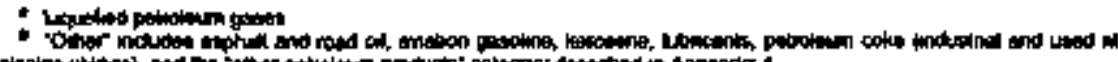

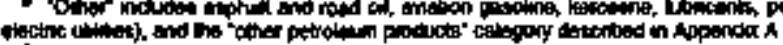

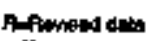

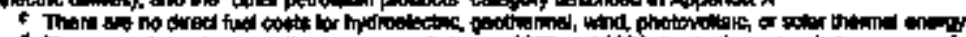

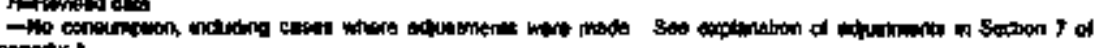
Apematix $A$

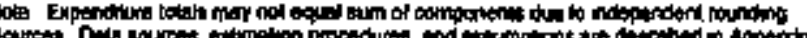

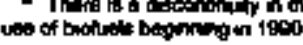




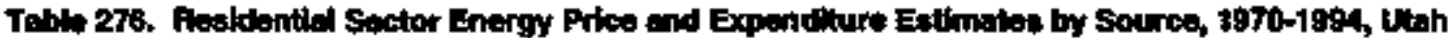

\begin{tabular}{|c|c|c|c|c|c|c|c|c|c|c|}
\hline \multirow[b]{4}{*}{ rer } & \multicolumn{8}{|c|}{ miting trowis } & \multirow[b]{3}{*}{ Einothen } & \multirow{3}{*}{ 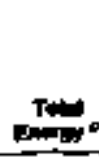 } \\
\hline & \multirow[b]{2}{*}{ 에 } & \multirow{2}{*}{ Mint } & \multicolumn{4}{|c|}{ Monguman } & \multirow[b]{2}{*}{ Elotwent } & \multirow[b]{2}{*}{ Vinte } & & \\
\hline & & & nin & Mrowan & 40: & Toter & & & & \\
\hline & \multicolumn{10}{|c|}{ 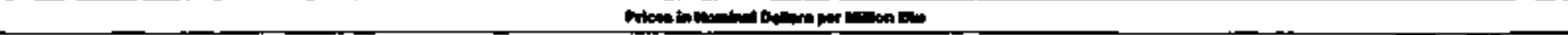 } \\
\hline \multirow[t]{2}{*}{ 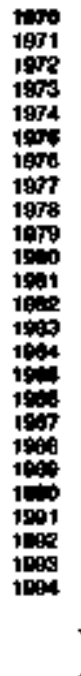 } & 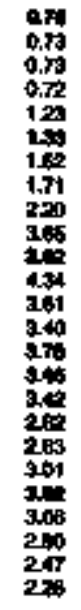 & 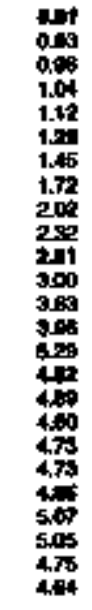 & 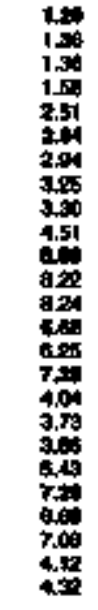 & 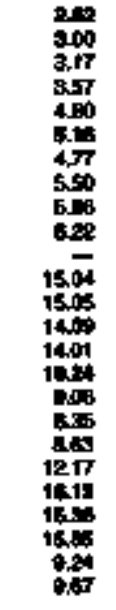 & 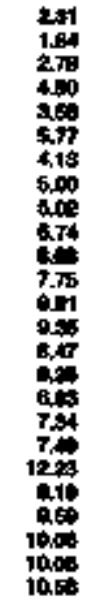 & 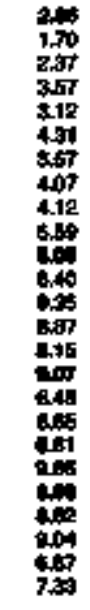 & $\begin{array}{l}= \\
= \\
= \\
= \\
= \\
= \\
= \\
= \\
= \\
= \\
= \\
= \\
0.75 \\
475 \\
4.75 \\
4.75\end{array}$ & 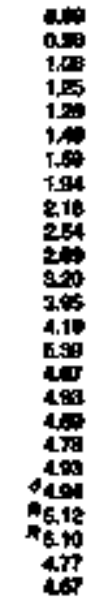 & 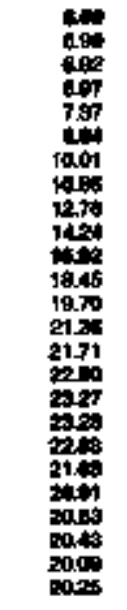 & 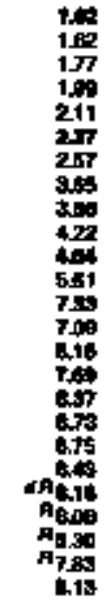 \\
\hline & \multicolumn{10}{|c|}{ 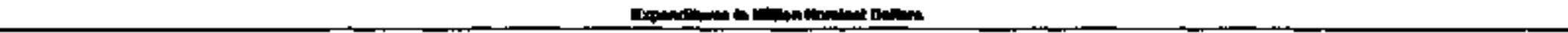 } \\
\hline 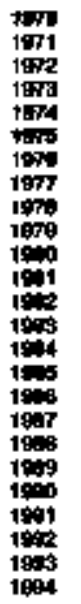 & 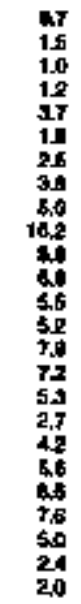 & 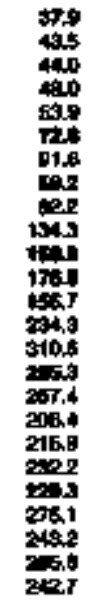 & 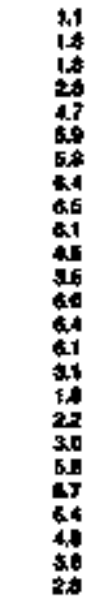 & 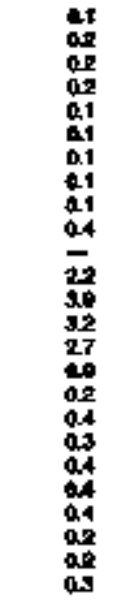 & 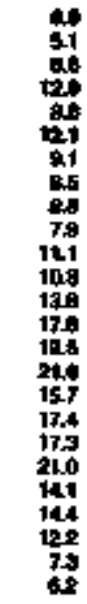 & 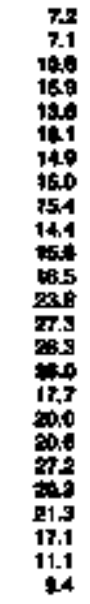 & $\begin{array}{l}= \\
= \\
= \\
= \\
= \\
= \\
= \\
= \\
= \\
= \\
=5 \\
46 \\
68 \\
6.1\end{array}$ & 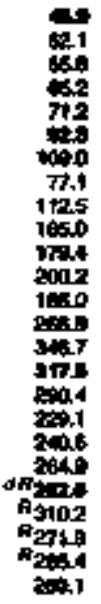 & 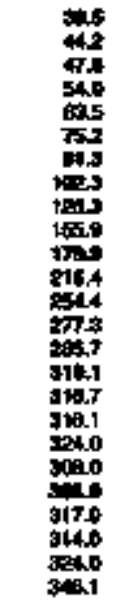 & 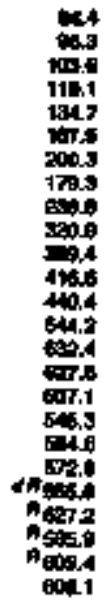 \\
\hline
\end{tabular}

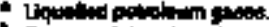

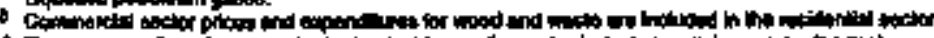

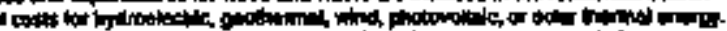

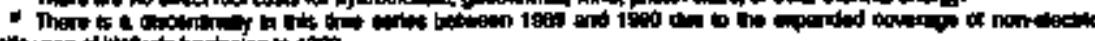

Anfomingen

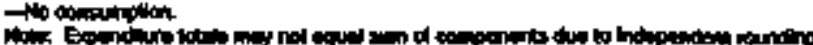

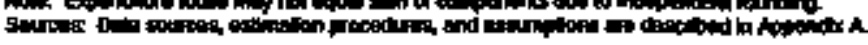




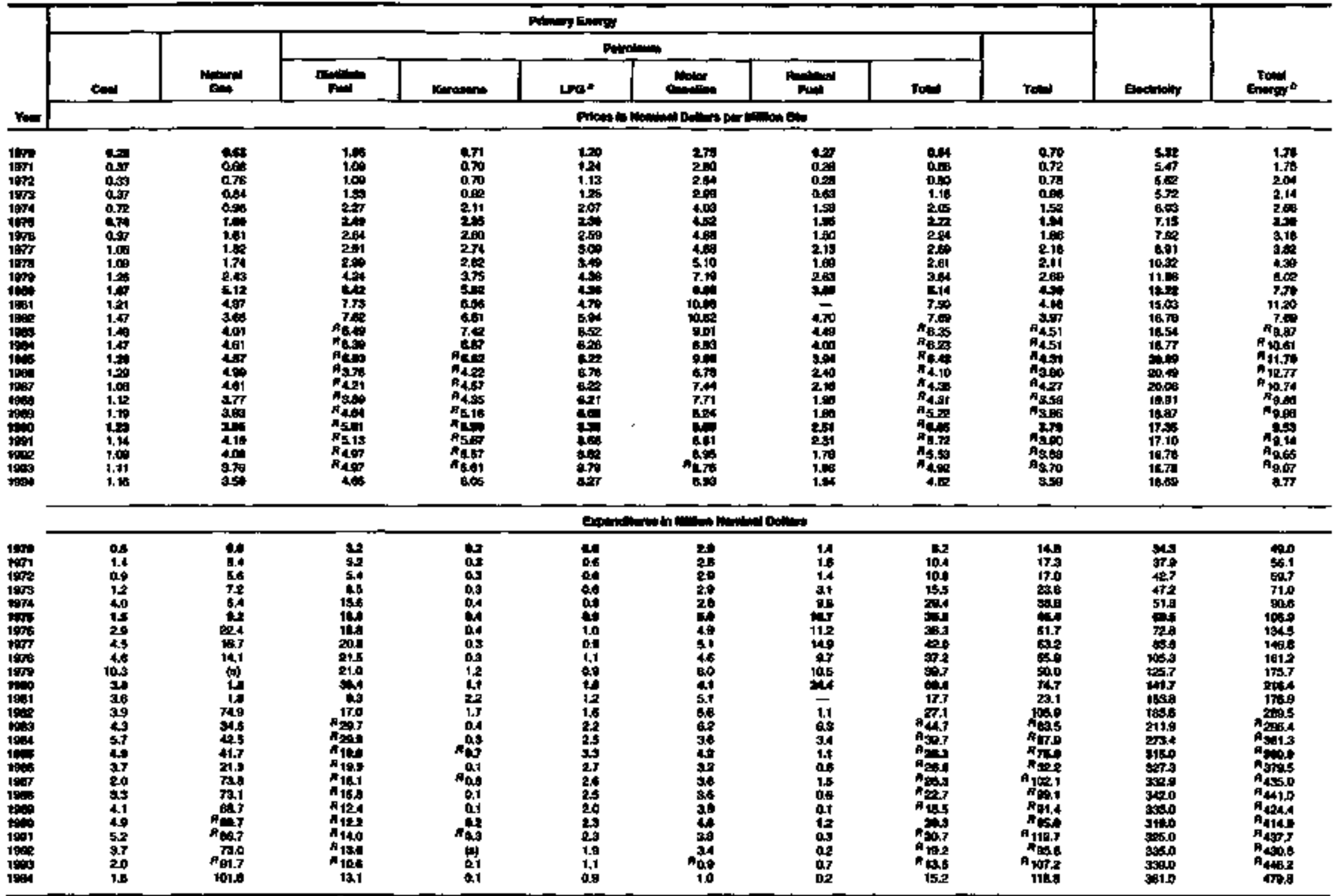

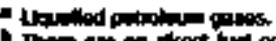

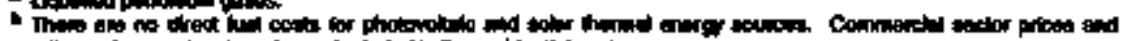

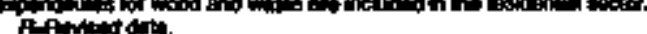

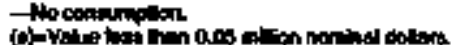

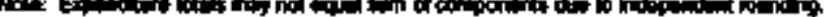

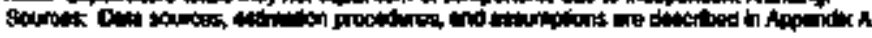


Table 278. fodustrial sector Energy Prich and Exponditure Eatimetes by Source, 1970-1994, Utah

A

\begin{tabular}{|c|c|c|c|c|c|c|c|c|c|c|c|c|c|c|c|c|c|}
\hline \multirow[b]{4}{*}{$r=$} & \multicolumn{15}{|c|}{ 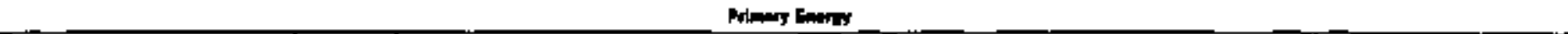 } & \multirow[b]{3}{*}{ entinging } & \multirow[b]{3}{*}{ Tomats } \\
\hline & \multicolumn{3}{|c|}{ cons } & \multirow[b]{2}{*}{ Natid } & \multicolumn{9}{|c|}{ Putrolin: } & \multirow[b]{2}{*}{ 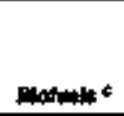 } & \multirow[b]{2}{*}{ Tot:m $c$} & & \\
\hline & collow & man & Tolv & & 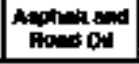 & 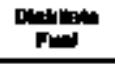 & Kimoasan & $\operatorname{trn} *$ & Lublouste & clapor & $\begin{array}{c}\text { Rostaved } \\
\text { Fand }\end{array}$ & Other 0 & Tow & & & & \\
\hline & \multicolumn{17}{|c|}{ 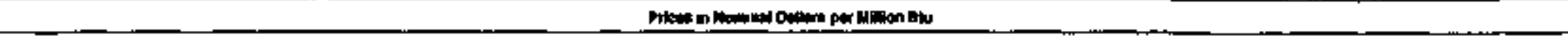 } \\
\hline \multirow[t]{2}{*}{ 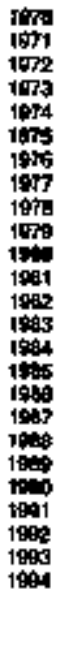 } & 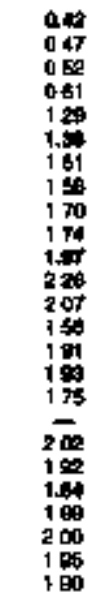 & 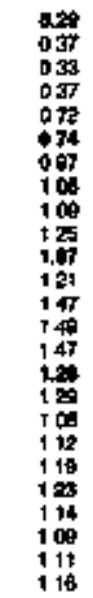 & 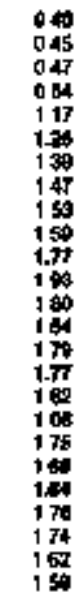 & 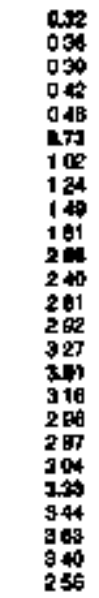 & 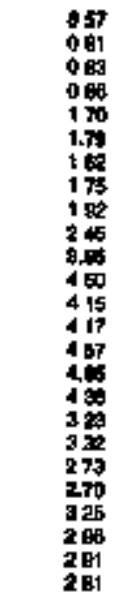 & 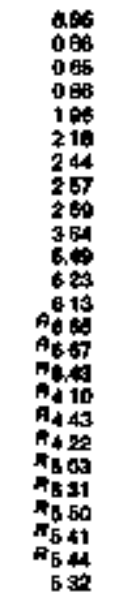 & 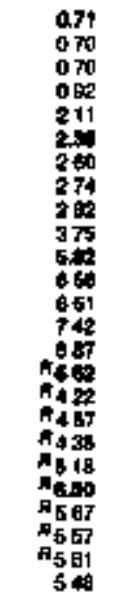 & 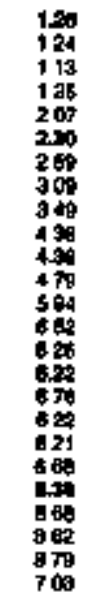 & 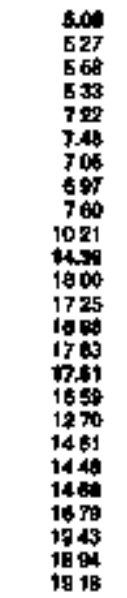 & 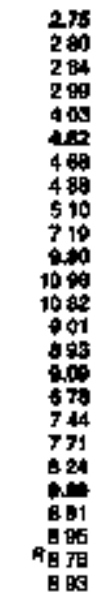 & 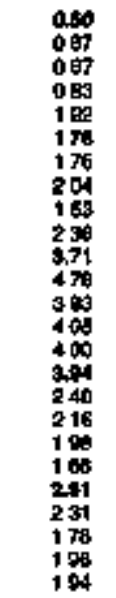 & 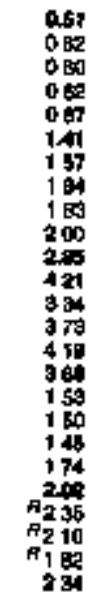 & 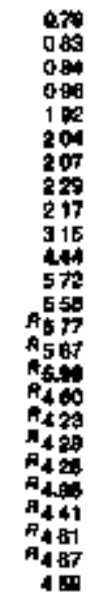 & $\begin{array}{l}= \\
= \\
= \\
= \\
= \\
= \\
= \\
= \\
= \\
= \\
= \\
= \\
= \\
1 \overline{17} \\
180 \\
178 \\
178 \\
176\end{array}$ & 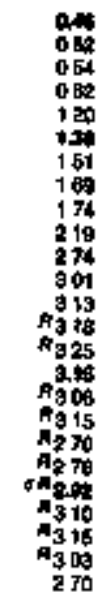 & 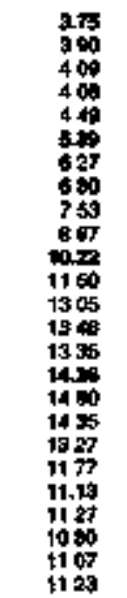 & 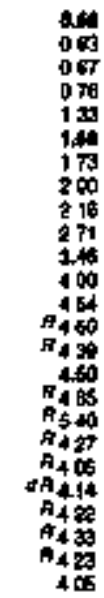 \\
\hline & \multicolumn{17}{|c|}{ 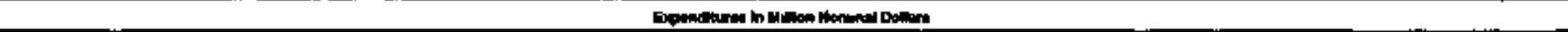 } \\
\hline 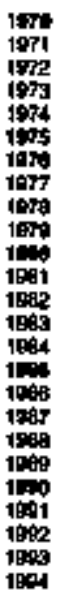 & 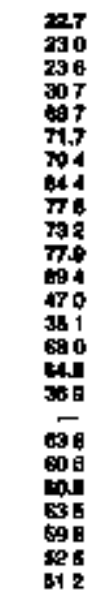 & $\begin{array}{l}34 \\
49 \\
47 \\
72 \\
108 \\
145 \\
146 \\
153 \\
204 \\
241 \\
120 \\
218 \\
273 \\
210 \\
109 \\
191 \\
107 \\
121 \\
153 \\
184 \\
193 \\
134 \\
132 \\
199 \\
222\end{array}$ & 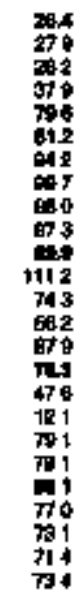 & 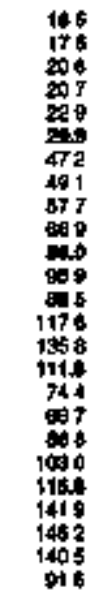 & 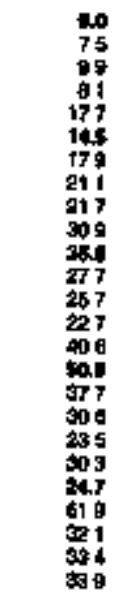 & 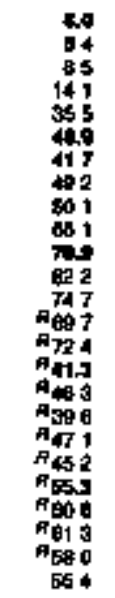 & 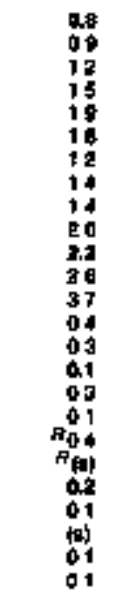 & 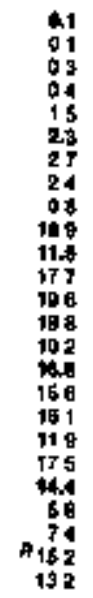 & 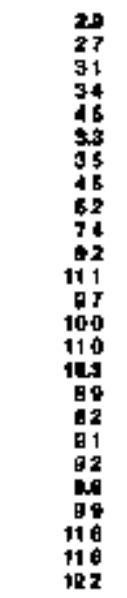 & 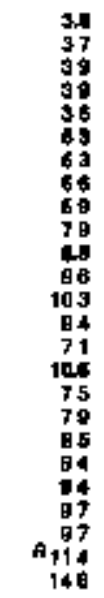 & 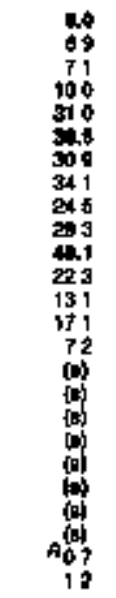 & 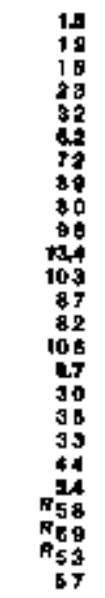 & 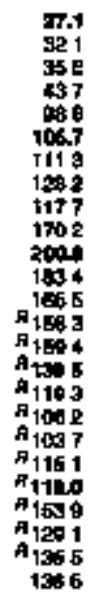 & $\begin{array}{l}= \\
= \\
z \\
z \\
z \\
= \\
= \\
= \\
= \\
= \\
= \\
= \\
0 \\
10 \\
10 \\
11 \\
11\end{array}$ & 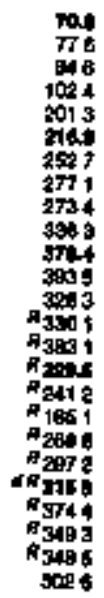 & 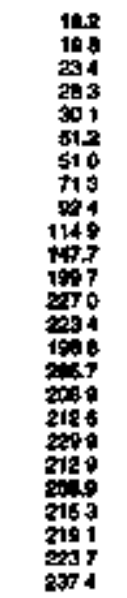 & 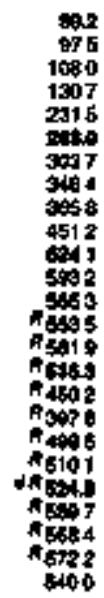 \\
\hline
\end{tabular}

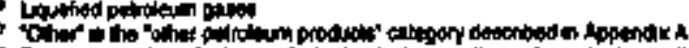

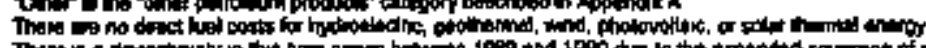

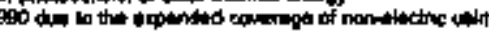

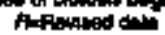

Apowion

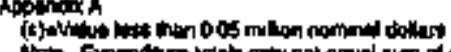

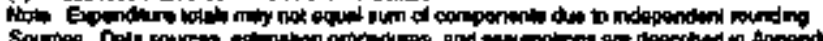


Tabls 279. Tranaportation Sector Energy Price and Expendllure Eetlmates by Source, 1970 1994, Utah

\begin{tabular}{|c|c|c|c|c|c|c|c|c|c|c|c|c|c|}
\hline \multirow[b]{4}{*}{ 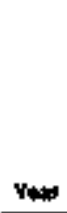 } & \multicolumn{11}{|c|}{ Priney Energy } & \multirow[b]{3}{*}{ Anetikety } & \multirow[b]{3}{*}{ Entist } \\
\hline & \multirow[b]{2}{*}{ Con } & \multirow[b]{2}{*}{ Rint } & \multicolumn{8}{|c|}{ Purrolvum } & \multirow[b]{2}{*}{ Totod } & & \\
\hline & & & Antion & $\begin{array}{c}\text { OAtilliane } \\
\text { fund }\end{array}$ & Fin & $\operatorname{PPG}$ & Lutrizanit: & 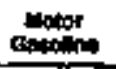 & (1) & Tot & & & \\
\hline & \multicolumn{13}{|c|}{ 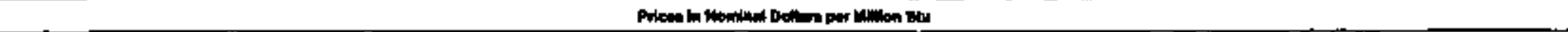 } \\
\hline \multirow[t]{2}{*}{ 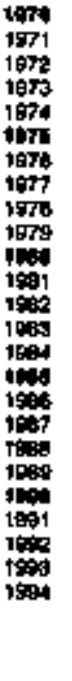 } & $\begin{array}{l}120 \\
037 \\
030 \\
037 \\
072 \\
674 \\
067 \\
106 \\
= \\
= \\
= \\
= \\
= \\
= \\
= \\
= \\
= \\
= \\
=\end{array}$ & $\begin{array}{l}= \\
= \\
= \\
= \\
= \\
= \\
= \\
= \\
= \\
= \\
= \\
= \\
= \\
09 \\
514 \\
509 \\
469\end{array}$ & 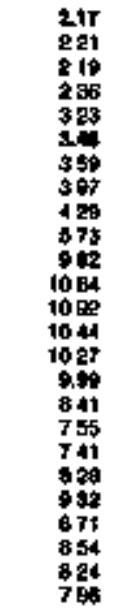 & 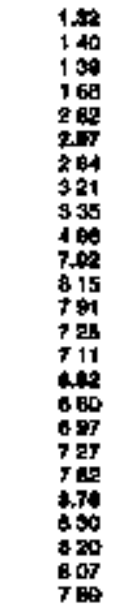 & 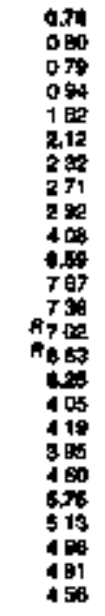 & 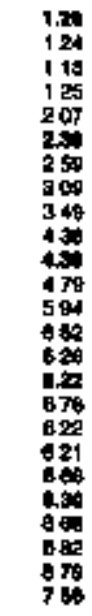 & 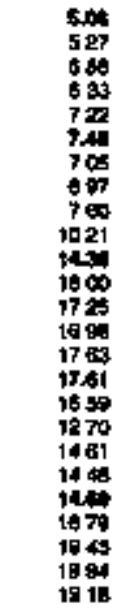 & 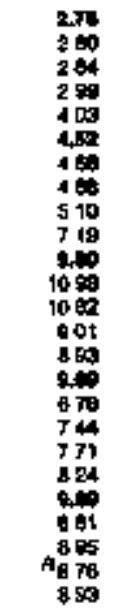 & 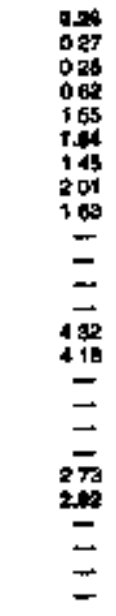 & 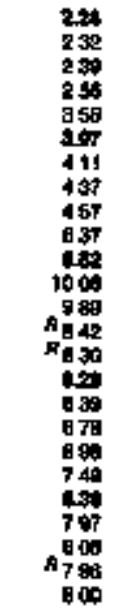 & 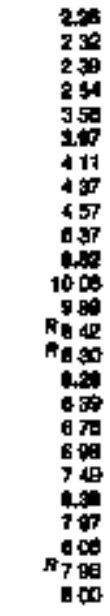 & $\begin{array}{l}= \\
= \\
= \\
= \\
= \\
= \\
= \\
= \\
= \\
= \\
= \\
= \\
=\end{array}$ & 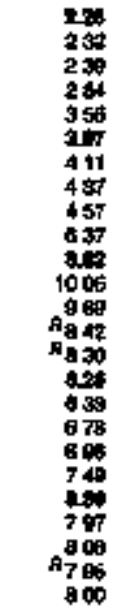 \\
\hline & \multicolumn{13}{|c|}{ 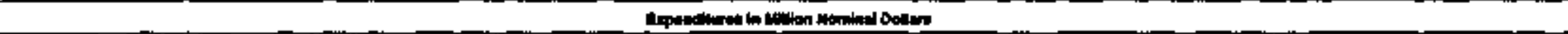 } \\
\hline 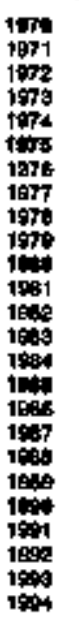 & $\begin{array}{l}00 \\
000 \\
0 \\
0 \\
= \\
= \\
= \\
= \\
= \\
= \\
= \\
= \\
= \\
= \\
=\end{array}$ & $\begin{array}{l}= \\
= \\
= \\
= \\
= \\
= \\
= \\
= \\
= \\
= \\
= \\
= \\
\bar{z} \\
0 \\
01 \\
01 \\
02 \\
0.9\end{array}$ & 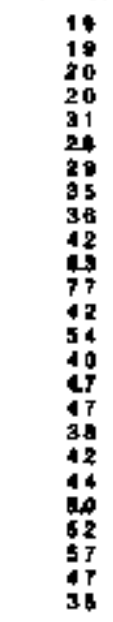 & 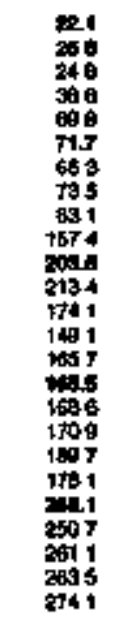 & 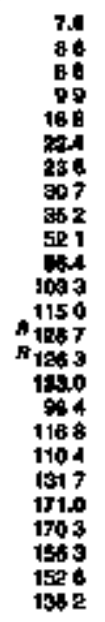 & 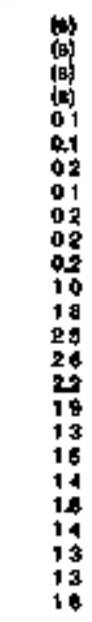 & $\begin{array}{l}9 A \\
62 \\
60 \\
64 \\
63 \\
72 \\
76 \\
82 \\
98 \\
195 \\
101 \\
203 \\
177 \\
185 \\
202 \\
184 \\
163 \\
150 \\
786 \\
109 \\
174 \\
181 \\
213 \\
212 \\
274\end{array}$ & 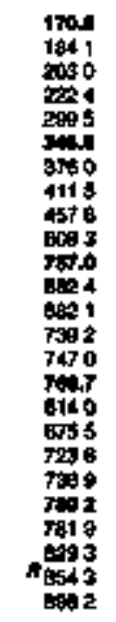 & $\begin{array}{l}0 \\
01 \\
01 \\
01 \\
05 \\
08 \\
00 \\
07 \\
00 \\
= \\
= \\
= \\
=0 \\
= \\
= \\
= \\
= \\
= \\
= \\
= \\
=\end{array}$ & 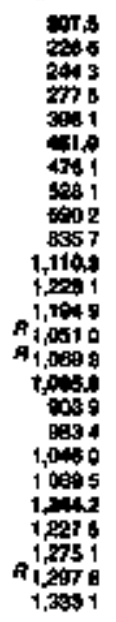 & 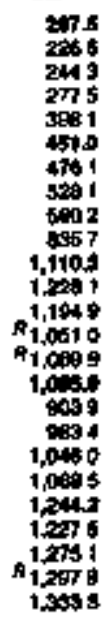 & $\begin{array}{l}= \\
= \\
= \\
= \\
= \\
= \\
= \\
= \\
= \\
= \\
= \\
= \\
= \\
=\end{array}$ & 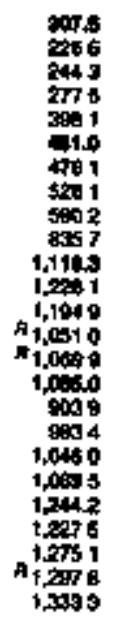 \\
\hline
\end{tabular}

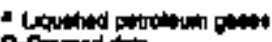

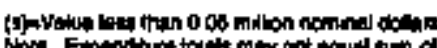

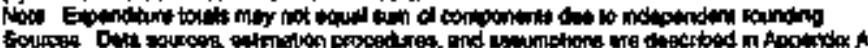

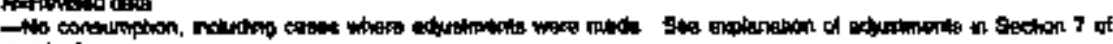




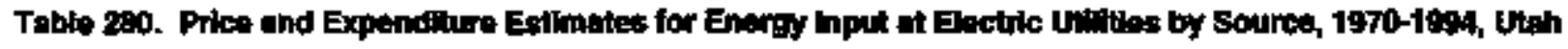

A

\begin{tabular}{|c|c|c|c|c|c|c|c|c|c|}
\hline \multirow[b]{3}{*}{ Vur } & & \multirow[b]{2}{*}{$\min _{n \rightarrow \infty}$} & \multicolumn{4}{|c|}{ 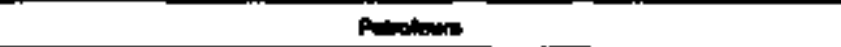 } & \multirow[b]{2}{*}{ finger } & \multirow[b]{2}{*}{ 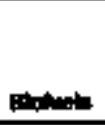 } & \multirow[b]{2}{*}{ " } \\
\hline & Cool & & tont & ond & 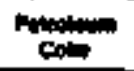 & Tots & & & \\
\hline & \multicolumn{9}{|c|}{ ניתו } \\
\hline 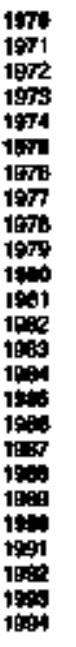 & $\begin{array}{l}0.24 \\
0.25 \\
0.29 \\
0.34 \\
0.40 \\
6.9 \\
0.94 \\
0.74 \\
0.85 \\
1,09 \\
1.14 \\
1.27 \\
1.47 \\
1.35 \\
1.34 \\
1.37 \\
1.44 \\
1.25 \\
1.27 \\
1.24 \\
1.17 \\
1.19 \\
1.24 \\
1.19 \\
1.14\end{array}$ & 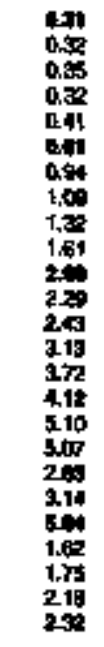 & 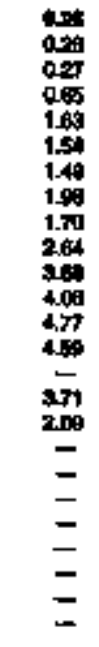 & 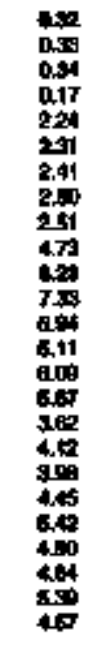 & $\begin{array}{l}= \\
= \\
= \\
= \\
= \\
= \\
= \\
= \\
= \\
= \\
= \\
= \\
=\end{array}$ & 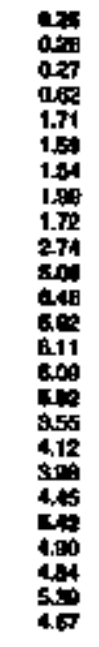 & $\begin{array}{l}\bar{z} \\
\bar{z} \\
\bar{z} \\
\bar{z} \\
\bar{z} \\
\bar{z} \\
\bar{z} \\
\bar{z} \\
\bar{z} \\
\bar{z} \\
\bar{z} \\
\bar{z}\end{array}$ & $\begin{array}{l}= \\
z \\
z \\
z \\
= \\
= \\
= \\
= \\
= \\
= \\
= \\
= \\
=\end{array}$ & 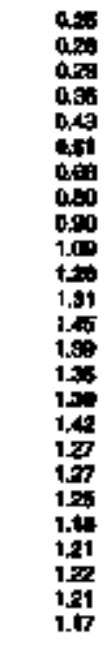 \\
\hline
\end{tabular}

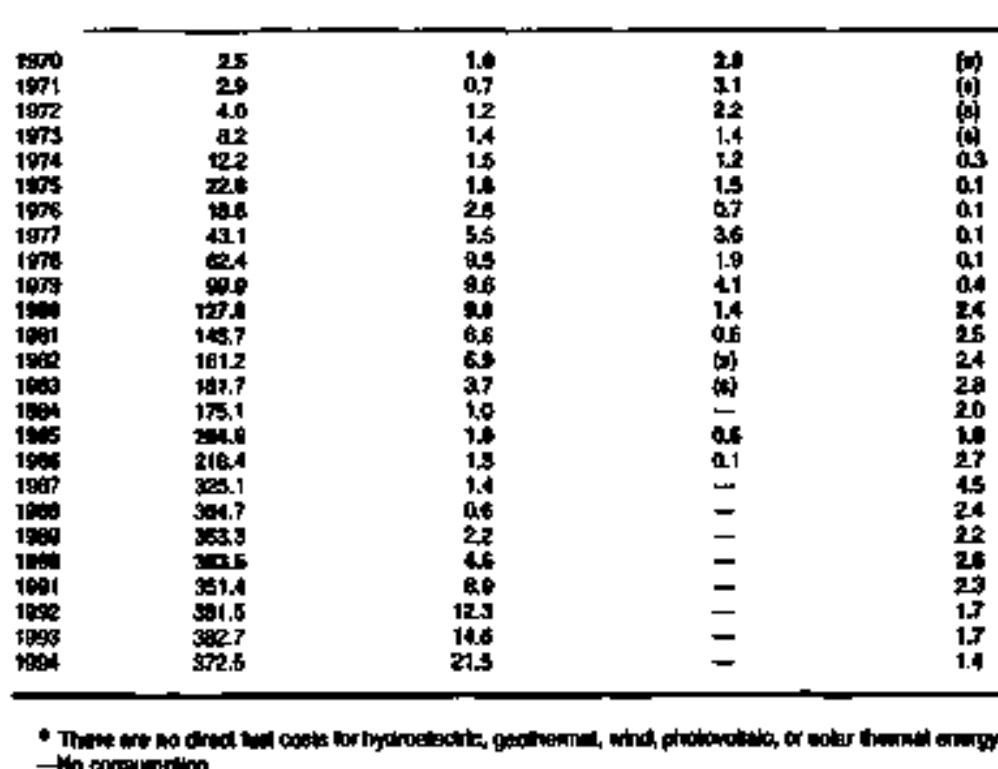

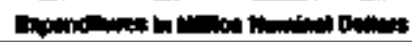

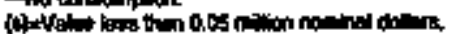

\begin{tabular}{|c|c|c|c|c|}
\hline $\bar{z} \bar{z}$ & 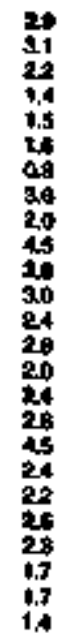 & $\begin{array}{l}z \\
z \\
z \\
z \\
z \\
z \\
z \\
z \\
z \\
z \\
z \\
z \\
z \\
z\end{array}$ & $\begin{array}{l}z \\
\bar{z} \\
\bar{z} \\
z \\
z \\
z \\
z \\
z \\
z \\
z \\
z \\
z \\
z\end{array}$ & 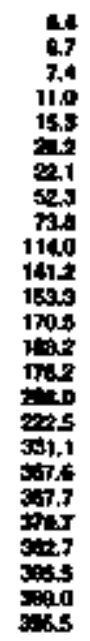 \\
\hline
\end{tabular}

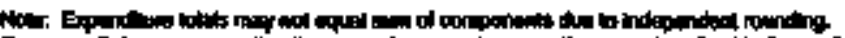

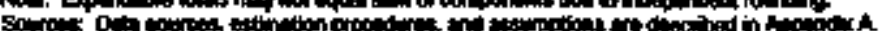




\begin{tabular}{|c|c|c|c|c|c|c|c|c|c|c|c|c|c|c|c|c|c|}
\hline \multirow[t]{2}{*}{ 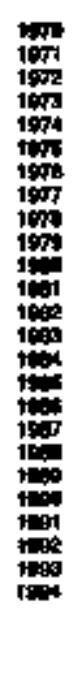 } & $\begin{array}{l}= \\
= \\
= \\
= \\
= \\
= \\
= \\
= \\
= \\
= \\
= \\
= \\
=\end{array}$ & 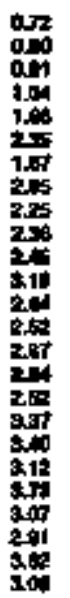 & 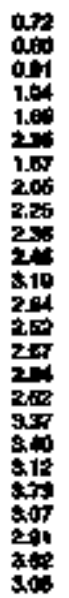 & 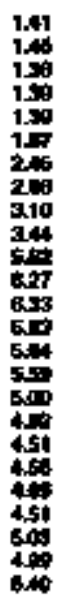 & 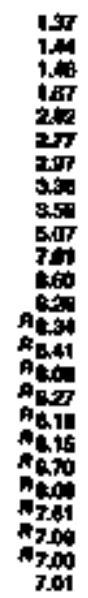 & 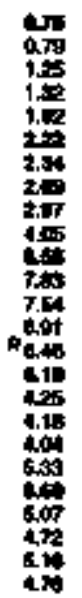 & 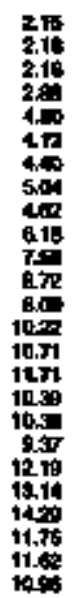 & 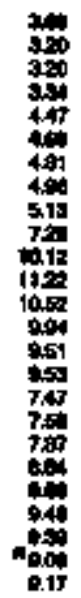 & 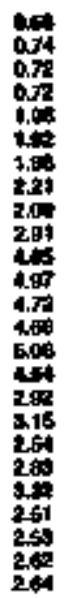 & 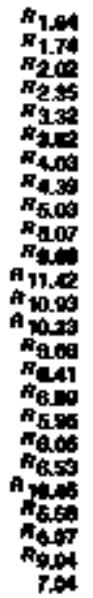 & 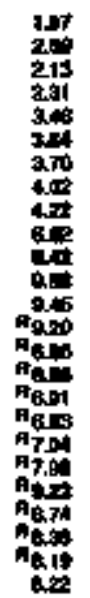 & 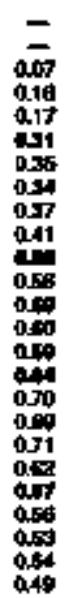 & 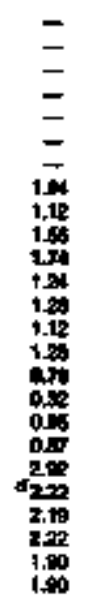 & 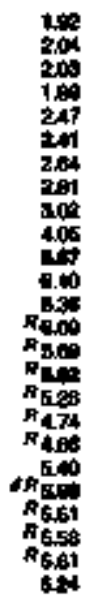 & 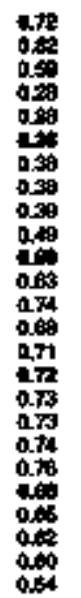 & 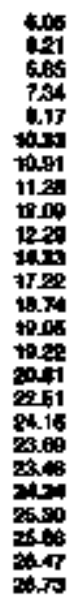 & 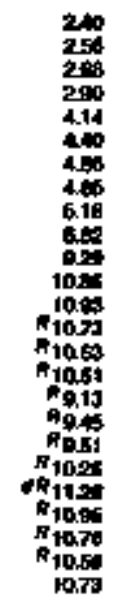 \\
\hline & \multicolumn{17}{|c|}{ 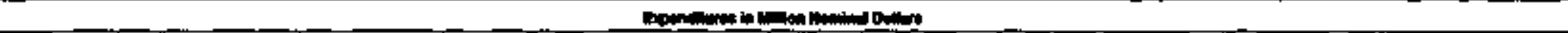 } \\
\hline 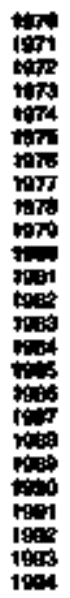 & $\begin{array}{l}= \\
= \\
= \\
= \\
= \\
= \\
= \\
= \\
= \\
= \\
= \\
= \\
=\end{array}$ & 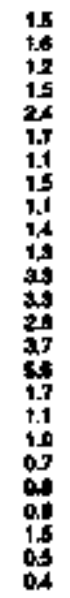 & 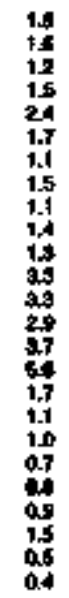 & 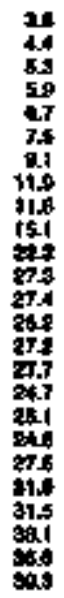 & 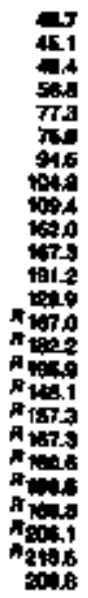 & 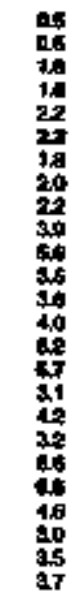 & 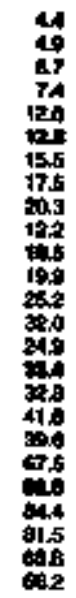 & 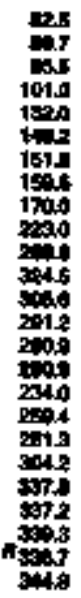 & 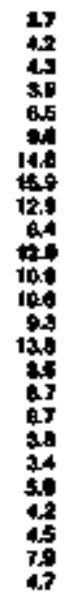 & 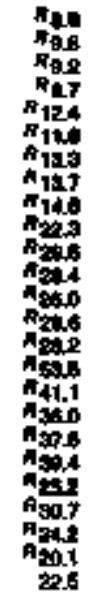 & 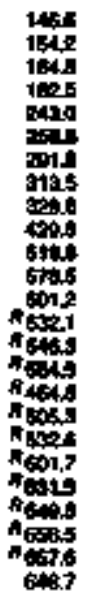 & 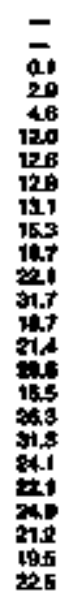 & 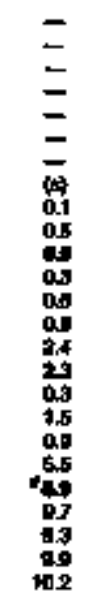 & 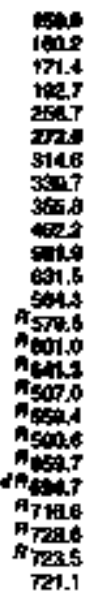 & 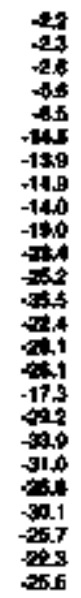 & 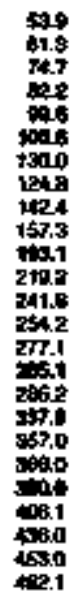 & 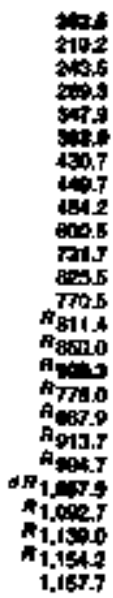 \\
\hline
\end{tabular}

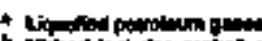

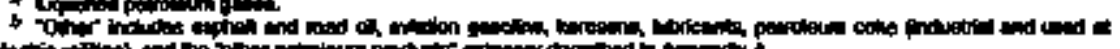

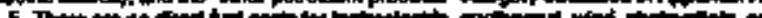


Toble 292. Ampidential swetor Energy Price and Expenditurt Etimales by source, 1970-1944, Vermont

\begin{tabular}{|c|c|c|c|c|c|c|c|c|c|c|}
\hline \multirow[b]{4}{*}{ Yor } & \multicolumn{8}{|c|}{ Primary Enengr } & \multirow[b]{3}{*}{ Equinoiny } & \multirow[b]{3}{*}{ 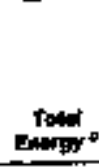 } \\
\hline & \multirow[b]{2}{*}{ cont } & \multirow[b]{2}{*}{ Mnowed } & \multicolumn{4}{|c|}{ Ancolown } & \multirow[b]{2}{*}{ Entuele * } & \multirow[b]{2}{*}{ Vited 5} & & \\
\hline & & & 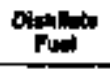 & Knrosine & Len & Tot: & & & & \\
\hline & \multicolumn{10}{|c|}{ 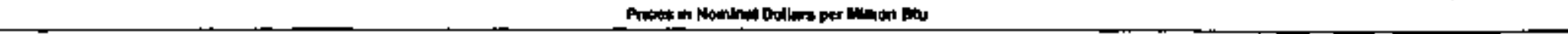 } \\
\hline \multirow[t]{2}{*}{ 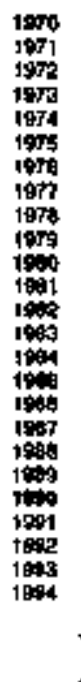 } & 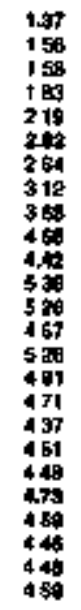 & 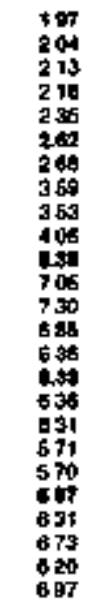 & 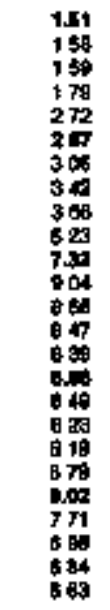 & 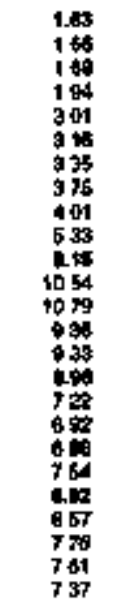 & 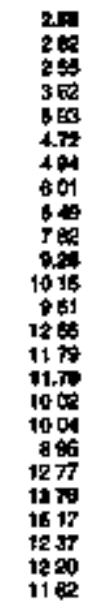 & 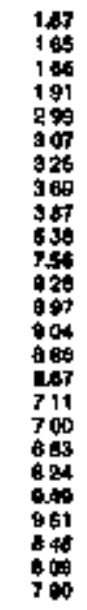 & 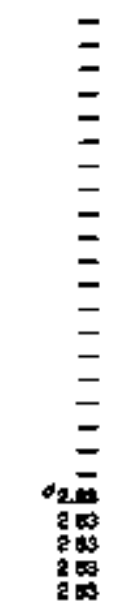 & 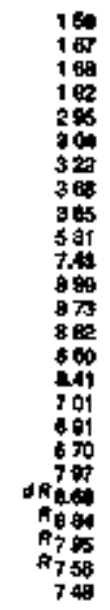 & 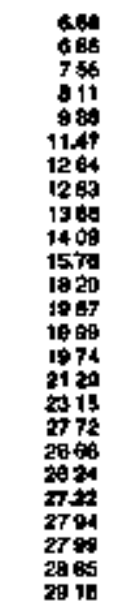 & 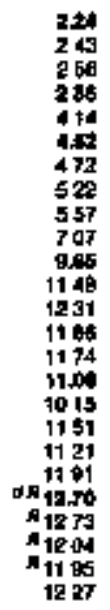 \\
\hline & \multicolumn{10}{|c|}{ 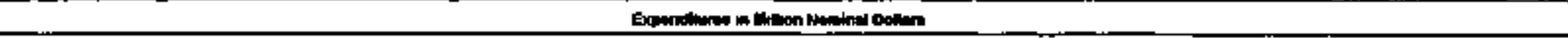 } \\
\hline 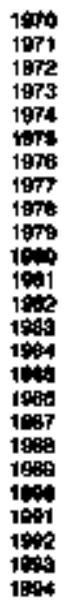 & $\begin{array}{l}06 \\
06 \\
08 \\
08 \\
05 \\
05 \\
05 \\
06 \\
06 \\
08 \\
07 \\
18 \\
12 \\
08 \\
12 \\
29 \\
07 \\
07 \\
08 \\
03 \\
05 \\
09 \\
04 \\
04 \\
02\end{array}$ & $\begin{array}{l}21 \\
23 \\
28 \\
28 \\
28 \\
10 \\
33 \\
42 \\
42 \\
46 \\
19 \\
90 \\
63 \\
65 \\
85 \\
81 \\
100 \\
104 \\
106 \\
119 \\
124 \\
137 \\
169 \\
197 \\
169\end{array}$ & 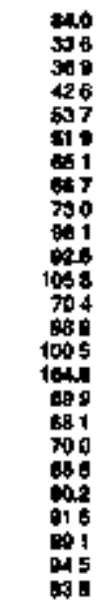 & 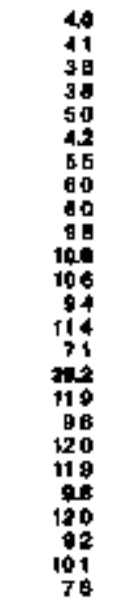 & 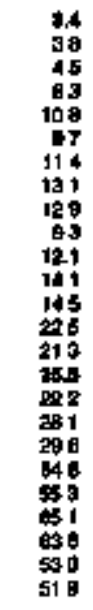 & 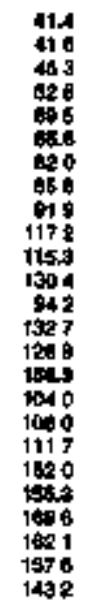 & $\begin{array}{l}= \\
= \\
= \\
= \\
= \\
= \\
= \\
= \\
= \\
= \\
= \\
=54 \\
36 \\
36 \\
36\end{array}$ & 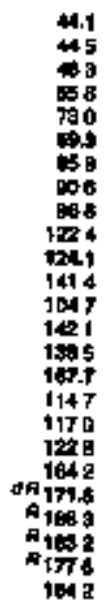 & 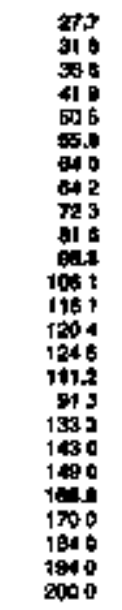 & 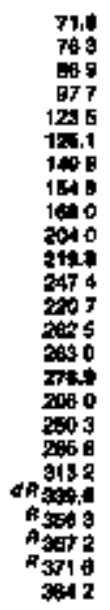 \\
\hline
\end{tabular}

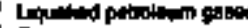

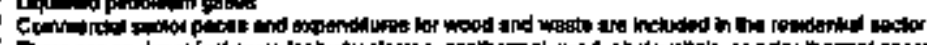

Anom dath

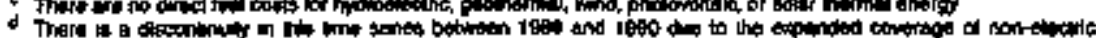

chrere a 


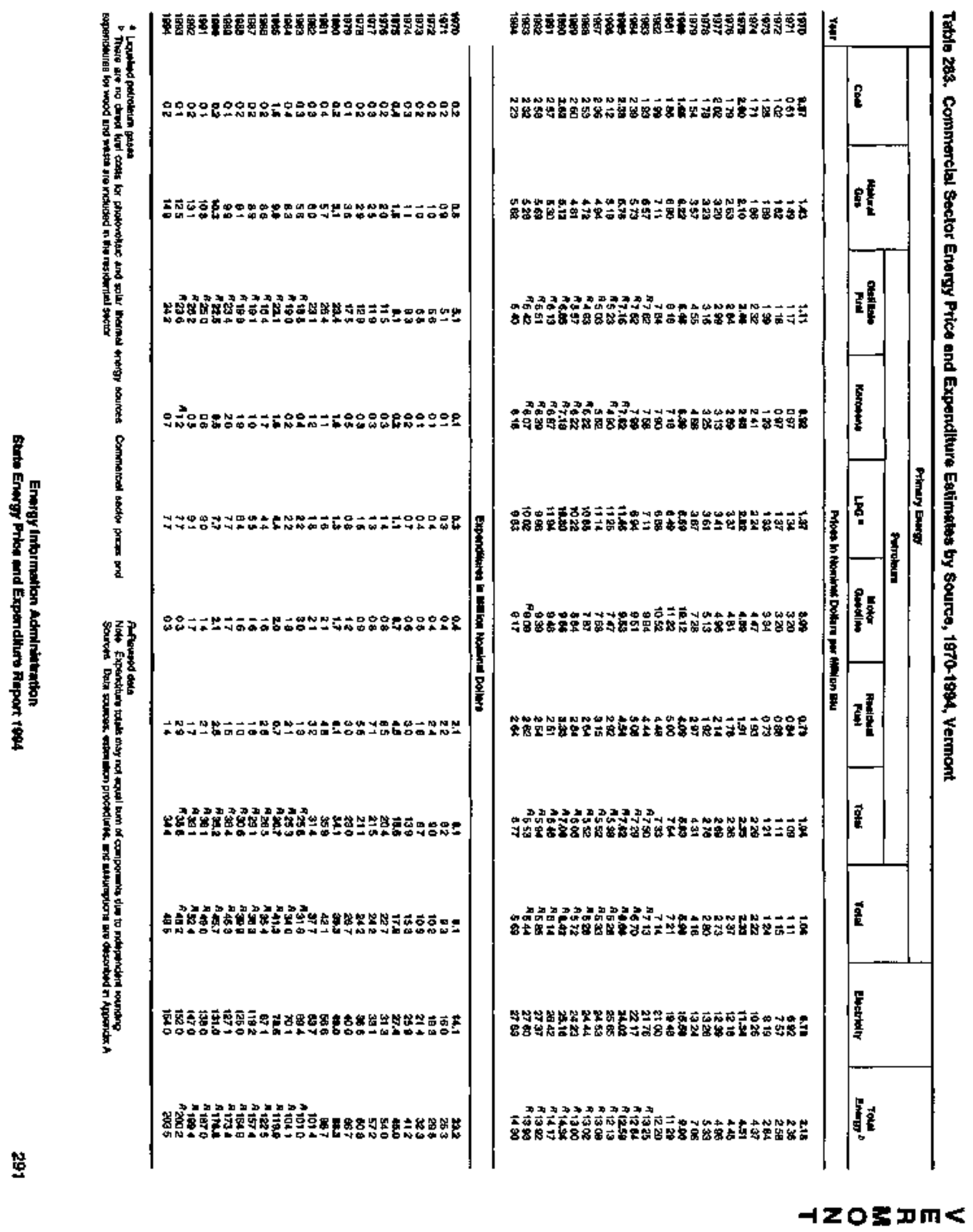




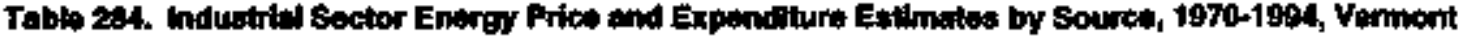

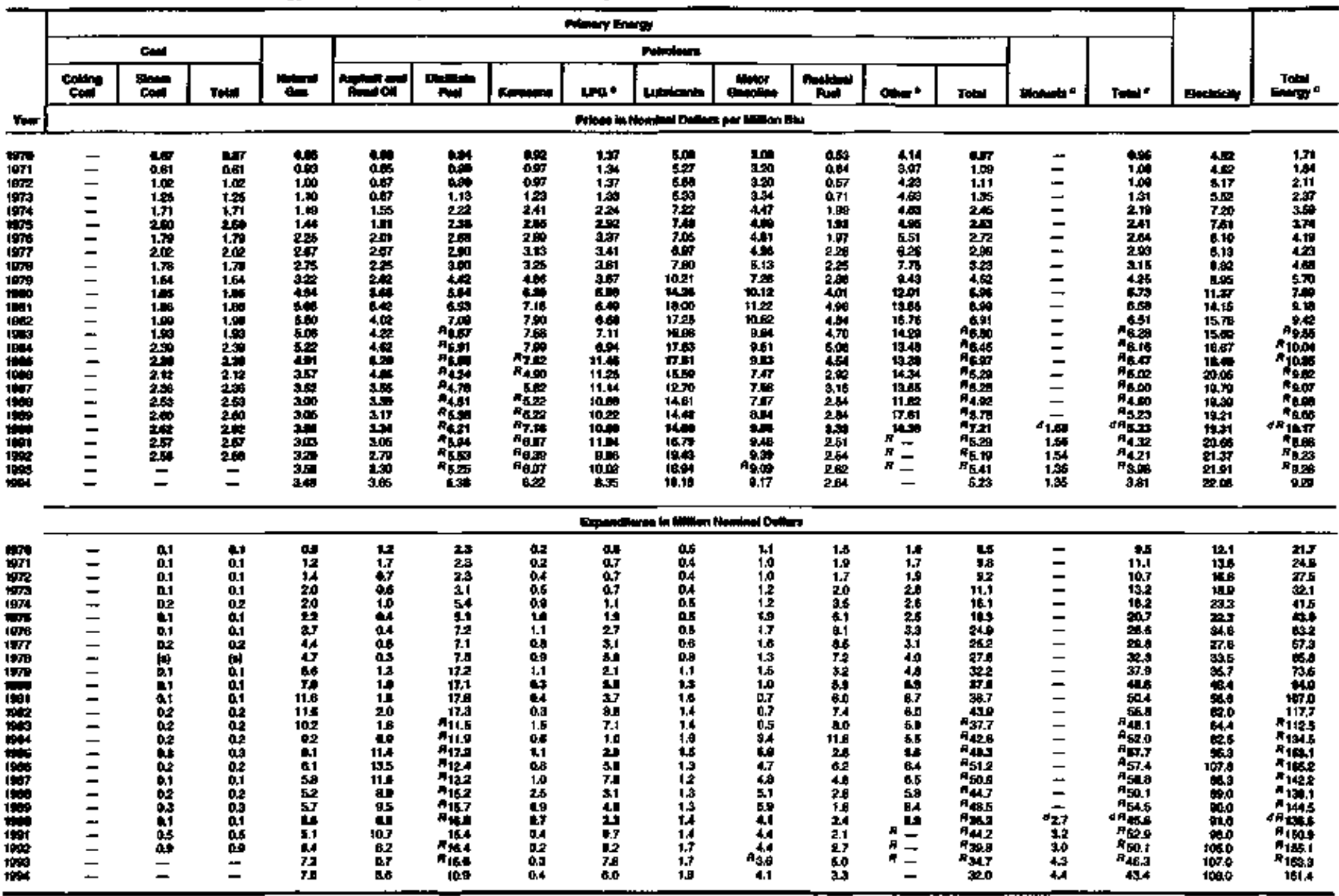

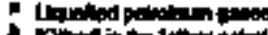

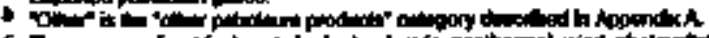

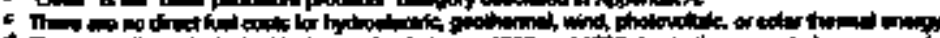

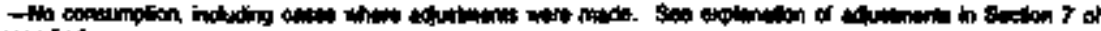

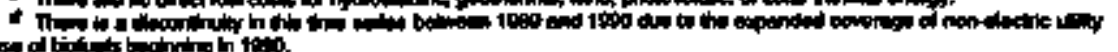
Noptidisen

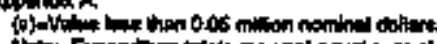




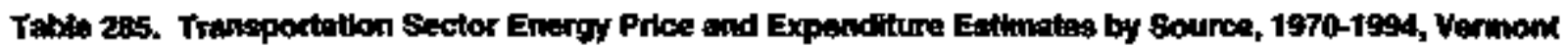

\begin{tabular}{|c|c|c|c|c|c|c|c|c|c|c|c|c|c|}
\hline \multirow[b]{4}{*}{$r$} & \multicolumn{11}{|c|}{ Phancy in ory } & \multirow[b]{3}{*}{ Endtery } & \multirow[b]{3}{*}{ En } \\
\hline & & & \multicolumn{8}{|c|}{ 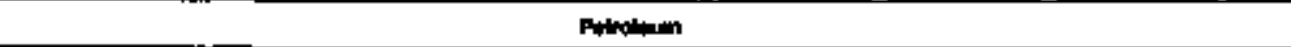 } & \multirow[b]{2}{*}{1} & & \\
\hline & - & "1 & 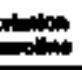 & Find & ind & IPth & 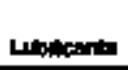 & 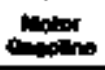 & 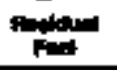 & Tote-1 & & & \\
\hline & \multicolumn{13}{|c|}{ 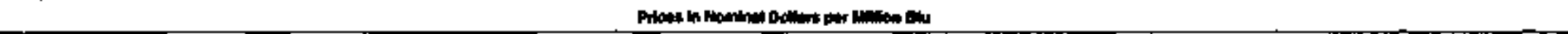 } \\
\hline 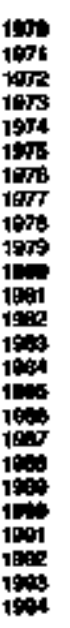 & $\begin{array}{l}0.91 \\
0.101 \\
1.20 \\
1.25 \\
1.71 \\
2.00 \\
1.79 \\
2.92 \\
= \\
= \\
= \\
= \\
= \\
= \\
= \\
= \\
= \\
= \\
=\end{array}$ & $\begin{array}{l}= \\
= \\
= \\
= \\
= \\
= \\
= \\
= \\
= \\
= \\
= \\
= \\
=\end{array}$ & 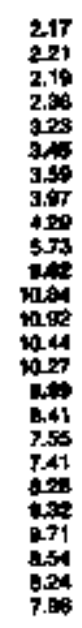 & 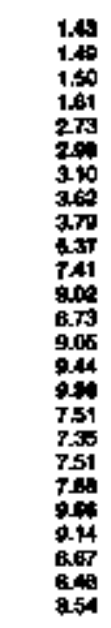 & 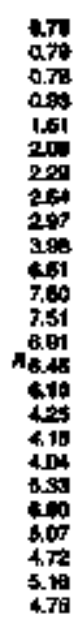 & 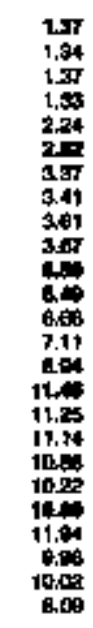 & 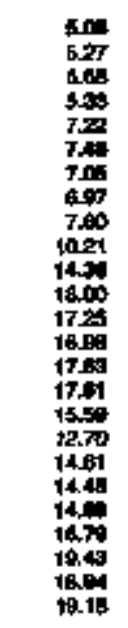 & 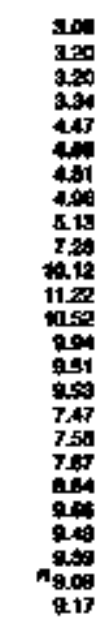 & 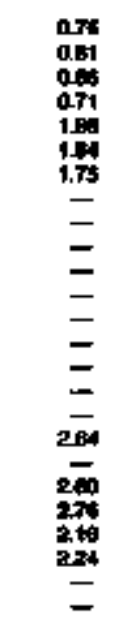 & 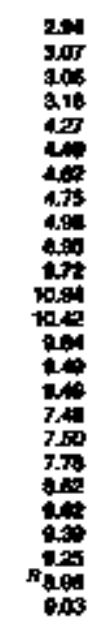 & 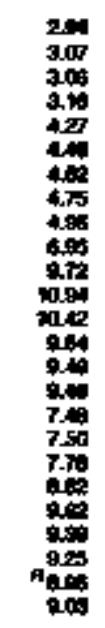 & $\begin{array}{l}= \\
= \\
= \\
= \\
= \\
= \\
= \\
= \\
= \\
= \\
= \\
= \\
=\end{array}$ & 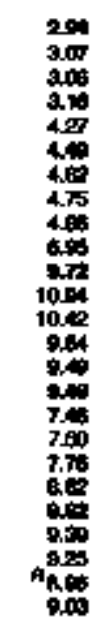 \\
\hline \multicolumn{14}{|c|}{ 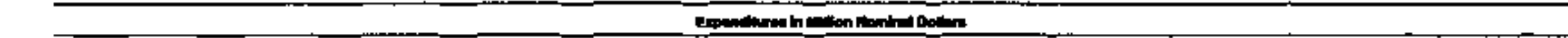 } \\
\hline 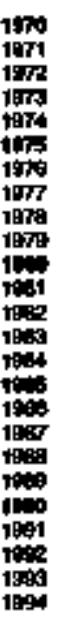 & 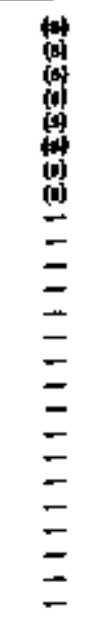 & $\begin{array}{l}= \\
= \\
= \\
= \\
= \\
= \\
= \\
= \\
= \\
= \\
= \\
= \\
= \\
=\end{array}$ & $\begin{array}{l}0.2 \\
0.1 \\
0.1 \\
0.1 \\
0.1 \\
0.2 \\
0.2 \\
0.2 \\
0.1 \\
0.1 \\
1.1 \\
0.0 \\
1.1 \\
1.3 \\
0.8 \\
1.1 \\
1.2 \\
0.5 \\
0.8 \\
0.7 \\
0.7 \\
0.7 \\
0.0 \\
0.5 \\
0.5\end{array}$ & 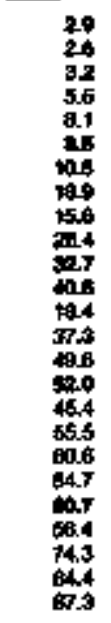 & 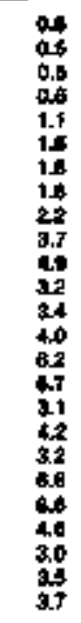 & 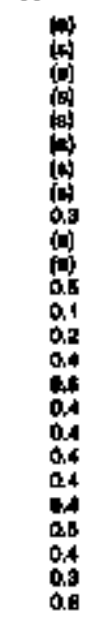 & 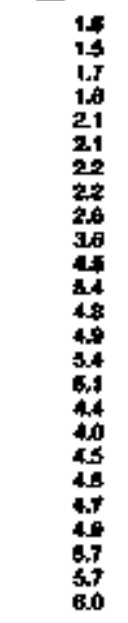 & 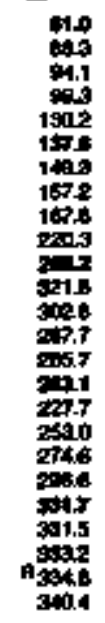 & 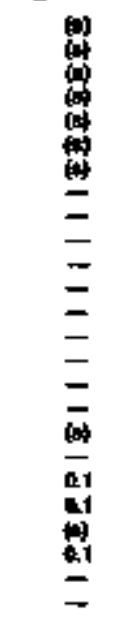 & 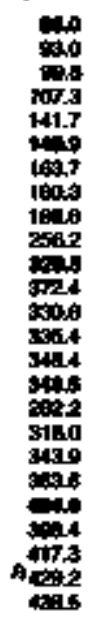 & 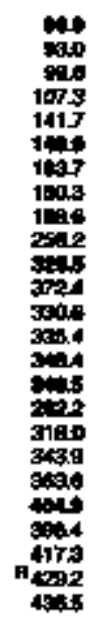 & $\begin{array}{l}= \\
= \\
= \\
= \\
= \\
= \\
= \\
= \\
= \\
= \\
= \\
= \\
=\end{array}$ & 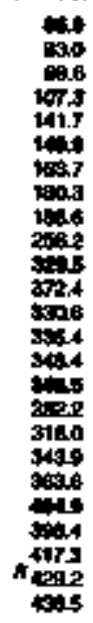 \\
\hline
\end{tabular}




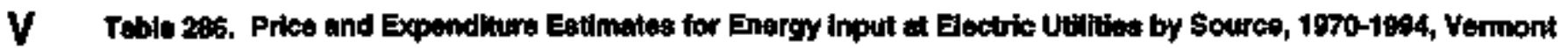

\begin{tabular}{|c|c|c|c|c|c|c|c|c|c|}
\hline \multirow[b]{3}{*}{ Ywr } & \multirow[b]{2}{*}{ Cod } & \multirow[b]{2}{*}{ 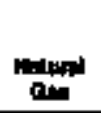 } & \multicolumn{4}{|c|}{ Arolen } & \multirow[b]{2}{*}{ 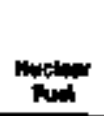 } & \multirow[b]{2}{*}{ Batuen } & \multirow[b]{2}{*}{ Tom } \\
\hline & & & ont & ut & 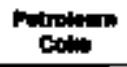 & Totd & & & \\
\hline & \multicolumn{9}{|c|}{ 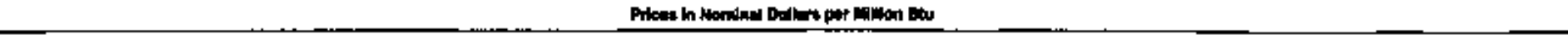 } \\
\hline \multirow[t]{2}{*}{ 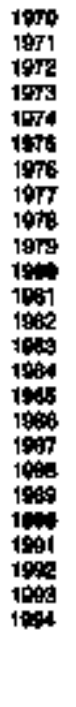 } & 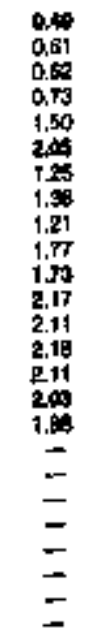 & 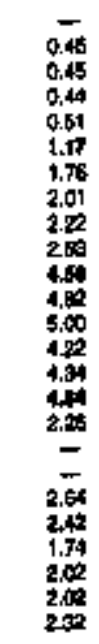 & $\begin{array}{l}0.09 \\
0.09 \\
0.01 \\
0.75 \\
1.97 \\
1.95 \\
1.90 \\
1.14 \\
1.06 \\
2.02 \\
= \\
= \\
= \\
= \\
= \\
= \\
= \\
= \\
= \\
= \\
=\end{array}$ & 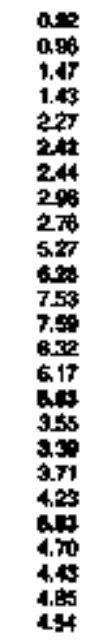 & $\begin{array}{l}= \\
= \\
= \\
= \\
= \\
= \\
= \\
= \\
= \\
= \\
= \\
= \\
= \\
=\end{array}$ & 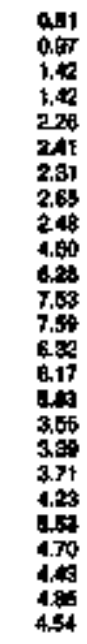 & 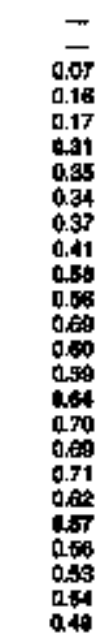 & 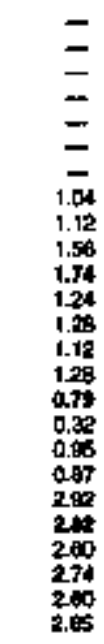 & 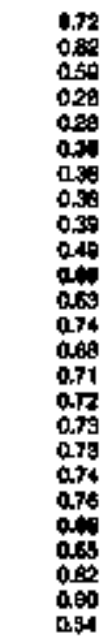 \\
\hline & \multicolumn{9}{|c|}{ 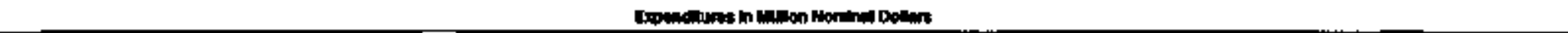 } \\
\hline 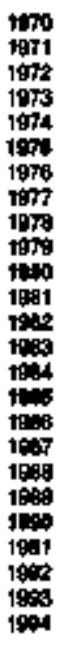 & $\begin{array}{l}07 \\
07 \\
03 \\
0.7 \\
1.4 \\
0.7 \\
03 \\
0.4 \\
02 \\
06 \\
04 \\
0.8 \\
1.7 \\
16 \\
20 \\
10 \\
06 \\
= \\
= \\
= \\
= \\
= \\
=\end{array}$ & 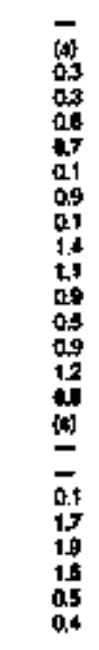 & $\begin{array}{l}0.1 \\
0.1 \\
0.1 \\
0.1 \\
0.1 \\
0.1 \\
0.2 \\
0.2 \\
0.1 \\
0.2 \\
= \\
= \\
= \\
= \\
= \\
= \\
= \\
= \\
= \\
=\end{array}$ & $\begin{array}{l}1.8 \\
1.8 \\
1.8 \\
1.8 \\
1.8 \\
1.8 \\
0.7 \\
0.3 \\
0.3 \\
1.0 \\
0.0 \\
1.1 \\
1.0 \\
0.8 \\
1.1 \\
1.1 \\
0.0 \\
1.4 \\
1.8 \\
1.2 \\
0.0 \\
0.4 \\
0.2 \\
0.5 \\
0.8\end{array}$ & $\begin{array}{l}= \\
= \\
= \\
= \\
= \\
= \\
= \\
= \\
= \\
= \\
= \\
= \\
= \\
=\end{array}$ & $\begin{array}{l}1.4 \\
1.8 \\
1.7 \\
1.8 \\
1.8 \\
1.2 \\
0.9 \\
0.7 \\
0.4 \\
1.8 \\
2.3 \\
1.1 \\
1.0 \\
0.8 \\
1.1 \\
1.1 \\
0.8 \\
1.4 \\
1.8 \\
1.2 \\
0.9 \\
0.4 \\
0.2 \\
0.6 \\
0.6\end{array}$ & 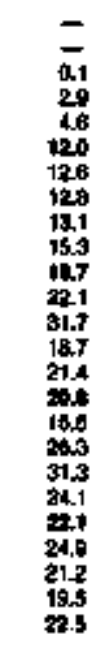 & 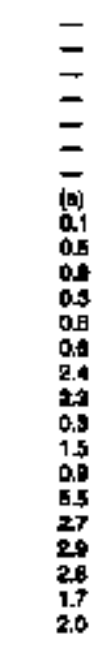 & 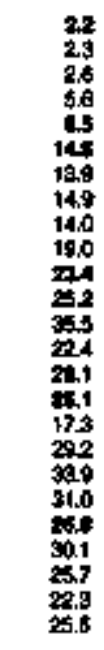 \\
\hline
\end{tabular}

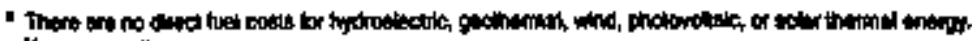

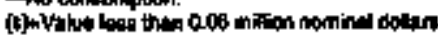

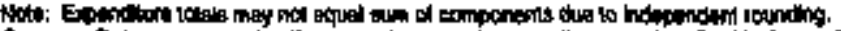

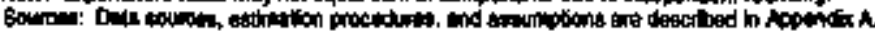


Table 287. Energy Price and Expendlitre Exulmates by Source, 1970-1994, Virglnti

\begin{tabular}{|c|c|c|c|c|c|c|c|c|c|c|c|c|c|c|c|c|c|}
\hline \multirow[b]{4}{*}{ Fon } & \multicolumn{14}{|c|}{ Phing tingeg } & \multirow{3}{*}{ 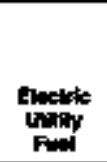 } & \multirow{3}{*}{ 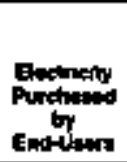 } & \multirow[b]{3}{*}{ Enety } \\
\hline & \multicolumn{3}{|c|}{ Fon } & \multirow[b]{2}{*}{ اسחה } & \multicolumn{7}{|c|}{ Panoinen } & \multirow[b]{2}{*}{ Nuserer } & \multirow[b]{2}{*}{ Oletwate } & \multirow[b]{2}{*}{ folla } & & & \\
\hline & Compen & $\cos$ & Tot & & $\begin{array}{l}\text { Ohathols } \\
\text { Fuel }\end{array}$ & m & $\mathbf{u a *}$ & שn & Mellot & at: & Told & & & & & & \\
\hline & \multicolumn{17}{|c|}{ 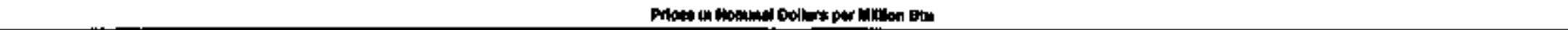 } \\
\hline \multirow[t]{2}{*}{ 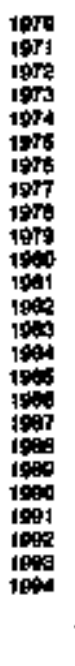 } & 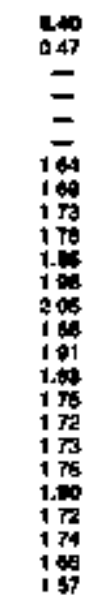 & 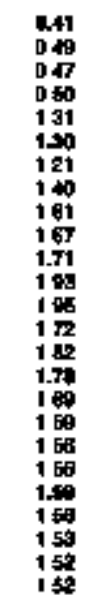 & 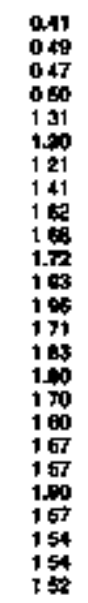 & 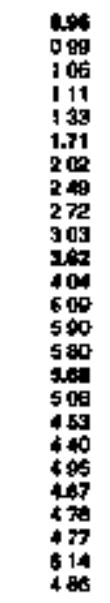 & 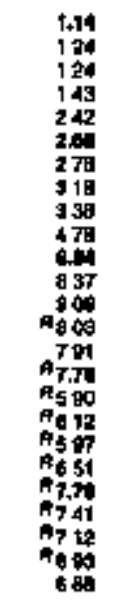 & 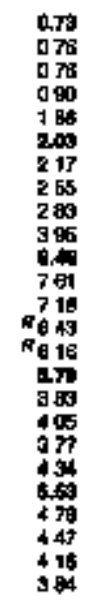 & 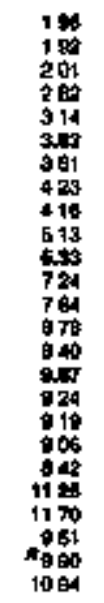 & 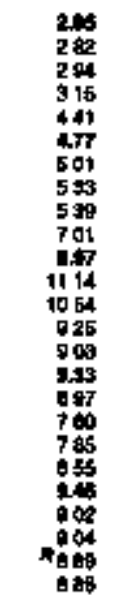 & 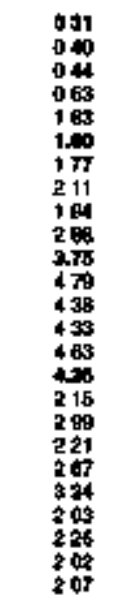 & 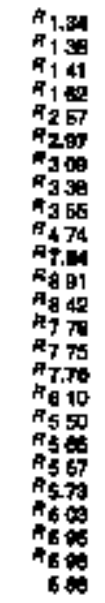 & 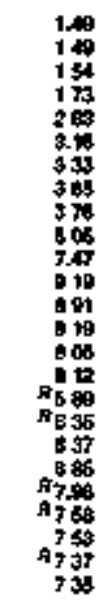 & 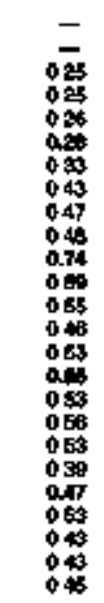 & 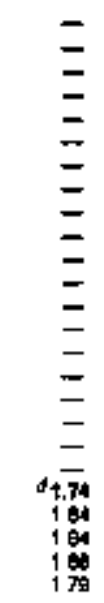 & 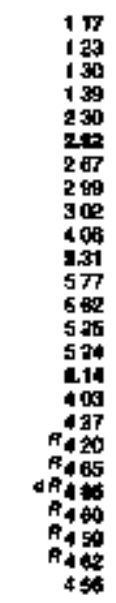 & 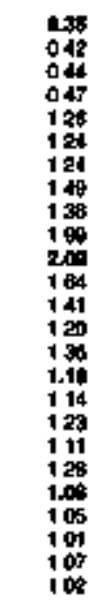 & 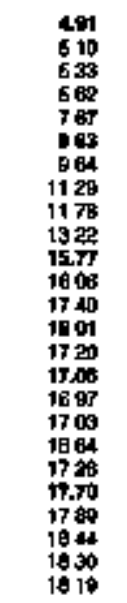 & 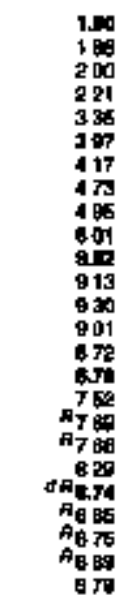 \\
\hline & \multicolumn{17}{|c|}{ 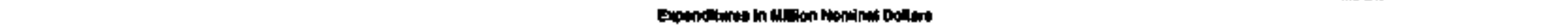 } \\
\hline 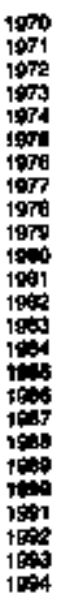 & $\begin{array}{l}93 \\
63 \\
= \\
= \\
= \\
03 \\
99 \\
243 \\
347 \\
330 \\
394 \\
897 \\
341 \\
610 \\
457 \\
421 \\
434 \\
428 \\
440 \\
427 \\
448 \\
484 \\
434 \\
407\end{array}$ & 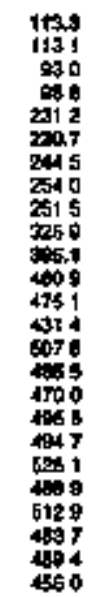 & 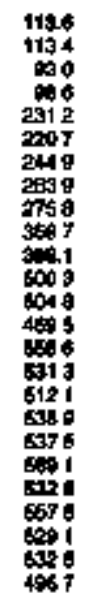 & 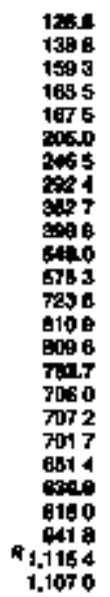 & 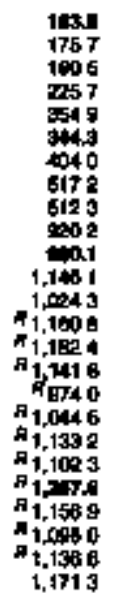 & 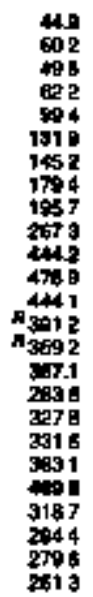 & 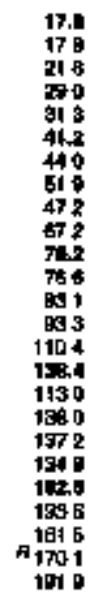 & 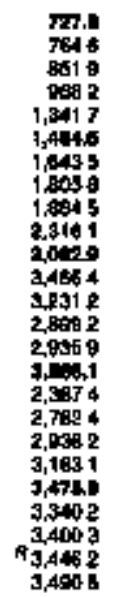 & 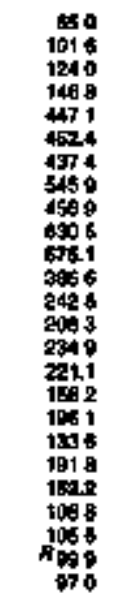 & 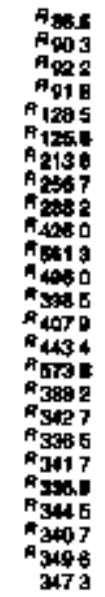 & 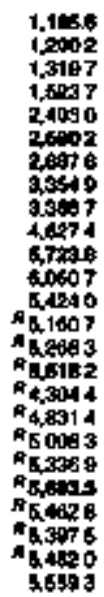 & 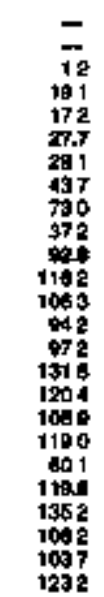 & $\begin{array}{l}= \\
= \\
= \\
= \\
= \\
= \\
= \\
= \\
= \\
= \\
= \\
= \\
4917 \\
417 \\
442 \\
465\end{array}$ & 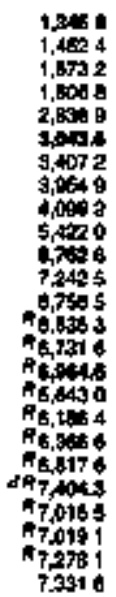 & 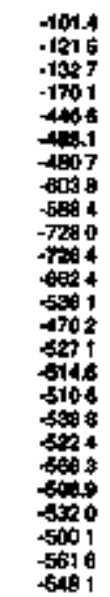 & 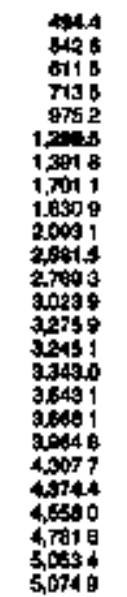 & 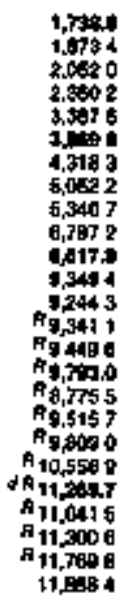 \\
\hline
\end{tabular}

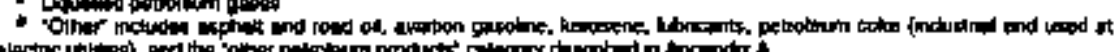

Anthersad dot

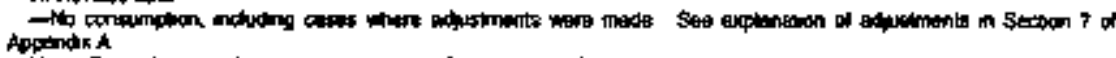

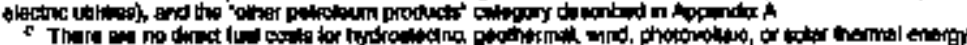

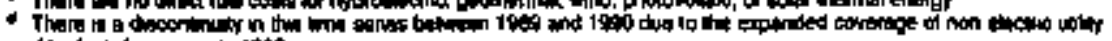

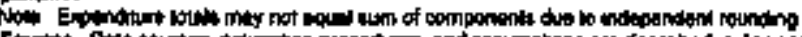

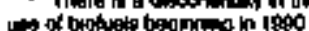




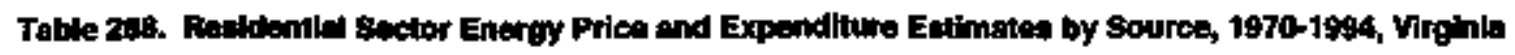

\begin{tabular}{|c|c|c|c|c|c|c|c|c|c|c|}
\hline & \multicolumn{8}{|c|}{ Prem Enoroty } & \multirow[b]{3}{*}{ 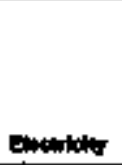 } & \multirow[b]{3}{*}{ 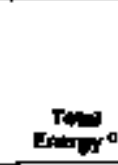 } \\
\hline & \multirow[b]{2}{*}{ 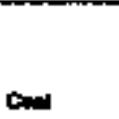 } & \multirow[b]{2}{*}{ (1) } & \multicolumn{4}{|c|}{ pand } & \multirow[b]{2}{*}{ "مسم:ت } & \multirow[b]{2}{*}{ 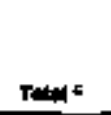 } & & \\
\hline & & & 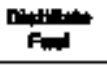 & 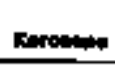 & LPG. & Tont & & & & \\
\hline 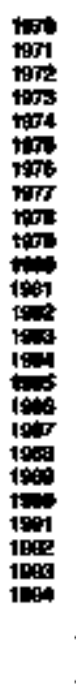 & 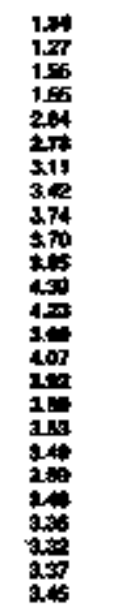 & 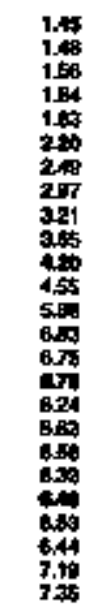 & 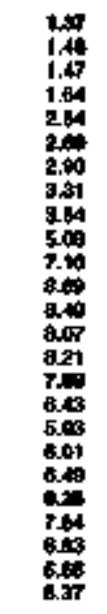 & 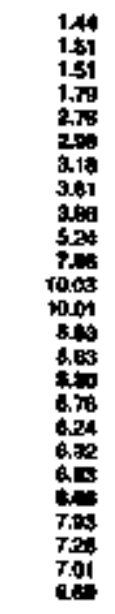 & 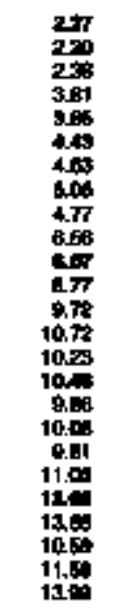 & 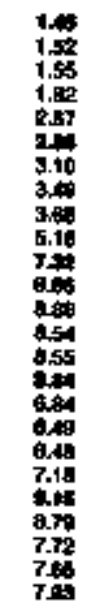 & 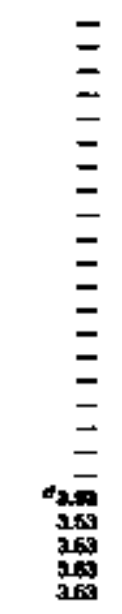 & 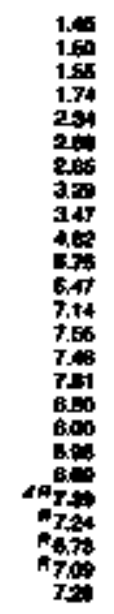 & 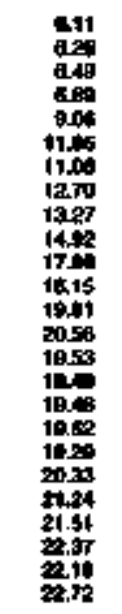 & 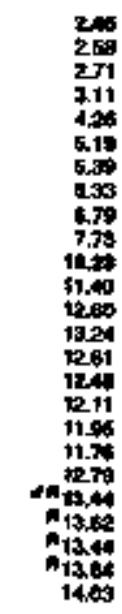 \\
\hline 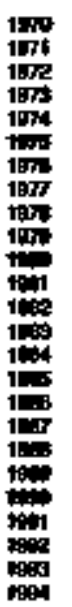 & 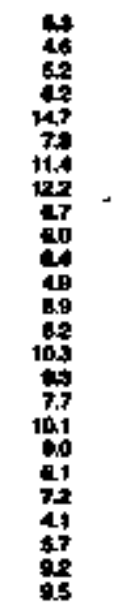 & 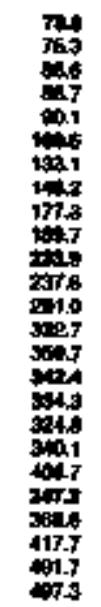 & 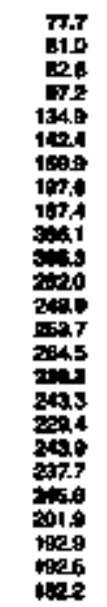 & 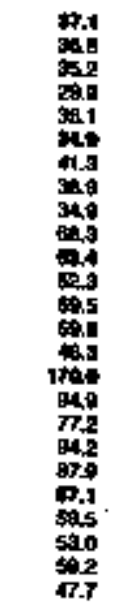 & 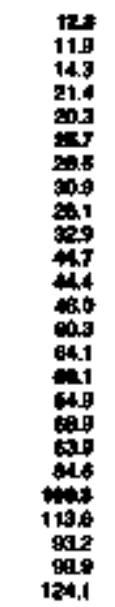 & 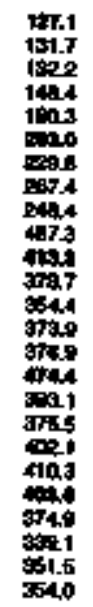 & $\begin{array}{l}= \\
= \\
= \\
= \\
= \\
= \\
= \\
= \\
= \\
\bar{z} \\
\overline{=} \\
0109 \\
1909 \\
2020 \\
2220\end{array}$ & 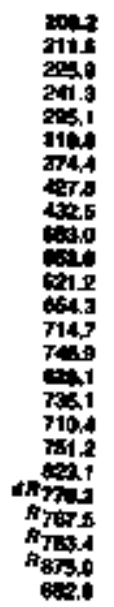 & 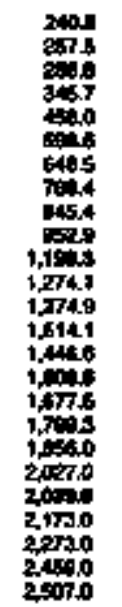 & 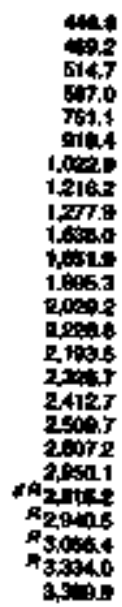 \\
\hline
\end{tabular}

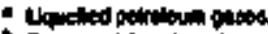

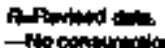

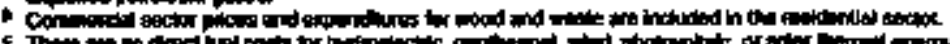

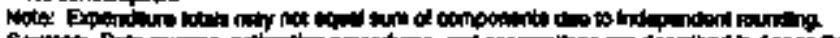

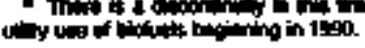




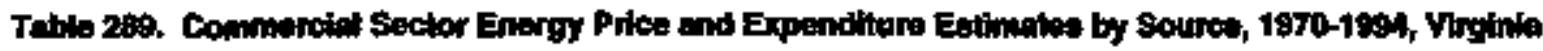

\begin{tabular}{|c|c|c|c|c|c|c|c|c|c|c|c|}
\hline \multirow[b]{4}{*}{$\mathbf{n}$} & \multicolumn{9}{|c|}{ 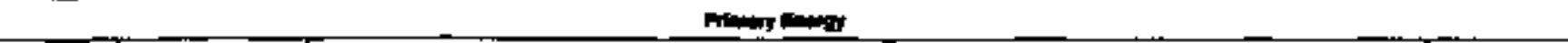 } & \multirow[b]{3}{*}{ andery } & \multirow[b]{3}{*}{ Ensts } \\
\hline & \multirow[b]{2}{*}{ Con } & \multirow[b]{2}{*}{ הים } & \multicolumn{6}{|c|}{ ntwing } & \multirow[b]{2}{*}{ Toket } & & \\
\hline & & & 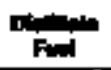 & 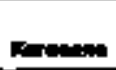 & $\mathbf{u n d}$ & tor & 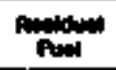 & $T=1$ & & & \\
\hline & \multicolumn{11}{|c|}{ 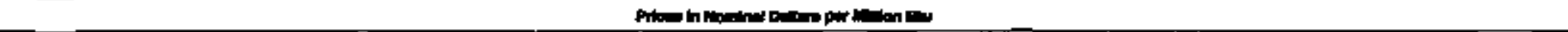 } \\
\hline \multirow[t]{2}{*}{ 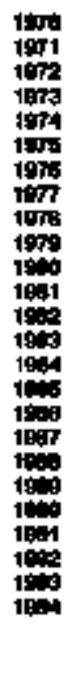 } & 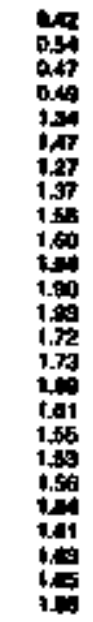 & 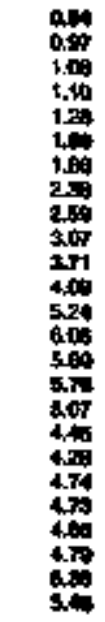 & 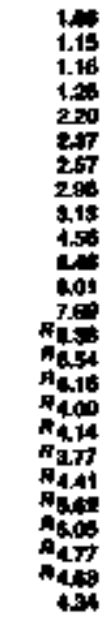 & 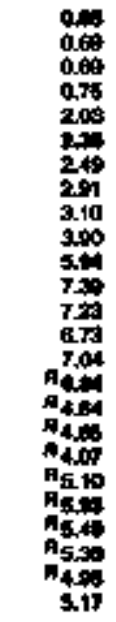 & 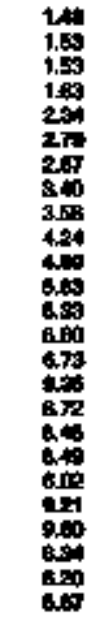 & 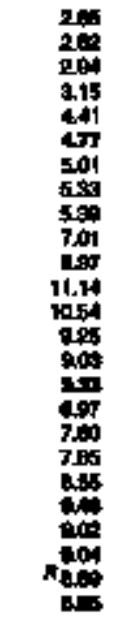 & 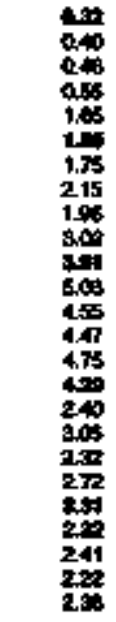 & 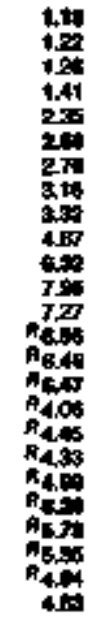 & 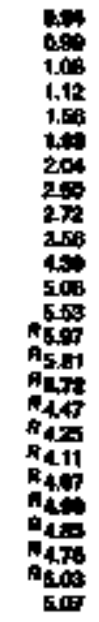 & 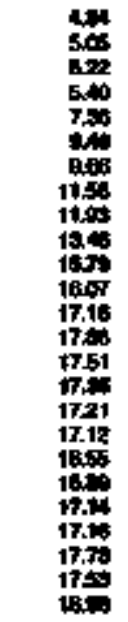 & 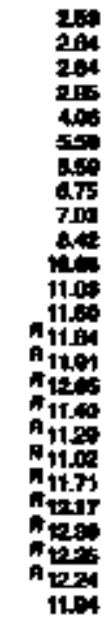 \\
\hline & \multicolumn{11}{|c|}{ A } \\
\hline 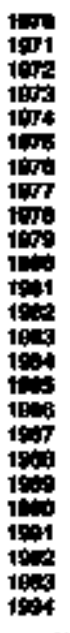 & 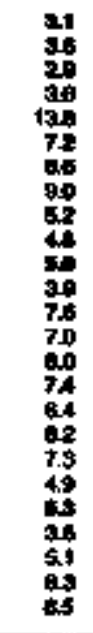 & 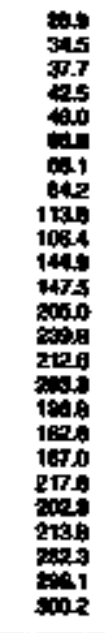 & 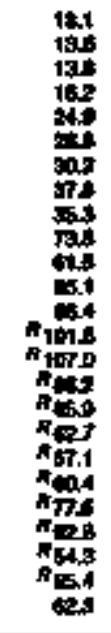 & 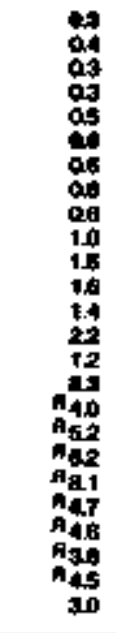 & 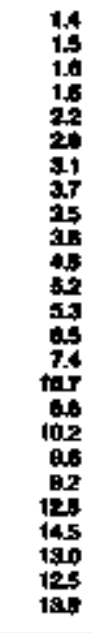 & 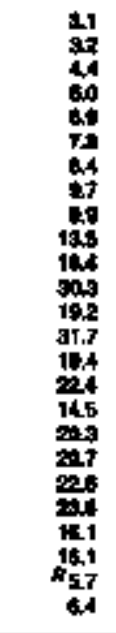 & 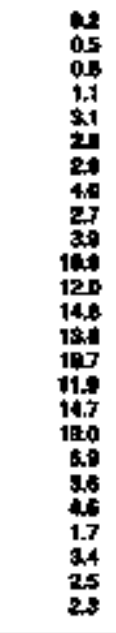 & 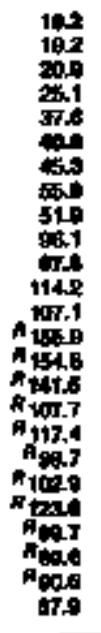 & 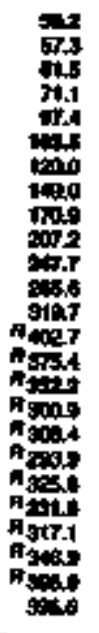 & 象 & 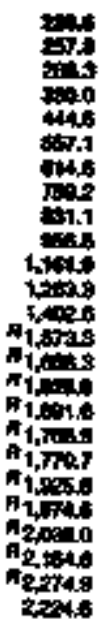 \\
\hline
\end{tabular}

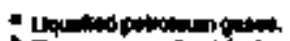

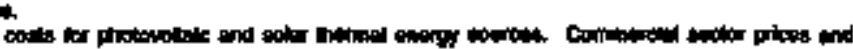

Aramend dom.

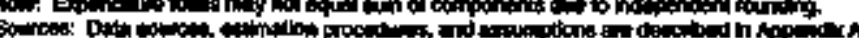


Tuble 290. Industrial Sector Energy Price and Expendillure Eotimates by Sourco, 1870-1984, Virginla

\begin{tabular}{|c|c|c|c|c|c|c|c|c|c|c|c|c|c|c|c|c|c|}
\hline & \multicolumn{15}{|c|}{ Prinery tinewy } & \multirow[b]{3}{*}{ Extholy } & \multirow[b]{3}{*}{ Twing } \\
\hline & \multicolumn{3}{|c|}{ cool } & \multirow[b]{2}{*}{ 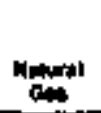 } & \multicolumn{9}{|c|}{ Abolonin } & \multirow[b]{2}{*}{ 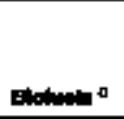 } & \multirow[b]{2}{*}{ Totis 6} & & \\
\hline & colven & sion & Tom & & $\begin{array}{c}\text { Auphat and } \\
\text { Roosd of }\end{array}$ & Pringut & 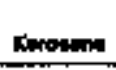 & LPG: - & Lubuttenth & 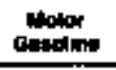 & 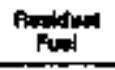 & onent" & 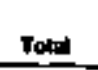 & & & & \\
\hline Your & \multicolumn{17}{|c|}{ 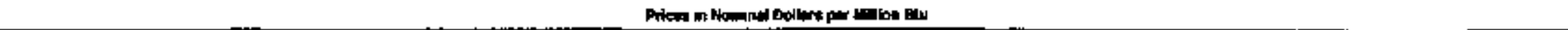 } \\
\hline \multirow[t]{2}{*}{ 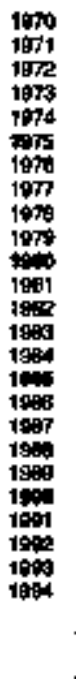 } & 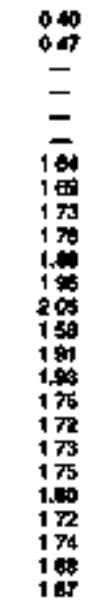 & 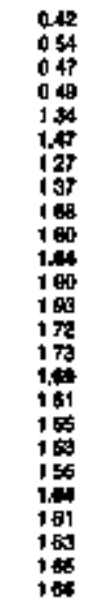 & 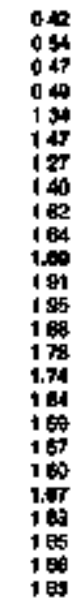 & 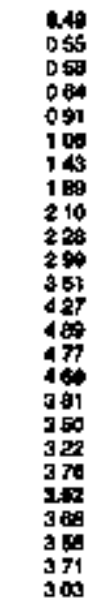 & 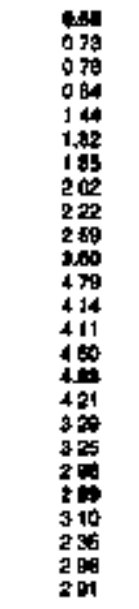 & 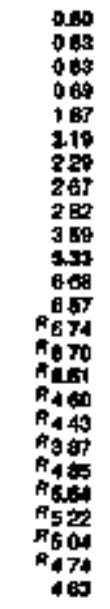 & 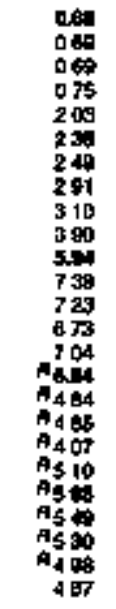 & 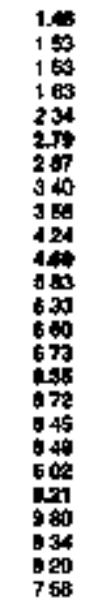 & 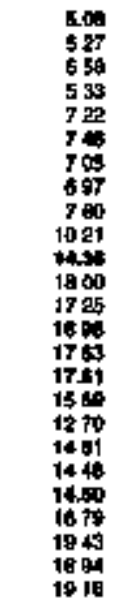 & 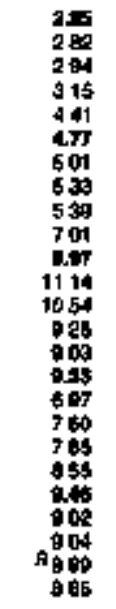 & 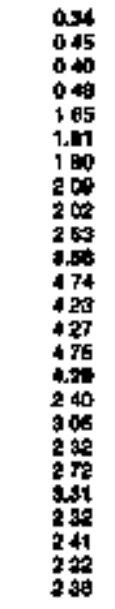 & 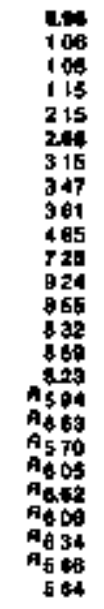 & 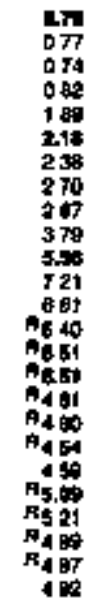 & 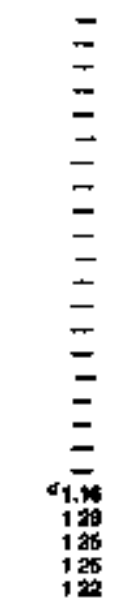 & 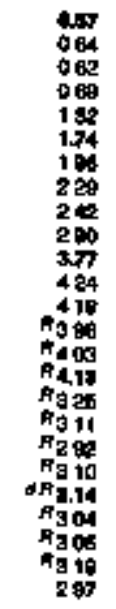 & 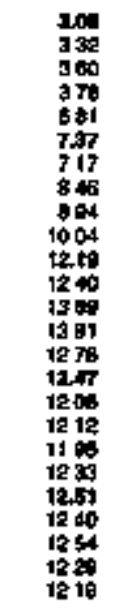 & 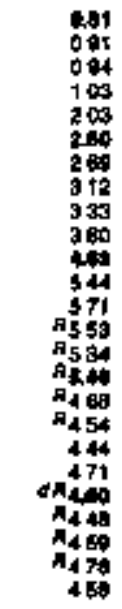 \\
\hline & \multicolumn{17}{|c|}{ 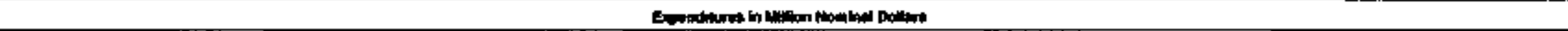 } \\
\hline 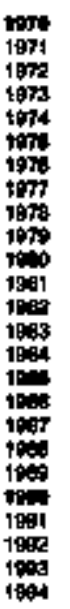 & 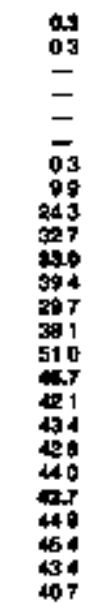 & 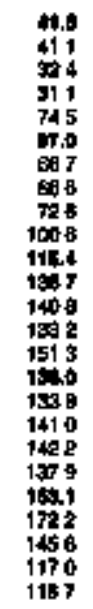 & 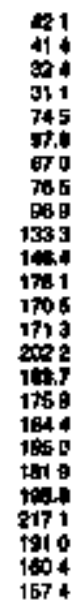 & 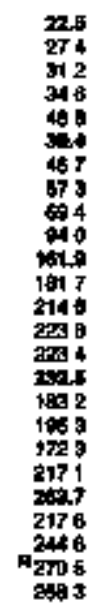 & 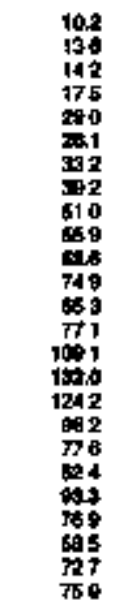 & 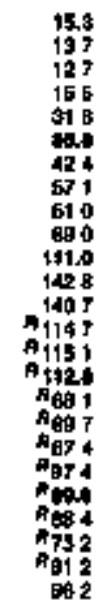 & 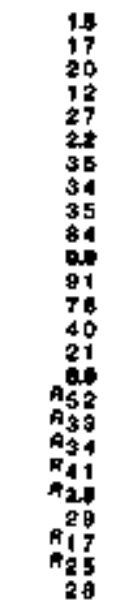 & 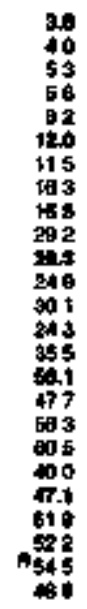 & 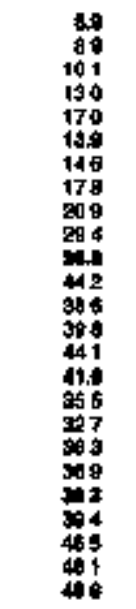 & 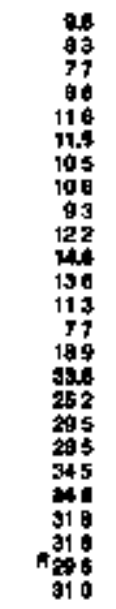 & 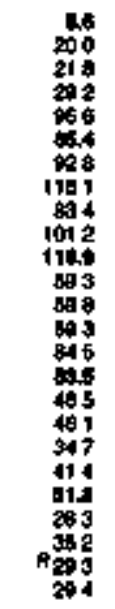 & 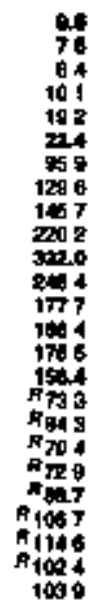 & 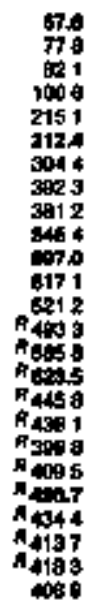 & $\begin{array}{l}= \\
= \\
= \\
= \\
= \\
= \\
= \\
= \\
= \\
= \\
= \\
= \\
130 \\
218 \\
218 \\
218 \\
203\end{array}$ & 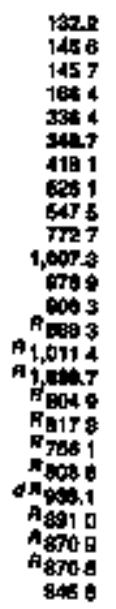 & 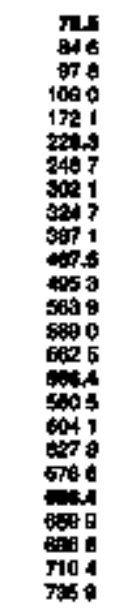 & 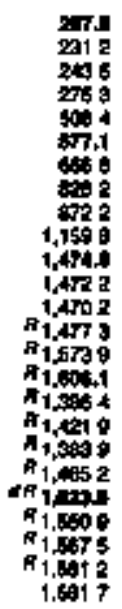 \\
\hline
\end{tabular}

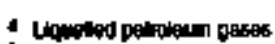

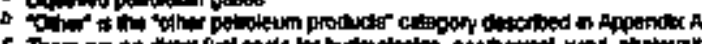

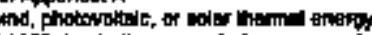

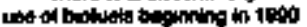

Amimand data

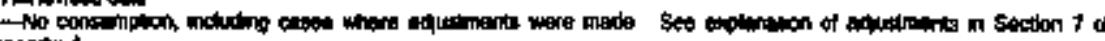

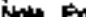

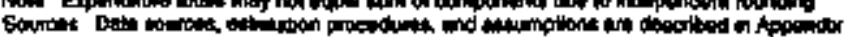


Tabie 291. Traneportalion Sector Energy Price and Expenditure Estimaties by Sourca, 1970-1894, Virglnia

\begin{tabular}{|c|c|c|c|c|c|c|c|c|c|c|c|c|c|}
\hline \multirow[b]{4}{*}{ row } & \multicolumn{11}{|c|}{ Orimary Eangy } & \multirow[b]{3}{*}{ Electitenty } & \multirow[b]{3}{*}{ Toled } \\
\hline & \multirow[b]{2}{*}{ Sod } & \multirow[b]{2}{*}{ ") } & \multicolumn{8}{|c|}{ Patronum } & \multirow[b]{2}{*}{ Total } & & \\
\hline & & & 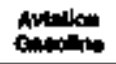 & Dinfan: & Funt & LP: & Lubpicants & Mortor & 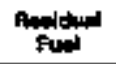 & Tom & & & \\
\hline & \multicolumn{13}{|c|}{ 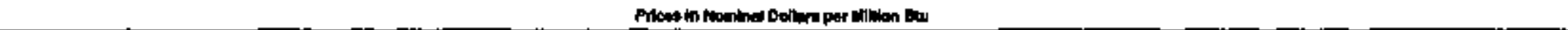 } \\
\hline \multirow[t]{2}{*}{ 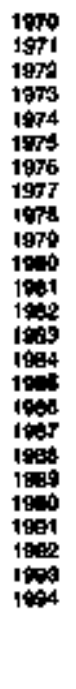 } & $\begin{array}{l}0,0 \\
0.54 \\
047 \\
0.43 \\
134 \\
1,47 \\
127 \\
137 \\
= \\
= \\
= \\
= \\
= \\
= \\
= \\
= \\
= \\
= \\
= \\
=\end{array}$ & $\begin{array}{l}= \\
= \\
= \\
= \\
= \\
= \\
= \\
= \\
= \\
= \\
= \\
= \\
= \\
586 \\
248\end{array}$ & 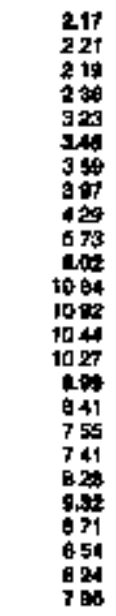 & 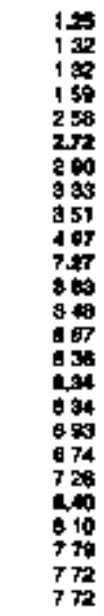 & 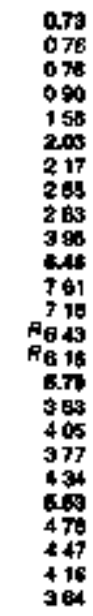 & 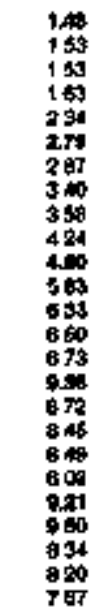 & 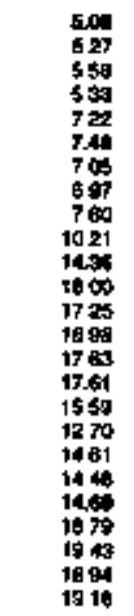 & 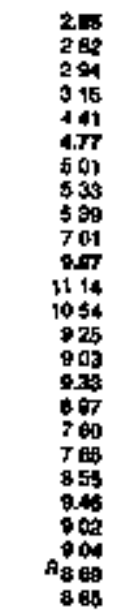 & 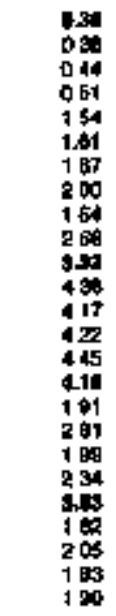 & 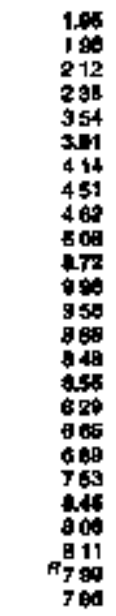 & 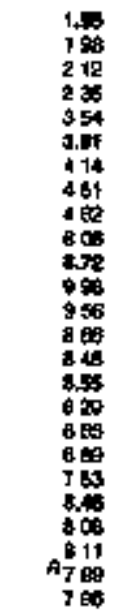 & 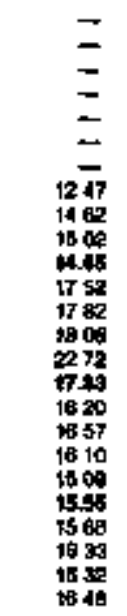 & 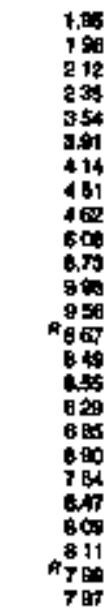 \\
\hline & \multicolumn{13}{|c|}{ 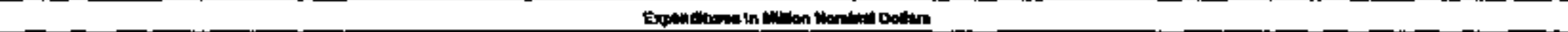 } \\
\hline 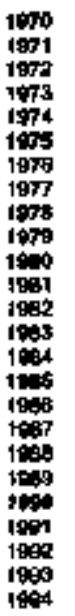 & $\begin{array}{l}0.1 \\
01 \\
001 \\
010 \\
00 \\
00 \\
0 \\
= \\
= \\
= \\
= \\
= \\
= \\
= \\
= \\
= \\
= \\
= \\
=\end{array}$ & $\begin{array}{l}= \\
= \\
= \\
= \\
= \\
= \\
= \\
= \\
= \\
= \\
= \\
= \\
= \\
0 ;\end{array}$ & 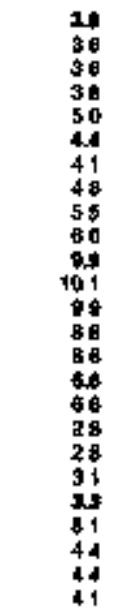 & 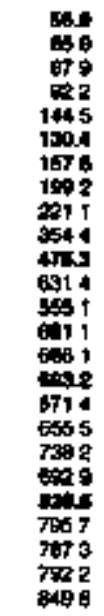 & 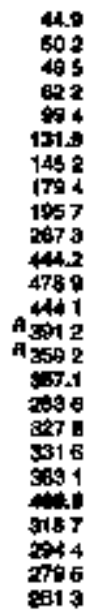 & $\begin{array}{l}03 \\
04 \\
04 \\
04 \\
04 \\
06 \\
03 \\
10 \\
10 \\
14 \\
91 \\
21 \\
10 \\
22 \\
34 \\
94 \\
18 \\
28 \\
30 \\
20 \\
21 \\
96 \\
31 \\
32 \\
82\end{array}$ & 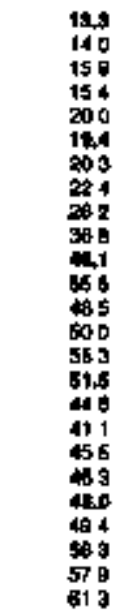 & 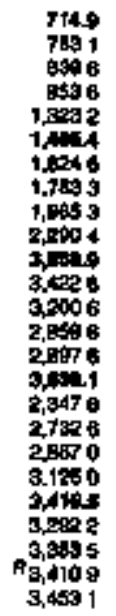 & 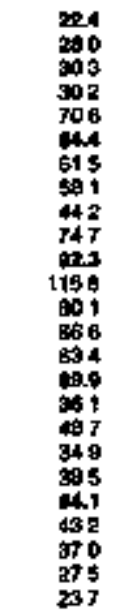 & 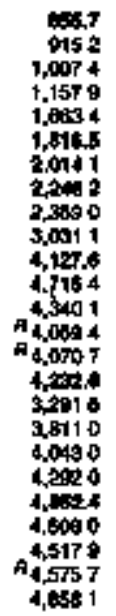 & 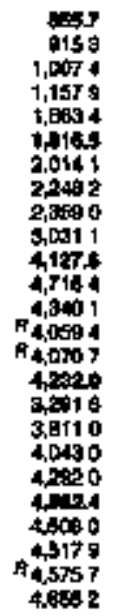 & $\begin{array}{l}= \\
= \\
= \\
= \\
03 \\
06 \\
16 \\
15 \\
22 \\
22 \\
23 \\
31 \\
32 \\
42 \\
12 \\
32 \\
40 \\
40 \\
43 \\
41 \\
40\end{array}$ & 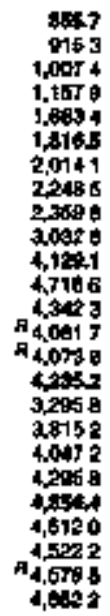 \\
\hline
\end{tabular}

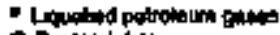

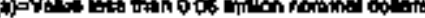

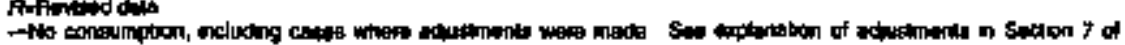

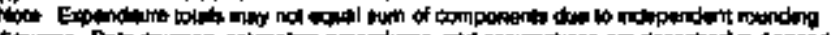
ipanitis $A$ 


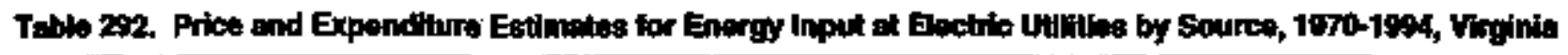

$\mathbf{I}$
$\mathbf{G}$
$\mathbf{I}$
$\mathbf{N}$
$\mathbf{A}$

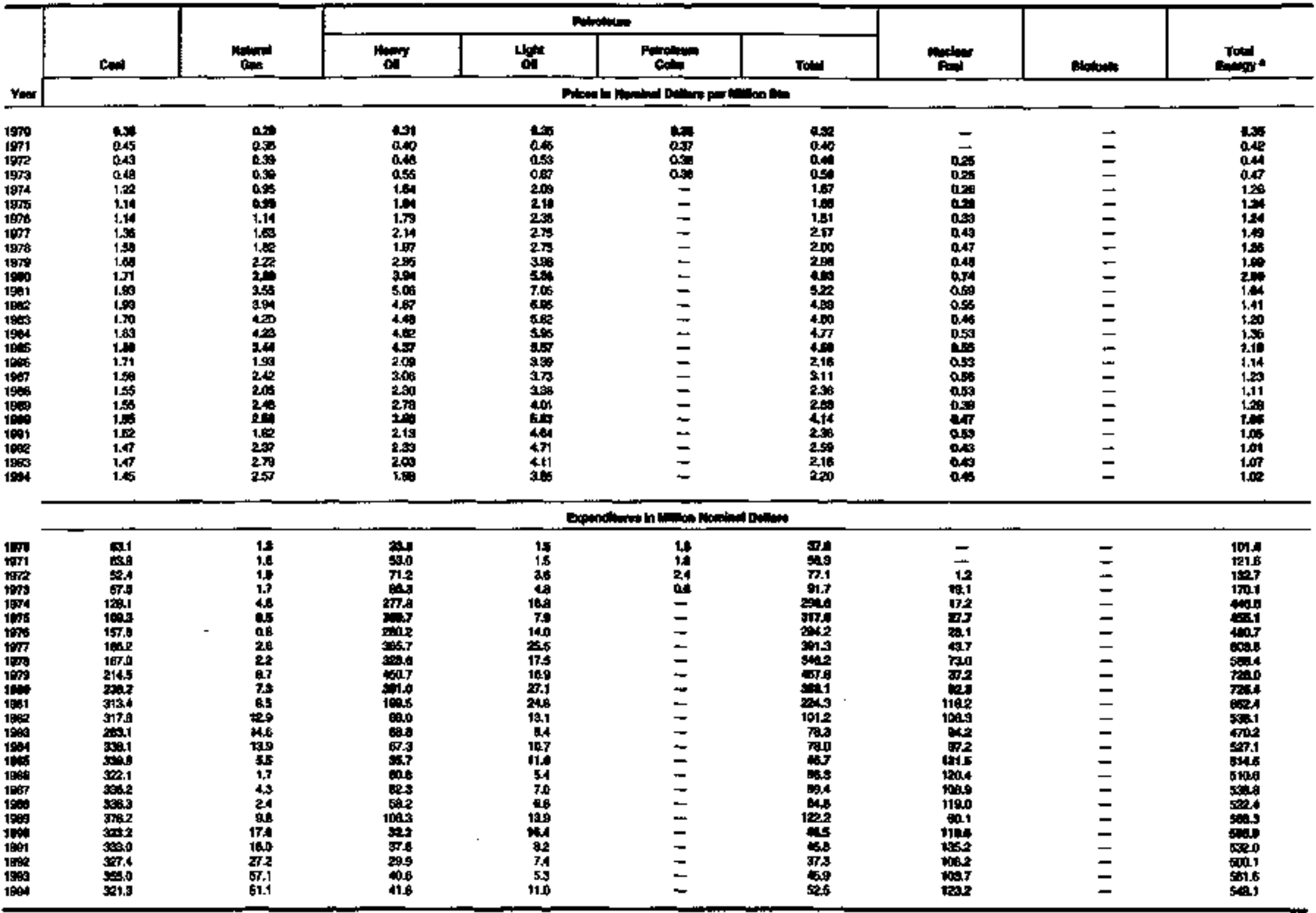

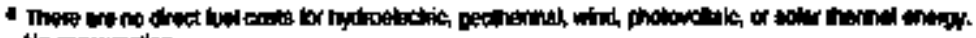

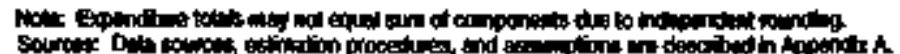

Howersunption. 


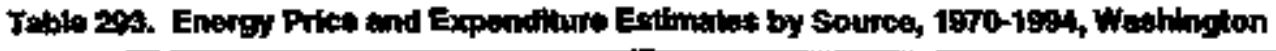

\begin{tabular}{|c|c|c|c|c|c|c|c|c|c|c|c|c|c|c|c|c|c|}
\hline & \multicolumn{14}{|c|}{ Miner tenow } & \multirow{3}{*}{ 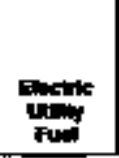 } & \multirow{3}{*}{ and } & \multirow[b]{3}{*}{$B^{T+\infty}$} \\
\hline & \multicolumn{3}{|c|}{ Com } & \multirow[b]{2}{*}{ (1) } & \multicolumn{7}{|c|}{ Peronem } & \multirow[b]{2}{*}{ rever } & \multirow[b]{2}{*}{ whot } & \multirow[b]{2}{*}{ Toteses } & & & \\
\hline & 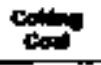 & cos & Topin & & Nam & \pm & LPG: & ats & الم= & Down". & Tots & & & & & & \\
\hline 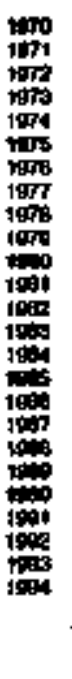 & $\begin{array}{l}= \\
z \\
= \\
z \\
z \\
= \\
= \\
= \\
= \\
= \\
= \\
= \\
=\end{array}$ & 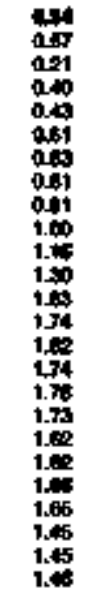 & 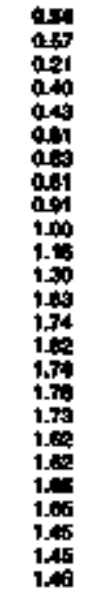 & 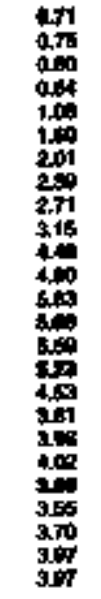 & 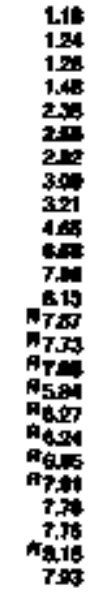 & 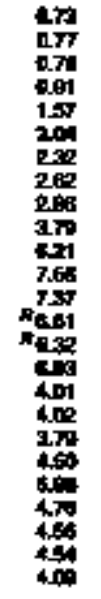 & 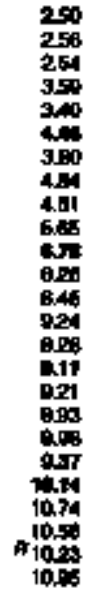 & 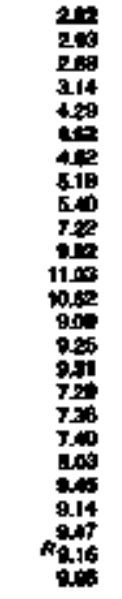 & 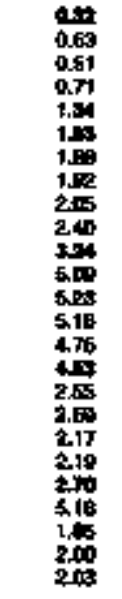 & 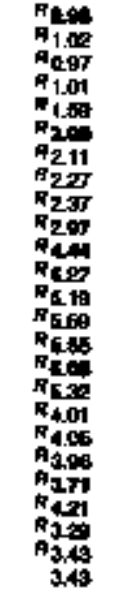 & 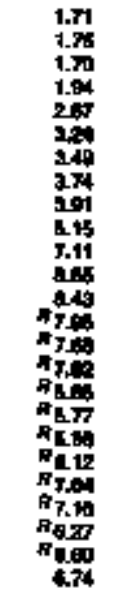 & 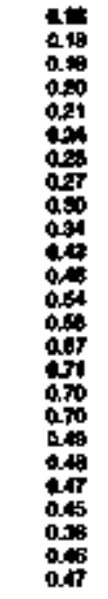 & 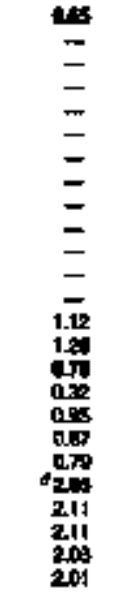 & 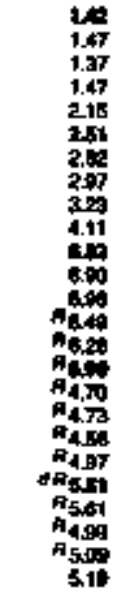 & 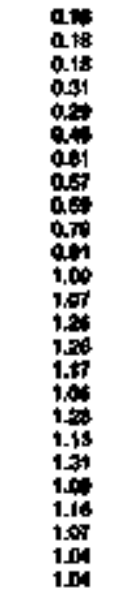 & 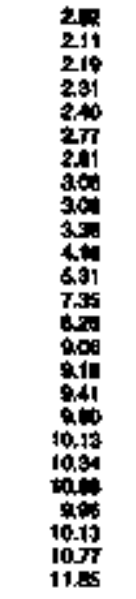 & 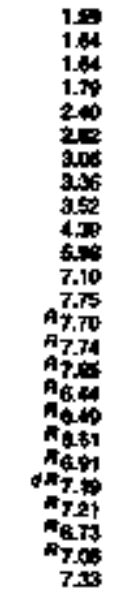 \\
\hline 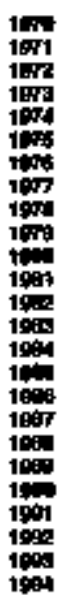 & $\begin{array}{l}= \\
= \\
= \\
= \\
= \\
= \\
= \\
= \\
= \\
= \\
= \\
= \\
=\end{array}$ & 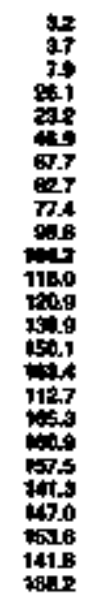 & 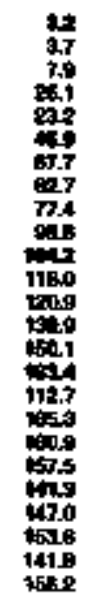 & 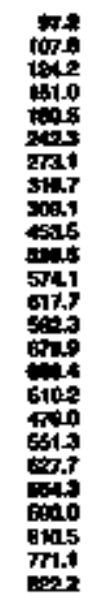 & 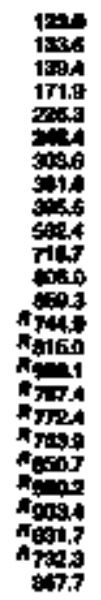 & 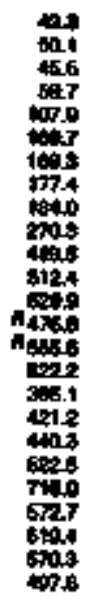 & 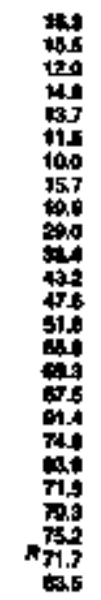 & 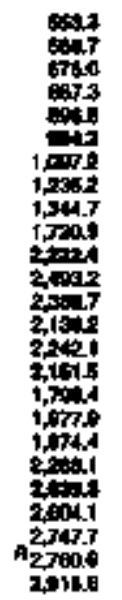 & 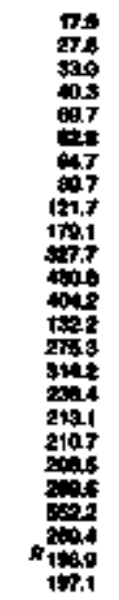 & 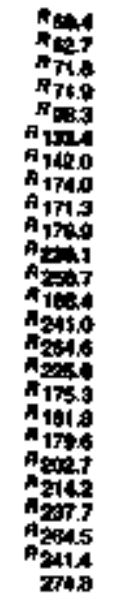 & 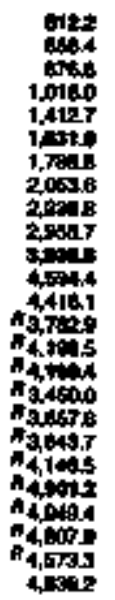 & 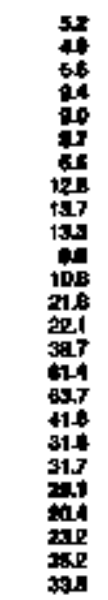 & 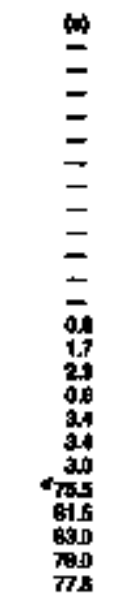 & 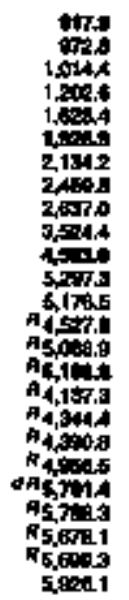 & 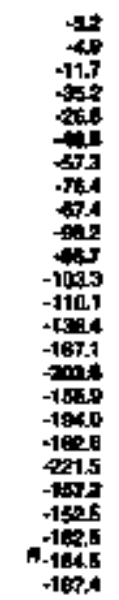 & 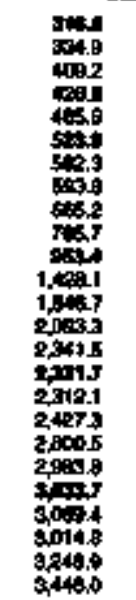 & 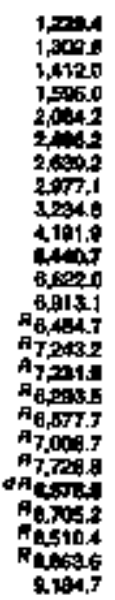 \\
\hline
\end{tabular}

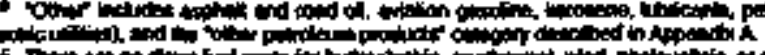
4 n

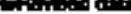

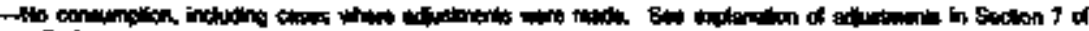

(1)

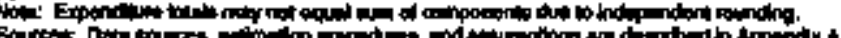


Table 294. Aeaidentiol Sector Enargy Price and Expmolture Eslimetea by Sourco, 1970-1994, Washington

\begin{tabular}{|c|c|c|c|c|c|c|c|c|c|c|}
\hline \multirow[b]{4}{*}{ Yhe } & \multicolumn{8}{|c|}{ mimary rineter } & \multirow[b]{3}{*}{ Euctidith } & \multirow[b]{3}{*}{ Tyory } \\
\hline & \multirow[b]{2}{*}{ Ceal } & \multirow[b]{2}{*}{ Anarid } & \multicolumn{4}{|c|}{ Putroluman } & \multirow[b]{2}{*}{ Boptathe } & \multirow[b]{2}{*}{ Tolen E } & & \\
\hline & & & 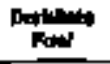 & 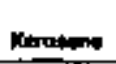 & LOA & 7 & & & & \\
\hline & \multicolumn{10}{|c|}{ 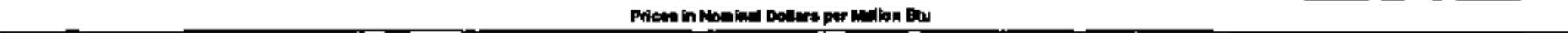 } \\
\hline \multirow[t]{2}{*}{ 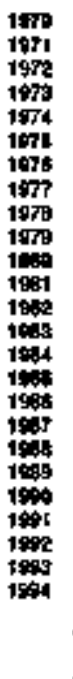 } & 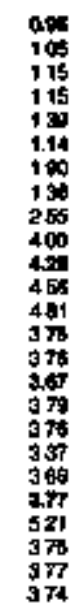 & 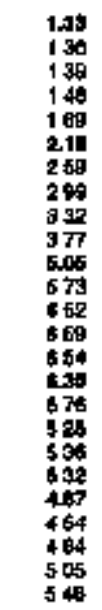 & 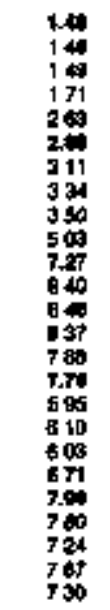 & 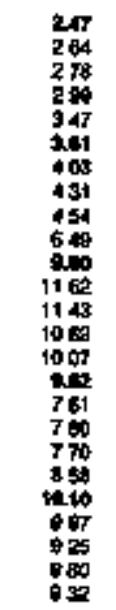 & 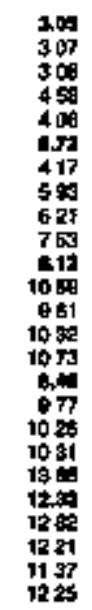 & 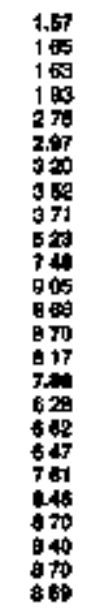 & $\begin{array}{l}= \\
= \\
= \\
= \\
= \\
= \\
= \\
= \\
= \\
= \\
= \\
= \\
478 \\
475 \\
475\end{array}$ & 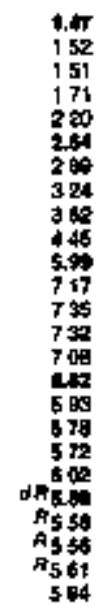 & 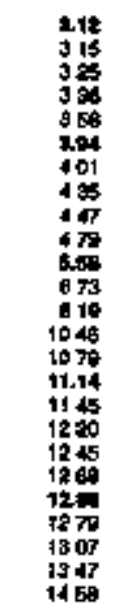 & 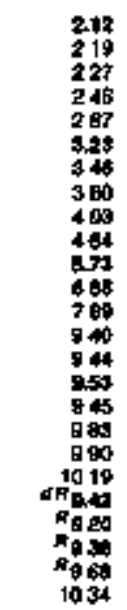 \\
\hline & \multicolumn{10}{|c|}{ 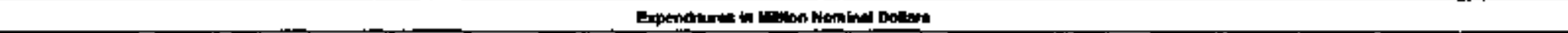 } \\
\hline 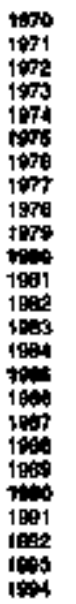 & $\begin{array}{l}13 \\
04 \\
03 \\
02 \\
01 \\
04 \\
03 \\
14 \\
35 \\
40 \\
54 \\
44 \\
65 \\
86 \\
63 \\
45 \\
26 \\
15 \\
30 \\
25 \\
1.0 \\
93 \\
27 \\
34 \\
25\end{array}$ & 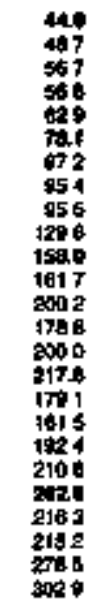 & 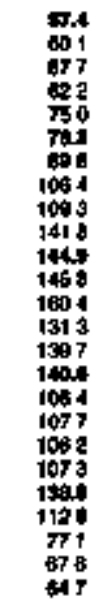 & $\begin{array}{l}1,4 \\
82 \\
18 \\
13 \\
15 \\
42 \\
68 \\
62 \\
91 \\
50 \\
16 \\
349 \\
47 \\
27 \\
42 \\
48 \\
28 \\
18 \\
26 \\
26 \\
28 \\
28 \\
18 \\
24 \\
36\end{array}$ & 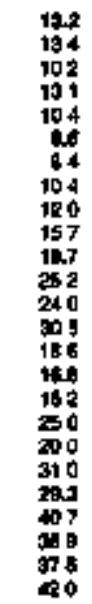 & 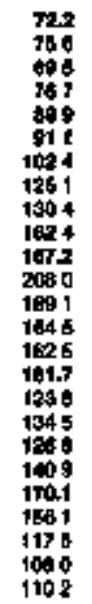 & 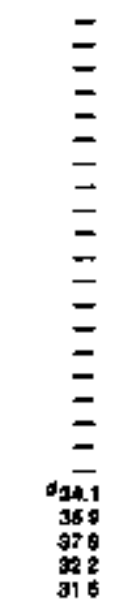 & 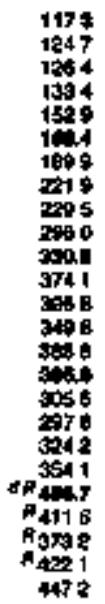 & 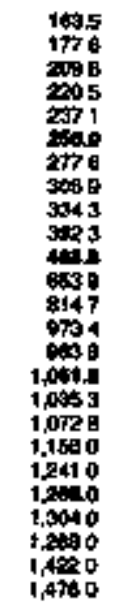 & 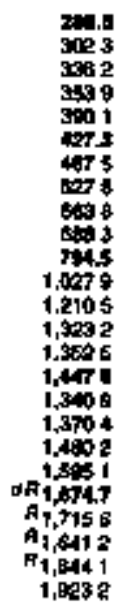 \\
\hline
\end{tabular}

- Landad potrotorem gans

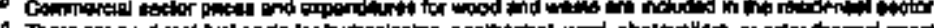

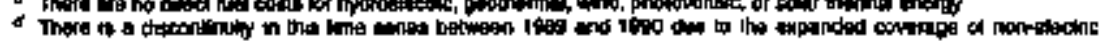

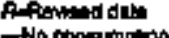

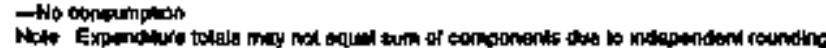

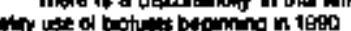

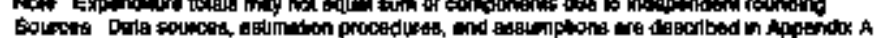

Energy Inlormontion Adiminletration 


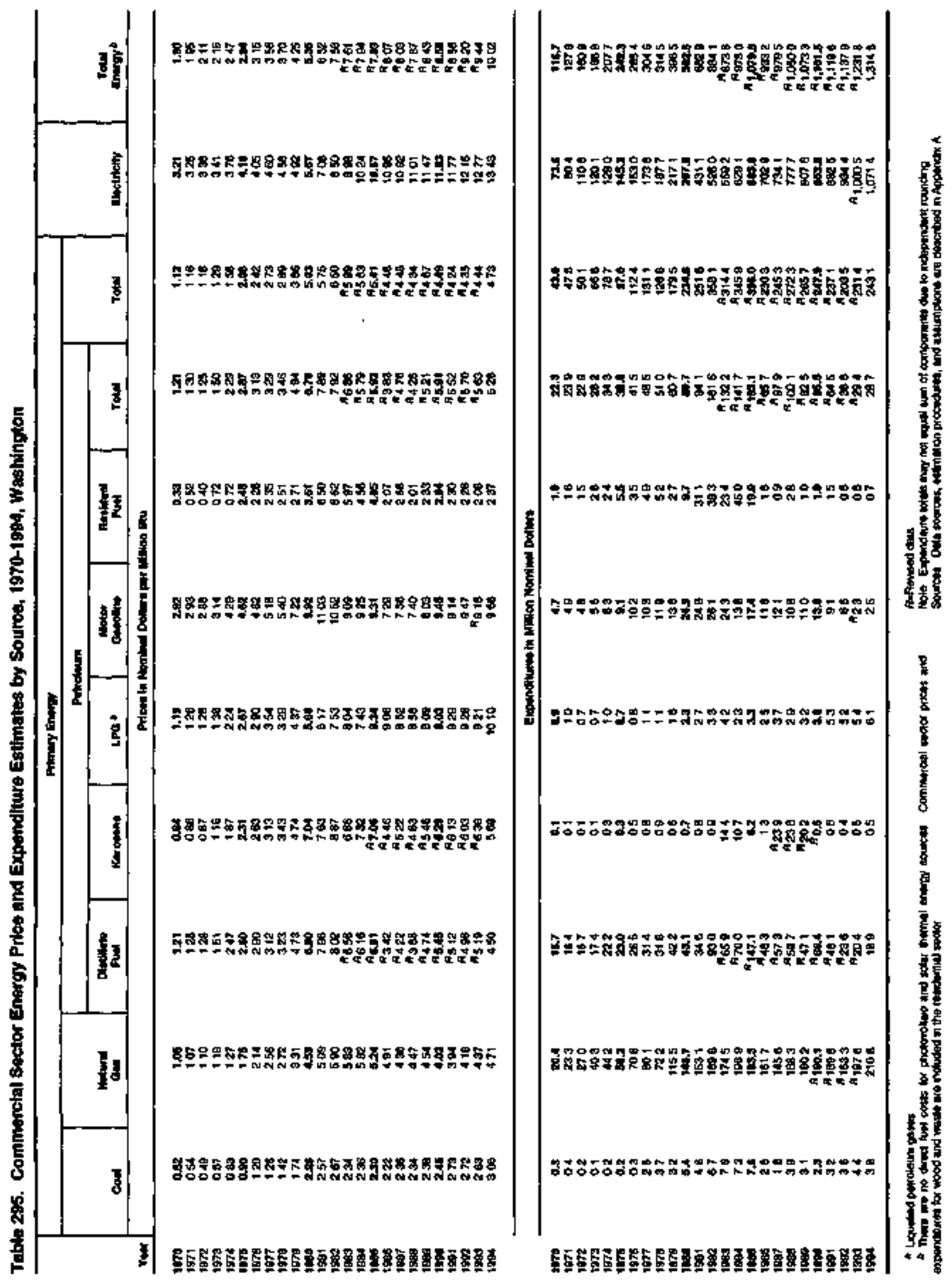




\begin{tabular}{|c|c|c|c|c|c|c|c|c|c|c|c|c|c|c|c|c|c|}
\hline & \multicolumn{15}{|c|}{ Moly tinesy } & \multirow[b]{3}{*}{ Enowitary } & \multirow[b]{3}{*}{ romeng } \\
\hline & \multicolumn{3}{|c|}{ con } & \multirow[b]{2}{*}{ 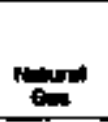 } & \multicolumn{9}{|c|}{ (1) } & \multirow[b]{2}{*}{ 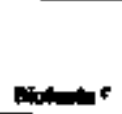 } & \multirow[b]{2}{*}{$T=\operatorname{Ta}^{\sigma}$} & & \\
\hline & collo & 1. & 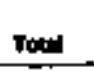 & & inding & 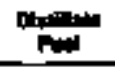 & Keptome & we: & 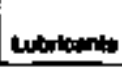 & 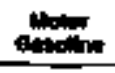 & 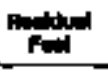 & 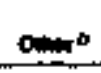 & 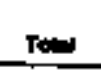 & & & & \\
\hline$x=$ & \multicolumn{17}{|c|}{ 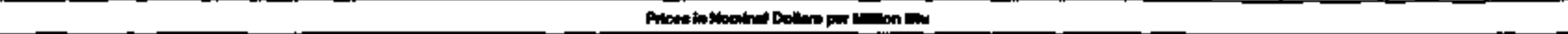 } \\
\hline \multirow[t]{2}{*}{ 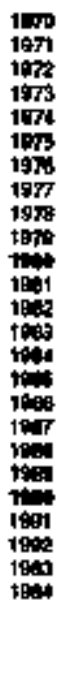 } & $\begin{array}{l}= \\
= \\
= \\
= \\
= \\
= \\
= \\
= \\
= \\
= \\
= \\
= \\
= \\
=\end{array}$ & 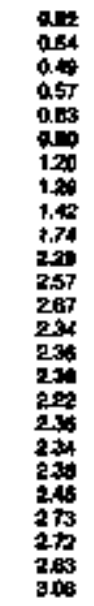 & 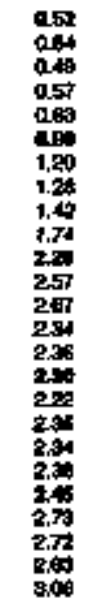 & 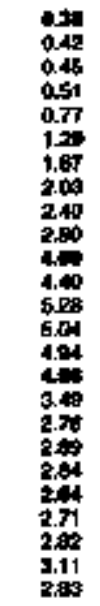 & 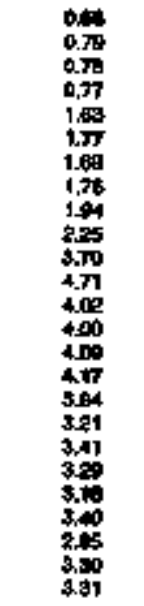 & 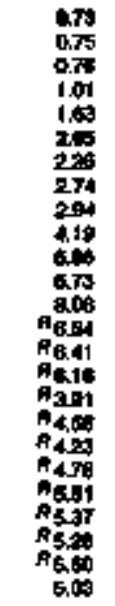 & 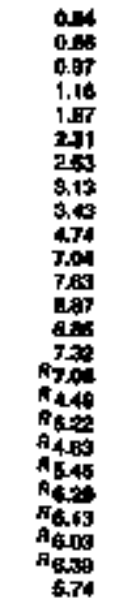 & 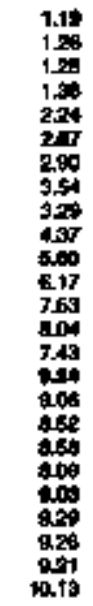 & 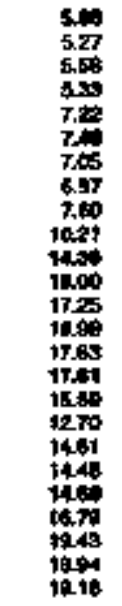 & 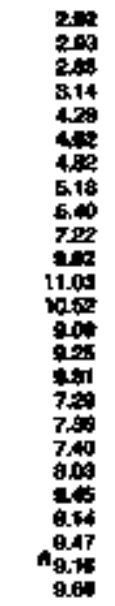 & 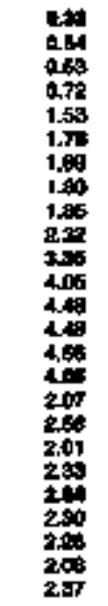 & 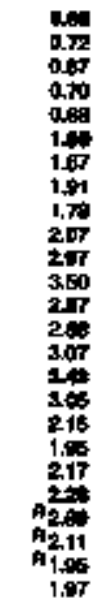 & 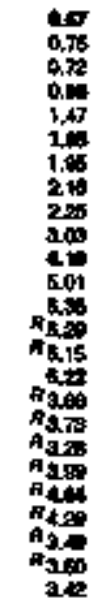 & $\begin{array}{l}z \\
z \\
z \\
z \\
z \\
z \\
z \\
= \\
z \\
z \\
z \\
= \\
11.50 \\
1.64 \\
1.54 \\
1.54\end{array}$ & 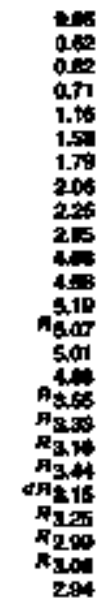 & 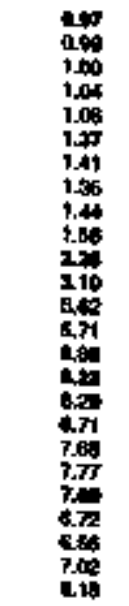 & 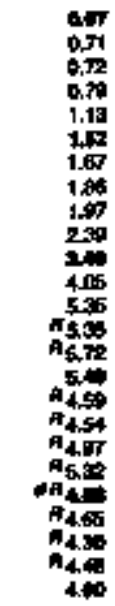 \\
\hline & \multicolumn{17}{|c|}{ 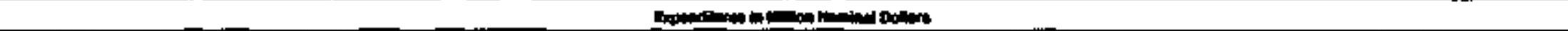 } \\
\hline 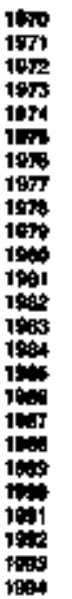 & $\begin{array}{l}\bar{z} \\
= \\
\text { z } \\
= \\
= \\
= \\
= \\
= \\
= \\
= \\
= \\
= \\
=\end{array}$ & 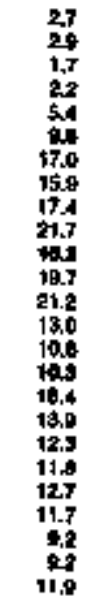 & 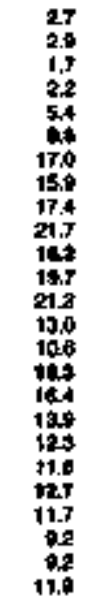 & 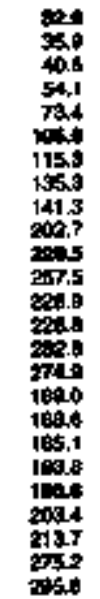 & 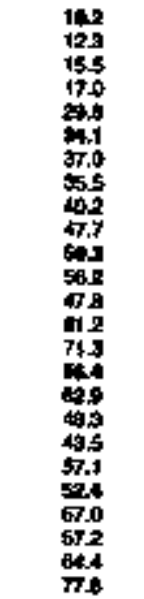 & 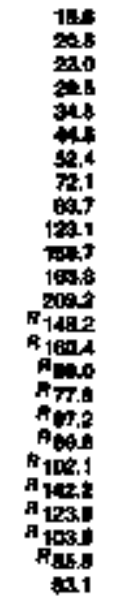 & 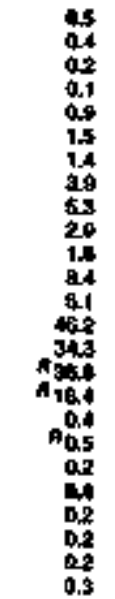 & 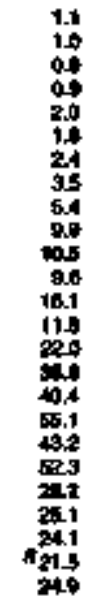 & 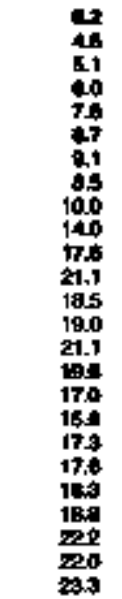 & 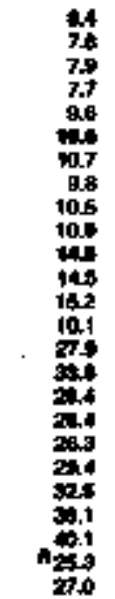 & 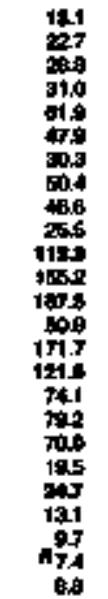 & 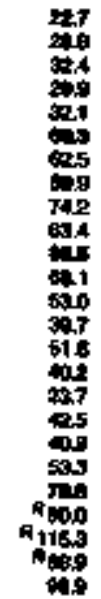 & 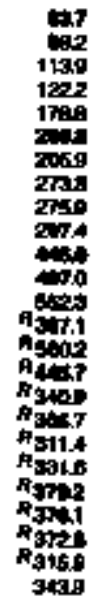 & $\begin{array}{l}= \\
= \\
= \\
= \\
= \\
= \\
= \\
= \\
= \\
= \\
= \\
045\end{array}$ & 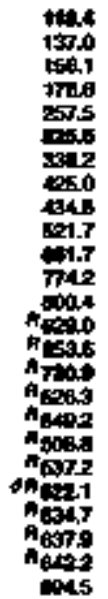 & 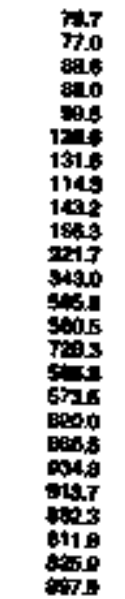 & 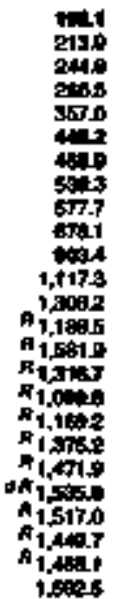 \\
\hline
\end{tabular}

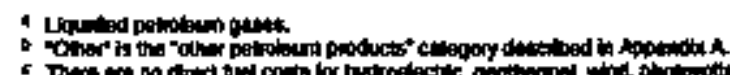

Promad ten

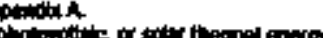

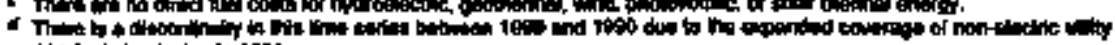
$\rightarrow+\infty$

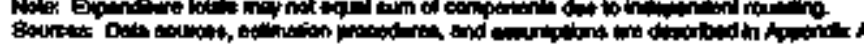

Eneroy Iniomalon Adininimantion 
Table 297. Transpontaiton Sector Energy Price and Expenditure Estimates by Sowce, 1970-1994, Washington

\begin{tabular}{|c|c|c|c|c|c|c|c|c|c|c|c|c|c|}
\hline & \multicolumn{11}{|c|}{ Pymartents } & \multirow[b]{3}{*}{ Elaviniatry } & \multirow[b]{3}{*}{ Tot } \\
\hline & & \multirow[b]{2}{*}{ الה } & \multicolumn{8}{|c|}{ תחmה } & \multirow[b]{2}{*}{ That } & & \\
\hline & cont & & 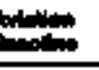 & 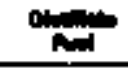 & مأم & Ine & 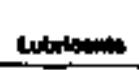 & . & Pinding & Tond & & & \\
\hline$\underline{\mathbf{n}}$ & \multicolumn{13}{|c|}{ 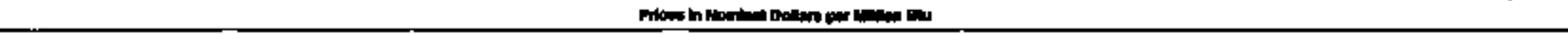 } \\
\hline \multirow[t]{2}{*}{ 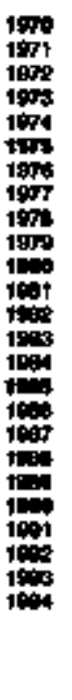 } & 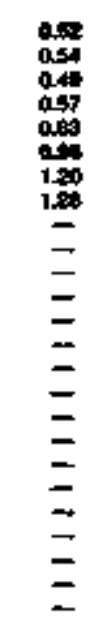 & $\begin{array}{l}= \\
= \\
= \\
= \\
= \\
= \\
= \\
= \\
= \\
= \\
= \\
3= \\
00 \\
00\end{array}$ & 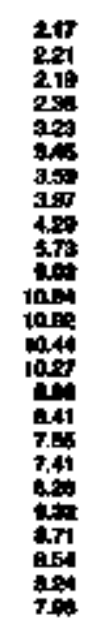 & 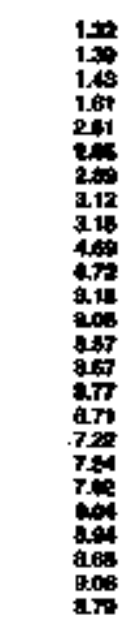 & 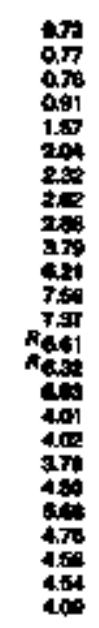 & 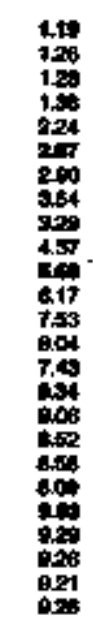 & 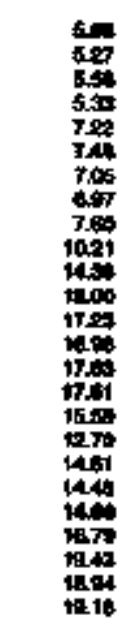 & 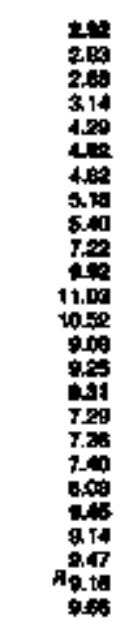 & 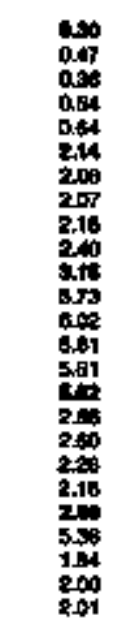 & 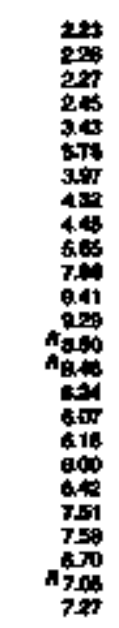 & 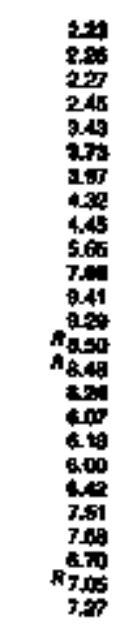 & 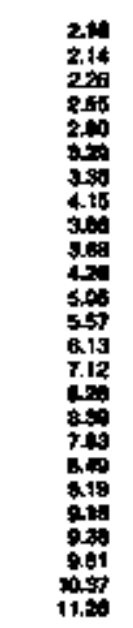 & 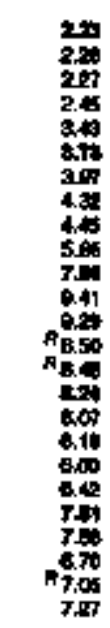 \\
\hline & \multicolumn{13}{|c|}{ 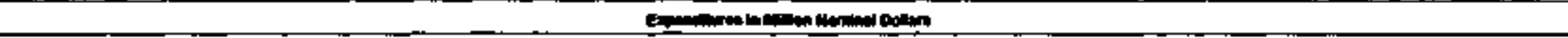 } \\
\hline 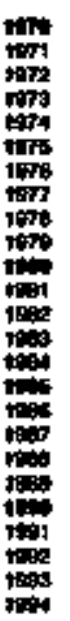 & $\begin{array}{l}8 \\
8 \\
8 \\
0 \\
= \\
= \\
= \\
= \\
= \\
= \\
= \\
= \\
=\end{array}$ & $\begin{array}{l}= \\
= \\
= \\
= \\
= \\
= \\
= \\
= \\
= \\
= \\
= \\
0 \\
03 \\
04 \\
0.4\end{array}$ & 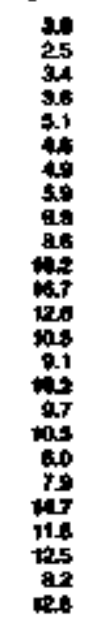 & 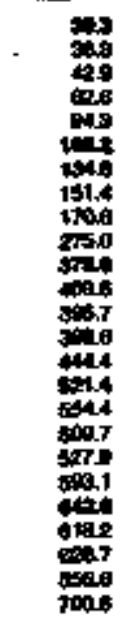 & 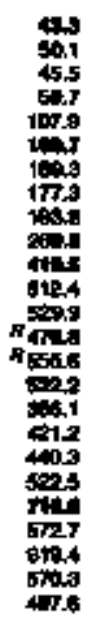 & 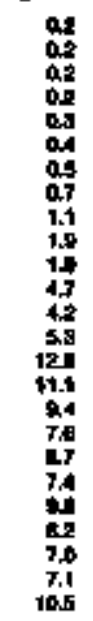 & 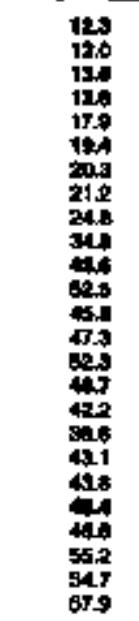 & 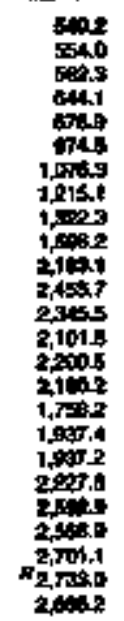 & 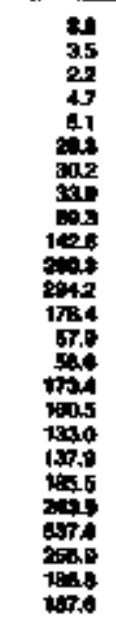 & 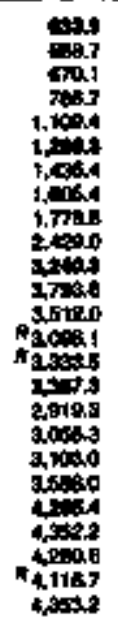 & 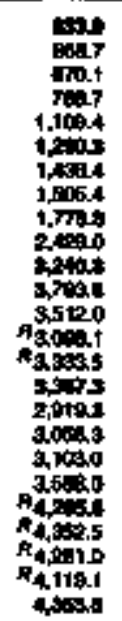 & 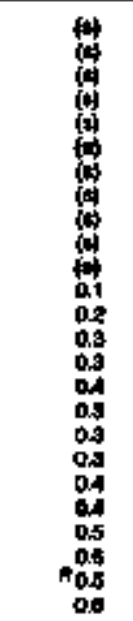 & 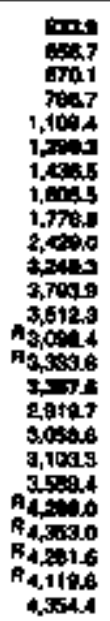 \\
\hline
\end{tabular}




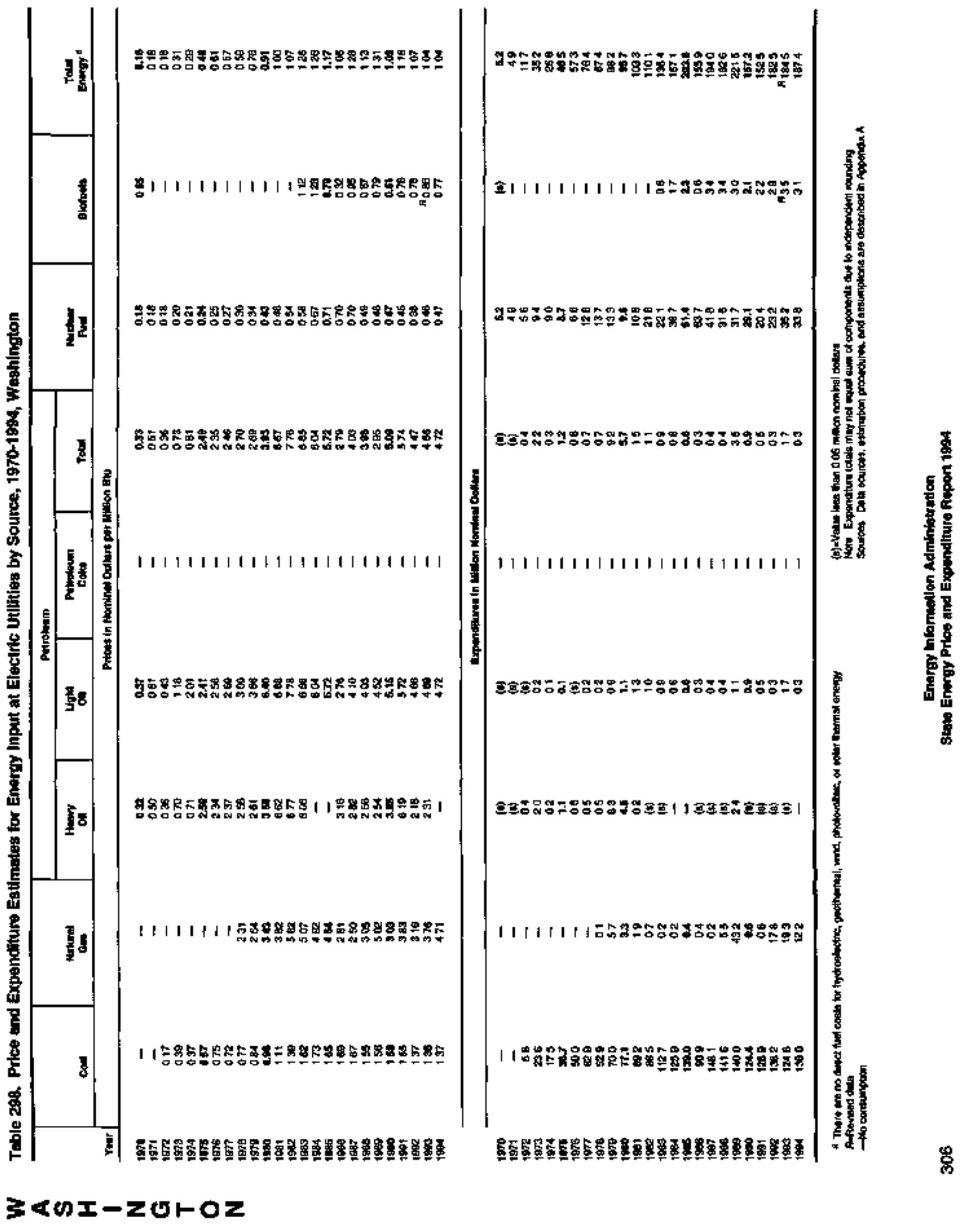




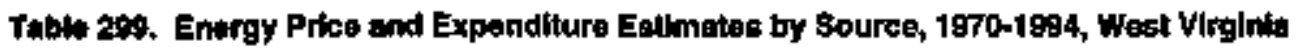

\begin{tabular}{|c|c|c|c|c|c|c|c|c|c|c|c|c|c|c|c|c|c|}
\hline \multirow[b]{4}{*}{ קט: } & \multicolumn{14}{|c|}{ 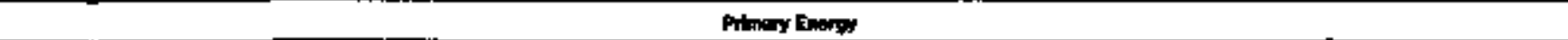 } & \multirow{3}{*}{$\begin{array}{l}\text { anders } \\
\text { ulinty } \\
\text { fund }\end{array}$} & \multirow{3}{*}{ 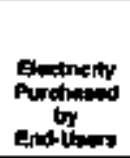 } & \multirow[b]{3}{*}{ Tow } \\
\hline & \multicolumn{3}{|c|}{ Cos } & \multirow[b]{2}{*}{ المبه| } & \multicolumn{7}{|c|}{ Prtolonim } & \multirow[b]{2}{*}{ Furing } & \multirow[b]{2}{*}{ 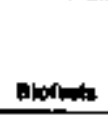 } & \multirow[b]{2}{*}{ Tobis 9} & & & \\
\hline & Colun & Inean & tיה & & Muman & Nat & LPGA & antor & Ronderd & Other & Tots & & & & & & \\
\hline & \multicolumn{17}{|c|}{ 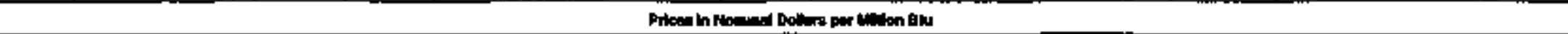 } \\
\hline \multirow[t]{2}{*}{ 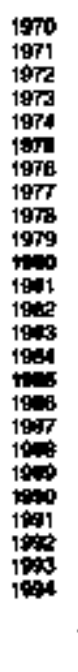 } & 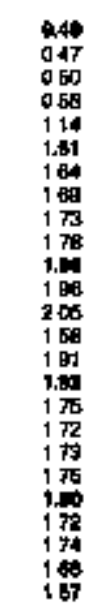 & 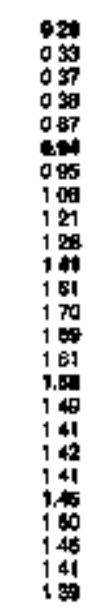 & 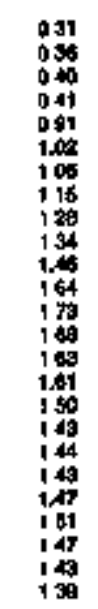 & 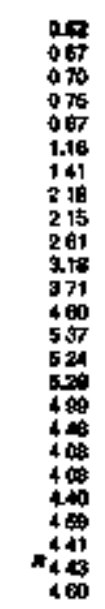 & 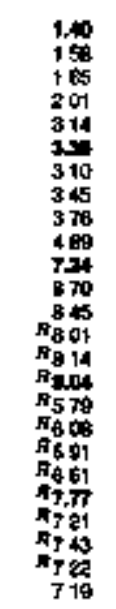 & 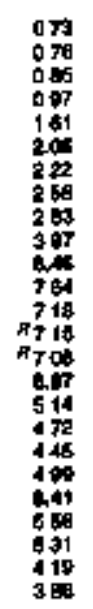 & 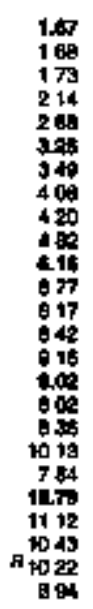 & 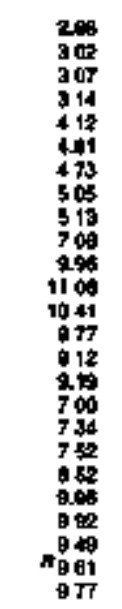 & 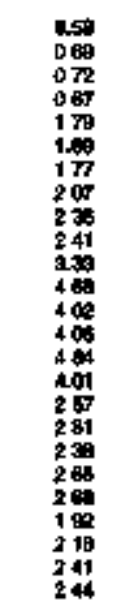 & 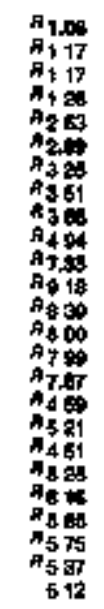 & 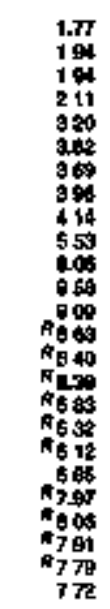 & $\begin{array}{l}= \\
= \\
= \\
= \\
= \\
= \\
= \\
= \\
= \\
= \\
= \\
= \\
= \\
= \\
=\end{array}$ & $\begin{array}{l}95 \\
= \\
= \\
= \\
= \\
= \\
= \\
= \\
= \\
= \\
= \\
= \\
\bar{z} \\
249 \\
245 \\
248 \\
244\end{array}$ & 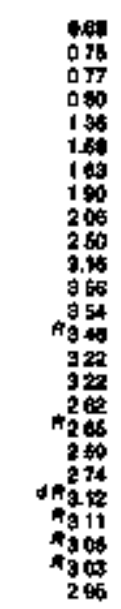 & 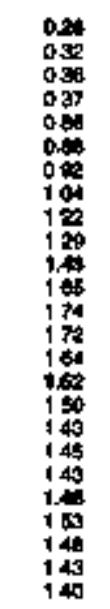 & 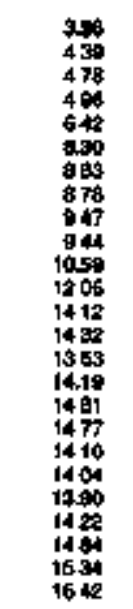 & 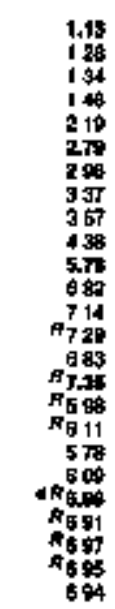 \\
\hline & \multicolumn{17}{|c|}{ 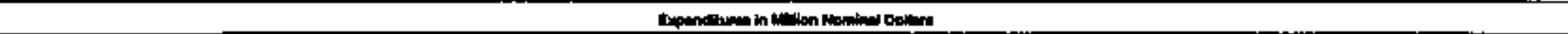 } \\
\hline 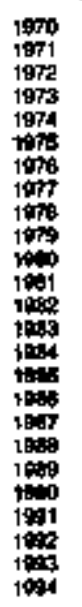 & 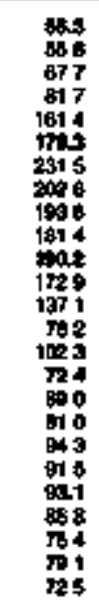 & 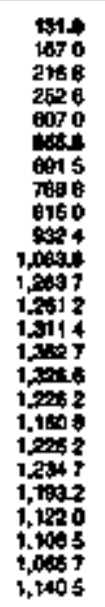 & 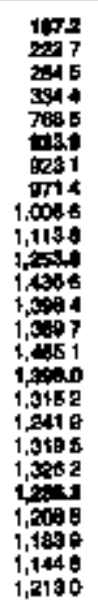 & 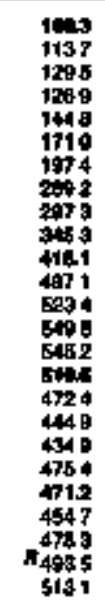 & 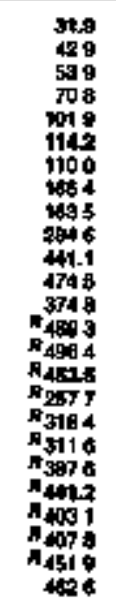 & 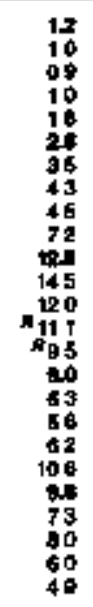 & 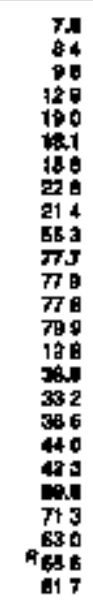 & 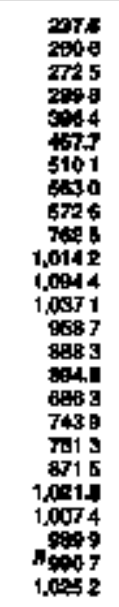 & 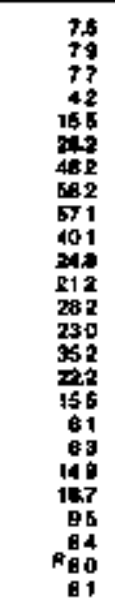 & 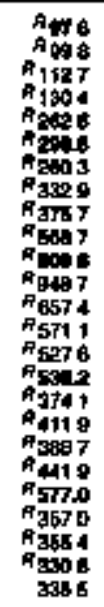 & 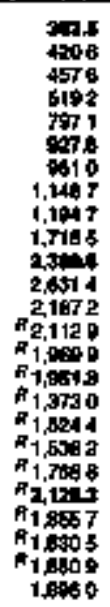 & $\begin{array}{l}= \\
= \\
= \\
= \\
= \\
= \\
= \\
= \\
= \\
= \\
= \\
= \\
= \\
=\end{array}$ & $\begin{array}{l}0 \\
= \\
= \\
= \\
= \\
= \\
= \\
= \\
= \\
= \\
= \\
=5 \\
109 \\
108 \\
108 \\
108\end{array}$ & 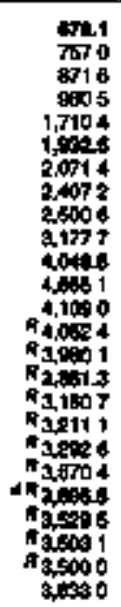 & 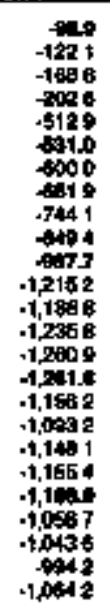 & 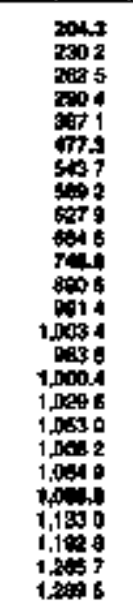 & 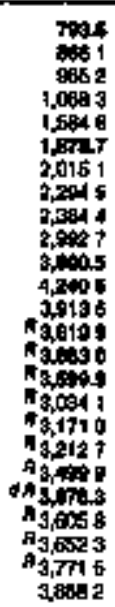 \\
\hline
\end{tabular}

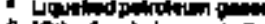

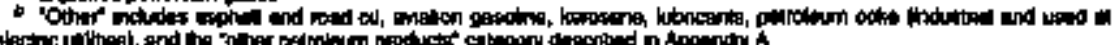

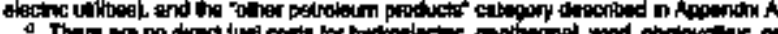

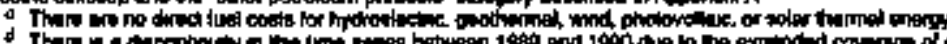

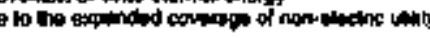

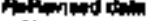

(1)

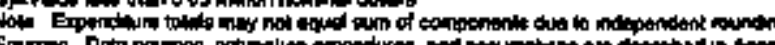

Energy Informetion Adminlotralion 


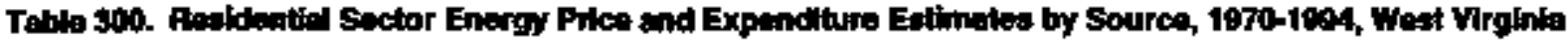

\begin{tabular}{|c|c|c|c|c|c|c|c|c|c|c|}
\hline \multirow[b]{4}{*}{ 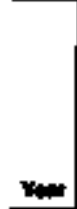 } & \multicolumn{8}{|c|}{ 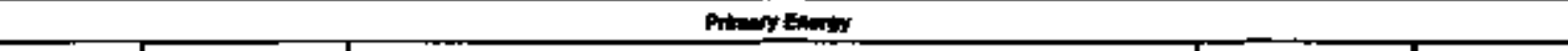 } & \multirow[b]{3}{*}{ Detrity } & \multirow[b]{3}{*}{$\underbrace{+\infty}$} \\
\hline & \multirow[b]{2}{*}{ (س) } & \multirow[b]{2}{*}{ 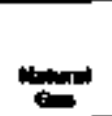 } & \multicolumn{4}{|c|}{ rim } & \multirow[b]{2}{*}{ 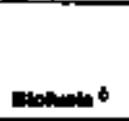 } & \multirow[b]{2}{*}{ 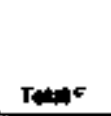 } & & \\
\hline & & & int & 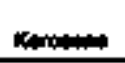 & Lma* & Tan & & & & \\
\hline & \multicolumn{10}{|c|}{ 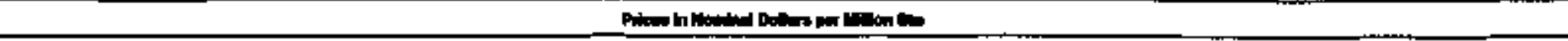 } \\
\hline \multirow[t]{2}{*}{ 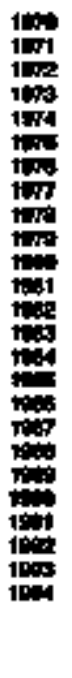 } & 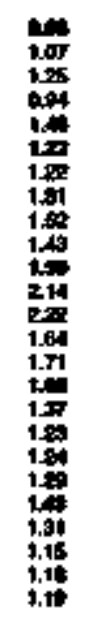 & 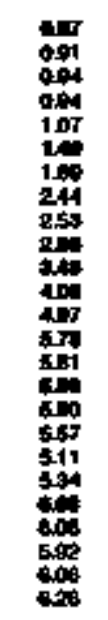 & 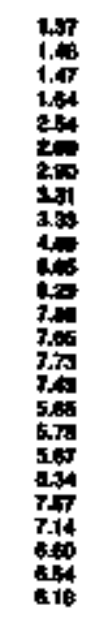 & 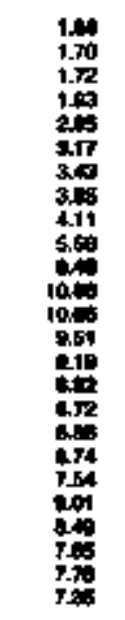 & 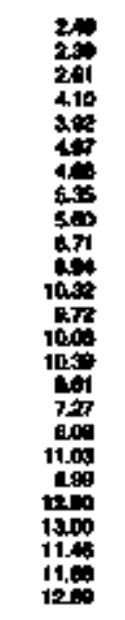 & 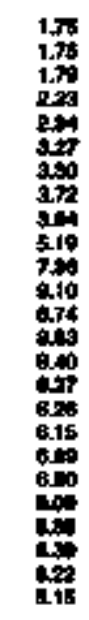 & 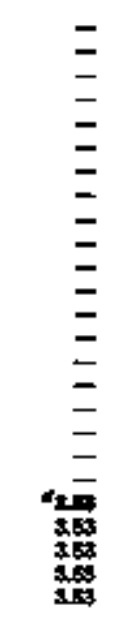 & 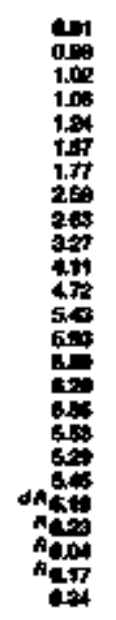 & 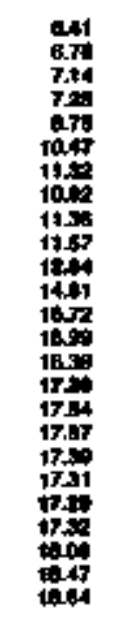 & 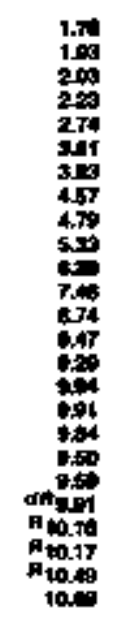 \\
\hline & \multicolumn{10}{|c|}{ 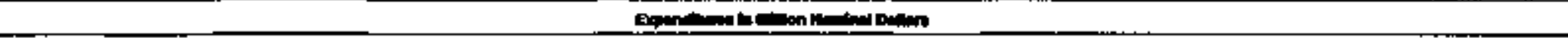 } \\
\hline 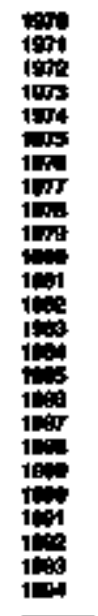 & 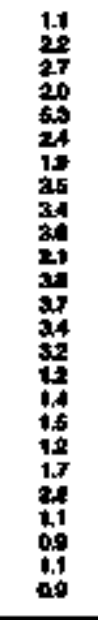 & 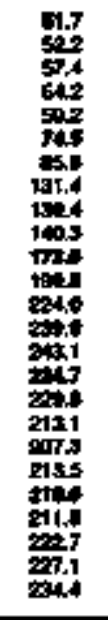 & 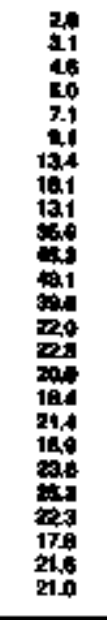 & 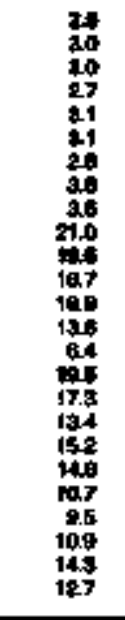 & 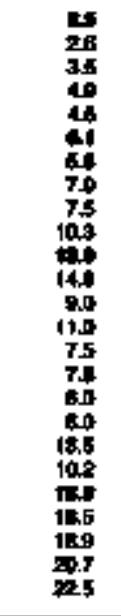 & 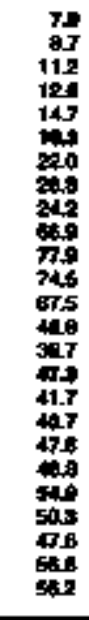 & 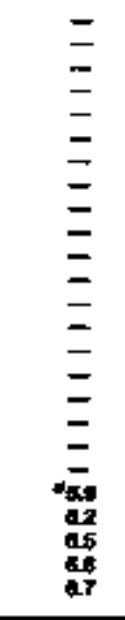 & 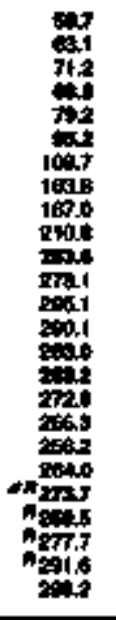 & 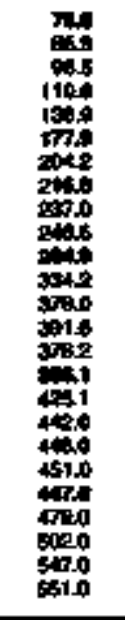 & 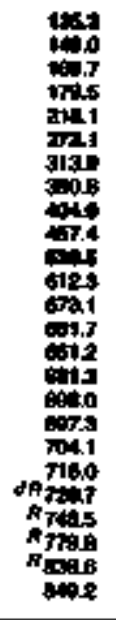 \\
\hline
\end{tabular}

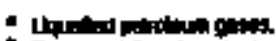

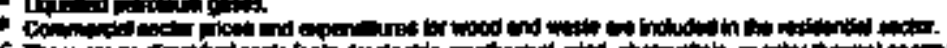

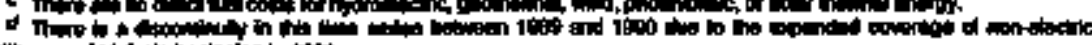

Aramind

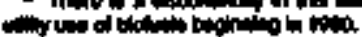

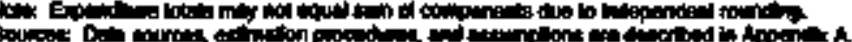




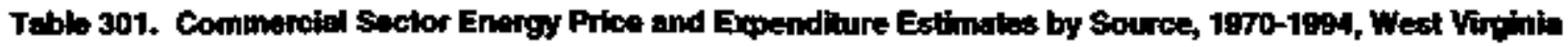

\begin{tabular}{|c|c|c|c|c|c|c|c|c|c|c|c|}
\hline \multirow[b]{4}{*}{ re } & \multicolumn{9}{|c|}{ 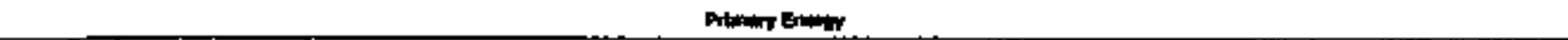 } & \multirow[b]{3}{*}{ Eestipy } & \multirow[b]{3}{*}{ 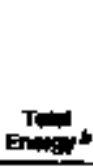 } \\
\hline & & \multirow[b]{2}{*}{ 些 } & \multicolumn{6}{|c|}{ 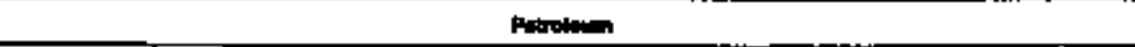 } & \multirow[b]{2}{*}{ Toun } & & \\
\hline & cold & & Find & Kminomo & LPA & 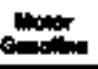 & Prathel & Intell & & & \\
\hline & \multicolumn{11}{|c|}{ 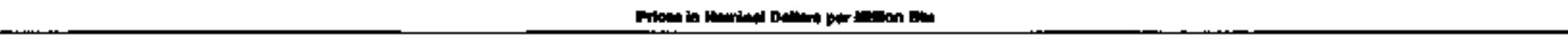 } \\
\hline \multirow[t]{2}{*}{ 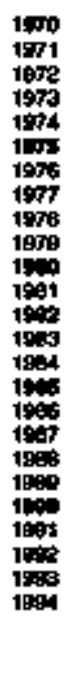 } & 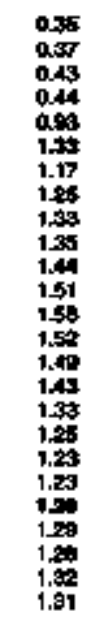 & 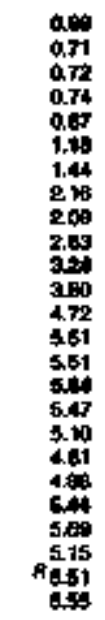 & 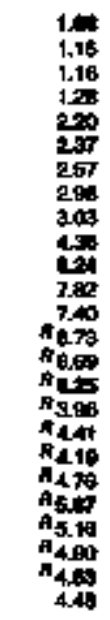 & 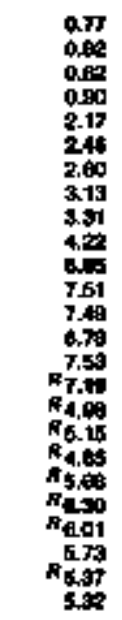 & 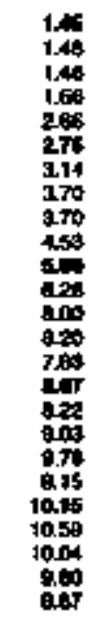 & 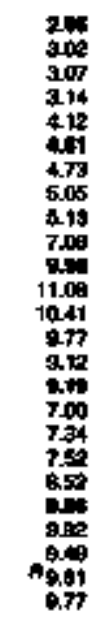 & 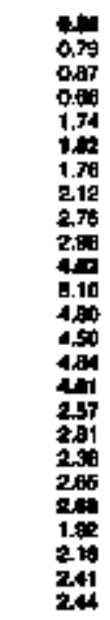 & 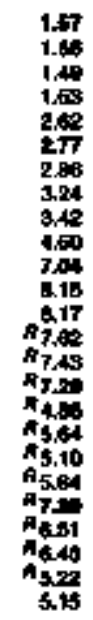 & 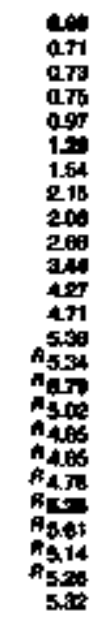 & 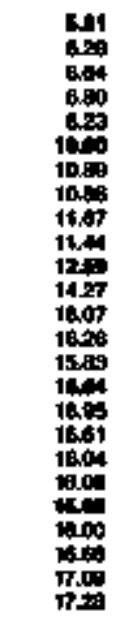 & 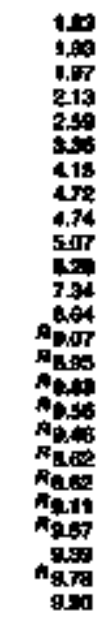 \\
\hline & \multicolumn{11}{|c|}{ 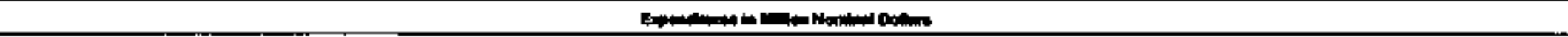 } \\
\hline 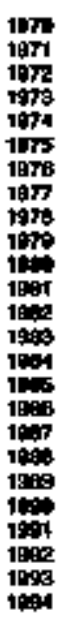 & 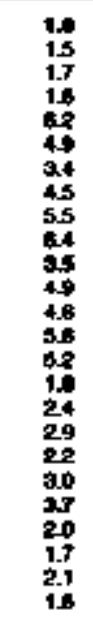 & 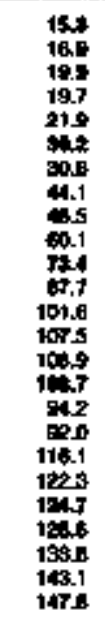 & 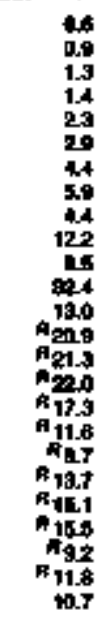 & 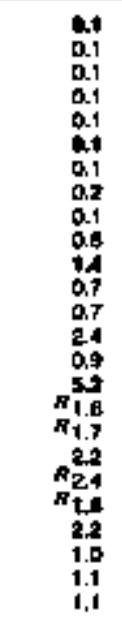 & $\begin{array}{l}04 \\
0.3 \\
0.3 \\
04 \\
04 \\
06 \\
0.7 \\
08 \\
09 \\
1.2 \\
1.5 \\
1.6 \\
1.3 \\
18 \\
1.0 \\
1.3 \\
11 \\
18 \\
2.1 \\
21 \\
2.7 \\
2.7 \\
29 \\
30 \\
2.6\end{array}$ & 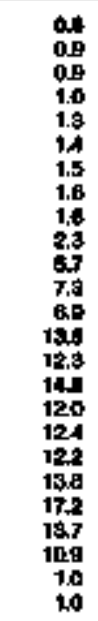 & 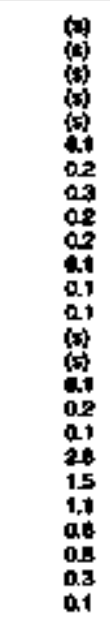 & 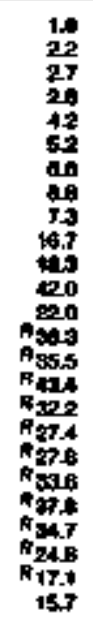 & 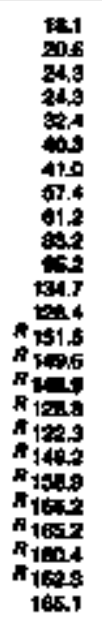 & 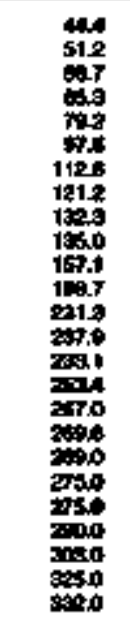 & 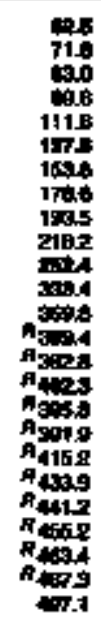 \\
\hline
\end{tabular}

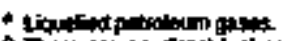

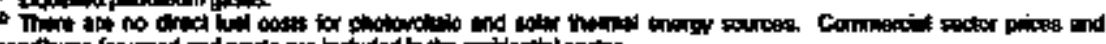

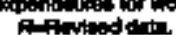




\begin{tabular}{|c|c|c|c|c|c|c|c|c|c|c|c|c|c|c|c|c|c|}
\hline \multirow[b]{4}{*}{$\mathrm{Yer}$} & \multicolumn{15}{|c|}{ 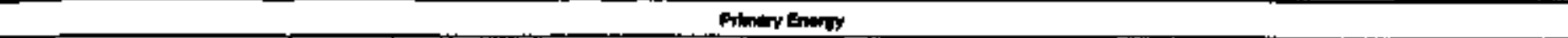 } & \multirow[b]{3}{*}{ mantent } & \multirow[b]{3}{*}{ Timions } \\
\hline & \multicolumn{3}{|c|}{ cons. } & \multirow[b]{2}{*}{ mand } & \multicolumn{9}{|c|}{ Mansinam } & \multirow[b]{2}{*}{ Alowena } & \multirow[b]{2}{*}{ 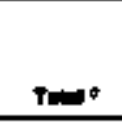 } & & \\
\hline & cown & Sold & Totol & & $\mid \begin{array}{c}\text { Anthat and } \\
\text { Hant on }\end{array}$ & Ful & Nerom & LPa* & 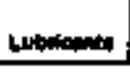 & mations & 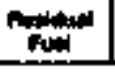 & and & Then & & & & \\
\hline & \multicolumn{17}{|c|}{ 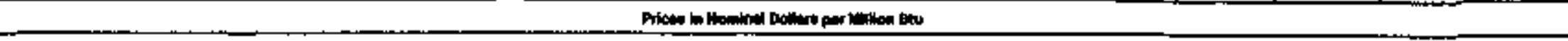 } \\
\hline \multirow[t]{2}{*}{ 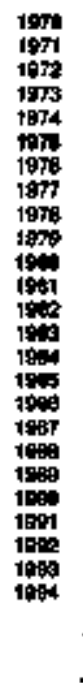 } & 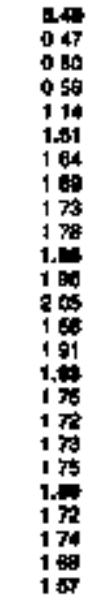 & 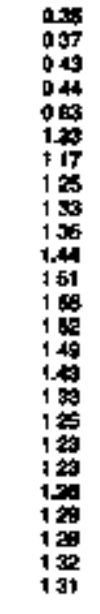 & 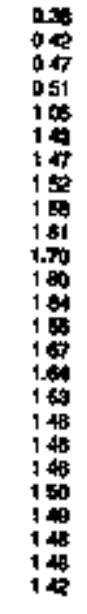 & 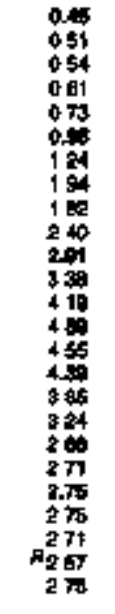 & 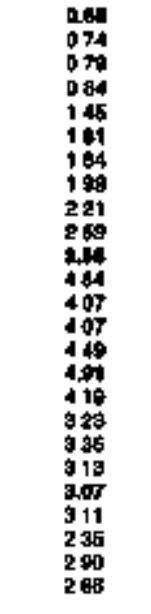 & 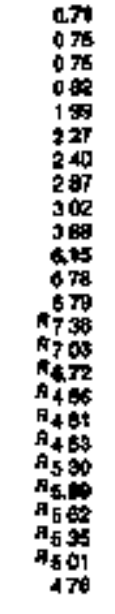 & 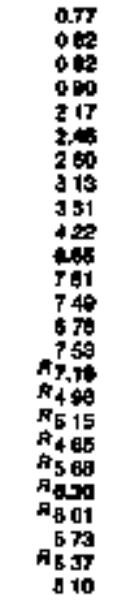 & 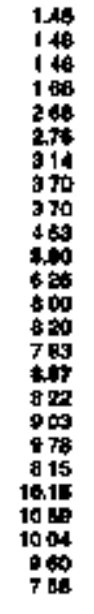 & 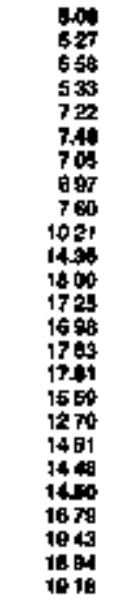 & 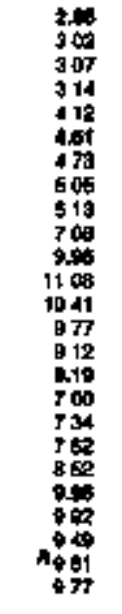 & 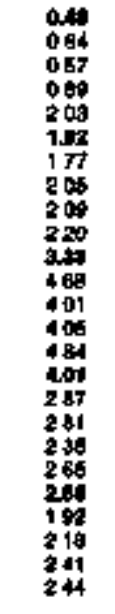 & 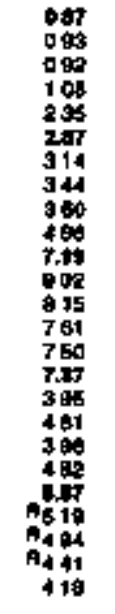 & 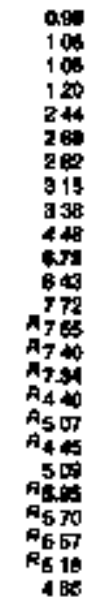 & 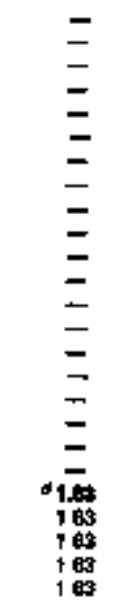 & 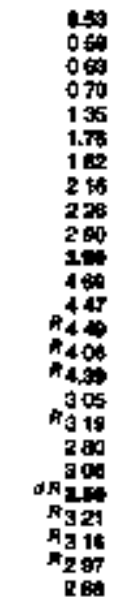 & 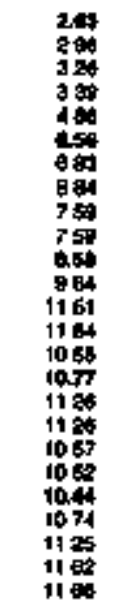 & 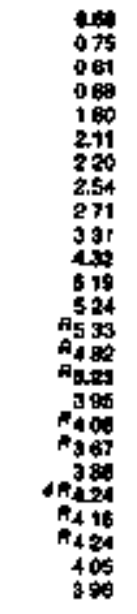 \\
\hline & \multicolumn{17}{|c|}{ 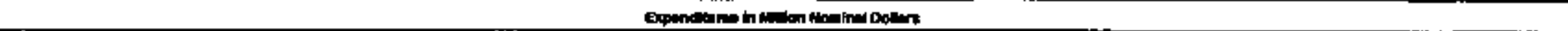 } \\
\hline 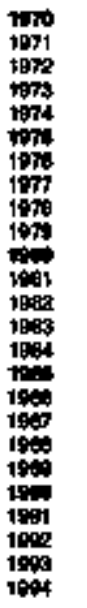 & 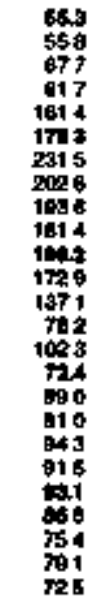 & 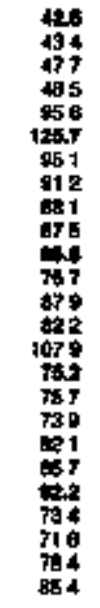 & 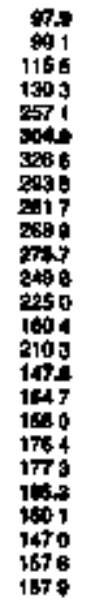 & 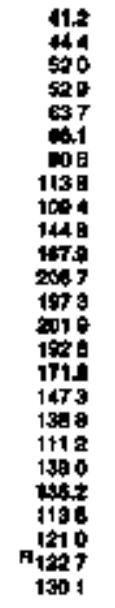 & 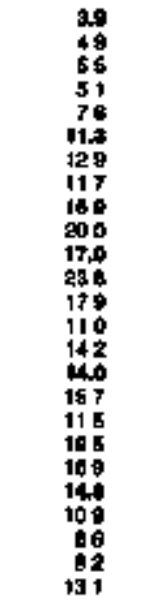 & 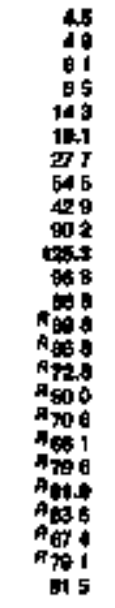 & 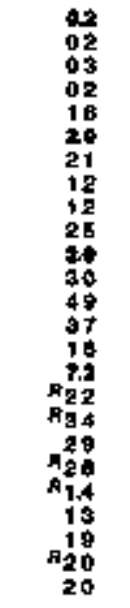 & 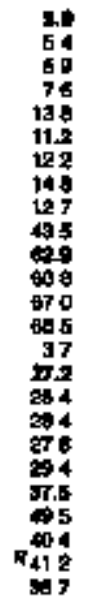 & 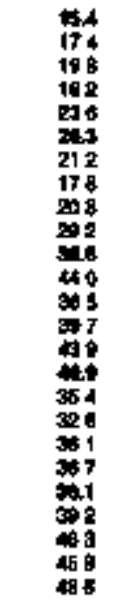 & 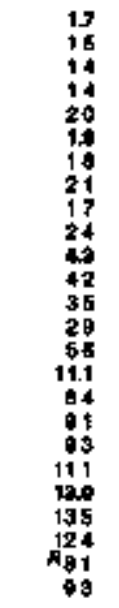 & 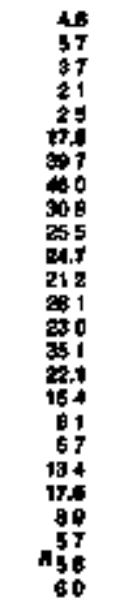 & 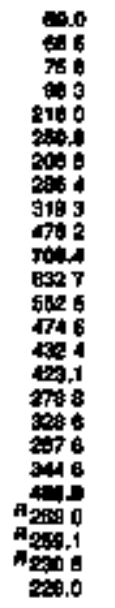 & 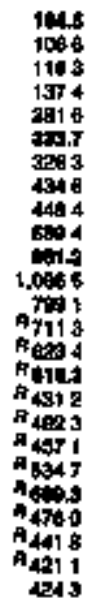 & 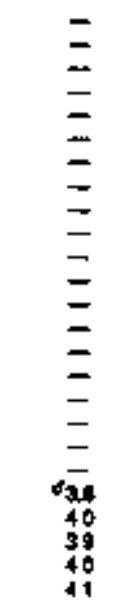 & 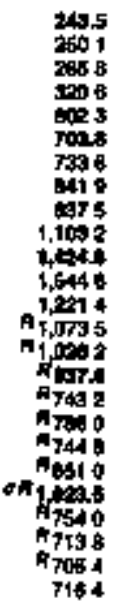 & 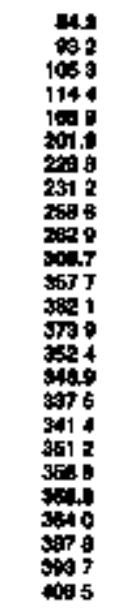 & 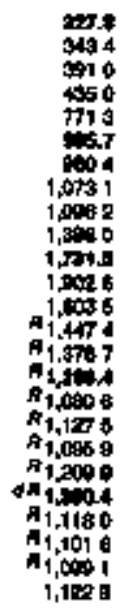 \\
\hline
\end{tabular}


Twbis 303. Tranaportation Sector Energy Price and Exponditure Eatimatos by Source, 1970-1994, West Virtinia

\begin{tabular}{|c|c|c|c|c|c|c|c|c|c|c|c|c|c|}
\hline \multirow[b]{4}{*}{ Vom } & \multicolumn{11}{|c|}{ Patmen Enarg } & \multirow[b]{3}{*}{ Enetiongy } & \multirow[b]{3}{*}{ 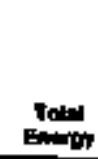 } \\
\hline & \multirow[b]{2}{*}{ Eo:al } & \multirow[b]{2}{*}{ Noned } & \multicolumn{8}{|c|}{ Fotming } & \multirow[b]{2}{*}{ Toled } & & \\
\hline & & & Antion & 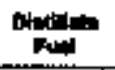 & Fint & Uat: & Leblom & 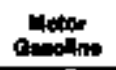 & Pridum & 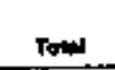 & & & \\
\hline & \multicolumn{13}{|c|}{ 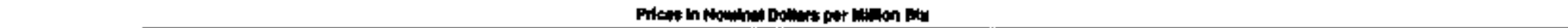 } \\
\hline \multirow[t]{2}{*}{ 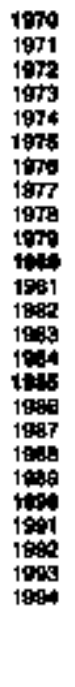 } & $\begin{array}{l}0.55 \\
0.37 \\
0.49 \\
0.40 \\
0.90 \\
1.17 \\
10 \\
= \\
= \\
= \\
= \\
= \\
= \\
= \\
= \\
= \\
= \\
=\end{array}$ & $\begin{array}{l}\overrightarrow{=} \\
= \\
= \\
= \\
= \\
= \\
= \\
= \\
= \\
= \\
= \\
272 \\
270\end{array}$ & 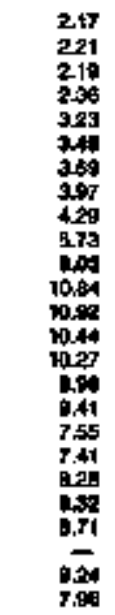 & 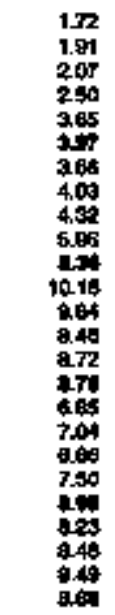 & 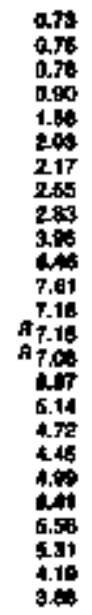 & 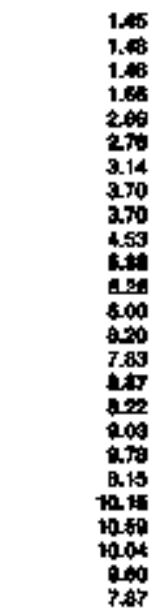 & 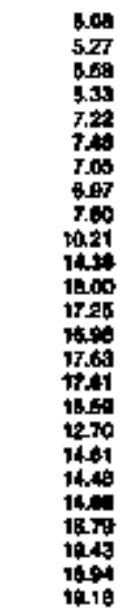 & 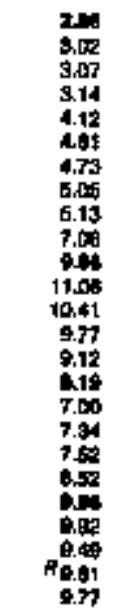 & $\begin{array}{l}= \\
= \\
= \\
= \\
= \\
2.81 \\
= \\
= \\
= \\
= \\
= \\
z \\
z\end{array}$ & 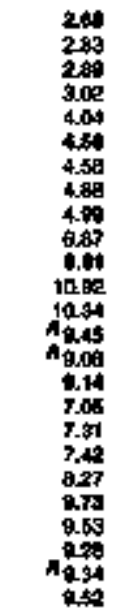 & 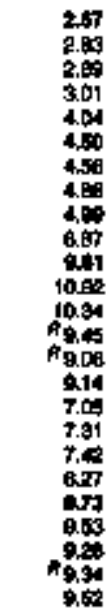 & $\begin{array}{l}= \\
= \\
= \\
= \\
= \\
= \\
= \\
= \\
= \\
= \\
= \\
= \\
=\end{array}$ & 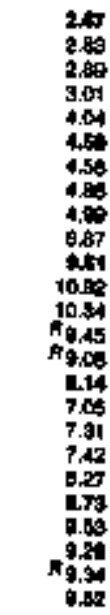 \\
\hline & \multicolumn{13}{|c|}{ 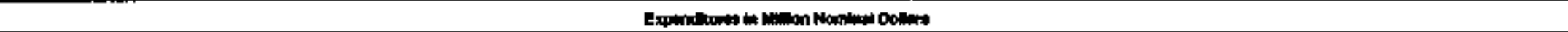 } \\
\hline 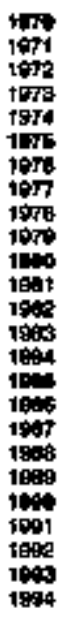 & $\begin{array}{l}0.1 \\
0.9 \\
0.1 \\
0.1 \\
0.1 \\
0.1 \\
0 \\
0= \\
= \\
= \\
= \\
= \\
= \\
= \\
= \\
= \\
= \\
= \\
=\end{array}$ & $\begin{array}{l}= \\
= \\
= \\
= \\
= \\
= \\
= \\
= \\
= \\
= \\
= \\
= \\
\vec{a} \\
(0)\end{array}$ & $\begin{array}{l}0.1 \\
0.4 \\
0.7 \\
0.7 \\
1.0 \\
1.1 \\
1.0 \\
1.2 \\
1.9 \\
1.7 \\
4.7 \\
1.7 \\
1.3 \\
2.3 \\
20 \\
1.8 \\
21 \\
1.3 \\
1.4 \\
1.8 \\
1.7 \\
1.5 \\
1.1 \\
1.0\end{array}$ & 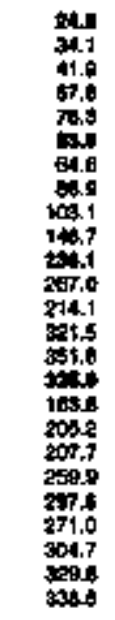 & 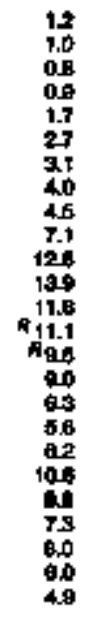 & $\begin{array}{l}1.1 \\
0.1 \\
0.1 \\
0.1 \\
0.1 \\
\$ 1 \\
0.2 \\
0.3 \\
0.3 \\
0.3 \\
0.0 \\
0.7 \\
0.6 \\
0.7 \\
0.6 \\
0.7 \\
0.0 \\
0.6 \\
0.0 \\
0.0 \\
0.7 \\
0.7 \\
0.8 \\
0.7 \\
0.0\end{array}$ & 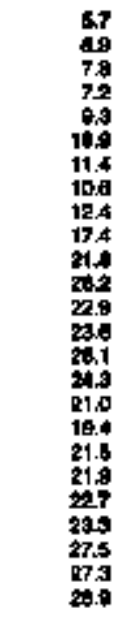 & 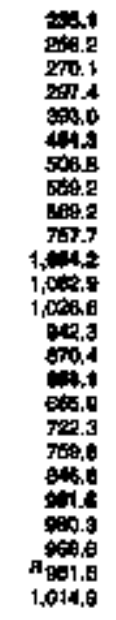 & $\begin{array}{l}\frac{\text { lib }}{=} \\
= \\
= \\
= \\
\bar{z} \\
= \\
= \\
= \\
= \\
= \\
= \\
= \\
= \\
=\end{array}$ & 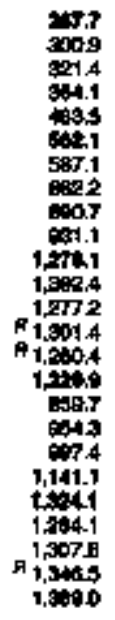 & 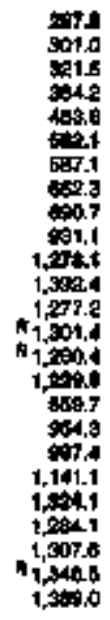 & $\begin{array}{l}= \\
= \\
= \\
= \\
= \\
= \\
= \\
= \\
= \\
= \\
= \\
= \\
=\end{array}$ & 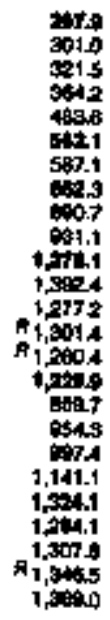 \\
\hline
\end{tabular}

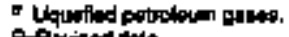

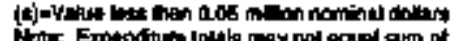

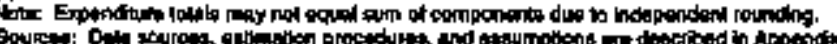

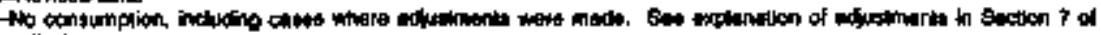

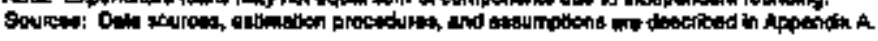




\begin{tabular}{|c|c|c|c|c|c|c|c|c|c|}
\hline \multirow[b]{3}{*}{ rest } & & \multirow[b]{2}{*}{$\min _{0}$} & \multicolumn{4}{|c|}{ Ponolnen. } & \multirow[b]{2}{*}{ 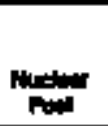 } & \multirow[b]{2}{*}{ 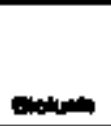 } & \multirow[b]{2}{*}{ " } \\
\hline & $c$ & & m & ond & 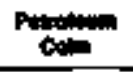 & Tone & & & \\
\hline & \multicolumn{9}{|c|}{ 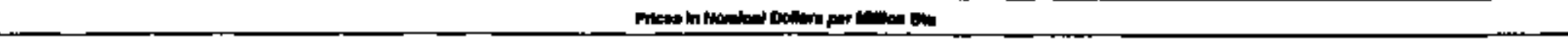 } \\
\hline \multirow[t]{2}{*}{ 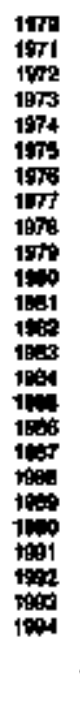 } & 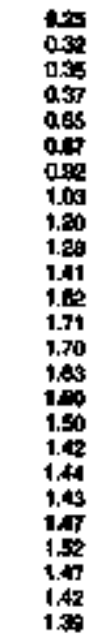 & 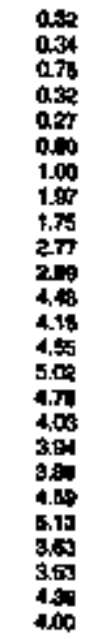 & 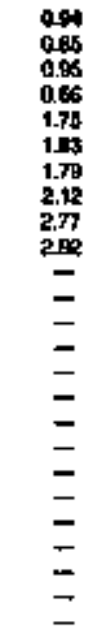 & 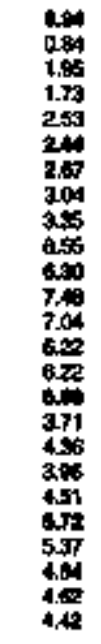 & $\begin{array}{l}z \\
z \\
z \\
z \\
= \\
z \\
z \\
= \\
\text { z } \\
= \\
= \\
= \\
=\end{array}$ & 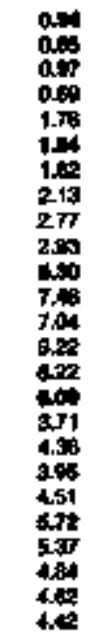 & $\begin{array}{l}= \\
= \\
= \\
= \\
z \\
= \\
= \\
= \\
= \\
= \\
= \\
= \\
z\end{array}$ & $\begin{array}{l}100 \\
= \\
= \\
= \\
= \\
= \\
= \\
= \\
= \\
= \\
= \\
= \\
= \\
=\end{array}$ & 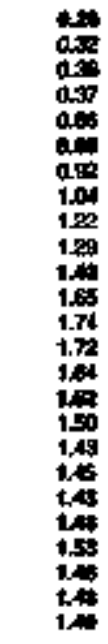 \\
\hline & \multicolumn{9}{|c|}{ 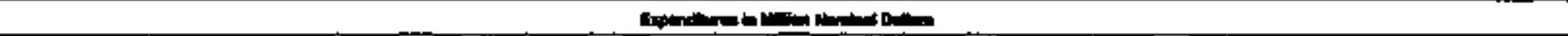 } \\
\hline 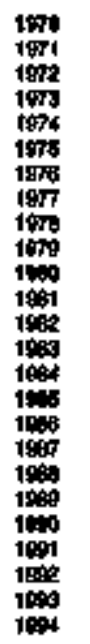 & 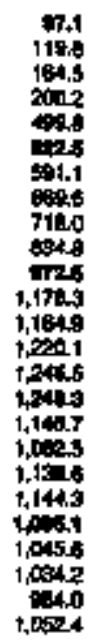 & 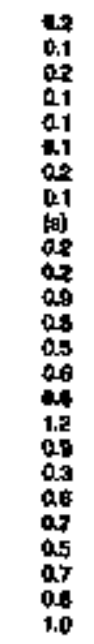 & 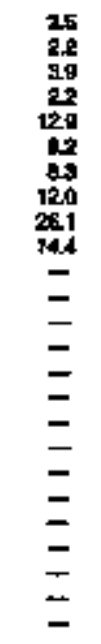 & 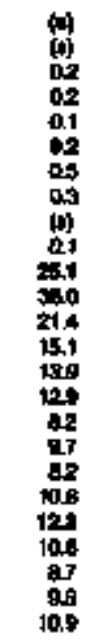 & $\begin{array}{l}z \\
z \\
z \\
z \\
z \\
z \\
= \\
= \\
= \\
= \\
= \\
= \\
=\end{array}$ & 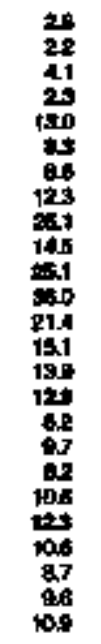 & $\begin{array}{l}= \\
= \\
= \\
= \\
= \\
= \\
= \\
= \\
= \\
= \\
= \\
z \\
=\end{array}$ & $\begin{array}{l}0 \\
= \\
= \\
= \\
= \\
= \\
= \\
= \\
= \\
= \\
= \\
= \\
= \\
=\end{array}$ & 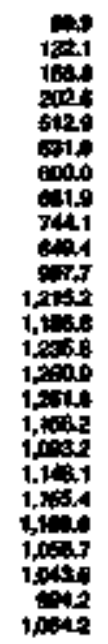 \\
\hline
\end{tabular}

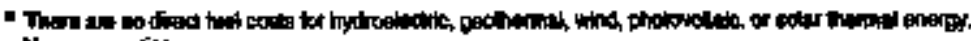
Mormination 
Table 303. Energy Price and Expendilure Entinetoo by Source, 1970-1994, Wigeonaln

\begin{tabular}{|c|c|c|c|c|c|c|c|c|c|c|c|c|c|c|c|c|c|}
\hline & \multicolumn{14}{|c|}{ |l } & \multirow{3}{*}{ 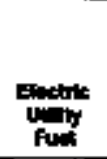 } & \multirow{3}{*}{ 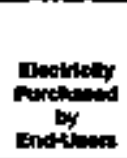 } & \multirow[b]{3}{*}{ 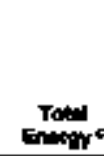 } \\
\hline & \multicolumn{3}{|c|}{ ct } & \multirow[b]{2}{*}{$\min$} & \multicolumn{7}{|c|}{ 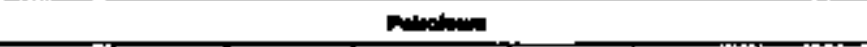 } & \multirow[b]{2}{*}{$\begin{array}{l}\text { Mucher } \\
\text { fuel }\end{array}$} & \multirow[b]{2}{*}{ notum } & \multirow[b]{2}{*}{ Tom } & & & \\
\hline & Cist & سمبc & Tot & & (1) & in & unad & 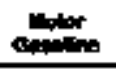 & 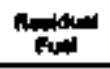 & Oners & Tolat & & & & & & \\
\hline Tit & \multicolumn{17}{|c|}{ 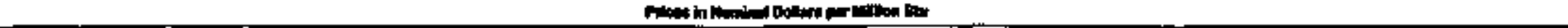 } \\
\hline \multirow[t]{2}{*}{ 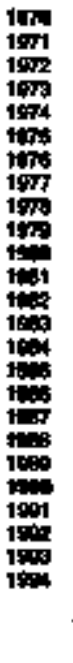 } & 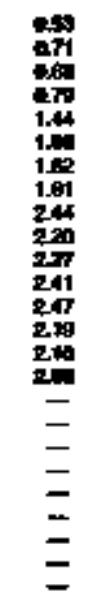 & 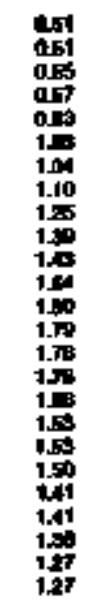 & 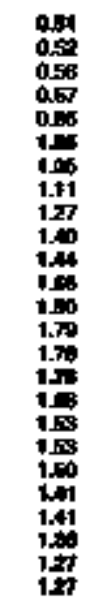 & 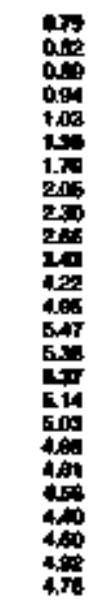 & 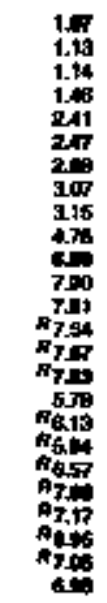 & 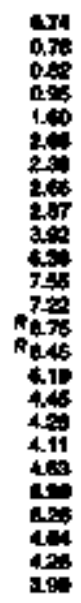 & 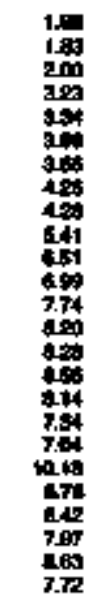 & 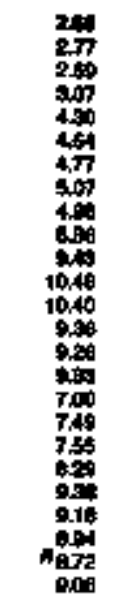 & 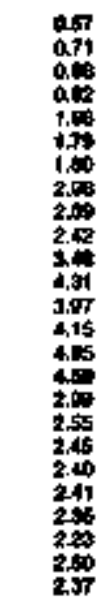 & 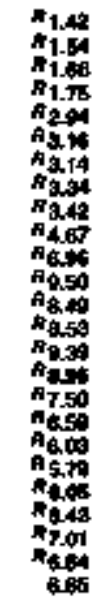 & 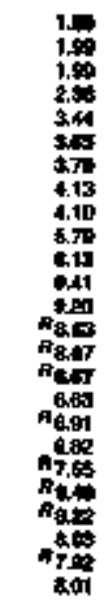 & 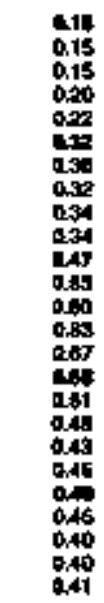 & 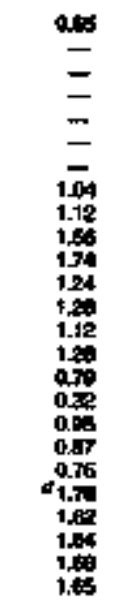 & 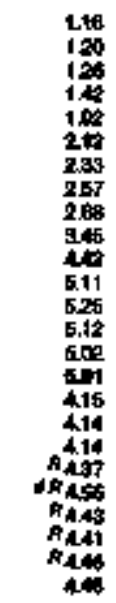 & 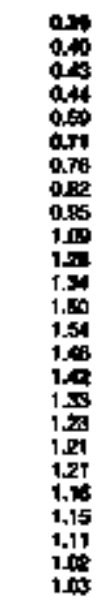 & 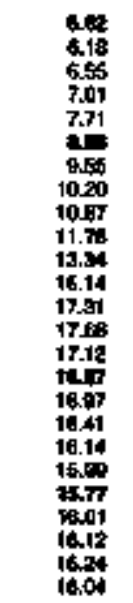 & 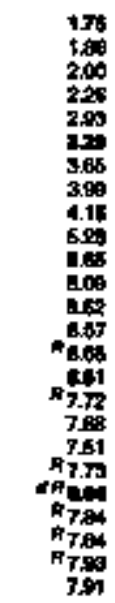 \\
\hline & \multicolumn{17}{|c|}{ 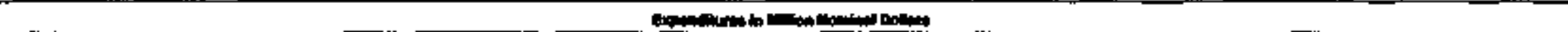 } \\
\hline 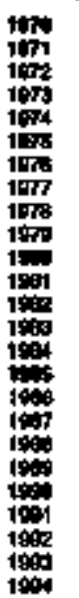 & 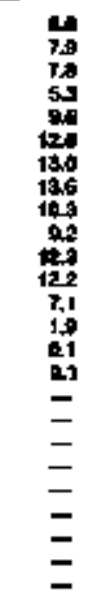 & 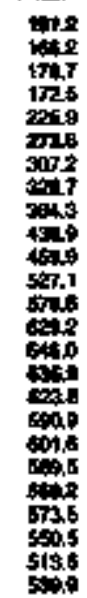 & 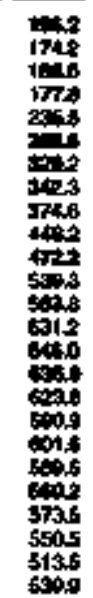 & 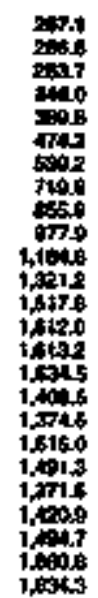 & 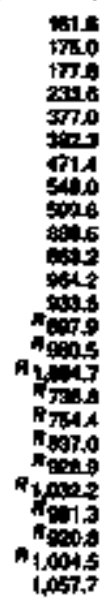 & 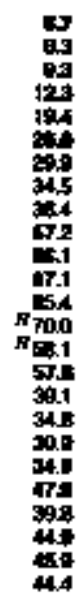 & 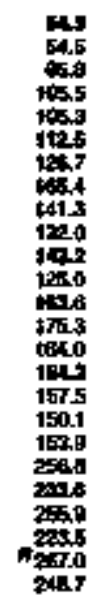 & 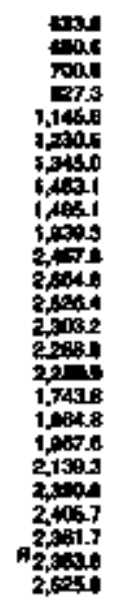 & 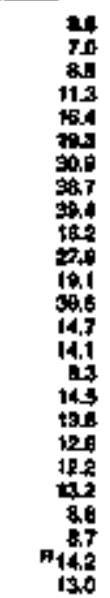 & 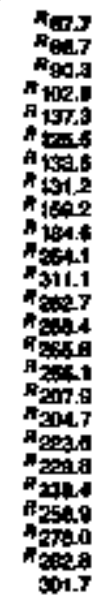 & 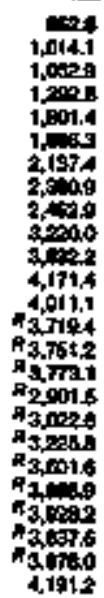 & 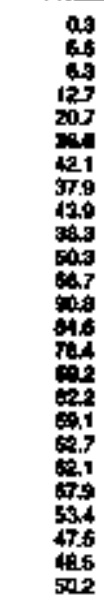 & 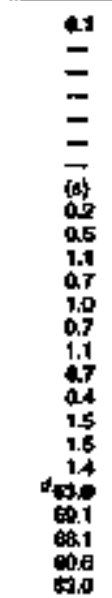 & 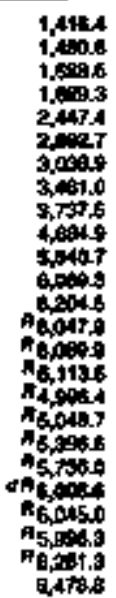 & 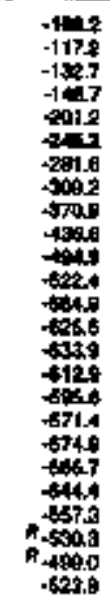 & 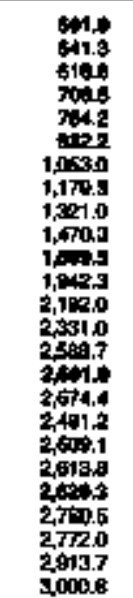 & 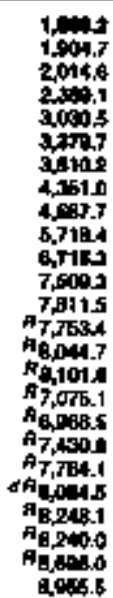 \\
\hline
\end{tabular}

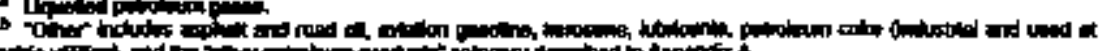

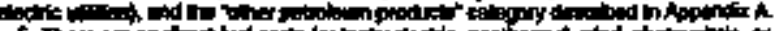

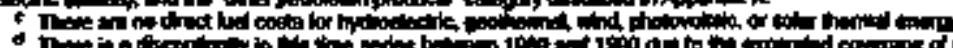

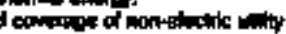

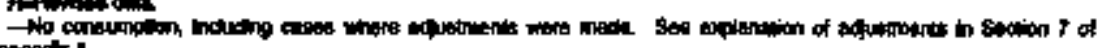
Apendik

1)

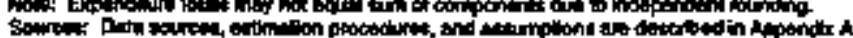

Enorgy Intormation Adminlatrellon 
Tabte 300. Fesidenthal Sector Energy Prion and Expenditure Estimatea by Source, 1970-1904, Wiaconsin

\begin{tabular}{|c|c|c|c|c|c|c|c|c|c|c|}
\hline \multirow{3}{*}{$r$} & \multicolumn{8}{|c|}{ Bimery Enety } & \multirow[b]{3}{*}{ Enstraty } & \multirow[b]{3}{*}{ Toted } \\
\hline & \multirow[b]{2}{*}{ con } & \multirow[b]{2}{*}{ " } & \multicolumn{4}{|c|}{ Pelroverian } & \multirow[b]{2}{*}{ Dohnes 6} & \multirow[b]{2}{*}{ Tans" } & & \\
\hline & & & Funt & 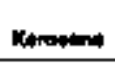 & $\angle P Q *$ & Totol & & & & \\
\hline 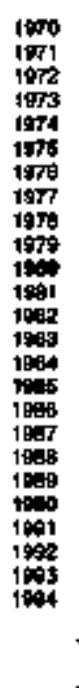 & 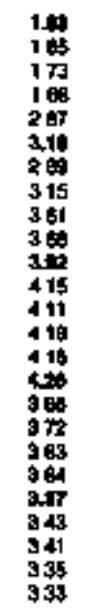 & 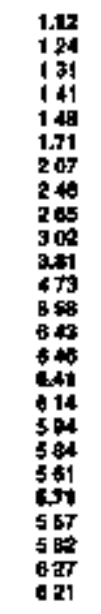 & 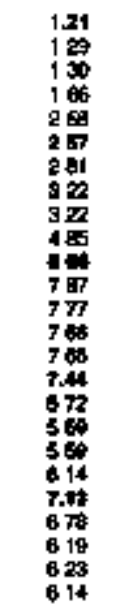 & 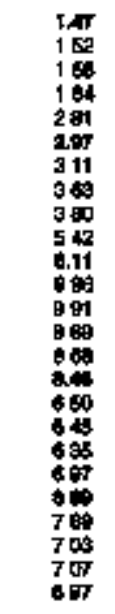 & 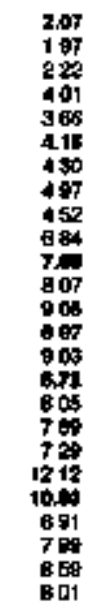 & 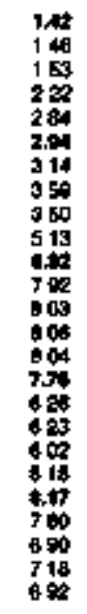 & 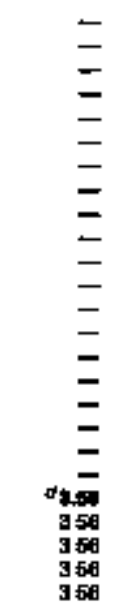 & 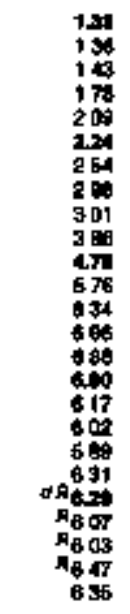 & 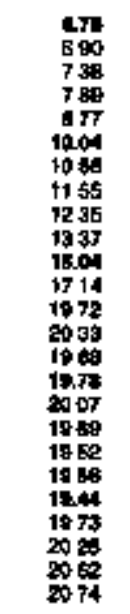 & 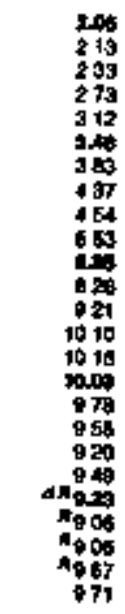 \\
\hline 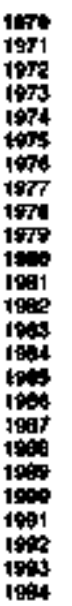 & 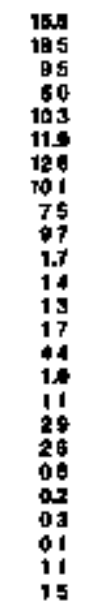 & 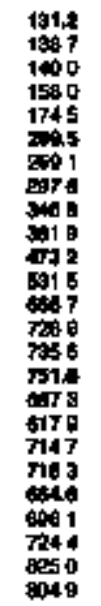 & 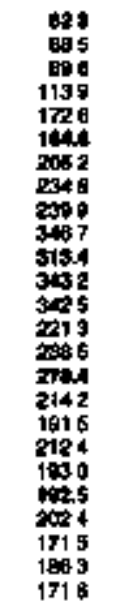 & 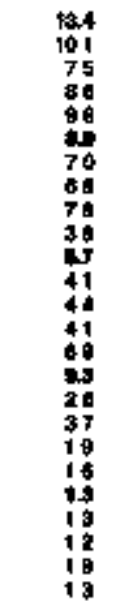 & 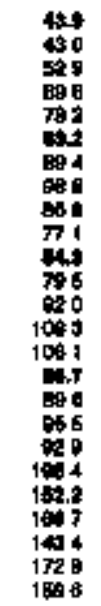 & 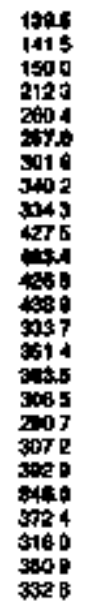 & $\begin{array}{l}= \\
= \\
= \\
= \\
= \\
= \\
= \\
= \\
= \\
= \\
= \\
\bar{z} \\
74 \\
183 \\
95 \\
93\end{array}$ & 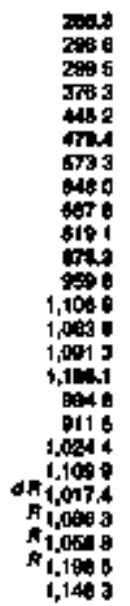 & 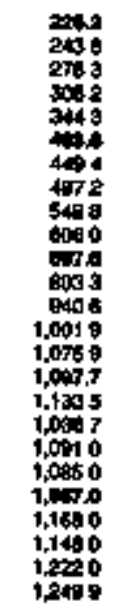 & 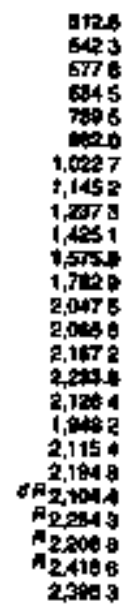 \\
\hline
\end{tabular}

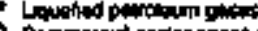

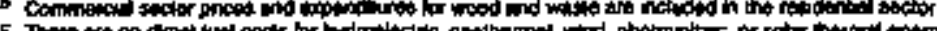

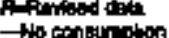

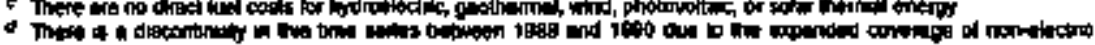

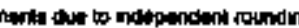

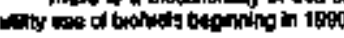


Table 307. Commercien Sector Energy Price and Expenchtume Estimatea by Sounce, 1970-1994, Waconain

\begin{tabular}{|c|c|c|c|c|c|c|c|c|c|c|c|}
\hline \multirow[b]{4}{*}{ Ver } & \multicolumn{9}{|c|}{ Prinery Enoty } & \multirow[b]{3}{*}{ Enefricity } & \multirow[b]{3}{*}{ 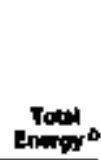 } \\
\hline & & \multirow[b]{2}{*}{ Mart: } & \multicolumn{6}{|c|}{ Peinoming } & \multirow[b]{2}{*}{ Total } & & \\
\hline & Cont & & 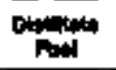 & Exroume & $\mathbf{L O}$ & Motor & Findoud & Tofis & & & \\
\hline & \multicolumn{11}{|c|}{ 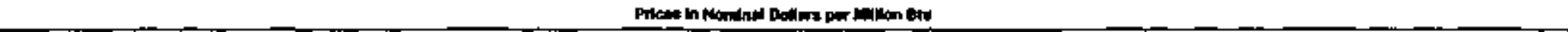 } \\
\hline \multirow[t]{2}{*}{ 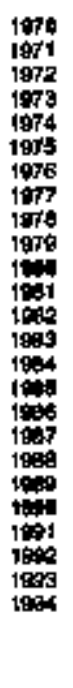 } & 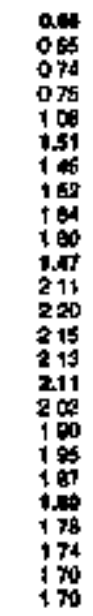 & 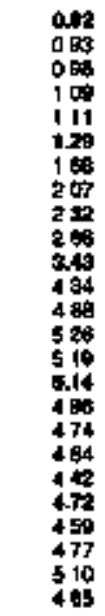 & 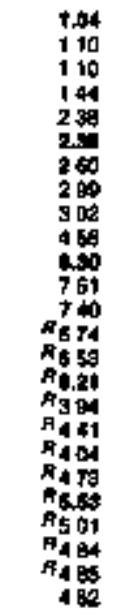 & 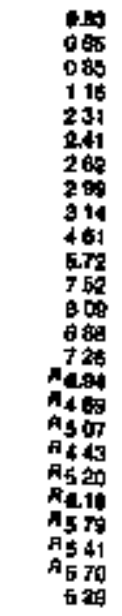 & 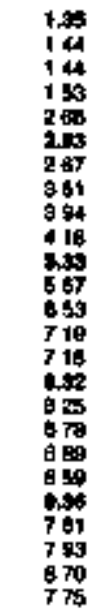 & 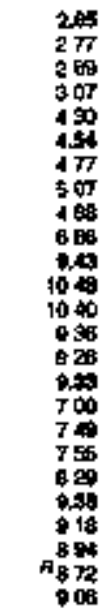 & 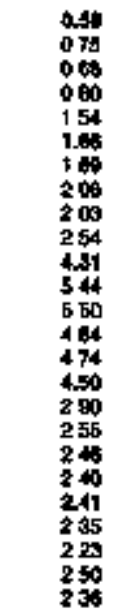 & 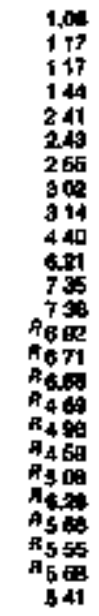 & 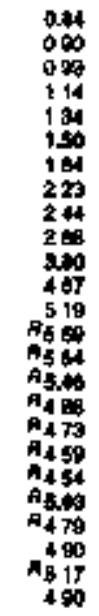 & 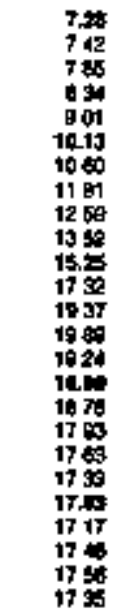 & 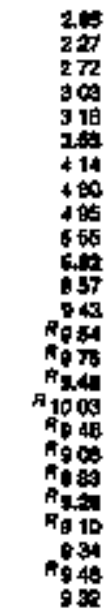 \\
\hline & \multicolumn{11}{|c|}{ 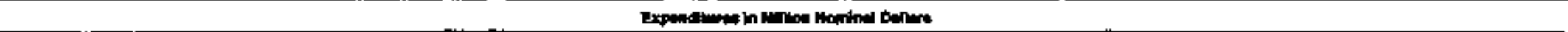 } \\
\hline 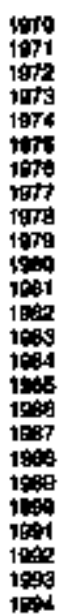 & $\begin{array}{r}11.7 \\
107 \\
75 \\
50 \\
71 \\
10.7 \\
117 \\
90 \\
63 \\
88 \\
1.3 \\
19 \\
19 \\
18 \\
42 \\
9.9 \\
11 \\
29 \\
24 \\
95 \\
0.1 \\
03 \\
01 \\
10 \\
14\end{array}$ & 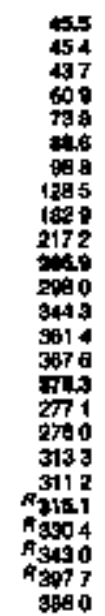 & 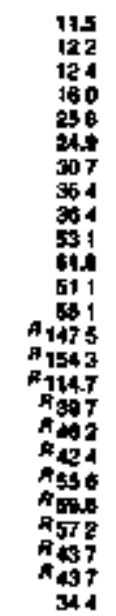 & $\begin{array}{l}06 \\
05 \\
03 \\
04 \\
00 \\
09 \\
05 \\
05 \\
05 \\
03 \\
14 \\
02 \\
00 \\
09 \\
03 \\
09 \\
01 \\
01 \\
02 \\
02 \\
03 \\
03 \\
03 \\
04 \\
02\end{array}$ & 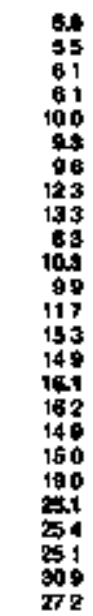 & $\begin{array}{c}04 \\
07 \\
06 \\
09 \\
12 \\
12 \\
13 \\
13 \\
16 \\
28 \\
91 \\
45 \\
47 \\
192 \\
124 \\
196 \\
193 \\
112 \\
114 \\
121 \\
457 \\
119 \\
90 \\
823 \\
42\end{array}$ & $\begin{array}{l}04 \\
09 \\
09 \\
09 \\
18 \\
14 \\
31 \\
41 \\
33 \\
34 \\
06 \\
04 \\
13 \\
03 \\
02 \\
30 \\
46 \\
18 \\
39 \\
45 \\
33 \\
28 \\
32 \\
31 \\
25\end{array}$ & 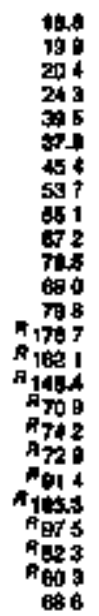 & 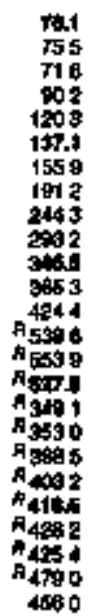 & 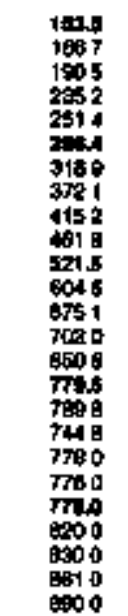 & 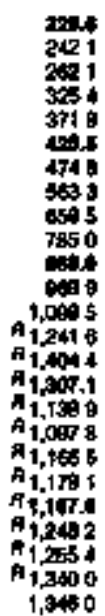 \\
\hline
\end{tabular}




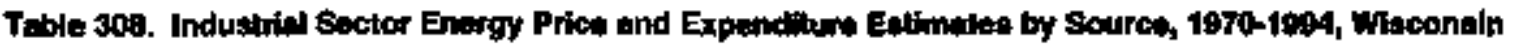

\begin{tabular}{|c|c|c|c|c|c|c|c|c|c|c|c|c|c|c|c|c|c|}
\hline & \multicolumn{15}{|c|}{ Pitmey Emery } & \multirow[b]{3}{*}{ nectionsy } & \multirow[b]{3}{*}{ reper } \\
\hline & \multicolumn{3}{|c|}{ cool } & \multirow[b]{2}{*}{ mond } & \multicolumn{9}{|c|}{ Pintropen } & \multirow[b]{2}{*}{ D: } & \multirow[b]{2}{*}{ Jolene } & & \\
\hline & Coline & Singed & rot & & Hond ond & Disulet & 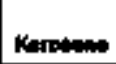 & LPG & Lubnewats: & : & 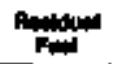 & othe & המה The & & & & \\
\hline Yoer & \multicolumn{17}{|c|}{ 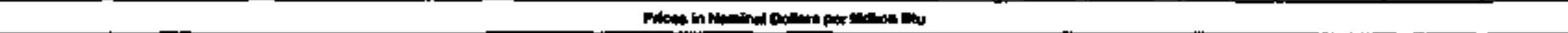 } \\
\hline \multirow[t]{2}{*}{ 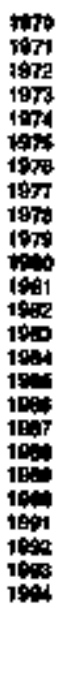 } & 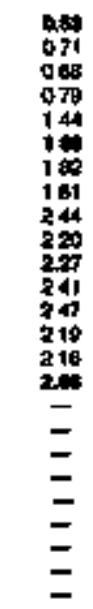 & 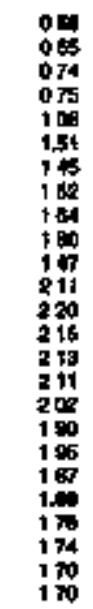 & 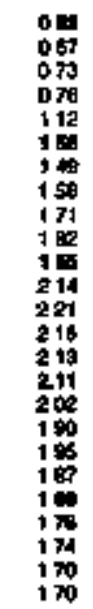 & 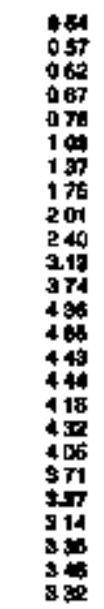 & 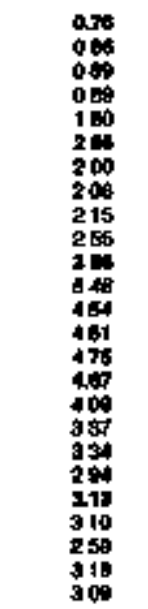 & 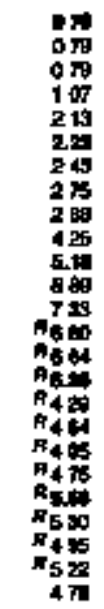 & 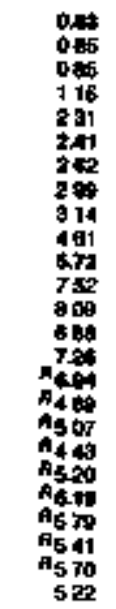 & 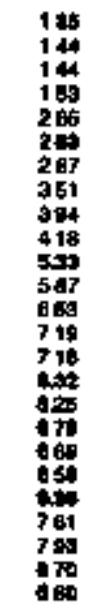 & 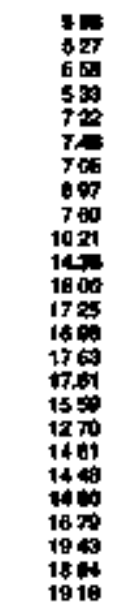 & 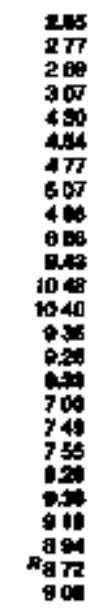 & 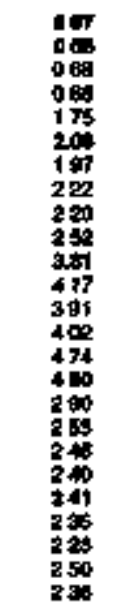 & 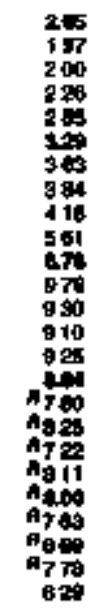 & 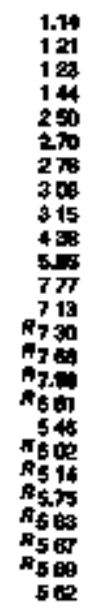 & $\begin{array}{l}= \\
= \\
= \\
= \\
= \\
= \\
= \\
= \\
= \\
= \\
= \\
\bar{z} \\
0 \\
100 \\
100 \\
100 \\
180\end{array}$ & 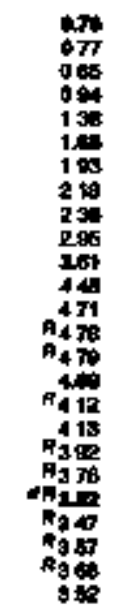 & 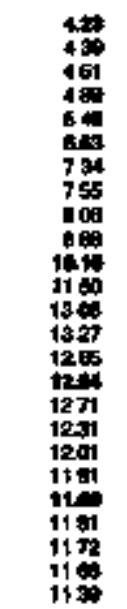 & 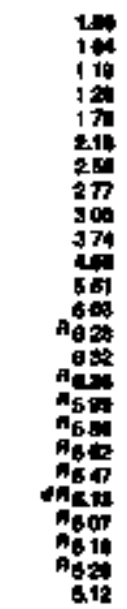 \\
\hline & \multicolumn{17}{|c|}{ 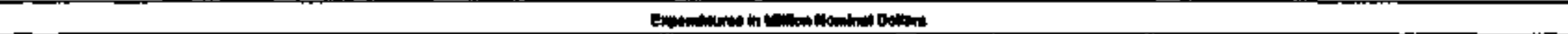 } \\
\hline 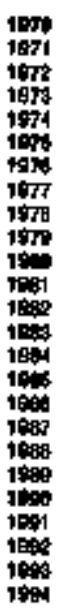 & $\begin{array}{l}50 \\
70 \\
70 \\
30 \\
00 \\
120 \\
150 \\
136 \\
103 \\
02 \\
123 \\
122 \\
71 \\
10 \\
01 \\
01 \\
= \\
= \\
= \\
= \\
= \\
= \\
=\end{array}$ & 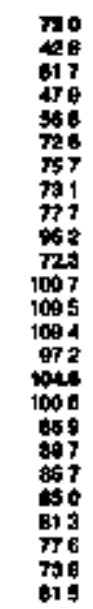 & 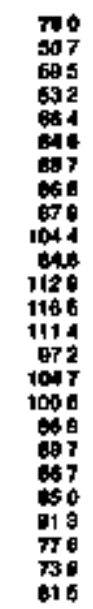 & 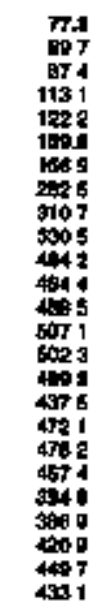 & 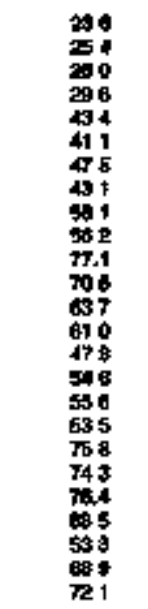 & 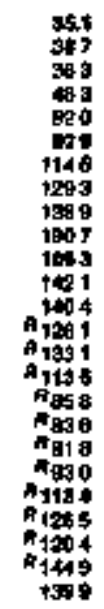 & $\begin{array}{l}59 \\
48 \\
31 \\
40 \\
59 \\
58 \\
52 \\
52 \\
59 \\
23 \\
19 \\
20 \\
15 \\
11 \\
09 \\
05 \\
05 \\
05 \\
02 \\
05 \\
04 \\
03 \\
04 \\
08 \\
05\end{array}$ & 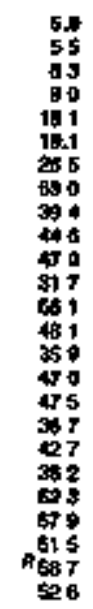 & 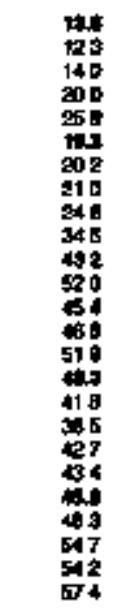 & 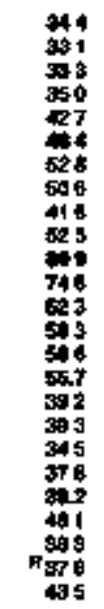 & 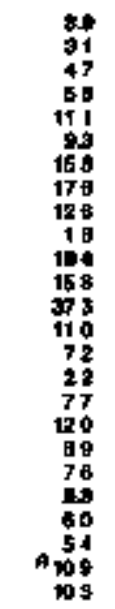 & 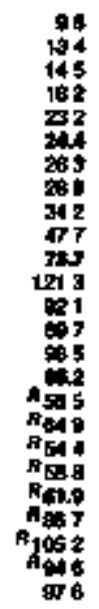 & 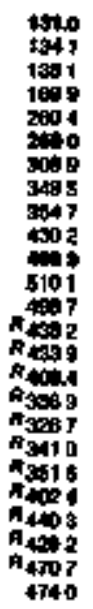 & 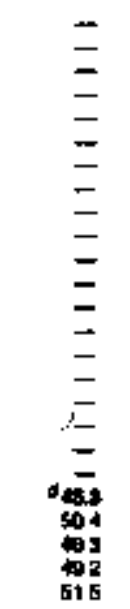 & 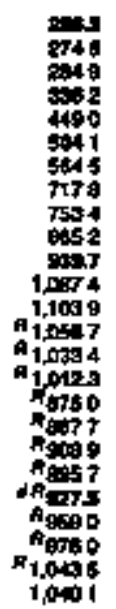 & 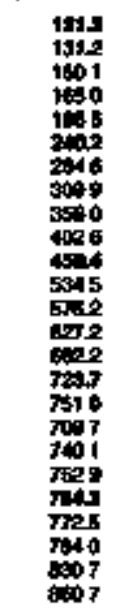 & 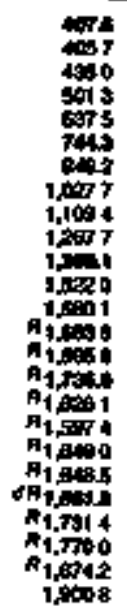 \\
\hline
\end{tabular}

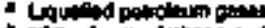

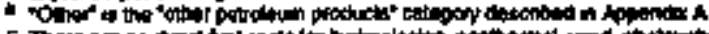

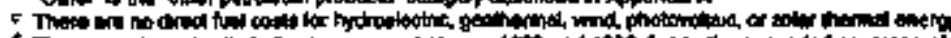

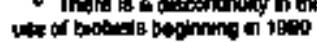

Aphomsed an

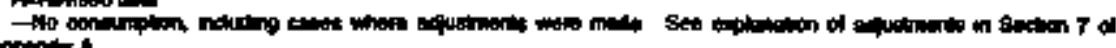
spenth

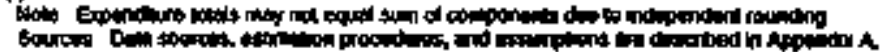




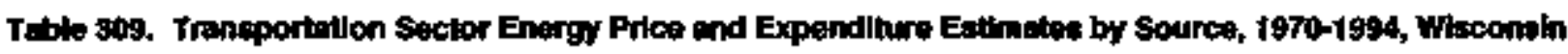

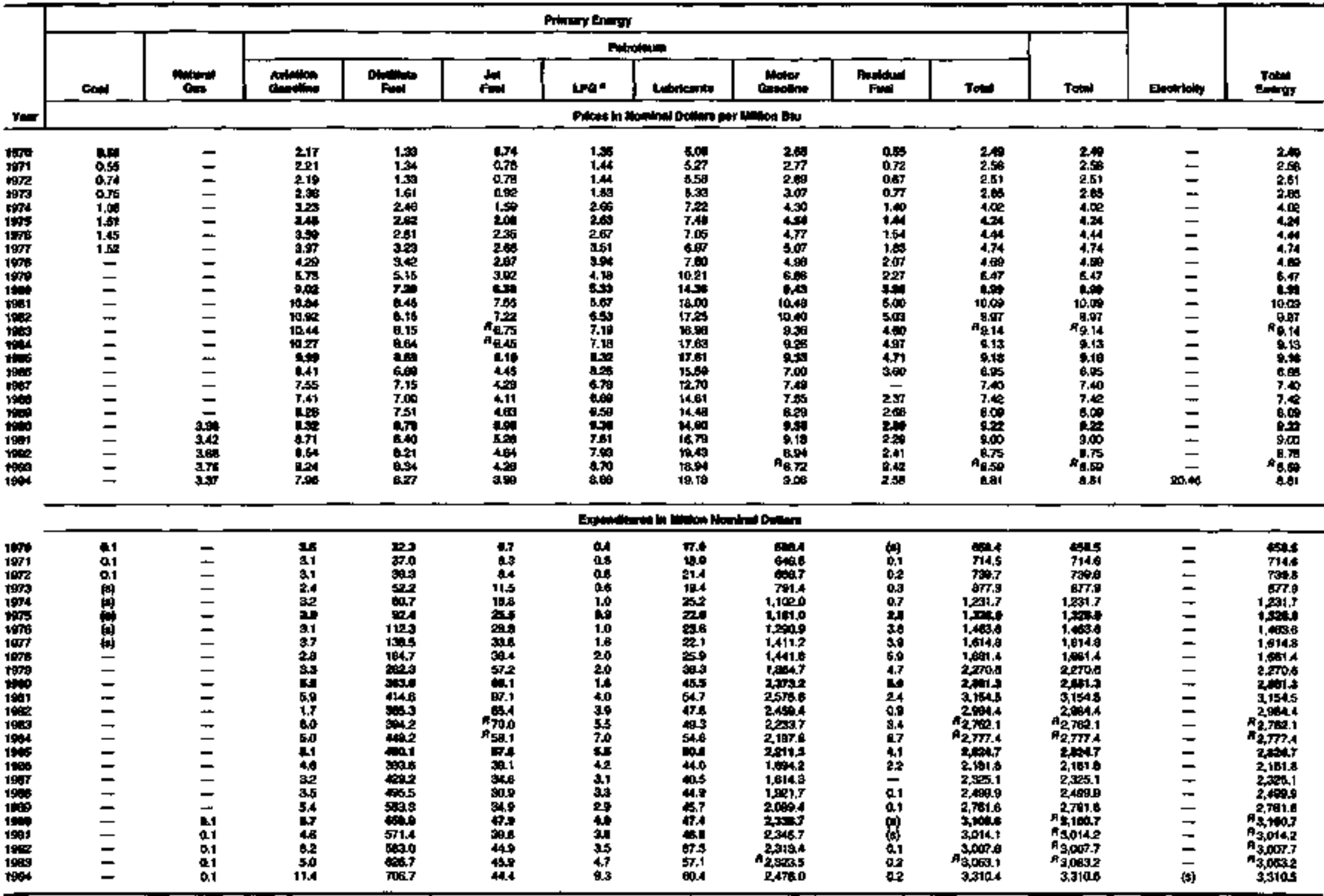

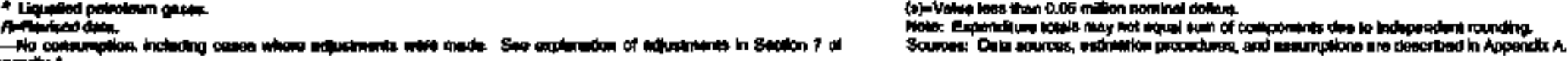

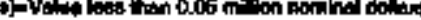

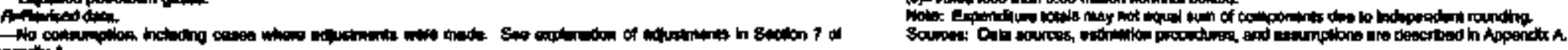
appention. 


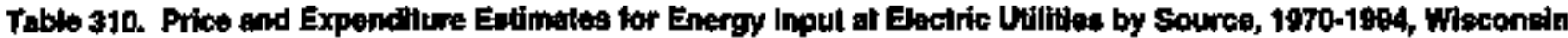

I
$\mathbf{C}$
$\mathbf{N}$
$\mathbf{S}$

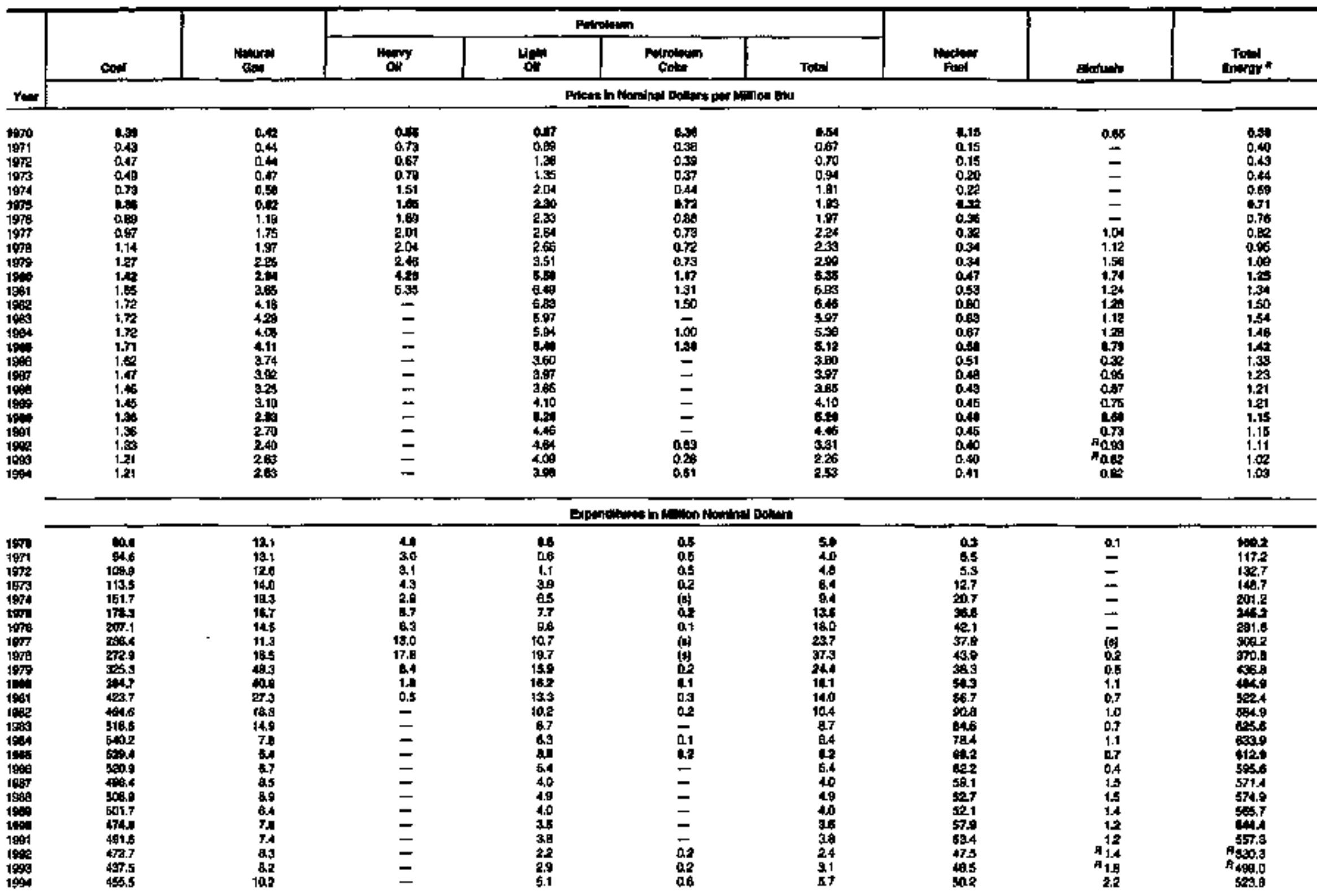

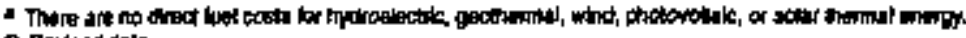

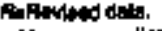

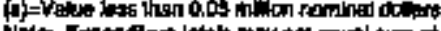


Tabl 311. Energy Pries and Expenditure Eetimetes by Source, 1970-1994, Wyom'ng

\begin{tabular}{|c|c|c|c|c|c|c|c|c|c|c|c|c|c|c|c|c|c|}
\hline \multirow[b]{4}{*}{ res } & \multicolumn{14}{|c|}{ Anevy tinerg: } & \multirow{3}{*}{ 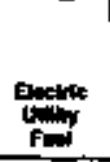 } & \multirow{3}{*}{ 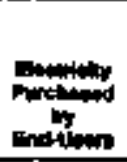 } & \multirow[b]{3}{*}{ 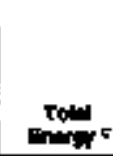 } \\
\hline & \multicolumn{3}{|c|}{ cons } & \multirow[b]{2}{*}{ Nater } & \multicolumn{7}{|c|}{ Patrovina } & \multirow[b]{2}{*}{ 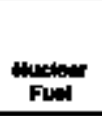 } & \multirow[b]{2}{*}{ (1) } & \multirow[b]{2}{*}{ Tot I I I } & & & \\
\hline & Cond & יוריל & Tow & & 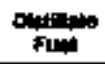 & Find & LPa* & moporing & $\begin{array}{l}\text { Ruspoull } \\
\text { Fuel }\end{array}$ & aners & Ton & & & & & & \\
\hline & \multicolumn{17}{|c|}{ 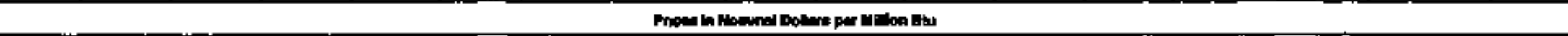 } \\
\hline \multirow[t]{2}{*}{ 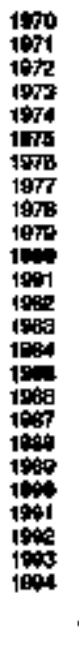 } & $\begin{array}{l}= \\
= \\
= \\
= \\
= \\
= \\
= \\
= \\
= \\
= \\
= \\
= \\
=\end{array}$ & 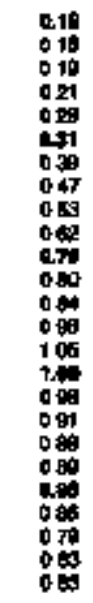 & 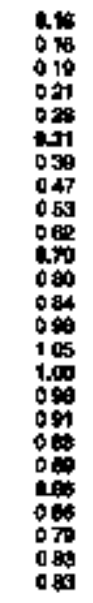 & 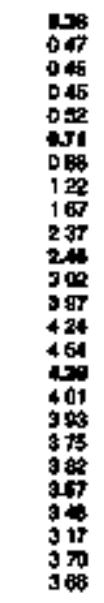 & 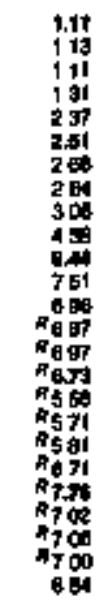 & 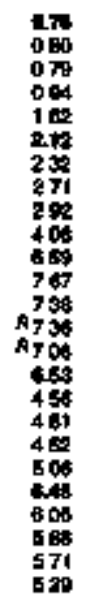 & 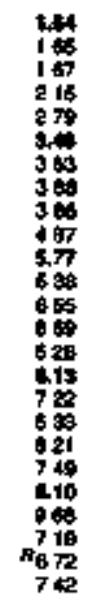 & 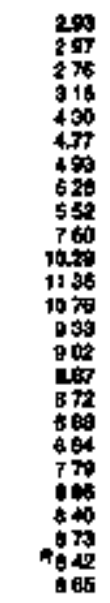 & 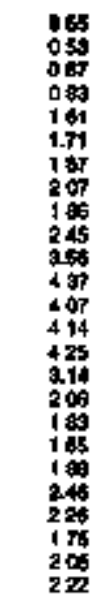 & 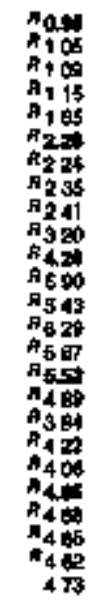 & 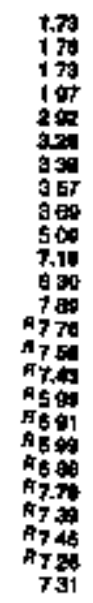 & $\begin{array}{l}= \\
= \\
= \\
= \\
= \\
= \\
= \\
= \\
= \\
= \\
= \\
= \\
= \\
=\end{array}$ & 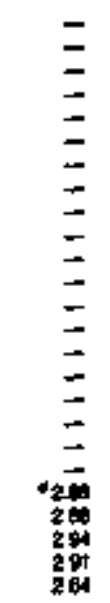 & 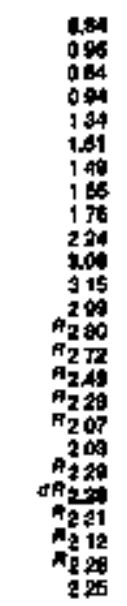 & 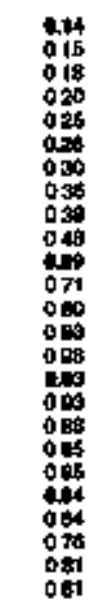 & 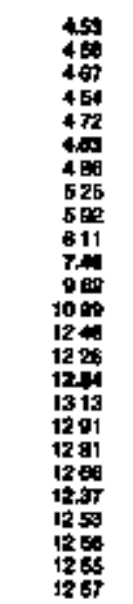 & 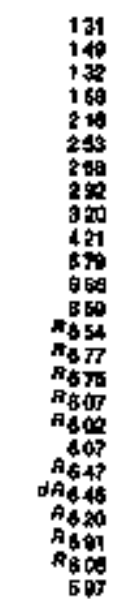 \\
\hline & \multicolumn{17}{|c|}{ 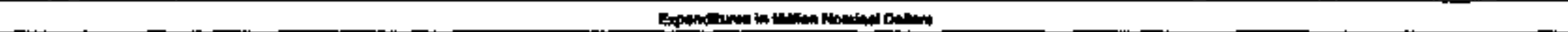 } \\
\hline 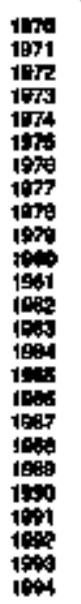 & $\begin{array}{l}= \\
= \\
= \\
= \\
= \\
= \\
= \\
= \\
= \\
= \\
= \\
= \\
=\end{array}$ & 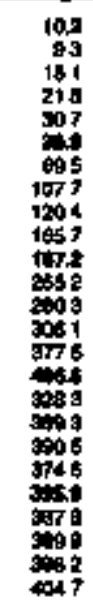 & 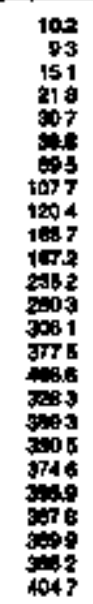 & 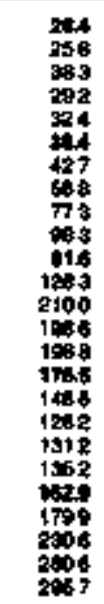 & 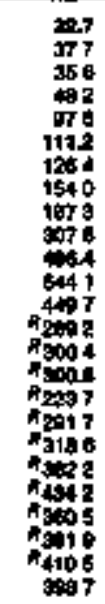 & 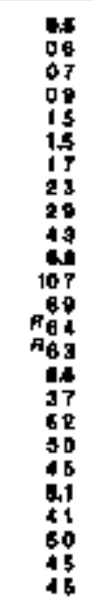 & 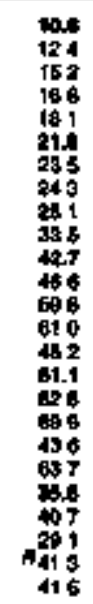 & 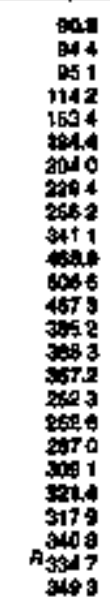 & 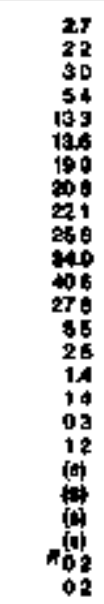 & 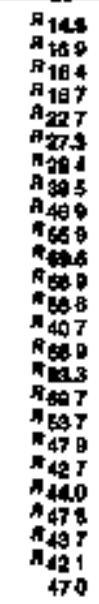 & 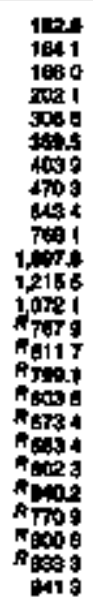 & $\begin{array}{l}= \\
= \\
= \\
= \\
= \\
= \\
= \\
= \\
= \\
= \\
= \\
= \\
=\end{array}$ & $\begin{array}{l}= \\
= \\
= \\
= \\
= \\
= \\
= \\
= \\
= \\
= \\
= \\
436 \\
33 \\
30 \\
32\end{array}$ & 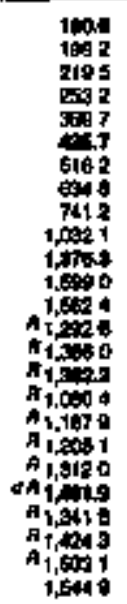 & 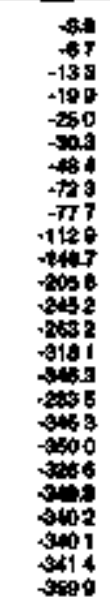 & 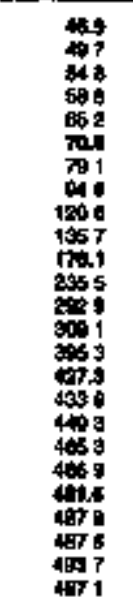 & 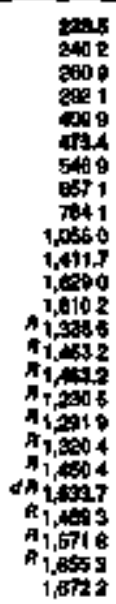 \\
\hline
\end{tabular}

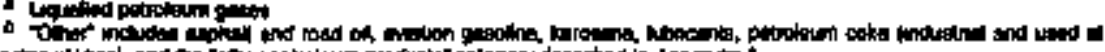

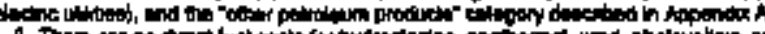

Antweddation

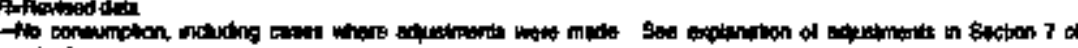
apenatis

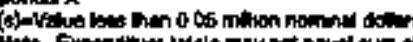

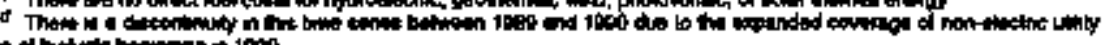

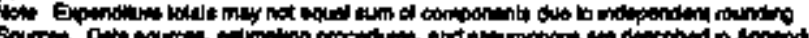

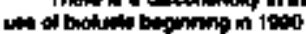




\begin{tabular}{|c|c|c|c|c|c|c|c|c|c|c|}
\hline \multirow[b]{4}{*}{$Y_{t}=$} & \multicolumn{8}{|c|}{ Phemer Enews } & \multirow[b]{3}{*}{ Enction } & \multirow[b]{3}{*}{ Entent } \\
\hline & & \multirow[b]{2}{*}{ Matin } & \multicolumn{4}{|c|}{ naming } & \multirow[b]{2}{*}{ 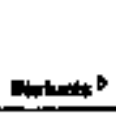 } & \multirow[b]{2}{*}{ Thide } & & \\
\hline & هat & & 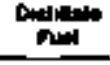 & Kmon: & LPG: & Tot & & & & \\
\hline & \multicolumn{10}{|c|}{ 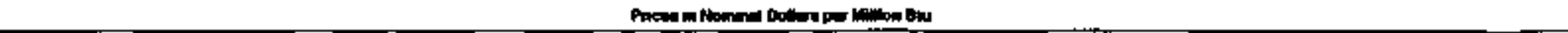 } \\
\hline 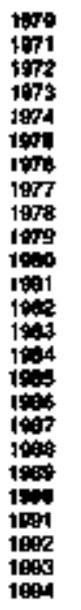 & 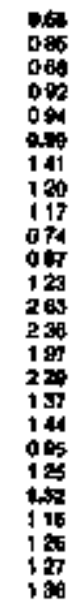 & 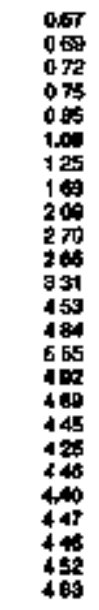 & 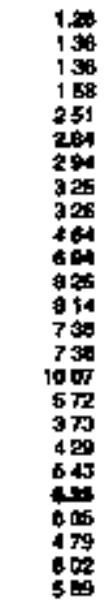 & 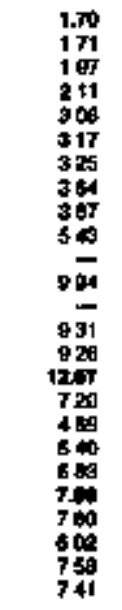 & 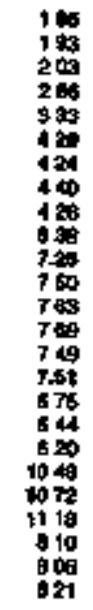 & 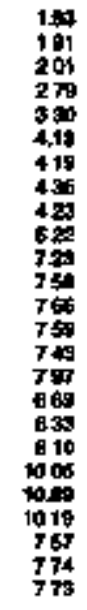 & $\begin{array}{r}= \\
= \\
= \\
= \\
= \\
= \\
= \\
= \\
= \\
= \\
= \\
0 \\
475 \\
475 \\
475\end{array}$ & 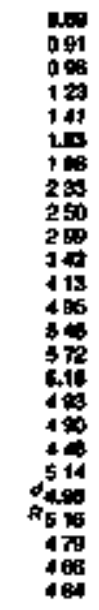 & 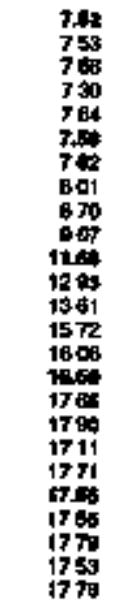 & 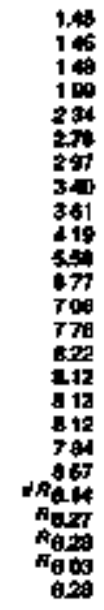 \\
\hline
\end{tabular}

\begin{tabular}{|c|c|c|c|c|c|c|c|c|c|c|}
\hline \multirow[b]{2}{*}{ 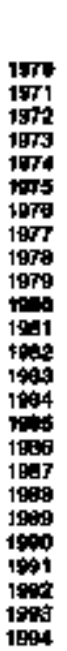 } & \multicolumn{10}{|c|}{ 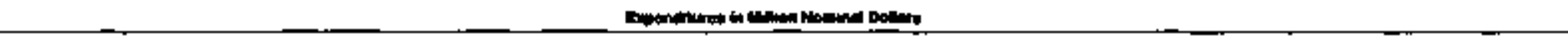 } \\
\hline & $\begin{array}{l}01 \\
02 \\
02 \\
01 \\
02 \\
03 \\
06 \\
10 \\
17 \\
17 \\
16 \\
08 \\
24 \\
28 \\
20 \\
15 \\
08 \\
08 \\
08 \\
10 \\
12 \\
13 \\
08 \\
15 \\
21\end{array}$ & 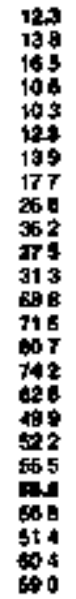 & 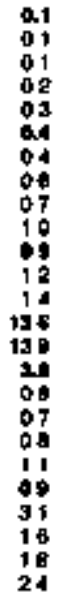 & 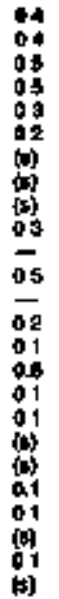 & 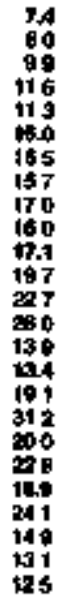 & 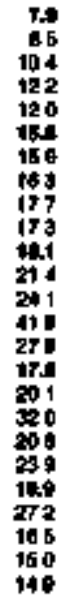 & $\begin{array}{l}\bar{z} \\
= \\
= \\
= \\
= \\
= \\
= \\
= \\
= \\
= \\
= \\
= \\
21 \\
21 \\
22 \\
20 \\
20\end{array}$ & 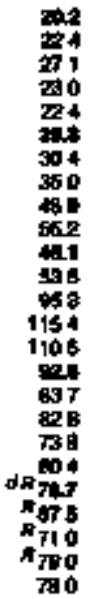 & 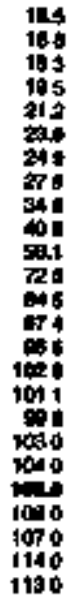 & 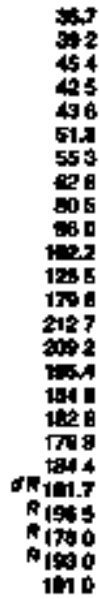 \\
\hline
\end{tabular}

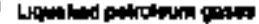

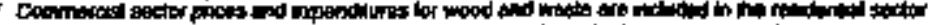

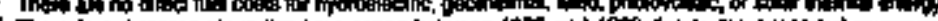

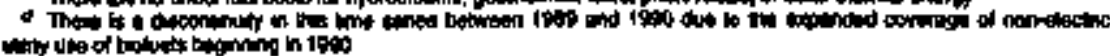

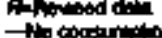

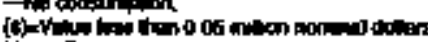

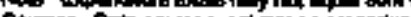

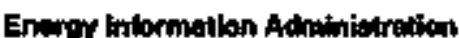




\begin{tabular}{|c|c|c|c|c|c|c|c|c|c|c|c|}
\hline \multirow[b]{4}{*}{$x=$} & \multicolumn{9}{|c|}{ Phangererg } & \multirow[b]{3}{*}{ 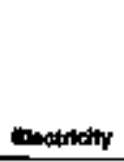 } & \multirow[b]{3}{*}{ Intory } \\
\hline & & \multirow[b]{2}{*}{ and } & \multicolumn{6}{|c|}{ RAtim } & \multirow[b]{2}{*}{ Tot } & & \\
\hline & con & & Fund & (1) & LPE" & م= & Fund & Totid & & & \\
\hline & \multicolumn{11}{|c|}{ 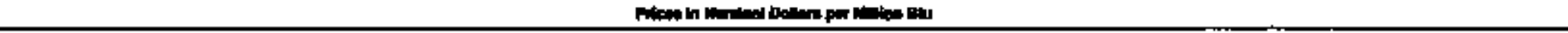 } \\
\hline \multirow[t]{2}{*}{ 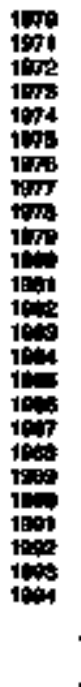 } & 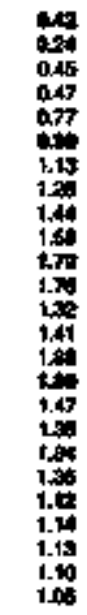 & 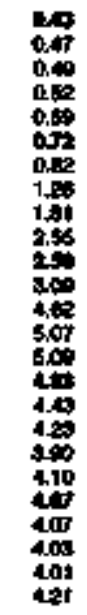 & 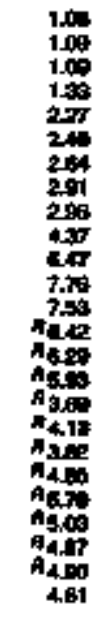 & 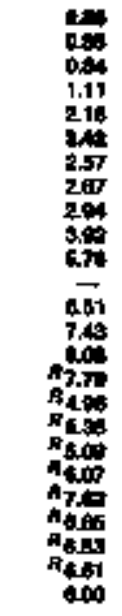 & 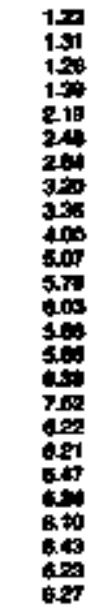 & 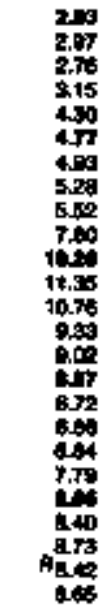 & 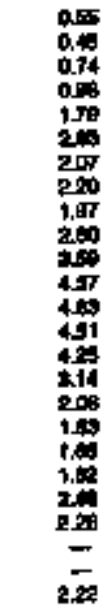 & 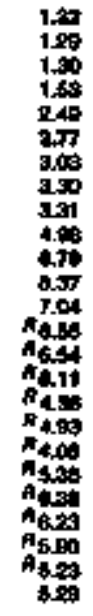 & 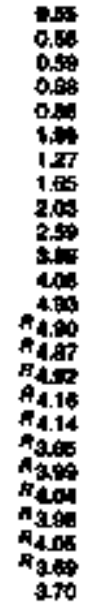 & 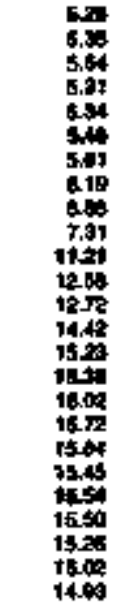 & 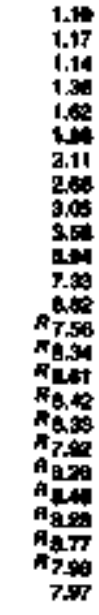 \\
\hline & \multicolumn{11}{|c|}{ Fin } \\
\hline 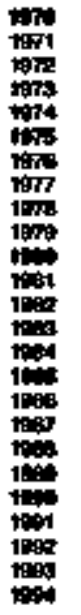 & 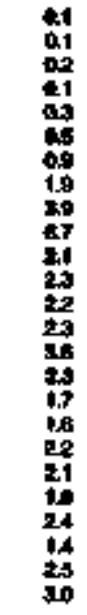 & 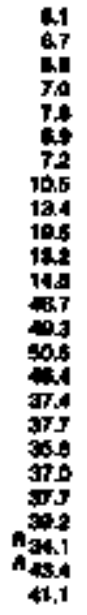 & 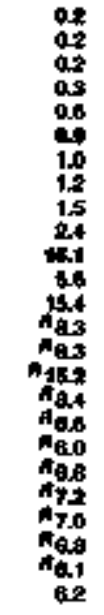 & 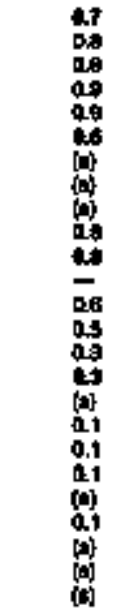 & 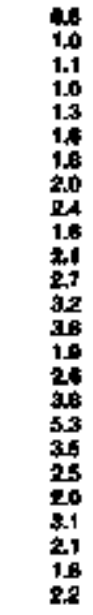 & 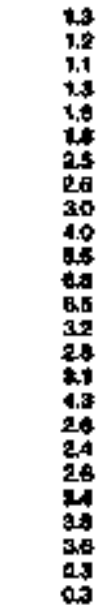 & 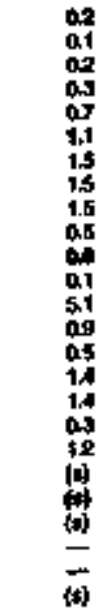 & 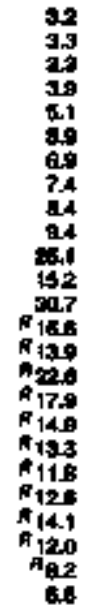 & 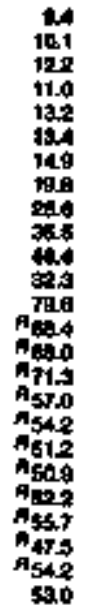 & 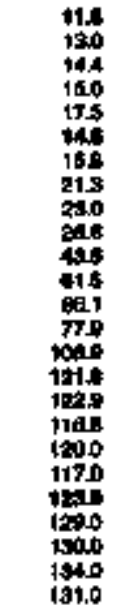 & 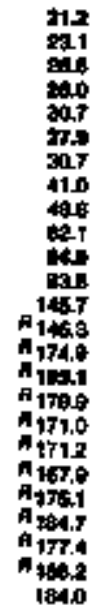 \\
\hline
\end{tabular}

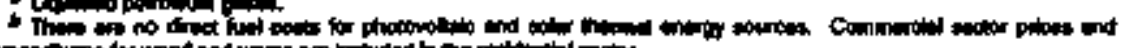

Hoortination

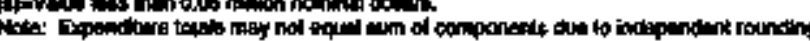

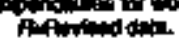


Tablo 314. Industrial Swetor Enwrgy Prkto and Expendfiture Eatimates by Source, 1970-1994, Wyoming

\begin{tabular}{|c|c|c|c|c|c|c|c|c|c|c|c|c|c|c|c|c|c|}
\hline & \multicolumn{15}{|c|}{ Primby Energs } & \multirow{2}{*}{ Bectikity } & \multirow{2}{*}{ Torom } \\
\hline & \multicolumn{3}{|c|}{$\cos$} & הatis & \multicolumn{9}{|c|}{ Antroberm } & Drotunest & Iot|l 6 & & \\
\hline ras & \multicolumn{17}{|c|}{ 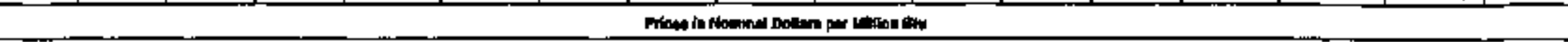 } \\
\hline 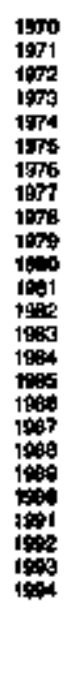 & $\begin{array}{l}= \\
= \\
= \\
= \\
= \\
= \\
= \\
= \\
= \\
= \\
= \\
=\end{array}$ & 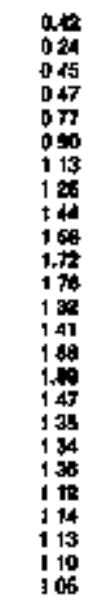 & 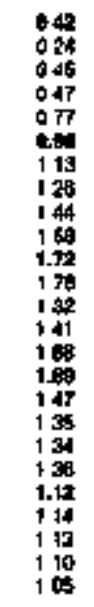 & 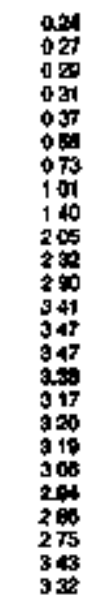 & 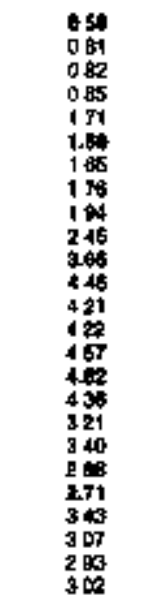 & 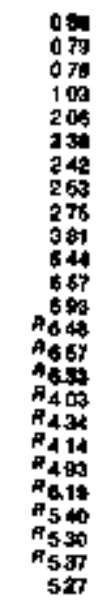 & 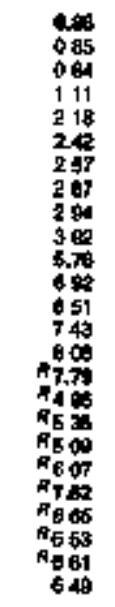 & 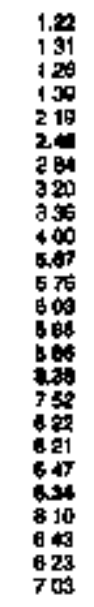 & 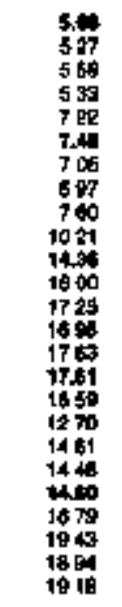 & 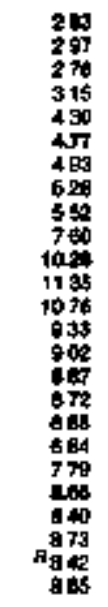 & 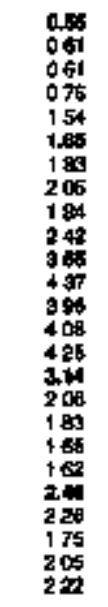 & 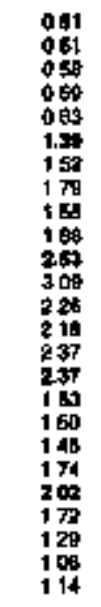 & 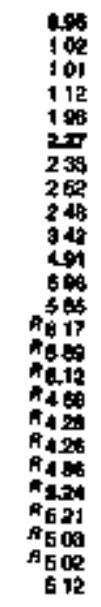 & $\begin{array}{l}= \\
= \\
= \\
= \\
= \\
= \\
= \\
= \\
= \\
= \\
z \\
15 \\
161 \\
169 \\
162\end{array}$ & 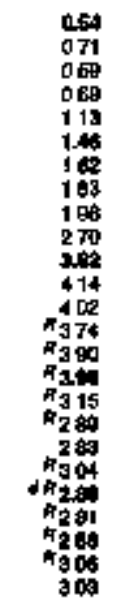 & 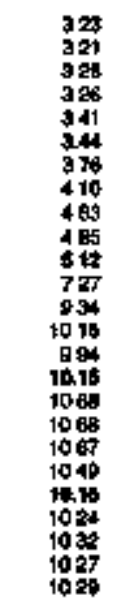 & 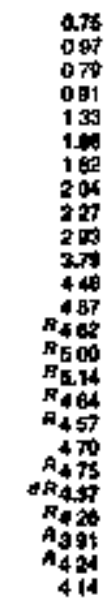 \\
\hline 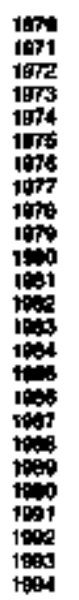 & $\begin{array}{l}= \\
\text { z } \\
\text { = } \\
\text { = } \\
= \\
= \\
= \\
= \\
= \\
= \\
= \\
= \\
=\end{array}$ & 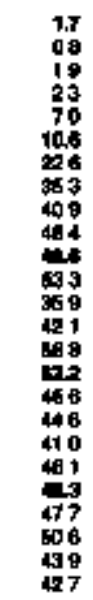 & 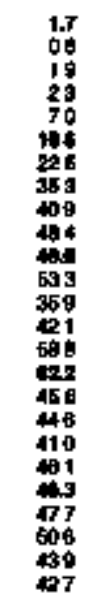 & 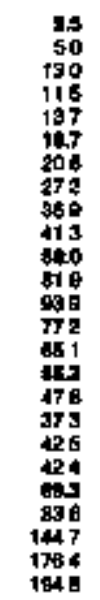 & 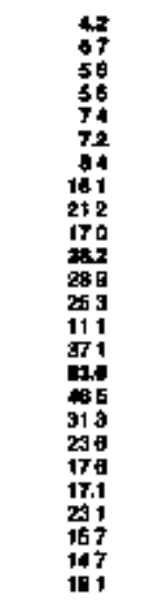 & 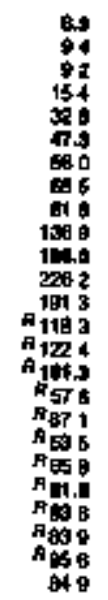 & 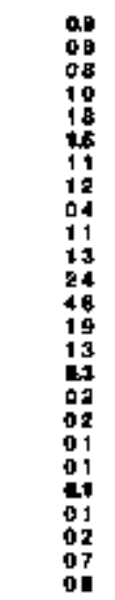 & 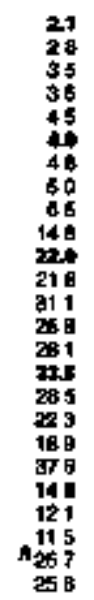 & $\begin{array}{l}5 \\
01 \\
01 \\
01 \\
02 \\
21 \\
21 \\
24 \\
20 \\
40 \\
80 \\
80 \\
62 \\
64 \\
60 \\
84 \\
41 \\
44 \\
48 \\
60 \\
52 \\
54 \\
63 \\
63 \\
68\end{array}$ & 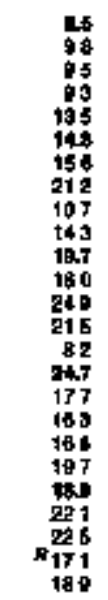 & 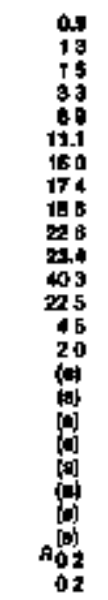 & 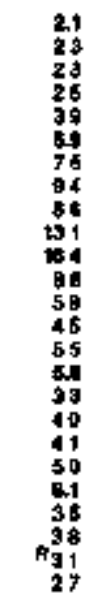 & 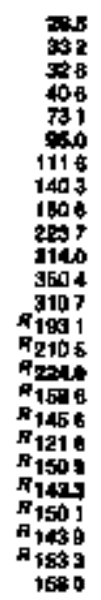 & $\begin{array}{l}= \\
= \\
= \\
= \\
= \\
= \\
= \\
= \\
= \\
= \\
\overline{10} \\
10 \\
10 \\
10 \\
12\end{array}$ & 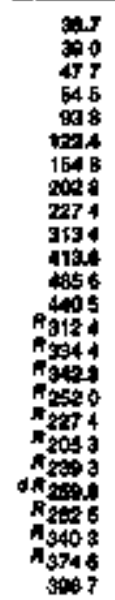 & 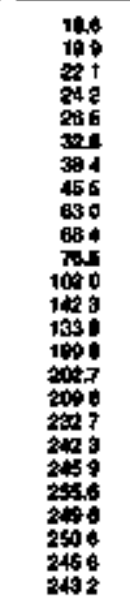 & 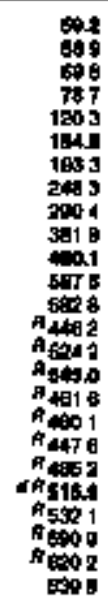 \\
\hline
\end{tabular}

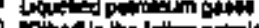

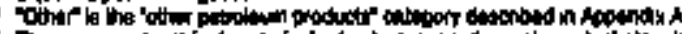

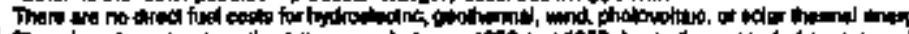

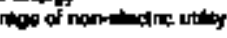
ropinita

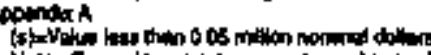


Teble 315. Trensportation Sector Energy Price and Expenditure Entimates by 8ource, 1970-1994, Wyoming

\begin{tabular}{|c|c|c|c|c|c|c|c|c|c|c|c|c|c|}
\hline \multirow[b]{4}{*}{ Yex } & \multicolumn{11}{|c|}{ Pintikg Giangy } & \multirow[b]{3}{*}{ andritity } & \multirow[b]{3}{*}{ Thow } \\
\hline & \multirow[b]{2}{*}{ Cod } & \multirow[b]{2}{*}{ Moncill } & \multicolumn{8}{|c|}{ Prowater } & \multirow[b]{2}{*}{$T_{0}$} & & \\
\hline & & & Antritan & Dinther & بـ & Lat. & Lubricants & ancor & Findurd & Tont & & & \\
\hline & \multicolumn{13}{|c|}{ 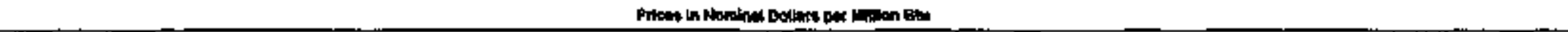 } \\
\hline \multirow[t]{2}{*}{ 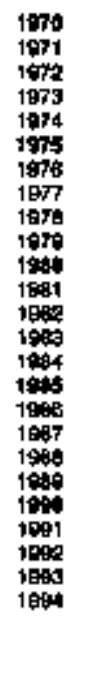 } & $\begin{array}{l}042 \\
024 \\
045 \\
047 \\
077 \\
094 \\
113 \\
120 \\
= \\
= \\
= \\
= \\
= \\
= \\
= \\
= \\
= \\
= \\
= \\
=\end{array}$ & $\begin{array}{l}= \\
= \\
= \\
= \\
= \\
= \\
= \\
= \\
= \\
= \\
= \\
= \\
53 \\
543 \\
536 \\
437\end{array}$ & 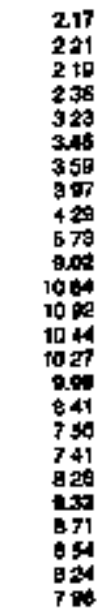 & 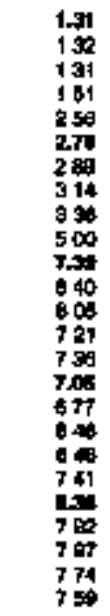 & 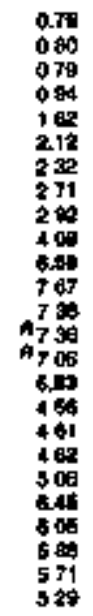 & 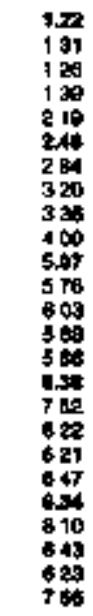 & 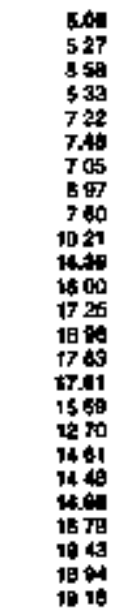 & 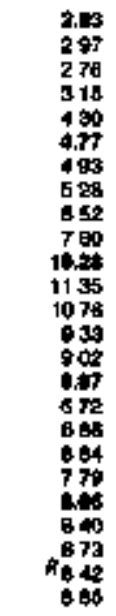 & 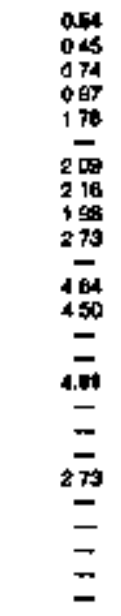 & 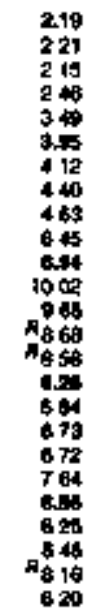 & 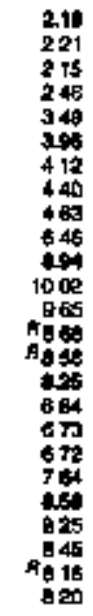 & $\begin{array}{l}= \\
= \\
= \\
= \\
= \\
= \\
= \\
= \\
= \\
= \\
= \\
= \\
= \\
=\end{array}$ & 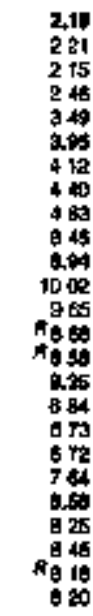 \\
\hline & \multicolumn{13}{|c|}{ 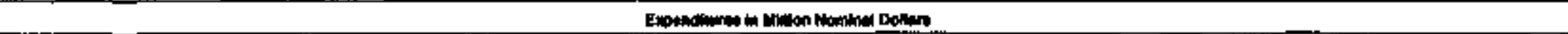 } \\
\hline 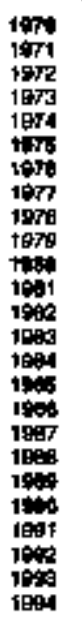 & 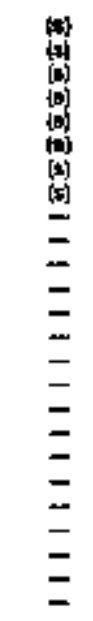 & $\begin{array}{l}= \\
= \\
= \\
= \\
= \\
= \\
= \\
= \\
= \\
= \\
= \\
= \\
= \\
061 \\
01 \\
01 \\
01\end{array}$ & $\begin{array}{l}2.0 \\
20 \\
28 \\
31 \\
10 \\
10 \\
40 \\
50 \\
64 \\
68 \\
40 \\
40 \\
33 \\
29 \\
39 \\
28 \\
21 \\
19 \\
20 \\
16 \\
1.7 \\
12 \\
19 \\
98 \\
19\end{array}$ & 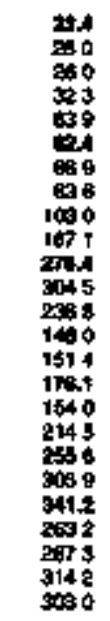 & 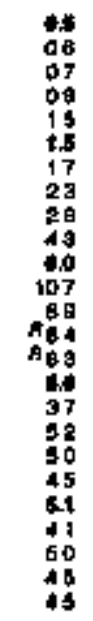 & $\begin{array}{l}04 \\
06 \\
07 \\
07 \\
10 \\
1.1 \\
14 \\
18 \\
29 \\
10 \\
1.4 \\
25 \\
28 \\
34 \\
18 \\
1.4 \\
14 \\
11 \\
12 \\
08 \\
48 \\
14 \\
08 \\
08 \\
10\end{array}$ & 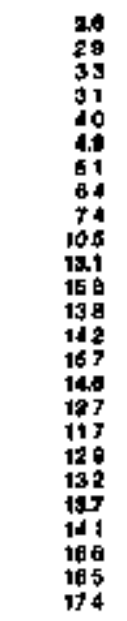 & 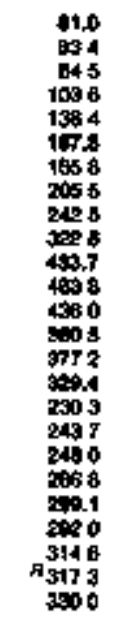 & 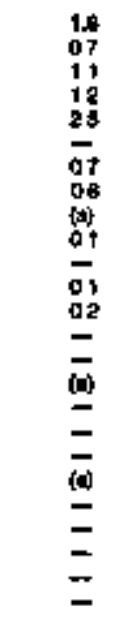 & 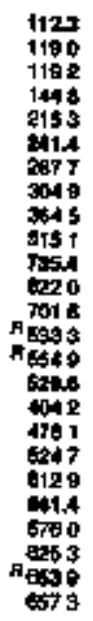 & 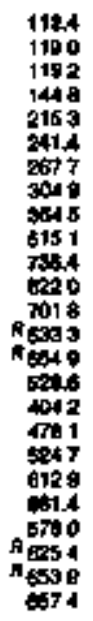 & $\begin{array}{l}= \\
= \\
= \\
= \\
= \\
= \\
= \\
= \\
= \\
= \\
= \\
= \\
= \\
= \\
=\end{array}$ & 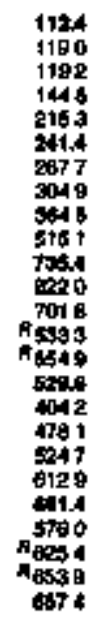 \\
\hline
\end{tabular}




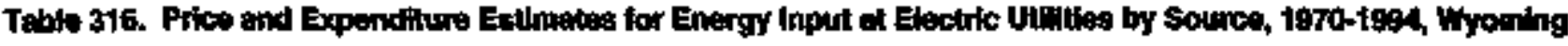

\begin{tabular}{|c|c|c|c|c|c|c|c|c|c|}
\hline \multirow[b]{3}{*}{$n=$} & \multirow[b]{2}{*}{ cont } & \multirow[b]{2}{*}{ 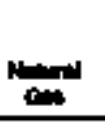 } & \multicolumn{4}{|c|}{ 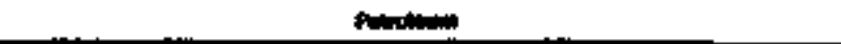 } & \multirow[b]{2}{*}{ Fid } & \multirow[b]{2}{*}{ 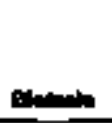 } & \multirow[b]{2}{*}{ 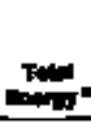 } \\
\hline & & & on & का & תוקר & Tow & & & \\
\hline & \multicolumn{9}{|c|}{ 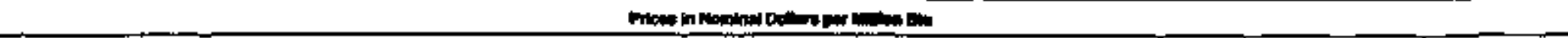 } \\
\hline \multirow[t]{2}{*}{ 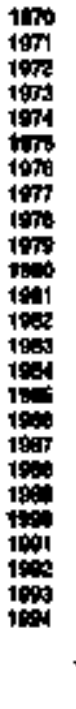 } & 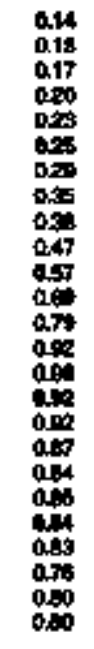 & 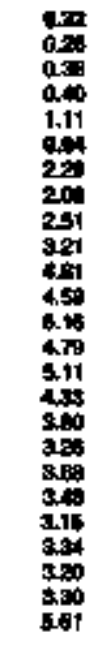 & 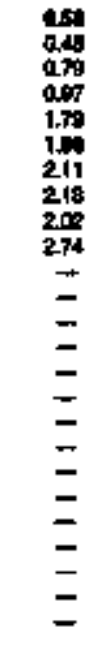 & 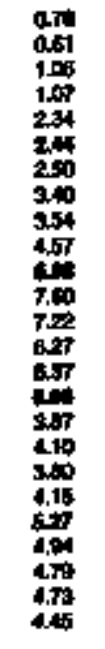 & $\begin{array}{l}z \\
z \\
z \\
z \\
z \\
z \\
z \\
z \\
= \\
z \\
z \\
z\end{array}$ & 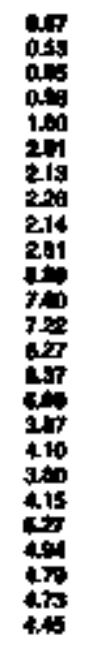 & $\begin{array}{l} \pm \\
z \\
z \\
= \\
z \\
= \\
= \\
= \\
= \\
= \\
=\end{array}$ & $\begin{array}{l}= \\
z \\
z \\
z \\
z \\
z \\
z \\
z \\
z \\
z \\
z \\
z\end{array}$ & 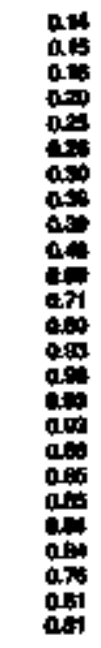 \\
\hline & \multicolumn{9}{|c|}{ 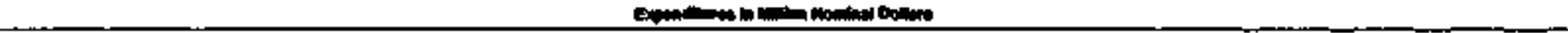 } \\
\hline 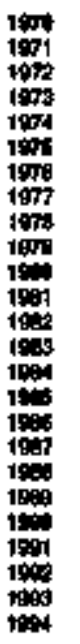 & 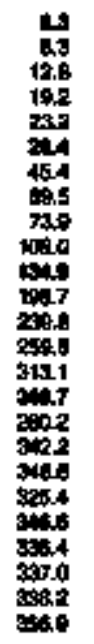 & 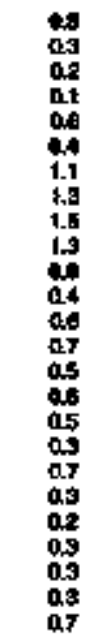 & $\begin{array}{l}0.1 \\
0.1 \\
02 \\
020 \\
12 \\
14 \\
1.7 \\
20 \\
20 \\
24 \\
= \\
= \\
= \\
= \\
= \\
= \\
= \\
= \\
= \\
=\end{array}$ & 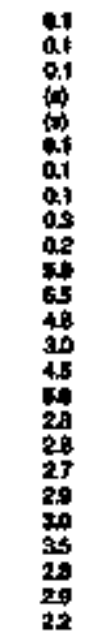 & 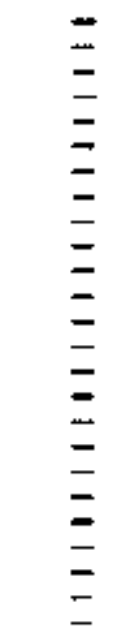 & 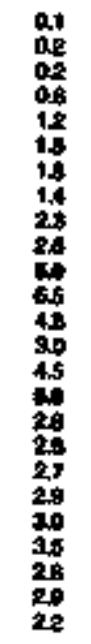 & $\begin{array}{l}z \\
\equiv \\
= \\
= \\
= \\
= \\
= \\
= \\
= \\
= \\
=\end{array}$ & $\begin{array}{l}z \\
z \\
z \\
z \\
z \\
z \\
z \\
z \\
z \\
z \\
z \\
z\end{array}$ & 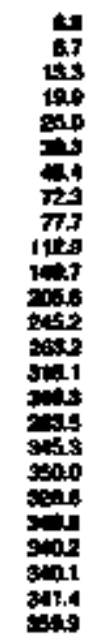 \\
\hline
\end{tabular}

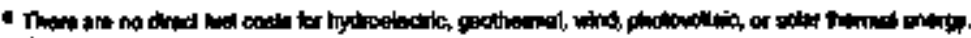

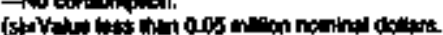

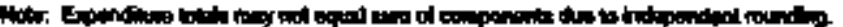

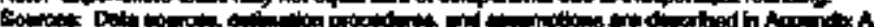


Energy Price and Expenmiture Estimetes by Sector and Souree, Each State and the United 8tater

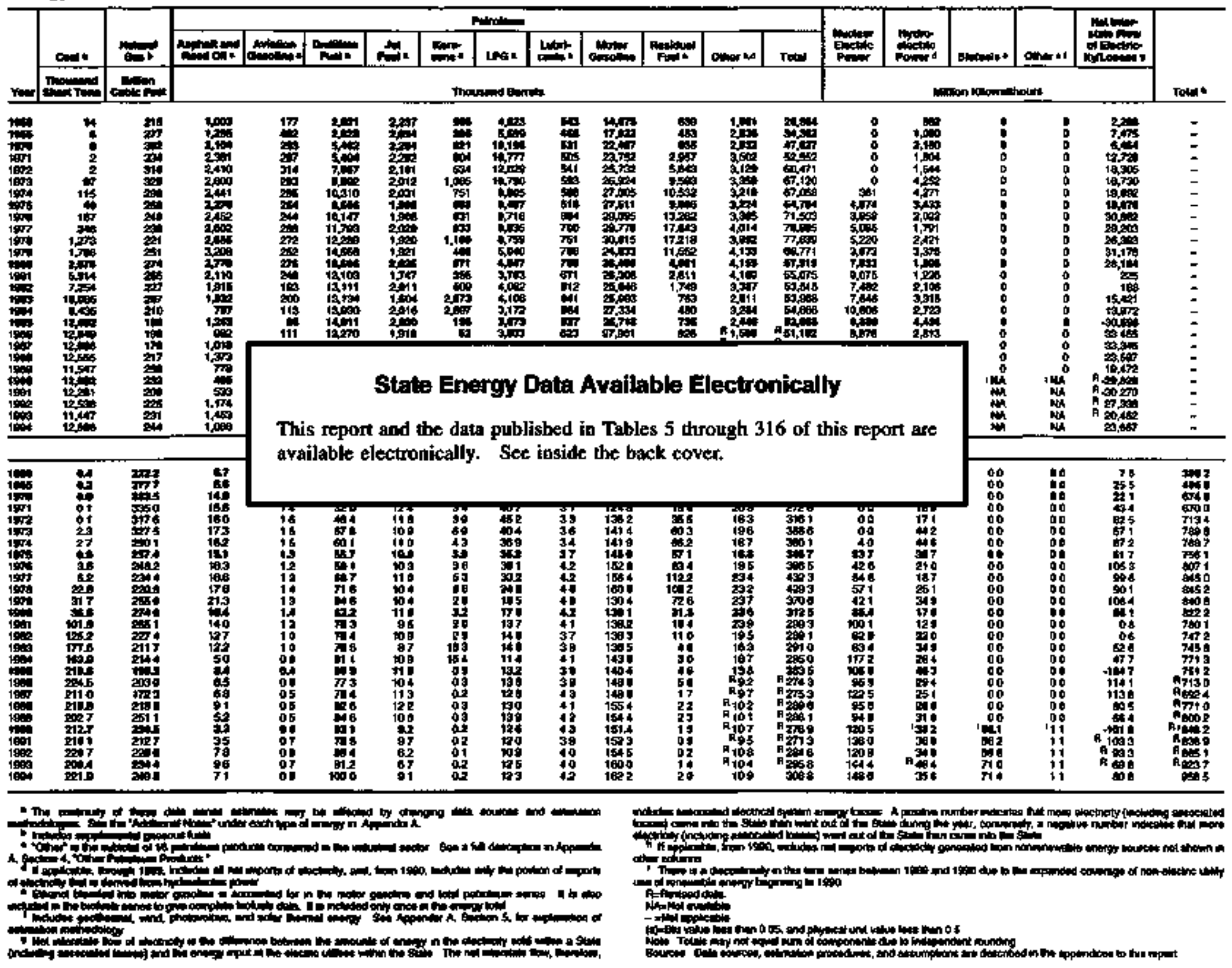


Appendix A

\section{Documentation}




\section{Documentation}

This appendix docurnents data sources and procedures used to develop the estimates in the State Energy Price and Expenditure Data System (SEPEDS) that are presented in the "Statistical Tables" of this report. Information is provided for each of the four major energy sources: coal. natural gas, petroleum, and electricity. The last section describes adjustEnents for cousumption of industrial process fuel and intermediate products and other uncosted energy sources.

\section{Price Estimation Wothodologies}

Price data in the State Energy Price and Experditure Report are expressed in dollars per million Btu. If the soutce data are in physical units, they are divided by the approptiate conversion factors to create the Bto prices. Estimated prices are used only when specific State-level prices are not available for a given energy source and sector. In some cases, prices for energy consumed in one sector in a State are assigned to another sector in the same State. Specific examples are: incustrial stean coal prices are assigned to the commercial and transportation sectors' stean coal use; industrial kerosene prices are assigned to commercial kerosene uses; industrial liquefied petroleum gases (LPG) prices are assigned to LPG used by the commercial and transportation sectors; industrial lubricants prices are assigned to transportition lubricants uses; and transportation motor gasolite prices are assigned to commercial and industrial use of motor gasoline.
In addition, there are a few cases where State-level prices could not be identified for any economic sector for a given energy source for some or all years. In these instances, a nztional-level price is used for all States for a given year. The procedures for estimating these national-level prices are presented in the body of the documentation under each energy source as appropriate. The cases where a national-level price is assigned to all states in all years are: transportation use of aviation gasoline; industrial and transportation use of lubricants; and some components of other petroleam products ased in the industrial sector.

Finally, within a given energy source and sector where price data are usually available, there are some cases of missing prices. Two general approaches are used to assign or estimate prices in cases where consumption occurs but no price is directly avajlable from the data sources. The first approach is to assign an adjacent State price or the simple average of adjacent States' prices. When this approach is not feasible, the consumption-weighted price from the Census division or region or the Petroleum Adrninistration for Defense district or subdistrict in which the State is kcated is assigned.

Three State groupings used in the report-U.S. Census regions and divisions, Federal regions, and Petroleum Administration for Defense districts-are shown in Figures A1, A2, and A3, respectively, on the following pages. Throughout the docnmentation, the term "State" includes 
the District of Columbia. States are often designated by the two-letter postal code abtreviations shown in the map legends.

\section{Exponditures}

Energy consuription estimates used to calculate expenditures in SEPEDS are taken from the Energy Information Administration (ELA) State Energy Data Report 1994, Consumption Estimates, DOEJEIA-0214(94), published in October 1996. The data system from which the consumption report is developed is called the State Bnergy Data System (SEDS). Full documentation of SEDS data sources and the methods used to estimate energy consumption are described in the consumption report.

To calculate energy expenditures, SEDS consumption is adjusted to remove quantities of process fivel and intermediate products used in the industrial and transportation sectors that are not purchased directly by end users. Residential/commercial use of solar energy and industrial use of hydroelectric, geothermal, wind, and solar energy sources are also removed from SEPEDS expenditure calculations since there are no direct fuel costs for those energy sonurces. SEDS consumption of wood in the residential and commercial sectors and biofuels consumption in the industrial sector are adjusted to cemove estimated quantities that were obtained at no cost. Energy expenditures, in million dollars, are calculated by multiplying SEPEDS prices for each fuel in dollars per mitlion Bta times the SEDS adjusted consumption in billion Btu.

\section{Endube Soctor, State, and National Consumption-Wolghted Avarage Prices}

Aggregated prices shown in this report are the consumption-weighted averages of the various entrgy sources included in the aggregatjon. The SEPEDS calculation is performed by sumping the expenditures for the energy sources and dividing by the sum of the corresponding consumption values. For example, the average price for residential petroleum is the sum of residential expenditures for distillate fuel oil, kerosene, and liquefied petroleum gas divided by the sum of residential consumption of those fuels.

Primary Energy and Electricity. Within each end-use sector, the sum of all fuels used directly as energy, i,e., coal, natural gas, petroleum products, and biofuels, is called primary energy. Electricity is considered a secondary energy source because it is generated from the primaty energy sources consumed by electric utilities. Within each sector, the primary energy price is calculated by summing the expenditures for coal (the sum of coking coal and steam coal), natural gas, petroleun (the sum of all petroleum products used by the sector), and biofuels and dividing by the sum of all the consumption for those fuels. The average price of atl energy consumed by each sector is the sum of the expenditures for primary energy and electricity divided by the sum of the consumption of the primary energy sources and olectricity.

Electric Utllity Sector. Electric utilities comprise a fifth energy consuming sector, although they are not considered an end-use sector. The average price paid by electric atilities for primary energy sources is the sum of their expenditures for coal, natural gas, perroleum products, nuclear fuet, and biomass fuels divided by the quantities consumed.

Prinary Eneray, Totel, and Total Energy. The average price for the "Primary Energy, Total" shown in the first SEPER table for each State and the United States is the sum of the four end-use sectors' and the electric utility sector's expenditures for coal, natural gas, petroleum, and other fuels divided by the sum of the five sectors' consumption of those fuels. The distinction between this average price and the "Total Energy" average price shown in the same table is that this price includes all fossil and other fuels consumed, including those used to generate electricity. The "Total Energy" price is the average price of fuels consumed directly as energy by the four end-use sectors and the much bigher price of the four sectors' electricity consumption. 
Region 1

Northenst

\section{Division 1}

(New Bungland)

Connecticut (CT)

Maine (ME)

Massachusetts (MA)

New Hampshire (NH)

Rhode lsland (RI)

Vermon (VT)

\section{Division 2}

(Middle Athontic)

New Jersey (ND)

New York (NY)

Peansylvania (PA)

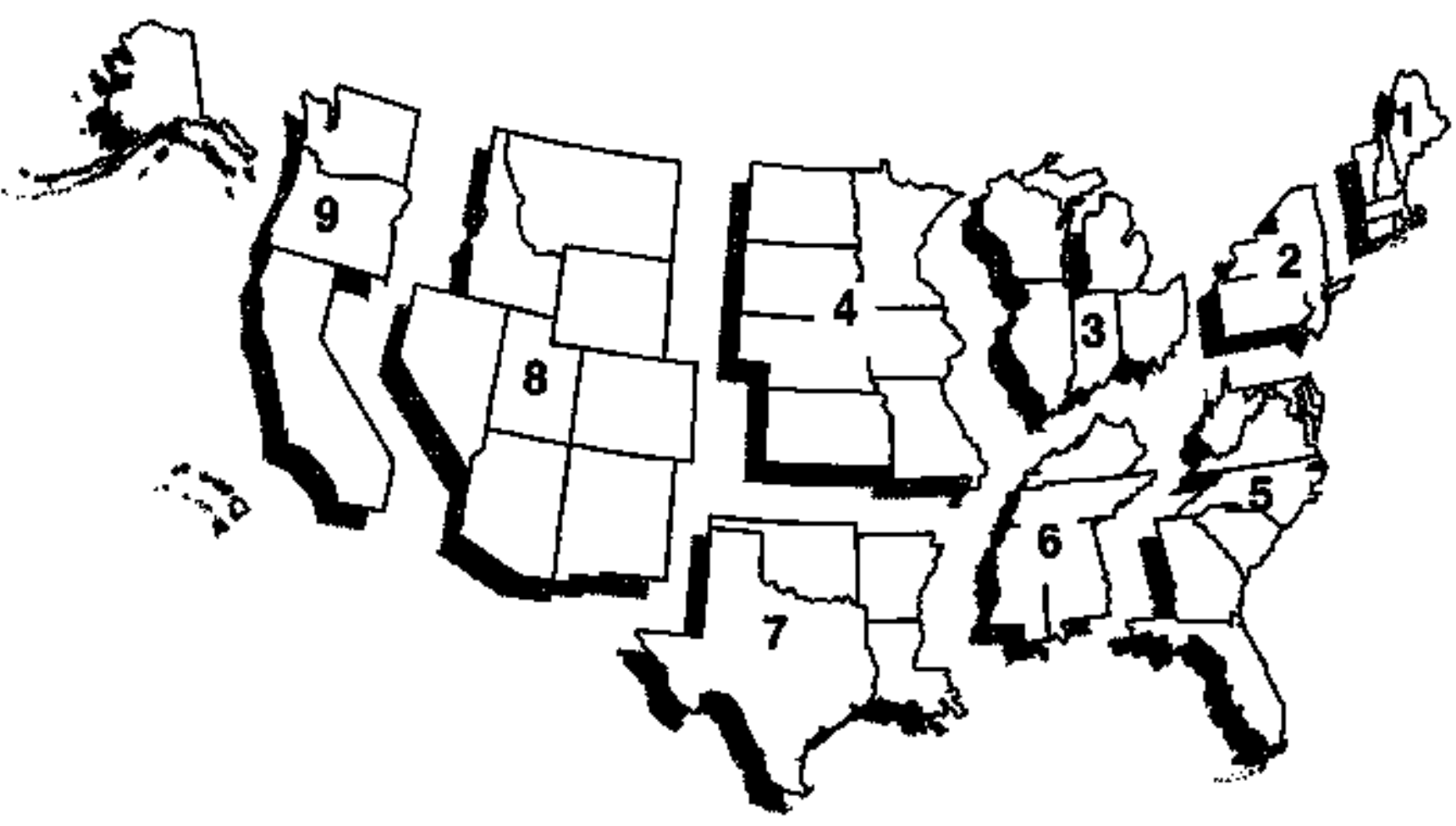

Region 2 Mtdwest

$\begin{array}{ll}\text { Divistion 3 } & \text { Division 4 } \\ \text { (East North Central) } & \text { (West North Central) } \\ \text { ILinois (IL) } & \text { Iowa (IA) } \\ \text { Indians (IN) } & \text { Kansas (KS) } \\ \text { Michigan (MI) } & \text { Minnesota (MN) } \\ \text { Ohio (OH) } & \text { Missouri (MO) } \\ \text { Wisconsin (WT) } & \text { Nebraska (NE) } \\ & \text { North Dakota (ND) } \\ & \text { South Dakota (SD) }\end{array}$

Region 3 South

Bivision 5

(South Atlantic)

Delaw日re (DE)

District of Columbia (DC)

Florida (FL)

Georgia (GA)

Marytand (MD)

North Carolina (NC)

South Carolina (SC)

Virginia (VA)

West Virginis (WV)

\section{Division 6}

(Enst South Central) Alabama (AL)

Kentucky (KY)

Mississippi (MS)

Tennessee (TN)

Divkiton 7

Arkansas (AR)

Louisiana (LA)

Oklahoma (OK)

Texas (IX)
(West South Central)
Region 4 West

Division $8 \quad$ Division 9

(Mountain) (Pacific)

Arizona (AZ) Alaska (AK)

Colorado $(\mathrm{CO}) \quad$ California (CA)

Idaho (ID)

Montana (MT)

Nevada (NV)

Hawaii (HI)

Oregon (OR)

Washington (WA)
New Mexico (NM)

Wyoming (WY) 


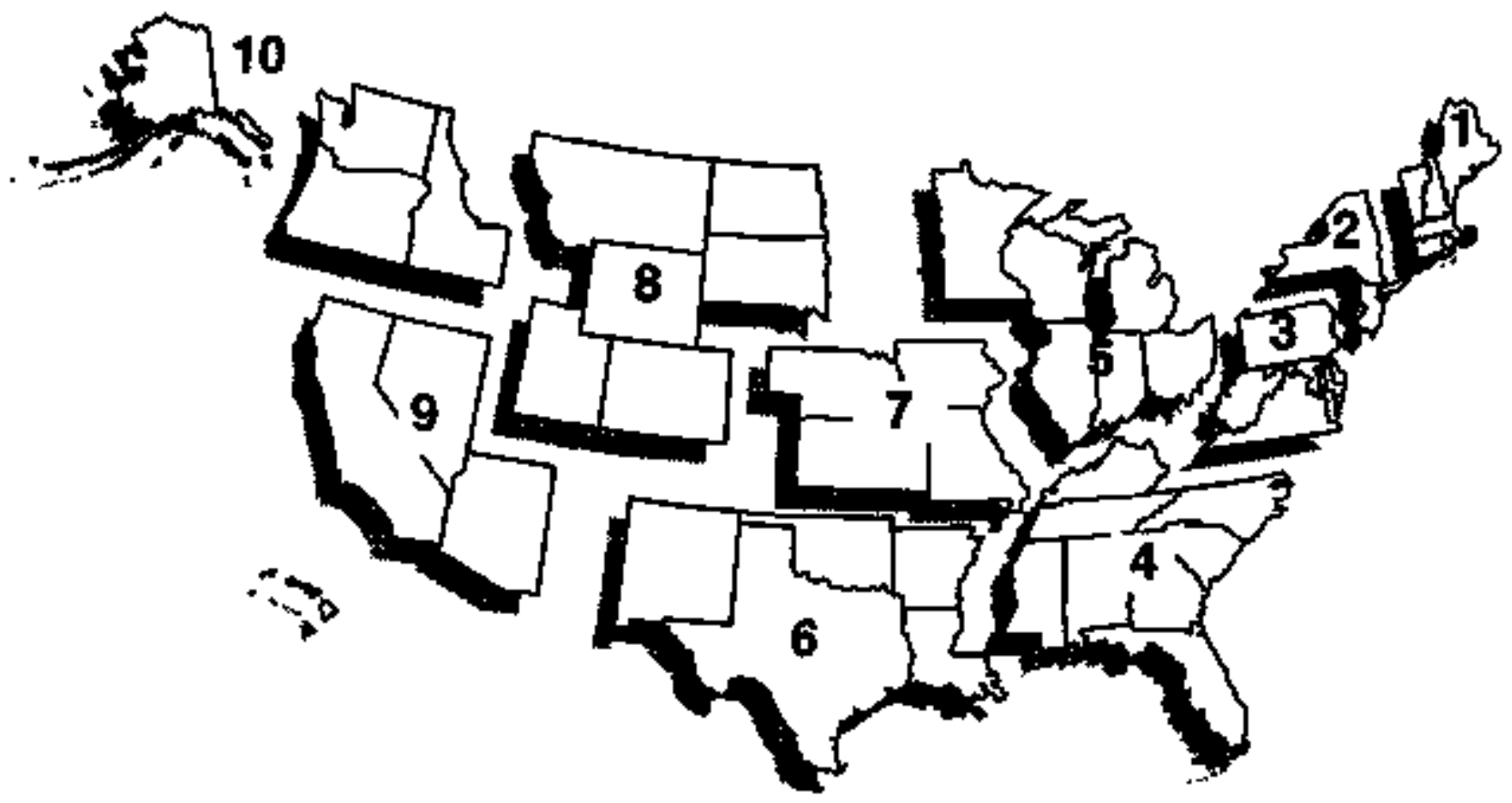

\section{Region 1}

New England

Connecticut (CT)

Maine (ME)

Massachusetts (MA)

New Hampshine (NF)

Rhode Island (RI)

Vermont (VT)

\section{Region 2}

New YorkNew Jersey

New Jersey (NJ)

New York (NY)

\begin{tabular}{|c|c|}
\hline $\begin{array}{l}\text { Region } 3 \\
\text { Midd Adtantic } \\
\text { Delaware (DE) } \\
\text { District of Columbia (DC) } \\
\text { Marylend (MD) } \\
\text { Pennsylvania (PA) } \\
\text { Virginia (VA) } \\
\text { West Virgina (WV) }\end{array}$ & $\begin{array}{l}\text { Rejon 4 } \\
\text { South Atlantlc } \\
\text { Alabsama (AL) } \\
\text { Florida (FL) } \\
\text { Georgia (GA) } \\
\text { Kentucky (KY) } \\
\text { Mississippi (MS) } \\
\text { North Carolina (NC) } \\
\text { South Carolina (SC) } \\
\text { Tenntssex (TN) }\end{array}$ \\
\hline
\end{tabular}

Redion 4

Region 5

Midwest

Illinois (iL)

Indiana (IN)

Michigan (MI)

Minnesota (MN)

Otio (OH)

Wisconsin (WT)

Reglon 6

Sopthwest

Actansas (AR)

Louisiana (LA)

New Mexico (NM)

OtJahoma (OK)

Texas (TX)

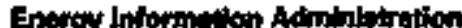
Stew Energy Price end Expinchiture Report 1998

$\begin{array}{ll}\text { Rezion 7 } & \text { Reglon 9 } \\ \text { Central } & \text { West } \\ \text { lows (IA) } & \text { Arizona (AZ) } \\ \text { Kansas (KS) } & \text { Califorria (CA) } \\ \text { Missouri (MO) } & \text { Hawaii (H) } \\ \text { Nebraska (NE) } & \text { Nevada (NV) } \\ \text { Region 8 } & \text { Rejion 10 } \\ \text { North Central } & \text { Northwest } \\ \text { Colorado (CO) } & \text { Alaska (AK) } \\ \text { Mootana (MT) } & \text { Idaho (ID) } \\ \text { North Darota (ND) } & \text { Oregon (OR) } \\ \text { South Dakota (SD) } & \text { Washington (WA) } \\ \text { Uteh (UT) } & \\ \text { Wyoming (WY) } & \end{array}$



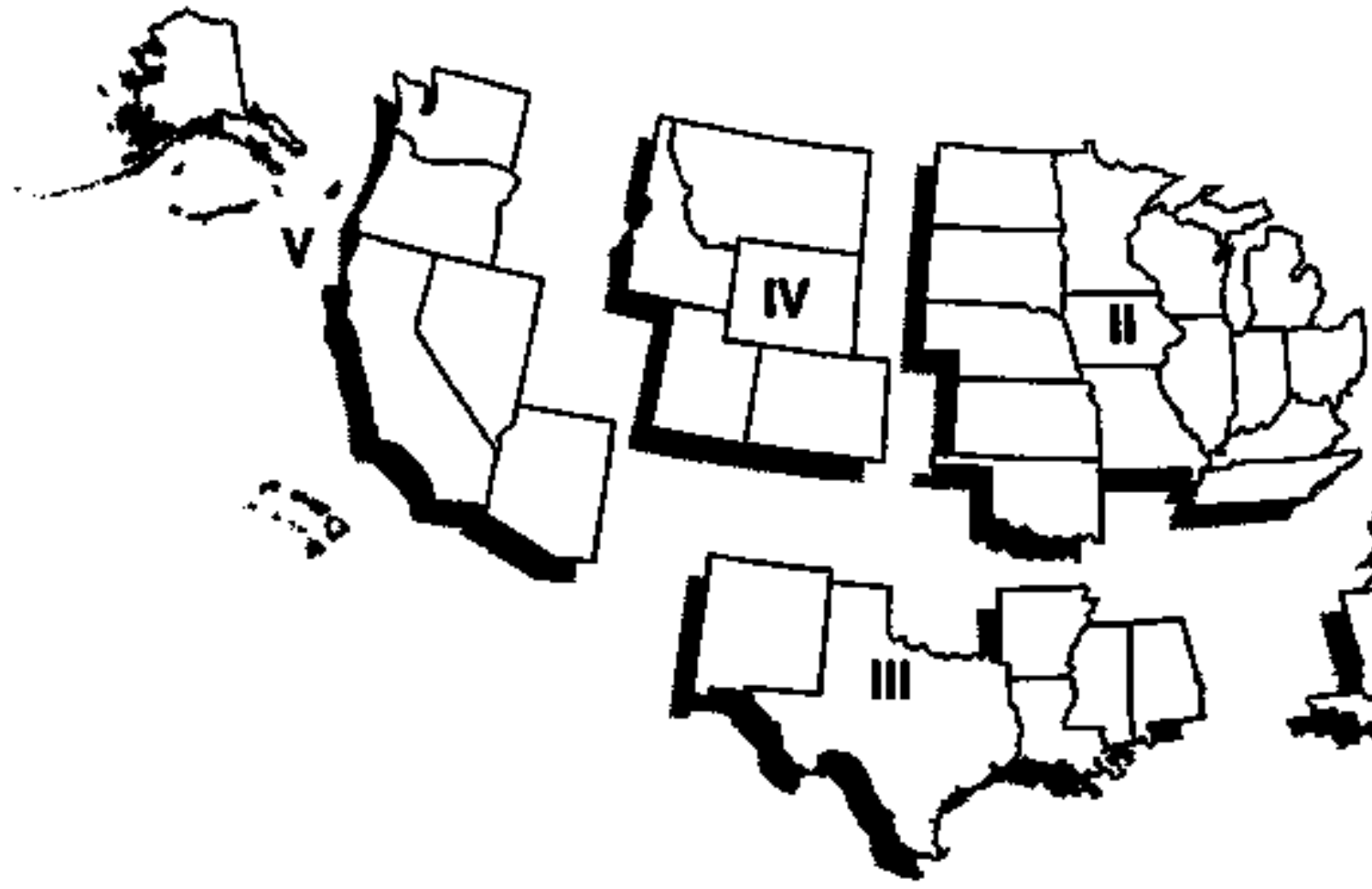

Dhariet II

Illinots (IL)

Indians (IN)

Iowa (IA)

Kanstas (KS)

Kentuxcy (KY)

Michigen (MI)

Minnesota (MN)

Missoari (MO)

Netroskls (NE)

North Dakota (ND)

Ohio (OH)

Olclahoma (OK)

South Dalote (SD)

Tennessee (IN)

Wisconsin (w)

\section{Dlatrict III}

Alcbamina (AL)

Arkapses (AR)

Louisians (LA)

Mississippi (MS)

New Mexico (NM)

Texas (TX)

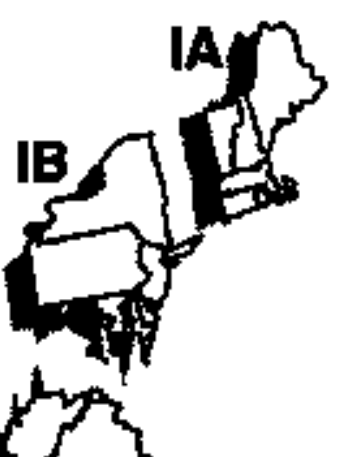

District of Cohmobia (DC)

Marylend (MD)

New lerecy (N)

Peansylvanin (PA)

\section{Diatrict IV}

Colorado (CO)

Idaho (ID)

Montana (MT)

Utah (UT)

Wyoming (WY)

\section{Distariet V}

Alaska (AK)

Arizoas (AZ)

Califonia (CA)

Hawali (Hi)

Nevida (NV)

Oregon (OR)

Washington (WA) 



\section{Section 2. Coal}

Coal prices are developed for the following categories: coking coal; steam coal (all noncoking coal); and coal coke, imports and exports.

Coking coal, used in the industrial sector only, is a high-quality bituminous coal that is used to make coal coke. Steam coal, which may be used by all sectors, includes anthracite, bituminous coal, subbitominous coal, and lignite. In the industrial sector, cosl consurnption is the sum of coking coal and steam coal. The industrial coal price is the quantityweighted average price of these two components.

Imports and exports of coal coke are available only on the national level and are accounted for in the industrial sector. Coal coke imports and exports are reported separately and are not averaged with other coal prices and expenditures.

Coking coal is generally more expensive than steam coal; therefore, it is jdentified separately in the development of the price estimates. Coking coal prices are those paid at coke plants for coal received and probably include taxes, although it is not clear from the source data collection forms. Analysts familiar with the survey believe that respondents would report the total cost of the coal (including cost, insurance, freight, and taxes) because that value is typically carried as the total expense in their bookkeeping systems.

\section{Physled Unit Prices: All Yeara}

Source publicatious contain physical unit prices for States or for groups of States. Individual State prices are used directly for their respective States. Group prices are assigned to each State within the group. Wherever individual State prices or State group prices are unavailable, prices are assigned from adjacent or neatby States or from States with similar coal use patterns as shown to Table AI.

\section{Blu Prices: All Years}

Btu prices for States are calculated from the physical unit prices and the constant conversion factor for coking ool. U.S. Btu prices are calculated as the average of the State Btu prices, weighted by consumption data from the State Energy Data System (SEDS).

\section{Dats Sourees}

\section{Prices}

1981 forward: Energy Information Administration, Quarterly Coul Report, October-December issue, Table A3 (1981-1991) and Table 39 (1992 forward).

1977-1980: Energy Information Administration, Coke and Cool Chemicols, Table 19 (1977), Table 15 (1978), and Table 7 (1979, 1980).

1970-1976: Burealu of Mines. U.S. Department of the Interior, Minerals Yearbook, "Coke and Corl Chemicals" chapter, Table 22. 


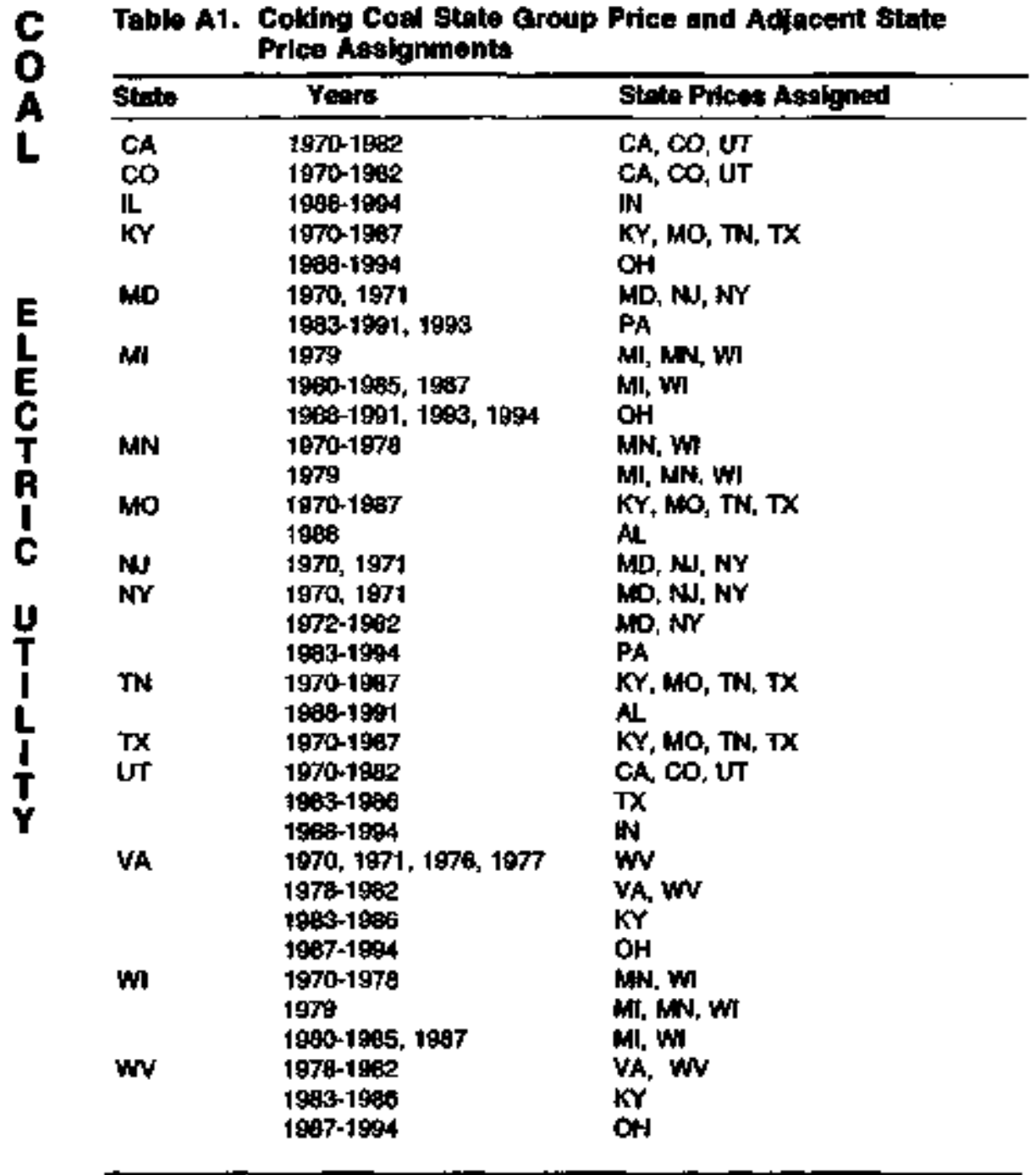

\section{Consumption}

1970 forward; Energy Information Administration, State Energy Date System, coking cosl consumption.

\section{Conversion Factor: Aft Years}

26.80 miltion Bton per short ton.

Steam coal is used in all sectors. Price dith are generally availsble in the electric utility, residential, and industrial sectors. However, no price data are directly arailable in the transportation and conmetcial sectors, and industrial sector stem coal prices are assigned to these two sectors. Data sources and computational procedures for extimating coal prices are discussed by sector. Estilnates of the amount of steam cosl covsomed by sector are taten from SEDS and are arfousted for process fuel consumption in the industrial sector. (See the "Consumption Adjustments for Calculating Expenditures" section on page 413.)

\section{Electric Utiltiy Sector}

\section{Btu Pricos: 1978 Fonwand}

State Btu prices, inchuding all taxes, are taken from Cast and Quality of Fuels for Electric Utility Plants (C\&O) for 1973 forward and are converted from cents to dollars per miltion Bto. Where individual State prices are onavailable, quantity-weighted Census division prices ane assigned as shown in Table A2.

\section{Btu Proes: 1970 Through 1972}

Btu prices for States are taken from the Edison Electric Institute Statistical Yearbook and are converted from cents to dollars. DE, DC, and $M D$ are each assigued the combined ptice for the three States. The steam coal electric utility sector AK price for 1971 is estimated as discossed bekw. 


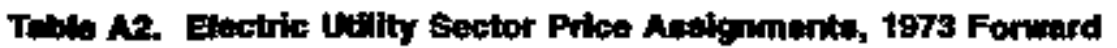

\begin{tabular}{|c|c|c|}
\hline 84t & Yens: & 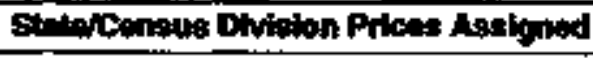 \\
\hline $\begin{array}{l}\text { CT } \\
\text { DC } \\
\text { OK } \\
\text { On } \\
\text { rid } \\
\text { VT }\end{array}$ & $\begin{array}{l}1975-1978 \\
1976 \\
1975,1974 \\
1975 \\
1903,1909 \\
1974 \\
1900,1093-1968\end{array}$ & 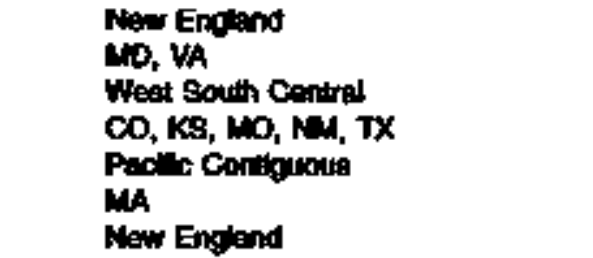 \\
\hline
\end{tabular}

\section{Allostre Prkes: Af Yours}

The AK price for 1994 is estimated from an informal survey of the single coel supplier in the Stite. Prior to that, Btu prices for AKwere taken from the Statititical Yearbonk because the $C \& Q$ does not publish pices for AK. For the years 1970, 1972, 1974, 1976, 1977, and 1979 forward, prices were taken directly from the Shotistical Yearbook. Prices for 1971, 1973. 1975, and 1978 are estimated from the Statistical Yearbook prices for the United States and the average ratio of AK-to-U.S, prices for the years when AK prices are available. The 1971 and 1973 estimated prices are based on the average ratio for 1970 and 1972, the 1975 price is besed on the average ratio for 1974 and 1976 , and the 1978 price is based on the average ratio for 1977 and 1979.

\section{Us. Prieos: All Vowrs}

U.S. Bth prices are calculated as the average of the State Bur prices, weighted by consumption deta from SEDS.

\section{Doite Sources}

\section{Pross}

1973 forward: Energy Information Administration, Cost and Quality of Fuels for Electric Utility Plants, Table 3 (1973-1979), Table 51 (19801982), Table 50 (1983, 1984), Table 40 (1985-1989), Table 7 (1990, 1991), and Table 2 (1992 forward).
1970 forward: Edison Electric Institute, Sratistical Yearbook of the Electric Utility Industry, table titled "Analysis of Fuel for Electric Genefation: Total Electric Utility Industry" (1970-1988), Table 29 (1989 forward).

\section{Consumption}

1970 forward: Encrgy Information Administration, State Energy Data System, electric utility sector coal consumption.

\section{Converston Fuctor: All Voars:}

Bou prices are taken directly from the data sources; no explicit conversion factors are used.

\section{Realdential Sector}

Residential sector steam coal prices are the average delivered prices for coal purchased by residential customers and include taxes.

\section{Prtoes: 1979 formard}

Residential stesm cool Btu prices for 1979 forward are not available. Electric etility coal spot prices from $C \& Q$ for 1979 forward are converted from cents per million $B t w$ to dollars per million $B t u$ and are used in a regression equation to estimate residential steam coal prices for 1979 forward. The residential steam coal prices calculated for 1974 through 1978 from Gas Househeasing Survey (GHS) and the average Btu spot prices from the Cost and Quality of Fuels for Electric Utitity Plants (C\&Q) for 1974 through 1978 are used to develop the regression equation. AK residential coal prices are estimared by using a different methodology, described on page 337.

Some States have GHS residential prices during the 1974 through 1978 period to use in the regression analysis, but are missiag prices in the 1979 forward data used to calculate prices. For these missing data. $C \& Q$ prices are assigned from other States for use in the regression, as show in Table A3. C\&Q prices for ND result in a negative price when 
C used in the regression; therefore MN spox prices are assigned to ND for 0 A

$\mathbf{L}$

Table A3. Residential Sector Cogl Spot Prlce Astignmentu from C\&Q, 1979 Forward

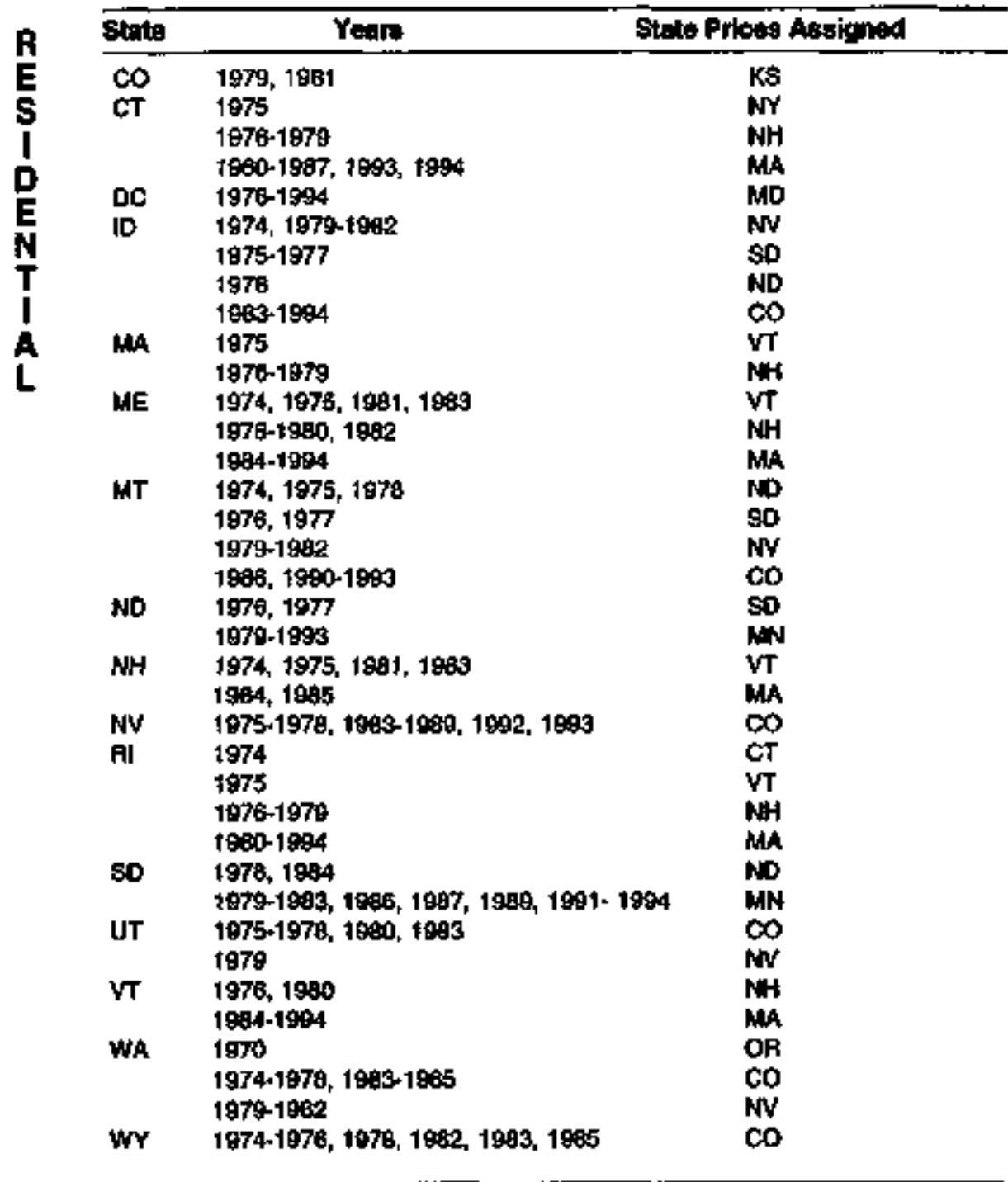

Price estimates for 1974 through 1978 for some States are not available because there was no consumption. To calculate prices for 1979 forward, these States are assigoed the final prices from selected States as shown in Table A4.

In addition, several States are assigned the simple average of the fithal prices of adjacent States as shown in Table A4.

\section{Prloes: 1971 Through 1978}

For 2971 through 1978 . Btu steam coal prices are calculated by using data from $G H S$. The price for a State is equal to the simple average of the city/utility price observations for that State. For 1971 and 1972, GHS reports physical unit prices rather than Btu prices (as published for 1973 through 1978) and, therefore, the State-level conversion factors for this sector from SEDS are used to convert to Btu prices for those years.

Table A4. Realdentiad Sector Spot Cosl Final Price Ausignments, 1979 Forward

\begin{tabular}{|c|c|c|}
\hline str:e & Yoara & 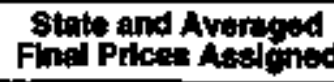 \\
\hline AP & $\begin{array}{l}1980,1982,1984,1985,1987+1994 \\
1981 \\
1983\end{array}$ & $\begin{array}{l}\text { AL } \\
\text { MO, OK, TN, TX } \\
\text { MO, MS, OK, TN }\end{array}$ \\
\hline$A Z$ & $\begin{array}{l}1982,1964,1905 \\
1967,1968,1990-1994\end{array}$ & GA $\mathrm{NH}, \mathrm{NV}, \mathrm{UT}$ \\
\hline CA & $\begin{array}{l}1979-1985 \\
1987+1904\end{array}$ & $\begin{array}{l}\text { NV } \\
\text { WA }\end{array}$ \\
\hline FL & $\begin{array}{l}1980-1994 \\
1960,1982,1904,1986,1988,1995, \\
1993\end{array}$ & $\begin{array}{l}\text { GA } \\
\text { AL }\end{array}$ \\
\hline MS & $\begin{array}{l}1979,1900,1903,1904,1906+14903 \\
1905\end{array}$ & $\begin{array}{l}\text { AL } \\
\text { AL, AR, TN }\end{array}$ \\
\hline ATT & 1966-1994 & WY \\
\hline $\begin{array}{l}\text { NM } \\
\text { OK } \\
\text { OA }\end{array}$ & $\begin{array}{l}1970-1094 \\
1970-1894 \\
1978,1980,1982-1894 \\
1991\end{array}$ & $\begin{array}{l}\text { CO } \\
\text { WO } \\
\text { CA, ID, NV, WA }\end{array}$ \\
\hline ix & 1990-1982, 1985-1994 & $\infty$ \\
\hline
\end{tabular}


AK residential coal prices are estimated by using a different methodology described below.

A simple everage of price observations in CT, MA, ME, NH, RI, and VT is assigned to each of these States. To impute other missing prices in the 1971 through 1978 period, States are assigned simple averages of adjacent State prices or are directly assigned the single price of an adjacent or nearby State as listed in Table AS.

\section{Prices: 1970}

Since State-level coal price data for 1970 are not available from either $G H S$ or $C \& Q$, the 1970 residential sector coal prices are calculated by tssing the 1971 through 1978 data from the Statistical Yearbook for the 39 States, with some reported coal use from 1971 through 1983 and regression analysis.

For estimating the 1970 prices, States missing Statistical Yearbook data are assigned prices as follows: ID/1970 through 1978 from MT; MA/1976 through 1978 from CT; ME/1970 through 1978 from NH; RI/1973. 1975 through 1978 from CT; and WA/1970 through 1972 from OR. DC, DE, and MD are all assigmed the comblned Statistical Yearbook price for those States. Wherever individual State prices are unavailable, prices are assigned from an acjacent or nearby State as follows: CA from NV; NM from CO; OK from CO; OR from WA; and TX from CO. AK residential coal prices are estimated by using a different methodology, described as follows.

\section{Alaskt Prices; All Yoars}

The AK residential coal price for 1994 is estimated from an informal survey of the single coal suppliter in the State.

The AK residential Bu prices for 1978 through 1993 are estimated from the WA State prices during that period. The average ratio of AK-to-WA prices during 1970 through 1977 is applied to the WA price each year that AK has consumption to estimate the AK price for the year.
Table A6. Residential Sector Spot Coal Price Assignments, 1971-1978

\begin{tabular}{|c|c|c|}
\hline State & Years & stlgned or Avaraged Prikes \\
\hline AL & 1971 & TN \\
\hline AP & 1977,1978 & $A \perp$ \\
\hline CA & $1971,1972,1974,1978$ & NV \\
\hline & $1971-1978$ & MD \\
\hline DE & 1971, 1972, 1974, 1976, 1977 & MD \\
\hline \multirow[t]{2}{*}{$\mathrm{GA}$} & 1971 & NC. TN \\
\hline & 1972 & $A L, N C, T N$ \\
\hline ID & 1977 & MT, UT, WY \\
\hline KS & 1971, 1972 & co, no \\
\hline \multirow[t]{2}{*}{ MN } & 1971 & $\mathbb{A}, \mathbf{N D}, \mathbf{W}$ \\
\hline & 1972 & $\left|A_{1}, W\right|$ \\
\hline MS & 1978 & Al. \\
\hline \multirow[t]{2}{*}{ нТ } & 1971 & ID, ND, WY \\
\hline & 1972,1973 & $10, W Y$ \\
\hline \multirow[t]{3}{*}{ ND } & 1972 & A $W$ \\
\hline & 1973 & MN, SD \\
\hline & 1974 & MN, MT, so \\
\hline NE & $\begin{array}{l}1971,1972 \\
1975\end{array}$ & $\begin{array}{l}\text { CO, IA, MO, WY } \\
\text { CO, IA, KS, MO, SD, WY }\end{array}$ \\
\hline NW & $1971,1972,1974,1977,1978$ & DE, NY, PA \\
\hline Aly & 1971 & co \\
\hline \multirow[t]{2}{*}{$\mathbf{N} \mathbf{N}$} & $1971,1972,1975$ & ID, UT \\
\hline & 1979 & ID, OR, UT \\
\hline OK & $1971-1978$ & $\infty$ \\
\hline on & $1 \oplus 71-1979$ & WA \\
\hline sc & 1971.7972 & $\mathrm{NC}$ \\
\hline \multirow[t]{2}{*}{ SD } & 1971 & IA, ND, WY \\
\hline & $1 \oplus 72$ & IA, WY \\
\hline$T X$ & $197:+1974,1977$ & $\infty$ \\
\hline UT & 1974,1978 & $C O, I D, N Y, W V$ \\
\hline \multirow{2}{*}{ WA } & $1971,1972,1974$ & \\
\hline & 1977 & MT, UT, WY \\
\hline WV & 1971, t972 & KY, MD, OH, PA, VA \\
\hline
\end{tabular}

AK physical unit prices for 1970 through 1977 are estimated by using the ratio of AK-to-U.S. electric utility sector prices. 
U.S. Btu prices are calcolated as the average of the State Bto prices, weighted by consumption data from SEDS.

\section{Dats Sounces}

Prices

I974 forward: Energy Information Administrition, Cass and Qwabity of Fuets for Electric Plonts, average spot cosl prices, Table 2 (1974-1979). Table 44 (1980-1982), Table 49 (1983, 1984), Table 39 (1985-1989), Table 8 (1990. 1991), and Table 3 (1992, 1993).

1971-1978: American Gas Association, Gas Househteating Sturey, table tidlod "Competitive Fuel Prices."

1970-1978: Edison Electric Institute, Statistical Yearbook of the Blextric Uhliny Industry, Table 435 .

\section{Consinptian}

1970 forward: Energy Information Administration, Stute Energy Data System, residential sector cosl consumption.

\section{Conversion Factors: 1971,1972}

Energy Information Administration, Stave Enersy Data Reporr 1994, Corsumption Estimates, Table D8.

\section{Commercial Sector}

Commercial sector prices are assigned from indestrial steam coal prices. States without Bh industrial steam coal prices were assignod the prices from adjacent States, as shown in Table A6. The AK price in 1994 is estimated from an informal survey of the single coal supplier in the State. U.S. Bto prices are calculated as the avernge of the State Btu prices, weighted by consumption data from SEDS.

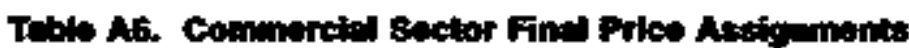

\begin{tabular}{|c|c|c|}
\hline 8tis & Vears & 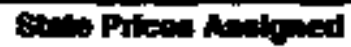 \\
\hline $\begin{array}{l}\text { Cr } \\
\text { DC } \\
\text { Mr } \\
\text { Ox } \\
\text { rit } \\
\text { vT }\end{array}$ & $\begin{array}{l}1990 \\
1800-1964 \\
1894 \\
1970 \\
1902,1904,1891-1994 \\
1990 \\
1994\end{array}$ & $\begin{array}{l}M r \\
M P \\
M \\
M H \\
M H\end{array}$ \\
\hline
\end{tabular}

\section{Indusetirial Sector}

For 1980 forwad, prices are basted on the average price of coul recejpts at insmufacturizg plentx. For 1970 through 1979, prices are based on the avefage const of coal solid to mannfacturing firms.

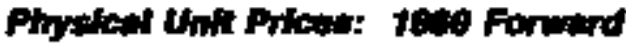

For 1984 forward, State prices ane tsken from the Qunterly Coal Report $(Q C K)$. The annual price is estitoated as the arerage of the quarterly prices weighted by the quarterly receipts. For 1980 through 1983 , pricts are taken difrectly from Form ELA-3 data. Publisted pricts in ayerage cost per ton are used directly when availnble, while missing prices are estimared by using simple averages or price assiznments from adjacent States. Although it is not clear from the datu collection forms, it is assomed that the prices include taxes. Analysts familitar with the survey believe thrt rexponden's would repont the weil cost of the conl (inchading cost, ingurance, freight, and taxes) because that value is typically carried os the total expense in their hooltreeping systems.

Miscing State datn are extimated by using simple avernges of the published data for adjacent States. In a few cases, only a single adjecent State or Centus division price is available for the estimation. The adjacent State and Census division price assignments used for estimations are shown in Thble A7. 


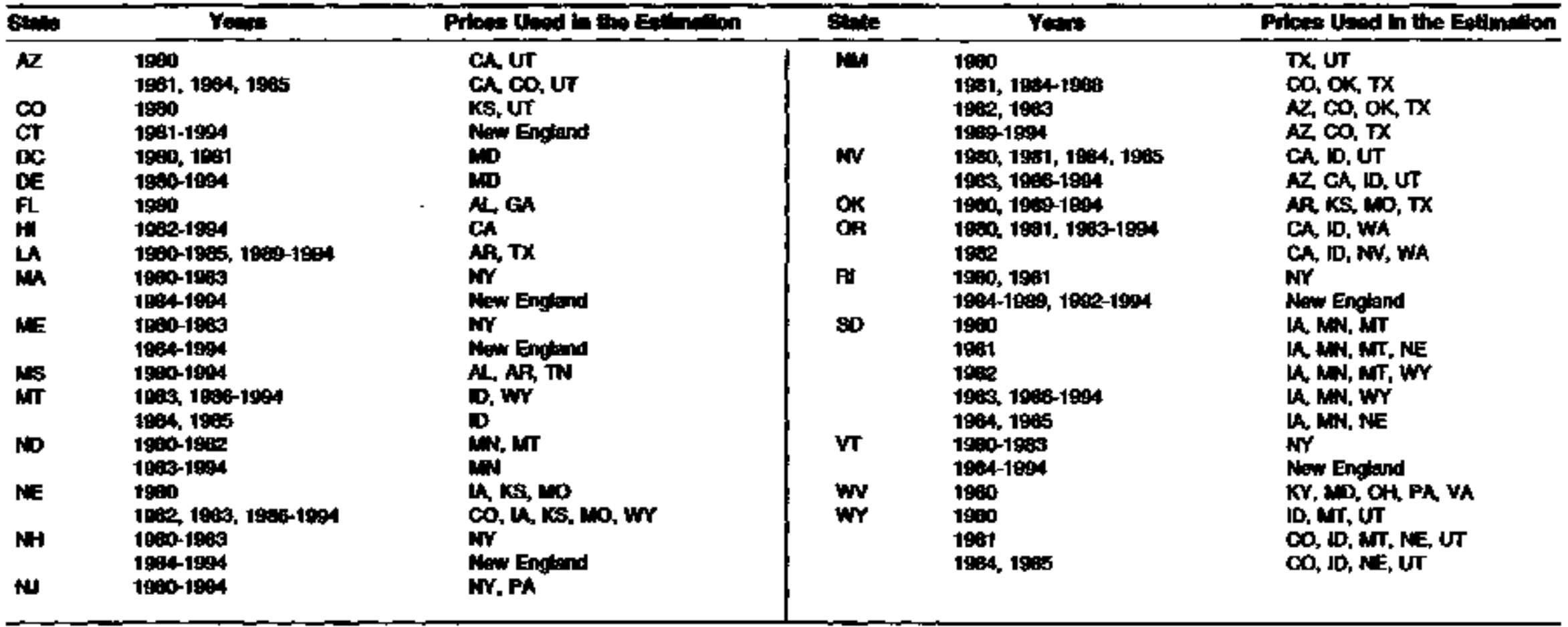

\section{Phyted unil Prices: 197, 1974 Throwgh 1979}

For 1971 and 1974 through 1979, avaibahle cost and quantity of biruninous coal, ligoite, and anthracite from the Animal Swrvey of Manesfactures (ASM) or Census of Mantigactures (CM) ant used to calculate prices as average cost per unit of sales for covertod States. (States with undisclosed data afe not considened covered.) Allangh it is not clear from the data sonrces, the prices probebly include taxes as explained for prices in the 1980 forward period.

For States with industrial steam codal use and for which ASU or CM data are not available in 1971 and 1974 throngh 1979, adjacent State simple averages of available ASMUCW dakn ane used to impute prices. The assigeed prices from adjacent Stutes are shown in Table A8.
Phyoked Unit Prlew: 1970, 1972, 1973

Ste-m cond industrial sector prices for 1970, 1972, and 1973 (years for which no ASW/CH prices are availgble) are estimated by using negresgion techniques. Values for the independent variable ane steam coal electric utility sector physical unit prices, and values for the dependent variable are the steam coal indostrial physical onit prices (from $A S W$ or estimated as described abowe) for 1971, and 1974 through 1977 . A few States are assigned electric etility prices tor the dependent variable in the regression, as shown in Table A9.

Wherever intividual State prices remath onvailable after the estimation by nsing tho above regression techniques, prices are assigned from auljacent or wearby States, as shown in Table Alo. 


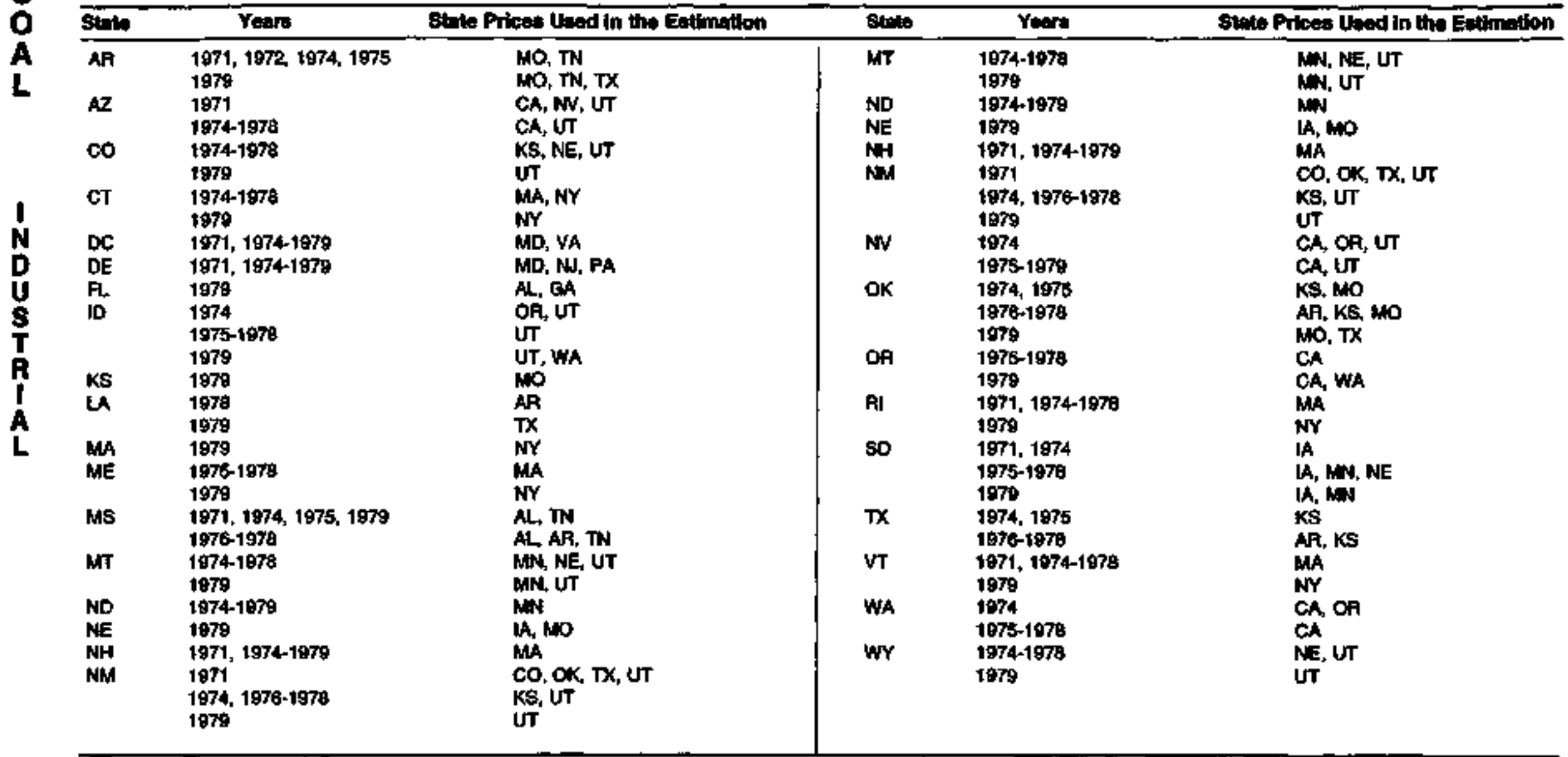

\section{Phyelow Unit Pricus: Alacks, All Yoars}

The AK steam coal industrial sector price for 1994 is estimated from an informal survey of the single coai supplier in the State. The 1993 price for Alaska is estimated by assuming that the ratio of the Alasks price to the WA price in the industrial sector is the same as the ratio of the AK and WA prices in the electric utility sector for 1993. For 1970 through 1992, the tatio of the AK price to the U.S. price in the electric tutility sector is used for the estimation.

\section{Bfu Prices: Afl Yoare}

Btu prices for States are calculated from the physical unit prices and the conversion factors, which vary by State and by year. U.S. Btu prices are calculated as the average of the State Btu prices, weighted by consomption data from SEDS, adjusted for process fuel and coking coal consumption. 
Table A9. industrial sector Price Asedinments Usod in the Regrepplon Equition for 1971, and 1974-1978

\begin{tabular}{|c|c|c|}
\hline Staila & Yoars & Stite Prione Actigred \\
\hline $\begin{array}{l}\text { AR } \\
\text { CA } \\
\text { CT } \\
\text { DC } \\
\text { ID } \\
M A \\
M E \\
\text { OK } \\
\text { OR } \\
\text { TX } \\
\text { WA }\end{array}$ & $\begin{array}{l}1973-1977 \\
1970-1977 \\
1975-1977 \\
1976,1977 \\
1970-1977 \\
1976,1977 \\
1970-1977 \\
1973-1975 \\
1973-1977 \\
1970 \\
1970.1972\end{array}$ & $\begin{array}{l}\text { Mo } \\
\text { NV } \\
\text { MY } \\
\text { MD } \\
\text { MTt } \\
\text { NH } \\
\text { KS } \\
\text { WA } \\
\text { NW } \\
\text { OA }\end{array}$ \\
\hline
\end{tabular}

\section{Data Sources}

\section{Prices}

1984 forward: Energy Information Adninistration, Qwarterly Cod Report. Tables 16 and 17 (1984 1st quarter through 1985 20d quarter and 1986, 1987, and 1988 1st quarters): Tables 18 and 19 (1985 3rd and 4th quarters, 1986, 1987, and 1988 2nd through 4th quarters, 1989-1991 lst through 4th quarters); and Tables 40 and 41 (1992 forward, 1st through 4th quarters).

Table A10. Industrial Sector Final Price As;ignmento for 1970, 1972, and 1973

\begin{tabular}{lll}
\hline Stat & \multicolumn{1}{c}{ Yoars } & Btike Prices Acilgned \\
\hline AR & 1672 & MO, TN \\
NH & $1970,1972,1979$ & MA \\
RI & $1970,1972,1973$ & MA \\
SD & $1970,1972,1973$ & IA \\
VT & $1970,1972,1979$ & MA \\
\hline
\end{tabular}

1980-1983: Energy Information Administration, "Quarterly Coal Con" sumption Report: Manufacturing Plants" (Form EIA-3). Only published data are used from Table 25 (1980). Table 11 (1981, 1982), and Table 2 (1983).

1971, 1974-1979: Bureau of the Census, U.S. Department of Cornmerce, Anntual Survey of Manufactures and Census of Manufactures. Table 4 (1971) and Table 3 (1974-1979).

1970, 1972, 1973: Steam coal electric utility physical unit prices.

\section{Consumption}

1970 forward: Energy Information Administration, State Energy Dats System, industrital sector coal consumption.

\section{Comveralon Factors: All Years}

Energy lnformation Administration, State Energy Data Report 1994. Consumption Estintates, Tables D10 and DII.

The conversion factor for CT/1990 is assigned from CT/1989; the NH/1976 conversion factor is assigned from NH/1975; the RU/1977 conversion factor is assigned from RV/976; and the VT/1986 conversion factor is assigned from VT/1985.

\section{Transportatlon Sector}

Transportation sector steam coal prices are assigned from industrial sector steam coal prices. U.S. Bti prices are calculated as the average of the State Btu prices, weighted by SEDS consumption data. 
Imports and exports of coal coke are components of total U.S. energy consumption and ane accourted for in the infustrial sector. Prices and

C values of imports and exports are developed only for the United States;

O no attempt is made to estimate State-level prices or expenditures. Prices

K are f.a.s. (free alongside ship) values and do not include taxes. The

E quantities of U.S. coal coke imports and exports are taken from SEDS.

\section{Physical Unk Pripes: AH rears}

For 1980 forward, the Coke Plant Report, the Quanterly Coat Report. and Bureau of the Census computer tapes provide physical unit coal coke import and export prices in dollars per short ton. For 1970 through 1979. Coke and Caat Chemicals, Intemational Coal, and the Winerals Yearbook provide coal coke impont and export physical unit quantities and values in short tons and dollars, respectively. Vahes are equivalent to expenditures.

\section{Btu Prices: All Yoars}

For 1980 forward, Btu prices are competed by dividing the physical onit prices by the conversion factor. For 1970 through 1979 , physical nnit prices are computed by dividing the import and export values by their respective quantities, and Bal prices are computed by dividing the physical onit prices by the conversion factor.

\section{Dola sownos:}

Prises

1989 forcyard: Burean of the Censos, U.S. Department of Commerce, electronic data from "Monthly Report DM 145" and "Monthly Report EiM 545."

1981-1988: Energy Information Administralion, Quarierly Coal Report, October-December issues, Tables A11 and A13 (1981-1985) and Tables A10 and Al2 (1986-1988).

1980: Energy Information Administration, Coke Plant Report, Tables 7 and 8.

1978-1979: Euergy Information Administration, Cote and Coal Chemicult 1979. Tables 5 and 6.

1977: National Coal Association, International Cod 1960, tables titled "U.S. Imports of Solid Fuels and Chestoms Value" and "U.S. Exports of Cake and Vhue."

1976: Energy Information Administration, Coke and Caal Chemicals, Tables 19 and 20.

1970-1975: Buread of Mines, U.S. Department of the Interior. Minerals Yearbook, "Coke and Coal Cbemicals" chapter, Tables 19 and 20.

\section{Cowsunpotion}

1970 forward: Enengy Information Administration, State Energy Data System, U.S. inports and exports of coal cole.

\section{Conversilon fuctors Alt Vors}

24.8 milliod Btu per short kon. 


\section{Section 3. Natural Gas}

Natural gas prices are developed for the residential, commectiol, industrial, trantportation, and electric utility seckors. Reported nataral ges prices are netail prices for sales of natural gas to ultimate users.

In general, taxes ane included in the prices. However, taxes collected by a utility from an end aser and tomed over to a Government antbority ficquently are not included it the revennes reported in the sonree documents and, therefore, are not included in the prices. Taxes paid by the nility (rather than the end nser) are considered operring costs and ane passed on to the end user as part of the rate. Therefore. Federal, State, business, and property taxes are typically inchaded in the prices, while sales and cther point-of-purctuse taxes typically are uot.

Estimates of the amount of natural gas consumed by the residential, commercial, industrial, and electric utility sectors are tilken from the State Energy Dan System (SFDS). Estimnstes for the indistrial sector are adjusted to remove estimated refinery consumpticn and lease and plast use of natural gas and estimattes of transportation sector use are adjusted to remove pipeline foel in each State. (See the discussion in Section 7, "Consamprion Adjustments for Calcularing Expendinges," on page 413.)

\section{Realdential, Commerclal, and Industribl Soctora}

\section{Prowas tos7 Fonwerd}

All mutural gas physical mit prices by State for the residential, commercial, and industrial sectors are taken from the Energy Information Administration (FIA) Noteral Gas Aruwal (NGA).

\section{Prices: 1970 Through toes}

All natural gas physical anit prices for the residential, commercial, and industrial sectors are calculated from value and muantity of sales data from the NGA or its predecessor report, Natural Gas Production and Consuniption. State prices are calculated directly from the data sources as average revenoe per unit of sales by natural gas utilities. Prices for each of the three sectors are calculated by dividing the value of natural ges, reported in thousunds of dollars, by the quantiry of nalmral gas sold, as reported in million cubic foet.

For 1970 through 1979 , woth the value and quantity of sales daca from the MGA are reported as composites for MD and DC and for ME. NH. and VT. In exch case, the corbbined prices ane assigned to each of the Strtes in the conposite.

\section{Btr Pilees: Af Vears}

Stote Btu prices for all years ane calculnted by using the physical unit price series and the State-level non-electric utility conversion factors. U.S. Btu prices are calculated as the average of the State Btu prices, weighted by consomption data from SEDS, adjosted for process fuel consumption in the industrial and transportation sectors.

\section{Deth souton}

\section{Frices}

1987 forwad: Enesgy Iuformstion Administration, Historical Natural Gas Anntal, 1930 Through 1995, Table 23 (residential); Table 24 
N (commercial 1987-1992) and Table 25 (commercial 1993 and 1994); and A Table 27 (industrial 1987-1992) and Table 28 (industrial 1993 and T 1994).

[980-1986: Energy Information Administration, Notural Gas Annual, Ylume 1. Table 11 (1980), Table 14 (1981-1985), and Table 15 (1986).

1970-1979: Bureau of Mines. U.S. Department of the Interior, Natural Ges Production and Consunption. Table 6 (1970 and 1979) and Table 7 (1971-1978).

\section{Consumption}

1970 forward: Energy Infomation Administration, State Energy Data System, residential, commercial, and industrial natural gas constumption.

\section{Conversion Factors: All Years}

Energy Information Administration, factors published rounded in State Energy Data Report 1994, Consumption Estimates, Tabkes D4 and DS.

\section{Transportation Sector}

Most of the natural gas used for transportation is consumet in pipeline operations and is discussed in Section 7. "Consumption Adjustments for Calculating Expendjtures," on page 413. A stuall but increasing portion is consumed by natural gas vehicles. Prices for natural gas consumed by vehicles are teported in the EIA Natural Gas Annual beginging in 1990. Much of the natural gas delivered for vehicle fuel represents deliveries to fueling stations that are used primarily by fleet vehicles.

In the first years of the vebicle fuel price series, the coverage of the reporting universe is not complete. Prices are assigned, when missing, from an adjacent State with the highest percentage of reported data. Natural gas vehicle fuel consumption in NE is assigned the SD price in 1992 and 1993, in NV is assigned the AZ price in 1992 and 1993, and in $\mathrm{DE}$ is assigned the MD price in 1994.

\section{Dafa Sources}

Prices

1990 forward: Energy lnformation Administration, Histarical Natural Gas Annual, 1930 Through 1995, Table 30.

\section{Consumption}

1990 forward: Energy Information Administration, State Energy Data System, natural gas vehicle cousumption.

\section{Conversion Factors: AN Years}

Energy Information Administration, unrounded conversion factors as pubtished rounded in the State Energy Data Report 1994, Consumption Estimates. Tables D4 and DS.

\section{Electric Utillty Sector}

\section{Prices: 1973, 1974, 1993 forward}

Prices for States are reported by Cost and Quality of Fuels for Electric Vtility Plants (C\&Q) for gas consumed at steam-electric plants only. Btu prices are taken frou $C \& Q$, converted from cents to dollars per million $\mathbf{B}$ t.

Where individual State prices are unavaitable from $C \& Q$, they are developed from the NGA. Physical unit prices prior to 1987 are calculated by dividing the value of natural gas, reported in thousands of dollars, by the quantity of natural gas sold, reported in million cubic feet. For 1987 forward, physical unit prices are taken directly from the NGA. Table All lists the States and years for which NGA data ate used. Btu prices are calculated from the physical unit prices by using State-level electric utility conversion factors.

Prices are not available from eithet $C \& Q$ or $N G A$ for CT/1973; ID/1974, 1987; NH/1983; and OR/1983, 1984, 1986, 1989, 1990. In these castes, 
Tablo A11. Naturel Gas Eloctric Utillty Sector Prices from NGA, 1973 Forward

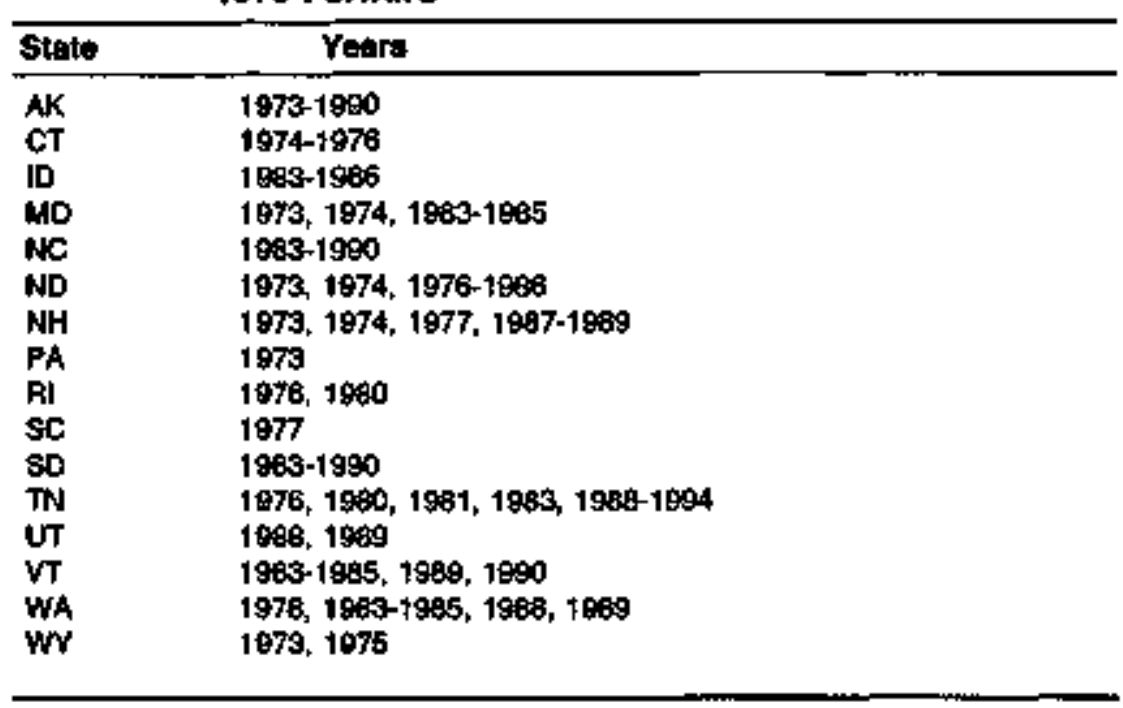

Nole: NGA holudie predecessor pubilealtars.

quantity-weighted Census division prices from $C \& Q$ are assigned. In addition, prices for VT/1986 and WAI986, 1987, 1990 use quantityweighted Census division prices from $C A Q$ for more consistent prices than those available from the NGA.

\section{Prices: 1980 Through 1982}

State Bw and physical unit prices for 1980 through 1982 are taken from $C \& Q$ for all reporting plants. Physical unit prices are taken directly from the data source, while Btu prices are converted from cents to dollars per million Btu.

\section{Prices: 1976 Through 1979}

State ptices are reported separately by $C \& Q$ for gas consumed at steamelectric plants and gas consumed at combustion turbine and intemal combustion units. Weighted-average Btu prices are calculated by using the two $C \& Q$ prices and the respective gas deliveries for steam-electric and corbustion use. The NH/1977 price is not available from $C \& Q . \mathrm{A}$ combised price is computed from value and quantity of sales data from the NGA for ME, NH, and VT and assigned to NH/1977.

\section{Prices: 1970 Through 1972}

State prices for 1970 through 1972 are taken from Natural Gas Production and Consumption and are calculated simjlarly to the way prices for the residential, commercial, and industrial sectors are calculated. Prices, as average revenue per unit of sales, are computed from value and quantity of sales data from the source reports. A combined price is reported for NH and VT for 197I and 1972, and each of these States is assigned the combined price. State Btu prices are calculated from the physical unit prices by using the State-level electric utility conversion factors.

\section{U.S. Pricus: All Years}

U.S. Btu prices are colculated as the average of the State Btu prices, weighted by consumption data from SEDS.

\section{Date Sources}

\section{Prices}

1973 forward: Energy Information Administration, Cost and Quality of Fuels for Electric Utility Plants, tables numbered as follows:

\begin{tabular}{|c|c|c|}
\hline Yoans & Price Data & Wohme Dita \\
\hline $\begin{array}{l}1973,+974 \\
1975-1979\end{array}$ & $\begin{array}{l}\text { Table } 10 \\
\text { Table 10, } 18\end{array}$ & $\begin{array}{l}\text { Table } 9 \\
\text { Tabble Q, t5 }\end{array}$ \\
\hline $1980-1982$ & Table 40 & - \\
\hline 1983, r9g4 & Table 53 & + \\
\hline 1965-1987 & Tath 43 & - \\
\hline 1998, 1999 & Tablo 44 & - \\
\hline 1990,1991 & Table 19 & - \\
\hline $1992-1994$ & Table 12 & . \\
\hline
\end{tabular}


1994: Energy Information Administration, Historical Natural Gas A Annual 1930 Through 1995. Table 31.

A 1980-1992: Energy Information Administration, Nafural Gas Annual L 1992, Volume 2, Table 23.

C 1976-1979: Energy Information Administration, Energy Data Reports, A Natural Gas Production and Consumption, Table 7 (1976-1978) and TaA ble 6 (1979).

1970-1975: Bureau of Mines, U.S. Department of the Interior, Natural Gas Production and Consumption, Table 6 (1970) and Table 7 (1971. 1975).

\section{Consunption}

1970 forward: Energy Information Administration, State Euergy Data System, electric utility natural gas consumption.

\section{Conversion Factors}

Btw prices that are calculated dinectly from Cost and Quality of Fuels for Electric Utility Plants (C\&Q) require no conversion factors. When Natural Gas Annual (NGA) data are used to develop prices that tre mitissing from $C \& Q$, conversion factors are used from the following source:

1970 forward: Energy Information Administration, State Energy Data Report 1994, Consumption Estimates, Tables D2 and D3. 


\section{Section 4. Petroleum}

averages of the two city prices are used. No States have prices from more than two cities. States with no prices are assigned a Census division simple average price. State average asphalt prices are calculated as the quantity-weighted average prices of the three products for each State. If there is no Census division price, the simple average of the prices for the other Census divisions within that Census region is used. Quantity data for 1970 through 1980 are taken from the Bureau of Mines and ELA reports on sales of asphalt. Quantity data for 1981 forward are taken from the Report on Sales of Asphalt in the U.S. published by the Asphalt Instinute. Non-paving asphalts are assumed to have the prices of paving asphalt cement.

\section{Physical Unit Pricos: Aff Years}

Asphalt prices in physical units are developed from monthly reports in the Engikeering News-Record, a comstruction industry weekly magazine published by MeGraw-Hil, lac. The source data consist of monthly reports from correspondents in 20 U.S. cities with price quotes for tank cars, dnums, or both, for the the major types of asphalt products: asphalt cement (AC-20), asphalt emulsion (rapid set and slow set), and asphalt cueback.

For 1986 forward, the tank car price is used. However, for 1986 and 1987. the drum price is used if a tank car price is not avajlable. For 1970 through 1985, when both tant car and dmm prices are available, a simple average of the two prices is osed. When only one price is available, that price is nsed.

Asphalt prices are developed by calculating a simple average annual price from the monthly prices for each city for the three prodocts. City prices are assigned to Stakes. CA, OH (1970 through 1983, 1992 forwad), and PA have prices from two cities; in these cases, simple

For 1970 through 1982, asphalt and road oil are estimated as separate data series. Asphalt prices are estimated as discussed above. Road olt prices are assumed to equal asphalt eutulsion prices because specific prices are not available from any source.

\section{Btu Prices: All Yoars}

Asphalt prices in dollars per ton are converted to dollars per gallon by dividing by 235 gallons pes ton for asphalt cement, 241 gallons per ton for emulsion, or 248.6 gallons per ton for cutback. These prices are then multiplied by 42 gallons per batrel and divided by 6.636 million Btu per barrel to get dotlars per million Bru. Road oil unit prices of dollars per ton are converted to dollars per million Btu by using the constant conversion factors of 5.5 barrels per ton and 6.636 million Btu per barrel. The average price of all asphalt and road oit is the consumption-weighted average of the individual product prices. 
A U.S. Btu prices are calcutated as the average of the State Btu prices,

\section{Data Sources}

Prices

1970 forward: McGraw-Hill, Inc, Engineering News-Record.

\section{Quantities for Calculating Weighted Average Prices}

1981 forward: Asphalt Institute, Asphatt Usage, United States and Canada.

1978-1980: Energy Information Administration, Energy Data Reports, Sales of Asphatt, Table 2.

1977: Energy Information Administration, Energy Data Reports, Asphalt Sales, Annual, Table 2.

1971-1976: Bureau of Mines, U.S. Department of the Interior, Mineral Industry Survey, Asphalt Sales, Annual, Table 2.

1970: Burcau of Mines, U.S. Department of the Interior, Mineral Industry Survey, Asphalt Shipments, Annual, Table 2.

\section{Consumption}

1970 forward: Energy Information Administration, State Energy Data System, industrial sector, asphalt and road oil consumption.

\section{Converojon Factors: All Yoars}

Conversion factors used are: $\mathbf{2 3 5}$ gallons per ton of asphalt cement; $\mathbf{2 4 1}$ gatlons per ton of emulsion; 248.6 gallons per ton of cutback; 42 gallons per barrel; 5.5 barrels per ton of rosd oil; 6.636 million Btu per barrel.
Aviation gasoline prices are developed for the transportation sector. Estimates of the amount of aviation gasoline conoumed by the transportation sector are taken from SEDS. Aviation gasoline prices are national averages, excluding taxes, developed from several sources, depending on the yeats. In all cases, physical unit prices are developed and then converted to But prices. Fecieral and State excise taxes, as well as State and local sales taxes, are not included.

\section{Phyolcal Unit Prlces: 1976 Forward}

Aviation gasoline prices for 1978 forward are assumed to be the national average refiners sales prices to end users published in the ELA's Annual Energy Review. The 1976 and 1977 prices are assumed to be the national average retaj] prices published in the ElA's Monthly Energy Review.

\section{Physical Unit Prices: 1970 Through 1975}

For 1970 through 1975, aviation gasoline prices are not available. Prices are derived by dividing the national motor gasoline prices for those years by the 1976 rational motor gasoline price and applying those percent changes to the 1976 national aviation gasoline price.

\section{Btu Prices: Afl Yoara}

Aviation gasoline Btu prices are calculated by converting the physical unit prices from cents per gallon to dollars per barrel ( 42 gallons per barrel) and then to dollars per million Bat (5.048 million Btu per barrel). 
Prices

1979 forward: Energy Information Administration, Annwal Energy Review 1995, Table 5.20, row titled "Sales Prices to End Users: Aviation Gasoline."

1978: Energy Information Administration, Annual Energy Review 1993. Table 5.2I, tow titled "Sales Prices to End Users: Avtation Gasoline."

1976, 1977: Energy Information Administuation, Monthly Energy Review. April 1984, page 106, column titled "Aviation Gasoline, Retail."

1970-1975: Energy Information Administration, Annual Energy Review 1989, Table 70, column titled "Motor Gasoline, Leaded Regular, Nominal."

\section{Consumptlon}

1970 forward: Energy Information Administration, State Energy Data System, transportation sector, aviation gasoline consumption.

\section{converston Factar: Afl Vourt}

5.048 million Btu per bajtel.

Distillate fuel prices are developed for all sectors. Distillate fuel in the transportation sector is assumed to be diesel fuel. Estimates of the amount of distillate fuel consimed in each sector are taken from the State Energy Data System (SEDS). Estimated consumption for the industrial sector is adjusted to remove the estimated refinery consumption of distillate fuel in each State. (See the discussion in Section 7, "Consumption Adjustments for Calculating Expenditures," on page 413.)
Residential distillate prices are developed by using a variety of data sources and several estimation methods, depending on the years involved. In all cases, physical unit prices for States are developed first, then Btu prices are calculated by osing the physical unit prices and the conversion factor. The prices contained in this series are the retail prices paid by consumers for residential heating oil, including taxes.

\section{Physloal Unit Pricas: 1983 Through 1990, 1992 forward}

For 1983 through 1990 and 1992 forward, physical unit distillate prices in cents per gallon (excluding taxes) are generally avallable for 24 States from the Petroleum Marketing Annual (PMA). For 1989 through 1993, prices represent No. 2 fuel oil, only. For 1994, prices inchude other No. 2 distillates. State-level prices for the States without PMA prices are estimated by using price data from the American Gas Association (ACA), SEDS consumption data, and PMA Petroleum Administration for Defense (PAD) district prices. The estimation procedures are described below and include the addition of State general sales taxes.

1. State prices in cents per gallon are generally available from the PMA for the following 24 States: AK, CT, DC, DE, ID, IL, IN, MA, MD, ME, MI, MN, NH, NJ, NY, OH, OR, PA, RI, VA, VT, WA. WI, and WV. Prices for these States are converted from cents to dollars per gallon, and State general sales taxes from the Bureau of the Census and successor sources are adderd.

2. For the States that do not have prices in the PMA, prices are estimated by using AGA fuel oil prices, SEDS consumption data, and PMA PAD district prices for Districts II, III, IV, and V and Subdistrict IC (all the States in PAD Subdistricts IA and IB have published prices). The following steps are used to estimate the prices:

a. Distiltate prices from the PMA for PAD Districts II, III, IV, and $\mathrm{V}$ and Subdistrict IC are converted from cents per gatlon to do]lars per gallon. 
b. The AGA lists fuel oil prices by company for the principal city served in dollars per million Bu including State sales taxes. A simple average of the city-level prices is used to derive a Starelevel price for each of the States without PMA prices. These AGA State averages are converted from dollars per million Btu to dollars per gallon by using the AOA convergion factor of 7.194 gallons per million Bu. State general sales taxes are subtracted to give State averages comparable to the PMA prices.

c. The AGA State prices derived in step $2 b$. are combined into PAD district averages by using SEDS consumption to weight each State's values. This procedure gives AOA consumplionweighted average prices for PAD Districts II, III. IV, and V and Subdistrict IC that are comparable to the volume-weighted PAD district prices published in the PMA. The AGA PAD district arerages are calculated by using only the availabte States; if a State does not appear in the survey, it is not included in the PAD cakculation.

d. Adjustment factors, ratios of the PMA PAD district price divided by the AGA derived PAD district price, are calculated for PAD Districts II, III, IV, and V, and Subdistrict IC.

c. Prices for the States not published in the PMA are calculated by multiplyting the AGA State prices derived in step $2 b$ by the appropriate PAD district adjustment factor from step $2 d$ and then adding State general sales taxes.

f. States that do not have prices in either the PMA of the AGA are assigned a PMA PAD district price, and State general sales taxes are added. The States with assigned PAD ptices are as skown in Table A12.

\section{Phyolcal Unit Pricos: 1991}

Physical unit distillate prices in cents per gallon (excluding taxes) are available for 24 States from the PMA. Because prices are not available from AGA for 1991, State-level prices for the remaining 27 States are estimated by using physical unit prices derived for 1990 in SEPEDS (de-

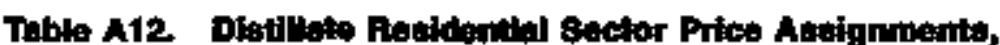
1903-1980, 19:2 Forwind

\begin{tabular}{|c|c|c|}
\hline 8tiplo & Yean & 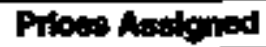 \\
\hline 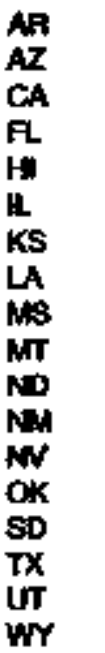 & 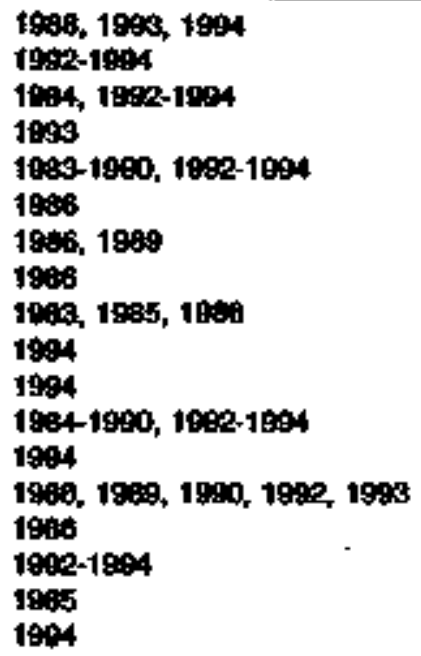 & 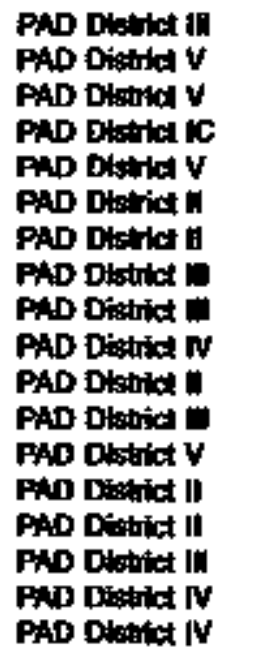 \\
\hline
\end{tabular}

scribed below) and the 1991 PMA Petroleum Administration for Defense (PAD) diatrict prices. The estimation procedures are described below and inclnde the addition of State general sales taxes.

1. Stabe prices in cents per gallon ane available from the PMA for the following 24 States: AK, CT, DC, DE, ID, IL, DN, MA, MD, ME, MI, MN, NH, NJ, NY, OH, OR, PA, RI, VA, VT, WA, W1, and WV. Prices for these States are converted from cents in dollars per gallon, and State general sales taxes from the Bureav of the Census' State Government Tax Collections (SCTC) are added.

2. For the remaining 27 States that do not have prices in the $P M A$, prices are estimated by asing the 1990 SEPEDS physical unit prices and PMA PAD district prices for 1990 and 1991. The following steps ane used to estimate the prices: 
a. For 1990, the Subdistrict IC price is withbeld in the PMA and the average of the VA and WV prices is used as the Subdistrict IC price.

b. The 1990 State prices derived from AGA and PMA, as described below, are adjusted by the percentage change in the 1990 and 1991 prices for each State's PHA PAD distriat or subdistrict.

c. The State generol sales taxes from SGTC are added.

\section{Phyolcal Unit Prloe: 1978 Through 1992}

Procedures for the 1978 through 1982 period are similar to those for 1983 forward except for changes in data sources. Annual physical unit prices are taken etther directly from the Monthly Energy Review (MER) or are calculated from monthly regional price dath, also from the MER. These data were collected on Form EIA-9A (formerly EIA Form 9 and FEA Form P112-M-1) and include raxes. Price dala from Platt's OII Price Hondbook and Oilnanac (PLort's) and SEDS consumption data for 1978 through 1982 are nsed to compute State prices when only regional deta are available. These calculations are described step-by-step below.

1. Annual State physical anit prices are generally available from the MER for the same 24 States covered by the PMA in 1983 and forward. These 24 States compose all of Federal Regions 1, 2, 3, 5, and 10 (Bee Figure A2 on page 330). Prices for these States exclude taxes and are converted to dollars per galloo.

2. Of the States without MER prices, the 22 in Federal Regions 4, 7 . 8 , and 9 have annali prices estimated from the monthly Federal regional prices published in the $M$ ER. No regional prices are available for Federal Region 6 for the 1978 through 1982 period, and some monthly prices are missing in regions 7,8 , and 9 in 1980, 1981 , and 1982 .

B. Missing monthly prices for Federal regions are estimated with assigned prices as follows: the Region 9 November 1980 price is exsigned to Decenber 1980; an average of the Region $7 \mathrm{July}$ and October 1982 prices is assigned to August and September 1982; an average of Region 8 June and September 1982 prices is assigned to July and August 1982; and an average of Region 3 Augnsl and October 1982 prices is assigned to September 1982. Imputation of missing Region 6 prices for 1978 through 1982 and missing Region 9 prices for 1981 and 1982 is discussed later.

b. The simple average of monthly State-level normal heating degree-day data is averaged for all the States within each of the 10 Federal regions and is used to estimate average Federal region heating degnee-days. AK, DC, and HI are assigned the monthly heating degree-days from MN, MD, and FL, respectively.

c. Weighted average annual physical unit distillate prices for the residential sector atre calculated for Federal Regions 4, 7, 8, and 9 (except for Region 9 in 1981 and 1982) by using the regional normal heating degrice-days and the monthly regional prices from the $M E R$.

d. In 1981, only March and May prices are available for Fedetal Region 9. To estimate the average annual price for this region, the relationshjp between the U.S. annual heating oil price (from the MER) and the U.S. March and May prices is expressed as a ratio and is used with the Region 9 March and May prices to estimate the 1981 annual Region 9 price.

e. City-level prices from Platr's are assigned to States as shown in Table A13. The assigned State-level Ptatt's prices for States are consumption-weighted into Federal regions by using tesidential sector consumption data from SEDS.

f. Adjustment factors, ratios of the regional $M E R$ distillate prices to the rogional Platt's-based distillate prices, are calculated for Federal Regions 4, 7, 8, and 9 (except for 1982).

g. Since there are no monthly regional distillate prices from the $M E R$ for Federal Region 6 for 1978 through 1982 and Federal Region 9 for 1982, the adjustment factors for these regions are based on the adjustment factors for previous time periods. The Region 6 adjustment factor for each of the years in the 1978 


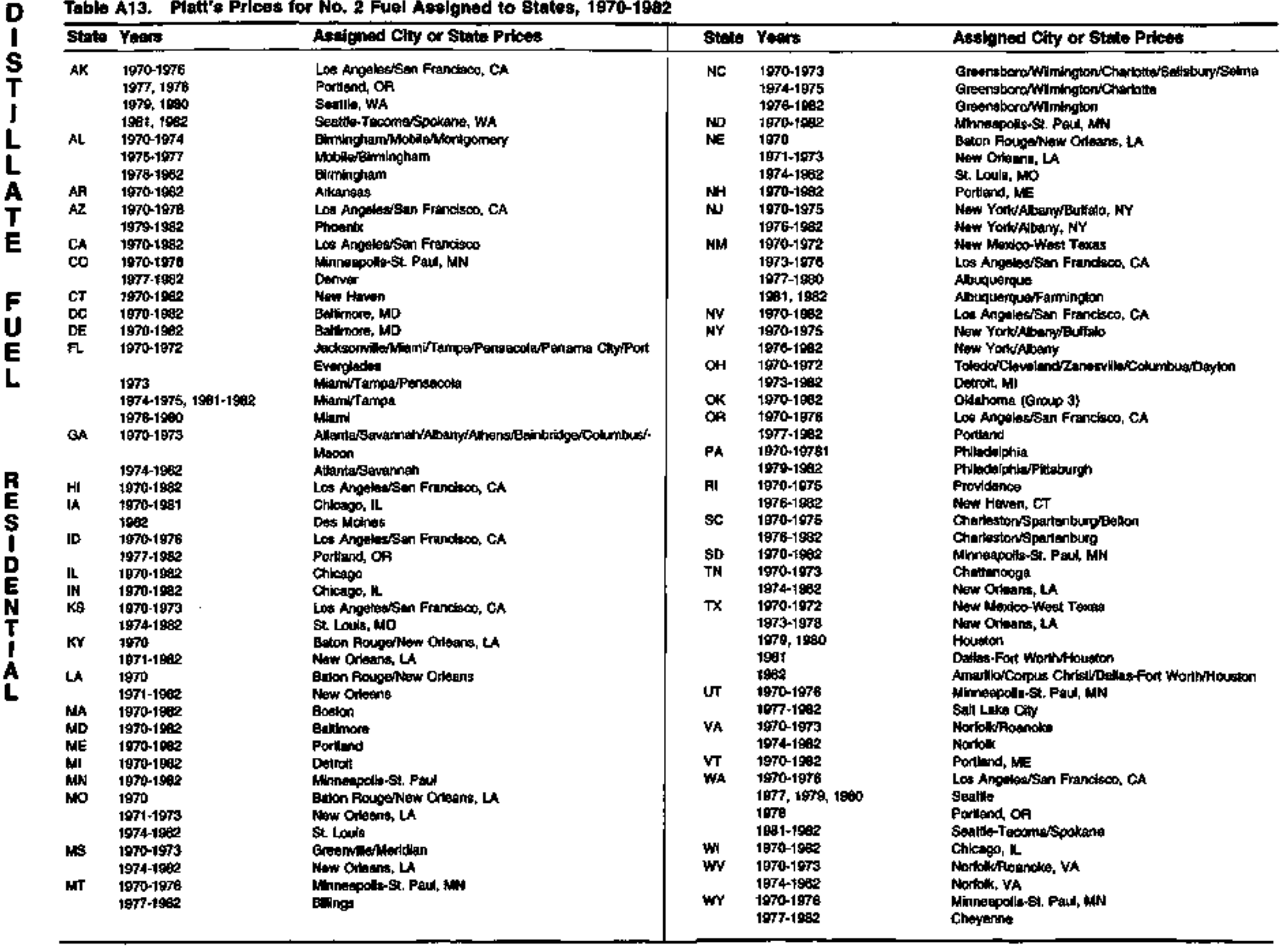


through 1982 period is equal to 1.1313 , which is the average of the adjustment factor for the West South Central Census Division for 1976 and 1977. The Region 9 adjustment factor for 1982 is equal to 1.1995, which is the average adjustment factor for Region 9 from 1978 through 1981 .

h. The residential sector distillate State prices for the 27 States in Federal Regions $4,6,7,8$ and 9 are calculated by multiplying the regional adjustment factors for each year and the State-level assigned Plart's prices.

\section{Physicel Unt Prices: 1976 Through 1977}

For the years 1975 through 1977, no State-level data are avaslable, and regional data from Forn EIA-9A are available only at the Census division kevel, except for Federal region prices for November and December of 1977. Using a methodology similar to that described above for the allocation of regional data to States, adjustment factors are calculated at the regional level add applied to Platt's price data assigned to States. The resulting prices implicitly include average regional taxes but do not reflect individual $\$$ tate differences.

1. Monthly regional price data for 1975 and 1976 are reported in the $M E R$ only for Ceasus divisions. In 1977 , however, monthly price data atc reported for Census divisions for January through October and for Federal regions for November and Decenber. The Federal region prices for November and December are assigned to their respective States and reaggregated into Census divisions in order to creata a consistent set of monthly Census division prices for 1977. Annoal residential sector distillate consumption data from SEDS are used to do the reaggregation.

2. The Census division monthly price data from the MER for 1975 , 1976, and the first 10 months of 1977 are used with the estimated Census division price data for November and Decerober 1977 to estimate State-level prices.

a. Missing monthly prices in the East South Central Divișion for June and November 1975 and the Mountain Division for March and July $197 \$$ are estigated by using an average of the prices for the month preceding and the month following the missing nonth. Missing November and Deceunber West South Central Division prices in 1977 are estimated with the assignment of the October price to both months. No monthly price data are available for the West South Central Division in 1975; step 2f. below dis* cusses how the calculations are handled for this division.

b. The monthly State-level notmal heating degree-day data are averaged for the States within each Census division to estimate regional monthly heating degree-days. AK, DC, and HI are assigned the monthly heating degree-days from $\mathrm{MN}, \mathrm{MD}$, and $\mathrm{FL}$, tespectively.

c. Weighted average annual distillate prices for Census divisions are calculated by using the monthly Census division price data from the MER and the normal beating degree-doys estimated for Centius divisions.

d. City-level No. 2 fuel oil refinery and terminal prices from Platt's for 1975 through 1977 are assigned to States as shown in Table A13. The assigned Platt's prices for States are consumptionweighted into Census divisions by using residential sector consumptiou data from SEDS.

e. Adjustment factors are calculared as the ratios of the MER distillate Census division ptices to the Plart's distillate Census division prices.

f. Since there are no 1975 MER price daca for the West South Central Division from which to calculate an adjustment factor, the 1975 adjustment factor for this region is assumed to be equal to the simple average of the West South Central adjustrment factors for 1976 and 1977 (le., I.1313).

g. The residential sector distillate State prices for all States are caiculated by nultiplying the regional adjustrient factors for each year by the State-level assigned Platt's prices. 
5. The residential sector distillate prices for all Stotes are calculated by multiplying the regionel adjustment factors for each year by the Stato-kevel assigned Platt's prices.

There are no regional or State-level distillate price data directly available for the 1970 through 1974 period. To estimate State prices, regional average prices are fjrst derived from the relationship between U.S. prices and Federal region prices for 1975 through 1980 . State prices are then estimated from the regional prices by using a methodology similar to that described for 1978 through 1982 . The resulting prices implicitly include average regional taxes bot do not reflect individual State differences.

1. The first step in the estimation of residential distillate prices for the 1970 through 1974 time period is to develop an equation that uses U.S. prices to estimate prices for Federal regions. Regression tecbniques are used for this purpose. U.S. prices for 1975 through 1980 from the Anuual Energy Review (AER) are used as the independent variable for developing the equation; annual Federal region prices we used as the dependent vasiable. Federal region prices for 1978 through 1980 are calculated above, but MER prices for 1975 through 1977 are for Census divisions. To convert these annual Census division prices into Federal region prices, the estimated State prices for 1975 through 1977 are aggregated into Federal regions by uaing SEDS consumption data.

2. Regression techniques are applied to the pooled Federal region price data (dependent variable) and the U.S. prices from the AER (independent variable) for 1975 through 1980. U.S. prices for 1970 through 1974 are input to estimate anmual Federal region prices for 1970 through 1974.

3. City-level prices from Platt's for 1970 through 1974 are assigned to States as shown in Table A13. The assigned State-level Platt's prices are consumption-weighted into Federal regions by using residential sector distillate consumption data from SEDS.

4. Adjustment fuctors, which are ratios of the regional MER distillate Federal region prices to the Platt's-based distillate Federal region prices, are calculated.

\section{Bat Pricoes: Afl Yeano}

Btu prices for States are calculated by converting the physical unit prices from dollars per gallon to dollars per barrel (42 gallons per barrel) and then to dollars per miltion Btor (5.825 million Btw per barret). U.S. Btu prices ane calculated as the everage of the State Btu prices, weighted by consumption data from SEDS.

\section{Ont source:}

Prices

1983 forward: Energy Information Administration (EIA), Petrolewn Marketing Annual 1985. Wolume 1. Table 25 (1983-1985) and annual issnes of the Pernotew Marketing Arnual, Table 36 (1986-1988), Table 38 (1989-1993), and Table 39 (1994). column titled "To Residential Consumers." Also available on the EIA Emergy InfoDisc, Volume I, No. 4, November 1996. See box on page 403 for instructions for locatfng the data series on the InfoDisc.

1983-1990, 1992 forward: American Gas Association, Residential Natural Gas Market Survey (1989, 1990, 1992 forward), and Gas Househeating Swrey (1983-1988), Appendix 2, "Competitive Frel Prices," cohumn tilled "Fuel Oit."

1970-1982: McGraw-ffil, Inc., Platt's Oil Price Handbook and Oitmanac, refinery and terminal prices for No. 2 foel oil, average of highs and lows.

1975-1982: National Ocesnic and Atmospheric Administration, U.S. Seasonal Heating Degree-Days Weighted by Population (1980 Cenfus). Historical Climatology Series 5-1, table titled "1951-80 State Pop. Wgt'd Heating Degree-Days." Department of Commerce. State, Regional, and National Bonthly and 
1975-1982: Energy Information Administration, Monthly Erergy Review, thble titied "Residential Heating Oil Prices by Rejion," February 1978, page 67 (1975, 1976); April 1980, page 83 (1977, 1978); July 1982. page 87 (1979-1982).

1970-1982: Enengy Information Administration, Annual Energy Review 1988, Table 67, "Motor Gasoline and Residential Heating Oil Prices, 1949-1988."

\section{Tuxes}

1994: U.S. Advisory Committee on Intergovemmental Relations, Sigmificart Features of Fiscal Federation, Tables 14 and 26.

1993: Bureau of the Census, U.S. Department of Commerce, Slate Tax Review, Volume 54, No. 31, map titled "State Gasoline, Sales and Cigarefte Tax Rates as of Joby 1, 1993"”

[983-1992: Burtau of the Census, U.S. Department of Commerce, State Government Tax Collections, table titled "Stale Governument Excises on General Sales, Motor Fuel, and Cigareftes, Begioning and End of Fiscal Year."

\section{Consenption}

1970 forward: Energy Information Administration, State Energy Data System, residential sector distrillate consumption.

\section{Corworston Factor: Aff yours}

5.825 million Bur per barrel

\section{Commercial Sector}

Commercivl sector distillate prices are estimated by using several differont data sources and estimation methodologies, depending on the years involved. For 1983 forward, retail prices paid by commercial/institutional establishments (exclnding taxes) for No. 2 distillate fuel are taken from the Energy Information Administration's Petrolewn Morketing An- nual (PMA). State general sales taxes from the Burean of the Census and successor sources are added. For 1970 through 1982, coinmercial distillate prices are based on refinery and terminal (wholesale) prices from Platt's and markups from Energy Prices: 1960-73 that include taxes. For both time periods, physical unit prices are catculated from the data sources. and Btu prices are computed by using the physical unit prices and the conversion factor.

\section{Phyaical Unt Prices: tows forward}

Physical unit No. 2 distillate prices in cents per gallon (excluding taxes) ane generally available for 24 States from the PMA. State-level prices for the remaining 27 States are estimated by using the PMA Petroleum Administration for Defense (PAD) district prices as shown in Table A14. State general sales taxes are then added.

\section{Phyalcal Unit Prlane: 1970 Throutgh 1902}

Commercial sector distillate physical unit prices for 1970 through 1982 are calculated by using Platt's prices assigned to States and commercial sector markips estimated from Energy Prices: 1960-73. The resulting estimates implicitly include State-specific taxes.

1. The first step is to compute the markups. Energy Prices contains single price estimates for small commercial users and two price es1tmates for large commercial users for 10 cities: Boston, MA; Albany, NY; New York, NY; Charlotte, NC; Washington, DC; Chicago, IL; Detroit MI; Minneapolis/St. Paul, MN; St. Lovis, MO; and Seattle, WA. First, a simple average of the two large commercial prices is calculated for each city except for Albany and New York. In this case, all four large commercial prices are areraged together, since cities are assigned to their respective States.

2. For the nine States covered by the Energy Prices data (noted in step 1), the inarkup of the reported prices from Energy Prices over the assigned Platt's prices (Table A13 on page 352) and the markup of the residential prices cakculated above for 1970 through 1972 over the Platt's prices is calculated. 
D Table A14, Dktillate Commercial Gector Prite Asalgnments, 1993 Forward

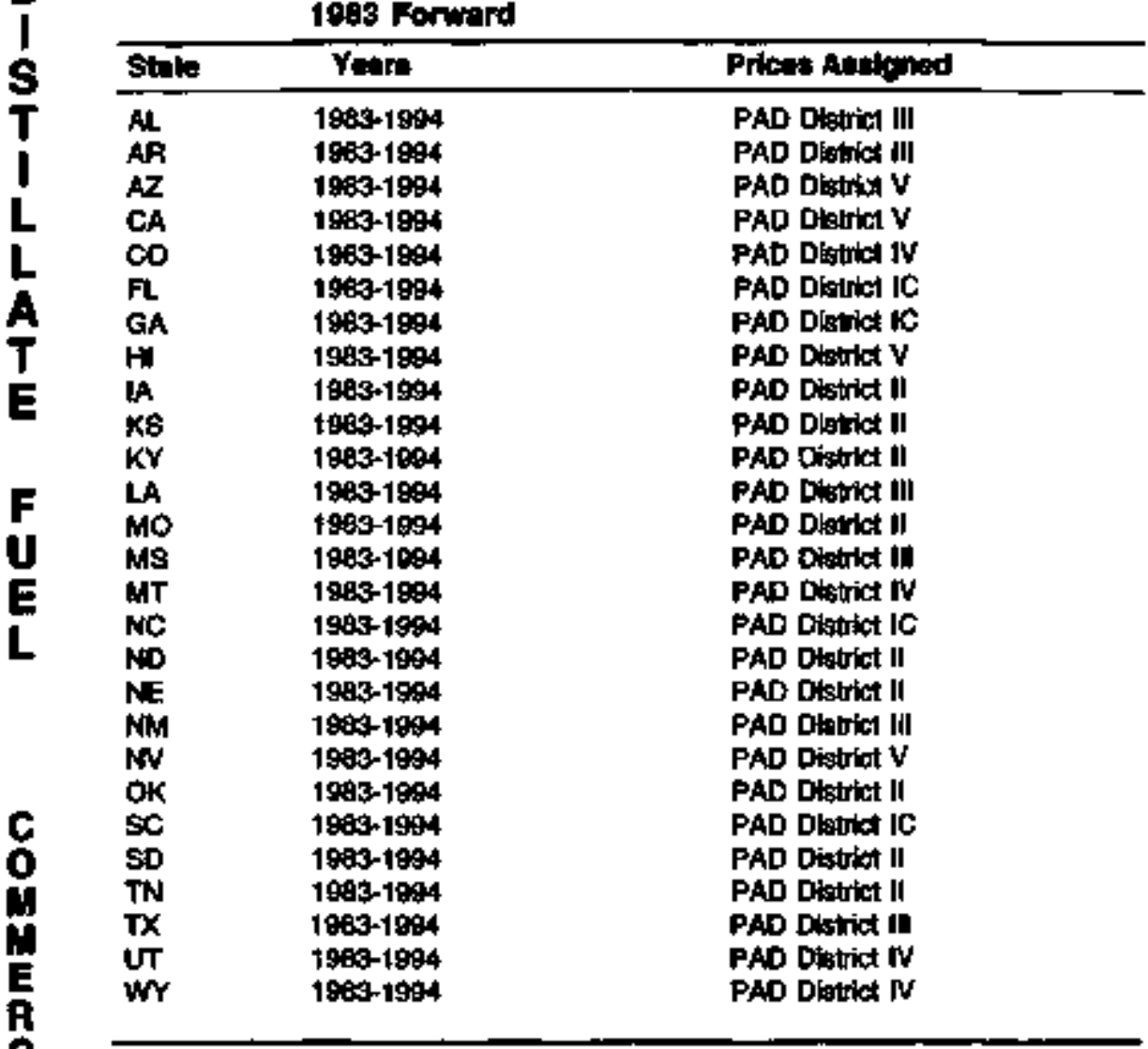

3. At this point, residential and commercial sector retail markups have been computed for nine States for each of the years 1970 through 1972. The next step is to calculate the average retail markup for the 3-year period for each sector. A simple average of the markup ratios is cakculated.

4. The average commercial and residential sector retail markups for the bine available States are assigned as shown in Table A15.

5. To translate the average commercial and residential markups for 1970 through 1972 into the estimated commercial sector retail
Tabie A15. Distillate Fusl Commurclal Sector Average Retail Markup Price Aasbgnmento, 1970-1972

\begin{tabular}{|c|c|}
\hline State & Cly Prlce Aoulpiminte \\
\hline 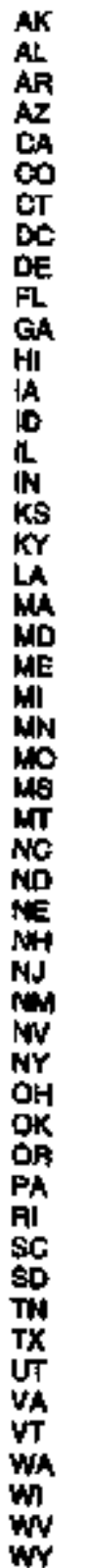 & 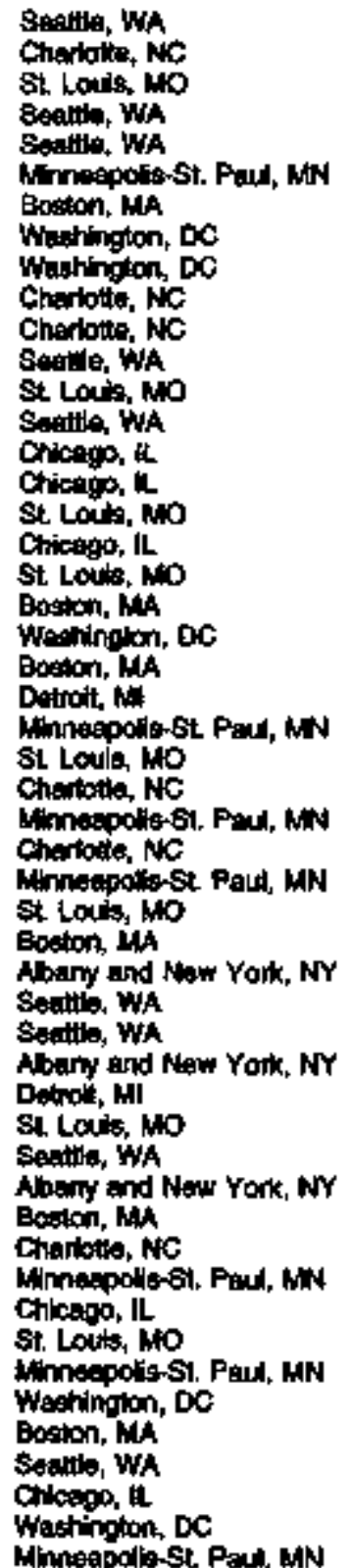 \\
\hline
\end{tabular}


markups to be used for 1970 through 1982, the relationship between these two markups is used, with the residential markups calculated for all States for each year. The calculation of the residential markups follows the same procedure used in step 2 . above.

6. The commercial sector adjustment factors for each State for each of the years 1970 through 1982 are multiplied by the corresponding Plott's prices for 1970 through 1982 to calculate the final commercial sector physical unit prices.

\section{Btw Prices: All Years}

Btu prices for States are calculated by converting the physical unit prices from cents to dollars per gallon, then to dollars per barrel (42 galJons per barrel) and, finally. to dollars per million Btu (5.825 million Bu per barrel). U.S. prices are calculated as the average of the State Btu prices, weighted by consumption data from SEDS.

\section{Dahu Sourcess}

\section{Prices}

1983 forward: Energy Information Administration (ElA), Energy InfoDisc, Volume 1, No. 4, November 1996 . See box an page 403 for instructions for locating the data series on the InfoDisc. Data are also published in the EIA Petroleum Marketing Annual 1985, Volume I, Table 25 (1983-1985) and annual issues of the Petroleum Marketing Anneal, Tabie 36 (1986-1988), Table 38 (1989-1993), and Table 39 (1994), column titled "To Cornmercial/Institutional Consumers."

1970-1982: McGraw-Hill, Inc., Plart's Oil Price Handbook and Oilmanoc, refinery and terminsl prices for No. 2 fuel oil, average of highs and lows.

1970-1982: Foster Associates, Inc, 1974, Energy Prices 1960-73, Tables 4-c and 5-b.

\section{Taxes}

1994: U.S. Advisory Committee on Intergovernmental Relations, Significant Features of Fiscal Federalism, Tables 14 and 26.

1993: Burear of the Census, U.S. Department of Commerce, State Tax Revlew, Volume 54. No. 31. tmap tited "State Gasoline, Sales and Cigarette Tax Rates as of Juty $1,1993 .^{\text {n }}$

1983-1992: Bureau of the Census, U.S. Department of Commerce. State Government Tax Collections, table titled "State Government Excises on General Sales. Motor Fuel, and Cigarettes, Beginning and End of Fiscal Year."

\section{Consumption}

1970 forward: Energy Information Adruinistration, State Energy Data System, commercial sector distillate consumption.

\section{Conventon Factor: All Years}

5.825 million Btu per barrel

\section{Eiectric Utillty Sector}

The electric utility price for distillate fuel is the average delivered cost of No. 2 fuel oil receipts at electric ntitities. (See Light Oil (Electric Utilities) on page 369.) For 1973 forward, these prices are taken from Cost and Quality of Fuels for Electric Utility Plants; for 1970 through 1972, prices from the Statistical Yearbook of the Electric Unility Industry are used with regression analysis. Btu prices are developed directly from the data sources and include all applicable taxes.

\section{Prices: 1973 Forward}

Btu prices for the years 1973 forward are based on the Btu prices reported in Cost and Quality of Fuels (C\&Q). For 1973, 1974, and 1980 forward, Btu prices are taken directly from the data source and are 
converted fron cents per million Btu to dollars per million Btu. For 1975 throngh 1979, consomption-weighted average Btu prices are calculated from prices and consumption reported separately for stearn-electric plants and for combustion turbine and internal combustion units. Wherever individual State prices are onavallable, quantity-weighted Censas division prices from $C \& Q$ are assiggued as shown in Table A16.

The $C \& Q$ does not bave prices for $A K$ in 1973 forward or HI in 1973 through 1982 and 1992 forward. Prices are estimated by calculating the ratio of the AK or HI prices from the Statistical Yearbaok to the Statistical Yearbook U.S. price and multiplying the ratio by the C\&Q U.S. price for each year. AK prices for 1973, 1975, 1978, and 1994 are not published in the Statisticol Yearbook and are estimated by calculating an average of the ratios of the AR to U.S. \$atistical Yearbook prices in adjacent years. The 1973 estimated price is based on the average ratio for 1972 and 1974, the 1975 price is based on the average ratio for 1974 and 1976, the 1978 price is based on the average ratio for 1977 and 1979 , and the 1994 price is based on the ralio for 1993. The average ratio is then applied to the U.S. C\&Q price for the missing year.

Table At6. Dietilate Electric Uttlity Census Division Pifice At: lignmente from CAO, 1973 Forward

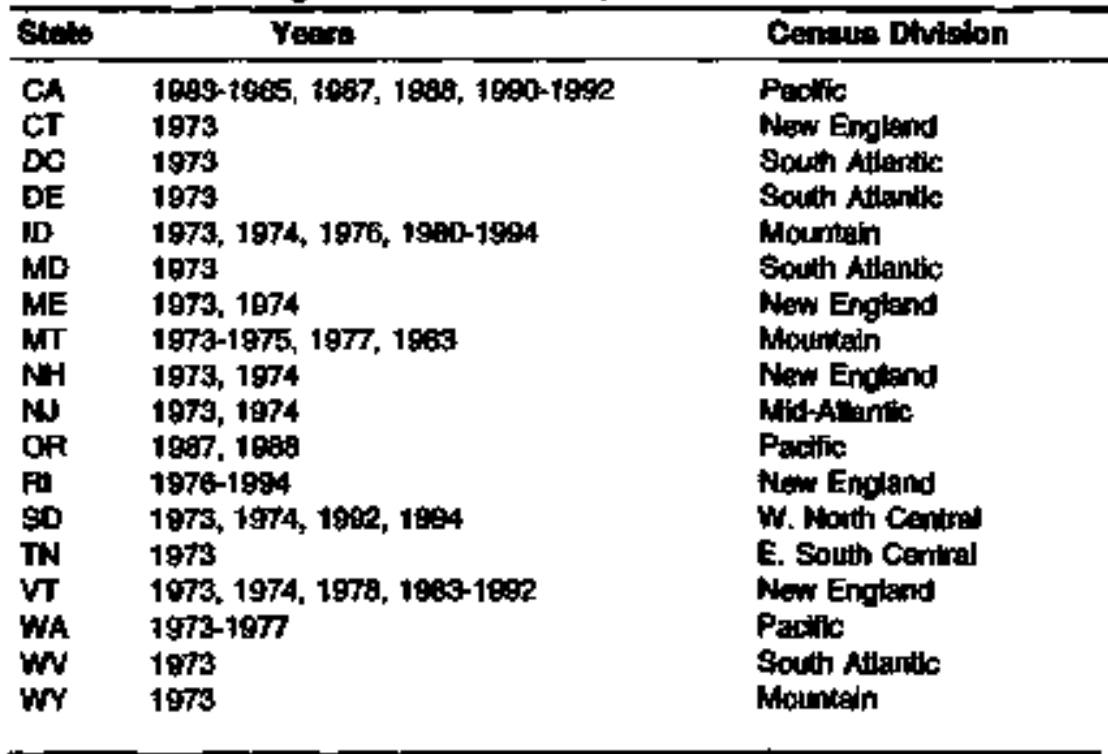

U.S. Bth prices for all years are calcolated as the twerage of the State Btu prices, weighted by consumption data from SEDS.

\section{Prlees: 1970 Through 1972}

Bu prices for 1970 through 1972 are estimated by uging data from Seatistical Yearbook of the Electric Utilty Industry. U.S. prices are then conputed by using the State-level prices and the eleciric otility distillate consumption data from SEDS.

1. Regression techniques are used to arrive at the equation for estimating electric utility sector distillate prices for the 1970 through 1972 period. AL is treated as the reference State. The regression equation uses Statistical Yearbook State-level prices for 1974 through 1980 as the independent variable and the State-level prices calculated abrove for 1974 through 1980 as the dependent variable. Substituting Btu prices for 1970 through 1972 fom the Statistical Yearbook into the regression equation yields the estimated electric utility sector State-level distillate prices.

2. Wherever individual State prices are unavaikable, quantity-weighted Census division prices are assigned as follows: ID in 1970 throogh 1972; TN in 1970; and WA in 1970 and 1971. AK in 1971 is calculated as the average of the AK price in 1970 and 1972.

3. U.S. Btu prices are calculated as the average of the State Btu prices, weighted by consumption data from SEDS.

\section{Datu Sounces:}

\section{Prices}

1973 forward: Energy Information Administration, Cost and Quality of Fuels for Electric Utiltry Planss, Table 6 (1973, 1974); Tables 5, 6, 12. 13 (1975-1979); Table 45 (1980-1982); Table 51 (1983, 1984); Table 41 (1985-1989); Table 14 (1990, 1991); and Table 8 (1992 forward).

1970 forward: Edison Electric Instinte, Statistical Yearbook of the Electric Uhilty Industry, table titled, "Analysis of Fuel for Electric 
Generation-Total Electric Utility Industry." (1970-1988); table titled. "Fossil Fuels Used for Electric Generstion Total Electric Utility Indus. try," (1990-1993); and Table 29 bitted "Receipts and Average Delivered Cost of Fossil Fuels Total Electric Utility Industry." (1994).

\section{Consaraption}

1970 forward: Energy Information Administration, Stale Enorgy Data System, electric ntility sector fistillate consumption.

\section{Converalon freton:}

Bu prices are calculated directly from data sonrces; no explicit conversion factors are needed for any years for the electric otility sector.

\section{Industrial Sector}

The industrial sector distillate prices ane developed by using a variaty of data sources and several estimation methods, depending on the years involved. For 1983 forward, prices of No. 2 distillate fuel (excloding taxes) are reported by the Petrolewn Marketing Annual (PMA). State general sales taxes from the Burealu of the Census and successor sources are added. For 1970 through 1982, prices are the average cost of distillate to manufacturing firms and implicity inclade taxes that reflect individual State differences.

\section{Phytial Unit Pinces: 1989 forward}

Physical unit distillate prices in cents per gallon (excluding taxes) are generally available for 24 States from the PMA. State-level prices for the remaining 27 States are estimated by using the PMA Petroleam Administration for Defense (PAD) district prices, as showo in Table A17. State geveral sales taxes are then added.
Phyoical Unt Prices; 1982

In 1984, the Bureau of the Census announced that State-itevel fuel cost and quantity information would no longer be published in either the Annual Survey of Manofactures (ASM) or Census of Manufactures (CM). In addition, the PMA, the source for 1983 forward industrial sector distillate price data, did not contain 1982 prices. Because of this lack of price data, the 1982 industrial sector distillate prices are estimated on

Tebfe A17. Distillate induatrial Sector PAOD Price Assionments, 1963 Forwerd

\begin{tabular}{|c|c|c|}
\hline نt. St. & Youts & Pritee Atolinged \\
\hline 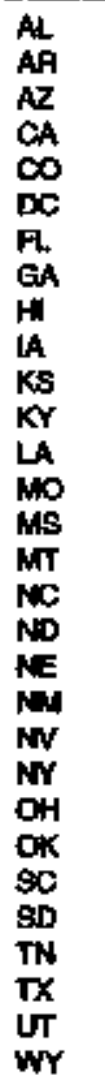 & 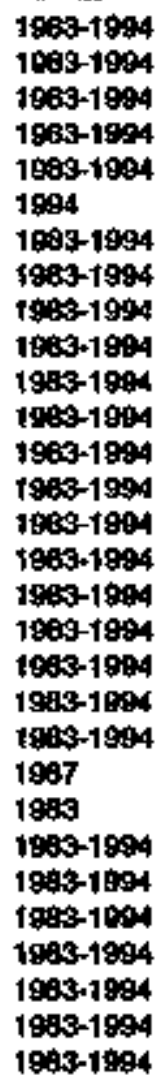 & 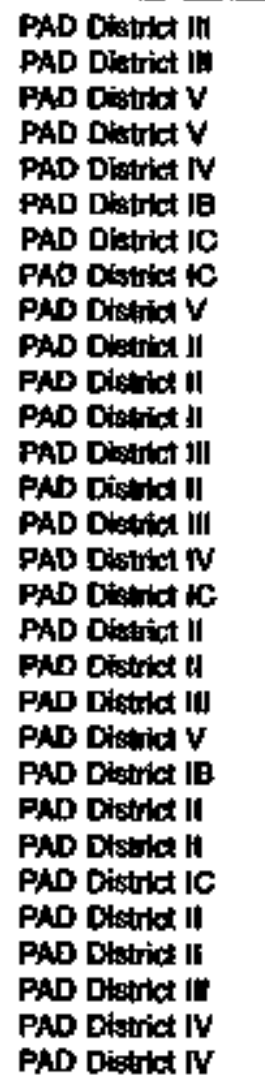 \\
\hline
\end{tabular}


D the basis of the relationship of industrial sector prices to electric utility sector prices for 1978 through 1981 . The 1983 prices are not used in the estimation because they exclude taxes. while the 1978 through 1981 prices include taxes.

1. In order to calculate the aretage ratios of industrial-to-electric utility distillate prices, electric utility price assignments are made for: AK in 1978 through 1982 from WA; ID in 1979 through 1982 from MT; RI in 1978 through 1982 from CT; and VT in 1978 from ME.

2. The average 1978 through 1981 ratios of industrial-to-electric utility sector distillate prices are calculated for each State.

3. Prices for 1982 are estimated by multiplying the average ratios by the electric utility data for 1982 .

\section{Physical Unit Pricos: t97t, t974 Through 1981}

For the years 1971 and 1974 through 1981 , industrial sector distillate prices are calculated directly from cost and quantity data from the Annual Sunvey of Martufactures (ASM) or Census of Mankfactures (CM) for all States where data are available. Taxes are included in the prices. There are no missing prices for 1971. Six States are missing some ASM cost and quantity data for the 1974 through 1981 period. Cost and quantity data for these States are estimated as the simple average of the cost and quantity data for their adjacent States. The States, the years for which data are estimated, and the adjacent States used to make the estimation are shown in Table Als.

\section{Phystcal Unit Prices: 1970, 1972, 1973}

Since $A S M$ and $C M$ data are not available for these years, the prices must be estimated. Physical unit prices are based on the ratio of 1971 CM prices to the 1971-assigned Plat's prices (Table A13 on page 352). The resulting ratios for each State are used with the Platt's assigned prices for 1970, 1972, and 1973 to ímpute prices.

1. The first step is to ealculate State-level ratios between prices calculated from the $1971 \mathrm{CM}$ cost and quantity data and the 1971
Table A18. Distillate industrial Sector Prico Assigmmenta, 1974-1981

\begin{tabular}{|c|c|c|}
\hline Shato & Yaame & Stalio Priced Wood \\
\hline $\begin{array}{l}\text { HI } \\
\text { ND } \\
\text { NW } \\
\text { NV } \\
\text { OK } \\
\text { WY }\end{array}$ & $\begin{array}{l}1979-1941 \\
1979-1981 \\
1974-1979 \\
1974-1981 \\
1974-1978 \\
1974-1981\end{array}$ & $\begin{array}{l}\text { CA } \\
\text { MN, MT, SD } \\
\text { AZ, CO, TX } \\
\text { AZ, CA, ID, OA, UT } \\
\text { AR, CO, KS, MO, TX } \\
\text { CO, ID, MT, NE, SD, UT }\end{array}$ \\
\hline
\end{tabular}

assigned Platts prices. There are no missing States in either of these two sets of prices.

2. State-level physical unit prices for 1970,1972 , and 1973 are estimated by multiplying the 1971 ratio by the assigned State-level Platt's prices for each respective year.

\section{Btu Prices: All Veare}

Btu prices for States are calculated by converting the physical unit prices from cents to dollars per gallon, then to dollars per barrel (42 gallons per barrel) and, finally, to dollars per million Btu (5.825 million Btu per barre]). U.S. Btu prices are calculated as the average of the State Btu prices, weighted by consumption data from SEDS, adjusted for process fuel consumption.

\section{Date Sources}

\section{Prices}

1983 forward: Energy Information Administration (E1A). Energy InfoDisc, Volume 1, No, 4, November 1996. See box on page 403 for instructions for locating the data series on the InfoDisc. Data are also published in the EIA Petrolewm Marketing Amual 1985, Volume 1. Tab]e 25 (1983-1985, and annual issues of the Petroleum Marketing Annual, Table 36 (1986-1988), Table 38 (1989-1993), and Table 39 (1994), column titled "To Industrial Consumers." 
1970-1982: McGraw-Hill, Inc, Plat's Oil Price Handbook and Oitmanac, refinery and terminal prices for No. 2 fuel oil, average of highs and lows.

1971, 1977, and 1981: Bureau of the Census, U.S. Department of Commerce, Census of Manufactures. Table 4 (1971) and Table 3 (1977, 1981).

1974-1976 and 1978-1980: Bureau of the Census, U.S. Department of Commerce, Annual Survey of Manufactures, Table 3.

\section{Taxes}

1994: U.S. Advisory Committee on Intergovernmental Relations, Significant Features of Fiscal Federalism, Tables 14 and 26.

1993: Bureau of the Census. U.S. Department of Commerce, State Tax Review, Volume 54, No. 31, map titled "State Gasoline, Sales and Cigarette Tax Rates as of July i. 1993."

1983-1992: Bureau of the Census, U.S. Departnent of Commerce, State Government Tax Collections, table titled "State Government Excises on General Sales, Motor Fuel, and Cigarettes, Beginning and Eed of Fiscal Year."

\section{Consumption}

1970 forward: Energy Information Administration, State Energy Data System, industrial sector distillate consumption.

\section{Converslon Factor: All Years}

5.825 million Btu per barrel

\section{Transportation Sector}

Consumption of distillate fuel in the transportation sector includes distillste fuel used for vessel bunkering and for milltary and railroad use. plus on-highway diesel fuel use. Because on-highway diesel fuel use accounts for the largest portion of this sector--increasing from 55 percent in 1970 to 81 percent in 1994-prices and expenditutes are calculated by using diesel prices. State physical unit prices for 1986 forward are taken from EIA's Petroleunt Marketing Antual (PMA). Physical unit prices for earljer years are calculated by using PMA prices and consumption data from the U.S. Department of Transportation $H$ ighway Statistics to weight monthly or quarterly prices from the U.S. Department of Agriculture Agrictultural Prices into ansual prices. Btu prices for all years are calculated by using the physical unit prices and the distillate conversion factor.

\section{Physicel Unit Prtess: 1986 Forward}

Diesel fuei physical unit prices for 1986 forward are based on the annual State-level price data available from the PMA for approximately 24 States and monthly tax rate information from Highway Statistics. Generally, the PMA provides prices in cents per gallon, excluding taxes, for AK, CT, DC, DE, ID, IL. IN, MA, MD, ME, MI, MN, NH, NJ, NY, OH, OR, PA, RI, VA, VT, WA, WI $I_{r}$ and WV. State and Federal excise taxes on diesel fuel are added to PMA grices to derive tinal physical unit prices, which are converted to dollars per gallon. In cases where the tax rate is not constant throughout the year, an annual average tax is calculated on the basis of the number of months each rate was in effect. State and local sales and other general taxes are nat included.

For the remaining States for which no prices are published, the PMA PAD district prices for diesel fuel and motor gasoline and State motor gasoline prices are used. The State diesel fuel price is estimated as the ratio of the PAD district diesel fuel price to the PAD district motor gasoline price times the State motor gasoline price. The use of the ratio assumes that the relationship between the motor gasoline State and PAD district prices is similar to that of the diesel fuel State and PAD district prices. Motor gasoline prices to end users at all refiners' company ontlets are used. When a State has no price available in that data series, the motor gasoline price to end users by all types of sellers through company outlets is used. States with no published diesel fuel or motor gasoline prices are assigned the motor gasoline price of an adjacent State. For 1991 forward, DC is assigned the MD price. State and Federal excise taxes are added as described above. 
Diesel fuel physical unit prices for 1983 through 1985 are based on the annual State-kevel price data available from the $P M A$ and monthly State and Federal tax rate information from Highway Statistics for 24 Strtes. The prices for the remaining 27 States are calculated by asing Agricultural Prices as outlined in the 1977 through 1982 methodology.

For the 24 States of AK, CT, DC, DE, ID, IL, IN, MA, MD, ME, MI. MN, NH, NJ, NY, OH, OR, PA, RI, VA, VT, WA, WI, and WV, the PMA provides physical unit prices, exchuding taxes. In 1983 through 1985, the $D C$ price is missing, and the MD price is assigned. In 1983, RI has no price and the PAD District IA average is assignted. A simple average of monthly State and Federal excise taxes is calculated as a combined average tax and added to the PMA price for a final physical unit price. State and local sales and other general taxes are not incladed.

\section{Physical Unit Prices: 1977 Through 1982}

Monthly prices from Agriculturat Prices and monthly special fuels consumption data from Highway Statistics are collected for the States. MD prices are assigned to DC. Prices include Stare and local per-gallon taxes. Federal taxes and State and local sales and other general taxes are not included.

The volume-weighted annual diesel physical unit prices for States and the United States are calculated by using the monthly Agrtcultural Prices price data, weighted by the monthly Highway Statistics consumption data. AK/1977 through 1982 prices are estimated on the basis of the assumption that the ratio of AK-to-U.S. diesel fuel price is the same as the ratio of the AK-to-U.S. motor gasoline price each year.

\section{Physical Unit Prioes: 1970 Through 1976}

Quarterly prices from Agricultural Prices and monthly special fuels consumption data from Highway Statistics are collected for the States. Prices include State and local per-gallon taxes. Federal taxes and State and local sales taxes and other general caxes are not included.
1. Prices for 1970 through 1972 are reported in cents per gallon and must be converted to dollars per gallon. Prices for 1973 through 1976 are alkeady reported in dollars per gallon.

2. For 1971 through 1973, Strte-level prices are not available for CT. $M A, M E, N H, R I$, and VT. Each is assigned the New England price for the 3 years.

3. The third quarter DE ptice is assigned to the missing fourth quarter DE price in 1972.

4. The combined MDNDE prices reported in 1973 are assigoed to each of the States.

5. For 1970 through $1976, \mathrm{MD}$ (or MDDE) prices are assigned to DC.

The woukty special fuels consumption for 1970 through 1976 are converred into quarterly consumption by summing the months for each quarter.

The consumption-weighted annual diesel physical unit prices for the States are calculated by using the quarterly weights and quirterly prices. For 1970 throngh 1972 . the quarterly ptices from Agricultune Prices are converted from cents per galion to dollars per gallon. For 1973 forwand, the prices are already in dollars per gallon in the source. AK/1970 throngh 1976 prices are estimated on the basis of the astumption that the ratio of AK-to-U.S. diesel fuel price is the same as the ratio of AK-toU.S. motor gasoline price each year.

\section{Btw Proag: Aff Yoars}

Btu prictes for States are calculated by converting the physical unit prices from cents per gallon to dollars per bomel (42 pallons per barrel) apd then to dollars per miltion Bto (5.825 million But per barrel). U.S. Btw prices are calculated as the average of the State Btu prices, weighted by consumption from SEDS. 


\section{Censumption}

1970 forward: Energy lnformation Administration, State Energy Data System, transportation sector distillate consumption.

\section{Converston Factor: AN Yeers}

5.825 million Btw per barrel

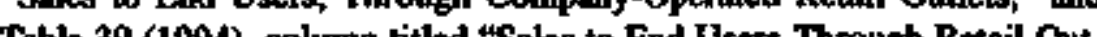
lets," for diesel fuel prices.

1986 forward: Energy Information Administration, Petrolewn Marketing Annwat. Table 29 (1986-1988) and Tabie 30 (1989-1993), columin titled "All Refiners, Sales to End Users. Through Company Outlets," and Table 35 (1994), column titled "All Grades, Sales to End Users, Through Retail Outlets," for mowor gasoline prices.

1986 forward: Energy Information Administration, Petrotewn Markering Annual, Table 28 (1986-1988) and Table 29 (1989 forward), column titled "Motor Gasoline Average. Through Company Ortlets," and Tahłe 31 (1994), colurne tided "All Grades, Sales to End Users, Through Retail Outlets," for additional motor gasoline prices.

1983-1985: Energy Information Administration, Petroleum Marteting Annual 1985. Volume 1. Table 25, column titled "Sales to End Users. Sales Through Company-Operated Retail Outlets."

1970-1985: Crop Reporting Board, U.S. Department of Agriculture, Agriculture Prices, tables generally titled "Motor Supplies: Average Price Paid by Farmers for Motor Fuel" for 1970-1979, and "Diesel Fuel: Average Price Paid by States" for 1980-1985.

\section{Thres}

1970 forward: Federal Highway Administiration, U.S. Department of Transportation, Highway Statistics, Table MF-121T for State tax rates, Table FE-101 (also EIA, Petroleun Marketing Amsuat, Table EN1) for Federal tax rales, and Table MF-25 for specinl fuels consumption dath. Tabte MF-25 is not included in the 1976 volume bot is poblicly available directly from the Federal Highway Administration. average cost of No. 6 fuel otl (residual fuel oil) as reported in Cost and Quality of Fuels for Electric Unilty Plants. (Ste Residual Fuel Electric Uallity Sector on page 388.)

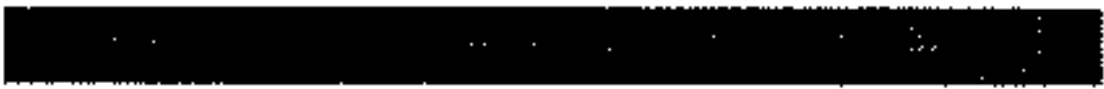

Jet fuel prices are estimated for all years for the transportation sector and for 1972 through 1982 for the electric atility sector. After 1982, electric utility use of jet fuel is reported as light oil. (See Llight OH (Wlectrk Utiltiles) on page 369.)

\section{Transportation Sector}

Consumption estimates from SEDS are developed for two types of jet fuel: terosene-type (used primarily by commercial aircraft and accounting for 97 percent of all jet fuel in 1994) and naphtha-type (tused primerily by military aireraft and acconnting for the remaining 3 percent in 1994). Prices are developed for kerosene-type jet fuel and are used 
as the price for bath types of jet furel. Taxes are not included in the E prices.

\section{Phyoics Unit Prices: 1989 forward}

$F$

Transportation sector jet fuel prices for 1983 forward are based on data from EIA's Petroleum Marketing Annual (PMA). Annual prices to end nsers are availabie for all States in most cases. Prices are converted to dollars per gallog. States without prices are assigned adjacent State or PAD subdistrict ptices as shown in Toble A19.

\section{Physical Unft Prices: 1976 Through 1982}

State-level jet fuel prices for 1976 through 1982 are calculated from the Producer Prices and Price Indexes (PPI) monthly indices for Census divisions and the jet fuel base prices by State for July 1975. The monthly price for each Census division is equal to the PPI monthly index times the jet fuel base price for July 1975 for that Census division. Census division monthly prices are assigned to each State within the Census division, and annual jet furel prices are computed as simple averages of the monthly State prices.

\section{Physical Unt Prices; 1970 Through 1075}

Jet fuel physical unit State-level prices for the 1970 through 1975 period are based on U.S. annual wholesale prices from the $P P I$ and the relationship of these prices to wholesale kerosene prices reported in Plat's. The U.S. prices are converted to Census division prices, which are then assigned directly to States.

Preliminary U.S. jet fuel prices from the PPI for 1973 through 1980 are calculated by using the annual jet fuel price indices, the jet fuel U.S. base price for July 1975 (0.276 dollars per gallon) and the U.S. index for July 1975 (235.8). The index for 1973 is assumed to be equal to a simple average of the 11 available monthly indices.

The calculated preliminary U.S. jet fuel prices from the PPI are used as the dependent variable in a regression equation for 1973 through 1980. where the wholesale kerosene prices from Platt's are the independent
Table A19. Jot Ful Tranoportation sector Price Actignments, 1983 Fonward

\begin{tabular}{|c|c|c|}
\hline State & Yenre & 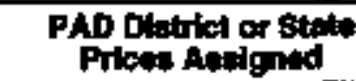 \\
\hline $\begin{array}{l}\text { DC } \\
\text { DE } \\
\text { ME } \\
\text { MH } \\
\text { PI } \\
\text { WT }\end{array}$ & $\begin{array}{l}1983-1968,1900,1893 \\
1987 \\
1985,1990,1991,1993,1994 \\
1987 \\
1980-1986 \\
1984-1988,1901,1902 \\
198,1994\end{array}$ & $\begin{array}{l}\text { MD } \\
\text { PAD subdlstrlat IB } \\
\text { PAD Subdistrict IA } \\
\text { PAD subdialint IA } \\
\text { PAD Subdisurict IA } \\
\text { PAD Subdistrict IA } \\
\text { PAD Subdistrict IC }\end{array}$ \\
\hline
\end{tabular}

variable. The regression equation is used to estimate U.S. annual jet fuel prices for 1970 through 1972.

Jet fuel prices for Censes divisions are estimated by using the preliminary U.S. prices derived above for 1970 through 1975 (calculated directly from the PPI data for 1973 through 1975 and estimated for 1970 through 1972). These prices are used as inputs to a regression equation which establishes a linear relationship between preliminary U.S. prices and Census division prices for the years 1970 through 1975. Census division prices are assigned to each State within the Census division.

\section{Stu Prices: All Years}

Btu prices for States are calculated from the physical unit prices and the Btu coaversion factor. U.S. Btu prices are calculated as the average of the State Btu prices, weighted by consumption data fron SEDS.

\section{Data Sourcos}

Prices

1985 forward: Energy Information Administration (EIA), Petroleum Marketing Annual, Table 21, column titled "Kerosene-Type Jet Fuel" (1985), Table 33, column titled "Kerosene-Type Jet Fuet, Sajes to End Users," (1986-1988), Table 35 (1989-1993), and Table 36 (1994). 
1983, 1984: EIA, Petroleum Marketing Annual 1994, Table A2, column titled "Kerosene-Type Jet Fuel, Sales to End Users." AIso available on the EIA, Energy InfoDisc, Volume 1, No. 4, November 1996. See box on page $\mathbf{4 0 3}$ for directions to locate the data series on the InfoDisc.

1973-1982: Bureau of Labor Statistics, U.S. Department of Labor, Producer Prices and Price Indexes, Supplenert, table titled "Proxtucer price indexes for refined petroleum products by region."

1970+1975: McGraw Hill, Inc., Plat's Oit Price Handbook and Ottmanac, 57th Edition, page 480.

\section{Comannption}

1970 forward: Energy Information Administration, State Energy Data System, transportation sector jet fuel consumption.

\section{Comveraion Factor: All Years}

5.670 million Btu per barrel

\section{Eloctric Utility Sector}

Jet fuel electric utility consumption estimates are taken from SEDS for 1972 throwgh 1982 only. For 1970 and 1971, no parallel series is available; ind for the years after 1982, the series is a part of tight oil. All applicable taxes are included in the prices.

\section{Etu Prices: 1975 Through 1962}

For the States that consumed kerosene-type jet fuel at electric utilities during these years, the Btu prices are taken directly from EIA's Cost and Qwaltity of Fuels for Electric Utility Plans (C\&Q).

\section{Bto Prices: 1972 Through 1974}

Becanse $C \& Q$ prices are not available for 1972 through 1974 , prices are estimated from $C \& Q$ prices for 1975 and 1976 and Agricultural Prices date for 1972 through 1976.

1. Simple annuad averages of Agricultural Prices quarterly values are calculated for 1972 through 1976. New England Census Division prices are assigoed to CT, MA, ME, NH, RI, and VT.

2. The average annual prices based on Agricuitural Prices values for 1975 and 1976 are used as the independent variables in a regression where the dependent variables are State-level prices based on $C \& Q$ prices for 1975 and 1976.

3. State-level price estimates for 1972 throngh 1974 are derived from the results of the regression analysis and the Agricultural Prices values for 1972 through 1974.

\section{U.S. Btu Proos: all Yoars}

U.S. Btu prices are calculated as the average of the State Btu prices, weighted by consumption data from SEDS.

\section{Date Sources}

Prices

1975-1982: Energy Information Administration, Cost and Quality of Fuels for Electric Utillty Plants, Tables 6 and 13 (1975), Table 13 (1976-1979), and Table 47 (1980-1982).

1972-1976: Crop Reporting Board, U.S. Department of Agriculture, Agriculture Prices, table titled "Household Supplies: Average Prices Paid by Farmers for Lawn Mowers and Petroleum Products." 
1972-1982: Energy Information Admitistration, State Energy Data System, electric utility sector kerosene-type jot fuel consumption.

\section{Converstion Factors: All Years}

Because Btu prices are available directly from the data sources, no conversion factors are used.

Kerosene prices are developed for the residential and industrial sectors. and the industrial sector prices are assigned to the commercial sector. Estimates of the amount of kerosene consumed by the residential, commercial, and indastrial sectors are taken from the State Energy Data System (SEDS).

\section{Residential Sector}

\section{Phyetod Unt Pricas: 1985 Forword}

In July 1985, the Burean of Labor Statistics ceased publication of the Producer Prices and Price Indexes (PPI) data series that is used to estimate State Energy Price and Expenditure Data System residential kerosene prices for 1970 throwgh 1984. The method used to estinate residential kerosene prices for 1985 forward is based on the observation that average U.S. Lerosene and distillate fuel prices from $19 \mathrm{BO}$ through 1984 wert highly correlated. Residential terosene prices for 1985 forward are estimated by applying the percentage changes in residential distillate fuel prices for each year to the residential kerosenc prices for the previous year in each State. Taxes are included in the distillete foel oil prices and are, therefore, reflected in the kerosene price estimates.
Becunse Agriculfural Prices kerosene price datn are not available after 1976, moathly Census divistion PPI prices and price indices are used as the basis for the residential kerosens series from 1977 through 1984. To motintain consistency in the seried, the PPI prices are anlitiplied by an adjustment factor that scocounts for the relationship between PPI and Agrictuliwal Prices datu for quarters in which the two series overlap. In the description of computational procedures below, the adjostmen factor is derived first, the PPI prices for 1977 through 1984 are estionted. and the final kerosene physical unit and Btu prices for States are calculated. The fioal residential sector kerosene prices approximate the average prices paid by farmers. Taxes ane inchuded in the source data from Agricullural Prices and are, therefone, neflected in the fingl price estimntes.

The first step is to compute the adjostment factor nelating $P P I$ and Agricylural Prices data.

1. Monthly PPI prices for the 18 months covered from Joly 1975 through December 1976 are calculated from the July 1975 base prices and monthly indices for Census divisions.

2. The calculated Census division monthly prices are assigned to each State within the respective Ceosus division.

3. Volume-weighted quarterly PPI- based prices for States are calculated by using the monthly volume weights developed from Retail Sales and muentories sales data for "other distillate fuel oil."

4. The adjustipent factor relaring PPI and Agriculturul Prices datu is calculated as the simple average of the ritios of the quartenty kerosene price by State from Agricultural Prices to the calculated quarterily PPI-based kerosene prices by State.

The next step is the calculation of monthly State-level prices from PPI kerosene Census division data for 1977 throngh 1984.

1. Monthly Census division PPI prices are calculated by ubing the July 1975 bast prices and the monthly price indices for 1977 tirongh 
1984. The missing montbly indices for Febnary. June, July, and October 1980 for the East South Central Division are assumed to be equal to the index for the preceding month.

2. Each State is assigned it's respective Census division monthly prices.

The next step is the celculection of annual physical unit Stote prices.

1. Annual PPI-bused physical unit prices for States are computed from the monaly PPI prices and the monthly consnmption weights.

2. Final residentital kerosene prices for States are estimated as the product of the anninil PPI-based Strte price and the adjustment factor calculated above.

\section{Phyalce Unt Prkon: 1970 Through 7976}

Physical unit prices for States are calculated from quarterly price intr from the U.S. Department of Agriculnure's Asricularal Prices and consumption weights derived from EIA's Retail Sales and Inventories of Fuel Oil. Tous are incladed in the sounce data.

The quatterly physical unit price dita from Agricultural Prices for 1970 through 1976 ane publishod in several different forms. The first step in the calculation of prices for these years is to organize the pablished $\mathrm{Ag}$ riculural Prices data into a consistent form.

1. For 197 I through 1973 , no quarterly prices are available for CT, MA, ME, NH, RI, and VT. Each of these States is sssigned the quarterly prices reported for the New Eughand Census Division.

2. For 1973, combined MDADE quarterly prices are reponted instead of separate State prices. For this year, the combined prices are assigned to both States.

3. No prices are neported for AK, DC/1970 through 1976. Quarterly weighted Census division prices are assigned to $\mathrm{AK}$, and $\mathrm{MT}$ prices are assigned to $D C$ for all 7 yents.
In order to weight the quarterly prices from Agriculural Prices into annual state prices, monthly quantity weights are calculated from ketail Sales and Inventories of Fuel OH. This assumes that the "other distillate oil" consumption data by PAD districts is kerosene.

1. Montbly weights are computed by using simple averaging of all available "other distillate oil" sales data for each month for each PAD district. Since data are available from November $197 \mathrm{~B}$ to Marcb 1981, some months have averages hased on three data points while others are based on one of two data points. For example, the average weight for March is the simple average of the 1979, 1980, and 1981 March volumes published in Retail Soles and Inventories of Fuel Oil.

2. Each month's share of average annual sales is calculated by PAD district from the average monthly sales figures. These shares, which become the monthly weighis, are then assigned to each State within its respective district.

Final State annual kerosene physical unit prices are calculated as the weighted average of the Agricuifural Prices quarterly prices. The monthly weights (shares) are converted to quarterly weights by summing the shares for months within a particular quarter. These same weights are used with the State-level price data for each year from 1970 to 1976 .

\section{Alaska Bur Prices: 1970 Through 1979, 1985, 1984}

Kerosene residential prices for AK are estimiated on the basts of the assumption that the ratio of AK-to-U.S. kerosente residential prices is the same as the ratio of AK-to-U.S. distillate fuel residential prices.

\section{Ban Prices: AH Vears}

Bto prices for States are calculated from the physical unit prices and the conversion factor. U.S. Btu prices are calculated as the average of the State Btu prices, weighted by consumption dara from SEDS. 


\section{Industrial Sector}

\section{Prices}

I985 forward: Residential sector distillate fuel price estimates (in physical units) from the State Energy Price and Expenditure Data System.

1975-1984: Bureay of Labor Statistics, U.S. Department of Labor, Producer Prices and Price Indexes, Supplement, table titled "Producer price indexes for tefined petroleum products by region."

1978-1981: Energy Infonation Administration, Retail Sales and Invensories of Fuel Oll, Table 2.

1970-1976: Crop Reporting Board, U.S. Department of Agriculture, Agricultural Prices, table titled "Household Supplies: Average Price Paid by Farmers for Lawn Mowers and Petroleum Products."

\section{Consumption}

1970 forward: Energy Information Administration. Stato Energy Data System, residential sector kerosene consumption.

\section{Converolon Factor: All Your:}

\subsection{0 million Btu per bartel}

\section{Commercial Sector}

State prices from the indastrial sector are assigned to the commercial sector. Although there is no industrial consumption of kerosene in DC in 1989. 1990, and 1992 through 1994, an industrial price is calculated to be assigned to the commercial sector consumption.
The industrial sector kerosene prices are based on wholesale price and price index data and on the industrial sector distiltate ptices. The procedures vary slightly for 1970 through 1974,1975 through 1984, and 1985 forward. In 1970 through 1984, physical unit prices are calculated first; then Btu prices are computed by using the physical unit prices and the conversion factor. Prices approximate an averuge kerosene price for the manuficturing sector. Taxes are included in the distillate fuel oil prices and are, therefore, reflected in the kerosene price estimtates.

\section{Physical Unit Prlces: 1986 Fonward}

The discontinuation of the Bureau of Labor Statistics' Producer Prices and Price Indexes, Supplement data affected the estimation of industrial sector kerosene prices. To estimate prices for 1985 forward, the percentage change in industral sector distillate fuel prices for each year is applied to the industrial sector kerosene prices for the previous year on a State-by-State basis.

\section{Phyolat Unit Pricos: 1975 Through 1984}

Physical unit industrial kerosente prices for 1975 through 1984 are estimated from $P P I$ base prices and indices for kerosene and No. 2 distillate oil, and from the industrial sector distillate prices in physical units. The ratio of $P P I$ kerosene prices to $P P I$ distillate prices is used as an adjustment factor to estimate kerosene prices.

Annual wholesale prices are calculated from PPI annual indices for kerosene and No. 2 distillate fuel oil and their respective July 1975 base prices for Census divisions. Annnal average distillate price indices for 1976 are estimated as the simple average of monthly indices. Census division prices for both kerosene and fuel oil No. 2 are assigned to each State within the respective Census divisions. The industrial sector physical unit kerosene prices for States are computed by using the distillate industrial physical unit prices and the ratio of PPI terosente prices to $P P I$ fuel oil No. 2 prices. 


\section{Physical Unit Prices: 1970 Through 1974}

Physical unit State-level prices for 1970 through 1974 are estimated from the distillate industrial prices and the average ratio of kerosene to distillate prices from PPI for 1975 through 1978 . The average annual wholesale price ratio between kerosene and frel oil No. 2 (distillate) is calculated from $P P$ J-based data for the years 1975 through 1978. Statelevel kerosene industrial physical unit prices are calculated as the profuct of the ratios and the industrial sector distillate prices for 1970 through 1974.

\section{Blu Pricen: All Yarrs}

But prices for States are computed by converting the physical unit prices in dollars per gallon to dollars per barrel ( 42 gallons per barrel) and then to dollars per million Btu ( $\$ .670$ million Btu per barrel). U.S. Btu prices are calculated as the average of the State Btu prices, weighted by consumption data from SEDS.

\section{Dath Sources}

Prices

1970 forward: Industrial sector distillate fuel price estimates (in physical units) from the State Energy Price and Expenditure Data System.

1975-1985: Bureau of Labor Statistics, U.S. Department of Labor, Producer Prices and Price Indexes, Supplement, tabie titled "Producet price indexes for refined petroleum products by region."

\section{Consamption}

1970 forward: Euergy Information Administration, State Energy Data System, industrial sector kerosene consumption.

\section{Converston Factor: All Yoars}

$\$ .670$ million Btu per bajrel
Beginning with 1980, the price of light oil consumed at electric utilities is the average delivered cost of No. 2 fuel oil as reported in Cost and Quality of Fuels for Electric Utility Plants. For earlier years, the price is the consumption-weighted avernge of the kerosene-type jet fuel price and No. 2 fuel oil. (See also Distillate Fuel Electric Utility Sector on page 357 and Jet Fuel Electric Utility Sector on page 365.)

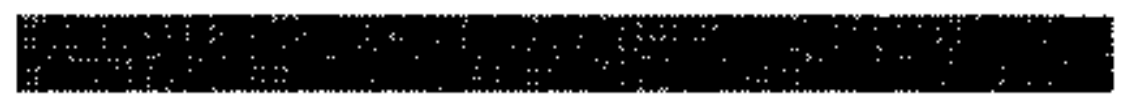

Liquefied petroleum gases (LPG) prices are developed for the residentis], conmercial, industrial, and transportation sectors. Estimates of the amount of LPG consumed by sector are taken from the State Energy Data System (SEDS) and are adjusted for process fuel and intermediate product consumption in the industrial sector. (See the discussion under "Consumption Adjustments for Calculating Expenditures" on page 413.)

\section{Residential Sector}

Residential sector EPG prices are estimated as the average prices of propane delivered to residential consumers in areas where natural gas is available as a competing fuel. Taxes are included in the prices. For 1973 forward, Btu prices are teported in the data source. For 1970 through 1972, physical unit prices are calculated first and Btu prices are calculated by using the physical uvit prices and Btu conversion factors. Prices for AK and HI in all years are estimated by a different methodology described in a separate section on page 371.

\section{Btu Prices: 1973 Through 1990, 1992 Forward}

Propane prices by city are reported by the American Gas Association (AGA) dicectly in dollars per million Btu, including taxes. The simple average of avajlable city prices is used as the State annual average. 
1 Prices that fall outside a reasonable range are omitted from consideraP tion for Central Hudson Gas and Electric for NY in 1979 through 1981; Artansas Louisiana Gas for AR in 1989; Public Service Electric \& Gas for NJ in 1989; Northwestern Public Service for SD in 1989; City of Long Beach for CA in 1989 and 1990; Orange \& Rockland Utilities for NY in 1989 and 1990; Pike County Light \& Power for PA in 1989 and 1990.

To estimate missing prices (other than AK and HI, which are described in a separate section that follows), simple averages of adjacent States' prices are used as shown in Table A20. Estimted date for one Stente are not used to estimate prices for another State.

\section{Btu Pricas: 1001}

Propane prices from the American Gas Association (AGA) are not available for 1991. Propane prices from the EIA Petroleum Marketing Annach (PMA) are uged to calculate the percentage change in propane prices between 1990 and 1991 for each PAD district or subdistrict. These pereentages are applied to the 1990 State residential LPG prices from the State Energy Price and Expenditure Datu System (SEPEDS) to estimate 1991 prices for the contiguous 48 States and the District of Columbia. Ptices for LPO in AK and HI are developed by using the methodology described on page 371.

Prices for PAD Subdistricts IA and IB and PAD District $V$ are not available for 1990 in the PMA, and prices for PAD Subdistrict IA and PAD District $Y$ for 1991 are not available. To estimate the missing PAD district prices, a tatio of the end-users price to the resale price for propane published for an adjacent district is calculared and applied to the known resale ptice for the PAD districts and subdistricts withon an end-users price. For 1990, the PAD District I end-users-to-resale ratio is multiplied by the PAD Subdistricts IA and IB reagle prices to estimate an end-nsers price for those Subdistricts. For 1991, the PAD Subdistrict IB end-users-to-resale ratio is multiplied by the PAD Subdistrict IA nesale prices to estimate an end-users price. For botb years, the U.S. end-users-to-resale price ratio is applied to the PAD District V resale price to estimate a PAD District V end-users price.
Table A20. LPG Reaidentiol Soctor Price Aoetgninents. 1973 Fonserd

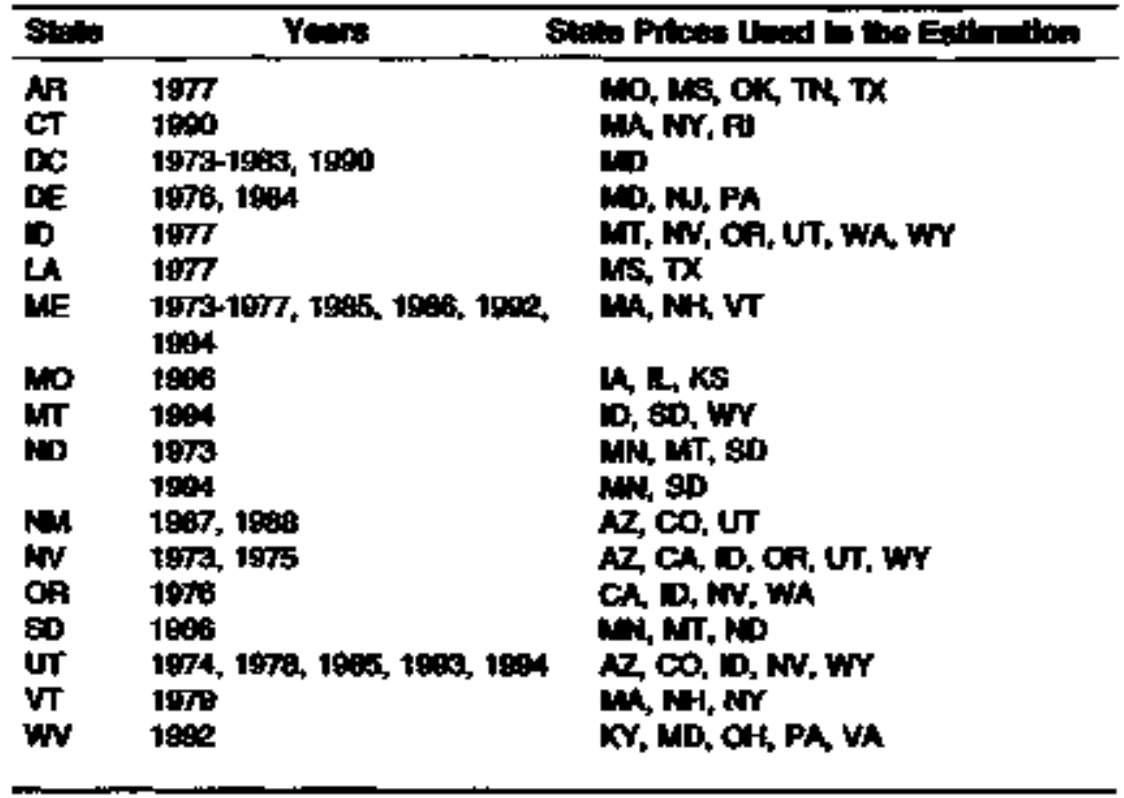

\section{Physted Unt Pries: 1971, 1972}

Physical nait residential L.PO prices are based on the city-level propante prices reported by AGA in cents per gallon. Prices for missing States are estimated. The AGA prices are the averape delivered prices for propane purchased by residential consumers as of December 31 .

l. City-level propane prices from AGA are assigned to their respective States. The Al/1971 price for the Fhoenix City Utilities System is onitted because it falls ontside a noasonable range.

2. Physical unit prices for a State are calculated directly from the available city/utility price observations reported by AGA. Final physical unit prices are equal to the simple average of the price observations for each State. 
3. MD prices are assigned for missing DC prices. AK aod HI pitces are discused in a separate rection that follows.

\section{Phydeal Unt Pucaks 1970}

Since AGA did nat poblish LPG prices prior to 1971, the residentid sector LPG prices for 1970 are estimited. To mutututp continuity wilh the AGA prices for 1971 forward, prices for 1970 are estimated by using simple regression analysis. The relationship between AGA data for 1971 and 1972 and corresponding Agricultural Prices data is the bakis for the estimation.

1. Before regnession analysis can be applied, Agricultural Prices data for 1970 through 1972 are prepared for 49 States (no AK or HI prices are available). These prices include tares. Development of AK and HI prices are destribed in a separate section on page 371.

2. State-level prices for small purchases, representing rexidential end users, for 1970 through 1972 are published by Agricultural Prices in cents per pound. When price per pound data are tot available, price per gallon data, repiesenting laxger volnme pirchases, are used. These prices per gallon are multiplied by 0.543, the average matio of price per pound to price per gallon for the United Statea for 1970 through 1972, to create nniform inpti deta in price per pooud.

b. For 1971 and 1972, the price reported for the New Engiand Region is assigned to CT, MA, ME, NH, RI, and VT.

c. Data in cents pes pound ane converted to dollars per gallon by multiplying by the propace conversion factor of 4.2 pourds per gallon, from the Petroleum Pnoducts Handbook and dividiug by 100.

d. Missing prices ose adjocteat Stales" average prices as shown in Table Az1.
2. The physical unit AGA prices and Agricultural Prices data for 1971 through 1972 (excluding AK and HiI) are used with simple regression antlysis to estimute final physical tenit LPG residential prices.

\section{Afakt and Hwmalf Prices; Aff Yoars}

Prices cannot be estimated for AK and fII by using adjacent State price assignments. Missing prices for these two States are estimated by compating ratios of the $\mathbf{A K}$ or HI prices to the simple average U.S. ptices calculated fiom the AGA data for yeirs when AK or Hil prices are available and applying these ratios to the U.S. simple average prices in years when pices need to be estimated.

1. AGA prices for AK are available in 1972 and 1980. The $1972 \mathrm{AK}$ to-US rotio is used to estimate prices for 1970. 1971, ant 1973 throangh 1979. The I980 AK-to-US price ratio is osed to estimate prices for 1981 forward.

2. AGA prices for HI are available in 1971, 1977 throegh 1979, and 1989. The 1971 HI-to-US AGA is used to estimate prices for 1970 and 1972 through 1974. The average ratio of the HI-to-US prices for 1977 through 1979 is used to estimate prices for 1975, 1976, and $19 \$ 0$ through 1984. The 1989 HI-to-US ratio is used to estimate prices for 1985 throngh 1988 and 1990 forward.

Tuble A21. IPG Reeidential Apolewhtwrol Prices Andigned to Eximite 1970 Prices

\begin{tabular}{|c|c|c|}
\hline stis & Yewe & Btis Pripas Wind \\
\hline $\begin{array}{l}\text { DC } \\
\text { Ny } \\
\text { Or } \\
\text { UT } \\
\text { Wh }\end{array}$ & $\begin{array}{l}1670-1072 \\
1970.1971 \\
1971-1872 \\
1972 \\
1990-1972\end{array}$ & 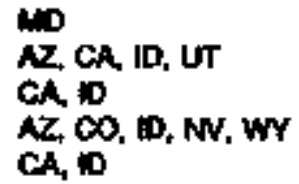 \\
\hline
\end{tabular}


C For 1973 forward, Btu prices for States are taken directly from the data sources. For 1970 through 1972, Btu prices for States are calculated by converting the physical unit prices by using the factors cited in Table A22 on page 372. U.S. Btu prices are calculated as the average of the State Btu prices, weighted by consumption data frotr SEDS.

\section{Data Sources}

Prices

1971-1990, 1992 forward: American Gas Association (AGA), Gas Househeeting Survey (1971-1988), Residential Gas Market Survey (1989 and 1990), and Residential Natural Gas Market Survey (1992 forward). Appendix 2, "Competitive Fuel Prices."

1991: Energy Information Administration, State Bnergy Price and Expenditure Data System, 1990 residential sector LPG prices.

1991: Energy Information Administration, Petroleum Marketing Annual, Table 35 (1990 and 1991), columns titled "Propane (Consamer Grade)."

1970-1972; Crop Reporting Board. U.S. Department of Agriculture, Agricultural Prices, table titled "Average Price Pajd by Farmers for Lawn Mowers and Petrolemum Products, Specified Dates, by State," column titled "L.P. Gas."

\section{Consumption}

1970 forward: Energy Infornation Administration, State Energy Data System, residential sector LPG consumption.

\section{Comverolon Factors:}

1970-1972: Energy Infornation Administration, State Energy Data Report 1994, Consunption Estimates, Table D1, as shown in Table A22.
1970-1972: 4.2 pounds per gallon from Guthrie, Virgil, ed. 1960. $P e$ trolewn Products Handbook. John Wiley and Sons, lnc., New York, New York: pages 3.5.

Conversion factors are not necessary for other years because Btu prices are available directly from the data sources.

\section{Commercial Sector}

Starting in 1994, commercial sector prices for LPG are estimated fiom PADD prices for consumer grade propane sold to commercial and institutional consumers published in the ELA, Petroleum Marketing Annual. PADD prices per gallon are assigned directly to ail States and converted to prices per Btu.

For 1970 throngh 1993. State LPG prices from the industrial sector are assigned to the commercial sector.

Tablo A22. LPG Btu Convertion Factors 1970 Formard (Million Gut per Barrel)

\begin{tabular}{|c|c|c|c|c|c|}
\hline Yan & Comprolion & Year & $\begin{array}{c}\text { Compinion } \\
\text { Fintor }\end{array}$ & Yow & $\begin{array}{l}\text { Comperalan } \\
\text { Fitotor }\end{array}$ \\
\hline $\begin{array}{l}1970 \\
1971 \\
1972 \\
1973 \\
1974 \\
1975 \\
1976 \\
1977 \\
1978 \\
1979\end{array}$ & $\begin{array}{l}3.779 \\
3.772 \\
3.760 \\
3.746 \\
3.750 \\
3.715 \\
3.711 \\
3.677 \\
3.669 \\
3.660\end{array}$ & $\begin{array}{l}1980 \\
1981 \\
1988 \\
1908 \\
1984 \\
1985 \\
1986 \\
1987 \\
1988 \\
1980\end{array}$ & $\begin{array}{l}3.674 \\
3.643 \\
3.615 \\
3.614 \\
3.504 \\
3.603 \\
3.640 \\
3.659 \\
3.652 \\
3.683\end{array}$ & $\begin{array}{l}1900 \\
1991 \\
1992 \\
1993 \\
1094\end{array}$ & $\begin{array}{l}3.625 \\
3.014 \\
3.424 \\
3.606 \\
3.635\end{array}$ \\
\hline
\end{tabular}


Prices

1994: Energy Information Administration, Petroleum Marketing Annest, Table 38, column titled, "Commercial/Institutional Consumers."

1970-1993: ELA, industrial sector LPG prices from the State Energy Price and Expenditore Data System.

\section{Consumption}

1970 forward: Energy Information Administration, State Energy Data System, commercial sector LPG consumption.

\section{Converalon Factors}

1994: Energy Information Administration, State Energy Data Report 1994. Consumption Estimates, Table DI, as shown in Table A22 on page 372 .

\section{Industrial Sector}

The industrial sector LPG prices are estimated from average LPG prices to manufacturing firms, average LPG prices to fammers, or refiner and gas plant operator sales to end users, depending on the data sources for the different years. Pricas for 1985 forward are taken from the Petrolewm Marketing Annual (PMA). Prices for 1978 through 1981 are taken from the Annual Survey of Manufactures (ASM) or the Census of Manufactures (CM) and prices for 1970 through 1977 and 1982 through 1984 are derived from Agricultural Prices and scaled to the $A S M / C M$ prices by using the ratio of $A S M / C M$ to Agriculitural Prices LPG prices for the years 1978 through 1981, when both price seties were available. Taxes are included in the 1970 through 1984 estimates but are not included in the prices for 1985 forward.
Starting in 1994, industrial sector physical unit prices are reported by PAD District, but not by State, in the EIA Petroleum Maketing Annual. Consumer grade propane prices are reported for three industrial sector egtegories-petsochemtcal plants, other end users (agricultural consurners), and industrial consumers. The prices for these three categories are consumption-weighted to develop PADD-level industrial sector price estimates that are assigned to the States in each PADD. Withleld prices for petrochemicals in PAD Subdistrict IB and IC are assigned the U.S. average price, and the withheld industrial price for PAD Subdistrict IA is assigned the PAD District I price.

\section{Physicd Unit Prlaw: 1986 Through 1093}

Industrial sector LPG physical unit State prices for 1985 forward are estimated by using physical unit annual prices in the Petrolewm Marketing Annual (PMA) for consumer grade propante sales to end-users. Where prices are not available, the PAD district price is assigned to the State as shown in Table A23. One exception is Arkansas for 1992 and 1993. Because the neighboring States in PADD III are LPG prodncers, the PADD III price is uncharacteristically lower than previously reposted prices for Arkansas. Therefore, the 3 monthly prices available for Arkansas in 1992 are averaged to derive an annual price. In 1993, the Missourl price is assigned to Atkansas.

When a PAD district price is not available, a consumption-weighted average price is calculated by using available prices for States within the district and the SEDS industrial sector LPG consumption for those States. A PAD District V price for 1985 is calculated as a consumptiolweighted average of $A K, C A, O R$, and WA prices; a 1986 PAD Subdistrict IA price uses the average of CT and NH prices; and PAD Subdistrict IA prices for 1987 through 1988 use the average of CT and MA prices.

When a PAD district price is not ayailable and there are no State data within the PAD district to develop a consumption-weighted average, a different methodology is used. The source table also contains resale prices. To estimate the missing sales to end-users PAD district price, a ratio of the end-users price to the resale price for an adjacent PAD 

A6signments, 1805-18es

\begin{tabular}{|c|c|c|}
\hline 8top & Years & PA \\
\hline 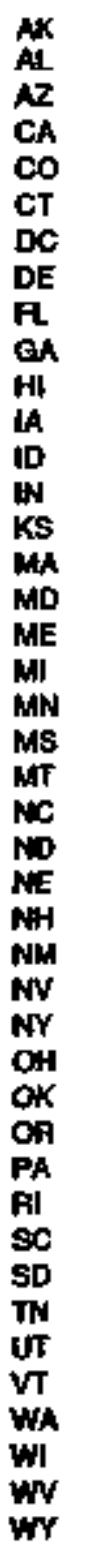 & 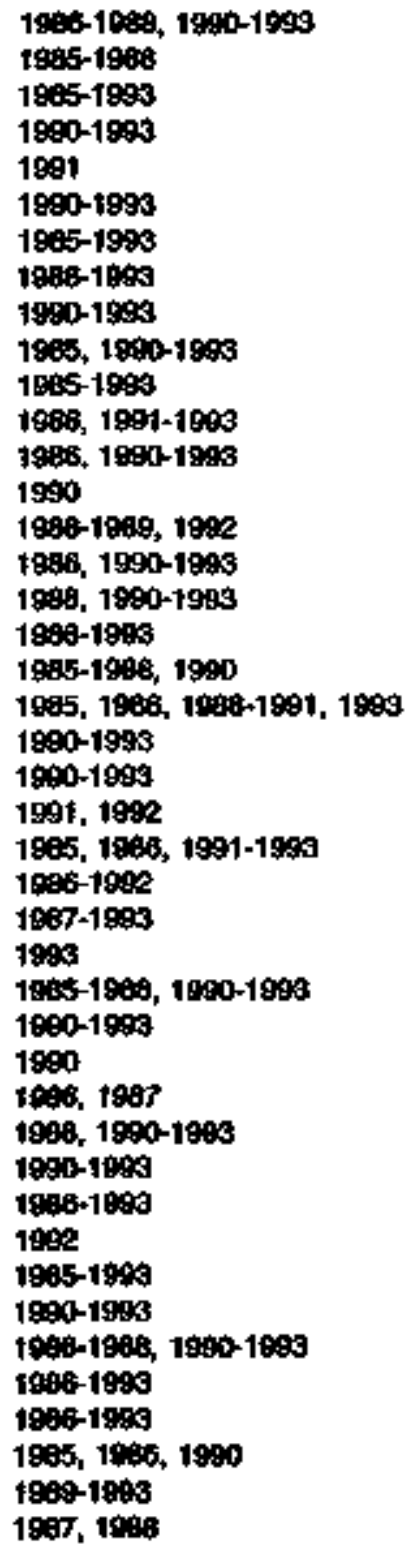 & 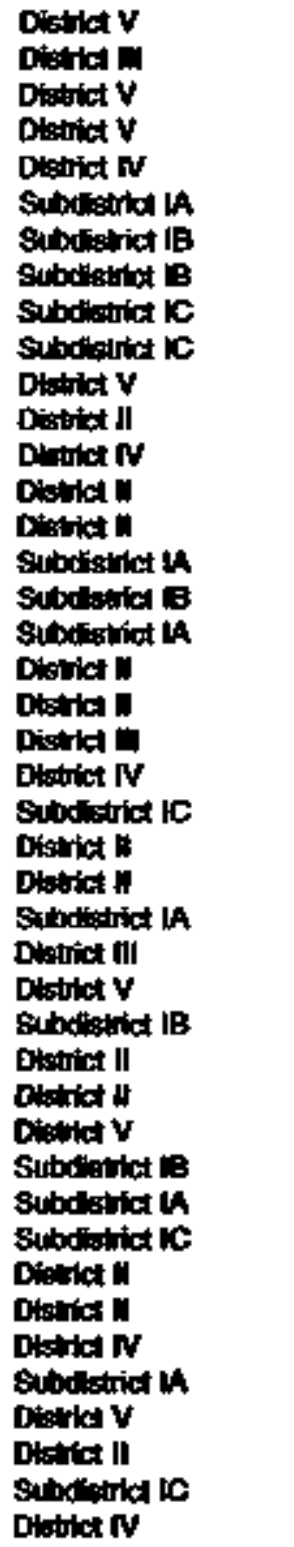 \\
\hline
\end{tabular}

district or subdistrict is calculated and applied to the known resale price for the PAD district or sobdistrice that does not have al end-usters price. PAD prices used is the estimations are shown in TabHe A24.

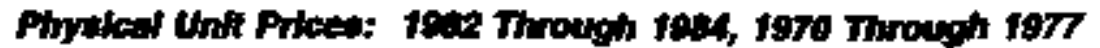

Induntrial sector L.PG physical nait prices for 1982 through 1984 and 1970 throngh 1977 are extimated on the basis of the relationship between State-level LPG prices from Agricultaral Prices and the prices calculated from Annwal Survey of Manufactures (ASM) or Cessus of Manufactures (CM) for 1978 through 1981.

1. Before the adjustment factor that relates Agriculturd Prices and ASH/CM data is compoted, monthly Agriculeural Prices data ane converted into annual prices and missing dath are estimated.

a. Annutl LPG prices are calculated as simple averages of the montbly prices from Agricultural Prices for the years 1977 through 1984. The only States missing data are WV in 1977 throngh 1981 and AK, DC, and HI in 1977 throogh 1984. WV is assigned the simple arerage of the $\mathrm{KY}, \mathrm{MO}, \mathrm{OH}, \mathrm{PA}$, and VA prices $\mathrm{AK}, \mathrm{DC}$, and HI pricess are discassed below.

b. The arerage ratio of ASWCH-based final prices for 1978 throngh 1981 and the 1978 through 1981 Agricultural Prices

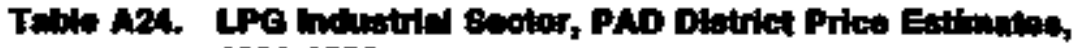
1990-189a

\begin{tabular}{|c|c|c|}
\hline$\overline{Y e m}$ & 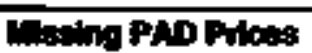 & 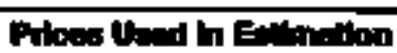 \\
\hline 1900 & 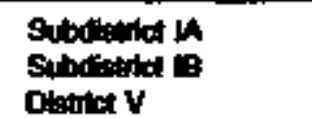 & $\begin{array}{l}\text { PAD Distifat I } \\
\text { PAD Dtitiat I } \\
\text { U.8. }\end{array}$ \\
\hline & 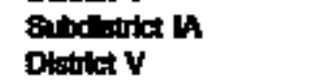 & $\begin{array}{l}\text { PAD Subdetikd is } \\
\text { U.S. }\end{array}$ \\
\hline 1992 & 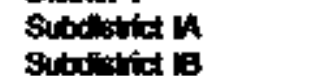 & 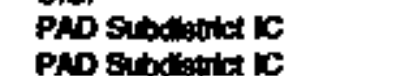 \\
\hline 1803 & 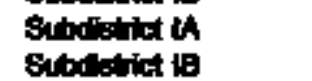 & $\begin{array}{l}\text { PND sub-itat ic } \\
\text { PND subsitict ic }\end{array}$ \\
\hline
\end{tabular}


Bnnual prices is calcultted for 48 States (excluding AK, DC, and HI) as the simple average of the ratio over the 4 years. This ayerage ratio is used as an adjustment factor.

2. Final industrinl sector LPG prices for 1982 through 1984 and 1970 through 1977 are estimated by using the State-level adjustment factors and ananal average LFG prices from Agricuttural Prices for these years.

a. Anmual sverage LPG prices are calculatod for 1982 through 1984 and 1970 through 1977 as the simple average of the monthly prices.

b. Agricultwrat Prices pnblished abaunl average prices in dollars per galion for all States in 1975 and 1976. For DE in 1970 through 1974, MD in 1970 through 1974, VA in 1970 throogh 1974 , and $W V$ in 1970 through 1972 , only prices for small volume purchases in ceuts per ponnd were pablished. These are coiverted to cents per gallon by multiplying by 1,96, the average ratio of cents por gallon to centr per pound for the United States for 1970 through 1974.

c. For 1970 through 1972, Agricultural Prices ane converted from cents per galloa to dollars per gallon.

d. For 1971 through 1973, the New England price per gallon reported by Agricultural Prices is assigned to CT, MA, ME, NH, RI, and VT.

e. MD prices are astigned to DC in 1970 through 1972,1974 through 1977, and 1982 through 1984. The combined MDME price in 1973 is assigned to $\mathrm{MD}, \mathrm{DE}$, and $\mathrm{DC}$.

f. Excluting AK and HI, States missing Agricultural Prices LPG prices are astigeed the simple average price of adjacent States. The Stotes with missing data and the adjactont State assigmments ve shown in Table A25.

8. Induatrial sector LPG physical unit prices for 1970 through 1977 and 1982 throogh 1984 for all States (except AK. DC, and HI) ane calcullated by using the estimated annual Agricultural Prices data for the respective year and the State-level average ratios as adjustment factors.

3. AK prices for 1970 through 1977 and 1982 through 1984 and $\mathrm{HI}$ prices for 1970 through 1977 and 1982 through 1984 are estimated by using the relationship between $A S H / C M$ based prices for these States and the U.S. price reported by Agricultural Prices (1979 through 1981 for $\mathrm{AK}$ and $197 \mathrm{~B}$ through $198 \mathrm{t}$ for $\mathrm{HI}$ ). The average ratio for the availabie years for the two States is calculaled and used with the Agricultural Prices U.S. prices for the years to be estimated.

\section{Physteg; Unit Prices: 1978 Through 1981}

For 1978 through 1981, the industrial sector LPG prices are either calculated dinectly from cost and quantity data from the $A S M$ or the $C M$ or are estimated by using the relationship of $A S H / C M$ date to LPG price dati from Asricultural Prices.

1. For 1978 through 1981 , industrial sector chysical unit prices for LPG are calculated as the average cost per unit from cost and quantity data published in $A S M / C M$. Since sales are reported in poutsds.

Tablo 425. LPG Indugtriel Sector Price Astignments, 1970-1976

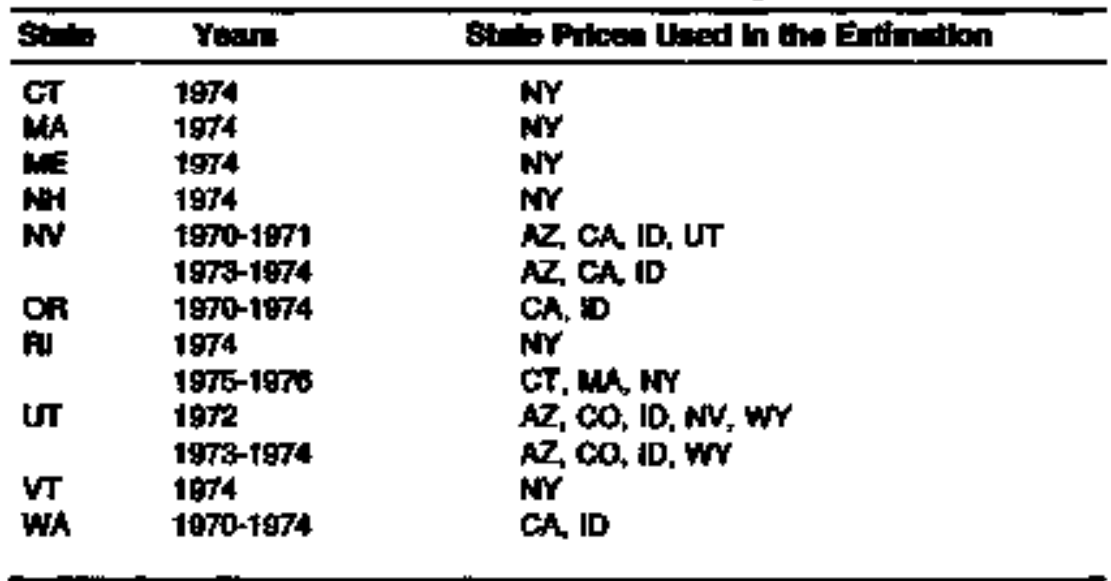


L the prices are converted to dollars per gallon. The conversion factor P of 4.5 pounds per gallon is from ASW/CM.

2. The AK price for 1978 is the consumption-weighted average Census division price. In addition, four States have prices estimated as the simple average of the prices of adjacent States, and DC is assigned the MD price as shown in Table A26.

\section{Btu Prices: All Years}

Btu prices for States and the United States are calculated from the physjcal unit prices and the conversion factors shown th Table A22. U.S. Btu prices are calculated as the average of the State Btu prices, weighted by cronsumption data from SEDS, adjusted for process fuel and intermediate product consumption.

\section{Daka Sources}

\section{Prkces}

1994: Energy Information Administration, Petroleumt Marketing Annual', Table 38, columns titled "Industria Consumers," "Petrochemical," and "Other End Users."

1985-1993: Energy Information Administration, Petnolewm Marketing Annual. Table 21 (1985), Table 33 (1986-1988), and Table 35 (19891993), colusans títled -Propane (Consumer Grade)," "Sales to End Users," and "Sales for Resale."

Table 428. LPG Indertrial Soctor Price A9ilgnmonts, 1978-1981

\begin{tabular}{|c|c|c|}
\hline Silnte & Yearte & Stalo Prices Utad \\
\hline $\begin{array}{l}\text { AR } \\
\text { DC } \\
\text { LA } \\
\text { NM }\end{array}$ & $\begin{array}{l}1978 \\
1978-1981 \\
1990 \\
1979-1981 \\
1978-1461\end{array}$ & $\begin{array}{l}\text { LA, HO, MS, OK, TX } \\
\text { MD } \\
\text { AR, MS, TX } \\
\text { AZ, CO, OK, TX } \\
\text { CO, IO, MT, ND, NE, SD, UT }\end{array}$ \\
\hline
\end{tabular}

1970-1984: Crop Reporting Board. U.S. Department of Agriculure. Agricultural Prices, tables titled "Average Price Paid by Farmers for Lawn Mowers and Petroleum Products, Specified Dates, by State," column titled "L.P. Gas, ${ }^{\text {" }}$ (1970-1976): "Household Supplies: Average Price Paid by Farmers" (1977-1979); "L.P. Gas: Average Price Paid by States" (1980); and "L.P. Gas: Average Price Paid by Months by States" (1981-1984).

1981: Bureau of the Census, U.S. Department of Commerce, 1982 Census of Manufactures, Fuels and Electric Energy Consumed, Part 2. States and Standard Metropolitan Statistical Areas by Major Industry Groups, Table 3, State-level quantity and cost of liquefied petroleum gases.

1978-1980: Bureau of the Census, U.S. Department of Commerce, Annual Survey of Manufactures, Fuels and Electric Energy Consumed, States by Industry Group and Standant Metropolitan Statistical Areas by Majar Industry Group, Table 3, State-level quantity and cost of liquefied petroleum gases.

\section{Consumption}

1994: Energy Information Administration, unpublished data collected from ELA-782B,

1970-1993: Energy Information Administration, State Energy Data System, industrial sector LPG consumption.

\section{Conversion Factors}

1970 forward: Energy Information Administration, Ssate Energy Data Report 1994, Constumption Estimates, Table D1, as shown in Table A22.

1978-1981: 4.5 pounds per gallon from Antual Survey of Manufactures, Appendix C. 


\section{Transportation Sector}

Starting in 1994, transportation sector prtices are estimated from PADD prices for consumer grade propane sold through retail outlets published in the ELA Petrolerm Marketing Annual. Physical unit PADD prices are assigned to all States within a PADD and converted to prices per Bta.

for 1970 through 1993, State prices from the industrial sector are assigned to the cransportation sector.

\section{Datis Sources}

\section{Prices}

1994: Energy Information Administration, Petroteum Marketing Annual. Table 38, column heading. "Thuough Retail Outlets."

\section{Consumption}

1970 forward: Energy Information Administration, State Energy Data System, transportation sector LPG consumption.

\section{Convertion Factors}

1994: Energy Information Administration, State Energy Data Report 1994, Consumption Estimates, Table D1, as shown in Table A22 on page 372.

1970- [993: Bu prices are assigned from the industria] sectot.

Lubricant prices are developed for the industrial sector and are assigned to the transportation sector. State-level prices are not available for either sector; national-kevel prices are assignted to all States and do not include end-user taxes paid at the time of sale. Estimates of lubricant consumption by the industrial and transportation sectors are taken from the State Energy Data System (SEDS).

\section{Physical Unft Prices: All Vears}

Prices of lubrtcants are estimated from U.S. Department of Commerce, Burean of the Census, data for three product cafegories:

1. Lubricating oils made in reftneries (SIC 29117.21) and not made in refineries (SIC 29920.21).

2. Lubricating greases made in refineries (SIC 29117.31) and not made in refineries ( $\$ 1 C$ 29920.31).

3. Lubricating oils and greases, not specifically known (n.s.k.), matte in refineries (SIC 29117,00) and not made in refineries (SIC 29920.00 for establishments with 10 employees or ntore and SIC 29920.02 for establishments with fewer than 10 employess).

For the years where Census of Manufactures (CM) data are available (1967, 1972, 1977, and 1982), total shipments are calculated by adding the shipments for the three product categories. Shipments for the third product category are withheld and estimated by dividing their value of shipments sum by the weighted average cost of the prodnct categories 29920.21 and 29920.31 .

Total shipments in each year for which $C M$ data are available is divided by the estimated SEDS total Jubricants consumption (in physical units) for that year to establish a shipments-to-consumption ratio. Ratios for the years not covered by the CM (i.e., 1968 throngh 1971. 1973 through 1976. and 1978 through 1981) are estimated by linear interpolation, and the 1982 value is used for 1983 forward. Total shipments for the years not covered by the $C M$ are estimated by multiplying SEDS consumption data by the appropriate shipment-to-consumption ratio.

Estimated shipment prices are calculated by dividing the value of shipments shown in the CM or the Annual Survey of Manufactures by the estimated shiponents for each product category. The shipment prices are used to represent wholesale prices. 
L End-user prices in dollars per barrel are estimated by multiplying the U shipment prices by trade ratio factors that represent the wholesale-to-teB tail markup. The trade ratio factors are developed from Burean of B Economic Antalysis (BEA) data for 1972 and 1977. For 1972, the sum R of data called "purchasers value" for the three prodoct categonies is diI vided by the sum of the "producers value" for the three categories wo C derive a trade ratio. A similar calculation is made for 1977, but the A terms "putchase value" and "basic value" are used in the source data.

The 1972 ratio is used for 1970 through 1972 , and the 1977 ratio is used for 1977 forward. The values for 1973 through 1976 are estimated by linear interpolation by using the 1972 and 1977 values. The trade ratios for 1982 and later years are not used because the range of petroleum products included in the ratios was expanded by BEA and the ratios no longer represent the specific mark-up for lubricants.

\section{Btu Prices: All Yesrs}

Btu prices are obtained by dividing the prices in dollars per barrel by the convergion factor 6.065 million Bty per barrel.

\section{Dota Sources}

Prices

1970, 1971, 1973 through 1976, 1978 through 1981, and 1983 forward: Bureau of the Census, U.S. Department of Commence, Annual Survey of Manufactures; Lubricating Oils and Greases (Industries 29117 and 29920).

1)

E 1972, 1977, and 1982: Burean of the Census, U.S. Department of Commerce, Censws of Manufactures, Petroleun Refining; Lubricating Oils and Greases (Industries 29117 and 29920).

1972 and 1977: Bureau of Economic Analysis, U.S. Department of Commerce, loput-Output Table Work Tapes for SIC Codes 29113 and 29920).

\section{Consunpation}

1970 forward: Energy Information Administration, State Energy Data System, lubricants consumption.

\section{Comvanion Funtor: Afl rome}

6.065 million Btu per barrel

Motor gasoline prices are developed for the traspoctation sector, and the transpontation sector pitces are assigned to the commercial and isdustrial sectors. Mator gasoline consamed in privately-owtred vehickes is acounted for in the transportation sector. Estinates of motor gasoline consumed by the transportation, commencial, and inchstrial sectors used in calcnlating expenditures are taken from SEDS. Prices in this series are retail prices (usually service station prices), inclading taxes.

\section{Phyoles Unil Priwes: 1903 Forwind}

Motor gasoline physical unit prices for 1983 forward are besed on annual State-level prices or assigned PAD Distriet prices from the Petrolem Marketing Anmal (PMA) except for prices for certain Staces and years, as noted in Table A27, that are derived from the Burean of Labor Statistics' Conswner Prices: Energy (CPI).

Strte and Federal motor gasoline taxes ane added to the prices from the PMA. Monthly State tax information and anousl Federal tax iuformetion are taken from Highway Shatistics. The monthly Stare taxes ane averaged to ereate an average auntal tax for each State which is combined with the Foderal tax to adjut the PMA price. Dee to the lack of uniformity in application, State and local general sales taxes tre not ibcluded. 
The PMA average sales pice (excluding taxes) of finished motor gasoline to end users though company ontiets is used, moler the assoumption that this price most closely approximates retail moior gasoline prices. Finished motor gasoline includes leaded and unleaded motor gasoline and gasohol.

In the States and yeurs (shown in Table A27) where prices are derived from the $C P I$, monthly CPI eity prices are weighted by monthly consumption from Highway Statistics. All tuxes are included in the CPI data.

Motor gasoline prices for HI are not collected or published in the CPI after December 1986. The following method is used to derive HI prices for 1987 through 1990, when PMA prices for HI becane availeble. The monthly HI CPI prices are osed to calculate ennual averages for 1983 throngh 1986. The annalal averages are divided by the PMA PAD District $V$ price (with HI State and Federnl taxes added) for ench year to develop annual ratios of the two prices. The four ratios for 1983 through 1986 are simple averaged to give one nato that is multiplied by the PMA PAD District V prices for the 1987 through 1990 to estimate Hl prices for those years. State and Federal taxes are added to the estimates.

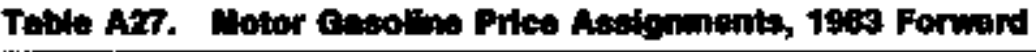

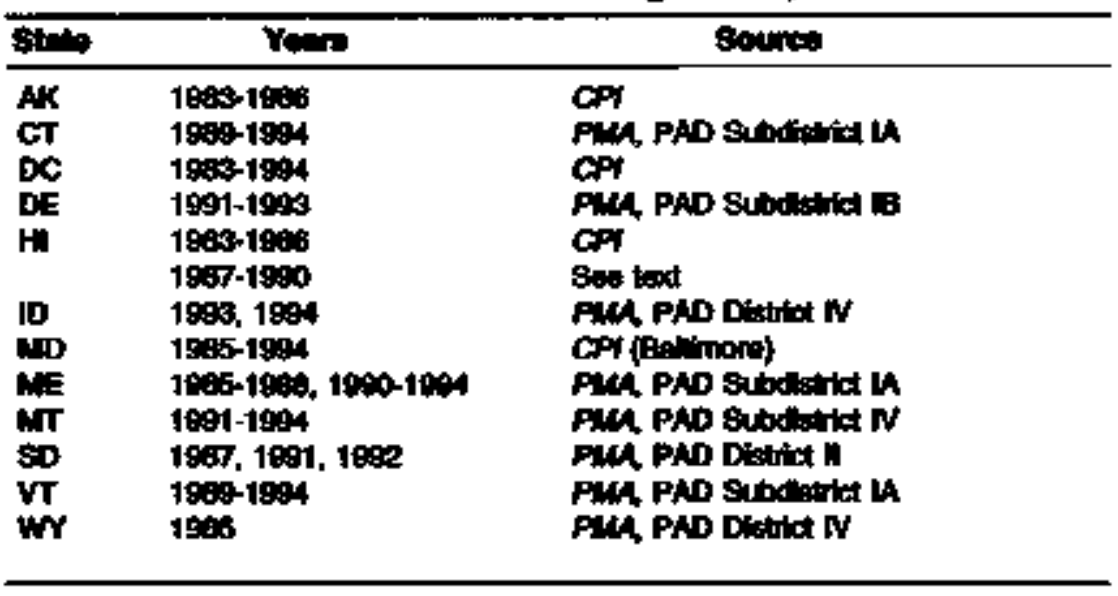

\section{Phystow Unit Prices: 1992}

Monthly physical unit motor gatoline prices for 1982 are taken from the Plan's Oil Price Handboot and Oilmanac (Platt's) table, "AAA 'Fuel Gauge' Report," the CPI, or both. Tabile A28 summarizes price data avai]ability by source. The Platt's prices are reported for both leaded and unleaded motor gasoline and for both full-service and self-service for all States except AK and HI. All avatiable Platt's prices for 1982 ane abed in the calculntion of motor gasoline prices. The continuity of these prices with prices published by Platt's in previous years suggests that taxes wre tachuded.

The available CPI monthly physical unit motor gasoline prices for 1982 are for all types of motor gasoline and cover 25 States, as shown in Table A29. The CPI prices are assigned to any State that has a county included in the Standard Metropolitan Statistical Ares (SMSA) definitions used by the Burean of Labor Statistics. These "all types" prices cover leaded regular, unleaded regular, and leaded premium, including taxes. All the ayailable CPI prices for 1982 are also used in the calcuIation of motor gasoline peices. Complete monthly data exist for the 25 States covered by the CPI. The CPI Detailed Report of April 1986 explicitly states that Federal, State, and local taxes are included.

To combine the product-specific Platt's prices with the "all types" prices published in the CPI, the Plot's prices are weighted into "all types" prices by using annoal U.S. data from the Monhly Energy Review (MEK) to calculate shares for leaded and onkaded motor gasoline (no breakdowns for regular and premium are possible because of data limitations).

Motor gasoline price deta reported by Platt's for 1982 cover the following months: February, April, June, Augnst, November, and December. The missing 6 months are assigned prices as follows: January is assigned the February price, and the other missing months are assigmed the averuge price of the preceding and succeeding months. A missing February price for MO is assumed to be equal to the April price, and a missing price for $O R$ is assumed to be equal to the average of the Apri] and Augnst prices. 


\begin{tabular}{|c|c|c|c|c|}
\hline Yans: & soume & Grades Covered & $\begin{array}{l}\text { Compostion } \\
\text { Price }\end{array}$ & Wieqing Statw \\
\hline 1982 & $\begin{array}{l}\text { Piatf's } \\
\text { CPI }\end{array}$ & 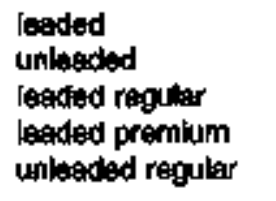 & $\begin{array}{l}\text { no } \\
\text { no } \\
\text { yes } \\
\text { yes } \\
\text { yes }\end{array}$ & none \\
\hline $1974-1981$ & CPY & 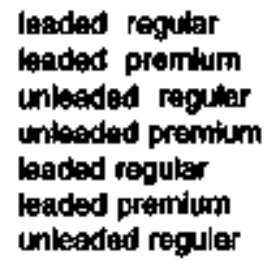 & $\begin{array}{l}\text { no } \\
\text { no } \\
\text { no } \\
\text { no } \\
\text { yes } \\
\text { yes } \\
\text { yes }\end{array}$ & $\begin{array}{l}\text { AR, DE, ME, MS, } \\
\text { MT, ND, NH, OK, } \\
\text { AI, SC, SD, VT, } \\
W V, W Y\end{array}$ \\
\hline 1978 & $\begin{array}{l}\text { Ptafl's } \\
\text { CPI }\end{array}$ & $\begin{array}{l}\text { leaded regular } \\
\text { leaded reguler } \\
\text { leaded prembum } \\
\text { undeaded regritar }\end{array}$ & $\begin{array}{l}\text { no } \\
\text { yes } \\
\text { yes } \\
\text { yes }\end{array}$ & none \\
\hline 1976,1977 & $\begin{array}{l}\text { Platt's } \\
\text { CPI }\end{array}$ & $\begin{array}{l}\text { laaded regular } \\
\text { loded regular } \\
\text { loaded premkin } \\
\text { unleaded regulgr }\end{array}$ & $\begin{array}{l}\text { no } \\
\text { no } \\
\text { no } \\
\text { no }\end{array}$ & AK \\
\hline 1974, to75 & $\begin{array}{l}\text { Platts } \\
\text { CPY }\end{array}$ & $\begin{array}{l}\text { beaded regular } \\
\text { boded regular } \\
\text { waded premium }\end{array}$ & $\begin{array}{l}\text { no } \\
\text { no } \\
\text { no }\end{array}$ & AK \\
\hline $1970-1973$ & Platf's & leaded regular & no & AK, HI \\
\hline
\end{tabular}

For States with data fron Platt's only, prices by product type (leaded and unleaded) are first calculated as the simple average of full-service and self-service prices for that product for each month and State. The resulting prices are then weighted into monthly composite prices by using U.S. leaded and unleaded shares of motor gasoline prodact supplied from the MER. The following 26 States have data oniy from Platt's: AL, AR, AZ, CT, DE, IA, ID, LA, ME, MS, MT, NC, ND, NE, NH, NM, NV, OK, RI, SC, SD, TN, UT, VT, WV, and WY.
Table A29. Motor Ostollne Prloo Agalgnments from Consumer Prices: Energy, 1976-1882

\begin{tabular}{|c|c|}
\hline Statis & Chy Pries Asalgnmerits \\
\hline 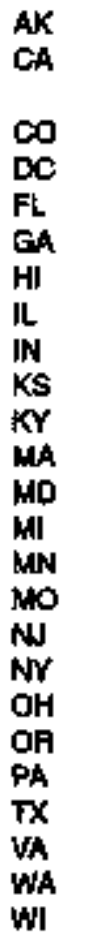 & 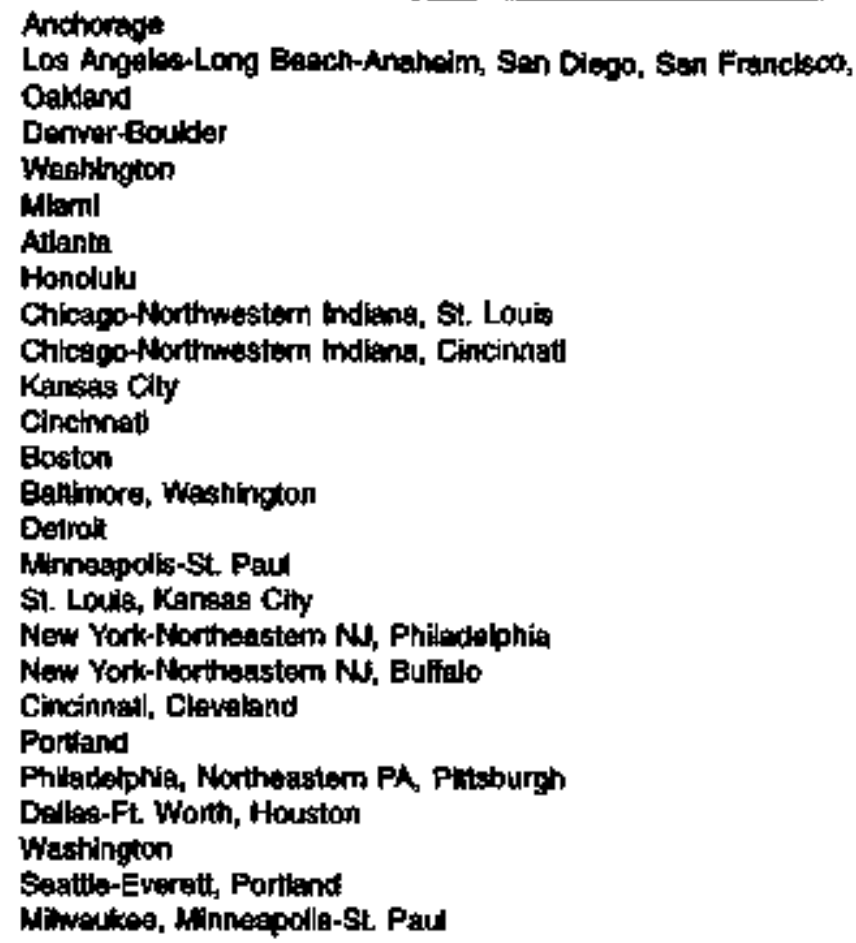 \\
\hline
\end{tabular}

Note: Al types of motor gasoline art included

Platt's repotts two prices for each motor gasoline product for each year: one full-service price and one self-service price. These two prices are combined by using a simple average into a single peoduct price for each State for each nonth.

The unleaded U.S. share of total motor gasoline consumption is reported in the $M E R$ as 52.1 percent in 1982. Assuming that the remainting motor gasoline consumption is leaded, the leaded portion of total consumption is 47.9 percent. These shares are used for all States and months to calculate the composite prices from the leaded and unieaded prices. 
For AK and HI, the only States with data only from the CPI, the "al] types" monthly prices reported are used directly as monthly composite prices.

For States with price data from both Platt's and the CPI. the Platr's data are first combined into product type prices and weighted with the $M E R$ shares. The resulting combized prices for all motor gasoline types are averaged together, with the combined $C P I$ city prices assigned to the respective month and State. The following 23 States have monthly composite prices computed in this way: $\mathbf{C A}, \mathbf{C O}, \mathrm{DC}, \mathbf{F L}, \mathbf{G A}, \mathbf{I L}, \mathbf{N N}$, KS, KY, MA, MD, MI, MN, MO, NJ, NY, OH, OR, PA, TX, VA, WA, and WI.

1. Leaded and unleaded gasoline prices are calculated as simple averages of full-service and self-service prices from Plart's and are then weighted into a composite price by use of $M E R$ shares of kaded and unlesited motor gasotine consumption.

2. Monthly "all types" motor gasoline prices covering leaded regular. leaded premium, and unleaded regular are taken directly from the $C P I$. If there is more than one $C P J$ price observation for a month and State, the $C P I$ prices are simple averages.

3. Using a simple average, the composite Platt's prices are combined with the "all types" CPI prices for each State. The resulting prices are the monthly composite prices for 1982 .

Annual physical unit prices for all States are calculated from the monthly motor gasoline prices calculated above and weighted by the monthly motor gasoline consumption volumes for \$tates from Highway Statistics.

\section{Phyolcal Unt Prices: 1979 Through 1981}

For 1979 through 1981 , Platr's monthly motor gasoline ptices are taken from a table titled "Platt' $\$$ /Lundberg Summary." Prices are available for cities by product-type, by grade, and by type of service (full service, self service). Four products and grades of motor gasoline ate covered: leaded regular, unleaded regular, leaded preminm, and unleaded premium. These data cover 37 States and taxes are included. The CPI reports "all types" prices, including taxes, for the cities listed in Table A29. Plats's city price assignments to States for 1979 through 1981 are shown in Table A30.

The computation of monthly composite prices for 1979 through 1981 varies, depending on the available data sources for each State. Monthly composite prices are estimated for the 14 States which do not have reported price data from either data soarce. If both Platt's and the CPI report prices for a city, the CPI price is used.

1. For States with city price abservations only from Platt's, prices for leaded and unleaded motor gasoline are combined by use of simple averaging, regardless of the type of service, and are converted to dollars per gallon. The leaded and unleaded prices are then weighted together into a monthly composite price. The following 12 States have prices only from Plas's for 1979 through 1981: AL, AZ, CT, IA, ID, LA, NC, NE, NM, NV, TN, and UT.

a. The Platt's prices for 1981 end in September of that year; monthly prices by grade and service type for October, November. and December are assumed to be equal to the corresponding September prices.

b. Leaded and unleaded prices are calculated for each State by simple averaging of all ptices avatlable for each product (leaded, unleaded), regardless of service type or grade of motor gasoline (regular, premium). All city prices for each State are averaged together.

c. Leaded and unleaded shares of total motor gasoline consumption for the United States are calculated from the $M E R$ for each year 1979 through 1981 . The monthly product type prices are weighted into composite prices by using the national leaded and unleaded shares as wejghts.

2. For States with city price observations only from the $C P I$, the monthly "all types" prices are used directly for States with only one price observation per month. For States with multiple observations, monthly prices are combined by simple averaging. States with CPI 


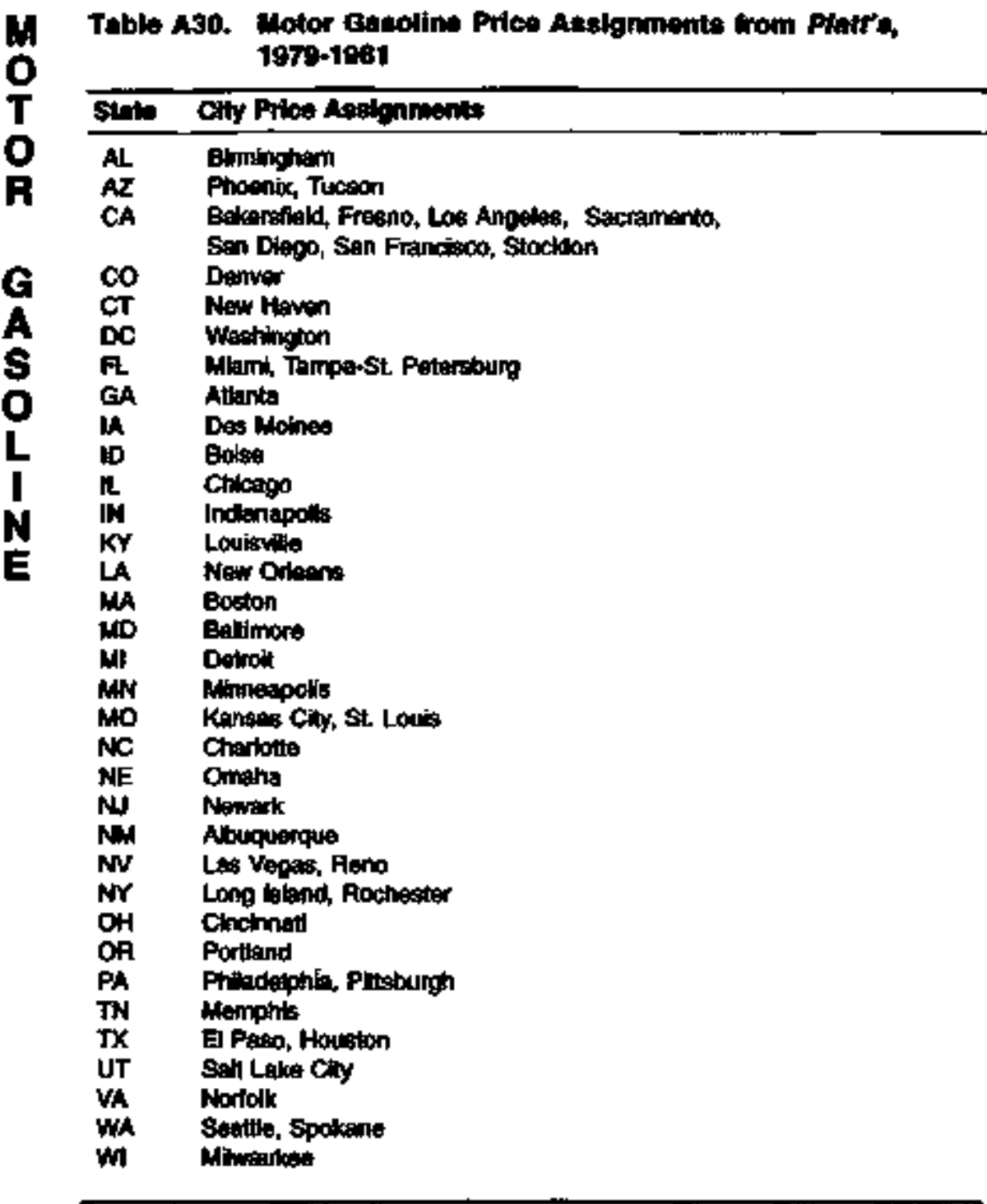

dath only are: AK, CO, DC, GA, HI, L, KS, MA, MD, MI, MN, MO, NI, OH, OR, PA, and WI.
3. Fon the eight States with price observations from both Plotr's and the CPI (CA, FL, IN, KY. NY, TX, VA, and WA), monthly composite prices for 1979 through 1981 are calcalated by using three steps;

a. The Platt's pricess ane combined into single "all types" prices as described above by using leaded and unleaded grades of motor gasoline shares as weights.

b. The CPI prices are combined by State.

c. Using simple averaging, the composite Platt's price for each State is combined with the "all types" CPI price for that State. The resulting prices are the monthly composite prices for 1979 through 1981 .

4. Fonrteen States are not covered by price data from either Platt's or the $C P I$ in 1979 through 1981. These Stalem are AR, DE, ME, MS, MT, ND, NH, OK, RI, SC, SD, VT, WV, and WY. Monthly composite prices for these States are estimated by nsing the monthly State-level composite prices for 1982 and Censas region monthly prices from the $C P I$ for 1979 through 1982.

a. The ratio between the 1982 State prices and the 1982 CPI Census region prices corresponfing to each $\$$ tate is calculnted for use as an adjustment factor in 1979, 1980, and 1981.

b. The monthly price for each of the 14 missing States is assumed to be the product of the 1982 Census region adjustment factor for that State times the monthly motor gasoline puice for that Censas region from the $\mathrm{CPI}$.

Anaual physical unit prices for all States are calculated from the monthly motor gasoline prices calculated above and weighted by the monthly motor gasoline coasnmption volumes for States from highway Statistics. 
The Plari's monthly leaded regular motor gasoline prices cover all States except AK and HI. The Platt's city assignments to States are shown in Table A31. In 1978, the CPI notor gasoline coverage was expanded froni 21 States to 25 States (28 SMSAs) and an "all types" price was published that covers leaded regular, leaded premium, and unleaded regnlar. The CPI SMSA assigmments to States for 1978 through 1982 are shown in Table A29 on page 224. Both the CPI and the Plati's prices include taxes.

Since both sounces report a single price for each city or SMSA, product weights are not needed to compute monthly composite prices. Instead, city price observations ane assigned to \$tates as shown in Table A29 and Table A31. Price observations are combined by asing simple averaging by State and month. If both Platt's and the CPI cover a city/SMSA, the $C P /$ price is used. Plart's prices are converted to dollars per gallon; the CPI prices are already expressed in dollars. All States are covered by the data sources, so no imputation is required for 1978. The following 26 States have prices only from Platt's: AL, AR, AZ, CT, DE, IA, ID, LA, ME, MS, MT, NC. ND, NE, NH, NM, NV, OK, RI, SC, SD. TN, UT, VT, WV, and WY. The following 19 States are covered only by the CPI: AK, CA, CO, DC, FL, GA, HI, IL, MA, MD, MI, MN, MO, NJ, NY, OH, OR, PA, and WI. Six States have price data from both sources: IN, KS, KY, TX, VA, and WA.

Annual physical unit prices for all States are calculated from the monthly motor gasoline prices calculated above and weighted by the monthly motor gasoline consumption volumes for States from Highway Statistics.

\section{Phytied Unf Prices: 1976,1977}

The calculation of monthly composite State prices for 1976 and 1977 depends opon the source of data. Different procedures are used for States with only Platt's data, States with only CPI data, and Strtes with both Platt's and CPI data. If both data sources cover a city, only the $C P I$ price is ased for that city. City price assignments to States are given in Table A31 for Platt's and in Table A32 for the CPI. Prices

\begin{tabular}{|c|c|}
\hline Stonto & 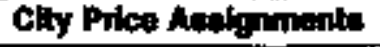 \\
\hline 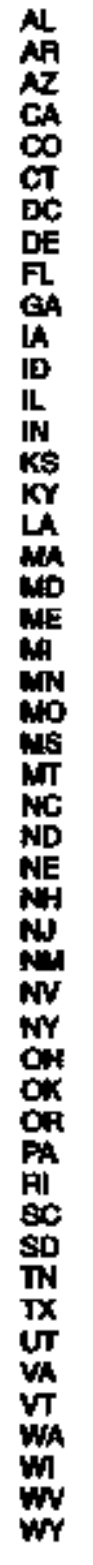 & 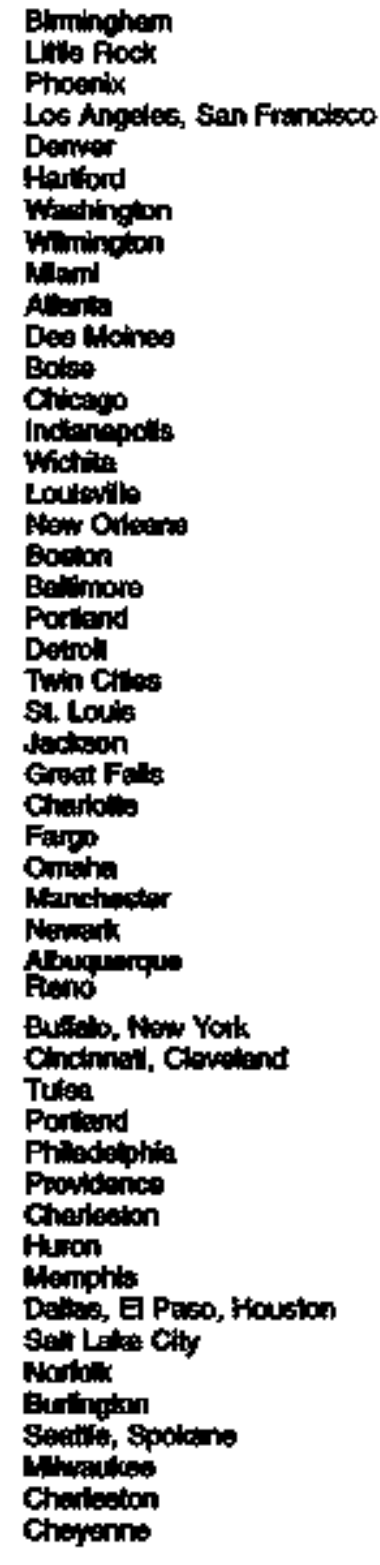 \\
\hline
\end{tabular}


$M$ from both sources include taxes. AK is the only State for which prices O need to be estimated.

O For States with data from Platt's only, the monthly prices reported in

R Platt's are used either directly or combined by simple averaging if there is more than one price observation for a State in a given month. The reported prices in cents per gallon are converted to dollars per galion.

G Prices for the following 29 States are cakulated by using this procedure

$A$ and cover only leaded regular motor gasoline: $A L, A R, A Z, C O, C T$,

3 DE, FL, IA, ID, LA, ME, MS, MT, NC, ND, NE, NH, NM, NV, OK, O OR, RI, SC, SD, TN, UT, VT, WV, and WY.

I If State-level motor gasoline prices for 1976 and 1977 are available only

N from the $C P I$, monthly composite prices are calculated as weighted av-

E erages of leaded and unleaded prices. Ptices for 15 States are calculated by using data only from the CPI: CA, DC, GA, HI, IL, MA, MD, MI, MN, MO, NJ, NY, OH, PA, and WI.

1. The weights used in this process are national-level shares of leaded and unleaded motor gasoline produet supplied. For 1977, the leaded and unleaded share of 0.725 and 0.275 , respectively, are taken from the MER. For 1976, MER data for 1977 through 1984 are used to estimate the unleaded share by using simple regression. The unleaded percentages for 1977 through 1984 are converted to shares and used to estimate leaded and unleaded shares of motor gasoline. The resulting 1976 leaded share is 0.744 and the unleaded share is 0.256 .

2. The next step is to calculate monthy composite leaded and unleaded prices for each state. If more than one CPI price observation is available for a particular grade of motor gasoline (leaded or unleaded) for a State in a given month, the CPI observations are combined by grade by using simple averaging. Regular and premium prices are averaged for an estimate of State-level leaxkd prices.

3. Final monthly composite prices for 1976 and 1977 are calculated by using the leaded and unleaded composite prices calculated above and the MER-based leaded and unleaded shares as volume weights.
Tabte A32. Wotor Gasoline Price Assignments from Connumer Prices: Energy, 1974-1977

\begin{tabular}{|c|c|}
\hline Stabio & Clty Price Aoplgnmants \\
\hline $\begin{array}{l}\text { CA } \\
\text { OCA } \\
\text { HI } \\
\text { IL. } \\
\text { IN } \\
\text { KS } \\
\text { MA } \\
\text { MD } \\
\text { WH } \\
\text { MO } \\
\text { NW } \\
\text { NY } \\
\text { OH } \\
\text { PA } \\
\text { TX } \\
\text { VA } \\
\text { WA } \\
\text { WI }\end{array}$ & 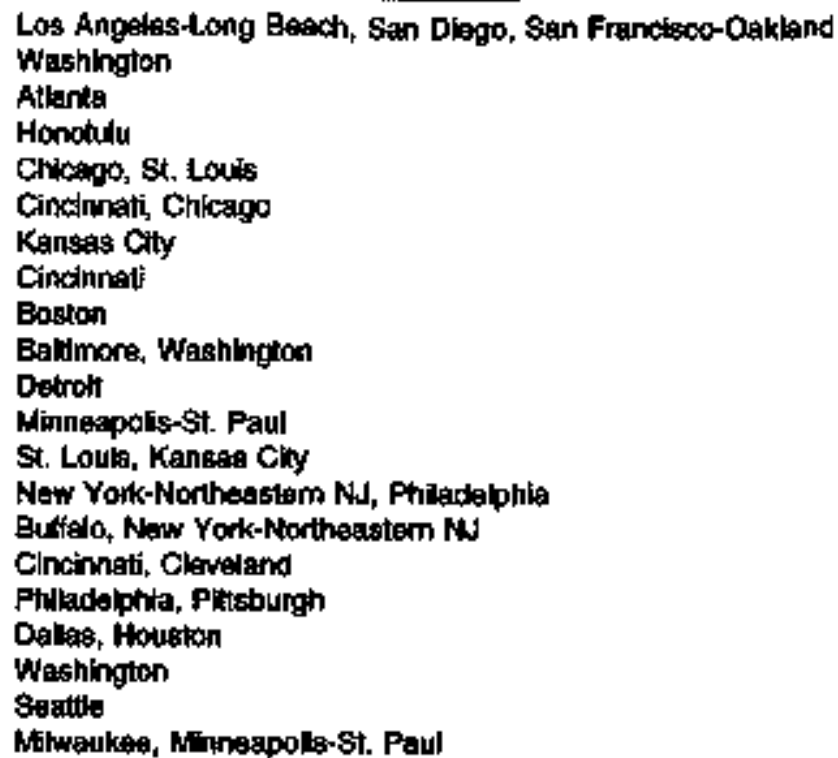 \\
\hline
\end{tabular}

Nale: Prices tre cyaileble soparately lor leaded repular, leadtad premium, and unleaded regular (1970, 1977f; cell bypes" prices and not avglatole.

For States with ptice data from both Plat's and the CPI, all price observations are averaged together by product type. If both sources report prices for a city, the $C P I$ price is used. Once composite leaded and unleaded prices have been calculated separately for each State, the leaded and unleaded consumption shares are used to weight the product-type prices into the final monthly composite motor gasoline prices. Six States are calculated with data from both Plat's and the CPI: IN, KS, KY, TX, VA, and WA.

1. Monthly leaded composite prices are calculated by combining Platt's prices with the $C P I$ prices for leaded regular and premium motor gasoline by month, since the Platt's prices cover only regular leaded fuel. If both data sources cover a city, the CPI prices are used. 
2. Since the CPI is the only source of unleaded gasoline price data for 1976 through 1977 , monthly unleaded composite prices are calculated from $C P I$ data only.

3. Final monthly composite prices for the six States with price data from both Platt's and the CPI are calculated by using annual U.S. leaded and unleaded shares and leaded and unleaded monthly composite prices.

Prices for 1976 and 1977 for $A K$, the only State not covered by price data from either data source, are estimated on the basis of the average relationship between the State and the national average price for years in which data are available. The national average price used for these estimations is a simple average of the prices of the 49 States for which data are available in all years (i.e., excluding $A K$ and $H I$ for all years). Annual prices for AK are estimated on the basis of the average AK-toU.S. price relationship for 1978 and 1979.

Annual physical unit prices (excluding AK) are calculated from the monthly motor gasoline prices calculated above and weighted by the monthly motor gasoline consumption volumes for \$tates from Highway Statistics.

\section{Phyatcal Unit Prices: 1974, 1975}

The Platt's price data for 1974 through 1975 cover only leaded regular motor gasolitne. Beginning in 1974, motor gasoline price data are also available from the $C P I$ for selected SMSAs. An SMSA price is assigned to each State with counties included in the definition of that SMSA; for the years 1974 through 1977, prices for 23 SMSAs cover 21 States. The State sssignments of SMSA prices for 1974 through 1977 are given in Table A32 on page 384. For 1974 and 1975, CPI prices are reported separately for leaded regular and leaded premium motor gasoline. According to the CPI Detailed Report, April 1986, these prices include taxes; the Platt's prices also include taxes. AK is the only State not covered by either of these two data sources; prices for AK are imputed for 1974 and 1975.

The Plat's regular leaded prices and the CPI regular and premium leaded motor gasoline prices, including taxes, are assigned to their re. spective States as shown in Table A31 and Table A32. If both sources cover a city, the $C P I$ price is used. The following 29 States are covered only by Plats's: AL, AR, AZ, CO, CT, DE, FL, IA, ID, LA, ME, MS, MT, NC, ND, NE, NH, NM, NV, OK, OR, RI, SC, SD, TN, UT, VT, WV, and WY. The following 15 \$tates are covered only by $C P I$ : CA, DC, GA, HI, IL, MA, MD, MI, MN, MO, NJ, NY, OH, PA, and WI. The following six States have bath Platr's and $C P I$ data for a particular city: IN, KS, KY, TX, VA, and WA.

All price observations assigned to a State, regardless of grade or data source, are added together and divided by the number of observations. As part of this calculation, Plott's prices are converted from cents per gallon to dollars per gallon.

Nejether Platz's nor the CPI reports price data for AK. The methodology of the estimation of annual AK prices is the same as used in 1976 and 1977.

Annual physical unit prices for the remaining 50 States (excluding AK) are calculated from the monthly motor gasoline prices calculated above and weighted by the monthly motor gasoline consumption volumes for States from Highway Statistics.

\section{Phyalcal Unit Pricos: 1970 Through 1973}

Monthly motor gasoline physical unit ptices for 1970 through 1973 are available only from Platr's, where city prices covering 49 States are reported in a table titled "Service Station Prices: Gasoline (Including Taxes)." These prices, as shown in Table A28, are for leaded regular gasoline only and include taxes.

Monthly average city prices from Platt's are assigned to the State in which the city is located. Platt ${ }^{t}$ city price assignments to States are given in Table A31.

Monthly composite prices for 1970 through 1973 are equal to the reported monthly Platt's prices or, if more than one city is available for a given State in a certain month, are a sinple average of the assigned city prices. The reported prices are converted from cents to dollars per gallon. 
M Platt's does not report data for either AK or HI for 1970 throngh 1973. 0 The methodology of the estimation of $\mathrm{AK}$ and $\mathrm{HI}$ prices is the same as that osed for 1976 and 1977 .

O Anmual physical unit prices (excluding AK and HI) are calculated from the monthly motor gasoltine prices weighted by the monthly motor gasoline consumption volumes for States from Highway Statilitics.

G

$\mathbf{A}$

5

\section{Btu Prices: AII Yeare}

Btu prices for States are computed by converting the physical unit prices in dollars per gallon to dollars per barrel (42 gallons per barrel) and then to dollars per million Btu (5.253 million Bth per barrel). U.S. Btu prices are calculated as the average of the State Btu prices, weighted by consamption data from SEDS.

\section{Data Bources}

Prices

1986 forwand: Energy Information Administration, Petrolewm Market ing Arnual, Table 29 (1986-1988), Table 30 (1989-1993), column titled "All Refiders, Sales to End Users. Through Company Outlets," and Table 35 (1994), colnmn titled "All Grades, Sales to End Users, Through Retail Qutlets."

1974 forward; Bureau of Labor Statistics, U.S. Department of Labot, Consunter Prices: Energy, computer printouts of monthly gasoline prices.

1983 forwand: Federal Highway Administration, U.S. Department of Transportation, Highway Statistics, Tables MF-26 (1983-1993) and MF33 BA (1994).

1983-1985: Energy Information Administration, Petroleun Marketing Anrual, 1985. Volume 1, Table 16, column titled "All Reftners and Gas Plant Operakors, Sales to End-nsers, Through Company Ovtlets."
1970-1982: MeGratw-Hill, Inc., Platt's Oil Price Hendbook and Oitmanac; table titled "AAA 'Fuel-gauge' Report" (1982); table titled "Platt's/Lundberg Summary," (1979-1981); and table titled "Service Station Prices: Gasoline (Includiog Taxes)," (1970-1978).

1974-1982: Bureau of Labor Statistics, CPI Detailed Report, Aprit 1986. Techmical Notes, page 110.

1982: Energy Iuformation Administration, Form EIA-25 "Prime Supplier Monthly Report" computer tape, unpublished data.

1976-1984: Energy Information Administration, Monthly Energy Review, January 1985, table titled "Petnotenril: Finisted Motor Gasoline Supply and Disposition."

Taxes

1983 forward (State Taxes): Federal Highway Administration, U.S. Department of Transpertation, Highway Statistics, Table MF-121T.

1991 forward (Federal Taxes): Energy Information Administration, Pe trokewn Marketing Annual, Table EN1.

1983 through 1990 (Federal Taxes): Energy Information Administration, Petnoleum Marketing Anunual, 1990, Table EN1.

\section{Censomptlon}

1970 forward: Energy Information Administration, State Energy Data System, transportation sector, motor gasoline consumption.

\section{Conversion Factor: Afl Years}

5.253 million Bta per barre] 
Petroleum cole is consnmed by the electric utility and industrial sectors. The portion of petrolenm coke consumed by the industrial sector $(97$ percent in 1994) is inckuded in the category "other petroleum products," which is discrused on page 399. The remaining petrolenn coke is used for electricity generation in 12 States over the period 1970 forward. A maximum of seven States reported consumption in any single year. Estimates of the annual consomption of petroleum coks by electric utilities are taken from SEDS. Estimates of the deliveries of petjoteum coke, used in the price calculation described below, are taken from the Cosf and Quality of Fuels for Electric Vitity Plants (C\&Q). The C\&Q prices are the delivered costs of the fuels and inclode all taxes, transportation, and other charges paid by the utilities.

\section{Btu Pricos: 1972 Fonwird}

The principal data sounce for petroleum coke used by electric utilities is an EIA public use computer tape covering the period 1972 forward. From 1972 throngh 1982, stenm plants with a maximum capacity of 25 megawats wene incladed. For 1983 and sthsequent years, the reporting threshold was raised to 50 megawattr capacity. In addition to the connputer tape, the data are also published, but not for the full period. From 1978 through 1982, $C \& Q$ was poblished mouthly and anmually; data for calculnting petrokum coke pricts ane in only the monthly reports. For 1983 and subseqnent years, $C \& Q$ was publisthed annually and includes petroleum coke prices for individual States and for the Nation.

The computer tape, Cost and Quality of Fuels for Electric Utility Plants (Form 423) Historic Files, shows quantity in chort tons, estimsted Btu per pound, and price in cents per million Bux. The data are presented by plant, by State, and by month. The Btu price is calcolated as the annual sum of the unit prices, weighted by the totel Bur in each reported delivery, divided by the annual som of the Btu delivered to all ejectric utility plants within the State.

Some States have electric utility petroleum cote consumption in SEDS but no deliveries of price data in the $C \& Q$. Those States are assigated prices from neighbaring States, as shown in Table A33. The petroleum coke consumed in LA in 1992 forward was obtained with no cost to the electric utility. The SEDS consumption is included with a zero price in calculating the electricity utility sector average price for LA and the US. The high DE prices prior to 1981 are actul teported prices.

\section{Gtu Prices: 1970, 1971}

For the years 1970 and 1971, prices are estimated by using the gross domestic product implicit price deflater. The deflator for 1970 or 1971 is divided by the 1972 deflator and the quotient is multiplied by the 1972 price for each State to develop the price estimates for 1970 and 1971. The deflators are 35.1 in $1970,37.1$ in 1971 , and 38.8 in 1972.

Although SEDS has a cousumption estimate for NJ in 1971, there are no NJ price data for any year in the Form 423 Historic Files. Form 423 data for PA in 1972 are used to estimate a PA price for 1971, which is assigned to NI. The Form 423 PA prices for 1972 and 1971 do nok appear in SEPEDS since SEDS has no petroleum coke contsumption in PA for those years.

\section{U.S. Btu Prices: Afl Voars}

U.S. Btu prices are calculated as the average of the State Btu prices, weighted by consumption data from SEDS.

Table A33. Potroleum Coks Elpetric Utifty Priog Assignmente, 1972 Forwand

\begin{tabular}{|c|c|c|}
\hline Stets & Yoats & \\
\hline $\begin{array}{l}\text { DE } \\
\text { KS } \\
M O \\
W Y\end{array}$ & $\begin{array}{l}1981-1992 \\
1975 \\
1993,1995 \\
1974 \\
1985\end{array}$ & $\begin{array}{l}P A \\
M 0 \\
M A \\
M A\end{array}$ \\
\hline
\end{tabular}


1972 forward: Energy Informtation Administration, electronic data from "Cost and Quality of Fuels for Electric Utility Plants (Form 423) Historic Files," as published in the following reports:

- 1983 forward: Energy Information Administration, Cost and Quality of Fuels for Electric Utijity Plents, Table 20 (1983, 1984), Table 12 (1985-7989), Table 40 (1990, 1991), and Table 28 (1992 forward).

- 1978-1982: Energy Information Administration, Cost and Quality of Fuels for Electric Utility Plants, table titled "Wood Chips, Refuse, and Petroleum Coke Used as Fuel by Steam-Electric Units."

1970-1972: Energy Information Administration, Annwal Energy Review 1992, Appendix C. Gross Domestic Product and Implicit Price Deflator.

\section{Consumption}

1970 forward: Energy Intormation Administration, State Energy Data System, electric utility petroleum coke consumption.

\section{Comveraton Fuctora: All Years}

No conversion factors are required; Btu prices are calculated directly from data sources.

Residual fuel prices are developed for the electric utility, industrial, commercial, and transportation sectors. Fstimates of the amount of residusal fuel consumed by sector are taken from SEDS and are adjusted for process fuel consumption in the industrial sector. (See the "Con-

\section{Electric Utility Sector}

The electric utility price for residual fuel (heavy oil) is the average delivered cost of No. 6 fue] oil receipts at electric utilities. For 1973 forward, Btu prices are developed directly from the data sources. For 1970 through 1972, prices are estimated by using simple regression analysis. All taxes, transportation, and other charges paid by vtilities are included in the prices for all years.

\section{Btu Pricus; 1979 fonwsird}

Electric utility sector residual fuel prices for 1973 forward are taken from Cost and Quality of Fuels for Electric Utility Plants (C\&Q). For 1973 through 1979, Btu prices are calculated as the weighted average of contract and spoc prices for No, 6 fuel oil. Table A34 lists the States and years for which consumption is indicated by SEDS but no price is shown in $C \& Q$. For these States, the Census division price, as slown in $C \& Q$, is assigned as the State price.

For 1983 forward, $C \& Q$ reports prices for steam electric plants of 50 megawatt capacity or greater. For 1980 through $1982, C \& Q$ prices cover all reporting plants of $\mathbf{2 5}$ megawatt capacity or greater.

The $\mathrm{C} \& Q$ does not have prices for AK in 1973 forward or $\mathrm{HI}$ in 1973 through 1982 and 1992 forward. Prices are estimated by calculating the ratio of the AK of HI prices from the Statistical Yearbook to the Statistical Yearbook U.S. price and multiplying the ratio by the C\& $Q$ U.S. price for each yeat. AK prices for 1973, 1975, 1978, and 1994 are not published in the Statisticel Yearbook and are estimated by calculating an average of the ratios of the AK to U.S. Statistical Yearbook prices in adjacent years. The 1973 estimated price is based on the average ratio for 1972 and 1974 , the 1975 price is based on the average ratio for 1974 and 1976. and the 1978 price is based on the average ratio for 1977 and 1979. The average ratio is then applied to the U.S. C\&Q price for the missing year. 
Table A34. Realdual Fuel Electric Wanty Cenaus Dlvision Price Assionments, 1970 Forward

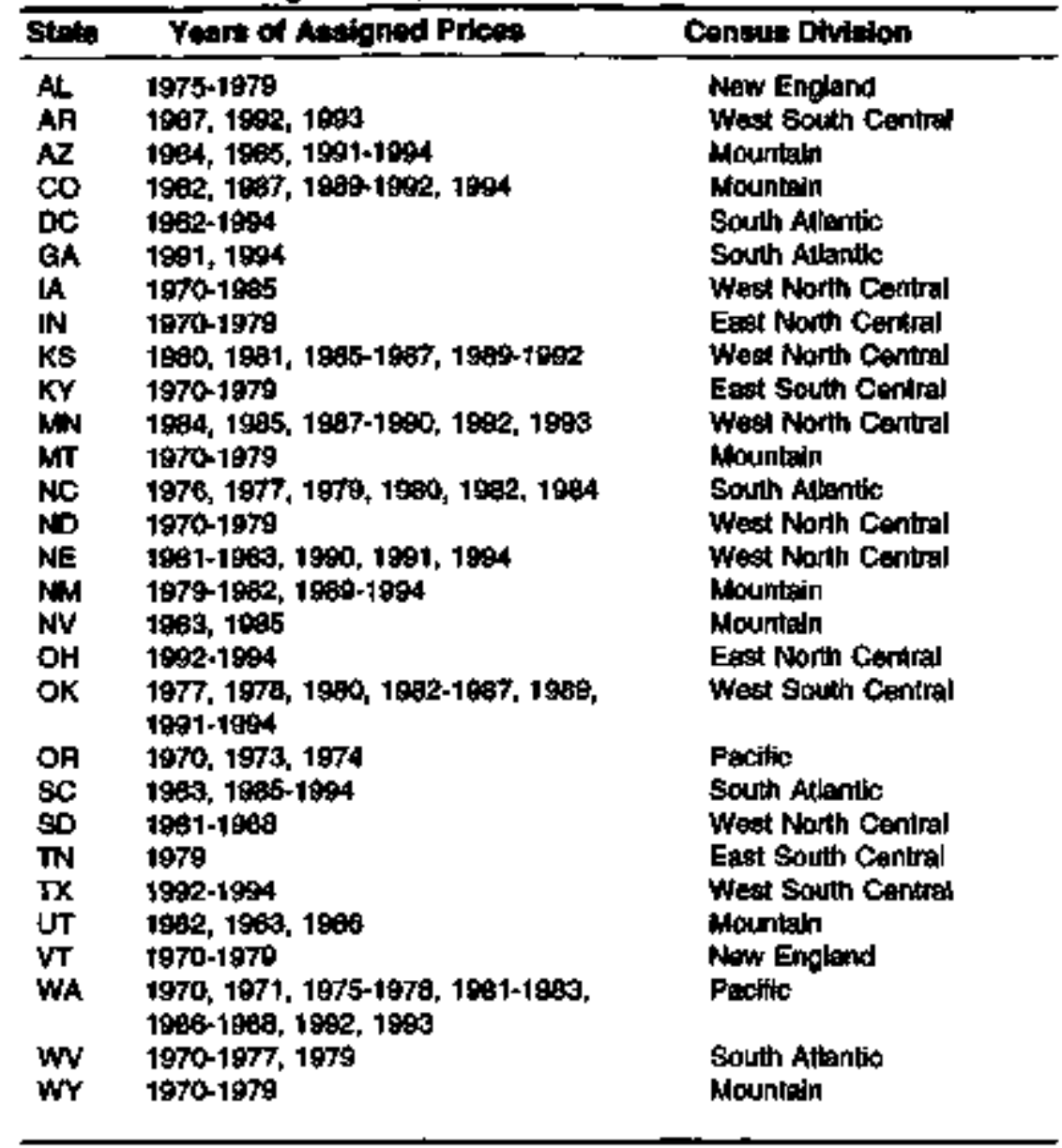

\section{Btu Prices: 1970 Through 1972}

State-level Bto prices for 1970 through 1972 are estimated by using regression techniques and price data from the Statistical Yearbook. The regression equations use Statistical Yearbook State-level prices for 1973 through 1980 as the independent variable and the State-level prices calculated above (inciuding the estimations for $\mathrm{AK}$ and $\mathrm{HI}$ ) as the dependent variable. Pacific regional price averages are assigned for the missing WA ptices in 1970 and 1971. The average of 1970 and 1972 AK Statistical Yearbook prices is substituted for the missing 1971 AK price.

\section{U.G. Btu Prices: All Years}

U.S. Bto prices are calculated as the average of the State Bun prices, weighted by consumption data from SEDS.

\section{Dota Sourcas:}

\section{Prices}

1973 forward: Energy Information Administration, Cost and Quality of Fuels for Electric Utility Plants, Table 6 (1973-1979), Table 45 (19801982). Table 51 (1983, 1984), Table 41 (1985-1989), Table 14 (1990, 1991), and Table 8 (1992 forward).

1970 forward: Edison Electric Institate, Stasistical Yearbook of the Electric Utility Industry, Table 43 (1970-1979), Table 26 (1980-1983). Table 28 (1984-1986), and Table 29 (1987 forward).

\section{Consumption}

1970 forward: Enetgy Information Adruinistration, State Energy Data System, electric utility sector heavy oil consumption.

\section{Convergion Factors: All Yeara}

Because Btu prices are avaitable directly from the data sources, no conversion factors are used.

\section{Industrial Sector}

The industrial sector residual fuel prices for 1984 forward are developed from refinerireselier prices of residual fuel as published in the Petroleun 
Marketing Annual. Residual fuel prices for 1970 through 1983 are calcufated or estimated by using average costs of residual fuel to manufactaring firms published in two Burean of the Census reports and Platt's OA Price Handboak and Oilnanac. Price data in these sources are available for the years 1971 and 1974 throngh 1981; prices for 1970, 1972, 1973, 1982, and 1983 are estimated. Prices for all years include taxes.

\section{Phystant Unit Pricos: 1004 Fonward}

Residual fuel inoustnal sector physical unjt prices are calculated by using refiner/reseller prices to end users from the Petroleum Marketing Annual (PMA). The States that do not have PMA prices are assigned their PAD district or subdistrict price as shown in Table A35, with the exception of AK. AK incustrial residual fuel price for 1984 forwatd is based on the WA industrial residual fuel price and the ratio of the AKto-WA industrial distillate fuel prices for each year. Tax data are added to develop final sector prices.

\section{Physical Unil Prices: 1982, 1083}

After 1981 , the U.S. Department of Commerce's Annual Survey of Honufactures and the Census of Manufactures (ASM/CM) ceased publication of fuel-specific State-level residual fuel data from which prices can be calculated. Prices for 1982 and 1983 are estimated from the average relationship between the ASM/CM-based prices generated for 1978 through 1981 and the assigned Platt's No. 6 foel oil prices for 1978 through 1981 (Table A36). These average ratios are calculated at the State-jevel for all States except AK, which shows no judestrial sector residital fuel use reported in SEDS for 1982 and 1983. Physical unit residual fuel industrial prices for 1982 and 1983 are calculated by using the assigined Platt's prices for 1982 and 1983 (Table A36) and the Statelevel average ratios. The resulting estimates implicitly include taxes that reflect individual State differences.

\section{Physical Unit Pricea: 1971, 1974 Through 1981}

For the years 1971 and 1974 through 1981, industrial sector residual prices are calculated directly from cost and quantity data reported by the

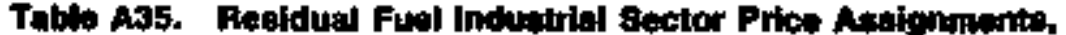
1994 Forwacd

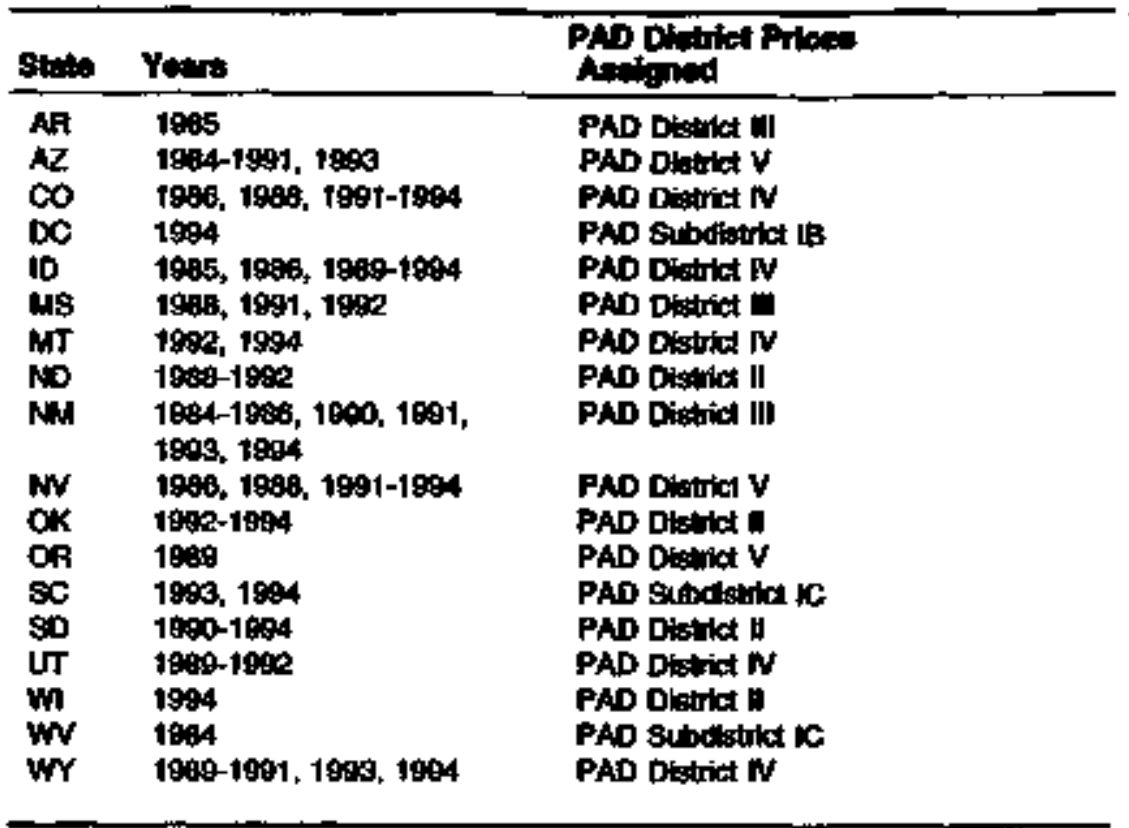

$A S M / C M$. For all States with avalable cost and quantity data, prices are equal to the average cost of residual fuel to manufacturers. Taxes are included in the publisted cost data. Missing data for these years are assigned from the average prices of adjacent States, as shown in Table A37.

Physical Unit Priced: $1970,1972,1973$

Since ASM/CM data are not available for 1970, 1972, or 1973, prices for these years must be extimated. Physical unit prices are based on the ratio of the $1971 \mathrm{CM}$ prices to the 1971 assigned No. 6 fuel cil prices from Platt's OA Price Handbook and Oilmanac (Table A36). The estimated $1971 \mathrm{CM}$ prices for NM and WY are used in the calculations. The resulting ratios for each State are used with the Platt's assigned prices for 
Table A38. No. 6 Fual Ol Price Ansignanente from Platt's, 1970-1983

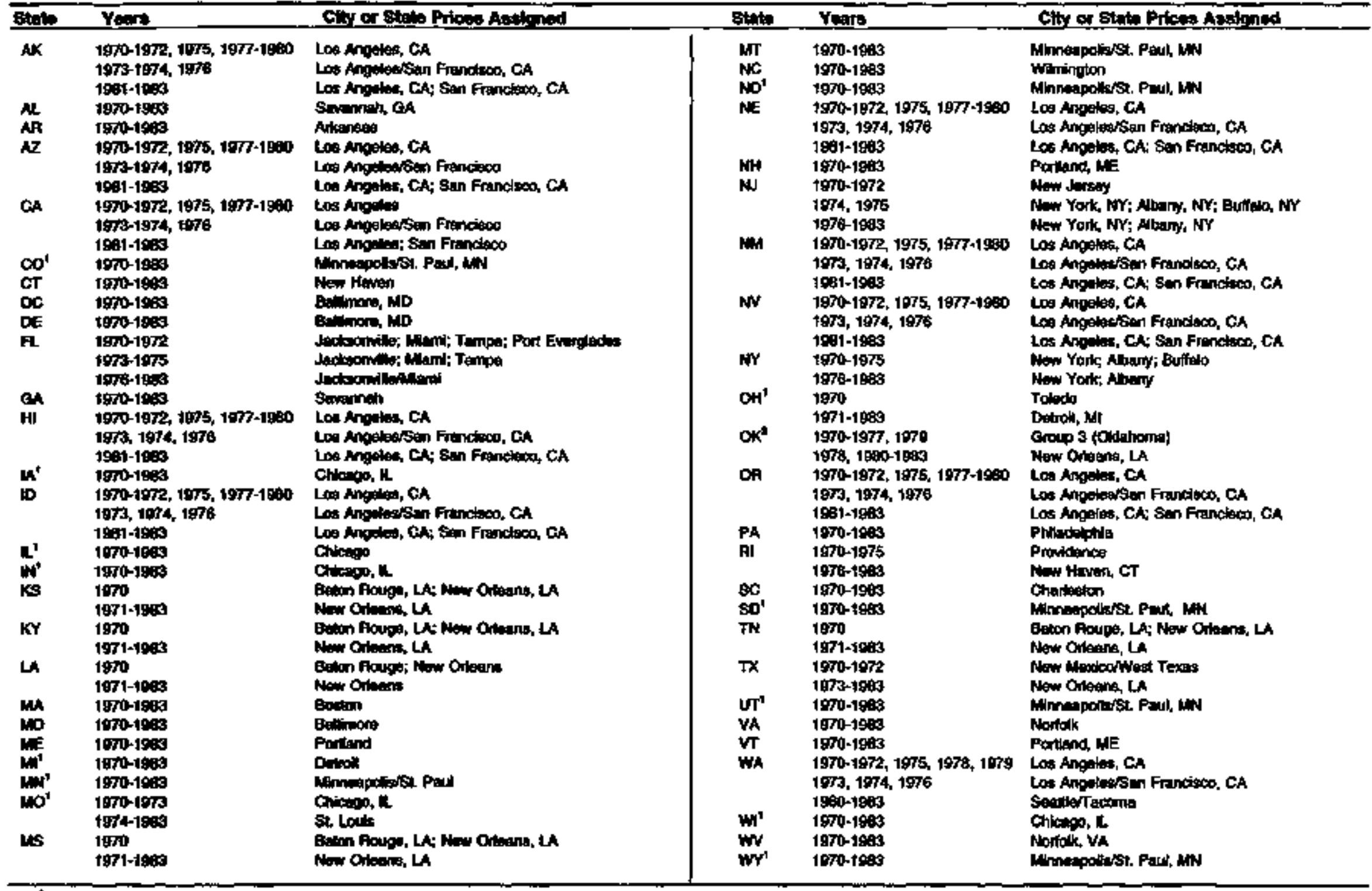

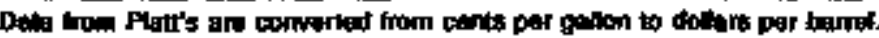

As ahown in Plolit: 
R 1970, 1972, and 1973 to estimate prices. The final estimates implicitly include State-specific taxes.

\section{Blu Prices: All Yours}

Btu prices for States are calculated from the physical unit prices and the conversion factor, U.S. Btu prices are calculated as the average of the

L State Btu prices, weighted by consumption data from SEDS, which are adjusted for process fuel consumption.

\section{Datg Sources}

L Prices

1984 forwad: Euergy Ibforration Administration, Petroleum Marketing Annual, Table A4 (1984-1990), Table A3 (1991 fotward).

1984 forward: Industrial sector distillate fuel price estimates from the SEPER data system (AK and WA only).

1970-1983: McGraw-Hill, Inc., Plat's Oil Price Handbook and OHmanac, refinery and terminal prices for No. 6 fuel oil, average of highs and lows.

Table A37. Restdual Fuel Induatrial \$ector Price Aasigrtments, $1971,1974-1981$

\begin{tabular}{|c|c|c|}
\hline Sinte & Yeas: & Strite Prlooe Uted \\
\hline $\begin{array}{l}\text { AK } \\
\text { DC } \\
M T \\
\text { NOD } \\
\text { NV } \\
\text { OK } \\
\text { SD } \\
W Y\end{array}$ & $\begin{array}{l}1980,1981 \\
1979-1981 \\
1974-1979 \\
1990 \\
1971,1974-1981 \\
1974-1978 \\
1974-1978,1980 \\
1985 \\
1071,1974-1981\end{array}$ & $\begin{array}{l}\text { HI, WA } \\
\text { MD, VA } \\
\text { ID, ND, SD } \\
\text { MN, MT, SD } \\
\text { AZ, CO, TX } \\
\text { AZ, CA, ID, OF, UT } \\
\text { AF, CO, KS, MO, TX } \\
\text { IA, MN, MT, ND, NE } \\
\text { CO, NE, UT }\end{array}$ \\
\hline
\end{tabular}

1971, 1977, 1981: Bureau of the Census, V.S. Department of Commerce, Census of Manufactures, Fuels and Electric Energy Consumed. Part 2, Table 3. (Dates shown on the report covers are, respectively, 1972, 1977, and 1982.)

1974-1976 and 1978-1980; Bureau of the Census, U.S. Department of Cornmerce, Annual Survey of Manufactures, Fuels and Electric Energy Consuned, Stotes by Industry Group, Table 3.

\section{Taxes}

1994: U.S. Advisory Committee on Intergovernmental Relations, Signifieant Features of Fiscol Federalison, Tables 14 and 26.

1993: Bureau of the Census, U.S. Department of Conumerce, State Tax Review, Volume 34, No. 31, map titled "State Gasoline, Sales, and Cigarette Tax Rates as of July 1, 1993," sales tax rates.

1987-1992: Bureau of the Census, U.S. Deparment of Commerce, State Government Tax Collections, Table 8, column titled "Percentage rate, September $1 .{ }^{\circ}$

1984-1986: Bureau of the Censas, U.S. Department of Commerce, Statistical Abstract of the United States, table titled "State Government Tax Collections and Excise Taxes," colnmn titled "Excise Taxes, General sales and gross receipts."

\section{Consumption}

1970 forward: Energy Information Administration, State Energy Data System, industrial sector residual constanption.

\section{Converton Factor: All Yosro}

6.287 million Btu per barrel. 


\section{Commercial Sector}

For 1984 forward, State-level commercial sector residual fuel prices are developed from refiner/reseller prices of residual fuel to end users published in the PMA. For 1970 through 1983, cornmercial sector residual fuel prices are estimated for all States from uational-ievel residual fuel prices and the State-level electric utility sector residual fuel prices. State and Federal taxes are included in the final prices for all years.

\section{Phyalest Unt Pricas: 1984 Fanward}

Commercial sector residual fuel physical unit prices are based on refiner/teseller prices to end users from the PMA. The States that do not have PMA prices are assigned their PAD district or subdistrict price (Table A38), with the exception of AK. The AK commercial tresidual fuel prices for 1984 forward are based on the WA commercial residual fuel price and the ratio of the AK-to-WA commercial distillate fuel prices for each year. Tax data are added to develop final prices.

\section{Phyofeal Unh Prices: 1976 Through 1983}

The commercial sector residual fuel physical unit prices for 1976 through 1983 are estimated from the electric utijty residual fuel prices and the U.S. average retail residual fuel prices (with taxes added) for each year. The resulting price estimates implicitly include taxes that teflect individual State differences.

1. The first step in the estimation of the commercial residual fuel physical unit State prices is to convert the State-level tax rates reported in the Bureav of the Census publications into the volume-weighted average U.S. sales tax rate by using commercial residual consumption data from SEDS.

2. A preliminary $U . \$$. residual fuel oil price, including taxes, is computed by using the average U.S. tax rate estimated above and the annual average U.S. residual fuel price to end users (average retail price excluding taxes) from the Monthly Energy Review (MER).
Table A38. Residual Fut Commercial sector Price Afalgnment*, 1984 Forward

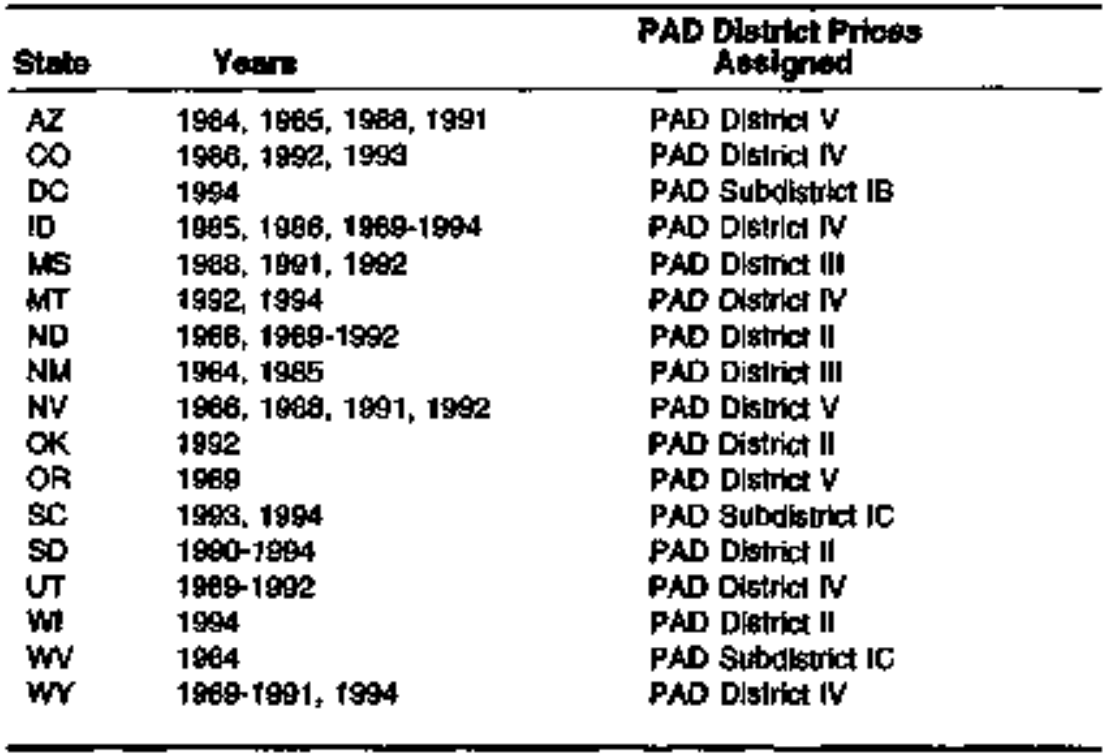

3. Commercial sector physical unit residual fuel prices for States are computed by using the electric utility sector tesidual fuel prices. To do this calcalation, the ratio of the State-level and U.S. prices in the commercial sector is assumed to be the same as the ratio of State and U.S. prices in the electric utility sector. Some States are missing electric utility ptices for 1976 through 1983 ; these are estimated by using adjacent States' average prices (Table A39).

\section{Phystcal Unit Prloss; 1970 Through 1975}

Because no national or State-level retail residual prices are available from published data sovices, commercial sector residual prices for 1970 through 1975 are estimated. The estimation method is based on the assumption that the average ratio of State-to-U.S. prices is the sarne in the commercial and electric utility sectors. The average ratio for 1976 through 1979 of the MER U.S. tax-adjusted prices to the electric utility sector U.S. prices is calculated and used as an adjustment factor with 
Table A39. Realdual Fuga Commonetal Sector Price Aestgnmemte, 1970-1893

\begin{tabular}{|c|c|c|}
\hline Etate & Yanre & $\begin{array}{l}\text { Stenc Pritoin Uated } \\
\text { in the Estingtion }\end{array}$ \\
\hline A & $\begin{array}{l}1 \$ 70-1974,1530,1602 . \\
1093\end{array}$ & FL, CA, MS \\
\hline ID & $\begin{array}{l}\text { 1900, 1961, } 1969 \\
1982\end{array}$ & $\begin{array}{l}\mathrm{CA}, \mathrm{CO} \\
\mathrm{CA}\end{array}$ \\
\hline IN & $1490-1960$ & IL, MA, OH \\
\hline KY & $1900-1989$ & IL $\mathbf{W O}, \mathbf{G H}, \mathbf{V A}$ \\
\hline $\mathbf{M T}$ & $\begin{array}{l}1980,1893 \\
1982\end{array}$ & Co, $\mathrm{m}$ \\
\hline $\mathrm{NC}$ & 1981. 1863 & Gh va \\
\hline No & 1980,1908 & $M N_{1}$ SD \\
\hline & 1931,1942 & MN \\
\hline OA & $1075-1983$ & Ch \\
\hline IN & 1970-1978, 1960-1983 & AR, GA, MO, MS, VA \\
\hline VT & $1880-1893$ & ME, MH, NY \\
\hline $\mathbf{w}$ & 1992,1993 & L, Ml, MN \\
\hline$w$ & $1990-1960$ & MD, OH, PA, VA \\
\hline$w r$ & $\begin{array}{l}1990 \\
1961,1999\end{array}$ & $\begin{array}{l}\infty 0, N E, \text { SD, UT } \\
C \infty\end{array}$ \\
\hline & 1902 & Mnt \\
\hline
\end{tabular}

\section{Bitu Pricos: Afl Years}

Btu prices for Strtes are calculated from the physical unit prices and the conversion factor. U.S. Btu prices are calkulated as the average of the Stute Btu prices, weighted by consurmption data from SEDS.

\section{Dats Sources:}

\section{Prices}

1984 forward: Energy Information Administration, Pefnoleum Marketing Annwal, Table A4 (1984-1990), Table A3 (1991 forward).

1984 forward: Commercial sector distillate fuel price estimates from the SEPER data system (AK and WA only).

197B-1983: Energy Information Administration, Monthly Energy Review, December 1988, table titled "Refiner Sales Prices of Rezidual Fuel Oil," column titled "Average Sales to End Users."

1976. 1977: Energy Information Administration, Morthy Energy Review, December 1983, table titled "Average No. 6 Residnal Fuel Oil Prices," cohumn titled "Average, Retail."

1970-1983: Electric utility sector residual foel price estimates (in physical units) from the SEPER data system.

Taxes

1994: U.S. Advisory Committee on Intergovernmental Relations, Significant Features of Fiscal Federaltsm, Tables 14 and 26.

1993: Bureau of the Census, U.S. Department of Commerce, Srate Tax Review, Volume 54, No. 31, map titled "State Gasoline, Sales, and Cigatette Tax Rates as of July 1, 1993," sales tex rates.

1987-1992: Burean of the Census, U.S. Department of Commerce, State Goverment Tax Collections, Table 8, column titled "Percentage rate, September 1." 
1976-1986: Bureav of the Census, U.S. Depertment of Commerce, Statistical Abstract of the United States, table titled "State Government Tax Collections and Excise Taxes," column titled "Excise Taxes, General sales and gross receipts."

\section{Consenaption}

1970 forward: Energy Information Administration, State Energy Data System, commercial sector residual fuel consumption.

\section{Convorsion Fuctor: AH Yous:}

6.287 million Btu per barrel

\section{Transportation Sector}

Residual fuel is consumed in the transportation sector for vessel bunkering, military use, and railioads. In 1970, vessels consamed 74 percent of the transportation use of residural fued, and the military and railroads accounted for 24 percent and 2 percent, respectively. By 1994, vessel use had grown to 99 percent, military use had fropped to 1 percent, and the railroads' share was insignificant. Prices are developed for vessel bunkering, and electric utility prices are assigned to the military and railroad uses. Tax adjustments ane made as described below. The transportation sector average price for each State and year is the consumption-weighted average of the prices of the three nses.

\section{Phyaled Unt Pricos: AH Years}

Vescel Bunlering. Fhysical unit prices are calcnlated from actual or estimated U.S. average bunker C prices and electric utility State and U.S. residual fuel prices for each year. The ratio of U.S. bunker C price to U.S. residal forel electric ulility price is multiphied by the State residal fuel electric utility price to obtain the State butrer C price. Taxes are calculated as described for the commercial sector (1976 throngh 1983) and added, so that final State vessel bunkering price estimates implicitly include State-specific taxes. Other procedures are described separately by groups of years:
1. For 1982 forward, national averaze prices for residual fuel with sulfur content greater than 1 percent are taken from the Anrual Energy Review and used as proxies for bunker $C$ prices.

2. For 1975 though 1981 , mational average bunker $C$ prices are available ftom the Monthly Petroleum Product Price Report (MPPPR). Annual average U.S. prices for 1975 and 1976 are calculated as the simple average of the monthly prices for each respective year because annial average prices are not shown in the MPPPR.

3. For 1970 through 1974 , no U.S. bunker C prices are available. To estimate State-level prices for these years, the average ratio of published bunker C prices and electric utility sector prices for 1975 trough 1979 is calculated and multiplied by the State-level electric ntility prices for 1970 through 1974.

Missing State prices are assigned adjacent States' average prices as shown in Table A40.

Mitiary und Railroad Use. For all years, electric utility sector residual fuel prices are assigned to military and raitroad uses. The electric utility prices include taxes. Since the military does not pay State taxes, their prices are adjusted to remove taxes. Estimation of the tax adjustment is described under the commercial sector.

In some cases, States have no reported consumption of residual fuel oil in the electric utility sector and, therefore, no final price in that sector. Since the electric utilities sector prices are used as a base in the calculation of prices in the other sectors, intermediate prices are calculated for $O R$ in 1971 and for all States that need prices for 1987 forward.

Average Prices. Transportation sector prices are the average of bunker fuel, military, and railroad prices, weighted by each category's share of cotal transportation consumption from SEDS.

\section{Btu Pricea: An Vears}

Btu prices for States are cakulated from the physical unit prices and the residual fuel conversion factor. U.S. Bto prices are calculated as the average of the State Btu prices, weighted by consumption data from SEDS. 


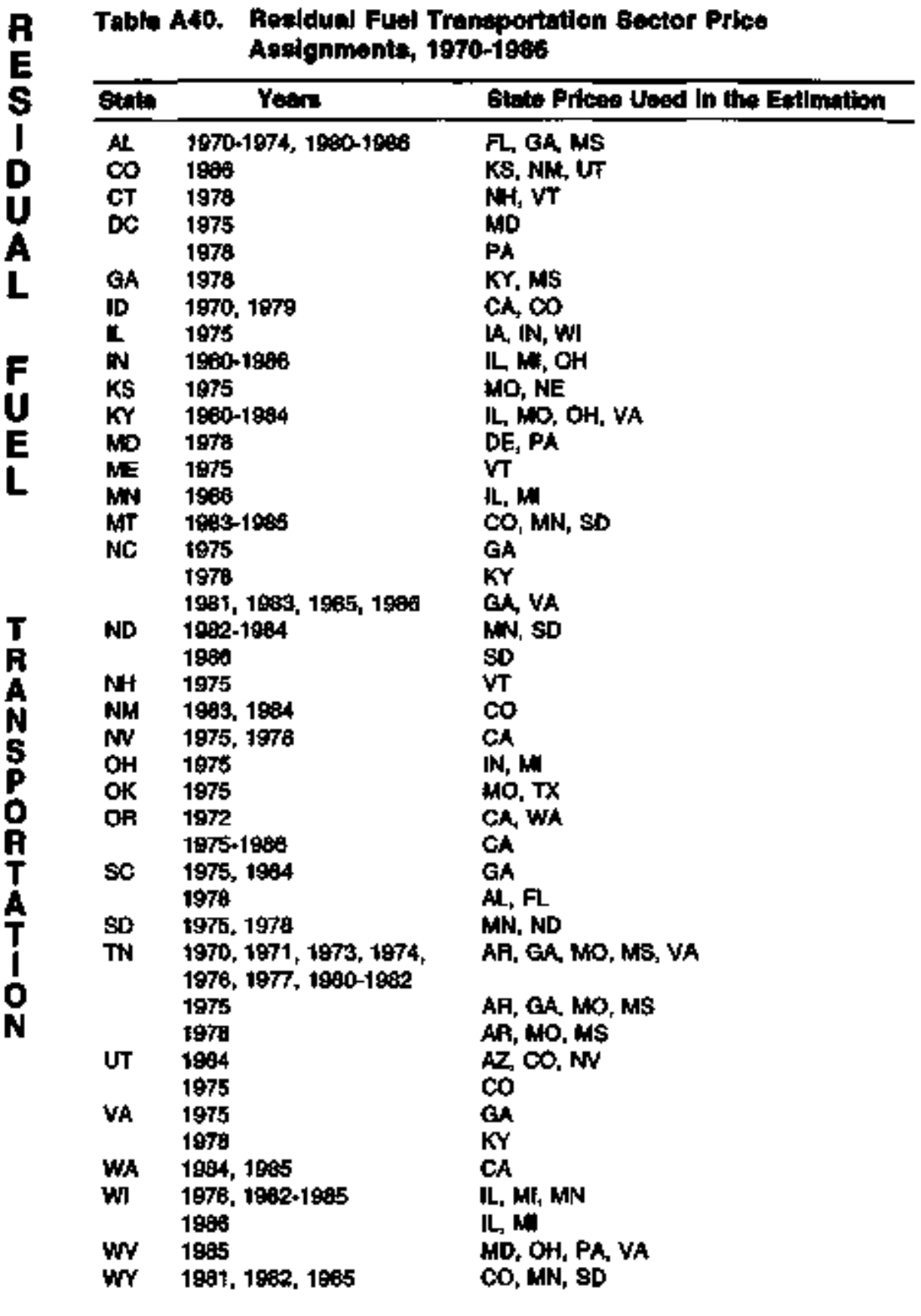

\section{Dita Bouroe:}

\section{Prices}

1982 forward: Energy Information Administration, Annual Energy Review 1995, Table 5.20, row titied "Sajes Prices to End Users, Residual Fuel Oil, Greater Than 1 Percent Sulfur Content."

1976-1981: Energy Information Administration, Monthly Petrolewm Product Price Report, Table 3.

1975: Federal Energy Administration, Monthly Petroleum Product Price Report, Table 3.

1970-1986: Electric utility sector residual fuel price estimates (in physical units) from SEPEDS.

\section{Taxes}

1994: U.S. Advisory Committee on Intergovemmental Relations, Significant Features of Fiscal Federallsm, Tables 14 and 26.

1993: Bureau of the Census, U.S. Department of Commerce, State Tax Review, Volume 54, No. 31, map titled "State Gasoline, Sales, and Ctgarette Tax Rates as of July 1, 1993," sales tax rates.

1987-1992: Bureav of the Census, U.S. Department of Commerce, State Government Tax Collections, Tabje 8, column titled "Percentage rate, September 1."

1976-1986: Bureaz of the Census, U.S. Department of Commerce. Statistical Abstract of the United States, table titled "State Govemment Tax Collections and Excise Taxes," colnmn titled "Excise Taxes, General sales and gross receipts."

\section{Consumption}

1970 forward: Energy Information Administration, State Energy Data System, transportation sector residual tuel consmomption, including the subcategories for vessel bunkering, military, and railroad uses. 


\section{Convarsion Fuctor: All Yarrs}

6.287 million Btu per barrel.

Sixteen separate products are included in the category called "other petroleum." Of the 16 products, prices are developed for the 7 noted with asterisks (*) below and described in the following paragraphs. All of these products are assigned to the industrial sector:

1. Aviation gasoline blending components

2. Crude oil

3. Miscellaneous products (*)

4. Motor gasoline blending components

5. Natural gasoline, including isopentane (1970- 1983)

6. Pentanes plus (1984 forward)

7. Petrochemical feedstocks, naphtha (*)

8. Petrochemical feedstocks, other oils $\left(^{*}\right)$

9. Petrochernical feedstocks, still gas (1970-1985) (*)

10. Petroleum coke (*)

11. Plant condensate (1970-1983)

12. Special naphthas (*)

13. Still gas

14. Unfinished oils

15. Unfractionated stream (1970-1983)

16. Waxes (*).

\section{Physical Unit Prices: All Years}

Only national-kvel prices are developed for the seven other petroleun products becanse State-ievel price information is not available, and taxes are not included in any of the estimates. Consumption for the other nine product 5 are completely removed as process fuel or intermediate products. (See the "Consumption Adjustments for Calculating Expenditures ${ }^{\text {tx }}$ section on page 413.)

Starting in 1984, three products-natural gasoline, plant condensate, and unfractionated stream-are dropped, and pentanes plus is added in the Energy Information Administration (EIA) reporting system that is the basis of the consumption estimates. Natural gasoline (including isopentane) and plant condensate are reported together as the new product, pentanes plus. Unfractionated stream is dropped because its components are reported separately as liquefied petroletum gases.

\section{Miscellsneous Products}

The products in this category vary from inexpensive (absorption oils similar to kerosene) to very expensive (bydraulic fluids). The price estimates are based on the evidence presented in the Bureav of Mines Mirerals Yearbooks of the $1970^{\circ}$ 's indicating that the greater part of the miscellaneous product line consists of finished petrochemicals, especially the aromatic hydrocabons: benzene, toluene, and the xylenes.

Price estimates for 1972, 1977, 1982, 1987, and 1992 are taken from Census of Mantufactures (CM) data on quantity and value of "aromatics" and "other finished petroleum products" shipped by petrolenm refining industries, i.e., Standard Industrial Code (SIC) 2911. The ratio of miscellaneous-products-to-crude-oil price tor these 5 years varies widely. The following ratios, shown rounded, are used to estimate miscellaneons products prices for the years indicated:

$$
\begin{array}{ll}
\text { 1970 - 1974: } & 1.91 \text { times the crude oil price } \\
\text { 1975 }-1979 ; & 2.42 \text { times the crude oil price } \\
1980-1984: & 1.56 \text { times the crude oil price } \\
1985-1989: & 1.99 \text { tomes the cride oil price } \\
1990-\text { forward: } & 1.86 \text { times the crude oil price. }
\end{array}
$$

Quantity data for 1992 are publistied in pounds and are converted to barrels by use of the conversion factors of 7.282 pounds per gallon and 42 gallons per barrel. 


\section{Frice Datis Somress}

1970 Forward: Eaergy Information Administration, Annual Energy Review 1995. Table 5.19, column titled "Composite, Nominat."

1972, 1977, 1982, 1987, 1992; Bnreau of the Census, U.S. Department of Commerce, Census of Marufactures, data for Standard Industrial Code (SIC) 2911 on "Quantity and Value of Shipments by All Producers" as shown in Table 6a from MC77-1-29A. Product Codes 2911054, 2911056 (1972 and 1977); Table 6a-1 from MC87-I-29A, Product Codes 2911DS5 and 2911D57 (1982 and 1987); and Table 6\#-1 from MC92-I29A. Product Codes 2911D 55 and 2911D 57 (1992) .

\section{Fryiegl Unit Conversion Fecters}

1992: Gas Processors Suppliers Association in cooperation with the Gas Processors Association, Engineertrg Duta Book, 9th Edition, 1972. 4th Revision, 1979, pages 16-2 and 16-3, lines 42-47.

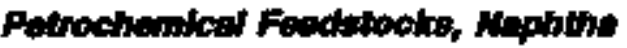

Naphthas for petrochemical feedstock use are those oils with boiling poings less then $401^{\circ}$ F. Consumer prices for 1978 through 1980 are derived from the special Antural Survey of Manufactures (ASM) series on "Hydrocarbon, Coal, and Coke Mrterials Consumed" by using data for industries in SIC 2869 (industrial organic chemicals) and SIC 2821 (plastics materials, synthetic resing, and nonvulcauizable elastomers). A price extimate for 1982 is obtained from the $C M$, and is based on data for SIC 2869 only. Since the ratio of petrochemical-niqutha-to-crideoil price is reasonibly constant in 1978, 1979, 1980, and 1982, the simple avernge of the foar ratios, 1.23, is used to estienate prices for petrochenical feedstocks, naphthes, for all other years.

\section{Prise Dato Secres}

1970-1977, 1981, 1983 forward: Energy Information Administration, Annual Enersy Review 1995. Table 5.19, column titled "Composite, Nominat."
1982: Bureau of the Census, U.S. Department of Commerce, $1982 \mathrm{Cen}$ sus of Mansfactures, M82-[-28F-3(P), page 6, SIC 2869.

1980: Burean of the Census, U.S. Department of Commerce, $1980 \mathrm{An}$ nual Survey of Manufactures, M80(AS)-4.3, page 9. SIC 2821.

1978, 1979: Bureen of the Census, U.S. Department of Commerce. 1979 Annual Survey of Morafactures, M79(AS)-4,3, page 8, SIC 2821 and 2869 .

\section{Petrochinical Fadotocks, Othar Oifs}

Petrochemical feedstocks referred to as "other oils" or "gas oils" are those oils with boiling points equal to or greater than $401^{\circ} \mathrm{F}$. Consumer prices for 3 years are obtained from the data on gas oils presented in the special ASM series on hydrocarbons consumed by psing data for industries in SIC 2865 (cyclic crodes and intermediates). The other-oils-tocnide-oil price ratio is quite stable, and the average ratio for the 3-year period, 1.607, is used to estimate prices for petrochemical feedstocks, other oils, for all other years.

\section{Price Date Serurtes}

1970-1977, 1981 forward: Energy Information Admituistration, Arnual Energy Review 1995, Table 5.19, column titled "Composite, Nominal."

1979, 1980: Bureav of the Censos, U.S. Department of Commerce, 1980 Antual Survey of Manufactunes, M80(AS)-4.3, page 9, STC 2865.

1978: Burenu of the Census, U.S. Department of Commerce, 1979 ARanal Survey of Maraffactures, M79(AS)-4.3. page 8. SIC 2865.

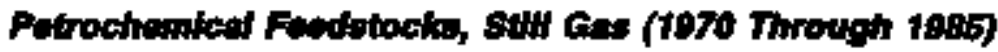

The source date for still gas is a mixture of consumer prices and producer prices for industries in SIC 2869 and SIC 2911 (petroleum refining). The still-gas-to-cnide-oil price ratio is somewhot variable becanse still gas is a highly variable gaseons mixture. Value and qunntity are available for 1972, 1977 through 1980, and 1982. In imputing prices 
for years when data from the CM or $A S M$ are not available, the average still-gas-to-crude-oil price ratio, 0.759, is used. After 1985, EIA data series no longer report feedstoct and refinery use of still gas separately and all SEDS industrial consumption is removed from SEPER. (See the "Consumption Adjustraents for Calculating Expenditures" section on page 413.)

\section{Price Dotw Sources}

1970, 1971, 1981, 1983-1985: Energy Information Administration, Annual Energy Review 1995, Table 5.19, "Composite. Nominal."

1982: Bureau of the Census, U.S. Department of Commerce. $1987 \mathrm{Cen}$ sus of Manufactures, MC87-1-29A. Table 6a, SIC 2911.

1979, 1980: Bureau of the Census, U.S. Department of Commerce. 1980 Anлwal Swrey of Maruffactures, M80(AS)-4.3, page 9, SIC 2869.

1978: Barean of the Census, U.S. Departmeat of Commerce, I979 Annual Survey of Marufactures, M79(AS)-4,3, pHge 28, SIC 2869.

1972, 1977: Bureau of the Census, U.S. Department of Commerce. 1977 Census of Manufactures, MC77-1-29A, page 29A-20. SIC 2911.

\section{Potroletw Colte}

Petroleum coke is consumed by the industrial and electric utility sectors. The portion of petroleum coke consumed by the electric atility sector (ahout 3 pencent in 1994) is described in the Petrolewm Cole (Utilities) section on page 387. The remaining petroknm cole is marketed to industrial consumers in two forms, calcined and uncalcined. Calcined coke is about three times as expensive as oncalcined. A quantityweighted U.S. average price is calculated by using U.S. Department of Commerce exports data and is assigned to all States with industrial petroleum coke consumption. The weighted average price is calculated by dividing the sum of the valoes of calcined and uncalcined petroleum coke exports by the sum of the two quantities exported. The physical vait conversion factor for petrolenm coke is $\mathbf{5}$ barrels per short ton.

\section{Price Date Sources}

1989 forward: Buread of the Census, U.S. Department of Commence, December issues of EM-345. Foneign and Domestic Exparts, For Petroleum Coke, Not Calcined, Commodity 2713110000 and Petroleum Coke, Calcined, Commodity 2713120000 .

1988: Bureab of the Census, U.S. Department of Commence, December issue of EM-522, U.S. Exports, Schedule B, Community by Coturery, PLtrolenm Coke. Except Calcined, Commodity 5213150, and Petroleum Coke, Calcined, Commodity 5175120.

1987: Burean of the Cengus, U.S. Department of Commence. December issue of EM-622, U.S. Exports, Schedule B, Conuradity by Cowatry, Petrolenm Coke, Except Calcibed, Commodity 5213150, and Petroleum Cole, Calcinod, Comnodity 5175120.

1986: Bureas of the Censos, U.S. Department of Commexce, Decensiber issue of EM-546, U.S. Exports, Schedule B, Cominodity by Country, Petroleum Coke, Except Calcined, Commodity 5213150, and Petrolenm Coke, Cakcined, Commodity 5175120.

1978-1985: Burean of the Cenpus, U.S. Department of Commerce, FT446, U.S. Exports, Schedule B. Commodity by Country, Petroleum Coke, Except Calcined, Contunodify 5213159, and Petrolean Coke, Catcined, Commotity 5175120.

1970-1977: Burean of the Census, US. Department of Commeree, December issnes of FT-410, U.S. Exports, Schedule B, Commodity by Country, Petroleum Coke, Except Caleined, Commodity 3329420, and Petroleum Coke, Calcined, Commodity 3329410.

\section{Spcoid Nophtho}

Prices for special naphthas are developed as the simple averages of the city ptices for "varmish makers and painters saphitan" and two types of "solvent naphtha" that are published in the Chessical Martefing Reporter. For 1984 forwant the prices are averaged from the first issue of each month; for 1974, 1979, and 1980, when petroletim prices were increasing rapidly, prices are averaged from 10 randomly-selected issues; 
O and for all other years, ptices are averaged from at least 5 randouly7 selected issues.

\section{Price Data Source}

1970 forward: Schnell Publishing Co., Inc., Chemical Marketing Reporter, selected monthly issues.

\section{Waxos}

Waxes data include fully refined crystailine wax, other refined crystalline wax, and microcrystalline wax. Price estinates for 1970 through 1973 and 1986 forward are obtained by dividing the value of exports by the quantity exported. For 1974 through 1985, prices are estimated by applying price indices to a representative base price. PTojucer prices for 1967 for the three waxes are available from data in the 1967 Census of Manufactures. A weighted-average price for 1967 of $\$ 15.75$ per barrel is obtained by summing the values of shipments of the three waxes and dividing the sum by the total quantity shipped. An annual composite price index for these three waxes is listed in the Bureau of Labor Statisrics publication Producer Prices and Producer Price Indexes for April 1974 through June 1985. Price estimates for 1975 through 1984 are derived by multiplying the pubitished price indices by the estimated 1967 base price. The indices for 1974 and 1985 are estimated as the simple average of monthly price indices that are available for that year. The physical unit conversion factors for wax are 280 pounds per barrel; and 1 pound equals 0.45359237 kjlograms.

\section{Price Data Sources}

1989 forward: Bureat of the Census, U.S. Department of Commerce. December issues of Report No. EM-545, titled Foreign and Domestic Exports for Paraffin Wax Less Than 0.75 Percent Oil (Commodity 2712200000 ) and Other Mineral Waxes NESOI (Commodity 2712900000 ).

1987, 1988: Bureau of the Cepsus, U.S. Department of Commerce, December issues of Report No. EM-546 (1987) and EM-522 (1988). titled U,S. Exports, Schedule B, Commodity by Country for Paraffin Wax and
Other Petroleum Waxes Unblended incl Microcrystalline Wax (Commodity 4925200 ).

1986: Bureau of the Census, U.S. Department of Commerce, Dexember issue of EM-546, U.S. Exports, Schedule B, Commodity by Country for Paraffin Wax, Crystalline, Pully Refined (Commodity 4925210), Paraffin Wax, Crystalline, Except Fully Refined (Commodity 4925220), and Petroleun Waxos, NSPF incl Microcrystatline Wax (Commodity 4925240).

1974-1985: Bureau of Labor Statístics, U.S. Department of Labor, Producer Prices and Producer Price Indexes, Annual Stpplement. Commodity Code 0577 .

1974-1985: Bureav of the Census, U.S. Department of Commerce, Censks of Manufactures, 1967, page 29 A-15, quantity and value of shipments of waxes in 1967.

1970-1973: Bureal of the Cersus, U.S. Department of Commerce, December issues of FT-410, U.S. Exports, Schedule B. Commodity by Country for Paraffin Wax, Crystalline, Fully Refined (Commodity 3326220), Paraffin Wax, Crystalline, Except Fully Refined (Commodity 3326230), and Microcrystalline Wax (Commodity 3326210).

\section{Btu Prices; All Years}

Btu prices for the seven petroleum products are calculated by converting physical unit prices from dollars per bartel to dollars per million Btu by using the conversion factors shown in Table A41. The U.S. average price that is developed for each product is assigned to the industrial sector of States in years where there is consumption. The State-level and U.S. "other petroleum" average prices are the average of the seven petroleum products, weighted by SEDS consumption data. The variable State average prices reflect the different mix of products consumed.

Table A42 shows national-level estimated prices and expendimures by the other petroleum product components for the years 1970 through 1994. 
Table A41. Other Potroleum Products btu Converslon Factors

Pefroleum Product

Mllion Btu

per barrot

Mlscellanions Procticts

5.796

Petrocthemiteal Feedsioxice

Naphtha

5.248

Other Ols

5.825

6.000

Still Ges

Special Naptilhas

5.248

Waxes

5.537 


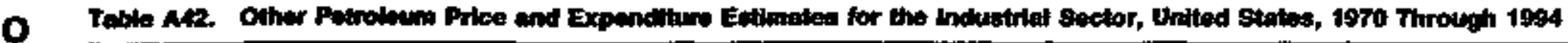

\begin{tabular}{|c|c|c|c|c|c|c|c|c|c|}
\hline \multirow[b]{2}{*}{ t } & \multicolumn{3}{|c|}{ 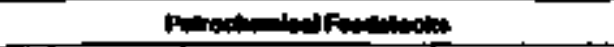 } & \multirow{2}{*}{ 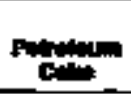 } & \multirow{2}{*}{ 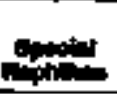 } & \multirow[b]{2}{*}{ Wane. } & \multirow{2}{*}{ Prodits } & \multirow{2}{*}{ Antos } & \multirow{2}{*}{ ctomenting } \\
\hline & ה" & chento & הeren & & & & & & \\
\hline \multicolumn{10}{|c|}{ 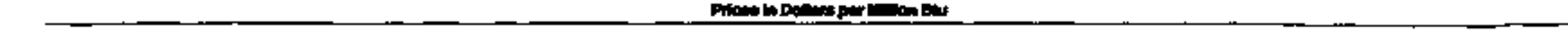 } \\
\hline 我 & 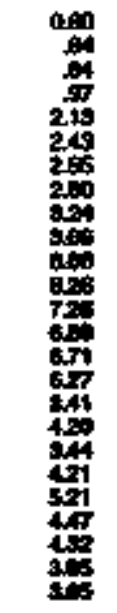 & 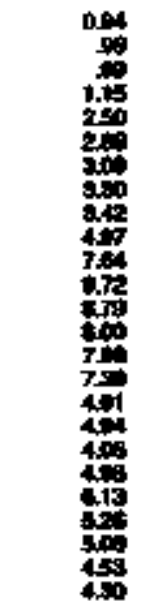 & 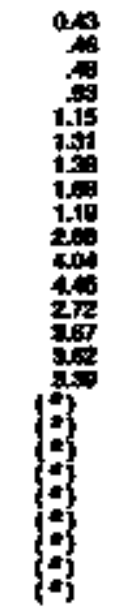 & 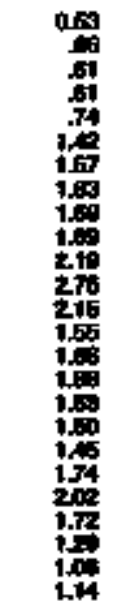 & 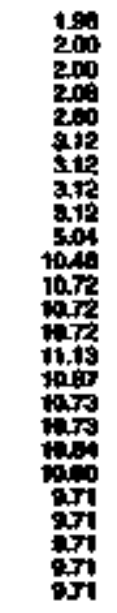 & 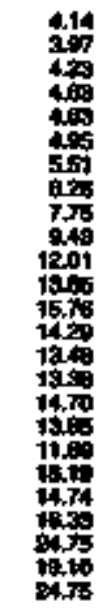 & 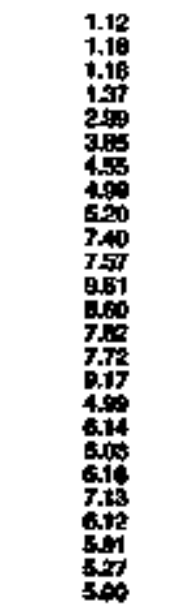 & 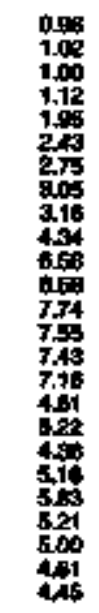 & $\begin{array}{l}= \\
= \\
= \\
= \\
= \\
= \\
= \\
= \\
= \\
= \\
= \\
= \\
=\end{array}$ \\
\hline \multicolumn{10}{|c|}{ 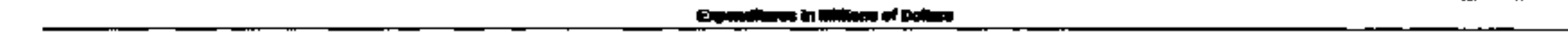 } \\
\hline 我 & 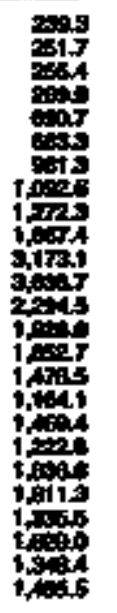 & 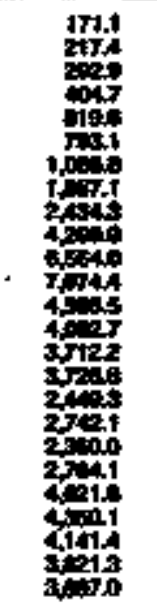 & 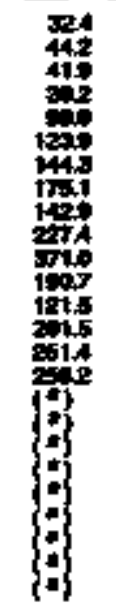 & 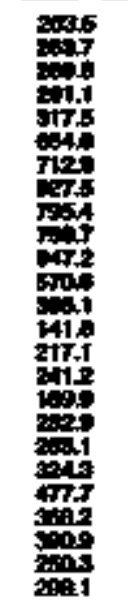 & 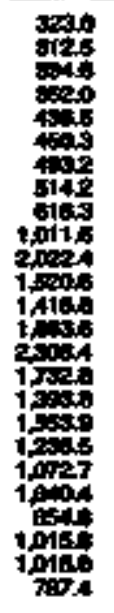 & 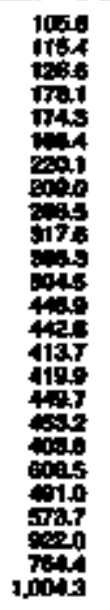 & 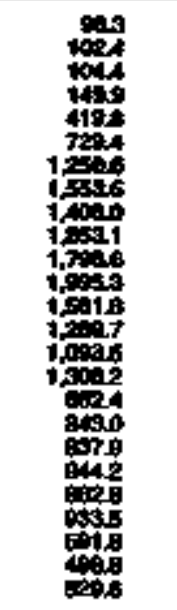 & $\begin{array}{l}= \\
= \\
= \\
= \\
= \\
= \\
= \\
= \\
= \\
= \\
= \\
= \\
=\end{array}$ & 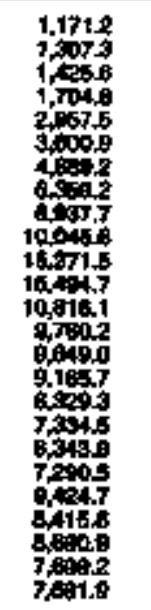 \\
\hline
\end{tabular}

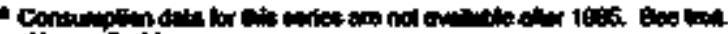
Tiation.

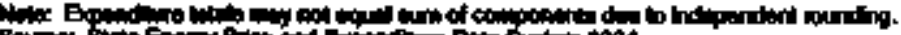

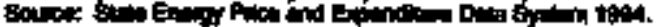




\section{Instructions for Locating Data in the Oll and Gas Information Resource System on the EIA InfoDisc}

Install the EIA InfoDisc following the instructions in the disk readme.txt file. Install the Oil and Gas Information Resonnce Systen application by (i) selecting (domble, not single, clicking) the "Energy Darabases Button" on the keft side of the /nfoDisc introdoctory menu screen and then selecting "Oil and Gas Dan" or by (2) selecting "Oil and Gas Data" in the "Instalt Smart Applications" screen.

To select the data used in SEPEDS as distillate fuel prices enter the following responses in the series of OGRS menu boxes:

- Main Menu prompt, "Run a saved request?" Euter "N"

- Petroleam Marketing

- Selection by Key

- EIA- and B

- Date Menu - Amula Avg. Select Dates

8300

$$
9400
$$

- Prodict - Total No, 2 Dist. (select using the space bar)

- Sales - Residential, Conmercial, and/or Indestrial

- Area - U.S. Total, U.S. Stpte, and/or All PADD Av.

- Data Type - Price

- F10 to run extract

- Select Output and Extract Dala

- Choose type of mutput and give filenarne
To select the data used for kertosene-type jet foel prices it SEPEDS, enter the following responses is the series of OGIRS meno boxes:

- Main Mean piompt, "Run a saved nequear? Enter "N"

- Petroleum Marteting

- Selection by Table

- Specific columo(s) from a Table

- Anapal Avg.

- All years (yields 1983-1994)

- Table 36

- Colamn 3

- Select Outpat and Ertract Data

- Choose type of cotpit and give filename. 



\section{Section 5. Renewable Energy Sources}

Prices and expenditures for tenewables energy sources in the State Energy Price and Expenditure Data System (SEPEDS) are based on consumption estimates from the State Energy Data System (SEDS). Retewable energy sources reported in SEDS include estimates of the residential (including commercial) sector's use of solar and wood energy for 1990 forward: industrial sector consumption of hydroelectric power for all yesrs; industrial use of solar, wind, geothermal, and biofuels from I990 forward; and electric utilities use of hydropower and geothermal, wind, wood, waste, photovoltaic and solar thermal energy for all years. Transportation use of ethanol is included in the motor gasoline volumes in SEDS for all years.

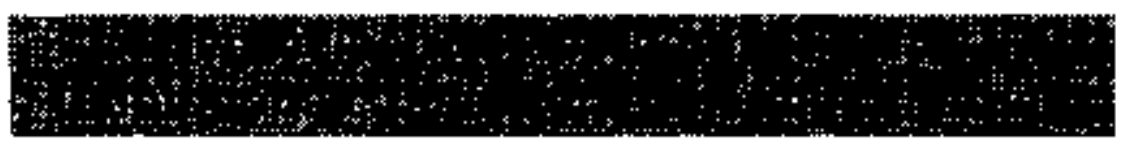

In SEPEDS, it is assumed that there are no direct foel costs for hydroelectric, geothermal, wind, photovoltaic, or solar thermal energy. SEDS consumption values are adjusted by removing these fuels before calculating SEPEDS energy expenditures as described in Section 7, "Consumption Adjustments for Calculating Expenditures," on page 413.

Prices are estimated for wood and waste in SEPEDS. It is assumed that taxes are included in the prices reported on the Energy Information Administration Residential Energy Consumption Survey and the Manufacturing Energy Consumption Survey, which are used as the basis for the SEPEDS price estimates.

\section{Residential and Commercial Sectors}

\section{Physical Unit Prices, 1990 Forward}

Prices paid for wood by the residential, including commercial, sector are based on unpublished data from the "1993 Residential Evergy Consumption Survey," Forto EIA-457. The four Census Region average prices for residential wood in 1993 are assigned to the States within each Census regton for all years, 1990 through 1994 . The prices are, in dollars per cord, for the Northeast region, $\mathbf{5 6 . 5 6}$, the South, $\$ 70.56$, the Midwest, $\$ 71.29$, and the West $\$ 94.95$.

\section{Dats Sourced}

\section{Prices}

1990 forward: Energy Information Administration, unpublished data from Fotm ELA-457, "1993 Residential Energy Consumption Survey," Census region compilation of the answers to questions $\mathrm{J}-28$, and $\mathrm{J}-33$ through $\mathbf{J}-36$. 


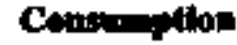

1990 forward: Energy Information Administration, State Eoerzy Data System, residential (including comneercial) biofuels consumption adjusted as described in Section 7. "Consumption Adjostments for Calculating Expenditures," on page 413.

\section{Commaton Factor}

20 million Btu per cord.

\section{Induetrial Sector}

\section{Btu Pices, 1900 Farwerd}

The incustrial sector biofuels consomption estimates in SEDS and the prices in SEPEDS are developed by dividing the industrial sector consniners into two groups-manofacturing indostries, specifically three Industriat categories, and nonutility power prodncers-and separating wood and waste consemption within the categories and gropos. The State-level industrial secior biofuels prices are cossanption-weighted averages of the consumption and prices of the individual components.

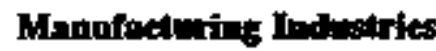

The ElA "1991 Manufacturitg Energy Consumption Survey" (MECS), FonI EIA-846, colkects unpublished data on quantities consumed in million Bm and expenditures in dollars for five types of biofuels-waste. pulping liquor, roundwood, wood chips, and biomass. At special request, a computer program calcolnted national average prices in dollars per nillion Btu for each of these fael types in 1991. The prices, in dollars per miltion Btu, are for waste, \$4.57; palping liguor, \$4.29; ronndwood, \$2.73; wood chips, \$1.59; and biomass, $\$ 1.59$.

MECS also identifies consumption of the different types of biofuels by the Sindard Industrial Clussification (SIC) codes 24, 26, and other (assumed to be mostly SIC 25). For each of the three SIC codes, an average biofuels price was calculated by using the consumption of each of the five types of biofuels to weight the price. These consumption. weigbted average prices for the three SIC codes in 1991 were applied to the SEDS estimates of biofuels cousumption by SIC code in each State to calculate State-level weighted average prices for 1990 forward.

\section{Noautility Power Prodacers}

No data are available on prices paid by nonutility power producers for wood and waste. The electric utility sector annual average prices for wood and for waste, separately. for 1990 through 1994 are assigned to the SFDS separate wood and waste consnmption estimates for the nonurility power producers is each State to calculate State-level weighted average pricess.

\section{Dote Sourees}

\section{Prices}

1990 forward: Enesgy Information Administration, unpublished data from Form ElA-846, "1991 Manufacturing Energy Consumption Survey," national average prices for waste, pulping liquor, roundwood, wood chips, and bionass.

1990 forwand: Energy Information Administration, SEPEDS electric utility sector prices for each year, sorted by fuel and consumptionweighted by fuel type.

\section{Consunpoption}

1990 forward: Energy Information Administration, State Energy Data System, indinstrial biofuels consumption adjusted as described in Section 7, "Consumption Adjustmenis for Calculating Expenditures," on page 413.

\section{Transportation Sector}

Biofuels consamed in the transportation sector as ethanol mixed in gasoline is inclatied in the motor gasoline volumes for all applicable years. 
Ethanol quantitjes and costs are included in the valumes and price of motor gasoline.

\section{Electrle Utility Sector}

State-level data on the amount of electricity generated at electric utilities from biomass fuels are taken from SEDS and are collected on the EIA "Monthly Power Plant Report," Form EIA-759. All electric utilities are required to report on EIA-759 but no price data are collected. State and national biomass fuels prices in dollars per million Btu are developed for the elsetric utility sector frond dath reported on other EIA and Federal Energy Regulatory Connuission (FERC) forms and from telephone surveys. Taxes are included in the prices for all years.

\section{Prices: All Years}

1989 Forward. State-level prices for biomass fuels used at electric utilities, in doltars per million Btu, are calculated from dota obtained from FERC Form 1 and Form EIA-412 and by follow-up telephone surveys of the electric utilities that are not required to submit thase forms. For States with more than one utility using biomass fuels, a consumptionweighted average price is calculated. There are anomalies that are unique to biomass fuels used at utilities. In some cases, there is to charge for the fuel. In other cases of municipal and industrial waste, the electric utilities charge a "tipping fee" for accepting the waste. That is, instead of paying for the fuel, the electric utilities are paid to talo the fuel. For Stales where all utilities pay nothing for the fuel or charge a

Table 443. Biomass Fugis Used at Eloctric Utilfiea at Mo Coat or For a Feo, 1969 Formard

\begin{tabular}{ll}
\hline State & Years \\
\hline Caliomb & $1084-1994$ \\
Connecticut & $1909-1994$ \\
Hawed & 1909,1990 \\
Montana & $1909-1994$ \\
Onio & $1989-1908$ \\
\hline
\end{tabular}

fee for recejving it (see Table A43), a price of zero is assigned. Although the corresponding consumption is inchuded in calculating the average price for foels consumed by electric atilities in the State and the United States, the expenditure inchuded is zero. The State and U.S. average electric utitity fuel prices ane, therefore, stightly lower because some of the foel did not cost anything.

1983 Throws 1983. A U.S. average price in dollars per million Btu is caiculated and assigned to all Siates. The oetional price is a consumption-weighted average price based os data obained from FERC Form 1 and Form ELA-412 and by follow-up telephoite surveys of the electric ntilities that report ase of biomass fuels for generating electricity.

Prices are enratic for biomass fuels used at utilities. In addition to the anotanlies of no change for the fuel and the "tipping fee" mentioned above, handling refuse-derived fuel is mone labor intensive than banditing conventional fossit fueis. The labor expenses are included in the plinu's operattug costs, not the fuel costs. Biomass fuels prices are also erratic because the demasd is relatively small and the pricing mechanism, even for a single facility. may change from year to year. A price or quantity change by a single major user affects the aational price more significantly than for any other foel.

1978 Through 1982. National avernge prices are derived from data collected on FPC Form $\mathbf{4 2 3}$ and published monthly by EIA in Cost and Quality of Fuels for Electric Utility Plonts (C\&Q). For these years, fousil-fueled plants with a combined capacity of 25 megawatts or greater were required to report on FPC Forn 423. Annual prices of biomass fuels sold to electric utilities are developed as quantity-weighted monthly prices for those plants where wood chips and refuse were used as fuel. Beginning in 1983, the reporting threshold was raised to 50 meigawatts, and very fer plants reported use of biomass fuels on the Form 423 in 1983 and sobsequent years.

A detailed neview of data in C\&Q showed that some eutries wene in ernor by factors of 10,100 , or 1,000 . Accordingly, the following corrections were mide. For 1982, the February, March, and April quantities for the Florida Power Corporation were divided by 1,000 to make them 80, 40, and 60 short tons, respectively. The March, April, and May costs for Northern Sutes Power were multiplied by 100 to mike 
R them $\$ 0.70$ per million Btu. For the 5 months from November 1979 E through March 1980, the reported quantities of wood delivered to Burlington Electric Co. were divided by 10 , in order to place them in the range of 7,980 to 9,390 short tons. For the 8 months from Jupe 1978

E through Jantuary 1979, seed corn delivered to the Logansport Indiena

W Electric Department were included in the biomass fuel. For February

A 1978 , the reported quantity of wood delivered to the Uatted Pawer AsB sociates was divided by 1,000 to ruake it 90 short tons.

E 1970 Through 1977. The annual prices for wood chips are derived by deflating the 1978 price by using the gross domestic product implicit price deflator based on 1987 dollars. The deflators are shown in Table A44.

\section{E Data Sources}

\section{G Prices}

1983 forward: Energy Information Administration, data reported on FERC Form 1, "Annual Report of Major Electric Utilities, Licensees and Others," and Form EIA-4 /2, "Annuad Report of Public Electric Utilities," and follow-up telephone surveys of the electric utilities that report use of biomass fuels for generacing electricity. 7able A44. Price Deflatore Uned for Blomass Fuela Prices, 1970-1977

\begin{tabular}{lccc}
\hline Yeare & Dofifator & Years & Defietor \\
\hline 1970 & 35.1 & 1975 & 49.2 \\
1871 & 37.1 & 1976 & 52.3 \\
$t 972$ & 38.8 & 1977 & 55.9 \\
1973 & 41.3 & 1978 & 60.3 \\
1974 & 44.9 & & \\
\hline
\end{tabular}

1978-1982: Energy Information Administration, Cost and Quality of Fuets for Electric Utility Plants, table titled "Wood Chips, Refuse, and Petroleum Coke Used as Fuel by Steam-Electric Plants."

1970-1978: Energy Information Administration, Annual Energy Review 1991, Appendix C, Gross Domestic Product and Implicit Price Deflator.

\section{Consumption}

1970 forward: Energy Information Administration, State Energy Data System, electricity generated from consumption of wood and waste. 


\section{Section 6. Electricity}

Electricity prices are retail prices for sales to ultimate users. Prices are developed for the residential, commercial, industrial, and transportation sectors. In general, taxes are included in the prices. However, taxes collected by a utility from an end user and hurned over to a government authority are generally not included in the revenues reponted in the Enengy Information Administration (EIA) Electric Power Annual or the Edison Electric Institute Statistical Yearbook and are not included in the prices. Taxes paid by the utility (rather thas the end user) are considered operating costs and are passed on to the end user as part of the rate. Therefore, Federal, State, business, and property taxes are typically included in the prices, while sales and other point-of-purchase taxes usually are not.

Consumption is based on sales by electric utilities to ultimate users. Electricity consumption data by State for the residential, commercial, industrial, and transportation sectors are obtained from the ELA State Energy Data System (SEDS). Consumption of electricity in the industrial sector is adjusted for estimated refinery use in each State. (See the discussion in Section 7, "Consumption Adjustments for Calculating Expenditures," on page 413.)

\section{Phyoleat Unit Prices: 1987 Fonward}

For 1987 forward, physical unit prices for States are calculated for all four sectors as the average revenue per unit of sales by all electric utilities reporting sales to a State. Revenues and sales data from the Electric Power Anneal are used to calculate physical unit prices. Data for the

residential and industrial sectors are drawn from their respective col umns. Data for the transpertation sector are taken from the eolumn titled "Other" that includes sales to railroads and railways. SEDS consumption is used to determine the postion of the data in the columin titled "Other" that is not sold to the transportation sector. These data are added to the column tited "Commercial" to arrive at the revenues and sales data for the commercial sector.

For 1987 forward, prices are based on preliminary revenues and sales data in the given year and are replaced with revised tata in the following year. There are no missing prices in any of the four sectors.

\section{Phystcal Unlf Prices: 1970 Through 1886}

For 1970 through 1986, preliminary physical unit prices for States are calculated for all four sectors as the average revenue per unit of sales by all electric utilities reporting sales to a State. The caiculation of physical prices uses the reventres and sales data from the Statistical Yearbook for each year in the series. Data for the residential sector and industrial sector are drawn from their respective columns. The conmercial sector is the sum of the columns titled "Commerctat." "Street and Highway Lighting." "Othet Public Authorities," and "Interdepartmental." The transpontation sector is the column titled "Railroads and Railways."

For 1980 through 1986, prices are based on preliminary revenues and sales data in the given year and are replaced with revised data in the following yeat. The only exception to this rule is the revenues data for AR in 198 I; preliminary data are used in this case because of an apparent errot in the revised data. 
For 1970 through 1981, MD prices are assigned to DC. There are no other missing prices for the residential, commercial, and industrial sectors.

In the transportation sector, numerous price assignments are made doe to the lack of sector-specific price data. Generally, electricity usage in the transportation sector is smill; the sector's electricity use ranged from 0.1 percent to 0.2 percent of total U.S. electricity consumption in 1970 hrough 1986. From 1970 through 1986, only 15 States used measurable amounts of electricity in the trabsportation sector (CA, DC, FL, GA, IL, LA, MA, MD, NJ, NY, OH, PA, TN, VA, and WA). A few individual State prices are unavajlable and are assigned the commercial sector prices: LA/1970 through 1986, and TN/1970 through 1986. (Prices are available for LA/1970, 1972, 1973, but those prices are replaced by commercial seclor prices to maintain a consistent series for the State.) In addition, MA/1985, 1986 transportation prices are estimated by multiplying the MA/1985, 1986 commercial prices by the average of the ratios of the commereial-to-transportation sector prices for 1980 through 1984. Similarly, the VAV1977 tratusportation price is estimated by multiplying the VA/1977 commencial price by the average of the ratios of the commercial-to-transportation sectors prices for 1978 through 1982.

In onder to reconcite national-level electricity prices based on the Statistical Yeartook with the EIA national-kvel electricity prices published in the Annwal Energy Review (AER), yearly adjusturent factors are calcolated for the residential, commertial, and industrial sectors as follows: a preliminary U.S. price for each sector is calculated as the average of the State pitces, weighted by SEDS consumption. These preliminary U.S. prices are divided by the national-level electricity prices publisbed in the $A E R$, and the quotient is used as an adjustment factor. The prellminary State prices are multiplied by the adjustment factor to produce the final physical unit State prices in those sectors. Since no transpontation sector prices are pnblished in the AER, no adjustruents are made to that sector and the fintl physical unit prices are derived solely from the Staistical Yearbaot sales and revenue dala. The annual adjustment factors for the residential, commercial, and industrial sectors are shown in Table A45.
Table A45. Annual Electrictity Prles Adjustment Factors

\begin{tabular}{|c|c|c|c|}
\hline Yoar & Residentid & Comberel: & indinatrils' \\
\hline 1970 & 1.05121 & 1.05712 & t. $\cos 28$ \\
\hline 1971 & 1,05832 & 1.05828 & $t .05504$ \\
\hline 1972 & 1.05271 & 1.05514 & t.0s7tos \\
\hline 1973 & 1.00626 & 1.00198 & $10 \leq 091$ \\
\hline 1974 & t.CEST2 & $1.0000 \mathrm{~s}$ & 1.04732 \\
\hline 1975 & 1.09257 & 1.08098 & 109732 \\
\hline 1976 & 1.07753 & 1.0775 & $1,0,6091$ \\
\hline 1977 & 1.06746 & 1,07675 & 1.0000 \\
\hline 1978 & 1,00654 & 1.06073 & 1.003851 \\
\hline 1979 & 1.09966 & 1.08349 & 1.08441 \\
\hline 1960 & 1.04457 & 1,05100 & $1.0678 t$ \\
\hline 1981 & 1.tox日ey & 1.48945 & $1,0,003$ \\
\hline tgot & 1.06054 & 1.00551 & 1.05497 \\
\hline 1990 & 1.05421 & 1.05301 & 1.05557 \\
\hline 1984 & 0.99293 & 1.01924 & 0.90015 \\
\hline 1985 & 1.00010 & t.0anod & 0.98555 \\
\hline 1996 & 0.99854 & 1.01518 & 0.90416 \\
\hline
\end{tabular}

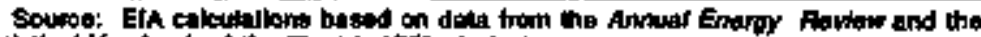

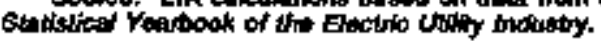

\section{Butu Prfoes: All Yaws}

But prices for States are calculated by dividing the physical unit prices by the conversion factor. U.S. Bto prices are calculated as the average of the State Btu prices, weighted by consumption data from SEDS, adjusted for process fuel consumption in the industrial sector.

\section{Dits Sounces}

Prices

1993 and 1994: Energy Information Administration, Electric Power Annual 1994 Volume $I$, Tables 4 and 6.

1987 through 1992: Energy Information Administration, Electric Ponver Annual 1988, Tables 19 and 21 (1987 data); Electric Power Annwal, Tables 27 and 29 (1988-1990) and Tables 26 and 28 (1991 and 1992). 
1970-1986: Edison Electric Institute, Statistical Yearboat of the Electric Utility Industry, tables titled "Revenues: Total Electric Utility Industry" and "Energy Sales: Total Electric Utility Industry."

1970-1986: Energy Information Administration, Asastal Energy Review I989, Table 95, "Retail Prices of Electricity Sold by Electric Utilities, 1960-1989."

\section{Cossumption}

1970 forward: Energy Information Administration, State Energy Data System, electricity consumption by end-use sector.

\section{Converston Factar: All Yours}

3,412 But per kilowathour level data on the amount of electricity generated from nuclear power plants are taken from SEDS. Utilties operating nuclear plants report fuel costs per kilowarthour to the U.S. Department of Energy anpually. These data are collected on a plant-by-plant basis and inciude all taxes, transportation, and handling costs paid by the utilities. The \$tate where the nuclear power plant is located is assigned the reported nuclear fuel cost. For States with two or more nuclear power plants, the average Iuel cost, weighted by generation, is attributed to the State.

\section{Physical Unit and But Prtect: All Years}

State-level nuclear fuel prices are estimated in two steps: (1) the total cost of fuels consumed at all nuckear power plants in a State is tivided by their total generation of electricity, and (2) the cost per output created in step 1 is divided by an annual U.S. average thermal conversion factor to create the price in dollars per million Bul. Oecasionally, the fuel costs at uuclear power plants inchude small amounts of non-nuclear foels, which are necessary to contioue essential plant operations doring refueling or maintenance of the reactor.

A price is estimated by another method for \$tates where generation and fuel cost dafa ate not avallable for years in which the State Energy Dota Report (SEDR) shows noclear electric power seneration. The ratio of the change in the national moclest fuel price is osed to estimate nocleat fuel prices for missing years within a State. The ratio of national prices osed in the estimation is the ratio before missing prices ane assigned. The States and years for which muclear prices are extimuted ane shown in Table A46.

Nuclear electricity generation levels are negative for $\mathrm{CO}$ in 1985, for TN in 1986 and 1987, and for $O R$ in 1993, inilicating that the nuclear power plants used more energy than they suppliect. In these cases, the fuel prices and expenditures are set to zero. In 1986, there is genteration in CO but price data are not available. The price for $\mathrm{CO}$ in 1986 is estimated by using the ratio of the 1984-to-1986 U.S. average prices and applying that ratio to the 1984 CO price.

Toble A46. Nuctear Eloctricty Fued Price Esimpitas, 1070 Through t9op

\begin{tabular}{|c|c|}
\hline Stante & Your: \\
\hline 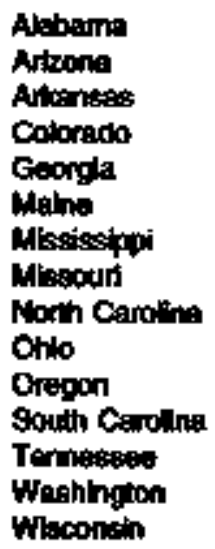 & 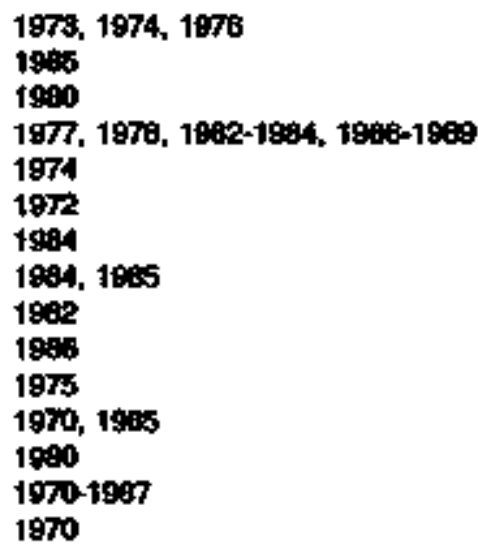 \\
\hline
\end{tabular}


E For $\mathrm{MO}$ in 1985 , a large credit resulting from litigation is assigned to fuel costs, creating an artificially low price. The 1986 MO uranium E price, which is in the range of the prices of other nuclear fuel plants, is C used to estimate the 1985 price by applying the ratio of the 1986-toC 1985 national prices.

The 1985 Historical Plant Costs and Annual Production Expenses for Selected Electric Plants has a footnote for the Duke Power Catawba plant in SC stating that the reported production expenses represent only 12.5 percent of the actual production expenses. The production expenses used in the calculation for the Catawba plant are adjusted accordingly.

Prices for $\mathrm{GA}$ in 1978, NC in 1982, and $\mathrm{OH}$ in 1986 are assigned because the prices calculated by using reported data are significantly different from prices for other years for these States.

$\mathbf{U}$
$\mathbf{L}$
$\mathbf{E}$
$\mathbf{A}$
$\mathbf{R}$
$\mathbf{F}$
$\mathbf{U}$
$\mathbf{E}$

There are no prices available for WA in 1970 through 1987 and national prices are assigted for those years.

\section{Data Sources}

\section{Prices}

1992 forward: Prices are developed by the Energy Information Administration, Office of Coal, Nuclear, and Alternate Fuels, from data compiled by the Utility Data Institute/McGraw-Hill. The data are collected on Federal Energy Regulatory Commission, FERC Form 1. "Annual Report of Major Electric Utilities, Licensees, and Others," and Energy Infornation Administration, Form EIA-412, "Annual Report of Public Electric Utilities."

1988-1991: Energy Information Administration, Electric P]ant Cost and Power Production Expenses, Table l6 (1988-1990) and Table 14 (1991).

1982-1987: Energy Information Administration, Historical Plant Costs and Annuol Production Expenses for Selected Electric Plants, Table 18 (1982-1984) and Table 20 (1985-1987).
1979-1981: Energy Information Administration, Themal Electric Plant Construction Cost and Annual Production Expenses, pages 267-279 (1979), Table 11 (1980 and 1981).

1975-1978: Energy Inforniation Administration, Steom Electric Plant Construction Cost and Annual Production Expenses, "Section II-Nuclear Plants."

1970-1974: Federal Power Commission, Stetm Electric Plant Construction Costs and Annual Production Expenses, data sheets for Nuclear Plants (1970-1973), and "Section I-Nuclear Plants" (1974).

\section{Conșumption}

1970 forward: Energy Information Administration, State Energy Data System, electricity generated by nuclear power.

\section{Comerwion Factors}

1970 forwad: Energy Information Administration, State Energy Data Report 1994. Consumption Estimates, Table D1, as shown in Table A47.

Table 447. Nuclear Steem-Electrle Thermal Conversion Factors (Thousand Bu per Kilowatthour)

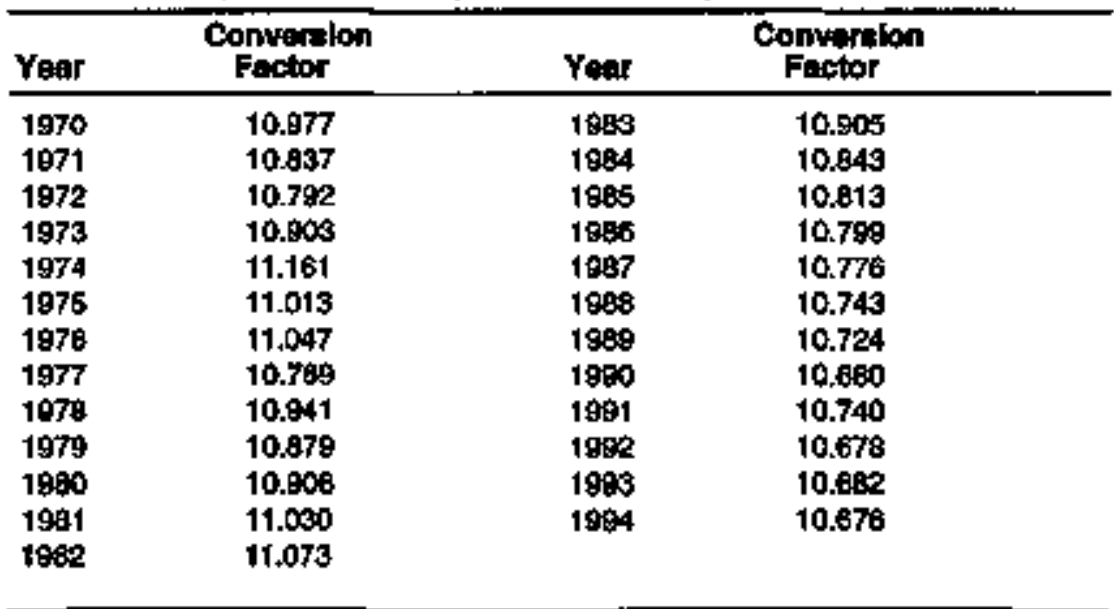




\section{Section 7. Consumption Adjustments for Calculating Expenditures}

Expenditures developed in the State Energy Price and Expenditure Data System (SEPEDS) and published in this report are colculated by using consumption estimates from the EIA State Energy Data System (SEDS) published in the State Erengy Dota Report 1994, Constumption Estimates (SEDR), October 1996. Expenditures are calculated by multiplying the price estimates by the consumption estimates, adjusted to remove process fuel, intermediate petroleum products, and other consumption that has no direct fuel costs, i.e., hydroelestric, geothermal, wind, solar and photovoltaic energy sources, and some biofuels.

Almost all aspects of energy production, processing, and distribution consume energy as an inherent part of those activities. SEDS industria! and transportation sector consumption estimates include energy consumed in the process of providing energy to the end-use consumer and are called "process fuel." Familiar examples inciude drilling for oil and gas, refining crude oil into finished petroleum products, transporting natural gas and petroleum by pipeline, and generating and delivering electricity to end users. Energy products that are subseguently incorporated into another energy product for end-use consumption are called "intermedjate products." Motor gasoline blenđing components are familiar examples of intermediate products that are consumed as part of the finished motor gasoline sold at service stations and other outlets.

Process fuel and intermediate products are not purchased by the end user and, therefore, do not have prices. Although the end user does not consume either process fuel or interuediate products directly, he does pay for them, because the cost to the processor or distributor is passed on to the end user in the price of the final end-user product. If their use was left in the consumption estimstes and was assigned prices, the expenditures would be conted twice, first as paid by the "processor" (producer. processor, or transporter) and again as included in the price to the end user.

Some renewable energy sources are not purchased. These include bydroelectric, gecthermal, wind, photovoltaic, and solat thermal energy. The consumption of these sources, which are measured in SEDS as kilowathours of electricity produced, are not included in SEPEDS expenditure estimates since there are no "fuel costs" involved. Biofuels (wood and waste) are sometimes purclased and sometimes obtained at no cost. Biofuels consumption estimates in the residential (including commercial) and industrial sectors in SEDS are adjusted to remove estimated quantities that were obtained at mo cost.

To estimate energy expenditures in SEPEDS, the consumption of process fuel, intermediate products, and some of the renewable energy sources are subtracted from the end-use sector in which they are included in SEDS, either the residential (including commercial). indnstrial, or transportation sector, because there are no prices associ. ated with them.

Process fuel consumption ajjustments include:

1. Fuel (petroleum, natural gas, cosl) and electricity consumed at refineries

2. Crude oil lease, plant, and pipeline fuel

3. Natural gas kase and plant fuel

4. Natural gas pipeline fuel

5. Electrical system energy losses (i.e., energy consumed in the generation, transmission, and distribution of electricity).

Intermediate product consumption adjustrneats irxlude:

1. Aviation gasoline blending components 
2. Motor gasoline blending components

3. Netural gasoline (1970 through 1983)

4. Pentanes plus (1984 forward)

5. Plent condensate (1970 through 1983)

6. Unfinished oils

7. Unfractionated stream (1970 through 1983).

Starting in 1984, natural gasoline (including iscopentane) and plant condensate ane reported together as the new product. pentanes plus, and the components of unfractionated stream are reported separately under liquefied petroleum gages.

Renowable energy consumption adjustunents include:

1. Photovoltaic and solar thermal energy in the residential (including commetcial) sector, the industrial sector, and at electric atilities

2. Electricity generated from hydropower, geothermal, and wind energy in the industrial sector and at electric utilities

3. Estimated portions of wood and waste consumption in the residential (inchuding commercial) and industrial sectors that wete obtained at no cost.

Table A48 shows the quantities of energy, by State, removed from SEDS consomption to calculate SEPEDS expenditures for 1994. State estimates for 1970 through 1994 are availabte on the State Energy Price and Expenditure Data System internet data files and personal computer diskettes.

Table A49 shows the adjustments made to SEDS national consutuption estimates for 1970 through 1994 to derive the net consumption data osed to ealculate the expenditures in SEPEDS.

\section{Adiustonont Procodure:}

Reffinery lowl. Refinery fuel consumption of distillate fuel, residual fuel, liquefied petroleum gases, ather petroleum (crude oil, petroleum coke, and still gas), nutural gas, coall, and electricity is individually estimated for each source and subtructed from its State Energy Data System (SEDS) industrial sector totul. Becense crude oil consumption is not an individual fitel in SEDS for 1970 through 1982, the small amoonts of crode oil that were used at refineties during those years were allocated to residual and distillate fuels consumed at refineries. The allocition from crude oil refinery use to residual and distillate fuels refinery use was made according to each fuel's share of the total crude oil used directly (including losses) as residual and distillate fuels (Petrotewn Supply Anmual Volume I, Table 2). Over this period, residual fuel ranged from a low share of 75 percent in 1971 to a high share of 92 percent in 1976. Refinery consumption of still ga6, exclndigg still gas consumed as petrochemical feedstocks, is subtracted from the SEDS industrial sector total for 1970 throngh 1985. Beginning in 1986, EIA data series no longer report refinery fuel and feedstock nse separately. and all industrial stall gas consumption is removed.

Refinery fuxel consumption data are available in the data sonrces by State or group of States (1970 through 1980) and by Petroleum Admintistration for Defense District (PADD) (1981 forward). Where State-level consumption data are not available, the State-level estimates are derived by allocating the district's or group's total consumption to the individual States within the district or gromp to the States that had operating refineries in a given year. Individual fuels are allocated to the refining States according to each State's share of the refining States" total industrial sector fuel consumption during the year. In some instances, estimated vefinery fuel consumption exceeds the SEDS estimate for total industrial consumption of a fuel within a State. When this occurs, the excess refinery fuel cossumption is reallocated as shown in Table A50.

Intermedlate Products. Aviation gasoline blending components, motor gasoline blending components, nahural gasoline (1970 through 1983), pentanes plus (1984 forward), plant condensate (1970 through 1983), unfinished otls, and unfractionaled stream (1970 through 1983) are used at refineries and blending plants to make end-use petrolenm products, particularly motor gasoline. Accordingly, consumption of these products is completely removed.

Residential (Including Con nerclal) Solar and Woad. There are no fuel costs for photovoltaic and solar thermal energy sources; therefore, all consumption is removed from the expenditure calculations. Some residential and commercial wood is purchased and some acquired at no cost. Based on responses to the ElA 1993 Residential Energy Consumption Survey. Census region percentages were developed. It is estimated that 61 percent of the wood consumed in States in the 


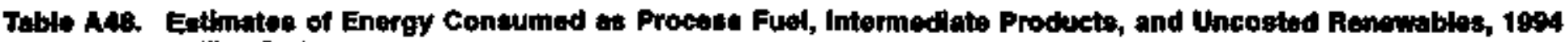
(Bilion Btu)

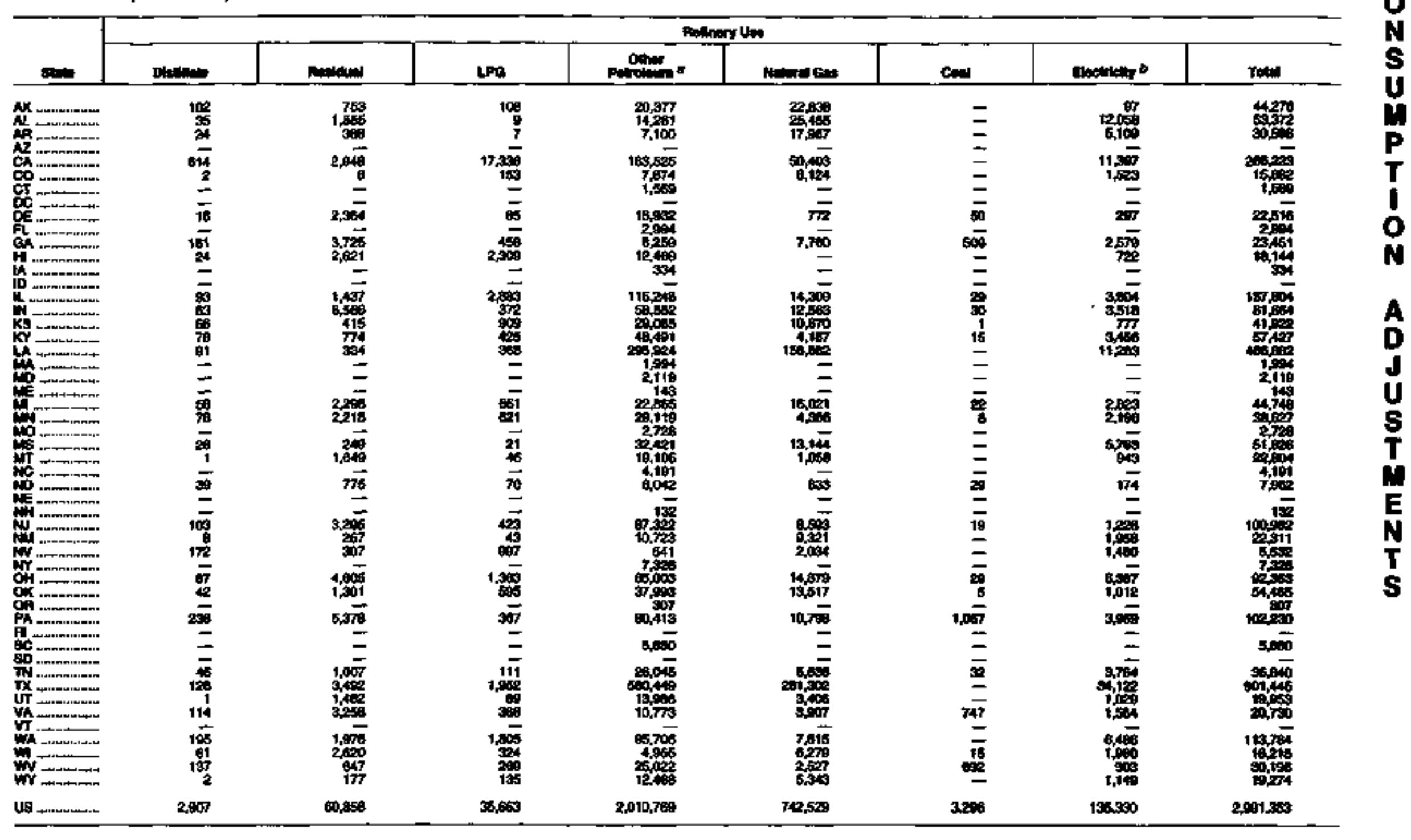

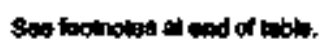




\section{Table A49. Estlmates of Enerey Consumed as Process Fuel, Intemedlate Producte, and Uncosted Ronewables, 1994 (Continued) (Billion Btu)}

\begin{tabular}{|c|c|c|c|c|c|c|c|c|c|c|}
\hline \multirow[b]{2}{*}{ 8int: } & \multicolumn{2}{|c|}{ 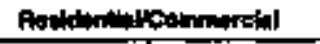 } & \multicolumn{5}{|c|}{ Inoluturital } & \multirow{2}{*}{ 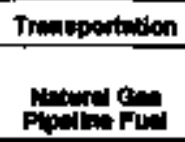 } & \multirow{2}{*}{ | } & \multirow[b]{2}{*}{ Tow: } \\
\hline & suren & wood & Predim & Ptond Puid & witery & soler & Blotual: & & & \\
\hline 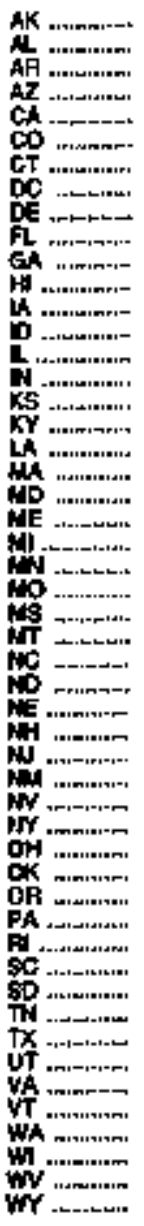 & 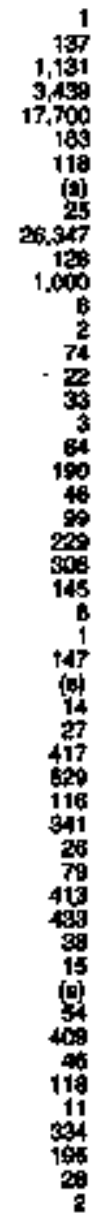 & 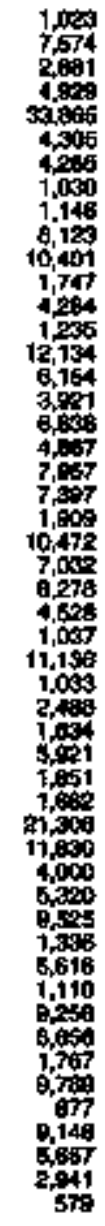 & $\begin{array}{r}= \\
= \\
= \\
= \\
= \\
= \\
= \\
= \\
= \\
= \\
= \\
= \\
= \\
= \\
= \\
= \\
= \\
= \\
= \\
= \\
= \\
= \\
= \\
= \\
= \\
= \\
= \\
= \\
= \\
=\end{array}$ & 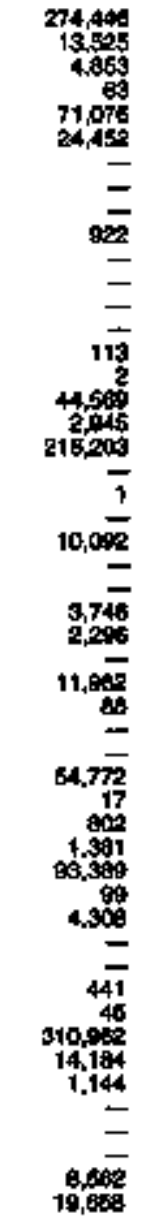 & 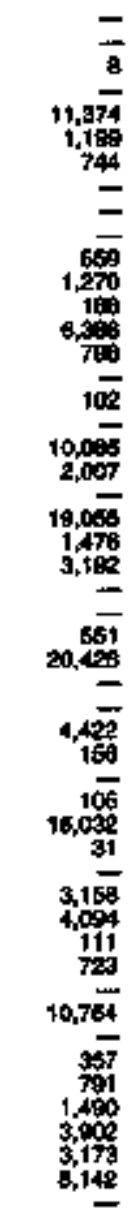 & 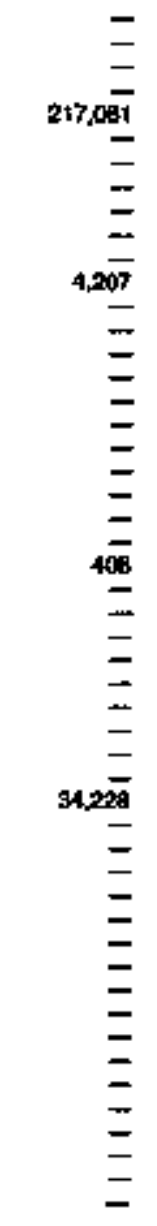 & 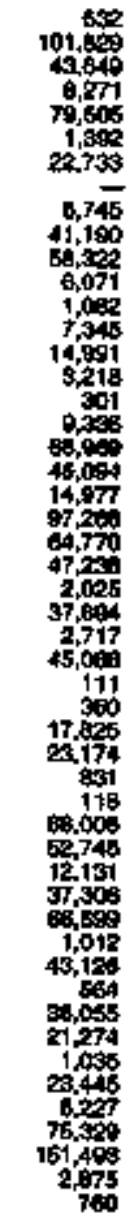 & 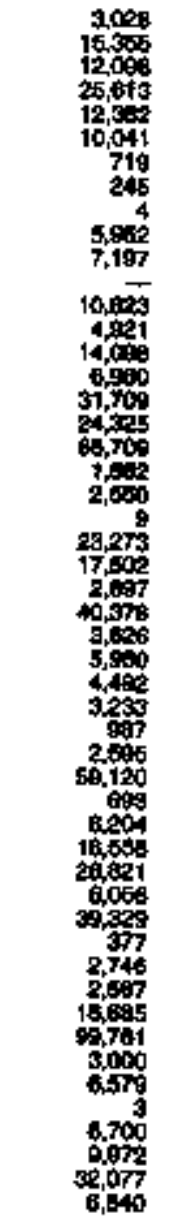 & 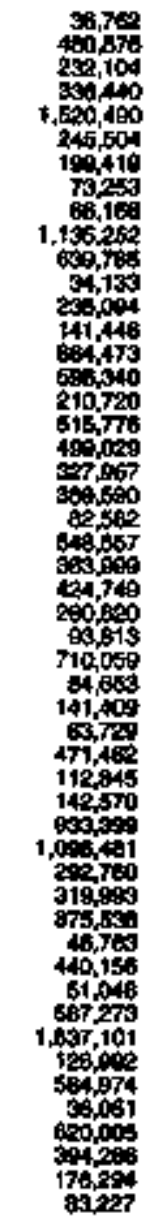 & 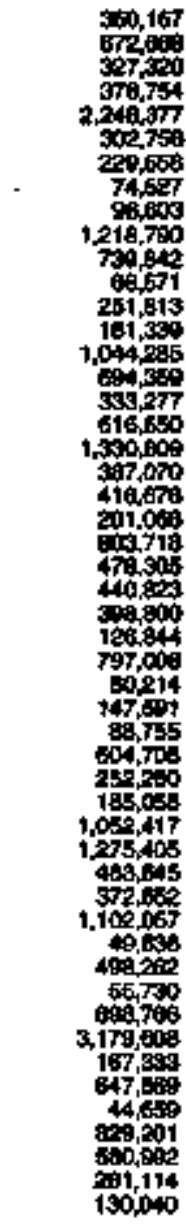 \\
\hline שתי-יו....... & 55,231 & 301,760 & 18,692 & $7,190,119$ & 135,468 & 256,294 & $1,475,460$ & 708,380 & $20,050,104$ & $27,096,458$ \\
\hline
\end{tabular}

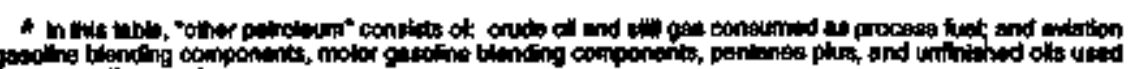

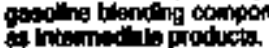

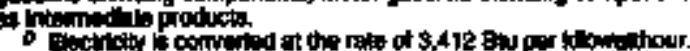

$\rightarrow$ weontumption.

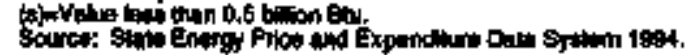


Table A49. Energy Consumption Adjustmente, 1970-1994 (Trillion Btu)

\begin{tabular}{|c|c|c|c|c|c|c|c|c|c|c|c|c|c|}
\hline \multirow[b]{3}{*}{ Varr } & \multirow[b]{3}{*}{ compingition } & \multicolumn{11}{|c|}{ Adjustownts } & \multirow[b]{3}{*}{ consininition } \\
\hline & & \multicolumn{2}{|c|}{ 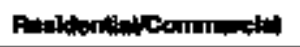 } & \multicolumn{6}{|c|}{ monetriel } & \multirow{2}{*}{ 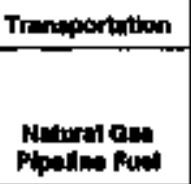 } & \multirow[b]{2}{*}{ 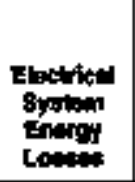 } & \multirow[b]{2}{*}{ Totel } & \\
\hline & & soter & Wood & Pufinety & 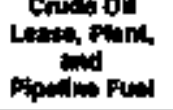 & 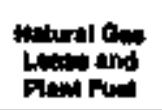 & 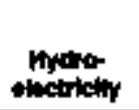 & 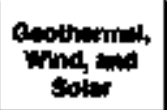 & elotumin & & & & \\
\hline $\begin{array}{l}1970 \ldots \\
1971 \ldots . . . \\
1972 \ldots- \\
1973 \ldots \\
1974 \ldots . . .\end{array}$ & $\begin{array}{l}66,334 \\
67,759 \\
71,276 \\
74,351 \\
72,509\end{array}$ & $\begin{array}{l}= \\
= \\
=\end{array}$ & $\begin{array}{l}= \\
= \\
=\end{array}$ & $\begin{array}{l}2,454 \\
2,442 \\
2,678 \\
2,690 \\
2,680\end{array}$ & $\begin{array}{l}= \\
= \\
=\end{array}$ & $\begin{array}{l}1,442 \\
1,450 \\
1,497 \\
1,589 \\
1,600\end{array}$ & $\begin{array}{l}34 \\
34 \\
34 \\
35 \\
35\end{array}$ & $\begin{array}{l}\bar{z} \\
= \\
=\end{array}$ & $\begin{array}{l}\bar{z} \\
\bar{z}\end{array}$ & $\begin{array}{l}740 \\
791 \\
768 \\
745 \\
684\end{array}$ & $\begin{array}{l}11,517 \\
12,127 \\
13,110 \\
13,899 \\
14,199\end{array}$ & $\begin{array}{l}18,197 \\
18,200 \\
18,000 \\
19,007 \\
19,119\end{array}$ & $\begin{array}{l}50,158 \\
50,098 \\
50,273 \\
55,344 \\
5,409\end{array}$ \\
\hline $\begin{array}{l}1975 \ldots . . . \\
1976 \ldots \\
1977 \ldots \\
1976 \ldots . . . \\
1979 \ldots\end{array}$ & $\begin{array}{l}70,569 \\
74,394 \\
76,317 \\
79,150 \\
76,90\end{array}$ & $\begin{array}{l}= \\
= \\
=\end{array}$ & $\begin{array}{l}\bar{z} \\
\bar{z} \\
=\end{array}$ & $\begin{array}{l}2,671 \\
2,690 \\
2,607 \\
2,854 \\
2,742\end{array}$ & $\begin{array}{l}\bar{z} \\
= \\
=\end{array}$ & $\begin{array}{l}1,454 \\
1,676 \\
1,709 \\
1,604 \\
1,534\end{array}$ & $\begin{array}{l}32 \\
32 \\
39 \\
32 \\
34\end{array}$ & $\begin{array}{l}\bar{z} \\
\bar{z}\end{array}$ & $\begin{array}{l}= \\
= \\
=\end{array}$ & $\begin{array}{l}596 \\
559 \\
544 \\
541 \\
613\end{array}$ & $\begin{array}{l}14,394 \\
18,250 \\
16,000 \\
18,030 \\
17,000\end{array}$ & $\begin{array}{l}19,017 \\
20,116 \\
21,030 \\
21,740 \\
21,905\end{array}$ & $\begin{array}{l}\$ 1,683 \\
54,278 \\
86,267 \\
58,410 \\
50,865\end{array}$ \\
\hline 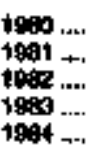 & $\begin{array}{l}75,905 \\
74,000 \\
70,000 \\
70,406 \\
74,090\end{array}$ & $\begin{array}{l}= \\
= \\
=\end{array}$ & $\begin{array}{l}= \\
= \\
=\end{array}$ & $\begin{array}{l}2,718 \\
2,204 \\
2,069 \\
2,122 \\
2,255\end{array}$ & $\frac{\overline{7}}{140}$ & $\begin{array}{r}1,059 \\
969 \\
1.149 \\
1.010 \\
t, 113\end{array}$ & $\begin{array}{l}30 \\
39 \\
393 \\
393 \\
393\end{array}$ & $\begin{array}{l}= \\
= \\
=\end{array}$ & $\begin{array}{l}\bar{z} \\
\bar{z}\end{array}$ & $\begin{array}{l}600 \\
609 \\
614 \\
505 \\
545\end{array}$ & $\begin{array}{l}17,307 \\
17,404 \\
17,100 \\
17,5 e 9 \\
16,157\end{array}$ & $\begin{array}{l}21,046 \\
21,320 \\
20,979 \\
21,304 \\
22,237\end{array}$ & $\begin{array}{l}54,140 \\
50,703 \\
40,807 \\
49,003 \\
51,817\end{array}$ \\
\hline 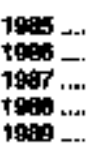 & $\begin{array}{l}74,054 \\
74240 \\
70,040 \\
80,200 \\
81,317\end{array}$ & $\begin{array}{l}= \\
= \\
=\end{array}$ & $\begin{array}{l}- \\
= \\
=\end{array}$ & $\begin{array}{l}2,048 \\
2,285 \\
2,495 \\
2,698 \\
2,711\end{array}$ & $\begin{array}{l}127 \\
109 \\
72 \\
165 \\
59\end{array}$ & $\begin{array}{l}1.001 \\
954 \\
6.194 \\
1,194 \\
5,103\end{array}$ & $\begin{array}{l}\mathbf{3} \\
\mathbf{3 9} \\
\mathbf{3 9} \\
\mathbf{3 3} \\
\mathbf{3 3}\end{array}$ & $\begin{array}{l}= \\
=\end{array}$ & $\begin{array}{l}\bar{z} \\
\bar{z}\end{array}$ & $\begin{array}{l}521 \\
501 \\
501 \\
630 \\
650\end{array}$ & $\begin{array}{l}18,631 \\
18,593 \\
10,150 \\
10,897 \\
24,256\end{array}$ & $\begin{array}{l}2,360 \\
22,470 \\
23,479 \\
24,460 \\
24,011\end{array}$ & $\begin{array}{l}51,004 \\
51,200 \\
63,531 \\
53,802 \\
50,606\end{array}$ \\
\hline 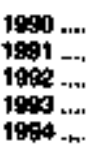 & $\begin{array}{l}83,9053 \\
83,990 \\
86,169 \\
87,007 \\
89,7 \% 9\end{array}$ & $\begin{array}{l}49 \\
\mathbf{5 0} \\
60 \\
5 \\
55\end{array}$ & $\begin{array}{l}337 \\
355 \\
374 \\
368 \\
347\end{array}$ & $\begin{array}{l}2,008 \\
2,688 \\
2,054 \\
2,676 \\
2,991\end{array}$ & $\begin{array}{l}51 \\
39 \\
27 \\
21 \\
19\end{array}$ & $\begin{array}{l}1,200 \\
1,184 \\
1,200 \\
1,190 \\
1,100\end{array}$ & $\begin{array}{r}92 \\
63 \\
97 \\
118 \\
128\end{array}$ & $\begin{array}{l}177 \\
197 \\
217 \\
244 \\
258\end{array}$ & $\begin{array}{l}1,394 \\
1,318 \\
1,426 \\
1,46 \\
1,476\end{array}$ & $\begin{array}{l}682 \\
622 \\
600 \\
649 \\
706\end{array}$ & $\begin{array}{l}20,227 \\
20,460 \\
20,111 \\
20,409 \\
20,060\end{array}$ & $\begin{array}{l}27,041 \\
25,976 \\
27,076 \\
2,543 \\
20,031\end{array}$ & $\begin{array}{l}58,012 \\
58,094 \\
59,089 \\
59,490 \\
60,767\end{array}$ \\
\hline
\end{tabular}

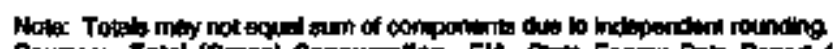

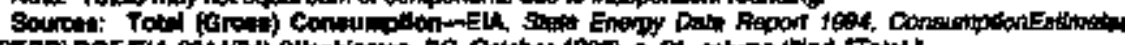

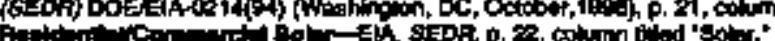

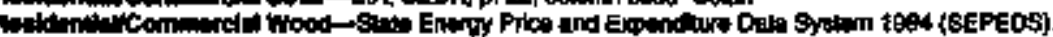

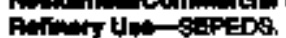

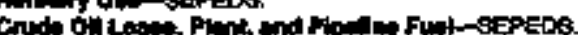

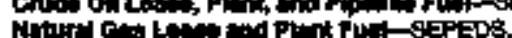

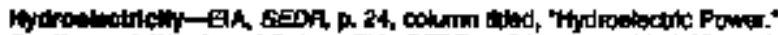

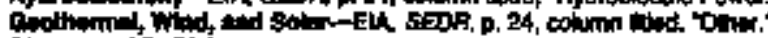

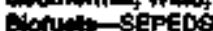

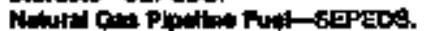

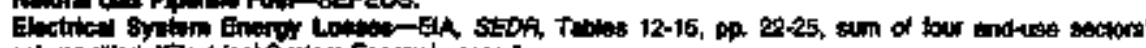

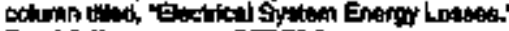

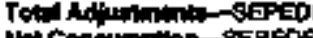




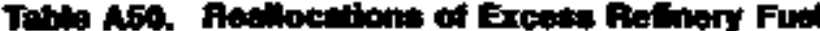
Conitipition

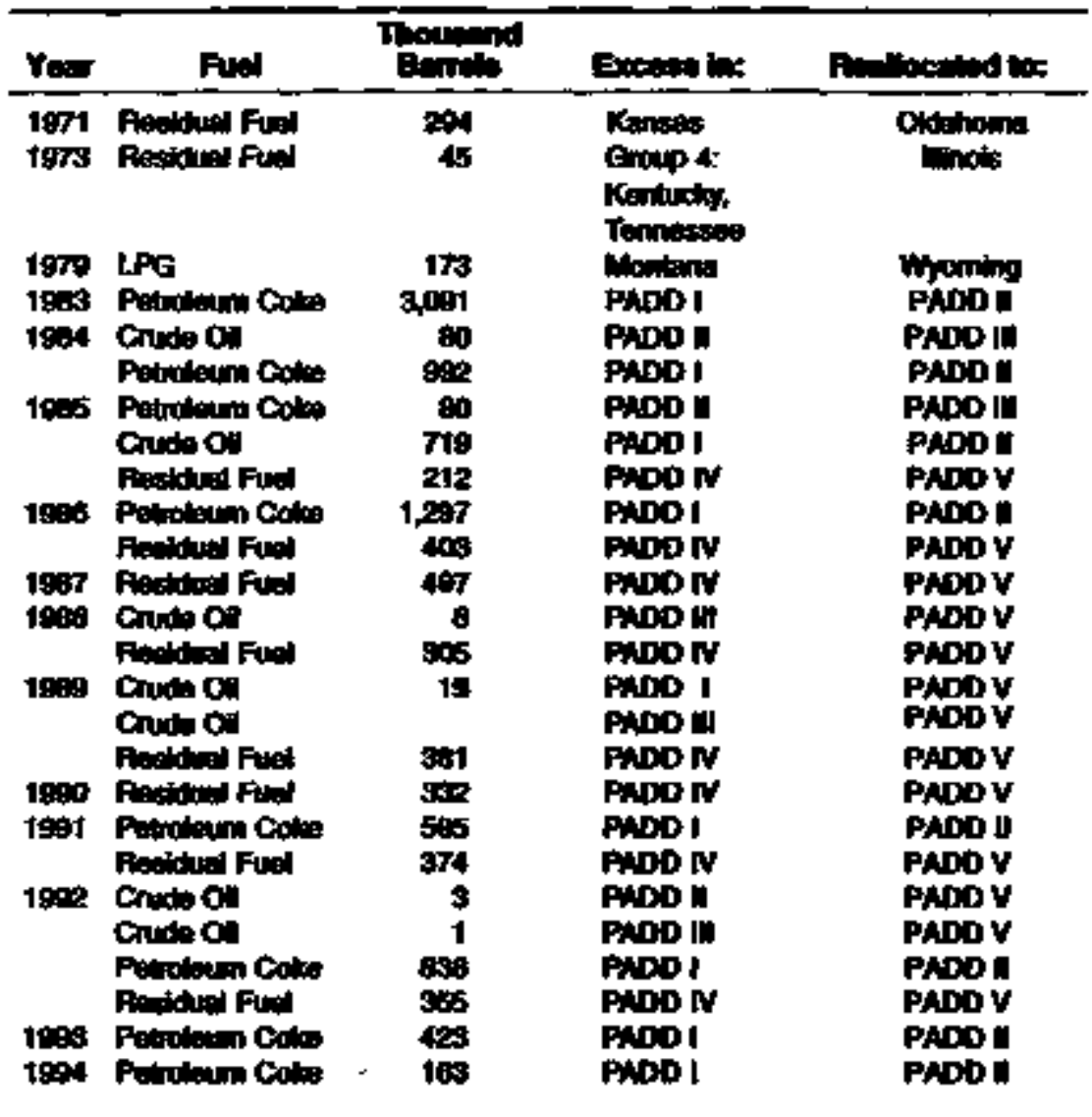

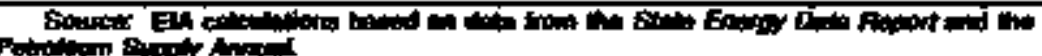

Nexthenst Censos Region is punchesed; 32 percent of the mood use in Midwest States is punchosed; 39 precen of the wood use in States in the South is panchased; and 42 percent of the wood chonemed in Western Strotes is pilichned.

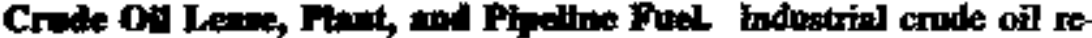
meining after refinery fuel consumption is removed is tsermed to be osed as besse, plaok, and pipeline fuel. Became theac are process fuel uses. the remaining conde oil is removed from SEDS industrial sector consunption.

Natural Gas Iease and Pant Foeh. Natural gas consumed as lease and plant fucl is process fuel and is aubtracted from SEDS industrial sector notural gas totalg by state atod year.

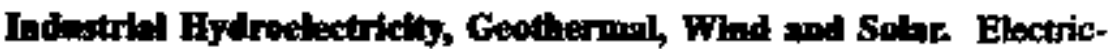
ity generated by indastries from hydropower and geothermal, wind, photovoltaic, and solar thenasl energy has no fuel cost. Operation and mrintenance costs associsted with these energy sonuces are inchuded indirectly in the prices of the industries' products. Therefore. SEDS Induatrial use of these renewable sources are removed from the expenditure celculations.

Indinotril Hiafolt. The cost of wood and waste products used for onergy vary widely from more expessive woods to free industrial waste poodncts. Based on infomation sathered from electric abilities on the EIA "Montly Power Ptant Report" (Form EIA-759), annual percentages were developed of wood consuned that was purchased and waste consuned that was purchnsed. Is is extimated that about 22 percent of the waste ind 75 percent of the wood consumed by industries are purchased.

Natural Gas Fipelite Fecl. Most of the aatural gas consumed in the trinsportroion sector in SEDS is usod to power pipelines. As such, it is - process find and is subtracted from SEDS conaumption in order to calculnue expenditures.

Eectried Syston Deers Losses. The amount of energy lost during generation, trensmission, and distribution of electricity (including plant use and unaccounted for electrical energy) is process fuel and is snbtracted from sectoral energy consunption catimutes nsed in SEPER. The energy losess ane "paid for" when nexidentinl, commercinl, indos. trial, and transportation sector constrmens buy the electricity produced at electric ntilities. 
1976-1980: Bnergy Information Administration, Energy Data Reports, Crude Petrolewn, Petrolewin Products, and Nanural Gas Liguids, table titled "Frels Consumed for All Purposes at Refineries in the United States, by States."

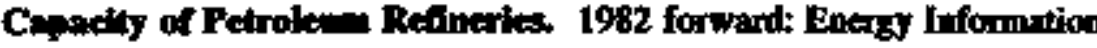
Administration. Petrolendm Supply Anaual Volume I. tables titled "Number and Capacity of Operable Petroleum Refineries," colnon heading, "Crude Capacty, Burrels per Cileudar Day. Opertints" (19821985), and "Atmospheric Crode Oil Distillation Capacity. Bartels per Calendar Day, Operating" (1986 forward).

1979-1981: Energy Information Administration, Enerby Duta Reports, Petroleum Refuneries in the Uniled States and U.S. Tervitories, table tithed "Number ant Capacixy of Petroleum Refineries," column headiag. "Crude Capacity, Bartels per Caleodar Duy, Operating."

1978: Enemgy Information Administration, Energy Data Reports, Petroleam Refineries in the United Stutes and Puerto Rico, table titled "Number and Capacity of Petnoleum Refineries." column heading. "Crude Capacity. Batrels per Calendar Day, Operating."

1970-1977: Burean of Mines, U.S. Department of the Interior, Mineral Indusiry Sorveys, Petrolewn Refineries in the United States and Puerto Rico, table titled "Number and Capacity of Petmolenn Refineries," column heading. "Crude Capacity, Birrels per Calewdar Day, Operating."

Foel Conponed at Ref ieries. 1981 forward: Energy Information Administration, Petnolew Supply Amunal Vofmme 1, table titled "Fuele Consumed at Refineries by PAD District." Data for 1991 are frotn a seperately published EIA ERRATA dated November 10, 1992, GPO Stock No. 061-003-00758-9.
1970-1975: Burente of Mines, U.S. Department of the Interior, Mineral Industry Surveys, Crude Petroleswa, Petrolewa Products, and Natural Gas Liquids, table titled 'Fuels Connumed for All Purposes at Refincies in the United Stries, by States."

1970 forward: Eutery Infon altion Adrinistration, Stute Bnargy Data Systej, industrial sector connunption estintetes for aviation gaeoline bfending companents, crude til, motor greoline hleadiag components, natural gasolice (1970-1963), pentanes phes (1984 forward), petroleum coke, phant condensate (1970-1983), still ges (excledins still tots co:sumed as petrochemical feedstocks, 1970-1985), anfinished oti, wat unfraxtionated stream (1970-1983).

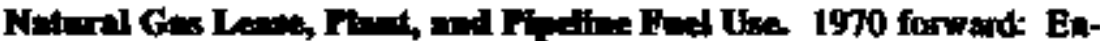

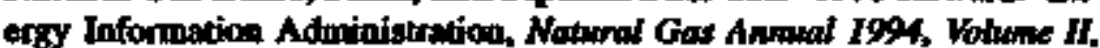
Table 14 (1970-1992), Table 15 (1993 and 1994).

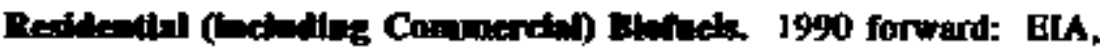
inpublished dutn fren the "1993 Residential Energy Consumption Survey," Fom EIA-457.

Indindinl Evifels, 1990 forwand: RIA, unpublished data from the "Moethy Power Piant Report" (Form ElA-759), the "Annal Report of Major Blectric Utilities Licensees and Other (FBRC Forn No.1), the "Anmual Report of Public Electric Utilities" (Form ELA-412), and the “Annapl Nontility Power Producer Report" (Rocm BIA-867). 

Appendix B

Metric and Other

Physical Conversion Factors 



\section{Metric and Other Physical Conversion Factors}

Data presented in this State Energy Price and Expendidure Report and in other Energy Infarmation Adrinistration pablications are expressed predominavely in onits that historically have been used in the United Stutes, such as British thermal units, barrels, cubic feet, and short tons. However, because U.S. commeree involves other nations, most of which nse metric units of meatore, the U.S. Goverument is commited to the transition to the metric system, as gtaled in the Metric Converaion Act of 1975 (Public Law 94-168), ameeded by the Omnibns Trade and Competitiveness Act of 1988 (Public Law 100-418), and Executive Order 12770 of Joly 25.1991.

The metric conversion factors presented in Tuble BI can be used to calculate the metric-unit equivalents of values expressed in U.S. customary anits. For example, 500 short tons are the equivaleat of 453.6 metric toes (500 shont tons $\times 0.9071847$ inetric kne/thort ton $=453.6$ metric toos).

In the metric system of weights and messures, the numes of multiples and subdivistions of any unit may be derived by combining the wame of the unit with prefixes, such as deke, becto, and tilo, mesning, respectively, 10, 100, 1,000, and deci, centi, and milli, meanieg, reapectively, one-tenth, one-hundredth, and one-thousandth. Common wetric prefixes can be found in Table B2.

The contersion fuctors presented in Thile $\mathrm{B} 3 \mathrm{can}$ be used to calculate equivalents in varions physiral nnits commonly used in energy analyses. For example. 10 burrels are the equivaleat of $420 \mathrm{VS}$. gallons (I0 bernels $\times 42$ gallonstharnel $=420$ gallons). 
Toble B1, Metric Converation Factors

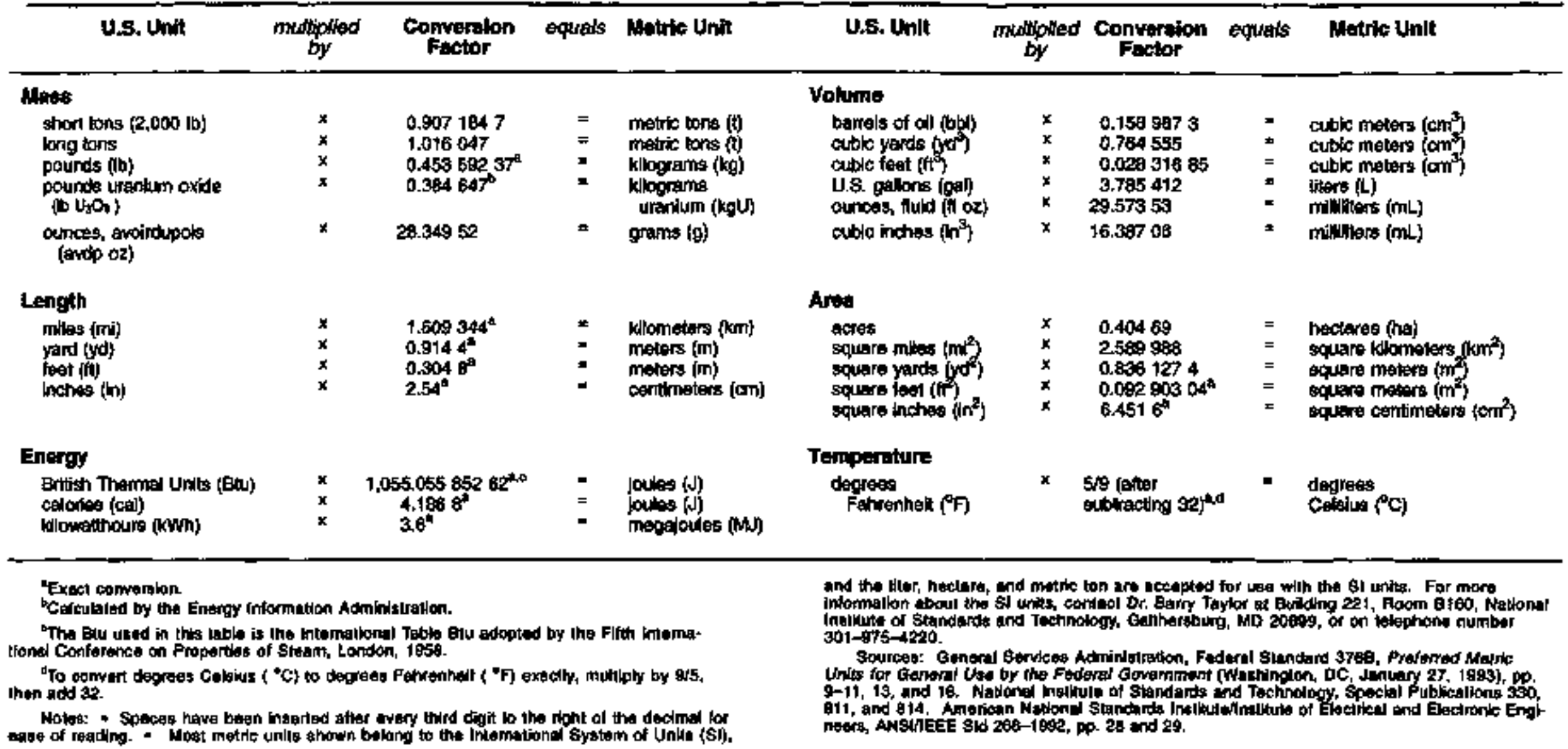


Tablo B2.

Motric Prefixes

\begin{tabular}{|c|c|c|c|c|c|}
\hline $\begin{array}{c}\text { Unit } \\
\text { Multiples }\end{array}$ & Preflix & \$ymbol & $\begin{array}{l}\text { Unh } \\
\text { Subdivistion }\end{array}$ & Pițfix & Symbol \\
\hline $10^{1}$ & deka & da & $10^{-1}$ & decl & d \\
\hline $10^{2}$ & hecto & h & $10^{2}$ & $\operatorname{cen} t$ & c \\
\hline $10^{3}$ & kllo & $k$ & $10^{-3}$ & mill & $m$ \\
\hline $10^{6}$ & moga & $M$ & $10^{6}$ & micro & $\mu$ \\
\hline $10^{\theta}$ & giga & $\mathbf{a}$ & $10^{*}$ & nano & n \\
\hline $10^{12}$ & tera & $\mathbf{T}$ & $1 \sigma^{12}$ & pico & $p$ \\
\hline $10^{15}$ & peta & $\mathbf{P}$ & $10^{-13}$ & femto & $\uparrow$ \\
\hline $10^{16}$ & exp & E & $10^{-18}$ & atto & a \\
\hline $10^{21}$ & zelta & $\mathbf{Z}$ & $10^{-21}$ & zepto & $z$ \\
\hline $10^{24}$ & yolta & $Y$ & $10^{-24}$ & yocto & $y$ \\
\hline
\end{tabular}

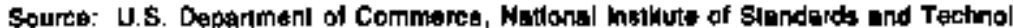

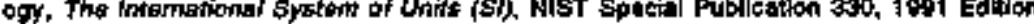
(Whasinglon, DC. Augurs 1991), p. 10.
Table E3. Other Phytical Convergion Factort

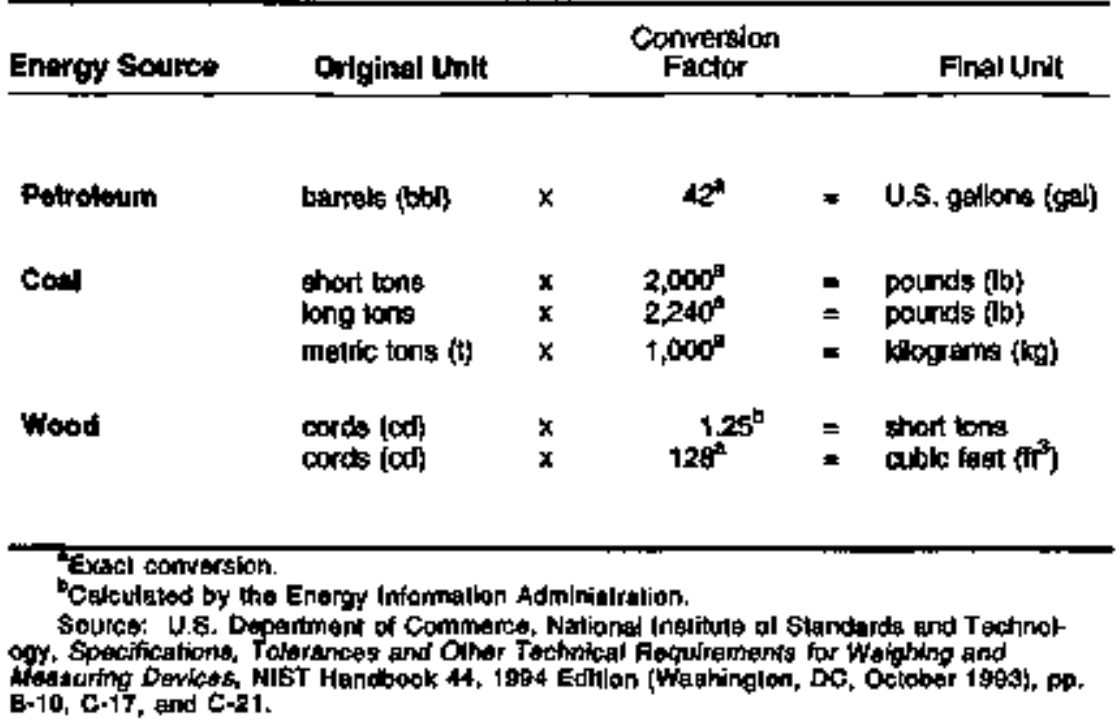



Appendlx C

Summary of Changes 



\section{Summary of Changes Since the State Energy Price and Expenditure Report 1993}

Revisions that are incorporated into the State Energy Price and Expenditure Data System (SEPEDS) since its previous release in Decertber 1995 are summarized in this appendix. All revisions appearing in the data tables of this edition of the State Energy Price and Expenditure Re. port (SEPER) are reported here. Users of the SEPEDS data files, available on personal computer (PC) diskettes and on EIA's Internet bome page, may notice data changes that do not appear in the report due to the level of rounding in the published tables. The full-precision data changes not evident in the report tables are also covered in this appendix.

The most abvious change in this edition of SEPER is the reformatting of the report, including the 90-degree rotation of the report pages that makes it possible to show date for all years instead of only selected years and presents the data in the same format as the consumption data in the State Energy Dato Report, 1994.

Price revisions occur for several reasons: new price series are added; data sources for prices change; price estimation methodologies are revised or price assignment and estimation procedures are updated; data entries are corrected; or consumption estimates are revised. The first four kinds of changes affect State-kvel and U.S. prices directly. The fifth, a revised consumption value, affects only the State prices that are estimated by consumption-weighted averaging of adjacent States and, similarly, affects U.S. average prices.

Consumption estimates used in SEPER are taken from the State Energy Data System (SEDS), publisbed as the State Energy Data Report 1994, Consumption Estimates (SEDR), which contains full documentation of the consumption estimation procedures. Consumption revisions are described in SEDR, Appendix G, "Summary of Changes." Since energy expenditure estimates depend on both the price and consumption estimates (including the consumption adjustments tor process fuel and intermediare products), nevision of either or both may canse revisions to the expenditures series.

\section{Biofuele}

Residentlal and Industrial Sectors, 1990 through 19\%4. The most significant data change in this edition of SEPER is the inclusion of two new ptice and expenditure seriestor 1990 forward-wood in the residential (including commercial) sector and biofiels in the industrial sector.

Residential pritiary energy expenditures for all States increased by at leask 2 percent and by as mich as 11 percent in Oregon and Washington in each yeat. Exceptional revisions occurred in the residential expenditures for primary energy sources in Hawaii. These increased between 20 percent and 40 percent each year when residential wood use was added. Residential wood consumption estimates for Census Region 4 are allocated to the States within the region by the number of honseholds; therefore, cach housethold in Hawaii is estimated to consume the same amount of wood as each housebold in Alaska. Because prices for blofuels are generally lower than those for other energy sources, the average price for all types of primary energy in the residential sector in 1990 forward decreased by 5 percent or less for most States, with the exception of Hawaii, where the average residential primary energy price decreased by 30 percent to 44 percent. 
The impact of the additional data on biofuels consumption in the industrial sector is more difficult to assess becanse of concurnent nevisions to distillate fuel and kerosene prices and expenditures. However, biofoels did contribute to the incresses in revised estimates of industrial expenditures for primary energy sources in Maine by 27 percent to 33 percent in 1990 through 1993, in New Hampshire by 5 percent to 13 percent, in Oregon by 5 percent to 11 percent, and in Washiagton by 4 percent to 16 percent. Because biofuels prices are generally lower than prices for other energy sources, the average price for industrial primary energy sources dropped in Maine by 21 percent to 32 percent in 1990 dinough 1993, in New Hampshire by 26 percent to 33 percent, in Oregon by 8 percent to 13 percent, and in Washington by 4 percent to 9 percent.

C Electric Utillties, 1992, 1983, 1965, 1\%3 throns 193. The average H price paid for biofuels by electric utilities in Minnesota for 1989 through A 1992 was revised by the inclustion of reclassified data. The Minnesat. A pricess decreased by $\$ 0.14$ to $\$ 0.30$ per million Btw in those years. WisC consin electric utilities' average price for biofuels was revised from C $\$ 0.76$ to $\$ 0.93$ per million Bto in 1992 and from $\$ 0.69$ to 0.82 per milE lion Bin in 1993 . The average price of biofuels prid by electric utilities $\mathbf{S}$ in Oregon in 1993 was revised from $\$ 2.48$ per milliou Bto to $\$ 1.13$ per million Btu in 1993.

Biofuels consumption estimates for electric ntilities were slightly revised in SEDS for several States in 1982, 1983, 1985, 1987 through 1990 and 1992 due to acquisition of more precise data and revised data for Califoruia in 1982 . These consumption changes caused expenditures to be revised by less thon 1 percent.

\section{Natural Gas}

Residential Sector, 1993. Rexidential sector natural gas consumption estimates for 1993 were revised in the data source publication, the EIA Natural Gas Anuwal 1994 (NGA), for Florida and lowa, and factors for corverting cubic feet of natural gas to Bw were revised for Lovisiana and West Virginia. Residential natural gas prices for 1993 were aleo revised in the NGA for Flordda, West Virginin, and Louisiann. The expenditures were revised by less than 1 percent in all four affected States.
Conmercial sector, 1995 throgh 1993. Natural gas consumed as vehicle fuel had been historically reported in the commercial sector consumption. In this update to SEPEDS, the auteral gas vehicte fuet, which could be identified separately for 1990 through 1993 , was removed from the commercial sector and shown in the transportation actor instead. Estiluates of commercial sector natural gas expenditteres were reduced by 0.3 percent or less for many Stutes in 1990 throngh 1993 causints small changes in the U.S. avernge price for commercial natural gas.

Other commercial sector changes occumbd becanoe the dats soance publication, the EJA Notumal Gas Ananal 1994, revised commercial sector natural gas consumption and prices for several Stanes in 1993. Smsil price revistions occurred for Florida, Lonisimns, and West Virginia. The most notnble was the increase in price for Texas from $\$ 3.8$ per million Bth to $\$ 4.33$ per million Btw, an increase which, compined with a 22 percent decrease in estimated consumption, caused an 11-percent decrense in Toxas commencial sector namural gas expenditures for 1993. All other nevisions in consunption and prices were by 1 percect or less.

Indmatrial Secter, 1933. The dach sonree publication, the EIA Natural Gas Anumal I994 (NGA), revised industrial sector untural gas prices in 1993 for Alabema, Florida, Iowa, Texas, and Weat Virginia. The Inrgest changes were Alabuma's price decrease from \$3.27 per million Bto to $\$ 3.18$ per million $\mathrm{Btw}$, and industrial astural gas price incresses in Florida from $\$ 3.49$ per million Btu to $\$ 3.53$ per million Blu and in West Verginia's from $\$ 2.50$ per million Btil to $\$ 2.57$ per million Bto. Those price nevisions, combined with NGA revioions to indestrial sabural gas coosumption, as describod in SEDR Appesdix G, cansed extimated iodustrial natural ges expeadicures to be revised in 15 States for 1993.

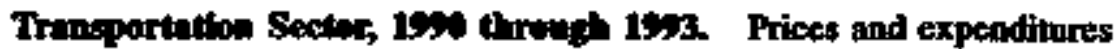
for netrral gas consumed as vehicle fuel in 1990 through 1994 are neported in the transportation sector of this edition of SEPER for the firat time. Vehicle fuel had been inciuded in the commercial sector natural gas consumption in previous editions. The adfition of antural gas used as vehicle foel increased total U.S. transportation expenditures by anoumts ranging from $\$ 1$ million in 1990 to $\$ 7$ million in 1994. 
Clectric Utilties, 1993. The price of ntural gas paid by electric atilities in Tennessee in 1993 was revised in the data sonce pablication, the EIA Naturat Gas Annual 1994 , from $\$ 2.69$ per million Btu to $\$ 1.07$ per miflion Btu, causints a 60-pencent decrease in estimated expenditures.

\section{Petroleum}

\section{Avbation cincoline}

Tramsertatien Secter, 143. Correction of data entry errors for aviation gasoline comsamption in the transportation sector of Alasta and Alabama for 1993 in the State Energy Data System cansed expendimres to decrease in Alasta and increase in Alabaena by amounts too small to be seen in the pablished tubles due to the level of roonding. Users of the SEFEDS date files may notice the small changes.

\section{Distible Fupl}

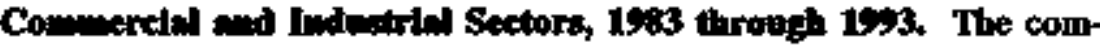
mercist and industrial price series for No. 2 fuel oil, which were ased for commercial and induatrial sector distillate fued prices in previons editions of SEPEDS. wers replaced by price series that represent weighied averages of all types of distillate fuel, inchading diesel fuels. consuried in those sectors. The incorporation of the new series coused prices to change for all States in all years, 1983 through 1993, except for eight isolated cases where the prices for both series are the stme. The Ingest price chunges toccurred for 1985, when the Lonigins commencinl sector price was changed frons $\$ 2.85$ per million $B$ to to $\$ 6.12$ per million Bm; the industrial sector price was revised from \$2.96 per million Bow to $\$ 6.09$ per enillion Btn; the Oklahoma commercial sector price was changed from $\$ 3.20$ per million Btw to $\$ 5.99$ per million Bto; and the indingtrial sector price was nevied from $\$ 3.34$ per million Btw to $\$ 6.24$ per miltion Bto. In 1987, the Kansas commencial sector distillete price was changed from $\$ 2.77$ per millien Beu to $\$ 4.21$ per million But and the Arizons industrial sector price was revised from \$2.64 per million Btu to $\$ 4.43$ per million Bw. All of the other retisions were by kss than 50 percent and nout were by less than 10 percent.
The revisions in prices caused proportional revisions in the expenditures for distillate fuel in the commercial and industrial sectors of al] States and the United States from 1983 through 1993. Industrial sector expenditures in 1993 were also affected by revisions to the consumption adjustmentes for distillate foel consumed by petroleum refiberits.

Electric Uallties, 1973, 1975, and 1978. The methodology for estimating the price of distillate fuel pait by electric atilities in Alastra for 1973,1975 , and 1978 was revised. The price changed from $\$ 2.15$ to $\$ 1.97$ per million Bip in 1973, from $\$ 3.11$ to $\$ 3.10$ per million Bun in 1975, and was increased by less than one cent per million Btu in 1978 . Expenditures for Alastan electric ufilities* distillate fuel were revised proportionally.

Wht Fund

Transportation Sector, $1 \% 33$ and 1984. Jet fuel prices for 1983 and 1984 in previous versions of SEPEDS were estimated from available monthly data. In the prepuration of this update of SEPEDS, 1983 and 1984 jet fuel prices became available from the database that is the source of the prices for 1985 forwant, as published in the EIA Petrotens Marketing Aarual. Frices and the resolting expenditures for jet fuel for all States were revised in 1983 by 10 percent or lests, with the exception of increases of 24 percent in Deleware, 15 percent in New Hampshire, 12 percent in West Virginia, and 11 percent in Idaho. Jet fuel prices and expenditures for all States were revised in 1984 by 10 percent or less, with the erception of 11 -percent increases in Delaware and West Virginia.

\section{Kenowne}

Commercial and Indastrial Sectors, 1985 through 1993. Because kerosene prices are estimated by using distillate fuel prices in the indos trial sector, and the industrial prices are assigned to the commercial sector, the revisions explained in the Dist?!nte Faf section above apply to commercial and industrial sector kenosene prices. 
Kesidentigl Sector, 1993. Estimated prices for residential use of liquefied petroleum gas (LPG) were reduced by the inclusion of additional data for five States in $\mathbf{1 9 9 3}$. The greatest revision occurred for Rhode Island, where the price decreased from $\$ 13.58$ per million Btu to $\$ 8.85$ per million Btu. The residential LPG price for Massachusetts was reduced from $\$ 12.32$ per million Bto to $\$ 10.50$ per million Bu. Prices for New Jersey decreased by $\$ 1.73$ per mitlion $B$ tu, for Hawaii by $\$ 0.27$ per million Btu, and for Alaska by $\mathbf{\$ 0 . 2 5}$ per million Btu. The reduced prices caused proportional reductions in estimated 1993 expenditures for residential LPG for the five States.

Industrial Sector, 1993. Revisions to the consumption adjustments for petroleum refinery consumption of liquefied petroleum gases in 1993 caused industrial sector consumption estimates to be revised in 1993 for alt 33 States with petroleum refíneries. Most States' industrial LPG expenditures increased by 2 percent or less. with the exception of targe increases (more than double) in Iowa, Ilinois, and Mississippi. Large decreases in industrial LPG expenditures occurred in Michigan (by 51 percent) and Kansas (by 39 percent), while smaller decreases ( 3 percent or less) occurred in Texas, Colorado, Montana, Utah, and Wyoming.

\section{Mator Gasoline}

Commercial, lodustrial, and Transportation Sectors, 1993. Revisions to the taxes added to the prices of motor gasoline in the commercial, industrial, and transportation sectors for all States in 1993 caused a \$0.26-reduction in all prices. These price revisions cansed a 3-percent reduction in motor gasoline expenditures for alt sectors in all States in 1993.

\section{Other Potrotown Producte}

Petroleum Coke, 1922 and 1993. Prices for petroleum coke consumed in the industrial sector were revised by less than one cent for 1992 and 1993 by the inclusion of additional data in the price estimation. The revisions caused expenditures for 1992 to decrease by less than one percent in the 34 States with petroleum coke consumption. Estimates of the amount of petroleum coke used by petroleum sefineries (not purchased) and, therefore, excluded from the expenditure calculations were also revised for 1993 . The 1993 price and consumption revisions caused expenditure decreases of nearly 100 percent for States in Petroleum Administration for Defense District (PADD) I, 41 percent for PADD III States, 11 percent for States in PADD II, and less than 1 percent in PADD V States. Petroleum coke expenditures for 1993 were increased by 3 percent in States in PADD IV.

Petroleum Feedstocks, Miscellaneous Products, and Special Naphthas, 1986 through 1993. Revisions to estimated consumption of petroleum feedstocks, miscellaneous products, and special naphthas for 1986 through 1993, as described in the SEDR Appendix G, caused revisions to expenditures for those three products in the 33 States with estimated consumption. The revisions are proportionally the same for the States in 1986 through 1990 because the same allocator for estimating consurnption is used for each of the produets each year. Expenditures, therefore, decreased by 21 percent for 15 States each year, increased by 2 or 3 percent for 10 States, and changed by an insignificant amount for 8 States. Expenditure revisions for 1991 through 1993 Were affected by a different consumption allocator that caused revisions for the 37 States ranging in magnitude from Kentucky's 634-percent increase each year to Pennsylvania and Iowa's 70-percent decrease.

Waxes, 1991 through 1993. Revisions to estimated consumption of waxes for 1991 through 1993, as described in the SEDR Appendix G, caused revisions to expenditures for waxes in the 44 States with estimated waxes consumption. Expenditures in Flotida, Indjana, and New Jersey more than tripled for each of the three years, while expenditure estimates more than donbled in Michigan, Ohjo, and Washington.

\section{Roskuat Fuot}

Alasken Cenmercial and Industrial Sectors and Electric Utilitles. Prices patid by commercial and todustrial customers in Alaska as well as by Alaskan electric utilities are estimated by using djstillate fuel prices. Therefore, the distillate fuel price revisions described in the Distillate Foel section cassed Alaska commercial sector residual fucl prices and expenditures to be rovised for 1984 and 1986 through 1988 and industrial residual fuel prices to be revised in 1985 through 1987 and 
1991 through 1993. A change in the methodology for estimating ptices paid by electric utilities in Alaska for residual fuel revised prices for 1973. 1975, and 1978. The U.S.-level average prices and expenditures were affected by these Alaskan revisjons by amounts too small to be seen in SEPER; however, they can be seen in the data ftles.

Industrtal Seetor, 1993. Corrections to estimates of the tefinery use of residual fuel in 1993 caused revisions to industrial sector expenditures in 1993 for the 32 States with petroleum refineries.

\section{Electricity}

Transportation and Commercial Sectors, 1992 and 1993. Snall revisions in electricity sales to the commercial sector of Nebraska, New York, and Oklahoma in 1992, as documented in SEDR, Appendix $G$, caused recalculation of the estimates of commetcial and transportation sector electricity expenditures for the District of Columbia and the 17 States with transportation electricity use. The 1992 revisions, in most cases, are ton small to be seen in the SEPER tables, and, in all cases, are by less than 1 percent. Electricity prices and expenditures for the commercial sector and transportation sectors in 1993 were also revised due to the routine replacement of preliminary consumption values used in the price estimations for the District of Columbia and the 19 States with transportation electricity use. The consunption revisions are dockmented in $S E D R$. Appendix G. Most of the commercial sector price revisions are not visible in the published tables due to the level of rounding but may be noticed in the data on the SEPEDS data files.

Residential Sector, 1992 and 1993. Estimates of electricity cossumption in the residential sector of 3 States in 1992 and 10 States in 1993 were revised in the source document as described in SEDR, Appendix G. In addition, prices of residential electricity in 1993 were revised for Alabama, Kentucky, Maryland, New Jersey, Ohio, Tennessee, and Texas by one cent or less. These consumption and price revisions caused proportional revisions to expenditures for the affected States for 1992 and 1993.

Industrial Sector, 1992 and 1993 . Estimates of electricity consumption in the industrial sector of seven States in 1992 and five States in 1993 were revised in the source document as described in SEDR. Appendix $G$. In addition, prices of industrial electricity in 1993 were revised for Alabama, Illinois, Kentucky, Maryland, North Carolina, and Tennessee, by one cent or less. The consumption revisions required estimates of refinery fuel use of electricity to be recalculated, affecting consumption estimates of other States within the Petroleum Administration for Defense Districts and causing small revisions is the industrial consumption of an additional 10 States in 1992 and 9 States in 1993. These consumption and ptice revisions caused proportional revistons to expenditures for the affected States for 1992 and 1993.

\section{Consumption Adjustments for Calculating Expendltures}

In general, changes in consumption estimates of process fuel and intermediate products are caused by revisions to industrial and transportation sector consumption estimates in SEDS. In this edjtion of SEPER, revisions to estimates of industrial consumption of natural gas and electricity for 1992 and 1993, and for several "Other Petroleum Products" for 1986 through 1993, as documented in SEDR Appendix G, were incorporated in the SEPEDS adjustments. Also in this edition of SEPER, corrections to estimates of the refinery use of distillate fuel, residual fuel, liquefied petroleum gases, and petroleum coke in 1993 caused revisions to industrial sector expenditures for the 32 States with petroleum refincries. Although the consumption adjustments for proceas fuel and intermediate products in 1994 are shown in Table A49, the revisions to previous years" data can only be seen in the data included on the SEPEDS data files. 



\section{Glossary}





\section{Glossary}

Asphalt: A dark-brown+to-black cement-like material containing bitumens as the predominant constituents obtajned by petroleum processing. The definition includes crode asphalt as well as the following finished products: cements, floxes, the asphalt content of emulsions (exclusive of water), and petroleum distillates blended with asphalt to make cutback asphalts.

\section{ASTM: The American Society for Testing and Materials.}

Avistion Gesoline Blending Components: Naphthas that are used for blending or compounding jnto finished aviation gasoline (e.g., straightrun gasoline, alkylate, and reformate). Excludes oxygenates (alcohols and ethers), butane, and pentanes plas.

Avtatton Gasoline: All special grades of gasoline for use in aviation reciprocating engines, as given in ASTM Specification D910 and Military Specification MIL-G-5572. Aviation gasoline includes blending components.

Barrel (petroleum): A unit of volume equal to $42 \mathrm{U}$. S. gallons. $^{\circ}$

Bífuels: Nonfossil biomass energy soturces and biomass-derived fuels, which together encompass all energy sources from recent-term organic (plant and animal) matter. Nonfossil biomass energy sources are essentially unpracessed; they are burmed or gasified, as received, to produce themal energy or electricity. Examples are fuelwood, waste wood, gatbage, and crop waste. Biomass-denived fuels, on the other band, result from the processing of biomass energy sources. They may be by-prod. ucts of industrial or agricultural processes of they may be fuels made from biomass feedstocks. Biomass-derived fuels generally have concentrated energy density and are more easily transported and used.
Examples are wood by-products (such as wood chips and dewatered wood liquors), pellets, briquettes, refuse-derived fuel (made frot garbage), ethanol (made from crops, such as corn), and methanol (made from wood). Different mixes of biofuels are used by eack consuming sector. The residential and commercial sectors burn wood and petlets for apace heating. The industrial sector's largest biofuel source is combustible by-products used for electricity generation and process stearn, followed in importance by wood chips. The transportation sector uses ethanol as an additive to motor gasoline. Some electric attilities use wood, industrial wood waste, and municipal waste as cofiring or primary fuels.

British Thermal Unit (Btu): The quantity of heat needed to taise the temperature of 1 pound of water by $1^{\circ} \mathrm{F}$ at or near $39.2^{\circ} \mathrm{F}$. See Heat Confent of a Quantity of Fuel, Gross and Heat Content of a Quantity of Fuel, Net.

Butane: A normally gaseous stratght-chain or branched-cbain hydrocarbon $\left(\mathrm{C}_{4} \mathrm{H}_{10}\right)$. It is extracted from natural gas or refinery gas streams. It includes isobutane and normal butane and is designated in ASTM Specification D1835 and Gas Processors Association Specifications for commercial butane.

- Isobutane: A normally gaseous branched-chain hydrocarbon. It is a colorless paraffinic gas that boils at a temperature of $10.9^{6} \mathrm{~F}$. It is extracted from nanral gas or refinery gas streams.

- Normal Butane: A normally gaseous straight-chain hydrocarbon. It is a colorless paraffinic gas that boils at a temperature of $31.1^{\circ} \mathrm{F}$. It is extracted from natural gas or refinery gas streams.

Butylene: An olefinic hydrocarbon ( $\left.\mathrm{C}_{4} \mathrm{H}_{8}\right)$ recovered from refinery processes. 
C Cosl: Includes all ranks of coal—anthracite, bituminous cos], subbituminotis coal, and lignite- conforming to ASTM Specification D388.

- Coking Coal: Coal that meets the requirements for making coal coke. It must be low in ash and sulfur and form a coke that is capable of supporting the charge of iron ore and limestone in a blast fumace. Coking coal is usually a blend of two or more bituminons cosls.

- Steam Coal: In this report, steam coal represents all noncoking coal.

Coal Coke: A hard, porous product made from baking bituminous coal in ovens at temperatures as high as $2,000^{\circ} \mathrm{F}$. It is used both as a fuel and as a reducing agent in smelting irow ore in a blast fumace.

Coke Plants: Plants where coal is carbonized in slot or behive ovens for the manufacture of coke.

\section{Coksing Coal: See Coal.}

Commercial Sector: The commercial sector, as defined economically. consists of business establishments that are not engaged in transportation or in manufacturing or other types of industrial activity (agriculoure. mining, or construction). Commercial establishments include hotels, motels, restaurants, wholesaje businesses, retail stores, laundries, and other service enterprises; religious and nonprofit organizations; health, social, and educational institutions; and Federal, State, and local goventments. Street lights, pumps, bridges, and public services are also included if the establishment operating them is considered commercial.

Constant Dollarg: Amounts expressed in constant dollars have been adjusted to remove the effect of changes in the purchasing power of the dollar. Prices expressed in constant dolkars usually refkect buying power relative to a base year. Prices in this publication are expressed in Neminal Dollars.

Conversion Factor: A pumber that translates units of one system into corresponding values of another system. Conversion factors can be uted to translate physical units of measure for various fuels into Btu equivalents.
Crade Oil Uged Drectly: Crude oil consumed as fuel by crude oil pipelines and on crude oil leases.

Cubic Foot (natural gas): A unit of volume equal to 1 cubic foot at a pressure base of 14.73 pounds standard per square inch absolute and a temperature base of $60^{\circ} \mathrm{F}$.

Degrte-Day Normals: Simple arithmetic averages of monthly or annual degree-days over a long period of time (the 30-year period 1951 through 1980 is used for the estimates in this report). The averages may be simple degree-day normals or populntion-weighted degree-day normals. Monthly, State-level simple averages are used for this report.

Degree-Days, Cooling (CDD): The number of degrees per day that the daily average temperature is above $65^{\circ} \mathrm{P}$. The deily averago temperature is the mean of the maximum and mintimum temperatores for a 24-hour period.

Degree-Days, Hetting (HDD): The number of degrees per day that the daily average temperature is below $65^{\circ} \mathrm{F}$. The datly average temperature is the mean of the maximum and minimum temperatures for a 24-hour period.

Degree-Days, Popalation-Weighted; Healing or cosling degree-days weighted by the population of the area in which the degree-days are recorded. To compute State popolation-weighted degree-days, each State is divided into from one to nine climatically homogeneous divisions. which are assigned weights based on the ratio of the popnlation of the division to the total population of the State. Degree-day readings for each division are multiplied by the corresponding population weight for each ifivision and those products are then summed to arrive at the State popalation-weighted degree-day figure. To compute national poppulation-weighted degree-days, the Nation is divided into nine Census regions, exch comprising from three to eight States, which are assigned weights based on the ratio of the population of the region to the total population of the Nation. Degree-day readings for each negion are moltiplied by the comesponding population weight for each region and those products are then summed to arrive at the national population-weighted degree-day figure. 
Difestel Fuel: Fuel used for intemal combustion in diesel engines; usually that fraction of crode oil that distills after kerosene. (See Diatllate Fuel ON1.)

Distillate Fuel Oil: A general classifieation for one of the petroleum fractions prodoced in conventional distillation operations. Inchuded are products trown as No. 1, No. 2, and No. 4 fuel oils and No. 1, No. 2, and No. 4 diesel fuels. It is used primarily for space heating, ontand off-highway diesel engine fuel (including railroad engine fuel and fuel for agricultural machinery), and electric power generation.

Electrical System Ruergy Lasses: The amount of energy lost during generation, transmission, and distribution of electricity, incloding plant and onaccounted-for nses.

Dlectrickty Geaeration: The process of protucing electric energy on transforming other forms of energy into electric energy. Also the amont of electric energy produced or expressed in wathours (Wh).

Electriclty Sales: The amount of kilowatthonrs sold in a given period of time; usually grouped by classes of service, such as residertial, commercial, industrial, and other. "Other" sales include sales for public street and highway lighting and other sales to public authorities, sakes to milnoads and railways, and interdepartbiental sales.

Mectric Power Ptint: A station containing prime movers, electric gen. erators, and auxilizry equipment for converting mechanical, chemical, andlor fission enersy into electric energy.

Electric Utillty: A corporation, person, agency, authority, or other legal entily or instmmentality that owas and/or operates facilities for the generation, transmission, distribution, or zale of electric energy, primarily for use by the public, and that files forms listed in the Code of Federal Regulations, Title 18, Part 141. Facilities that qualify as cogenerators or small power producers under the Public Utility Regulatory Policies Act are not considered ntilities.

Electric Uttlity sector: The electric utility sector consists of privately and publicly owned establishments that generate, transmit, distribute, or sell electricity primarily for use by the public and that meet the definition of an electric utility. Nonutility power producers are not included in the electric utility sector.

Eud-Uise Sectors: The residential, commercial, industria, and transpottation sectors of the economy.

Energy: The capacity for doing work as measured by the capability of doing work (potential energy) or the conversion of this capability to motion (kinetic energy). Energy has several forms, some of which are easily convertible and can be changed to anotber form useful for work. Most of the world's convertible energy comes from fossil fuels that are burned to produce heat that is then used as a transfer medjum to mechanical or other means in order to accomplish tasks. Electrical energy is usually measured in kilowathours, while heat energy is usually measured is British thermal units.

Ethane: A normally gaseous straight-chain hydrocarbon $\left(\mathrm{C}_{2} \mathrm{H}_{6}\right)$. It is a colotless, paraffinic gas that thoils at a temperature of $-127.48^{\circ} \mathrm{F}$. It is extracted from namral gas and refinery gas streams.

Ethylene: An olefinic bydrocarbon $\left(\mathrm{C}_{2} \mathrm{H}_{4}\right)$ recovered from refinery processes of petrochemical processes.

Expenditures: In this report, "expenditures" refers to the money directly spent by contumers to purchase energy. Expendiures equal the aurount of energy used by the consumer times the price per unit paid by the consumer. In calculating the amount of energy used, process fuel and intermediate products are not included.

Exports: Shipments of goods from the 50 States and the District of CoIumbia to foreign countries and to Puerto Rico, the Virgin Islands, and other U.S. possessions and torritories.

\section{fass: See Free Alontgdde Ship.}

Federal Energy Regulatory Commission (FRRC): The Federal agency with jurisdiction over interstate electricity sales, wholesale electric rates, hydroelectric licensing, nauural gas pticing, oil pipeline rates, and gas pipeline certification. FERC is an independent regulatory 
G agency within the Departzont of Energy and is the successor to the Federal Power Commission.

Federal Power Commission (FPC): The predecessor agency of the Federal Energy Regulatory Commission. The Federal Power Commission was created by an Act of Congress under the Federal Water Power Act on June 10,1920. It was charged originally with regulating the electric power and natural gas industries. It was abolished on September 30, 1977, when the Deparment of Energy was created. Its functions were divided between the Department of Energy and the Federal Energy Regulatory Commission, an independent regulatory agency.

Fossil Fuel: Any naturally occutring organic fuel, such as perroleum, coal, and natural gas.

Free Alongside Ship (f.a.s.)! The value of a commodity at the port of exportation, generafly including the purchase price, plus all charges incurred in placing the commodity alongside the carrier at the port of exportation.

Gasohat: A blend of finished motor gasoline (leaded or unleaded) and alcohol (generally ethanol but sometimes methanol) limited to 10 percent by volume of alcohol. Gasohol is included in fintished leaded and unleaded motor gasoline.

Geothermal Energy: Hot water or steam extracted from geothema] reservoirs in the Earth's crust and supplied to steam turbines that drive generators to produce electricity.

Heat Content of a Quantity of Fuel, Gross: The total amount of heat released when a fuel is burned. Coal, crude oil, and natural gas all include chemical compounds of carbon and bydrogen. When those fuels are burned, the carbon and hyorogen combine with oxygen in the ais to produce carbon dioxide and water. Some of the energy released in buming goes into transforming the water into steam and is usually lost. The amount of heat spent in transforming the water into steam is counted as part of gross heat content but is not counted as part of net heat content. Also referred to as the higher heating value. Btu conversion factors typically used in ElA represeat gross heat content.
Heat Content of a Quantity of Foel, Net: The amount of useable heat energy released when a fuel is burned under conditions similar to those in which it is normally used. Also referred to as the lower heating value. Btu conversion factors typically used in EIA represent gross heat content.

Heavy Oil: The fuel oils remaining after the lighter oils have been distilled off during the refining process. Except for statt-up and flame stabilization, virtually all petroleum used in steam-electric power plants is heavy oil.

Hydroelectric Power: The production of electricity from the kinetic energy of falling watet.

Imports: Receipts of goods into the 30 States and the District of Columbia from foretgn countries and from Puerto Rico, the Virgin Ișlands, and other U.S. possessions and territories.

Industrial Sector: The industrial sector comprises manufacturing industries, which make up the largest part of the sectof, along with miring, construction, agriculture, fistieries, and forestry. Establishments in the sector range from steel mills, to small farms, to companies assembling electronic components.

Jet Fuel: The term includes kerosene-type jet fuel and naphtha-type jet fuel. Kerosene-type jet fuel is a kerosene-quality product used prizaarily for commercial turbojet and turboprop aircraft engines. Naphtha-type jet fuel is a fuel in the heavy naphthas range used primarily for military turbojet and turboprop airctaft engines.

Ketosene: A petroleum distillate that has a maximum distillation temperature of $401^{\circ} \mathrm{F}$ at the 10-percent recovery point, a final boiling point of $572^{\circ} \mathrm{F}$, and a minimum flash point of $100^{\circ} \mathrm{F}$. Included aro the two

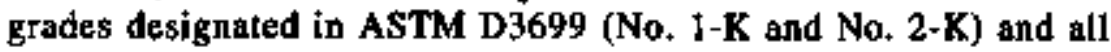
grades of kerosene called Jange or stove oil. Kerosene is used in space heaters, cook stoves, and water beaters; it is suitable for use as an illuminant when bumed in wick lamps. 
Lease and Plant Fuel: Natoral gas used in well, field, and lease operations (such as gas used in drilling operations, heaters, debydrators, and field compressors) and used as foel in natural gas processing plants.

Light Oil: Lighter fuel oils distilled off during the refining process. Virtually all petrolemm used in interal conbestion and gas-turbine engines is light oji.

Liquefied Petroleum Gases (LPG): Ethane, ethylene, propane, propylene, normal butane, butylene, and isobutane produced at refineries or natural gas processing plants, including plants that fractionate new natural gas plant likuids.

Lubricants: Substances used to reduce friction between bearing surfaces or as process materials either incorpotated into other materials used as processing aids in the manufacturing of other products or as carriers of other uaterials. Petroleum lubricants may be produced either from distillates or residues. Other substances may be added to impart or improve certain required propertics. Excluded are byproducts of iubrjcating oil refining. such as aromatic extracts derived from solvent extraction or tars dorived from deasphalting. Inchuded are all grades of lubricating oils from spindle oil to cylinder oil and those used in greases. Lubricant caltegories are paraffinic and naphthenic.

Miscellaneous Petroleam Products: Alj finished petroleum products not classified elsewhere-for exanale, petrolatum, lube refining byproducts (aromatic extracts and tars), absorption olls, tam-jet fuel, petroleum rocket fuels, synthetic natural gas feedstocks, and specialty oils.

Motor Gasollne: A complex mixture of relatively volatile hydrocarbons, with or without smalt quantities of additives, obtained by blending appropriate refinery streams to form a fuel suitable for use in spark-ignition engines. Motor gasoline includes both leaded and unleaded grades of finished motor gasoline, blending components, and gassohol.

Motor Gasolime Blending Components: Naphthas that will be used for blending or compounding into finished motor gasoline (e.g., straightrun gasoline, alkylate, reformate, benzene, toluene, and xylene).
Excluded are oxygenstes (alcohols and ethers), butane, and pentanes plus.

Naturat Gas: A mixture of tydrocarbons (principally methane) and small quantities of various nonbydrocartons existing in the gaseous phase or in solution with crude oil in underground reservoits.

Natural Gasoline: A mixture of hydrocarbons, mostly pentanes and beavier. extracted from natural gas that meets specifications for natural gasoline set by the Gas Processors Association. Includes isopentane.

Nominal Dollars: Amounts that have not been adjusted to remove the effect of changes in the purchasing power of the dollar.

Nomingl Price: The price paid for a product or service at the time of the transaction. The nominal price has not been adjusted to remove the effect of changes in the purchasing power of the dollar.

Nuclear Electric Power: Electrictiy generated by an electric power plant whose turbines are driven by steam generated in a reactor by heat from the fissioning of nuclear fuel.

Nuclear Electric Power Plant: A single-unit or multiunit tacility in which heat ptoduced in one or more reactors by the fissioning of nuclear fuel is used to drive one or more steam turbines.

Nuclear Fuel: Fissionable materials that bave been enriched to a composition that, whon placed in a nuclear reactor, wil] support a self-sustaining fission chain reaction, producing heat in a controlled manner for process use.

Pentanes Pius: A mixture of hydrocarbons, mostly pentanes and heavier, extracted from natural gas. Includes isopentane, natural gasoline, and plant condensate.

Petrochemical Feedstocks: Chemical feedstocks derived from petrolevm principally for the manufacture of chemicals, synthetic nbber, and a variecy of plastics. The categories reported are naphthas less than $401^{\circ} \mathrm{F}$ endpoint and other oils equal to or greater than $401^{\circ} \mathrm{F}$ endpoint. 
C Petroleam: A generic term applied to oil and oil products it all forms, such as crude oil, lease condensate, unfinished oils, petroletm products, natural gas plant liquids, and nonhydrocarton compounds blended into frnished petroleum products.

Petroleam Coke: A residue that is the final prodact of the condensation process in cracking. The product is either marketable petralenm coke or catalyst petrokum coke.

Petrolemm Coke, Catalyst: The carbonaceons residue that is deposited on and deactivates the catalyst used in many catalytic operations (e.g. catalytic cracking). Carbon is deposited on the caralyst, thus deactivating the catalyst. The catalyst is reactivated by borning off the carkon, which is used as a fuel in the refining process. That carton or coke is not recoverable in a concentrated form.

Petroleum Coke, Marketable: Those grades of coke produced in delayed or fluid cokers that may be recovened as nelatively pure carbon. Marketable petroleum coke may be sold as is or may be further purified by calciging.

Petroleum Products: Products obtained from the processing of crude oil (including lease condensate), natural gas, and other hydrocarbon compounds. Petroleum products include unfinished oils, higuefied petroleum gases, pentanes plus, aviation gasoline. motor gasoline. naphtha-type jet fuel, kerosene-type jet fuel, kerosene, distillate fuel oil, residual fuel oil, petrochemical feedstocks, special naphthas, lubricants, waxes, petroleum coke, asphalt, road oil, still gas, and miscellaneous products.

Photoroliatic and Solur Thermsl Energy: Energy radiated by the sun as electromagnetic waves (electromagnetic radiation) that is converted at electric utilities irto electricity by means of solar (photovoltaic) cells or concentrating (focusing) collectors.

Pant Condensate: One of the natural gas liquids, mostly pentanes and hesvier hydrocarbons, recovered and separated as liquids at gas inlet separators or scrubbers in processing plants.
Primary Energy: All energy consumed by end users, excloding electricity but inchuding the evergy consumed at electric utilities to generate electricity. (In estiunating energy expendinures, there are no fuel-associated expenditures for hydroelectric power, geothermal energy, solar euergy, or wind energy. and the quantifiable expenditures for pnocess fuel and intermediate products are exchuded.)

Process Duel: All energy consumed in the acquisition, processing. and transportation of energy. Quantifiable process fuel includes threec calegories: notural gas lease and plant operations, natural gas pipeline operations. and ofl refitery operations.

Propane: A nomally gaseous straight-chain hydrocarbon $\left(\mathrm{C}_{3} \mathrm{~F}_{8}\right)$. It is a colorless paraffinic gas that boils at a temperature of $\mathbf{4 3 . 6 7 ^ { \circ }}$ F. It is extracted from natural gas or refinery gas streams. It includes all products designated in ASTM Specification D1835 and Gas Processors Association Specifications for commercial propane and HD-5 propane.

Propylene: An olefinic hydrocabon $\left(\mathrm{C}_{3} \mathrm{H}_{6}\right)$ recovered from refinery of petrochemical processes.

Real Prices A price that bas been adjusted to remore the affect of changes in the purchasing power of the dollar. Real prices ussually teflect baying power relative to a base year. Prices shown in this poblication are Nominal Prices.

Refinery (petroleum): An installation that manufactures finished petrolean products from crude oil, unfinished oils, antnral gas liquids. other bydrocartons, and alcohol.

Residential Sector: The residential sector is considered to consist of all privale residences, whether occupied or vacant, owned or rented, including single-family homes, maltifamily howing unils, aud mobik bomes. Stcondary homes, such as suminer homess, are also included. Institutional housing, such as school dormitories, hospitals, and military barracts, generally are not incleded in the rexidential sector; they are incloded in the coinmercial sector.

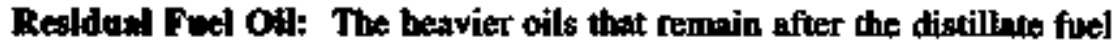
oils and lighter hydrocarbons are distilled away it refinery operations 
and that conform to ASTM Specifictitions D396 and 975. Included are No. 5, a residual fuel oil of medium viscosity; Navy Special, for use in steam-powered vessels il government service and in shore power plants; and No. 6, which inchudes Bunker $C$ fuel oil and is used for commercial and industrial heating, for electricity seneration, and to power ships. Imports of residaal fuel oil include imported crode oil bumed as fuel.

Road OAl: Any heavy petroleum oil, including resjdual asphrltic oil, used as a dust palliative and surface treatment on roads and highways. It is generally produced in six grades, from 0 , the most liquid, to 5 , the most viscons.

Short Tbe (coit): A tenit of weight equal to 2,000 pounds.

\section{SIC: See Strotand Industrial Closeificadion.}

Specinl Naphthes: All finished products within the naphtha boiling range that ane used as paint thinners, cleaners, or soivents. Those products are refined to a specified flash point. Special naphthas include all commereial hexane and cleaning solvents conforming to ASTM Specifications D1836 and D484, respectively. Naphthas to be blended or marketed as mokor gasoline or aviation gasoline, or that are to be used as petrochemical and synthetic natural gas (SNG) feedstocks are excluded.

Standard Industrit Clessicication (SIC): A set of codes developed by the Office of Management and Bulget which categorizes industries into groups with similar economic activities.

\section{Steare Call: See Corl.}

Soll Cas (refinery gan): Any form or mixture of gas prodnced in nefineries by distillation, cracking. reforming, and other processes. The principal constituents are methane, thane, ethylene, acrual butane, butylene, propane, and propylene. It is used primarily as refioery fuel and petrochemical feedstock.

Thtal Energy: All energy consumed by end users, including electricity but excluding the energy consumed at electric utilities to gentrate electricity. (In estimating energy expenditures, there are no fuel+associated expenditures for hydroelectric power, geothermal epergy, solar power, or wiod energy, and the quantifiable expenditures for process fues and intermediate products are excluded.)

Transportation Secter: The transportation sector consists of privare and public vehicles that move people and commodities. Included are automobiles, trucks, buses, motorcycles, railroads and railways (inclading streetcars), gircraft, ships, barges, and natural gas pipelines.

Unfinished Otls; All oils requiring further refinery processing, except thase requiring only mechatical blending. Included are naphthas and lighter oils, kerosene and light gas oils, hesvy gas oils, and residuum.

Unfroctionsted Strenns: Mixtures of unsegregated natural gas liquid components, excluding those in plant condensate. This product is extracted from natural gas.

Uafted States: The 50 States and the District of Columbia.

Wanes: Solid or semisolid materials derived from petroleum distillates or residues. Wares are light-colored, mote or less transilucent crystalline masses, slightly greasy to the touch, consisting of a mixture of solid hydrocarbons in which the paraffin series predominates. Included ase all marketable waxes, whether crude scake or fully refined. Waxes are used primarily as industrial coating for sorface protection.

Wind Enerta: The kinetic energy of wind convented into mechanical energy by wind turbines (i.e., blades rotating from a hub) that drive generators to produce electricity for distribution. 


\title{
Publication Order Form for State Energy Data Report 1994
}

Consumption Estimates

Published: October 1996

Energy Information Administration

GPO Stack No. 061-003-00972-7

Price per copy: $\$ 44.00$

Personal Name or Attention Line:

Company Name, if applicable:

Street Address:

City, State, Zip Code:

Daytime Phone Number (area code furst):

Purchase Order No:

May we make your name and addreso aveileble to other mailere?

yes

no

Please include payment with this order form.

Allow a rinimum of 4 weeks for domestic delivery and an additional 6 woeks for international delivery.

Quantity $x \$ 44.00=$ total due: (Intemational customers add 25\%)

$\square$ Check payable to Superintendent $\alpha$ Documents $\square$ CPO Deposit Actount No.

TVISA or Mastercand Account No.

Authorizing Signoturte Credit Card Expiration Date

Noto: Price includes neguler domestic postage and handing. It is subject to change.

\author{
Malf onder fom to: U.S. Govemment Printing Ofice \\ P.O. Box 371954 \\ Pitt:burgh, PA 15250-7954 \\ Or fax order form to: \\ $202-512-2250$
}

Thank you for your orderl 


\section{Publication Order Form for State Energy Price and Expenditure Report 1994}

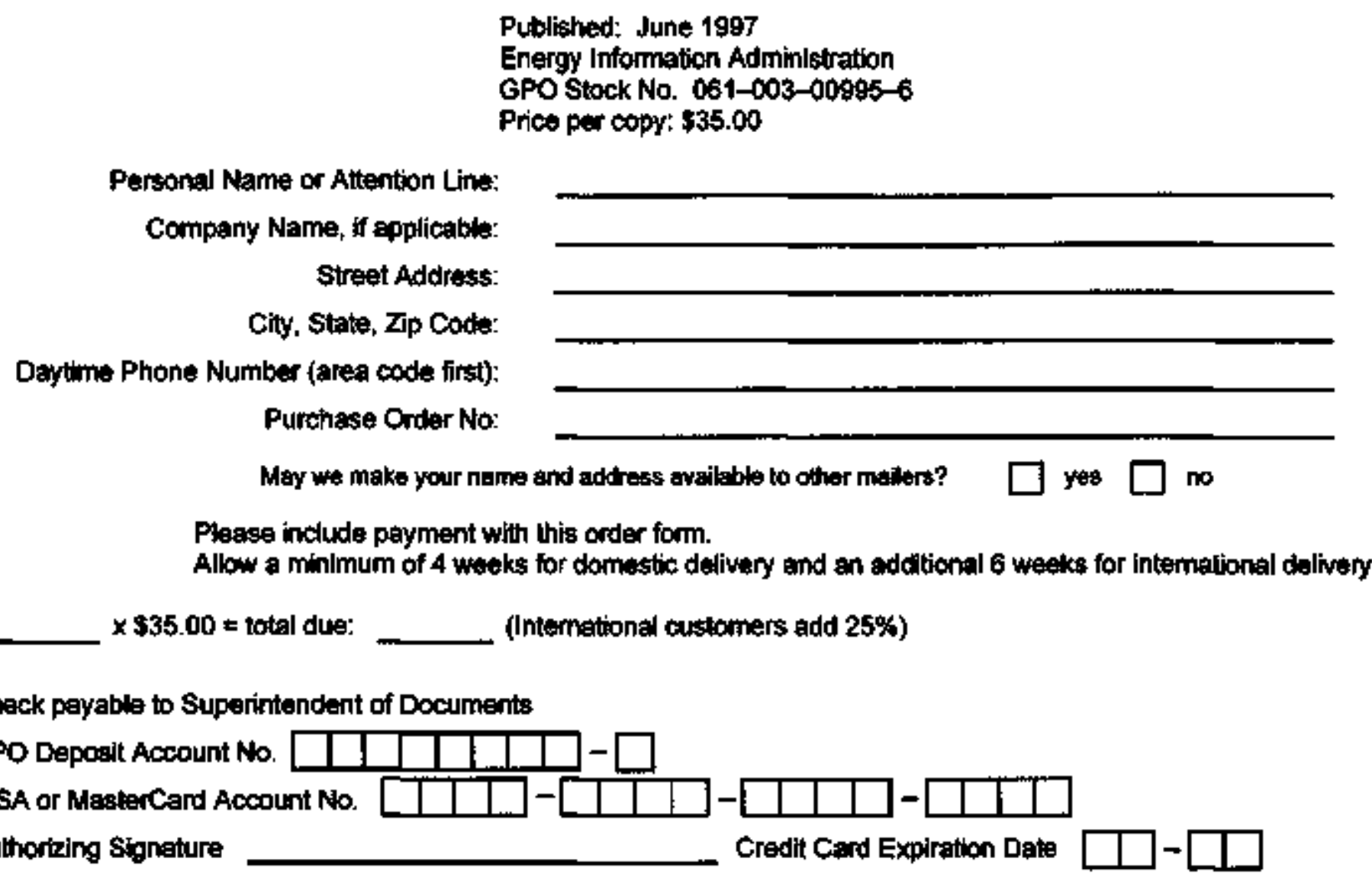

Note: Price inctudes regular domestic postage and handing. It is subject to change.

$\begin{array}{cl}\text { wat ondiar fom to: } & \text { U.S. Gowomment Printing Ofice } \\ & \text { P.O. Box 371954 } \\ \text { Pittsburgh, PA 15250-7954 } & \text { 202-512-2250 }\end{array}$

Thank you for your order! 


\section{Integratiad Misterieal terergy Doto Reports sofrom the Inergy Information Adninistration}

\section{Monthly Energy Rewtew}

Current monthly date on production, consumption, stocks, trade, and prices of the principal energy commodities in the United States.

Amunal Rnergy Review

Long-term historical anmual data on U.S. energy production, consumption, stocks, trade, and prices. Most series begin in 1949.

\section{State Energy Date Report}

Annual energy consumption estimates at the State and national levels by energy source and by major sector (i,e,, residential, commercinl, imdustrial, transportation, and electric utilities), beginning with 1960 .

\section{State Energy Price and Euxpenditure Report}

Annual energy price and expenditure extimates at the State and national levels by energy source and by major sector, (i.e., residential, commercial, industrial, transportation, and eloctric utilities), begimning with 1970 .

\section{Intemational Energy Amumal}

Annual data for production, consunption, and trade of primary energy commodities in more than 220 countries, dependencies, and areas of special sovereignty. Aliso included are prices of crude oil and petroleum products in selected countries.

\section{Intemaxtional Petrolosm Statisfics Report}

Current monthly international petroleum data on production, consumption, imports, and stocks.
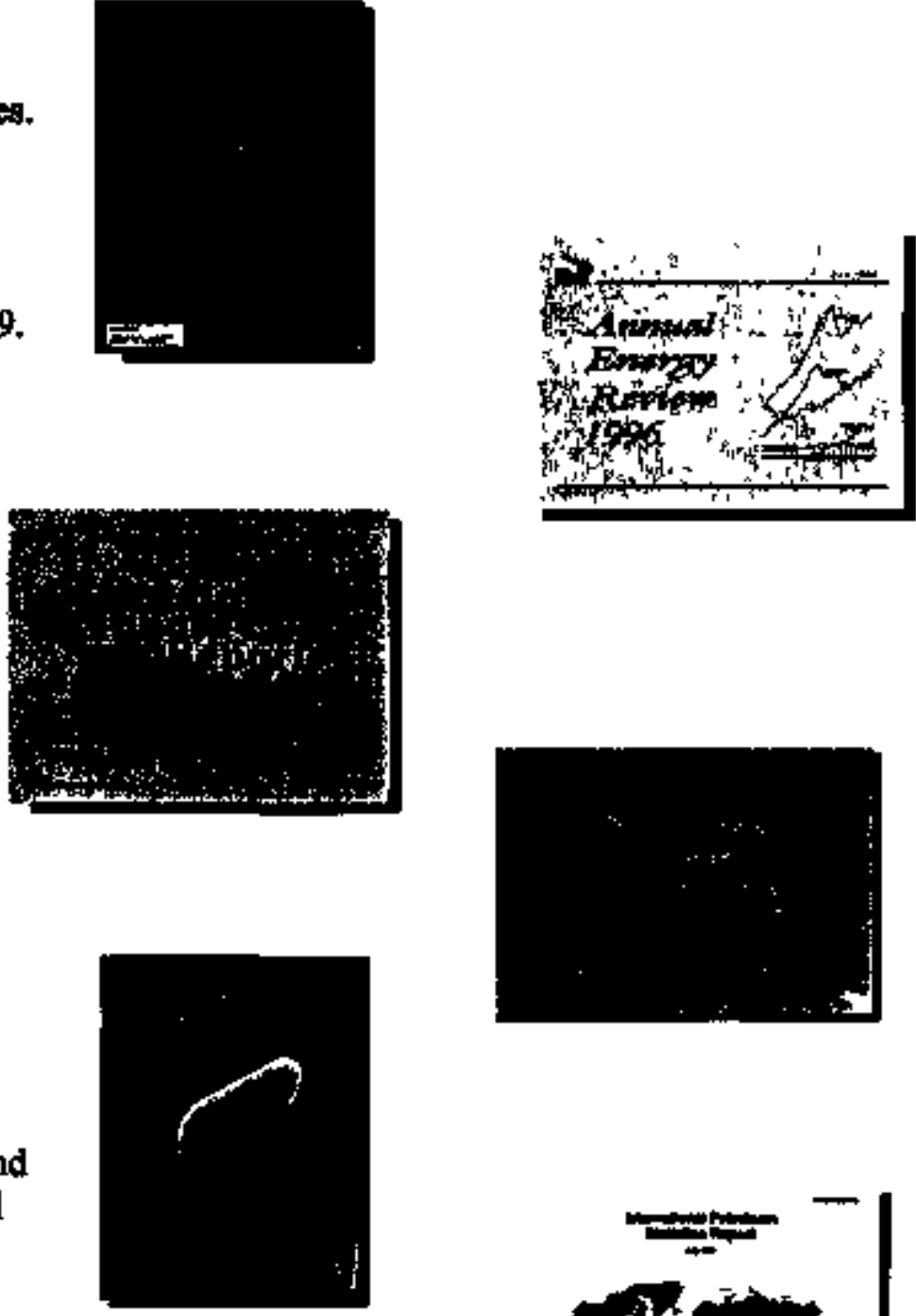

ELA also publishes many other reports. Fo mote informution, contut the National Enemy lnformation Center ot

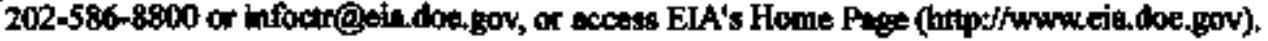




\section{From the \\ Energy Information Administration...}

\section{comes the most comprehensive collection of energy information and analysis tools available today on CD-ROM...}

Advanced search capability and Web browser

Instant access to EIA's wide range of national energy databases and special analysis publications

A must for anyone needing current or historical energy information.
The most comprehensive collection of energy information and anelysis tools available today

Information from nearly 200 of the most current periodicals and anaIytical publications of the Energy Information Administration (EIA)

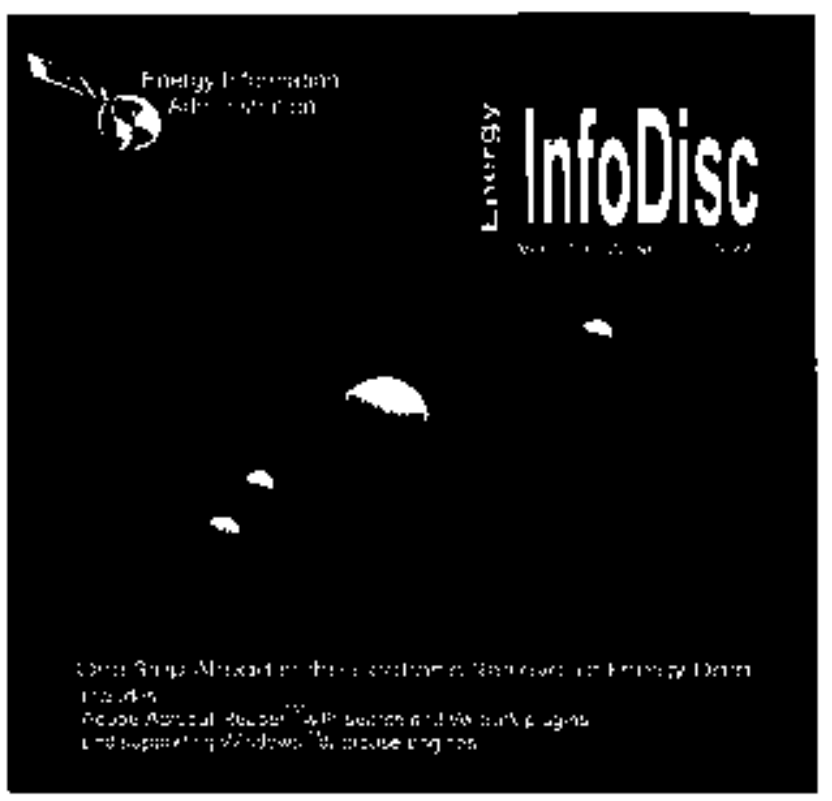

NOTE: Documents cen be soonesed using elther Microselt Wndows or Mingintogh Brstem 7 operating eysteme.

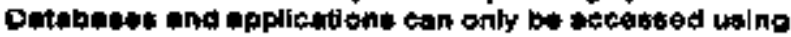
Microugeft Windowe

\section{Don't be without this Important resource. SubscribeToday!}

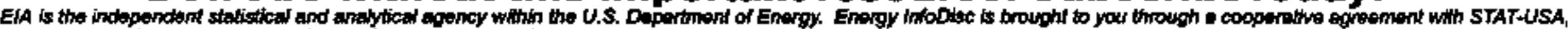
the business information senver of the U.S. Departinest of Compresce.

\section{STAT-USA Order Form}

Yes, enter my order for the Energy InfoDisc CD-ROM: Mumber of copies:

D Single bsue (U.S., Caneda, \& Mexdepi): SA5

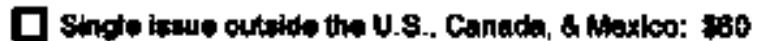

Duarterly subseription in the U.S., Canada, \& Itextes: $\$ 100$

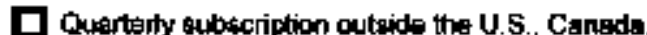
\& Mexico' \$180

Conlactor pertonal notive

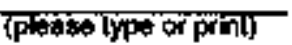

cómbany

Strest adrés

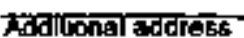

City, sigle, Zlp Code

Daybime phone

Fax number

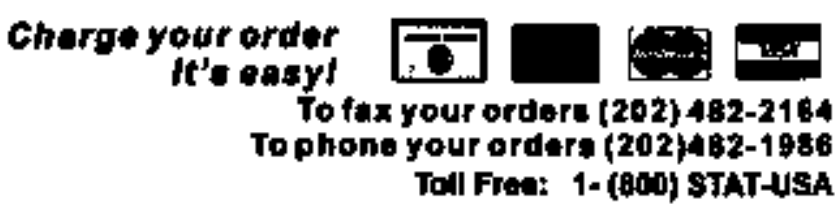

For privecy provection, check the box bulow.

Do not meke my name avallable to requesters.

Check method of payment:

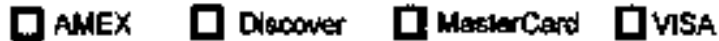

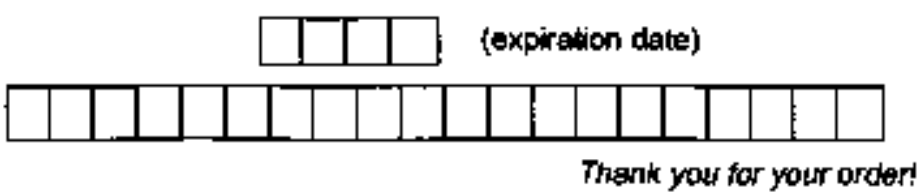

Authorizing signature

D Chack payablo to STAT-USA

Ma: to: U. S. Depertunent of Commerce

STAT USA ROOT H-4885

Wadhington, DC 20230 
Forrustal Bullding, Er-30

Washington, D.C. 20585

OFFICGIAL BUSINESS

Address Correction Requested

Do Not Fonward, Do Not Return

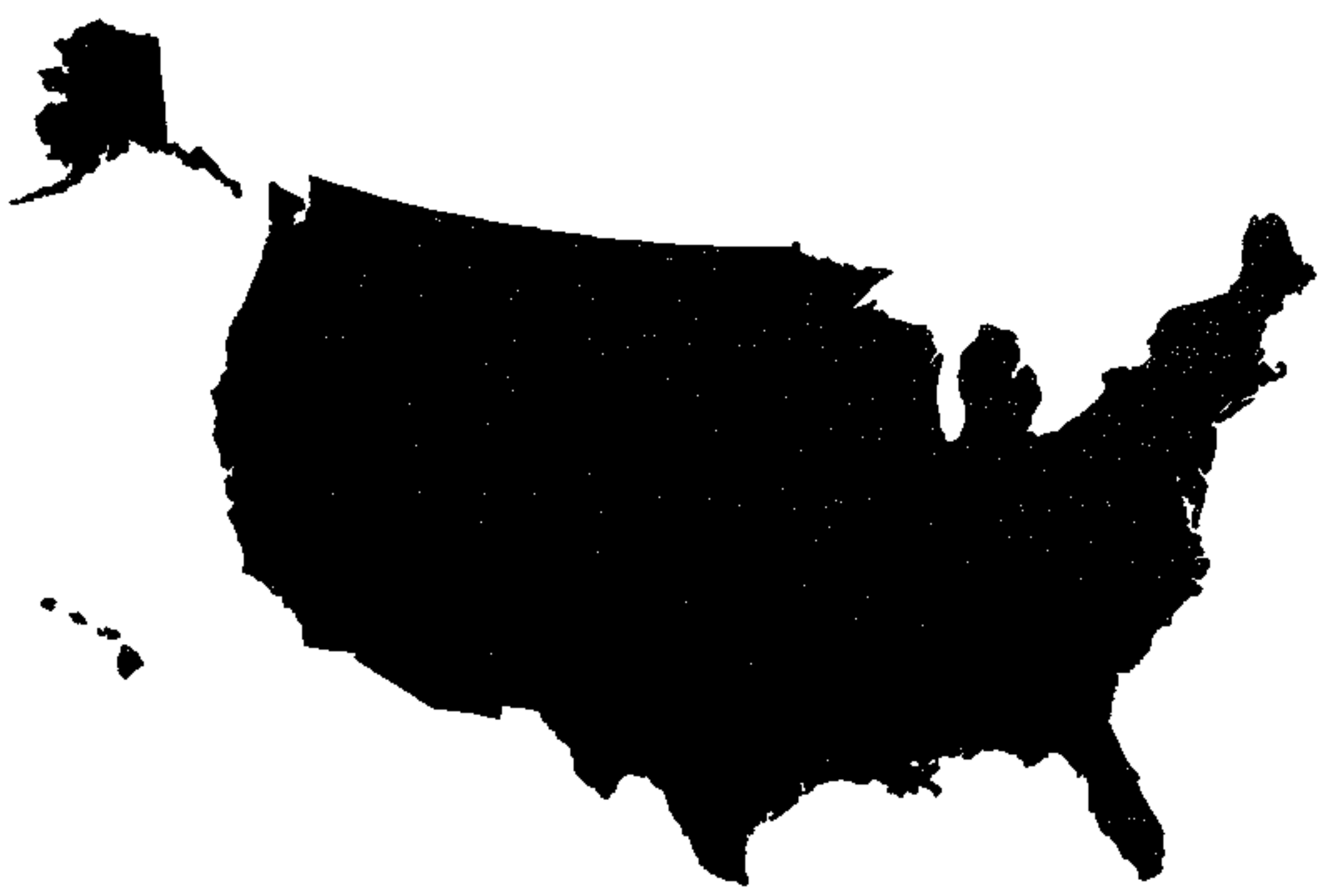

